




\section{Smithsonian Institution \\ Libraries}
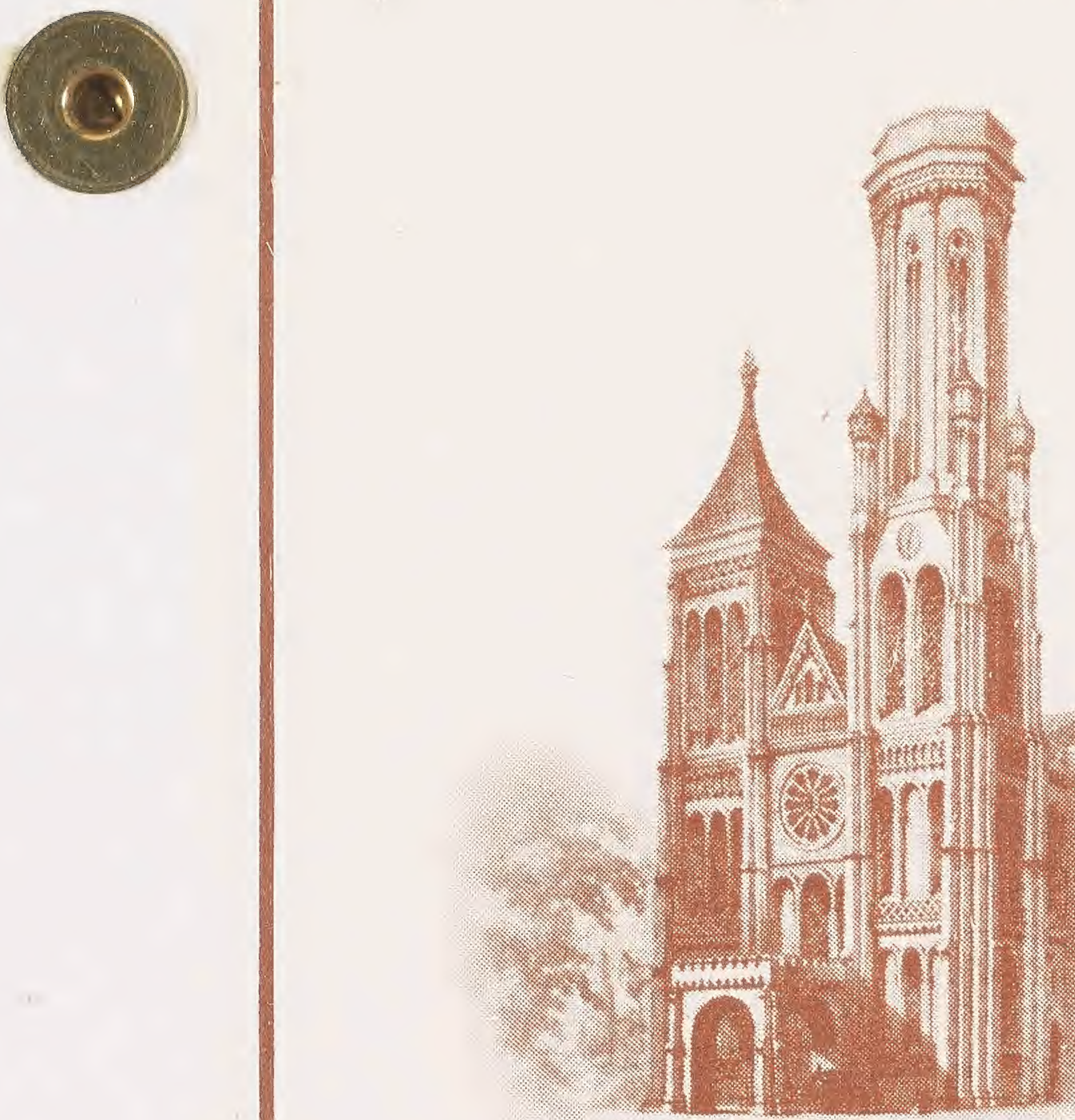

Archival box provided through a gift from The Women's Committee of The Smithsonian Associates 


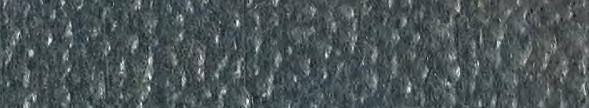

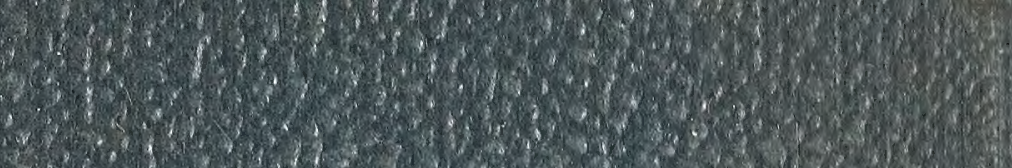

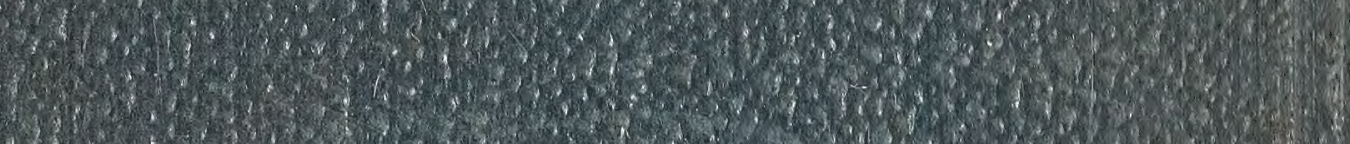

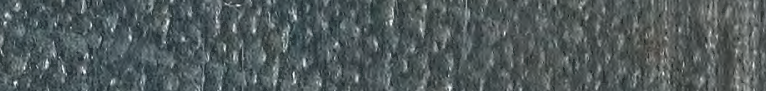

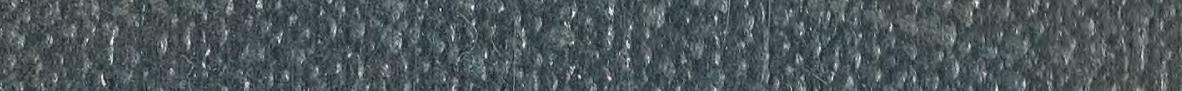

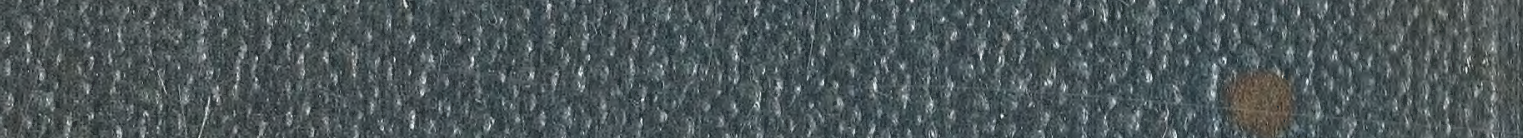

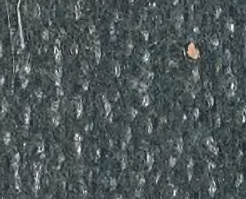

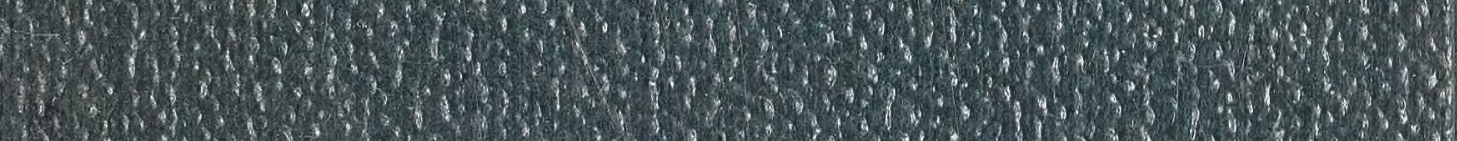
(1) 8.

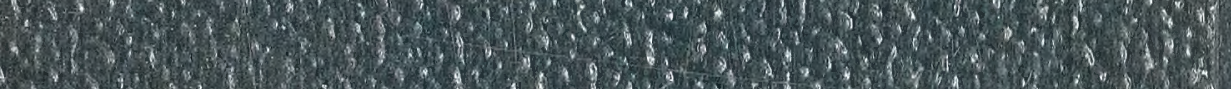
1. 

fang. 

for. $x x^{2}$

(2)

$3 x y^{2}$

20.0

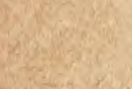

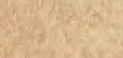




\section{ENGLISH MANUAL/}

OR

\section{PRAYERS AND CATECHISM,}

IN ENGLISH TYPOGRAPHY

E lean-Marie Raphael de Jerne 7

WITH THE APPROBATION OF

RIGHT REV. PA DURIEN, D.D., O.M.I.

Bishop of Nere Westminster.

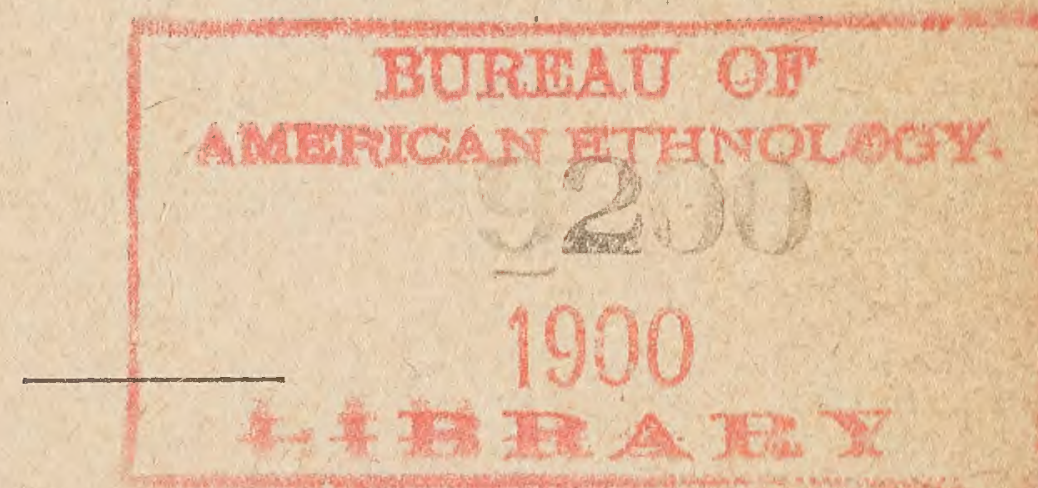

KAMLOOPS, B.C.

1896. 


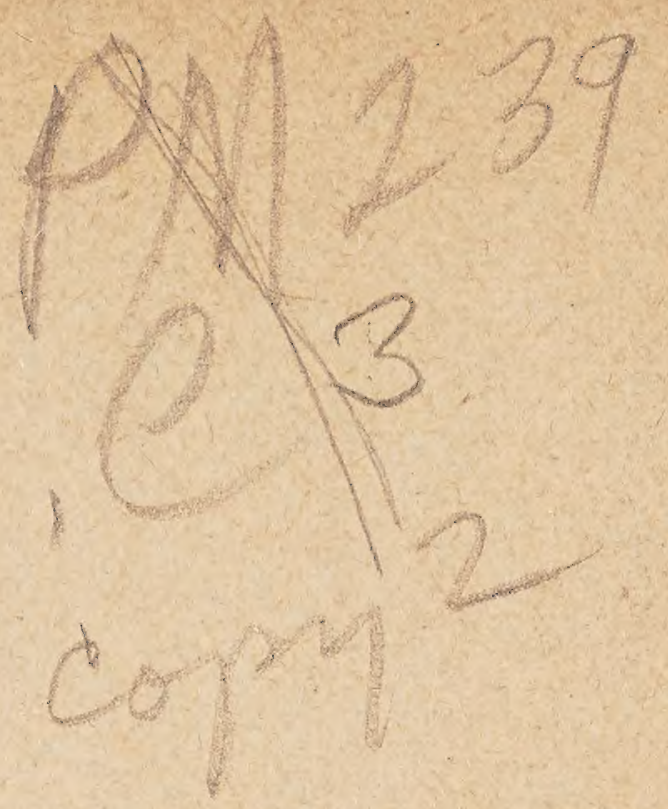

November 3 OTh, I 896.

Imprimatur.

$$
\text { + P. DURIEN, O.M.I., }
$$

Bishop of New Westminster: 


\section{Mrapers Catechism}

IN ENGLISH TYPOGRAPHY

․ 중

\section{PRAYERS}

\section{The Lord's Prayer}

Our Father, who art in heaven, hallowed be Thy name; Thy kingdom cone ; Thy will be done on earth as it is in heaven. Give us this day our daily bread; and forgive us our trespasses as we forgive those who trespass against us; and lead us not into temptation, but deliver us from evil.Amen

\section{The Angelical Salutation}

Hail Mary, full of grace ! the Lord is with thee: blessed art thou amongst women, and blessed is the fruit of thy womb, Jesus. Holy Mary, Mother of God, pray for us sinners, now and at the hour of our death.Amen.

\section{The Apostles' Creed}

$\mathrm{I}$ believe in God, the Father Almighty, Creator of heaven and earth; and in Jesus Christ, His only Son, our Lord; who was conceived by the Holy Ghost, born of the Virgin Mary, suffered under Pontius Pilate, was crucified, died, and was buried. He decended into hell; the third day he arose again from the dead : He ascended into heaven, sitteth at the right hand of God, the Father Almighty; from thence $\mathrm{He}$ shall come to judge the living and the dead. I believe in the Holy Ghost, the Holy Catholic Church, the communion of Saints, the forgiveness of sins, the resurrection of the body, arid the life everlasting,-Amen.

\section{The Confiteor}

I confess to Almighty God, to blessed Mary, ever Virgin, to blessed Michael the Archangel, to blessed John the Baptist, to the holy Apostles Peter and Paul, and to all the Saints, that I have sinned exceedingly in thought, word and deed, through my fault, through my fault, through my most grievous fault. Therefore I beseech blessed Mary, ever' Virgin, blessed Michael the Archangel, blessed John the Baptist" the holy Apostles Peter and Paul, and all the Saints, to pray to the Lord our God for me. 
May the Almighty God have mercy on me, and for give me my sins, and bring me to everlasting life.Amen.

May the Almighty and merciful Lord grant me pardon. absolution, and remission of all my sins.Amen.

\section{An Act of Faith}

O my God! I firmly believe that Thou art one God in three Divine persons, Father, Son and. Holy Ghost; I believe that Thy Divine Son became man, and died for our sins, and that $\mathrm{He}$ will come to judge the living and the dead. I believe these and all the truths which the Holy $\mathrm{Ca}-$ tholic Church teaches, because Thou hast revealed them, who canst neither deceive nor be deceived.

\section{An Act of Hope}

O my God! relying on Thy infinite goodness and promises, I hope to obtain. pardon of my sins, the help of Thy grace, and life everlasting, through the merits of Jesus Christ, my Lord and Redeemer.

\section{An Act of Love}

O my God! I love Thee above all things, with my whole heart and soul, because thou art all-good and worthy of all love. I love my neighbor as myself for the love of Thee. I forgive all who have injured me, and ask pardon of all whom I have injured,

\section{An Act of Contrition}

O my God! I am heartily sorry for having offerded Thee, and I detest all my sins, because I dread the loss of heaven and the pains of hell, but most of all because they offend Thee; my God, who art all-good and deserving of all $\mathrm{my}$ love. I firmly resolve, with the help of Thy grace; to confess my sins, to do penance, and to amend my life.

\section{The Blessing Before Meals}

+Bless us, O Lord! and these Thy gifts, which we are about to receive from Thy bounty, through Christ our Lord.-Amen.

\section{Grace after Meals}

+We give Thee thanks for all Thy beirefits, O Almighty God, who livest and reignest for ever : and may the souls of the faithful departed, through the mercy of God, rest in peace,--Amen

\section{The Manner in which a Lay Person is to Bapm tize in Case of Neees sity.}

Pour common water on the head or face of the rerson to be baptized, and say while pouring it:

"I baptize thee, in the name of the Father, and of the Son, and of the Holy Ghost."

N.B.-Any person of either sex who has reached the use of reason can baptize in case of necessity. 


\section{- Catechísm =}

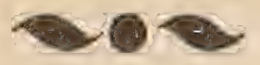

\section{]. $\mathrm{GOD}$}

1. Who made you?

God made me. you?

$\therefore$ Why did God make

God made me to know Him, to love Him and to serve Him, and by that means, to be happy with Him for ever in heaven.

3. What is God?

God is a spirit, eternal, infinitely good, all powelful : He sees all things.

4. Why do you say that God is a Spirit?

Because God has no bory.

5. Why do you say God. is eter"nal?

Because. God ever was and ever will be.

6, Why do you say Gad is infinitely good:

Because all good is in God: he is supremely good above all things.

\%. Why do you say God sees all things?

Because God sees all things past, present and to come, and even what we conceal in our hearts,

8. Where is God?

God is everywhere.

\section{I. TRINITY}

9. Are there several Gods?

No, there is but one God.

10. Are there several persons in God?
Yes, there are three persons in God; the Father, the Son and the Holy Ghosi: this is called the Blessed 'Irinity.

11. What is the Blessed Trinity?

The Blessed Trinity is one God in three persons.

1\%. Is the Hather God?

Yes, the Father is God.

13. Is the Son God?

Yes, the mon is God.

14. Is the IIoly Ghost God?

Yes, the Holy Ghost is God.

15. Are the Father" the Sron and the Holy Ghost three Gods?

No, they are three persons, but one God.

16. Why are the three Persons only one God!

Because the three Persons are equal in all things.

17. Which of the three Persons is the best, the oldest, the most powerful?

None; the thiee persons are equal in all things.

18. Do the three persons differ from each other?

Yes, they differ: the one is not the other.

\section{JESUS CHRIST}

19. Did one of the three Persons become man?

Yes, one of the three Persons became man. 
20. Which of the three Persons became man?

God the Son.

21. How did God the Son become man?

God the Son became man by taking a body and soul like ours.

22. Whence did the Son of God take his body?

The Son of God took his body from the Womb of the Virgin Mary by the operation of the Holy Ghost.

23. Did the Son of God, become man, cease to be God?

No; the Son of God is now God and man.

24, Why did the Son of God become man?

The Son of God became man to save us from evil and to give us life everlasting.

25. What is the name of the Son of God made man? Jesus Christ is the name of the Son of God made man.

26. Who is Jesus Christ?

Jesus Christ is the Son of God made man.

27. How did Jesus Christ redeem us?

Jesus Christ redeemed us by dying on the cross.

28. Did Jesus Christ rise again from the dead?

Yes, the third day Jesus Christ came to life again.

29. Did Jesus Christ remain long here on earth after His Resurrectión?

No: He remained here on earth only forty days, and then went into Heaven.

30. Will Jesus Christ come on earth again?

Yes: Jesus Christ will come on earth again on the last day, to judge tho living and the dead,
31. What will our Lord Jesus Christ then do?

He will take the good to Heaven; He will cast the wicked into everlasting fire.

\section{THE CHURCH.}

32. Who will teach men the doctrine "of Jesus Christ and show to them the vay to heaven?

Those Whom Jesus Christ elected to establish and maintain His Church.

33. What is the Church?

The Church is the society of all who believe and obey those whom Jesus Christ has elected to teach his doctrine, and show to men the way to Heaven.

34. Who are those whom Jesus christ has elected to preside over His Church?

They are the Pope, the Bishops and the Priests.

35. Did Jesus Christ establish several Churches?

No: Jesus Christ established only one Church, out of which there is no Salvation.

36. Which is the true Church of Christ?

The Catholic, Apostolic and Roman Church.

37. Who is the head of the Church of Christ?

Jesus Christ is the head that we do not see, and the Pope is the head that we see.

38. Is the Church always right in what she teaches $u s$ ?

Yes: the Church is always right in what she teaches us, because the Holy Ghost directs her that she may not err". 
39. Does the commit a great sin, he who rejects the teaching of the Church?

He who rejects the leaching of the Church commits a sin of pride, which is called heresy; and which lears to Hell.

\section{THE CREED}

40. What must we do to go to Heaven?

We must act as good children of God.

41. Who is a good child of Gind?

He who is baytirg and believes and acts accorciing to the true word of God.

42. Where is what we aive to beliere?

What we are to believe is in the Apostles' Crefr.

43. Say the Apostles' creed.

I believe in God, etc.

44. Do you believe all that is contained in the Apostles' Creed?

Yes, I believe all that is contained in the Apostles' Creed, because in Baptism God gave me faitll to believe what he has revealed.

\section{THE SIGN OF THE} CROSS

45. By what do we vecognize the children of God?

By the sign of the Cross.

46. How do you make the Sign of the C'ross?

I sign $t^{t} \mathrm{e}$ forehead, then the breast, then the left shoulder, then the right, and say: "In the name of the Father, and of the Son, and of the Holy Ghost, Amen."
47. What do you remember when you make the Sign of Cross.

I remember the Blessed Trinity, and Jesus Christ dying on the Cross.

48. How do you remem. ber the Blessed Trinity?

Because I name the Father, the Son; and the Holy Ghost.

49. How do you remember the death of Jesus christ on the Cross?

Because I form on myself the Cross on which Jesus Christ died.

50. When shall we make the Sign of the Cross.

Always when we pray, and when we do anything, and when we are tempted to do any evil,

51. Why do we make the Sign of the Cross betore we pray or do anything?

T'o offer our prayer and our work to God, and that He may help us to do them well.

52. Why do we make the Sign of the Cross when we are incited to do evil?

To ask God to help us not to do evil.

\section{THE COMMAND- MENTS}

53. Where do we find what God wants us to do?

In the Ten Commandments of God.

54. How shall we keep exactly the Ten Commandments of God?

We shall keep exactly the Ten Commandments of God. if we love God with all our hearts, and our neighbor as ourselves. 
55. And what about the precepts of the Church?

God wants us to obey the precepts of the Church.

56. Say the precepts of the Church.

\section{PRAYER}

57. Are we able alone to do what God commands?

No: we must have the help of God.

58. What must we do to obtain the help of God?

We must pray and receive the Sacraments.

59. Which is the best among all prayers?

That prayer which Jesus Christ taught us, and which is called "The Lord's Prayer."

60. Recite this prayer.

Our Father, etc.

61. Which is the second prayer which we must know?

The Salutation of the Angel in which we speak to the Blessed Virgin Mary, Mother of Jesus Christ.

62. Recite that prayer.

Hail Mary, etc.

\section{SIN}

63. What is sin?

To disobey God, that is called sin.

64. How many kinds of sin are there?

Two. One, original sin, that is in our hearts when we are born; the other, actual sin, that we commit ourselves.

65. Which is that sin that is in our hearts when we are born?

It is that sin which Adam committed when he disobeyed God.

66. And which is that sin which we commit ourselves?

It is that which we commit when of our own accord we disobey God.

67. How many kinds of actual sin are there?

There are two kinds of actual sin: one giving death, mortal; one not giving death, venial.

68. What does mortal sin do to us?

It separates us from God, kills our souls, and subjects them to the punishment of Hell.

69. How many mortal sins will lead us to hell?

A single mortal sin will send us to hell, if it is not forgiven before we die.

70. What does venial sin do to us?

It lessons the love of God in our hearts, and makes us less worthy of his help.

71. In how many ways do we commit sin?

In four ways: in thought, word, deed and omission.

72. How many capital sins are there?

Seven : pride, coveteousness, lust, envy, gluttony, anger and sloth.

\section{BAPTISM}

73. Why did Jesus Christ institute the seven sacraments?

Jesus Christ instituted the seven sacraments to sanctify our souls.

"4. Name the seven sacraments?

Baptism, confirmation, holy eucharist, penance, 
extreme unction, holy orders and matrimony.

75. What is Baptism?

Baptism is a sacrament which cleanses us from original sin, and from the other sins we committed before baptism; and it makes us children of God and of the Church.

76. Is baptism necessary to salvation?

Baptism is necessary to salvation, because without it we cannot enter into the kingdom of Heaven.

7\%. Who can administer" baptism?

The priest is the ordinary minister of baptism; but in case of necessity any one who has the use of reason may baptize.

78. How is baptism given?

Whoever baptizes should pour water on the head of the person to be baptized, and say, while pouring the water, "I baptize thee, in the name of the Father, and of the Son, and of the Holy Ghost."

79. What dispositions are required in adults for baptism?

They must know and believe what God has revealed us; they must be sorry for all their sins, and they must be determined to live forever as true Christians.

80. What do- we renounce at baptism?

Werenounce three things: we renounce the devil, his works and his pomps.

\section{CONFIRMATION}

81. What is confirmation?

Confirmation is a sacra- ment through which we receive the Holy Ghost to make us strong and perfect Christians, and soldiers of Jesus Christ.

82. Who administers confirmation?

The Bishop alone administers confirmation.

83. How does the Bishop give confirmation?

The Bishop extends his hands over those who are to be confirmed, prays that they may receive the Holy Ghost, and anoints the foreliead of each with Holy Chrism in the form of a cross, and then gives a slight blow on the cheek.

84 What preparation should be made to receive confirmation?

'l'o receive confirmation one inust be in the state of grace; must know and believe in the chief mysteries of Faith, and the duties of a Christian, and must yearn for the coming of the Holy Ghost in his heart.

\section{HOLY EUCHARIST.}

85. What is Holy Eucharist?

Holy Eucharist is a sacrament which contains the body and blood, soul and divinity of Jesus Christ.

86. Who instituted the Holy Eucharist?

Jesus Christ instituted the Holy Eucharist at the last supper, the night before $\mathrm{He}$ died.

87. How did Jesus Christ institute the Holy Eucharist?

At the Last Supper, Jesus Christ took bread, blessed it, and gave it to His 
Apostles, saying, "Take ye and eat, This is my body ;" then he took the Chalice in which he poured some wine, blessed it, and gave it to His Apostles, saying to them, "Take and drink ye all of this." This is my blood. Do this for a commemoration of me."

88. What happened when Jesus Christ said, This is my body, This is my blood?

When Jesus Christ said This is my body, the substance of the bread was changed into the substance of his body; when $\mathrm{He}$ said, This is my blood, the substance of the wine was changed into the substance of His blood.

89. When Jesus Christ changed the substance of bread and wine into the substauce of His body and blood, did the substance of bread and wine disappear?

When Jesus Christ changed the substance of bread and wine into the substance of His body and blood, there remained only the appearances, that is the figure, color and taste of bread and wine.

90. When Jesus Christ broke what he had changed into the substance of His body, was His body also broken?

When Jesus Christ broke what $\mathrm{He}$ had changed into the substance of His body, His body was not broken, only the appearances of bread were broken.

91. When Jesus Christ changed the bread into His body, was there only His body under the appearances of bread?
When Jesus Christ changed the bread into His. body, Jesus Christ was whole and entire under the form of bread, His body, His blood, His soul and His divinity.

92. Why did Jesus Christ institute the Holy Eucharist?

Jesus Christ instiuted the Holy Eucharist to continue the sacrifice of the Cross, where He atoned for our sins, and to nourish our souls.

93. Where now is the substance of bread and wine changed into the substance of the body and blood of Christ?

At Mass the priest changes the substance of bread and wine into the substance of the body and blood of Christ through the power he received from Christ.

94. Has Mass, then; a great power with God?

Yes, Mass is the most powerful of all prayers; at Mass we duly adore God, we duly thar.k Him, we duly satisfy for our sins, and we obtain from God all graces and blessings.

95. Does Mass help the souls in purgatory?

Yes, Mass is very profitable to the souls in purgatory.

96. Why do you say Jesus Christ instituted the Holy Eucharist to nourisin our souls?

Because Jesus Christ instituted the Holy Eucharist to increase sanctifying grace and all virtues in our souls, to lesson our evil inclinations, to unite us with Himself. 
97. Do all who make Holy Communion receive the same graces?

Those only who receive Holy Communion with the due dispositions receive those graces. Those who make Holy Conmmunion without the due dispositions, do not receive those graces, and commit a great sacrilege.

98. How shall we prepare to receive Holy Communion?

To receive Holy Communion we must be well prepared in soul and body.

99. How shall we prepare our soul?

By purifying our soul from all sins in a good confession, and by kindling in our hearts an ardent love for Jesus Christ.

100. How shall we kindle in our hearts an ardent lovefor Jesus Christ?

By acts of a lively faith, of firm hope and ardent love: by fervent contrition and firm purpose to sin no more, and by a burning desire to receive Jesus Christ in Holy Communion.

101. How shall we pre. pare our body?

We must abstain from midnight from everything which is taken as food or drink, we must have our body clean and decently dressed, like persons who are incvited to the presence of a great king.

102. What should we do after Holy Communion?

After Holy. Communion. we should solely think of Jesus Christ present in our hearts. After Mass we should not leave the church with the people who did no receive : we should remair sonie time to entertain Jesus Christ.

103. How shall we entertain Jesus Christ after Holy Communion?

We must adore Him present in our hearts; we must thank him for all His blessings; we must ask Him to help us to persevere in His grace; we must renew our resolution to resist all temptations, and to avoid all occasions of sin; we must offer Him our heart, body and soul, to be consecrated entirely to His service; then we pray for the living and the dead.

104. Is it good to desire to receive Holy Communion when we cannot actually receive it?

Yes, it is very good to desire to receive Holy Communion when we cannot actually receive it. Jesus Christ answers our desire by coming 10 us and by pouring abundant graces in. our hearts.

\section{PENANCE}

105. What do' you do when you want to receive the Sacrament of Penance?

When I want to receive the Sacrament of Penance, I do five things; 1st. I examine my conscience; 2 nd. I excite myseif to contrition. and firm purpose; 3rd. I confess my sin's to the Priest; 4 th. I receive absolution; 5th. I perform the Penance of Satisfaction imposed by the Priest.

106. What do you do when you want to examine you censcience? 
When I want to examine my conscience, I repair to the Church, where I kueel down, and after making the Sign of the Cross, I pray to God to enlighten my soul that I may know my sins.

107. How do you examine your conscience?

To examine my conscience, I first repass in my mind all the places where I have been since my last Confossion; then I examine myself on the Ten Commandments of God, the Precepts of the Church, the Seven Capital Sins, and the duties of my state; last of all I find out how many times I committed each of my sins.

108. After you hare exainined your conscience what do you do?

After I have examined my conscience, I excite myself to a lively contrition of my sins, and to a firm purpose to sin no more.

109. What do you do to excite yourserf to a lively contrition and :o a firm purpose?

To exi ite myseis to a lively contrition of $m y$ sins, and to a firm purpose to sin no more: 1st. I pray God to grant me the grace oi zontrition and firm priroose; 2nd. I consider heaven which I have lost by my sins; 3rd. I consider hell, which I have deserved; 4 th. I consider the goodness of God whom I have offended; .5th. I consider the sufferings of Jesus Christ, and His death upon the Cross, caused by my sins, then I say the act of contrition, and I recite the Confiteor.
110. How do you confess your sins to the Priest?

I kneel down by the side of the Priest, and after making the Sign of the Cross, I say: "Bless me Father, for I have sinned. I confess to Almighty God and to you Father." Then I tell the Priest how long it is since my last confession, whether or not I received absolution and performed my penance, then I confess my sins.

111. After confessing all your sins what do you say?

After confessing all my sins, I say: "For these and all the other sins I do not remember, and all the sins of iny past life, I am heartily sorry, and humbly ask pardon of God, and of you Father, penance and absolution,"

112. What do you do when the Priest speaks to you in' con fession?

I answer simply and clearly all his questions; I listen in silence, with great attention and respect to his advice and directions; I see what penance he imposes upon me, and whether or not he gives me absolution.

1I3. What do you do when the Priest gives you absolution?

When the priest gives me absolution I bow down $m y$ head, I recite the ant of contrition, and make the Sign of the Cross.

114. What do you do when the Priest tells you "Go in peace and pray for" me?"

I go and kneel down at some distance, where I re- 
turn thanks to God; I renew my resolution to resist the temptations and avoid all occasions of $\sin$; I remember what advice the Priest gave me, and how I am to conform myself to it; I ask God to Help me with His grace; then I make the penance the Priest imposed upon me; I then leave the Church, and keep myself for some time yet in recollection.

115. What is the absolution which the Priest gives at confession?

Through the power which he received from Christ, the Priest gives me absolution, by which my sins are forgiven, my soul is healed, and the grace of God is conferred upon me.

116. Does the Priest always give absolution?

The Priest cannot always give absolution ; only to those whom he sees well disposed does he give absoIution; but to those whom he does not consider well disposed he cannot give absolution; he dismisses them with his blessing.

11\%. What do you do when the Priest does not give you absolution?

Wiien the Priest does not give me absolution, I excite myself to a greater sorrow for my sins, and I follow the advice the Priest gives me, that I may soon enter into the true dispositions $x e-$ quired to receive absolution.

118. Is it a great sin to conceal a mortal sin in confession?

Yes, lt is a great sin to conceal a mortal sin in con- fession; the confession becomes null, and the sins that have been confessed are not forgiven. All subsequent confessions are also null, and the sins are not. forgiven, until the mortal sin which has been concealed, has been confessed, as well as the number of confessions made since the sin was concealed, which confessions must be all made over again.

119. Is it a sin to neglect to diligently examine one's conscience before contession?

Yes, it is a sin to neglect to diligently examine one's conscience before confession; it is a contempt of the Sacrament of Penaince, it exposes one to omit sins in confession, in which case it is equal to the concenling: of one's sins in confession.

120. Is it a great sin not to excite oneself to true: contrition and firm pur' pose of amendment?

Yes, it is a great sin not to excite one elf to contrition and to firm purpose of amendment, and to avoid the occasions of sin; it is a sin of contempt of the Sacrament of Penance. It is equal to the concealing of one's sins in confession.

121. What do you do if you forget a sin in your confession?

If I forget a sin in confession without any fault of mine, it is forgiven with the other sins which have been told in confession, but I confess it to the Priesti in my next confession.

122, Does the Priest make known to anybody 
what he hears in confession?

The priest can tell nobody what he hears in confession; he must keep it an inviolable secret.

123. What is that penance which the Priest gives you when you go to confession?

That penance is sacred which the Priest gives me in confession; we shoult not forget that it is a cin to neglect it, but we should be anxious to perform it as soon as possible, and in the manner prescribed by the Priest.

124. Why does the Priest impose a penance in confession?

The Priest imposes a penance in confession as an atonement for our sins. God wants it; it is tne will of God that we should atone for all our sins.

125. What becomes after death, of those who have not fully atoned for their. sins in this world?

When chose who have not fully atoned for their sins in this world die, they go to Purgatory, where they finish the atonement for their sins.

126. Will they remain forever in Purgatory?

They will not remain forever in Purgatory. When they will have completed the atonement for their sins they will go to Heaven.

\section{EXTREME UNCTION}

127. What is Extreme Unction?

Extreme Unction is a sacrament instituted for the spiritual and corporal relief of the sick in danger of death.

128. What are the effects of Extreme Unction on the soul?

Extreme Unction cleanses the soul from the effects of sin, and strengthens it against the last assaults of the devil.

129. What are the effects of Extreme Unction on the body?

It soothes the pain of the patients, and restores them to health, if it be expedient for their salvation.

130. How should we preparefor Extreme Unction?

We should be truly sorry for our sins, and make a good confession, if it be posssible.

131. How does the Priest administer Extreme Unction?

The Priest anoints with the Holy Oil of the Sick, the eyes, ears; nostrils, mouth, hands and feet of the perperson in danger of death, asking God to remit him the sins which he may have committed through each of those sen ses.

\section{HOLY ORDERS}

\section{What is Holy Or- ders?}

Holy Orders is a Sacrament by which Priests are ordained, and receive the power and grace to dispense the Word of God and administer the Sacraments, and perform their other sacred duties.

133. When did Jesus Christ institute Holy Orders? 
When He instituted the Holy Eucharist, Jesus Christ instituted, also, Holy Orders when He said, "Do this in commemoration of Me." 134. Who confer's the Sacrament of Holy Orders:

Only the Pope and Bishops confer the Sacrament of Holy Order's.

135. How does a man become a Priest?

He receives the Sacrament of Holy Orders at the hands of the Bishop; there he receives the power and grace to dispense the Word of God and administer the Sacraments.

XVI. MATRIMONY

136. What is Matrimony?

Matrimony is a Sacrament which sanctifies the union of man and woman.

137. What are the effects of the Sacrament of Matrimony?

The effects of the Sacrament of Matrimony are-1st. To sanctify the love of husband and wife; 2nd. To give them grace to bear with each other's weaknesses; 3rd. To enable them to bring up their children in the fear and Inve of God.

138. What should young people do in order to make a good marriage?

They should tirst pray to
God to find their proper consorts; they should also consult their parents.

139. How should parents act. when tihey know their children's ontentions?

They sh,uld consult the will of God ard advise their children in accordance with that divine will.

140. What does God forbid to parents in regard to their children's marriage?

God expressly forbids parents to sell their children in Matrimony; to prevent them, unreasonably, from marrying the party they have chosen, and to compel them to marry another party against their will.

141. When a couple have agreed to get married, what shall they do?

They must not live together until they are married before the Priest; their banns should be published on three consecutive Sundays; they should duly prepare for marriage by learning all their prayers and the Catechism.

142. Does the Church forbid marriage between certain persons?

Yes; the Church forbids marriage between certain person who are prevented from maxrying by some impediments. 


\title{
Prayers and Catechism
}

\author{
IN ENGLISH
}

(PHONOGRAPHY)

KAMLOOPS, B.C.

I 896. 

English Prayers.

The Sign of the Cross.

I Believe in Good

of $-2 a-2$ to - oh?

$a-9,-a-d \alpha \cdot d c^{2}, \mu a$ <.

$r-, x, \cdots \cdot$

s, di:

our Father. "ul-odiys

ord oo tin.

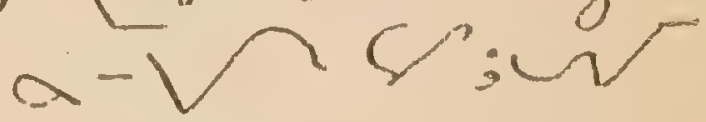

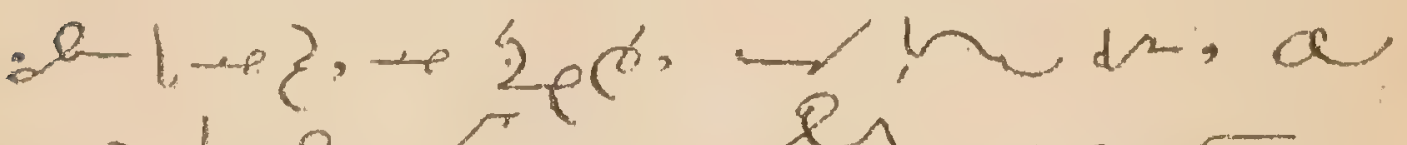

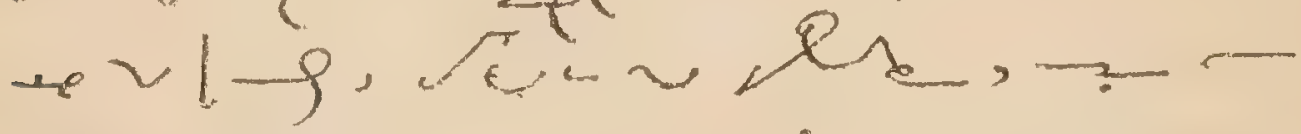

$\cdots \cdot\langle c+2, V$ " w

$\theta=r V^{2}=-3<c$

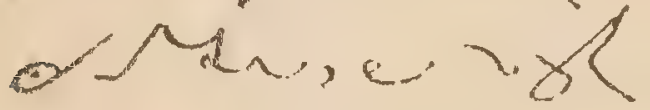

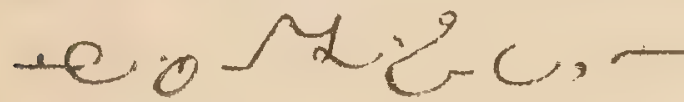

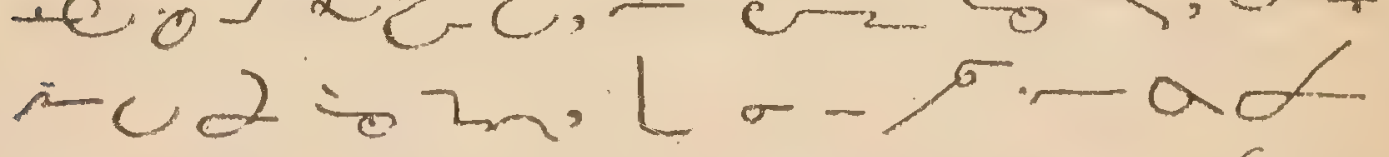

$\rightarrow \mathcal{S} \cdot S$.

- set ob.

rot sin

Hail Mary.

R. - ?... =

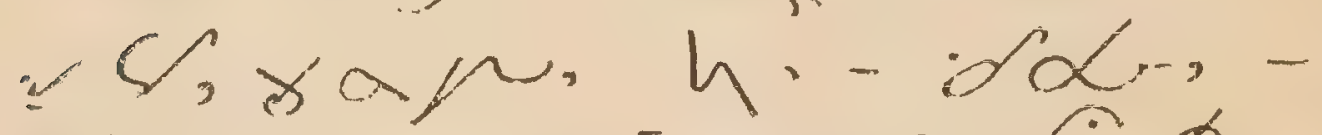

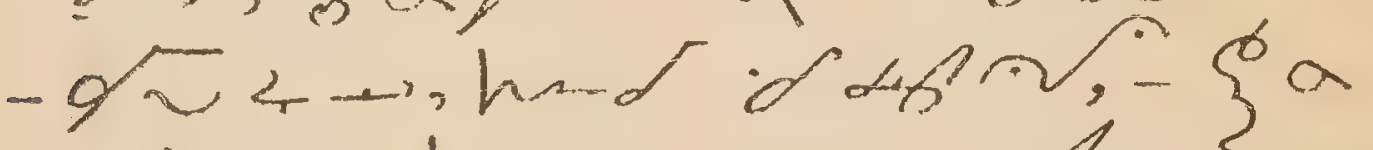

- $35,-h w \sim-i,-\gamma a^{2} w$,

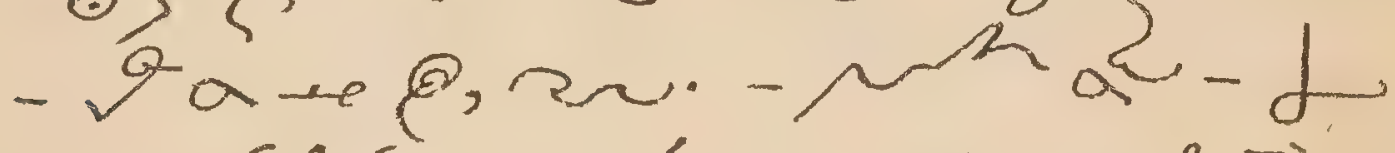

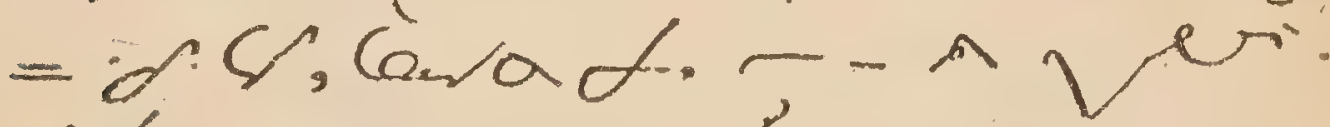

SC, य, $2-\zeta$.

$\sigma-d a d+2$.

<. 
I Confess.

$0<00 \mathrm{C}_{0}, 0$

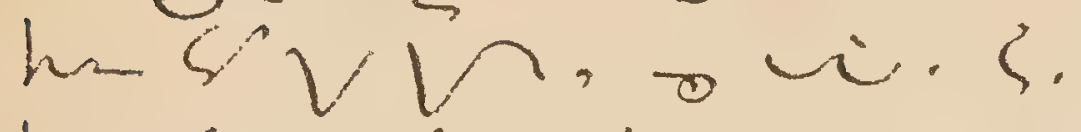

hes -2 , olus b) $q_{2}, \infty-\infty$

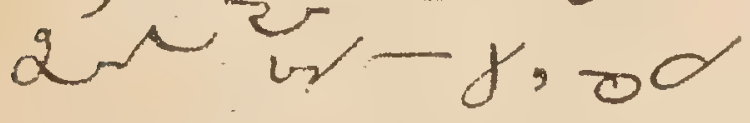
- i, - o der?

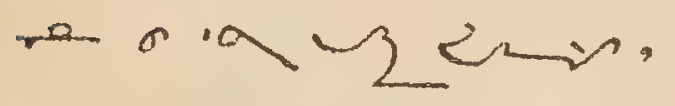

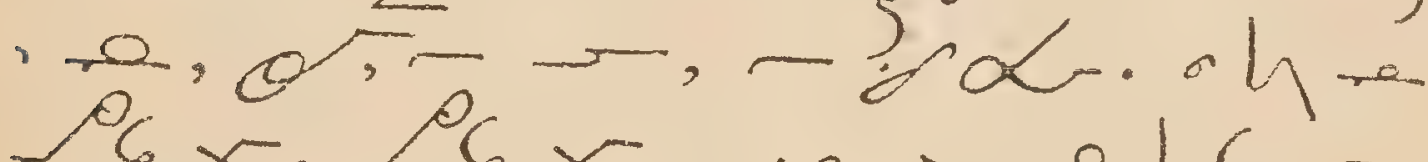

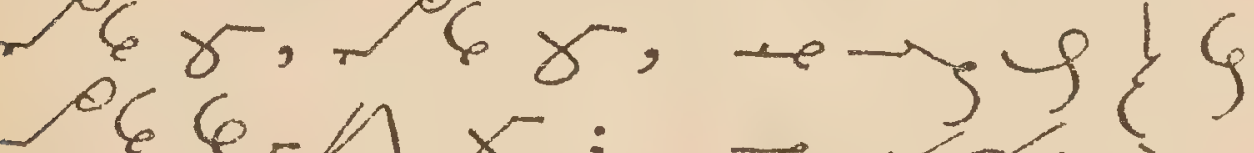

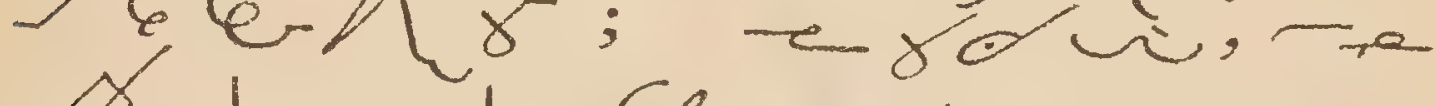

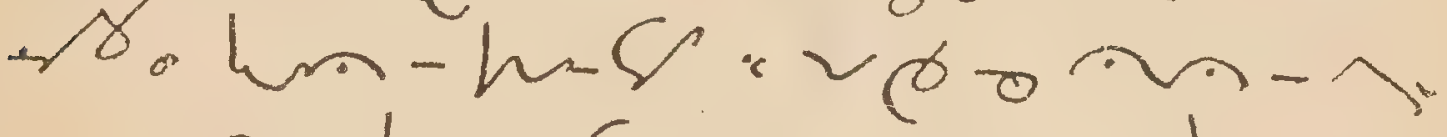
VN, he \& - - inh

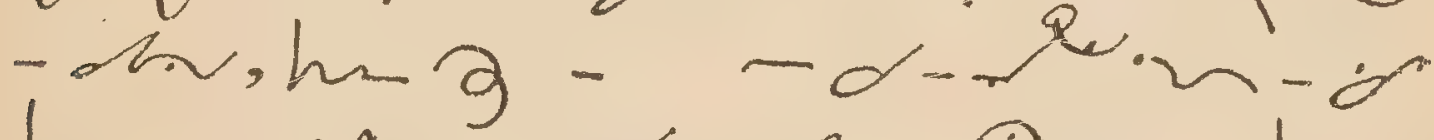

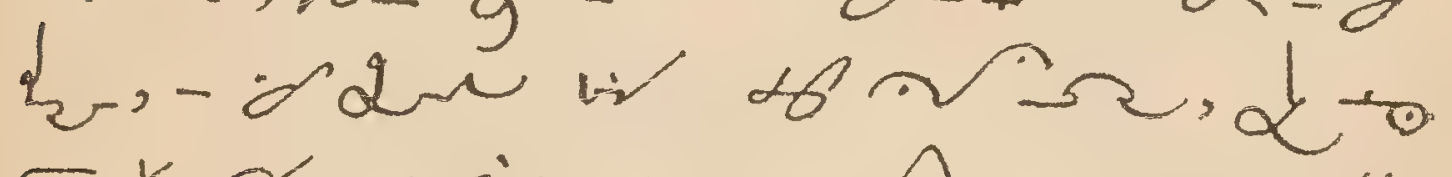

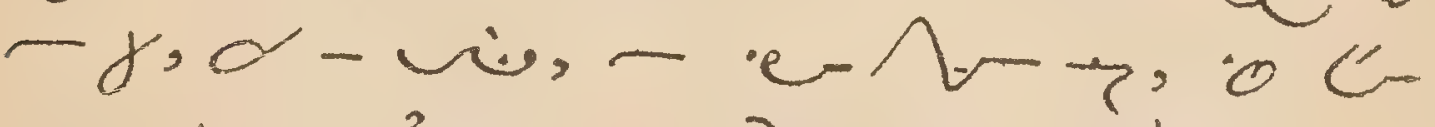

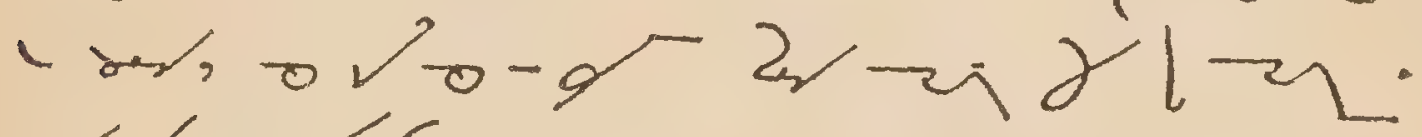
$\alpha \alpha \sigma C$.

$$
\text { . Act of Hope. }
$$

Misereatur. $S_{-}$de $06 \alpha$ ist.

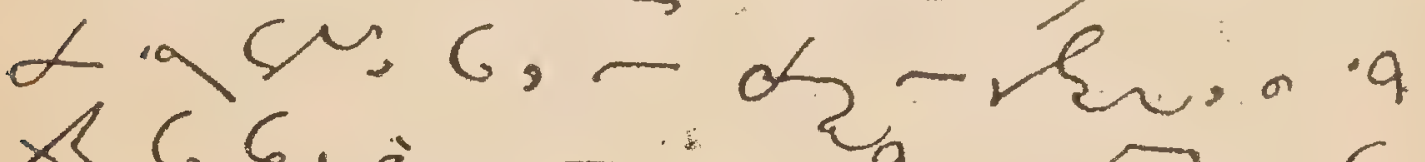
oCGvi, - $0 q_{3}-\gamma$ a 6

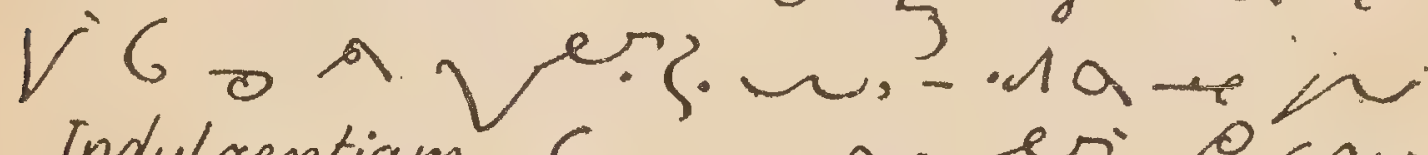
Indulgentiam. $S_{-}-A v$ ris o- - wat ax 
PRAYERS.

25

Act of Love. $\quad$ II. $0 \gamma^{2}>-3 a$

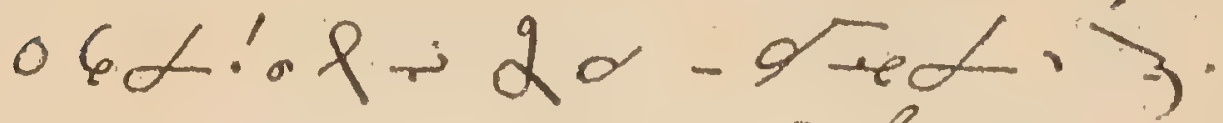

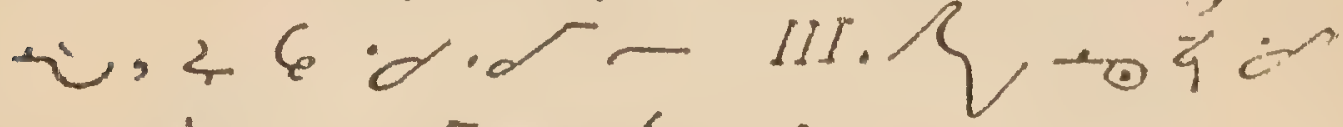

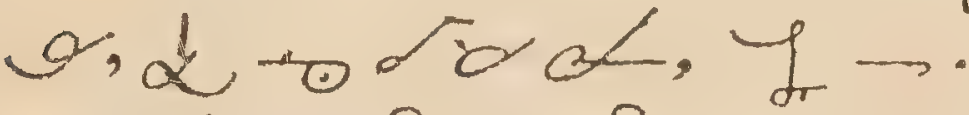

-gadf. of IV.q we rar -

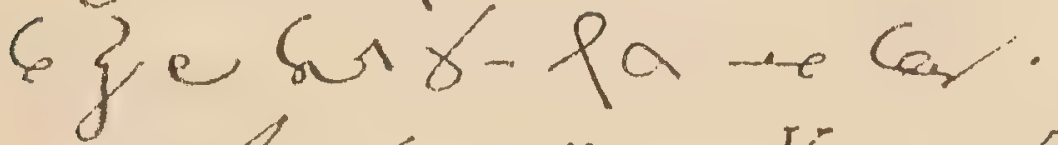

w. . r o $\alpha$.

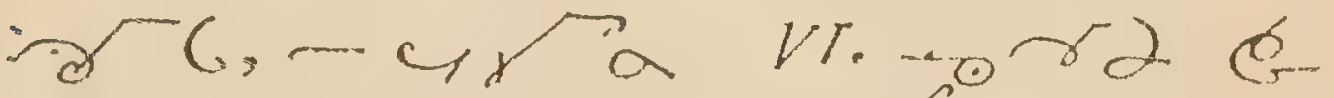

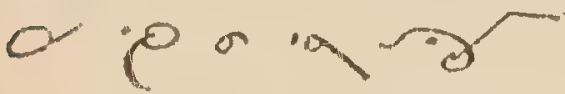

VIT.

Act of contrition. wil. $\odot$ rd lo

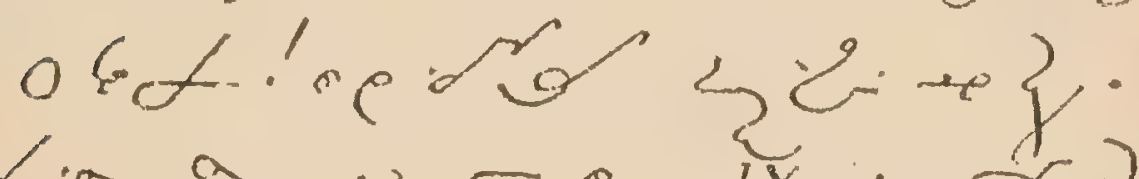

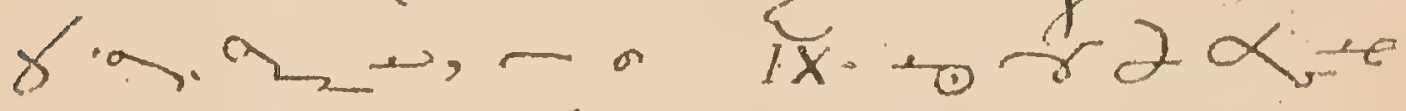

-2, or 6 i. 2 ?

$-2 \alpha,-3,-r \alpha \alpha_{-}+$

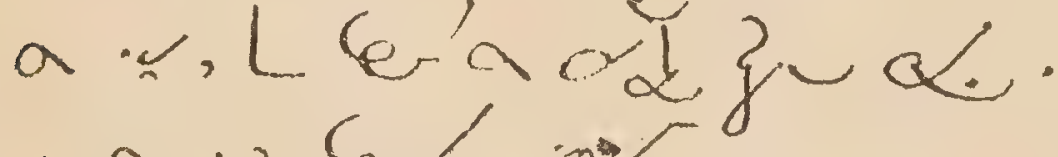

$\rightarrow a \rightarrow, 6 \alpha, \infty$

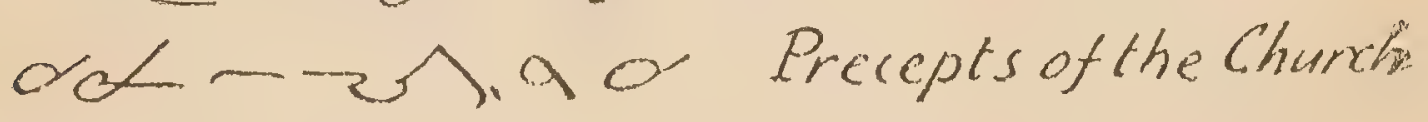

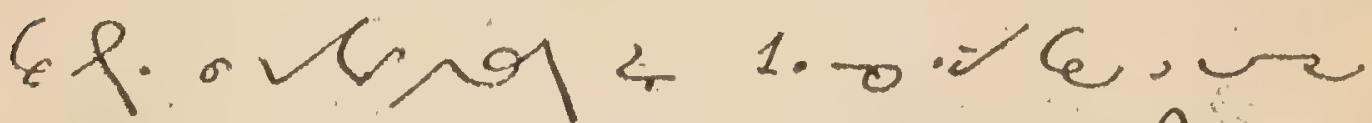

- .1 a wep, o -orea h.

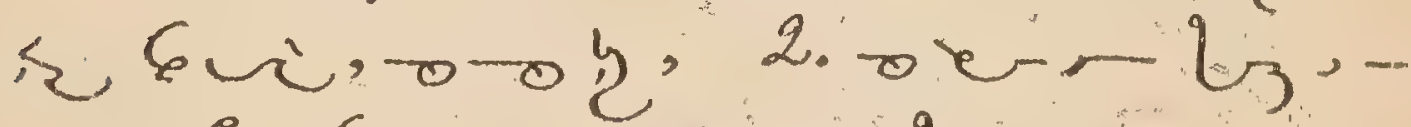
-

The Commandments. 3. o.

I. a.e.dred, to. al.

$r 2 \cdot \sin$ b. 6 . 
ii.

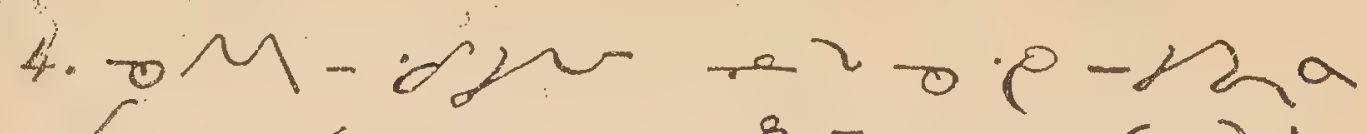

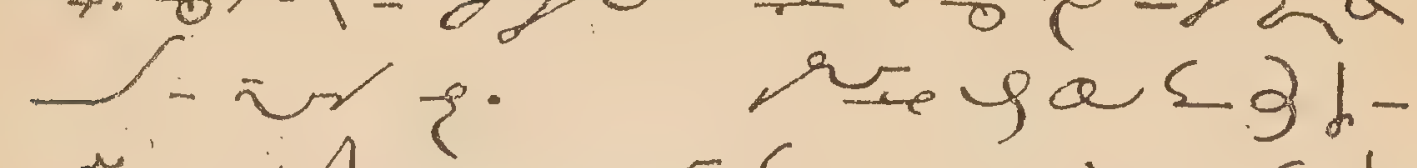

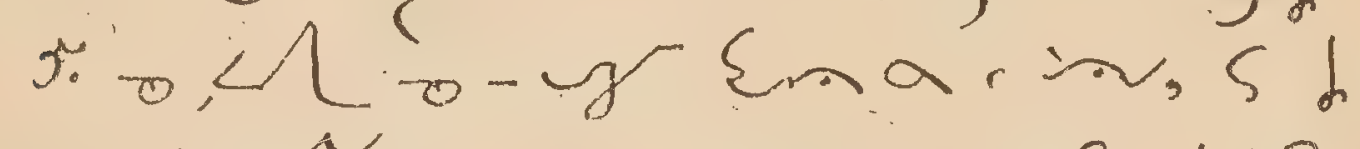

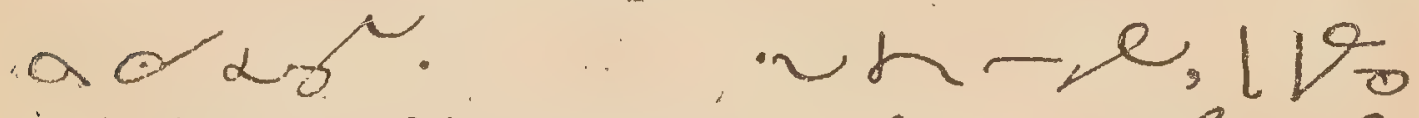

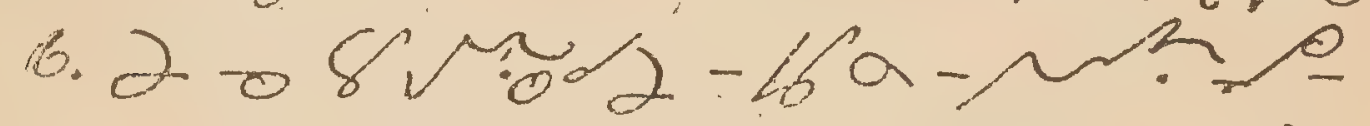

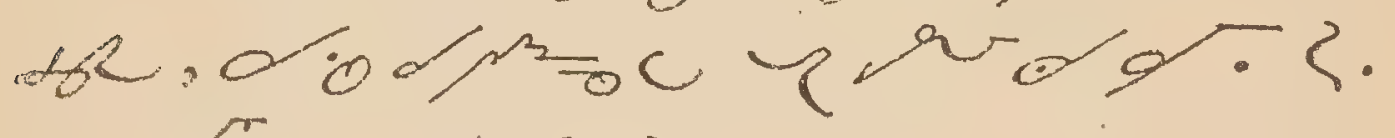

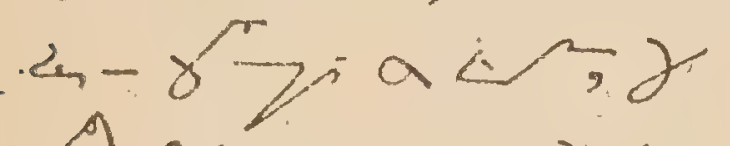

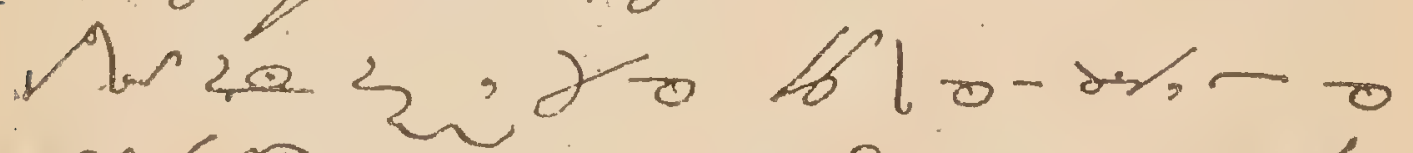

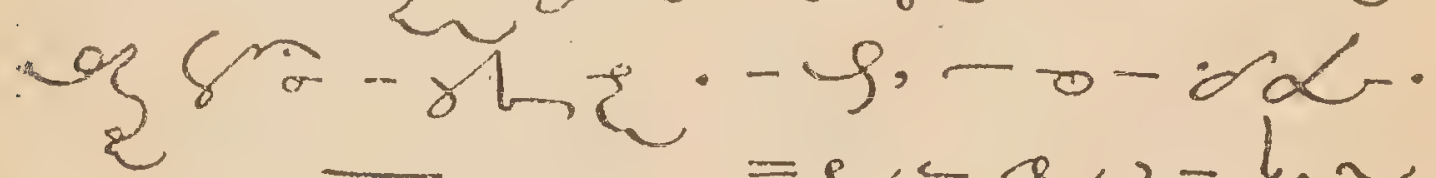
- $=e-a,-j, \approx$ The Angelus, $\partial,-V^{2} l, \theta$

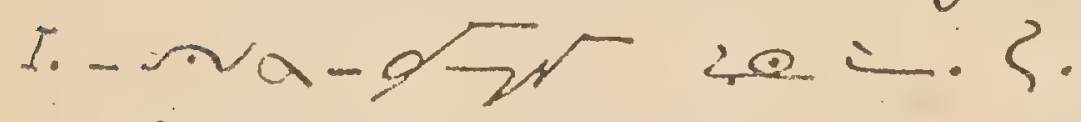

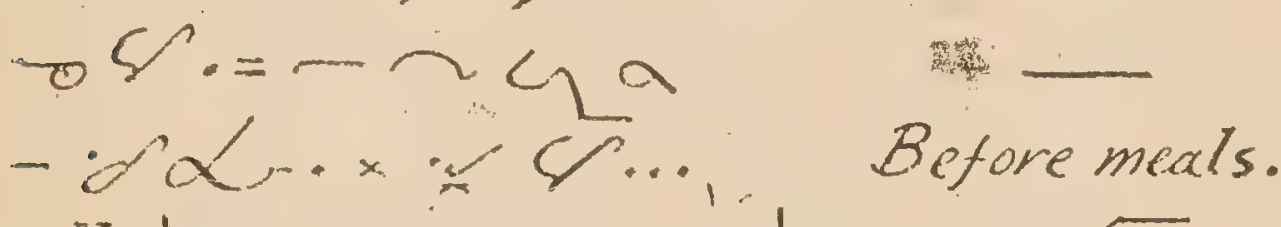
IT. 100-ia-"hco9, = $\rho_{i}=l-\rho_{0} 6+<, m \sim d g$

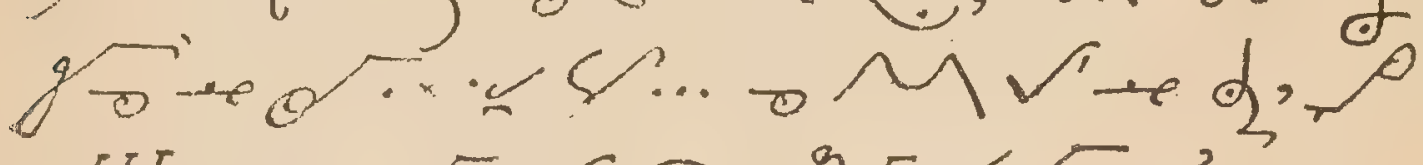

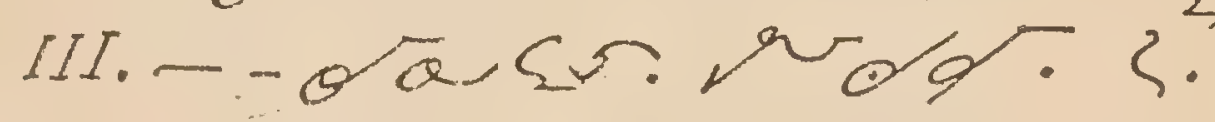
$=-x-2-3 C \cdot x<$. $\times \forall b c . j 6 a j a j$ Aftermeals.

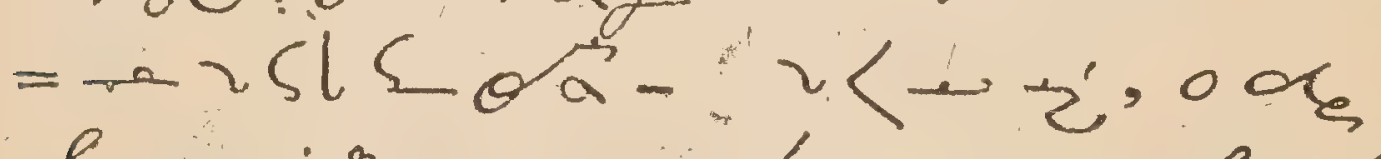
karr, $\alpha$, o $2=k-b$ oremus. \& V. Vi-s- aू-

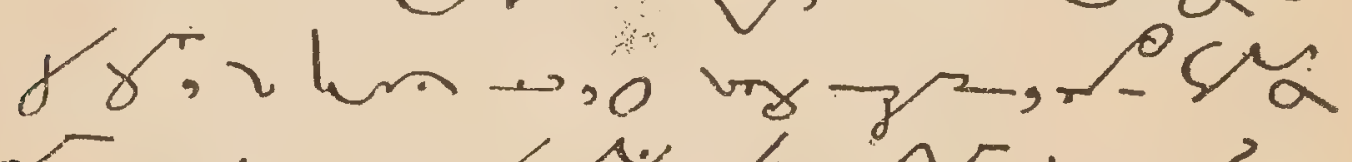
s.tentol. 


\section{English Catechism.}

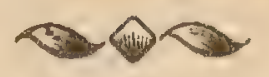

\section{God.}

8. $\sim \sim \mathcal{L} ?=\alpha$

1. $0 \leq c ?=\alpha \leq C \cdot \sim V^{2} \cdot$

2. $0,-\angle S L$ ? $=\alpha$

II. Trinity.

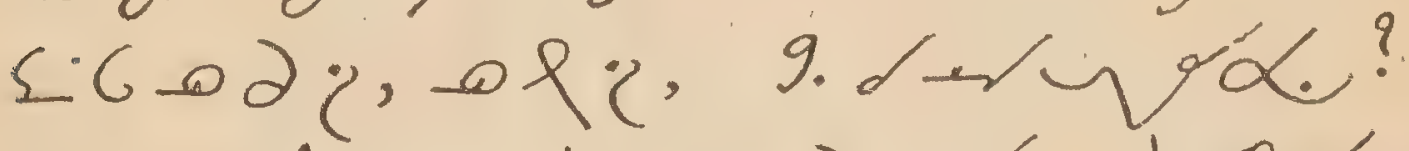

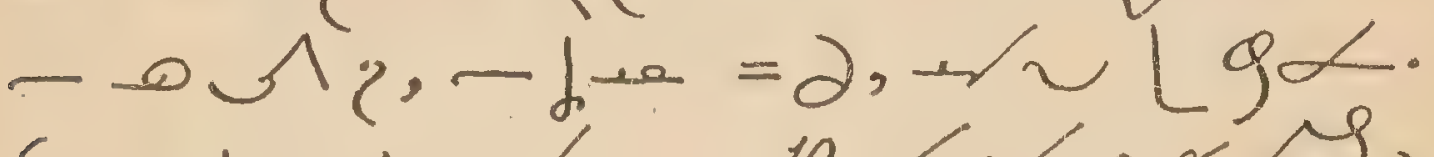

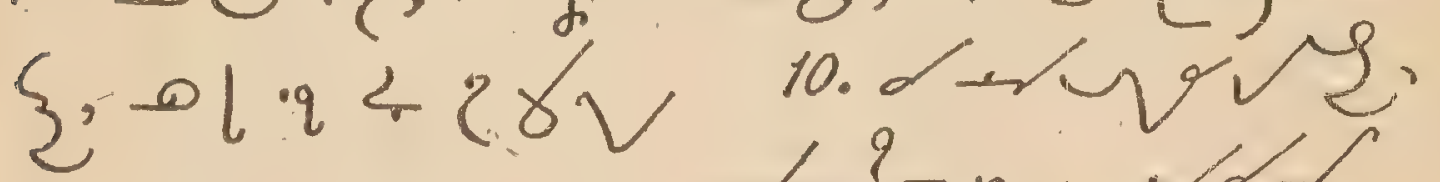

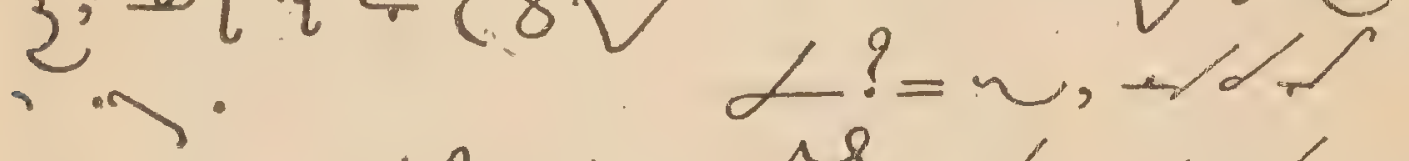

3. $\theta \sim \mathcal{L} ?=\mathcal{L} \sim \mathcal{S}, \mathcal{L}:-\mathrm{s}$. -

- v, r, y, ${ }^{2}, \varphi,--\delta \alpha$;

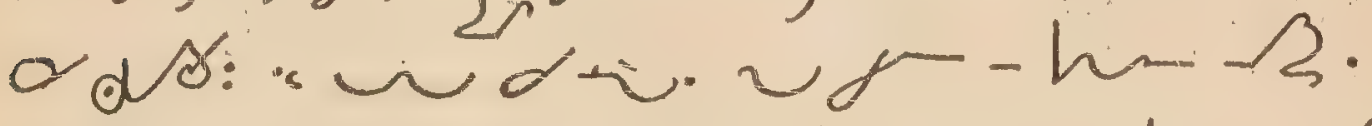

4. $\theta \rightarrow \omega \log 11 \cdot \theta v-h-\beta$ ?

$\sim \cdot \mu^{?}$ ? $=$ L $\angle \cdot e=-\ln \beta \sim g \alpha$

$\partial \mathrm{L}$.

rs.

5. $\theta-0 \mathrm{c}, \mathcal{L}$

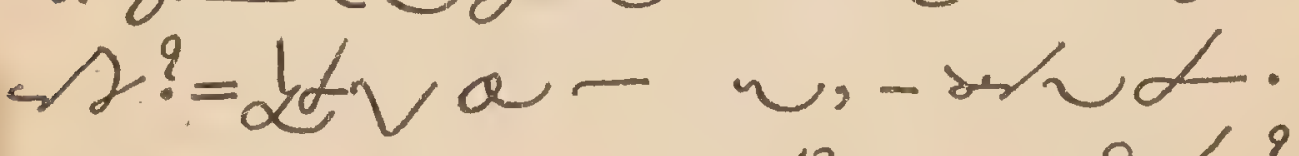

$\checkmark v 1$

13. $\sim-9 \alpha$ ? $=\sim$

6. $\theta-\infty \cup \cup d \sim-\varphi \sim d$.

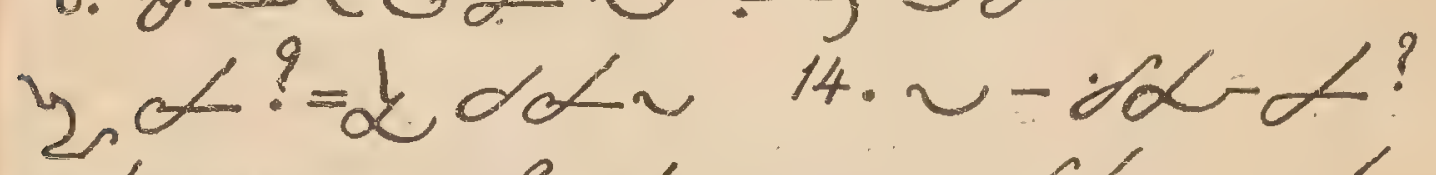

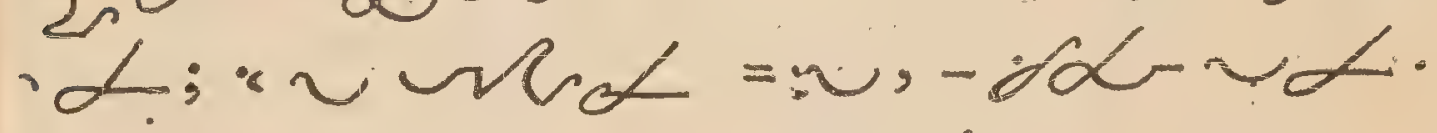

$2 \dot{\alpha} \sim$. $15 \cdot d-2 \pi,-9-1$

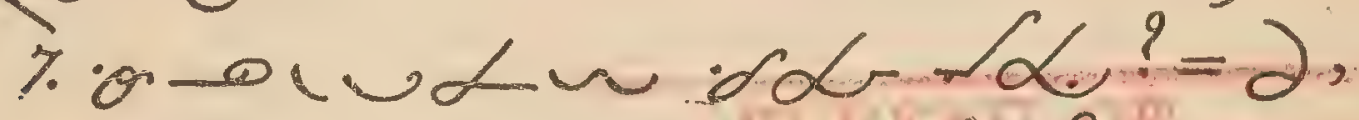

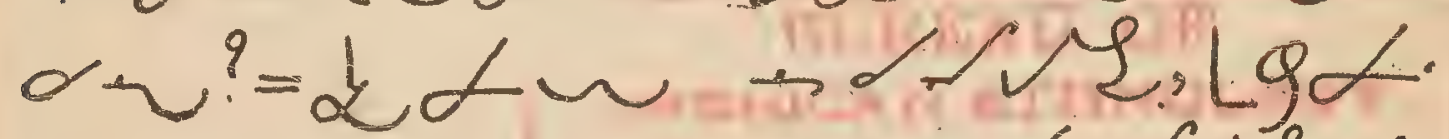
$\alpha+d, \Omega=016 \cdot 0 d-r i s$

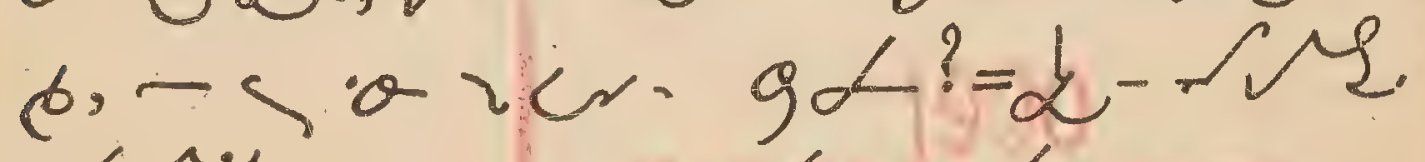

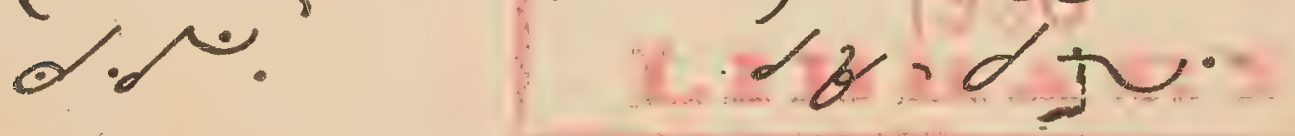


1\%.na-s 24.0--gad $\sim-L,-\delta \sim,-\{\xi ?=-9 a \alpha \xi \xi$

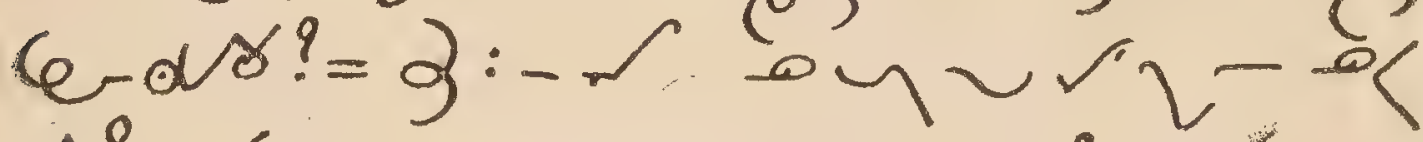
$r d z \cdot d \sim \cdot \sim A v e r$

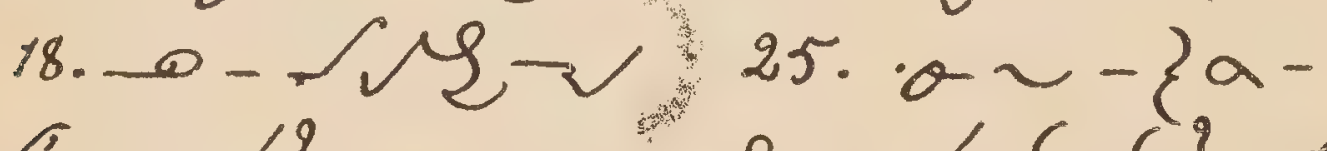

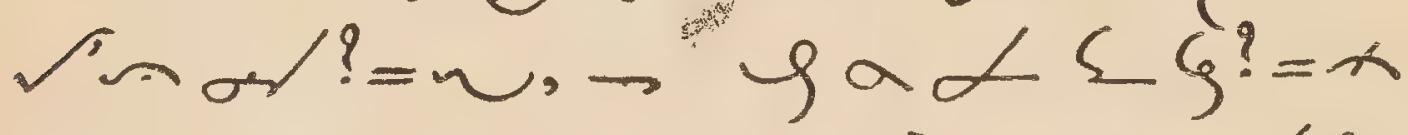
$\sim:-g \sim \alpha-\sigma r \sim-\{a-\varphi a \alpha \leqslant \xi$. III. Tesus Christ.

19. $\%$ * $q a-\sqrt{ } \mathcal{L}-g a \alpha \leq \xi$.

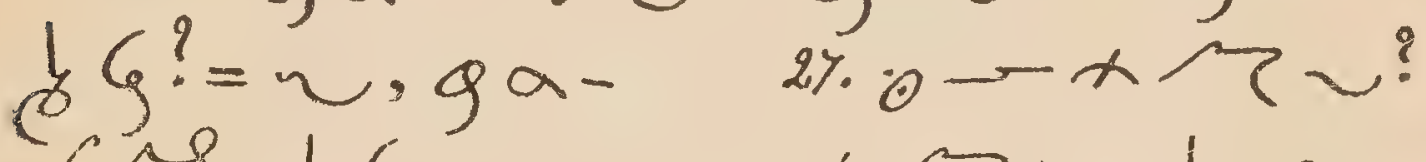

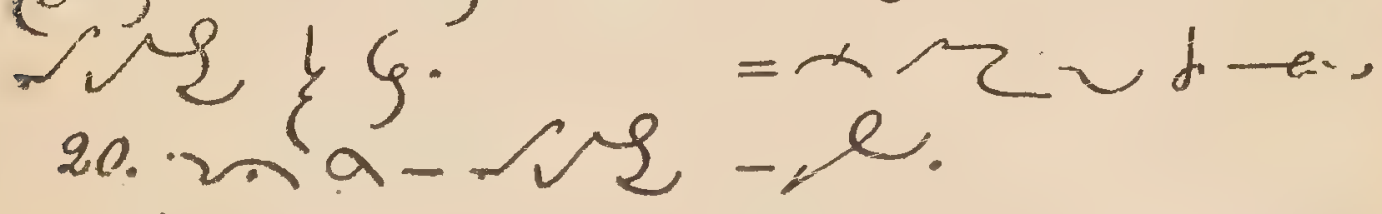

$\xi \xi ?=\alpha-\varphi$. 28. $\rightarrow$ - $r 2 \Omega$

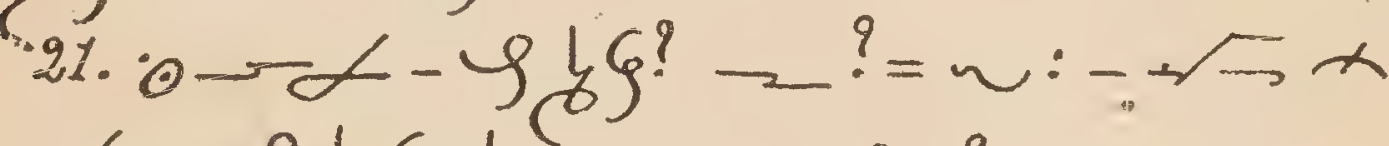
$=\alpha-9\} \xi \alpha$ 不 h-.0. 22: $\sim \ldots-9 a \alpha<\alpha \sim \sim \beta ?=2$ :

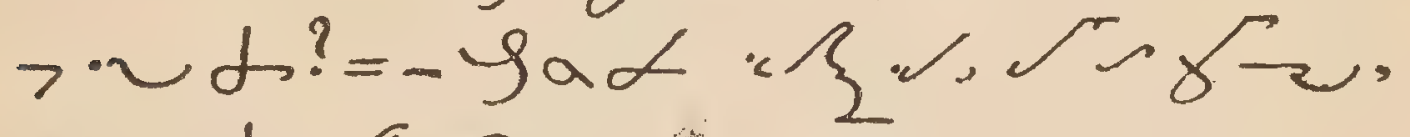

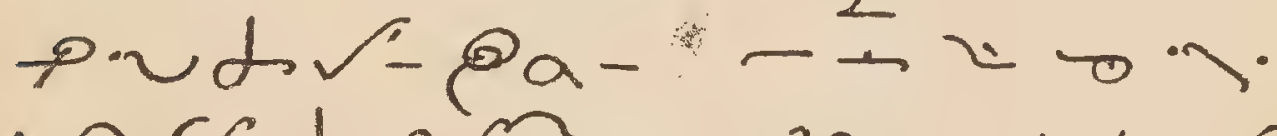

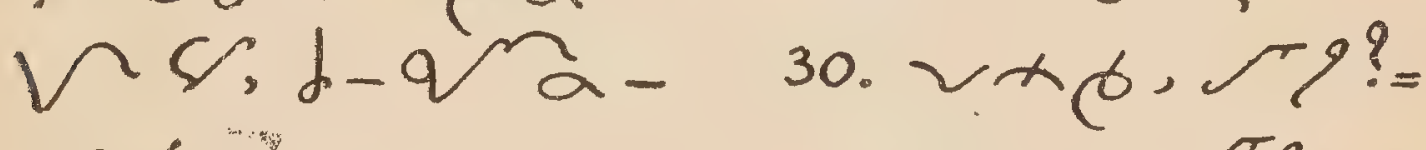
'd 2 .".

23. $\sim: x \sim \phi, r 9, \cdot$

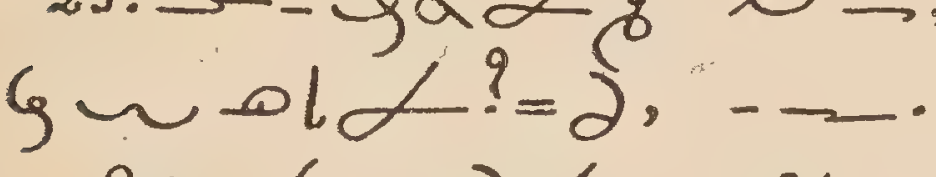

$-9 a \alpha \sim \partial \alpha \quad 31 . \theta 2 d \theta x$. -G. $\quad 0 ?=" v>-\alpha<0$ 
ก;" $2 \alpha-2$ - $0-q \sim-"$ verel.

38.

IV. The Church.

, $\sim \sim \sim \sim ?$

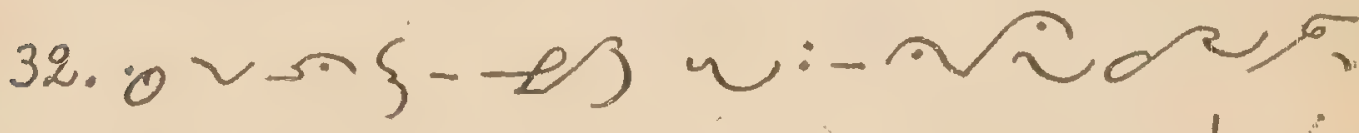

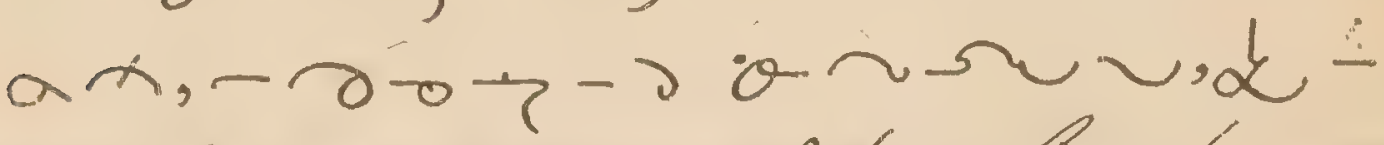

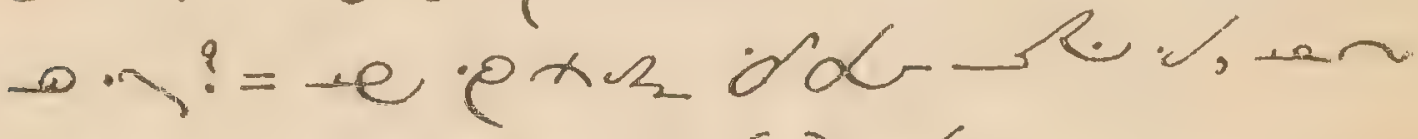
ovp- $\varepsilon_{3} \sim \Omega s \%$

33. $\theta \sim-\pi \cdot=-39 \cdot e \cdot b \cdot v^{2}-3$. ri-veradoh "ork-ria- geenen n'?="

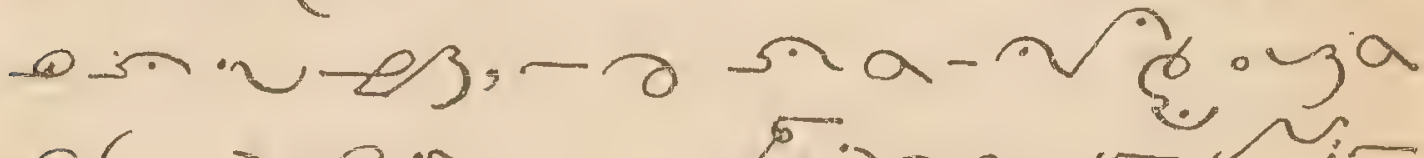
- $\{-\partial-\infty \cdot$ 34. od te ex.e n r.

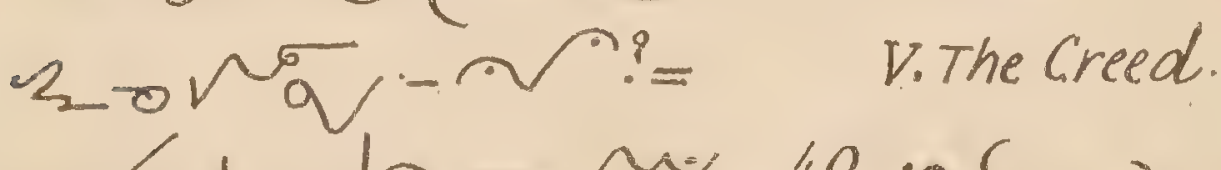
$\rightarrow d-d,-b-r$ m $40.0<-20-0$

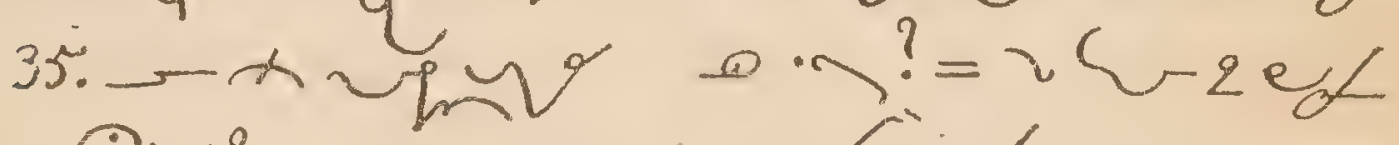

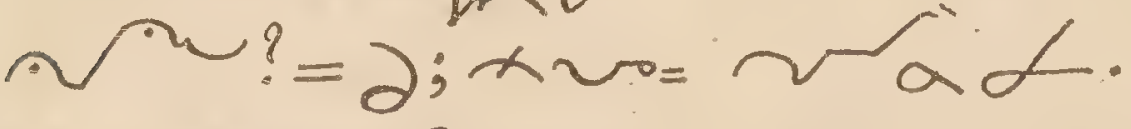

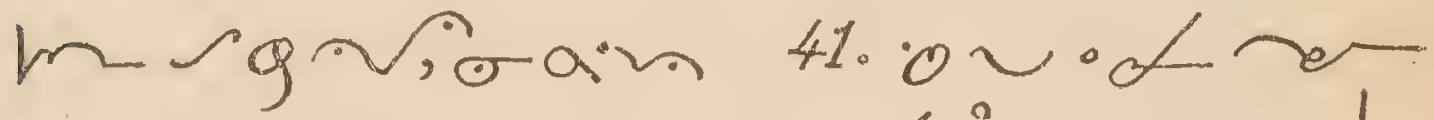
-rid an. $\alpha \mathcal{L} \cdot=\cdot O \sim d=$

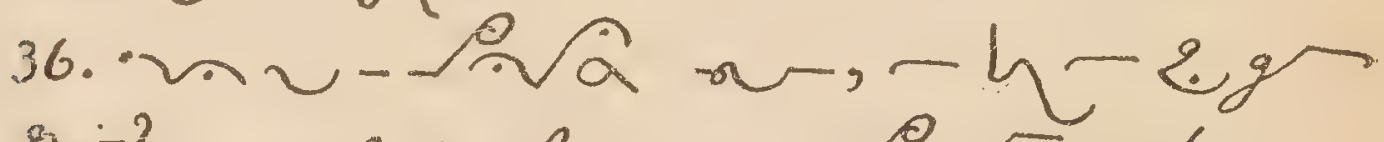

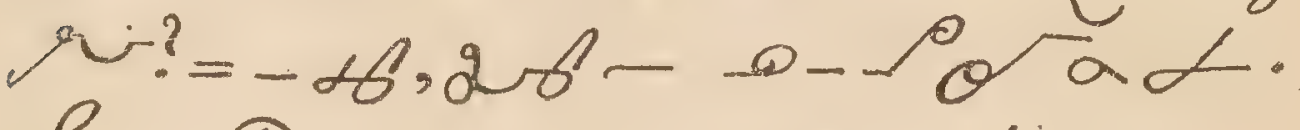

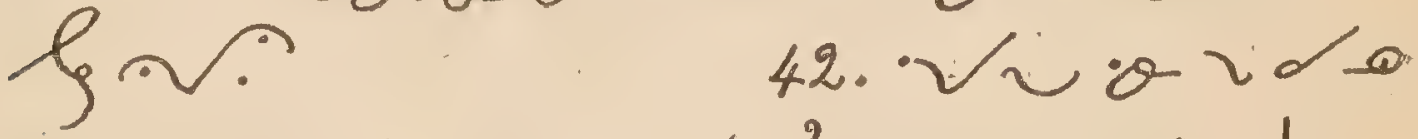

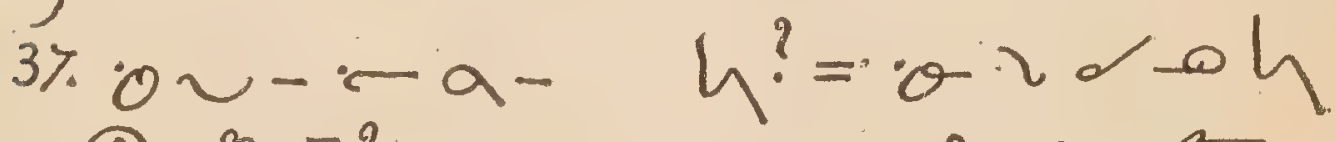

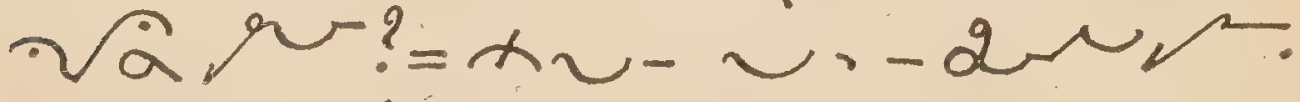

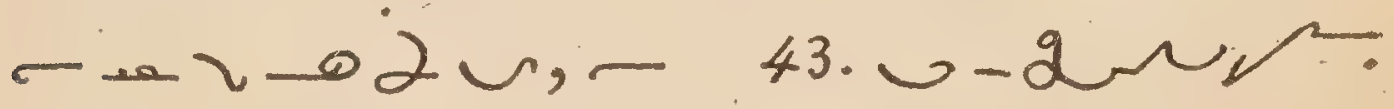




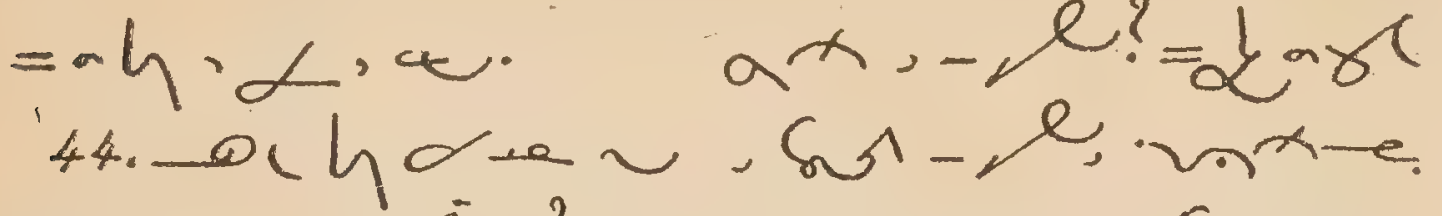
$\{, 2 \sim<==50.4 \gamma \geq 5-9$

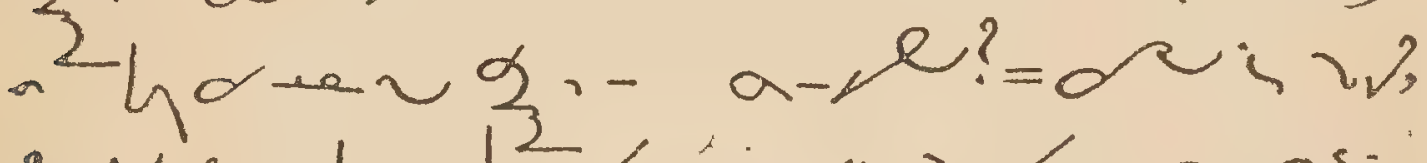

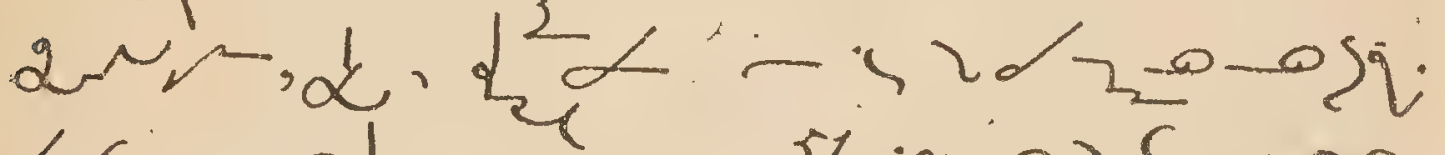

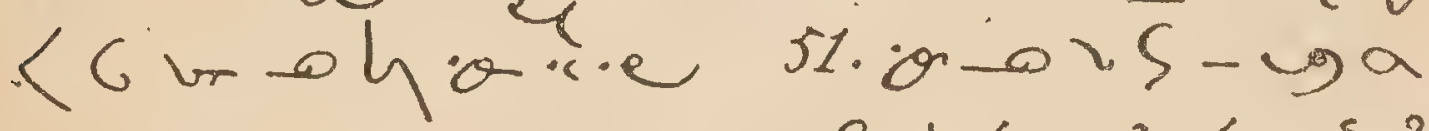
w. $-e b u d-0$ in? $V I$. The Sign of the Cross. . = - a d I t do to $45 . j \theta-02 \beta-\alpha, \ldots,+\sin c$

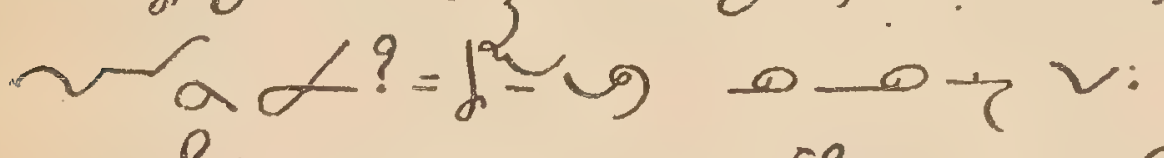
$a-l$

$$
52.0-0<5-9 a
$$

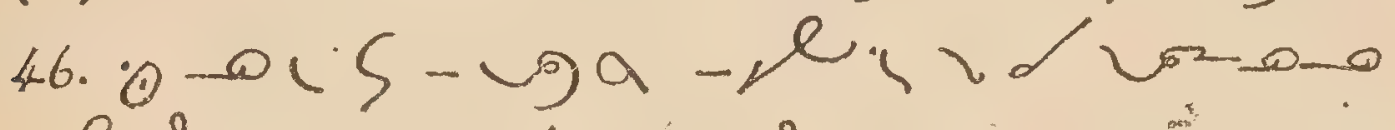
$-\ell ?=a-g-\gamma k, V ?=-e_{e} \alpha=0.1 c$ $-1,-,-1 \alpha, 2002$

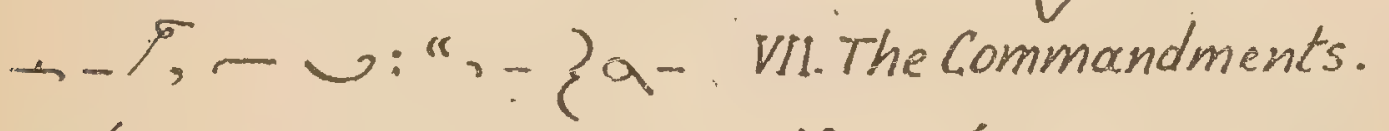

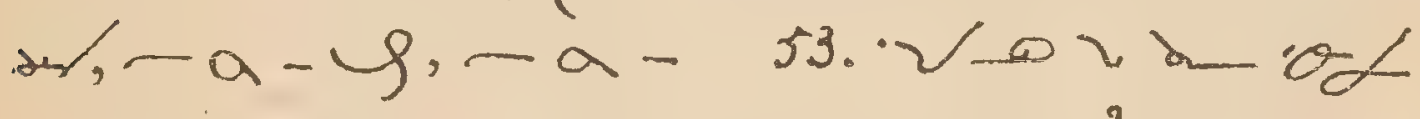
$\therefore \alpha, \zeta . "$

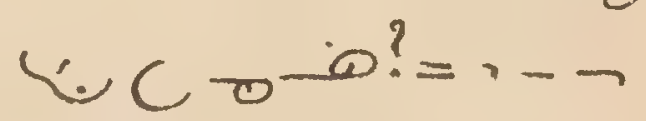
4\%o-in he bad.

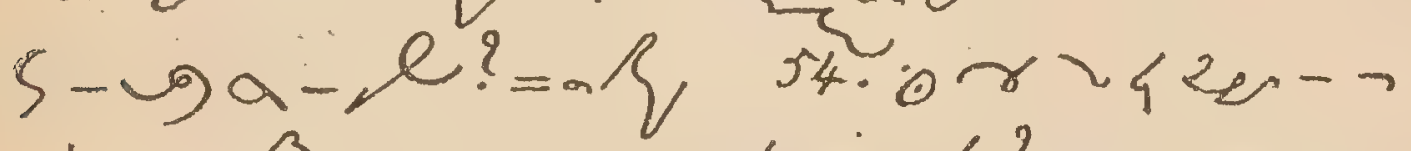
- hm $\beta,-x \ldots, c a \alpha ?=2$ razes $-r$.

4.8. o- a $\{-\ln$ $1 ?=2 \cdot z-2 x-9 \cdot d$. $-\dot{d} \alpha-\dot{s}$

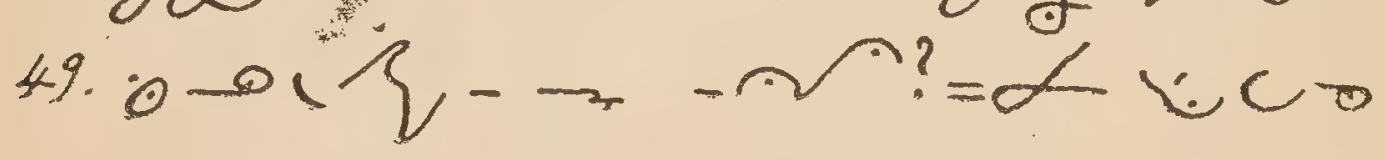




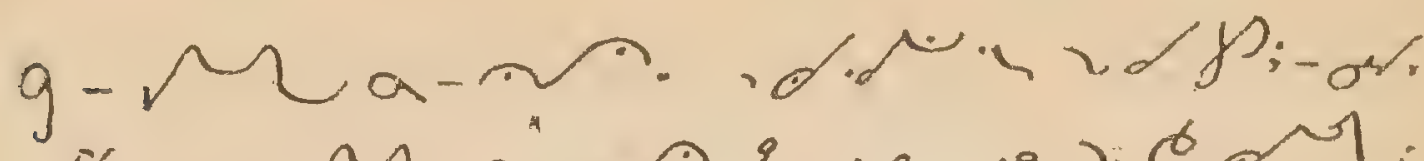
56. ง- M.

VIST. Prayer. 65. 2nutay yev

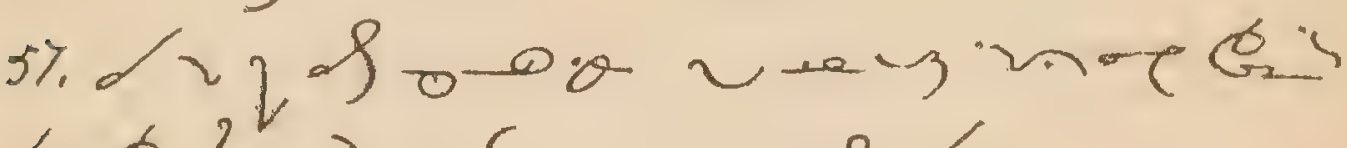

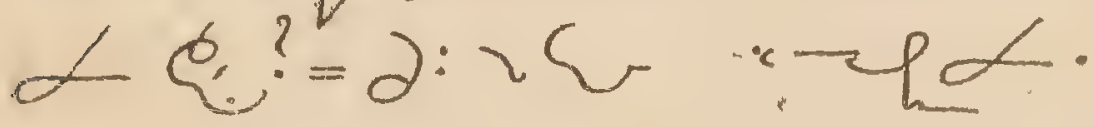

$\alpha-1 a d$.

66. $-2 \cdot \pi+\frac{1}{3}$

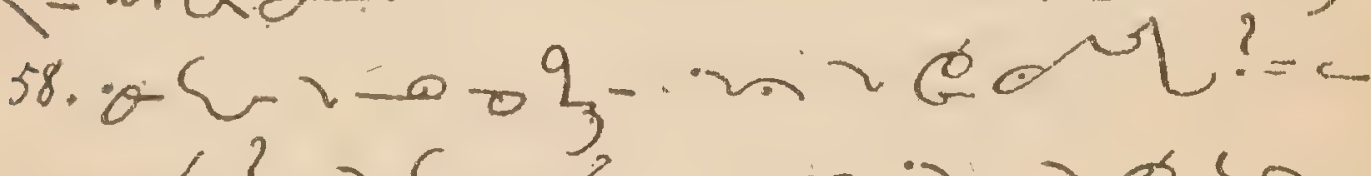

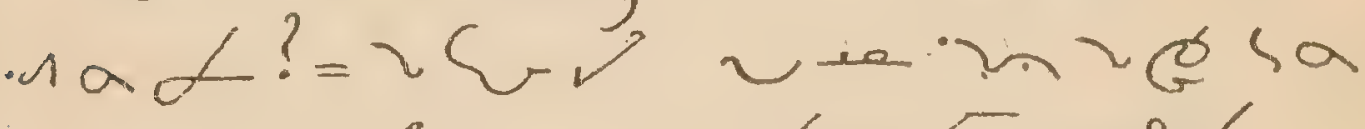
$\sim \sim$ - $r$ é. ogg $y+p t$. s9.ma-he 6). $0\left\{\alpha \alpha^{2},\right\}^{\circ}$ $r^{?}=\pi / d \omega \alpha 2$

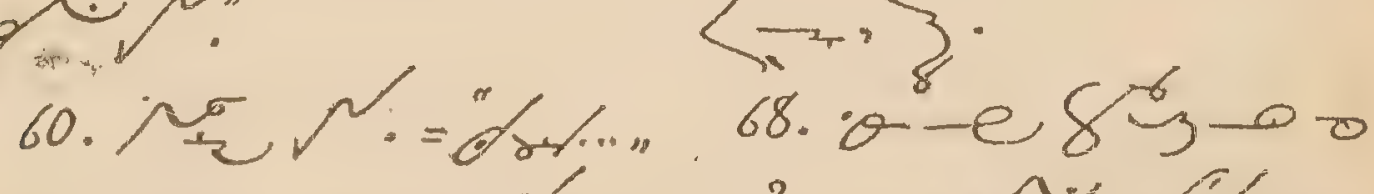

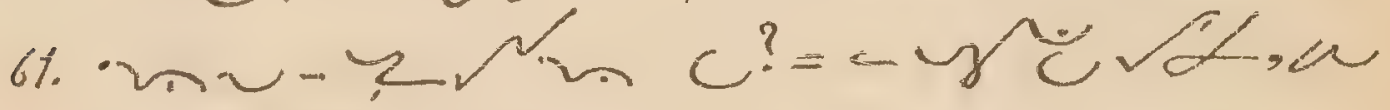

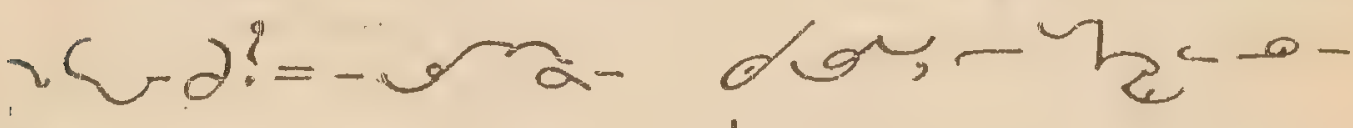

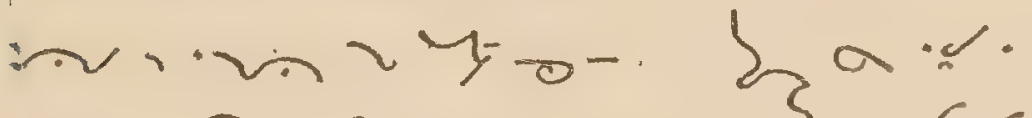

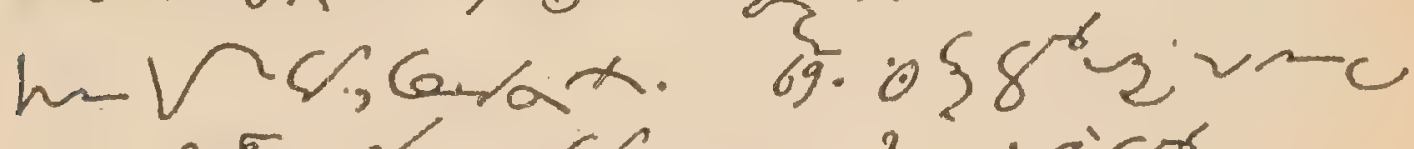

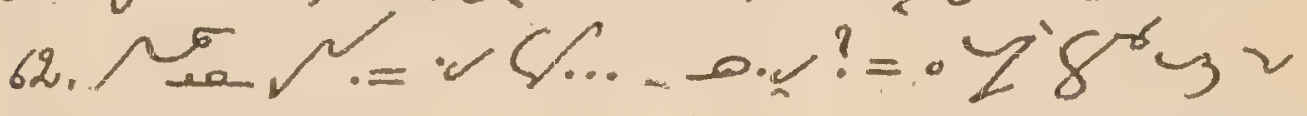
IX. $\sin$. iccosis

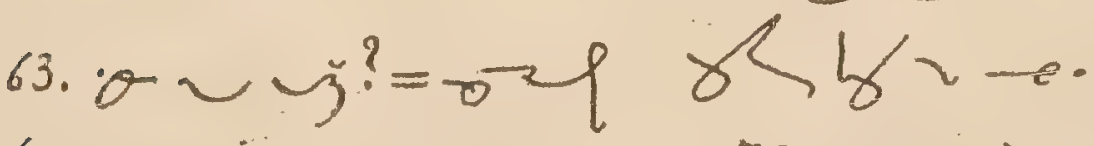

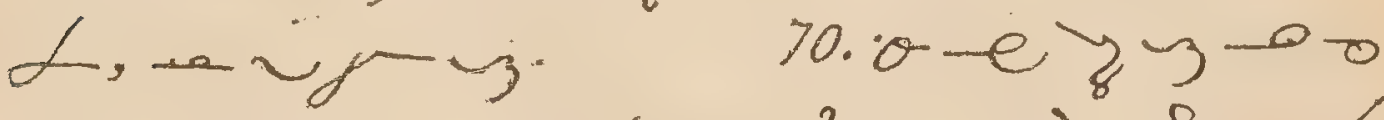

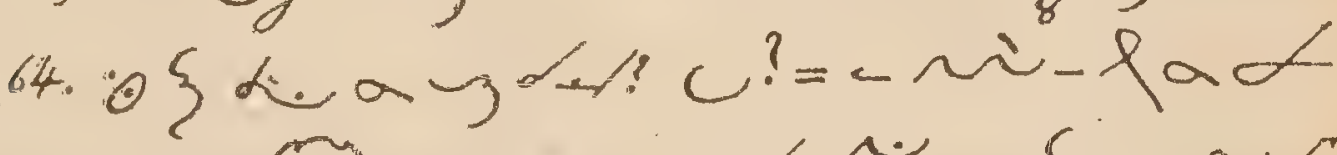

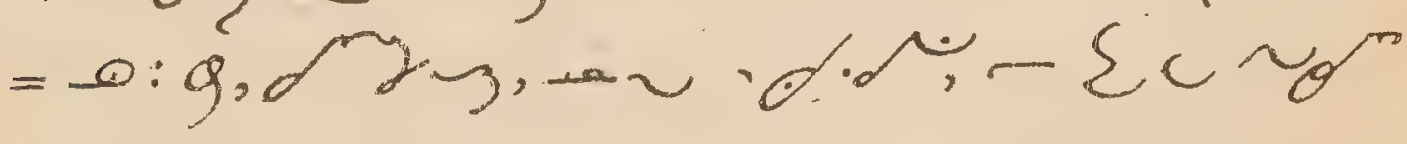




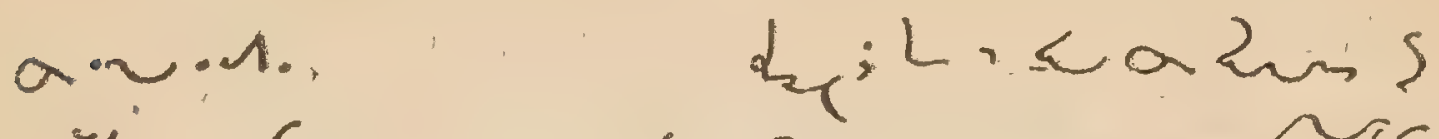

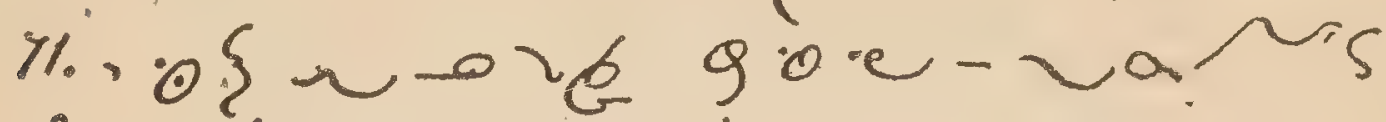

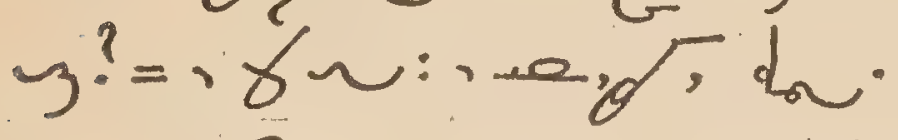

$=-G$. $\quad 78.0 \sim b_{2}<$ ? $=q$

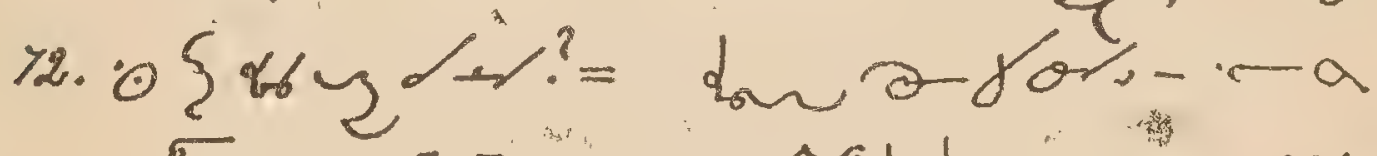

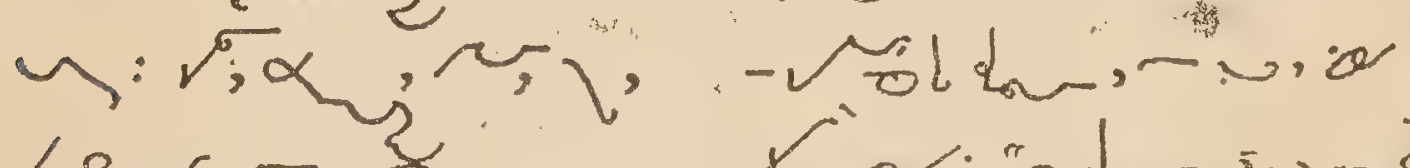
He.

f-or: "a $\alpha_{\alpha}=, 1-$ \} X. Boptism. $a-\operatorname{ser} s-a-g,-a$ 13.0.

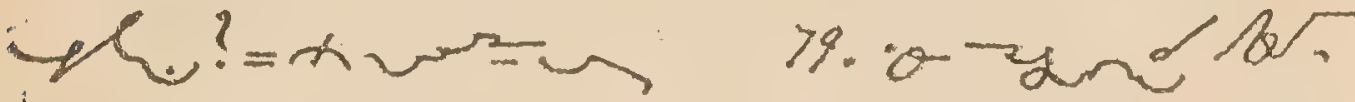

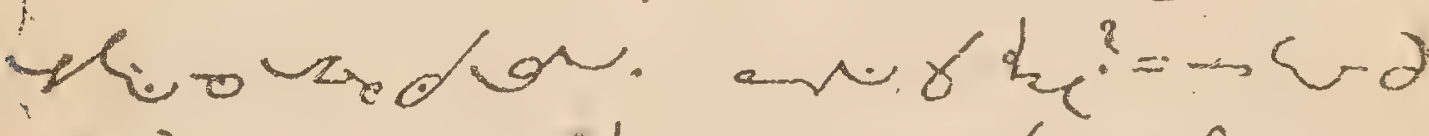

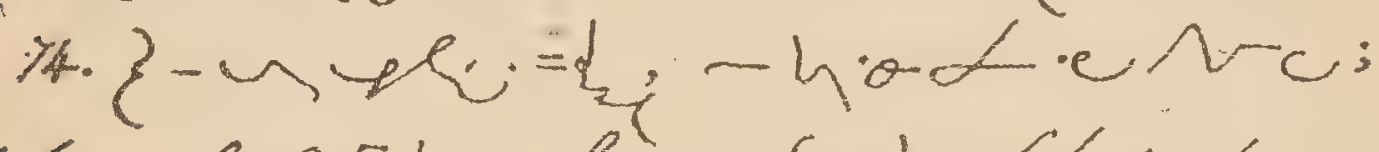

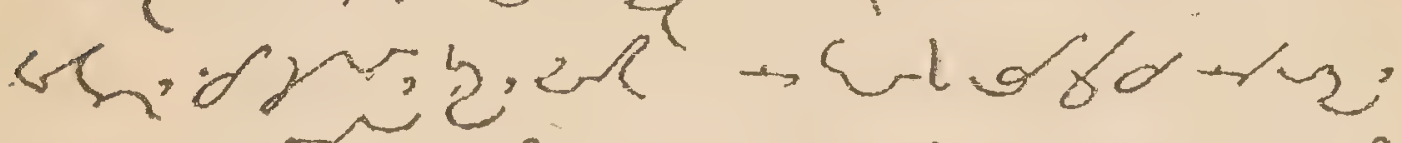
aidr carg.

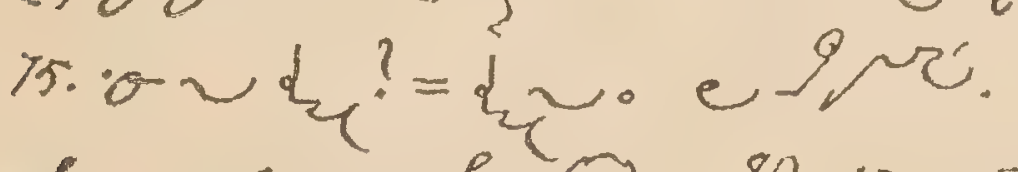

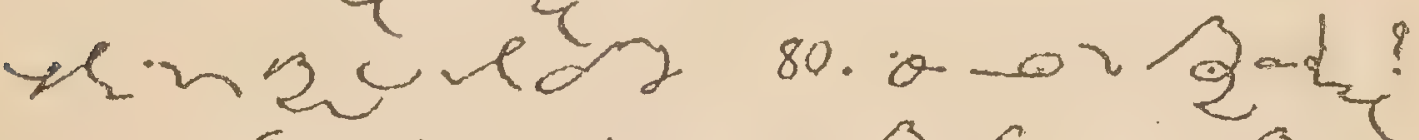

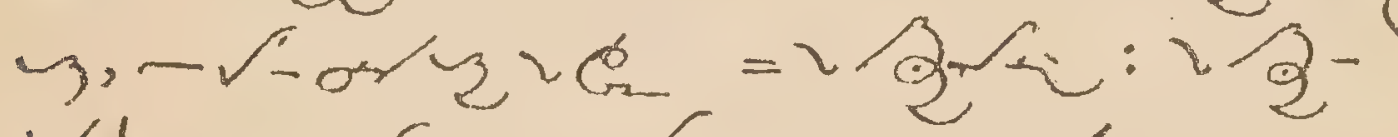

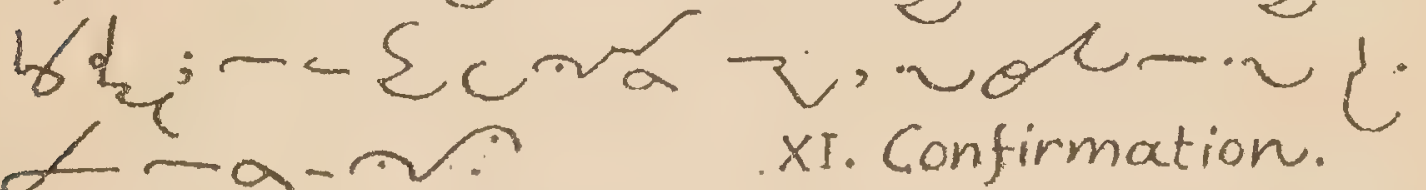

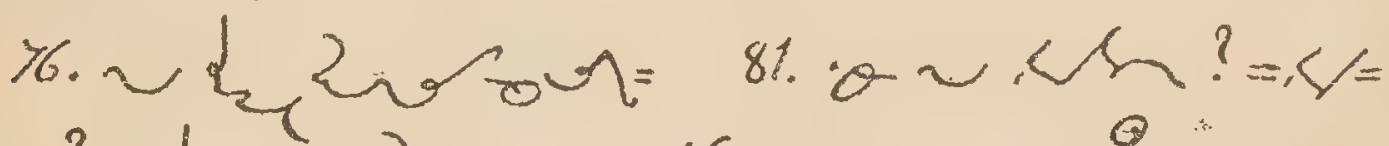
$n ?=d_{2} \sim 2$ so huovkrind

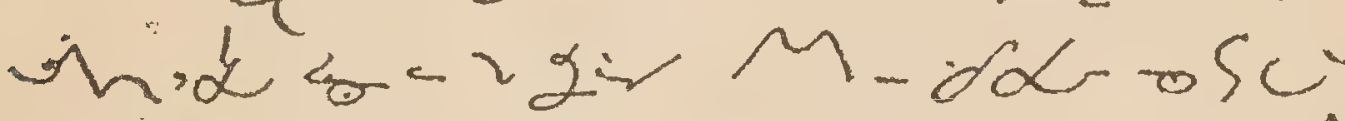
- - $e_{e} \alpha \cdot$.

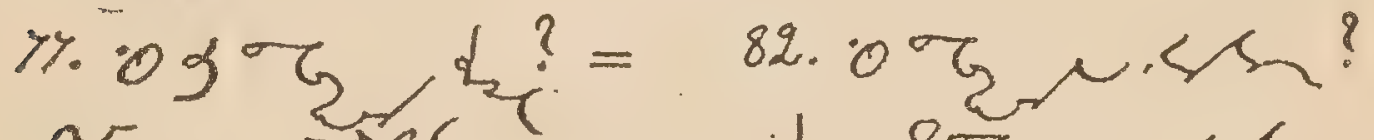

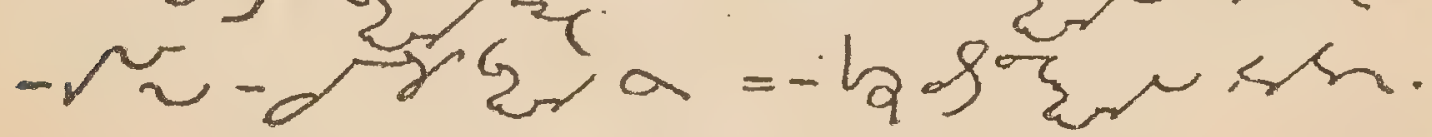




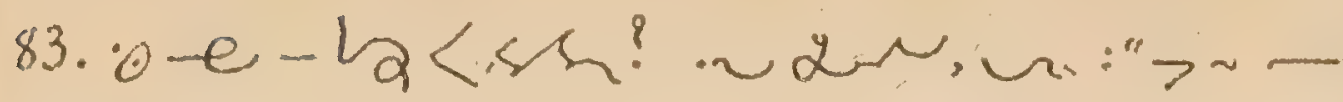

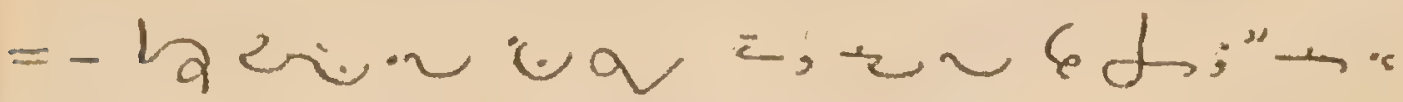

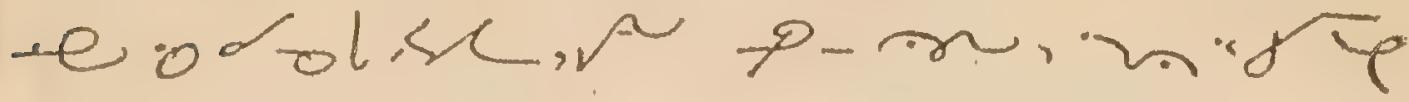

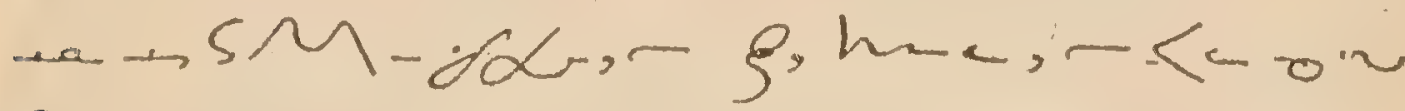

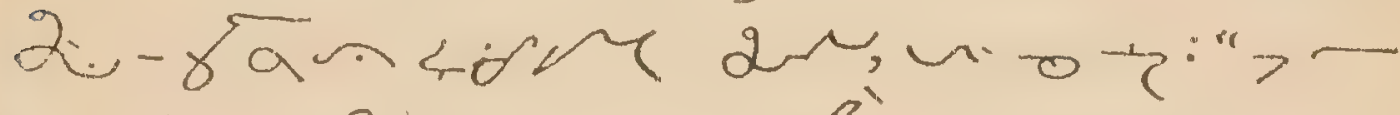

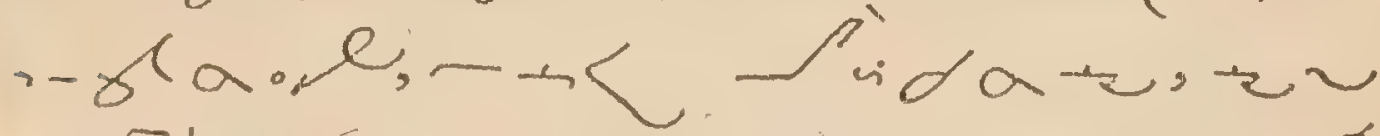
- 5 b. 84. $\theta$ औ

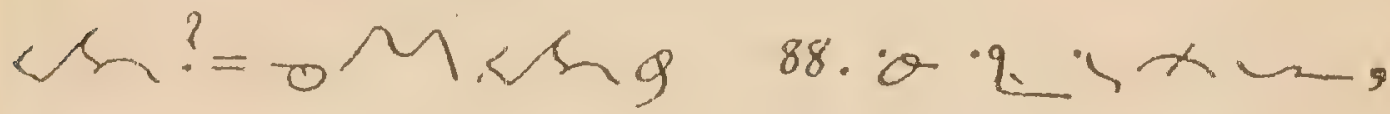

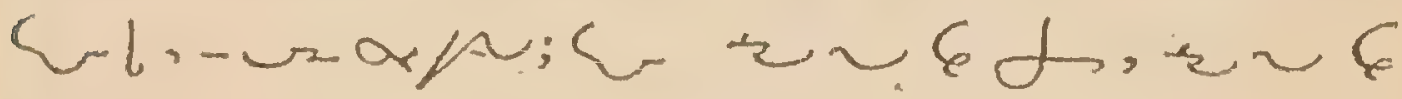

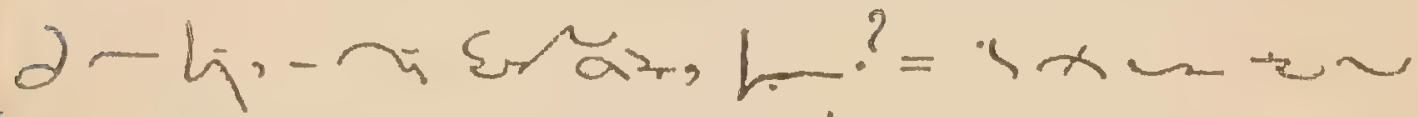

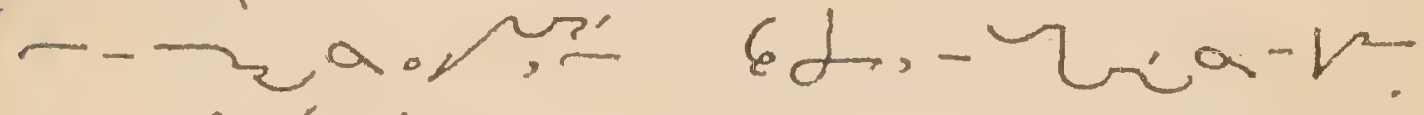
unb-ba-dol a wa- o- Uia . $\downarrow d$; : is «

X11. Holy Eucharist.

85. $\theta \sim$ of $2 \sim$ ? =

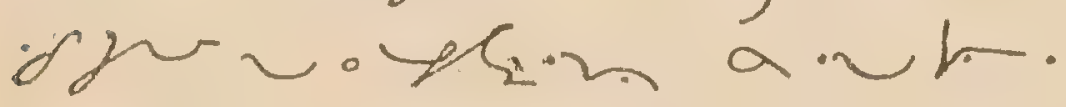

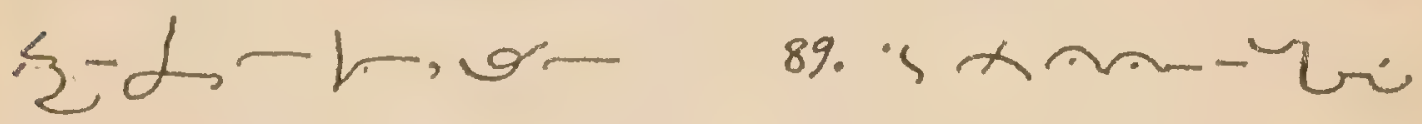
र,ax. ar-go- Li 86. 0 w. d J ? a d d - - . . -

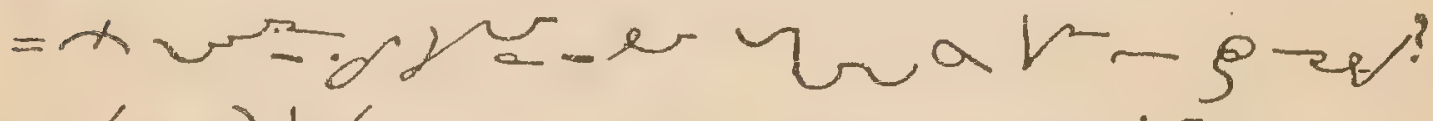

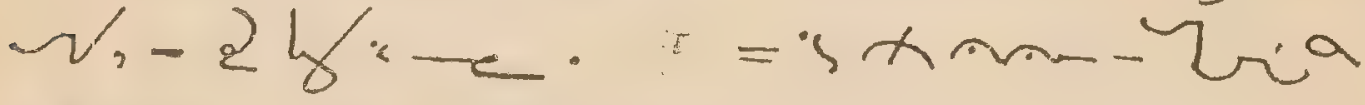
8\%. $0 \rightarrow x w=d V^{2}-9$ o- Lia.u or? $=\sigma-e-v,+$ d, -

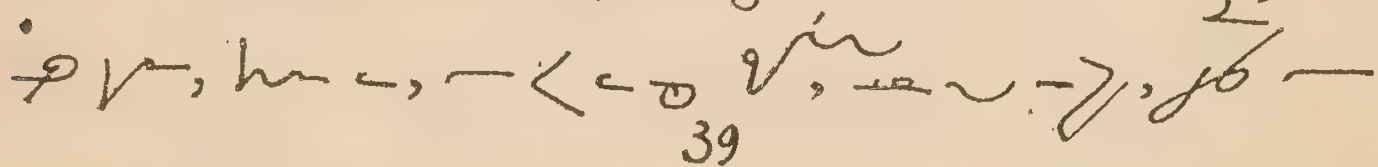


34

ENGLISH

wav $-9 \cdot m-d+m a r=9$

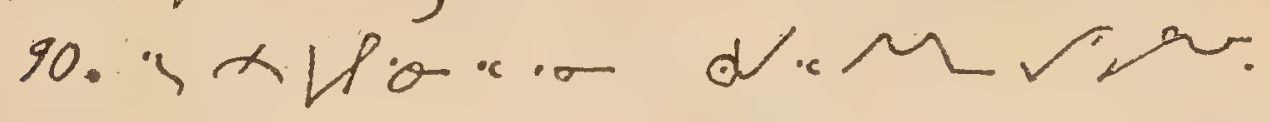

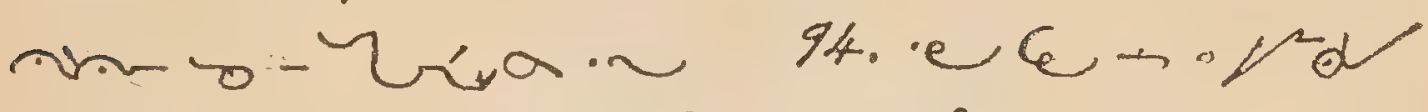

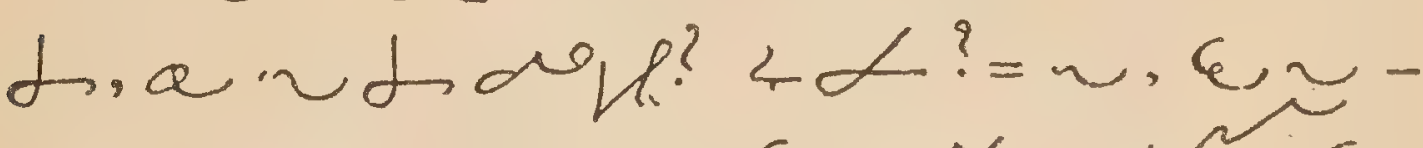

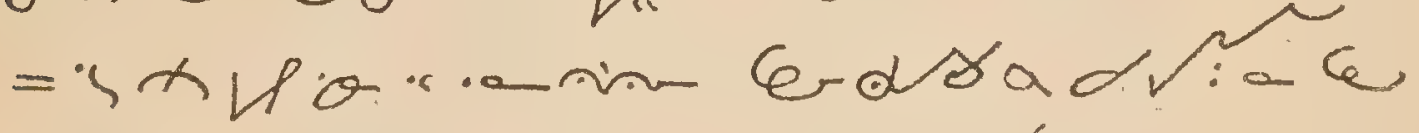

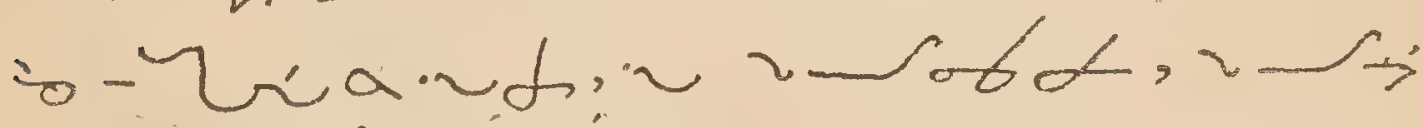

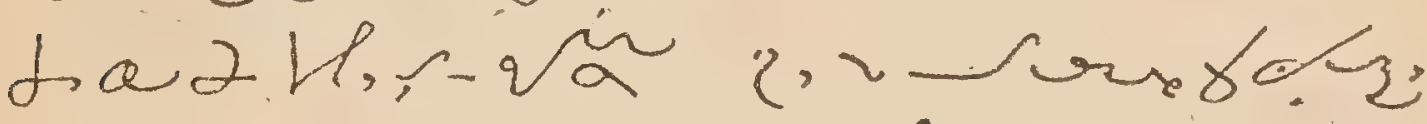

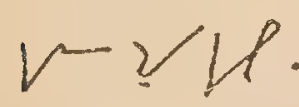

$-29,2 d \mu$

91. sxin- vo - hi

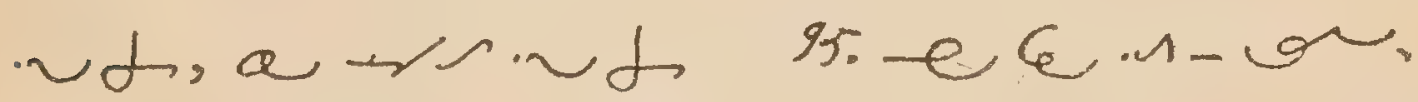

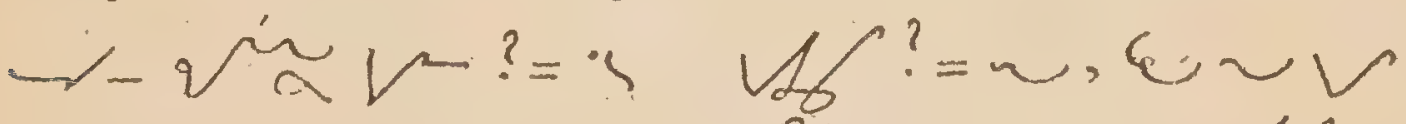

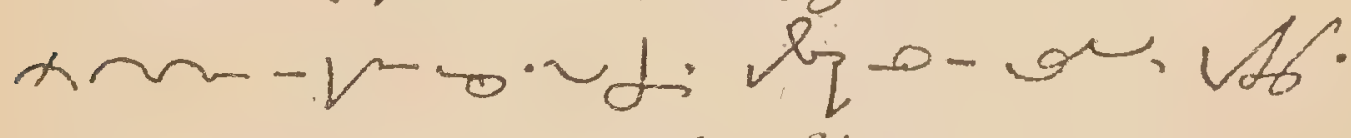

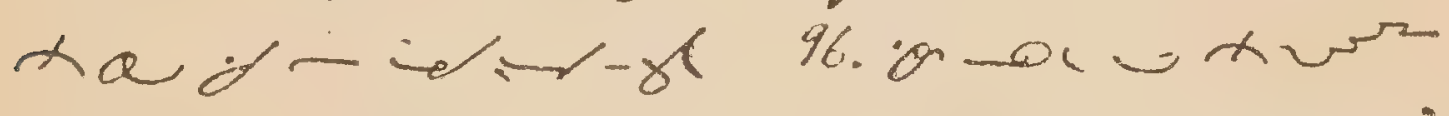

ar, ud, uh, - d foren dou?

$=2 \times w=\cdot d \%$

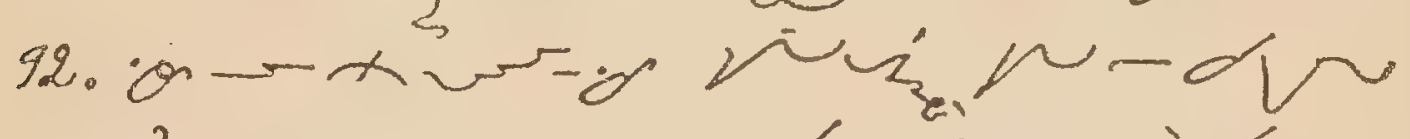

$2 \sim$ ? =xw=.d 'dorisonid

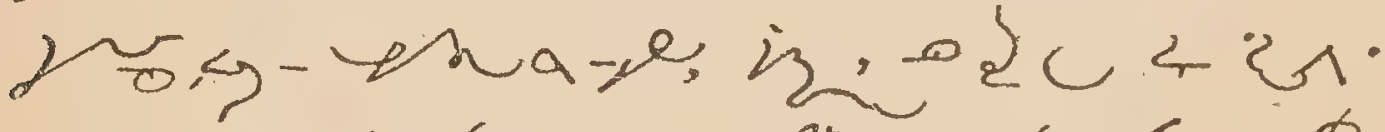

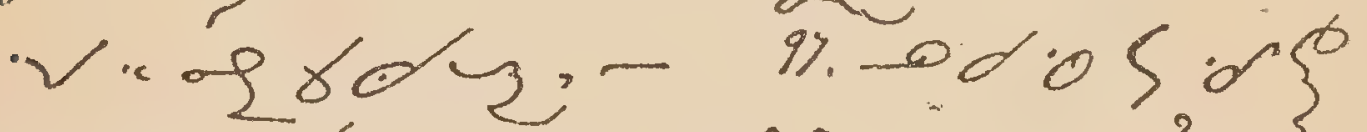

order. M-im?=ier

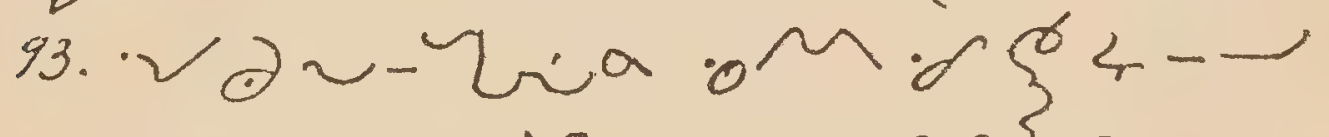

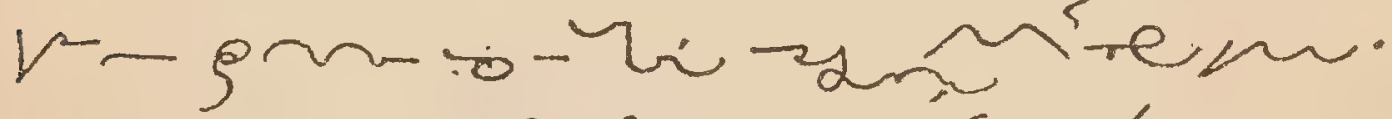

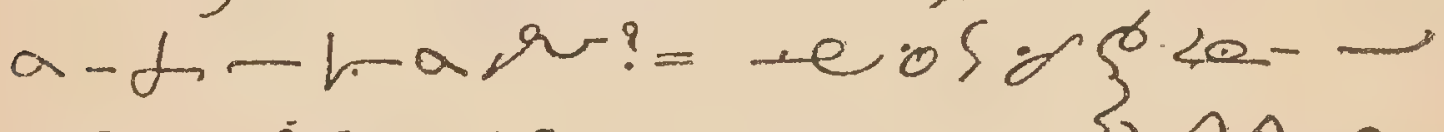

-

$a r-90-\tau^{2} a_{40} r .-6 \cdot r^{2}$. 


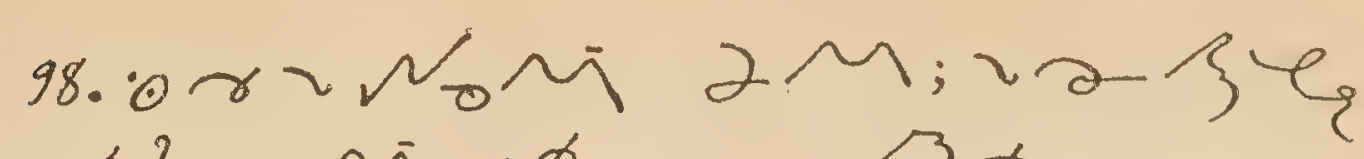

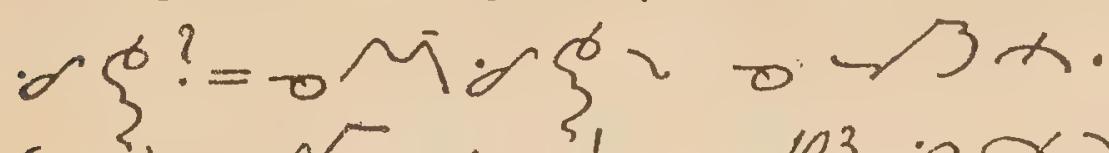

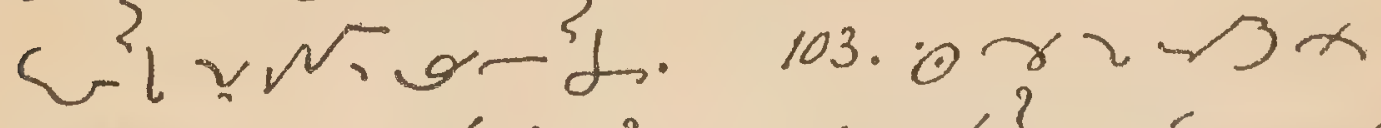

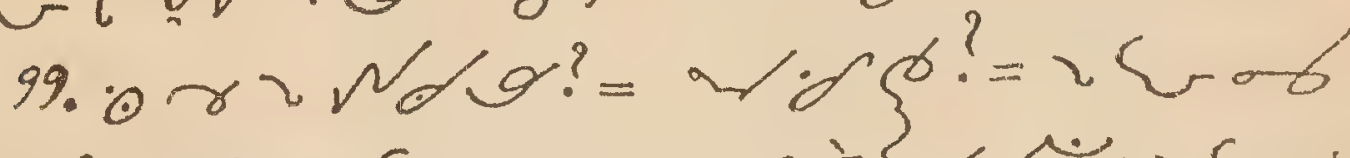

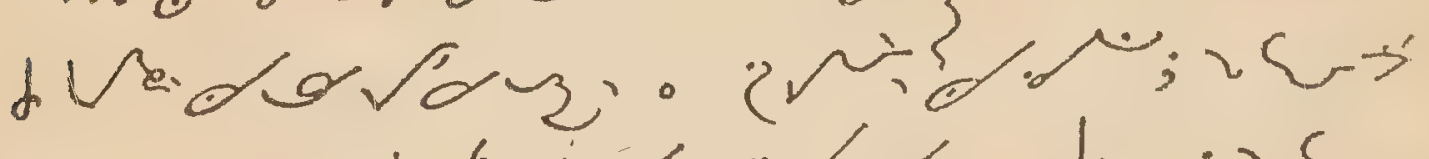

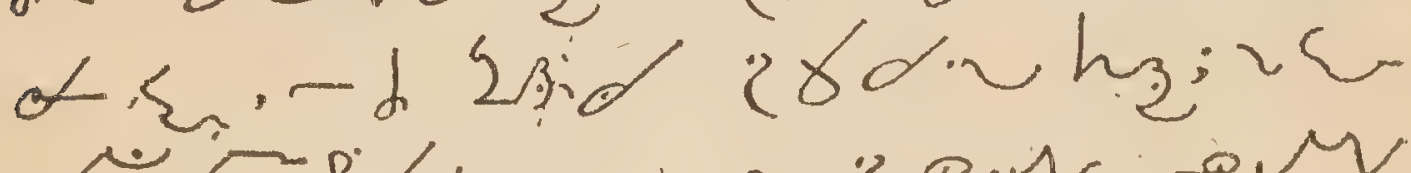
. 100. orरLind wer wild

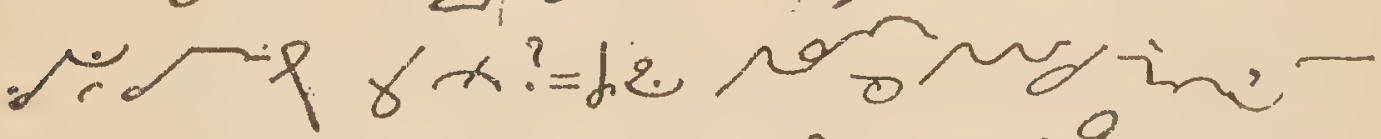

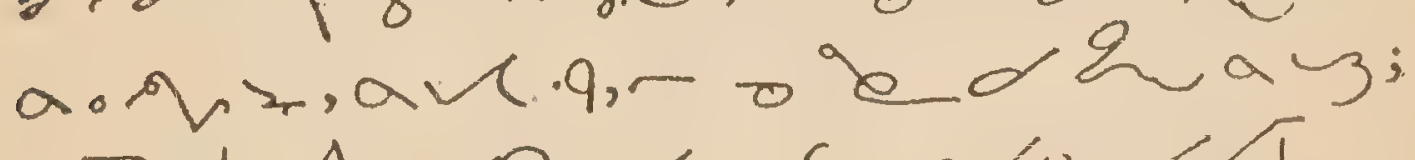

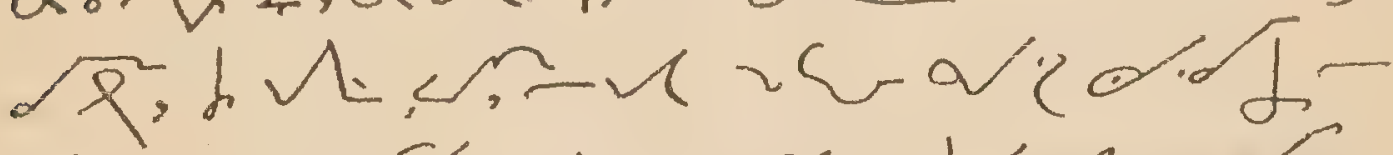

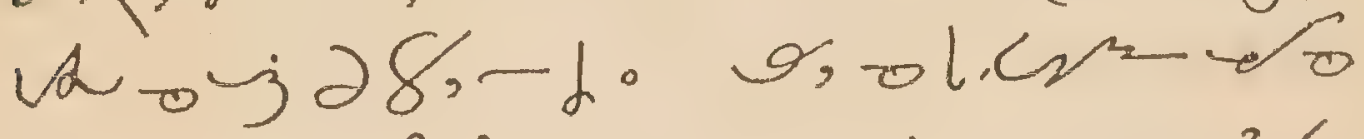

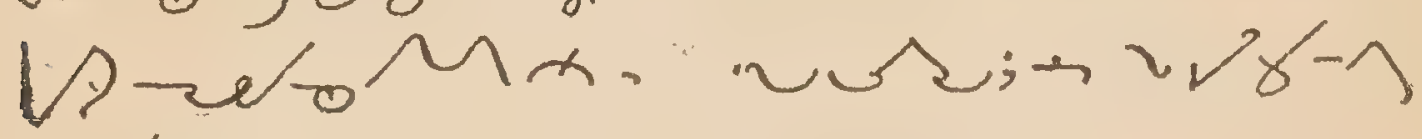
$\delta \xi$.

101. orvNo/d? 104. unct on 2el

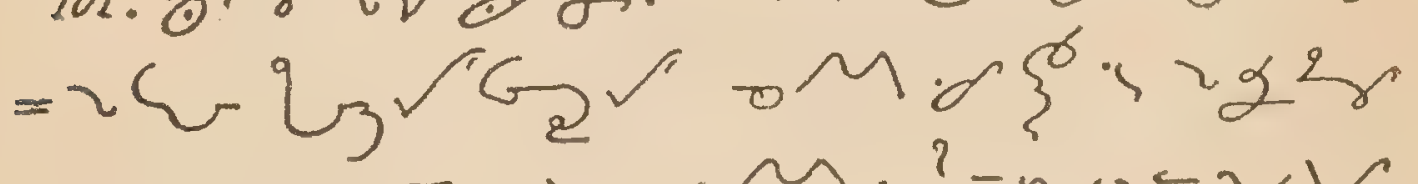

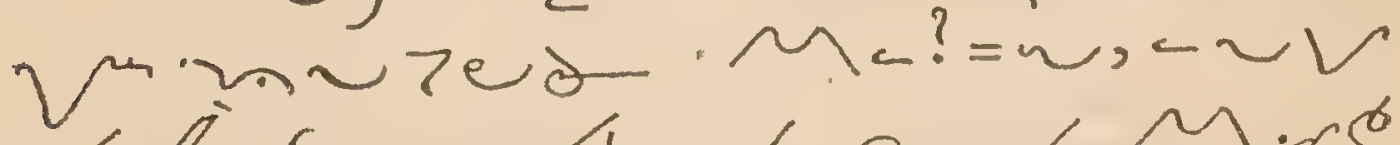
diverigd of o-voM.d?

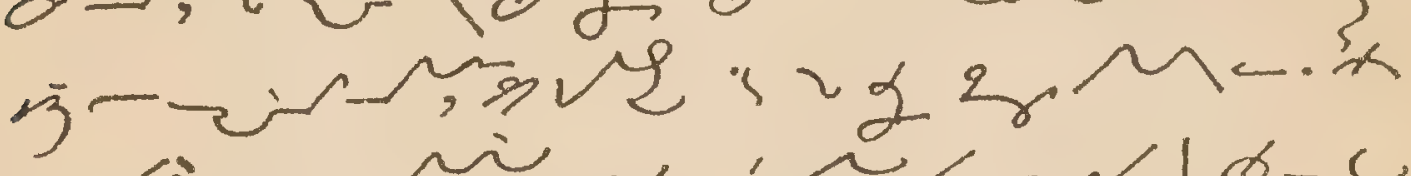
od 102. 0 - $202=2$

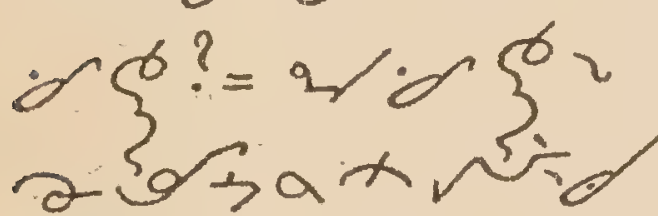
-driquid

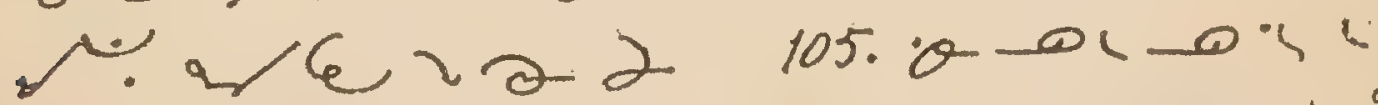

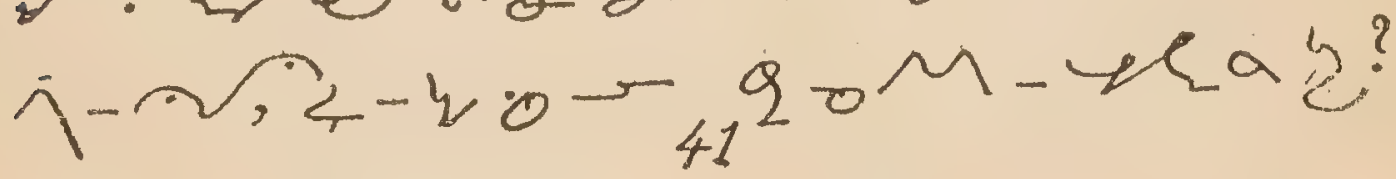


36

ENGLISH

$=\operatorname{sog} q_{0}$ - yh $\theta-0<-0 ?=2 / 0$

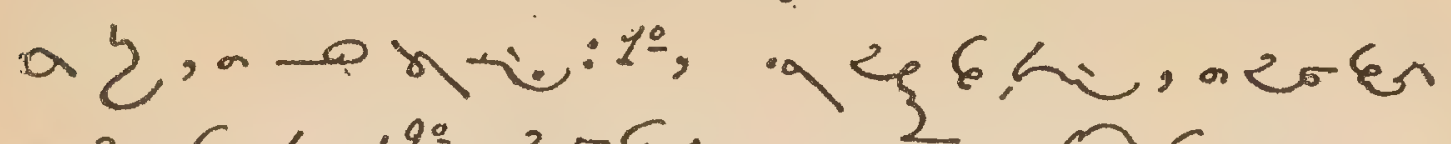

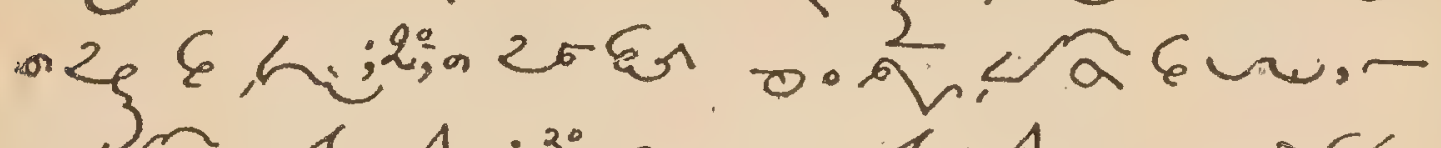
of

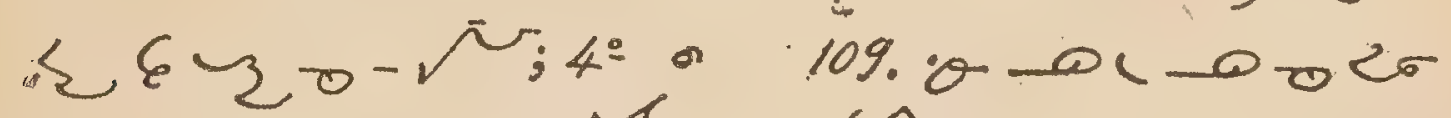

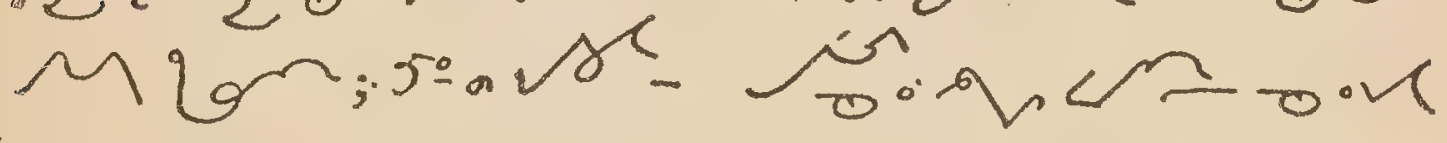

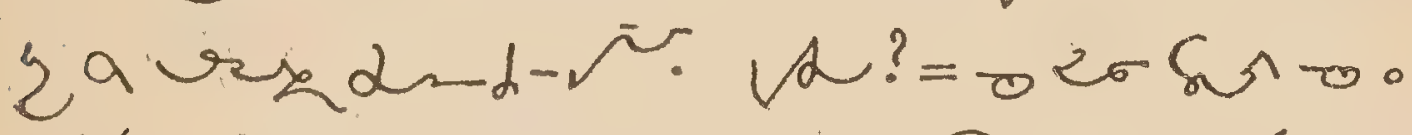
106. 0 - 0 -

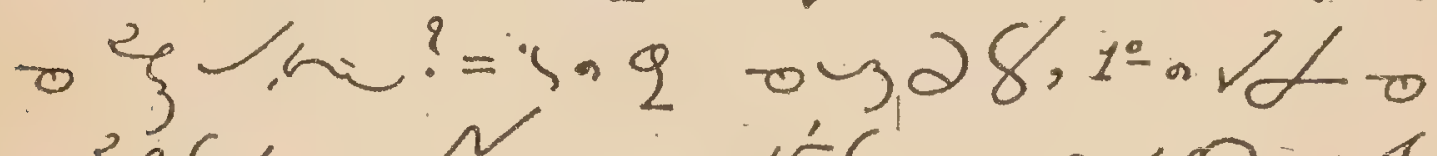

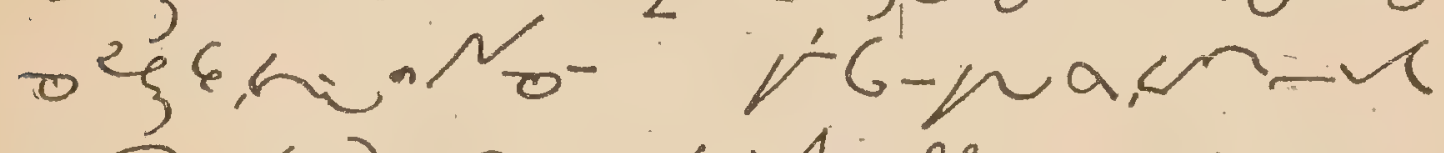

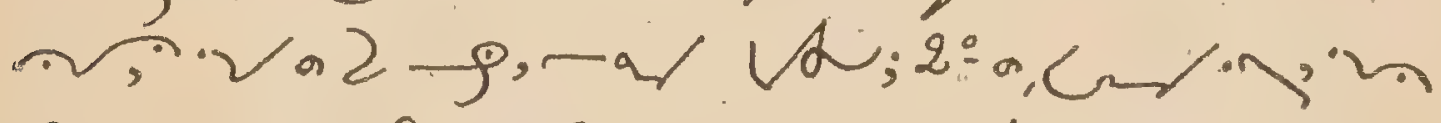
s-ma-e,

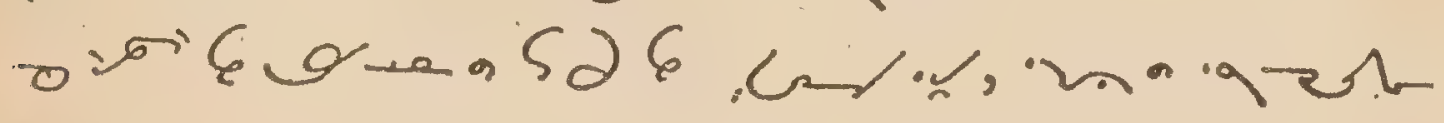
ic.

$$
\text { 4: }
$$

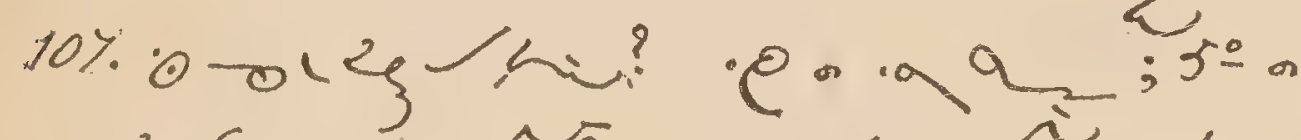
$=0$ zद, hiv

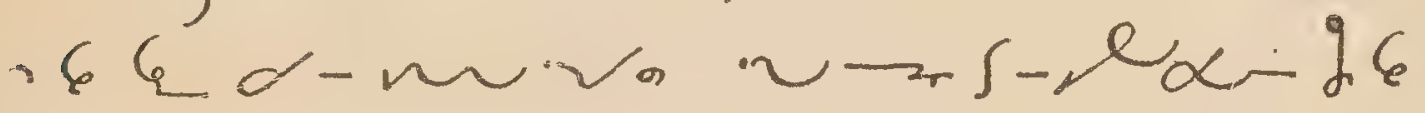

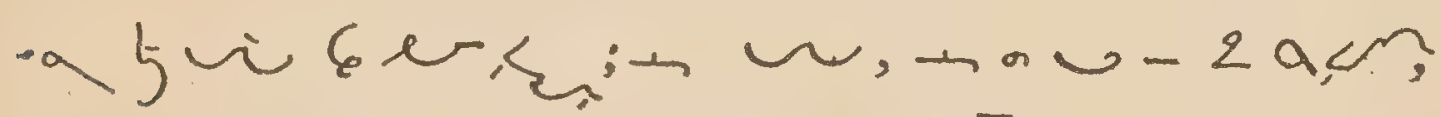

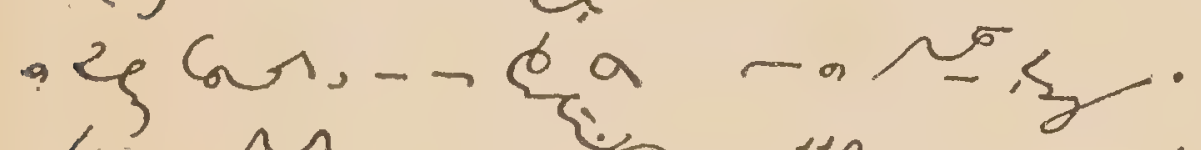

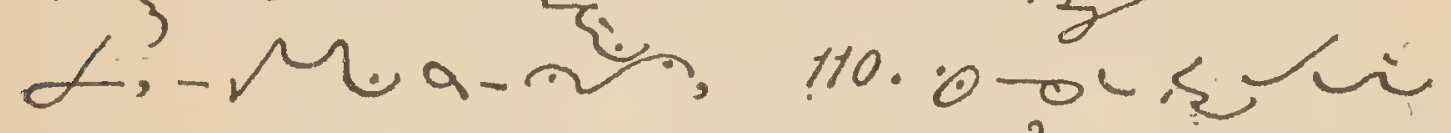

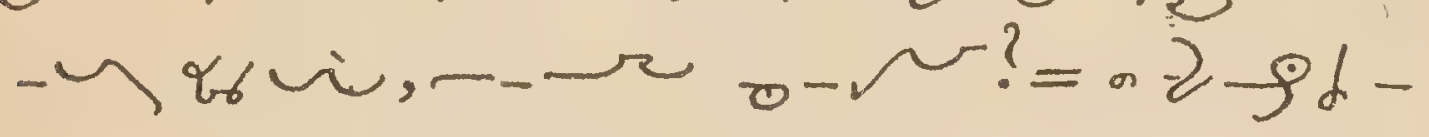
a cuseradaj wa-ri-ars

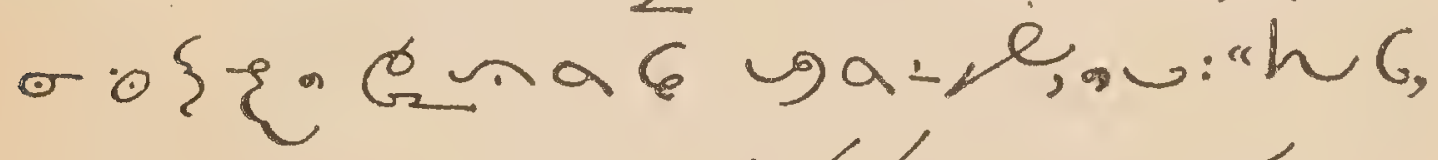
n.

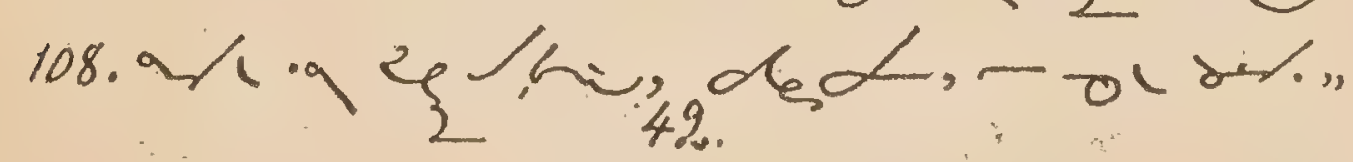


-

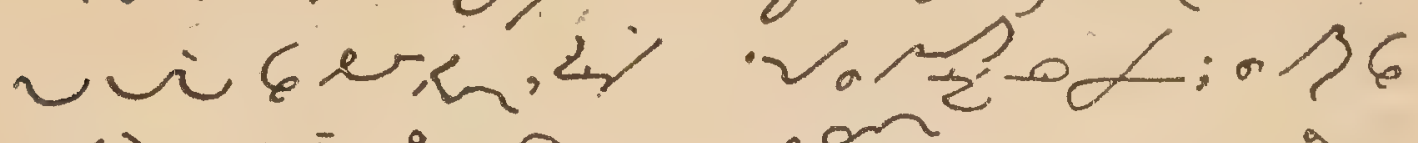

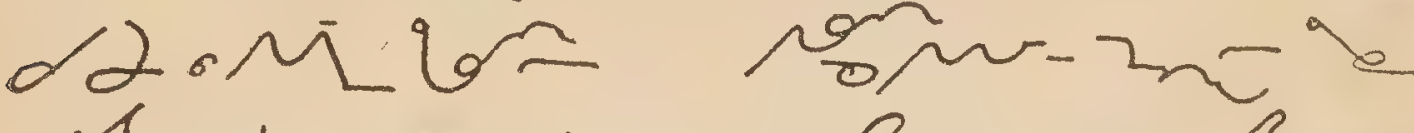

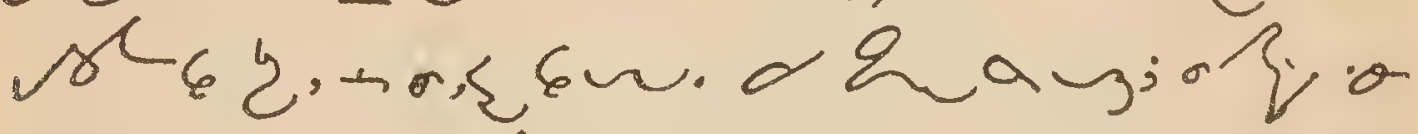

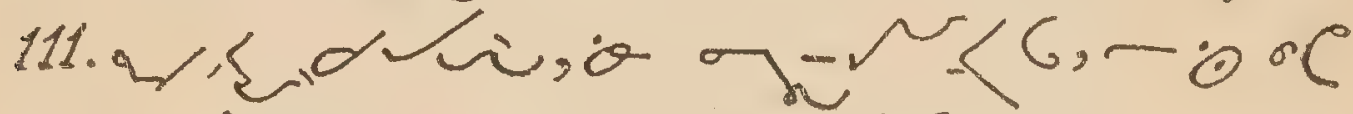

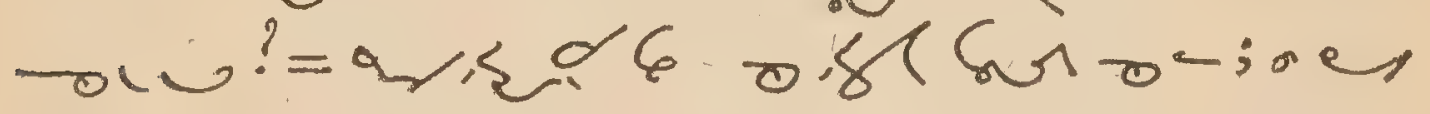
i,

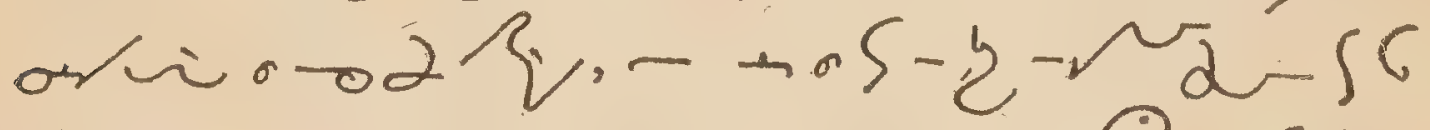

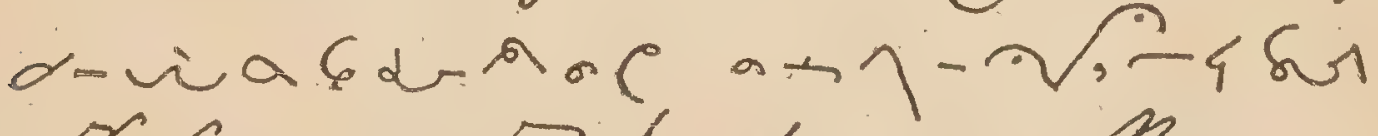

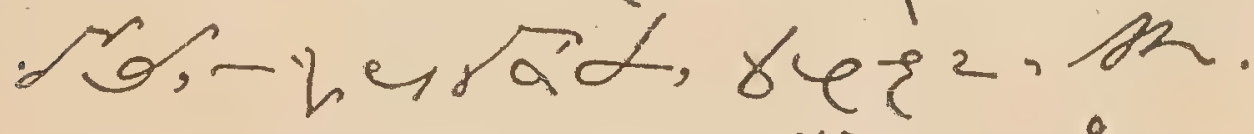
-a人ds

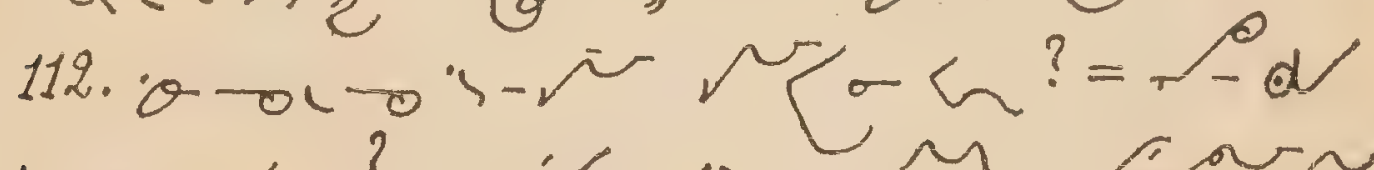

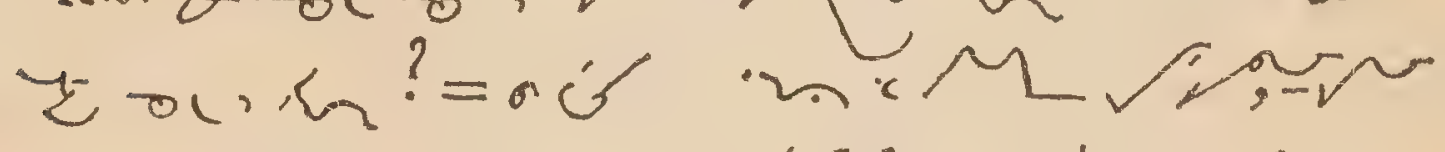

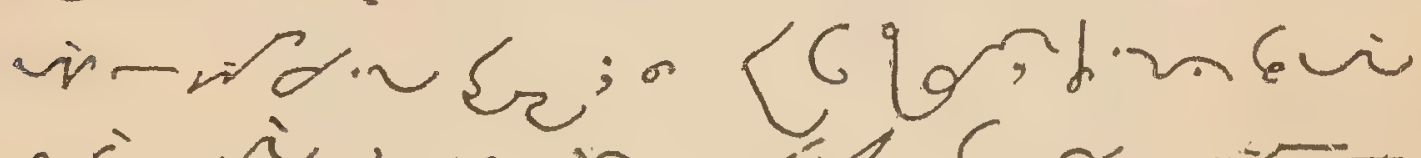

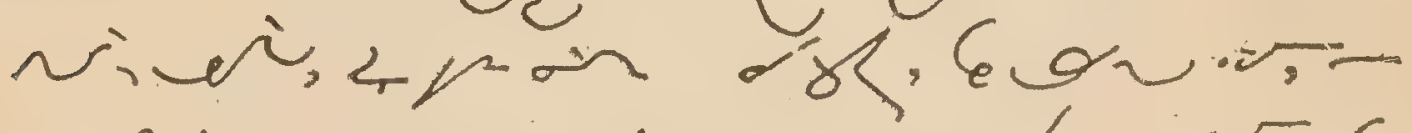

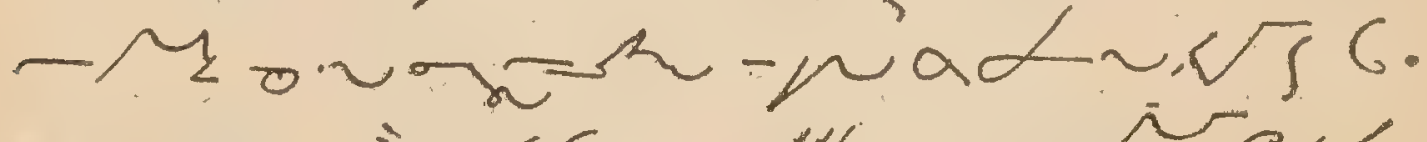

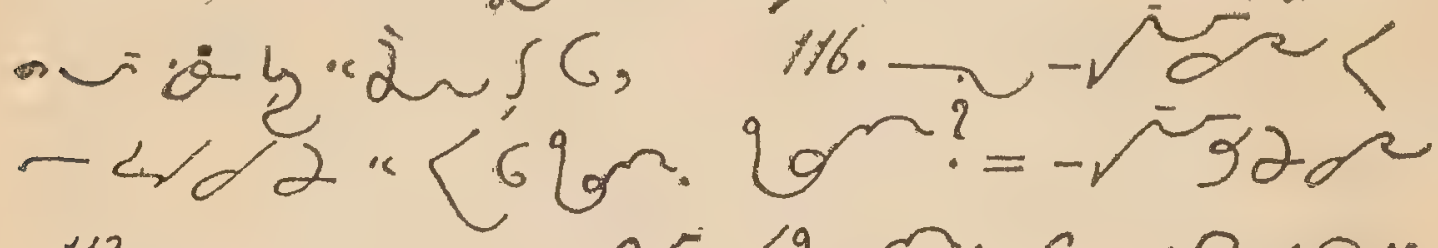

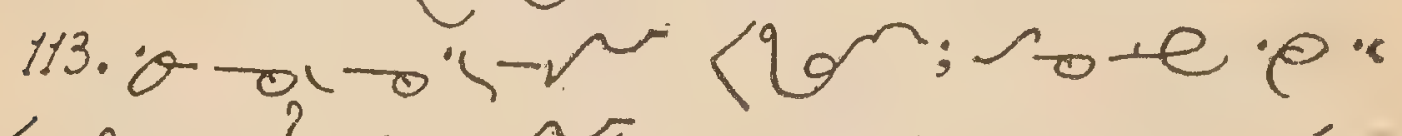

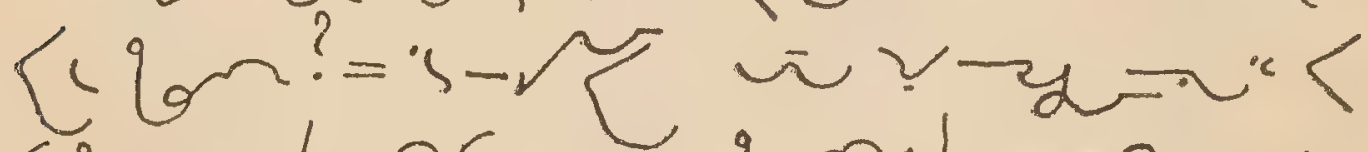

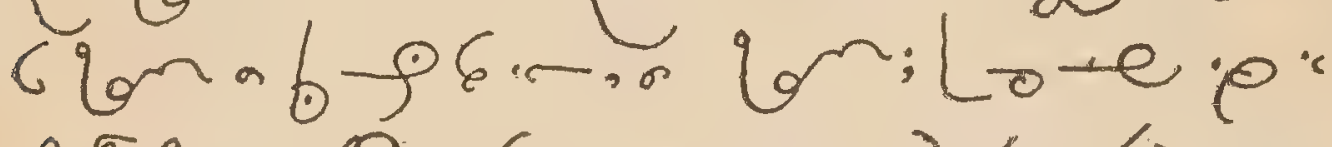

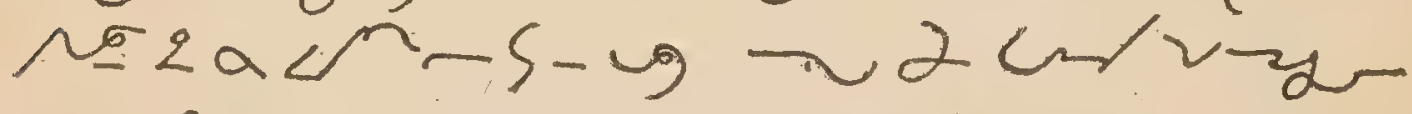
$a-l$.

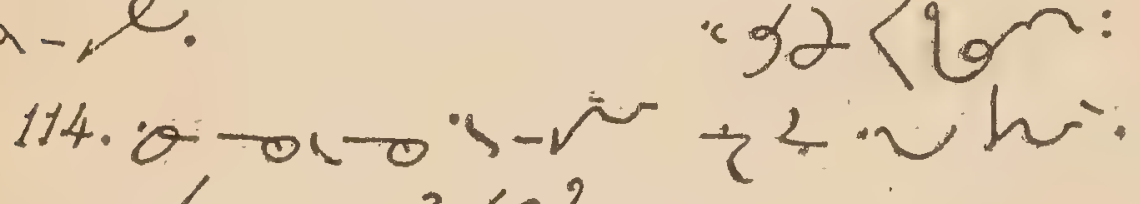
$\sim c \mid f k-\sqrt{\gamma} C^{?}=43$ 
38

ENGLISH

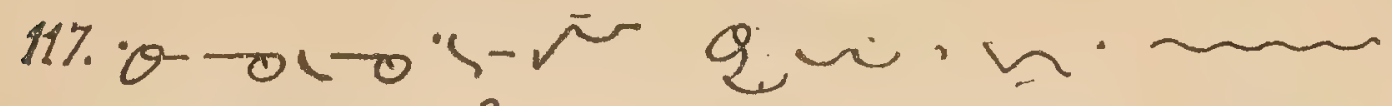

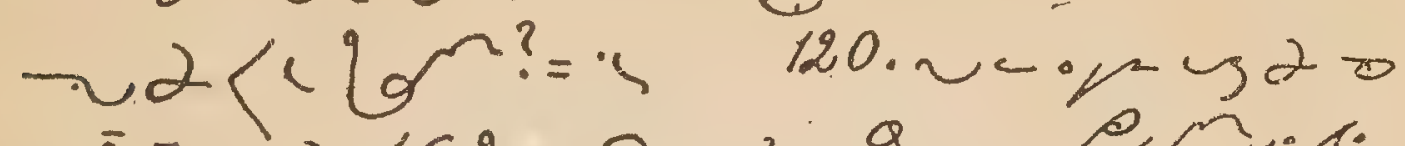

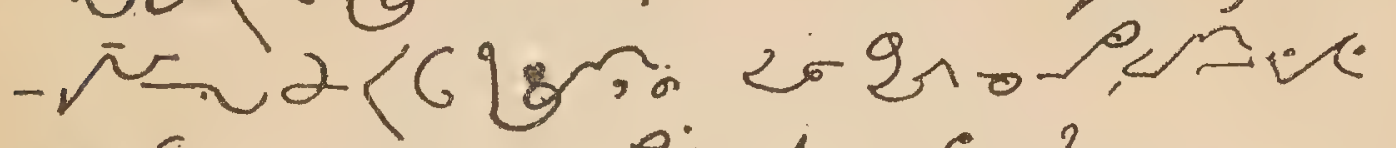

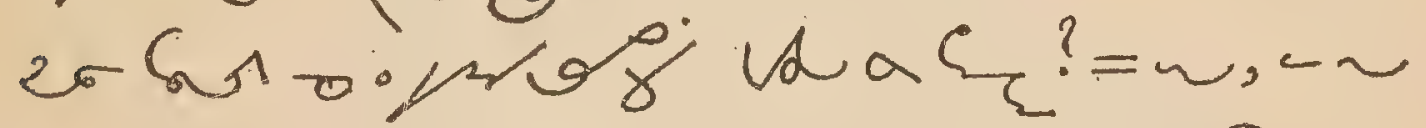

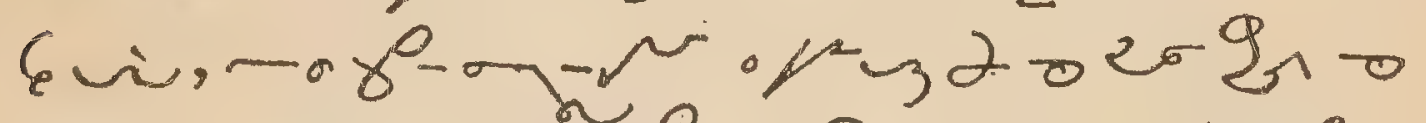

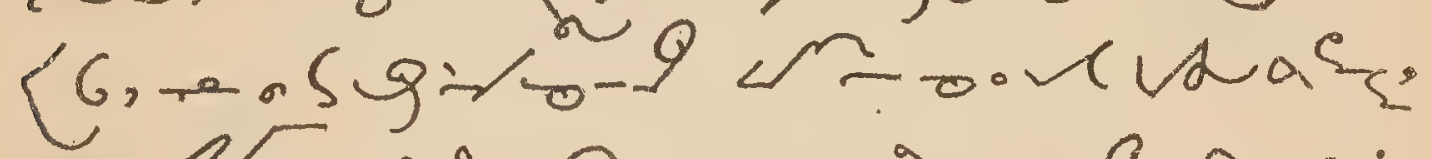

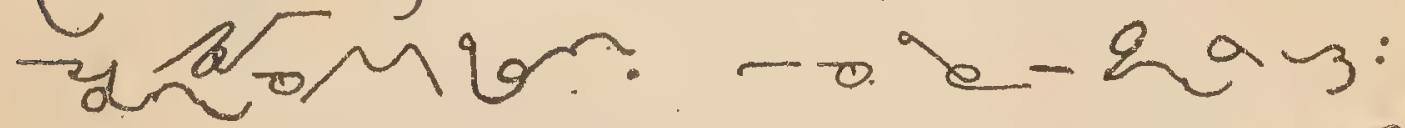

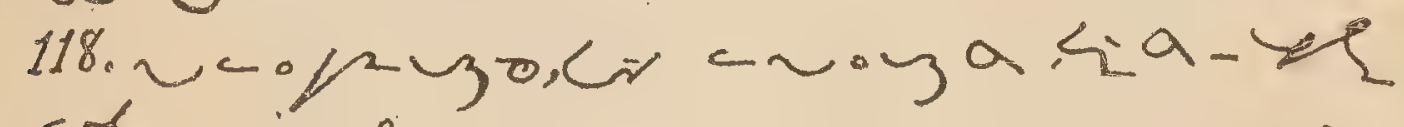

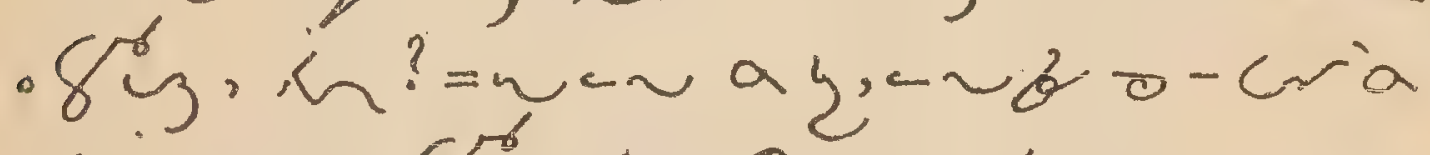

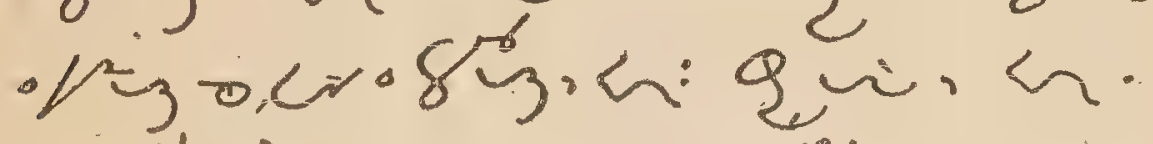

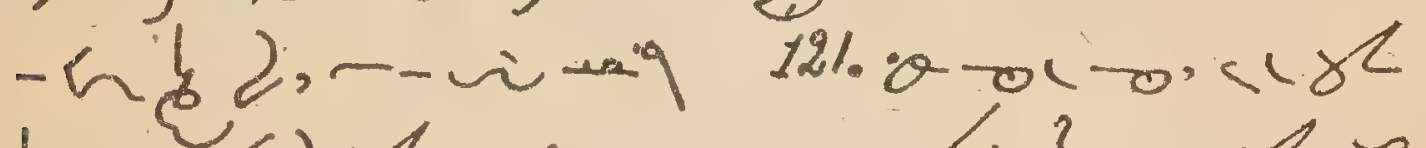

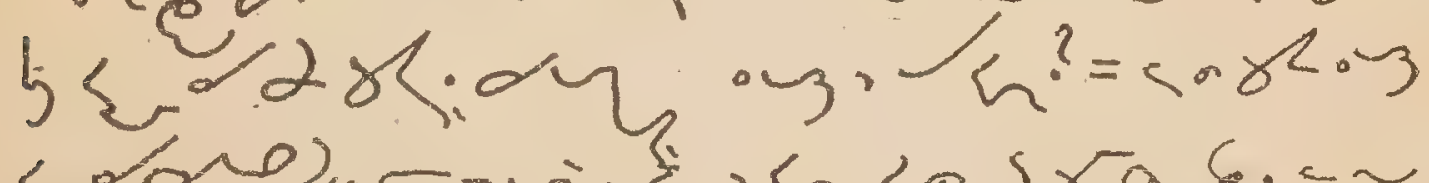

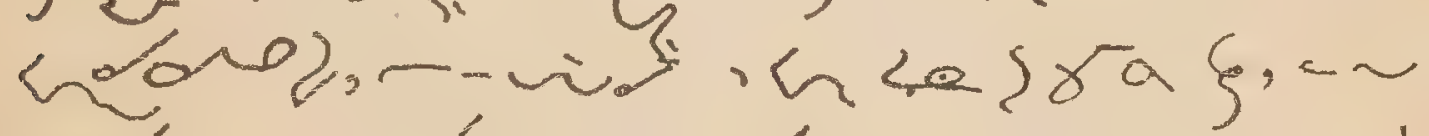

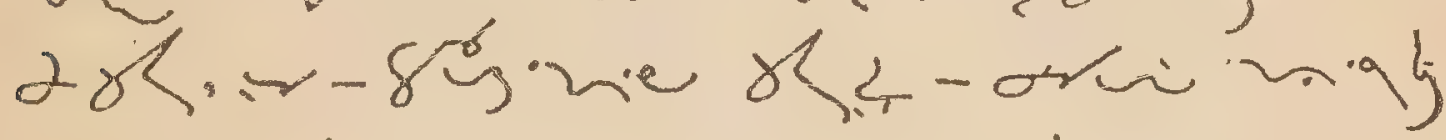

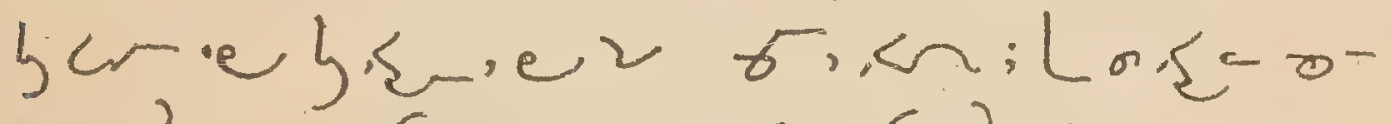
e-dakizus wiken.

a cin."nhal 122.e-iso o

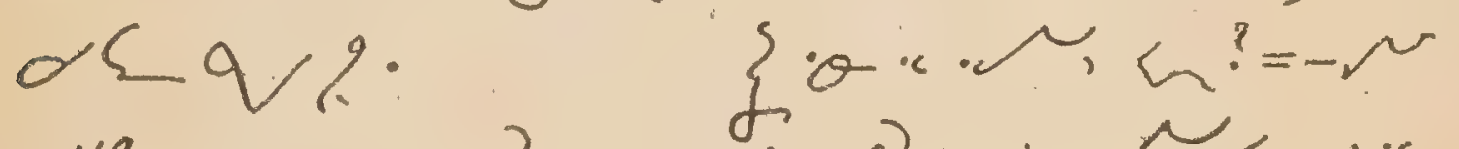

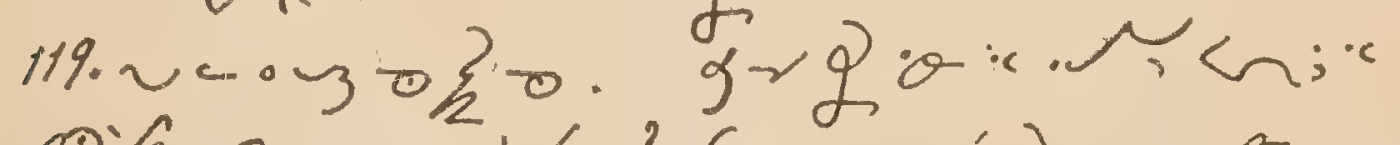

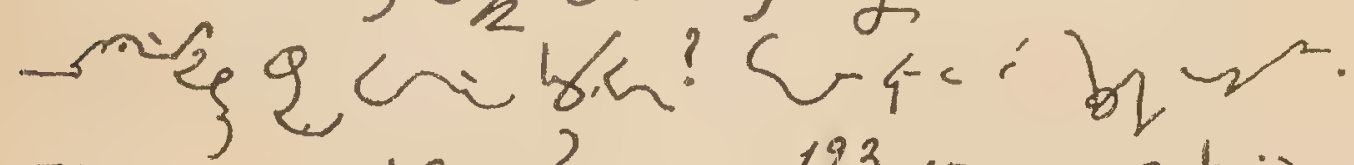

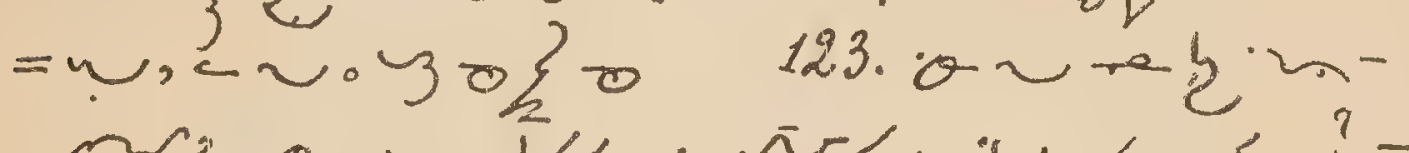
-

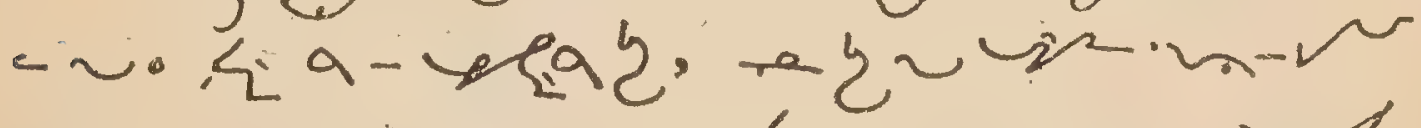

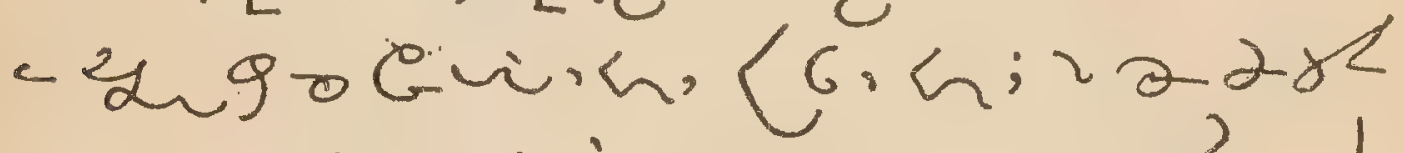

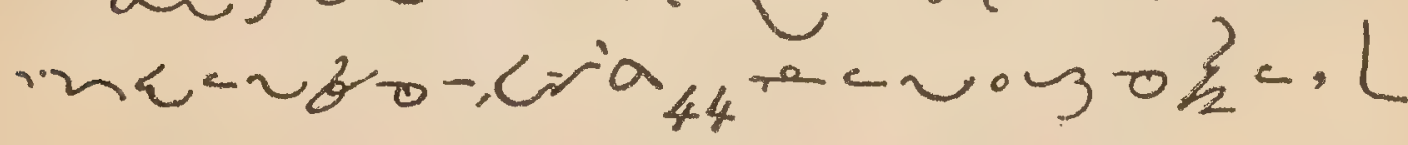


CATECHISM.

39

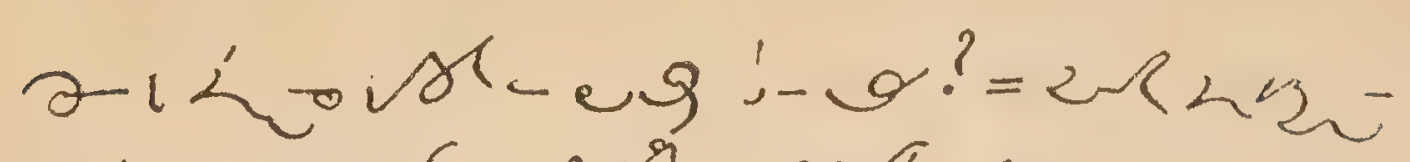

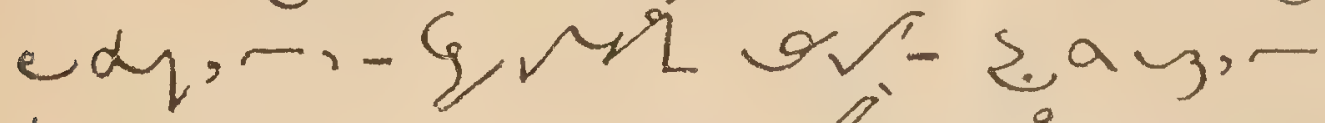
$d-\sqrt{2}$. 位-i-e - ere

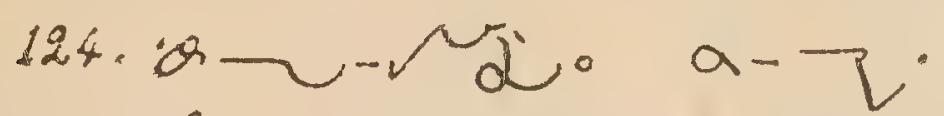

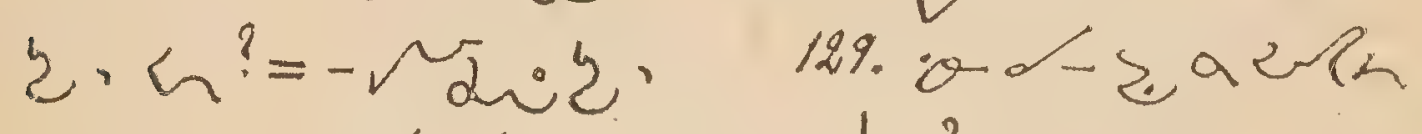

viergroli: $\alpha,-\alpha ?=c$ or $-3 a$

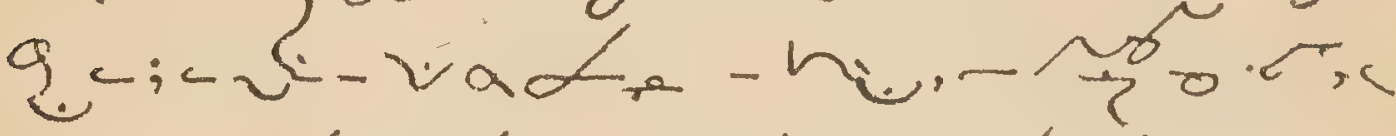

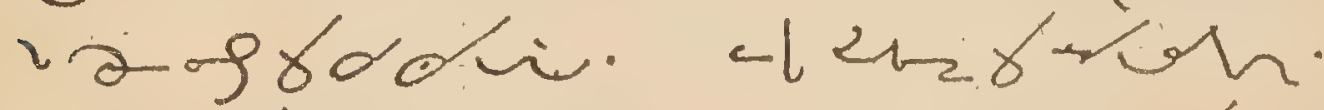

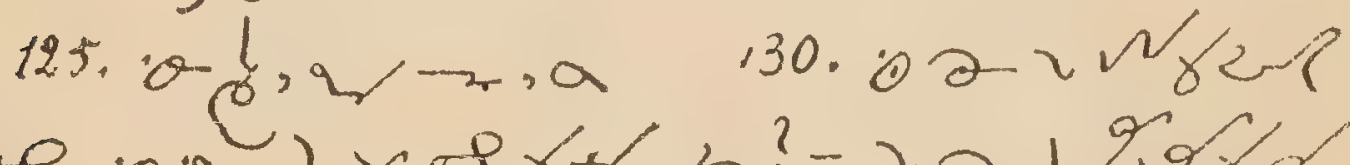

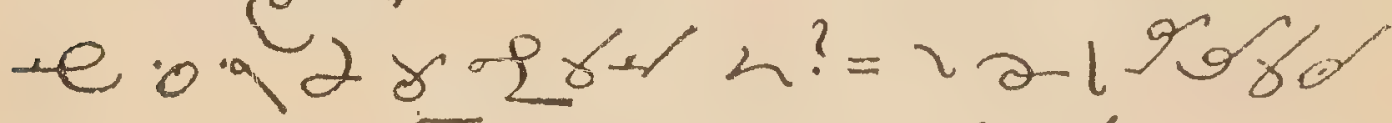

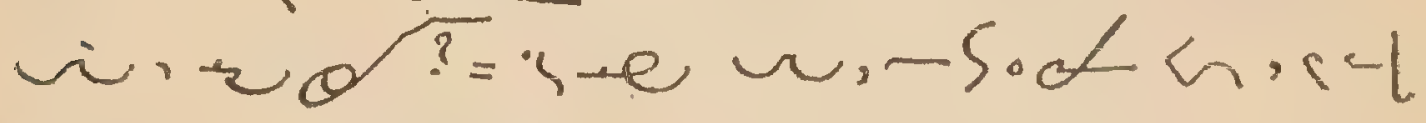
ordogrtiv $d$.

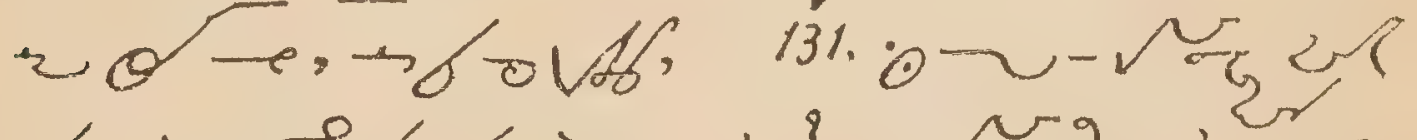

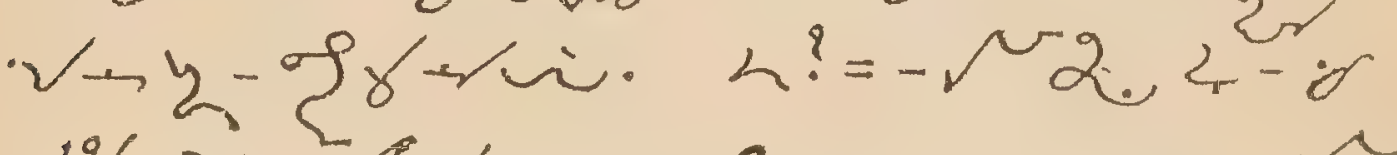

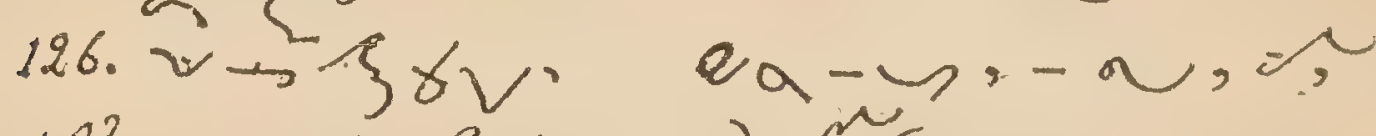
$14 f^{\circ}=-2 \alpha \xi \gamma \quad 2, \sigma_{0}, \ldots-v$

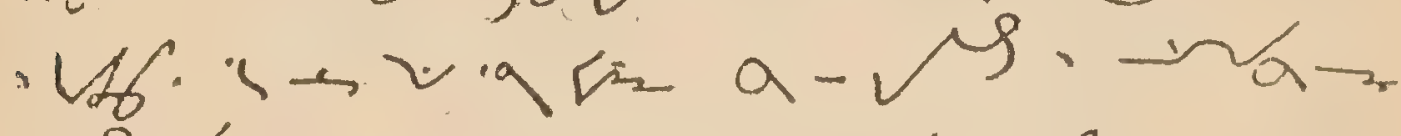

$\rightarrow\{\gamma+v i, \rightarrow \sigma$ erdokipi

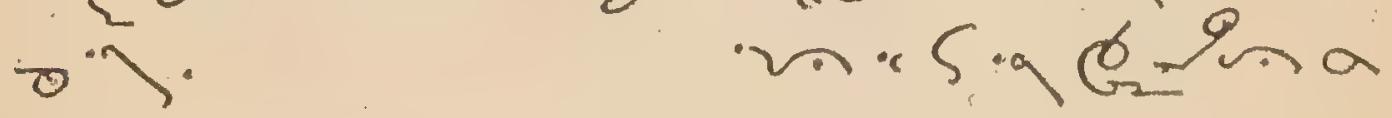
xir. Extreme Unction. e in.

19\%. oचयर八? = xill. Holy Orders.

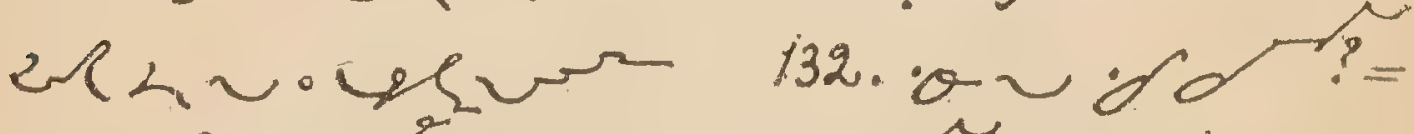

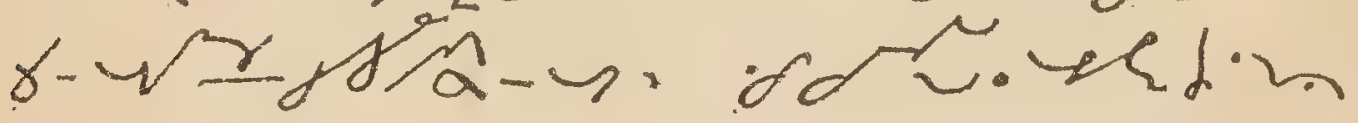
inam. ridz, riv

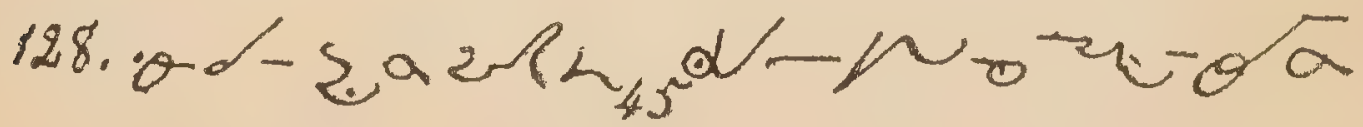


ortariser.

133. '

\&d $r=i x w$

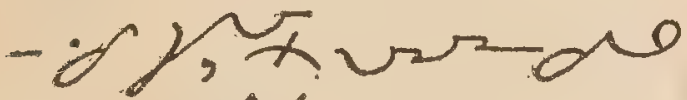

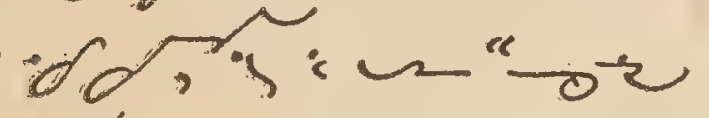

- Smac."

$8134.0 .4 \sim$.era

dor $r=-\alpha-h$

ot - tradory.

135. $0 \sim{ }^{\circ} \mathrm{g} \cdot \mathrm{c}$ ?

"riceraron

do- ${ }^{\circ} a-b a ;+$

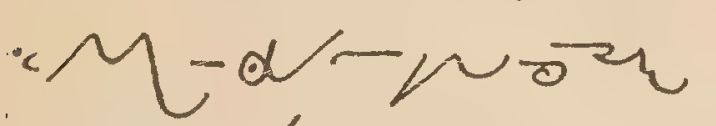

$-\theta$ ar -2

- er

XVI. Matrimony.

136. $\theta \sim 6 \mathrm{G}$ ? $=\mathrm{Gg}$

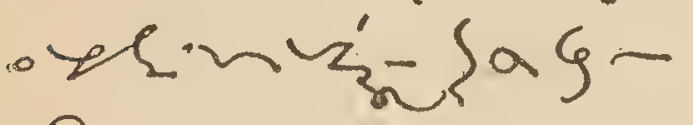

乌.

137. $\theta d \rightarrow 2 a-y$

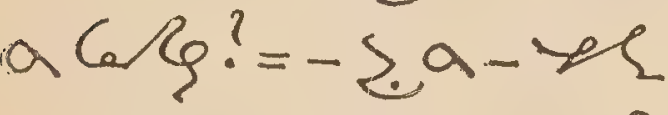

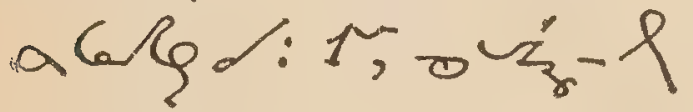

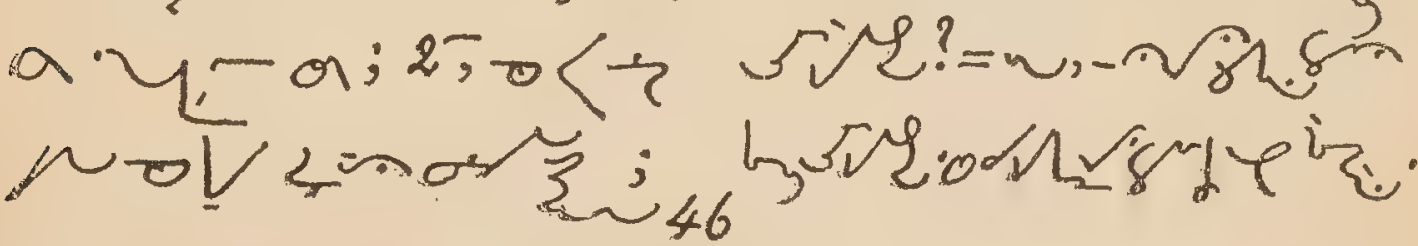

3, o $\{$ o o

138.0 क 24 -

'dosoct $8 m ?=$

or $\sqrt{ }$ oo od

P.corarar $+\sqrt{2}$

139. $0 \curvearrowright \sqrt{2} \cdot+0$

s in? $=+\infty$

$c r-v a d, r o$

vig 2 a 3$\}^{2}$

140. $\theta \sim \alpha \gamma$ L 0 ive or

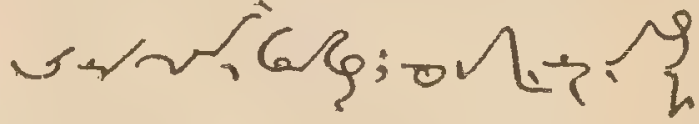
$\sqrt{6}-8 \rightarrow 2-10$ $082 / 8: 2+w$

141. ' $8,0 \gamma \rightarrow-0 ?=\rightarrow(-2$

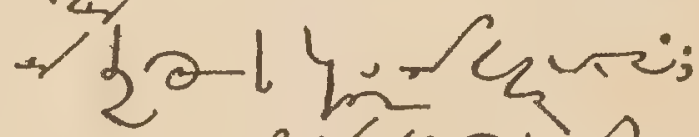
$\rightarrow \sigma-N^{2} 8 \mathrm{~cm}$

$\alpha+N-\alpha^{2}$. 142. - $\sqrt{2}<5$

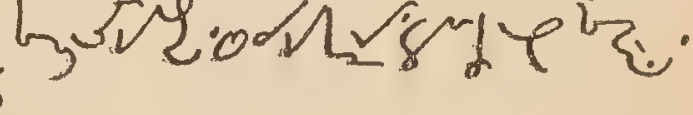

- $-1-\rho a \alpha$. 1thired $8-16-\sqrt{5}$;

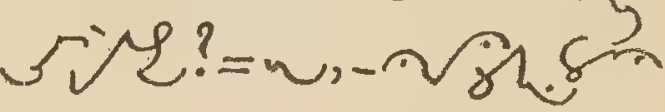






\title{
CHINOOK MANUAL
}

OR

\section{Prayers, Hymns and Catechism}

\author{
IN CHINOOK
}

WITH THE APPROBATION OF

RIGHT REV, P. DURIEN, D.D., O.M.I,

Bishop of New Westminster.

KAMLOOPS, B.C.

I 896. 

Chinook Manual.

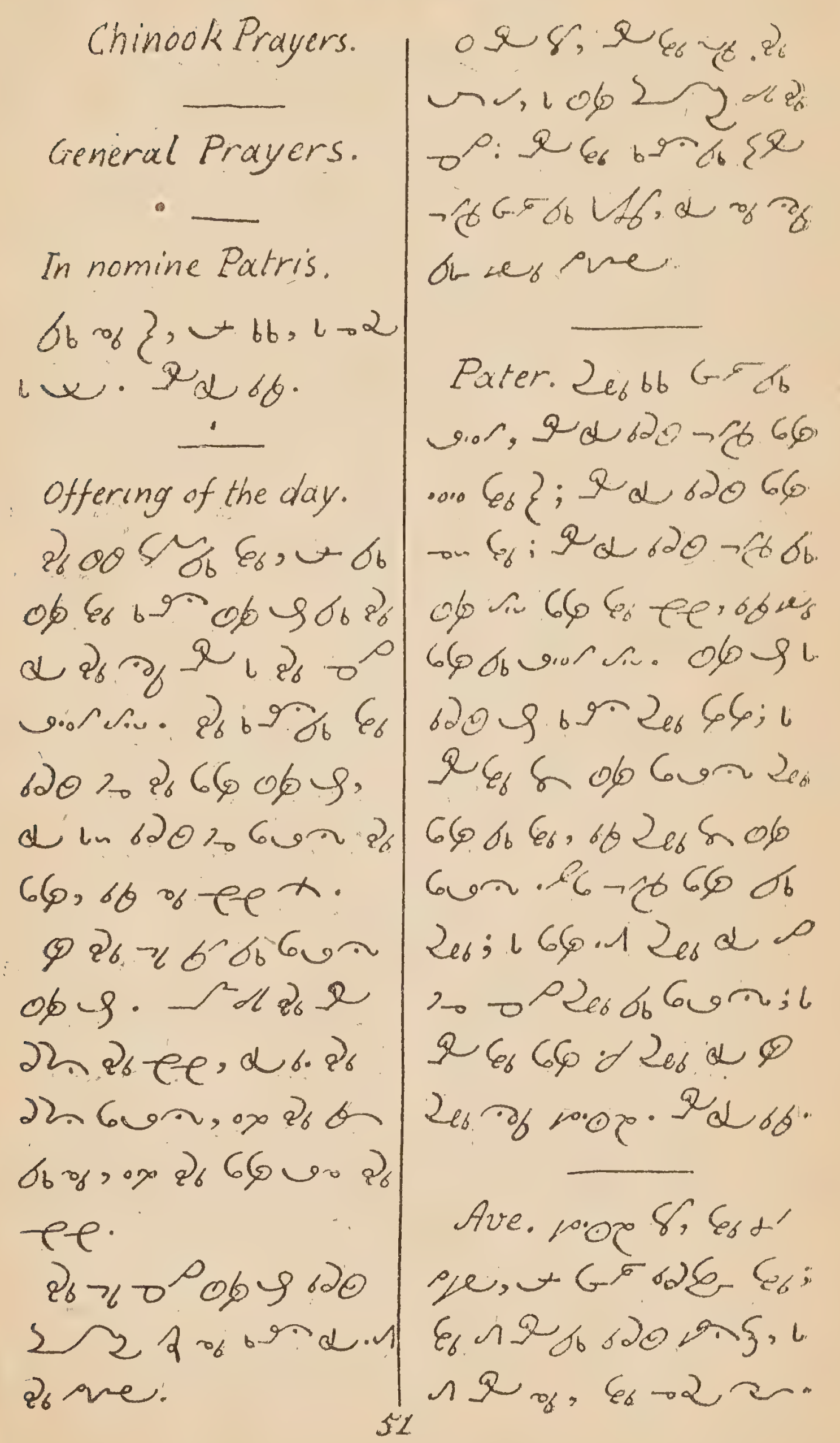




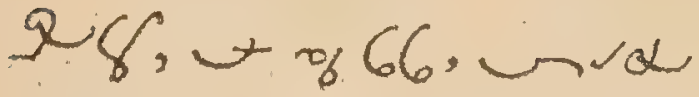

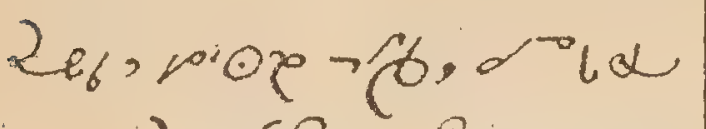

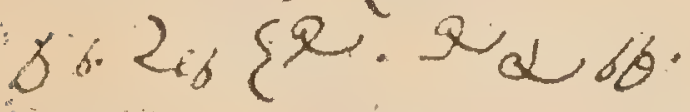

Credo. 2e6 600207 $\checkmark 66,566662010 ; 0$

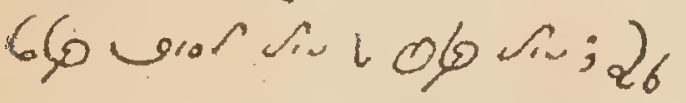
$66008 x, 6$. - क रeson; सw 660 alig

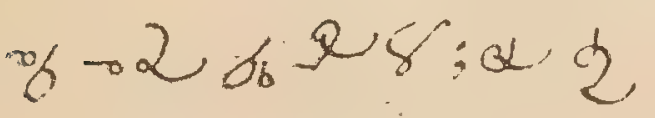
vo of ting $x_{6} 660$ ore

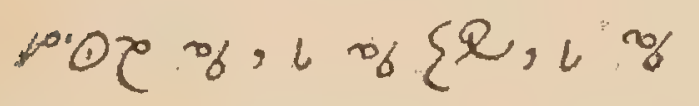

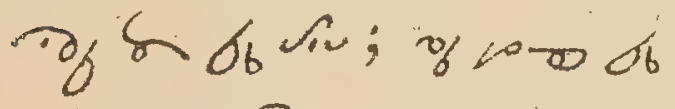
ib , l. $9 y$ \% $\angle a \sigma_{b}$

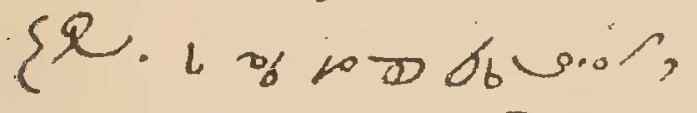

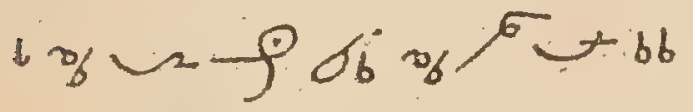

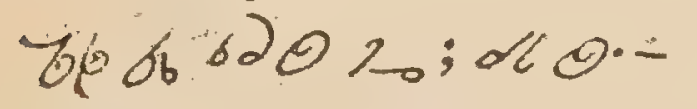

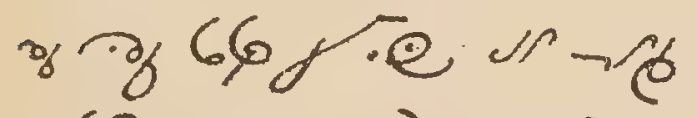

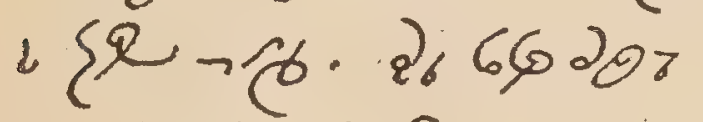

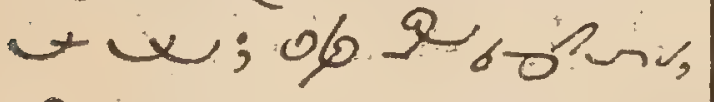

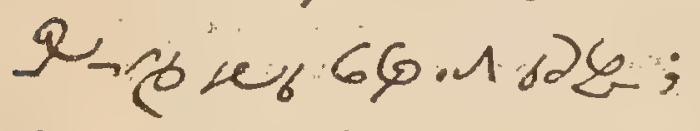
6थ कु

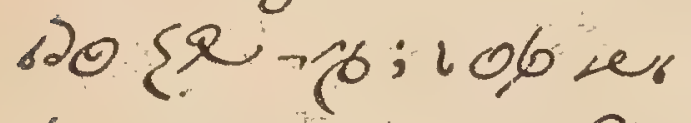
$\angle 9.62\} \alpha G \%$. a) 18 .
Confiteor. $8_{6} 0.006 \mathrm{pec}$ $\sigma_{6}+b_{6} 66020 \% \sigma_{6}$

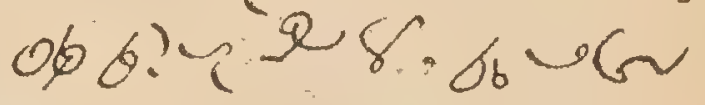
ox in, 不 3 के $\sigma_{6} \mathrm{H} / \mathrm{y} / \mathrm{z} \gamma, \sigma_{b}$ $620 x-{ }_{0} G 506$ war su, $166 \varepsilon_{6} 2_{6} \quad b 6, \alpha z_{6}$ 66 Gion, ob as ep.

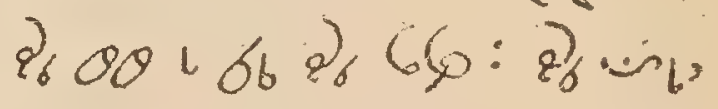

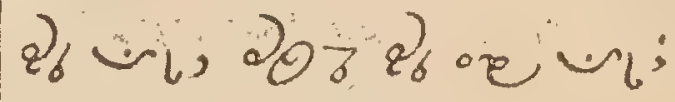
$66,2600060082 y^{2}$ $8.6 b \mathrm{c}$ are Nob

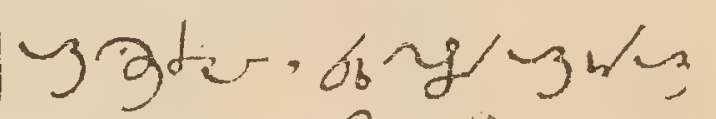

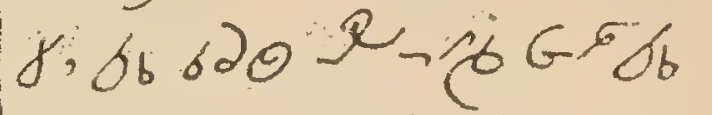

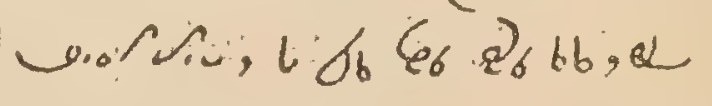
Lenutal Qे $_{6}$.

Misereatur. \&og66 por 2e6, a $w 606$ $120>, x_{0}$ siolo

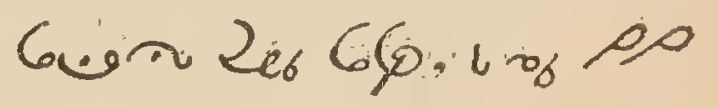

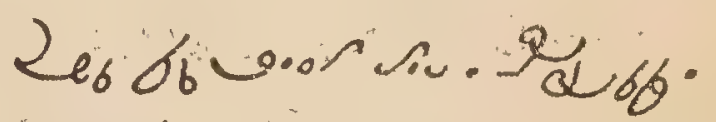
Indulgentixm. Q 66 mor $206,2 \%$ lo 60020600 , $520+0606620 \%$. 
MANUAL.

47

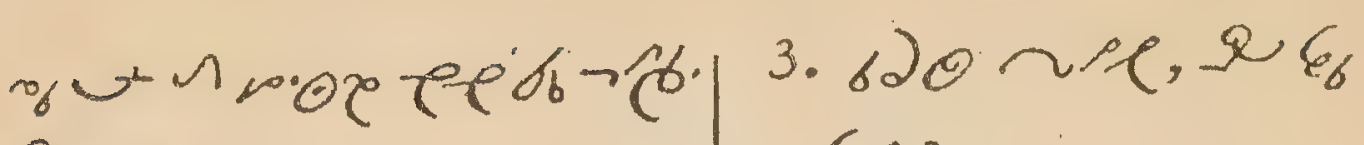
sec 68 . .over.

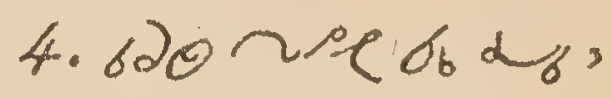

The ten Commandments.

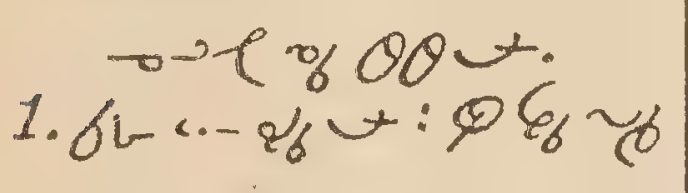
a $\varepsilon_{b} \sim \theta$.

s. ob rot os rife. a $6_{6} 6600 \varepsilon_{6}+9 \%$. To a $660 \mathrm{~s} g$.

6.6669996665

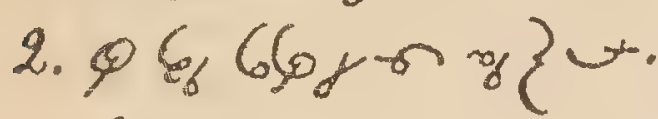

3. $2 \pi y$.

4. 922268 it 16866 .

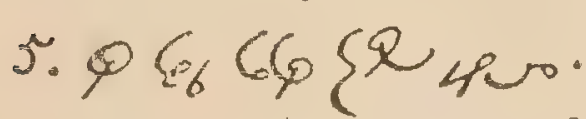

600.

6.96660602626

9.5 .

$7 . \phi \varepsilon_{6} \alpha \sigma^{\circ} 10$.

8. $9 \xi_{b}$ \}on.

9. 96.660 eld 660 wan bas-gns.

Seven Sacraments.

10. $\theta \varepsilon_{6} 66$ fed $\varepsilon_{6} \mathrm{~L}=203 \mathrm{G}$.

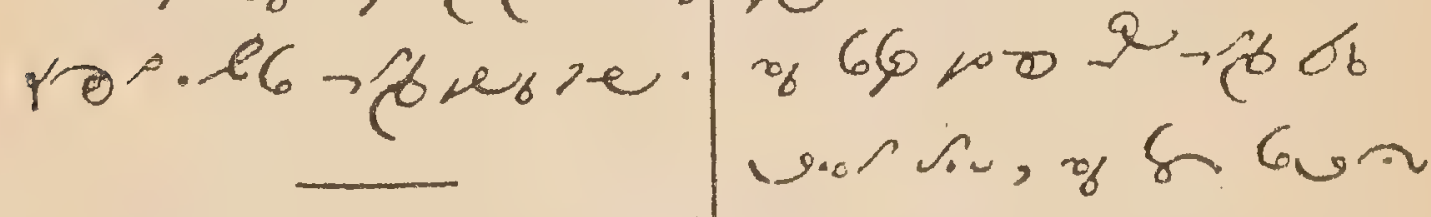

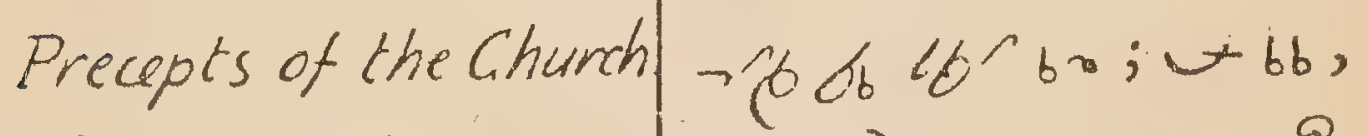

- of oOh

1. 222 are 986

\&.

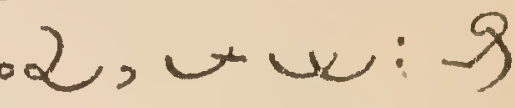

$\varphi_{u_{b}}$, ib law;

$\rightarrow-2 \%$ क $606 \%$

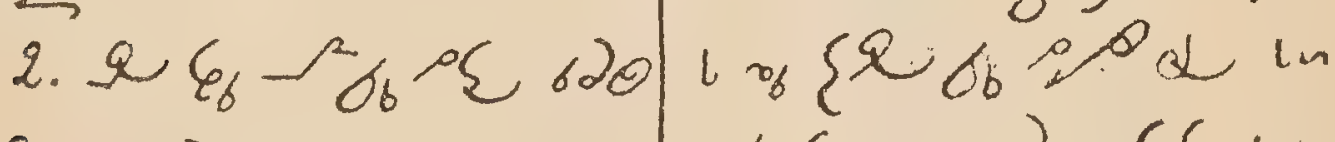

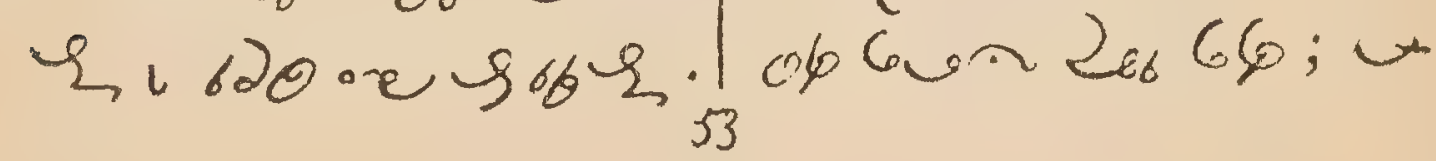


MANUAL.

$49^{\circ}$

$066,+\% 66,66$ m After meals.

$\alpha 2_{66}=\alpha 2_{66} \%$

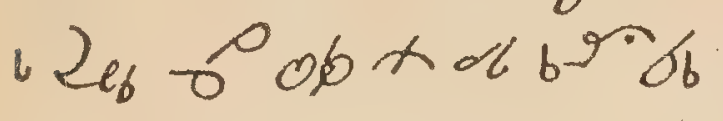

26 .

remus. ow! Q $6_{6}$

ot 1 Q 1 be.

s $\sigma_{b} 6 \partial \theta 1046090$

$\sigma_{6} 2_{6} ; 0 . \sin _{6} 06$.

$66 \varepsilon_{6} \sin \sigma_{6} 2 e_{6} \alpha$

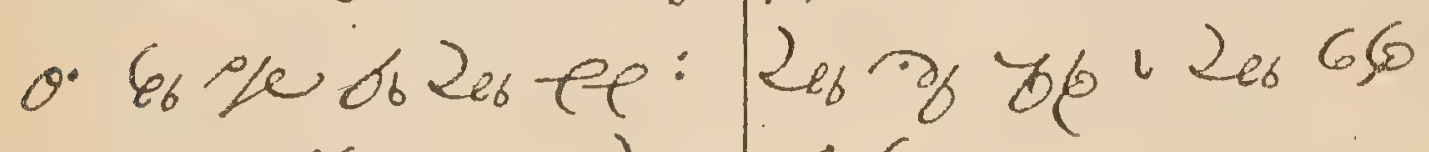

nog 660 be 60 26 $\sim 66.00$.

Log $g \varepsilon_{6}-02 ; x$

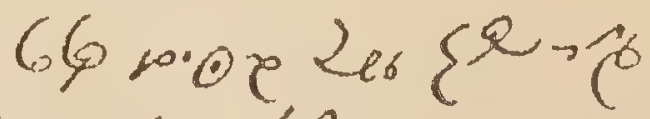

$\varepsilon_{6}$ \% 06 x

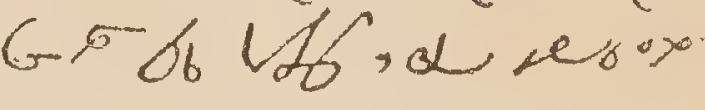

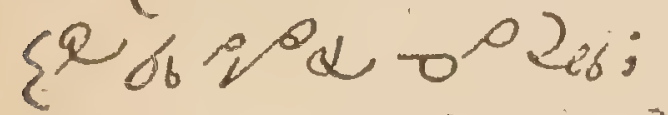

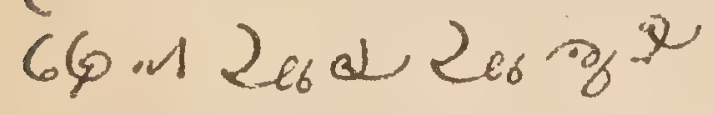

1206000 sion s. =

Q 60

100 b 0.0 NS.

Private Baptism:

N... \& 650 on 6 .

$\sigma_{b}$ of $\{+b 6<-2$

Subtuim. O 8,6

a, $6_{6}$ \% 66$\lrcorner ; 206$

$b^{\prime} b_{6} 6_{6}, 9 \varepsilon_{6}, 22 \mathrm{n}$

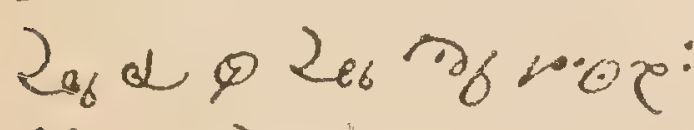

66 d Les br Gun.

Morning Prayers.

+66 of $\$ 66<-2$,

Before meals.

L. Id 68 .

$0+66 \mathrm{~h} 2 \mathrm{l} 6 \mathrm{O}$

Vent Samite. $O+$

0668 on $206 \alpha 26$

$w, g_{0}$ or $\mathrm{Le}_{6}, \alpha$ \%

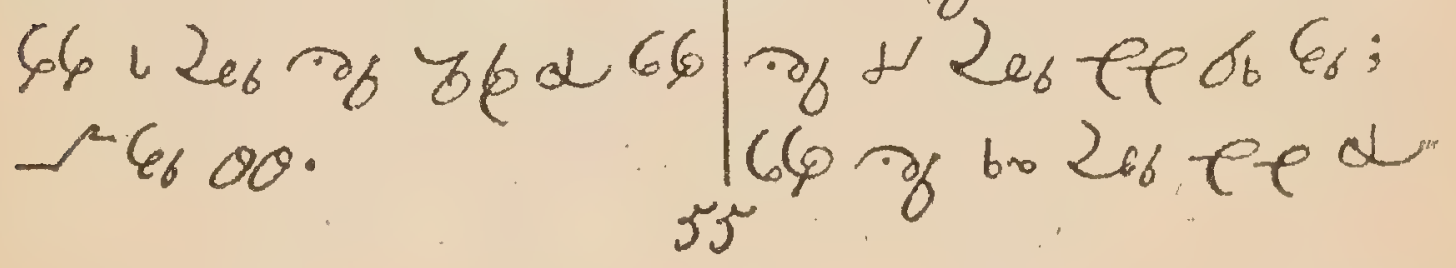


50

CHINOOK

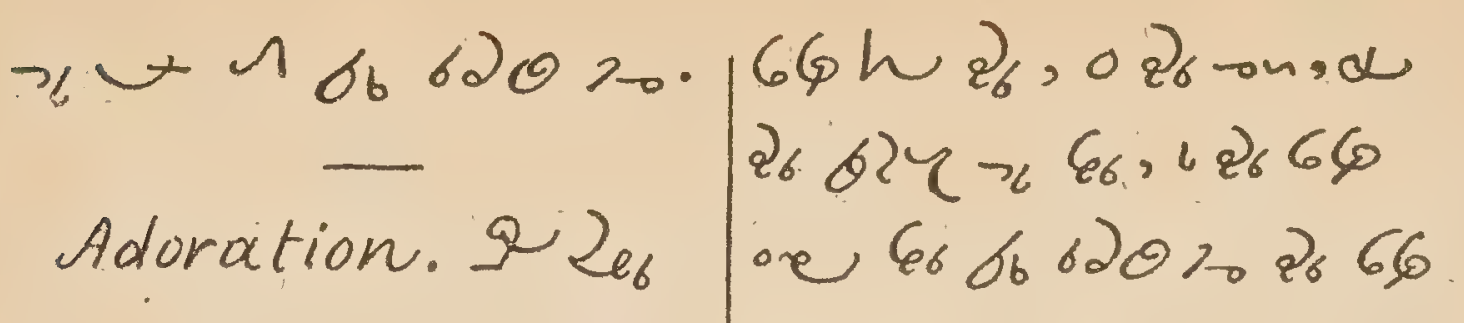

2) $6 b+12 e_{6} 660.0$ of $\varphi$.

r. $=0 \wedge \Omega, \Lambda$ ore

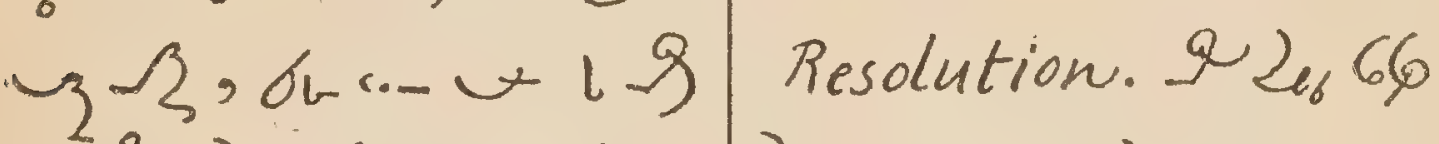

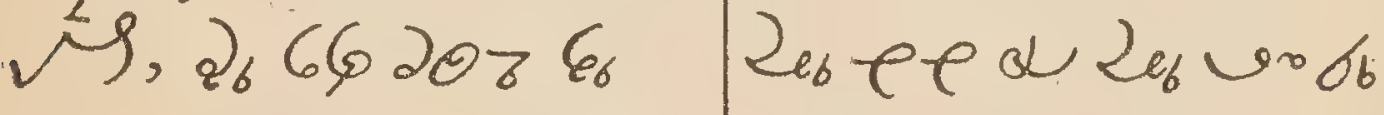

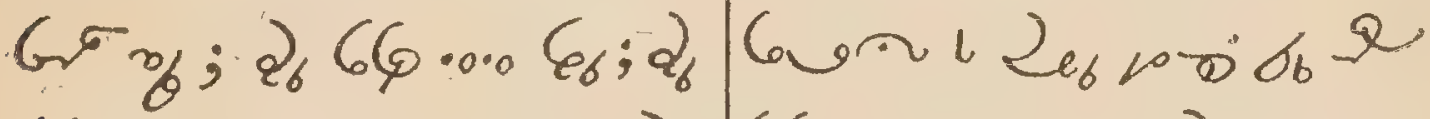

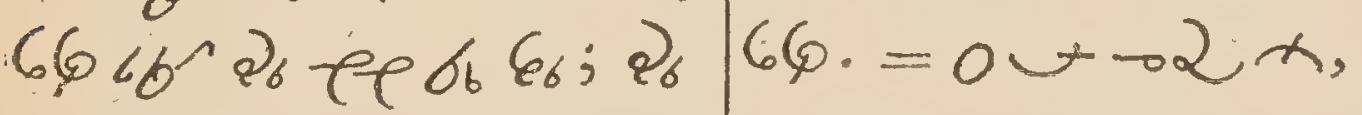

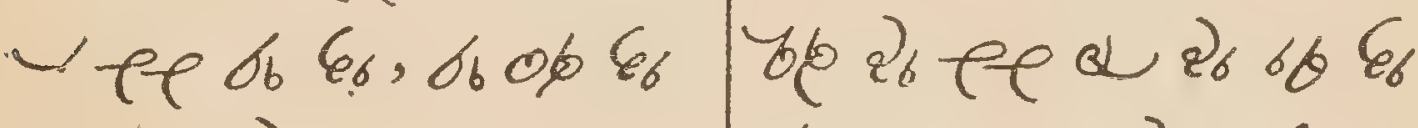

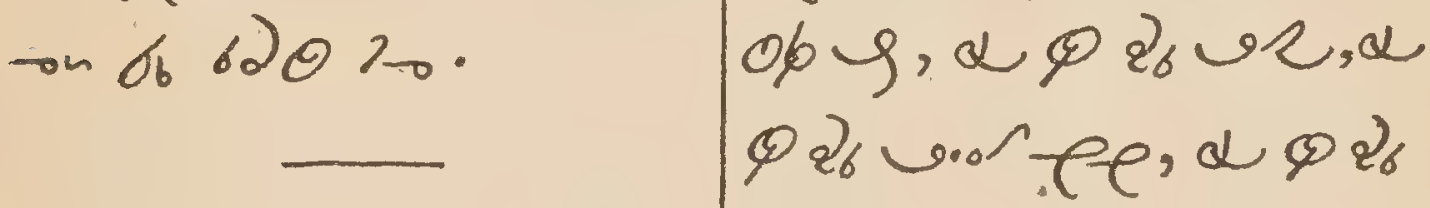

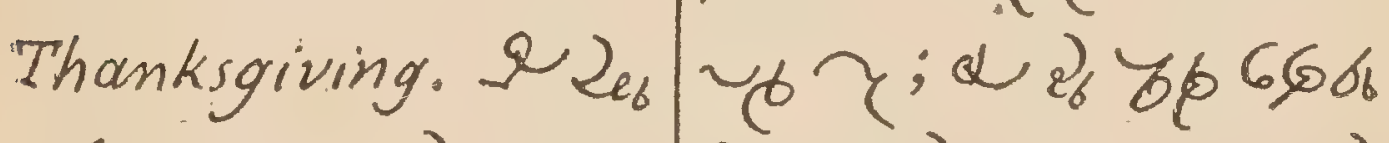

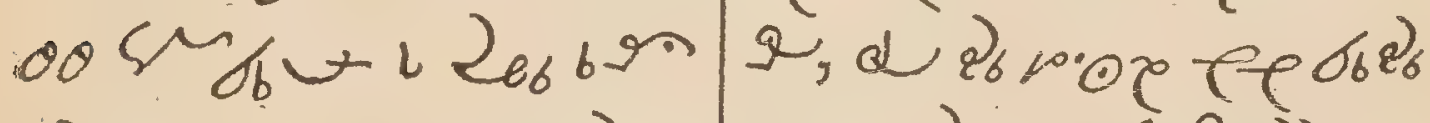

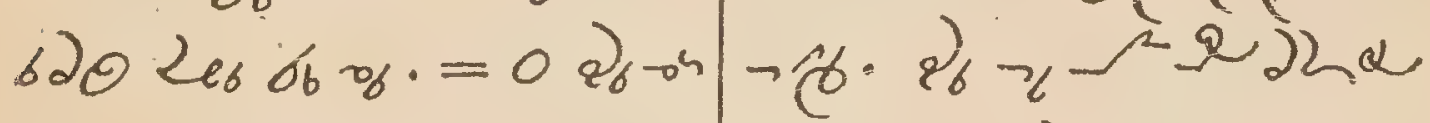

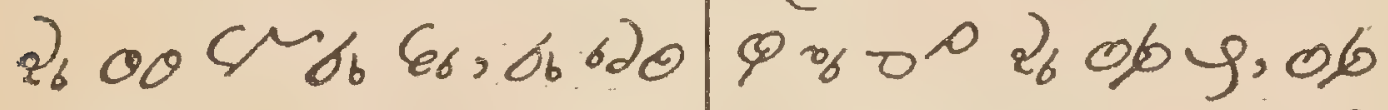

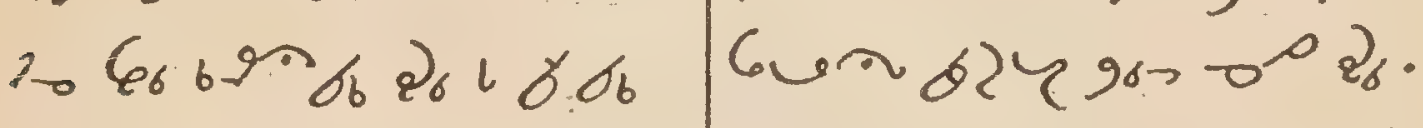

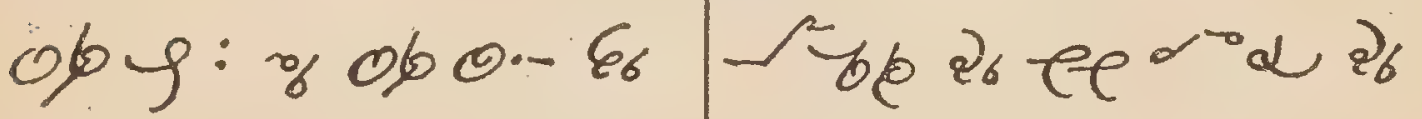

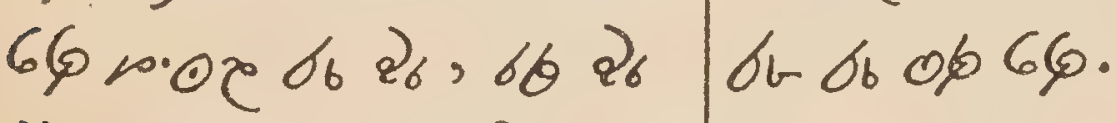

$2 \sin 069: 66$ Qt $266=$

Nob 66. 020 op $9:$ Petition. Q 2 $2_{6} 006 b$

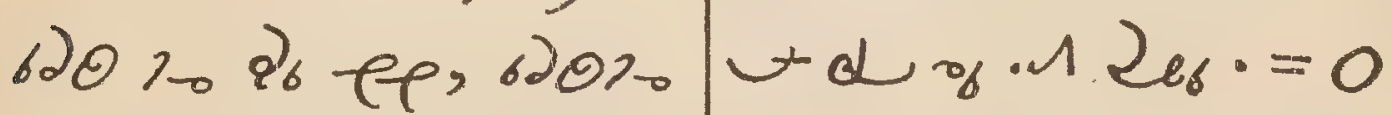

$2_{6} 00,620102_{6} 66, q_{6}$ on, 66220086

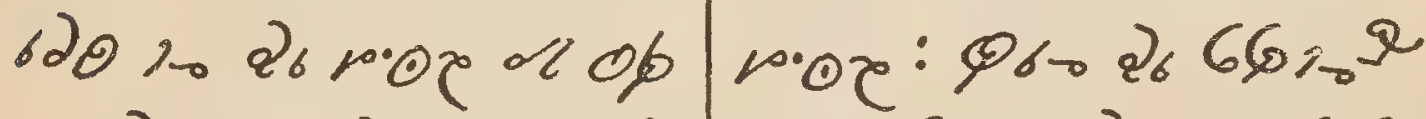

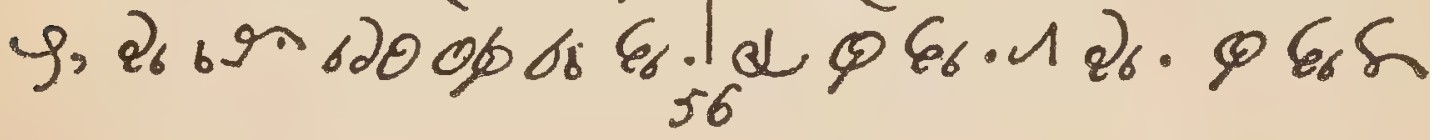


MANUAL.

$5 \mathrm{I}$

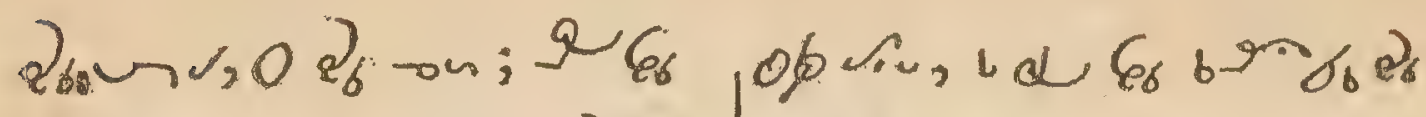

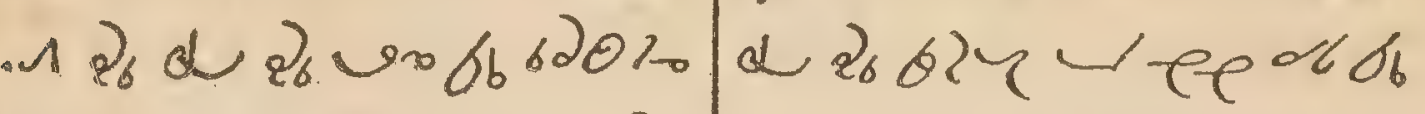

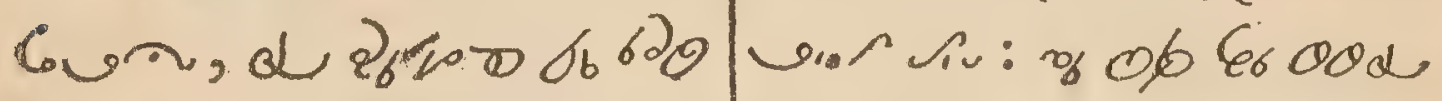

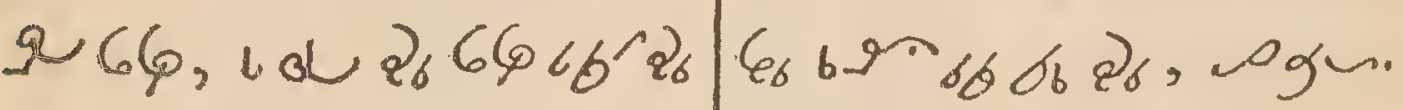

teb 600 . op 9 , d. 66 2 6 in 010 Guत शे 66 . $\varepsilon_{6}$ oror $\varepsilon_{6}<e$.

Love. 22er $\rightarrow$ ज.

$$
=0 w, Q_{\sigma} \rightarrow \varepsilon_{6} \sigma_{b} \sigma \partial \theta
$$

2 ee, $1 \sigma_{b} \sigma \partial \Omega_{0}, r_{0}$

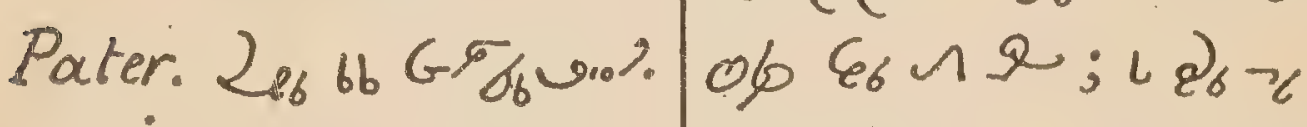

Ave: pore 8 .

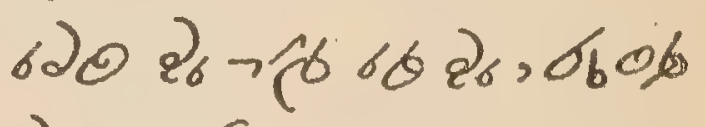

Credo. 2866208.

$2_{6} \rightarrow \varepsilon_{6}$.

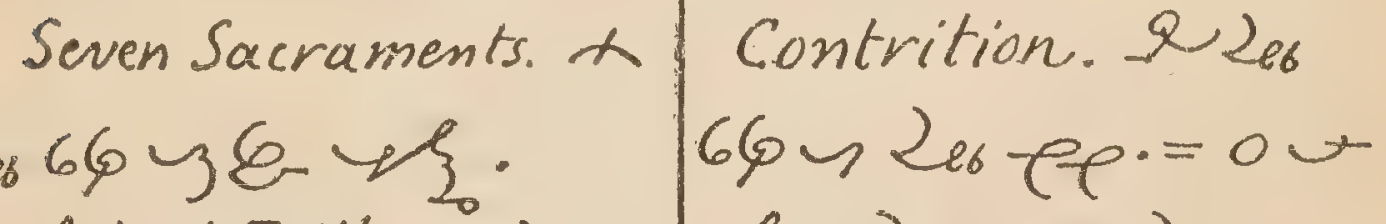

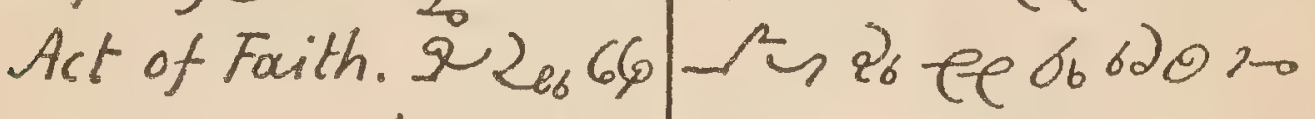

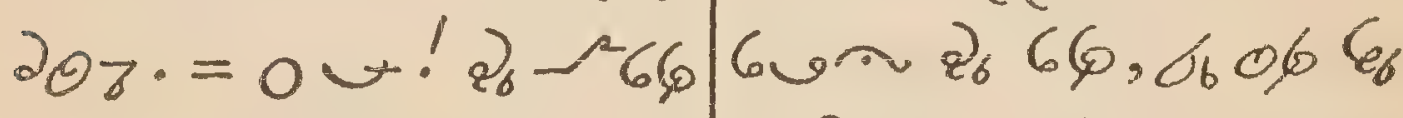

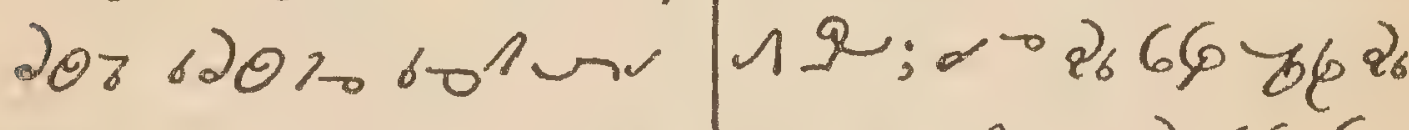

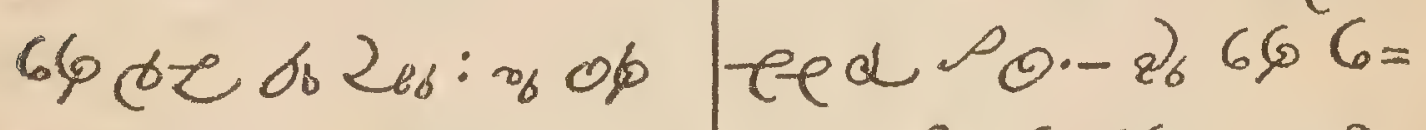

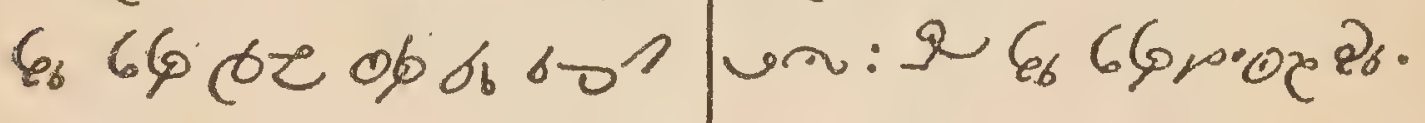

जu, b2\% o $\varepsilon_{8}$ oo.

Hope. Q 2es 66 be Invocation. To the B. Virgin.

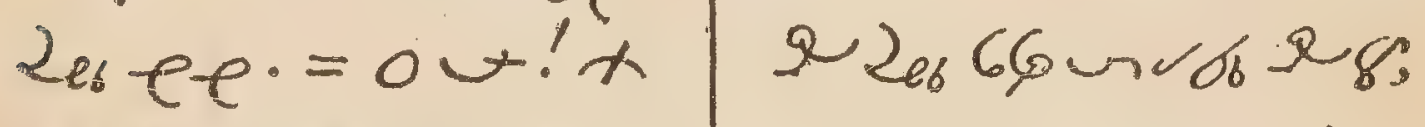

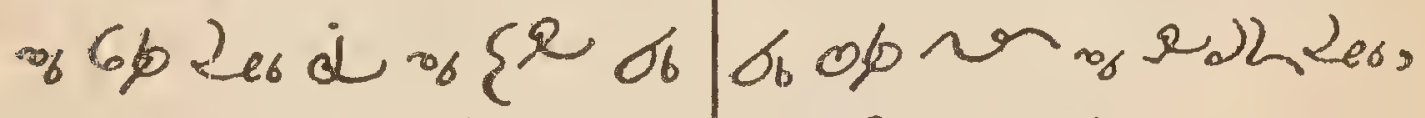

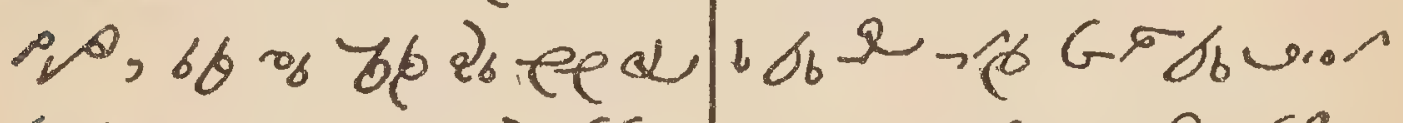

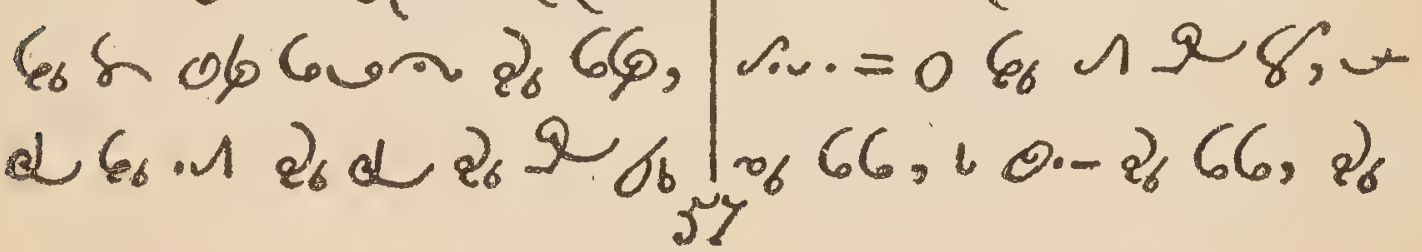


52

CHINOOK

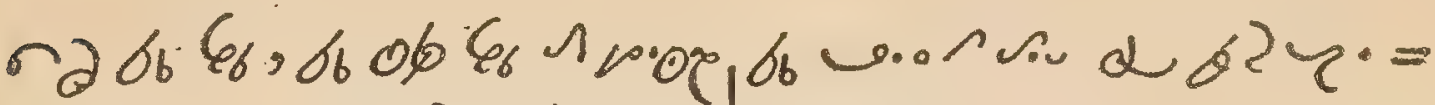

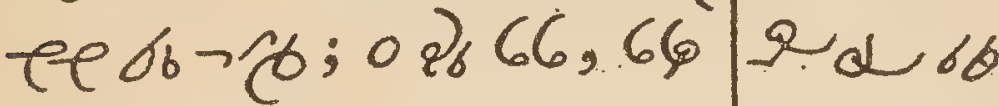
rov $2_{6}, a_{6}$ oreror; $2_{6} \cdot$ a ee, 60 b6 $2_{6}$ ee; $660 \sim 7 \alpha$ d 86 66 6. or $\Omega-2, d=0$ $\varphi, 8 b 620 \varphi d l \theta_{6} G=$ $6606 \Omega, 100660026$ \{x.

To the Guardian Angel. 066 ore d n 6866

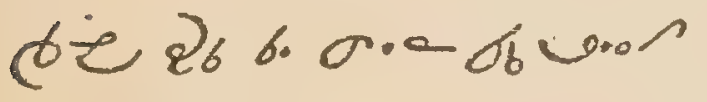

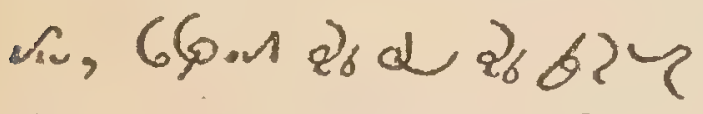

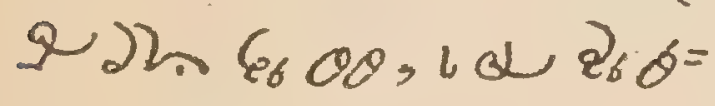
$2 y 22 \pi 10266506$

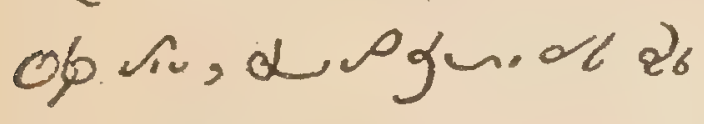
Sito 00 .

To the Patron Saint.

- keb eato Gob

worsin, $66.1 q_{6} ; 1 \varepsilon_{6}$

The Angelus, wer.

Sub tuum. o 8 .

Night Prayers.

$+ \sigma _ { b } 0 \longdiv { c _ { c } }$

Veni Sancte. o

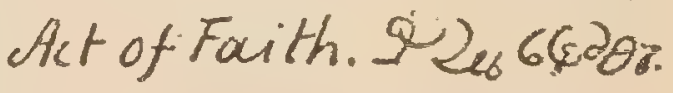

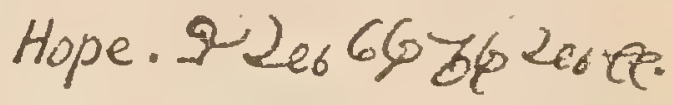

Tove. Q \& 2ab 2 \&.

contrition. Q 2es60

$y$ Leb ee.

Adoration. Q 2 tera

b6+1206660\%\%. =

ow, $2_{6} 65000068,2_{6}$.

$66060^{\circ} a_{6}$ ee $\sigma_{6} \varepsilon_{6}, 2_{6}$

$e^{2 \pi \varepsilon_{6}}\left(\sigma_{00}, \varepsilon_{6}\right.$

ore on $\varepsilon_{6} b$ on $\left.\varepsilon_{6}\right\} b_{6} q_{6}$

Q $\left.a_{6}\right\}, 60 u-b_{b} w$

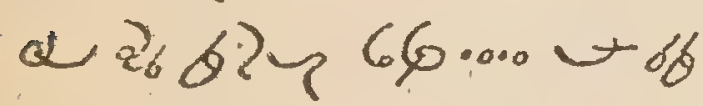

norew; $\varepsilon_{6} 660208$

68,0606 \% $624{ }^{2} 6_{8}$

$66.669976606 \pi, \alpha$

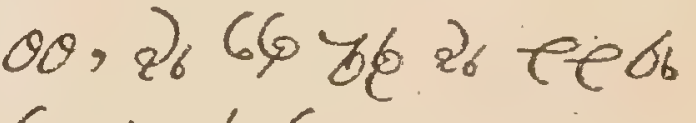

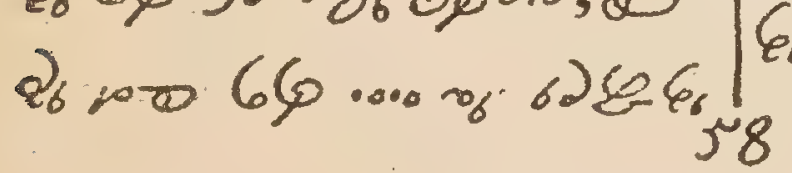

6r b6 ob 66 orenope 
MANUAL.

53

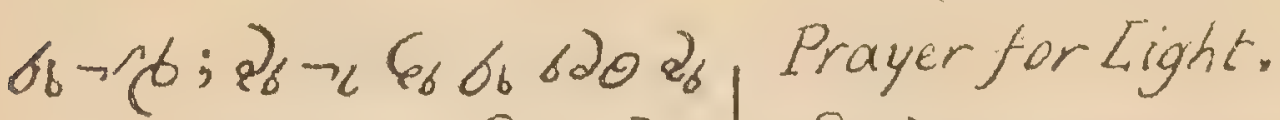

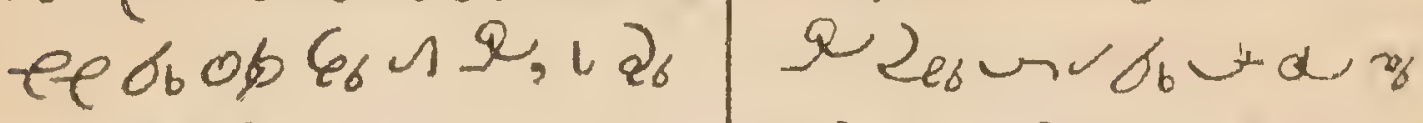

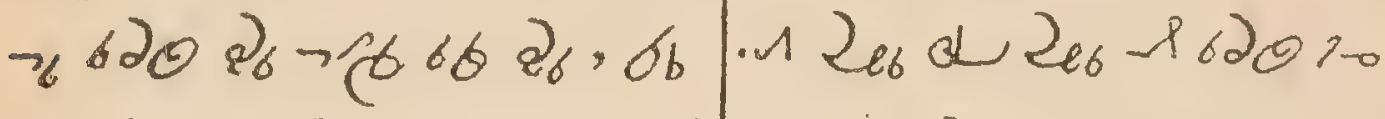

06 qu $^{2} \cdot 68$.

6on 2 e6 $66006 .=$

Thanksgiving. Q2e6

oww, 6860 Fis

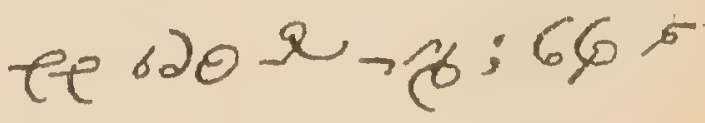

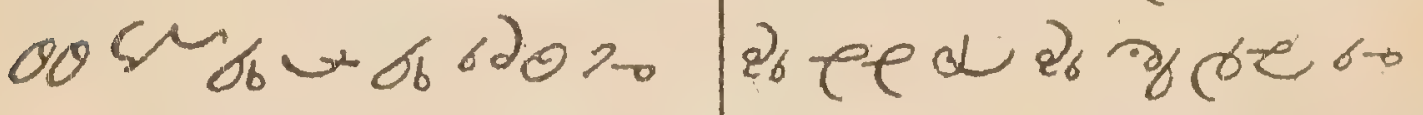

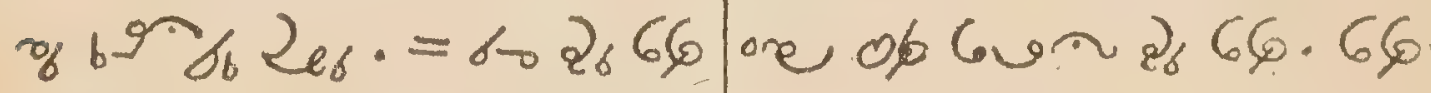

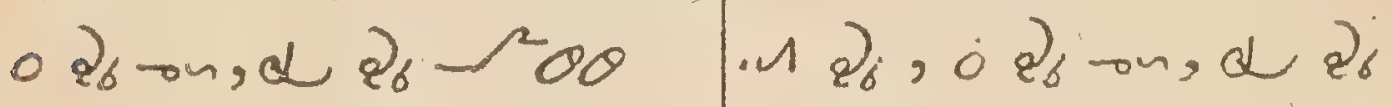

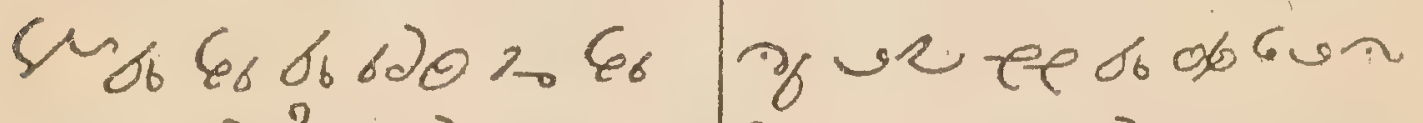

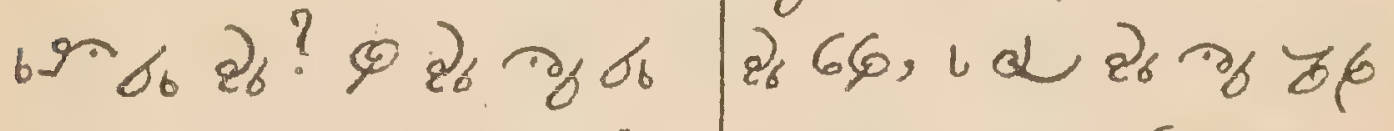

$0,0 \wedge, 4624956$

eealom-6ur o

ee or qे: $\varepsilon_{6} 660$ qे, 68

Po ob of $x$.

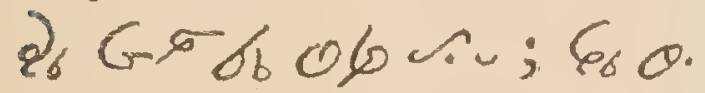

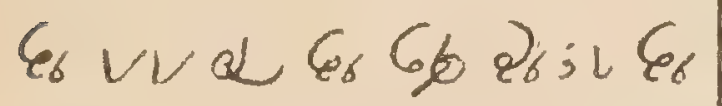

Examen. $22_{66} 66$

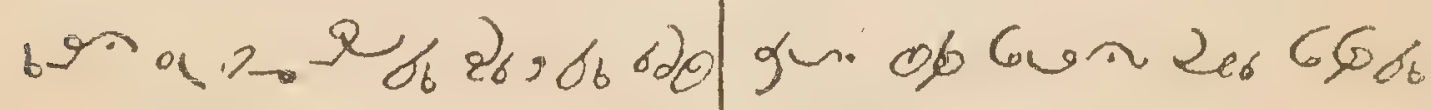

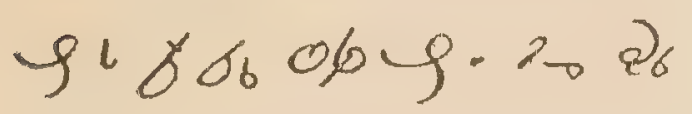
$0,9 \ldots . .$.

$66,028 \rightarrow \pi$ in 620 .

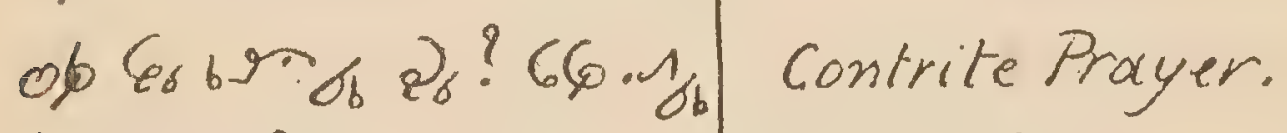

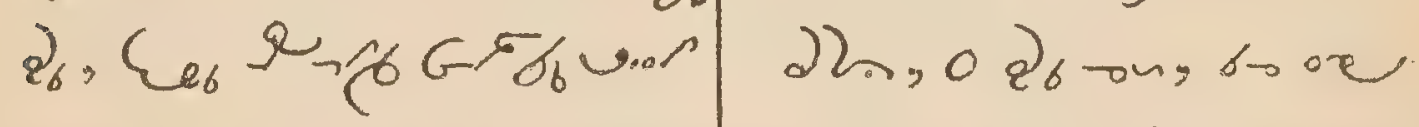

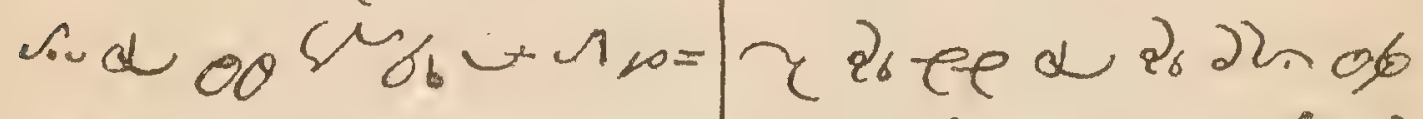

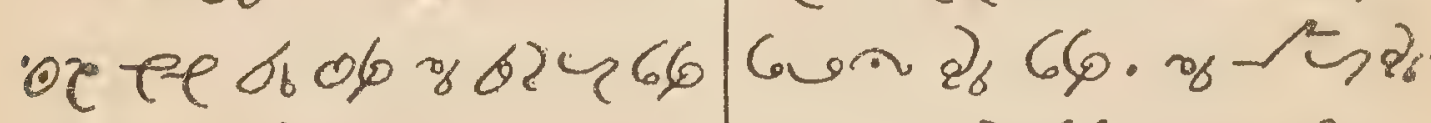

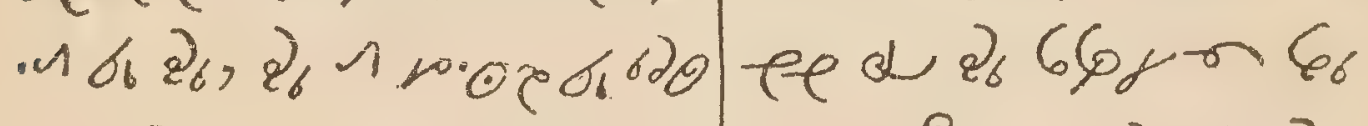

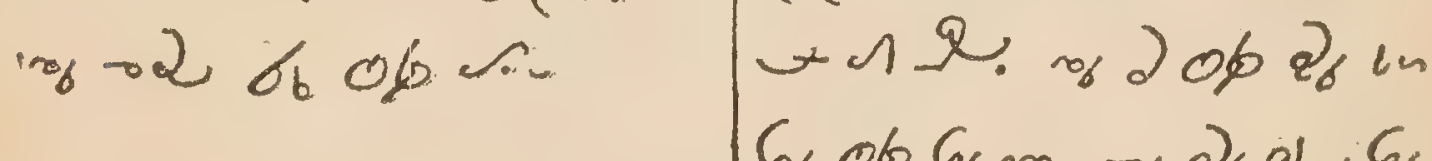

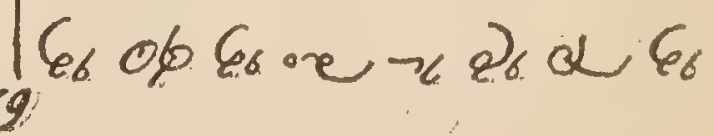


54

CHINOOK

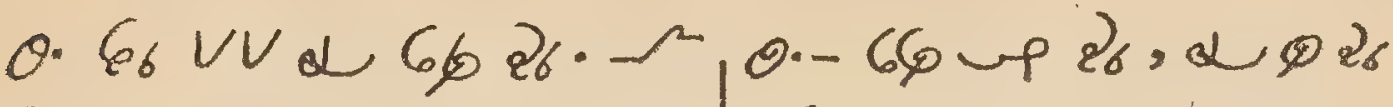

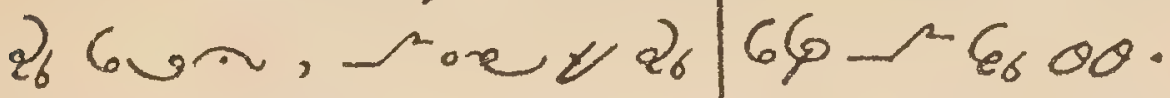

ep. $\log ^{\rightarrow}$ Q $_{6} 66061$

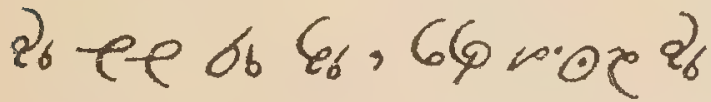

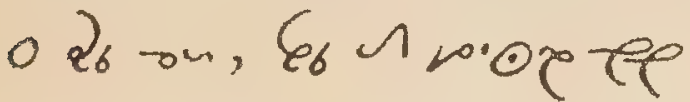

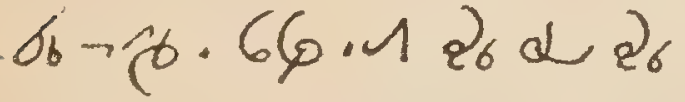
- 660 \& $z_{6}-2 \omega 066=$ ur के 660 .

Confiteor. o.oceeinn Ten Commandments.

tate of 00 w Precepts of the Church. 200 \% 00 . Capital sins. 36

Firm Purpose. 22 26 of 60 a 660:v=

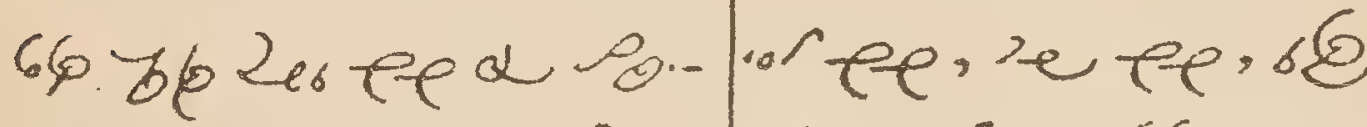

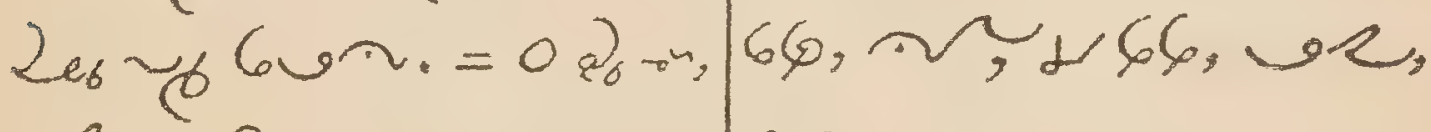
Sores agu.gon $\sim$.

2. $660 \rightarrow 66 \varphi^{: 0 \%, 06}$ Gun Pf 65 , g, 0665

Night offering. Q Les

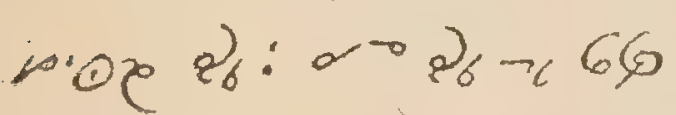
i $\sigma_{6}+, \sigma_{6}$ \& $84 \sigma_{6}$

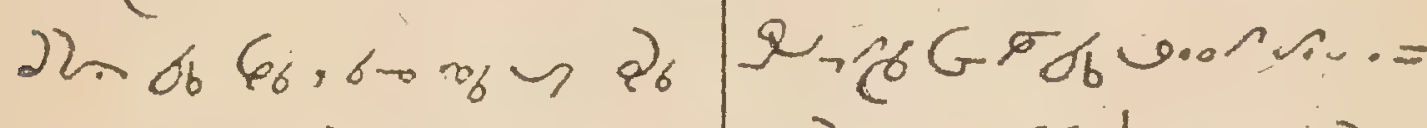

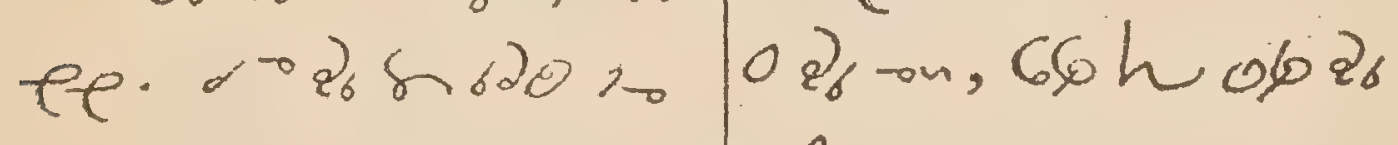

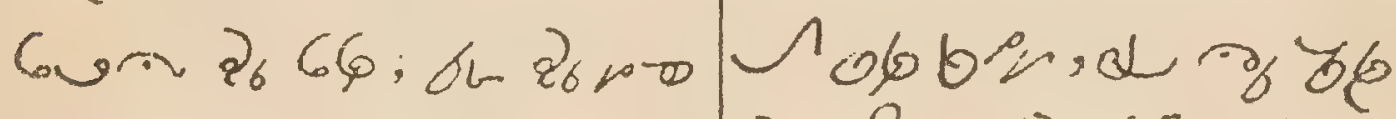

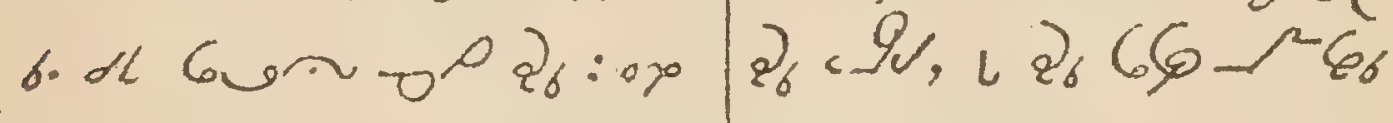

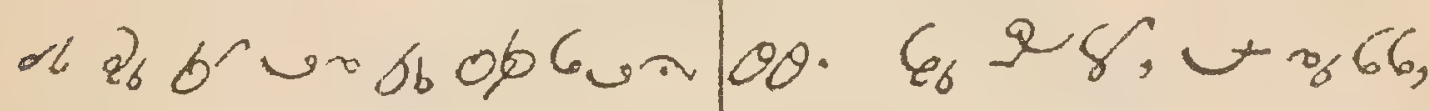

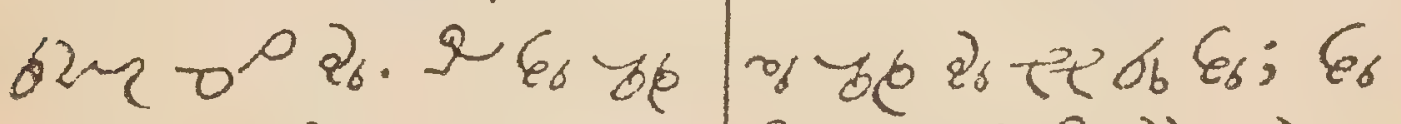

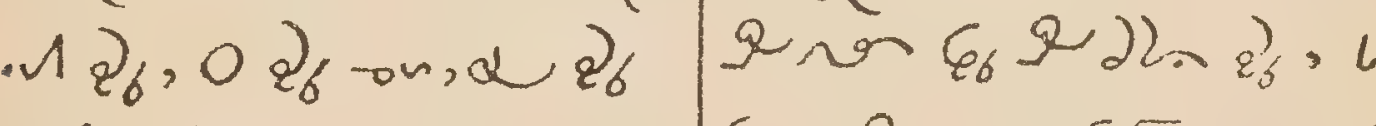

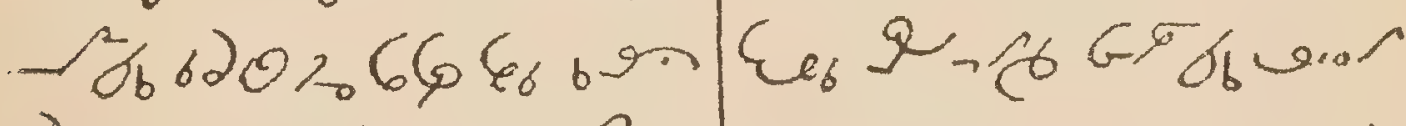

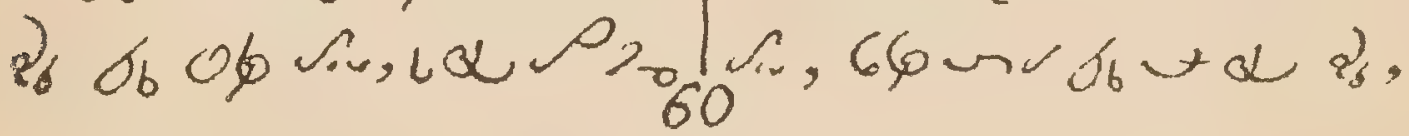


MANUAL.

55

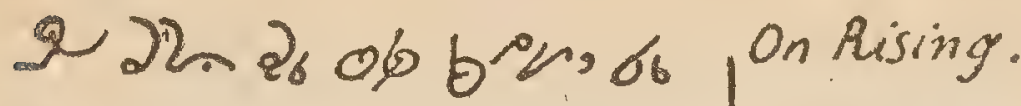

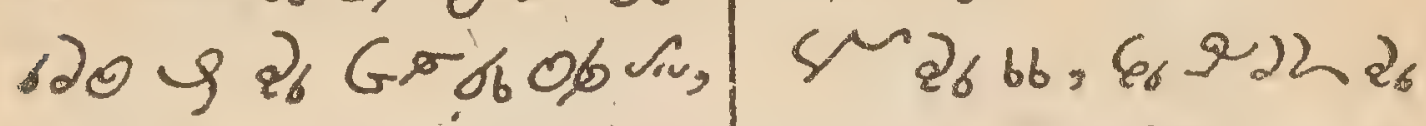

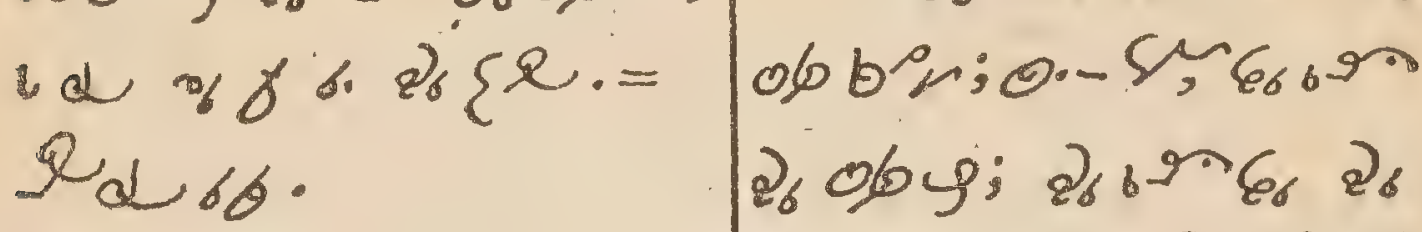

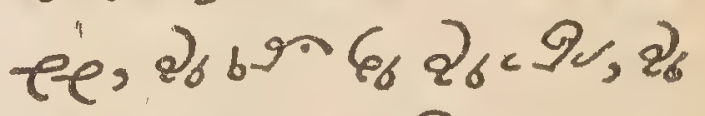

For the living and the dead.

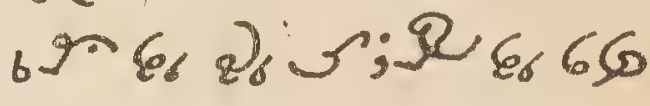

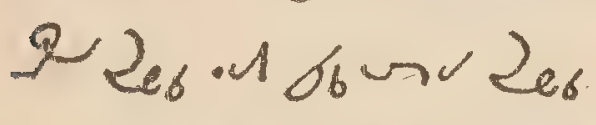

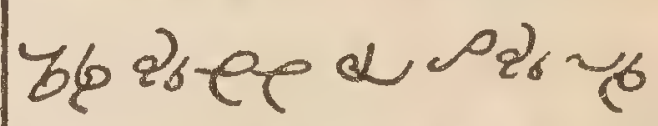
织. $=0 \mathrm{w}, 66000 \mathrm{0r}$ $12_{6}$ o P. ora.

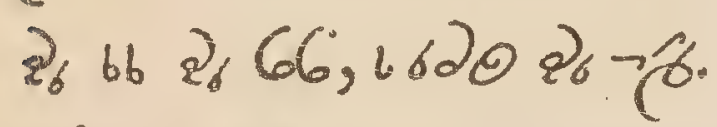
66.1 of $10 \% 1002906$ $\mu_{6} G$ by 2821

$\rightarrow$ te, older bran, Ongoing to bed. $0 u \varepsilon_{6} 6602_{6} ! 8_{6} \sigma^{20}$

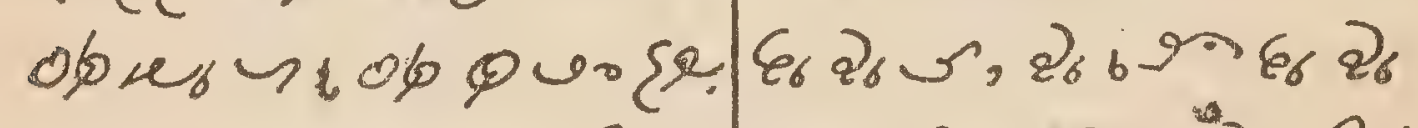

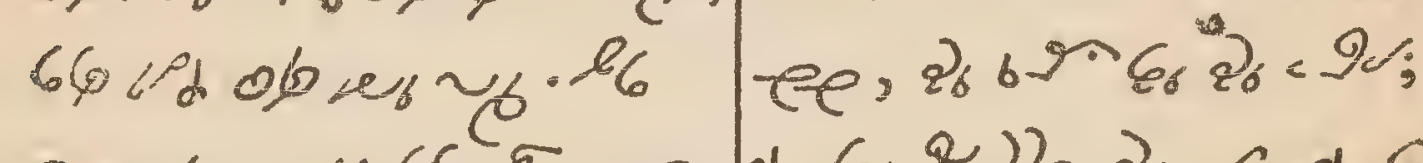

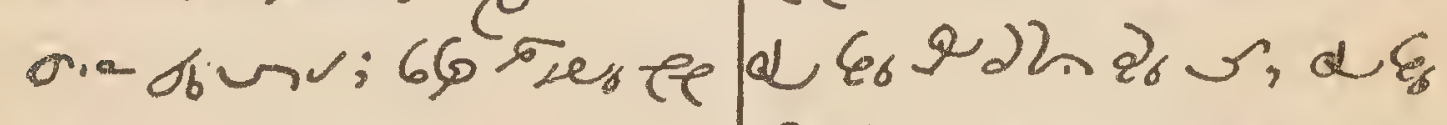
op-cens.

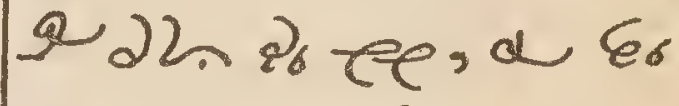

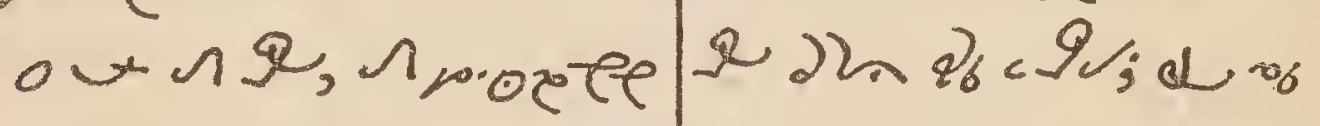

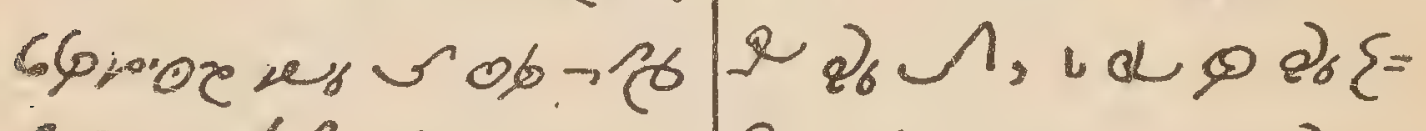

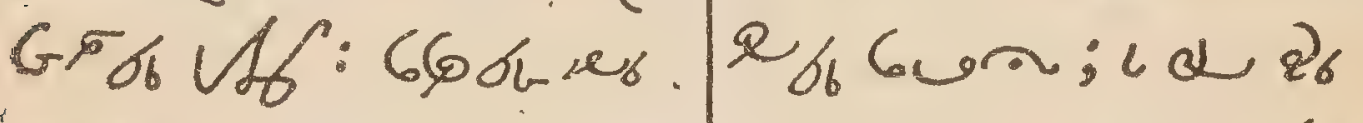
eve, 1 of ? $\rightarrow$ if G ore e bo $\varepsilon_{6}$ al a $_{6}<9$

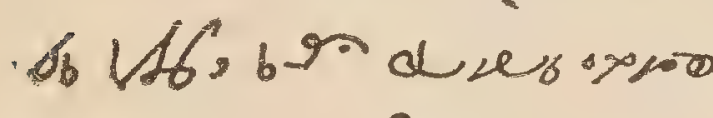

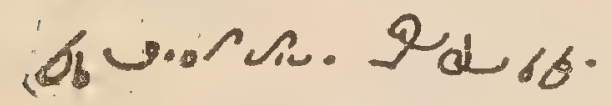
$-29.2 \alpha 6$. The Angelus. On. sub tum. 08 .

61 
56

CHINOOK

Preparation. Uत $2_{6} 660,66,060$ 2. for Confession. $66 \gamma \varepsilon_{0} \cdot \alpha \partial_{b} \partial_{0}$

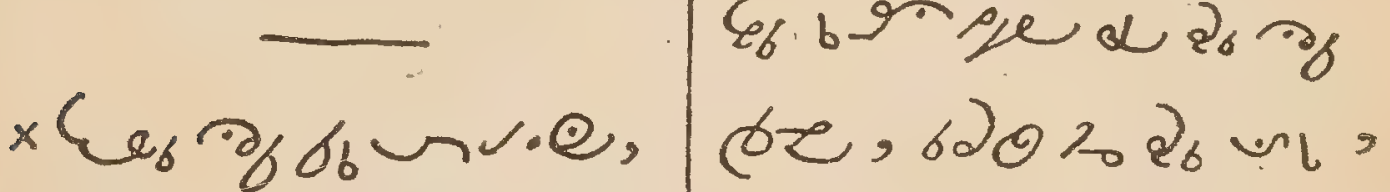

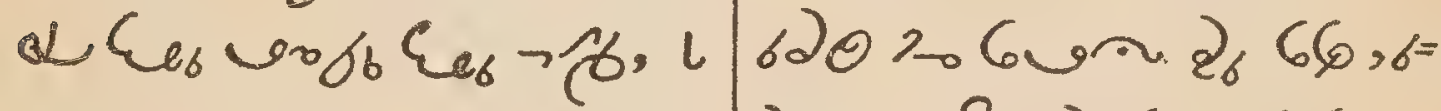

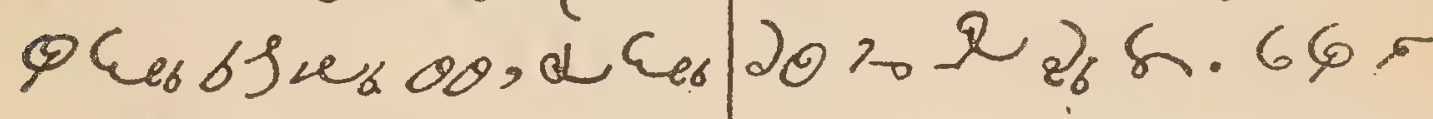

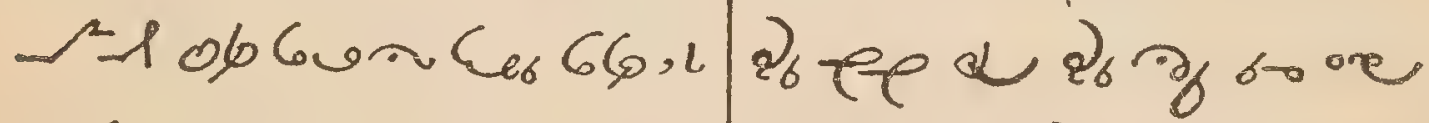

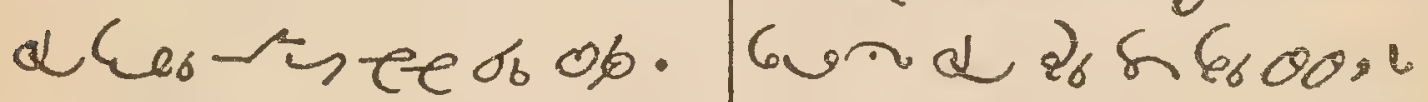

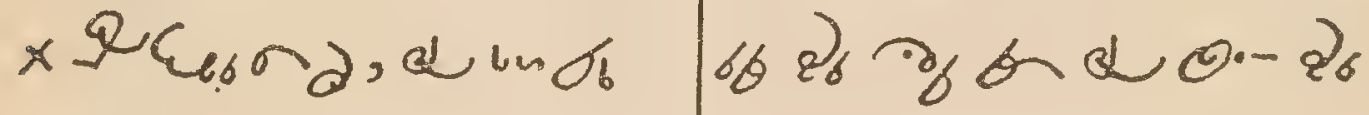
$\varepsilon_{6} 6 u+2060101$ Leserbu.

x Res Lara 660 an $\times 66$ sur. Y QQ Les .006 pe.

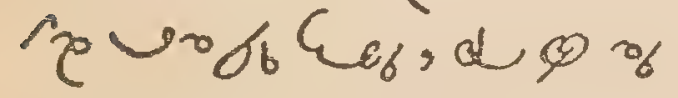

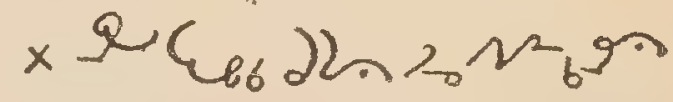

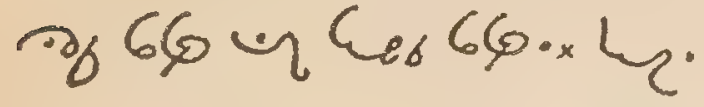
$=66,0\} \backsim 66,602, \imath$

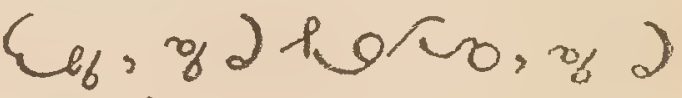
hi?

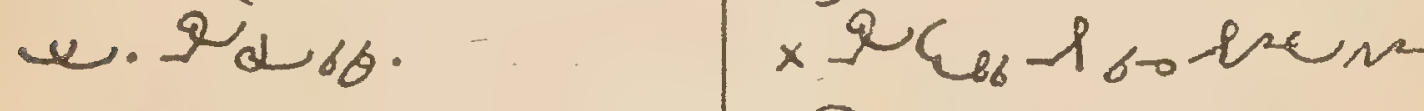

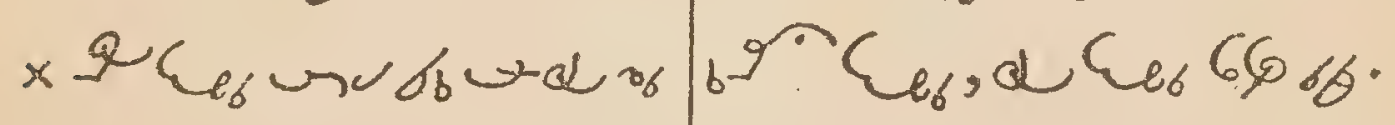

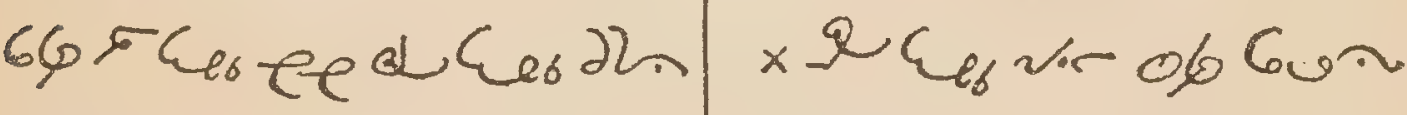

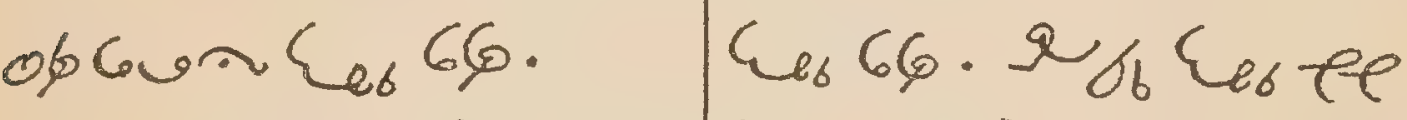

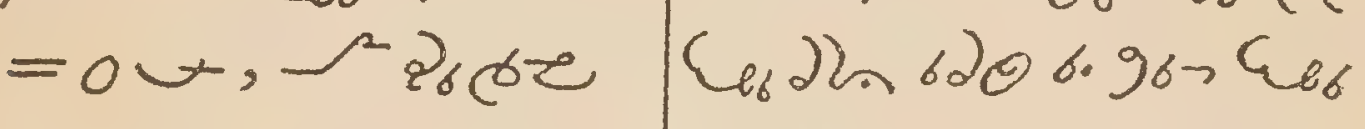

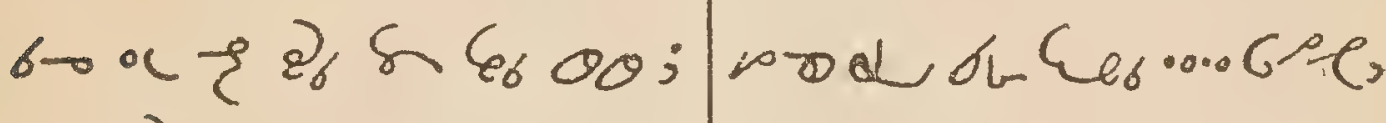

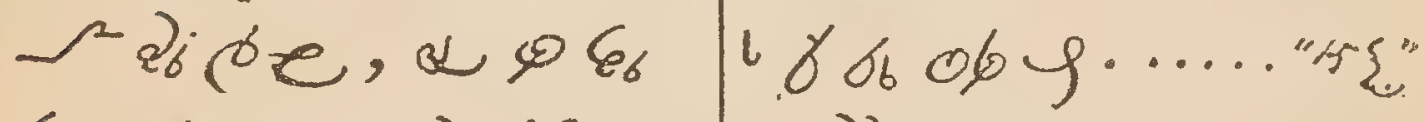

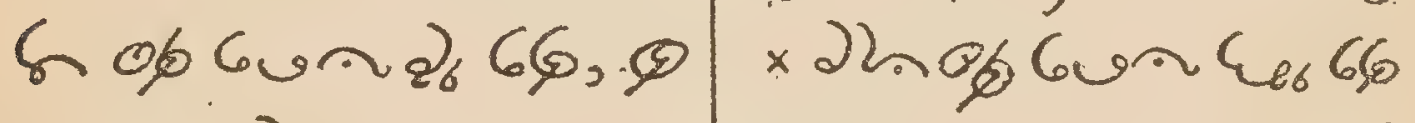

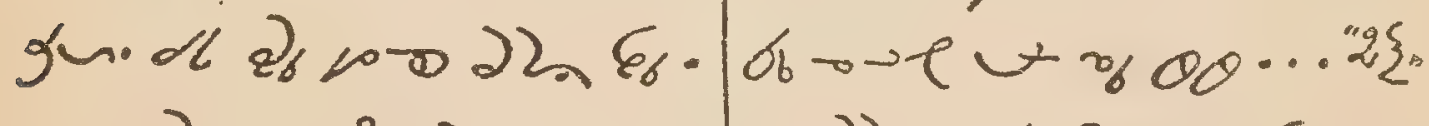

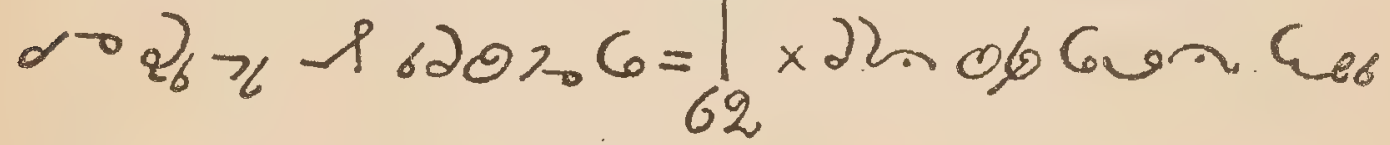




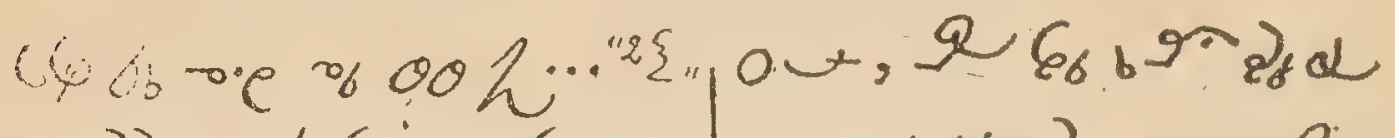

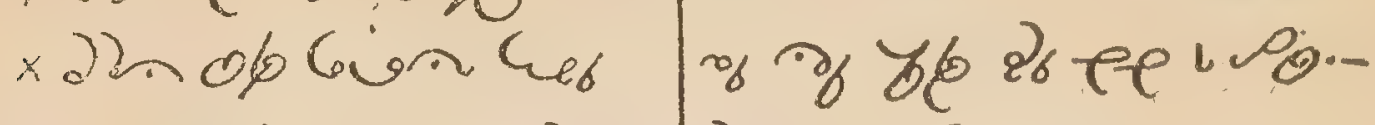

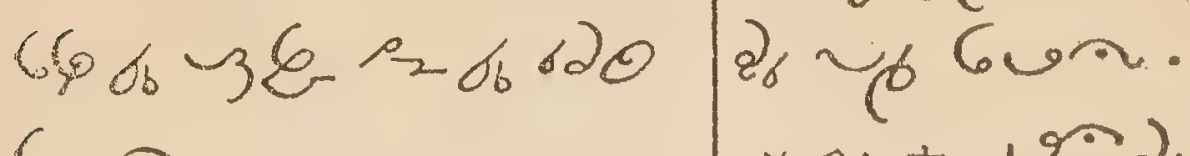

Gur...

$x \cdot \pi$ ol $\mathrm{Ce}_{6} \mathrm{C} 6$ $60006+6$ giced $\varepsilon 66 \ldots$

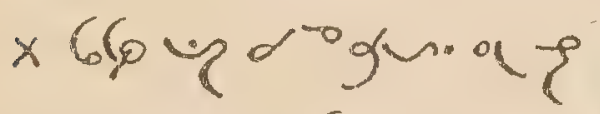
Ee66\% c.. Gun...

$x Q c_{66} 69 \backsim \varepsilon_{66}+9$ bo $0660 \pi C_{6} 6 \%, \mathrm{~L}$ r. to ber te ol P. $\varepsilon_{06} 6 \%$ Gง ... $\times r_{2} \ldots$ $\times a C_{26} \cup \sim$ b $\circlearrowleft d$

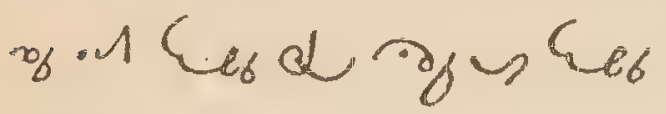
eeblab 60 , dig bock ee ol $\rho_{0}-c_{6}$ 66 Gun...

Prayer for Contrition. ow, \& $\varepsilon_{6}$ b $22_{6} \alpha$ भक्ष प वे ee br 0, 6 ⿶凵 $2666 ; 0 \uplus, \Omega$

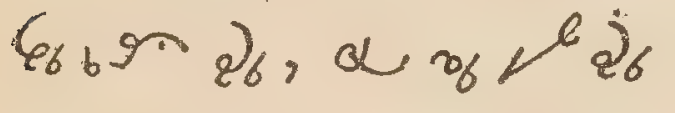
$\times 0 w, 6$ शु

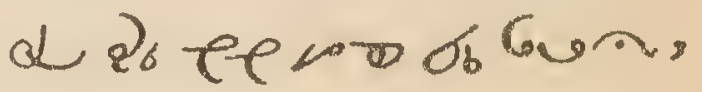
- $x$ शे $6.50 \mathrm{csh}$. $\times O b^{2} \alpha$ al bra ee bran. x 4 क a $627 \%$ ¿ 66 wa $\times 0\}$ b d d $20 \%$

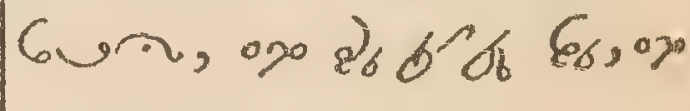
2) und b6 68 : $\times 0\} 6^{2} \alpha 160 Q_{6}$ ee ob $68 \div \sigma_{b} 600 \rightarrow c^{6}$ First Consideration.

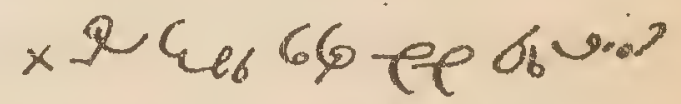
$\therefore$ bo \% ore, al $c_{\text {es }}$

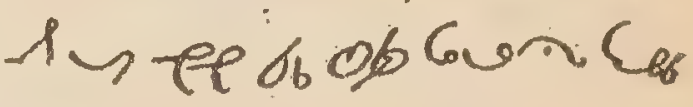
66.

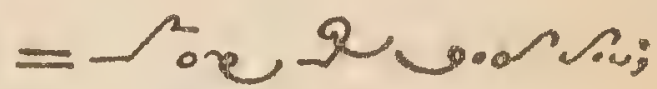
a non GFo vor s.;

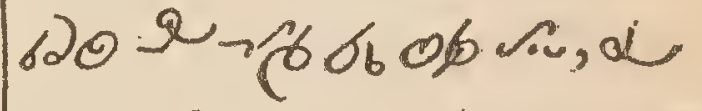

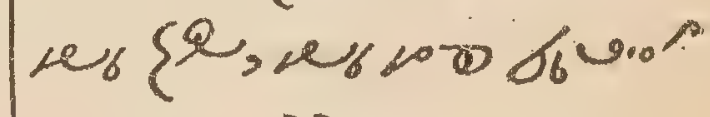

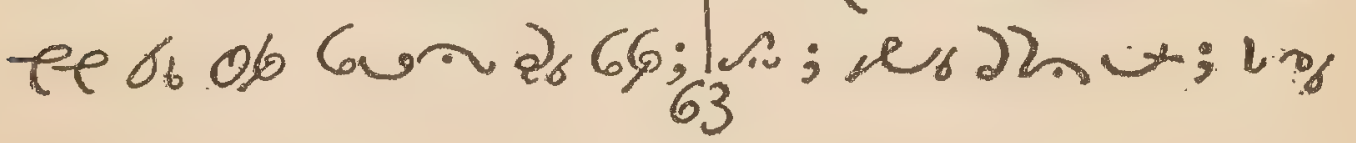


58

SHINOOK

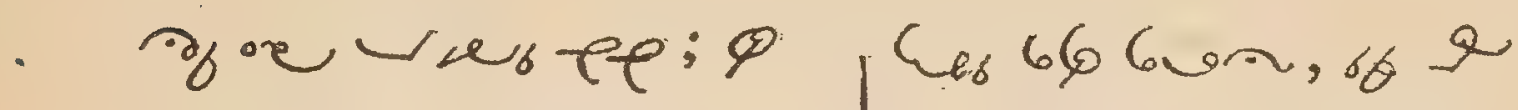

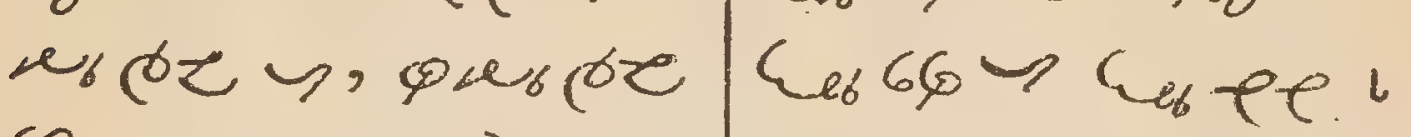
\{

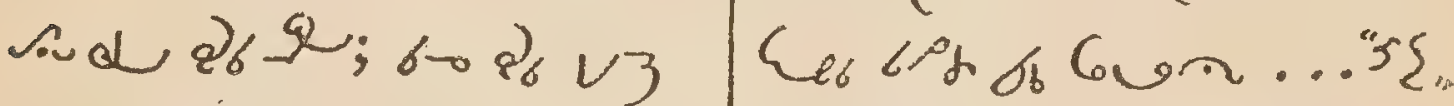
Q 2866 Gun; 00 \%

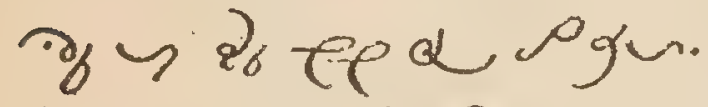

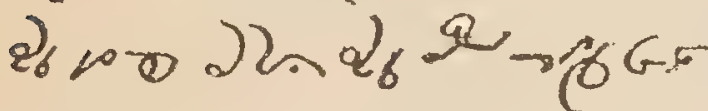

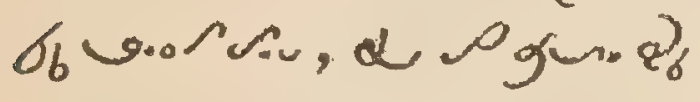

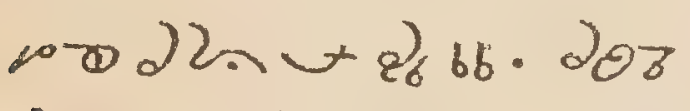

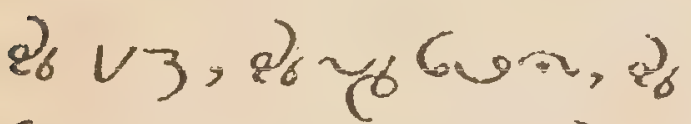

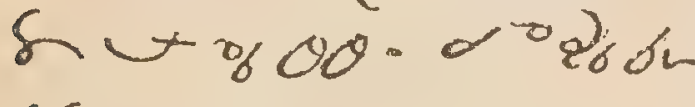

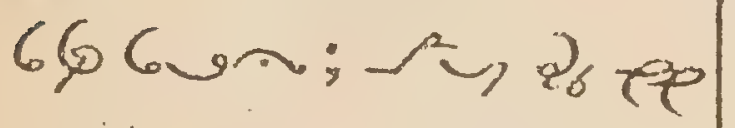

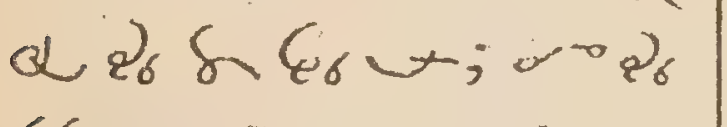

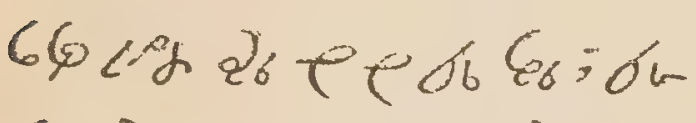

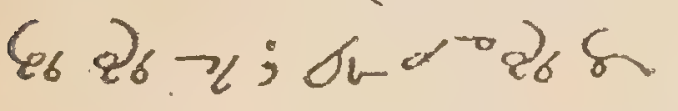

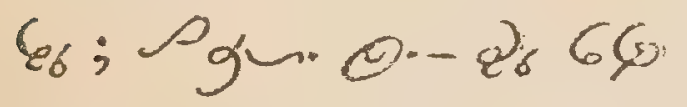

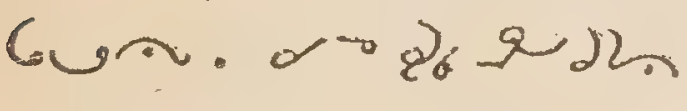
$2_{6}$ ee $\alpha$ Q $_{6} 100 \sigma_{6}$ Gun; 0 के करे कर

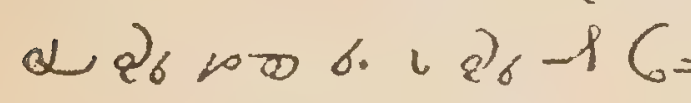

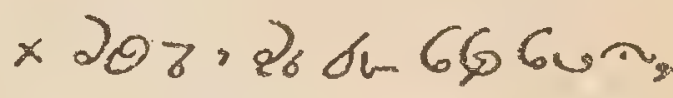
$z^{2}$ y

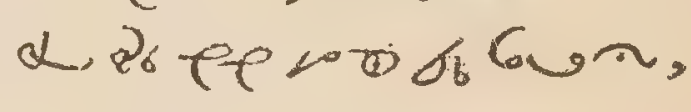
ox $P_{6} 66 \mathrm{cs}$...

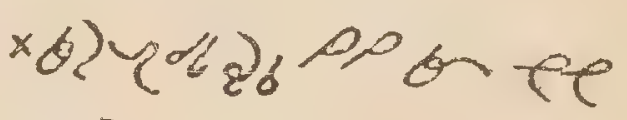
$600201060 \pi 650$ $\times 824$ वl $82_{6} e_{6} 6=$ ?ח.

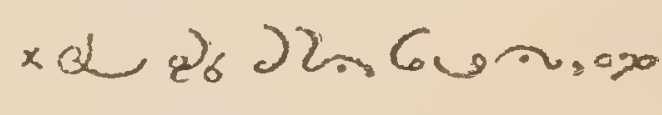

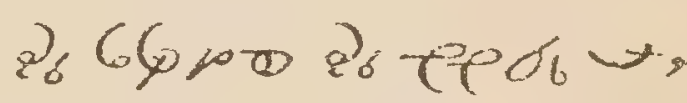
के जu x bरप्र al के 69060 26 Te ᄀît.

Stcond. Cionsideration. x CGova les ee á

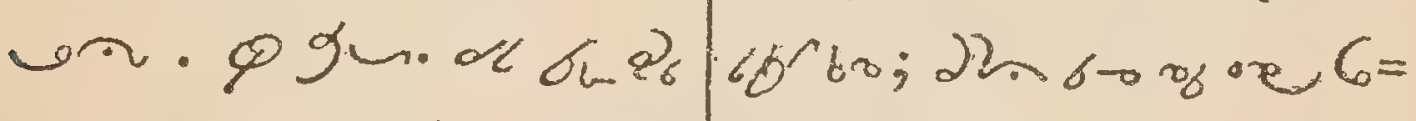

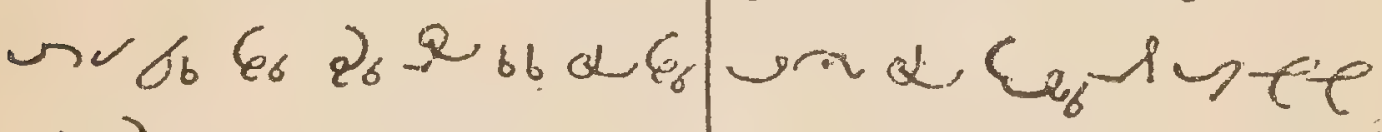
$12 b_{6}$.

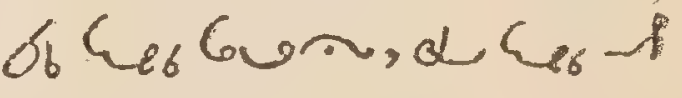

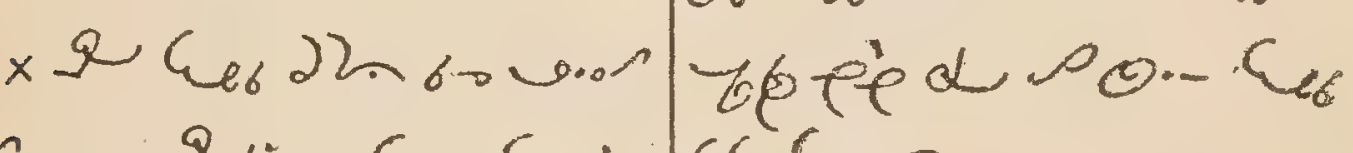

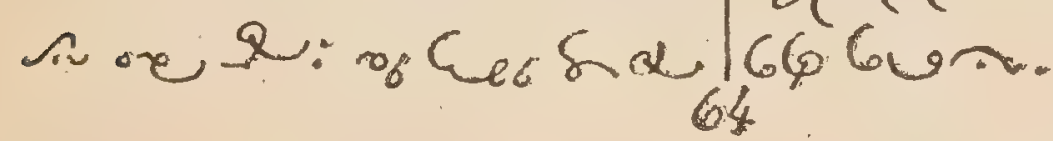


MANUAL.

59

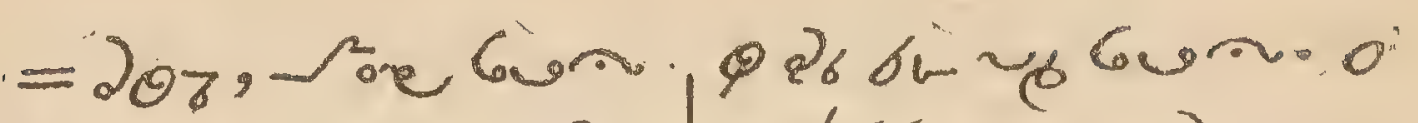

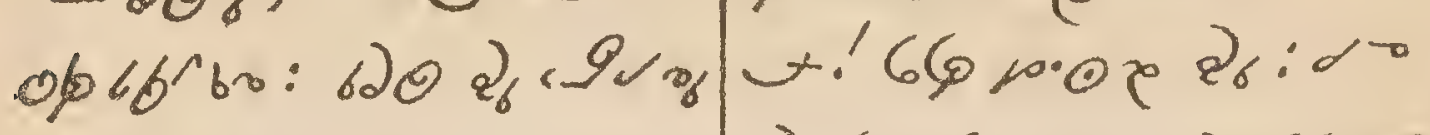

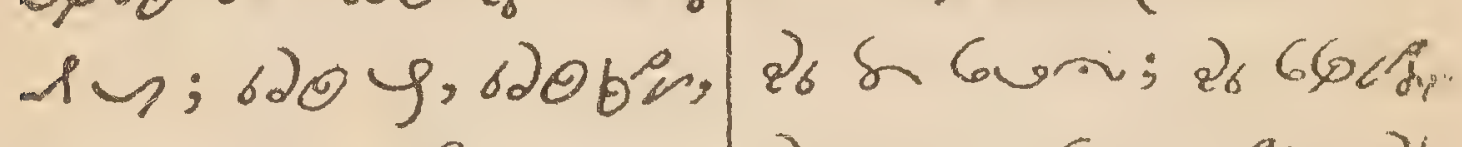

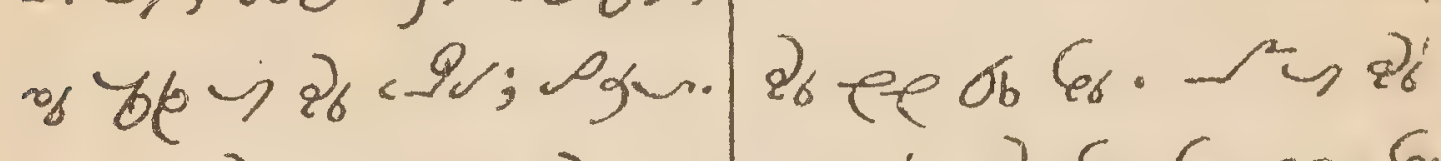

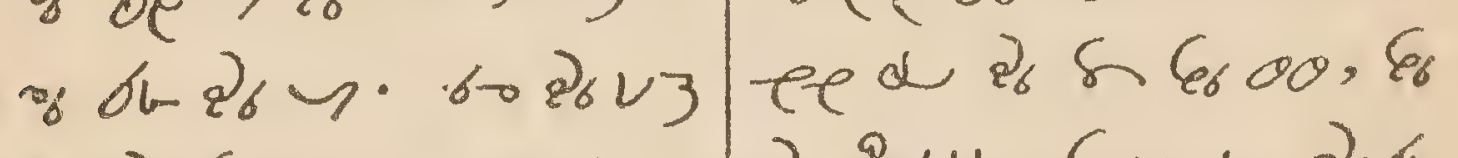

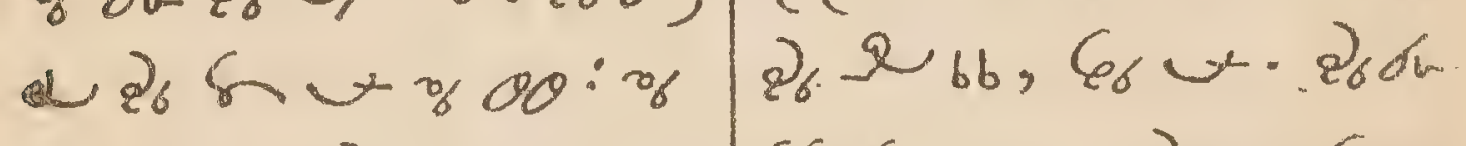

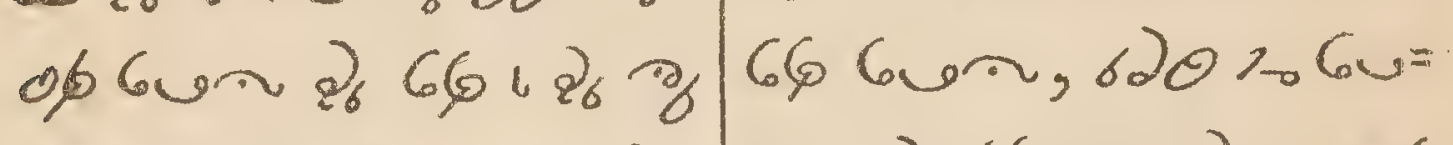

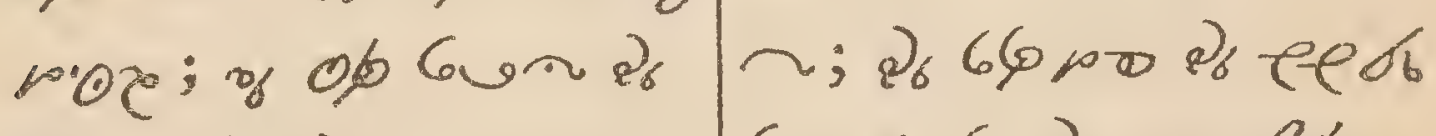

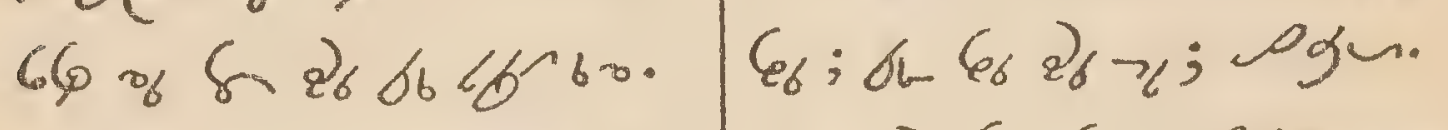

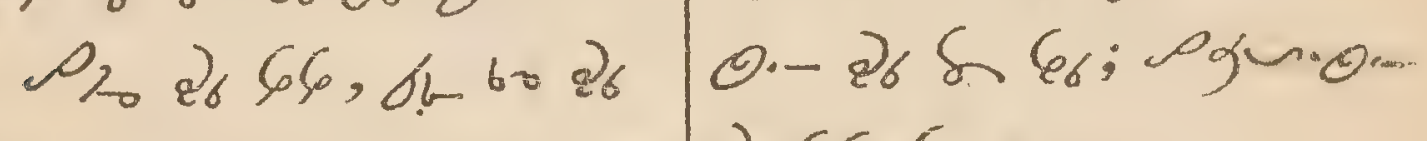

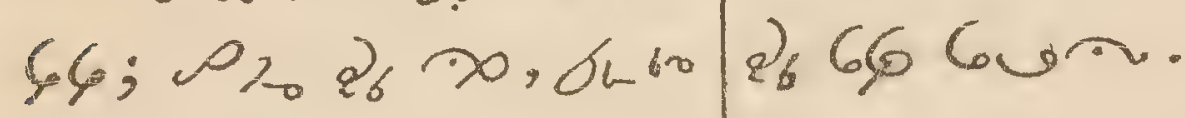

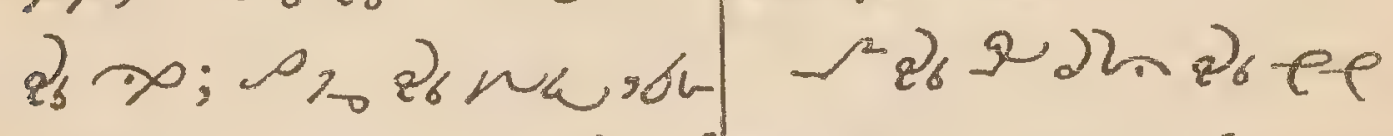

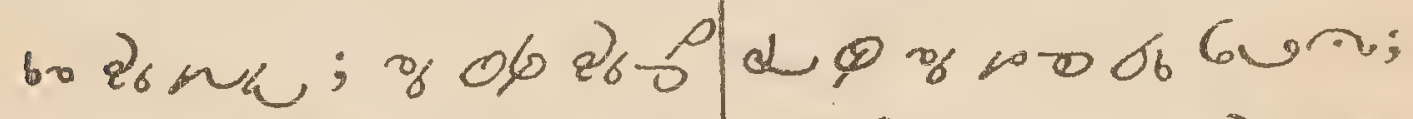

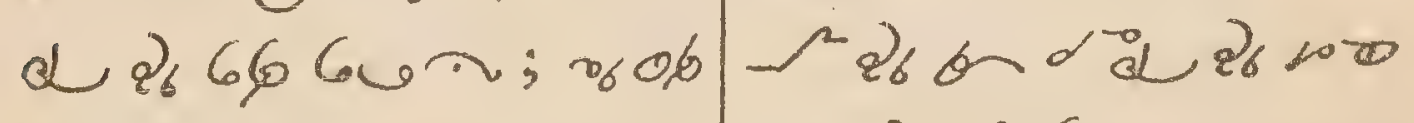

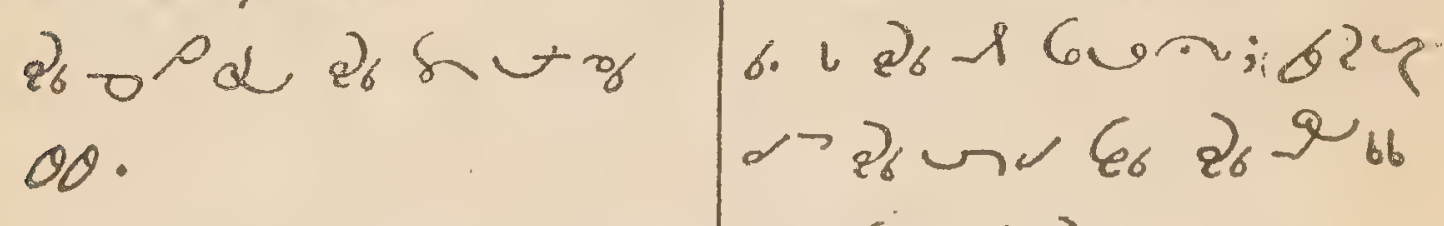

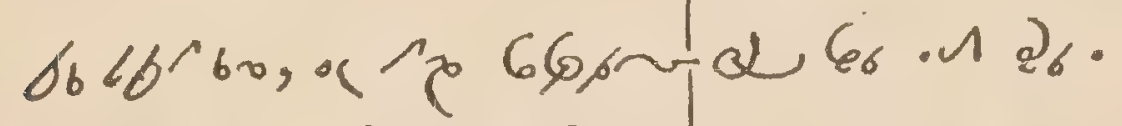

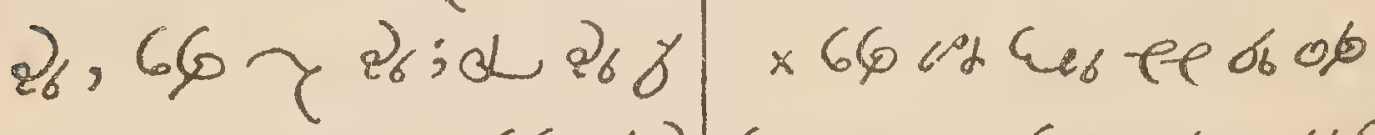

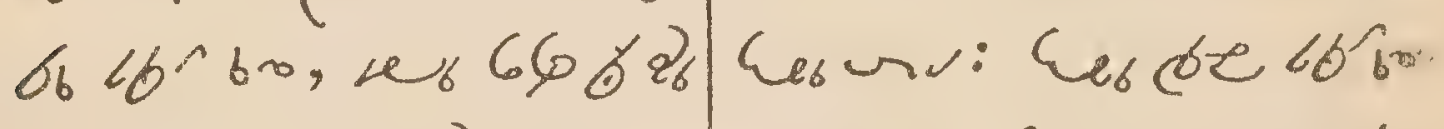

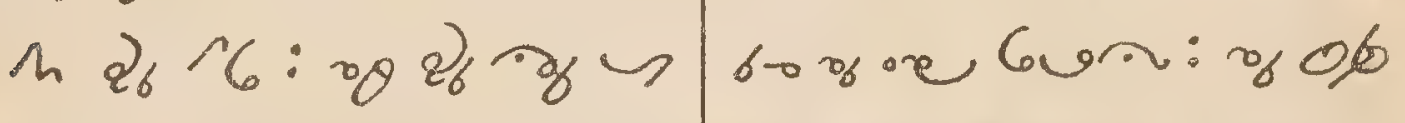

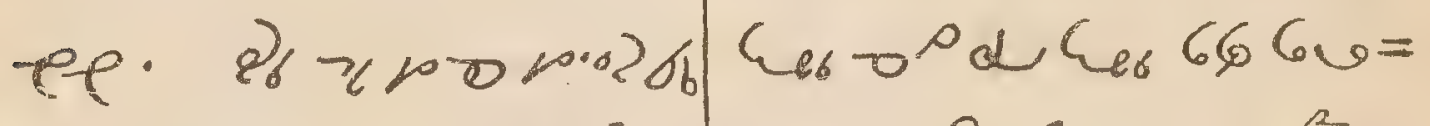

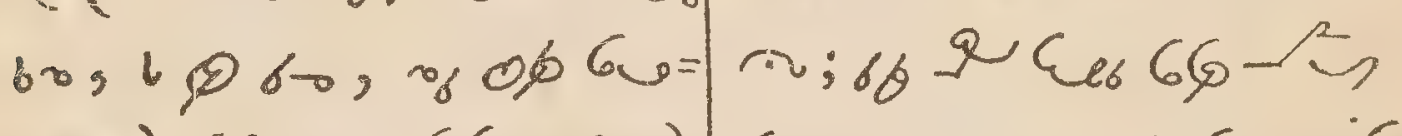

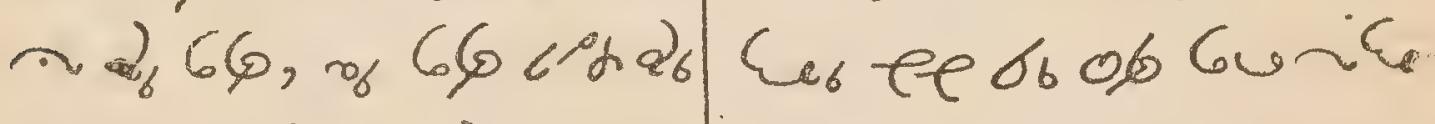
o6 60. 208 26 v3: $c_{06} 60 ; 66$ bo her te

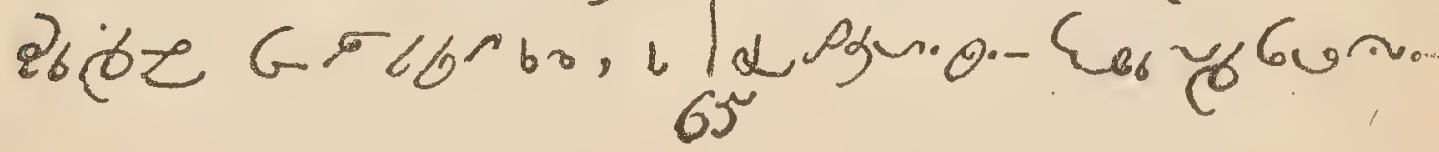


.60

CHINOOK

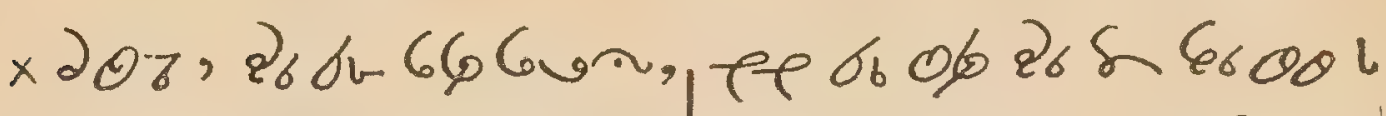

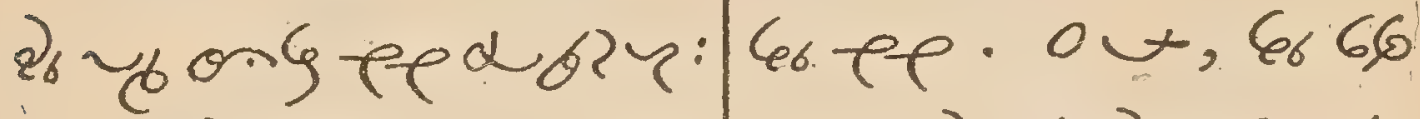

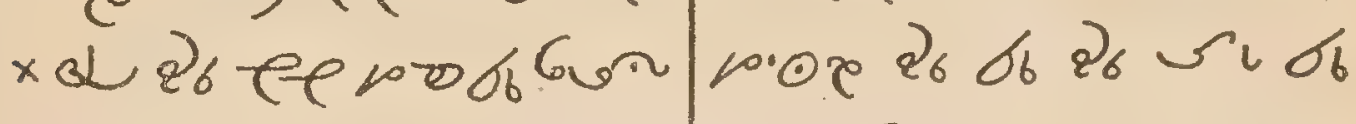

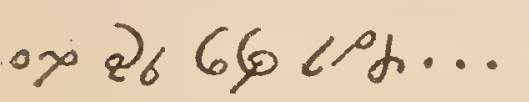

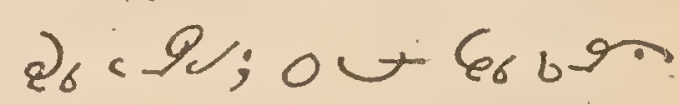

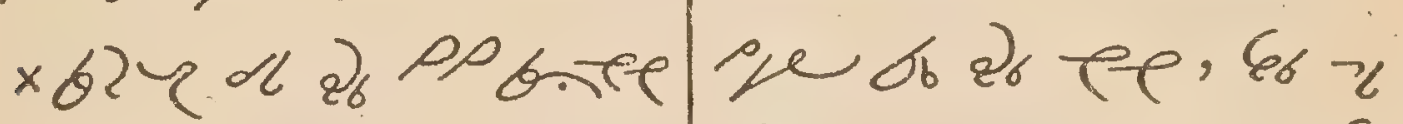

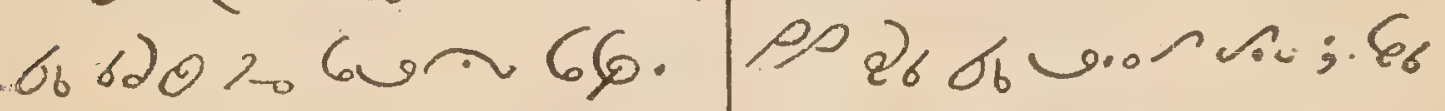

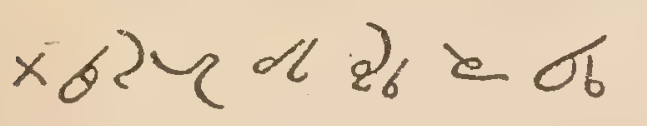

Gur.

$x$ Q $262260 \pi, 00$

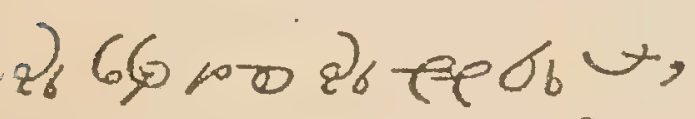

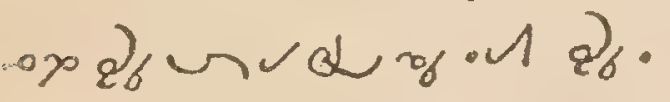

$\times 62 \tau$ d \& 6606

2o te of $41 \sigma_{6} 620$

$\neg$ zo.

Third Consideration.

xecureerso

.ro on, on top, d

Les 14 ee bo $066=$

wa $\varepsilon_{66} 66, d C_{e 6}$

1 bo fed Po.

Ler 666 an

x 0 ' G or 2 ,

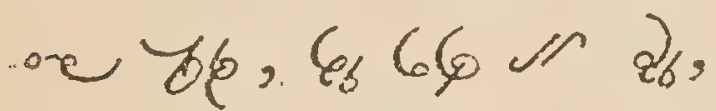

66 650 1000 2े 60 26 606

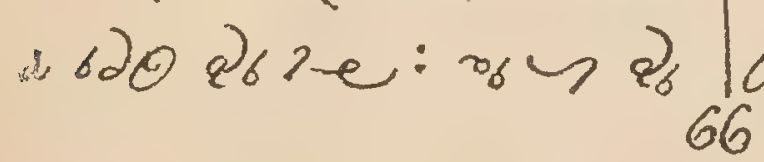

66 on 286 ó $16160: 1$

$\checkmark 8_{6}$ ee d शे $_{6} 66.6=$

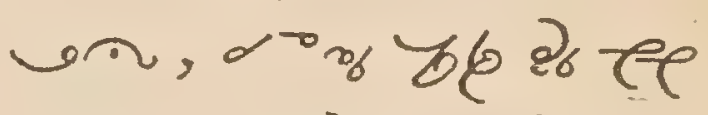

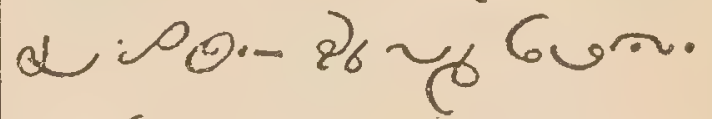

× Cer be trom

ar 9 ore 70 : $w$

Ge6 66 r;

$\rightarrow$ if eb 0660 a

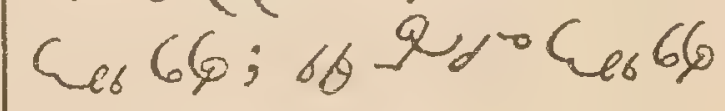

यदer te ob ob Gu

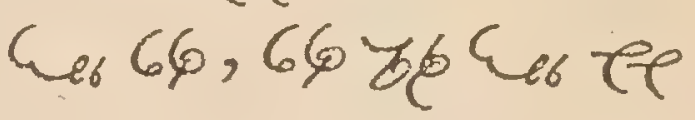

L.

6 6几

x 203,26 б च б6न,

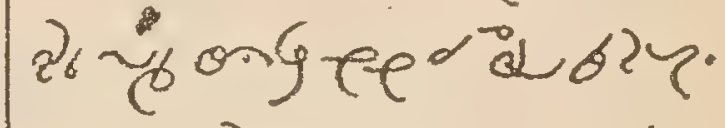

x. Q Q $_{0}$ ef

$6 u \pi, 0 \times$ \& $650^{\circ} \mathrm{ph} . .$.

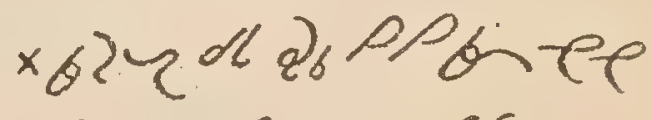


MANUAL.

61

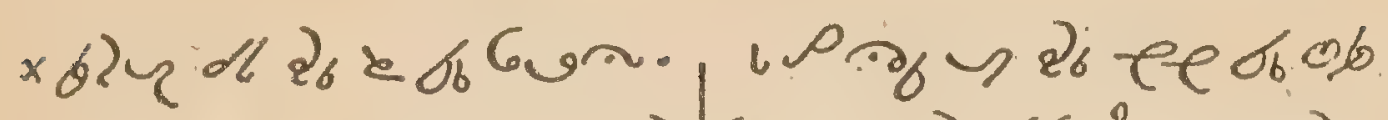

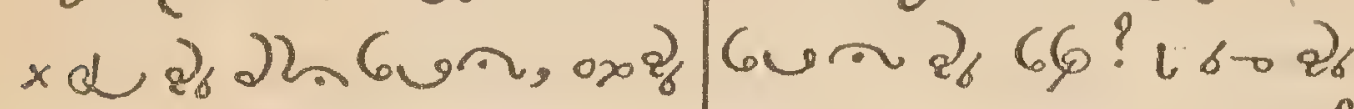

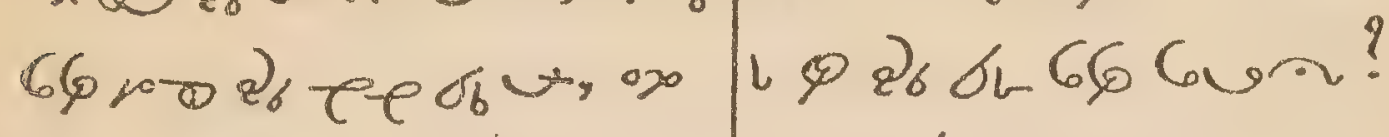

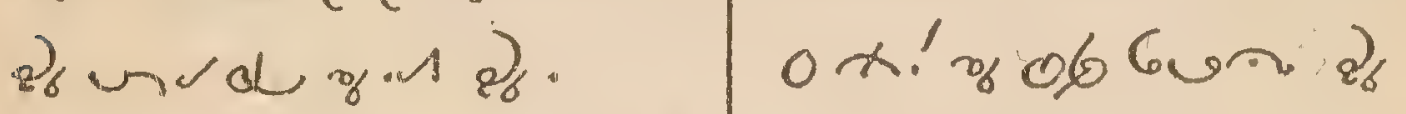

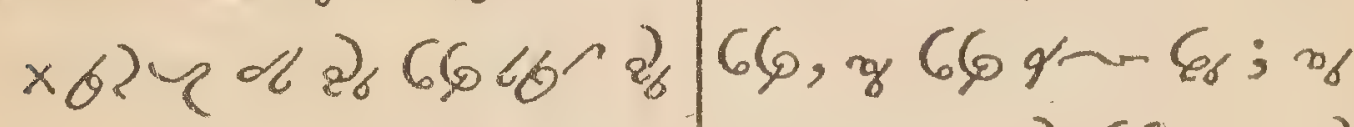

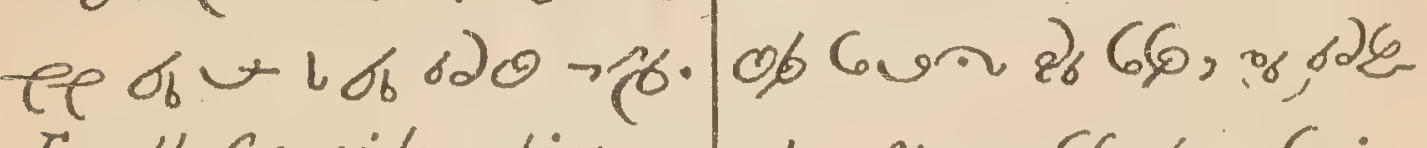
Fourth Consideration. $06 \rightarrow$ The 650 or $\varepsilon_{6}$ : x lab efor 6 :

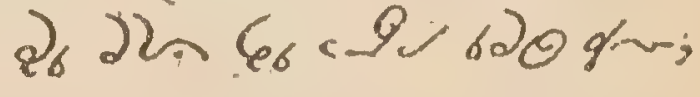
gr weredrs, $2_{6} 22$.

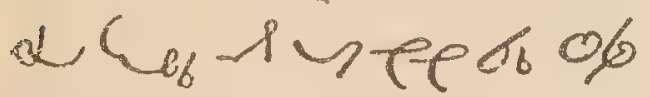
86 $2 \pi \varepsilon_{6} s_{2} \alpha d$ Gur Ce6 6oral Ceb res 650 \& 68 6r yor, stoped Po. se6 6ron. olo a b8 y 00006

$=208$ or wer6 $650 \%$ \% , 650 - $Q$ of, res 660 d xy $r$, $x_{8} 650$ ro,

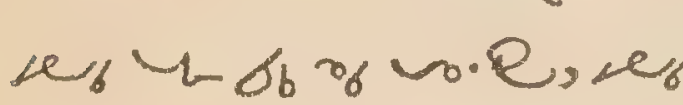

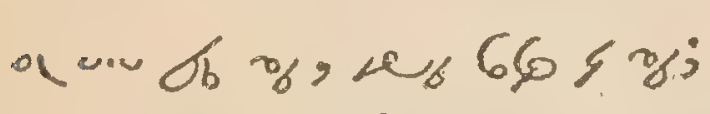
\% ob Gurn? 650, \% $660606 x ; \% 06$ 26, 2

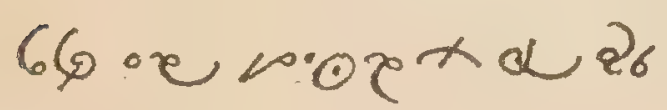
तु 6 m.

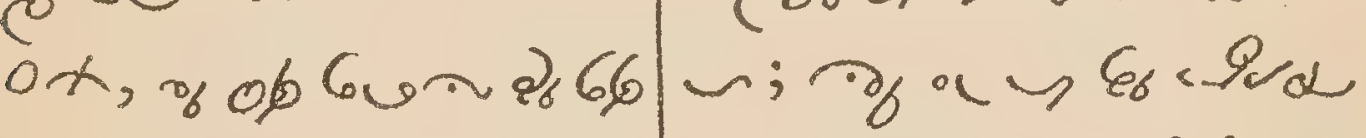

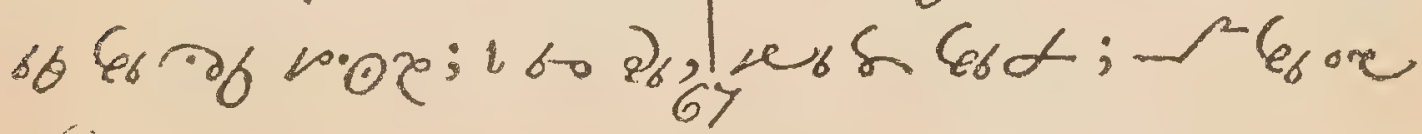

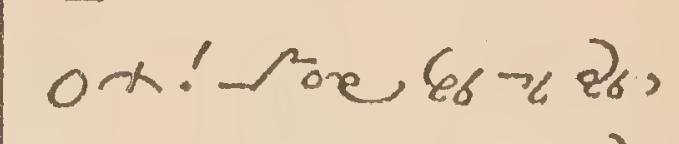

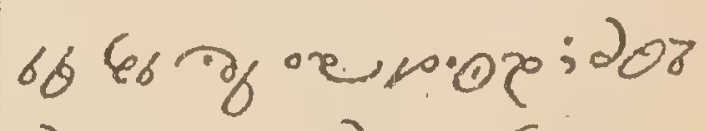

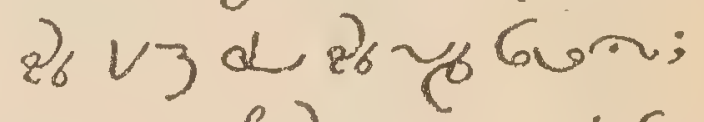

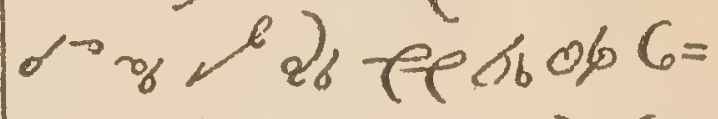

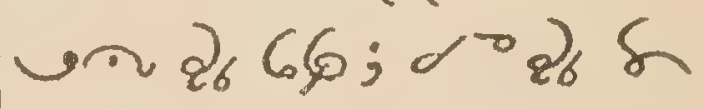

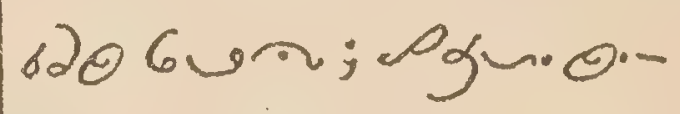
के 6 और.

$0 x ! 2_{6} 22-66606$ orepor ob pa: $\zeta_{6} 1$

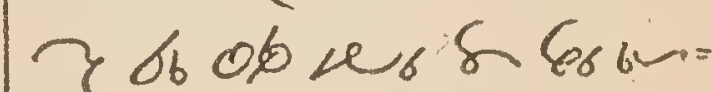




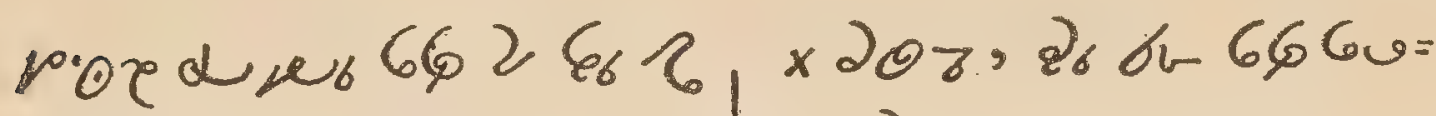

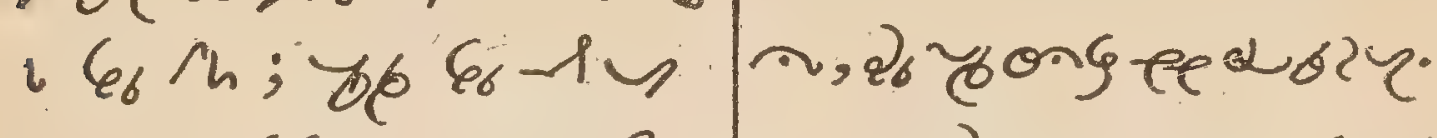

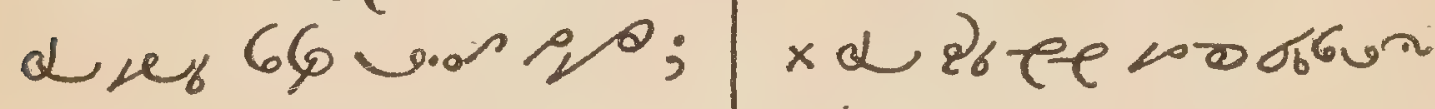

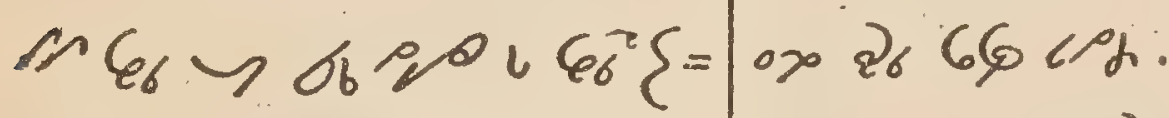

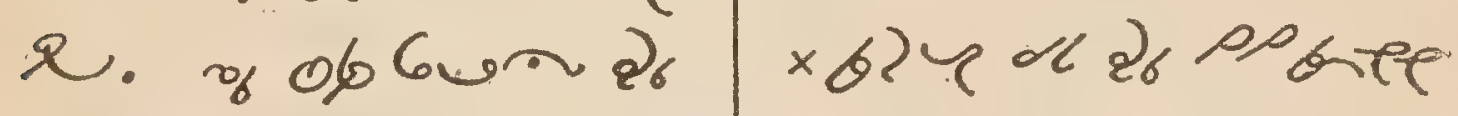

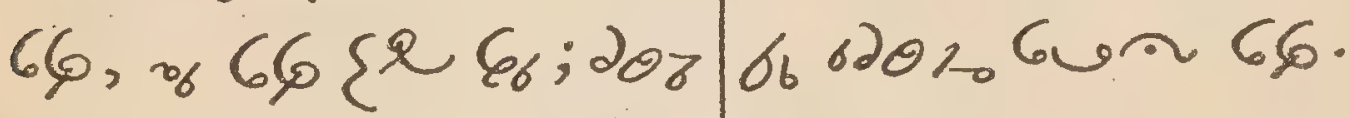
$q_{6} \cup 3, q_{6} \sim$ चुन.

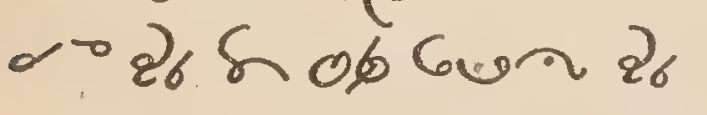
66. 9 g $\backsim \cdot 0 \cdot-26$

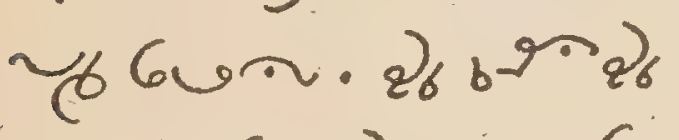
ef $\sigma_{6} \varepsilon_{6} \cdot 2_{6}{ }_{6}$ in $\varepsilon_{6}$

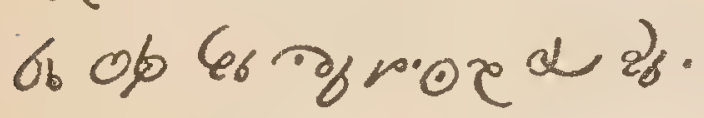

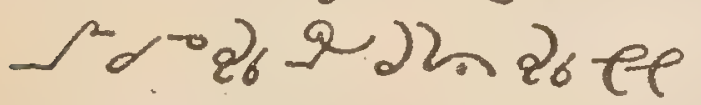
स्व

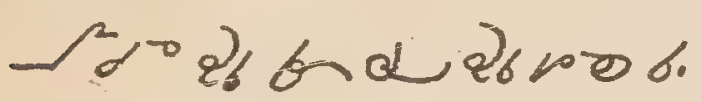

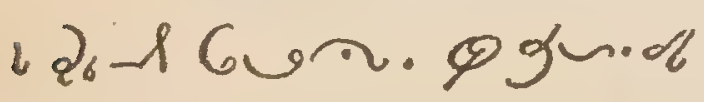
or $q_{6}$ जu br $\varepsilon_{6} \cdot q_{6} \cdot 2$ फक्य द. $\varepsilon_{6}$.

$x$ Les betroyare rop: pob Gun Eer $66, \pi 66$ व 4 x; 68

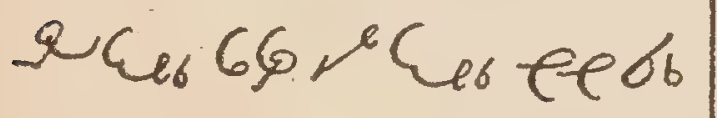
ob6un Ceb 66, 660 Tok lo ed ogn.o. cer 6 w. $\times 62$ पdl $2_{6}$ e 666 \%。

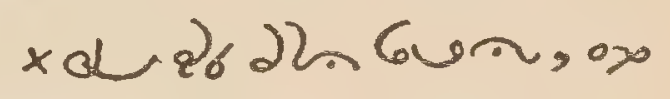

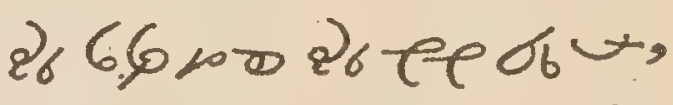

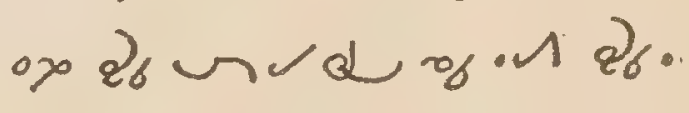

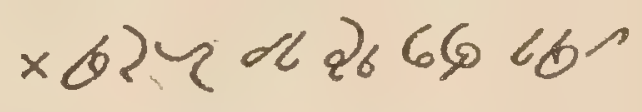
26 ee $\sigma_{6}-466620$ $\times \mu \rho \Omega$. x.0.0 Gre nu.

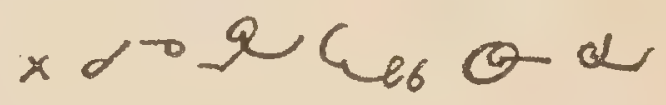
\% 86. Ceb $_{0} 0.0$ Gee; 6. Las tebaron. wrobure, b2? ee6rob Gua 6 er 65

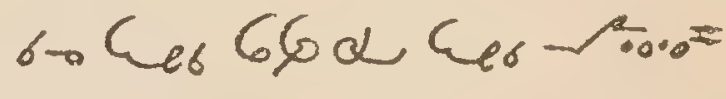
Ge; biy 66y ueb eebrob Gun Ger6. bly00 b $4 \alpha$

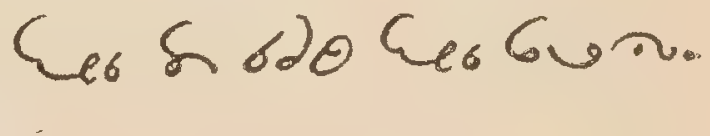


MANUAL。

63

xd Geb dw.0.0.6Pe,

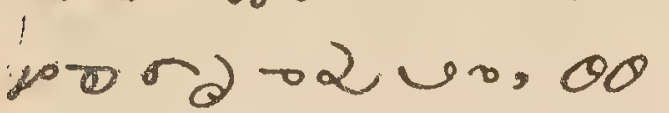

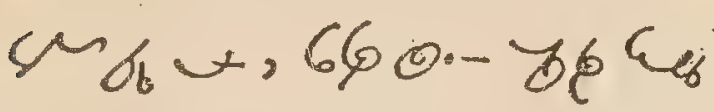
redd Le wor ob $6=$ un; 2hit; 200 ob

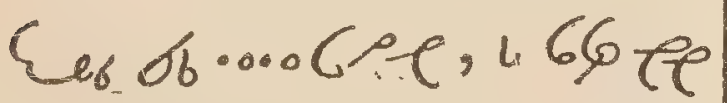
d Ce, $6506 \%$ \%00;65

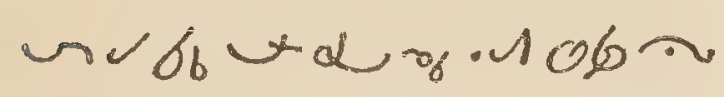
ep leb 1 ; 6600 he mard Cer;d Cer

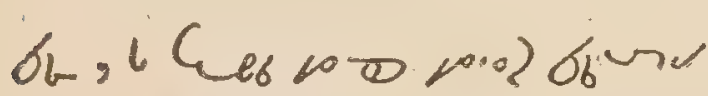
-e, e 2) al so ox Ces एक

offering
of the Mas.s.
o ins.
owb, 662560

$2_{6}$ or 1000 ; $6 \mathrm{~d} 2 \mathrm{n}$

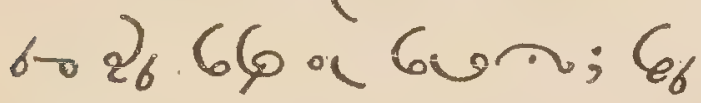

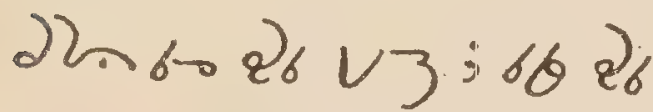
on a $26 b_{6} \sigma_{6} \varepsilon_{6} 06$ द; 86 \&6 $6601002_{6}$ ee $\sigma_{b} r^{2}, r_{2}$. \% 50 व 66 .

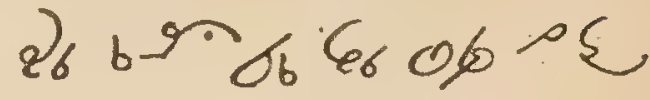
68 ol 2600: Juc. - $\varepsilon_{6}$

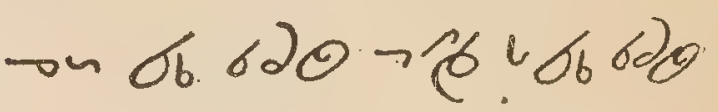
N; wor siv lobsi. Go te ob:

26 b' 0660005 .

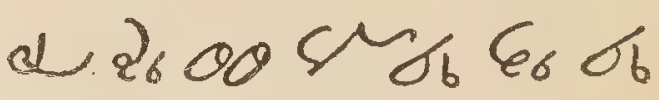
$820>68 b^{2} \sigma_{b} \varepsilon_{\sigma}, \mathrm{L}$

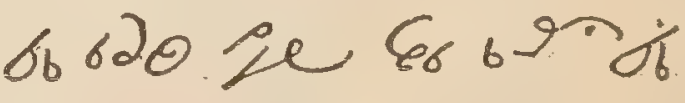
$\varepsilon_{6}=$

$26 \cdot 206 \varepsilon_{600} 0 \%$ d 26 in oloun 26 66 .

$26{ }^{2} 26 \varepsilon_{6} 06 \mathrm{ps}$ d $\varepsilon_{6} 66 \mathrm{Nop} \varepsilon_{b}$, d

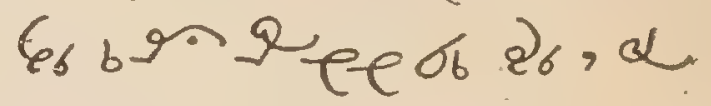

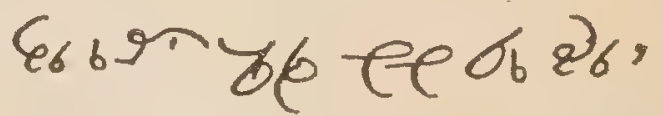
a $0 .-z_{6}$ कु $\backsim=l$

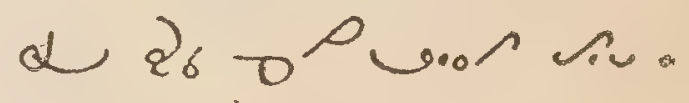


64

CHINOOK

Before Communion. $z_{26} 1000, m_{0} 66$

Faith. $22_{26} 66003$

1000: के 660 \&.

xar $G_{F} \sigma_{b} \oplus=0$ Contrition. A zer

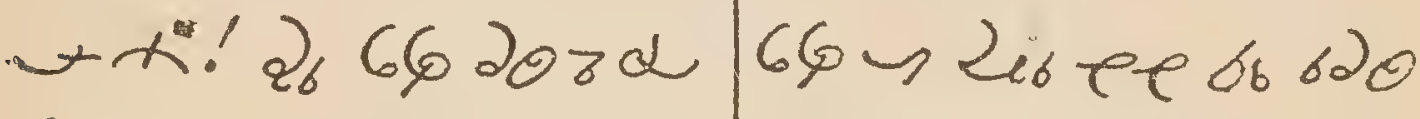

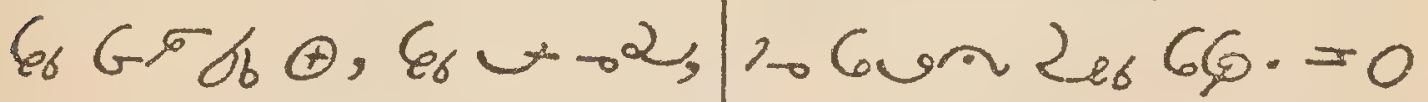

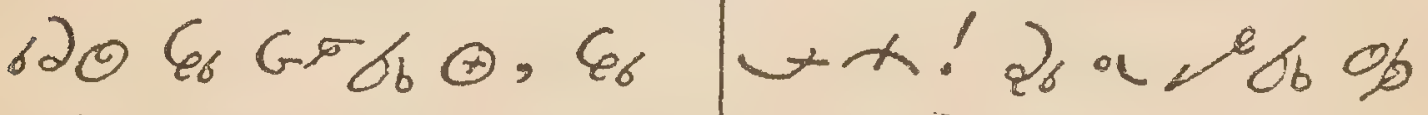

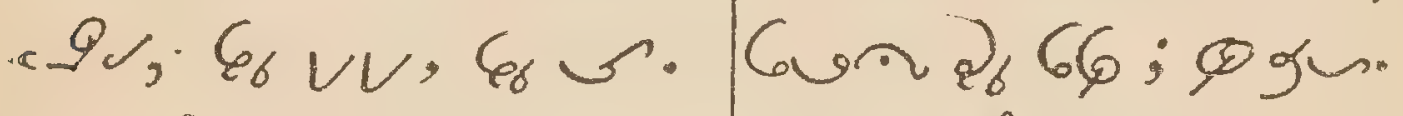

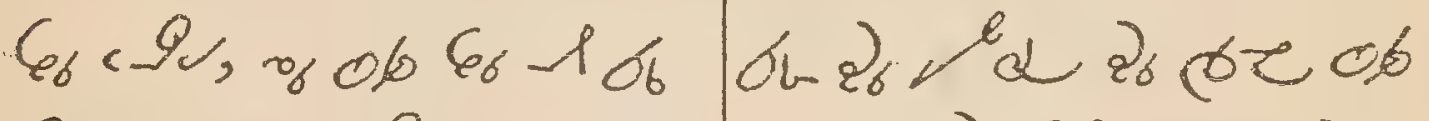

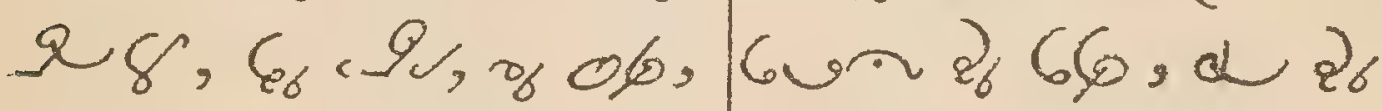

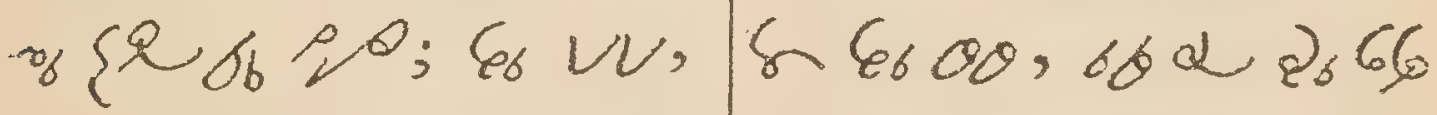

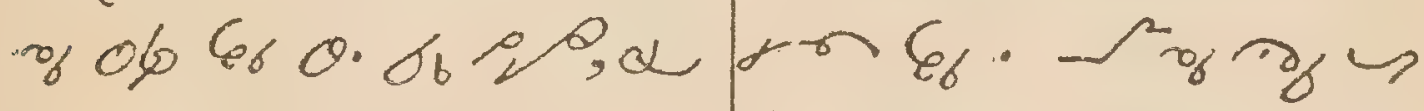

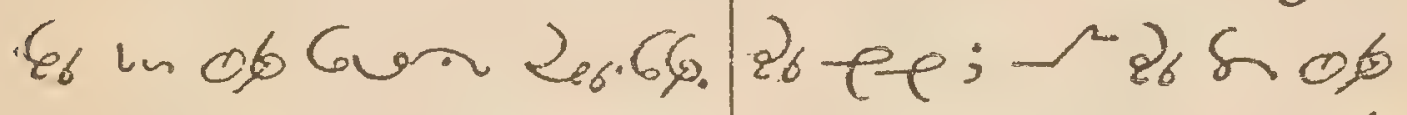

6 Gon $86.66 \cdot 0 \times 166$.

Humility. Q $2_{66} 66100022_{6}, 6600006$

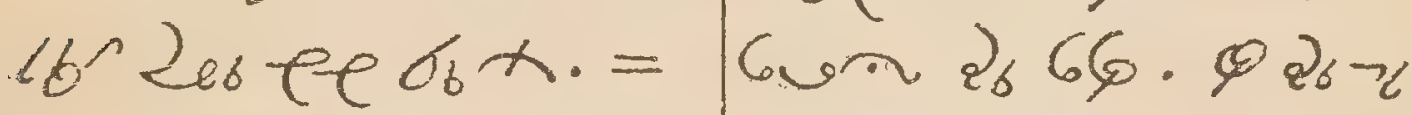

owx! $602_{6}, i a_{6} \cdot \alpha q_{6} \tau_{6} \varepsilon_{6} \sigma_{6} a_{6}$ ee

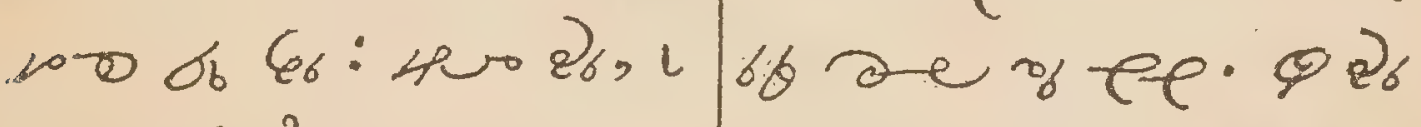

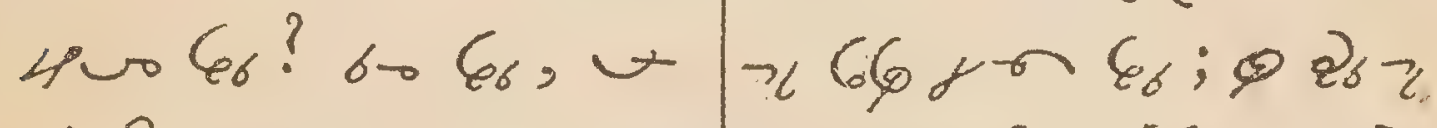

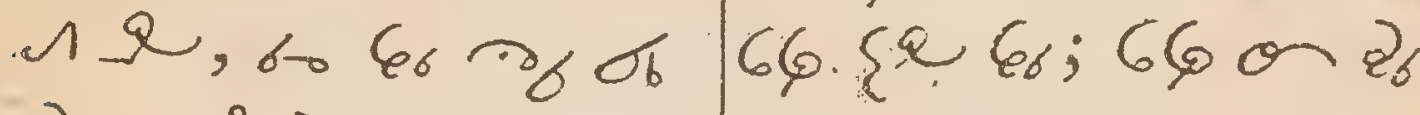

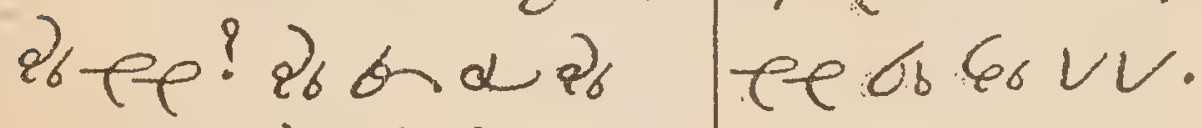

तु $6_{6}, 20 \%$, 26 b :

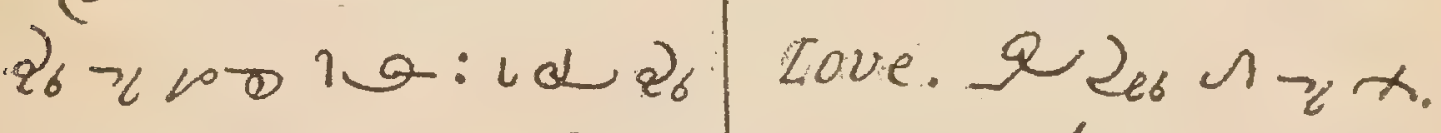

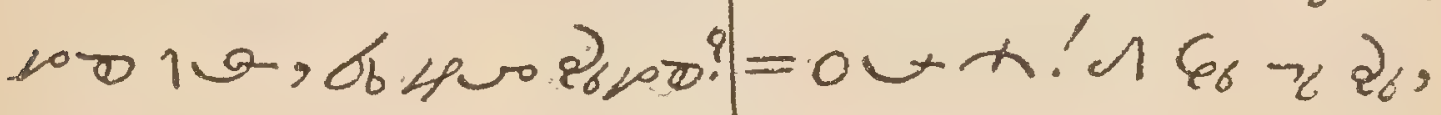

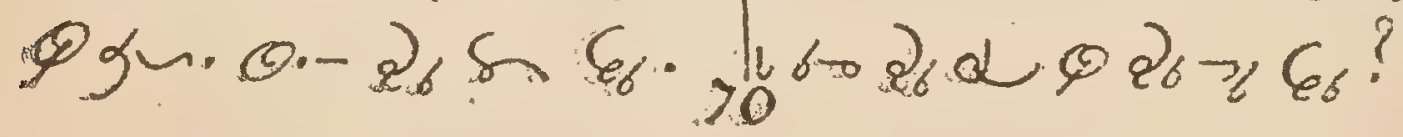


MANUAL.

65

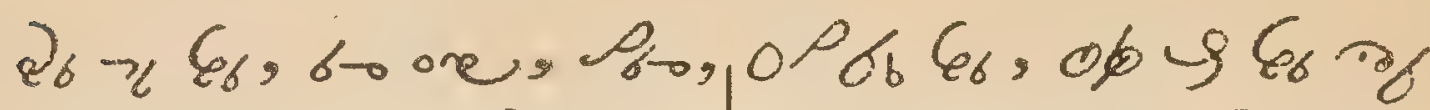

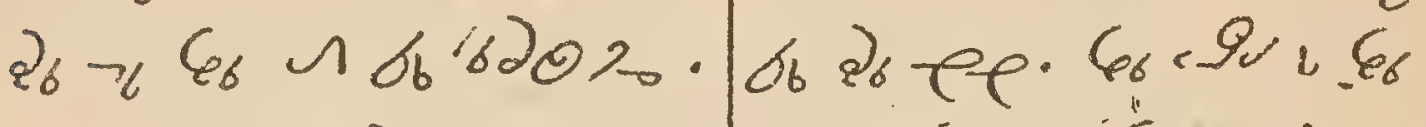

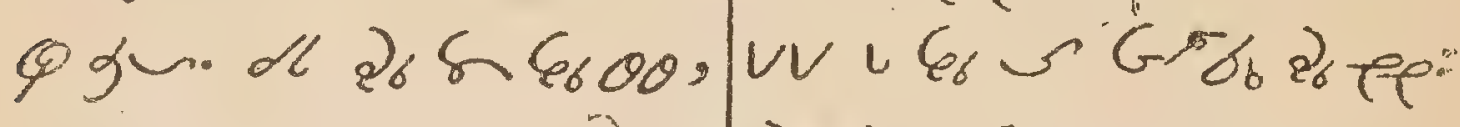

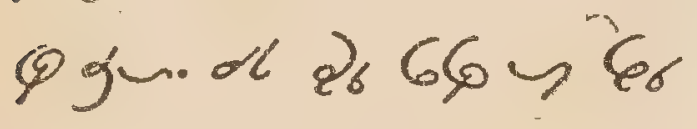
er.

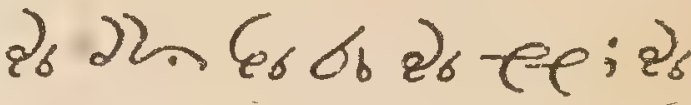

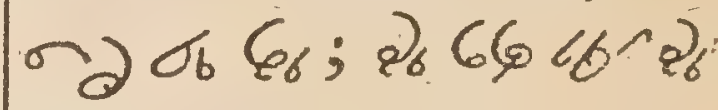
Pe $\sigma_{6} 68 ; 2866$ ore on Desire. Q L er o.

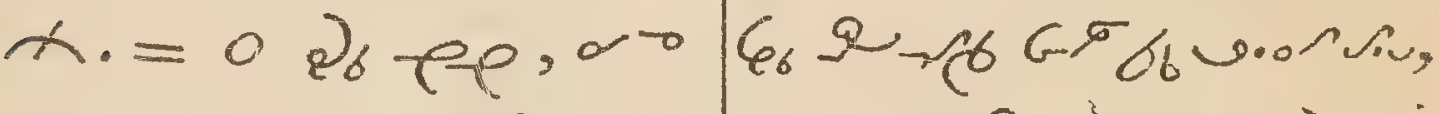

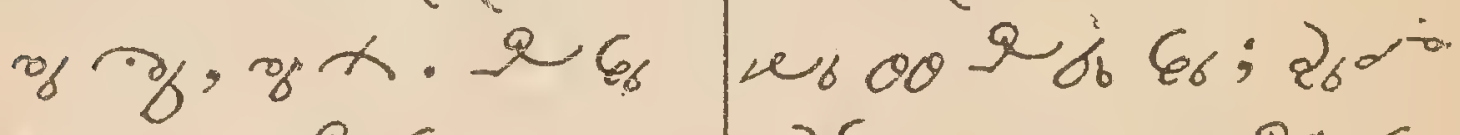

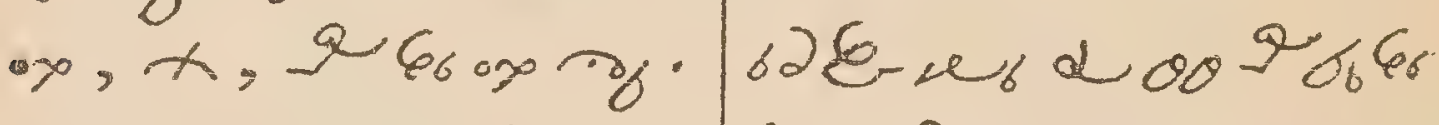

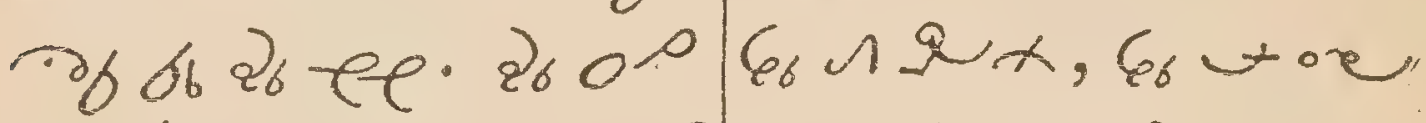
$\sigma_{6} 68,666000609.00,686682010$.

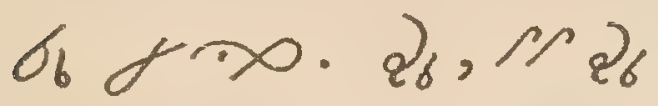
Q o ob $\varepsilon_{6}$.

Thanksgiving. Q $2{ }_{6}$ $028, w 066$, $00 \operatorname{cu}_{6} x_{0}=0 \varepsilon_{6}$ 10.- $2666,660 \cdot 12_{6}$ L. कु 60 Q ax, $\varepsilon_{b} \partial_{\sigma} \sigma_{b} \theta_{6} e_{0}$ $\rightarrow \varepsilon_{6} \partial_{0} \sigma_{b} \varepsilon_{6}, q_{\sigma}$

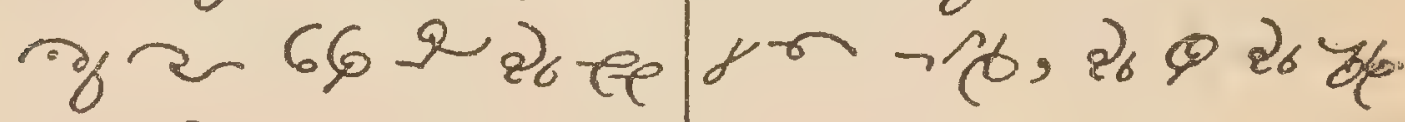

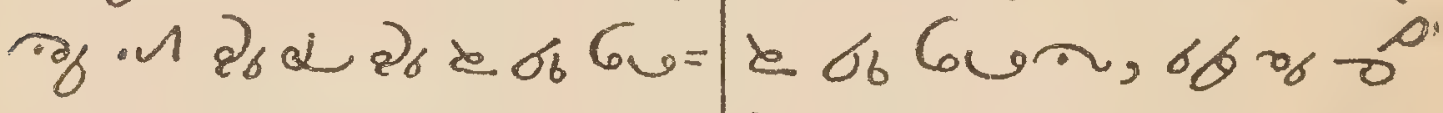

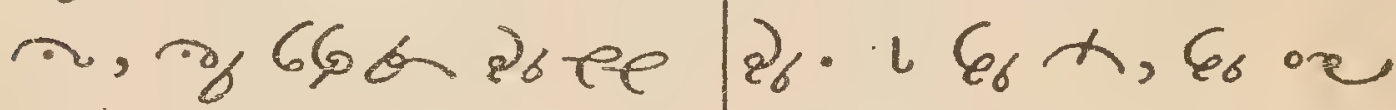
$\sigma_{6} 6$ 等。

$.000,1 \varepsilon_{b}$ go $\sigma_{b} \varepsilon_{b}$ ep. $G_{6}$ ore $Q$ on, $\xi_{6}$ ore

After Communion. $\Longrightarrow 66000 \varepsilon_{6} \cdot \varepsilon_{6} Q$ Adoration. ox!' $\varepsilon_{6}$ x., $2_{6}$ oo in $\sigma_{b} \varepsilon_{6}, \sigma_{6}$

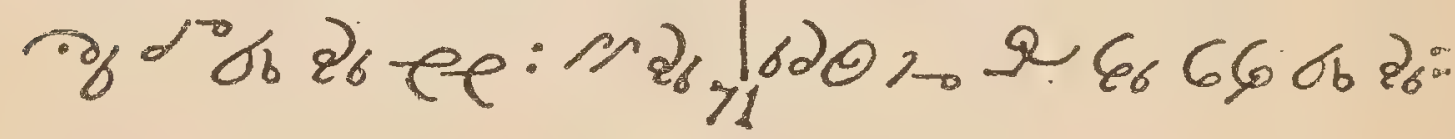


$\left.4_{6} \partial_{6}\right\}, 6_{6}\left\{Q_{66} \rho_{0}\right.$, al in of 6 un 26 66 ; द.

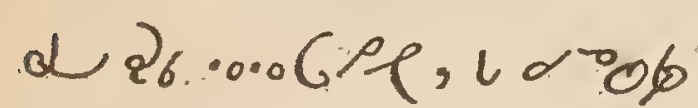
$9,66.6 .9066=9166.86$.

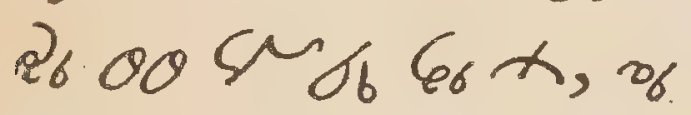

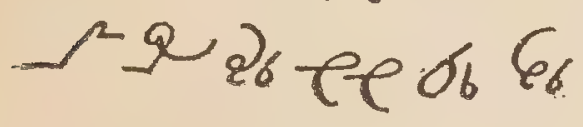

Petition. $2_{26}$ ur

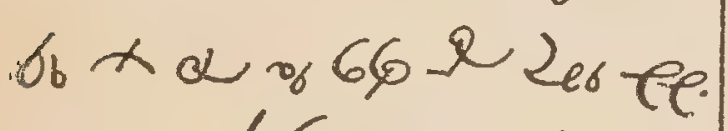

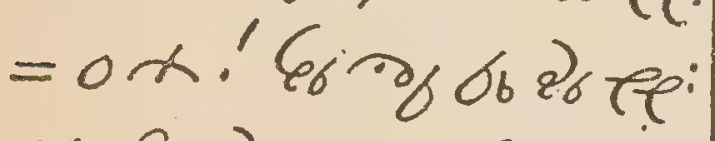

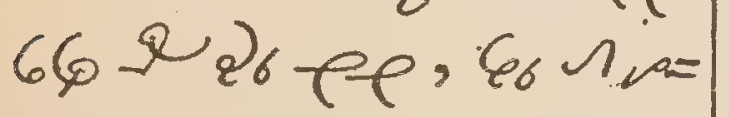

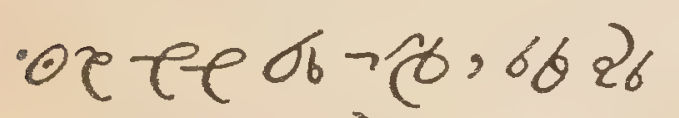

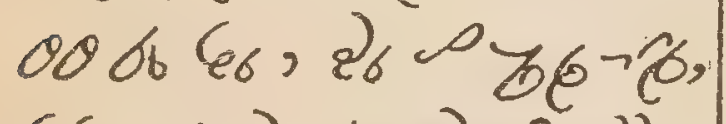

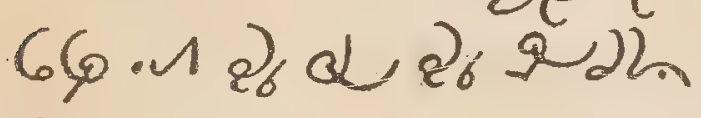
26 eeal bun so 26; 660 be 2b eed

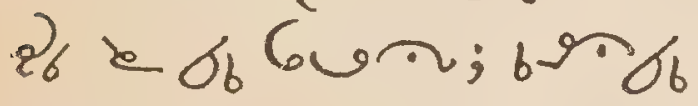

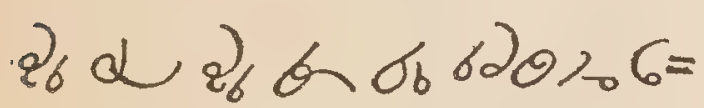
un; al 6. 20 $22 \pi \sigma_{6}=$ $\sim, 0 \times 2610006 \varepsilon_{6} ; \Omega$

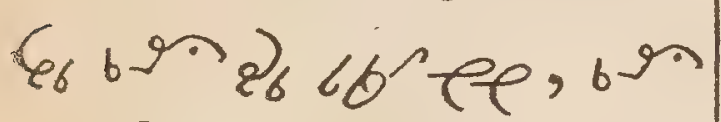
ol $2_{6} \theta_{0} 36 \sigma_{b} a ; 66$

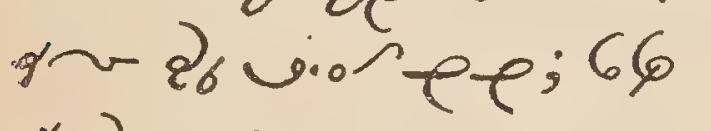
bror ee; 66 us

\section{$\gamma^{660 ; 66<480}$}

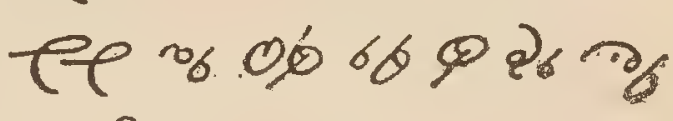
1 \&.

Resolution. Q2e660 arn $\sigma_{b} x \sigma_{0} \alpha$ 2as 2 66 6n: $=0 x .22 n$

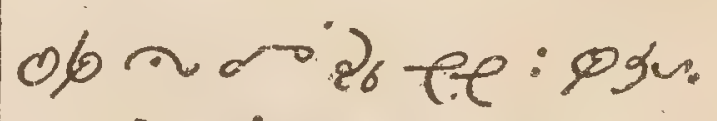
on $q_{6}$ \& bob $6 u n$ ? $66 ; 9 g \sim 0.2860 \gamma$ Go; 9 gu' bu $a_{6}$ < ob

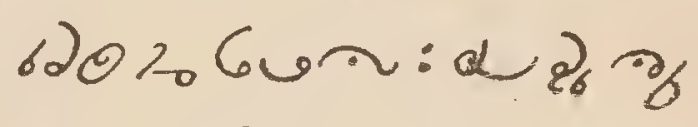
ee $6660 \pi, 0 x 2_{6} 66$ us ar ee; Mroodo op 26 bunibin

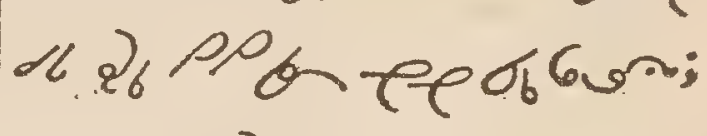

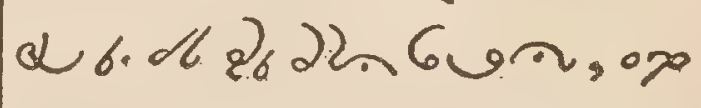
$z_{6} \backsim \sim \sigma_{b} \varepsilon_{6} ; \phi g u \cdot d$.

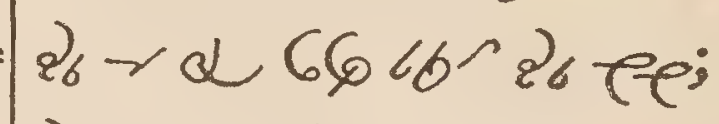

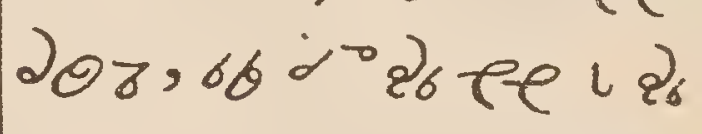
$\Omega^{2} 80.0 \times 660.100$ $\sim \alpha a_{6} 66$ a b ee;

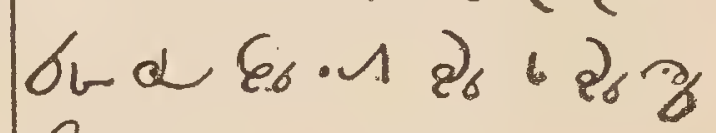


MANUAL。

67

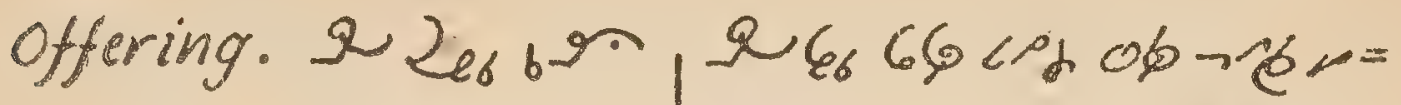

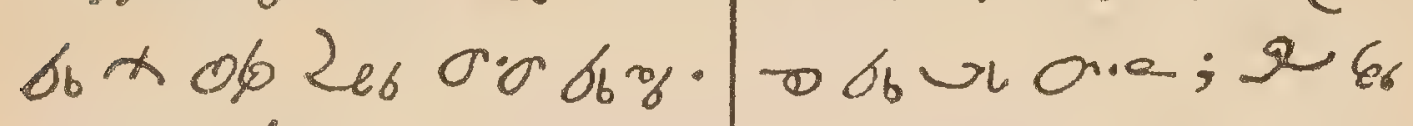

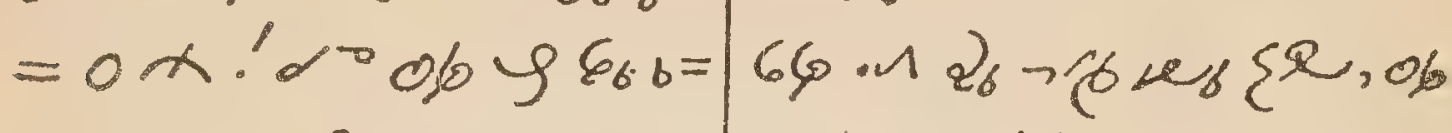

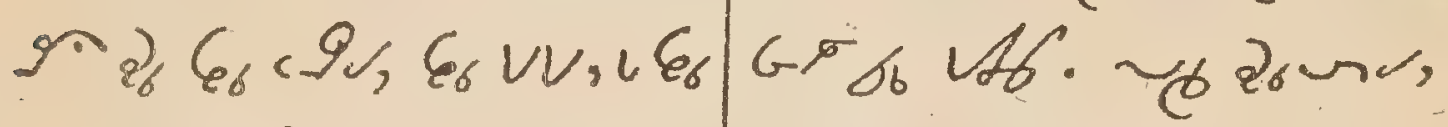

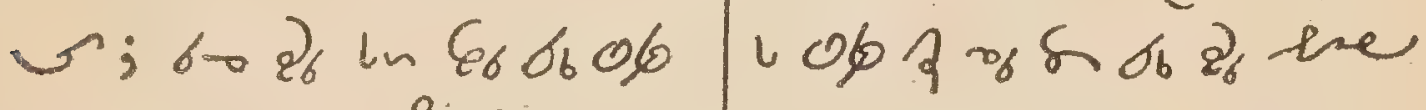

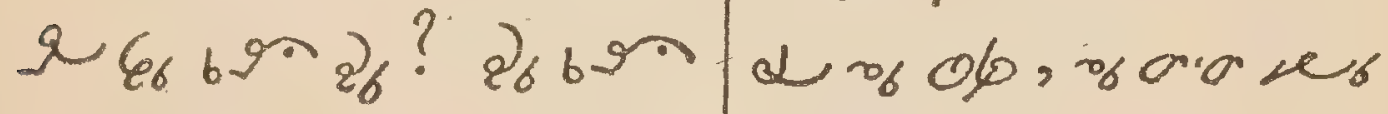

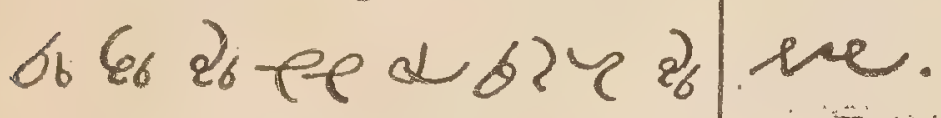

efob 6, a d कर के?

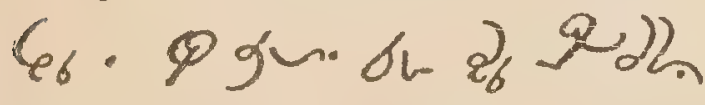

दo do do ep. 2b bra

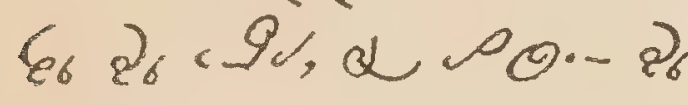

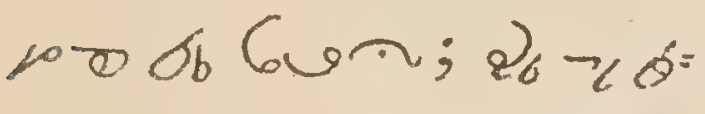

$2 \tau$ श $2266 \sigma_{b} \oplus ; P_{6}$

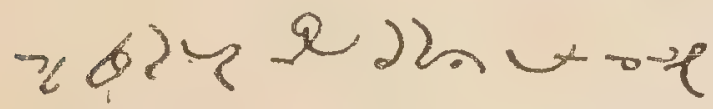

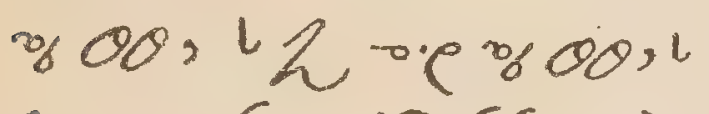

$20 .-\varepsilon_{6} b^{20} 66068 \%$.

The Rosary.

1. Joyful Mysteries.

1.6. - sn: : 2or te

$6+-2 \%$ क 8

-2:

य ग 2 कु कुष 9

$\sigma_{b} \propto 8$

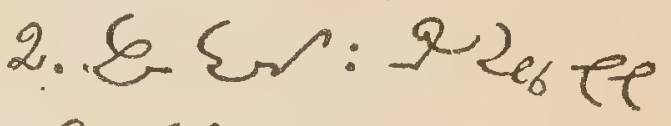

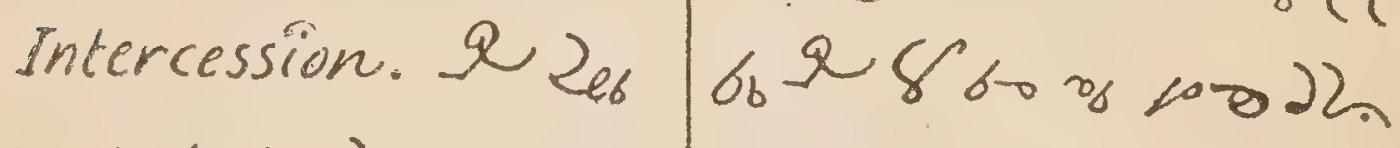

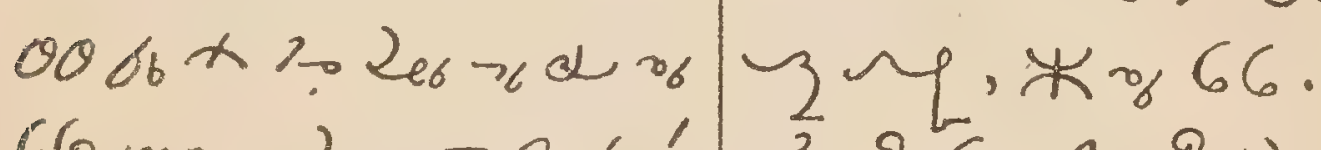

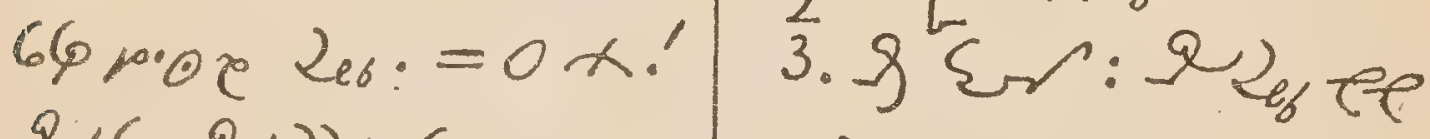

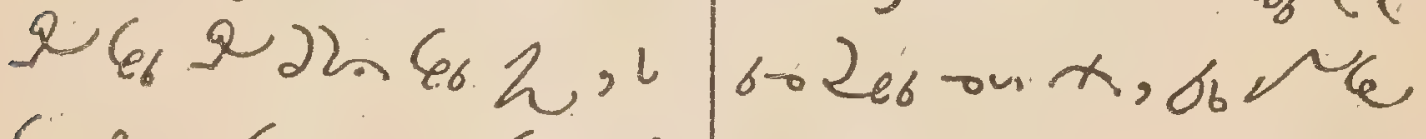

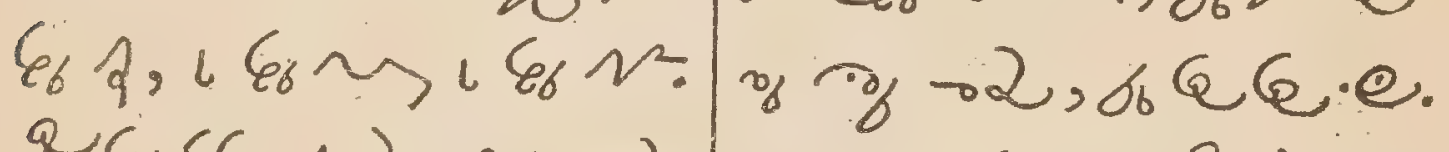

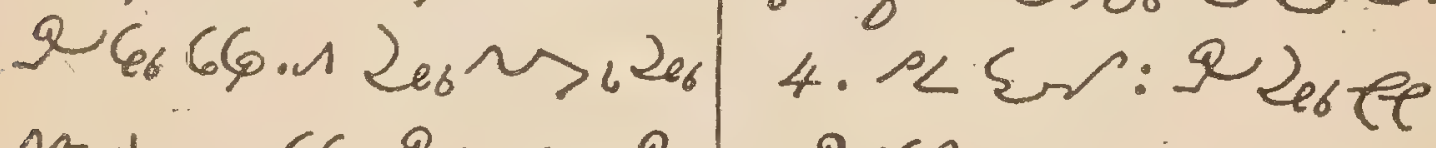

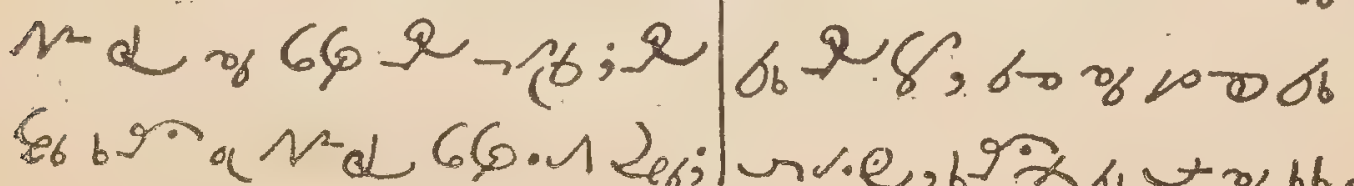

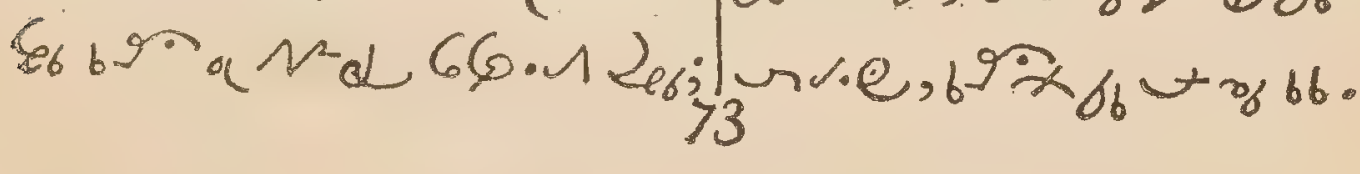




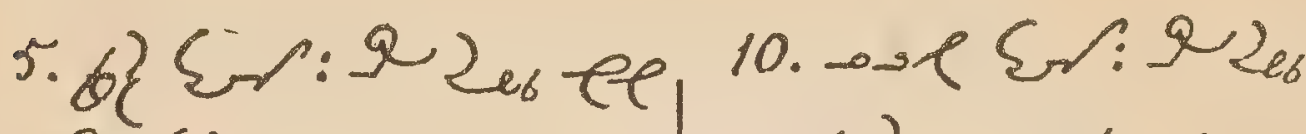

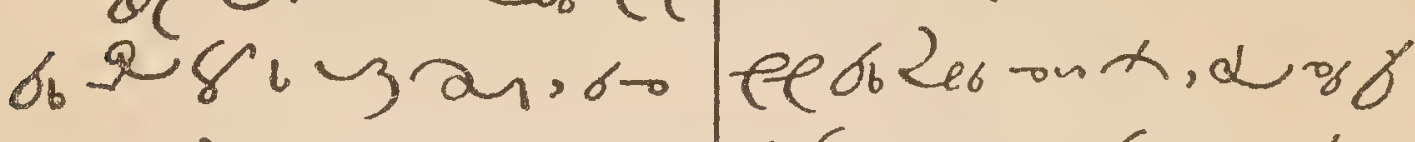

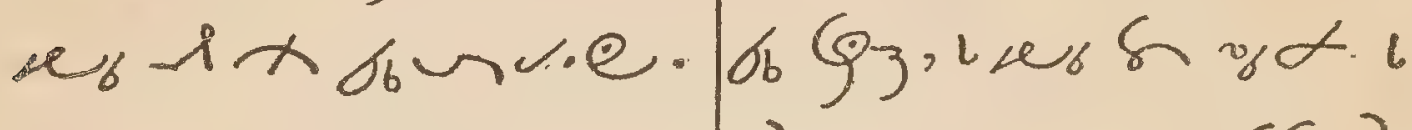
20\% re;us 662

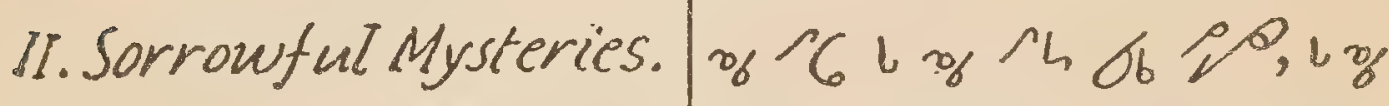

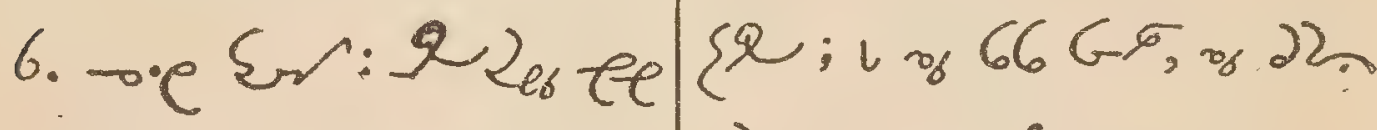

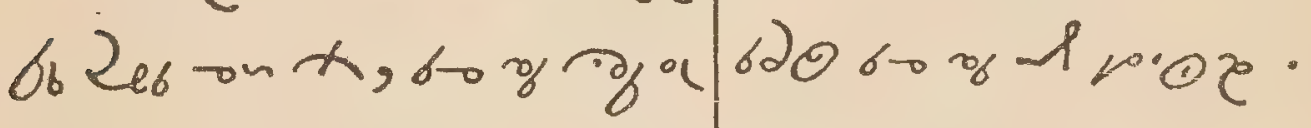
$\rightarrow$ ee ob ${ }_{3}$ : 10.0) bo oc 94,1000 bo s.

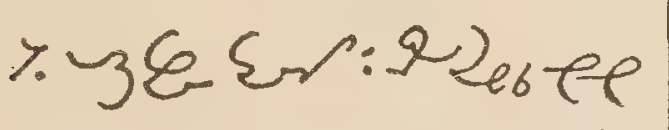
$6626 \sin x, 60$ og

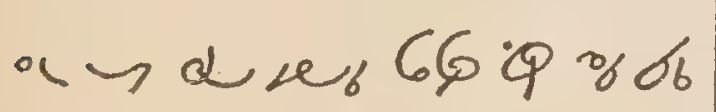
¿ 2 ro.

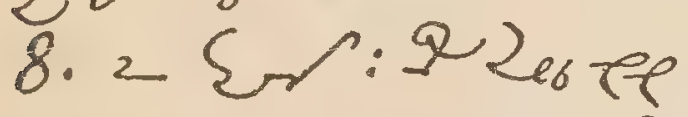
6r 2es on \% b y以lgay s.

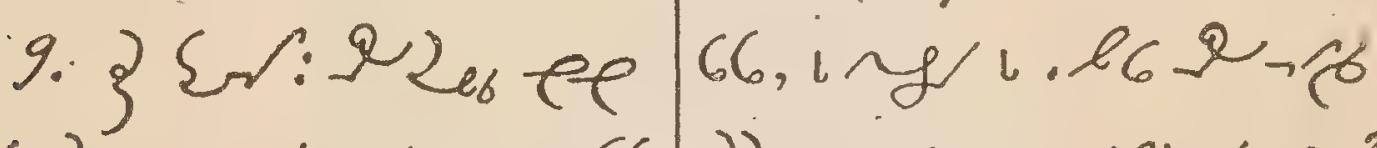

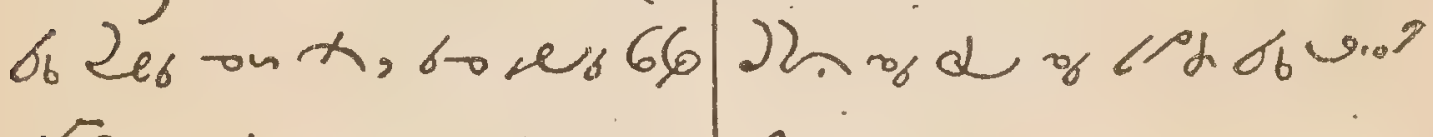
g.e \% l

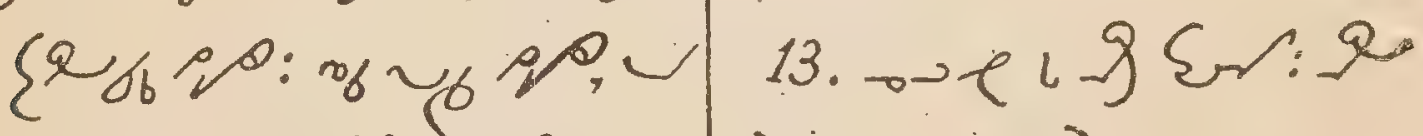

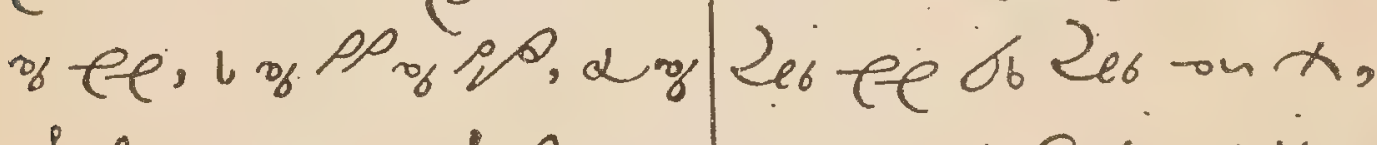

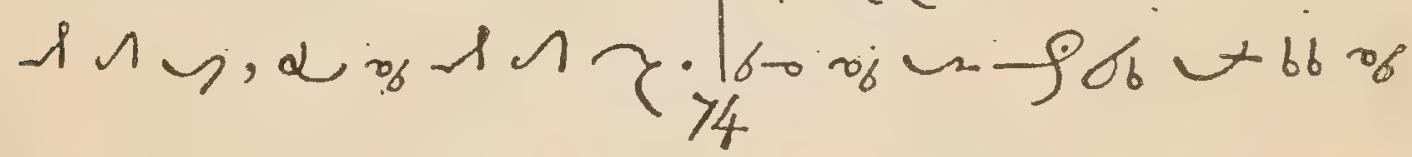


MANUAL.

69

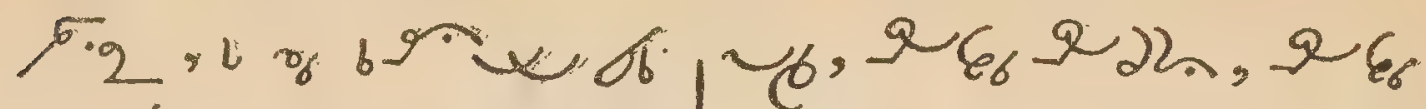

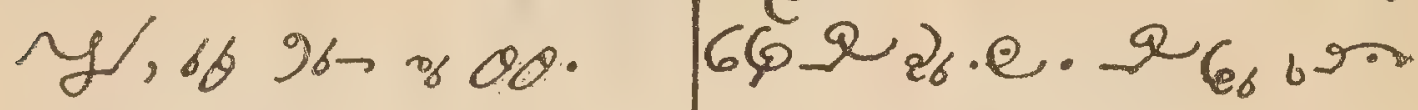

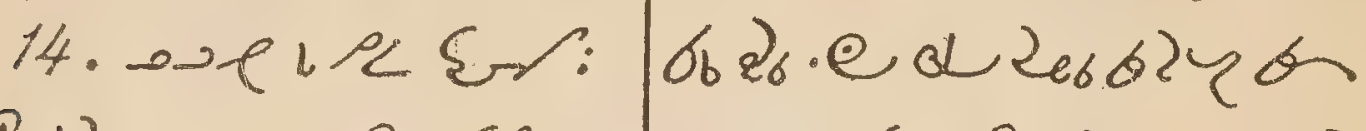

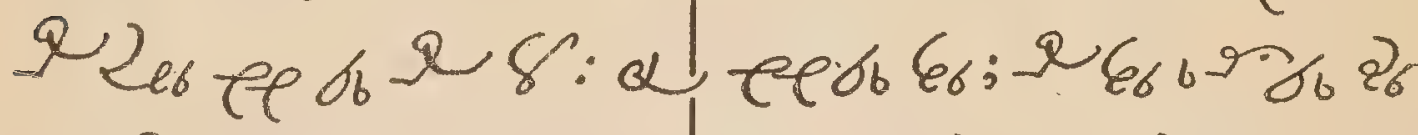

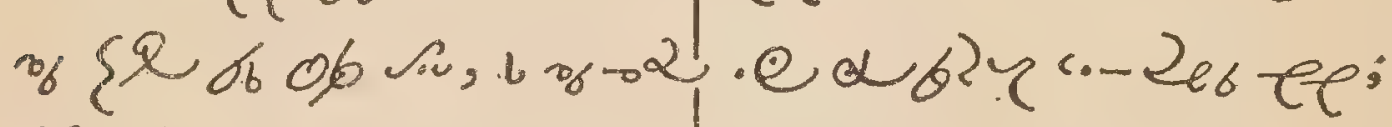

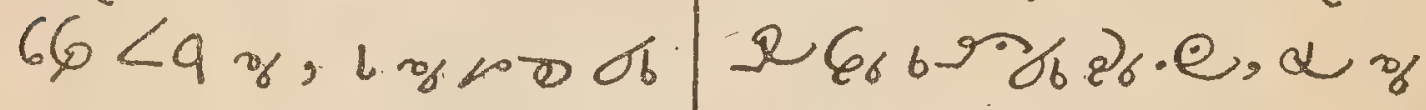

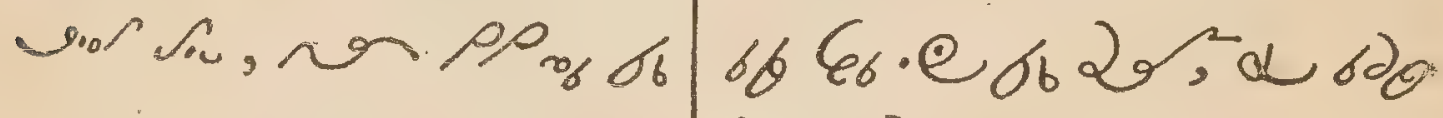
vis

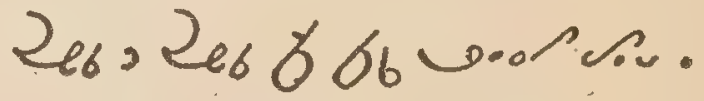

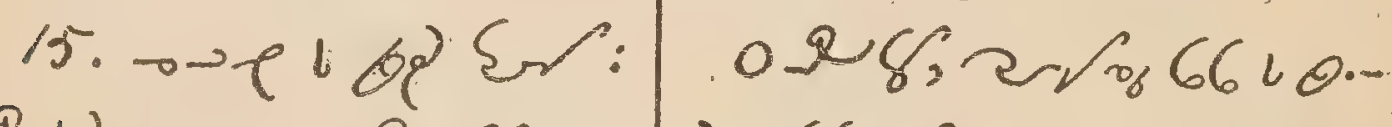

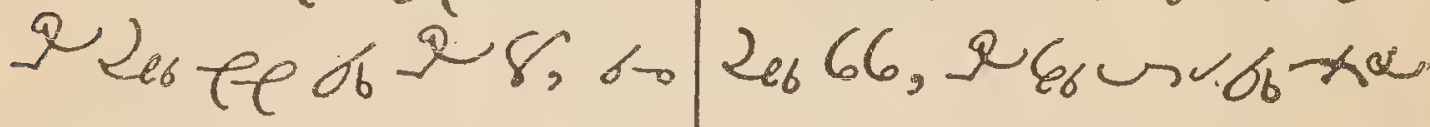

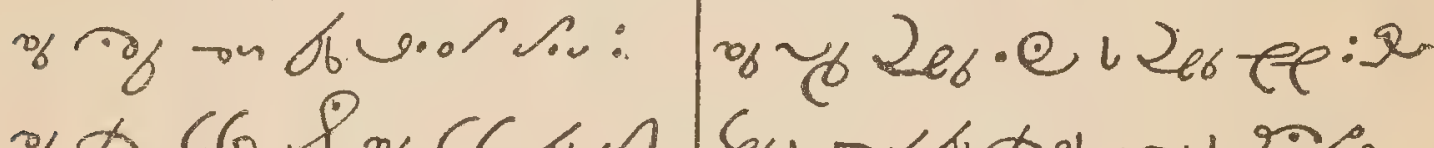

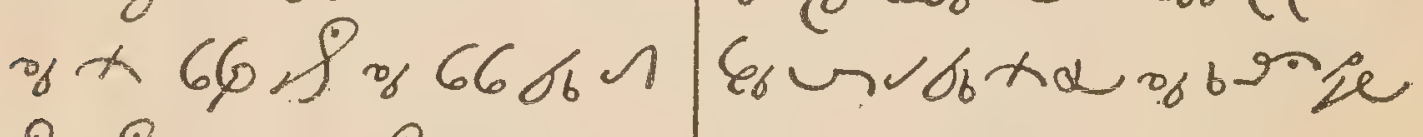

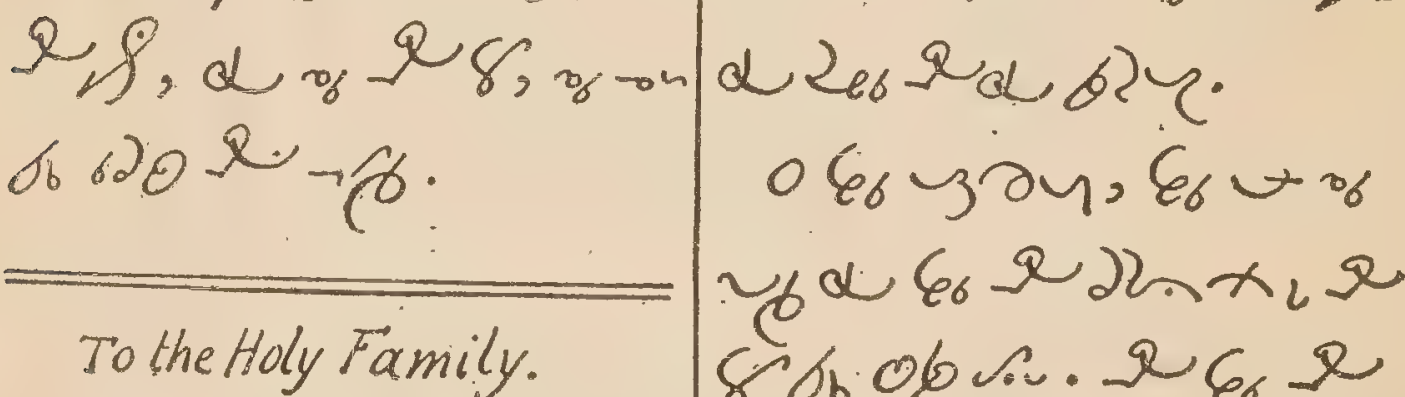

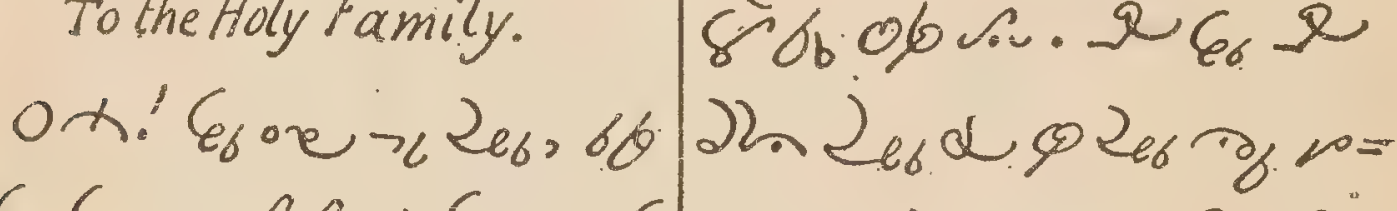

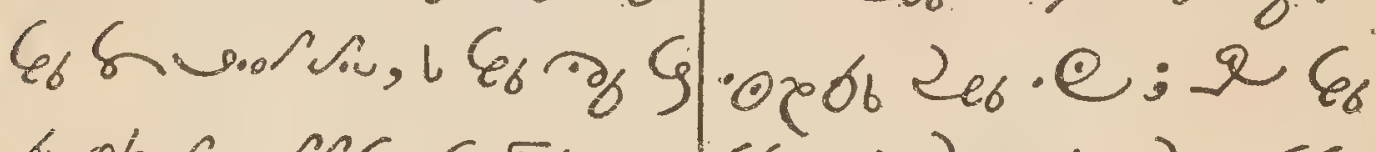

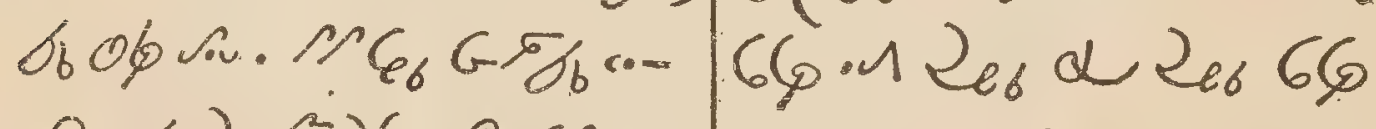

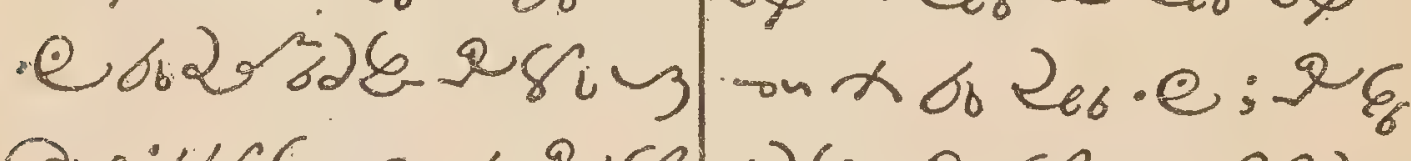

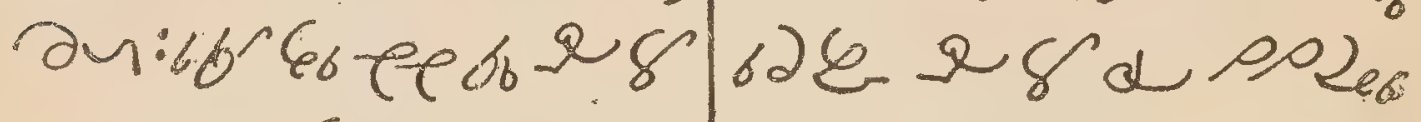

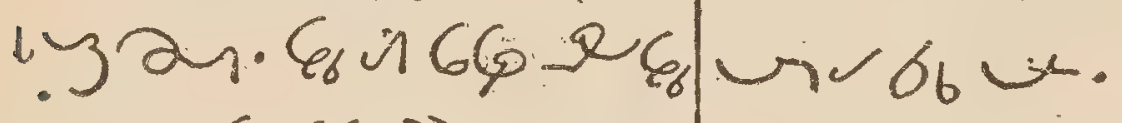

- e, a $\varepsilon_{6} 66022 . \sigma_{6} q_{6}$

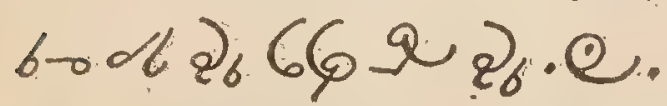

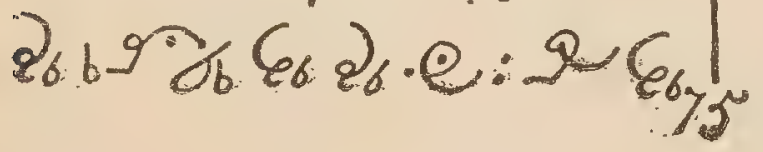


70

CHINOOK

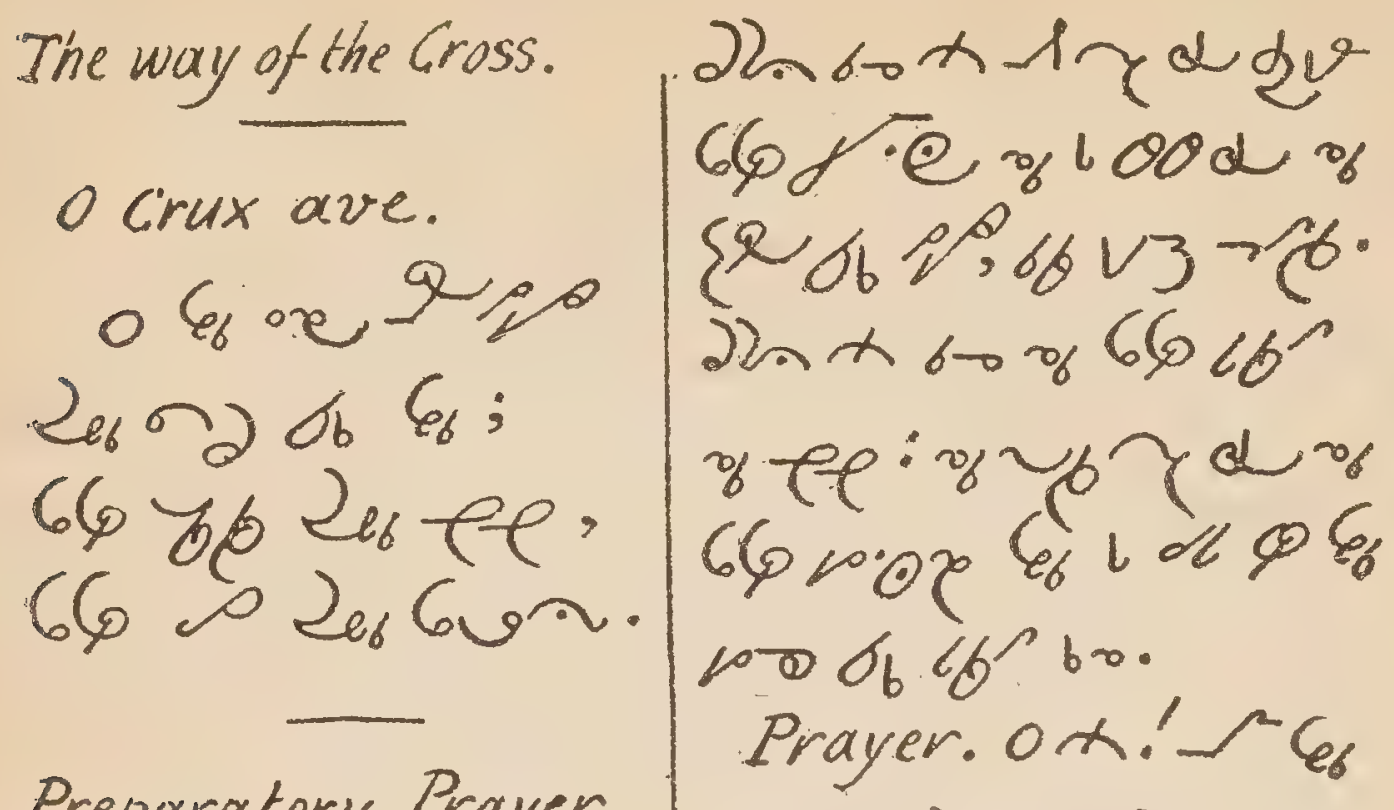

Preparatory Prayer.

ou.' $6_{6}$ o ${ }^{\circ} 2_{6} \cdot 2_{6}$

$n_{2} \sigma_{b} \varepsilon_{6} \sigma: \mathrm{S}_{0}$

ee b6 $60 \nu_{0} 6 u n a_{6}$

66. 207,2666 a

$\sim \varepsilon_{6}$ el. $1 \varepsilon_{6}-v_{0}$

Q) $\varepsilon_{b}$ ore $\geqslant q_{6} \cdot \phi$

gu. 0. $2_{6} 6506 \mathrm{am}$.

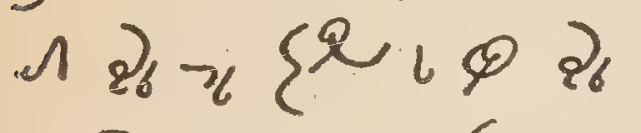

"9 $\rightarrow$ ef ob 6.

I. Station.

Adoramus te. 2egro

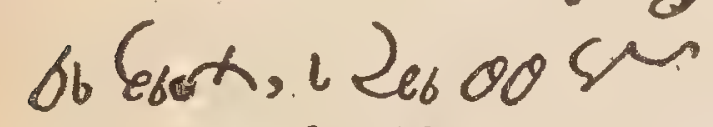

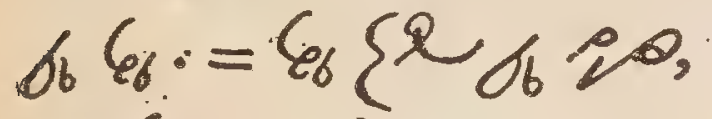

168 6 in $620-C_{0}$.

Consideration. ¿v

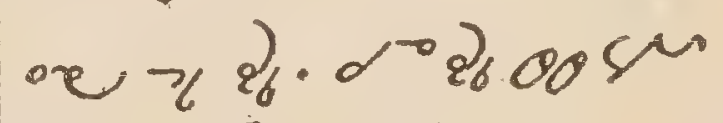

666.2666010006

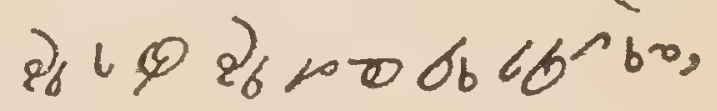

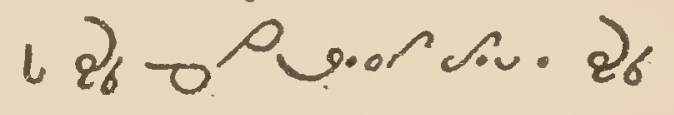

चु बी $\mathrm{fe}^{\alpha}$ a

Gun $0 .-102$.

Pater. Ave. Gloria.

Miserere. Ox 66 rog

$2_{6} .=660$ r.0 $22_{6}$.

Fidelium. $O \Psi 66$

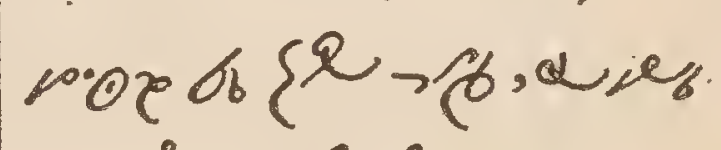

ox 1 v.or א..

II. Station.

$e_{6} 66$ G590, $\alpha$ rog $\rho \rho=22 n x$.

$004 \times 528 \% .76 \%$ 
MANUAL.

$7 \mathrm{I}$

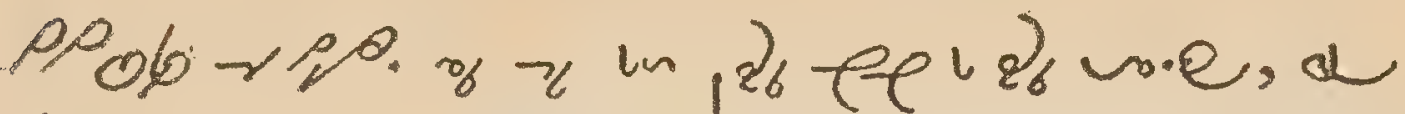

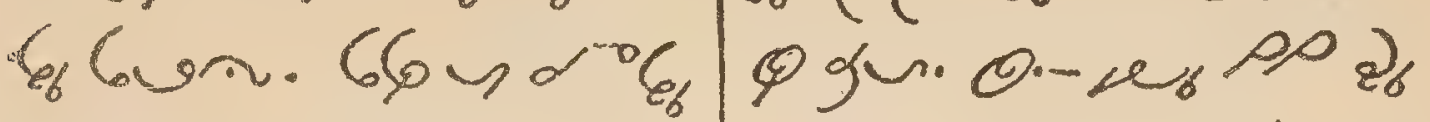

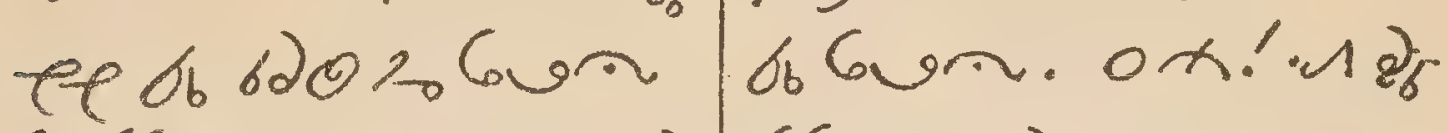

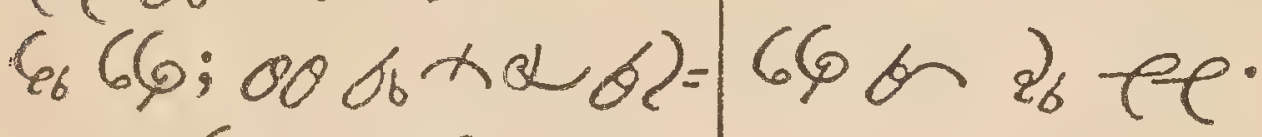

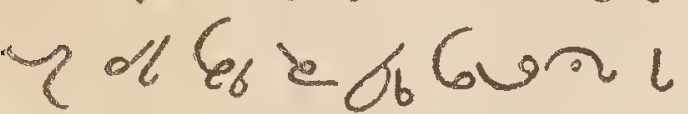

Q gin. 0. - \% $\nabla^{\rho} \varepsilon_{6}$.

Pr. ox. 660 pore

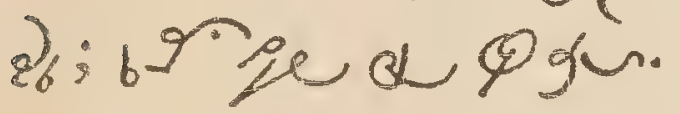

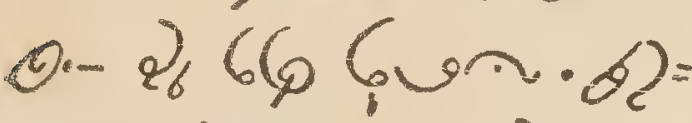

$\tau$ do $q_{6} 66<6$ क

(

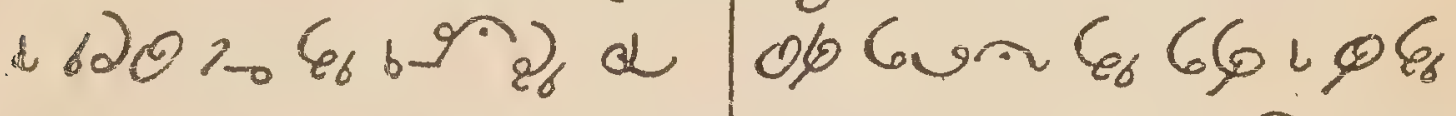

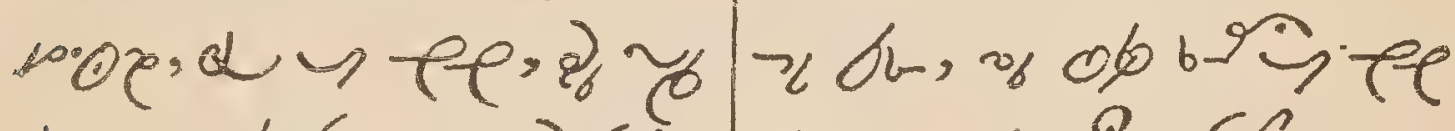

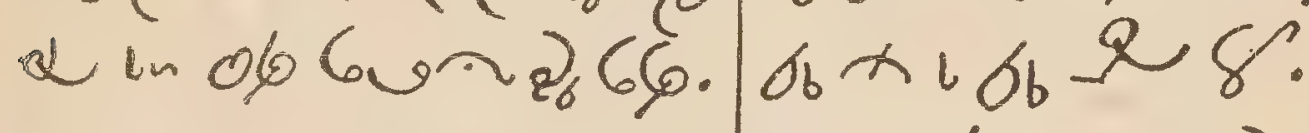

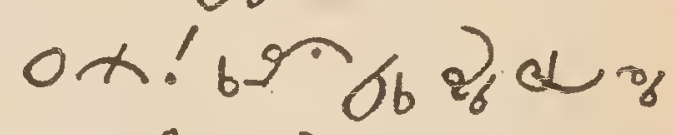

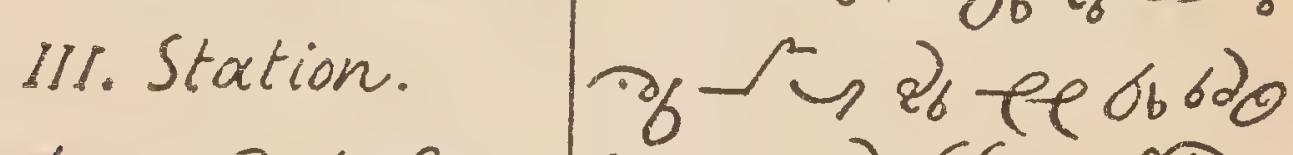

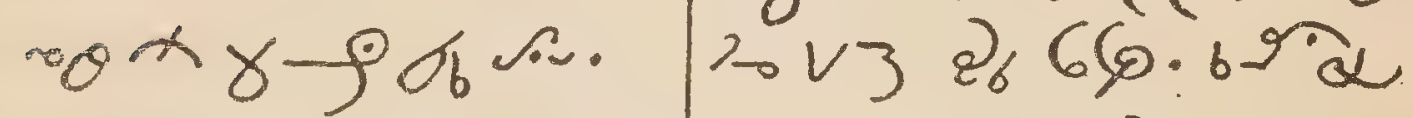

$=22$ 人。

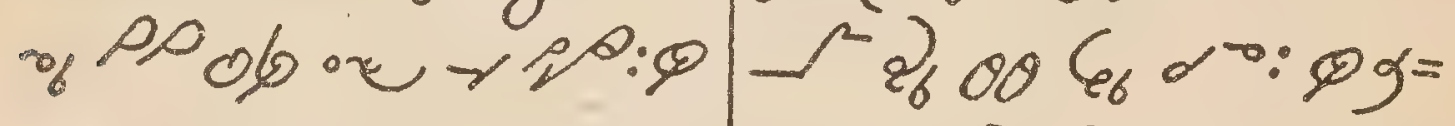

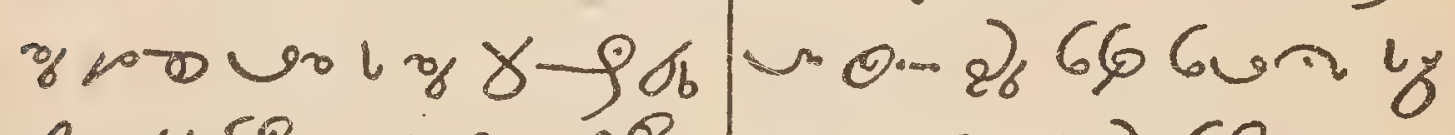

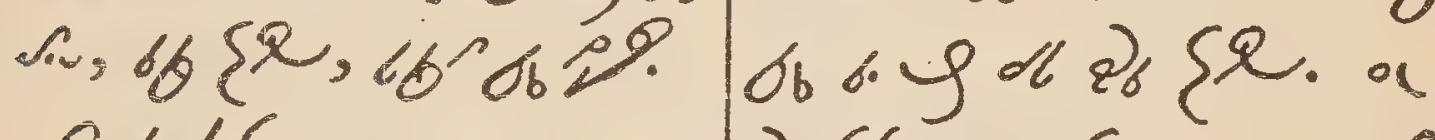

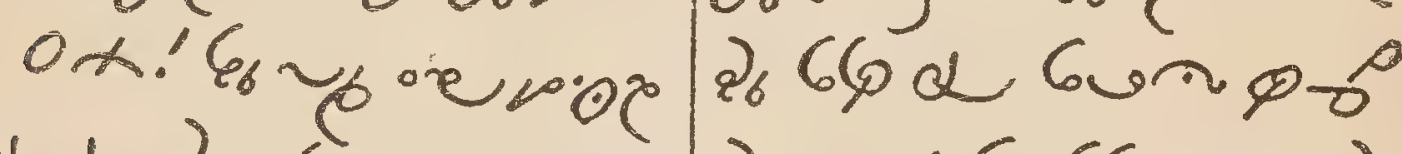

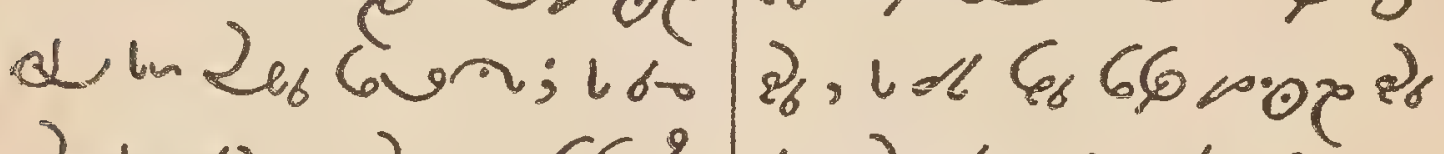

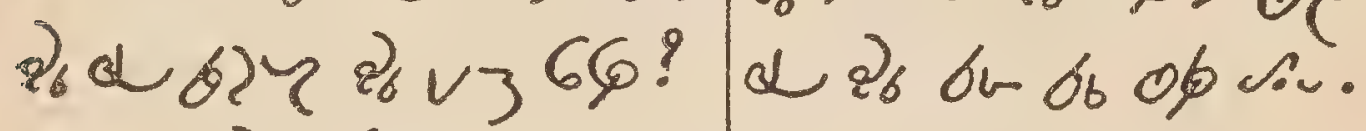

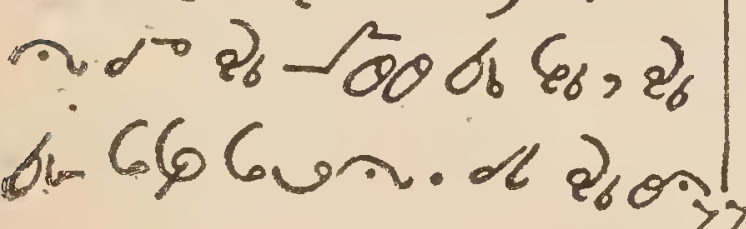

- 


\section{Station.}

r.1x a ppos.

$=2 \sin 000 \sim-6$

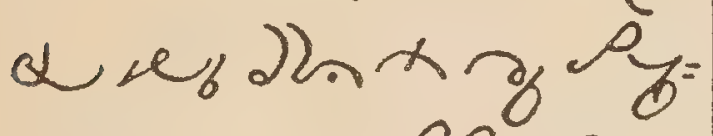

एᄂQ6\%

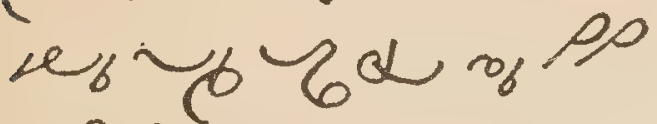
P. $28 x$.

$0 \times ! q_{6}>P \rho P \theta_{\sigma}=$

$\varepsilon \varepsilon_{6} \cdot Q_{6}>\ln \sigma_{6} 06$ Gun $2_{6} 66 \cdot \alpha q_{6}-1$

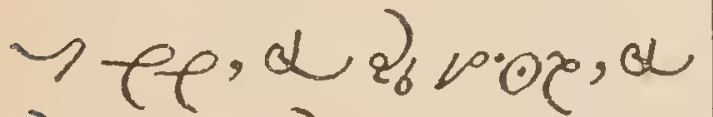

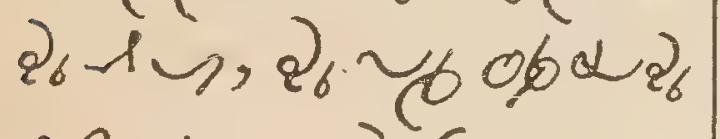

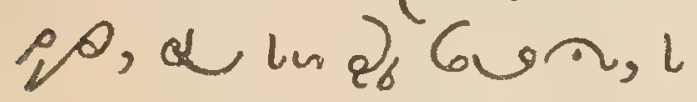

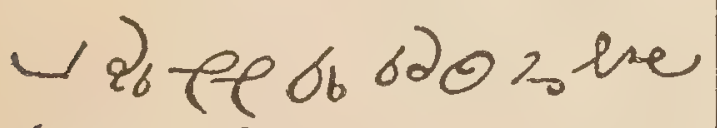
$\varepsilon_{6} 6{ }^{2} 2_{6} \sigma_{6}$ ol $\Omega$.

\section{vr. Station.}

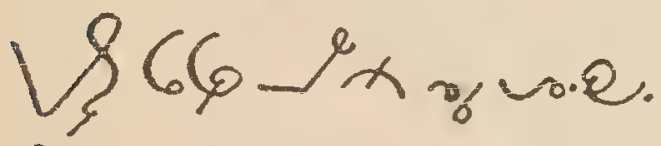
$=2$. 0,0 on $\xi \partial \varphi$ \% efardh

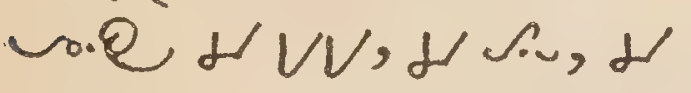
Gon. \% क्याओ

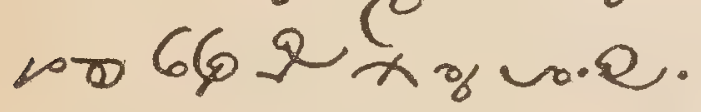

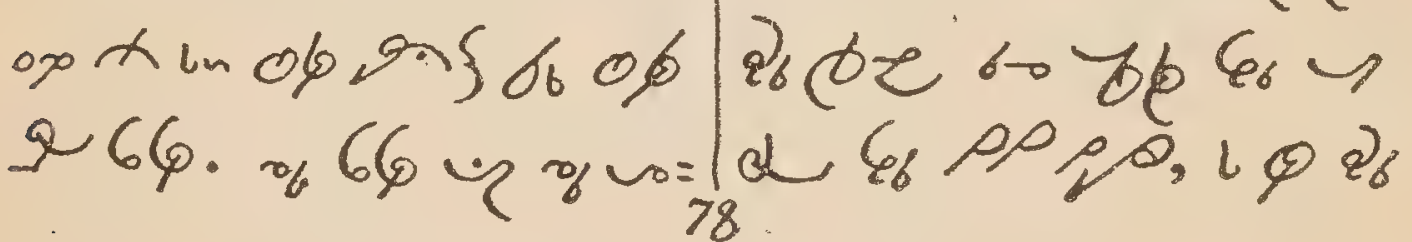

$.26648 \% 40=$ ox! \% \&6 Gun, $6 \theta$

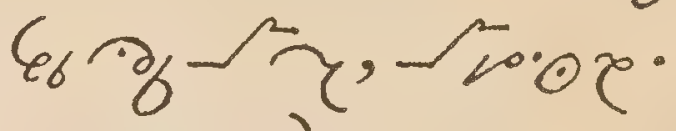
of be a eda 2) - 24660 6un, 1 $G_{6}$ ג, $6600002 z_{6} ; 66$ 066 - 2 26 66 ; 66 on 26 ee br war

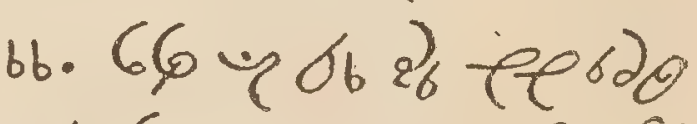
of $6.496 \rightarrow$ al $\varepsilon_{6}$ Po

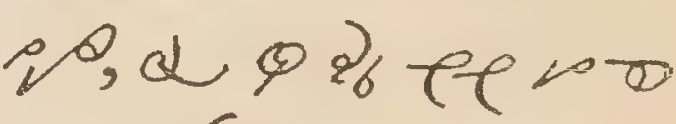
bo bun.

VIf. Station. $x$ E- or $\gamma-\rho$. $=2 \pi x 6$ का

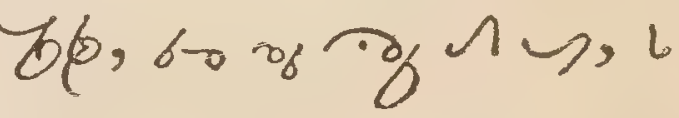
\% $\theta-\gamma-\rho \sigma_{b} \alpha, 8 \phi$

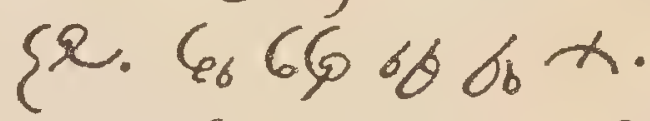

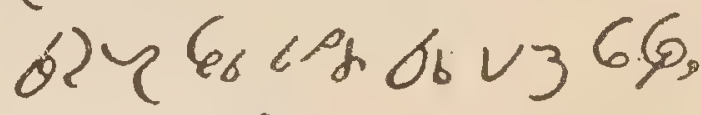
by 1 1 1 ee. ing $\gamma-906 \Omega$. ox.'s sqa ee.

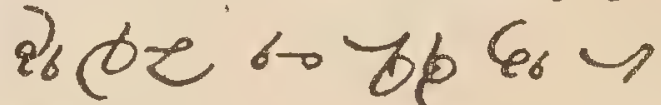
78 
MANUAL.

73

- $a_{6}$ e

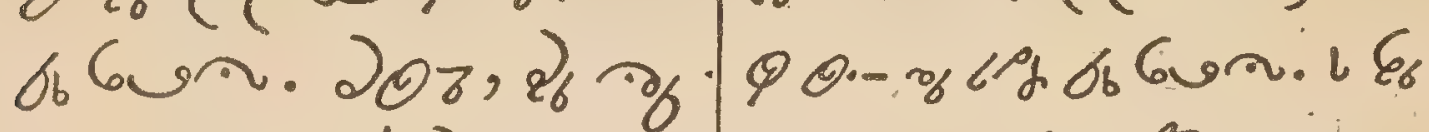

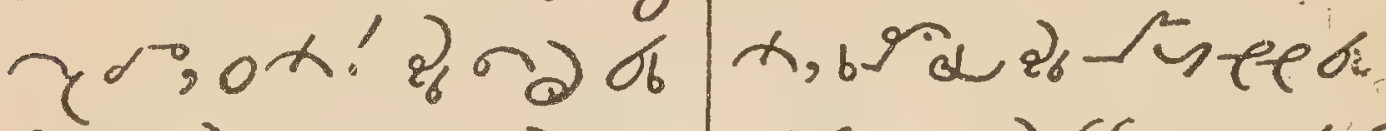

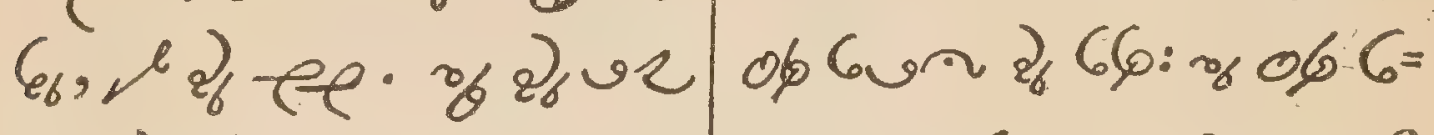

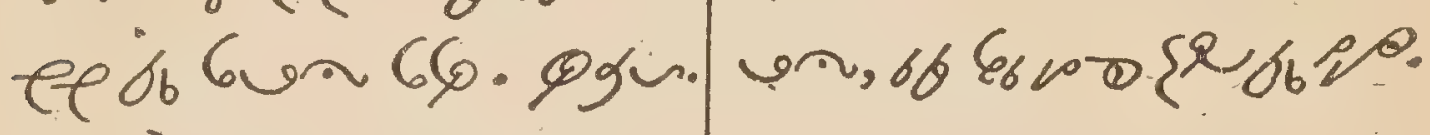

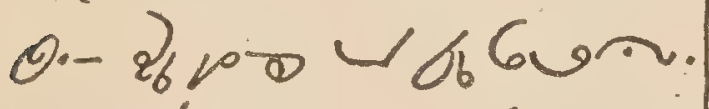

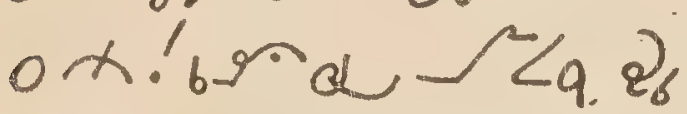
cedis $66,199 \mathrm{gm}$. IX. Station:

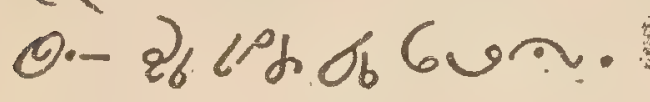
$x-9 \sigma_{0 y} \gamma-\rho=2200$

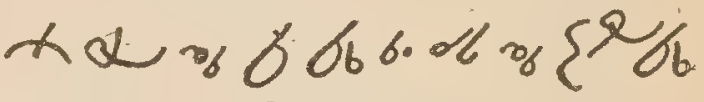

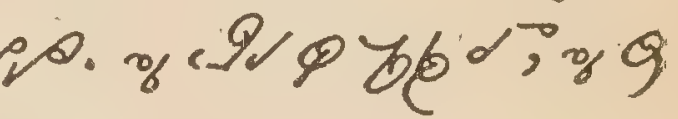

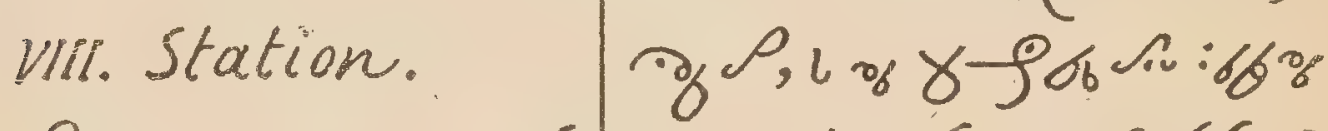

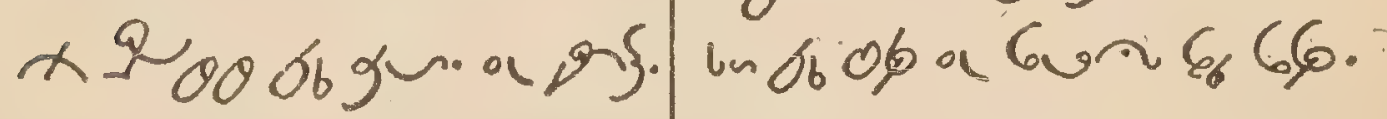

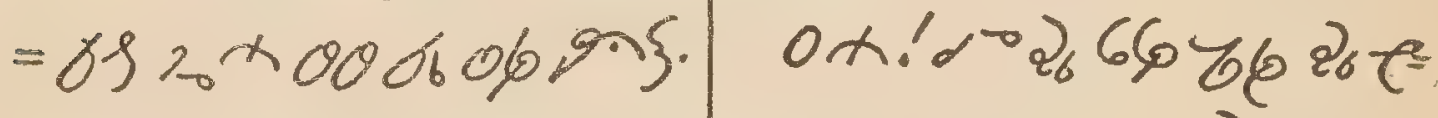

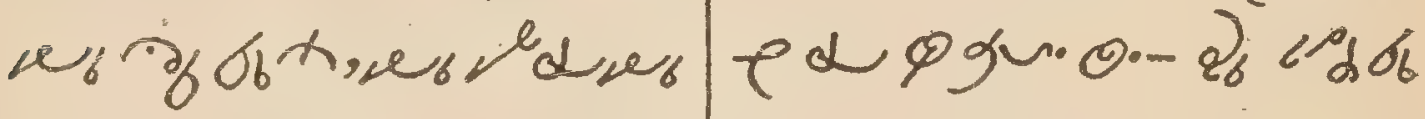
In bor or

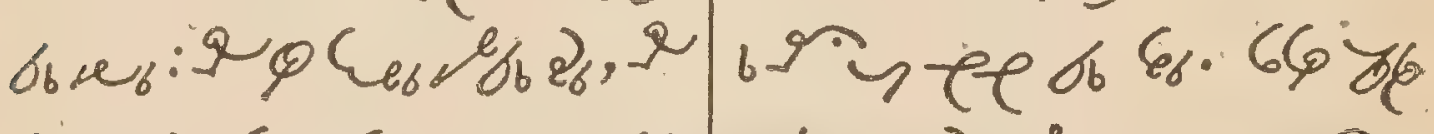

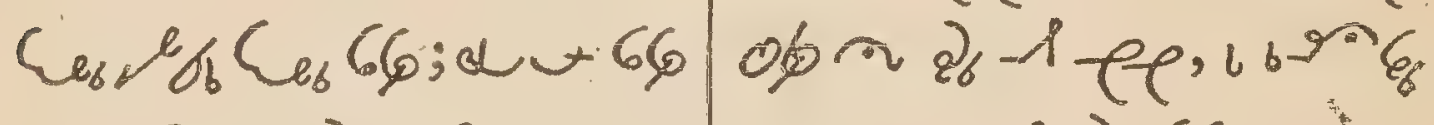

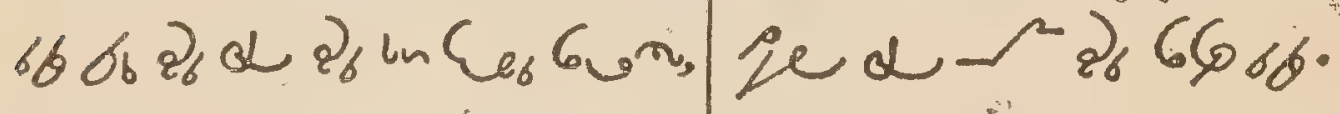

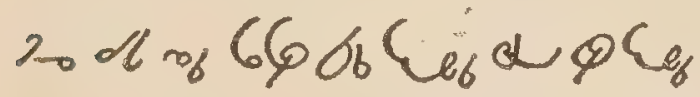
心666u几?

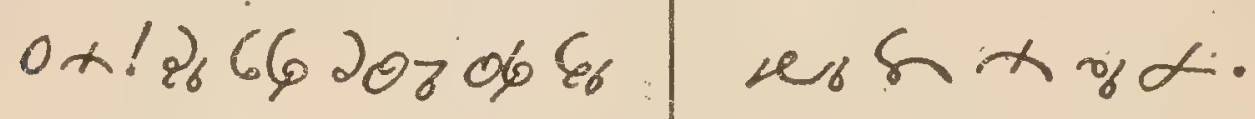

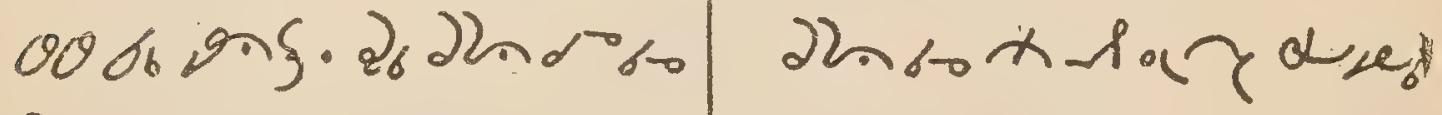

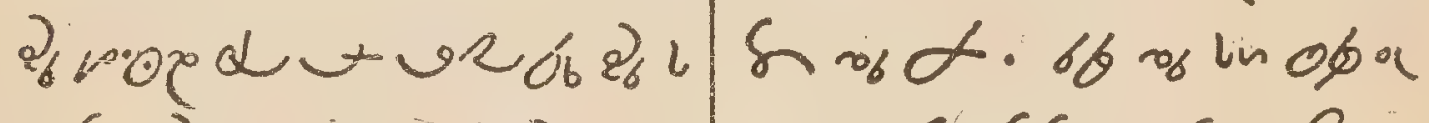

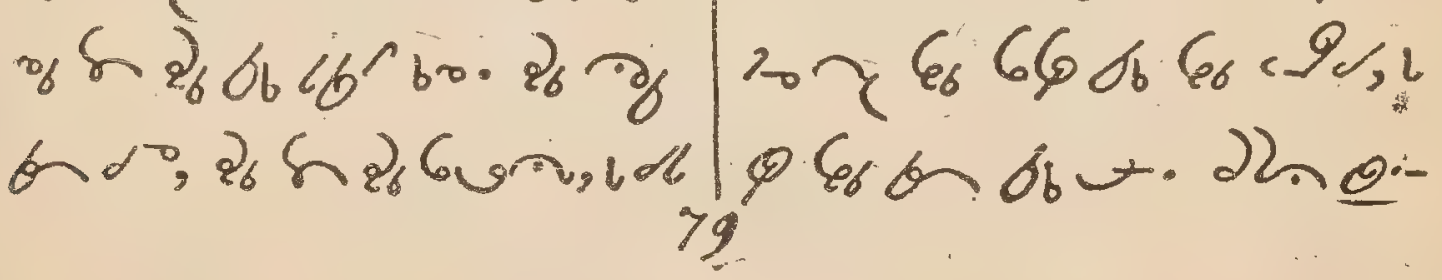




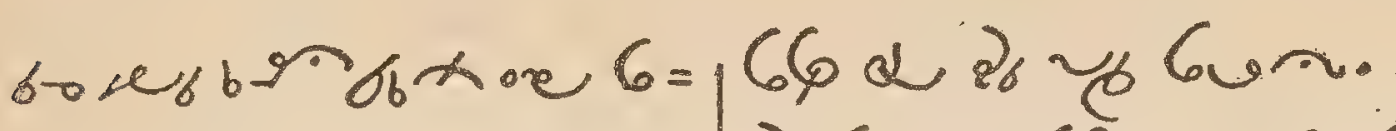

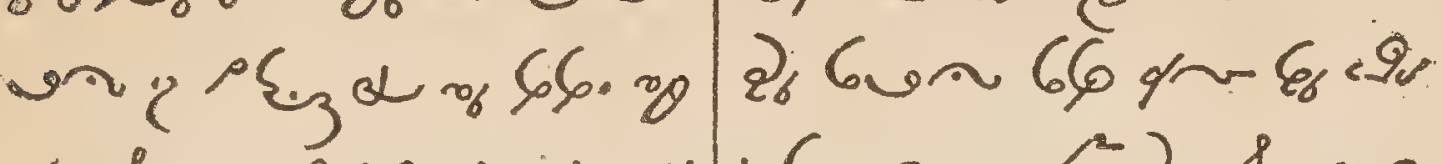

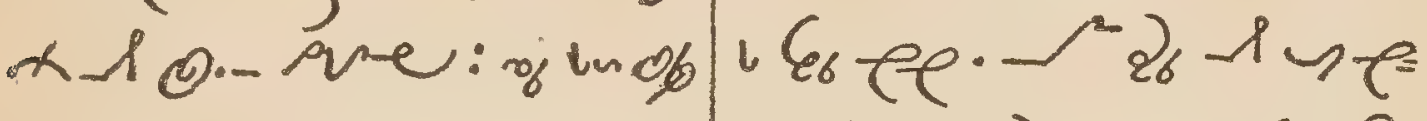
$\varepsilon_{6} \partial_{0} v 3 \sigma_{6}$ bo $0106 \varepsilon_{6} e^{\alpha,}, 12_{6} 0006 \varepsilon_{6}, \Omega$

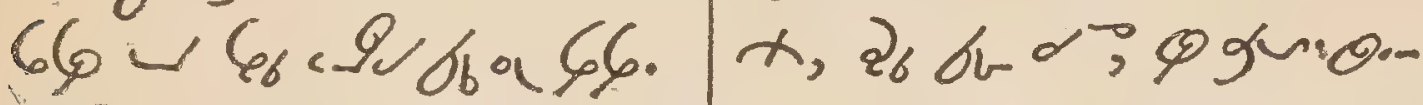

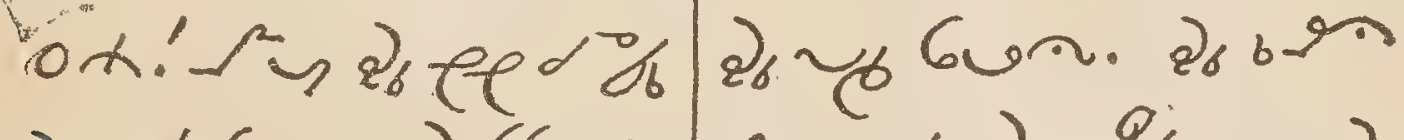

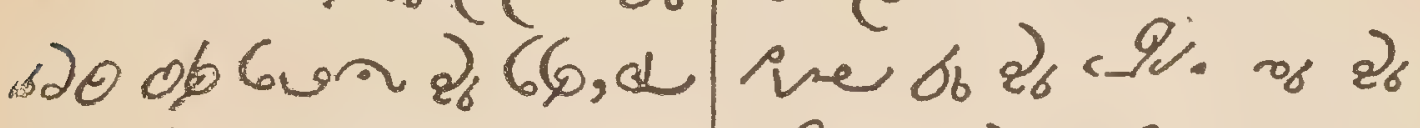
2.86. 66.8605028 - od 9 gui. O.- $z_{b} b^{\circ}$

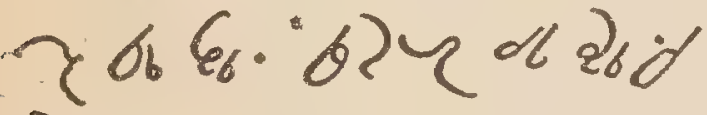
$2_{6}$ eed $\phi$ gu. 0 - r br bua.

c9) 28 br Gun $2_{6} 66 a_{6} e^{-0} \alpha \phi$

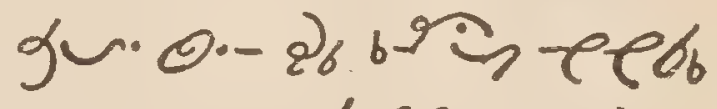
6. ox! 66 or $2_{6}$

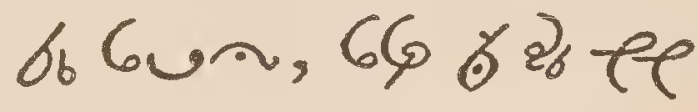
6) 6 6 b P

XI. Station.

eb $6620 \times$ bo so.

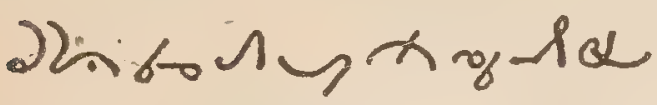
किण б Gur.

XII. Station.

$x_{6} 66$ po ore 206 \% of r T i r thaser

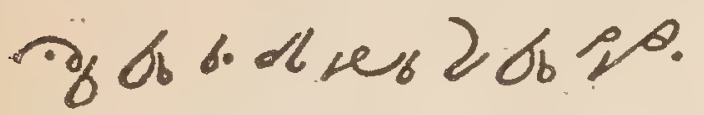
O-x-lor $y$ efa $\% 22 \pi \%$ \% \% $66 \%$ efol $C / 69$ pos of $206.0 \% 166 \% 14$.

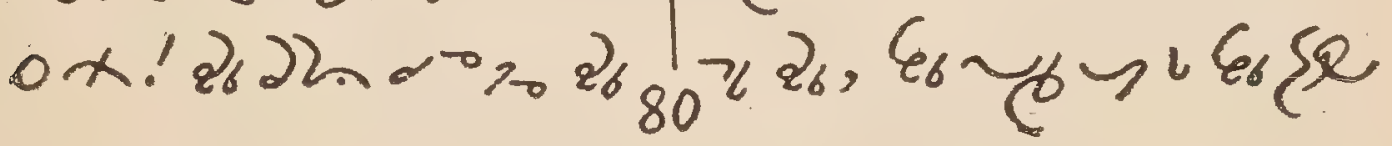


MANUAL.

75

d $692 q_{2} \cdot 2$ are

$a_{6}$ ee $\sigma_{6} \varepsilon_{6}, a_{6}>\varepsilon_{6}+\Rightarrow$

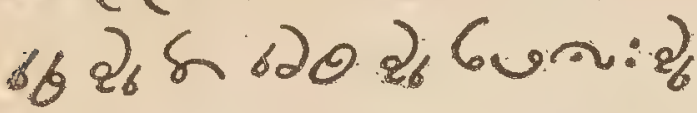

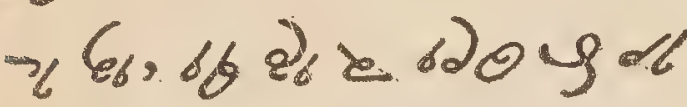

b6 6un; $\varepsilon_{6} q_{6}>0,06 a_{6}$

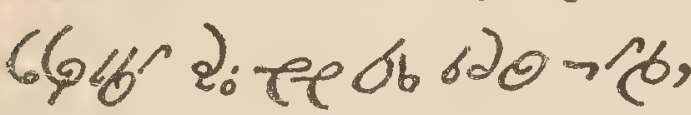

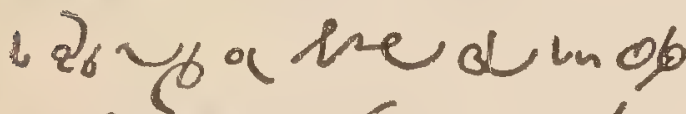

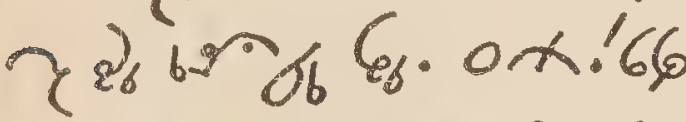

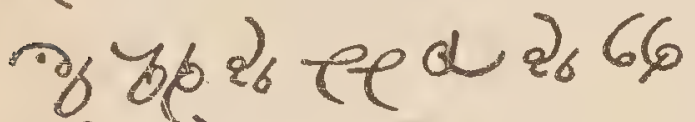

sy 20000.

xiII. Station.

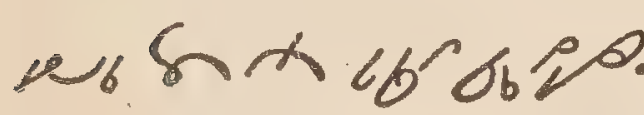

2h to वर्यक्ष

a 8 a y

HWV $\{\Omega$.

060 , 28 are \& 66 .

ni $\sigma_{6} \varepsilon_{6}-2 \alpha \propto 2_{6}-1$

be tel $\phi$ gu. O.- $a_{6}$

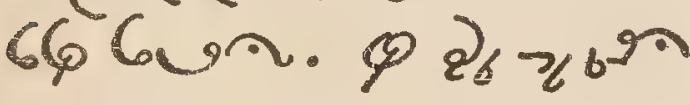

4 ee ob $x . d(286)=$

$\tau^{2} 2 \pi$ al $x x^{2}=$

soton ob a ee.
XIV. Station. ces PPx of as ob $\varepsilon^{R}$. $=22000=$

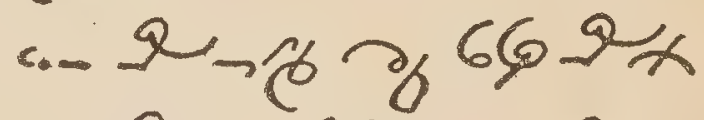

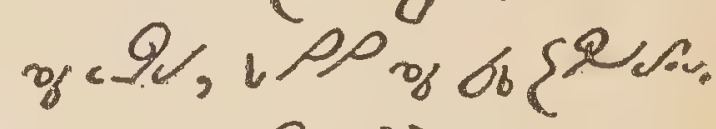
res $Q$ 2hrops

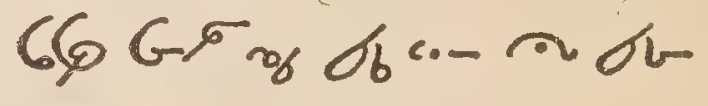
se-g.e do s. $0 \times$ ' 260054b $\varepsilon_{6}$ $666202_{0} 6660$ al $6 \%$ 26. 26 $000066_{6}$ :

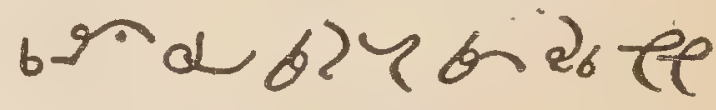
brun; brong te

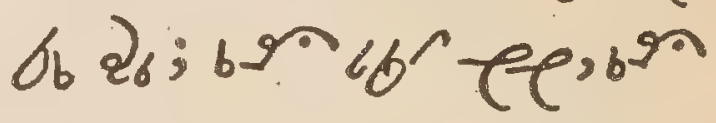

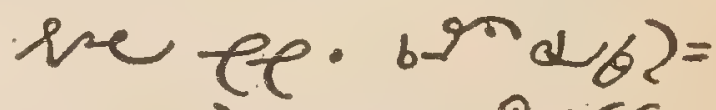
7 b० के ec 06 \% 60 ,

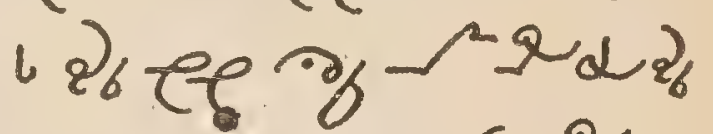
$\sim \oplus \oplus \circ$ de $\varepsilon_{6} \varphi_{G}$ ob gro 68 a 9496506

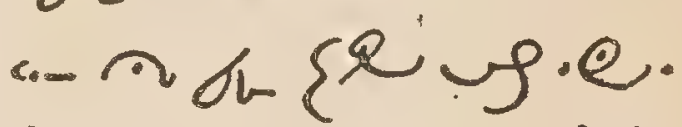

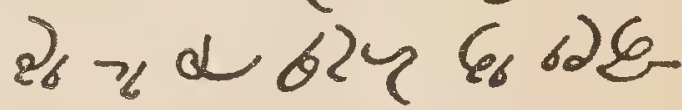

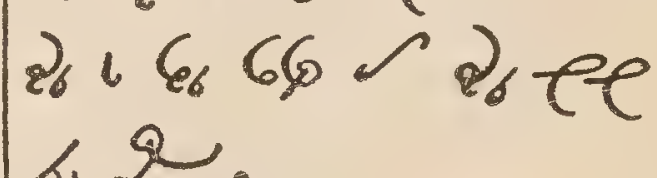

81 
76

CHINOOK

1. O tloos St Joseph.

"Air. paye 182."

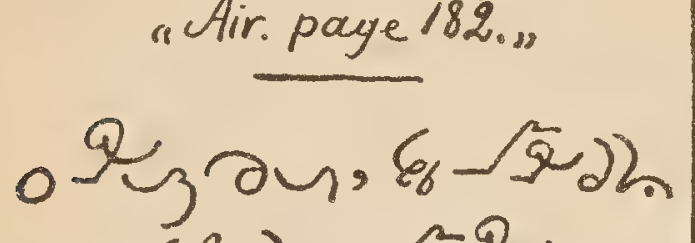

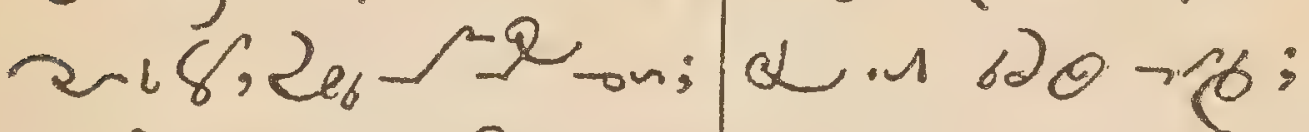

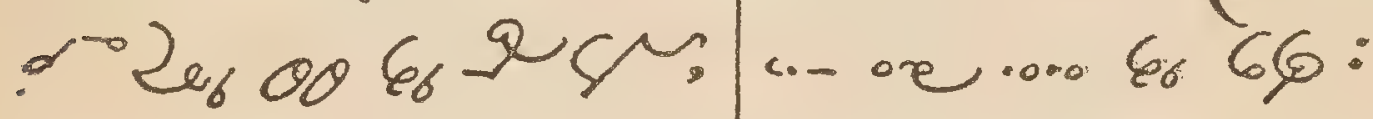

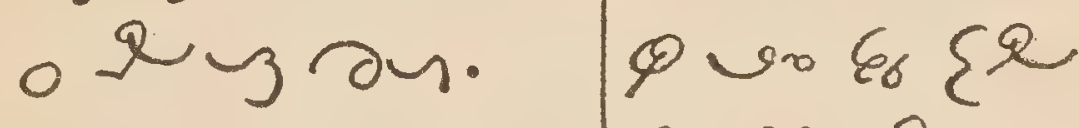

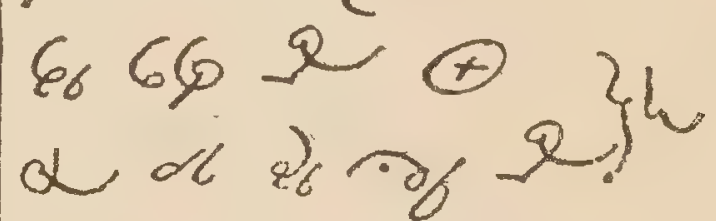

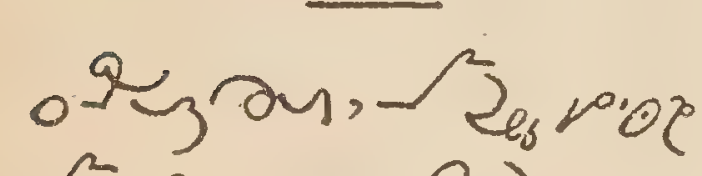

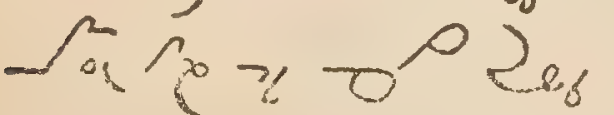

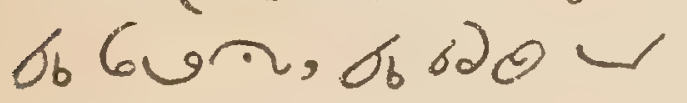
olezวu.

Es Sore $\rightarrow D_{6}$

og 4666 ee $\alpha$ dl $\mathcal{N}^{2} \delta \theta$ G

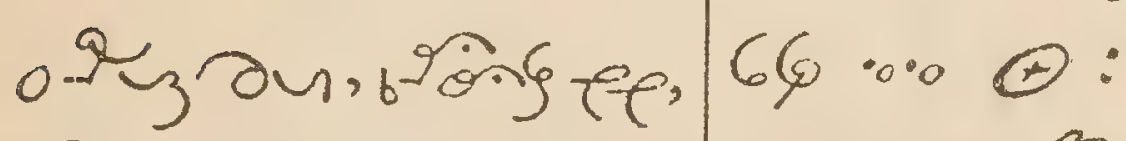

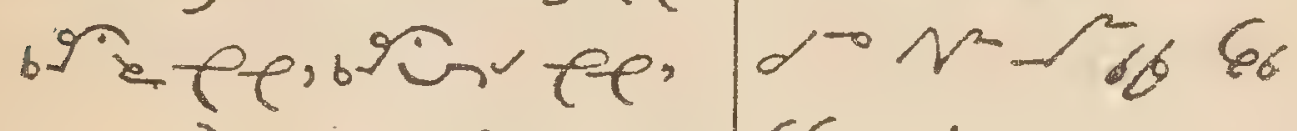

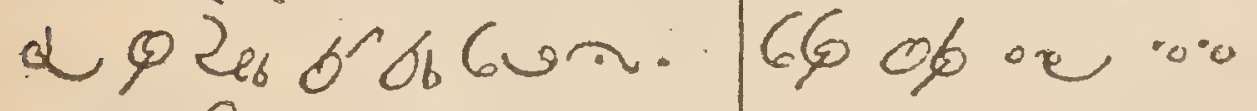

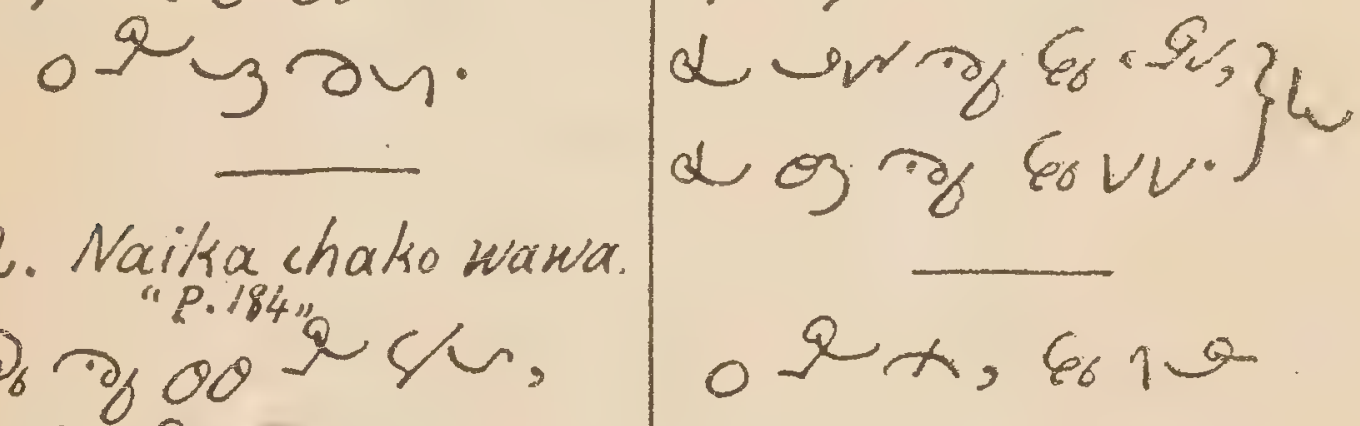

$\sigma_{6} \varepsilon_{6}$ \& $\bar{x}$;

2) 200 ore 2

b6 $\varepsilon_{6}, z_{6}$ แ6.

$P_{0}>66 \sim \varepsilon_{6}$,

$2_{6} 66$ oin $\varepsilon_{6}$

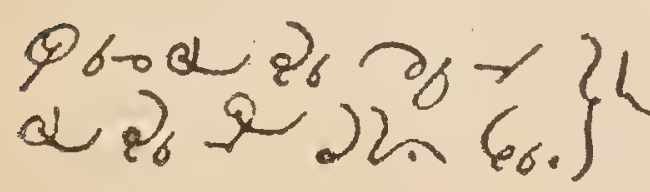

ob cole bo ar:

Q v.e $922,6 \%$

$\left.i_{6} e_{e}\right)_{6}$.

ग07 ${ }^{2} \mathrm{G} G$

tá of ob or:

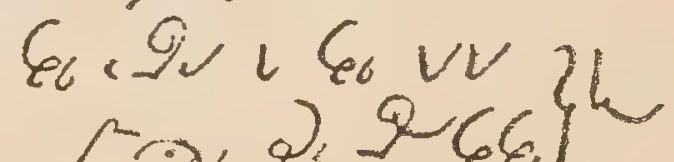


MANUAL

77

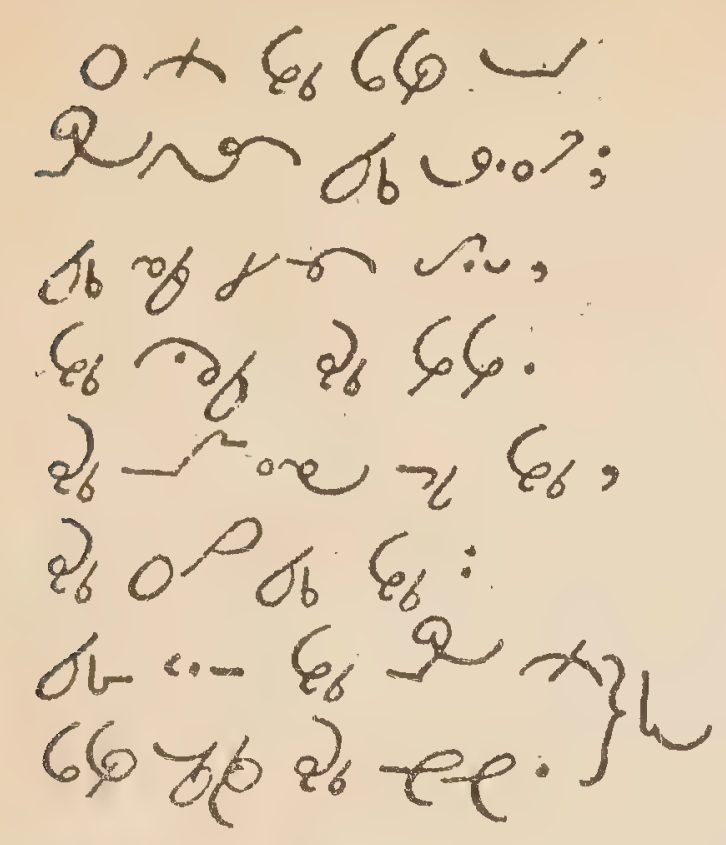

3. O maika Hloos J.C.

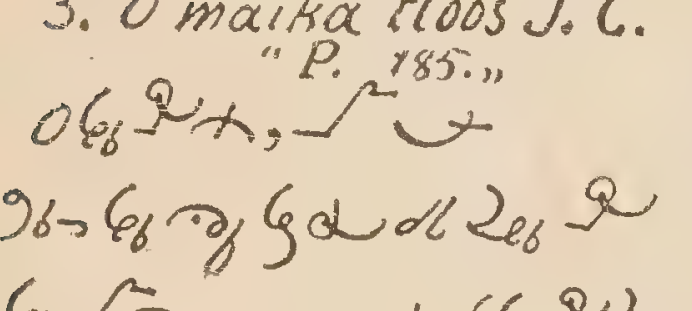

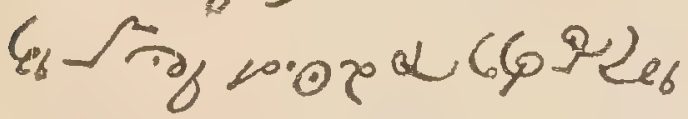

$662 \varepsilon_{6} \geqslant 660$ Sore on 68 .

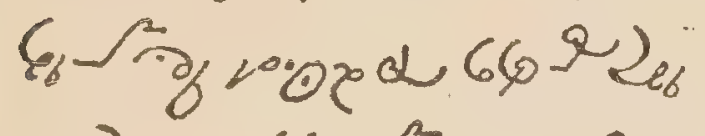

66206 $\rightarrow 66$ Sores on 68 .

$966_{6}+29-862_{6}:$

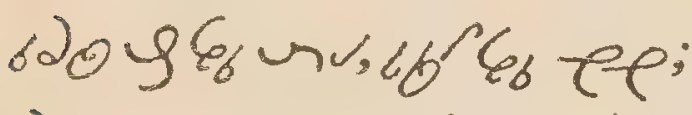

$2007606066^{\wedge} 206$

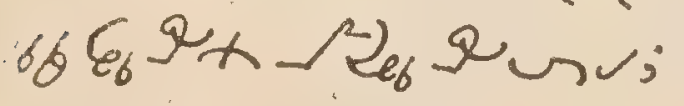

206 7606616 2 26 ee.

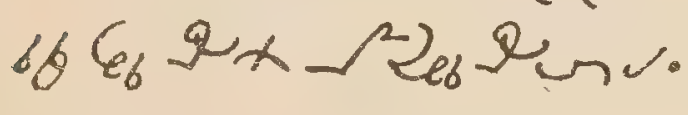

4. Jakwas alta. "P.190i"

$$
\text { of } 0 \text { as } a \text { ee }
$$

$2_{6} 3-6068$ :

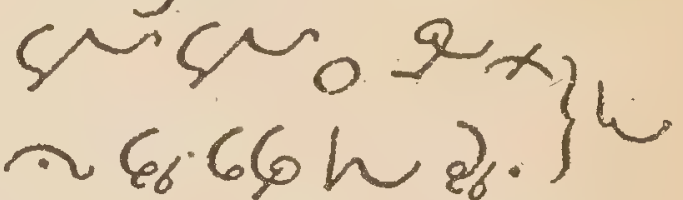

$68 \approx 2660$ el

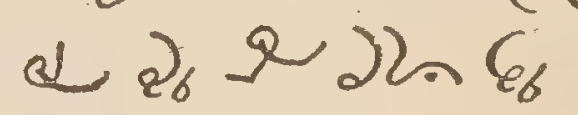

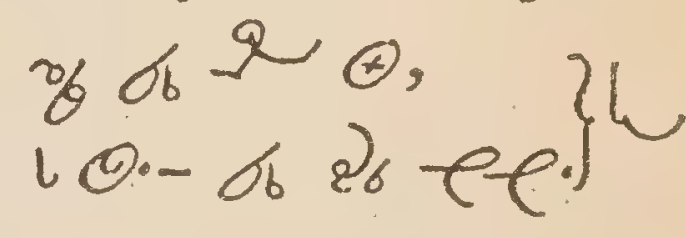

3: Sahale Taye Tanaz. "Wo 82.".

$\forall \rightarrow 2$ क क of 0.0 bon, thestep: ore be rd o $P_{b}<0$

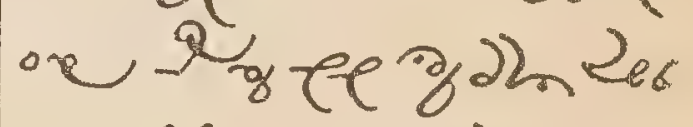
6610002 26.

-

6. OJ.C. Nawitka. "No90.", $0 \times 203$ ore $\varepsilon_{6} \rightarrow$ यe 
78

CHINOOK

a in 266 ar.

$\sim 02_{6}>\varepsilon_{6}$,

रान यु $\varepsilon_{6} 00$;

Q to a d d 2er

lpa bu Gu.

\%. Ankate ookook J.C.

"s: 83.",

8. Naika, O.J.C.

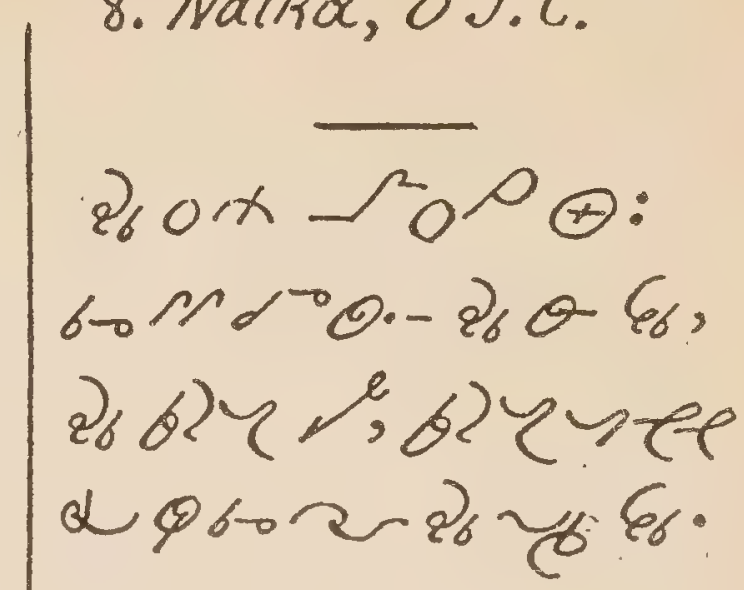

के थ, क्षक

601000 2);

के य, दो be

ob $\varepsilon_{6} a_{6} \geqslant$,

or $\begin{array}{llll}\varepsilon_{6} & 2_{b} & \tau\end{array}$

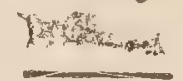

$96600-2 \%$

Quoxक $z_{\sigma}$ ee

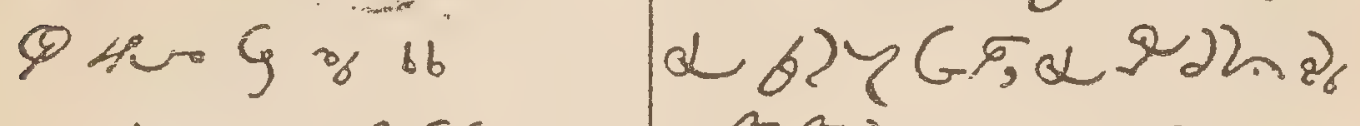

‥ 0, ב च $660 \%$

of op e 8 \% 66:

er! 9 r.

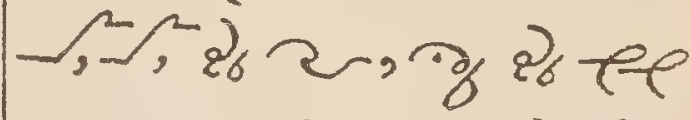

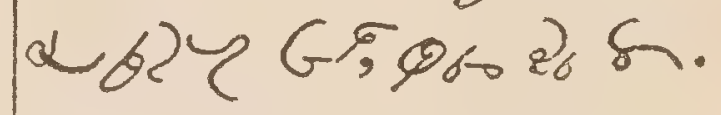

9. Tloos alta nsaita.

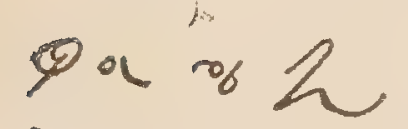
"P.183."

624 … \% 0.0

Q 20 ,

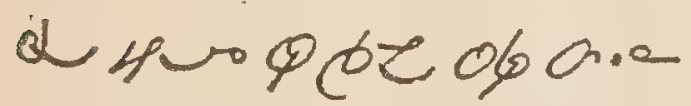
$\phi$ bol 22 n.or s:

208.' 208. ob 98 :

oy,

84 or, 列, of. 
MANUAL.-

79

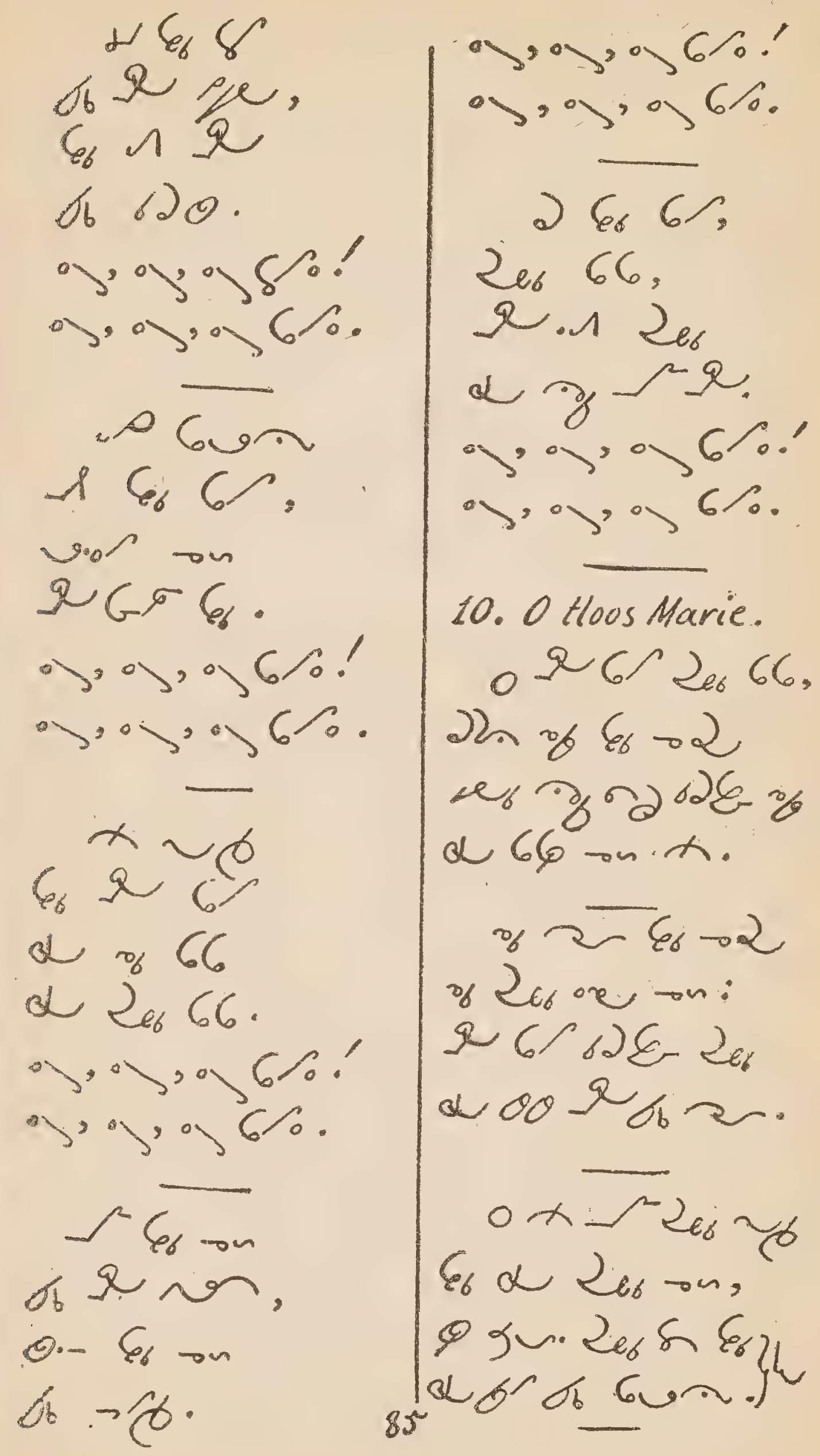


11. Kanawe son OT. Mulie.

82090 a 8

$\varepsilon_{0} 92 \pi 2 \omega_{6}$

$\varepsilon_{0} \geq \dot{\alpha} \varepsilon_{0}-\alpha$

b v.or S.

b) บ 206 $\rightarrow 68$

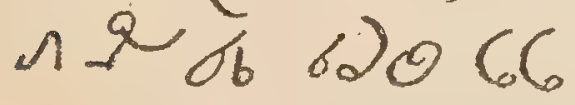

b6 $2666 \mathrm{ee}$

0.8 o 9 gu. 万ह

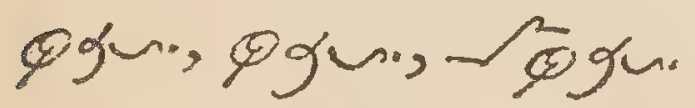

26 und $\varepsilon_{8} \circ 8^{\circ}$

$2{ }_{6}$ on, 2eb 66;

$\rho_{0} \geqslant \mathrm{o}^{\rho}$.

पुद है $66 \%$

12. Ookook Son iles Hlous. oben? volge o 2 r ore te शr yo 8 यन 66 की के 66 . $66020,5>$ की

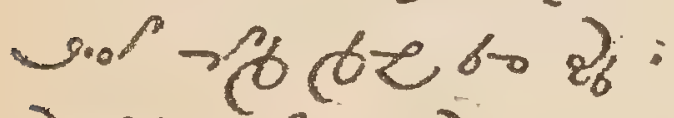
86 $666 \%$ \% 8 ef; 86
- Gonáchine

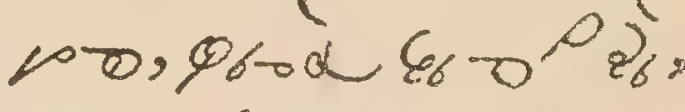
10,8 पु 28 et

13. Na Jesus...

2 2, -2 2 "y6s"

$\varepsilon_{6} \partial \varepsilon_{b}$ on

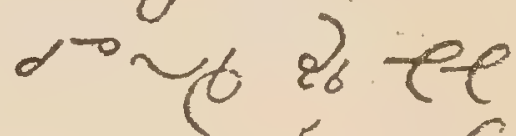

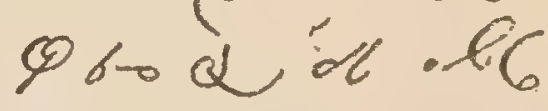

on Grob a fe.

22-2 "

$\varepsilon_{6} \partial_{0}$ \& के $_{6}$ on:

Q6 od a ? ef

$b^{\prime} d_{6} \cdot \ell_{6}$ tin.

-d 2 laton,

$\leadsto$ य \&

$\circ \sim$ at $f e$

14. Dret nsaika mamooks. "w: $9 \%$ ",

- 2660 ee

2es $\times \operatorname{an} 9$

bicind odoy

रो un ab 2a. 
MANUAL.

$8 I$

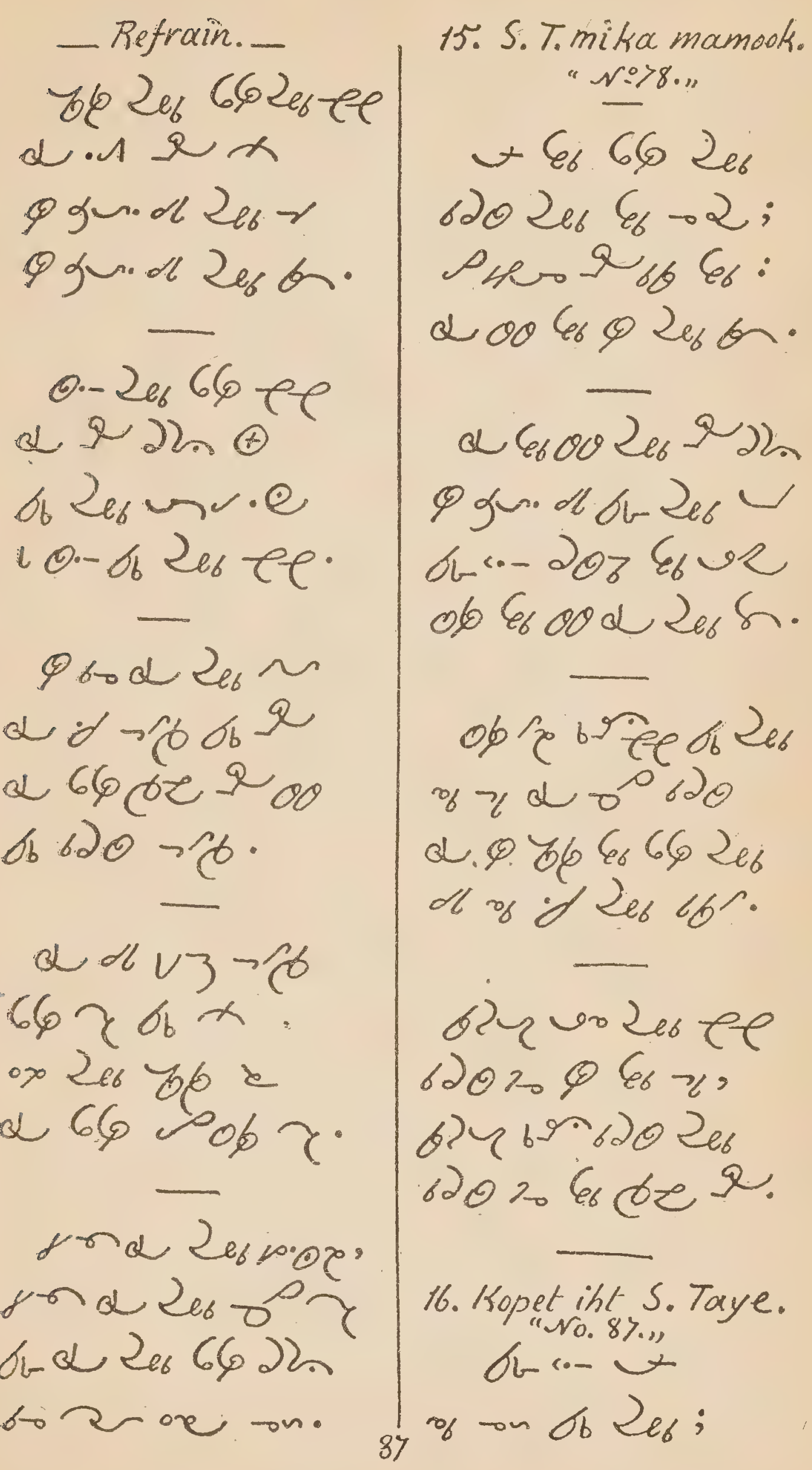


82

CHINOOK

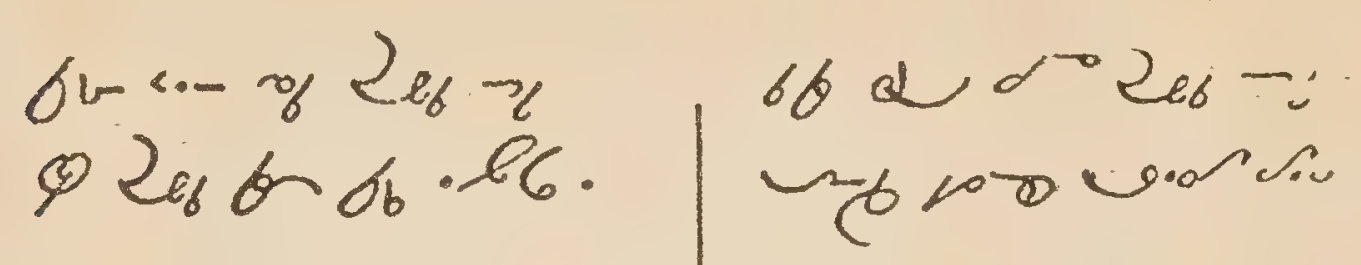$$
\text { Q } 62026628
$$$$
66 \text { yo 20 te }
$$$$
600200 x
$$

d d 66060 .

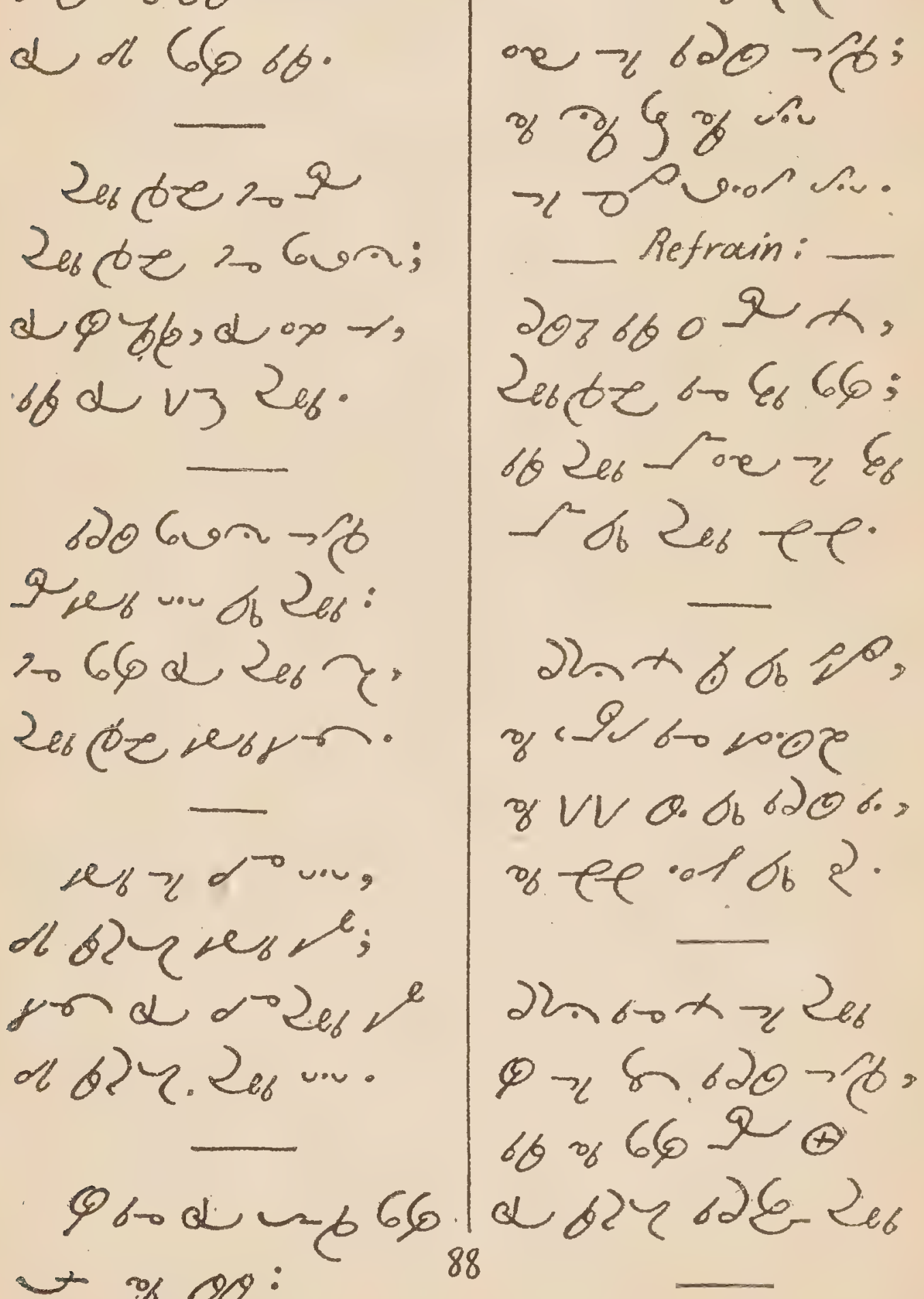


MANUAL.

83

18. Dret aias Hoos..

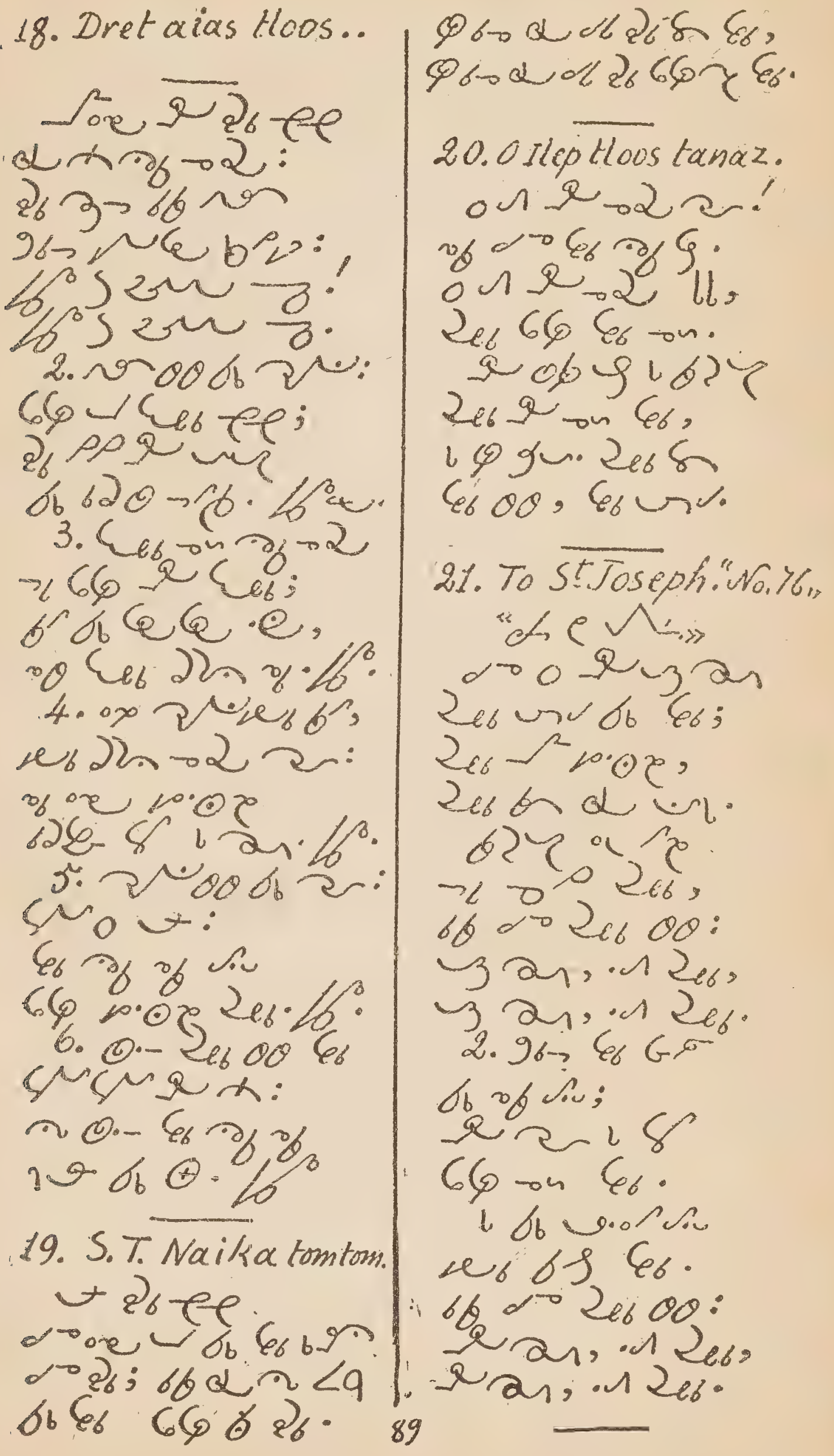


84

CHINOOK

I. God.

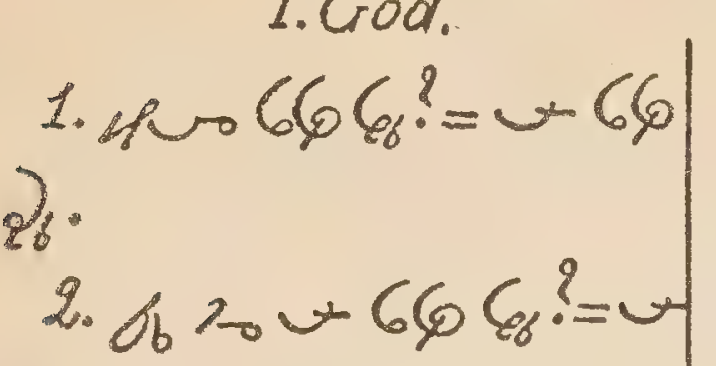

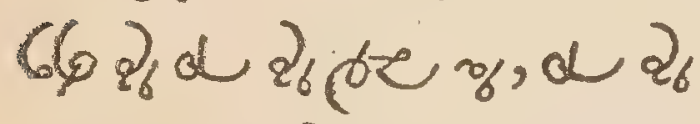

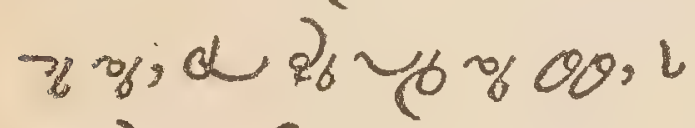

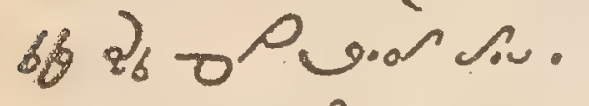

3.6心? = tate

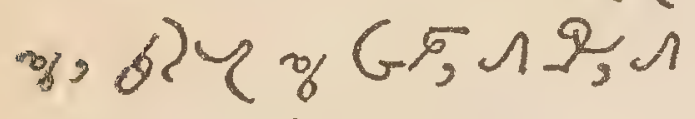

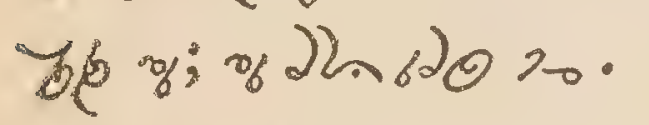

4.10006800:6ute

$\omega ?=$ \% $06, \alpha \rho_{r j}, 9 u$

․

$520066000: v 624$

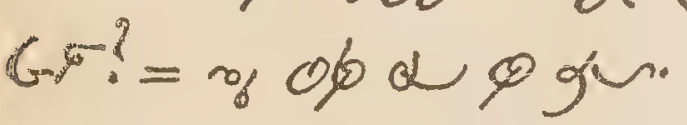

grw $\cap$ g, lөgu.

$\alpha$ का $\rho$.

6. $10086600: 41$

Q? $=\% 06,462010$

Q G F b w, low

Q 66 620 20.

$\%$ 万od $\varepsilon_{6} 00:$ : v2

$120 \pi_{0} ?=$ \% of $\alpha w$

$22.620106 .596,6=$

गे $206.500,16 \partial \theta=20$

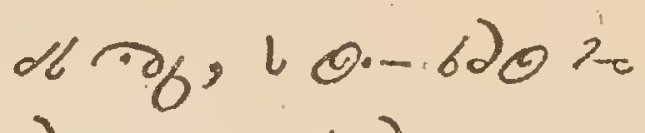

26r 10 b6 le el.

8. 6. $\backsim ?=\cdots G 5006$.

II. Holy Trinity.

9. a $\partial w ?=\varphi ; w$ buc.. \%.

10.0 $\left.2 \lg _{6}\right)^{?}:=$ $208,-9 \cup 9_{\%}, w:$

$\checkmark 6, w-2, w \times ;$

of $\sim_{2} \Omega$.

11. 20 of $\sim \Omega ?=-2$

Brob ac... Wh

$\checkmark$.

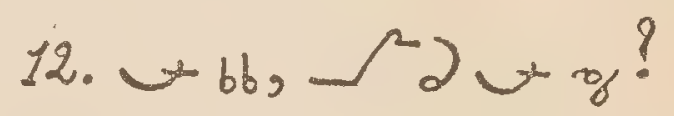

$=207, ज 4, \Omega \%$.

13. ज-2, $/ 2)$ ?

$=207,4-2, \Omega$, s.

14. ज世, 2)

=208, we, א\%。

15. ज66, ज-2, $儿$

$w,-92 \omega ?=9 ;-9,9$

\%以, 1 b...

16. 6od 949, , 6h...

? $=$ of, d of 9 , 9 
MANUAL.

85

$\mu_{6}-S_{66} 600 \mu_{6}, 06$

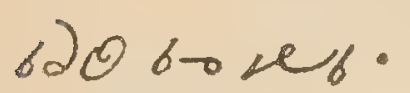

17.6b06-9 9 , ,

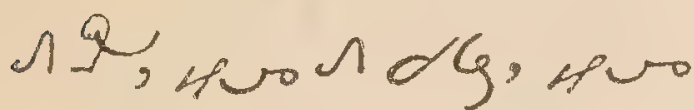

A 68 ? = PAv; of -9

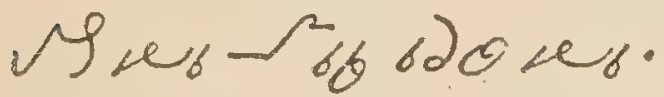

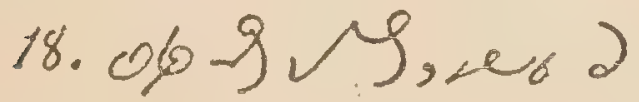

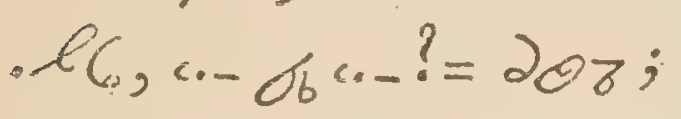

er.

$000 .-\cdots$

\% 2 bw? $=\varphi_{0} \lll$ -2, ⿶roglo. $24: 06 \nu_{0} \Downarrow-2 m_{6} \zeta$ ?

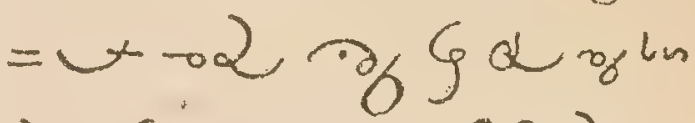
266un, i. 90 रe6 660.052.

$25.60 \%\}-2 \alpha$

\% $\xi ?=\longleftarrow-2 \alpha$ क $\}$

$x \approx\{$.

26. 4pur $x ?=$ 中r $2 \%$ \%

$2 \%$ os $x \ln 266$ 6on?

III. Jesus Christ.

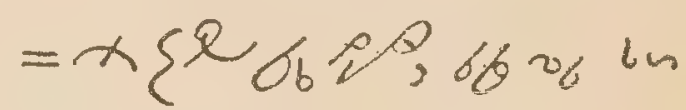

19. $0600-9 \vee 9$, , . 2

$\partial\} 96, ?=20 z, \cdots \sigma_{b}$

206 on.

of 9 Y $^{2} \xi 960$.

28. $2 \times G 5\{x ?$

$20.4000606-949$

$=\varphi r ; \sigma_{6}-990 . x$

क $\zeta ?=$ \% $九 2:$ क

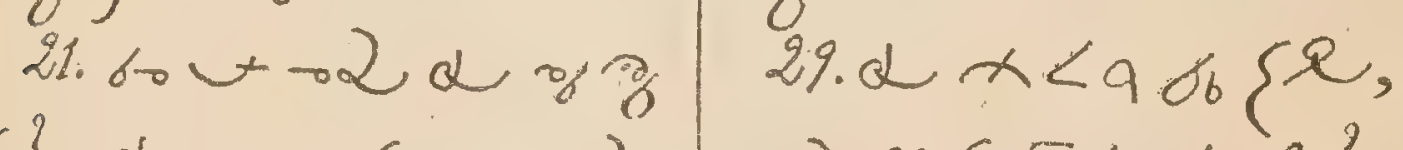

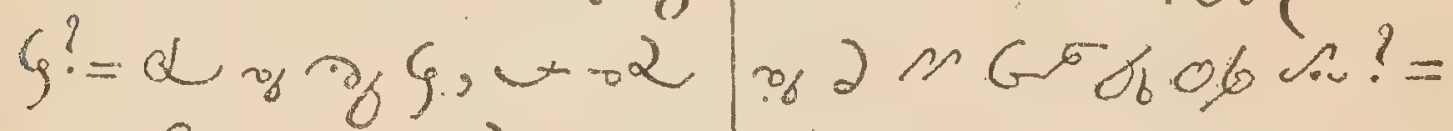

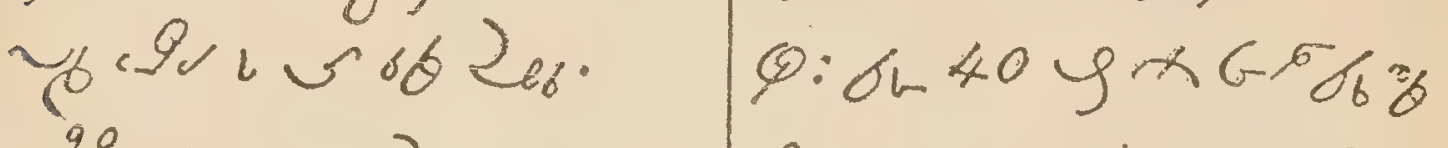

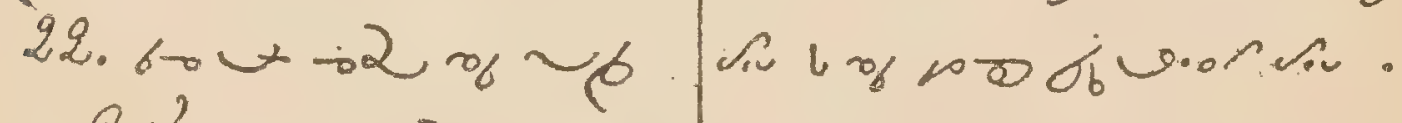

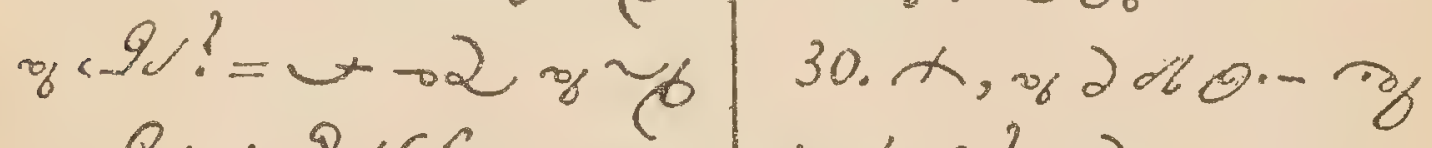

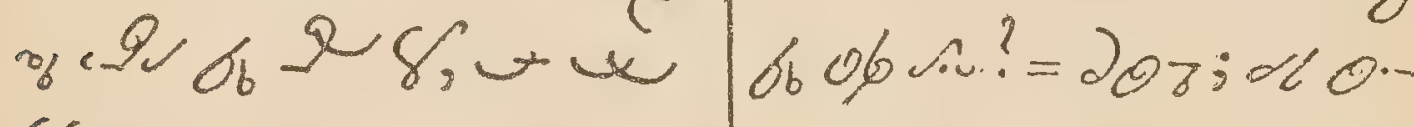

660168 .

$x y_{6} 66060 \sin 06$

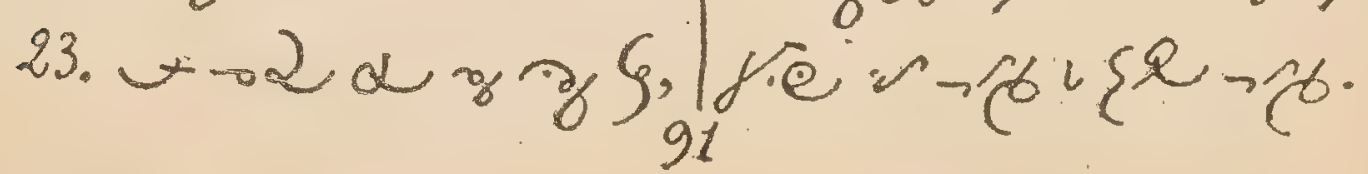


86

CHINOOK

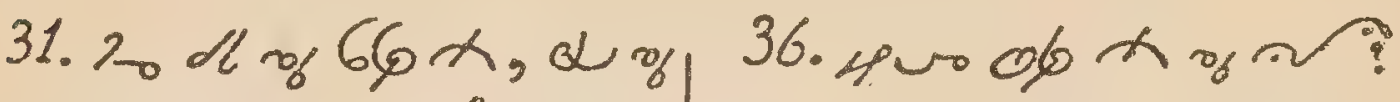

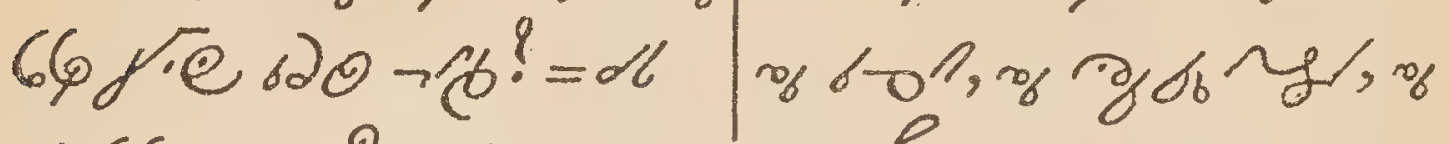

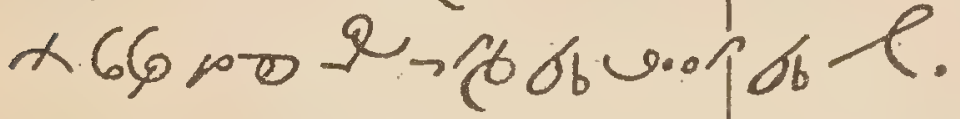

s, log 60 6.

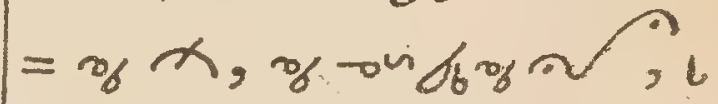

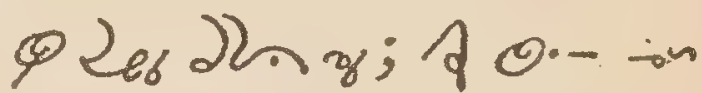

IV. The Church.

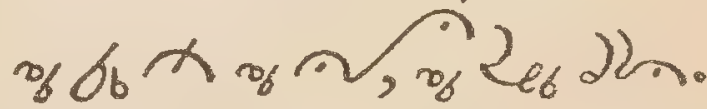

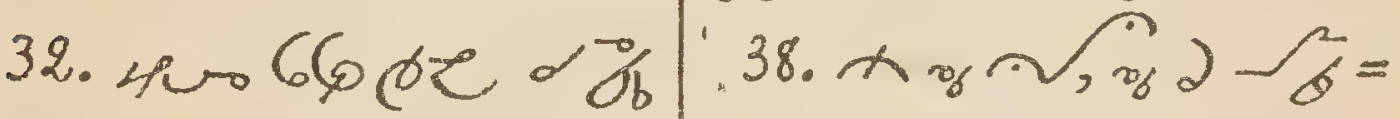

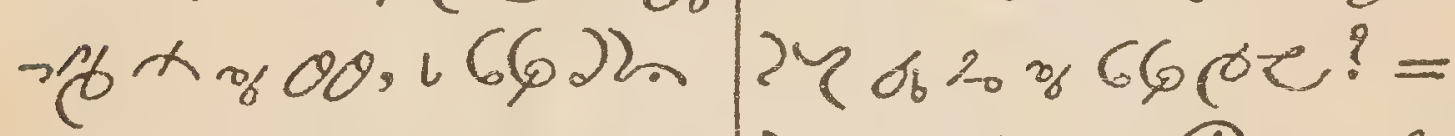
bus

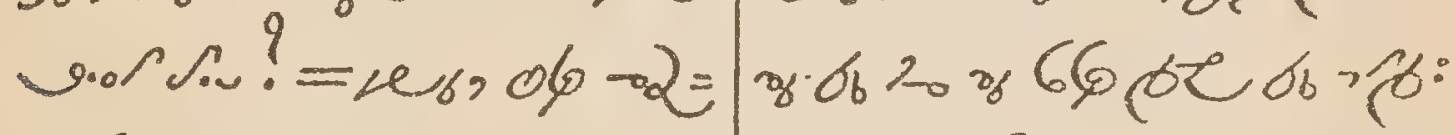

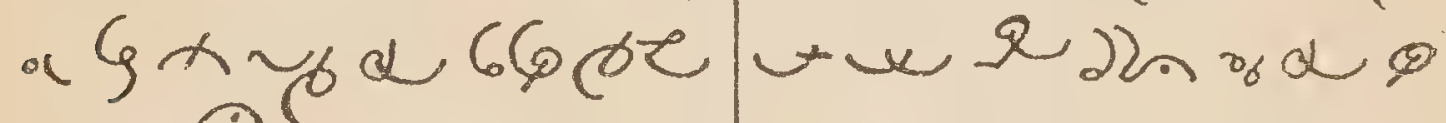
\% 2 . m un.

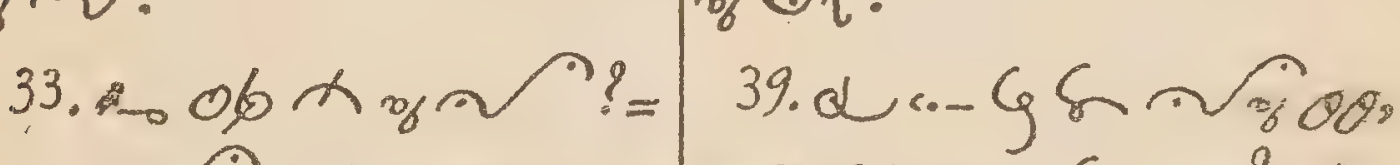

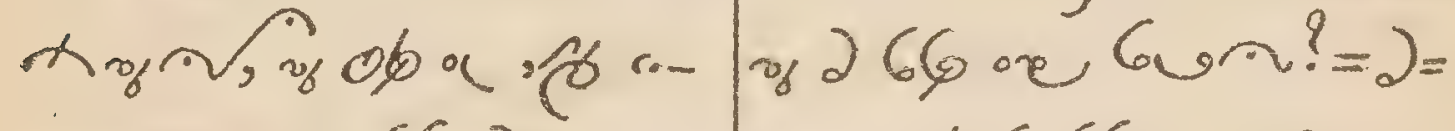

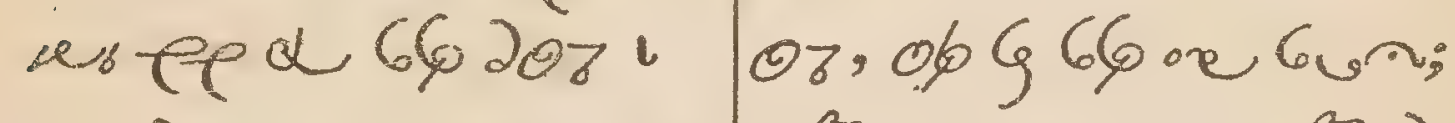

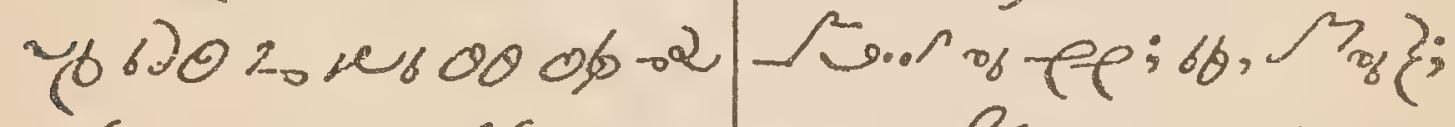

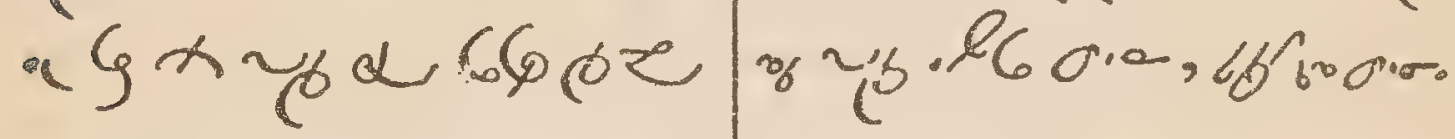
अ 00 ᄂ का 0 . $34.40006-2 \times 9 \mid V . T h e$ Creed.

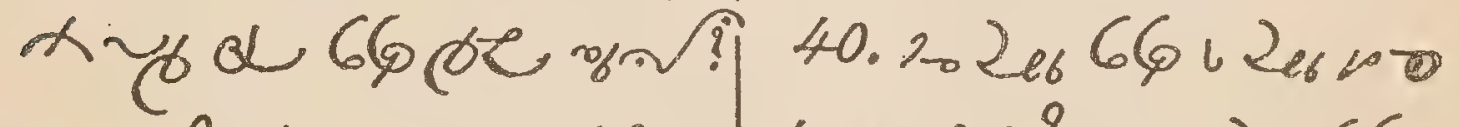

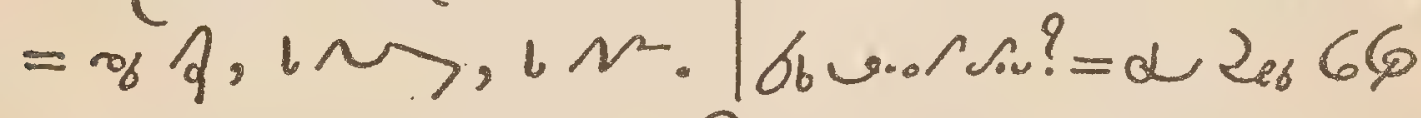

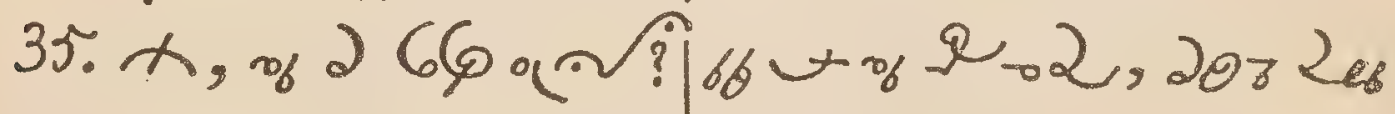

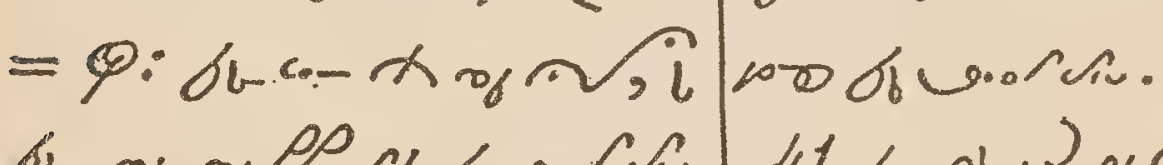

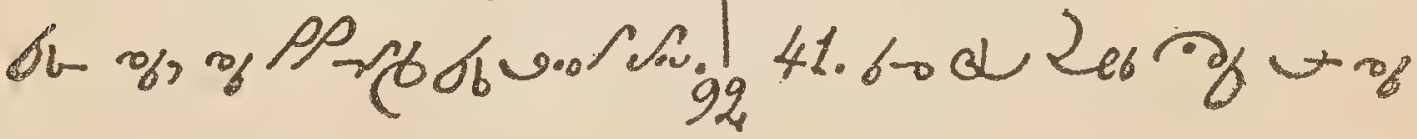


MANUAL.

87

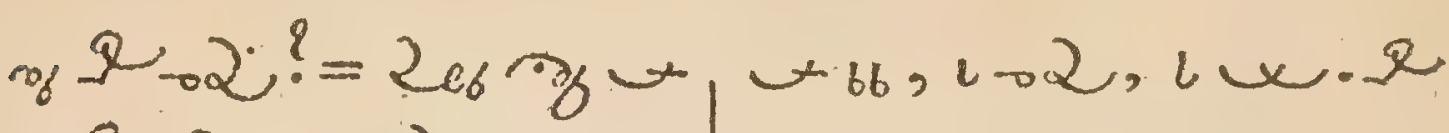
of $2, \alpha, 26 \operatorname{tg}_{0}$ on 86 .

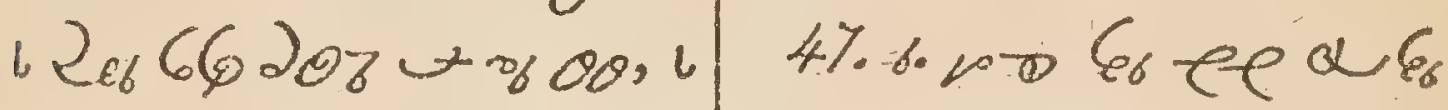

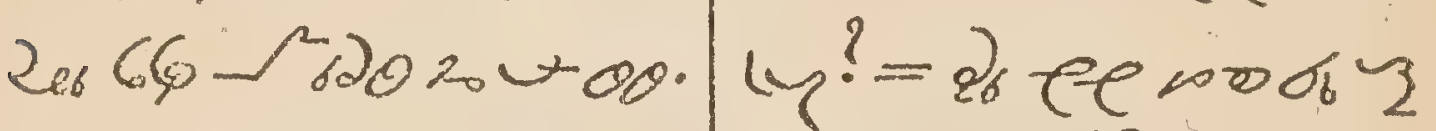

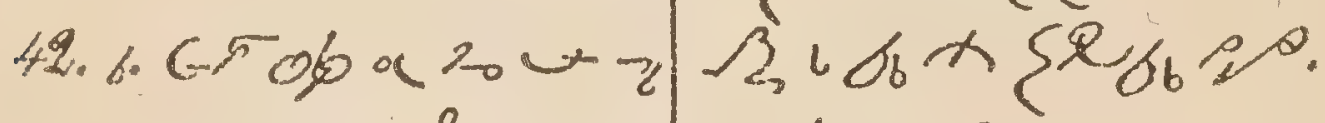

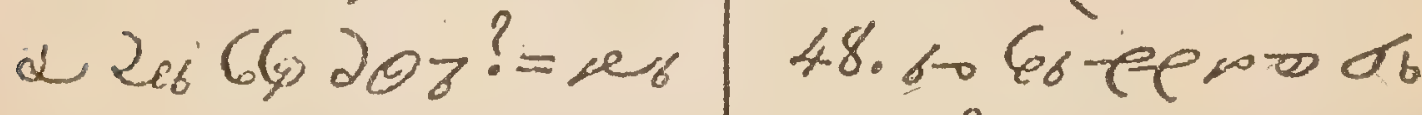

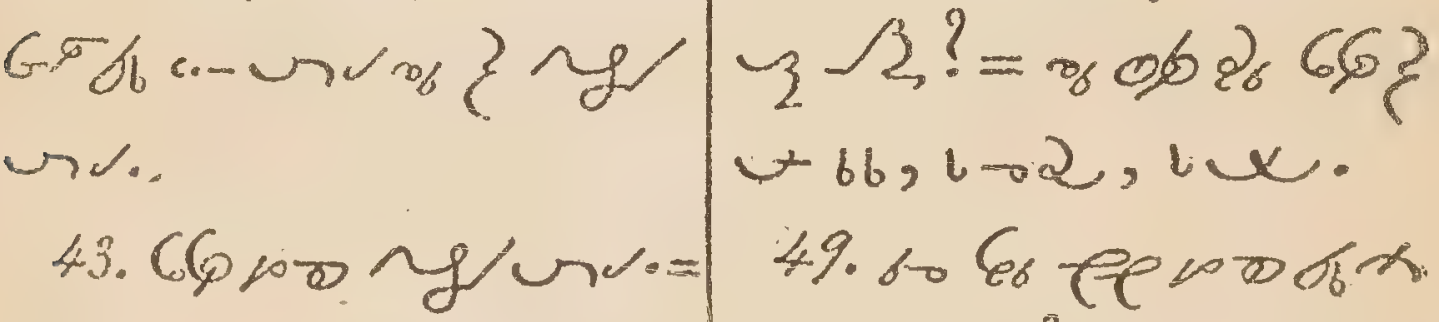

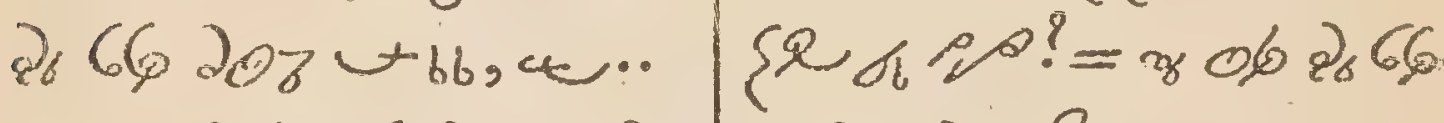

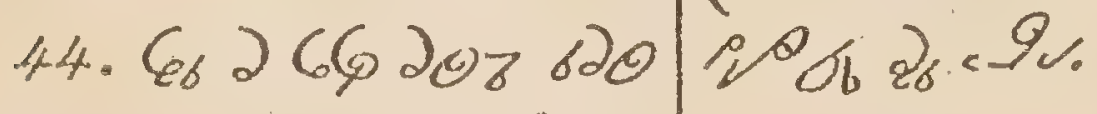

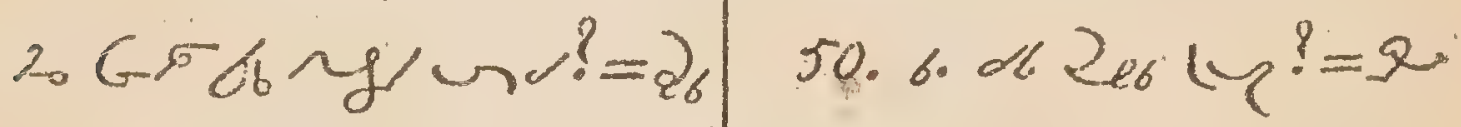

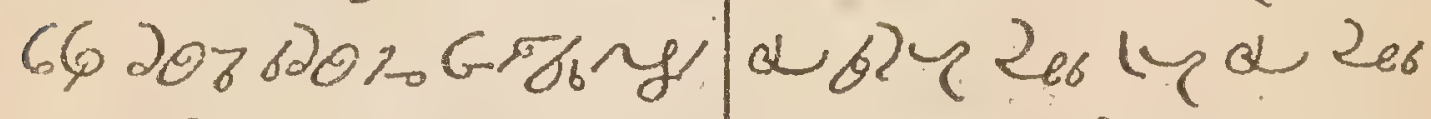

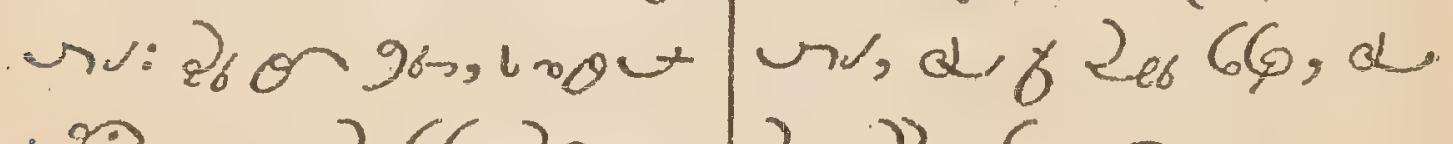

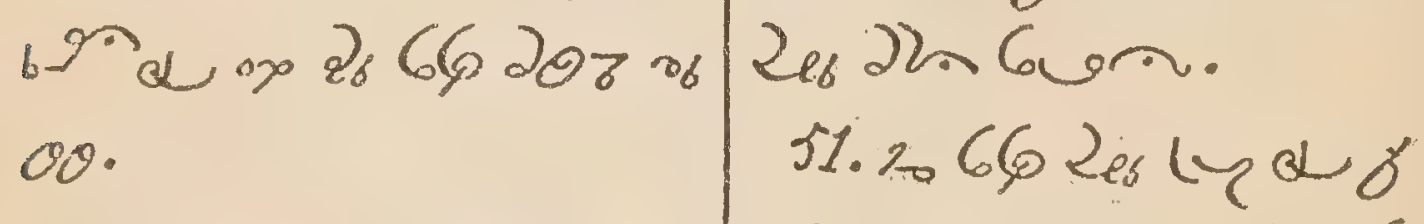

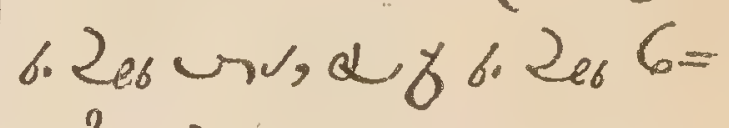

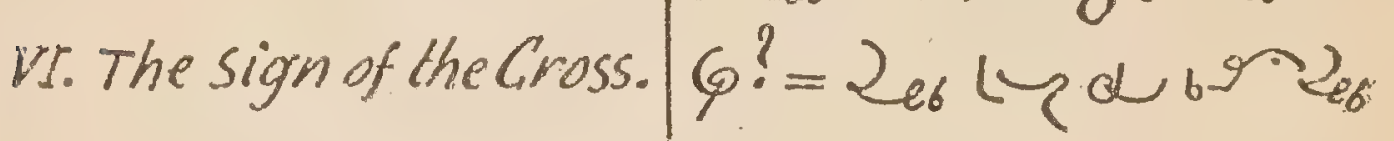

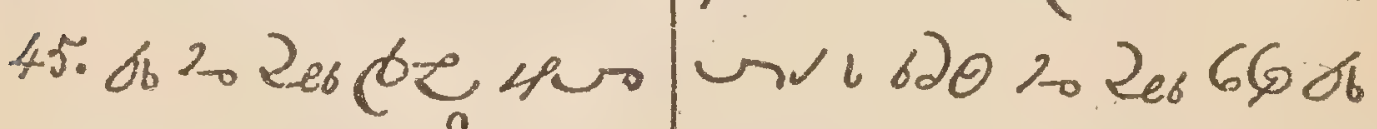

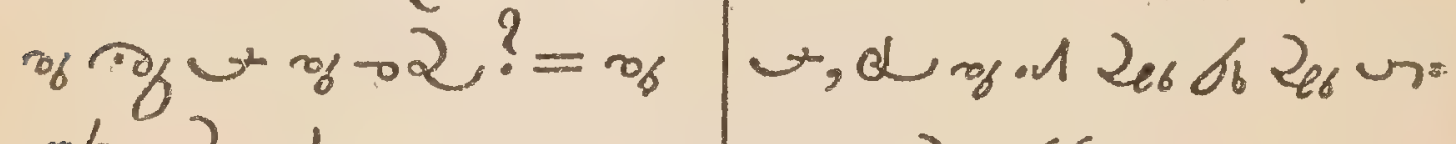

$06,206 \mathrm{ly}$.

$\checkmark, 6620669$.

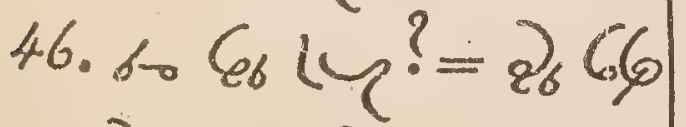

52. 3066.206 (y) 28

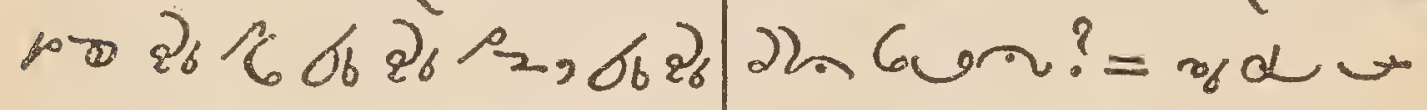

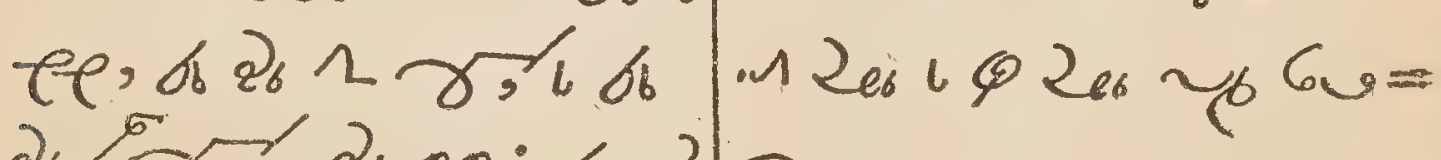
2. 
88

CHINOOK

VII. The Commandments. $620 u r ?=0 \%$ of $\times 660$

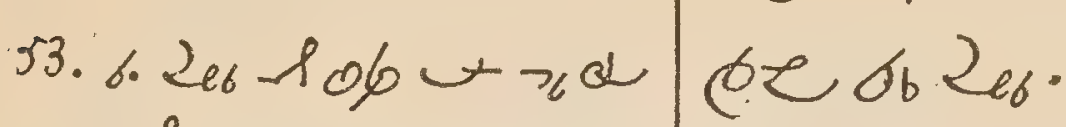

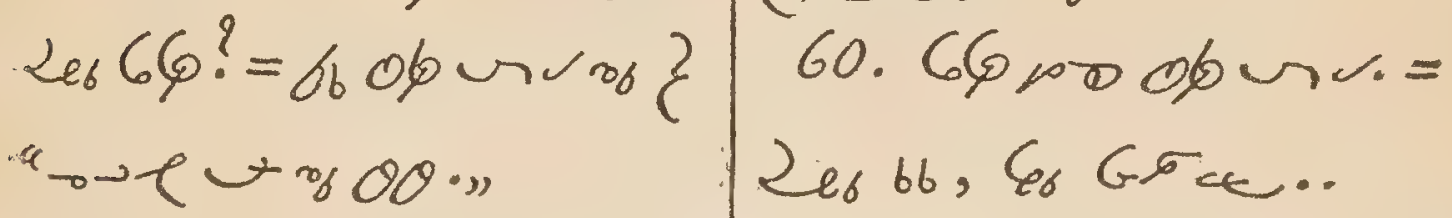

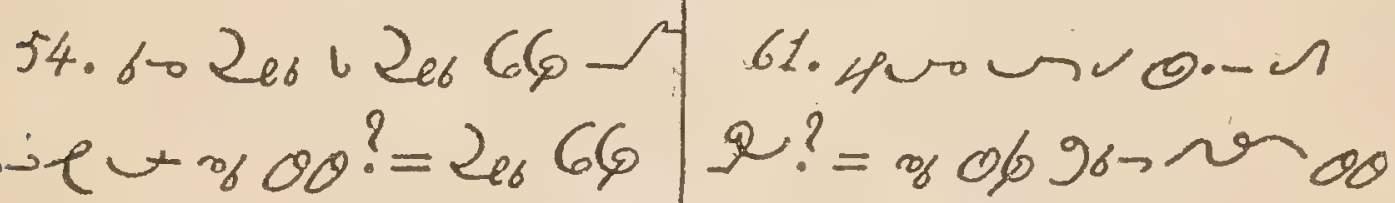

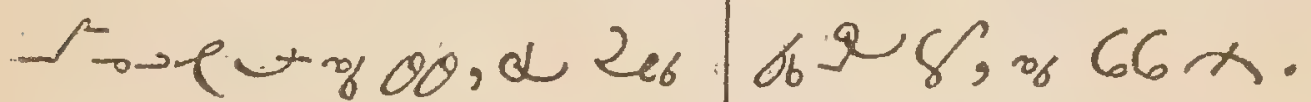

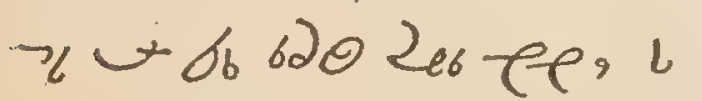

62. 660000007 .

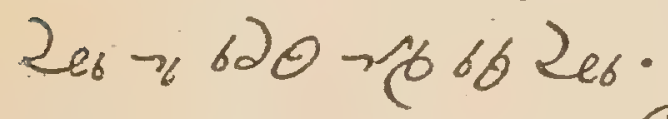
$=08,4$.

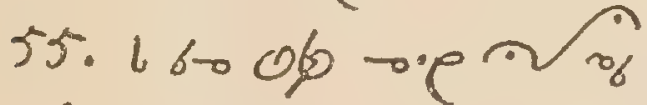

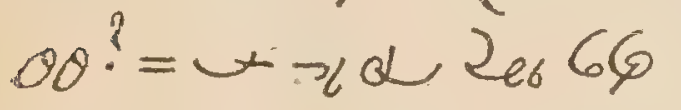

Ix. Sin.

rs $\sqrt{\text { rig }} 00$.

$63.2006 \varepsilon_{66} 60$ ?

56.660 rod of see $\Omega$

6 . 66 ? = oo 2 206

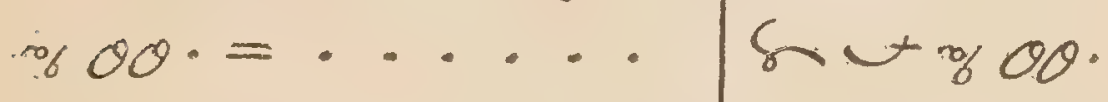

64. gun. a. 66 on $G=$

VIII. Prayer.

$5 ?=6 \cdot 966 u \cap G 5:$

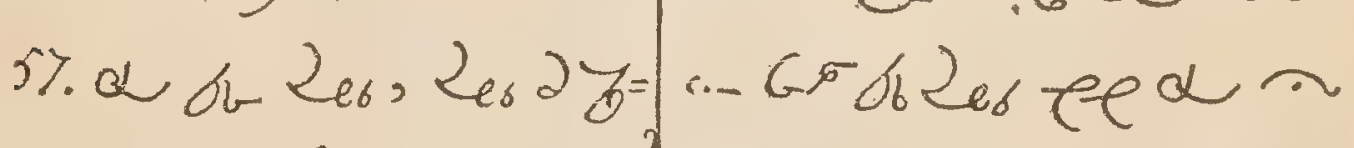

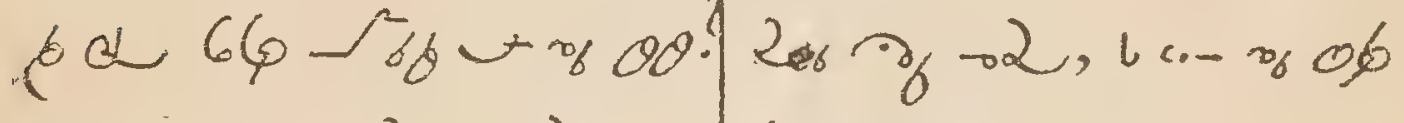

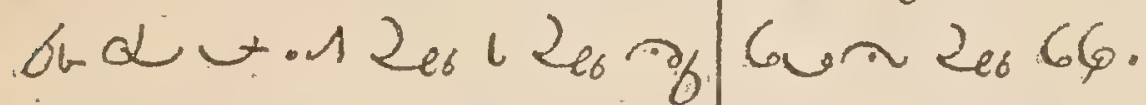

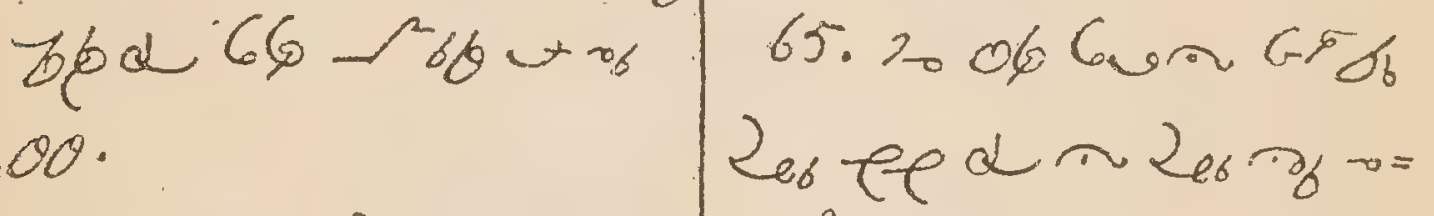

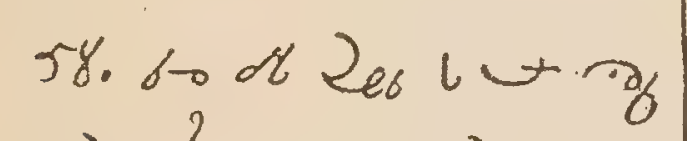

$2 ?=0,0066 \pi z_{6}$

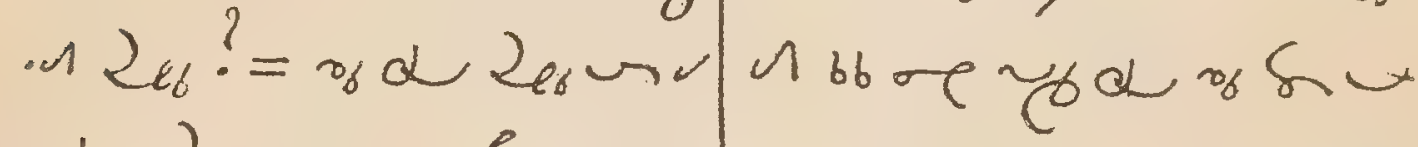

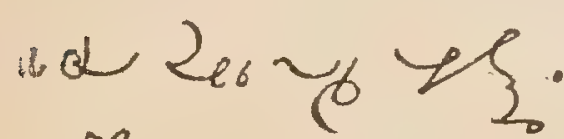

$\because 80$.

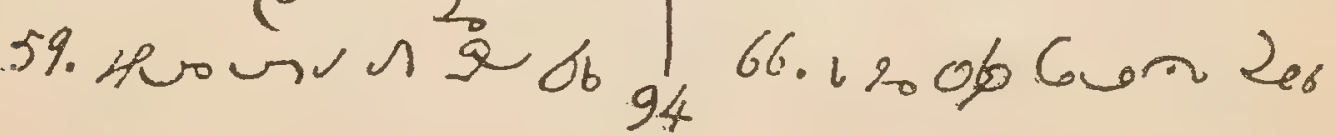


MANUAL

89

$66 ?=\operatorname{rog} 00 \alpha$ 2 26 ee $72.94 \cdot 0 s_{2} 6660 \pi$ 26 6\%.620066un 2066 660: w.0ree, he ter

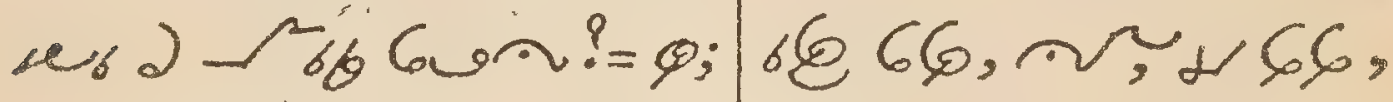

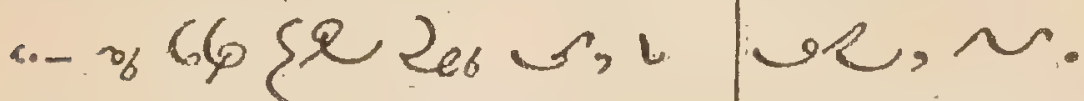

‥ 9 \% 66 \{ \{ 2es 3 .

68.600660 a 6058

2e6 $\backsim ?=0$ of of $w$ ve

60206,66 or $51206=10 \%$ 600020606164600

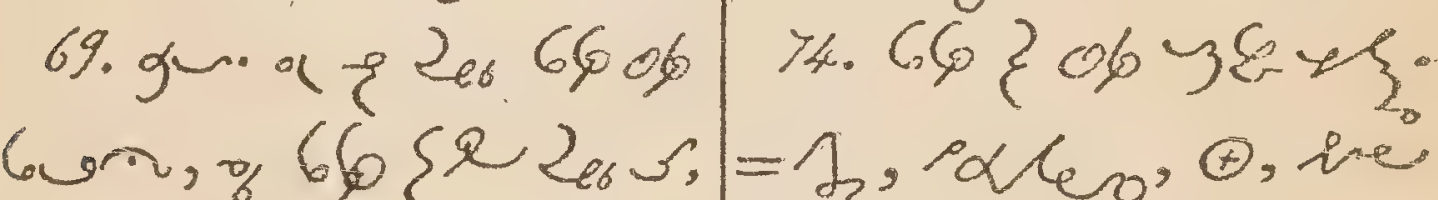

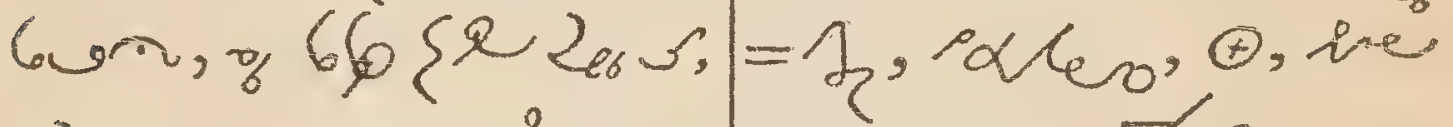

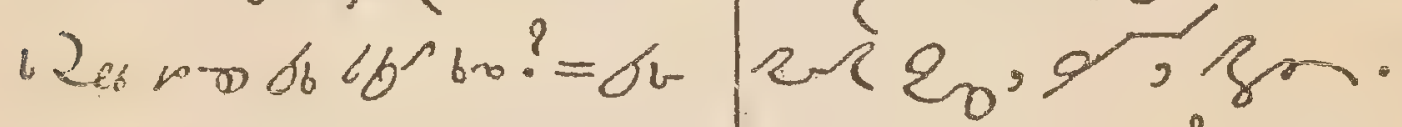
… $\{, \alpha \phi$ or $\partial \delta$ \& 8 Les $\{$.

$70.600660 \pi 966$ \{R 2er $\checkmark ?=$ \% of $\alpha$ $\varphi+\mu$ र 26 , or a 9 og a 1126 .

7t.gur. are.eples 66 bon? = 2 a.a.:1\%, $\because$ ob, a b 2les re; 20

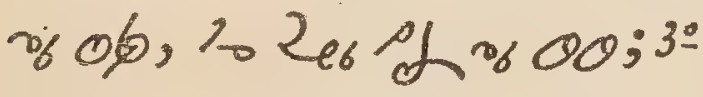

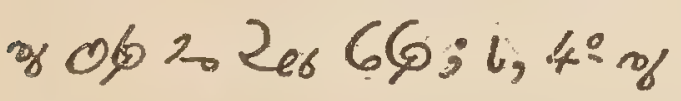
of 20 206 $6606 \% 00$
X. Baptism.

73.600 fex, 2 os $660-36-2 \xi=x 66$

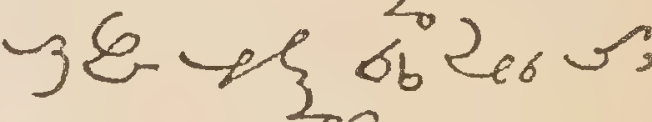
Q का का

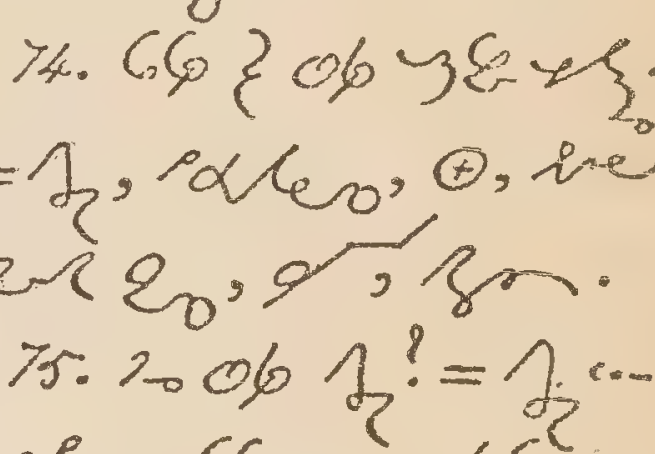

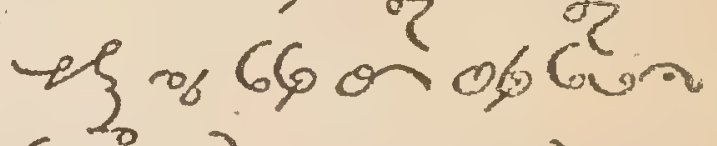
Gob 206 epal $26 \%$ -2, 1066 un 2e6 66 $\alpha \phi 260$ on; los 6606

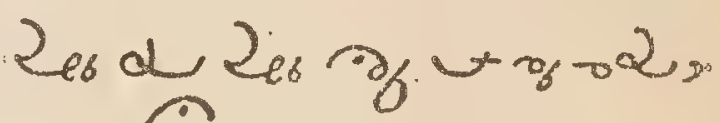
$\ln \hat{v}_{6}-2$.

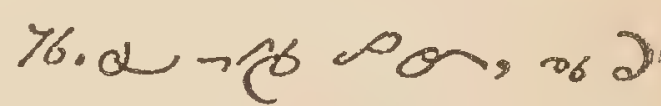
100 bo v.0 1. S ? $=1960$

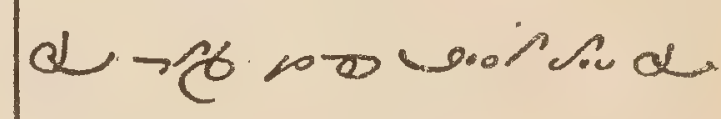
Oron.

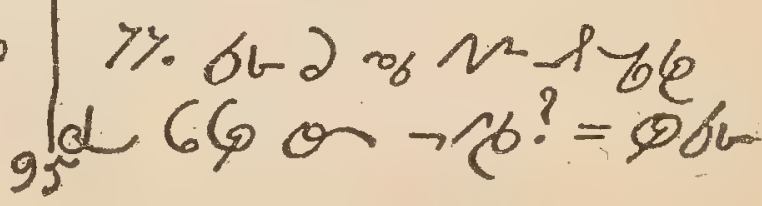




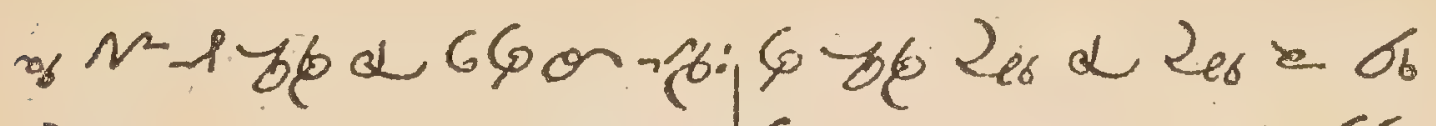

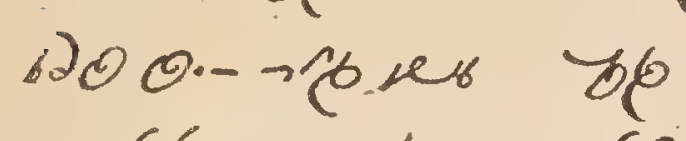

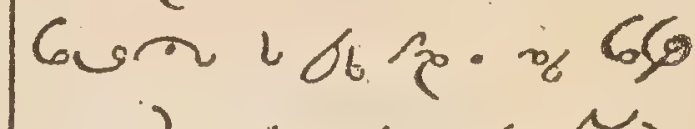

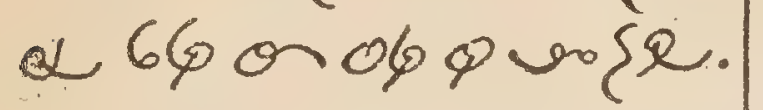

$78.601 .-966 \alpha$ or 2 66 on $\neg \beta^{\circ} ?=\mathrm{g}^{\circ} 0.20 \%$

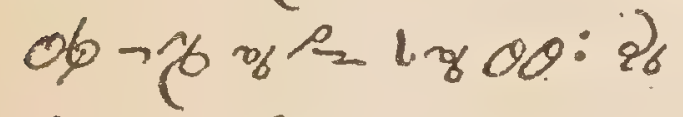
66 on $66,6666,1-2,1$ wis ?.

49.60\% $d, 06-\not{z} \geqslant$ mon? = $e_{\alpha}$ कु क Lw ob00; को 650 प re 66206 on ro 650 ; 660 \%

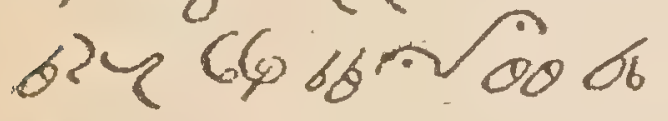
on $\rightarrow$ ph.

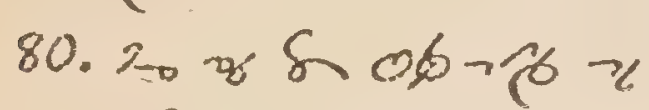

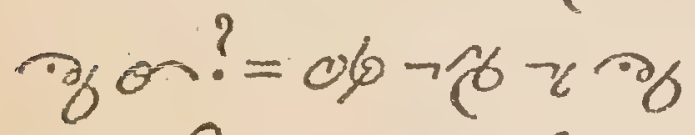
on, 9 \% 8 : कर

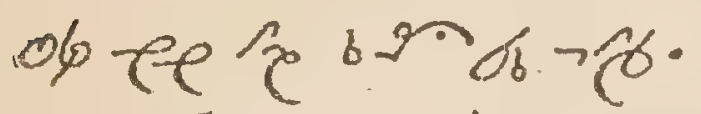
xr. Confirmation.

81. 2o "ater ? $=2 \alpha=$

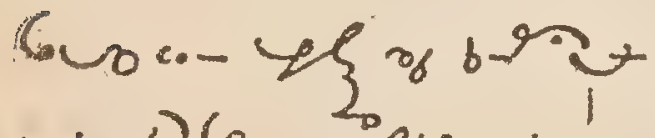

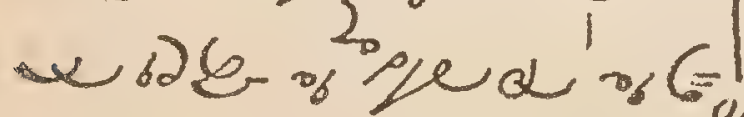

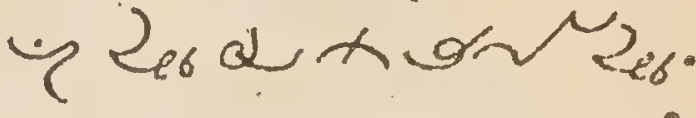
82. Hor ba क्रler?

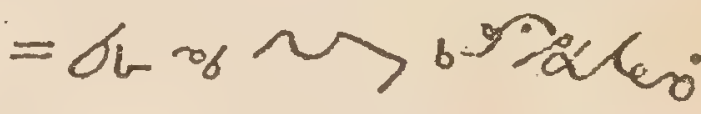
83. 60 $\sim \alpha$ of $62^{\circ}$

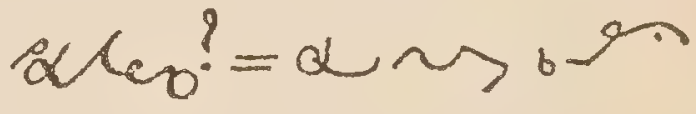

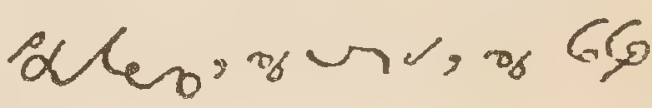

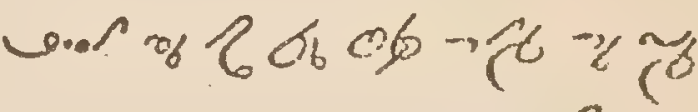
करen, y यर

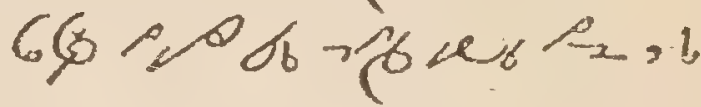

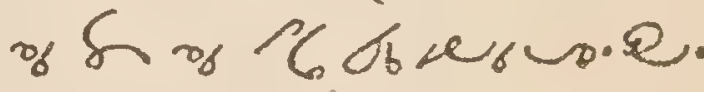
84.06 of $\rightarrow$ o d oleo 60 क $660, \alpha$ कु $T T^{?}=Q_{\text {og }} 506 \sigma_{0}=$

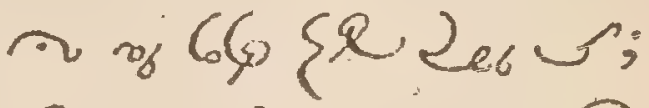
ब. कर 2 ब 65

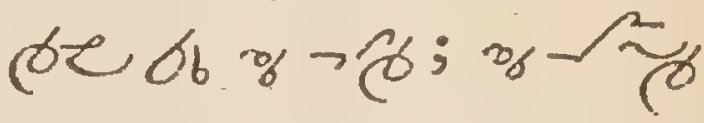

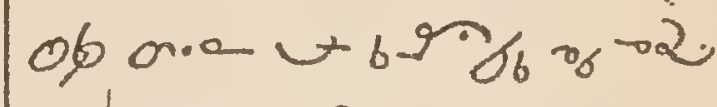
las 660 opr ef al u $\partial_{\sigma} \sigma_{6}$ r. $^{\circ}$

XII, Holy Eucharist. 85. $100 ?=\Theta i_{0}-y \xi_{\text {. }}$ p20 GF brow, 
MANUAL.

$9 I$

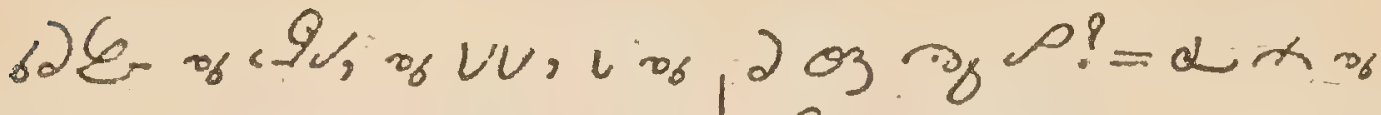
$\checkmark$.

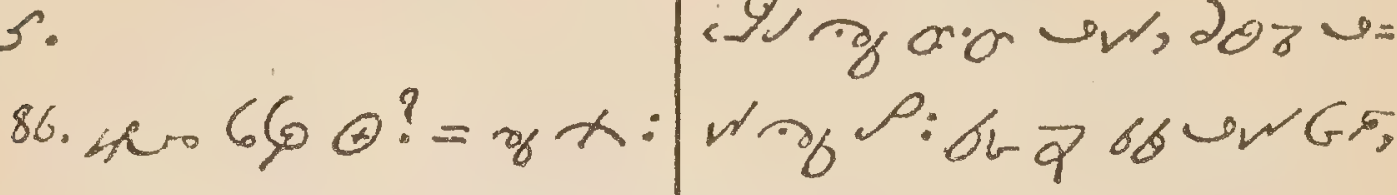

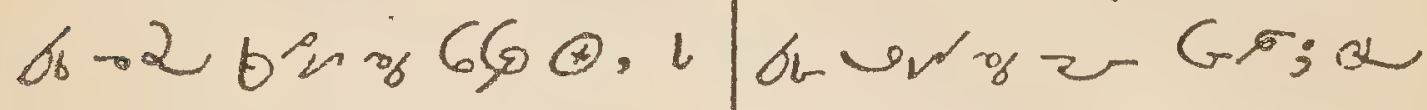

क 9 क

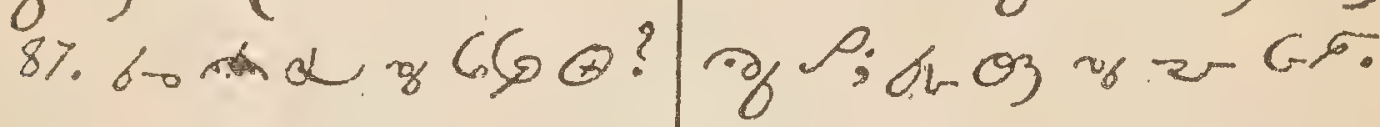

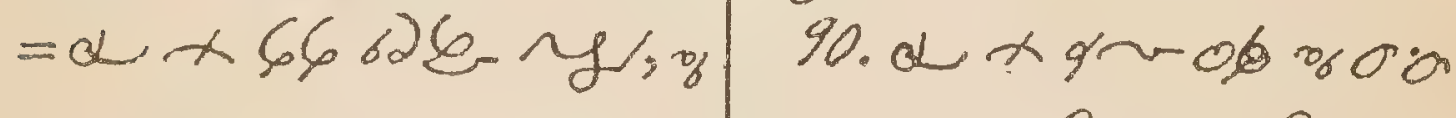

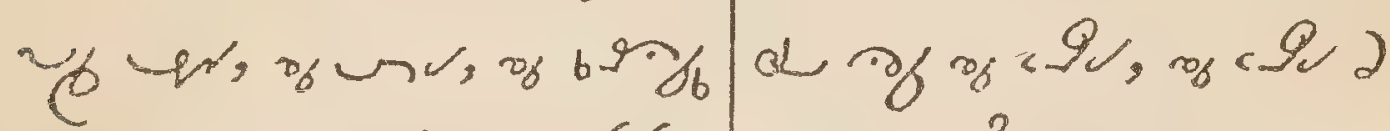

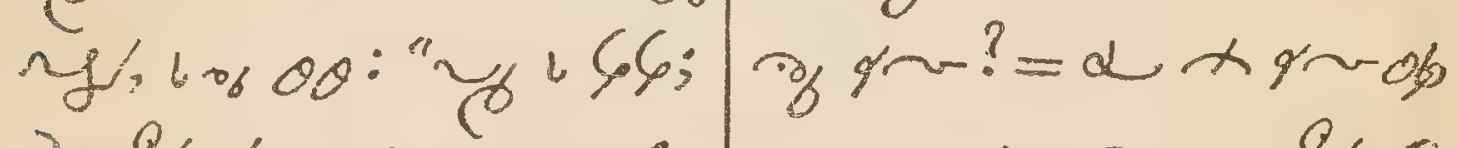

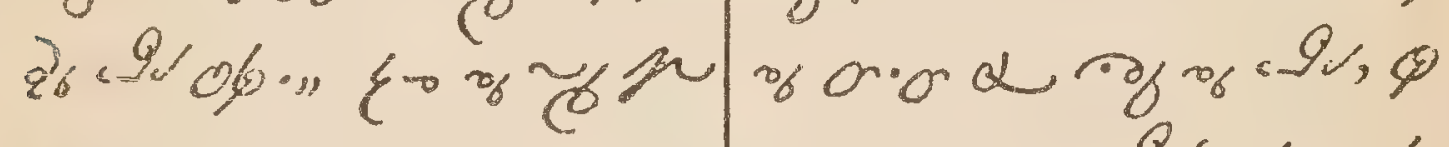

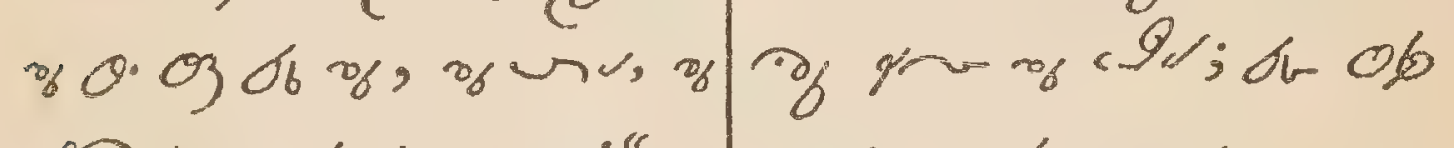

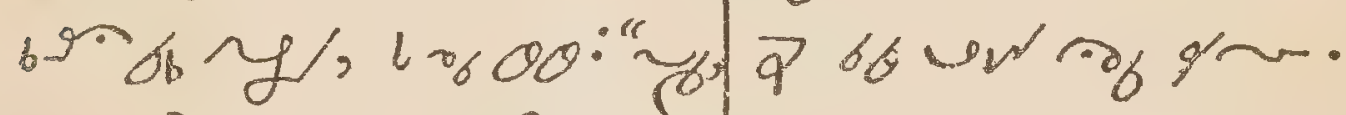

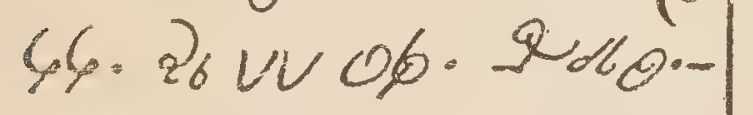

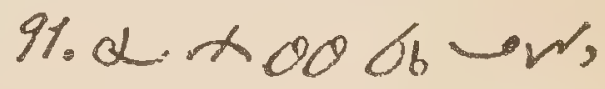

$c_{66} 6606, \alpha c_{06}$ b? $?$ obe $26 . "$

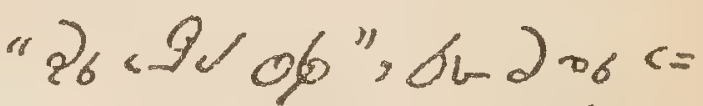

$94 G_{2}+\rho_{\text {wo }} W$ ? =

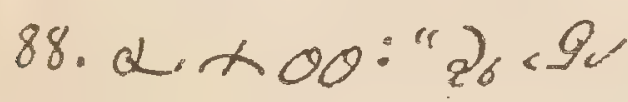

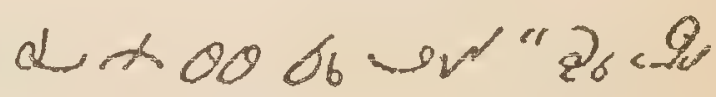

of, 26 vv 06, of 26006

ob", 9 br + ro $490 \mathrm{GH}$

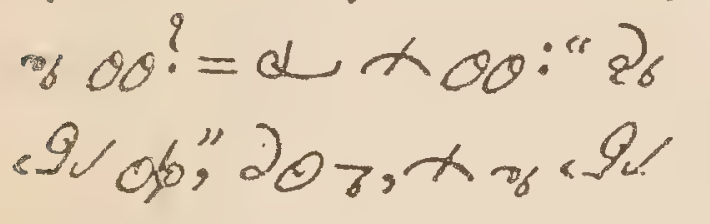

bo ob bo en, Ond

UV $6.5,0.0$, viG.

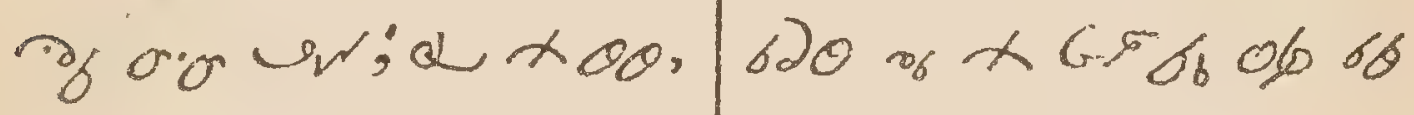

"Q6 V of ", 20r $x$.

wor.

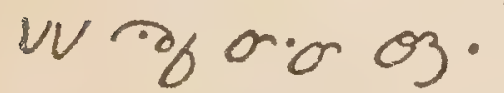

92. 60 me tex 10066

8\% a $x$ og 94 कु 0 .0

$\theta ?=x \operatorname{CGO} \theta, \alpha v_{\sigma} \dot{\theta}$

ur, a $x$ चv

b) $6(5) 22 \times 62_{66}+1 \%$

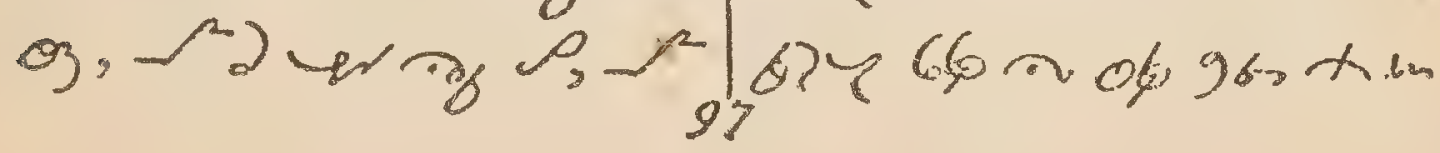


92

CHINOOK

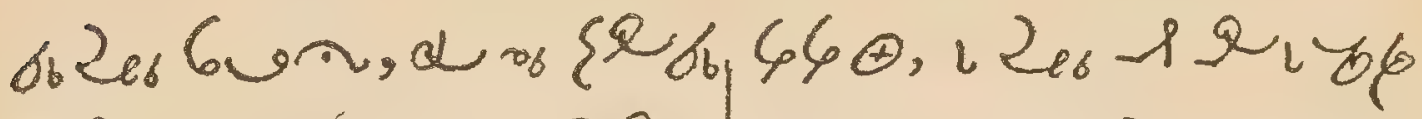

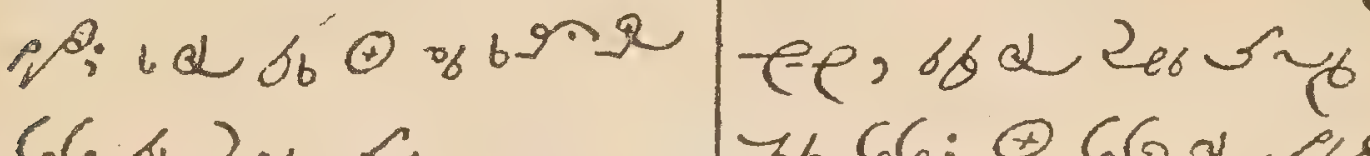

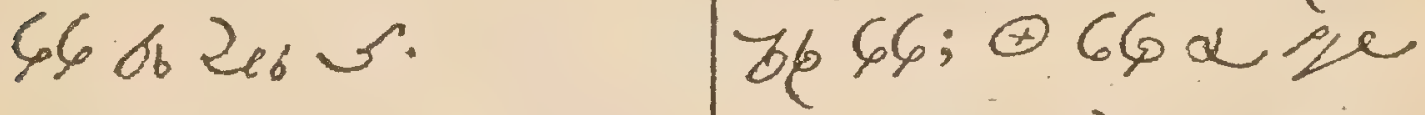

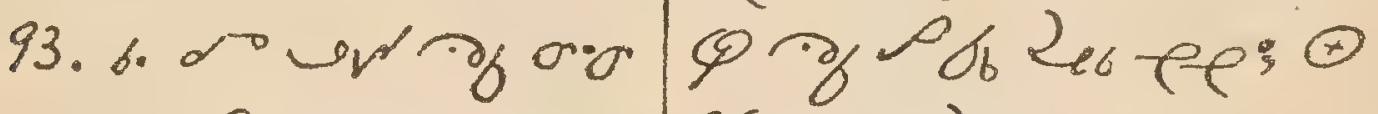

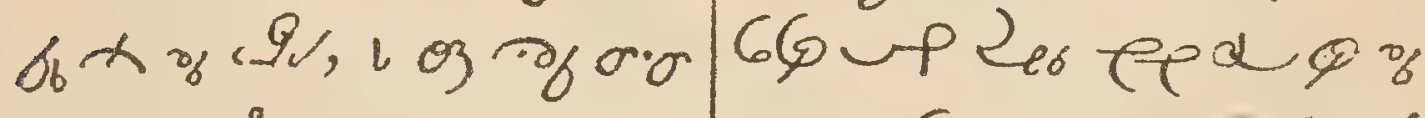

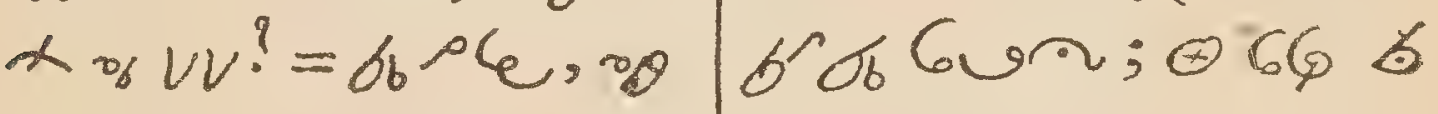

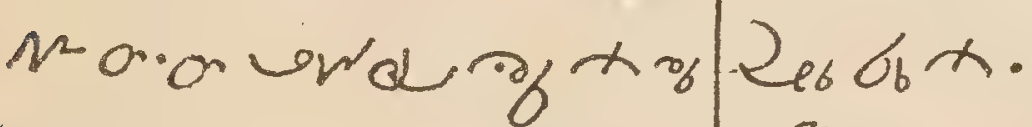

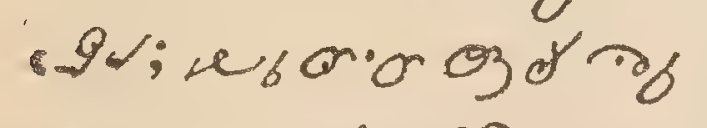

$$
\text { 从rvV: }
$$

ry o d w 69 so.

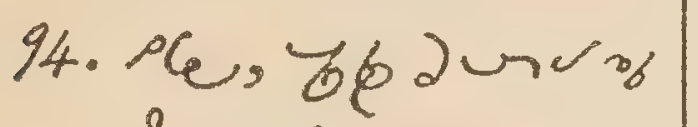

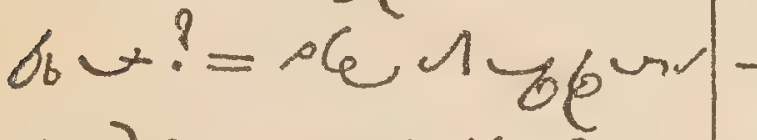

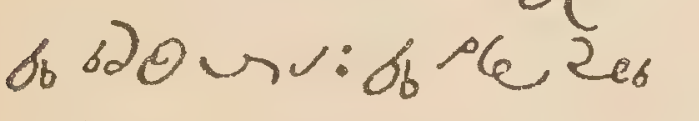

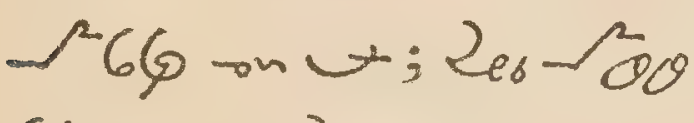
seng, $\sigma_{b} ; 2_{e_{6}} \ln 6 b_{6}$ ob 6 an $9602666 ; 2060$ Q

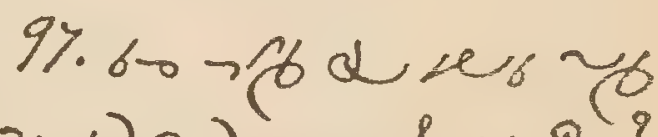
$\oplus ; 6202 x_{6}-166$ ?

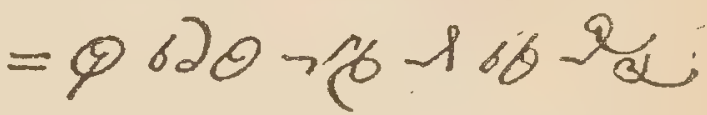

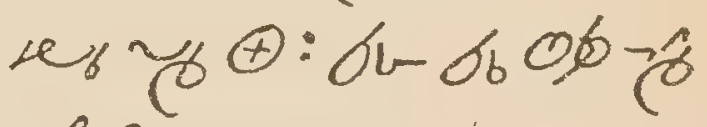
sex felis fo Q, bubses $\otimes 66$ ob Q - e .66 vore $V \oplus 66$ br ob 189 arete

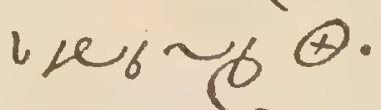

98. $60266661266 \%$

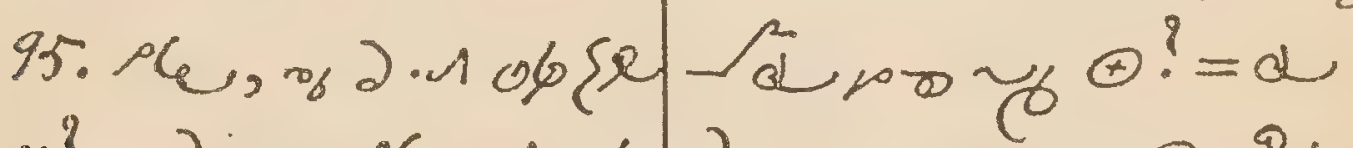
$-\mu^{\circ}$ ? = 20r,

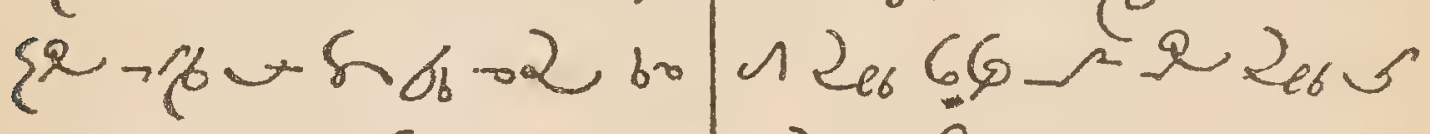

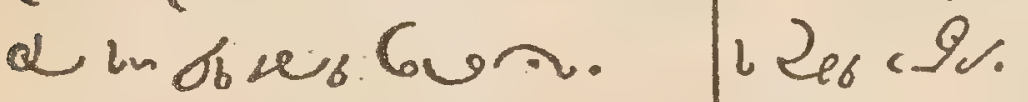

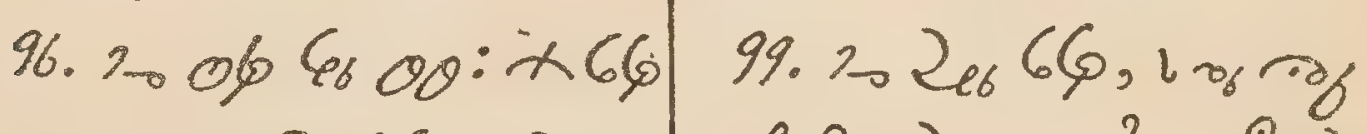

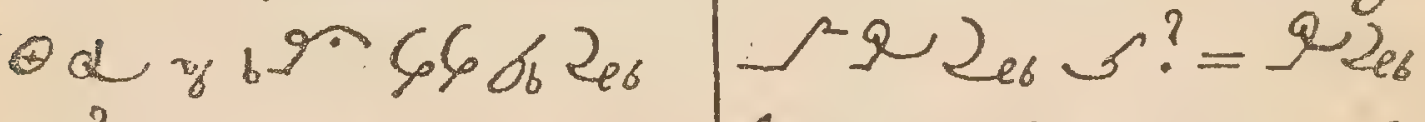

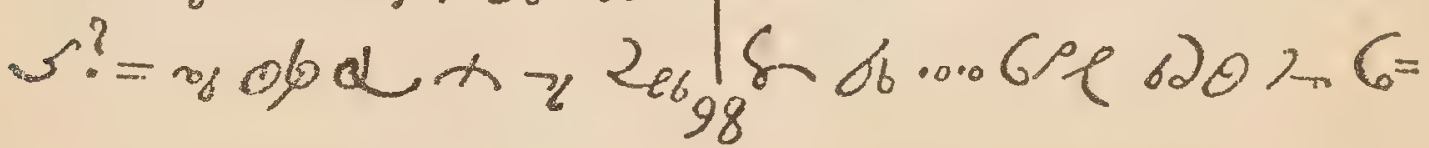


MANUAL.

93

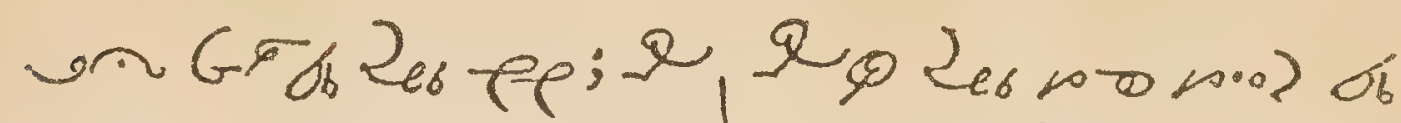

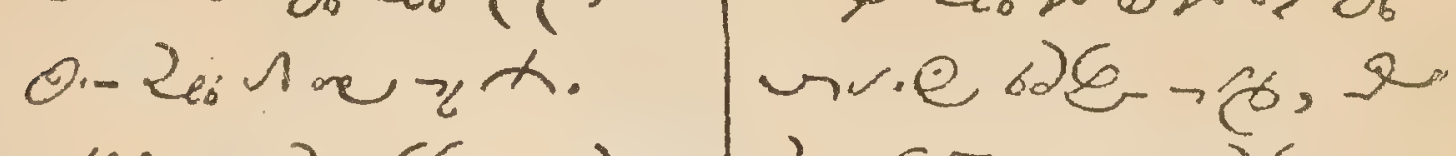

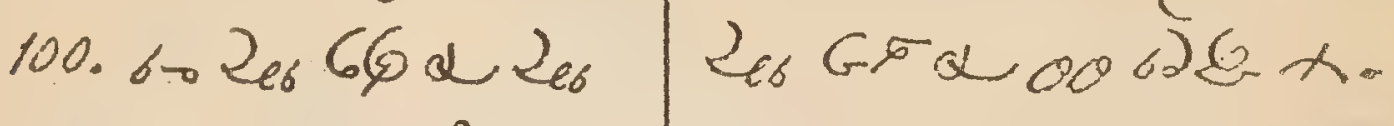

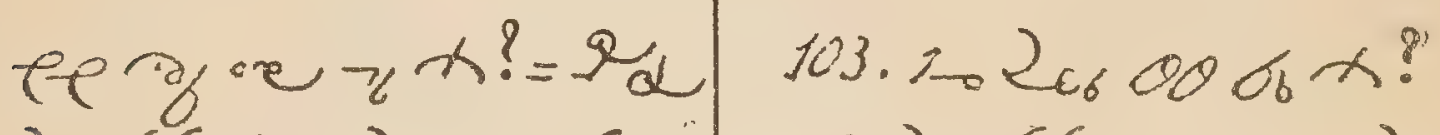

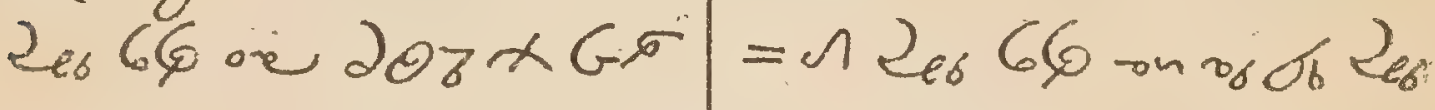

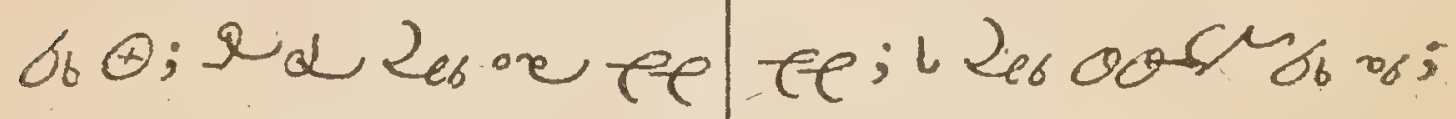

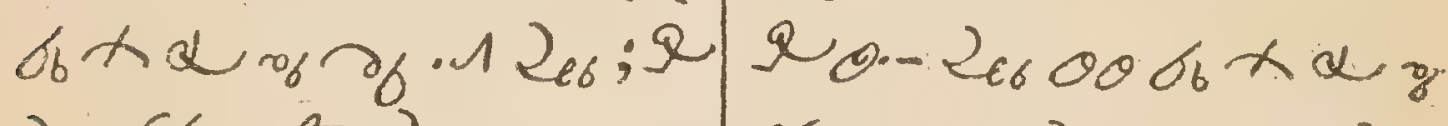

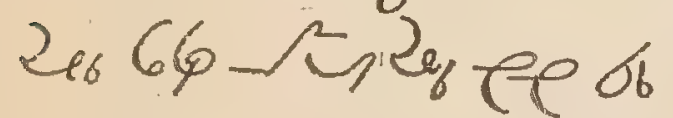

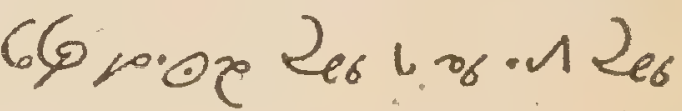

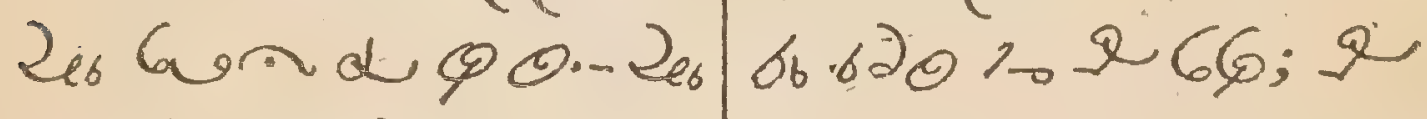

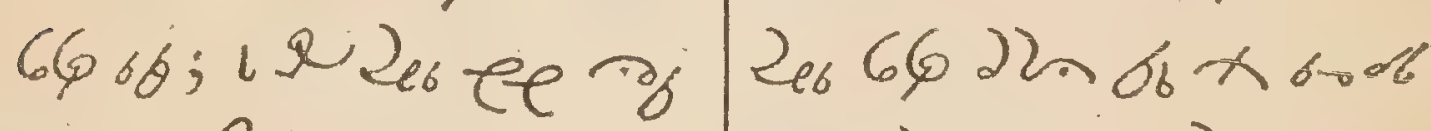
ore of bt.

101.602066601206 36206 e $6602060=$

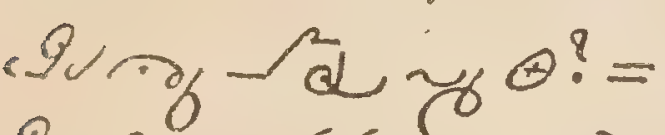

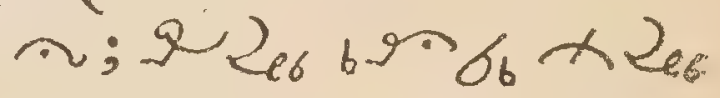
ell 2ac9u $600 \%$

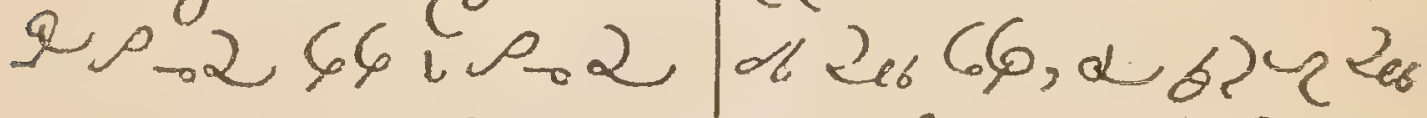

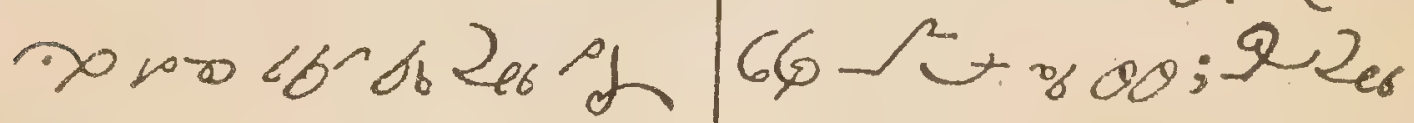

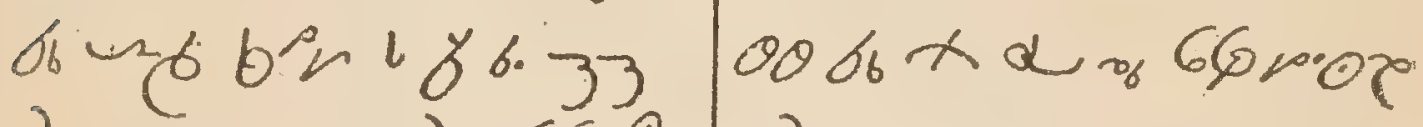

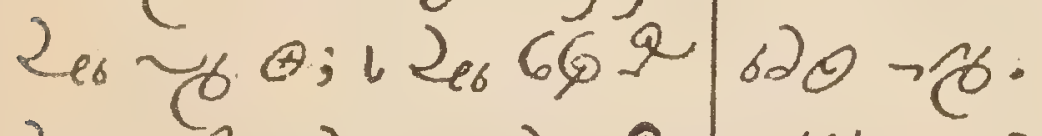

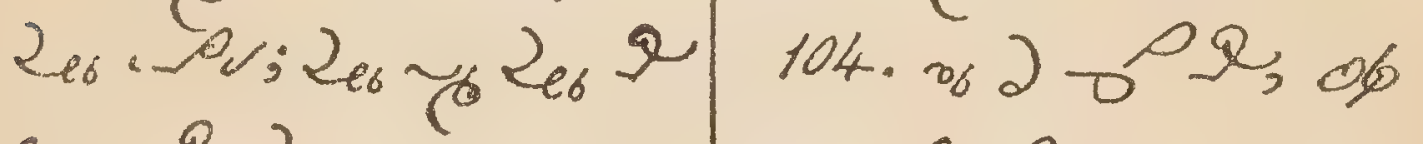
he; Q Qt

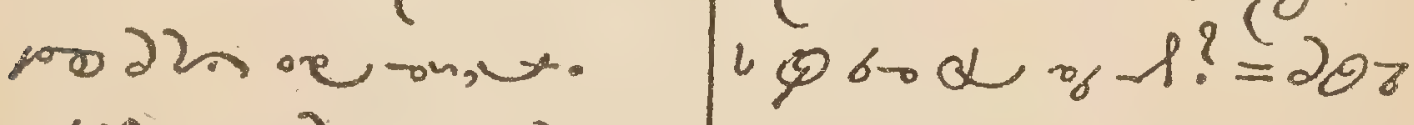

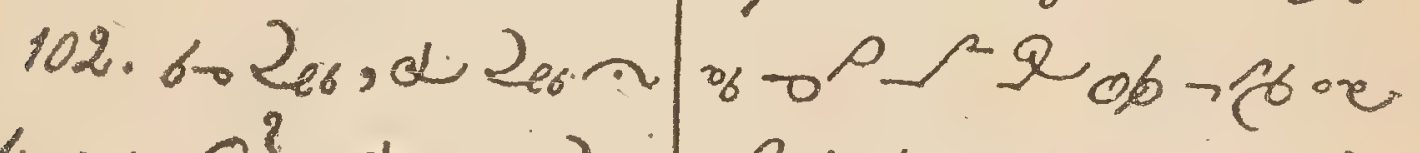

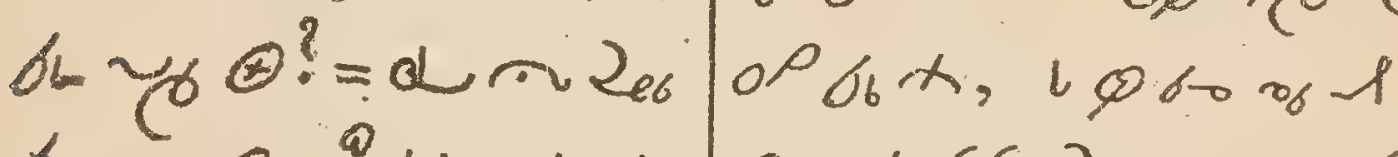

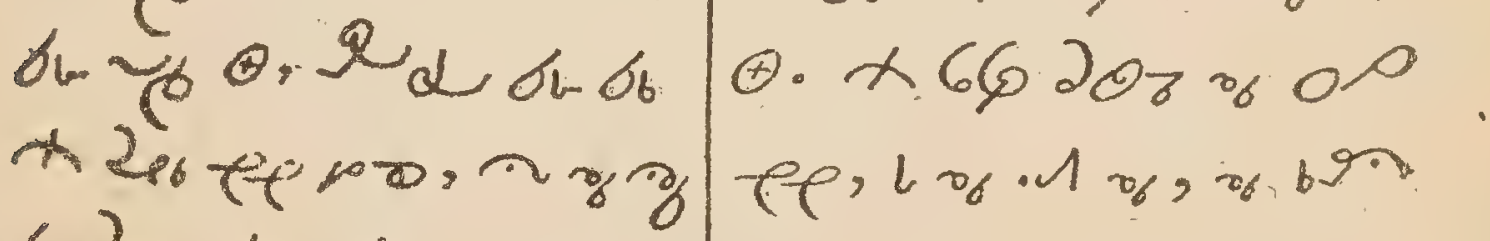

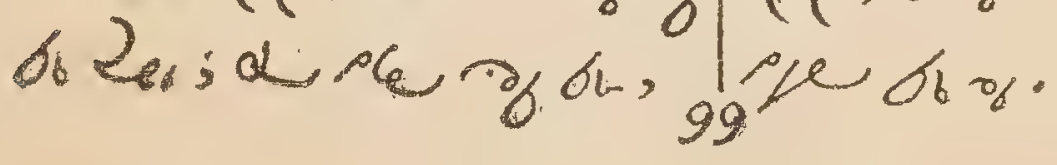




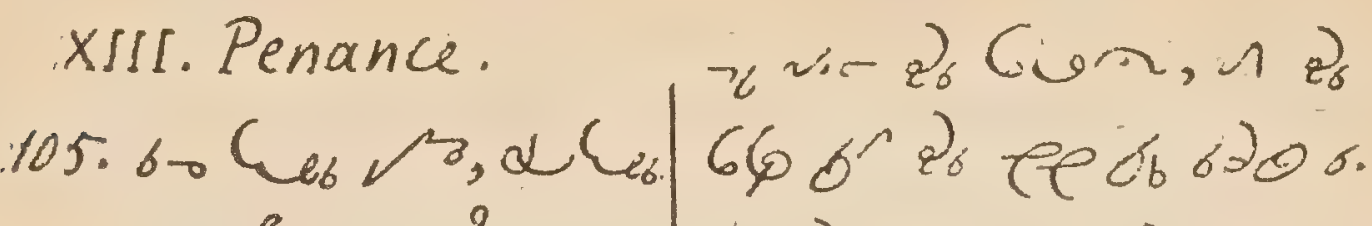

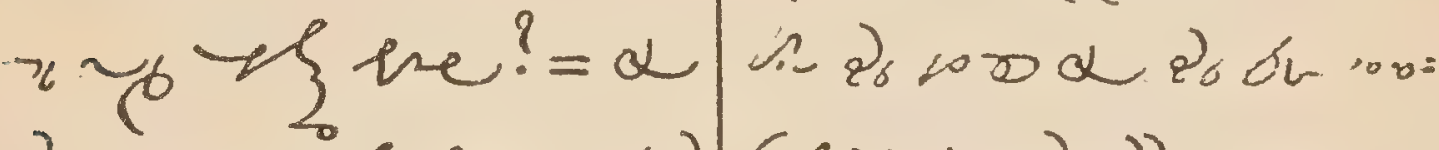

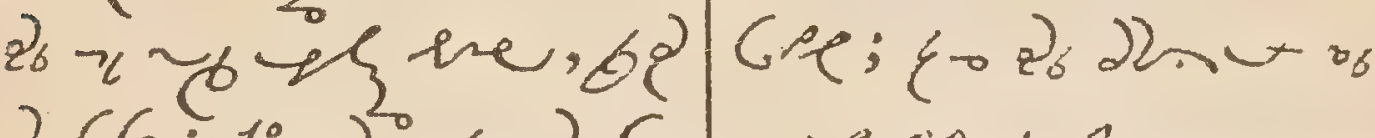

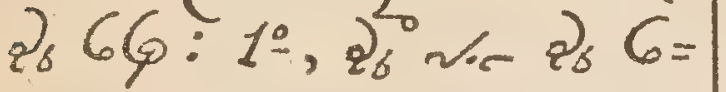

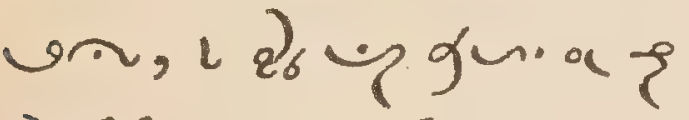

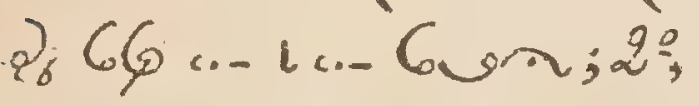

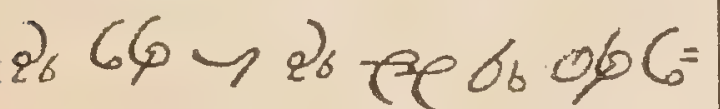
an $2_{6} 1,122_{6} 660$ 26 $\operatorname{ted} 90.286660$ त; 3․, 2. 100 650 be 2. Guत $6 N^{2} ; 4^{\circ}, 26$

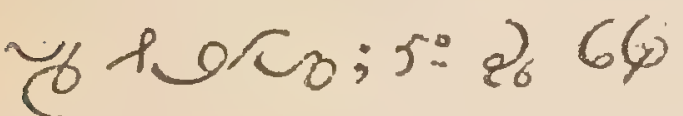
ere $M_{6} T^{\circ} \sigma_{b}$.

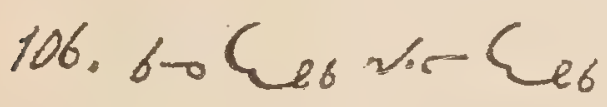

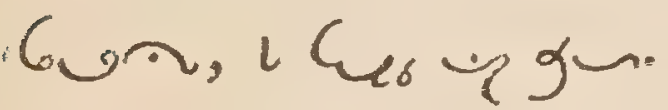

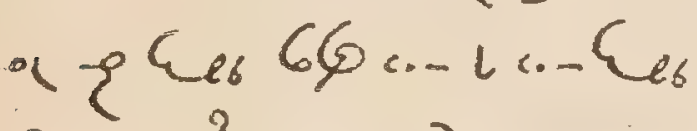

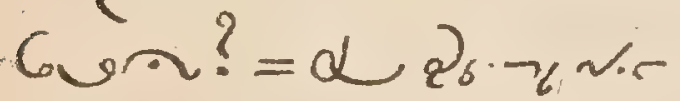
Qे Gun, 2े poobrur

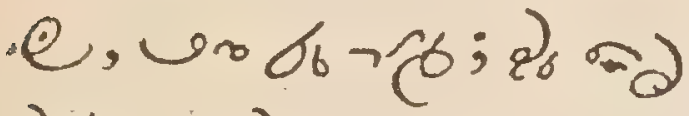
$2_{6}\left(y ; z_{6} \sim \sim b_{b}+\alpha\right.$

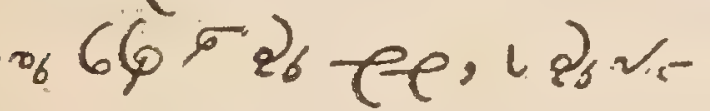
2.6un.

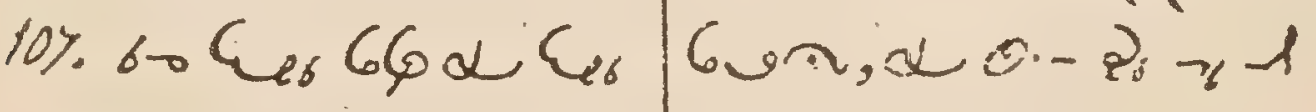

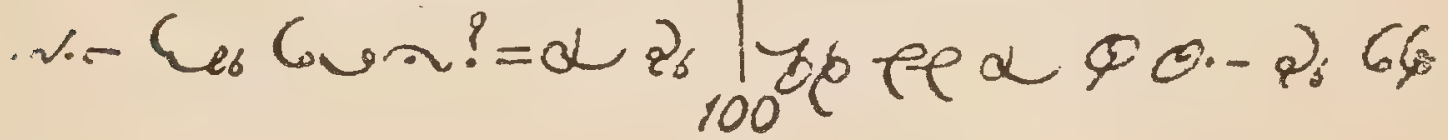

ᄂ $3 E_{-} r_{2} \sigma_{6} 500 \sigma_{3}+$

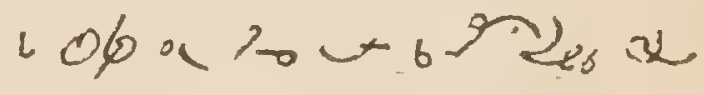
Lis $66 ; \xi_{0} 26500$ 2. $\operatorname{con} \alpha)_{3} 6 \sigma_{0}$ gu a ₹ 2. $66 \ldots, 1$ gu. a 8 zे 660 ... 108. Q ces be 65? $\mathrm{Ces}_{6} \mathrm{G}$, $10 \mathrm{Co}-\mathrm{Ces}_{\mathrm{s}}$ $66 ?=\alpha$ 23 bu C60 -

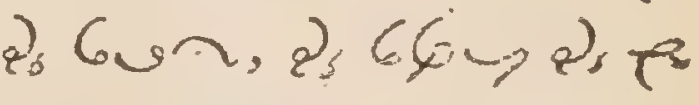

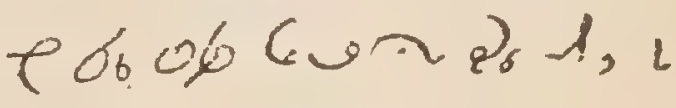

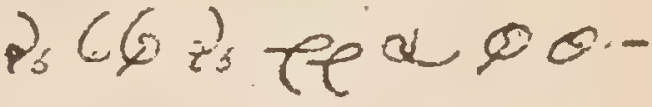

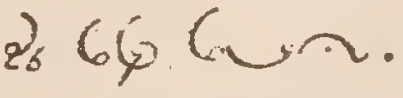

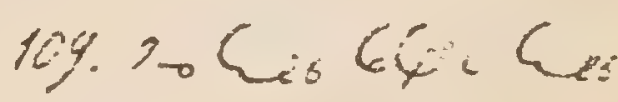

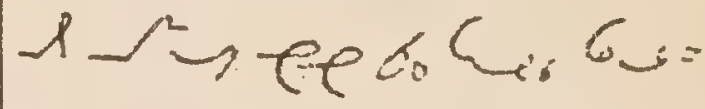

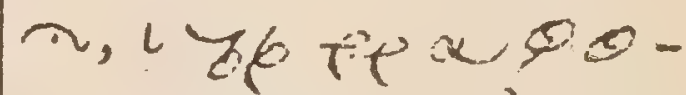

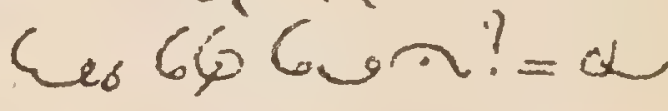
8) $\rightarrow 66 \leftrightharpoons$ \&'s fe $C_{0}$ ? 
MANUAL.

95

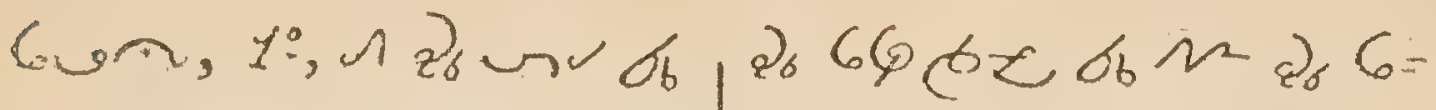

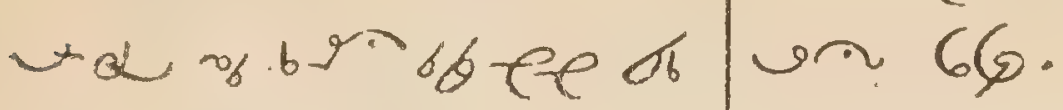

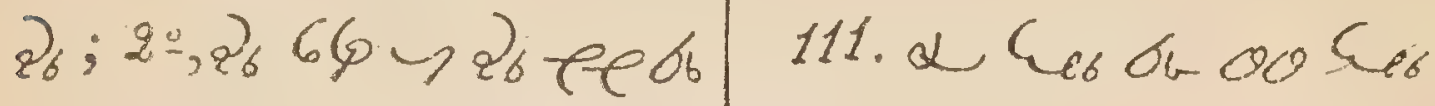

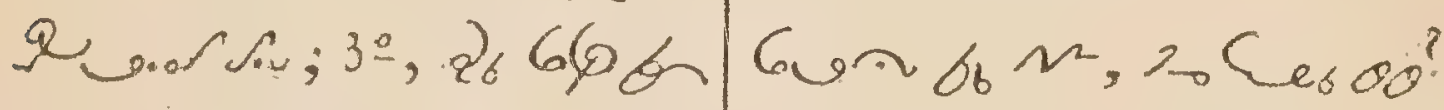

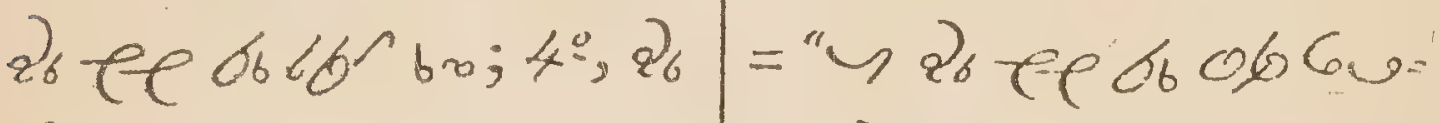

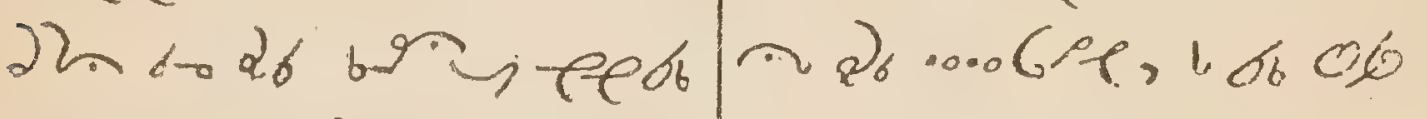

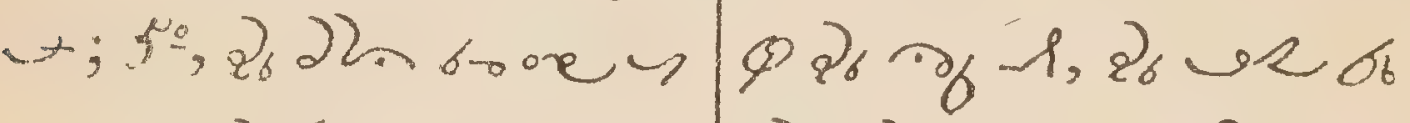

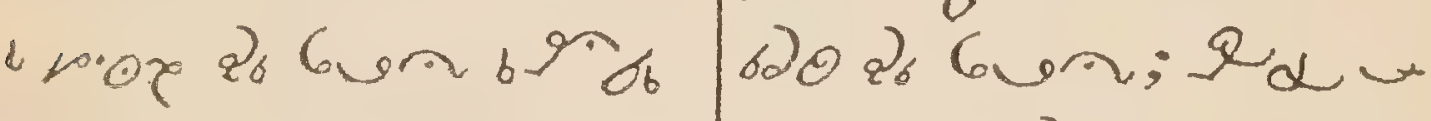
स⿻上丨

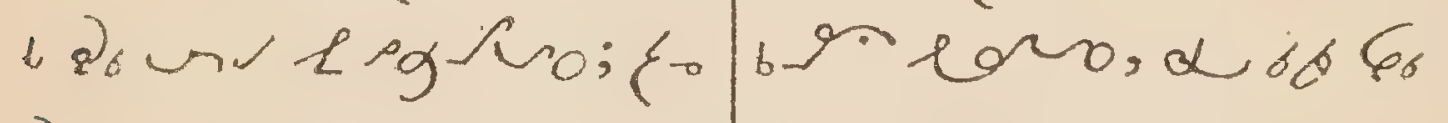

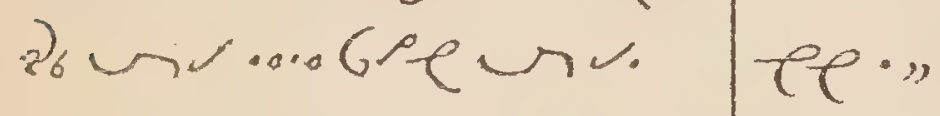

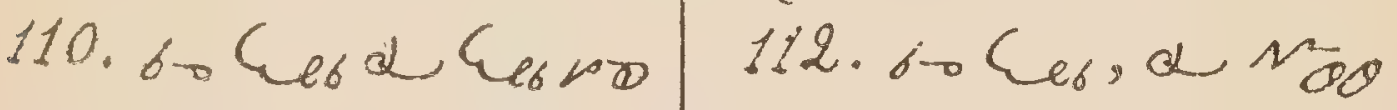

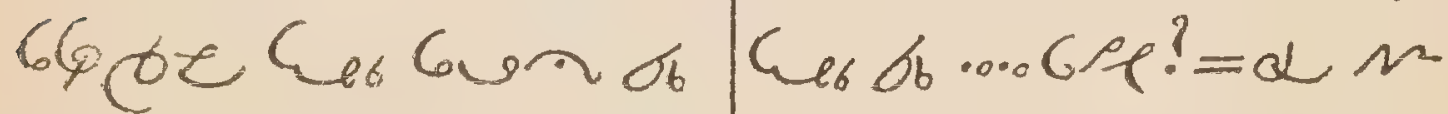

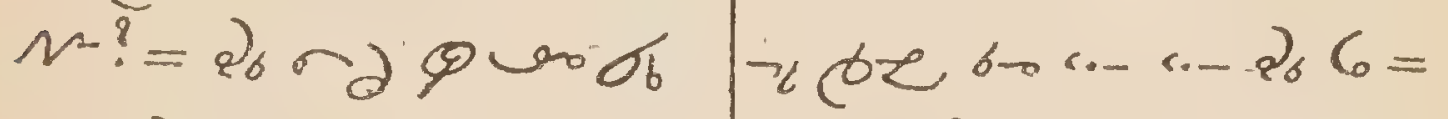

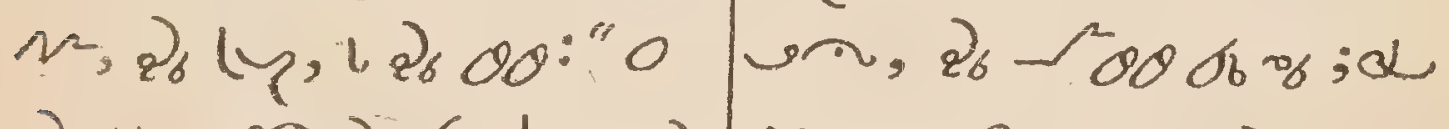

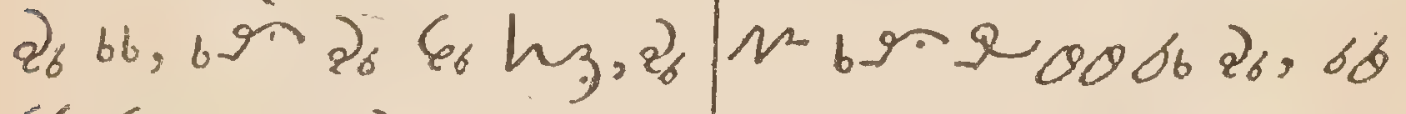

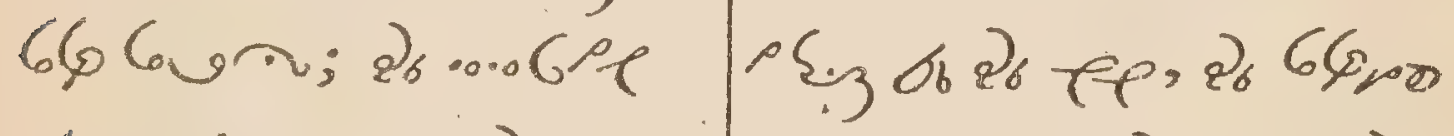

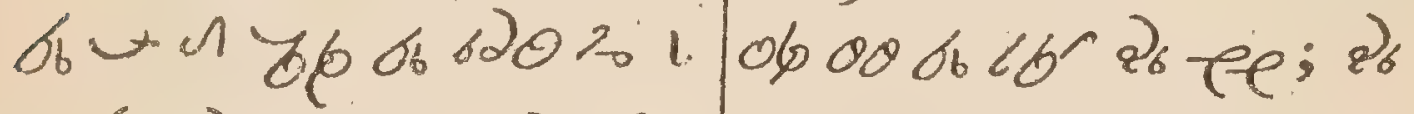

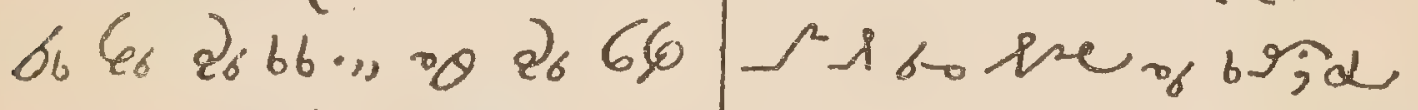

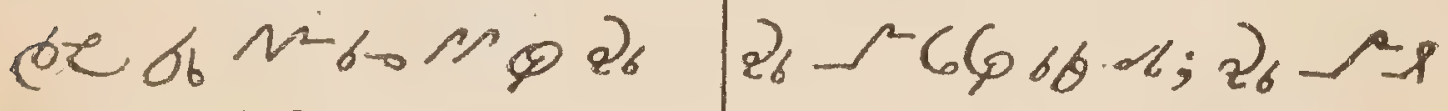

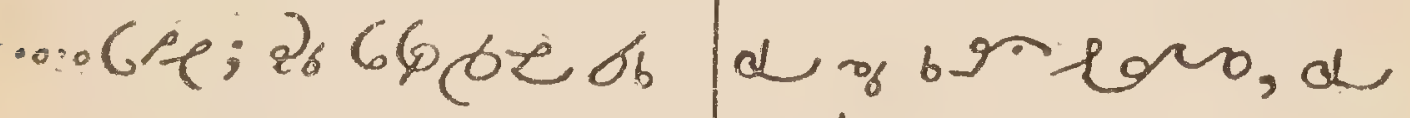

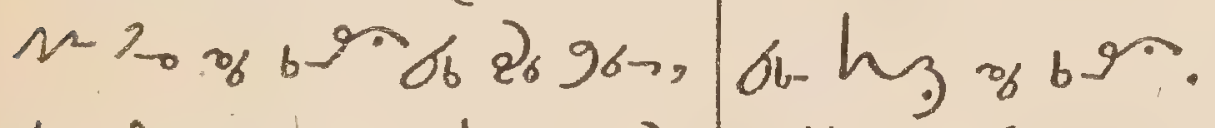

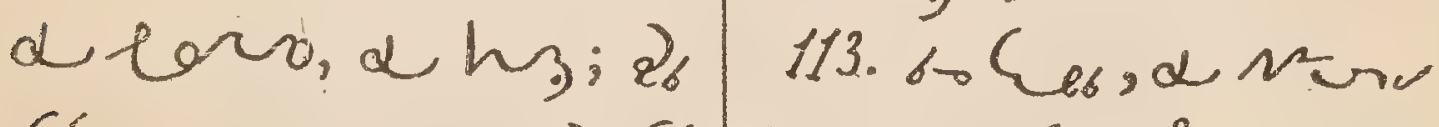

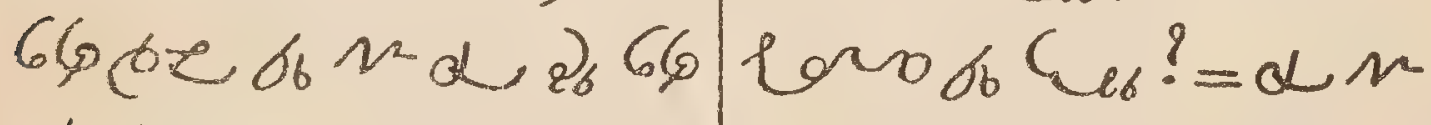

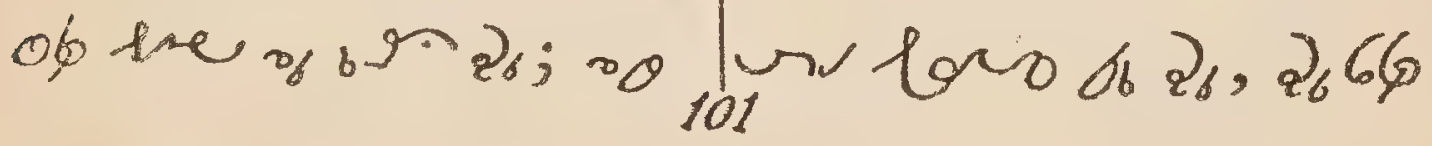




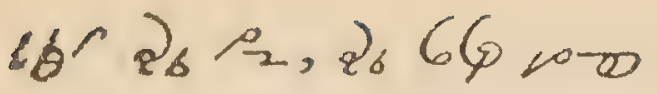
Lforr. $\mathrm{s}$ ? e

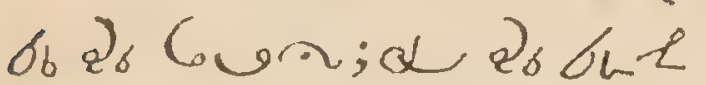

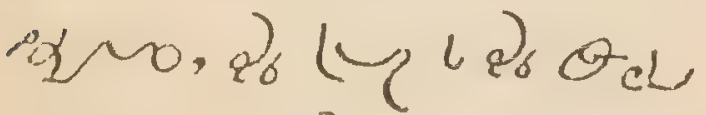
रू०० के मका

$114 \cdot 6-C_{e r} 66, \alpha, N^{2}$ 00: "w⿻, lun b \&6."?

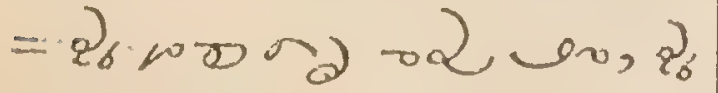

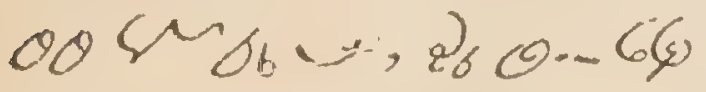

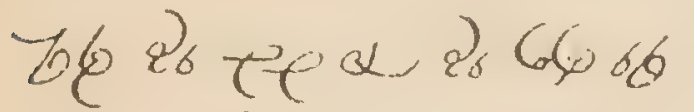

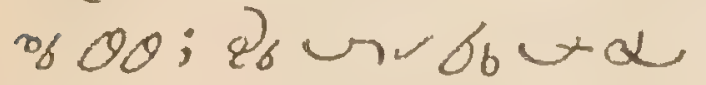

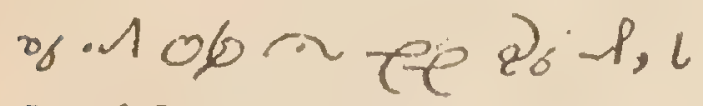

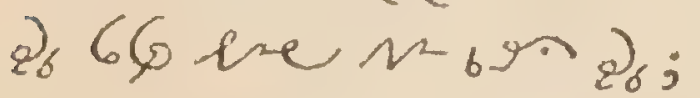
Q शे की, शे po 10.0206 us.e, l gar as pov. 115. . 06 tour 12 b9. $66 \cdot 0.0 \mathrm{CP} ?=0606$

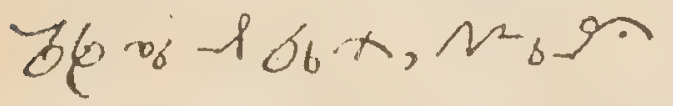

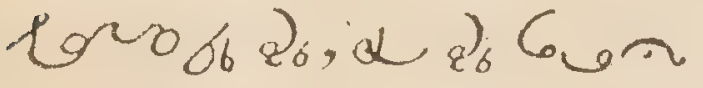
ig $5, \alpha, a_{6}$ सe

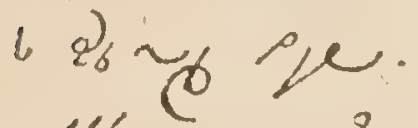

116. 6ro $\mu^{2}$ ? of 2 \&? $?$

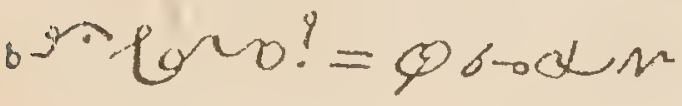

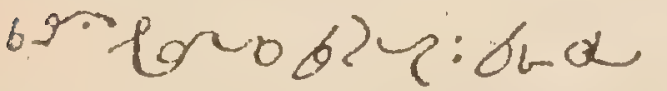

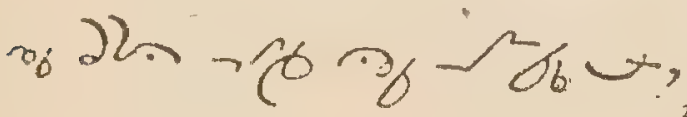

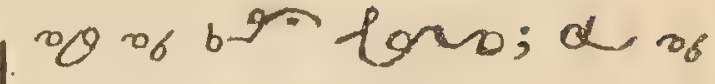

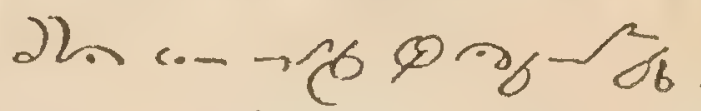
t, bu hy of 6 g.

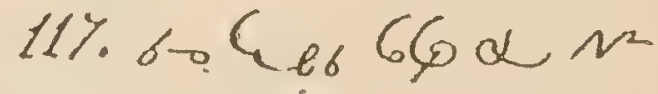
Q9 6an lovo br Eer,

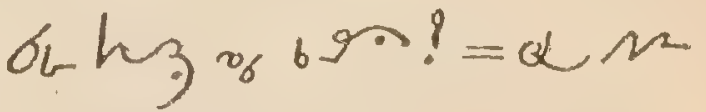

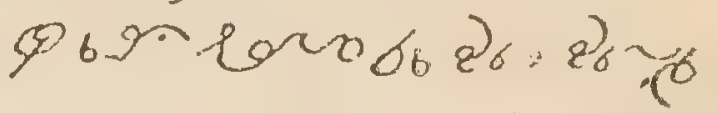
Nox y e of 2o $69=$ n, $\varepsilon_{b}-60682^{2}$

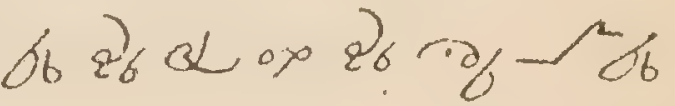

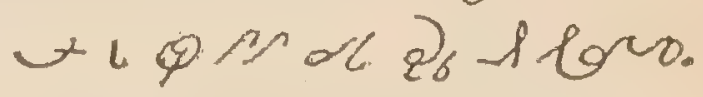

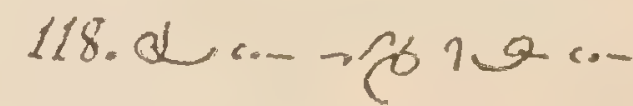
og Gom 66.0.0P, lo

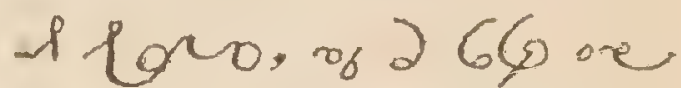
Gun? = 203; ore $60=$ थ \% 600 ob न

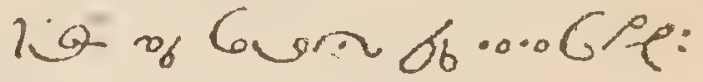
of ... Cregfor;ob

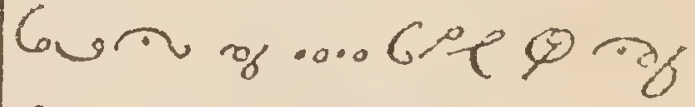
S; $\alpha, 0$. ong $^{\circ} 00.0=$ cre, grad ag sh Goribu al of of 6. be bracologa 1.9 960, lo o8 00 gu. ?

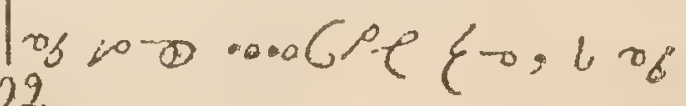
102 
$000-066 u$ ang gr wa, 19208 \% 66

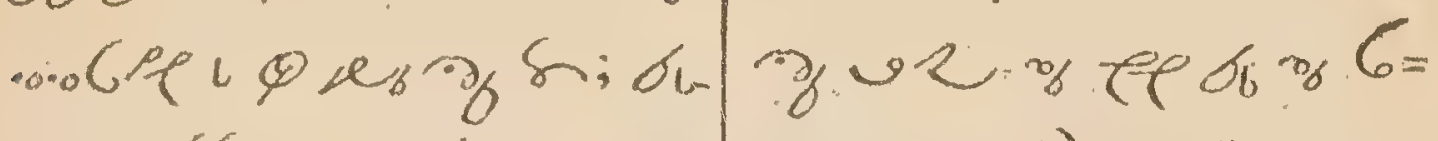

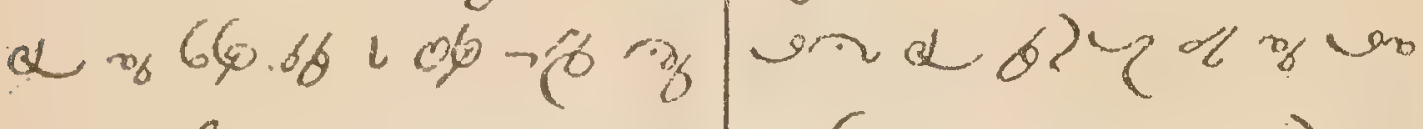
(c).- $\Omega_{\text {ob }}$.

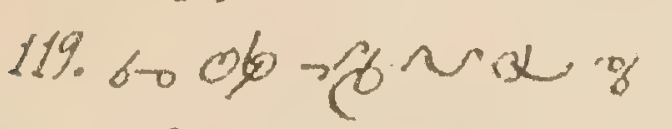

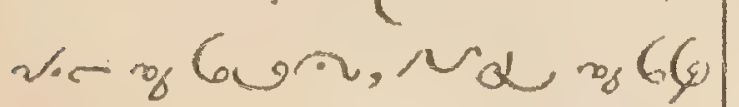

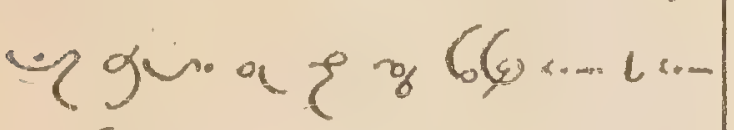
च6 6ur, of 2600 ore Gun? $=208$, or $6=$

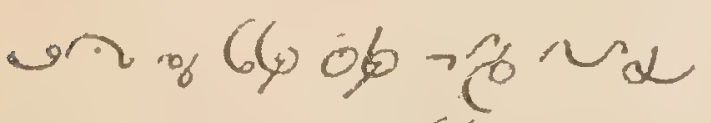

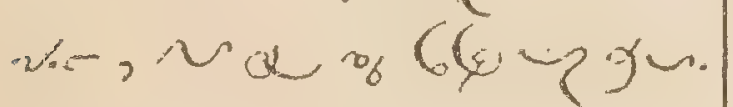
$a+v_{0} 6 \sigma_{0} \cdots-1 \cdots-\eta \sigma_{v}=$

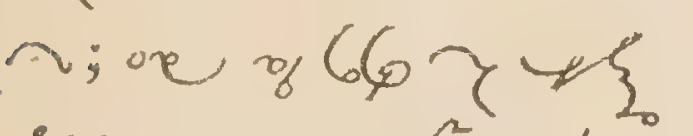

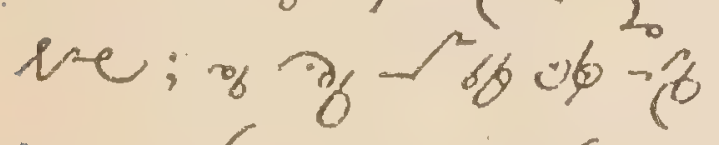
$10 \% 60$ \% 60.006.

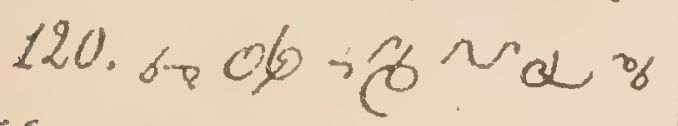

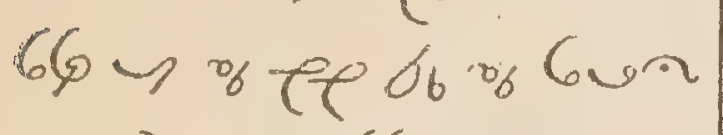

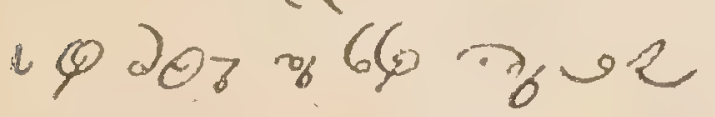
m elobroura

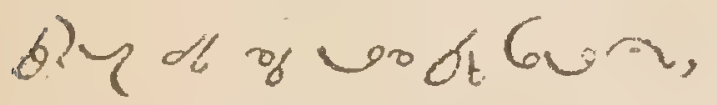
160620061.06600060

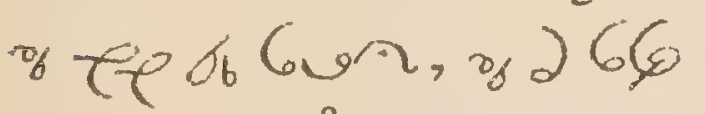

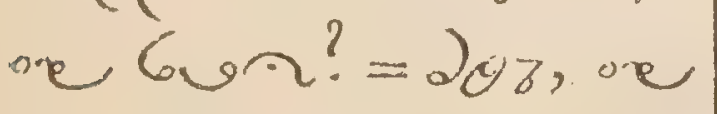

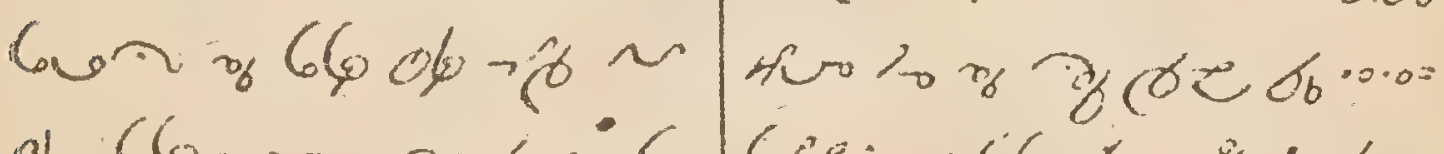

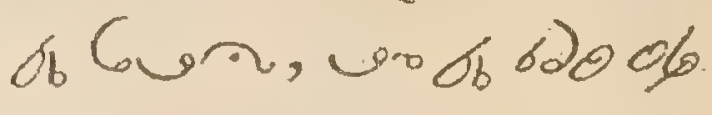
1060 m bu in ted

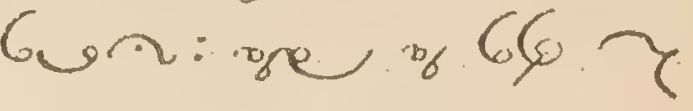
there; of $x_{0}{ }^{2}$ ob ob 伤 karg lowa ...che

$121.60606600 \propto$

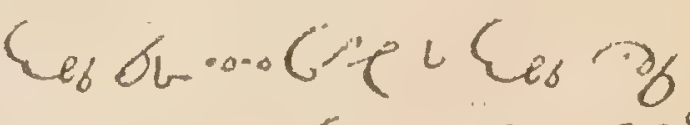

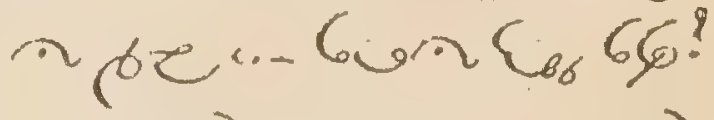

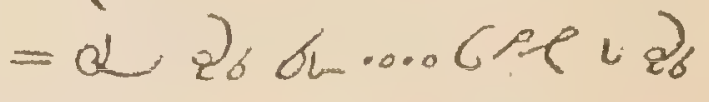

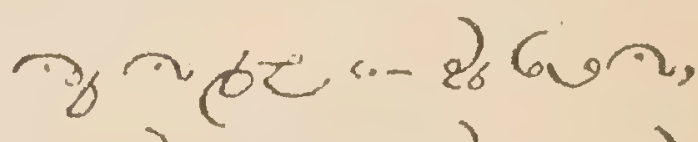

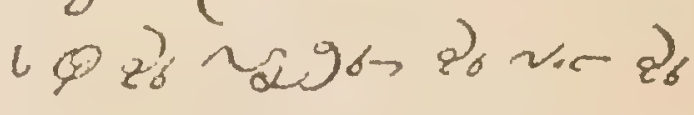
Gua, uhioblua Q 26+1806-066un

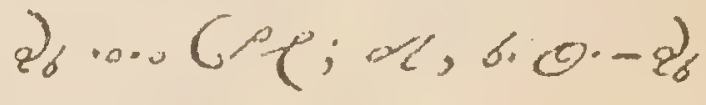

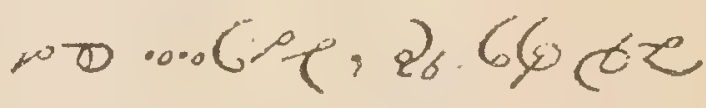
cologin ob 2

122.6012 ? गु 20006

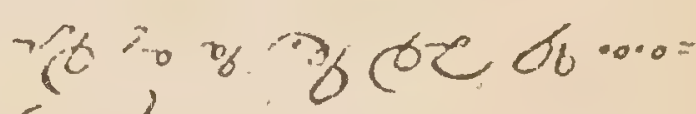
Cipe $=460 \alpha N^{2} 0806$

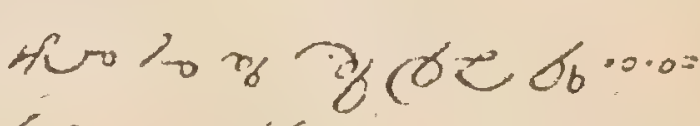

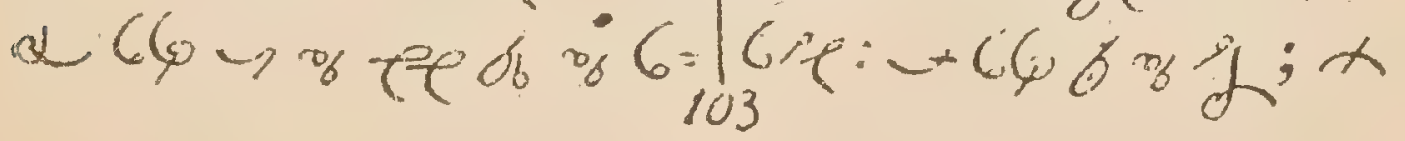


98

CHINOOK

ofeably w60\% Mrg be bb a.o. Ge

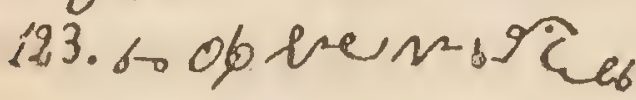

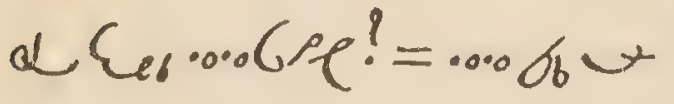

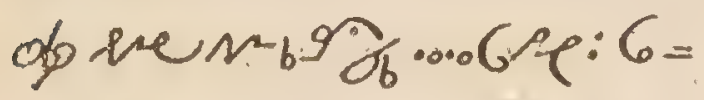
and lab hog, Gin ol

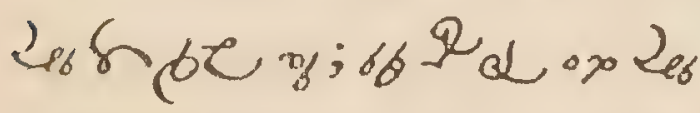
66 ob the, I s og oo $\mathrm{N}$.

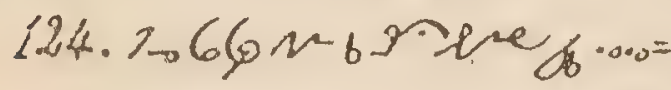

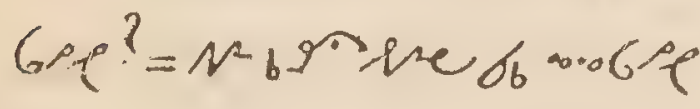

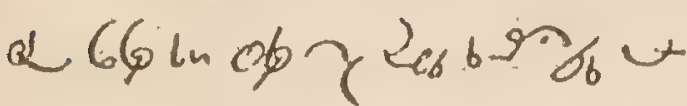
d 20666an; gew Ge a d 2 2 in bo ob bor रes 66.

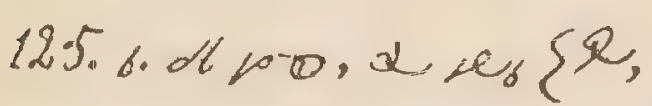

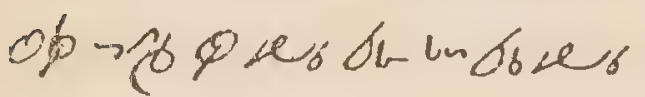

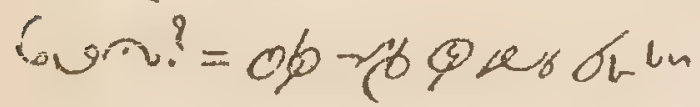

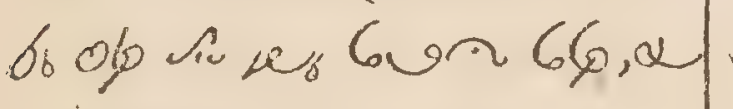

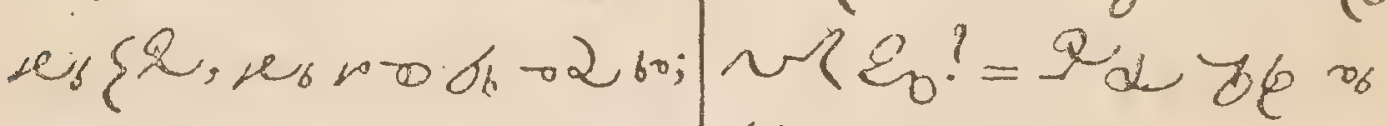

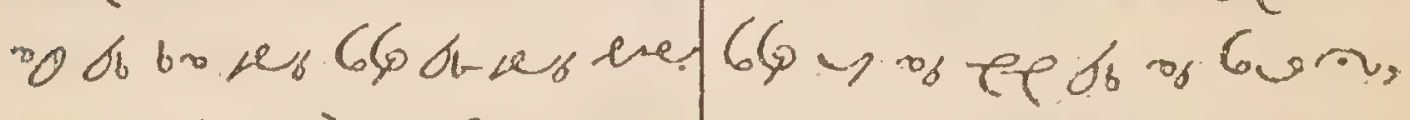

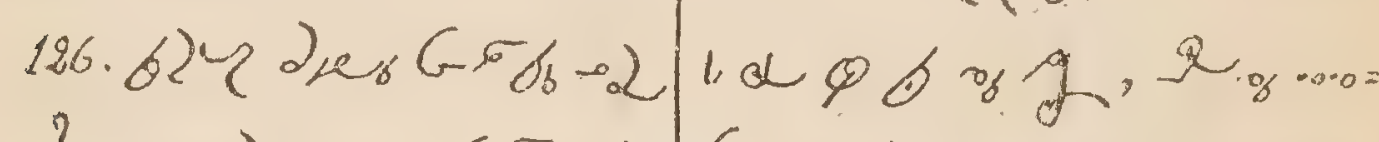

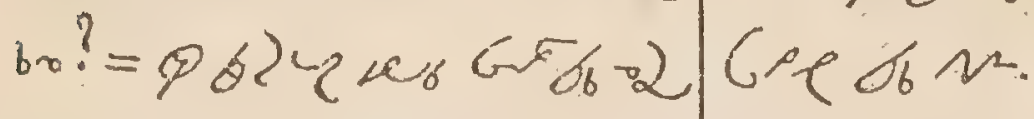

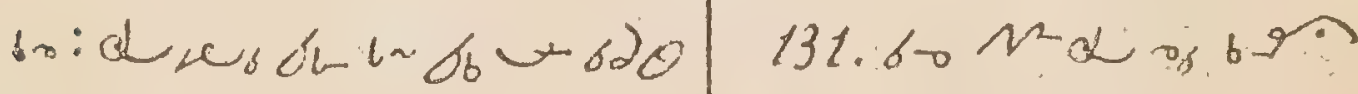

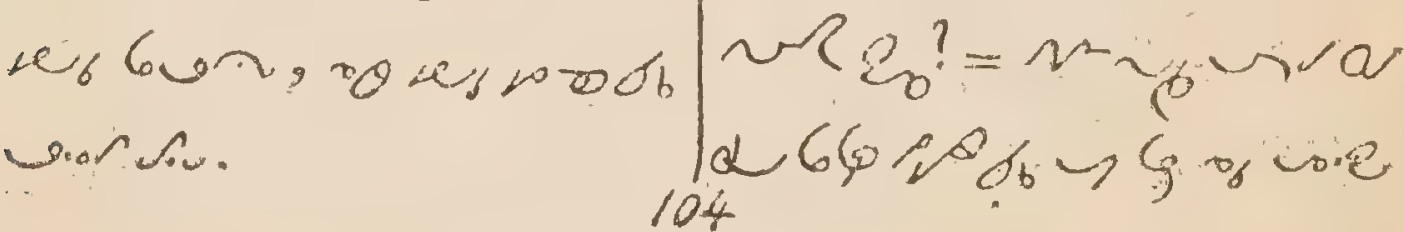

XIV. Extreme Linction. 12\%. $2 \mathrm{R}\left(Q_{0} ?=\sim \mathcal{E}_{0}\right.$

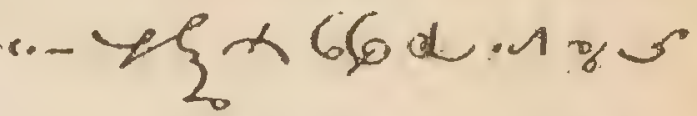

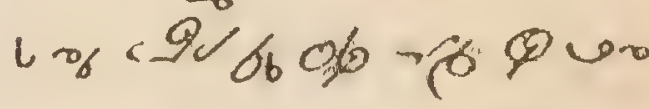
\{ 2.

128. $602122_{0.1} 060$

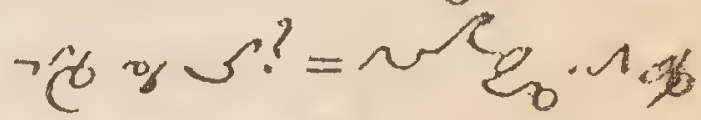

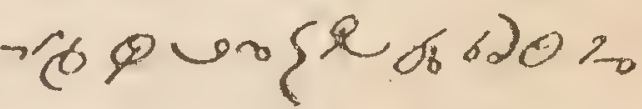

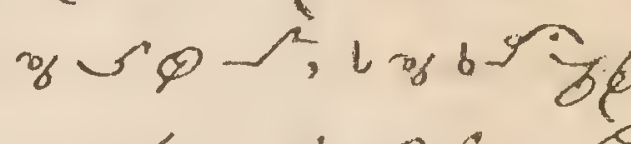
ee $\sigma_{6} \%, \alpha \phi ; 0$ br 6ur.

$129.8-2-2 \varepsilon_{0} \cdot 10 \%$

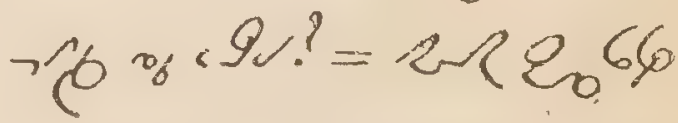

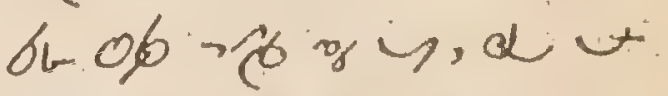
$2 \pi$ al $e_{\text {or }}$ of $\rightarrow 9$ ro

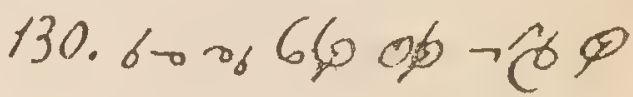

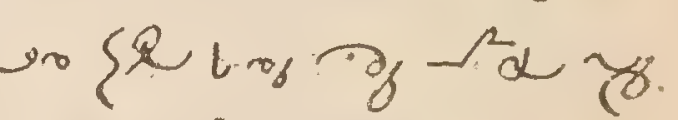


MANUAL:

99:

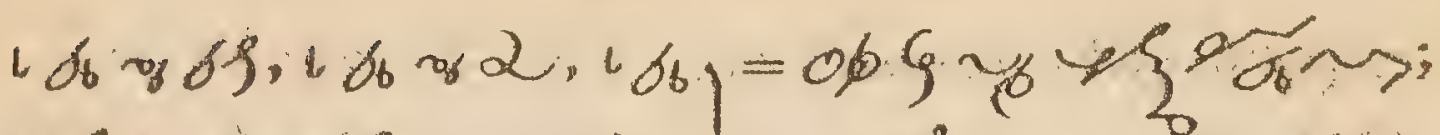

कर

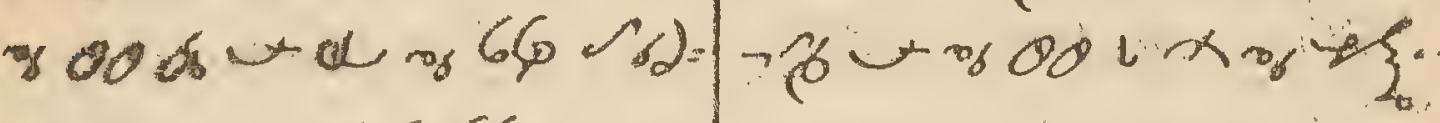

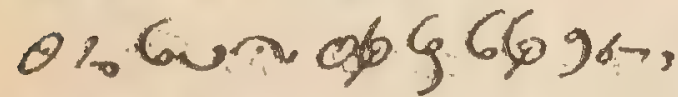

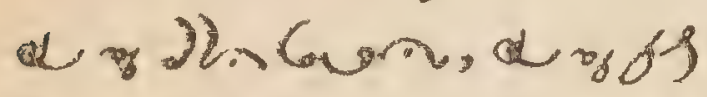

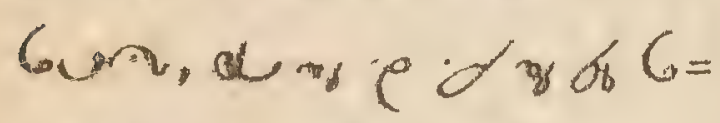

an, al g 20060 ,

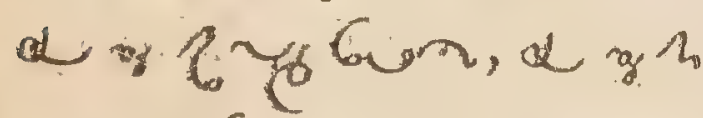
moblon.

Xv. Holy Order.

132. $.0 q^{\prime} \cdot=q^{\prime}$

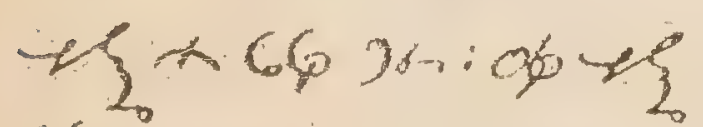

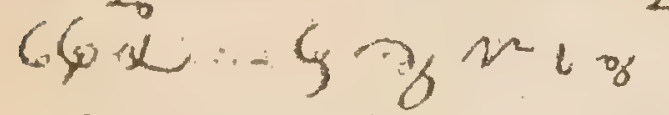

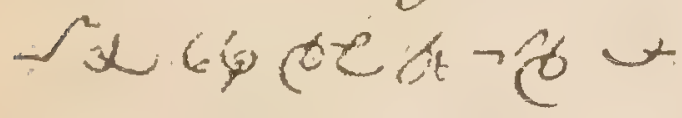

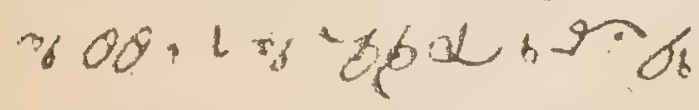

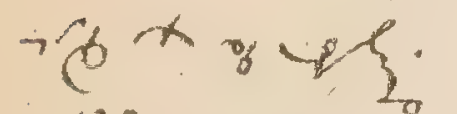

13.3.6. $\times 64^{\circ} 9^{2} 9_{6,} ?=$

$\alpha+6600,+8 \tau_{j} 66,0$,

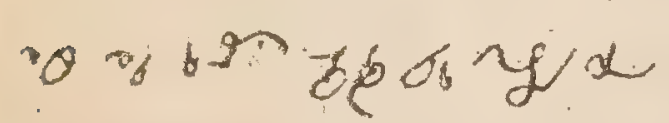

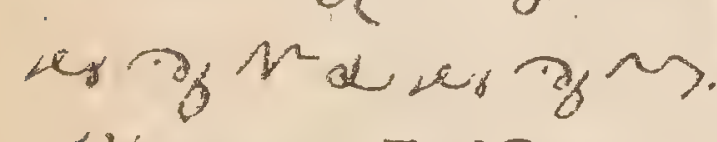

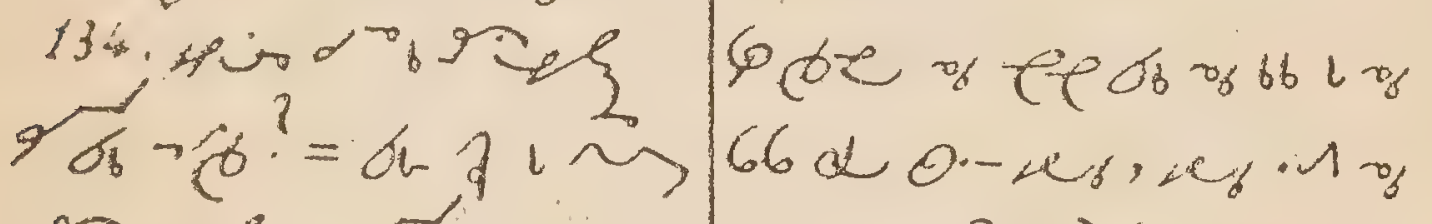

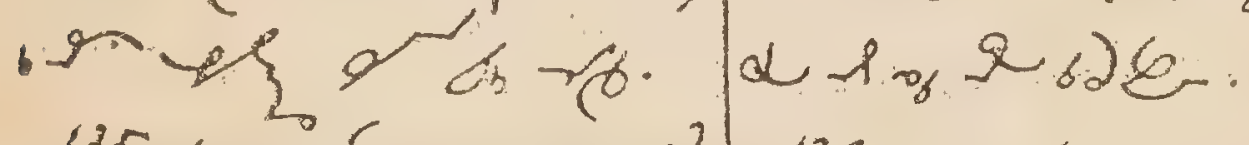

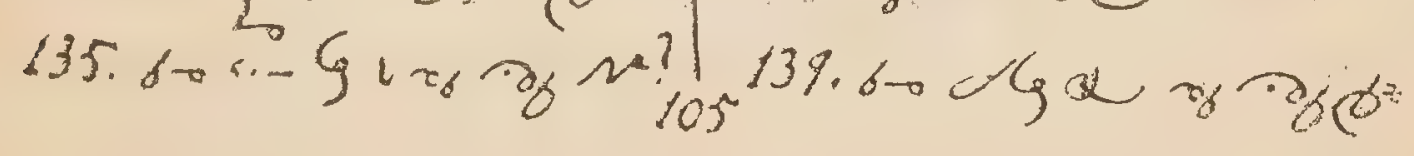

XVI. Matrimony.

136. $10 \mathrm{chon} ?=\mathrm{rm}$.

‥ 2$\}$ of 6,6 al ... $\}$.

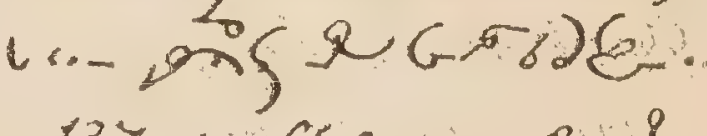

$13 \%$ - $\rightarrow$ ring ge?

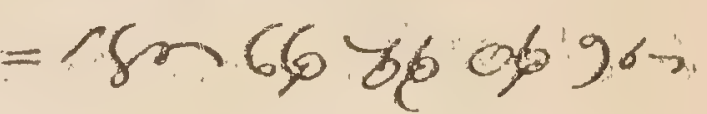

+66 a clipafie.

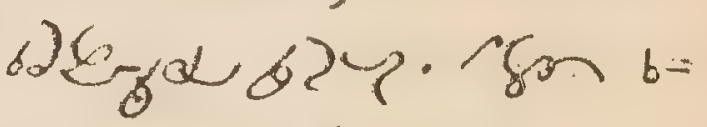

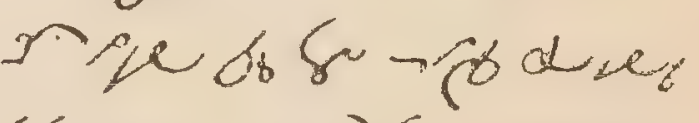

65000 6) 6 ; $18 \mathrm{~m}$

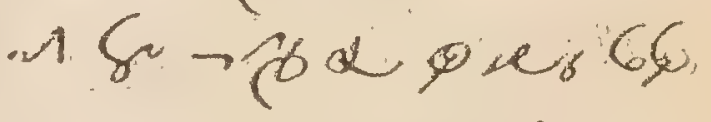

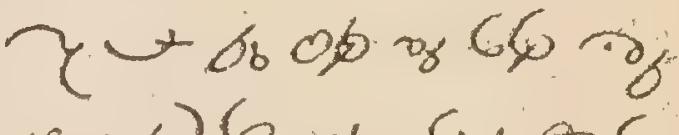

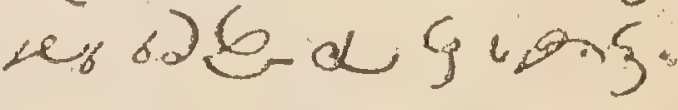

138. d ...- - क力

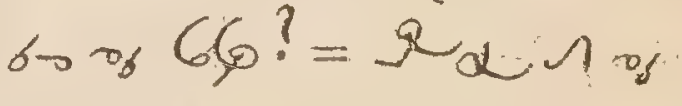

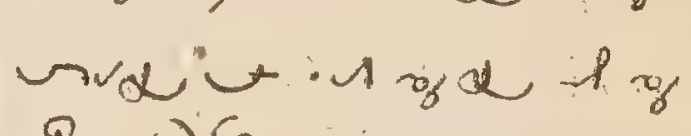

Q 62 E, 1 a

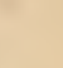


roo

CHINOOK MANUAL.

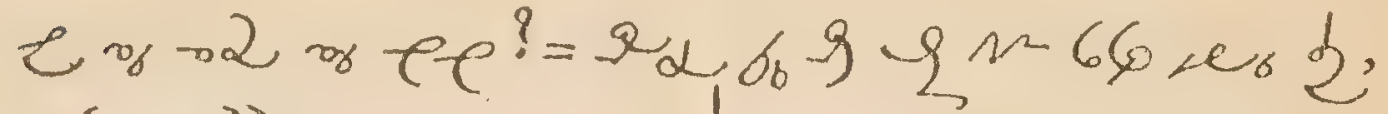

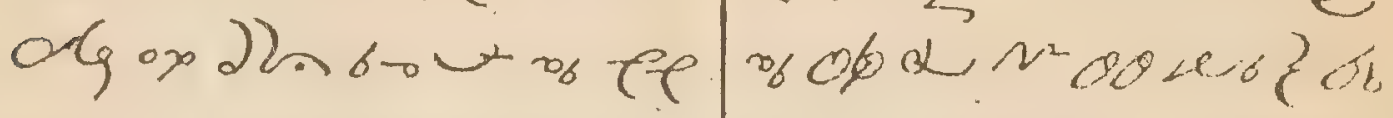

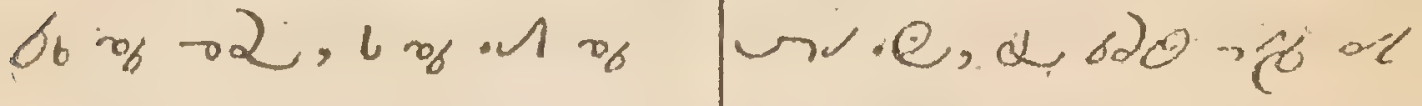
$\rightarrow 2$ al $406 a^{2}$ o) 6

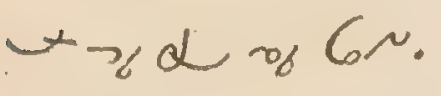

of be res $\Rightarrow$ b ives

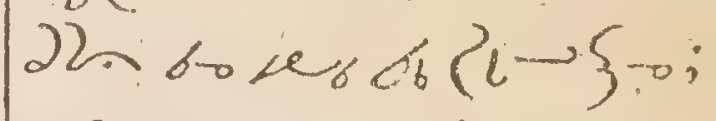

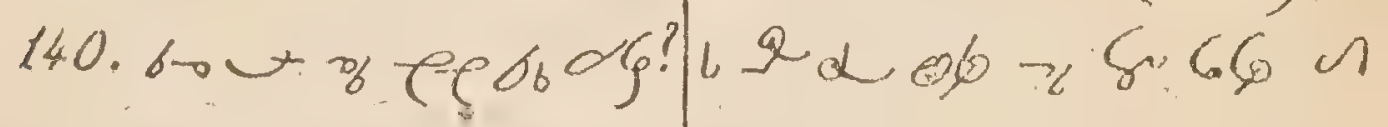

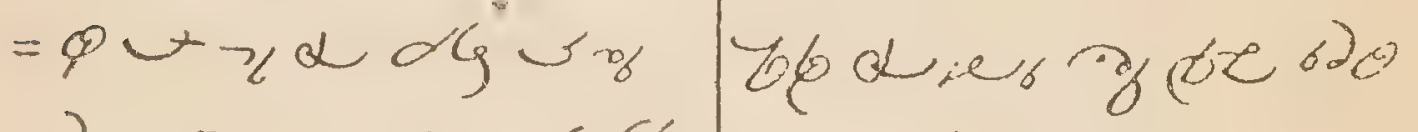

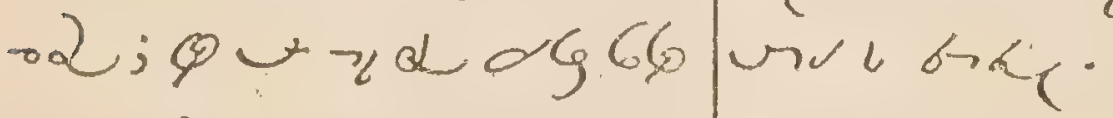

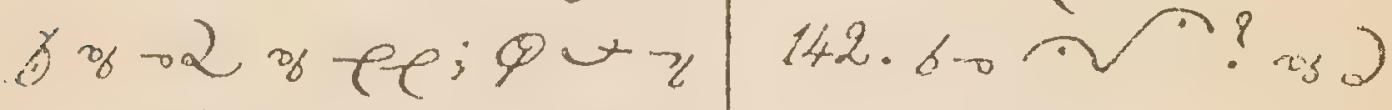

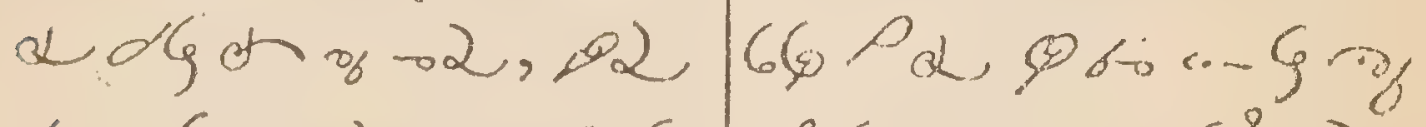

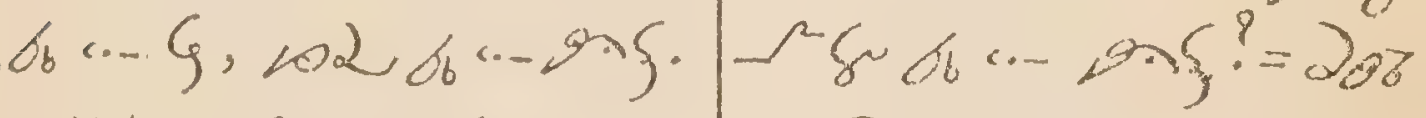

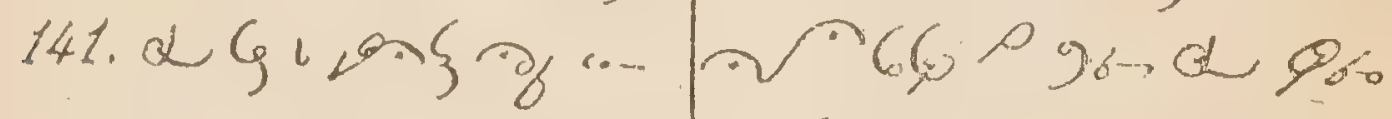

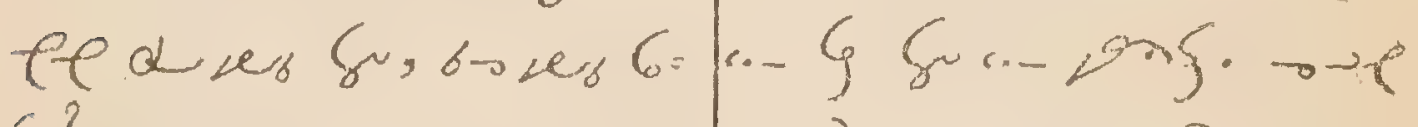

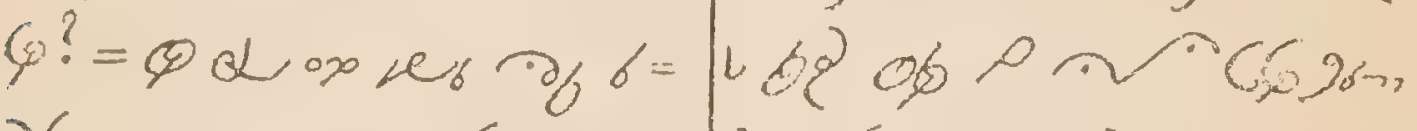

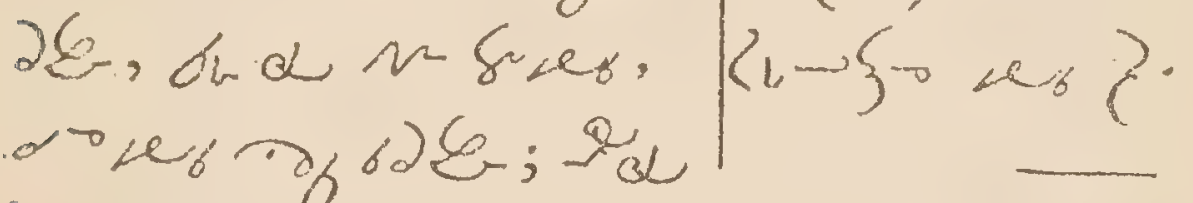

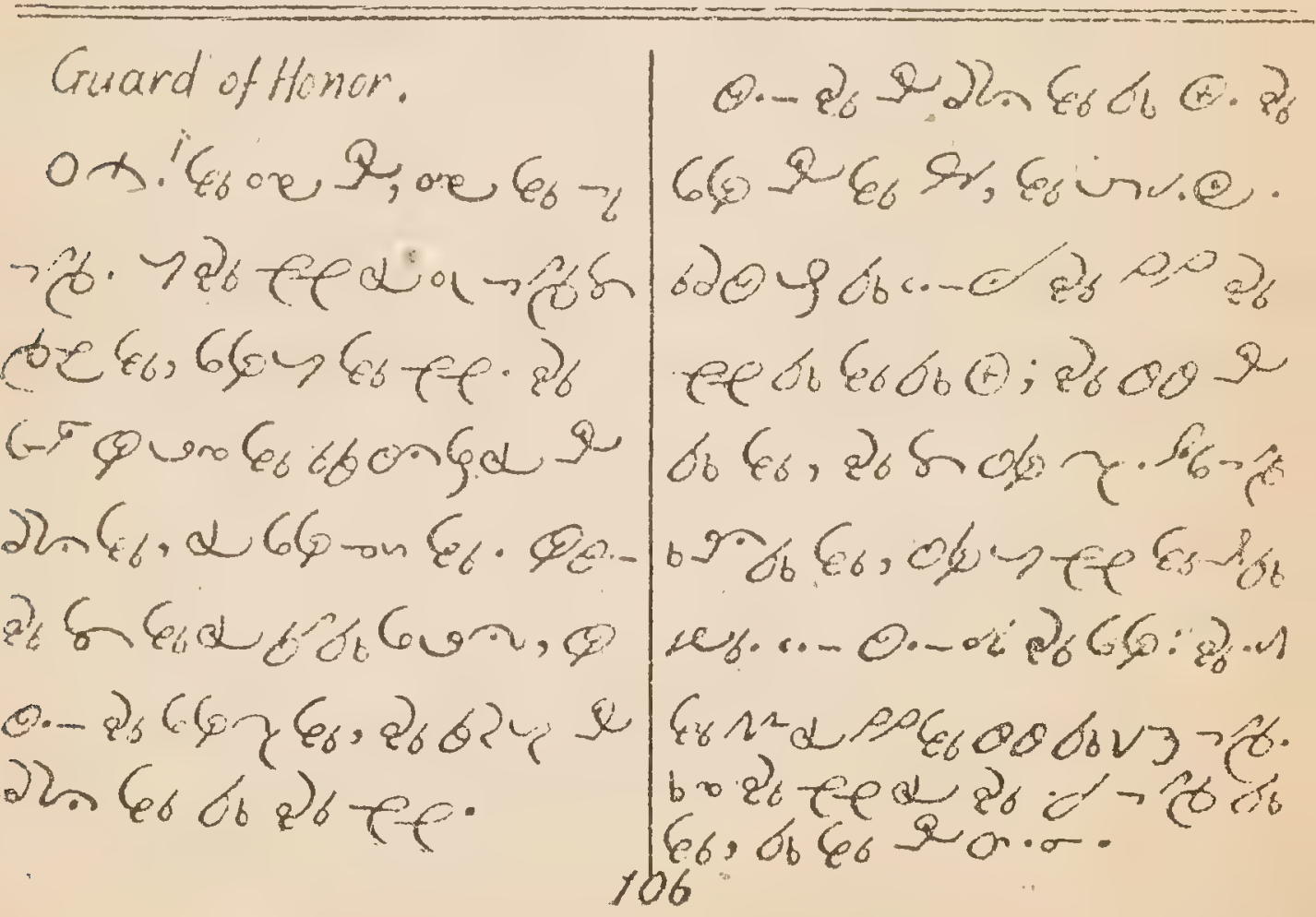






\section{LATIN MANUAL}

OR

\section{HYMNS AND CHANTS}

WN USE BY THE INDIANS OF BRITISH COLUMBIA

WITH THE APPROBATION OF

RIGHT REV, P, DURIEN, D.D., O.M.I.

Bishop of Ner Westminster.

KAMLOOPS, B.C.

1896. 
Thangh mest 


\section{Latin Manual.}

- 10

Pater noster. $+12 \%$

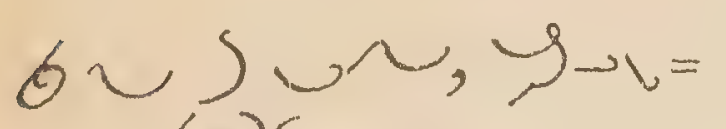
credo. Sos $\checkmark \partial \xi-e, a_{j}=$ - $18-e, v a-8=$ e s, us S U? - S IP b?2h $6 \rightarrow \cdot q \rightarrow \partial L_{0}=$ $\rightarrow, r \rightarrow G, \partial$ $\rightarrow-2 \%-c-2$ $-G \times-15<2=$ $\mu-2 \alpha s-\alpha$ s $3-\mathrm{aro}, \mathrm{np}$ $2 \cdot 6.9 .5$.

Ave Maria. on $6 \%$,

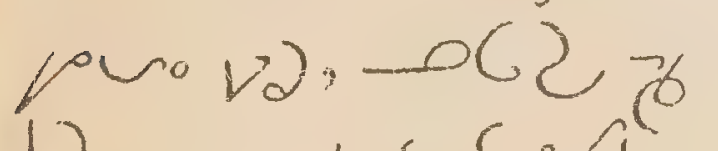
$l_{i \rightarrow \infty} \rightarrow$ S S $-(2>2 \Omega 3 \Omega$ $-2 \sim=y_{0} 80$, Gr $\rightarrow$ o o $\rho d L$

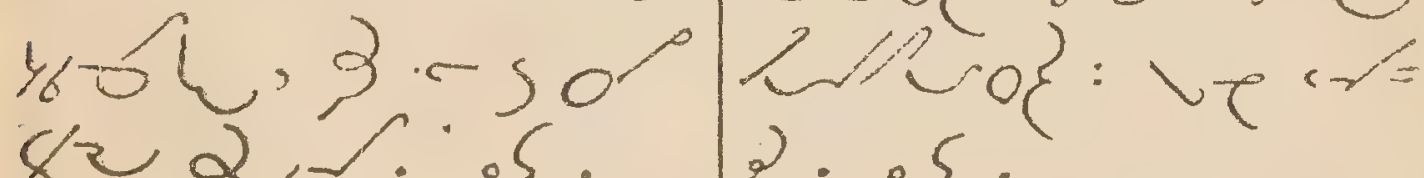
$82 \alpha 2 \cdot 0\} .12 \cdot 0 \xi$.

re, a cabzr.

s ser ye = us, -0 , 2. $\sim \rightarrow-A Y 0$. 2几ट6\% 2 ; durgaro fo. Exu, - Uve; 2uz- si l, ro wo chescicy

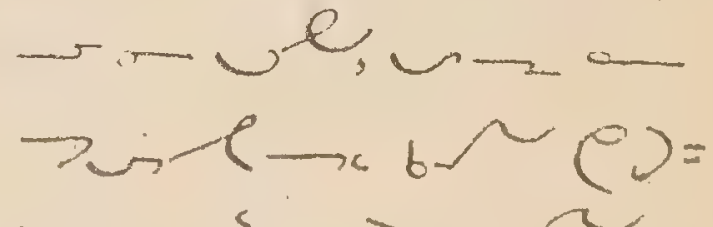
b32; $3-3-\approx$ n $\rightarrow$ he - gue. = Ye mas ofo, Yote esoo; 1 Evor to zinof: ve ra 
Responses for Mass.

at) 262 b r r w w

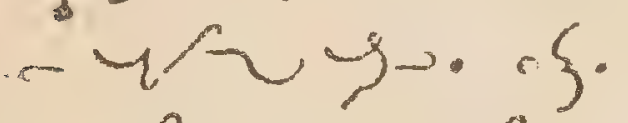

sfib - dof $x$.

$=--e b^{2 x}$

$23-2 b_{e}$

$+\overbrace{-6} C, \cdots, \cdot$

चuर ब4

9) $9+9: 9062 \cdot 36$

-

$=60-2, \sigma i s$,

$\sim \rightarrow,-\sigma$ )

$-0, e a r n s c=$

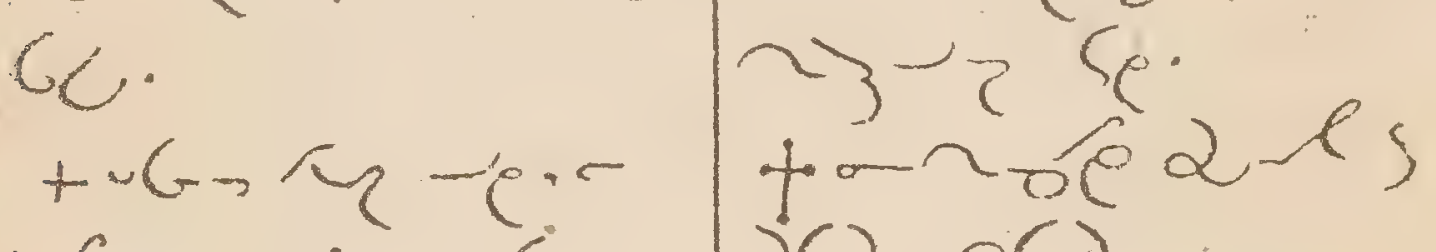

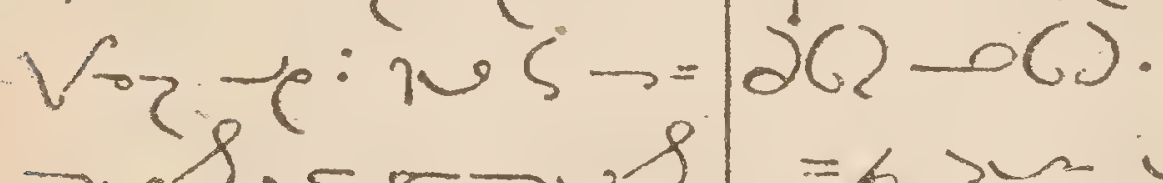
> vk, - - suk

s grese-pers

$$
\begin{aligned}
& \text { vale } 0 . \\
& =-s \rho_{b}-d a r
\end{aligned}
$$

$+x \rightarrow y+15 \sim 09$

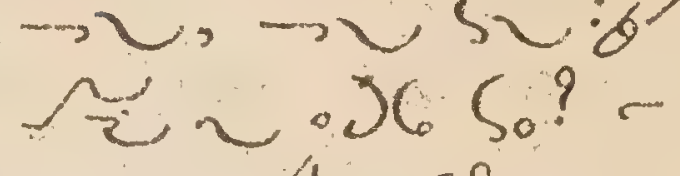
क) $\alpha \zeta$ ?

$\left.=y{ }^{3}-0,6\right)_{C}$

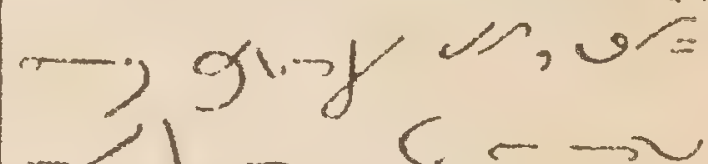
Sथ.

$+60 \mathrm{~b}-\mathrm{f}-\mathrm{w}$, - - 14 - yo.

$=u_{2}=2 \sqrt[3]{3}=$ $n_{0},-9,-r, \ldots$ s yp ypel. of. $+5 \rho b=\alpha$ $=a-e^{2} e^{2} \alpha$ $\mathrm{C}_{3}-\mathrm{r}^{2} \mathrm{C}^{\circ}$ tan ales $=6$ m ver + ound ... - $=$ co pe al. =culat a e e = b之一, $\rightarrow \varepsilon=$

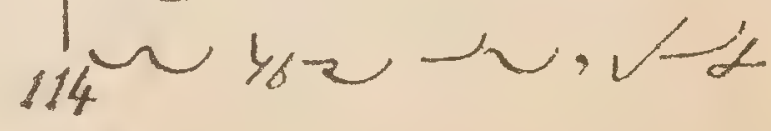


ste a d 2 .

$+\circ$.

=gund $\rightarrow 0 e b_{b}=$

$3, \operatorname{los} 6 \%$

$V(), \log$

of, loo

$C_{6}>d y=$

d) des.

Y.

bes exyer

br, bo 4h $\partial \xi, \sigma=$

aros. Vb-al:

$S_{0} C_{b}$, So $C_{b}$, So $S_{0}=$

$r_{6} u_{b} \cdot \rightarrow 0$ 舟 be

ce yvir, lop

undartiote

ogk tre, $y e=$ aol inebe.

eq yer- br,

- $P S a-0,0$ )

-eare

$+60 / 2-(e)=$

bz $\sim, r-\varepsilon \sim$

br $2 \sim, 4<$ e

$=\gamma_{c} \cdot 2 l$.

$=0\}$.

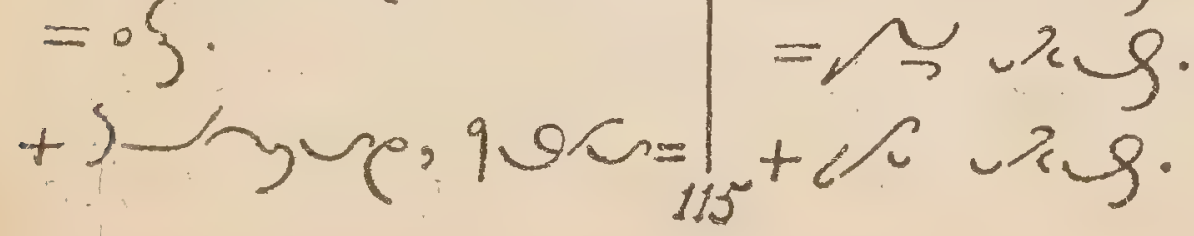

of:- jervok tro= -2.e. lad

Cobz-Gufa.

Q2.

$$
=\circ \text {. }
$$

$+\rightarrow-9 v \sim$

Moh 2 .

$=-\eta$ o o o s s $\Rightarrow$.

$+e_{3} \rightarrow d h,-(6)$ ",

untere

$=-\sqrt{-1}-0 \rightarrow$ d

$+-0(2) 20-02=$ of $5 e$.

$=-\infty 85(-2)=$

+or dup.

$=r$. 4 工 0 .

ta vig.

$=$ is $x^{2} g$.

$+c$ iseg.

$=\approx$. 9 .

$+\simeq$. 
$=i f-r g$.

$+4 \pi \geqslant s$.

Gloria in excelsis.

Lots en $\rightarrow 0$.

- 5 Ite oGulb?

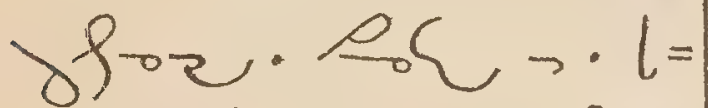

rur s.o. of

- bet a pere

o $2(2-1,4) 60$

loce-e. 0()$-2$,

Run , $\rightarrow$

b esbz: - oc w

(马)

$062 \rightarrow \cup, 9 \rightarrow$

$\sim 6 \sim 6-6 \sim$

$\% 0 \varphi,(4) 2 h$.

$6-x^{\sim} y 09 \rightarrow, n=$

$\sim \rightarrow \sqrt{6} \sim 0\} \alpha \Lambda$.

bur a sus

b G

ury〜.-

a $\triangle \mathrm{G} \cdot \Omega=$

rauk, su

भi क

s.6\% $\rightarrow$ с 6. 0. $+-a C 2 \partial h b$.

$$
\text { - rus jo. }
$$

+ork vis upl.

$=\circ\}$.

$+\cdots \cdots$

= To pave.

+002 dug.

$=-\phi u_{1}<\tau_{0}$.

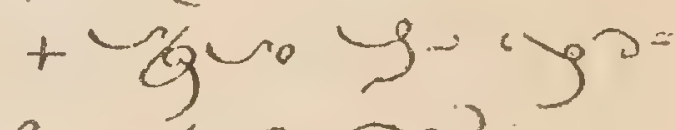

a vore $\partial\}$ :

$=t^{\circ}-t-0,2$

$+5 \cdot \rho^{\prime} \mathrm{Cb}^{\prime} \cdots$

$=l-l \varkappa$.

\section{credo.}

tos (q) R. erb3r. uravine

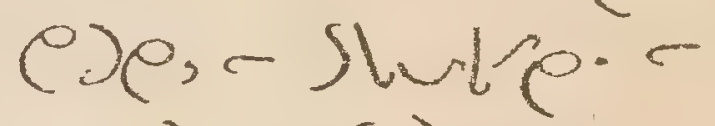
$s(q-a)$ - >e

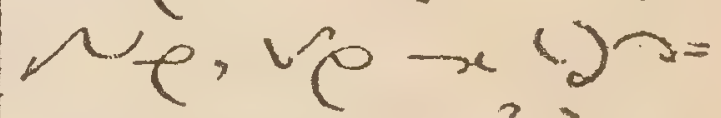
Je. -2 d de.

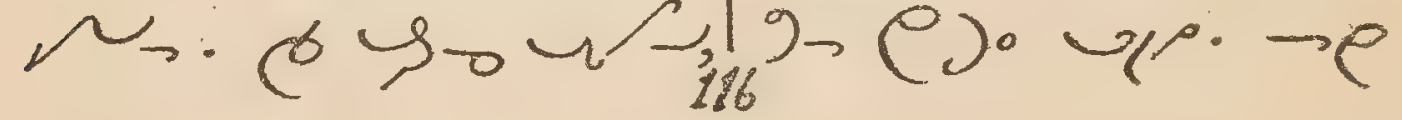


$\rightarrow \rightarrow 0,-\xi \rightarrow-62$, rejeray see 9 xe, gyus=

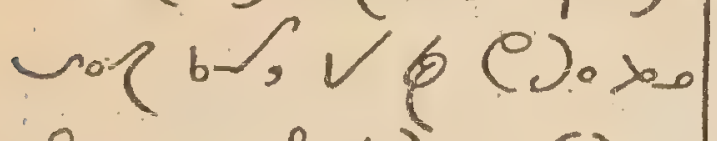
q. 6 . 12 ord. $-4 / 2 K$ परि, $=$ $3 \rightarrow \rightarrow u \sim-S_{8}=$

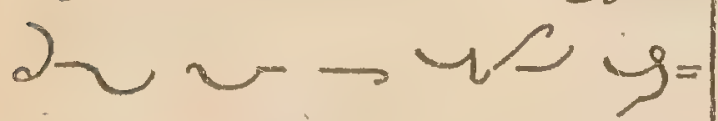
- , 2 $6 \%$ V 2, 06 × $\sim \cdot \mathrm{Cls}_{3}=$ uve poh, 7 guro le, du -

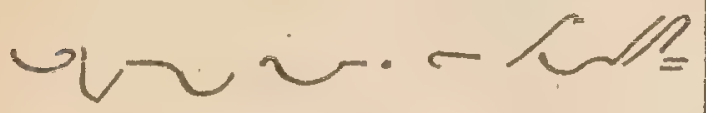
n ko $\rightarrow$ u, uge rue - enz s we, u- a $=$

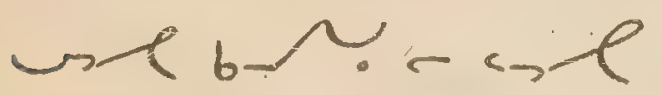
$3-\approx 66^{\circ} \Omega=$ $\rightarrow$ We, - 8e: $=$

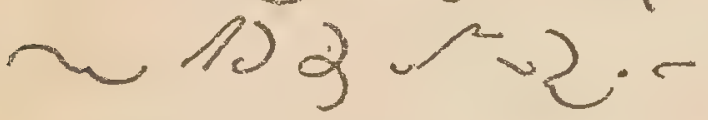
sufe Ye, og? - $\operatorname{lig}_{2}: \sigma<, b 1$, vob h-. - vo us ore, $910 \mathrm{~N}_{6}: 6$ \% w

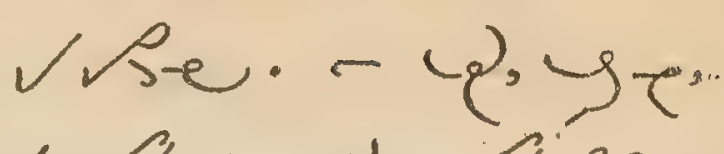

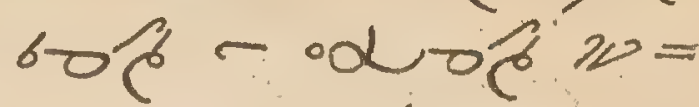
re gund \& duc. 5 suak yo - 2yo inkoz sole. - ve is. of. $+\infty \overline{2} h_{6}$.
$=-6$ s 0. +os $2 \sim .$. inva old whe $\rightarrow$ 6oh $\sim-\mu-6 \hat{c}$

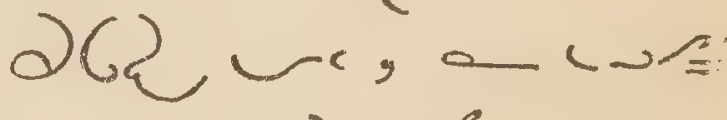
- $20621,0 n=$

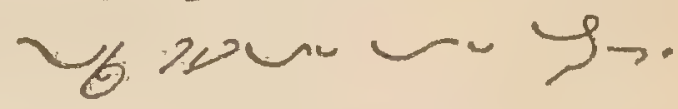

trejo up upl.

$$
=0\} \text {. }
$$

+ - or a d

$=-\infty$ U U - 0 .

+ukgo.

$=$ ok $-0 Q_{q}$. 

+ pue ok -62
-0 $2 \rho$.

$=>q-\sim e \sim$.

Sanctus.

$Y \sim, Y \sim, Y=$ $\sim-62 \rightarrow q=$ o. v) $q$ vrdt 10. - . वरू ह un = 1) $\sim 0$ $-2, \partial(2-0)$ प्रू s यू.

+veso vo upl. $=\circ\}$. +ok...s jounos. $=-1 \rho 2.6 \rho$ $+\checkmark e j \cdot$ up upl.
$=0\}$.

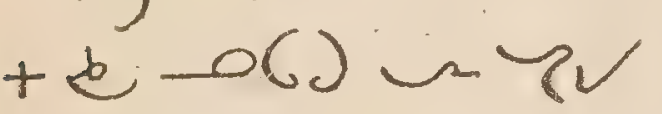
ohe. $=-b u<-0$. $9-x, 6 b^{2} y=$ Q, Gul dh.

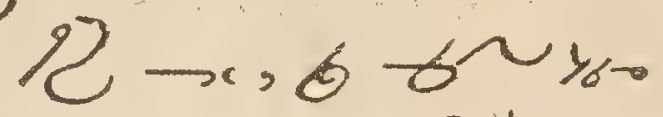

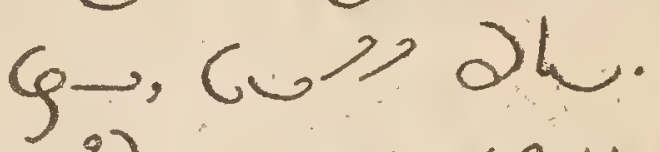

, $-6,6$ tr $\varphi \rightarrow,-0$ dh w.

+-06 dhe. $=-c$ c 40 +ok...up ual. $=\circ\}$.

+062 bh 6 .

$=-64 / 0$

+ «u

= -o pue

$+12-\alpha<\cdot \ldots$

$=\circ\}$.

+-oce due.

$=-6$ y -0 .

+ jue \&...

$=160-1-06$ ?.

+ S Mulo $9 .$. $118=-0$ pure. 
MANUAL.

III

Vespers of the B. Virgin.

062 . orin: *

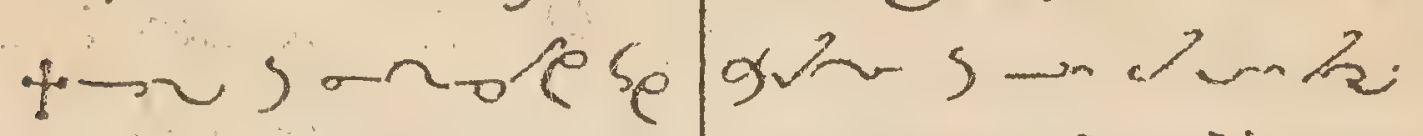

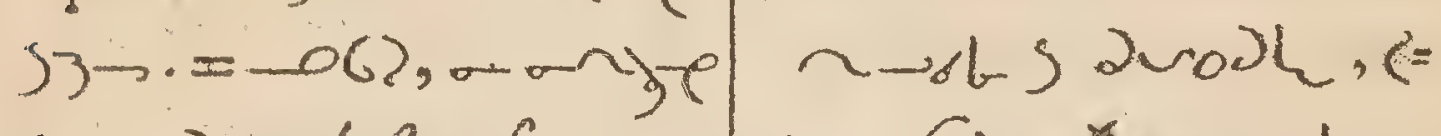

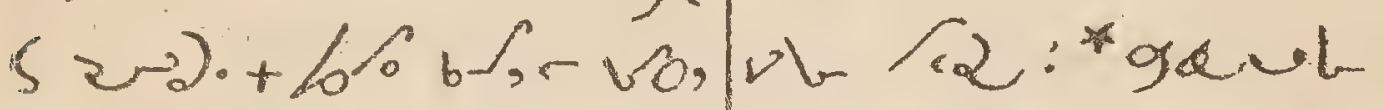

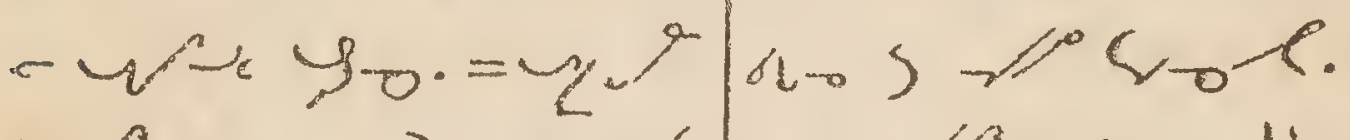

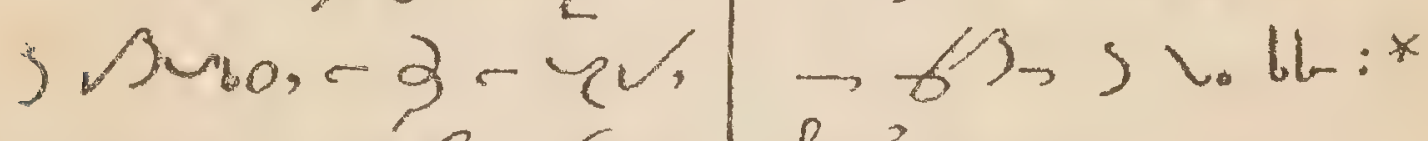

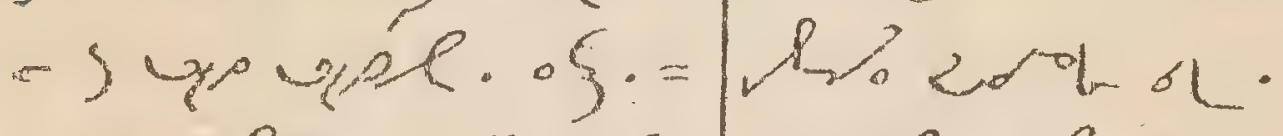

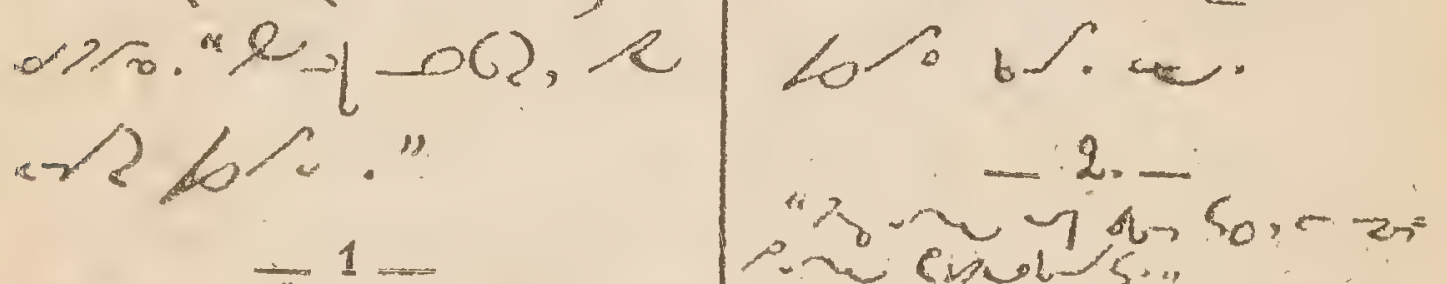

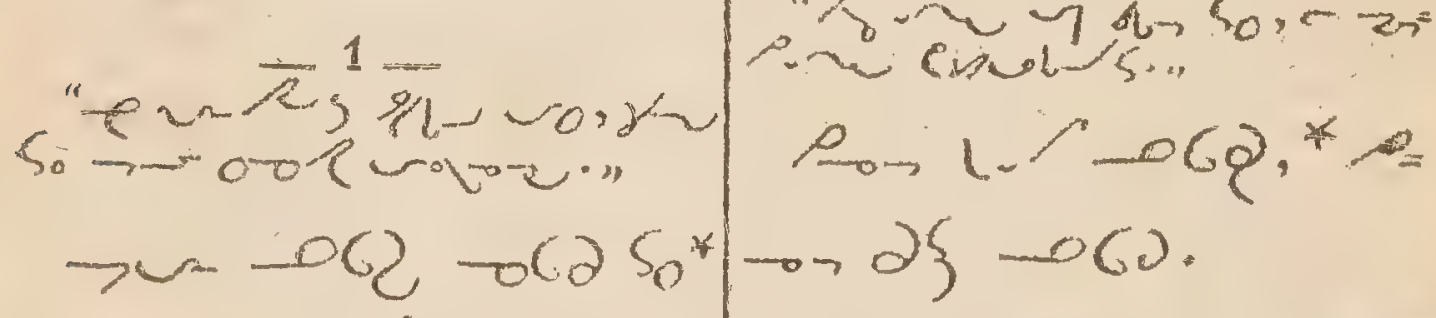

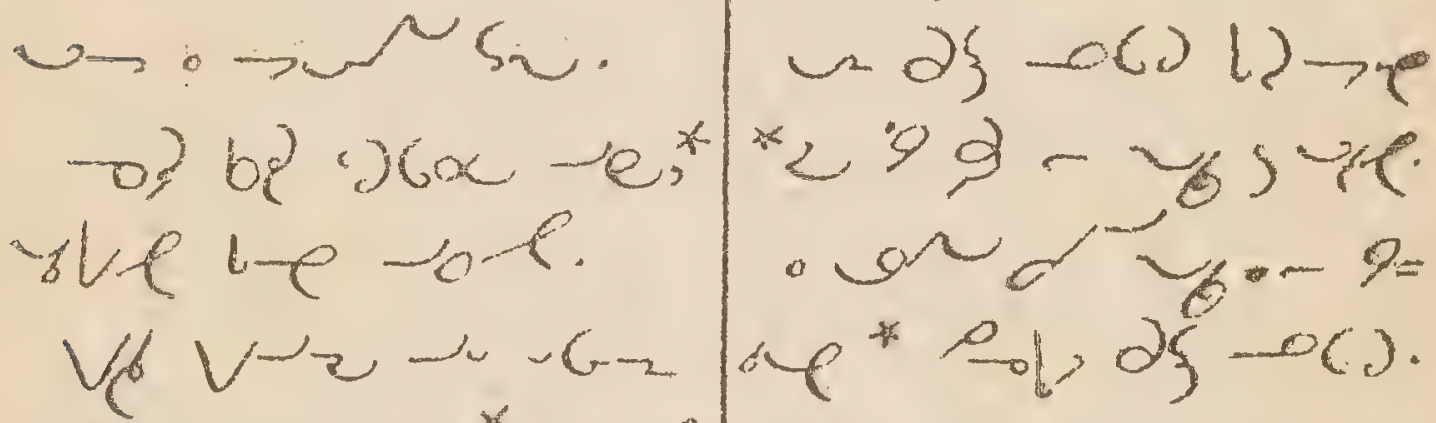

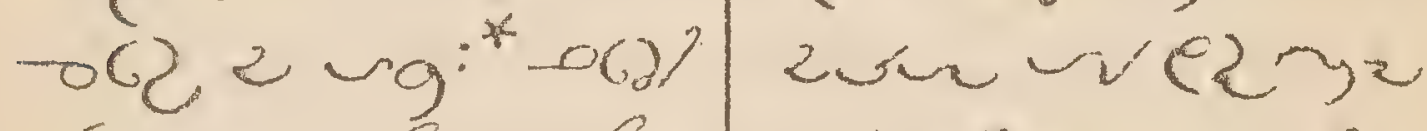

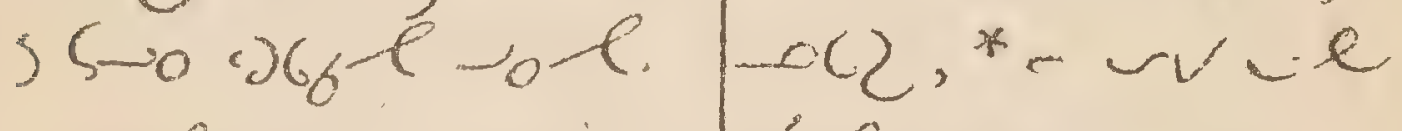

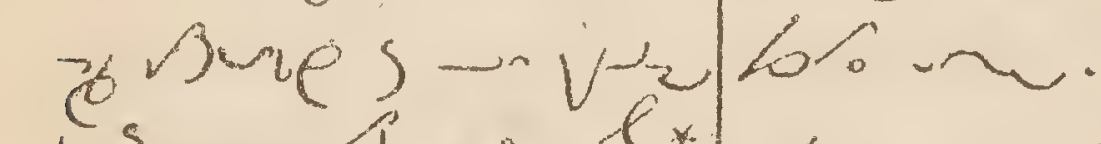

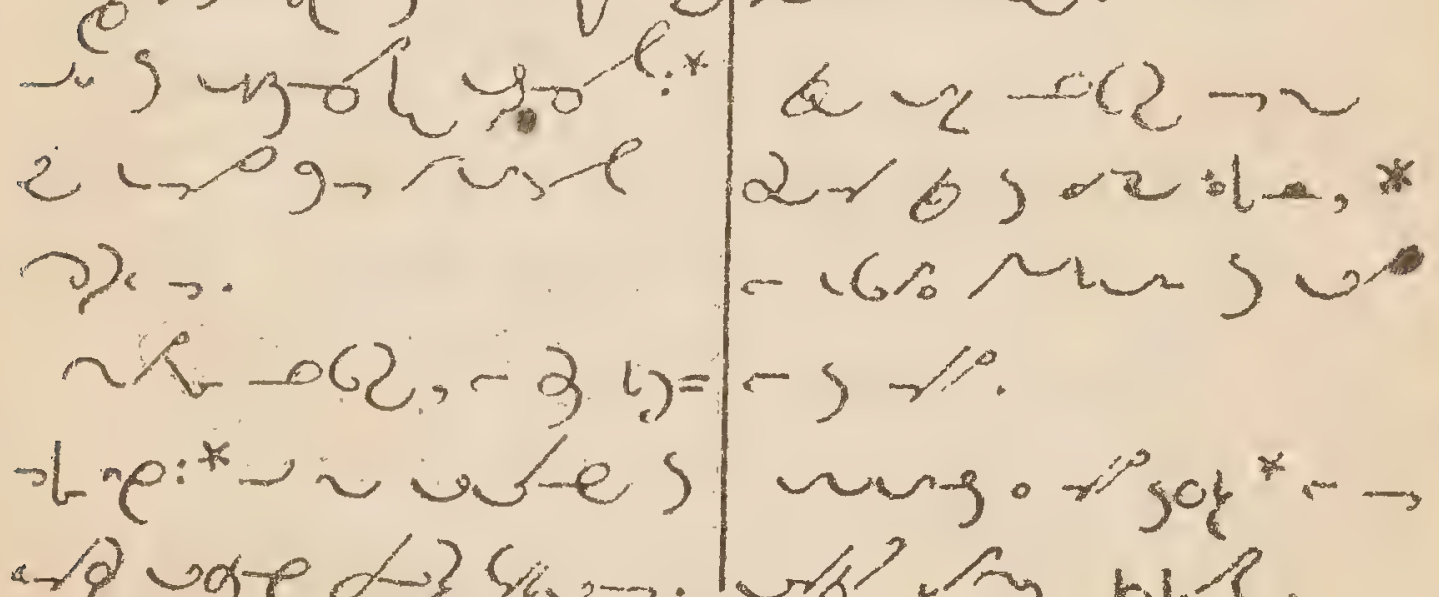

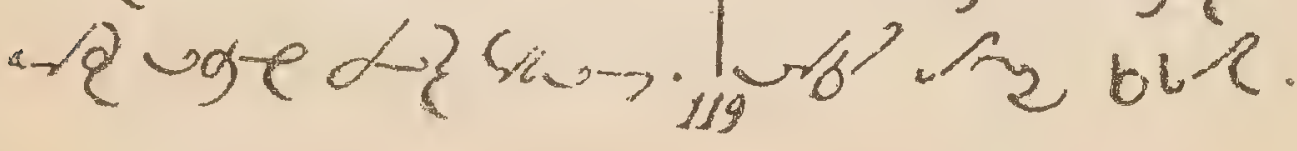




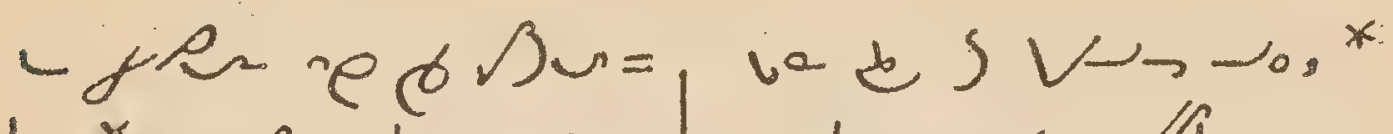

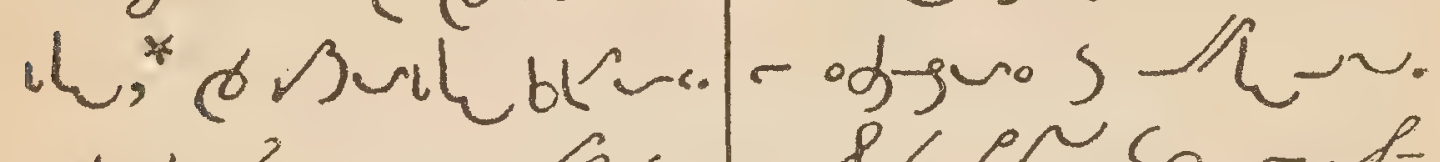

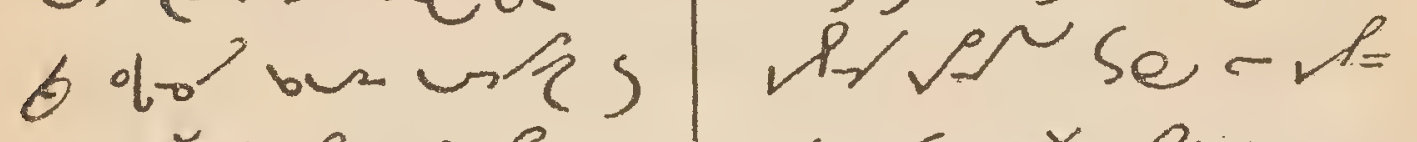
66:*6rvorts7. ve se * gy by $6 \%$ \%

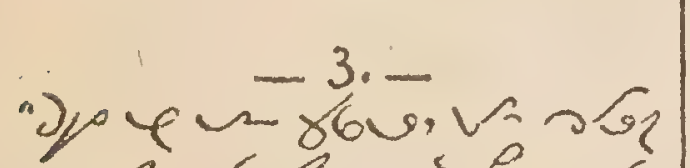
$\rightarrow 0 \sin 52-5 \sin 5$ stieve:"

son TS $\sim 6>0$ $q G_{i}: * j-06-0()$ is.

q2 $-21-2 \alpha N^{*}$ Jan 2 ar. sor 6 - wo veo* he furvo ns re. n z enz- 9 , $1-00)^{*} z-6 \partial e$

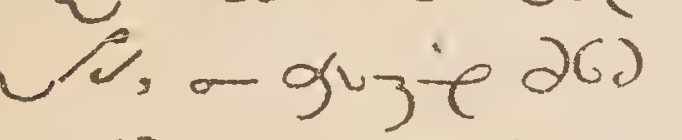
-62 .

6. $n \rightarrow R \rightarrow$, $\sim \rightarrow \sim_{0}: * \backsim 2 \sim$ $-0,0$.

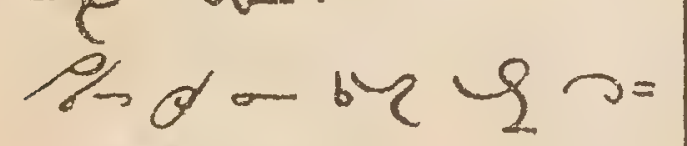

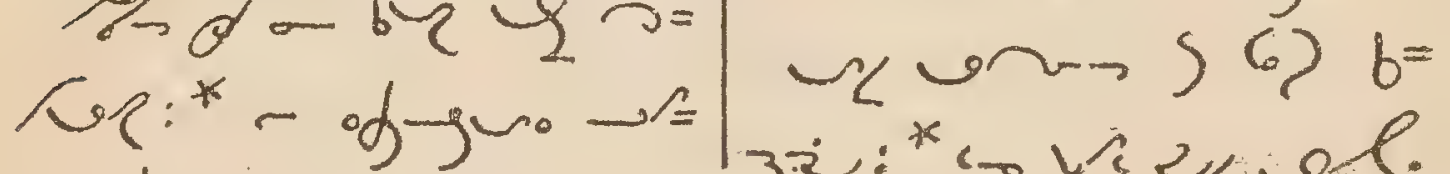

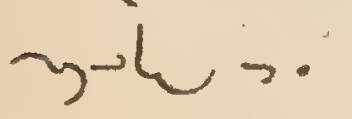

$\vec{P}-06-0(x)$ $2,{ }^{*} b u l b^{2}-1$. $10 \% \cdot 6 \%$

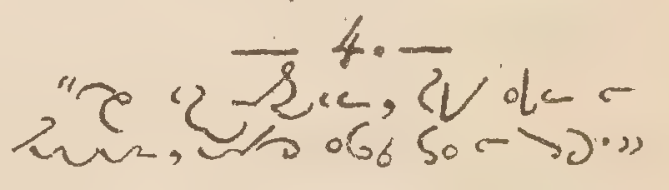

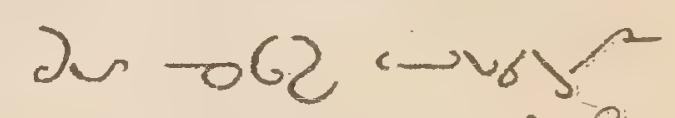

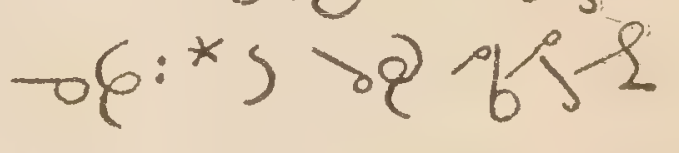
bing re.

$x-0,2<-0.0 h^{2}$ M०r, ${ }^{\circ \rho}$ ur Lom

re du $r$ r wh; ${ }^{*}$ 你 $\rightarrow\left\{, b G-e^{2}\right.$ b or: 6 $\rightarrow-\mathrm{RTh}_{2} \sim$ eq: *2 uter old 山 以 $120^{2}:{ }^{*}$ - vis zull. 
MANUAL.

I I 3

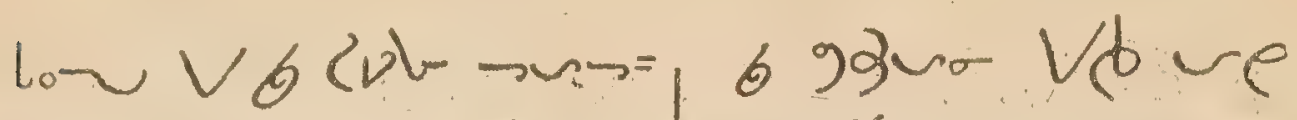
evezm:*2 aq: 2 ivire- $=$

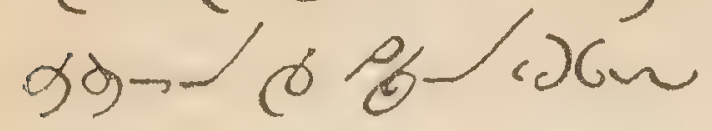

$\sim 2 \gamma$ \%

$10 \%$ a 1 .

$$
\begin{aligned}
& \text { a sus or ed ed d= } \\
& \text { noj, * n unovo }
\end{aligned}
$$

"ywa

3 $6 \partial_{2} \rightarrow-10$.

3 -

$\rho_{0} s G_{p}-g_{Q}, * \rho_{0}$

-e tevin.

be oftow. ugre

- o.

$\sim \rho s$

6 bur 2 -

$\sim 0, \sqrt{\xi}$, uram.

bug abeve

Capit." q dvorg getp

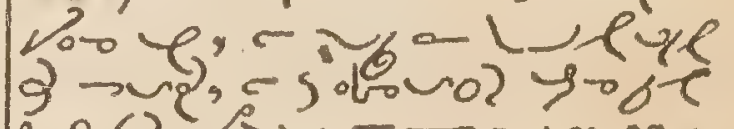

10 62.

Hymn.

Ave maris stella.

ditivent viso

- $6 \sim$ vis

$\rightarrow 62 d 6$.

ob v v V o

o- $x \sim_{2} p_{0}: * x|c|$

zic) yho.

Gor wevers

be: * go wing for

$\sim$ 我 $\sim \rightarrow \rightarrow$.

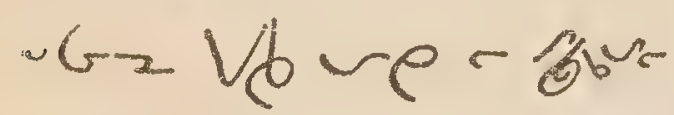

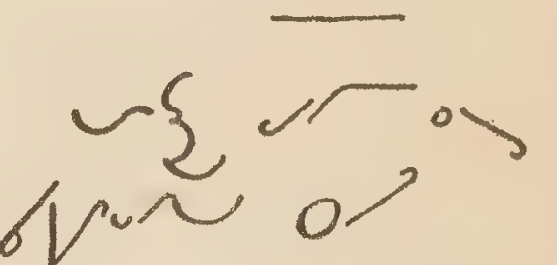

g $\rightarrow 2 \cdot 5$ w.

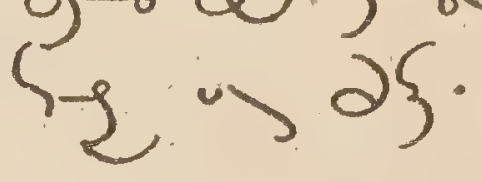

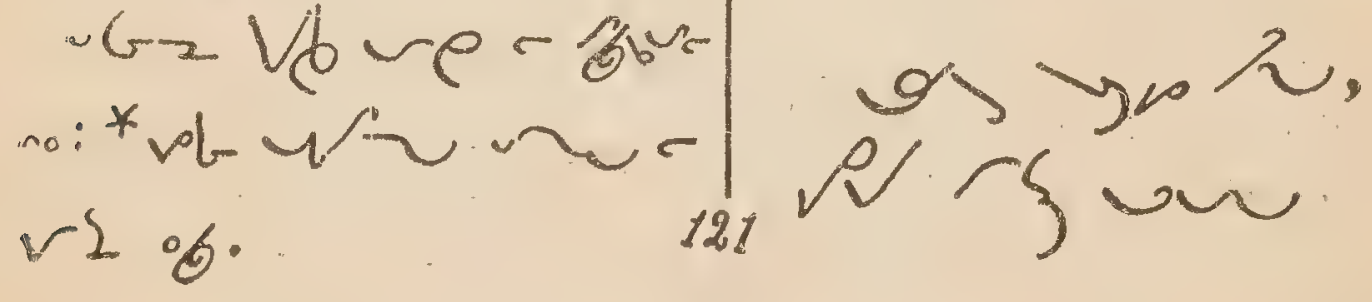


$62 \mu v=$

b) $\alpha_{0} \alpha u$.

\&sw6 $\sim 6 \checkmark a \lambda$ 6 $\rho$ dr

N $\sim \sim \sim$

Vo $3 / a 八=$ srez Ge, 2 un ire $c_{2}$ x $-\alpha \dot{e}$

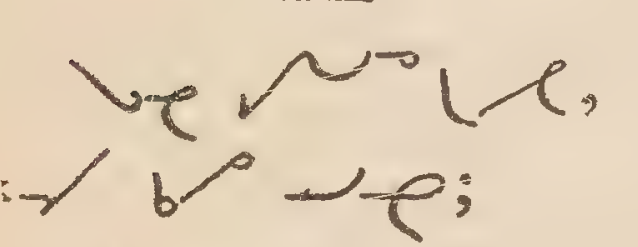
- vze see.

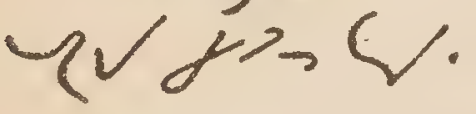

$\sim e \rightarrow 0$ e $\sim$ 은 y. Yo L of 2 . of.

* $725 P^{2}$ $V_{B} \circlearrowleft \mathscr{S}_{0}=R_{0}-G_{\mathrm{C}}$ $v$ raperen

Ad Magn. "Yo $6 \%$,

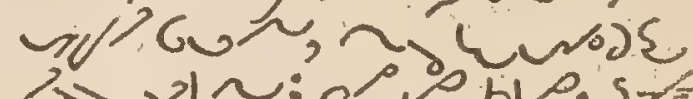

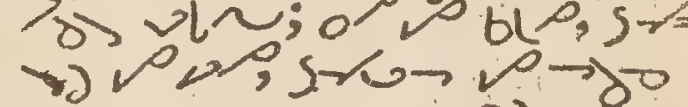

$\rightarrow C 20$ 2:-3ज2 el दe

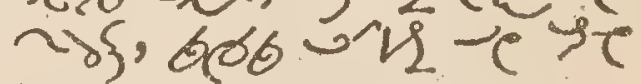
यगतर"

bout* od6 so -

$-002 \overline{d h}$. - ou< 0 .

oremus. OCL. gum 2 bse e, ouc 西 on $6 \pi$ r v

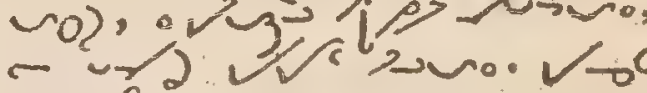

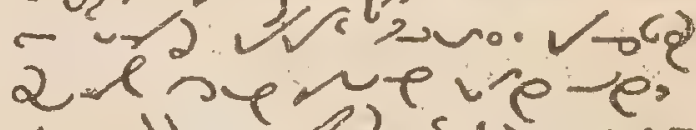
b-6.lW- 22 sesere up se. $=$ of ved. y.

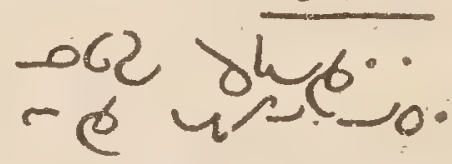
$\sqrt{2} \rightarrow 0<-062$.

$\rightarrow$ fine.

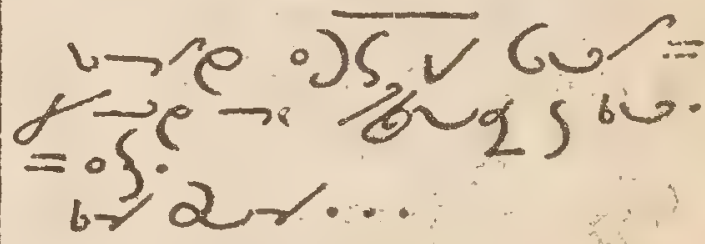
$-06 c^{\circ}=0$. 
Greator alme.

Dof os un te. ro ensue, sur zobes $33 \rightarrow>x^{2}$ vindo. $6-62 ? 25$

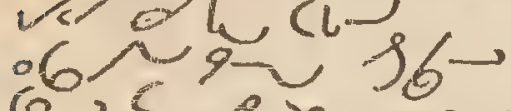

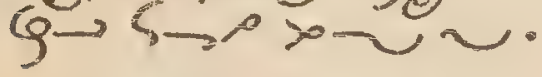

त42 $69 \rightarrow 2$ -2n की $3>0.0-i>C$.

in bze, los. गदक ति 62 : चान 25

$\overrightarrow{3} \rightarrow \sqrt{d} S \alpha S$ So $\rightarrow$,

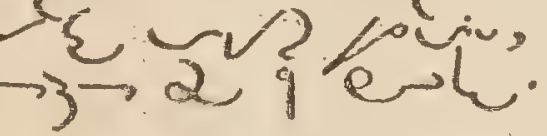

vu ore lo.

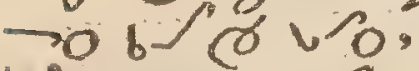

Sions yis. of.

Jesu, Redemptor.

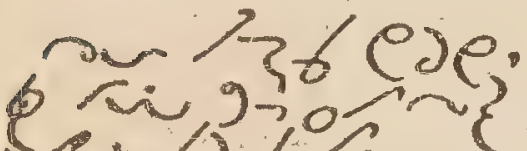
(2, $62,5 \%, \frac{1}{2}$

b)

- $-5,-3-6 a$ rysere.

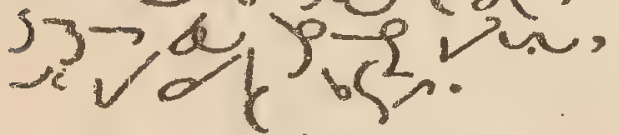

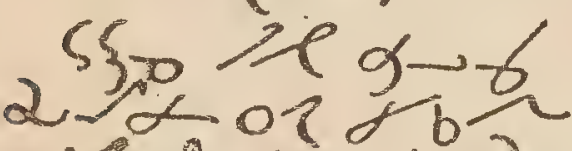

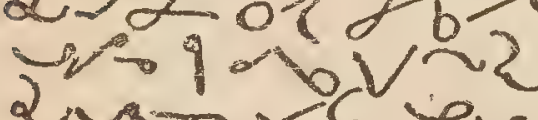

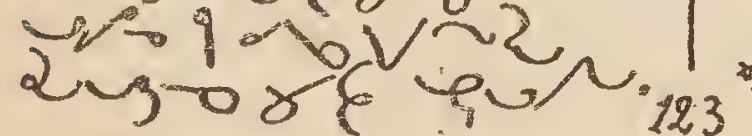

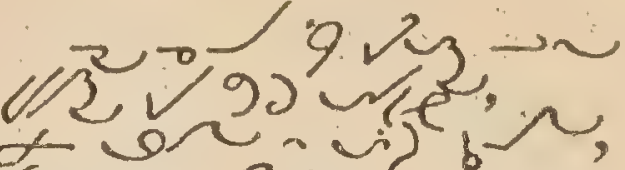
क्ष an w?

- gen, thizs? जर 90 रेग, ग) wor a $\alpha+6$.

- 2 , lor $\alpha$, en 20 90962.

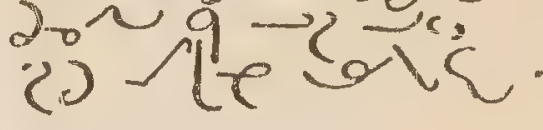
6 $2 \sim-1 \sim 60$. क

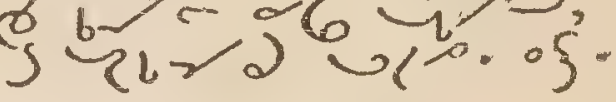
Adeste fideles.

$$
\text { ouna }
$$

racera.

ae $>>00<$. * a-j.

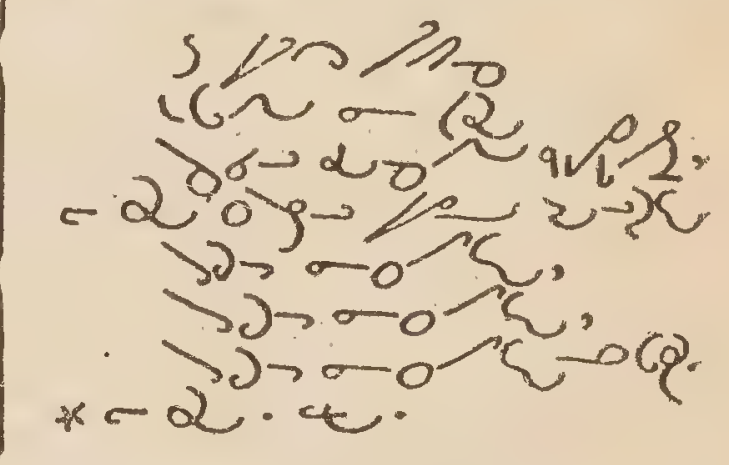

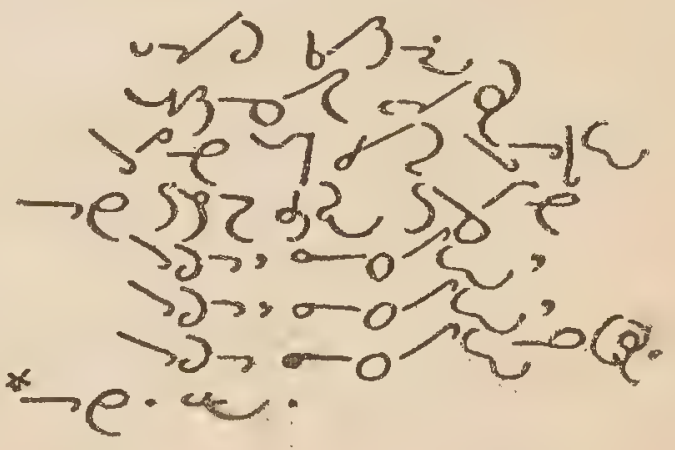


म गू मूरे

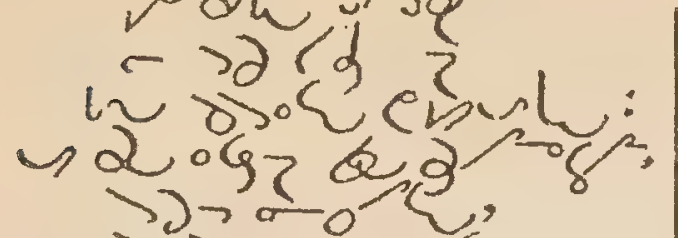

$\rightarrow \rightarrow 0$

* येग

Audi benigne.

2a J/2 $9 \rightarrow b$. 3.9 y. מon

ve o s) 10.

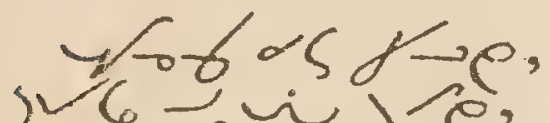
- $\rightarrow$ vise, उ

se br w

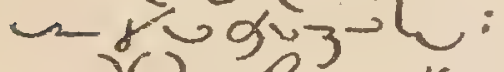
- $262, \rho-\pi$, gu sie 96 i.

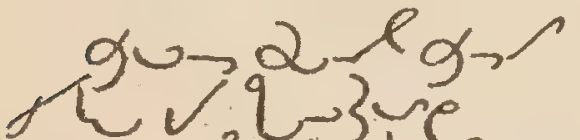

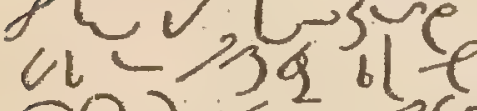
का $\mathrm{x}$, 58 . voloo See, 'gu? 72 . We. sno-esiso. of.

Vexilla Regis.

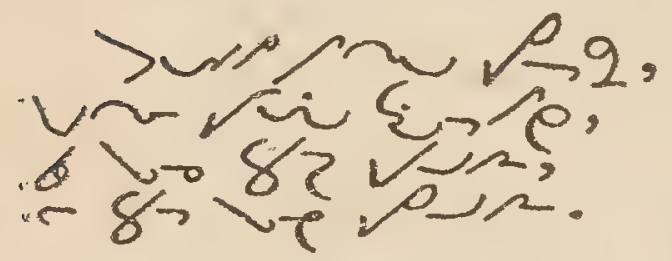

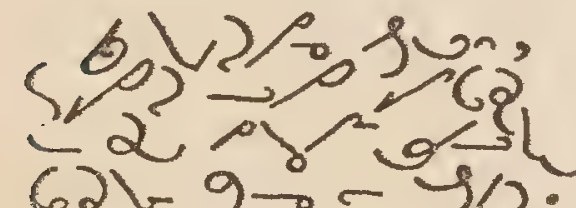
$62 k 9 \rightarrow-9 / 2$. $\therefore v-9 \& 9()^{2}$ करत

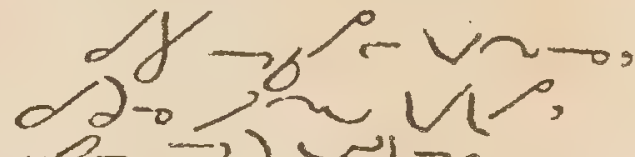
$-0,2 \sim u$ es? ll sol?

loo ina $\mathrm{ke}$ vely $y$ la Ine

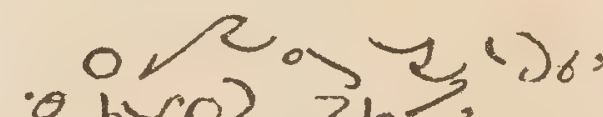
9 brok $2 b$,

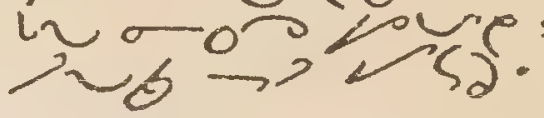

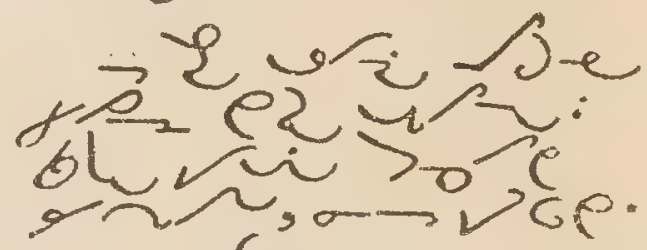
०\}.

Stabat Mater.

$\rightarrow d 6-2$ op

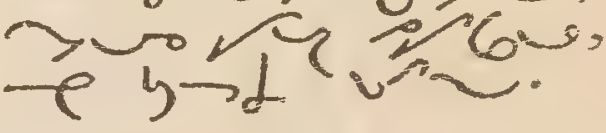

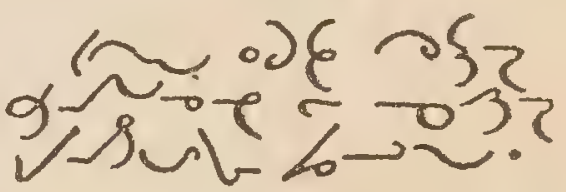

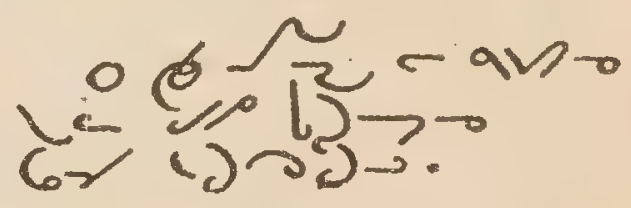

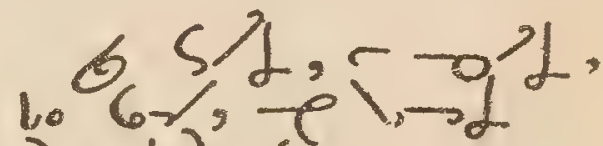
)- 62 sen $\rightarrow$.

$60 \sim 06690 / 2$ s 90 nuno. 


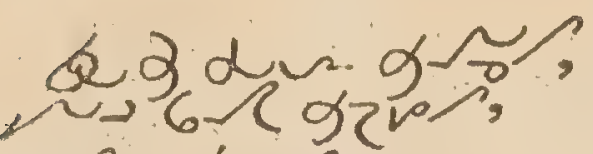
- 32 < 0 -

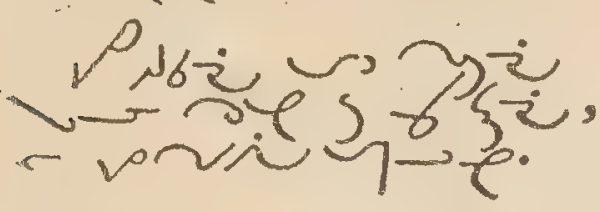
sis vere de busme.

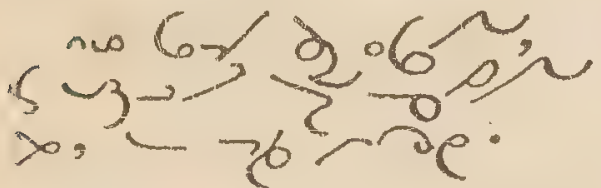

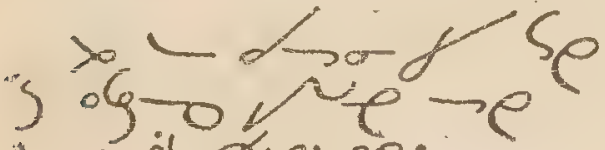
uiprove 3o6r/ avid $y$ so 3 .

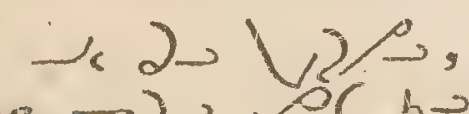

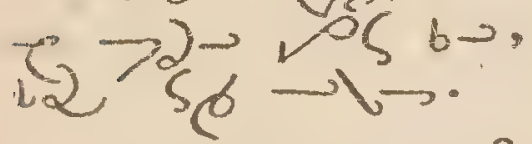
x 5 - 6 h

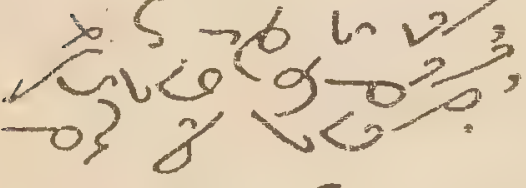
-

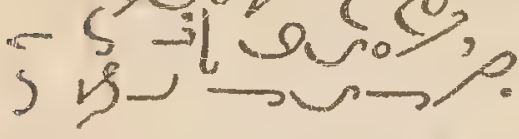
cis V 92 ? ×5

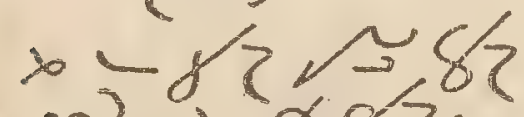

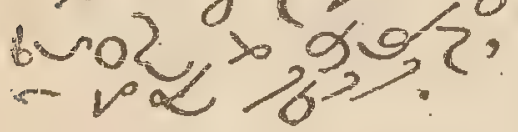
+o s wow Wh?

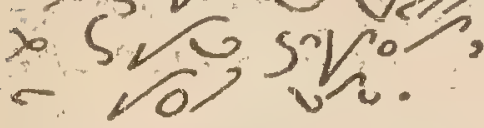

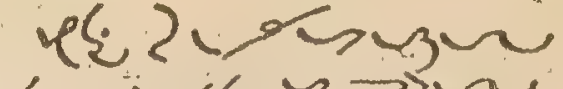

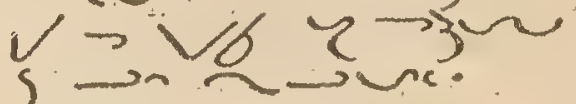
न 06 \%

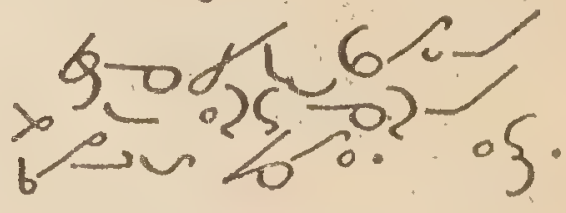
0 . Filii et filide. dorso, dista, ditio. OU一, - Un,

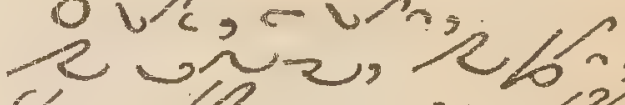
8 - whom,

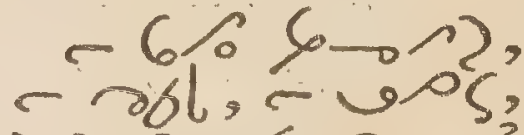

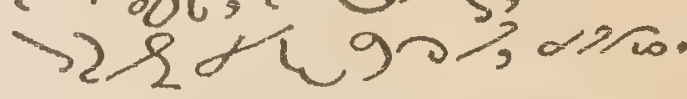
- 6-a 60

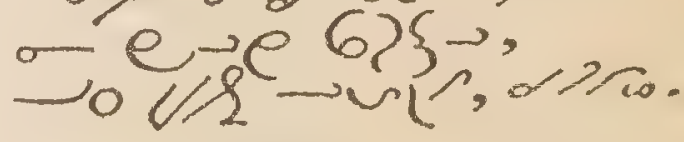

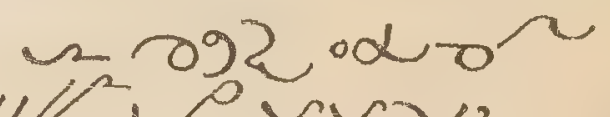
"IV) solusin? 6. , $\rightarrow \longrightarrow 0$,

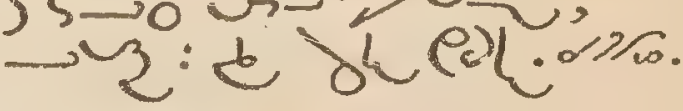
dept $\rightarrow$ र boung su? Gen -h:ar.

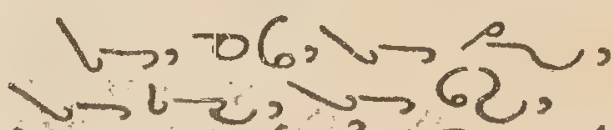
125 


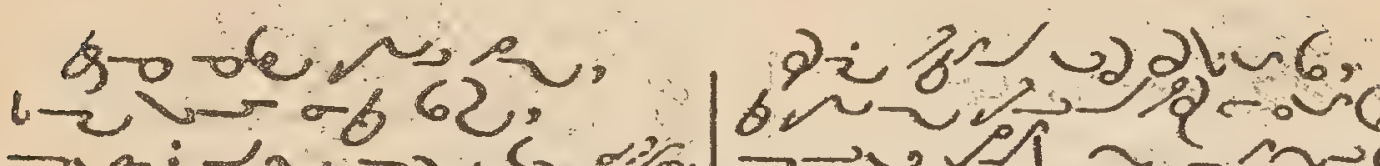
政 $\log _{0}, 0,1, k$,

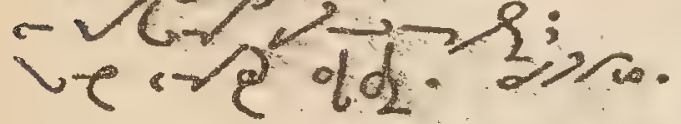
$3 \cdot 920 y+6$.

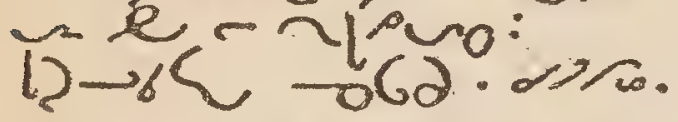

- oh 2 ince,

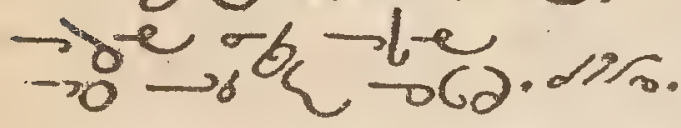
Salutis humana Sator.

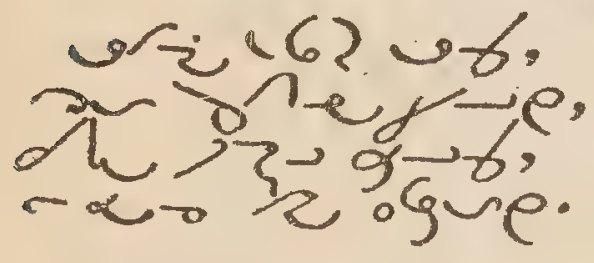

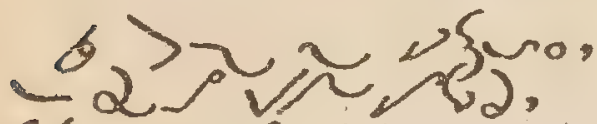
$827 \%$ ग3 - $852-6 \geqslant 2$. Yh, ju? be.

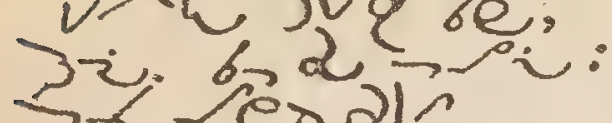
so pra - >us-

$=6052$ fुñ (2) anes

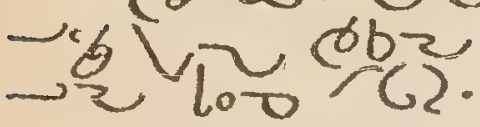

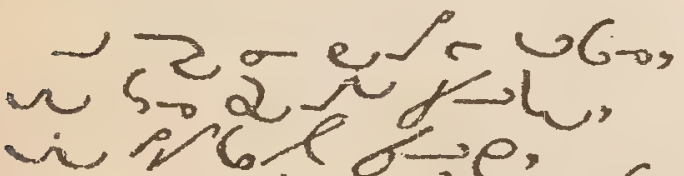
i

sacris Solemniis.

woro, $30-3.6$ -2,

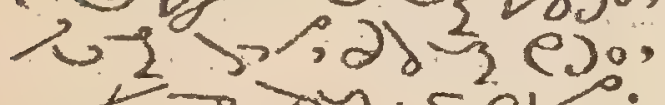
च为

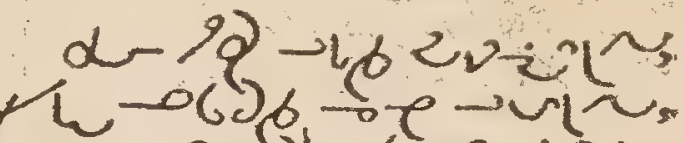

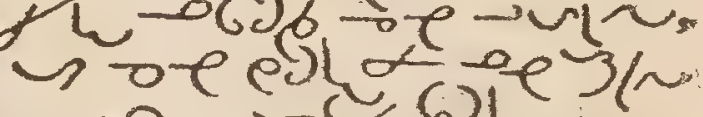
un ons 6 ?L.

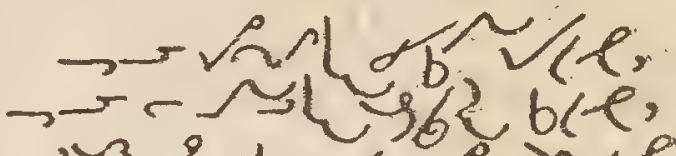

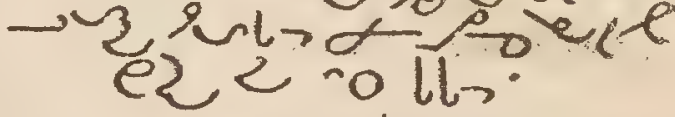
añus B $90<-62069$ - a b or or GPl $-\beta$ - 2 agd $72-$ hom ${ }^{2}$, I the jole. 3 . Verbum Supernum.

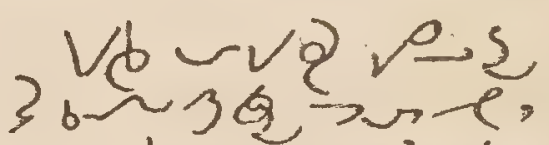

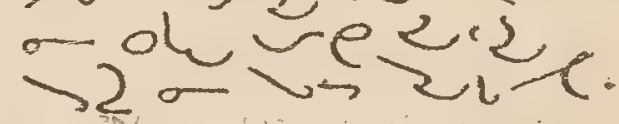

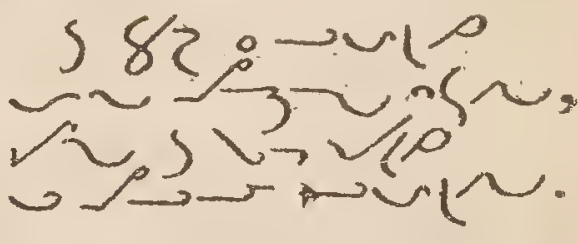

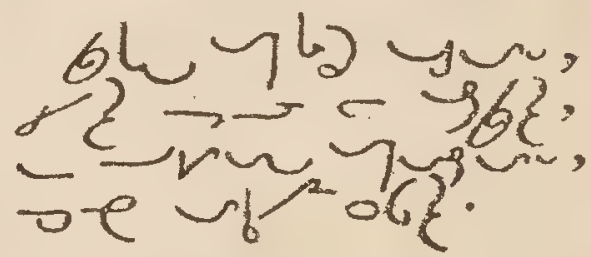
क्ये zare, are, 


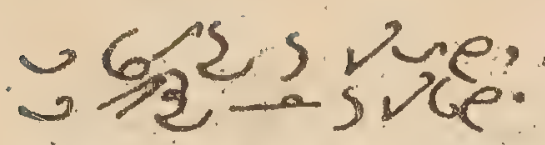

ousres. orydie.e. phevare.

(a) $3 y-0$ a

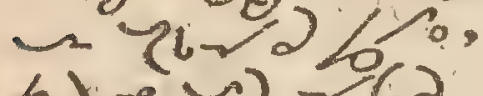

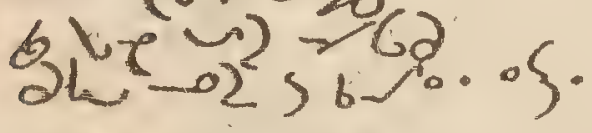

Aeterne Aex.

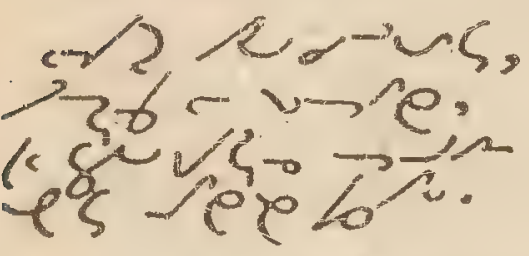

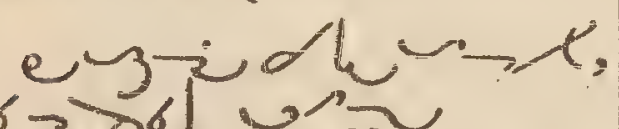

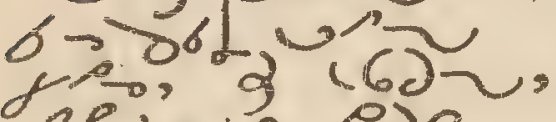
se bue exe.

$$
\text { - } 3 \text { AR 602. }
$$
unde ande - sulf $x \rightarrow 0$

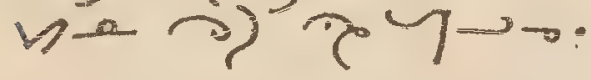

16 年 Velo, $800:$ पo.

\%nd-be $62020,26 e$

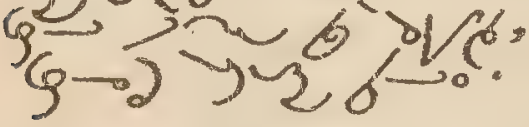

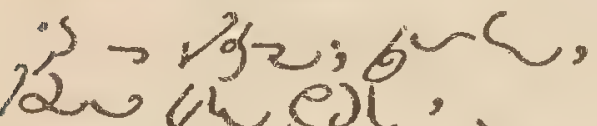
-dondp.

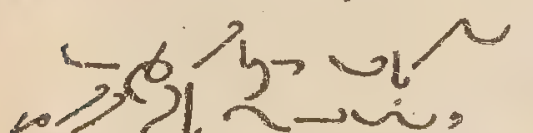
12 ie 8 tive. b)

Auctor beate seculi.

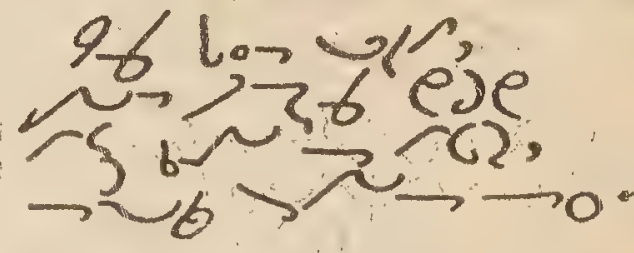
\&08.

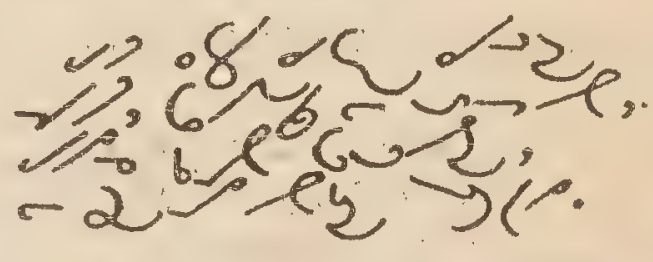

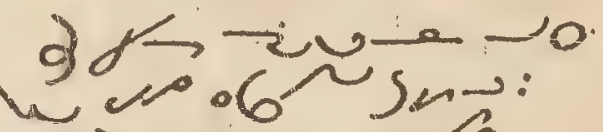

- 9,97 पर 0 -

"cior y ye.

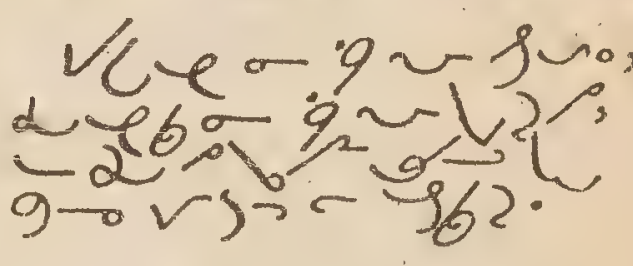

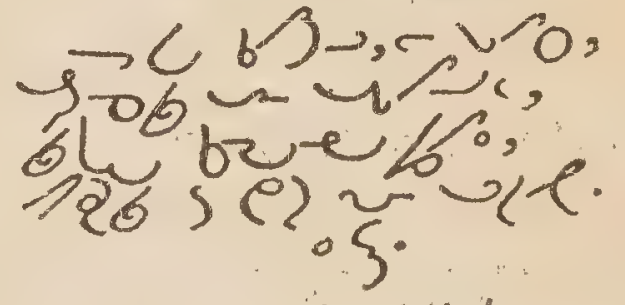

Te Joseph celebrent.

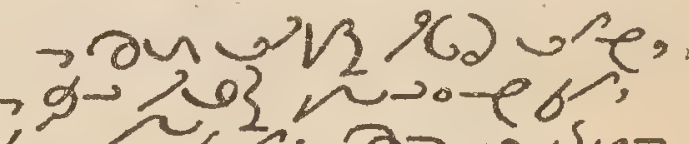
6 csis

-6e-cercign-

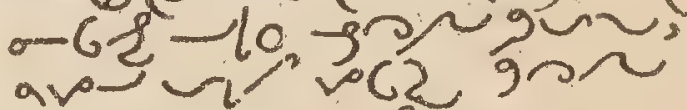
gne be an. 


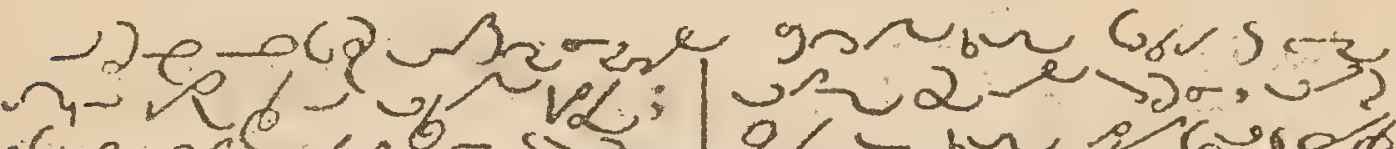

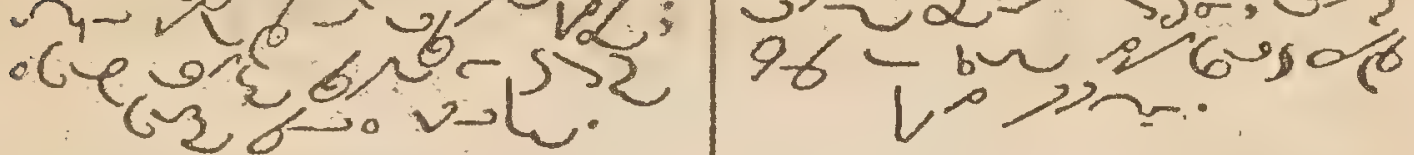
$\alpha-82 \geqslant 8$ lo 939

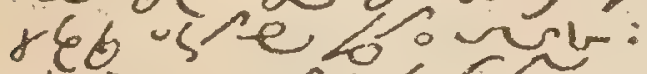
entorol.

2her es soigh, - onsinusis

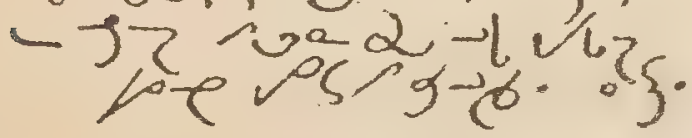
Calitum Joseph.

vie on $=\operatorname{cog}^{2} \alpha$

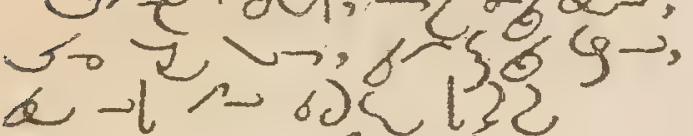
a-l $=100<2$ sitile rawe

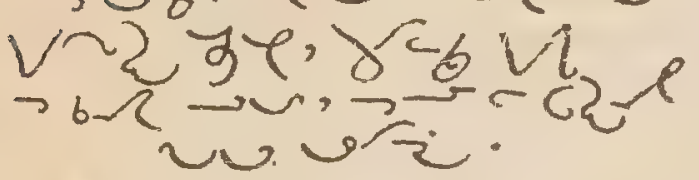

is rompar?

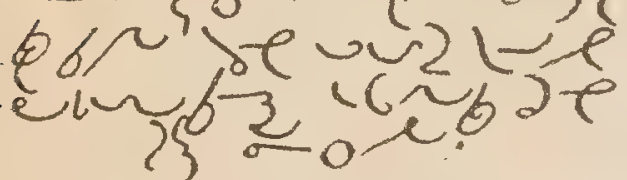

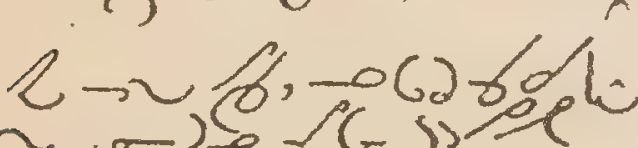

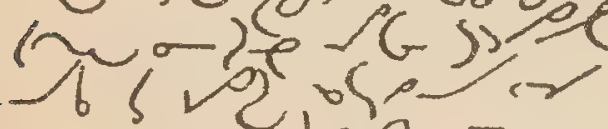
- 14 .

enखutars

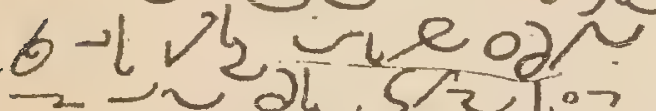
6 . $512 \xi^{10}$

christe sanctorum.

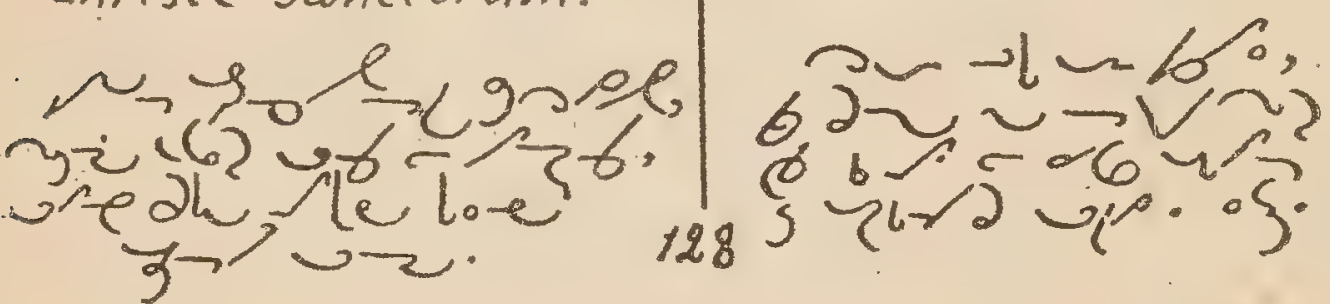
gar 25 s.cus onvus bulez $12 y-2,100$

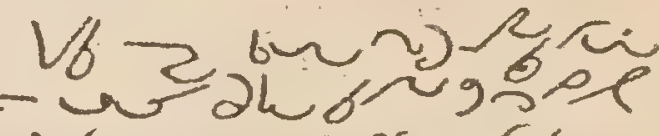

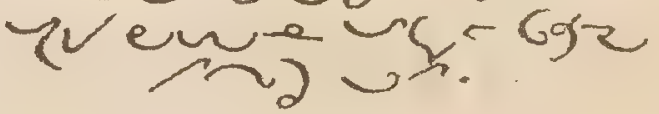
更goh b $\sim 9,2 \rightarrow, 6=3-3 \rightarrow$

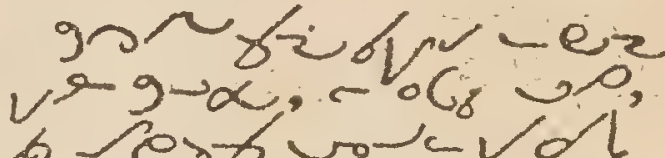
o crovicivat 4 бo Adoro te devote.

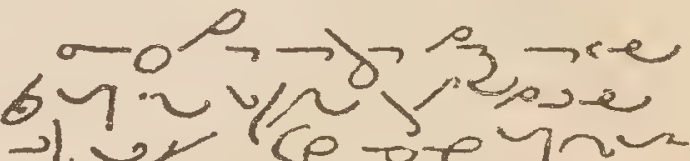

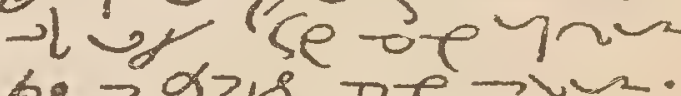
$60-925$ of twa.

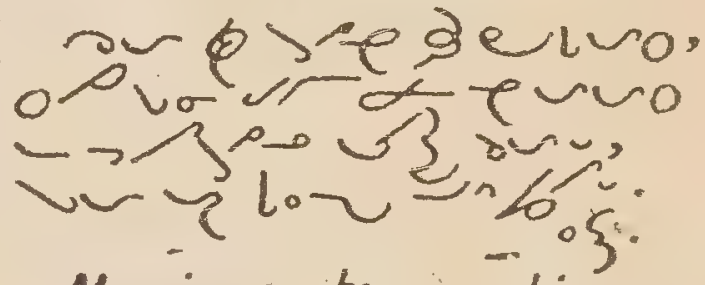
Maria mater gratice. $6 \% 6-1$ pun, 6rave 6 - is - 820 o nul. 


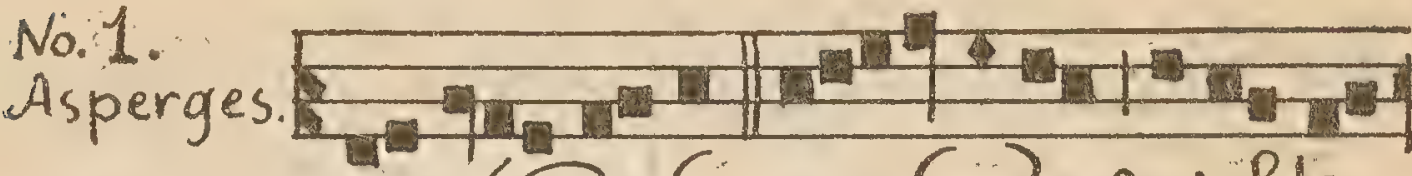
e $25,-0 G 2,296$

구룰

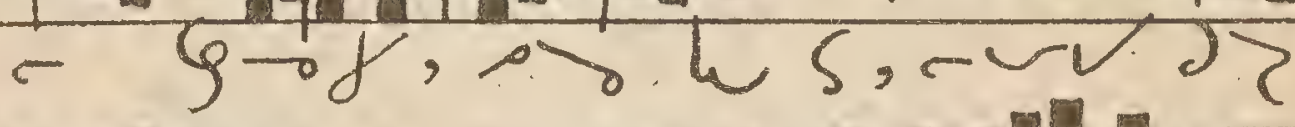

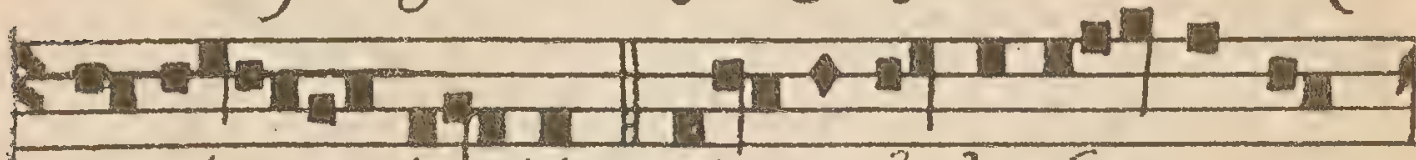
$\rightarrow d \cdots b \gamma \cdot 6 \circlearrowleft \Longrightarrow S<\longrightarrow=$

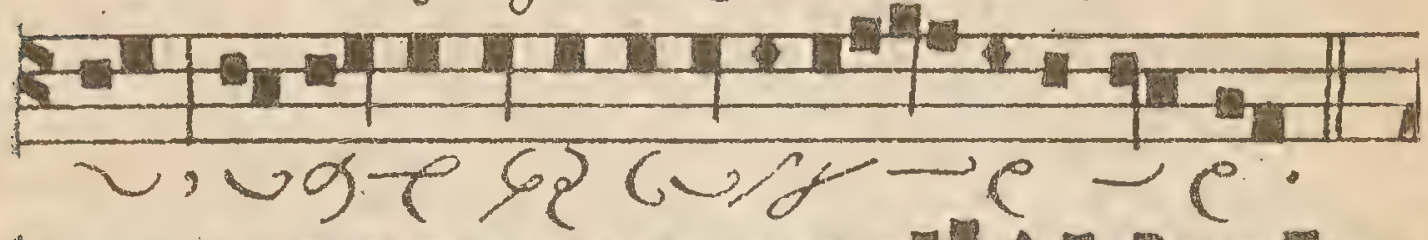

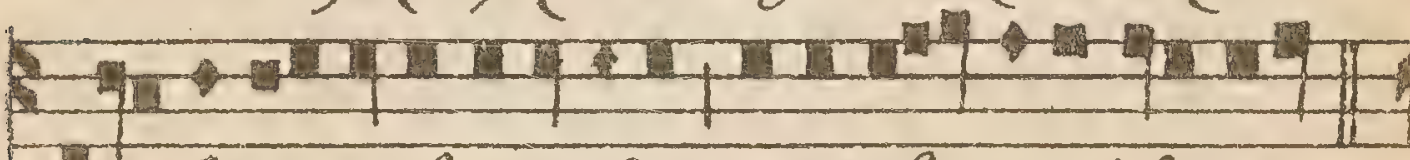

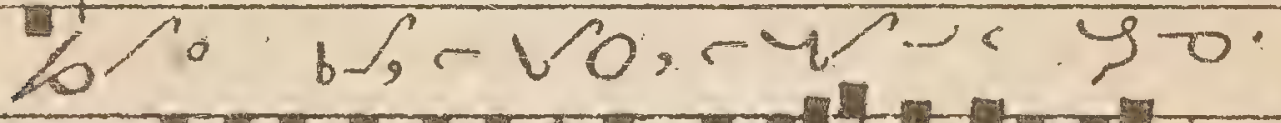
End

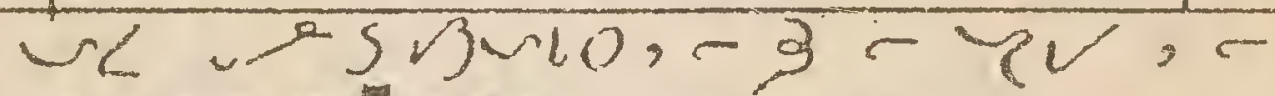

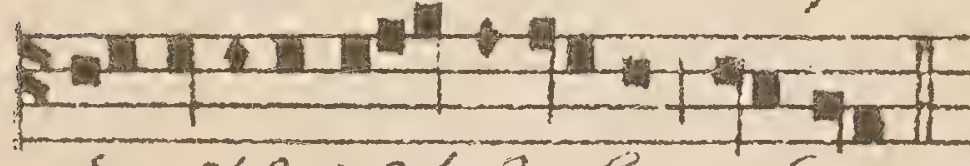

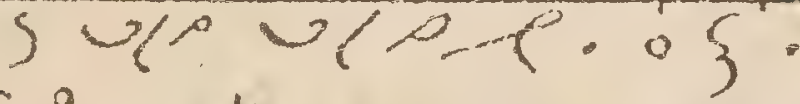

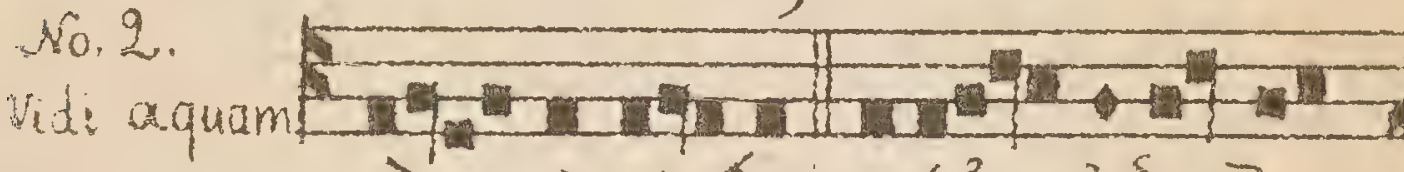

$$
\longrightarrow \rightarrow \& \geq P\}
$$

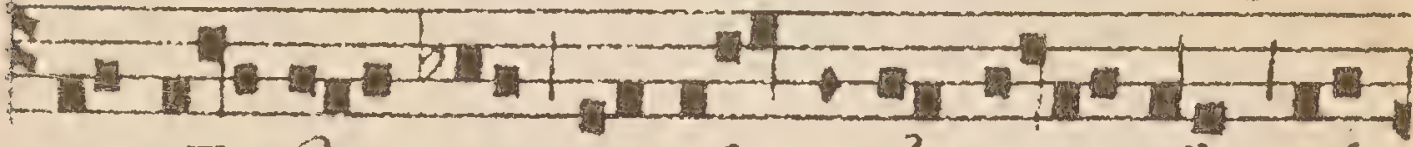

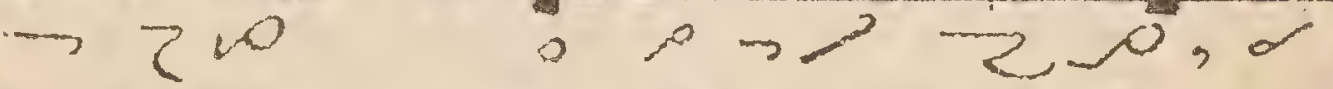
$r r_{0}-e \cdot 2 \sigma \alpha \alpha_{2}$

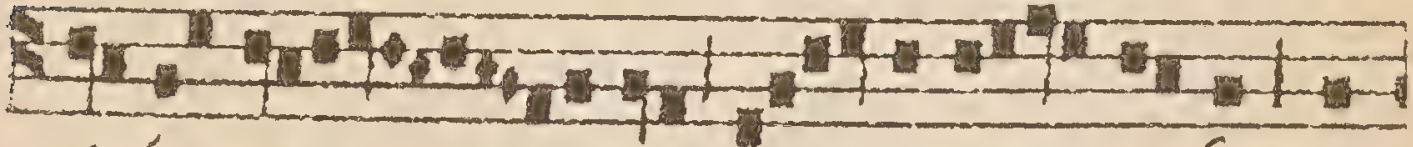

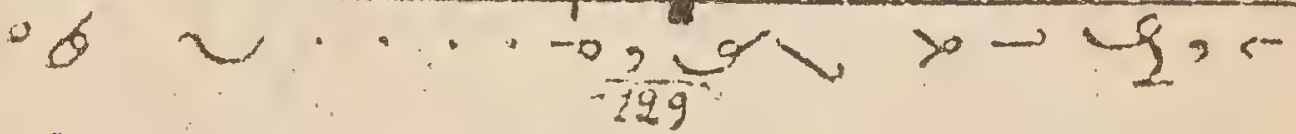




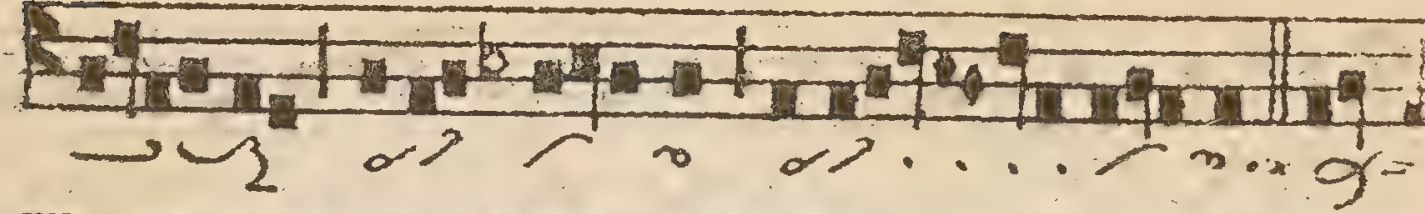

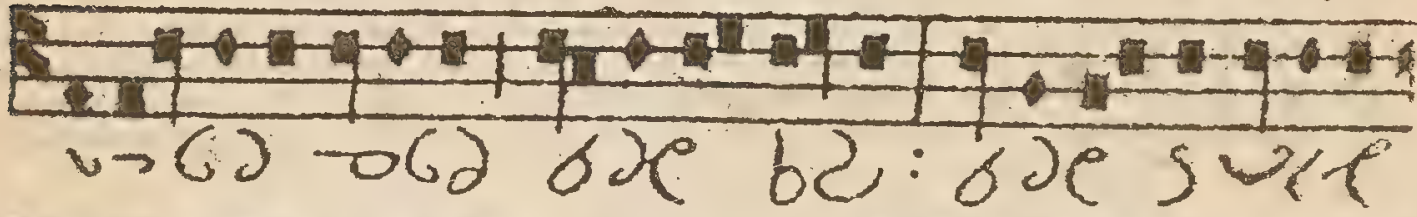

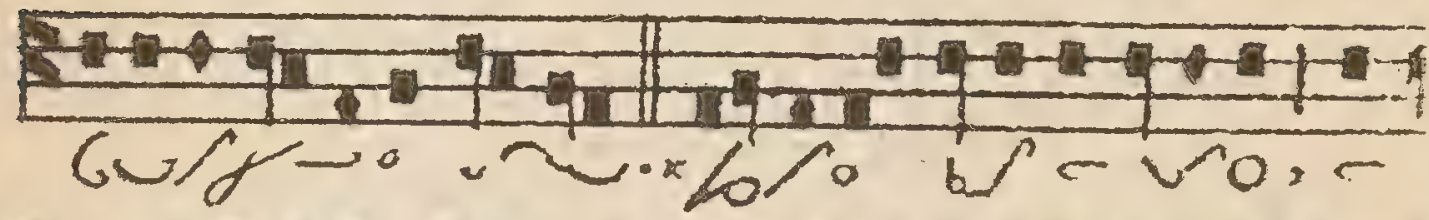

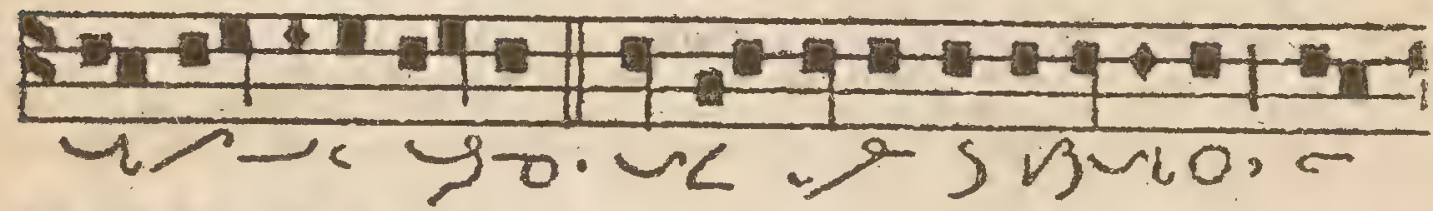
हल

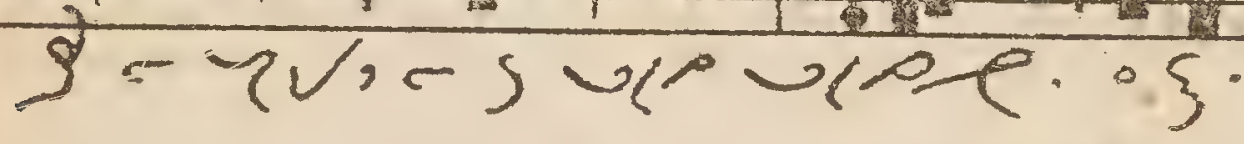
Responses for the Preface.

No. 3.

Responses. 整 Vej. us us p.ok

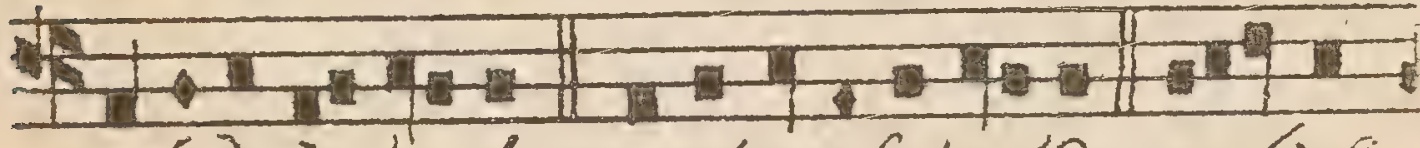

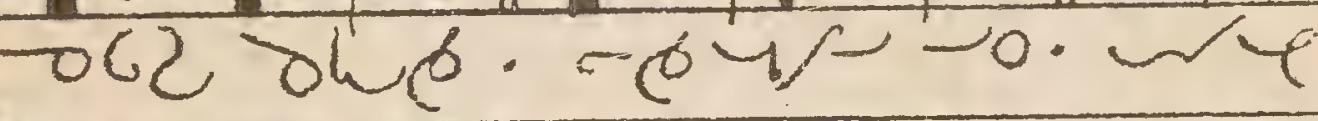

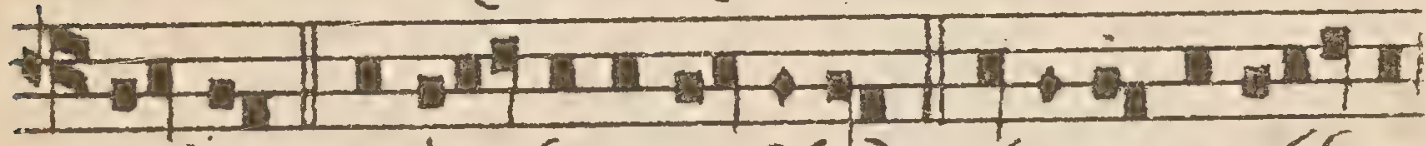

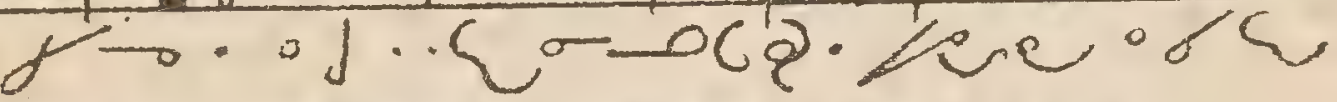

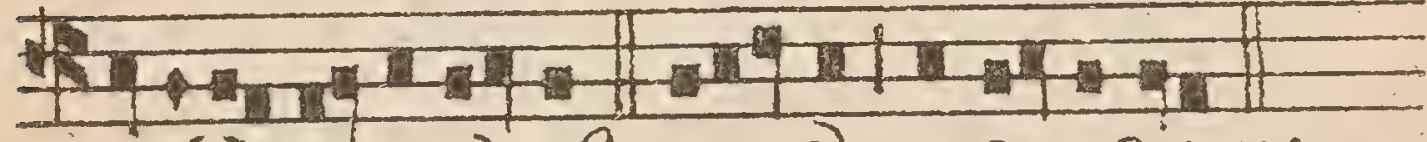

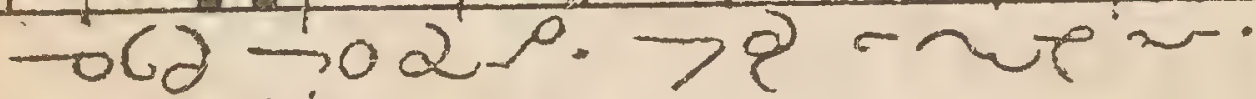

No. 4. Do ferial. 等 2 trin

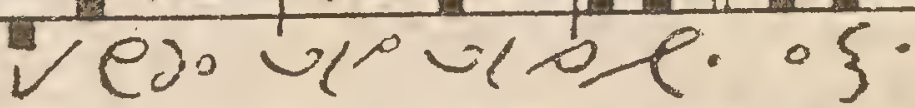

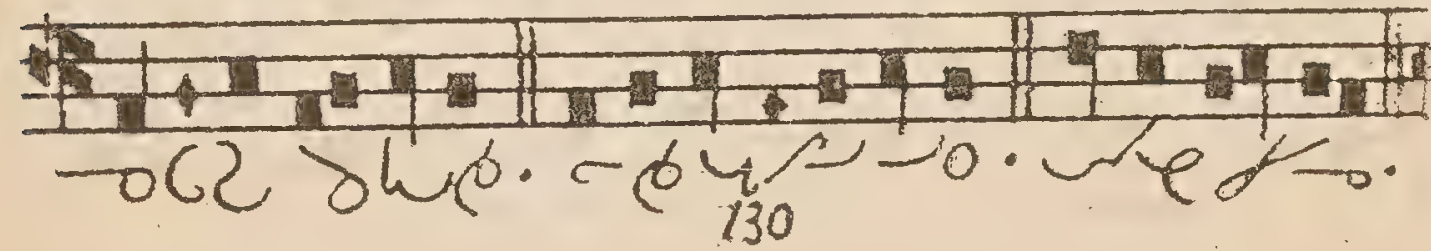




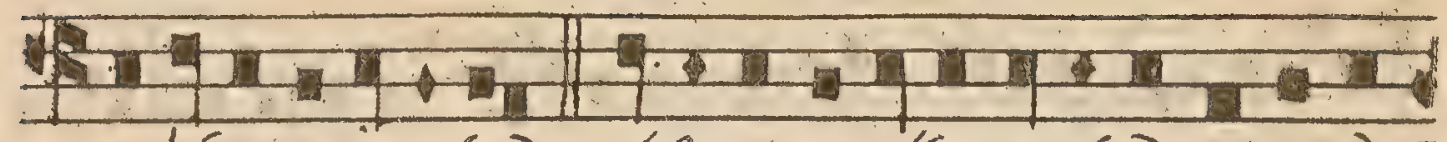
- Jर $\sigma-062$. liee of $/ 062 \rightarrow 02=$

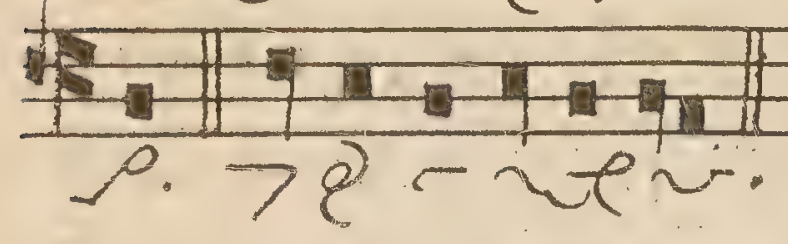
Messe Royale.

No.5. Kyrie Royal.

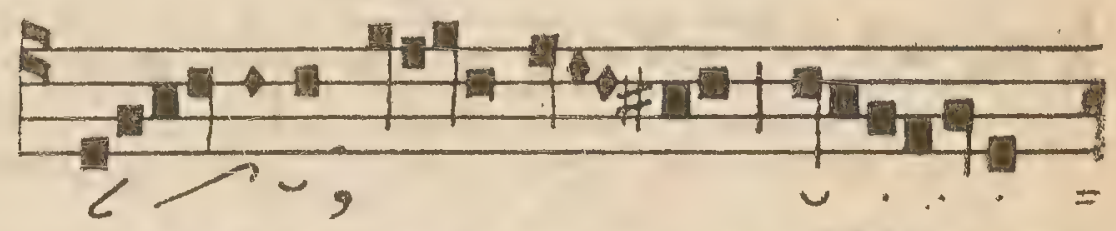
s

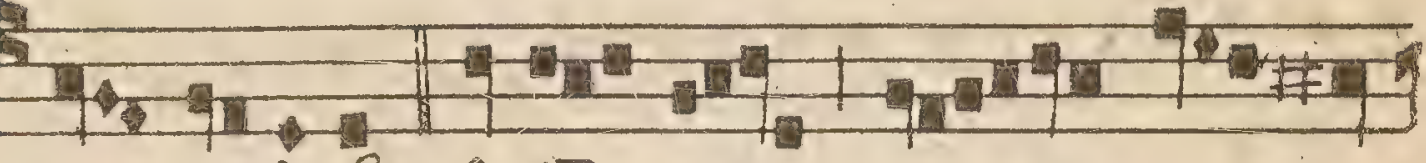

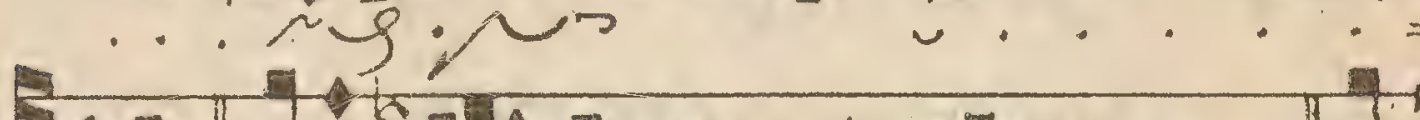

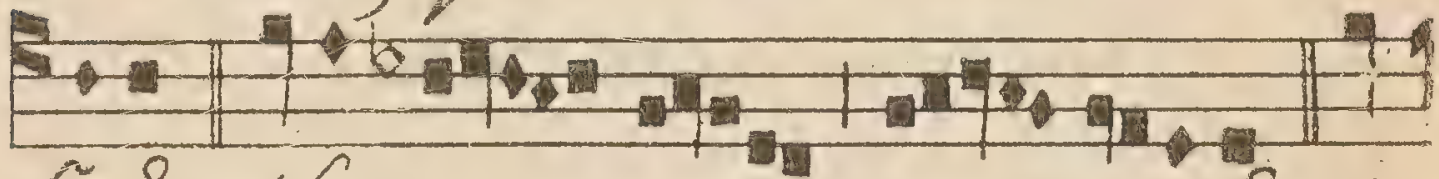

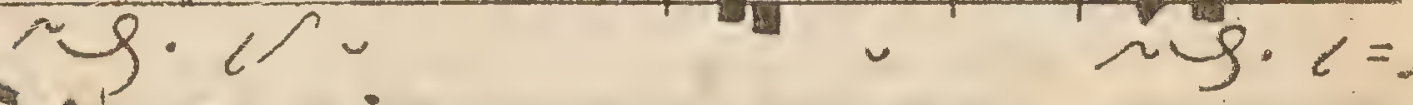

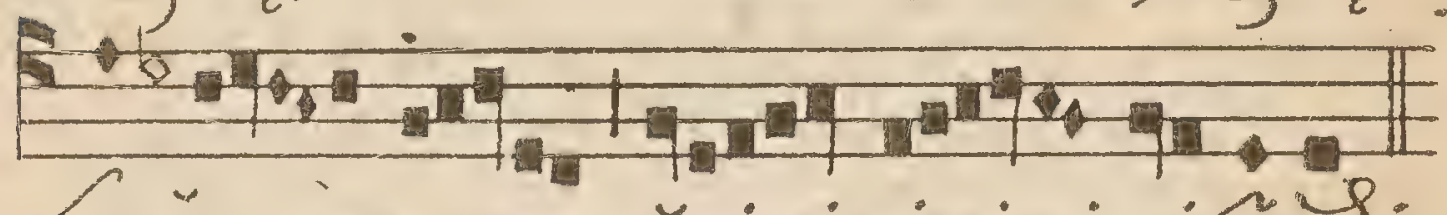
No.6.

Gloria Royal.

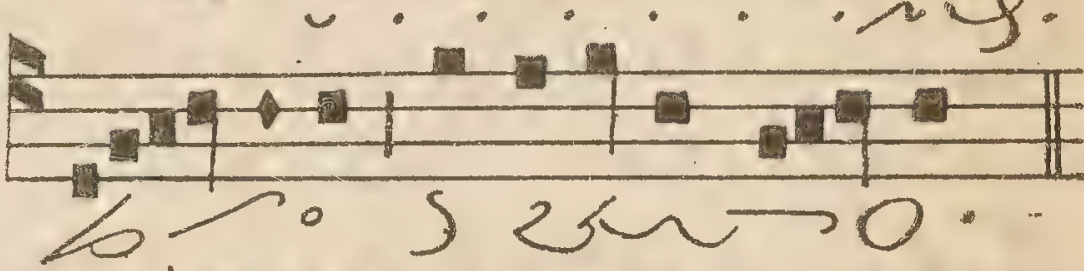

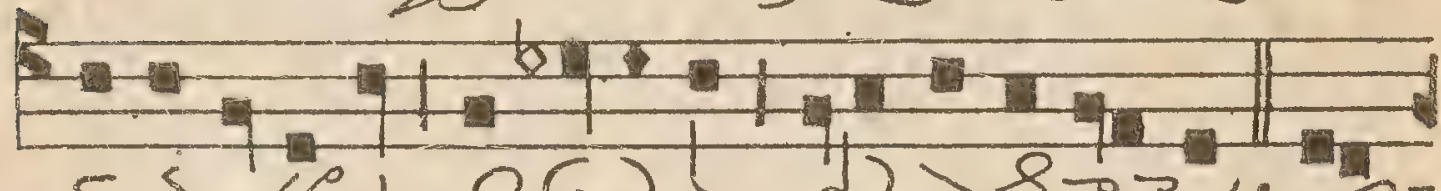

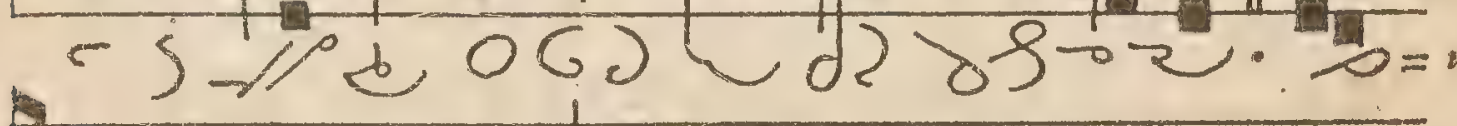
E-

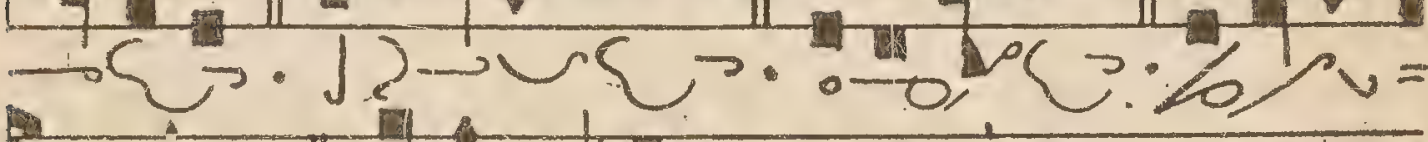

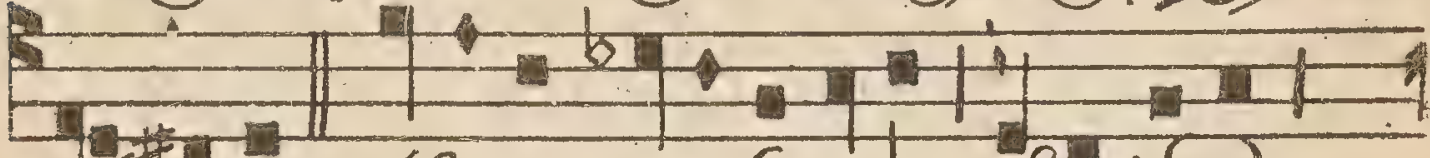

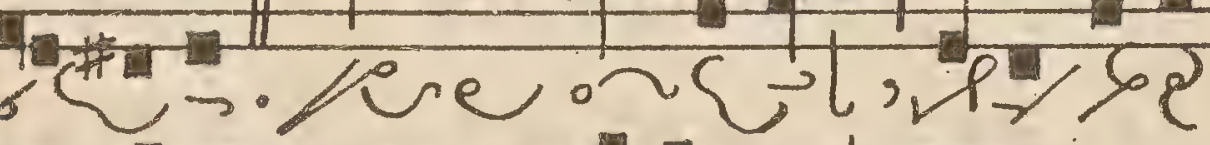

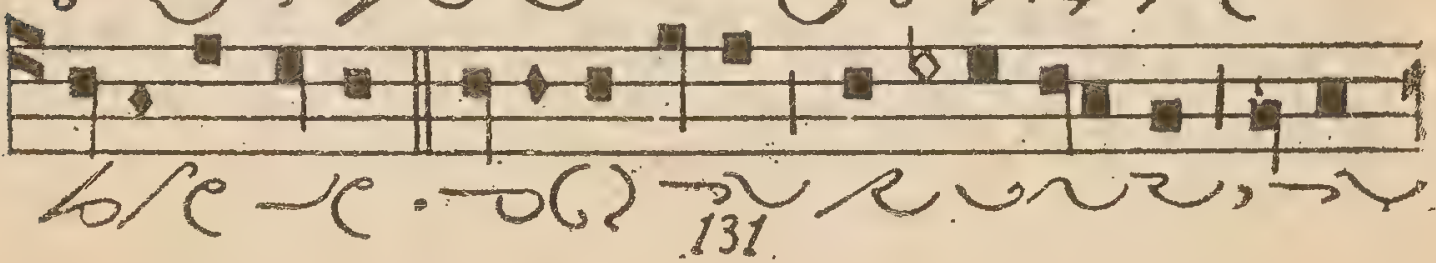




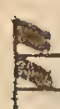
br ejb3.0G v' (J)

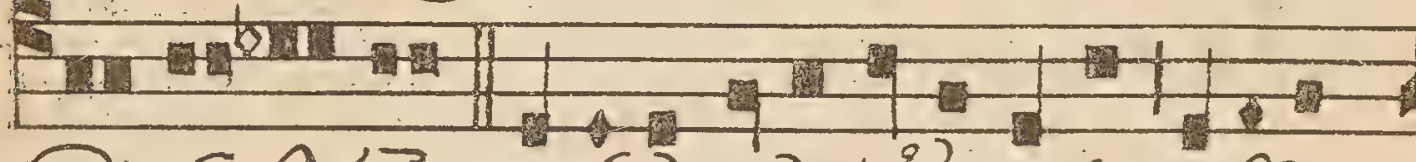
Dur $\sim 20$ OQ

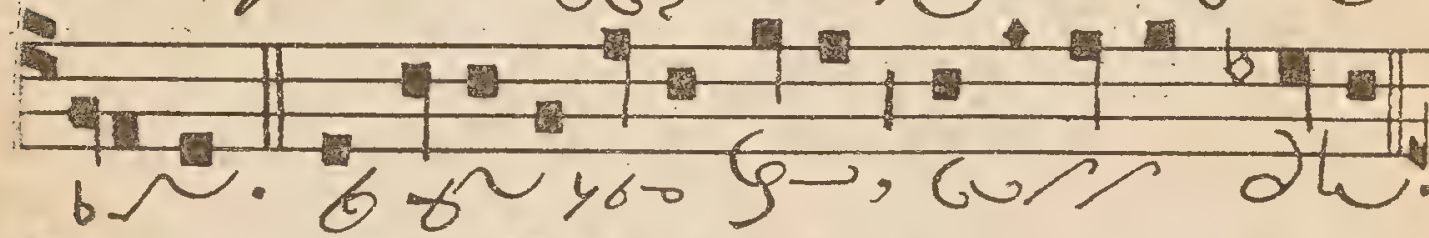
(2) 1

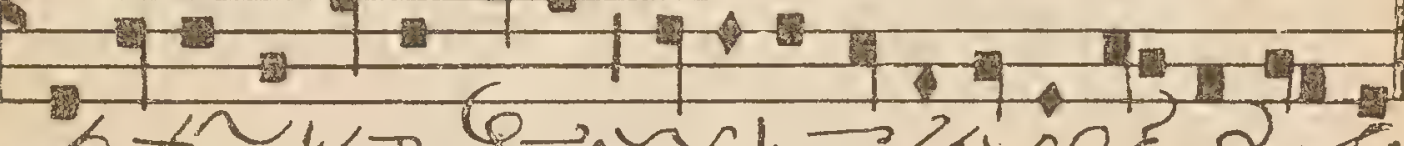

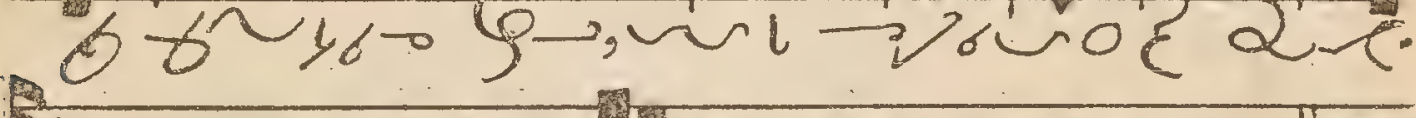

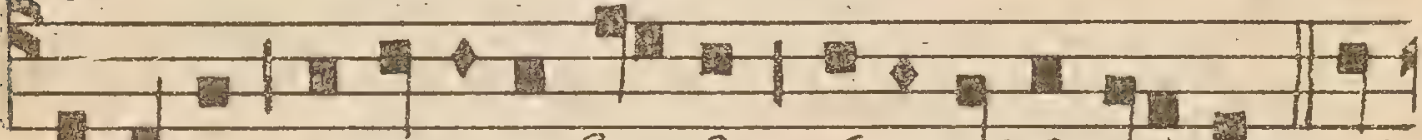

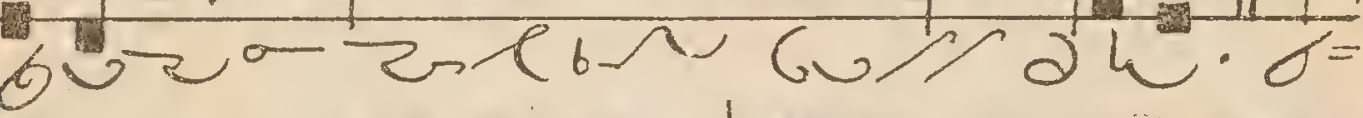

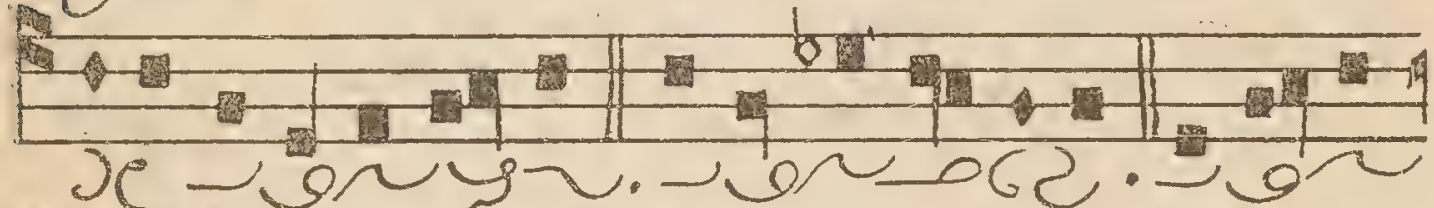

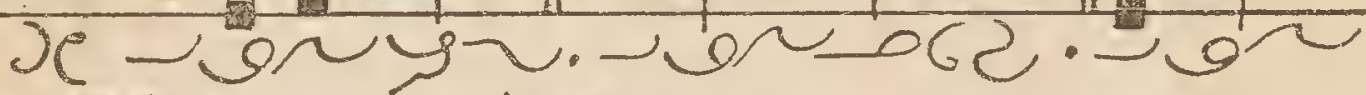

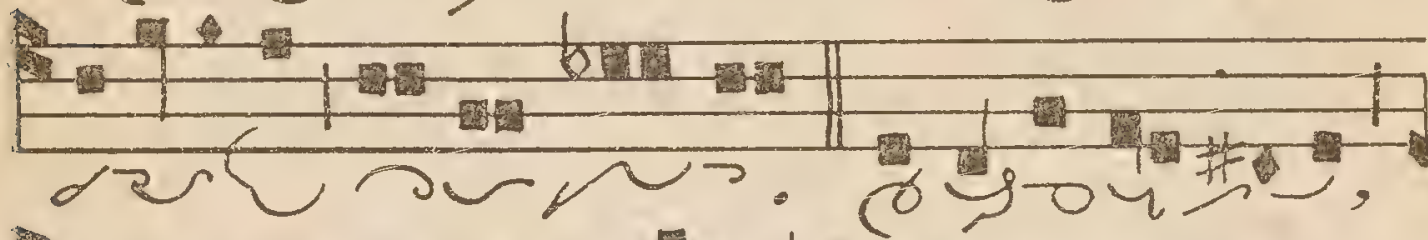

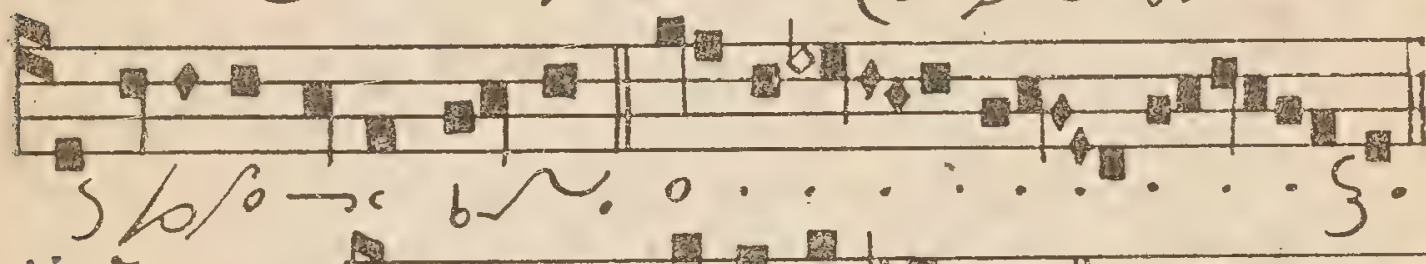

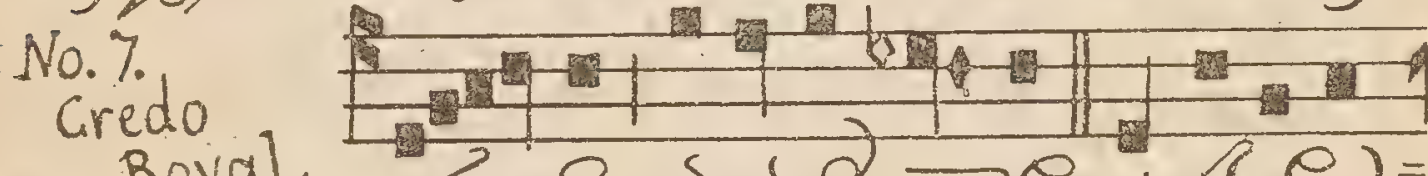

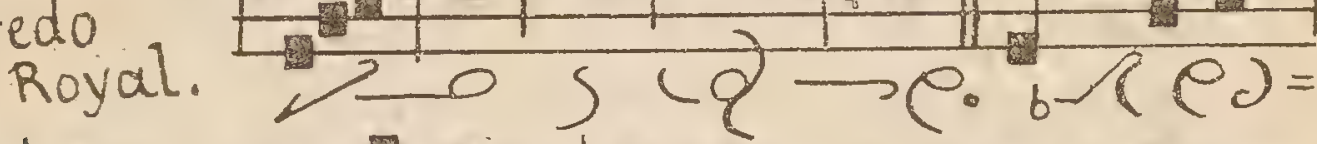

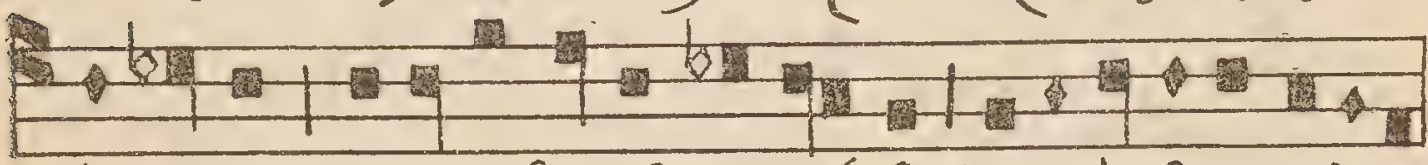
b35 rokurandwhereb -

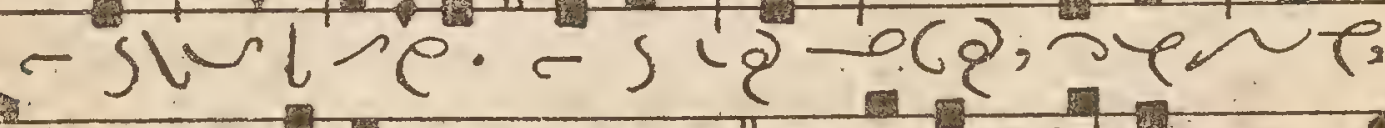

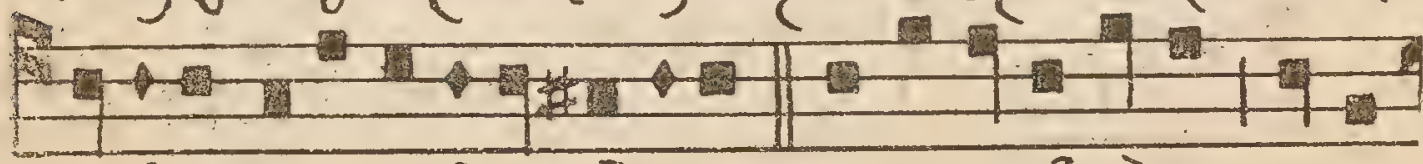

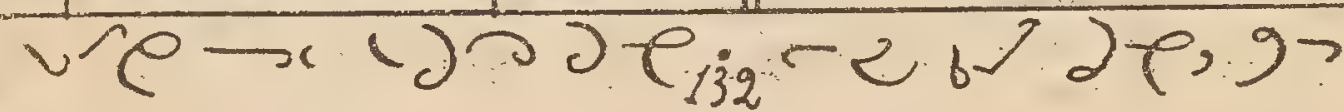




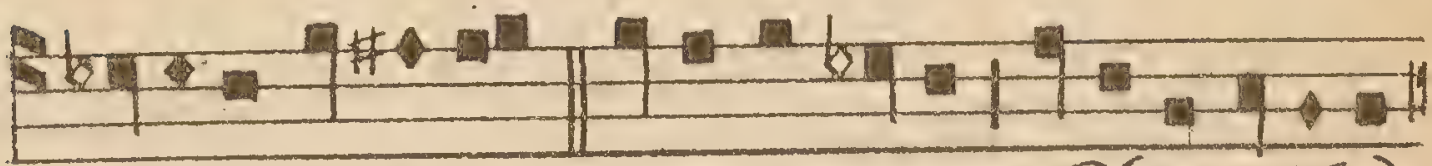

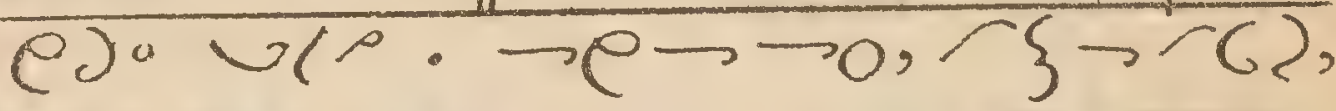

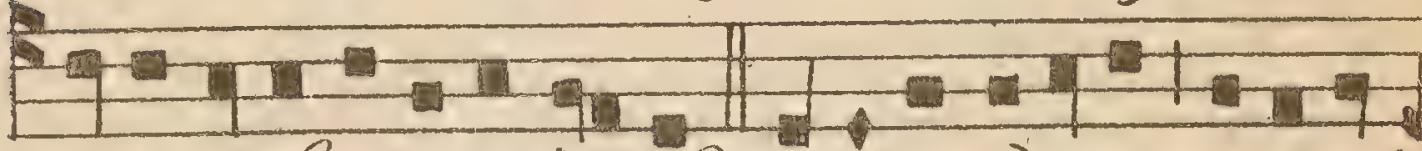

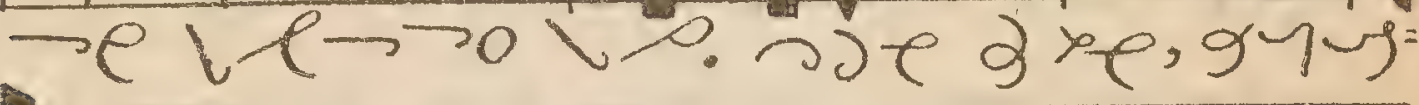

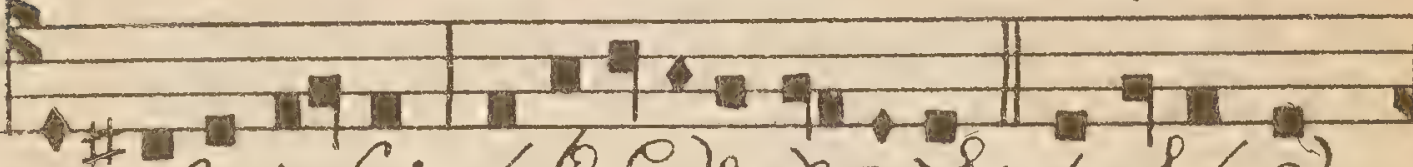

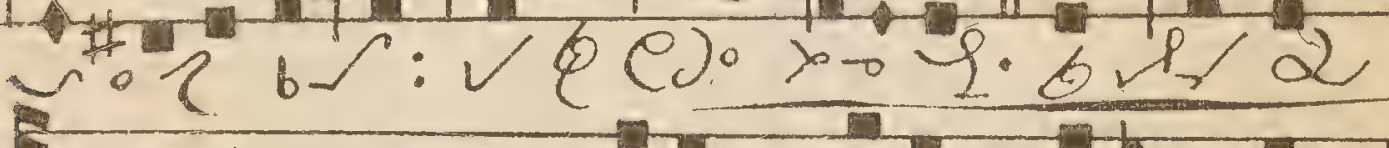

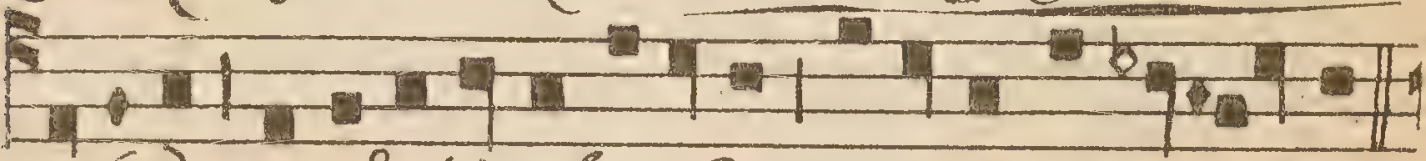

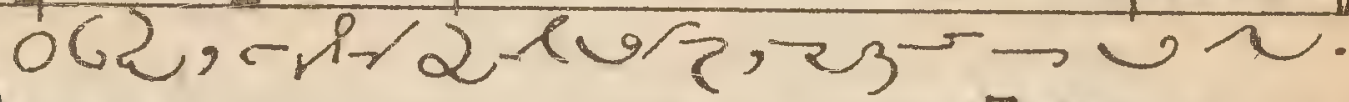

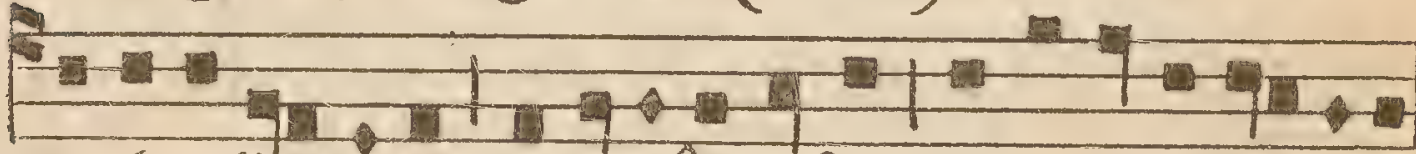
$-\int \gamma d v w \rightarrow 4=90.26 \% \mathrm{~V} 2$ ),

sents

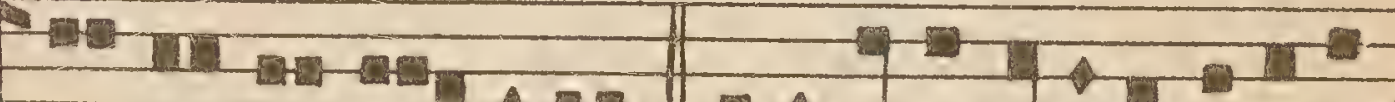

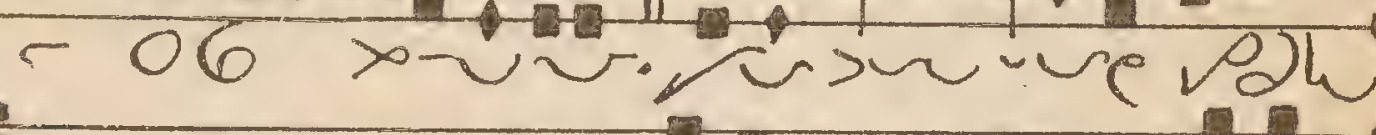
s

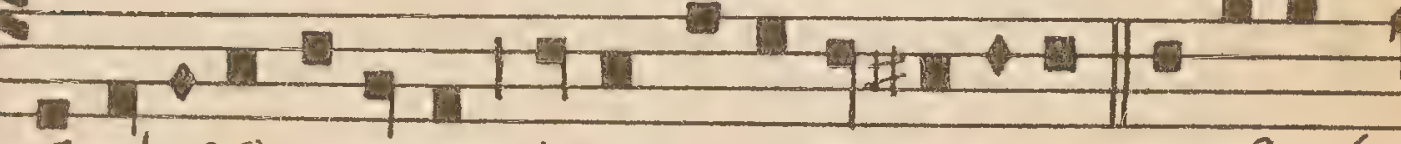

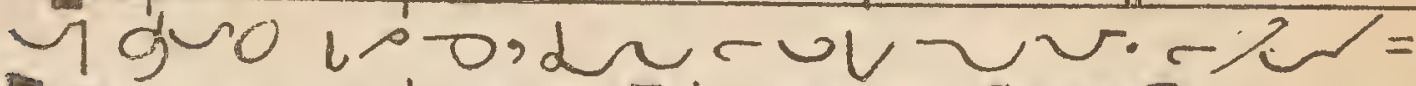

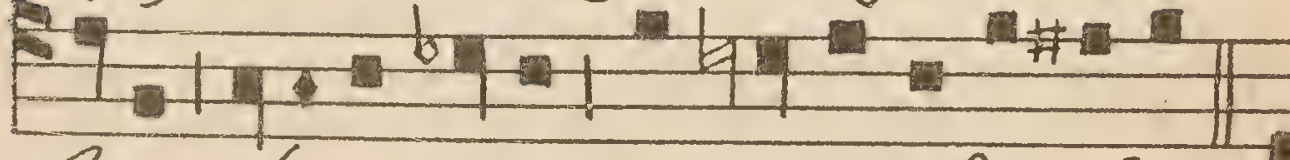

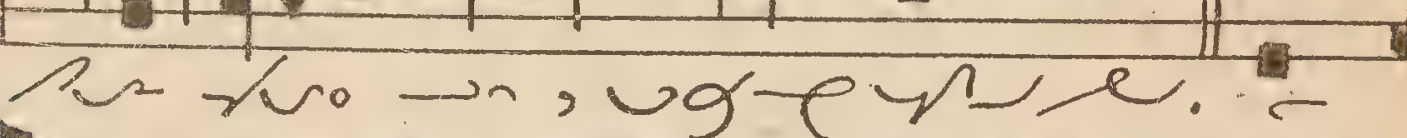
1

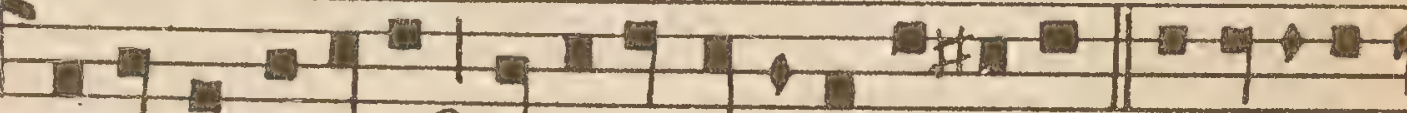
enssul,

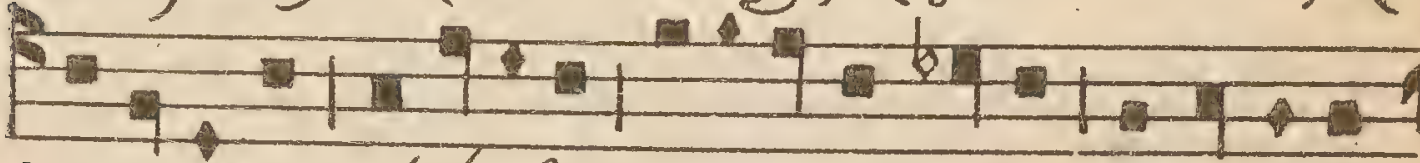

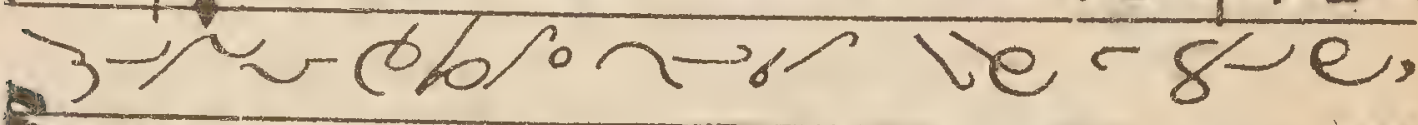

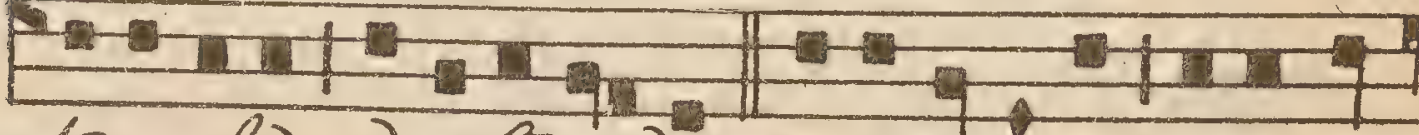

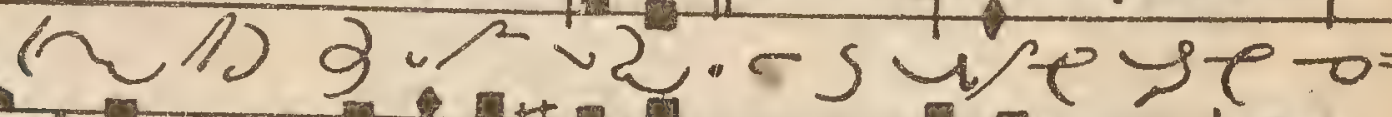

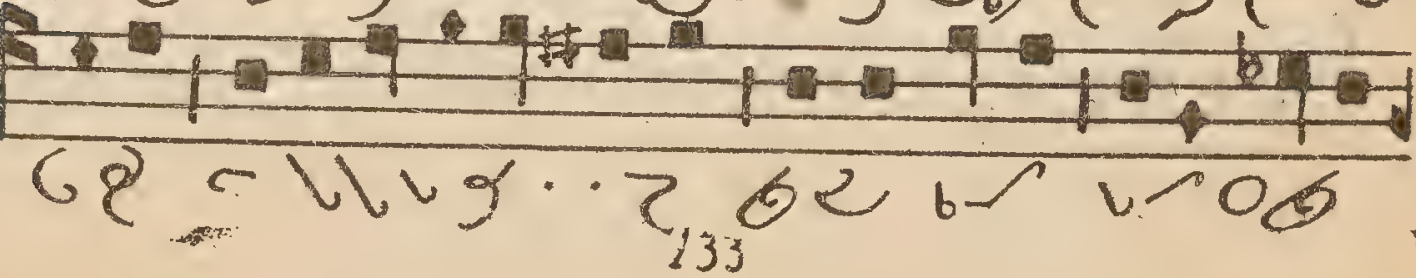


-1.1.

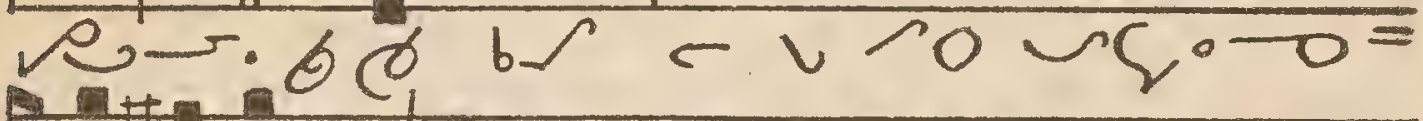

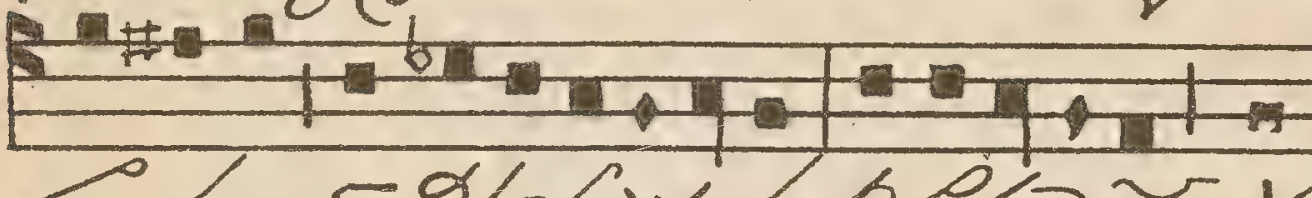
- $-96 / v 6$ ohu

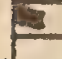

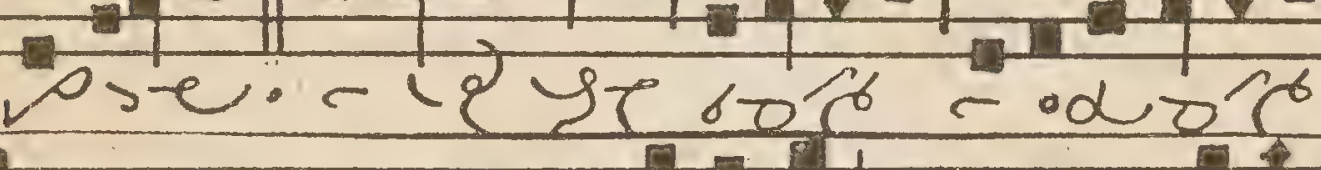
-

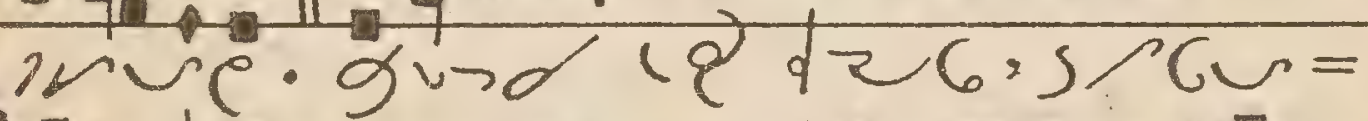

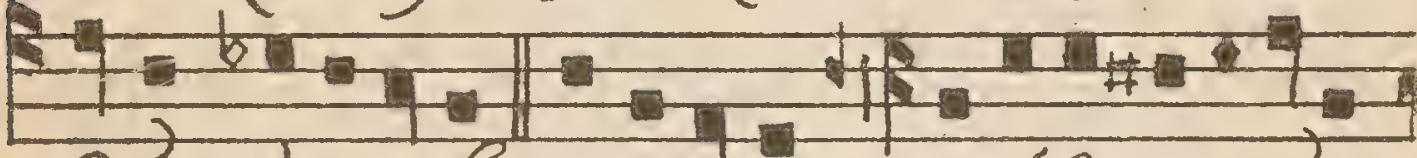

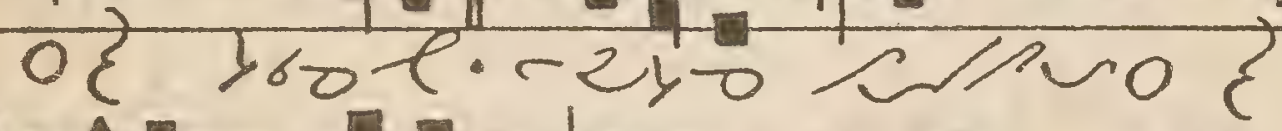

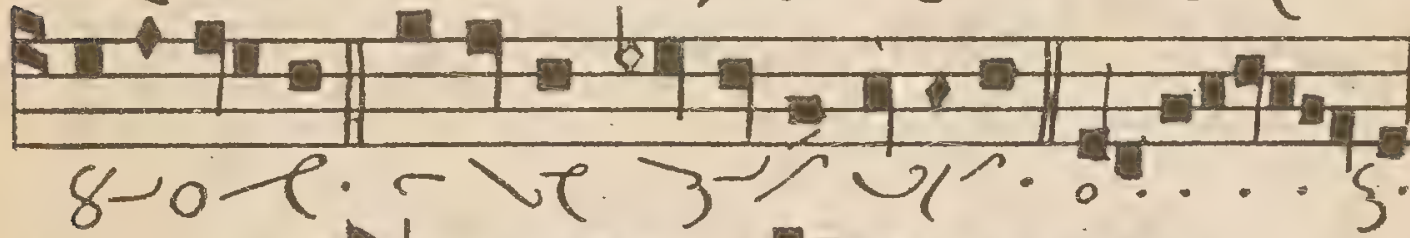
No. 8. Sanctus Royal. to

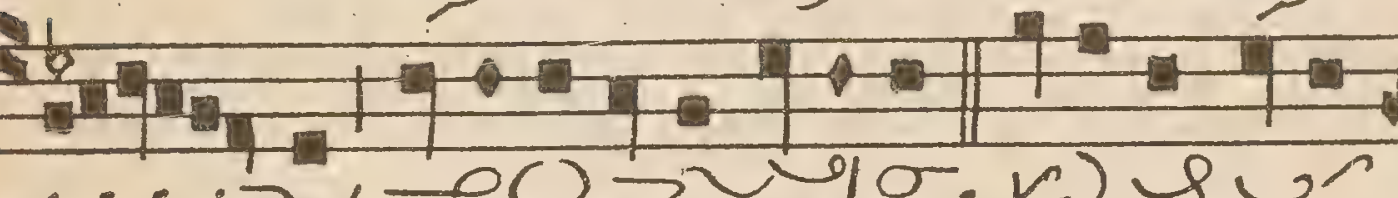

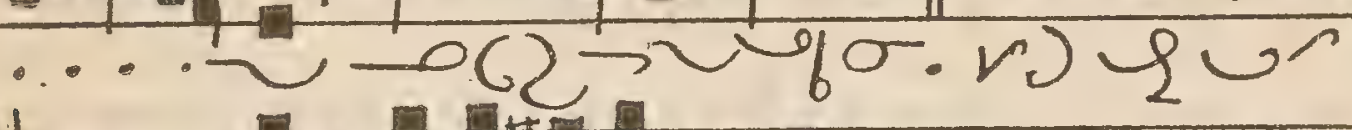

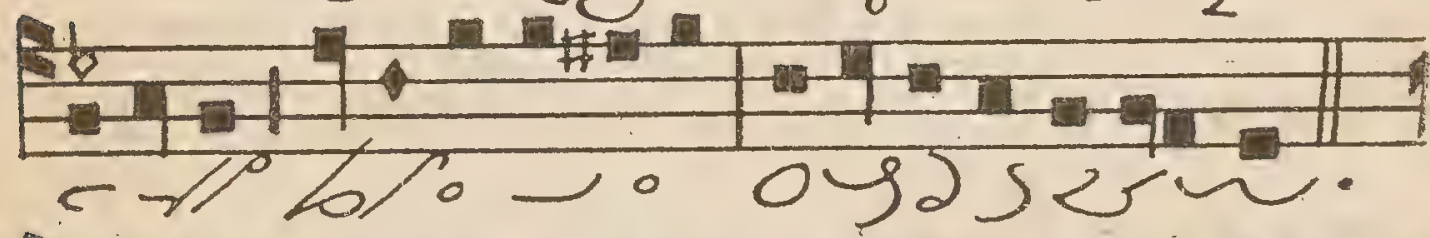

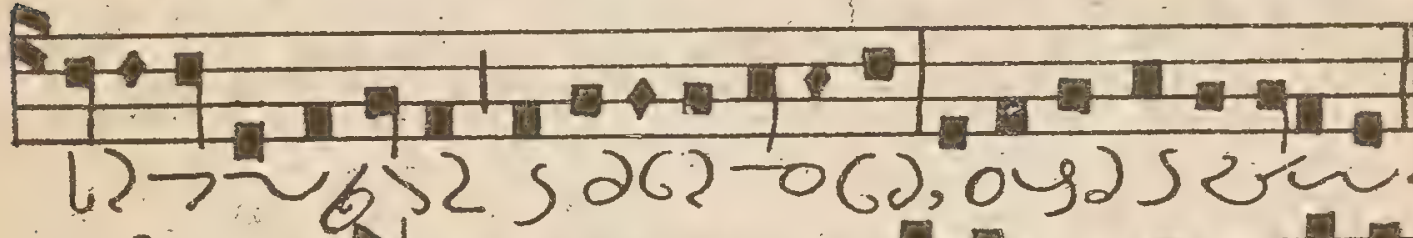

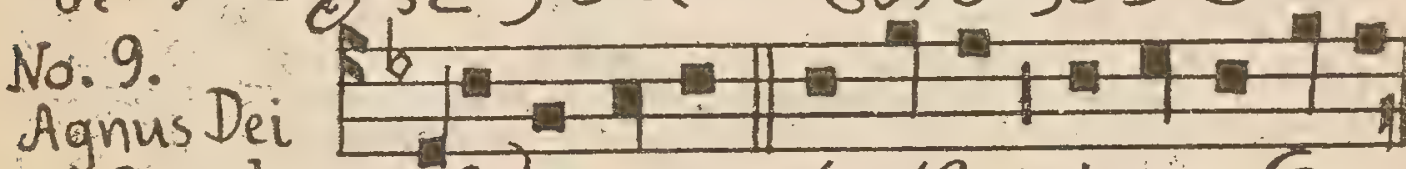

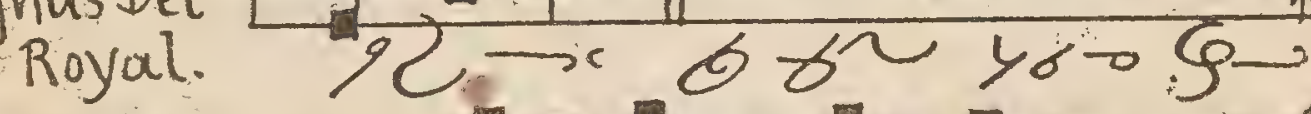

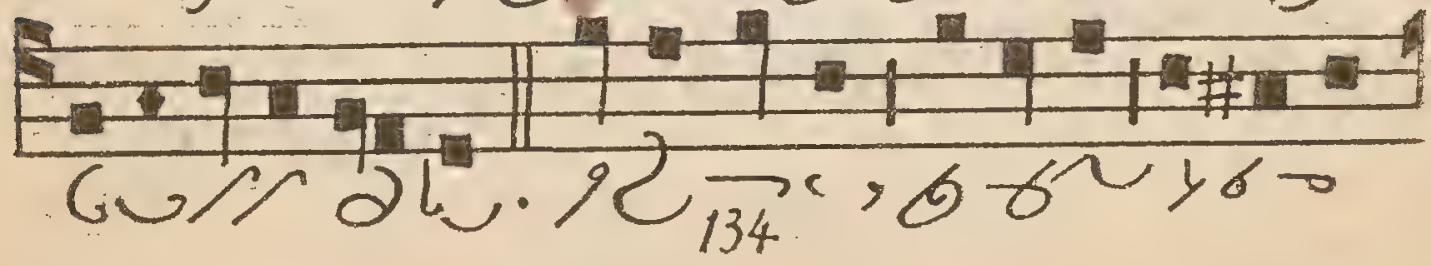




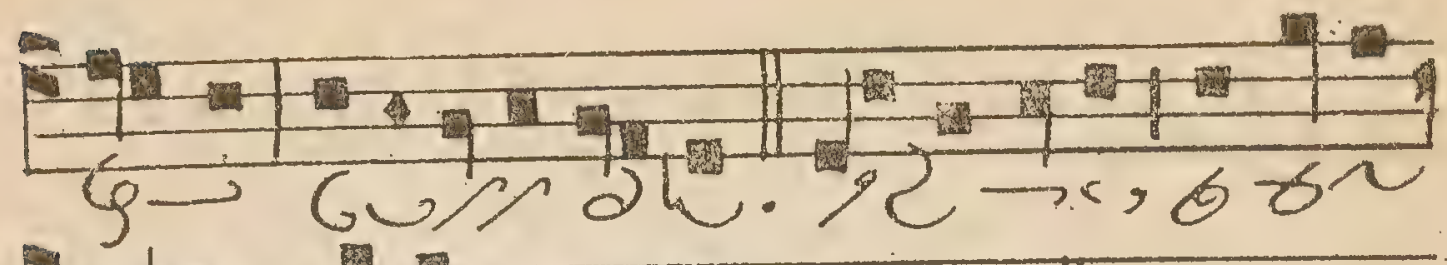

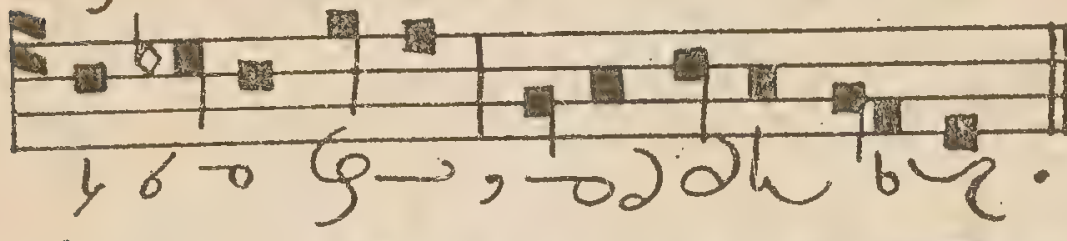

No. 10.

Kyrie

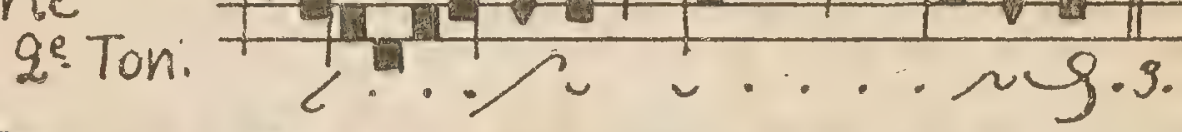

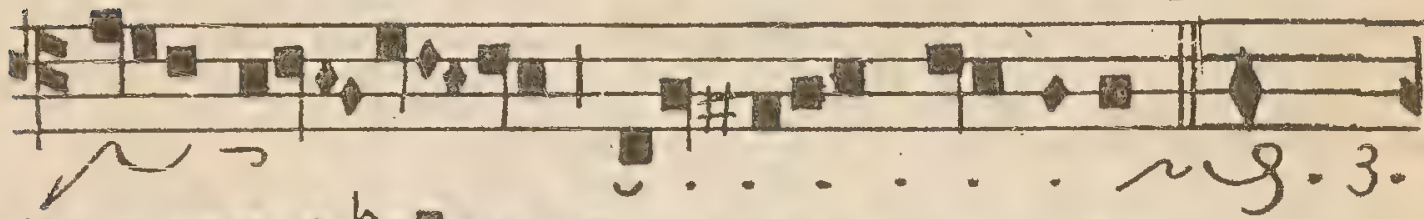

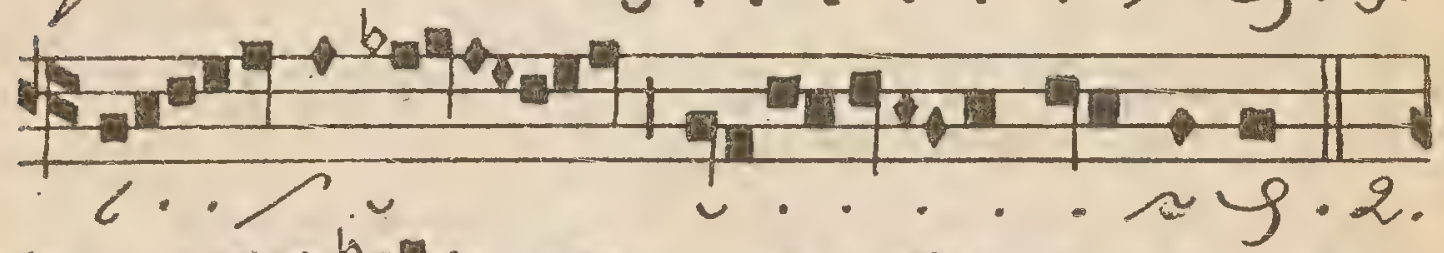

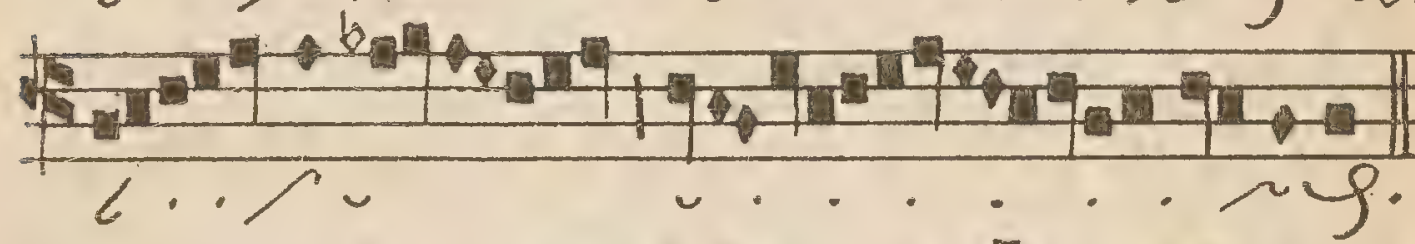

No. 11. Ko.11.
Kyrie Anges.
des. Cu

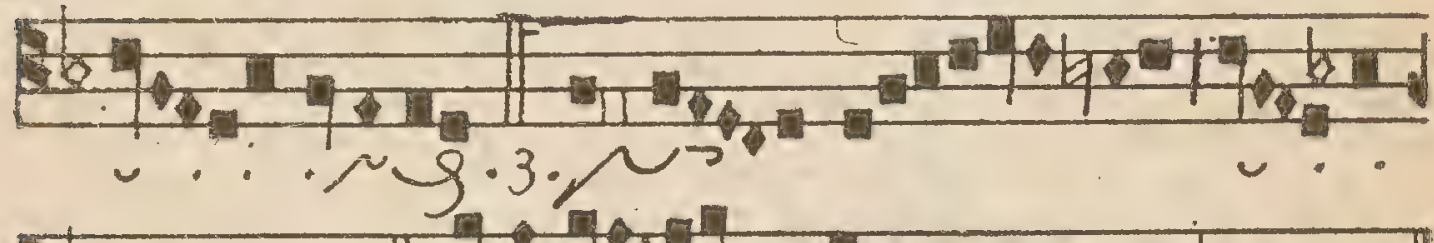
36. $\sim 9.3 .</ 2 \sim g^{2}$

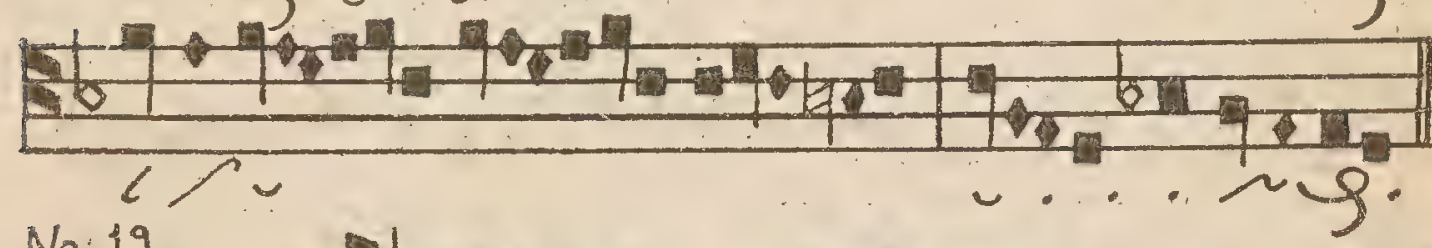
No.12. $\underset{\text { Kominical }}{\text { Ko }}$

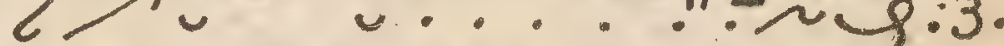

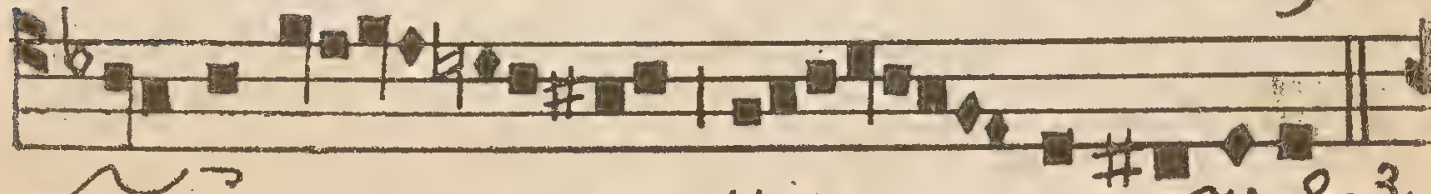
$135 \cdot \cdots \cdot 3.3$. 


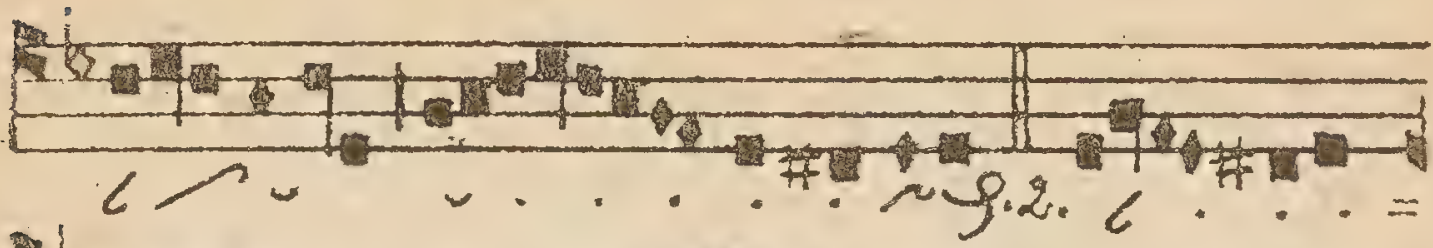

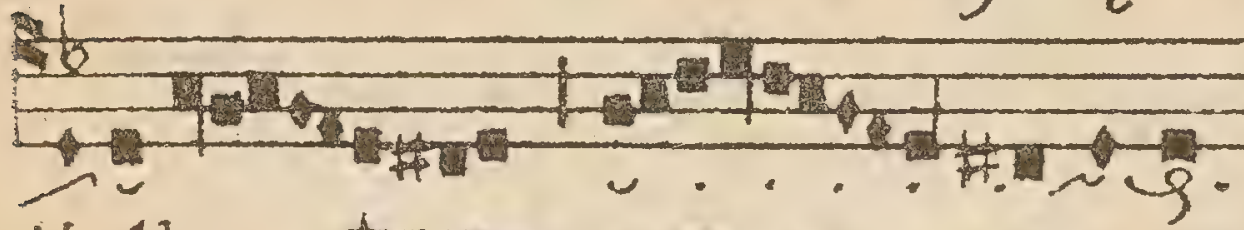

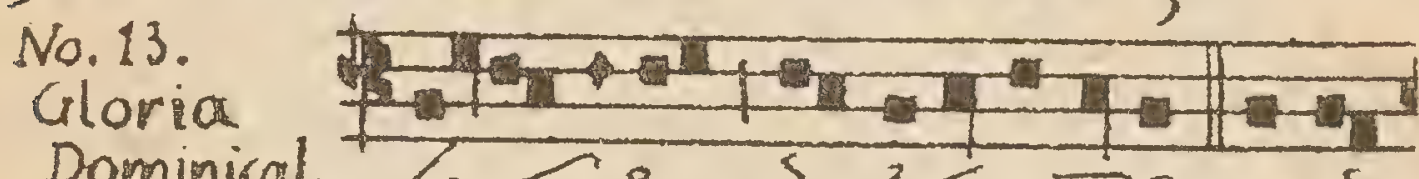
Dominical. lo 7 S 2un 0.05 4. $\checkmark \rho \& 0 G)(b) \delta 802 \cdot 0 c=$

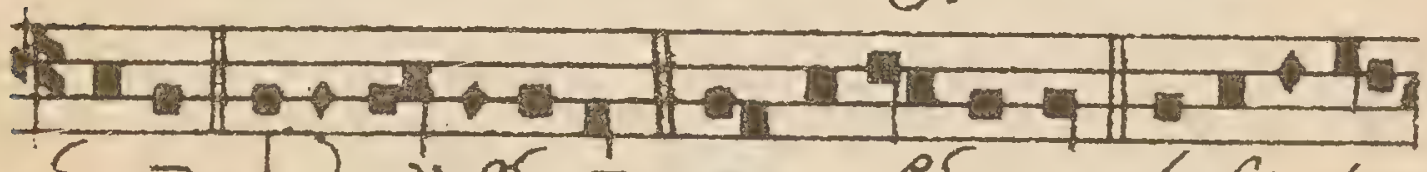

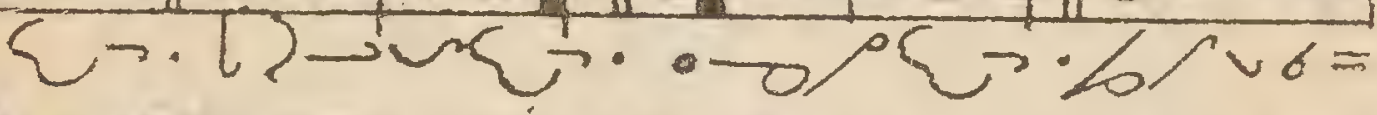

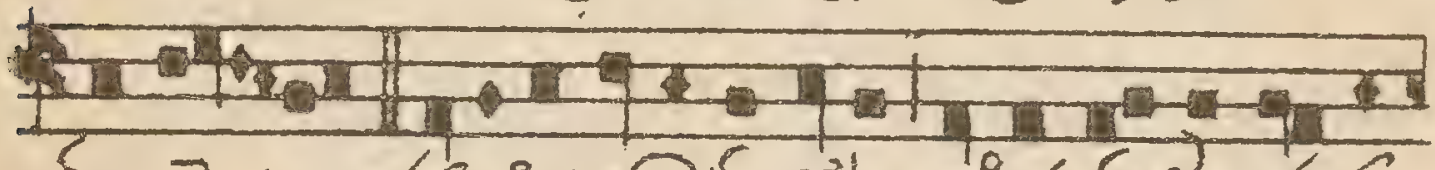

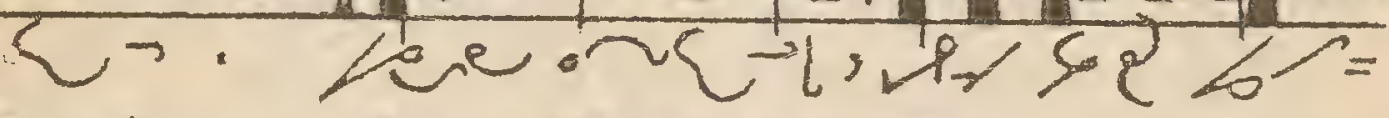

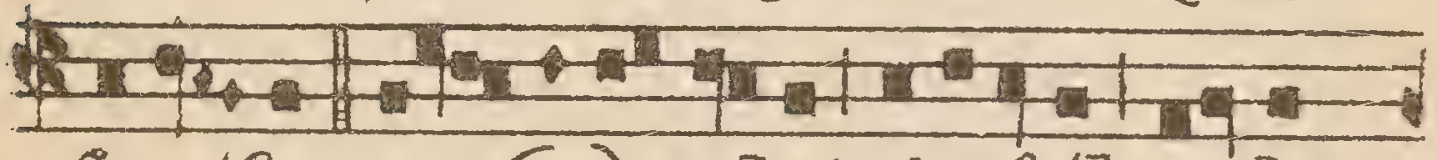
$(-6 \cdot 0 G) \rightarrow \sim 2 v^{2},-$ 4.

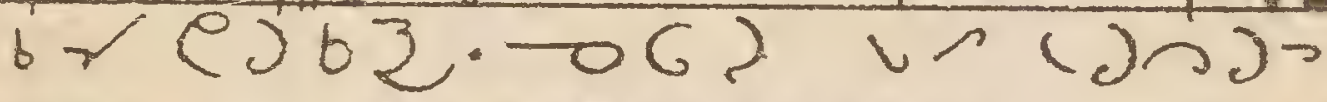

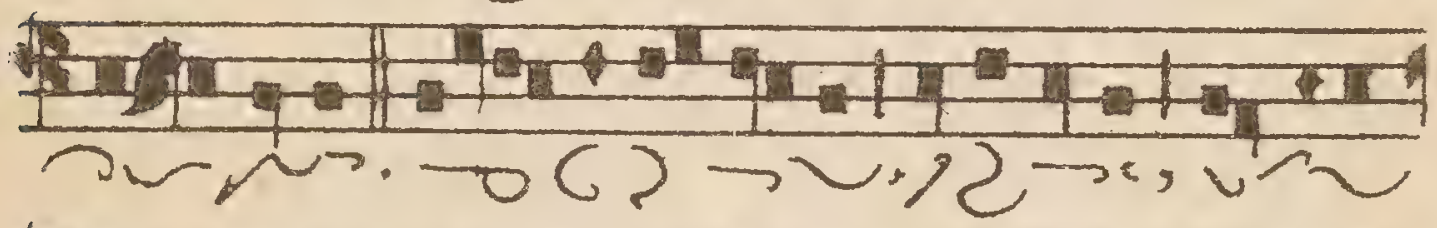

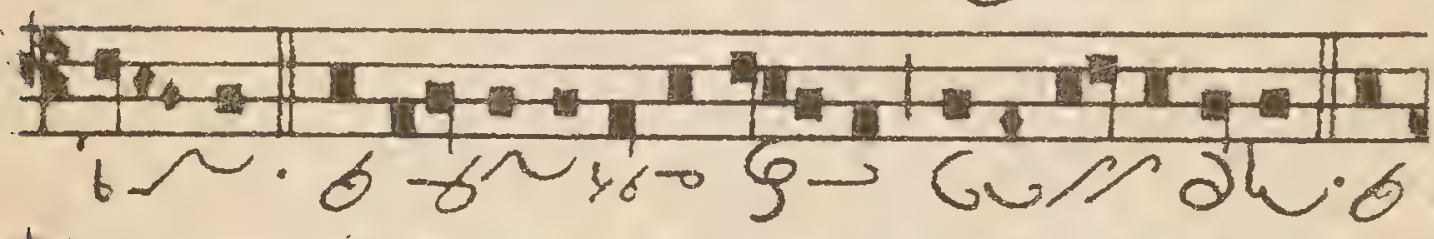

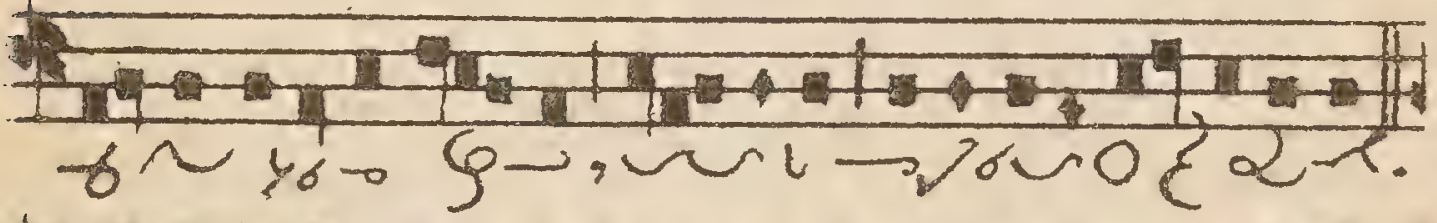

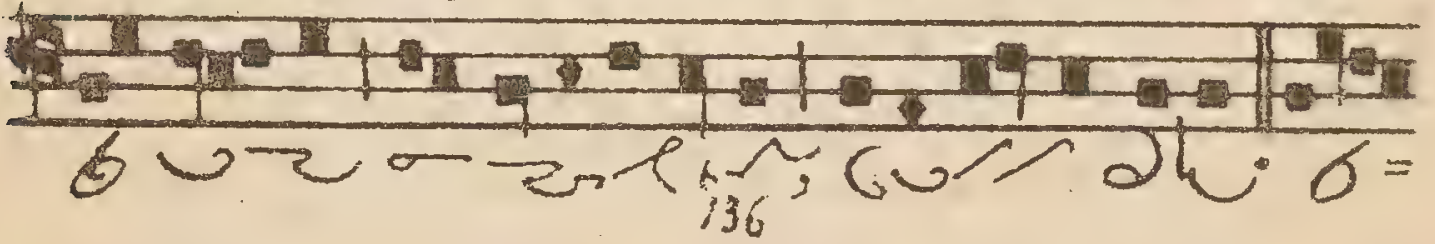


然-

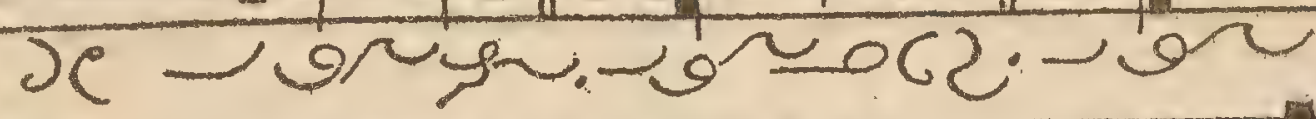

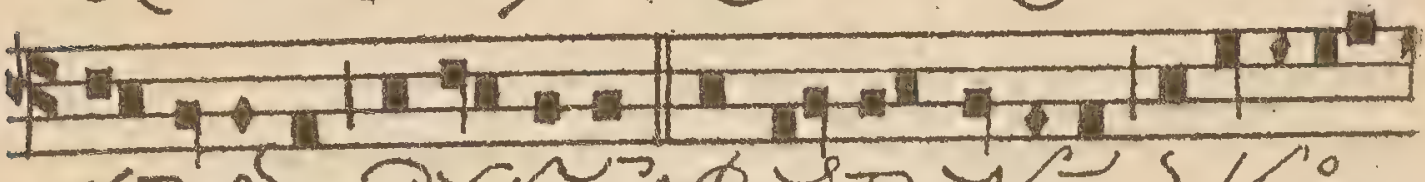
वर्य suñ.

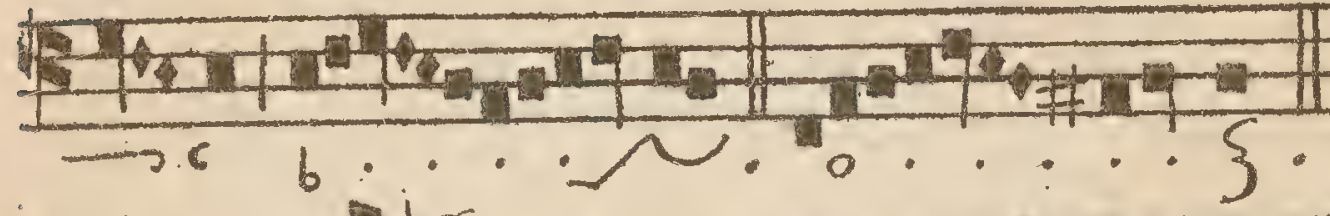
No14.jo eredo

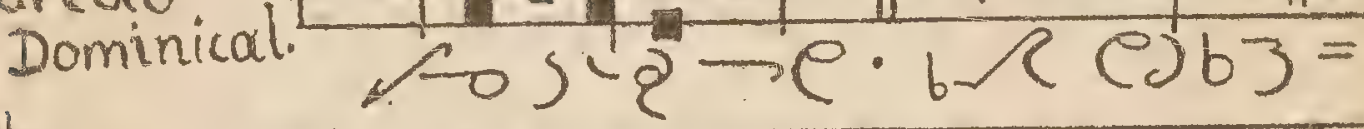
50

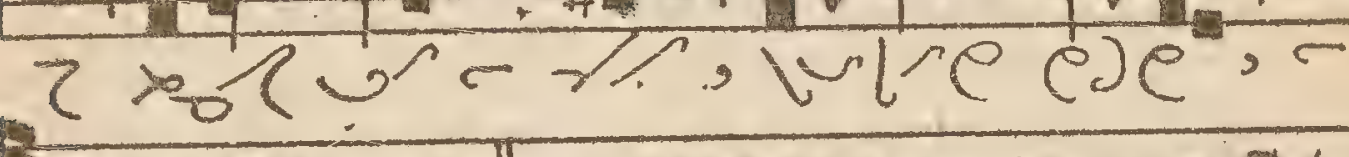

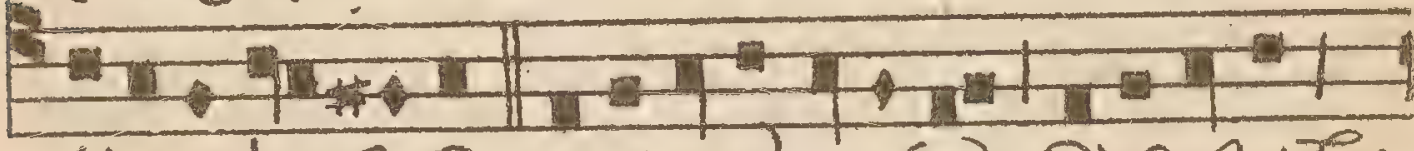
sul re.reg oce, sene.

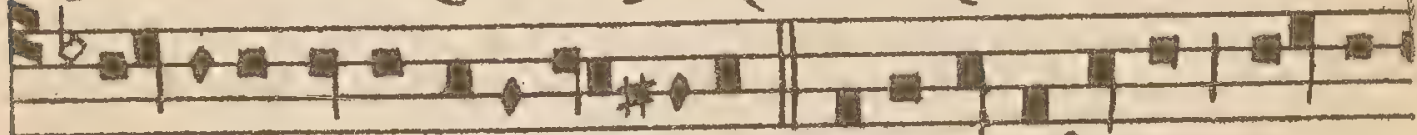

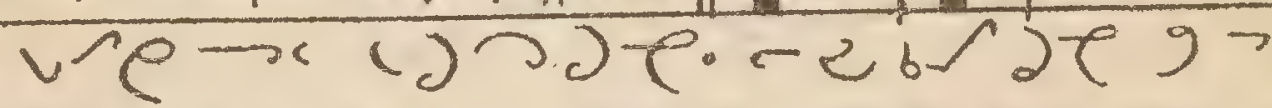
t

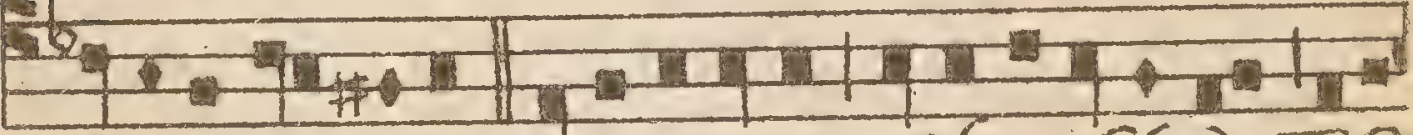
ej. we. 36

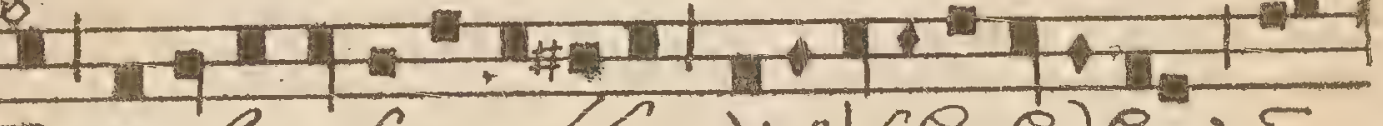
3 - 1 le $\rightarrow 0$ lo. soegre,gurjur? e-

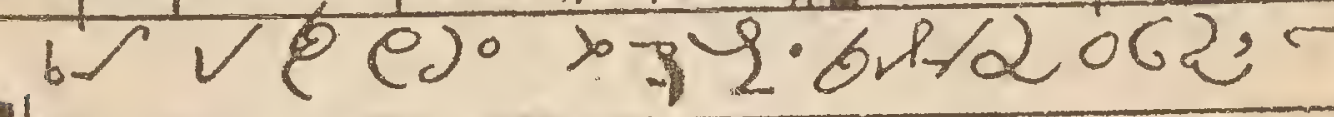

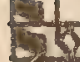

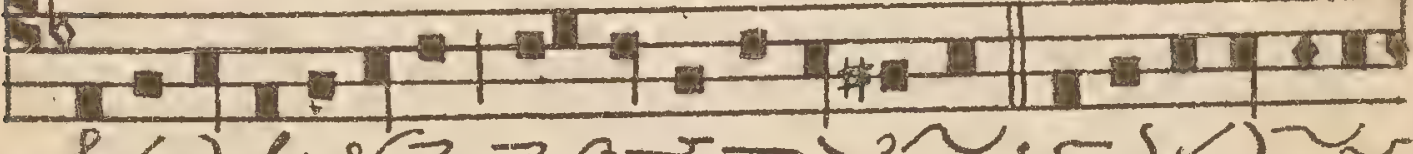

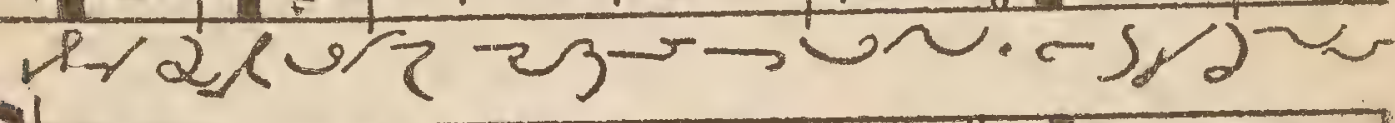

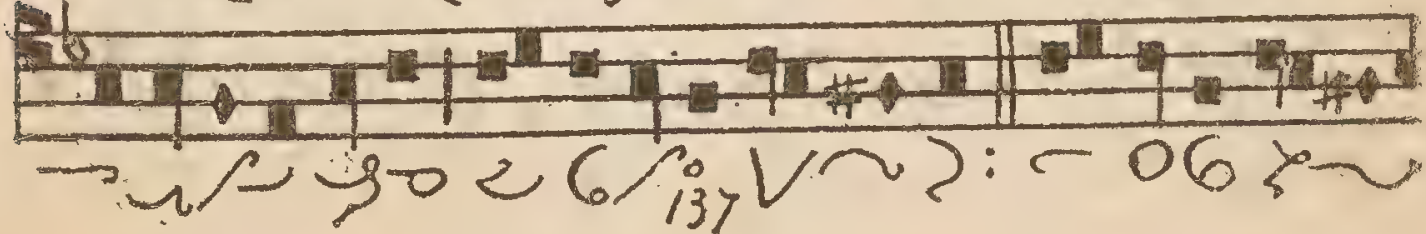




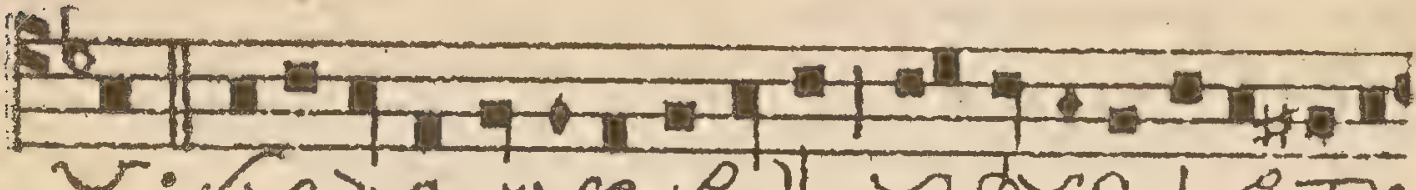

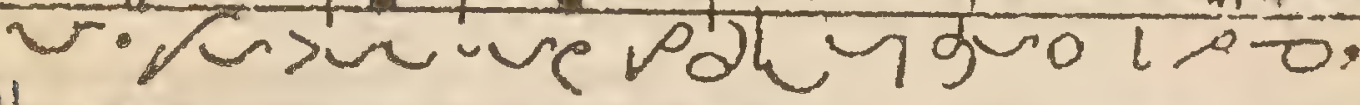
. एक

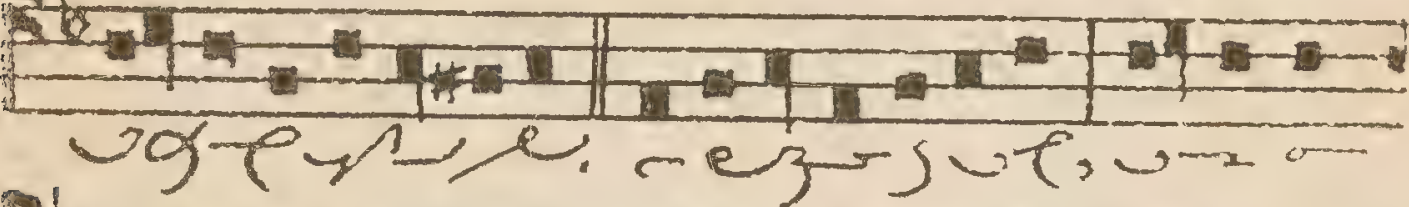

2)

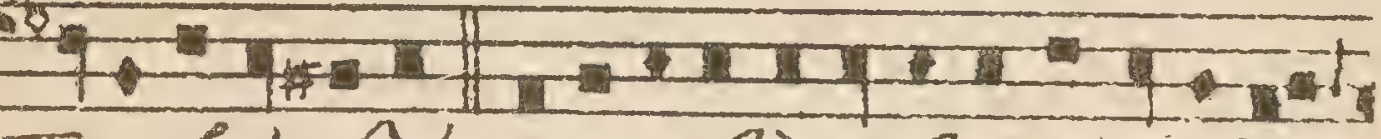

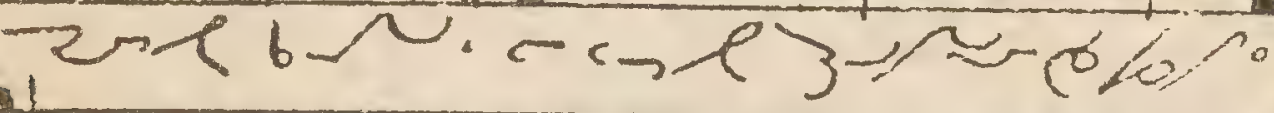

16

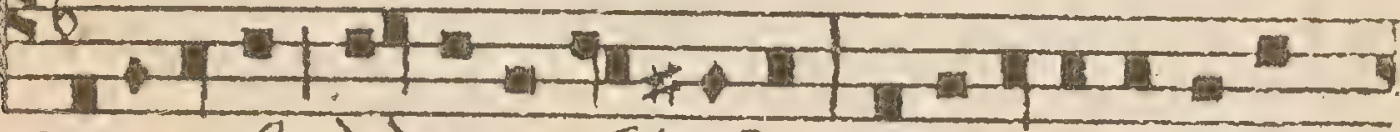

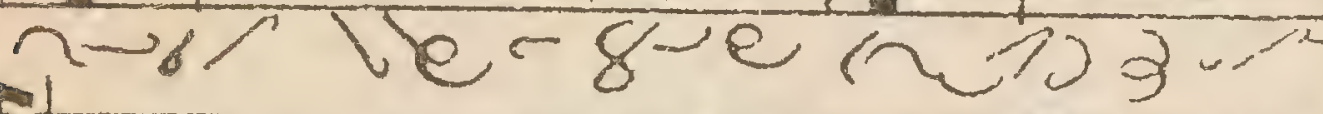

,

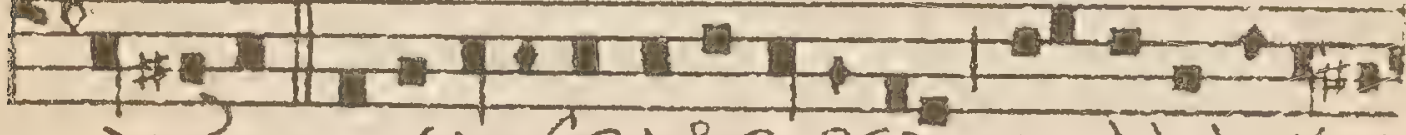

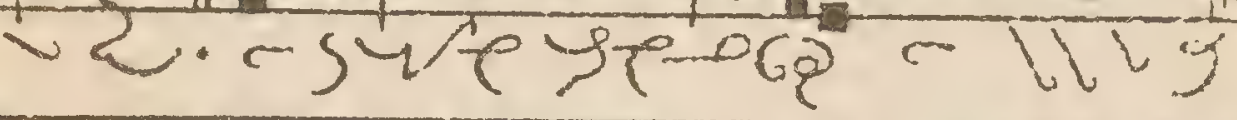

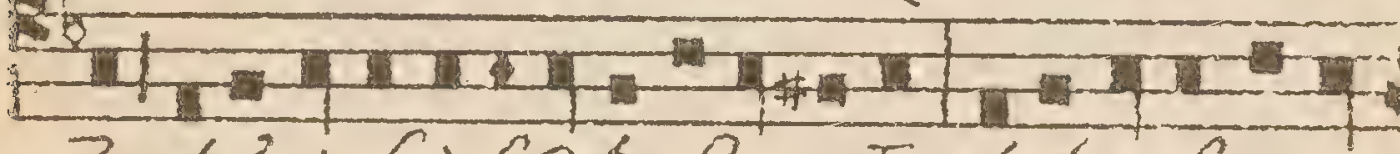

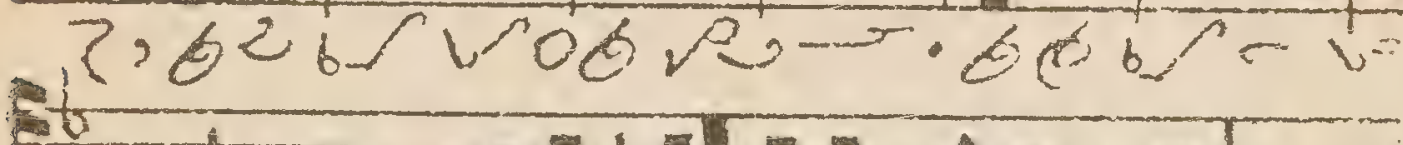

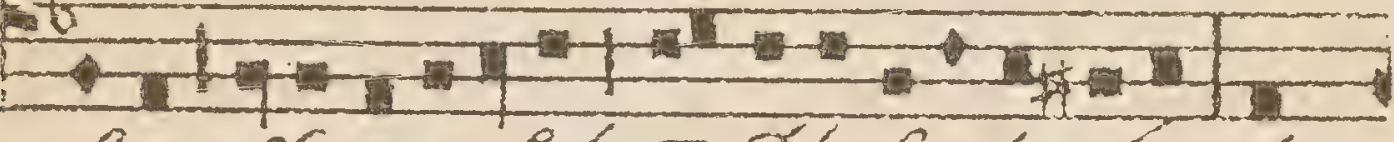
1

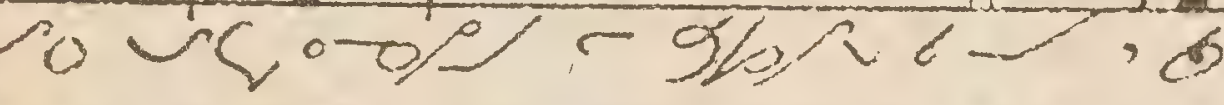

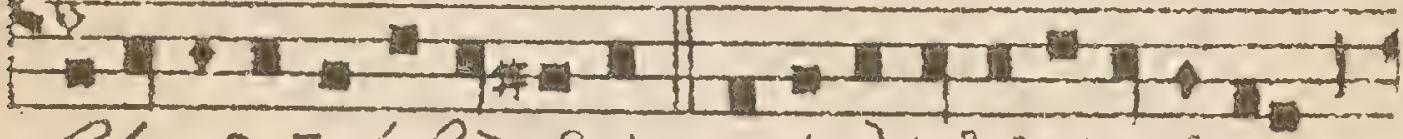

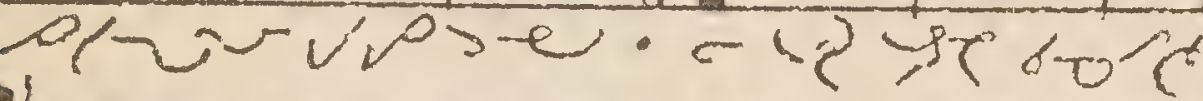

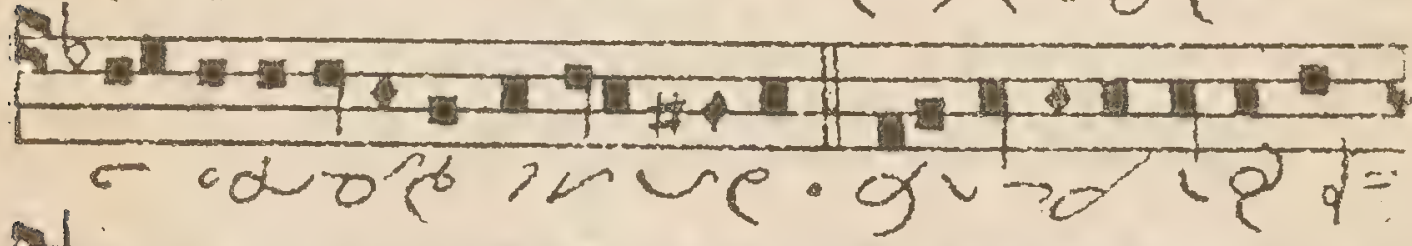
4

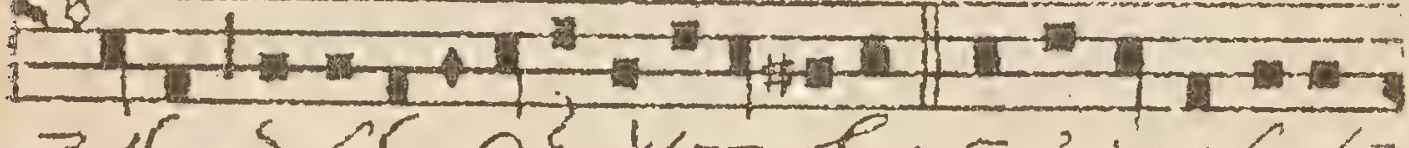
. $265 / 5,0\} 16-0+5 \cdot 240,2=$

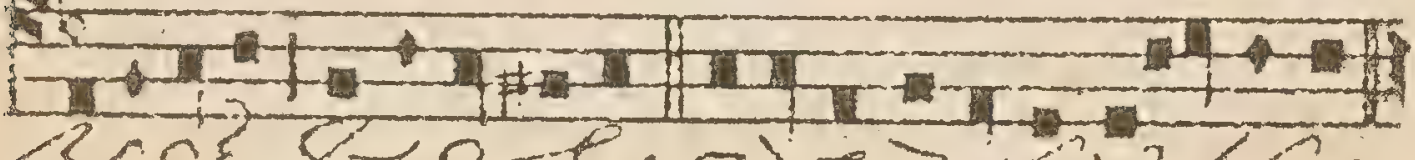

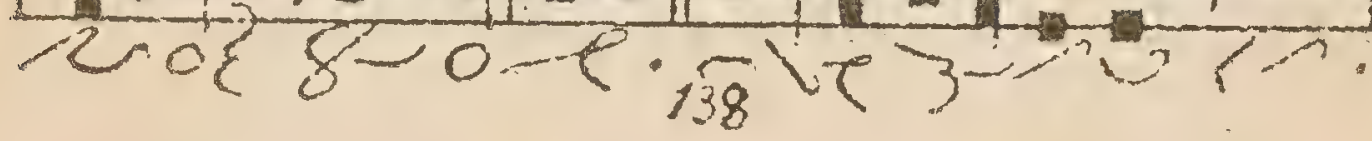




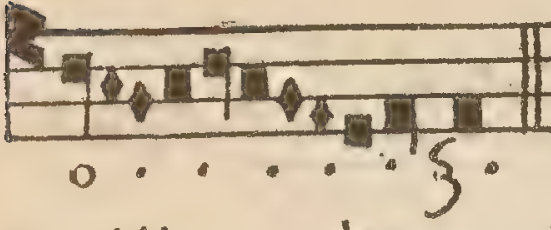

No. 15.

Sanctus.

Dominical.

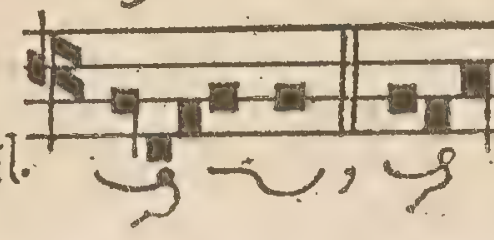

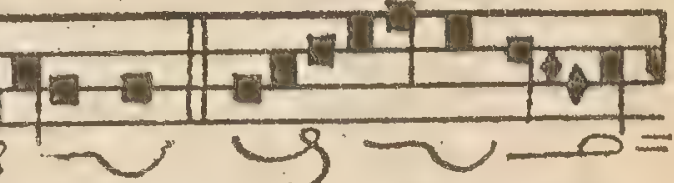

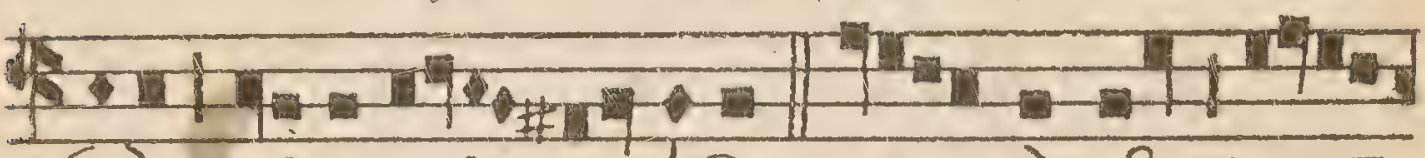

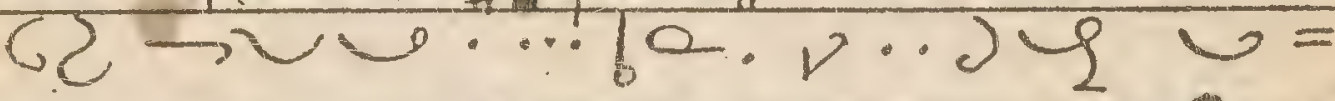

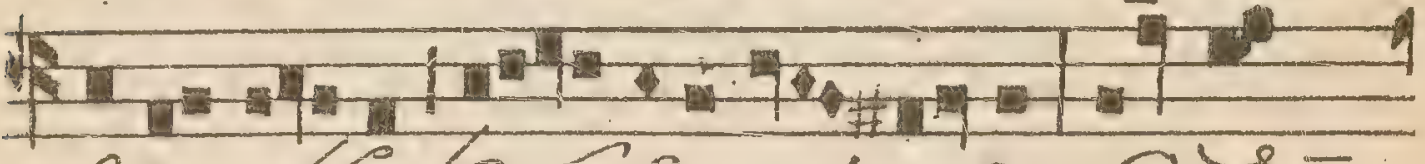

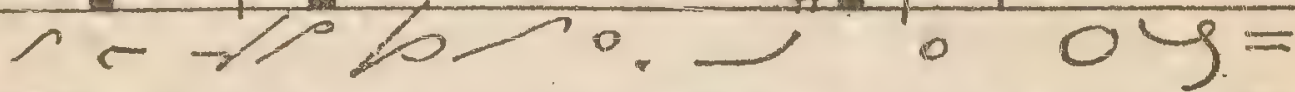

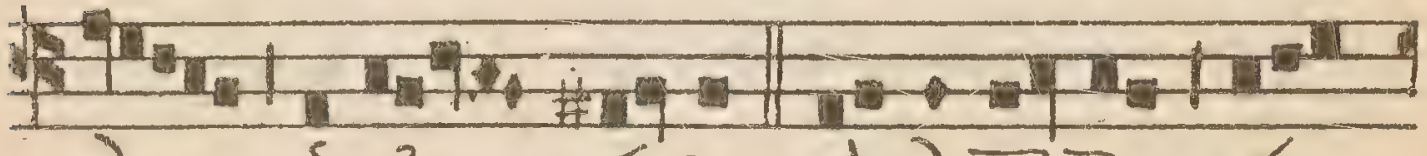

d $52 \cdots u \cdot 12>\sim 6$

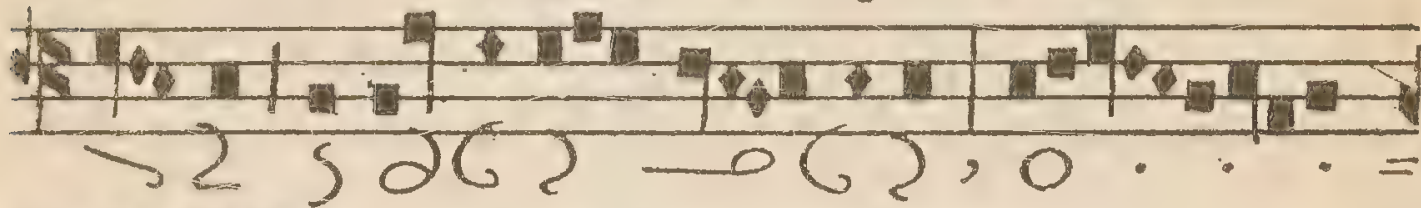

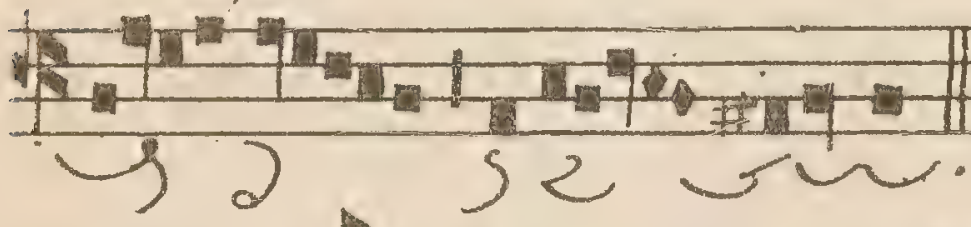

No. 16.
Agnus Dei
Dominical.

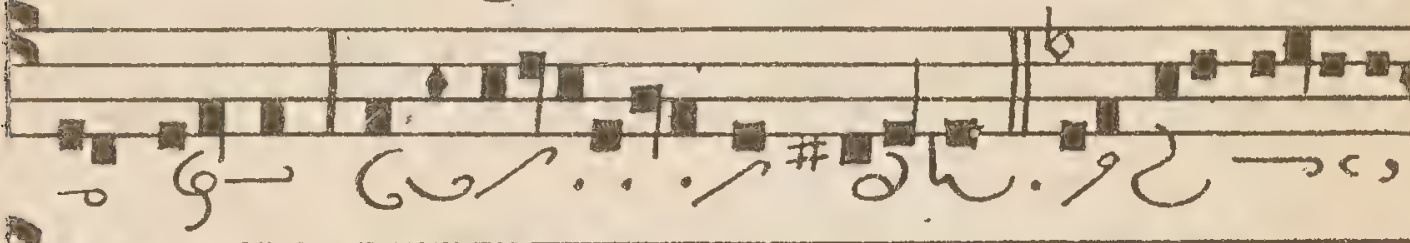
(17)

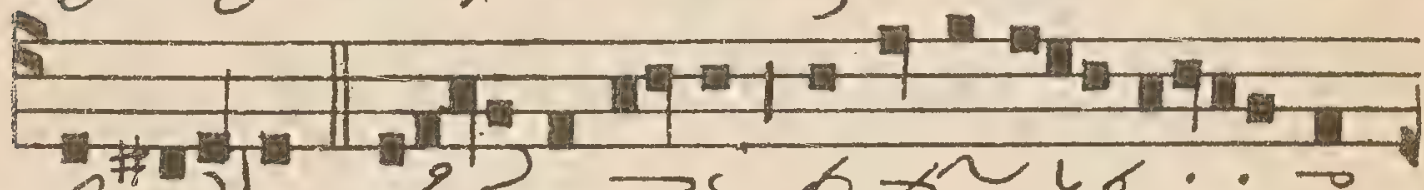

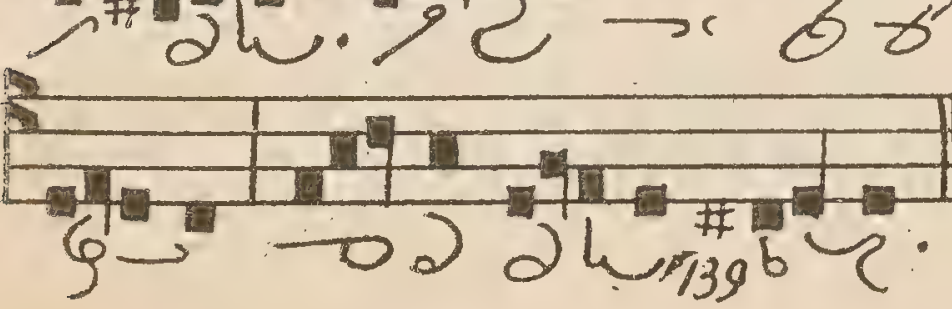


No. 1\%. Missa 6

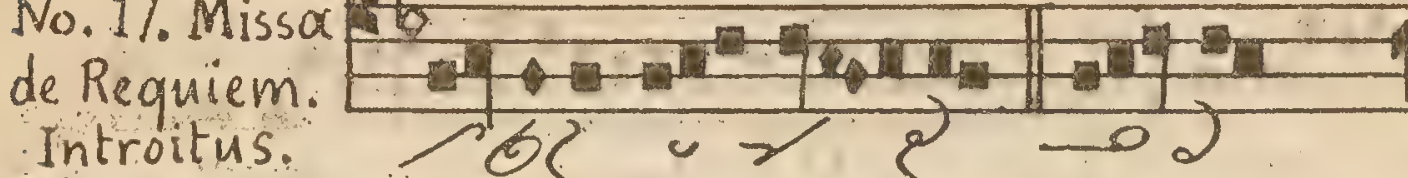

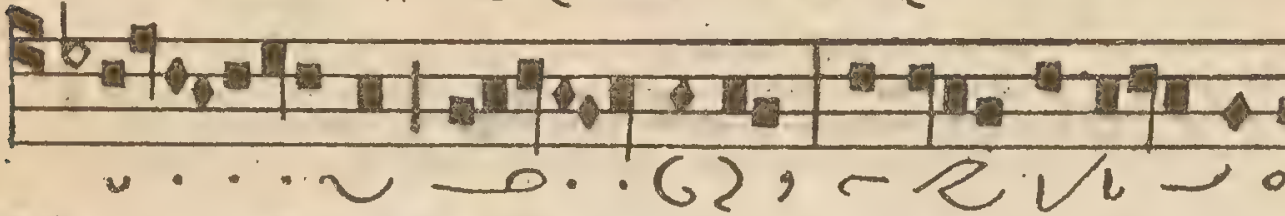

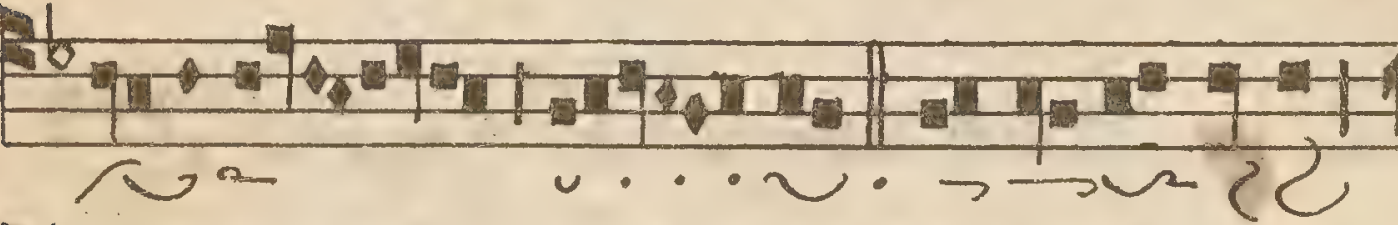

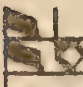

7- 1 - in $\rightarrow$ Jig $\rightarrow$ r despor:

sto

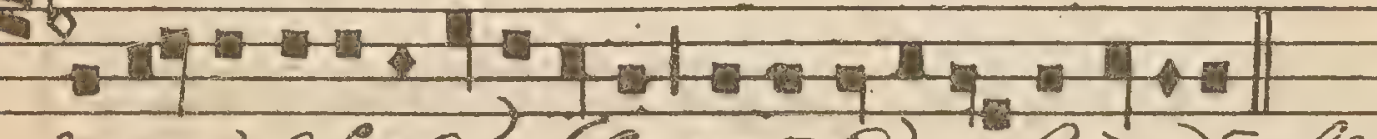

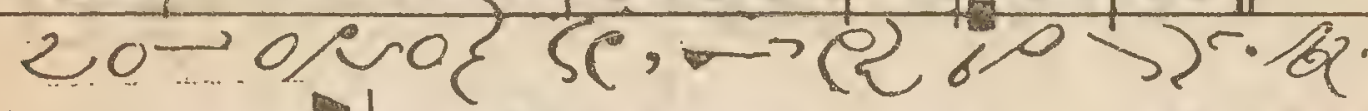
No. 18. Kyrie
de Requiem Kyrie
de Requiem

16

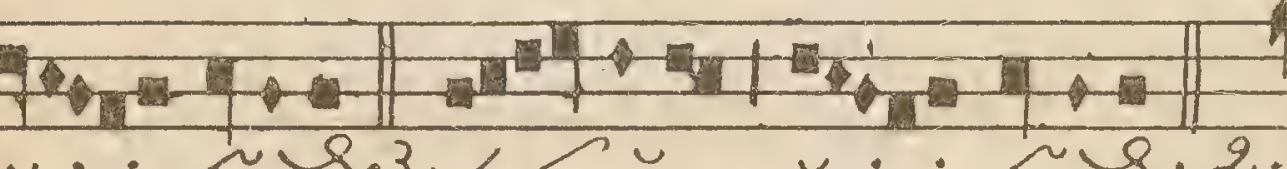

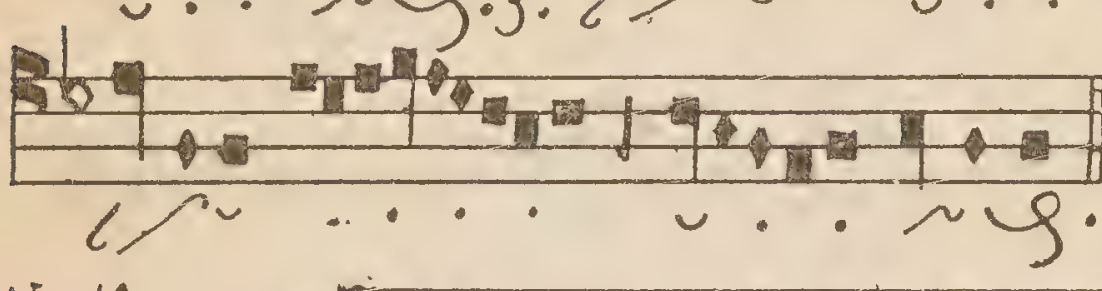

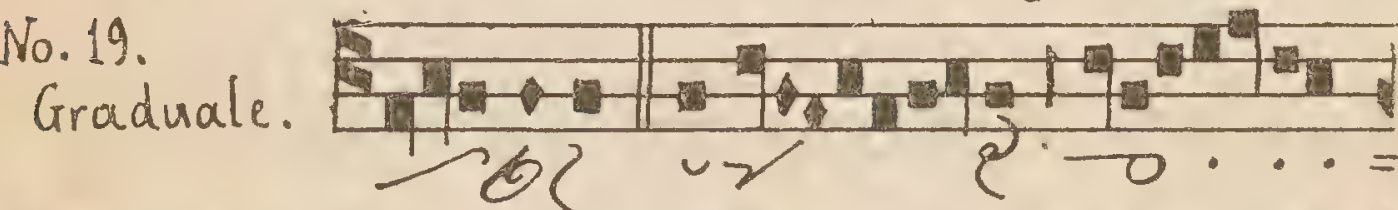

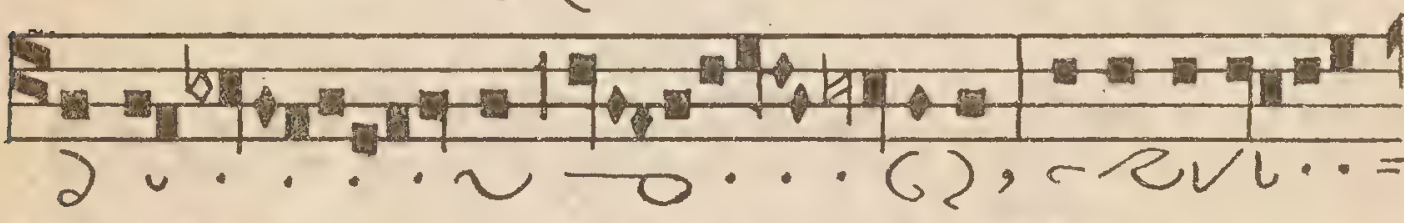

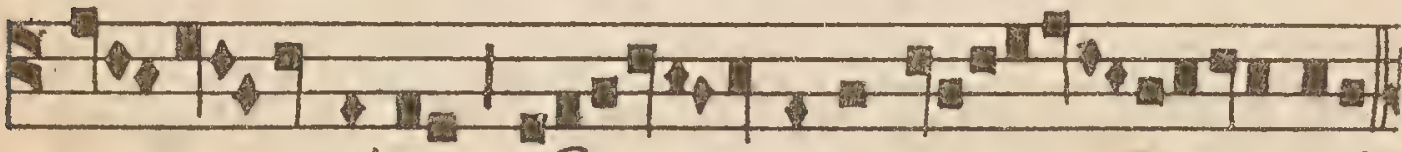

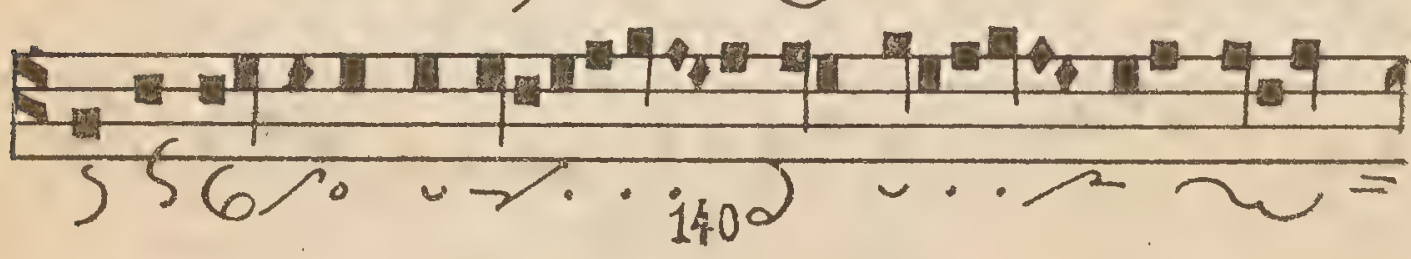




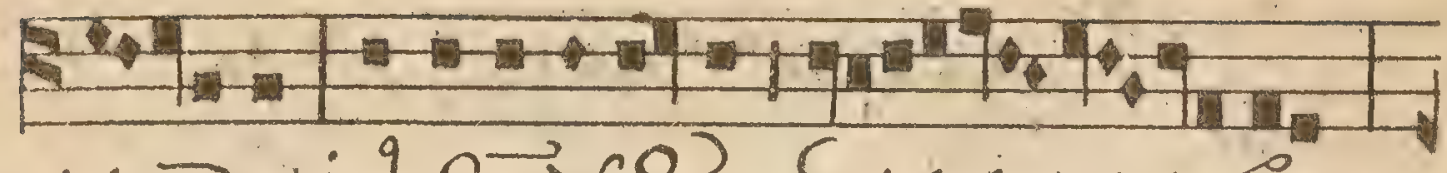
ㄱ ح:90 $2026 \ldots . . .0$

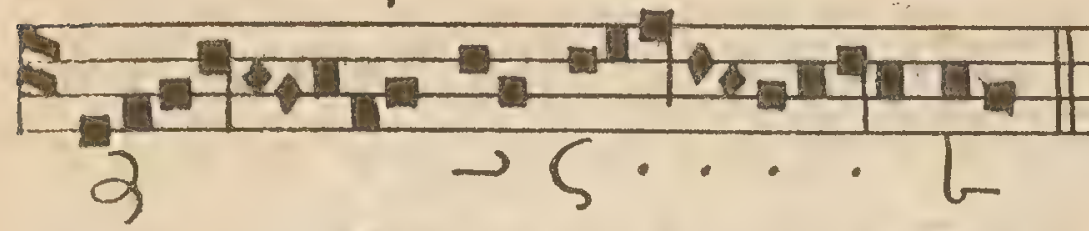

No. 20. Absolve.

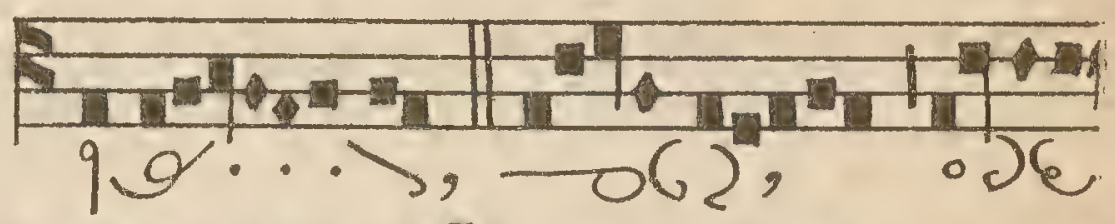

5.6. cre $\rightarrow 50 \rightarrow y_{0}$

3.7 ing

qej $\rightarrow \cdot(\rho \rightarrow 10 \cdots \cdot \cdot$

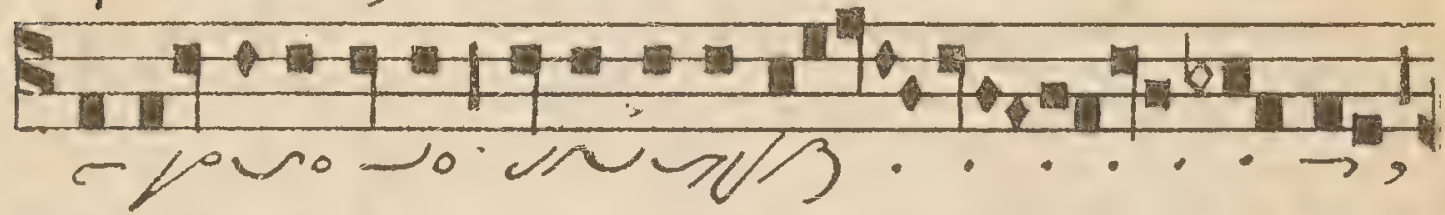

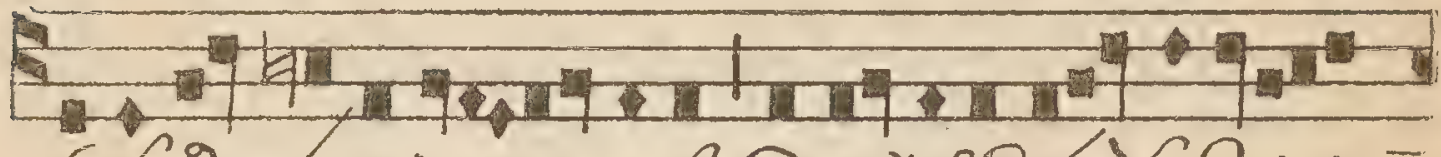

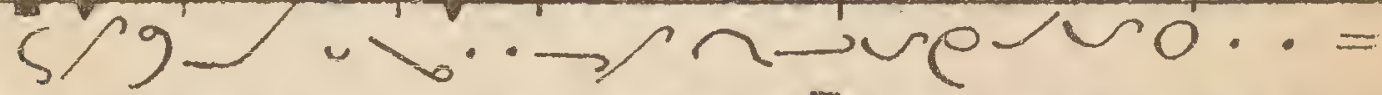

8-40,

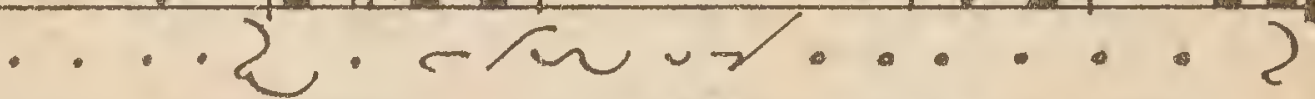

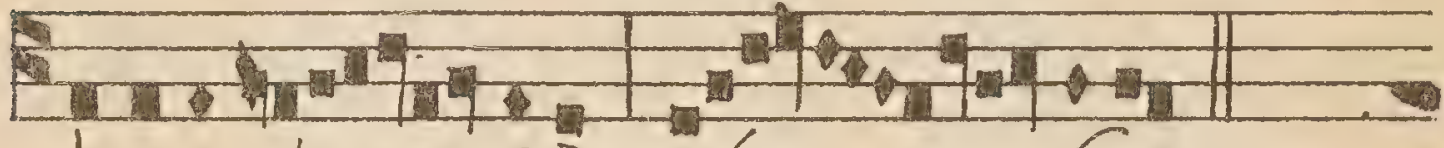

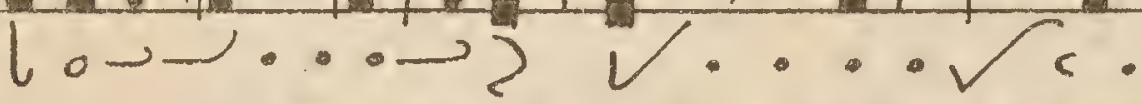

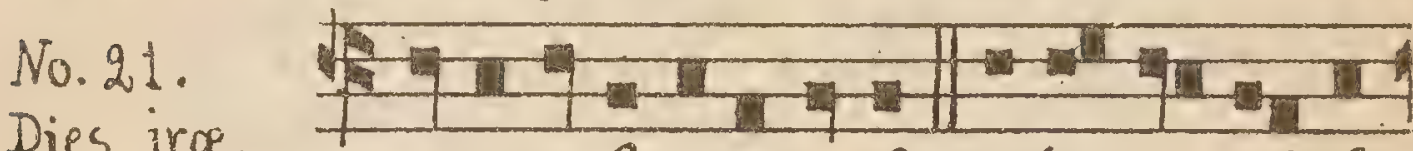

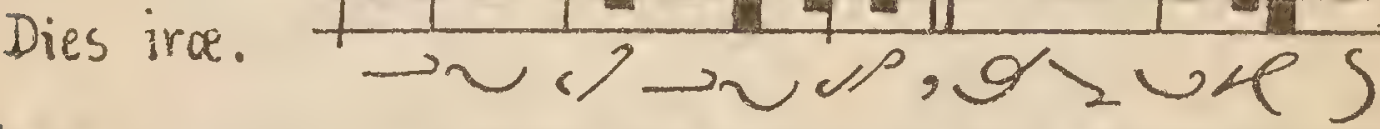

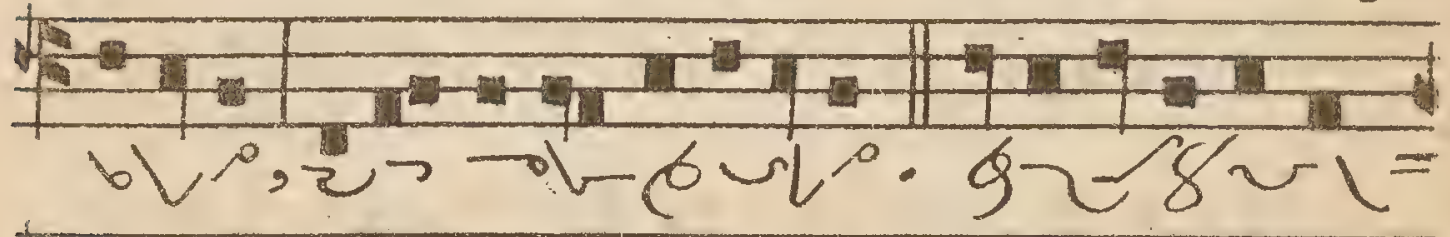

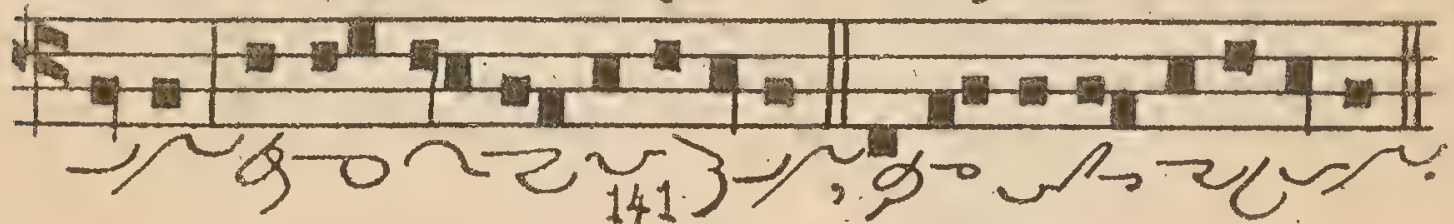




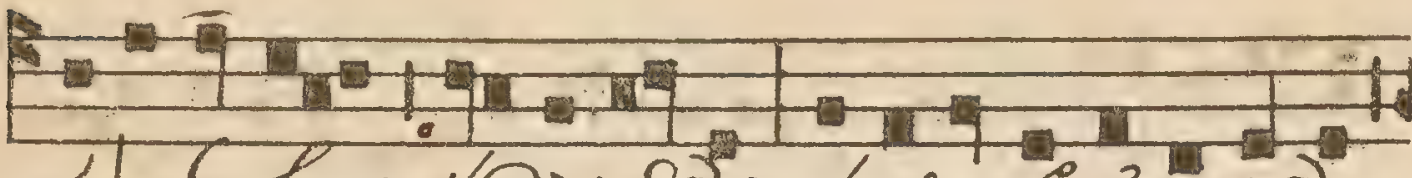

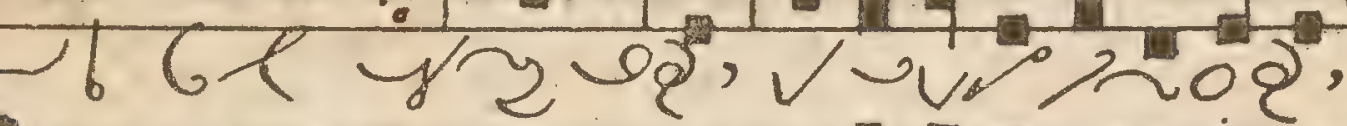

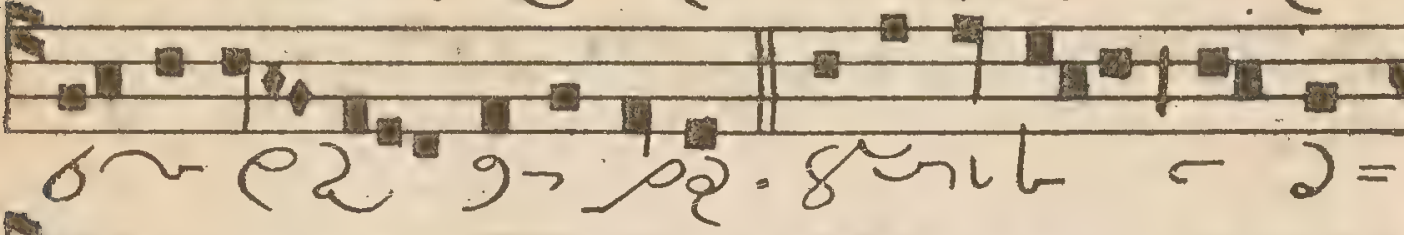

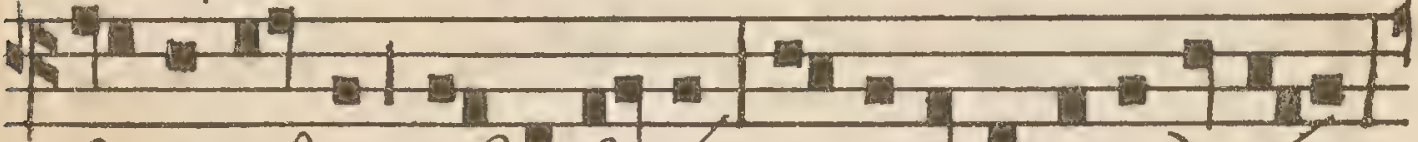

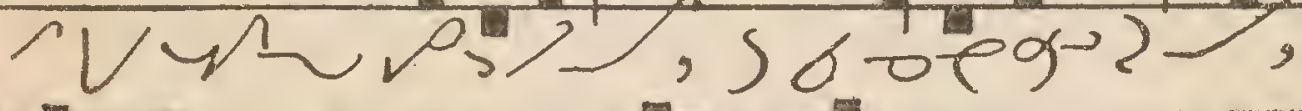

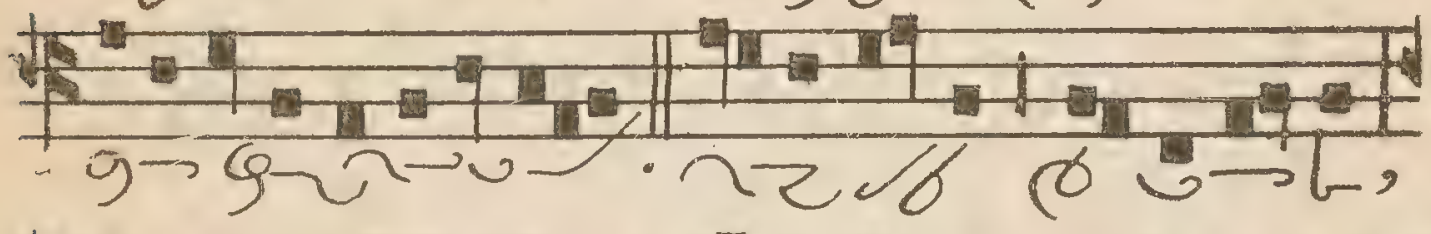

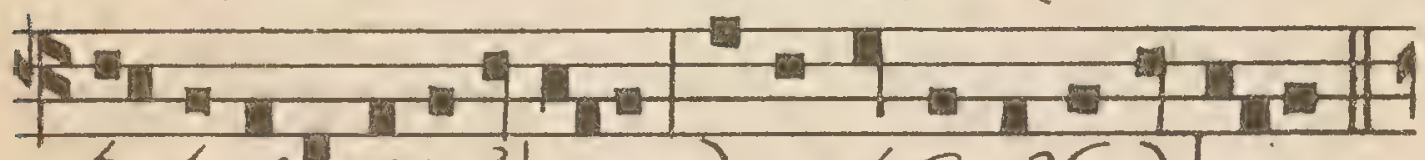
boplab a s le >62l.

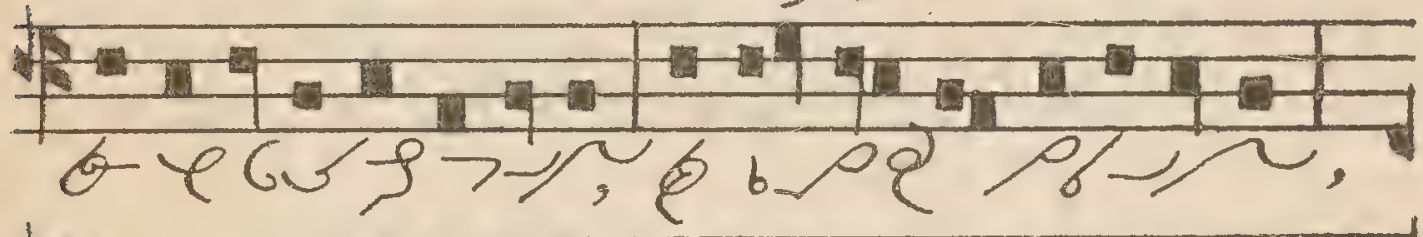
$\frac{1}{b 2}$ 5. "

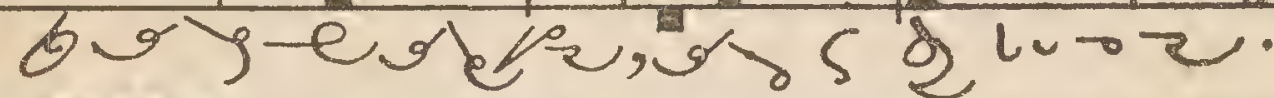

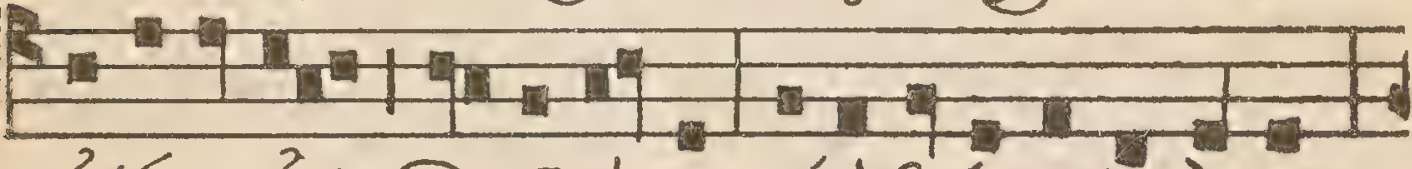

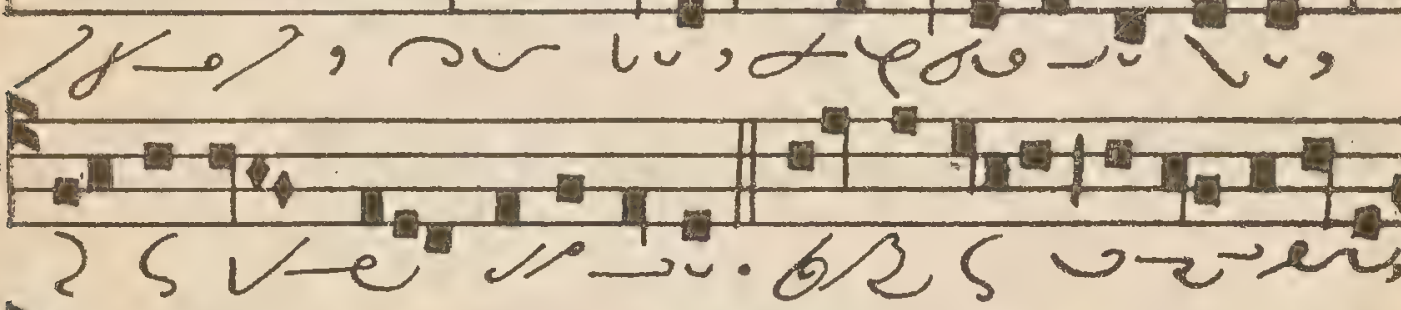
s - 1 - 1 -

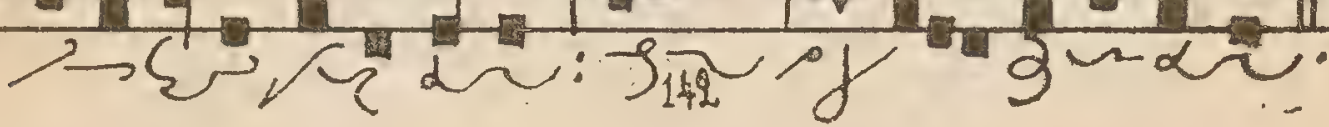




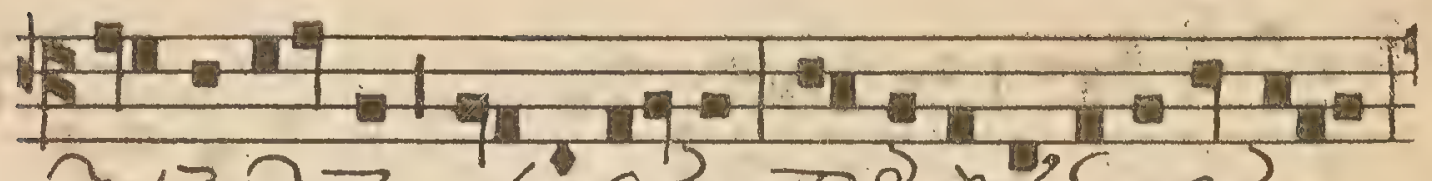

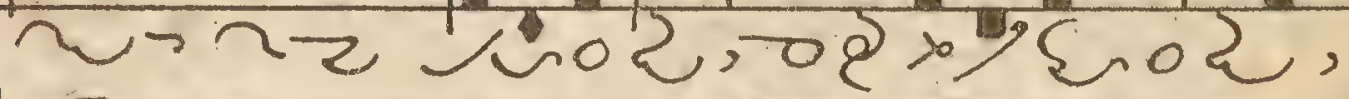

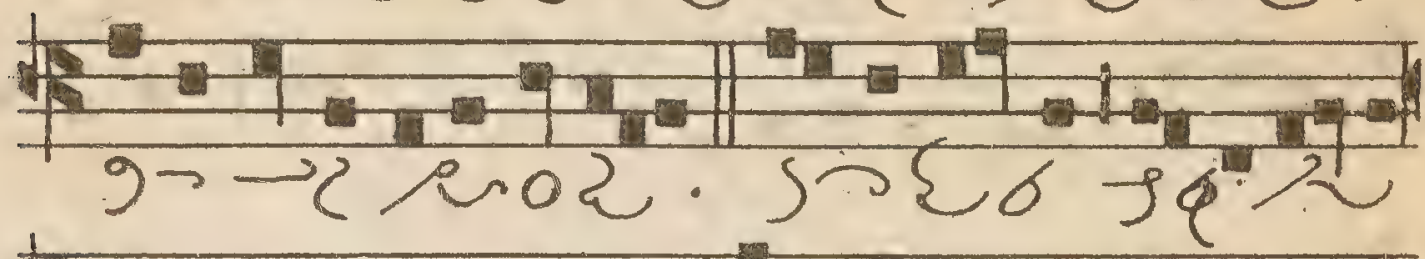

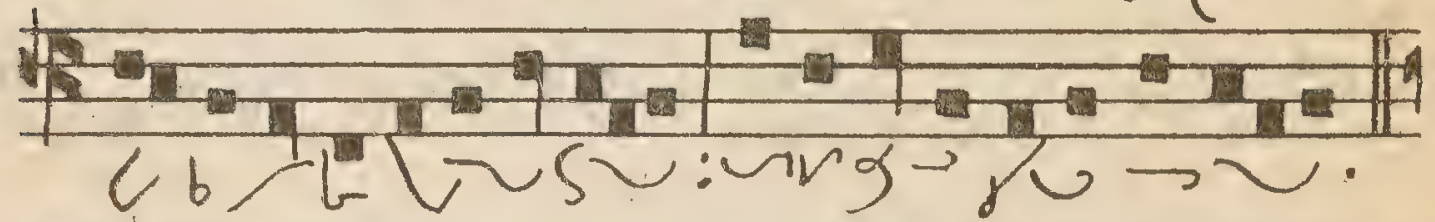

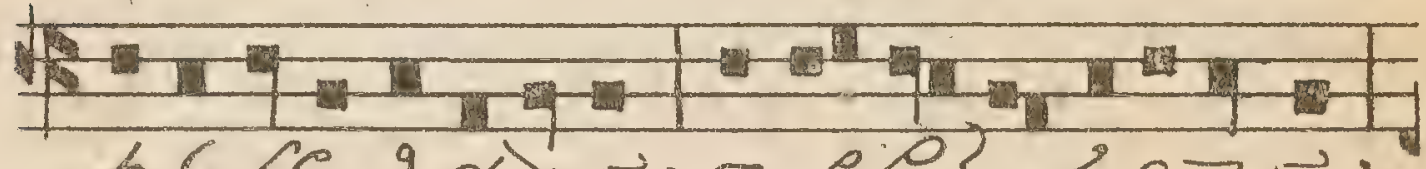

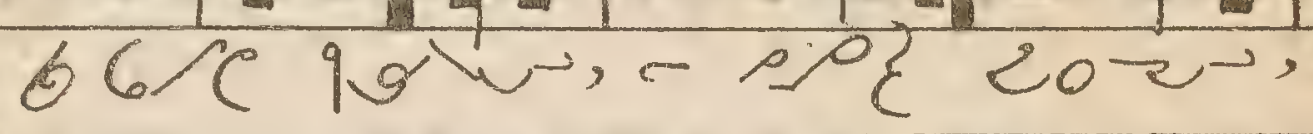

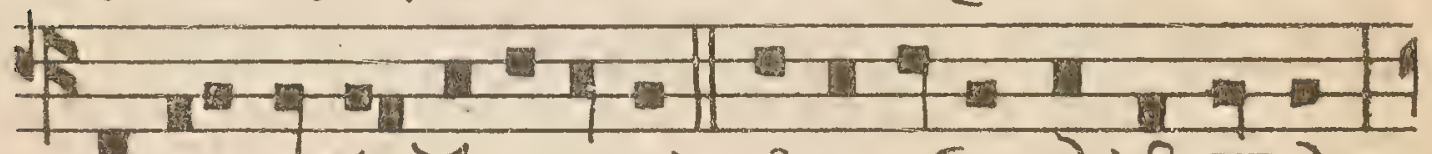

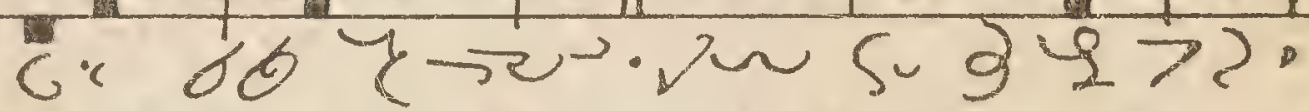

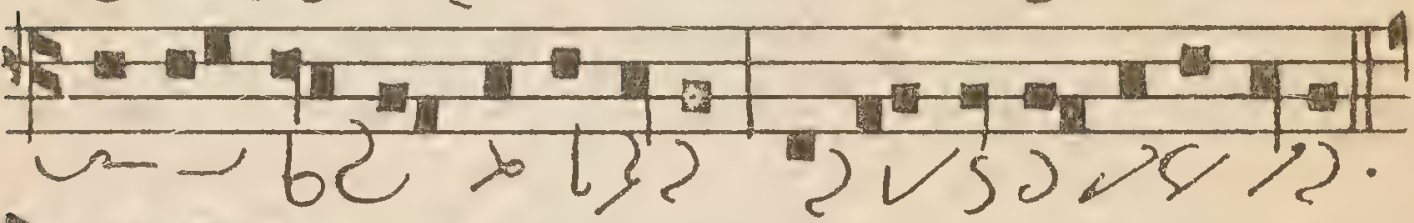

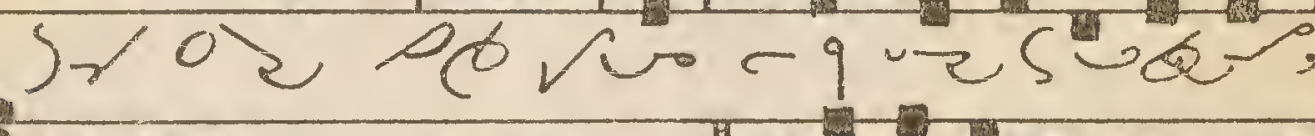
ह.ं

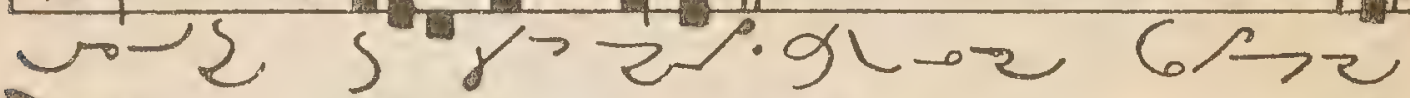
Bis

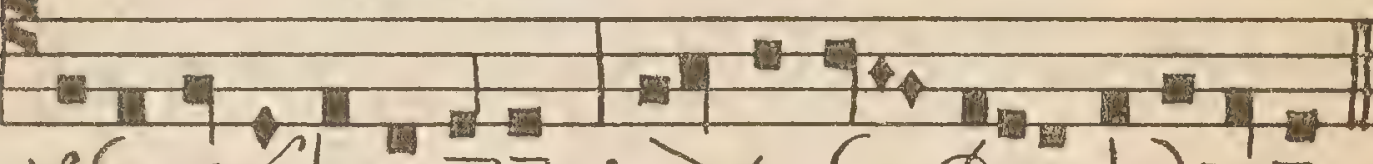
ve of 底需 1 0 1

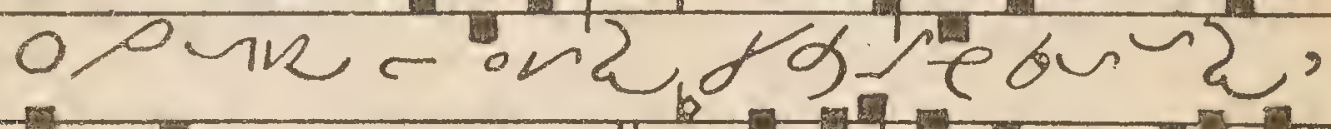

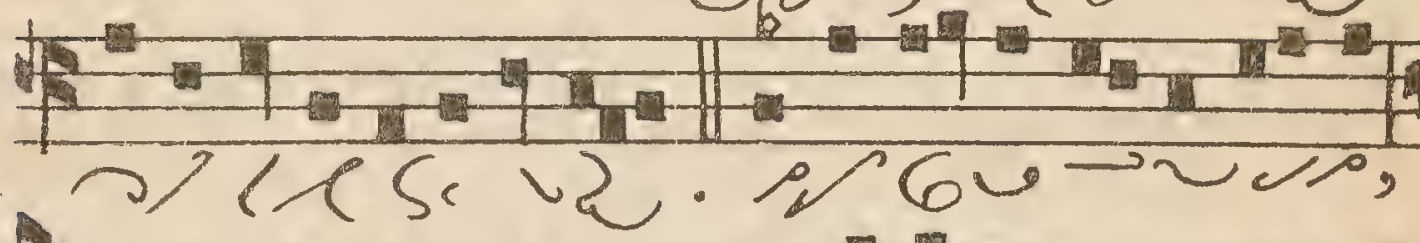

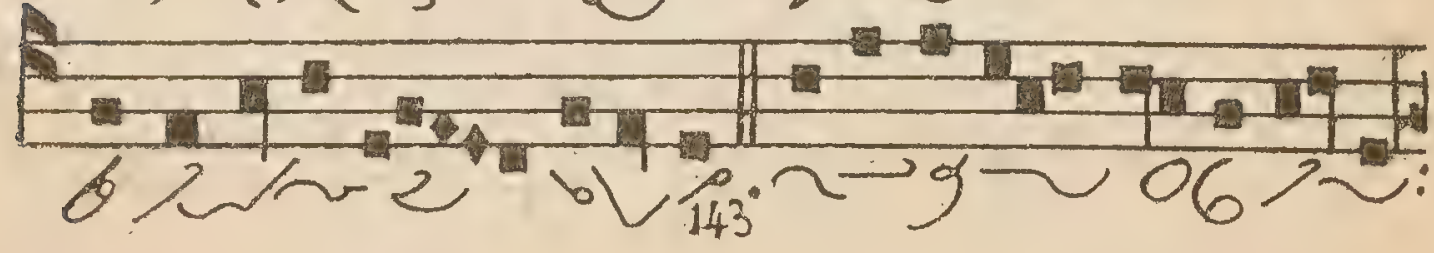




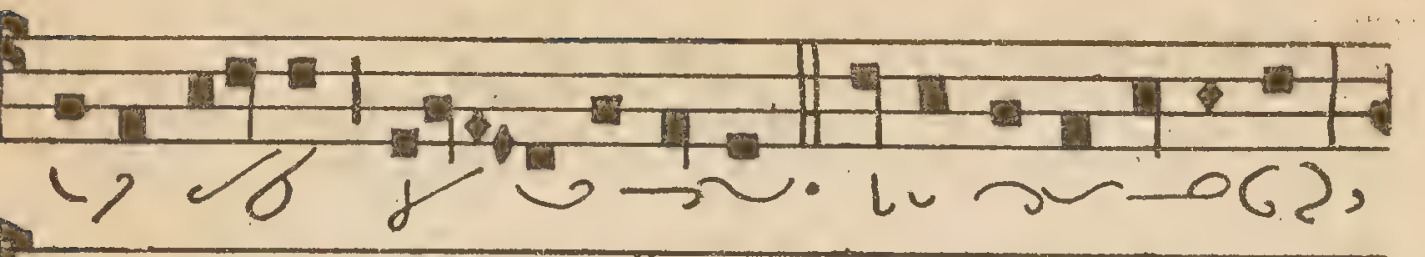
Eq $.02 \sim 200.5$.

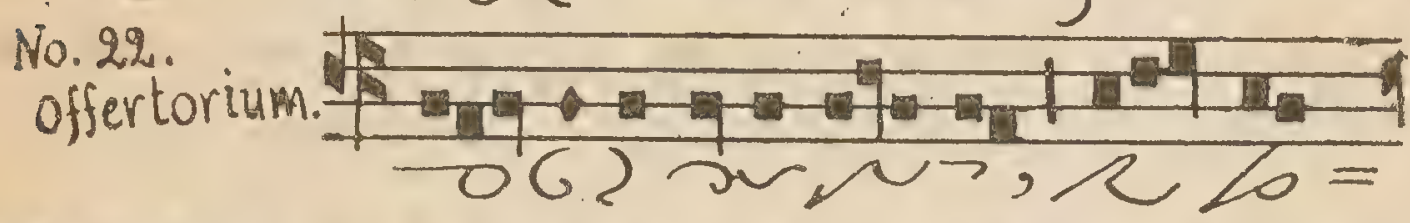

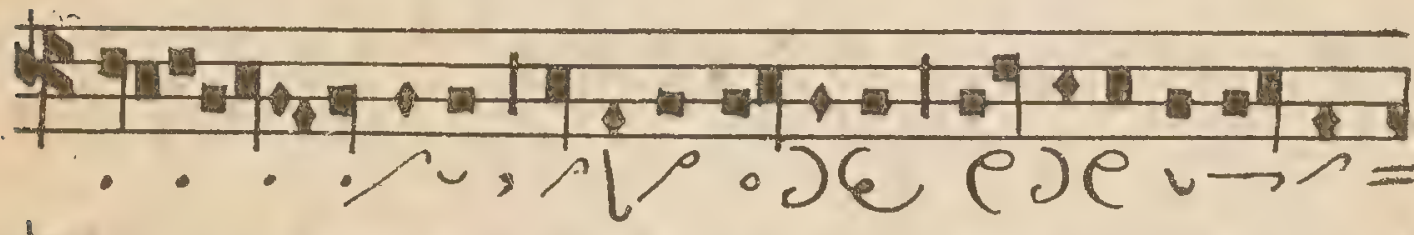

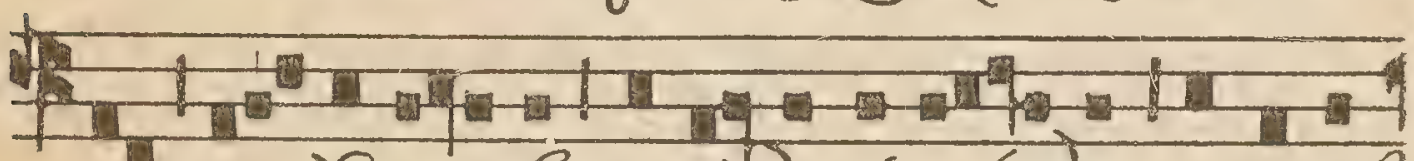

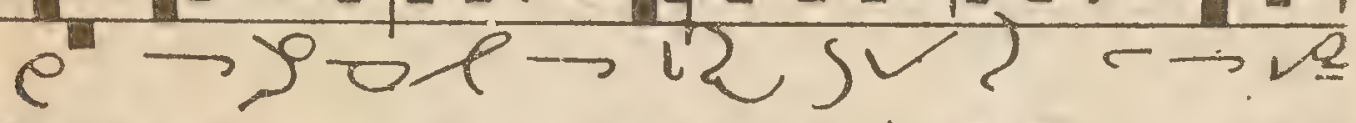

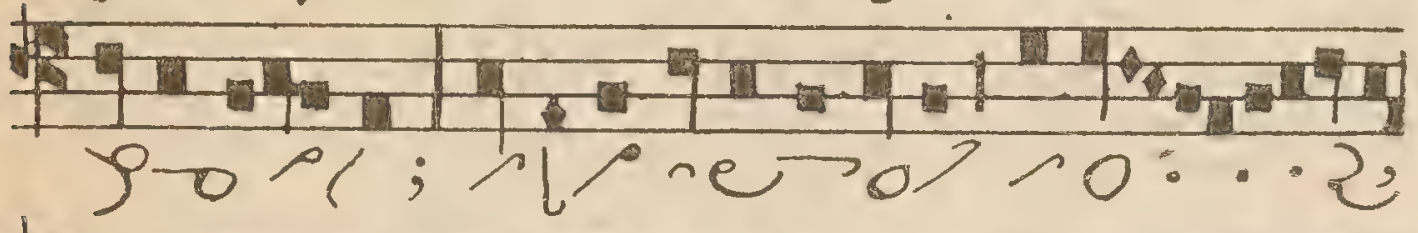

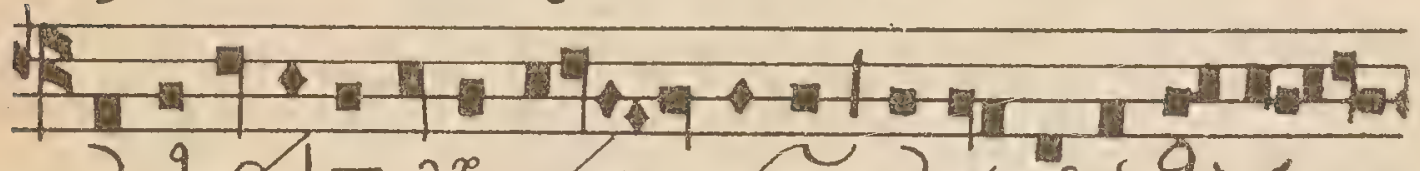

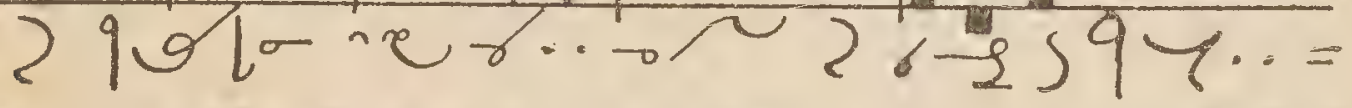

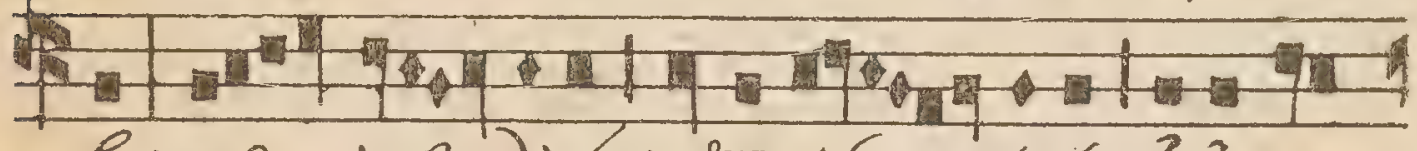

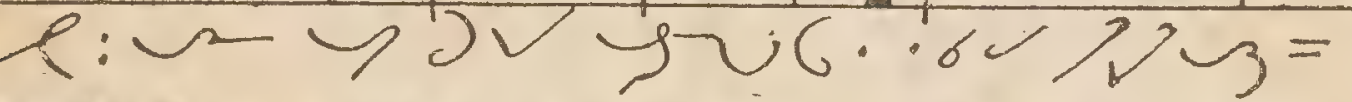

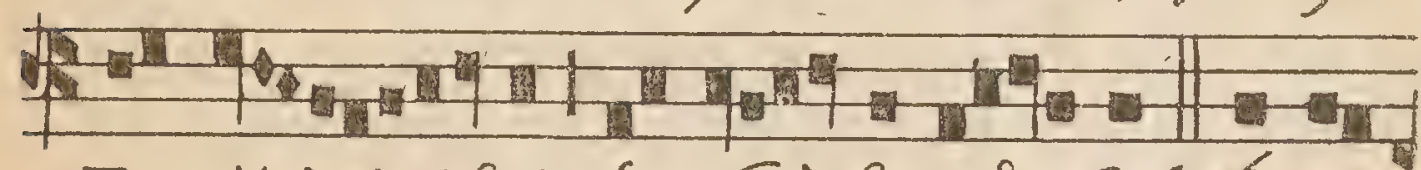
$z$ z..e s r y r: $40=$

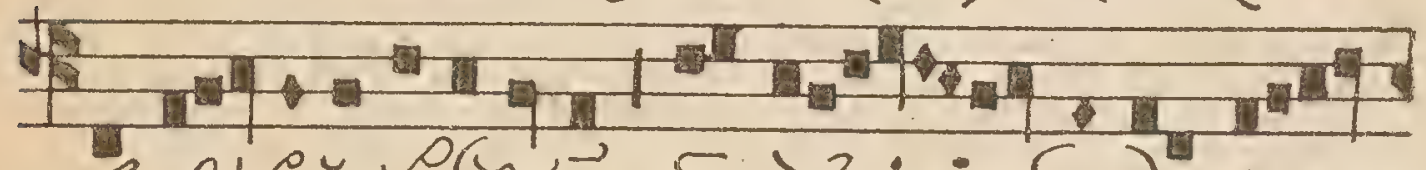

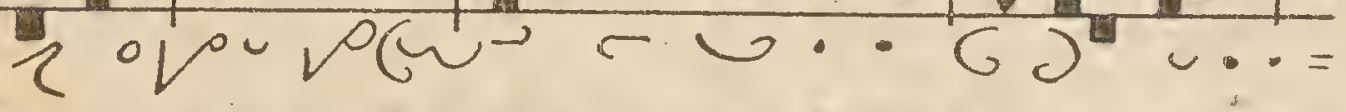

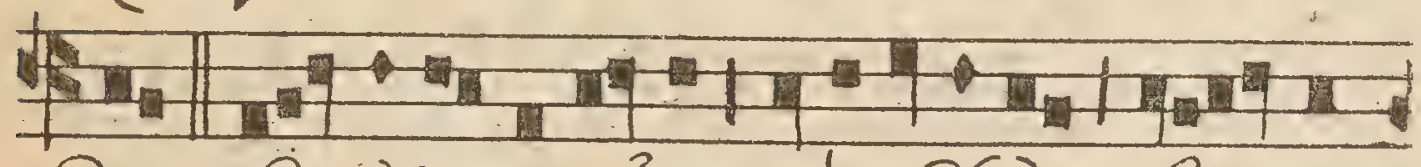
we ever $\sim \rightarrow[-O G), \mathrm{O}_{2}$

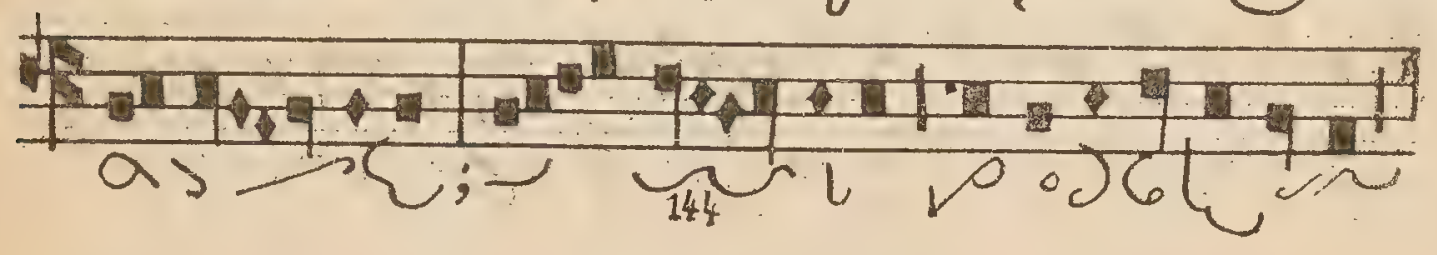




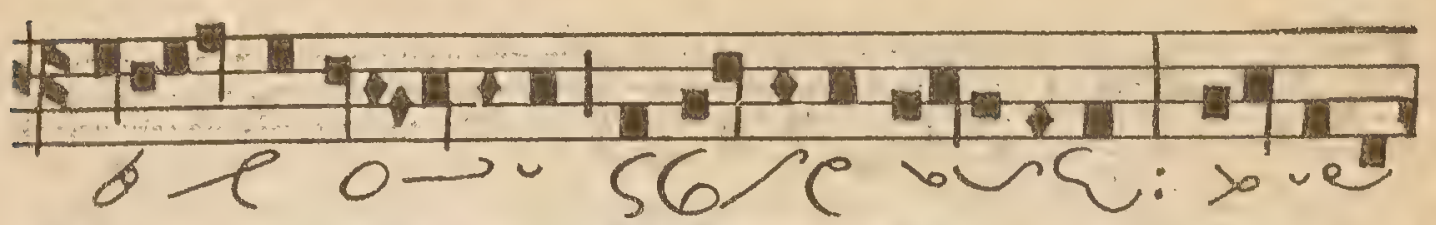

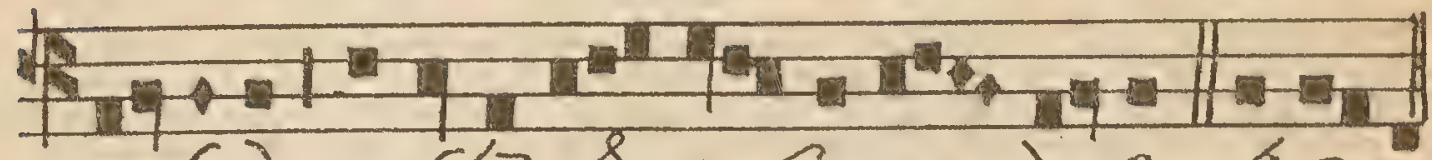

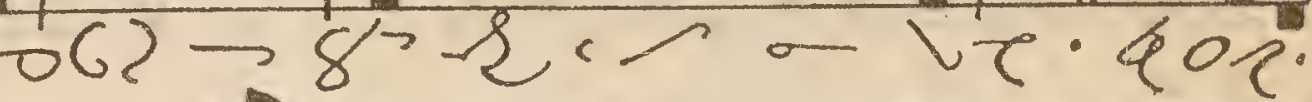
No. 23.

Sanctus.

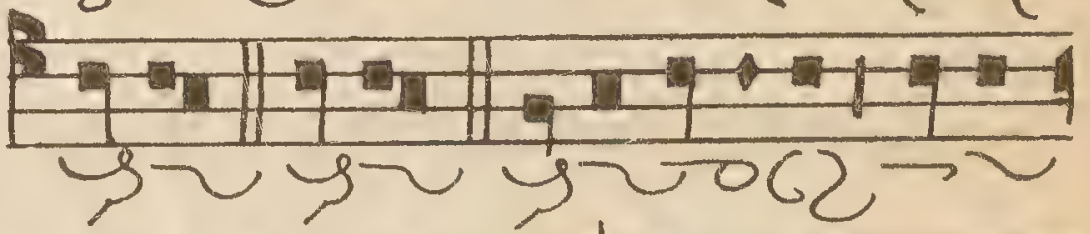

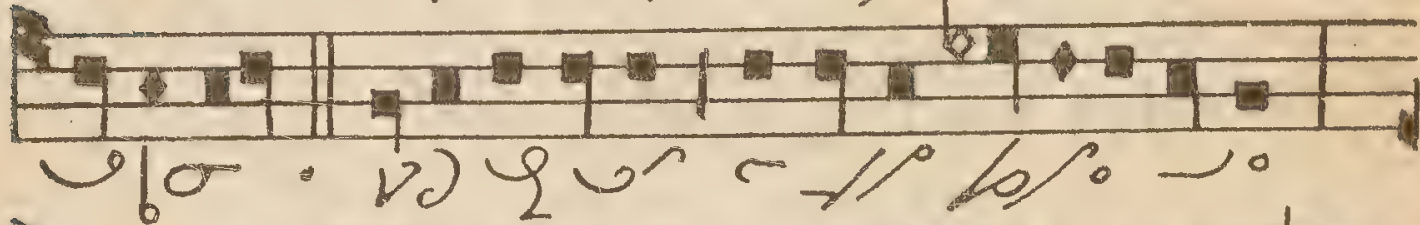

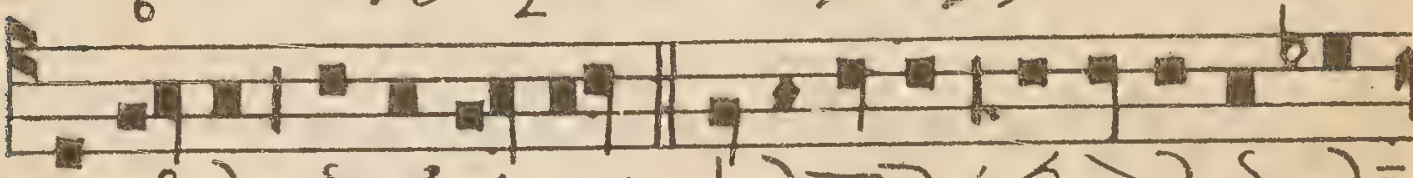

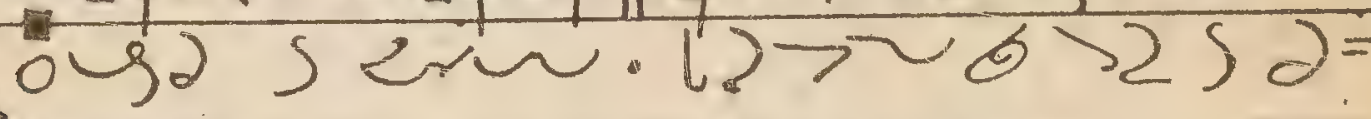
ह. No. 24 . Pie Jesu Domine.

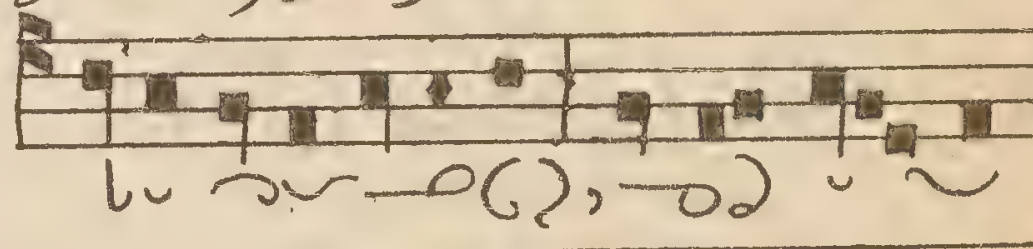

E.

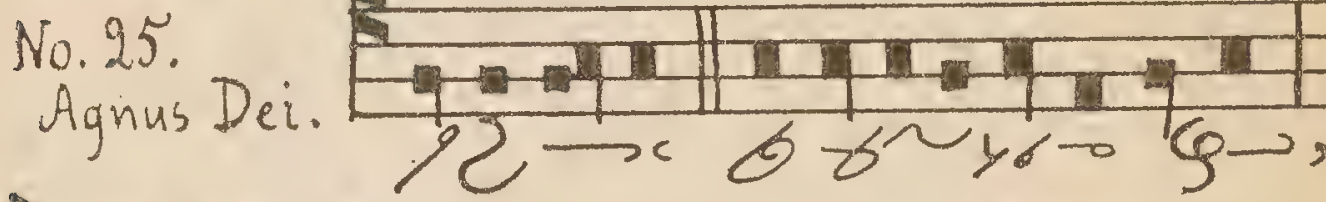

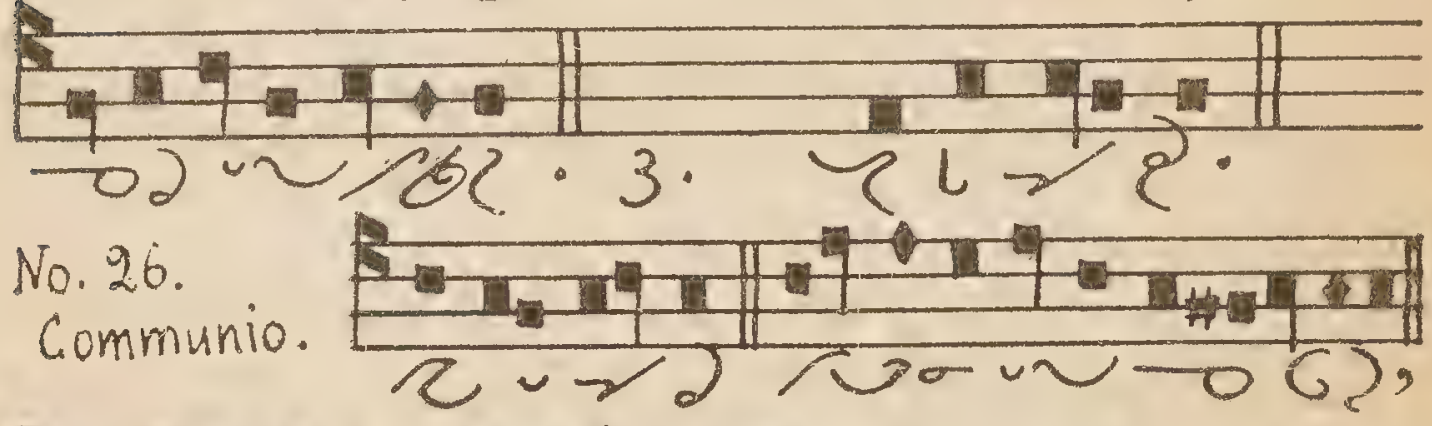

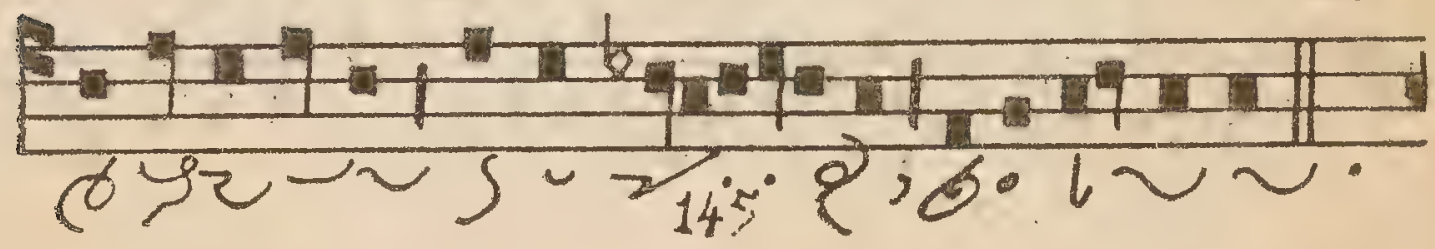




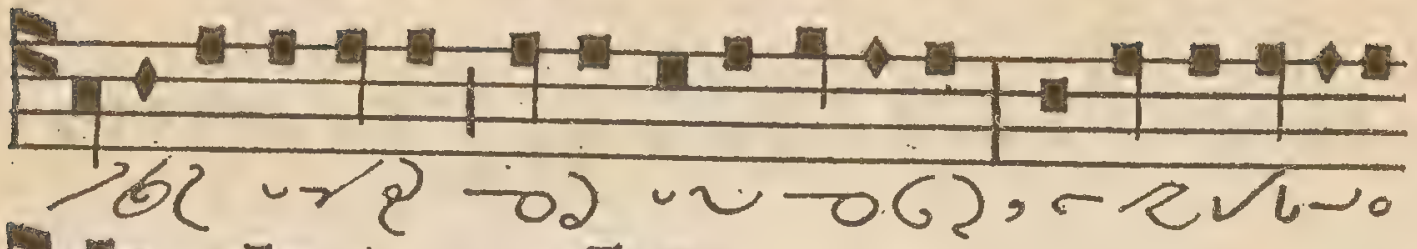

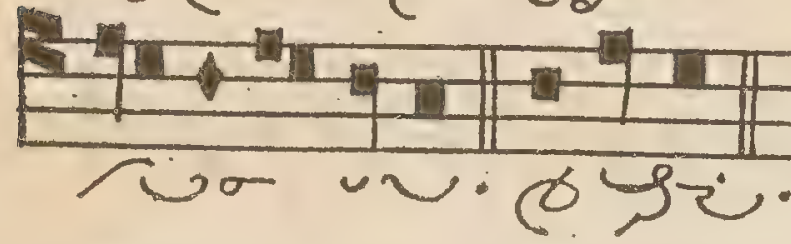

ㄴำ $2 \%$

Exuittabunt

Miserere

At a funeral.

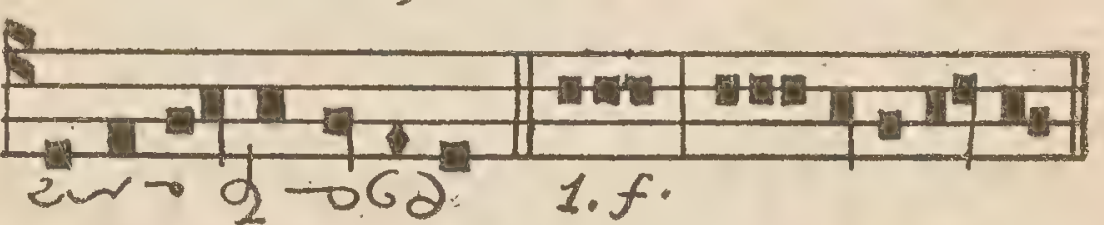

Gulpsciw, uge 4. Gugke

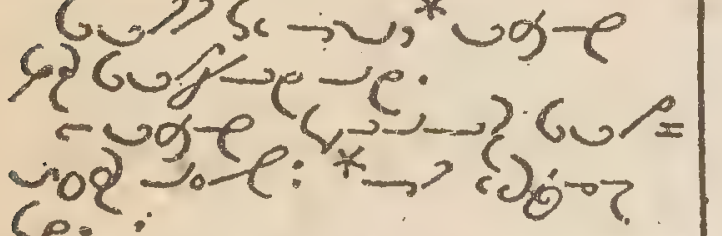
se.

cm R 59 J J o o s So:* - 15050 G 5 .

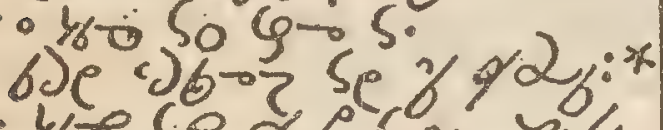
- be se gas sor.

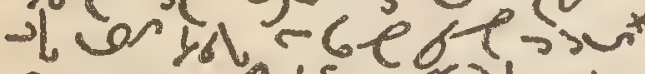

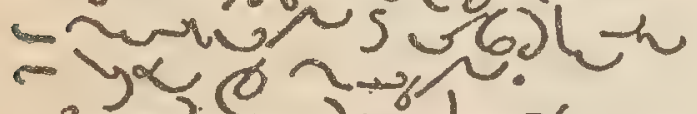

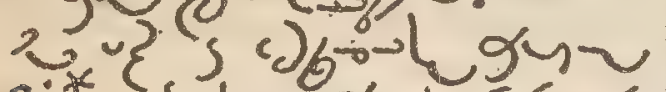

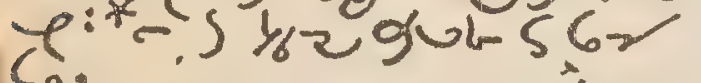
so.

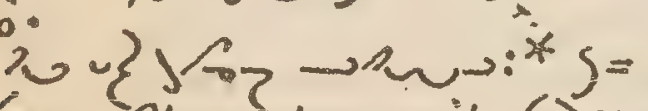

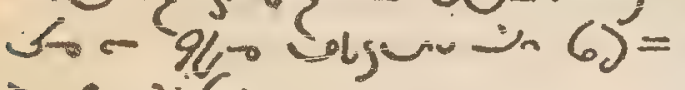

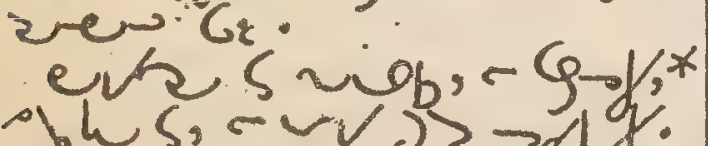

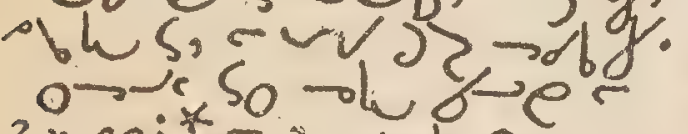

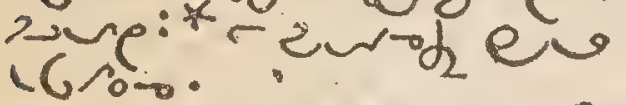

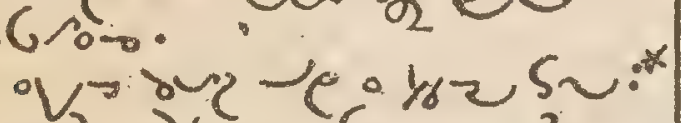

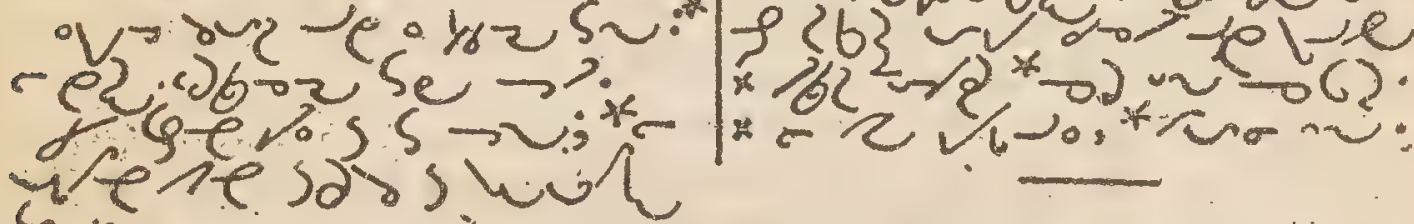
suevesassuoh

2 True soinu do: ineyeresare

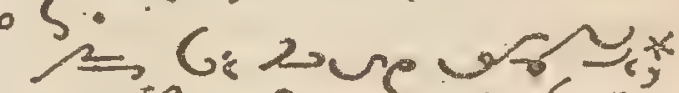
-4य $3 \backsim 69565$ Toub sol ve -e; - che o $-9 y 3$. ifs $\rightarrow$ sol $\rightarrow$, $\rightarrow$ or sn, sed

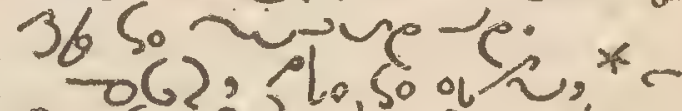
e se gुज s o ojevir w vive

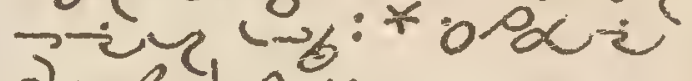
$9 \rightarrow$ cl $4<\alpha=$

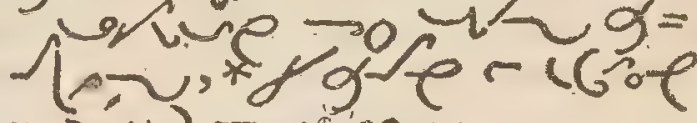

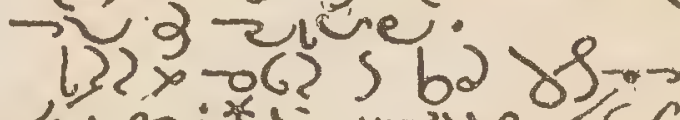

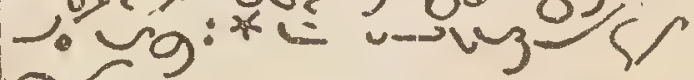
गुरे

39 iol y ure w= $8263 \sim 2 \%$ d - 2 र

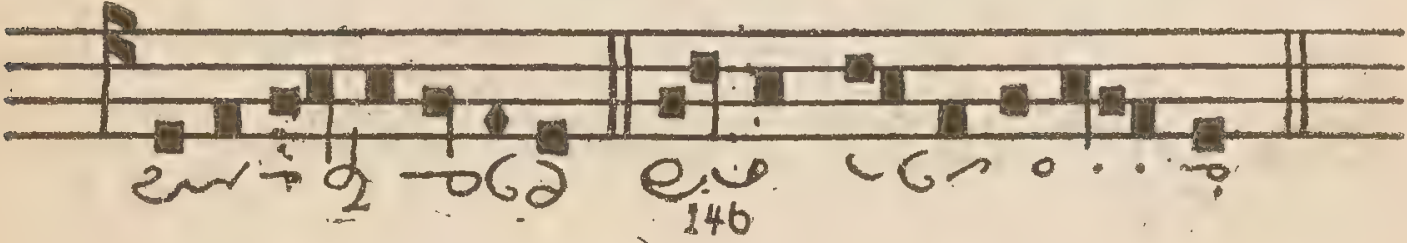




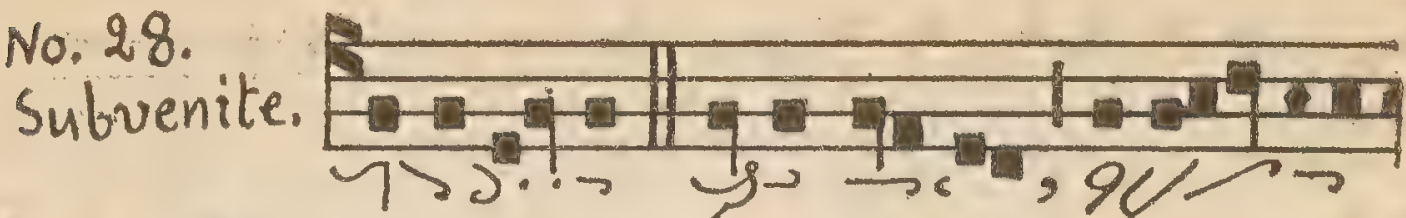

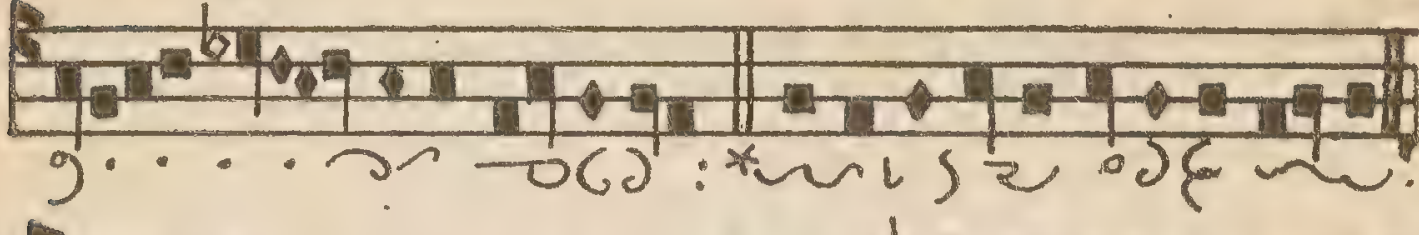

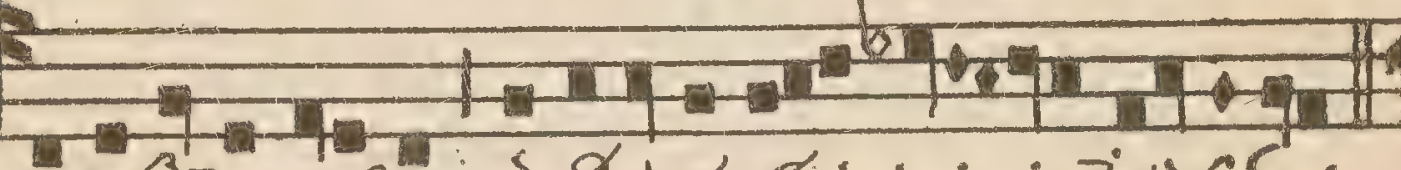

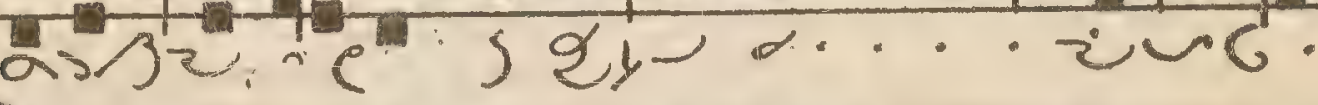

a.

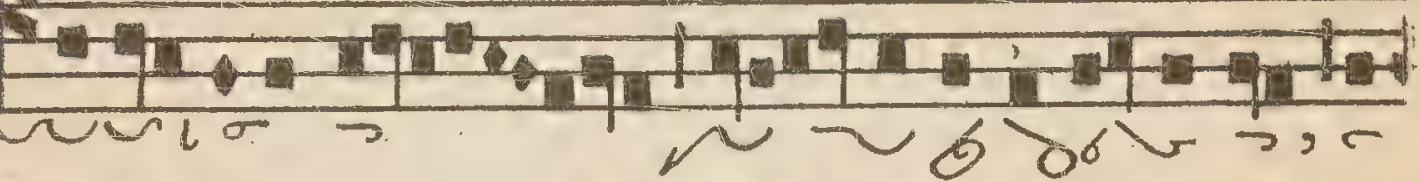

s

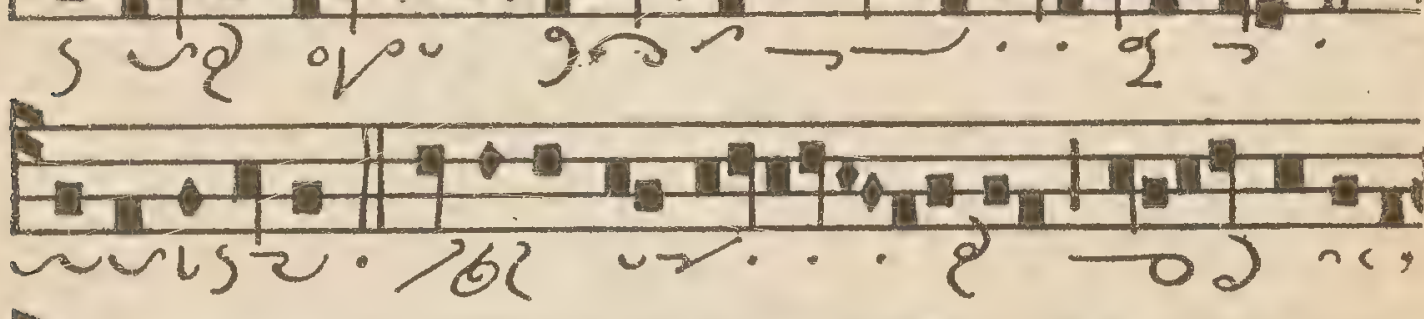

ㄴ.

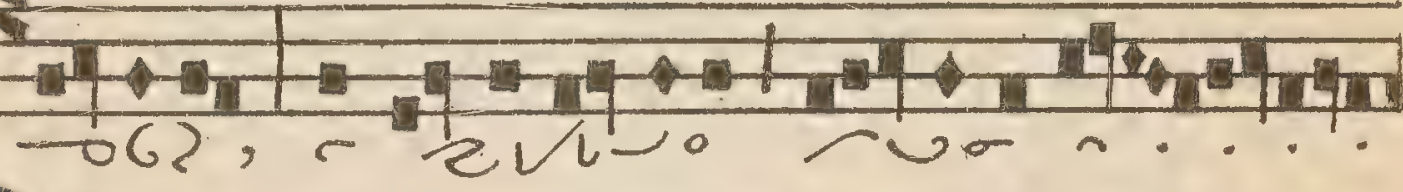

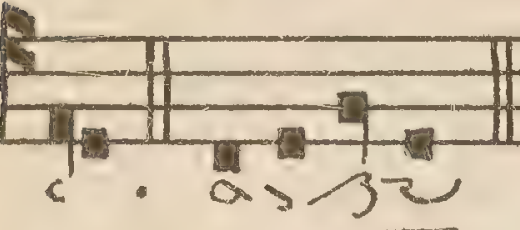

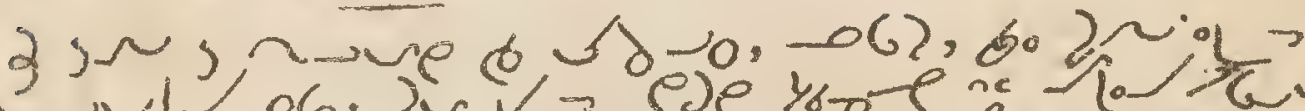
nud ob. 2u $=$ ese

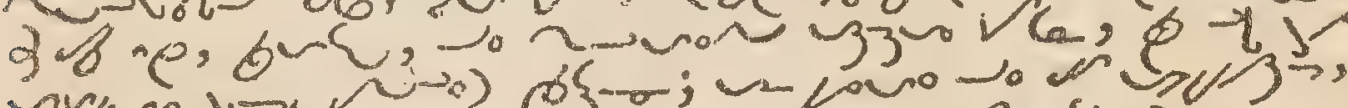

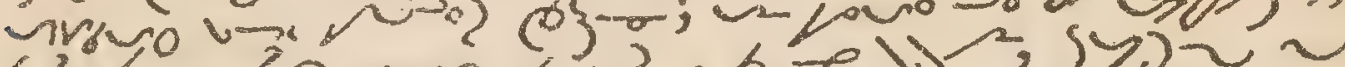
$\rightarrow 210$ gy 130 . No.29.

Liberame

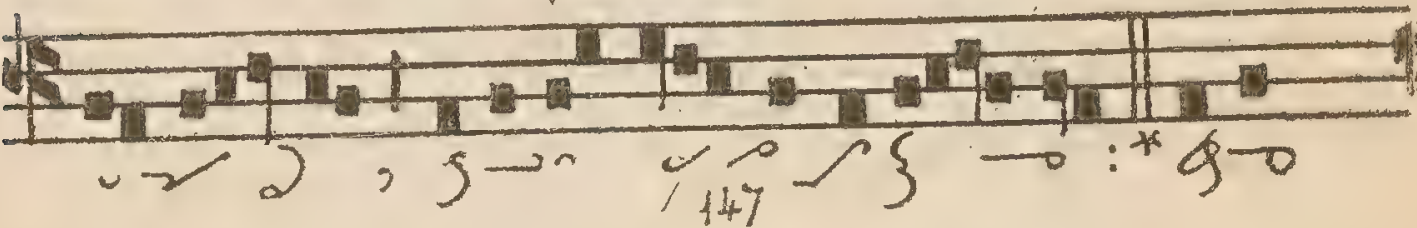




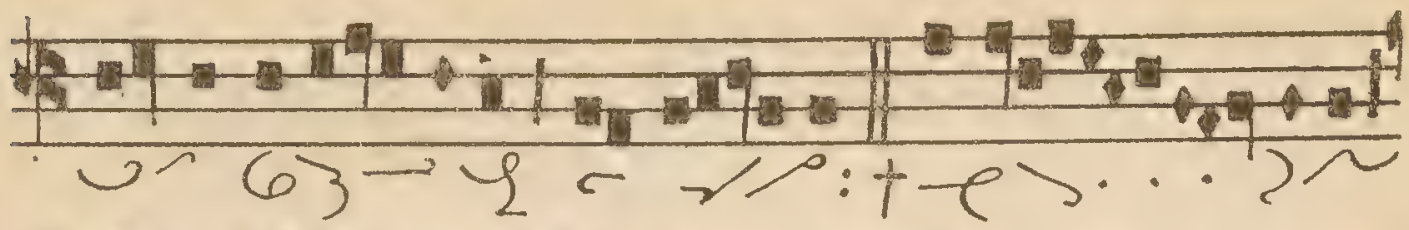

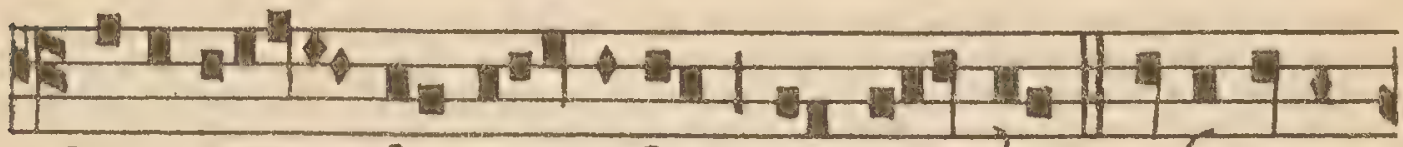

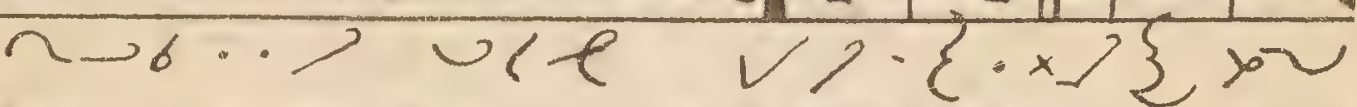

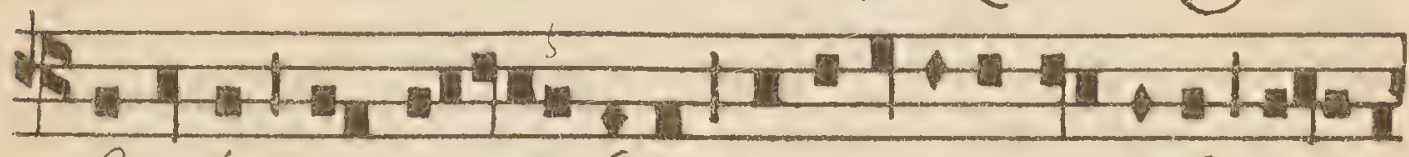

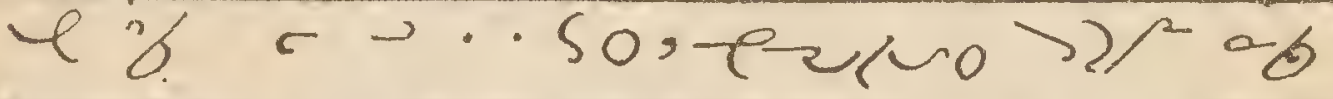

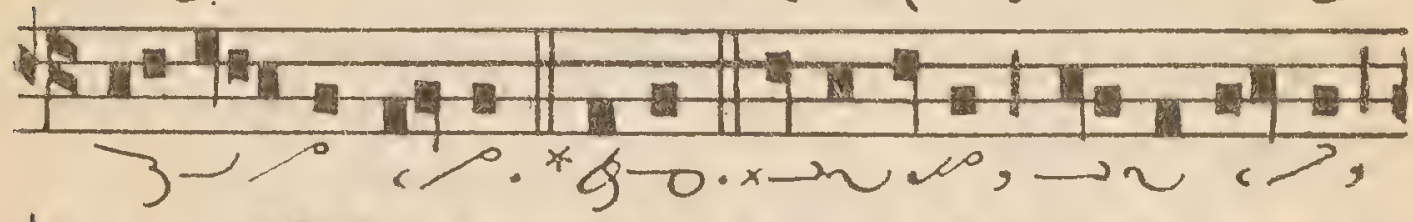

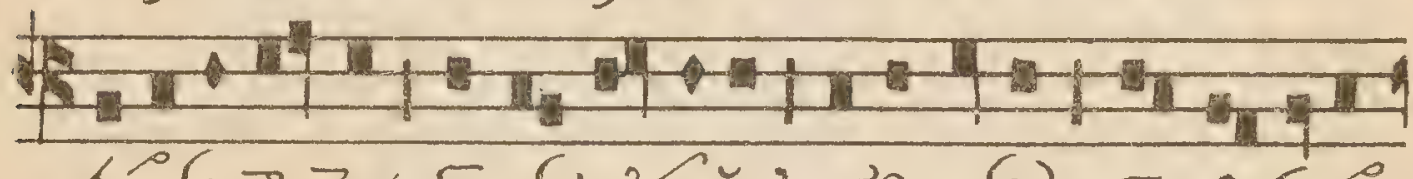

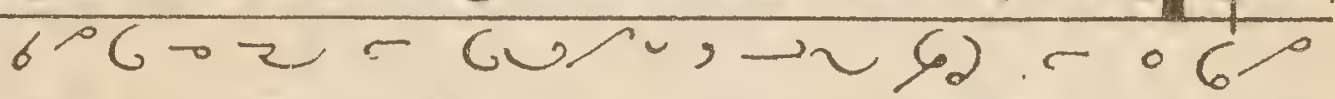

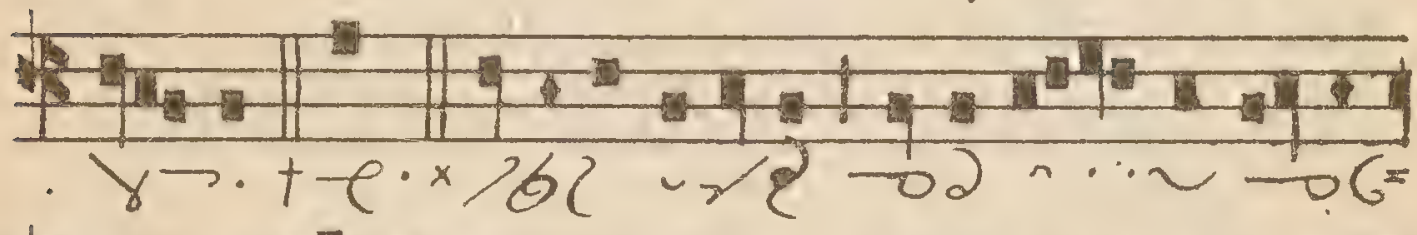

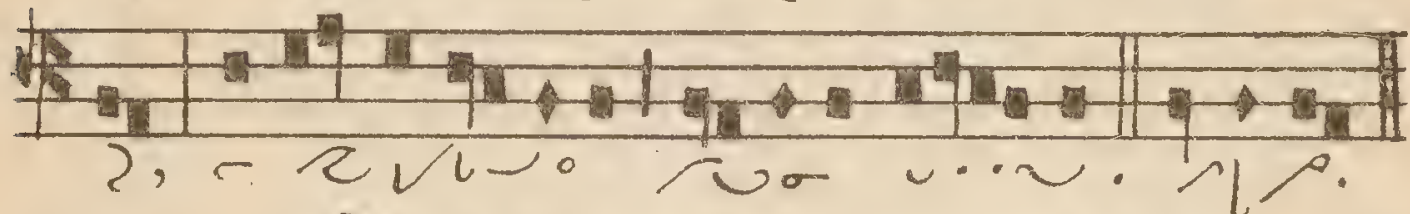
No. 30. Libera me. other air.

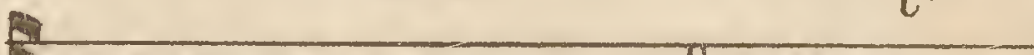

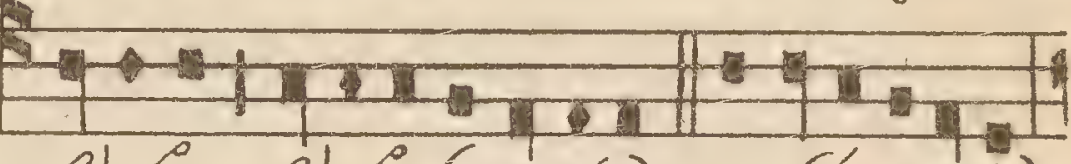
l. 1. 5 -62, $\rightarrow 83$ - 2 A.

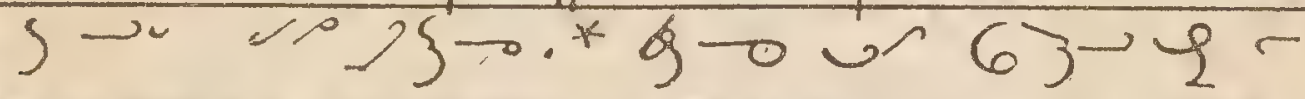

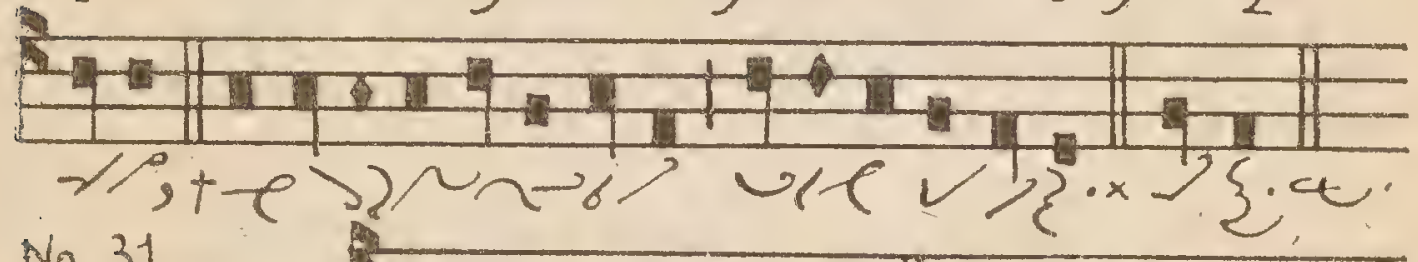
No. 31.

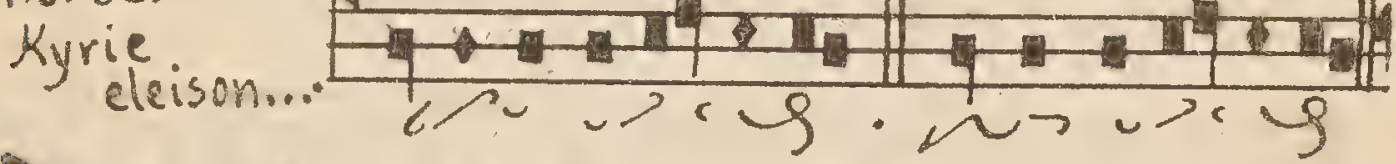

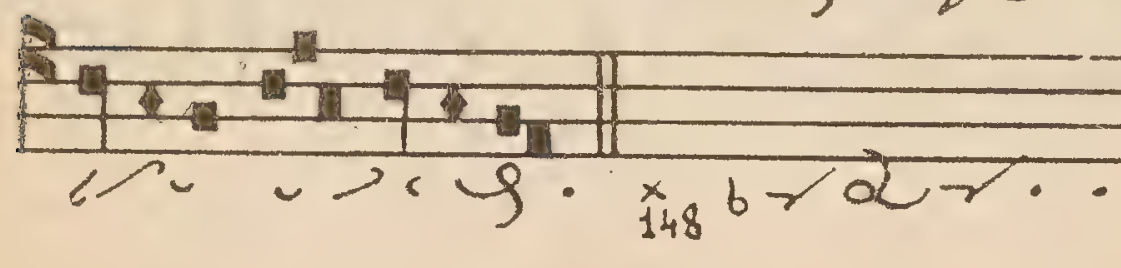




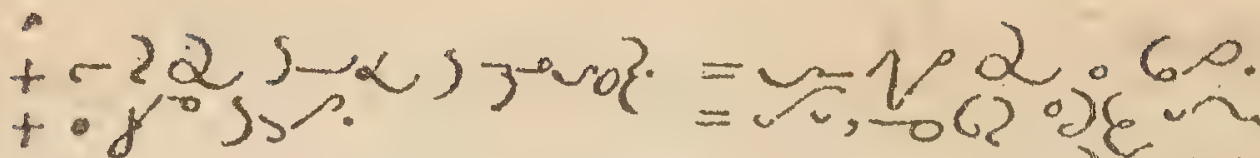

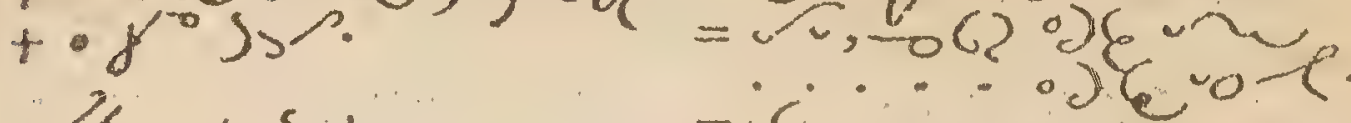

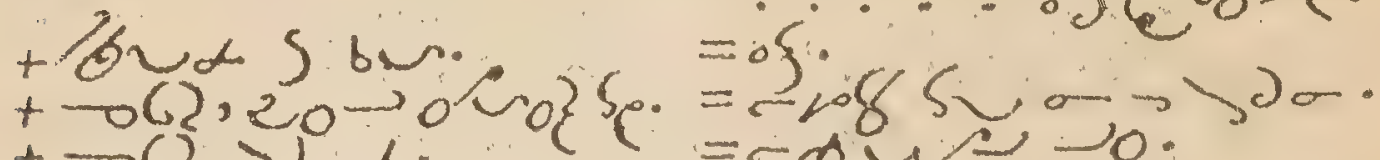

+062 dup. or $=-\phi L_{1}=-0$. oremus. of

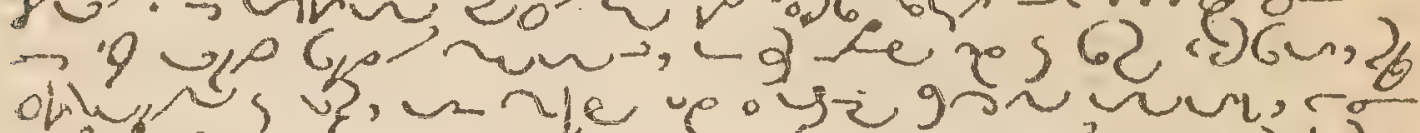

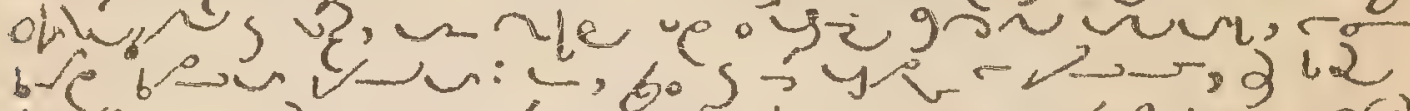

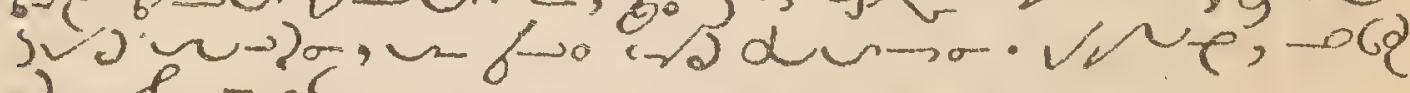
d $-e_{0}=0 \xi$.

No. 32 .

In Para $=$ disum.

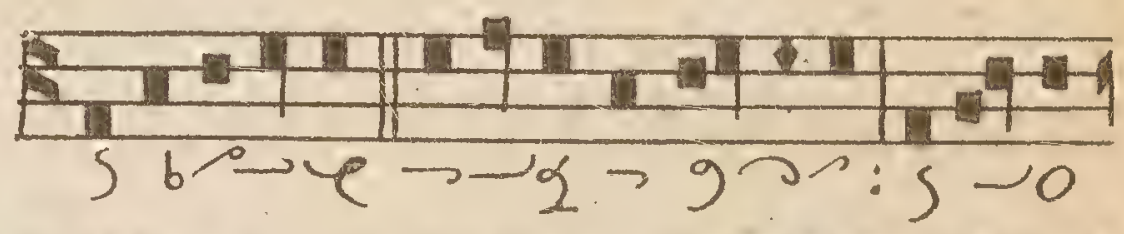

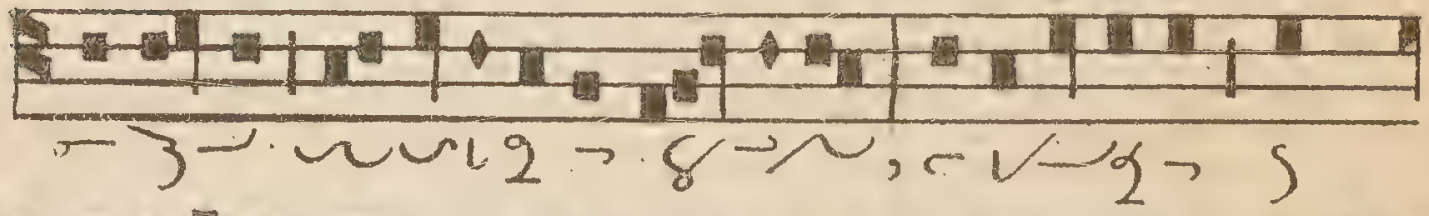

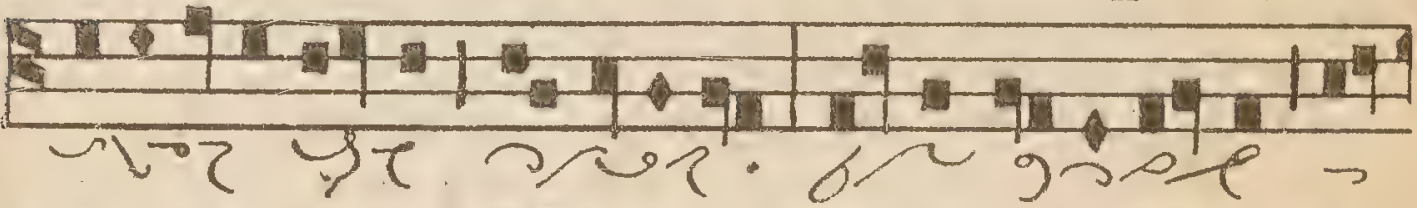

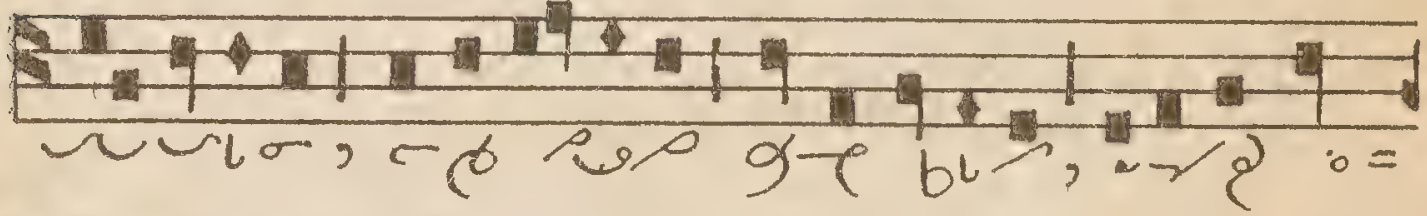

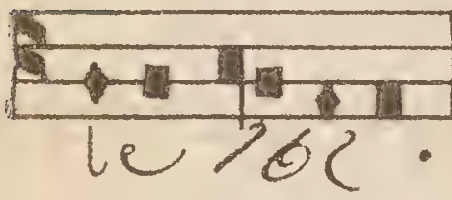

Blessing of a grave. +or $\rightarrow$ W cus $=$

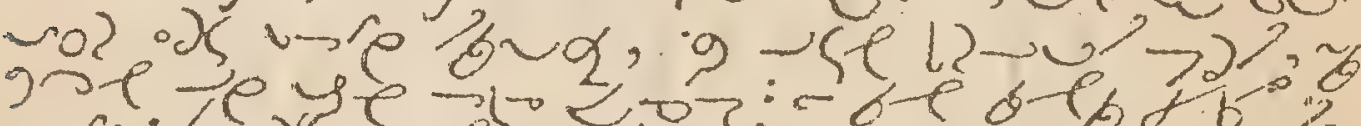
va-

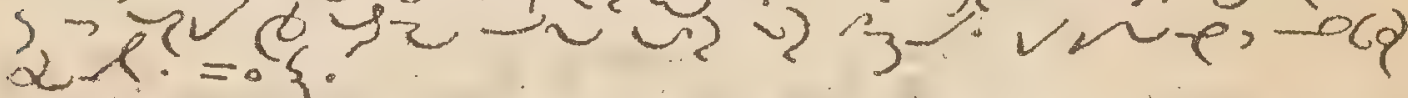

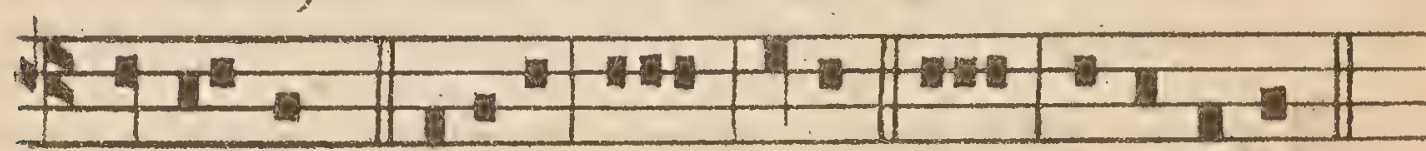
bye 2. d. 149 . 

Benedictus. l?

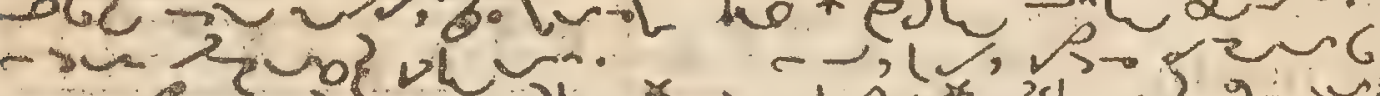

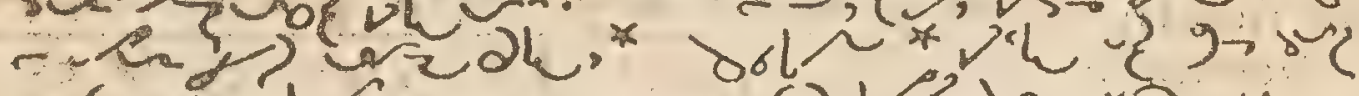
$3-06-1-r 4$.

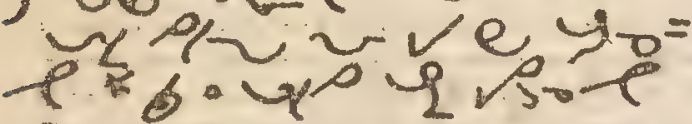
060 b le le ini.

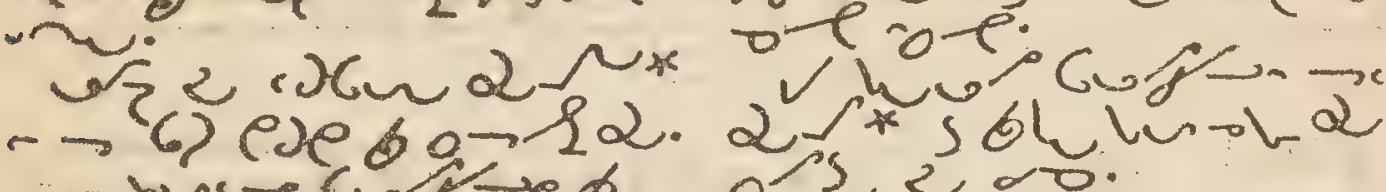

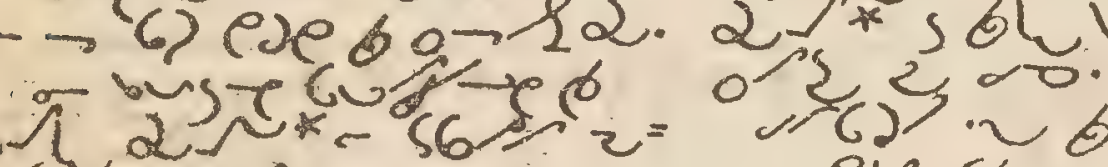

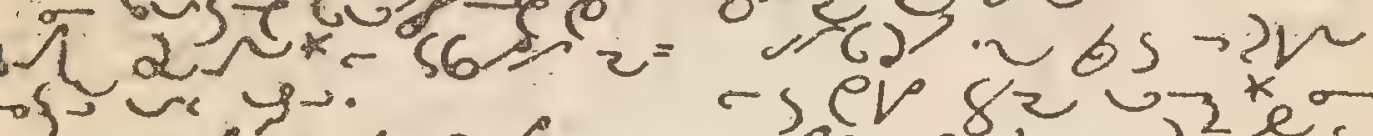

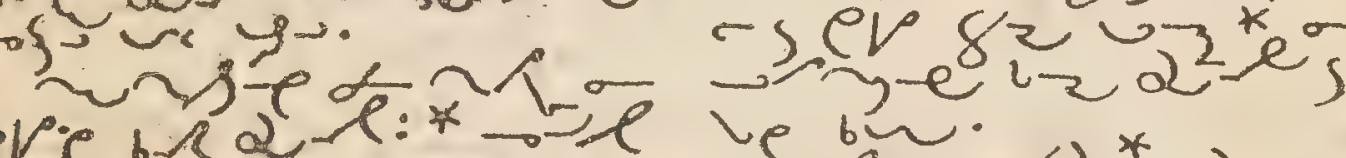
-

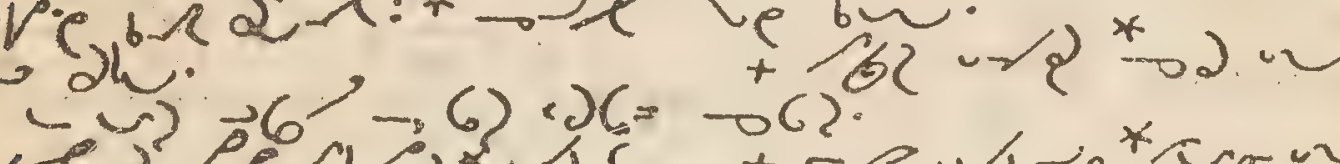

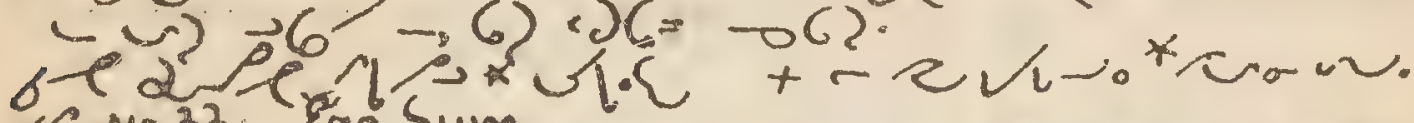

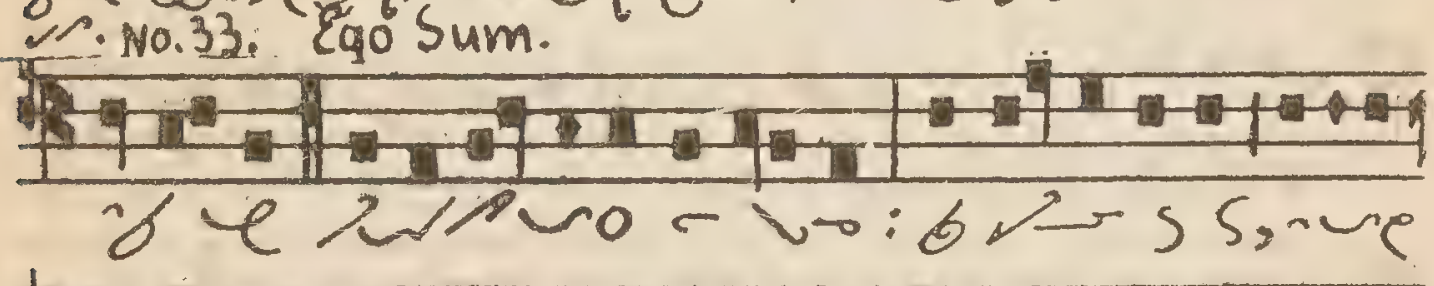

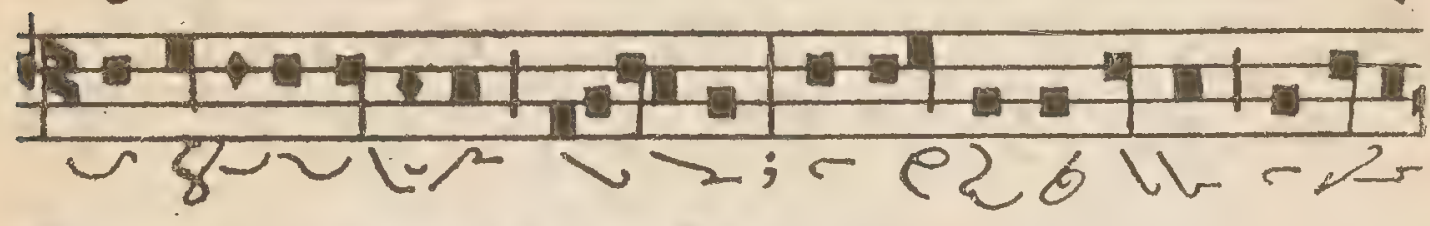

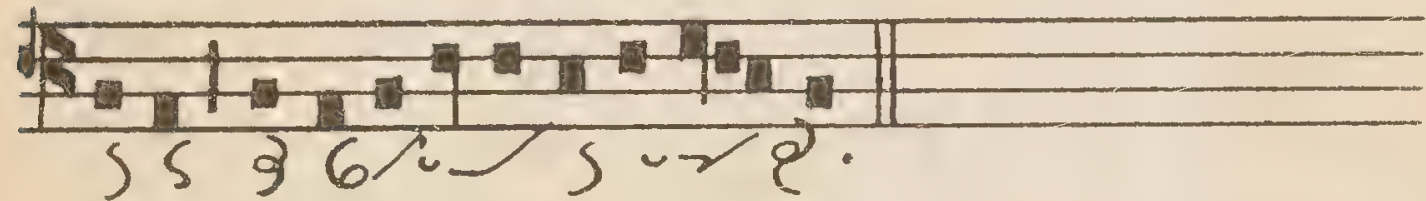
(6)

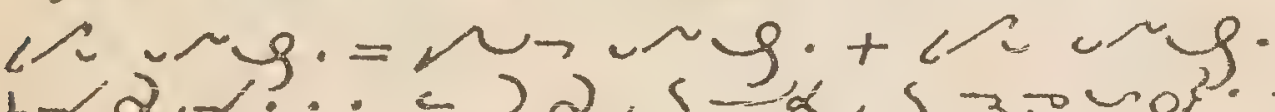

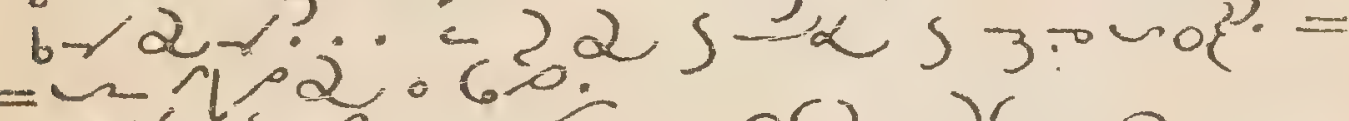
$+\gamma-3>=0$

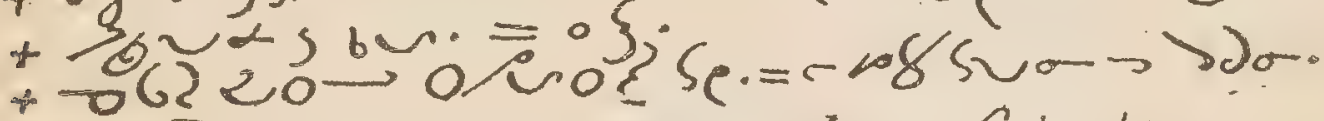

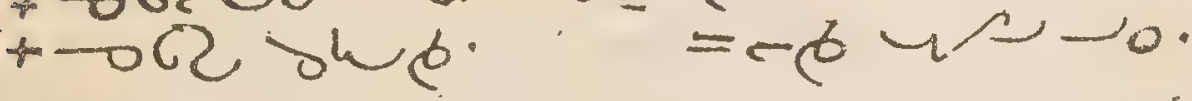

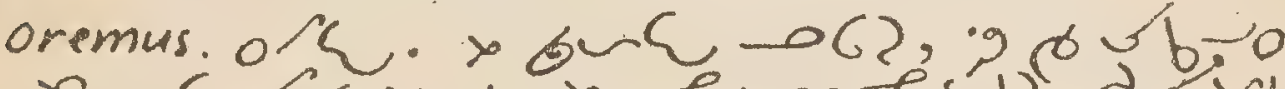

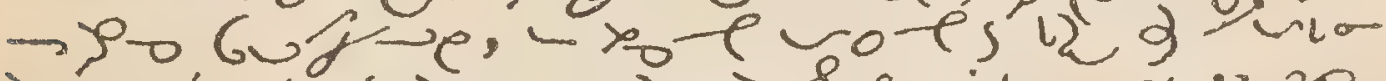

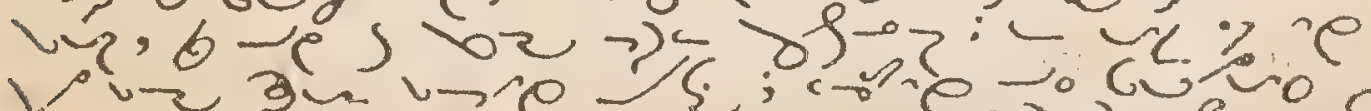

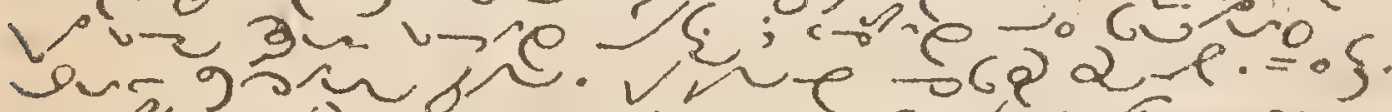

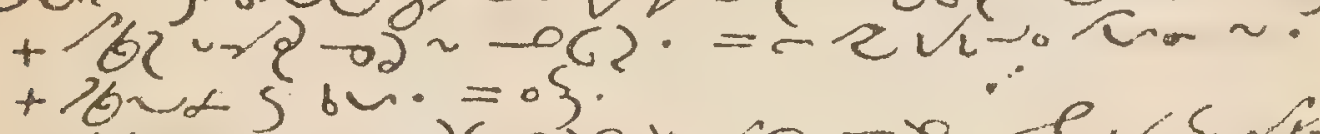

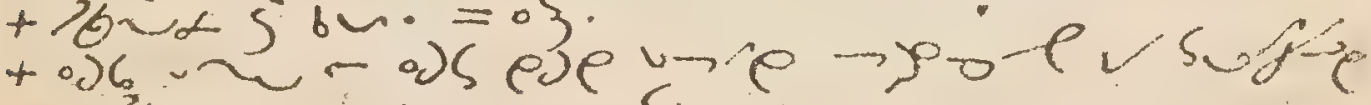
$\rightarrow b \sim 2.5$ bu. $=0.50$. 
child's funeral.

No.34.

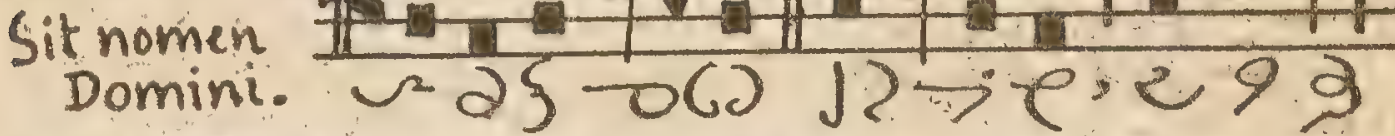

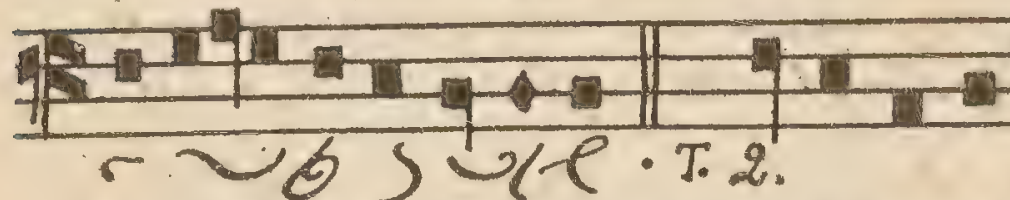

\section{Laudate} Pueri.

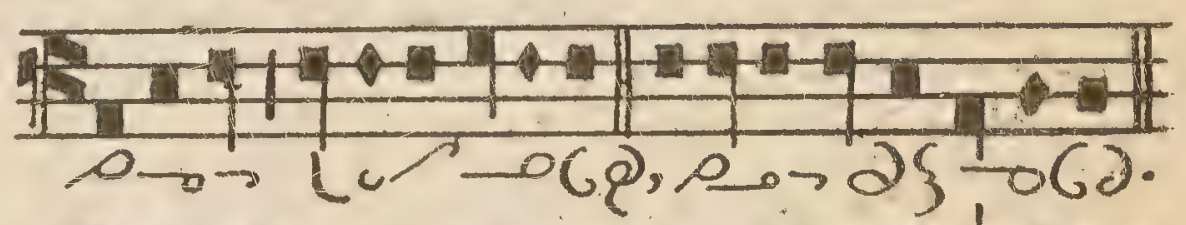

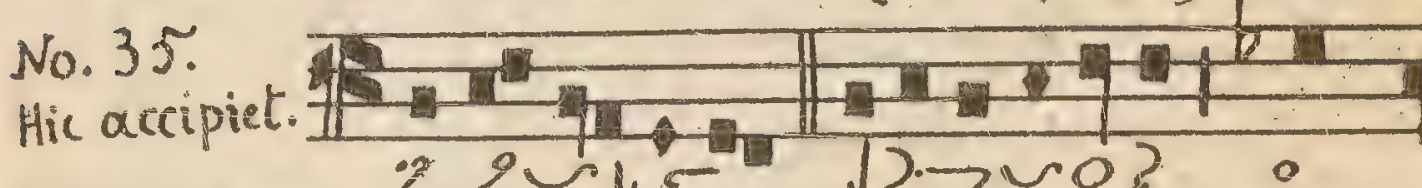

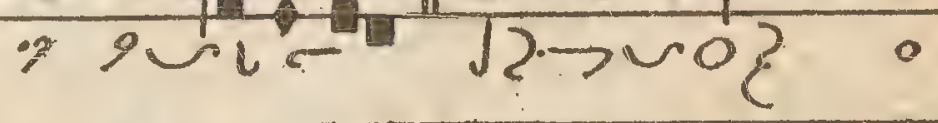

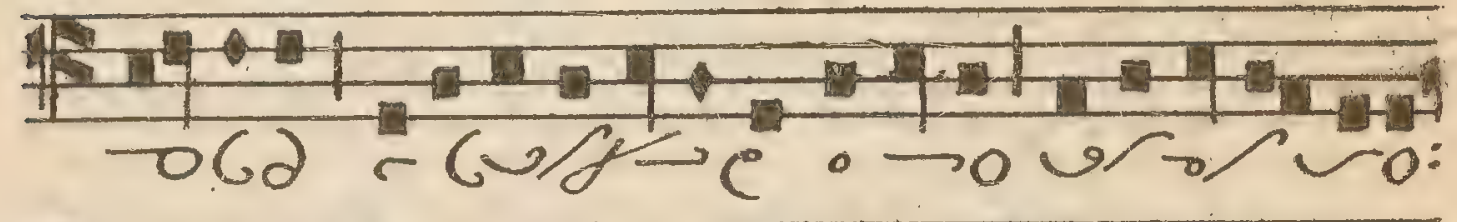

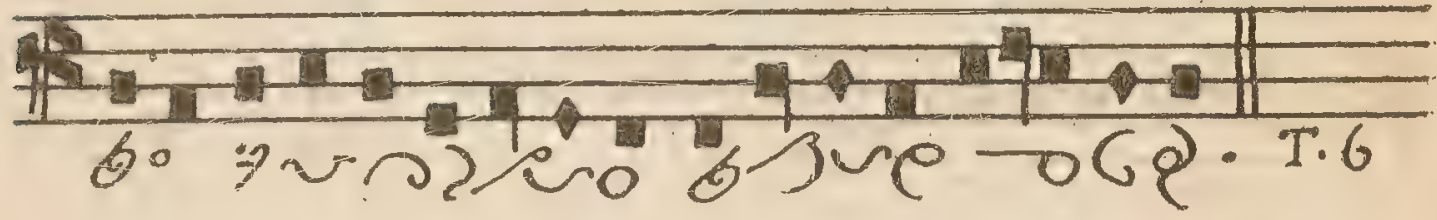

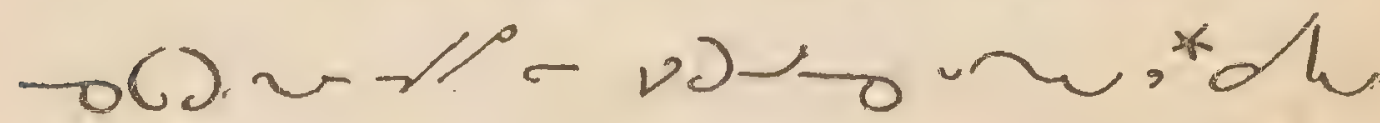

$$
\begin{aligned}
& \text { rife-ezo oly } 0 . . .
\end{aligned}
$$

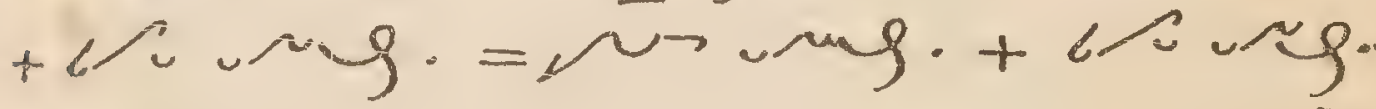

$$
\begin{aligned}
& +b-2-1 . .-22,5-\alpha \text { s } 3 \text { - } 10 \text { ? } \\
& =-1 / 2 \cdot 6 \% \\
& \text { + } 5 \text { or h/ saure num-. } \\
& =-g \sqrt{6}+5.59 y-t_{0} \text { s u } 2 \text {. }
\end{aligned}
$$

No. 36 .

Juvenes it virgines. $\left(\frac{2}{2}-V^{2}\right.$, , $620=$

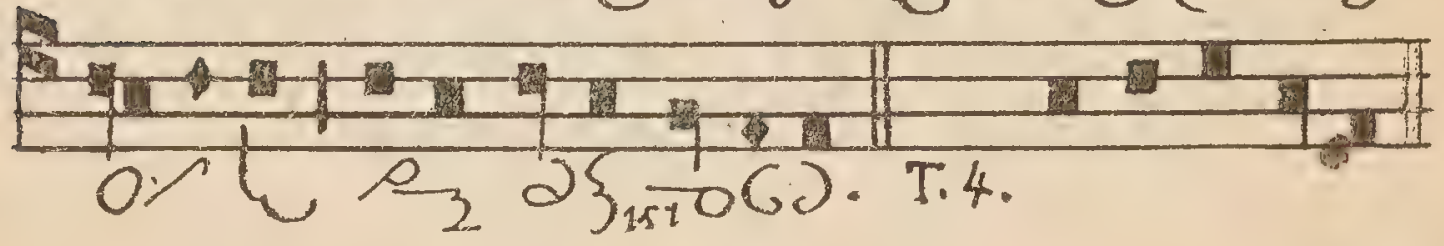




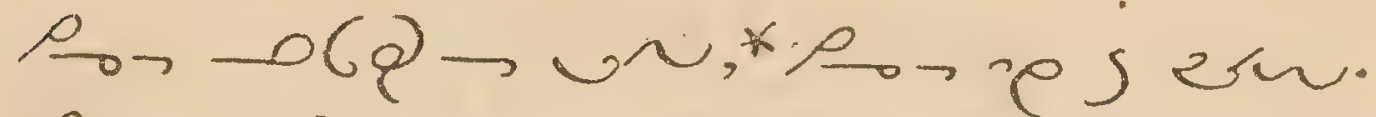

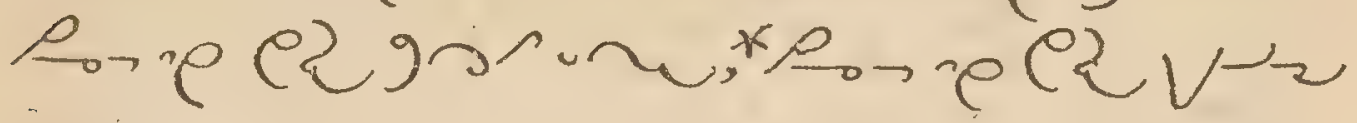

$$
\begin{aligned}
& \text { 口. }
\end{aligned}
$$

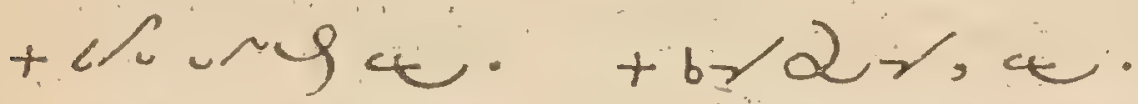

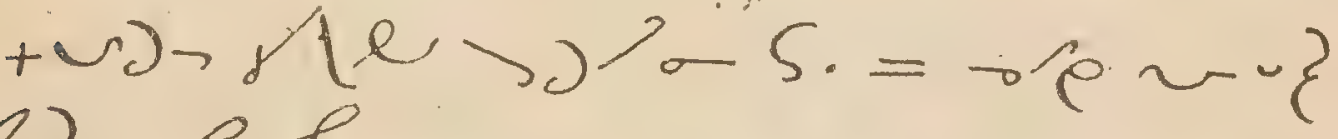

$$
\begin{aligned}
& \text { lowel. }
\end{aligned}
$$

No.3\%.

Benedicite.

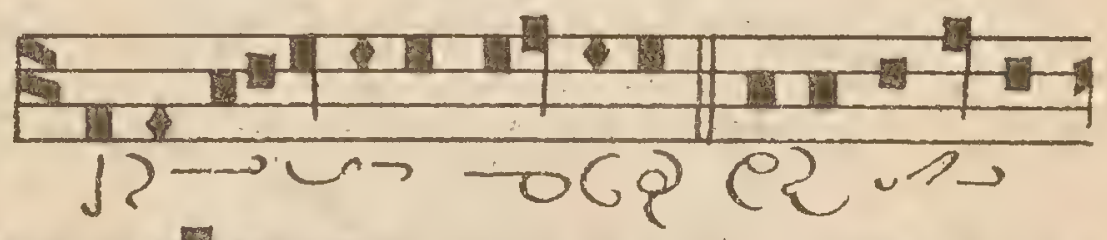

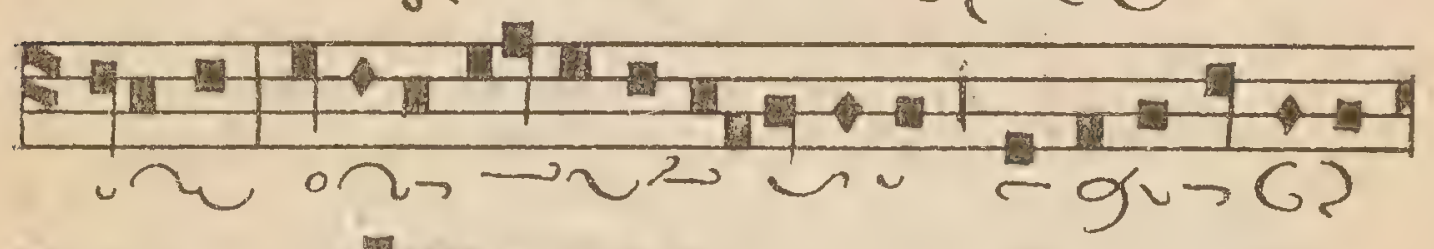

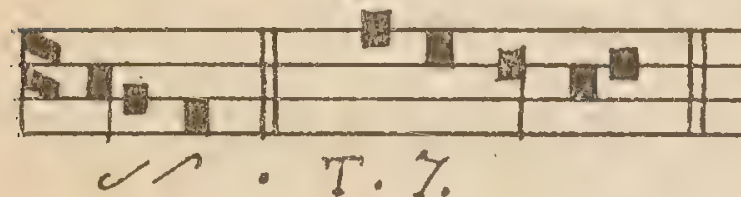

12 us ejo a $-0(0)$ o $(2), * p_{0}=$ nulvath re s we.

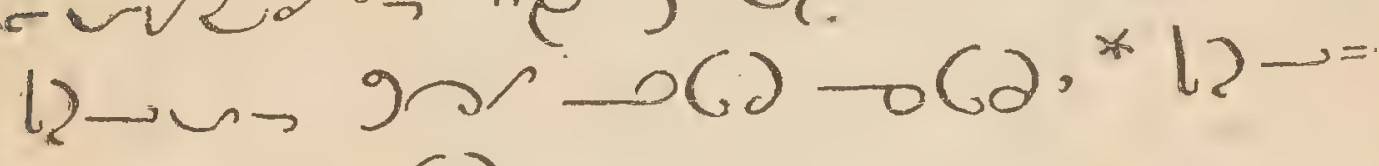
$n \rightarrow-r-O C \partial$.

No.38. Toni Psalmorum.

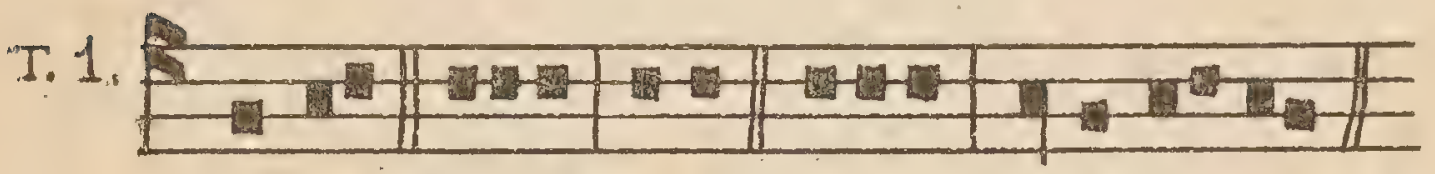

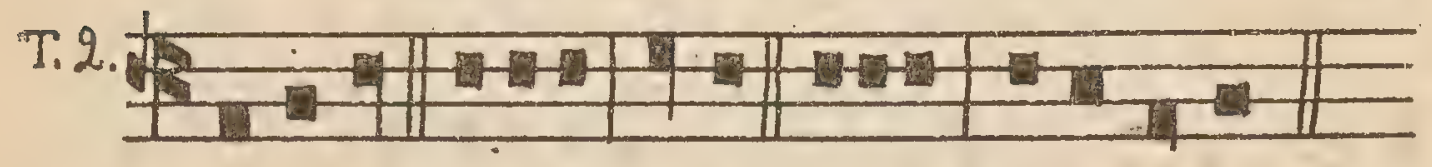

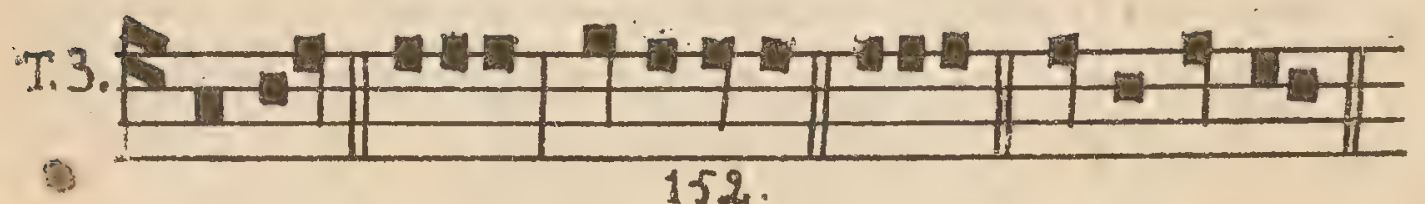




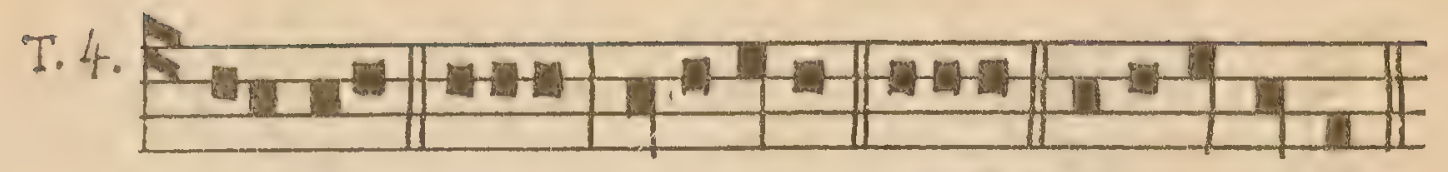

T.5.

T.6.

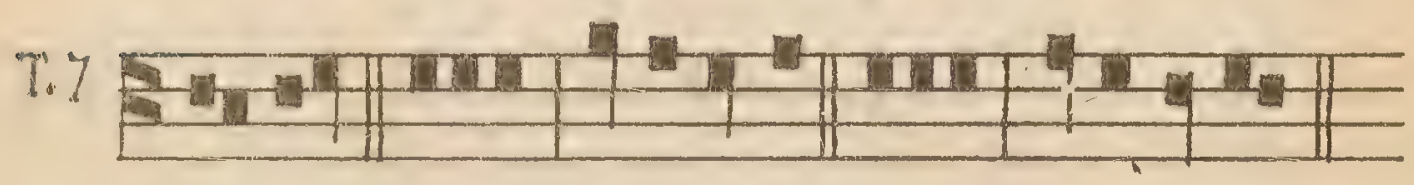

T.8
Cantus per annum.

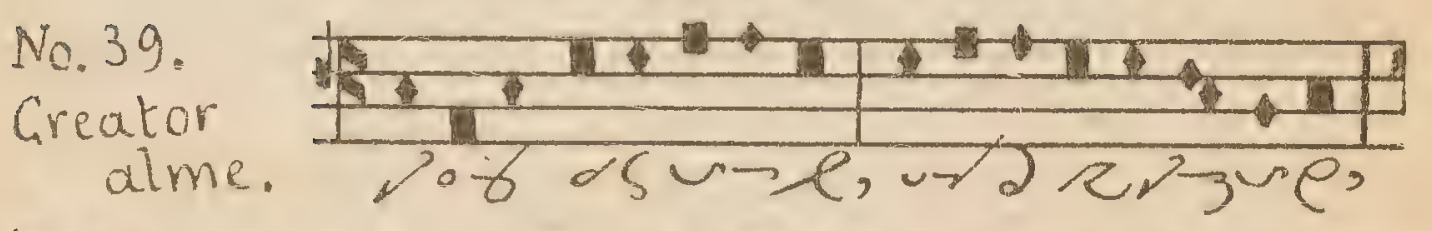

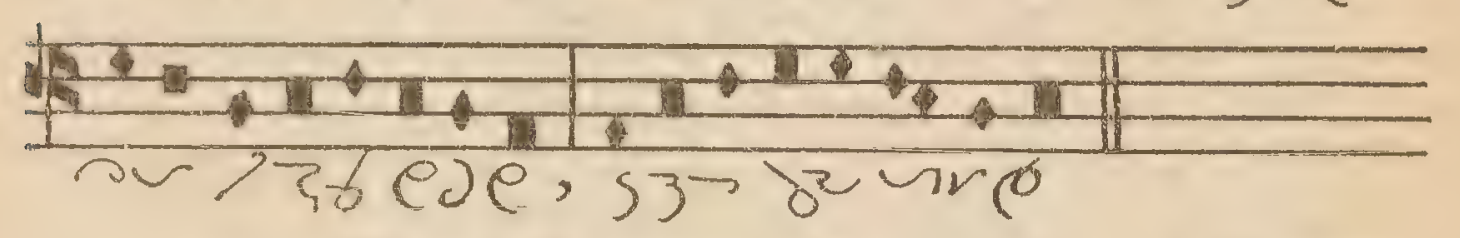

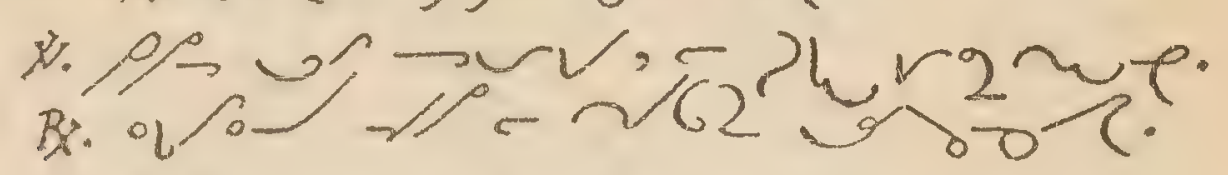

No. 40.

Rorate.

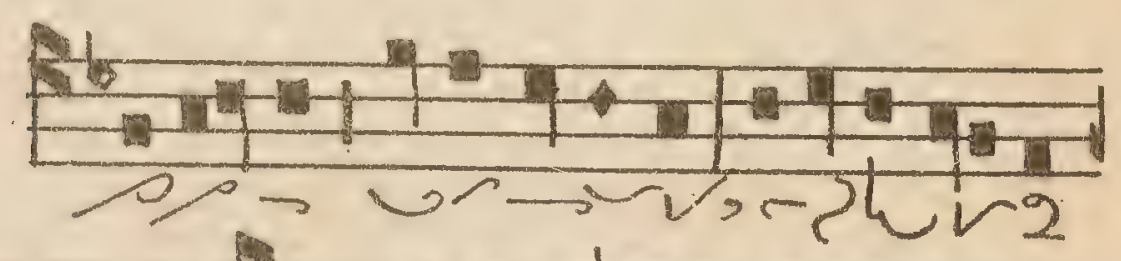

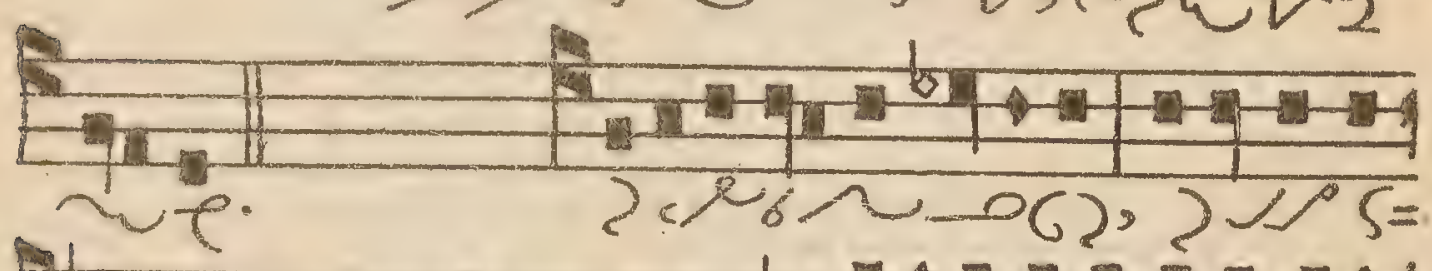

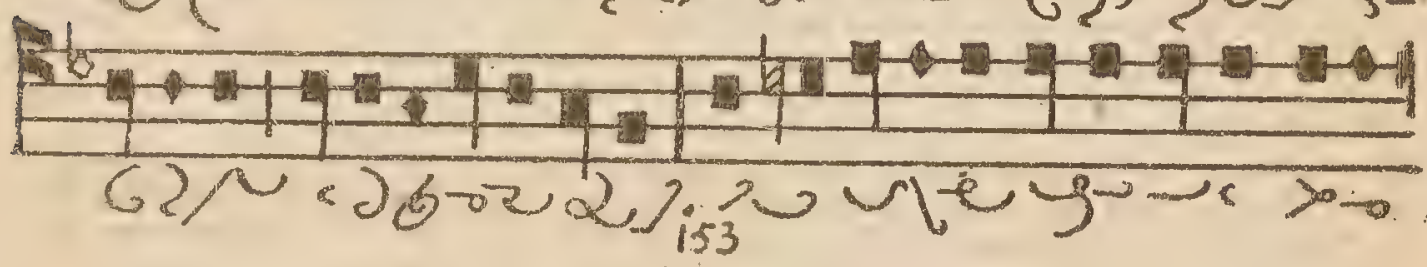




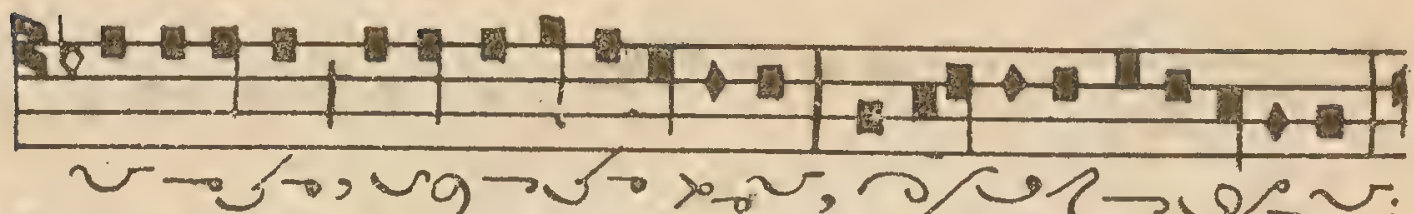
है।

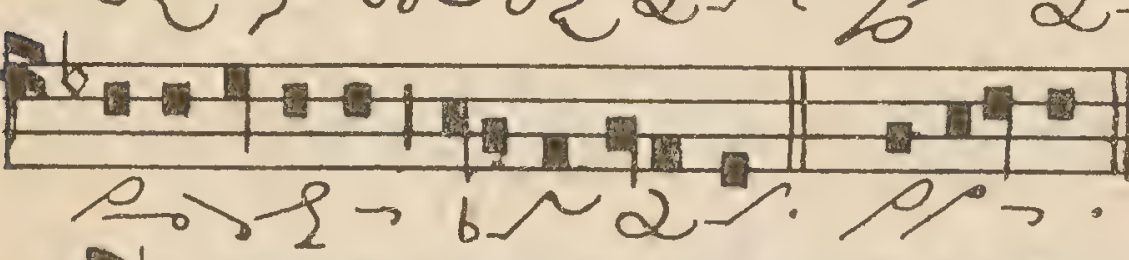

oर $9-46022 \% \div 6 / 2 \pi, 1$

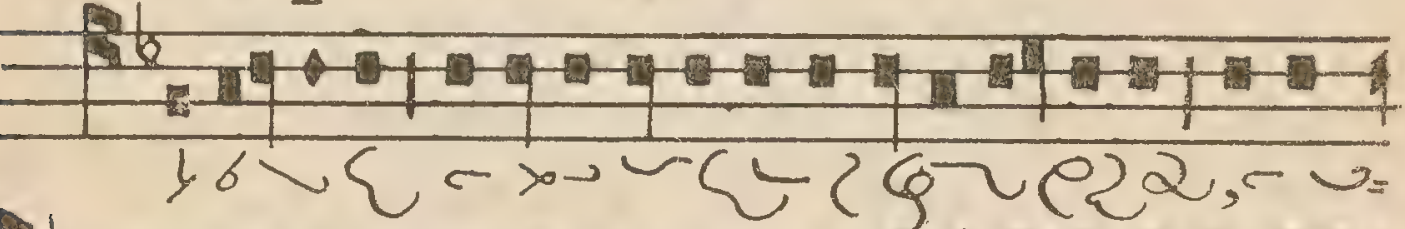

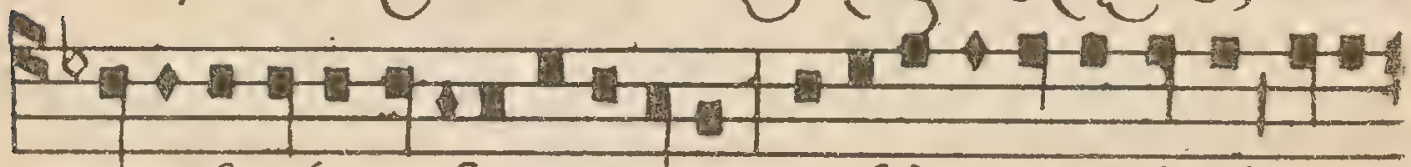

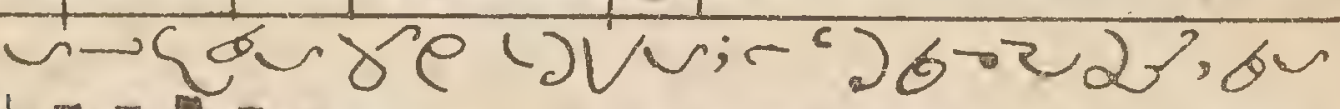

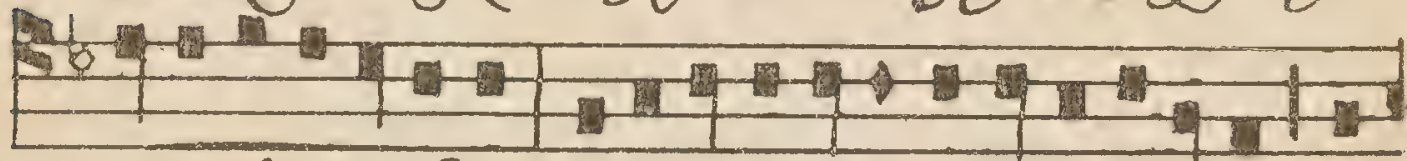

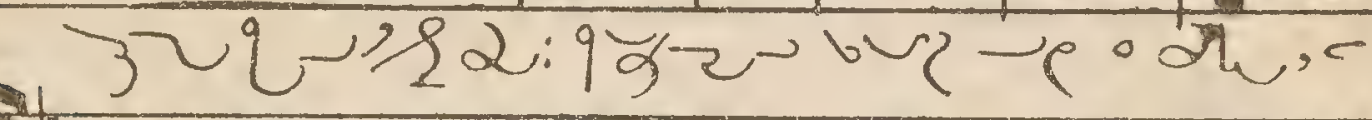
ह1

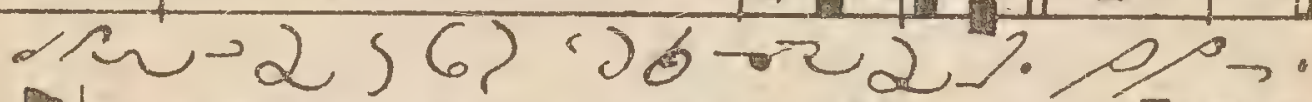

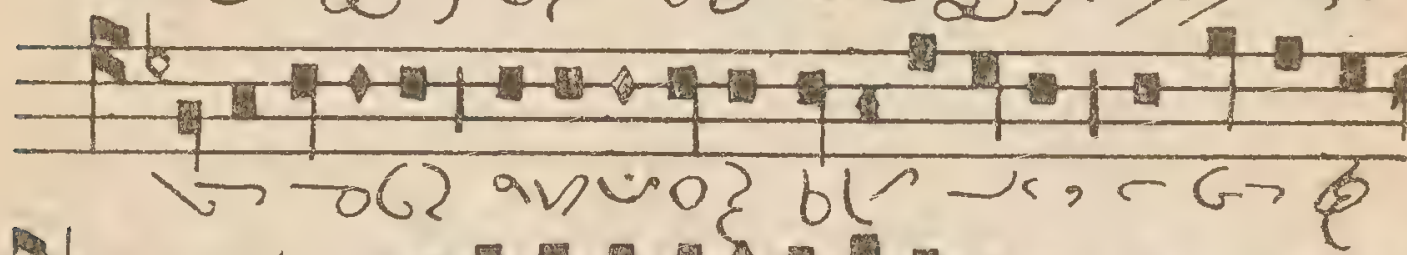
है।

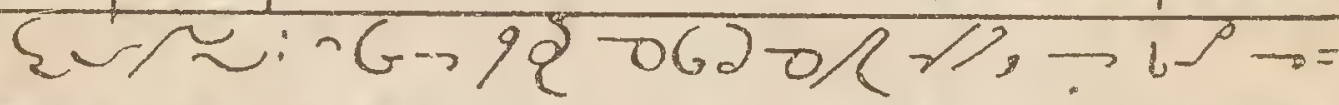
A.

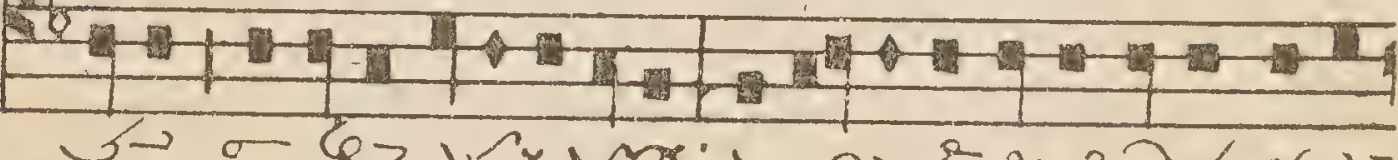
क grveng:

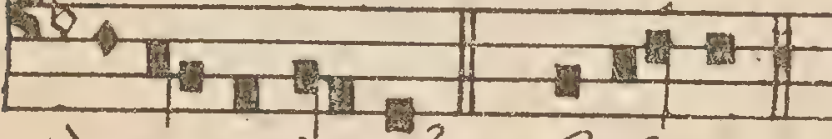
$\downarrow 22 \% .0 \% 5$.

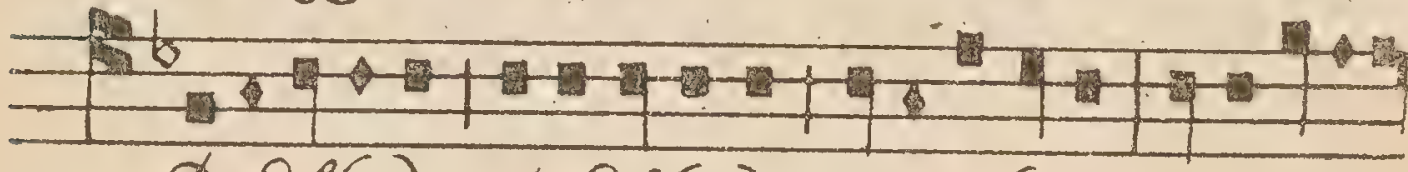

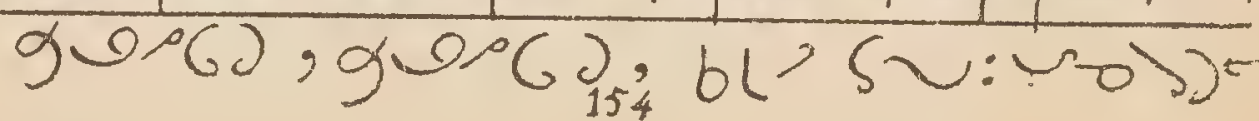




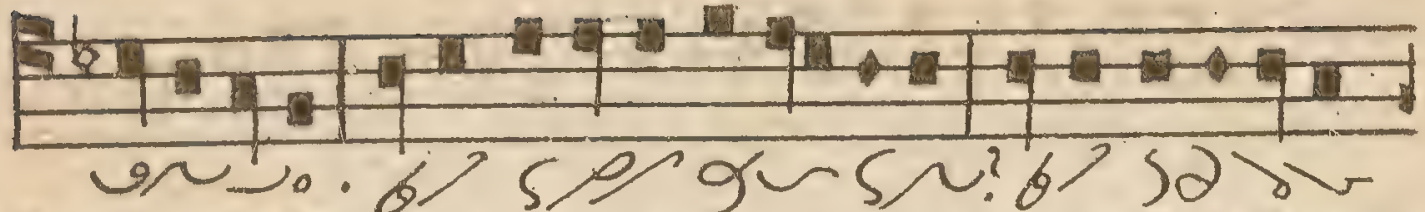
Eb

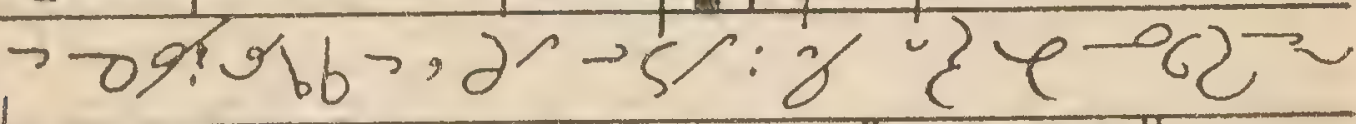

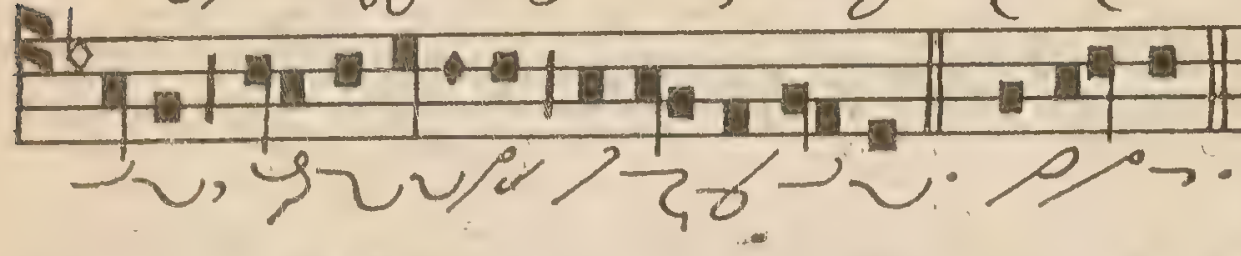

No. 41 .

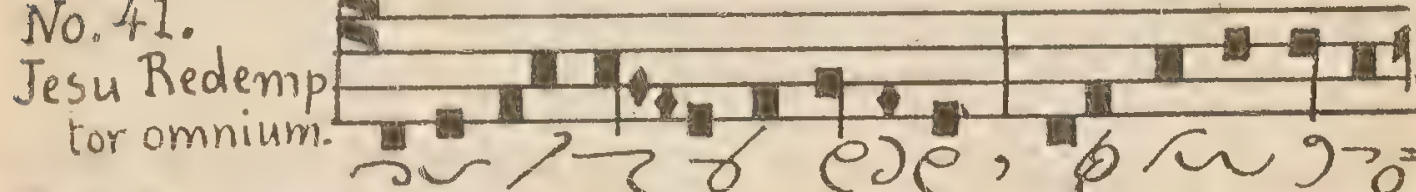

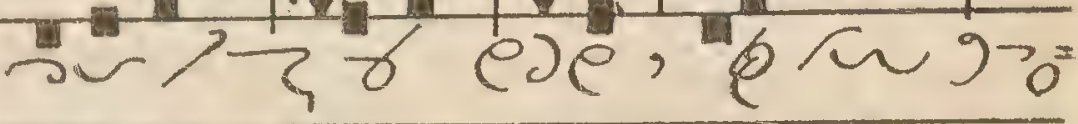

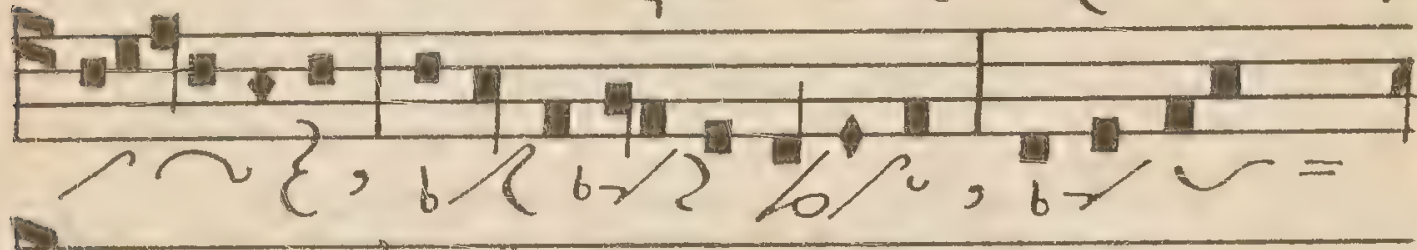

tin

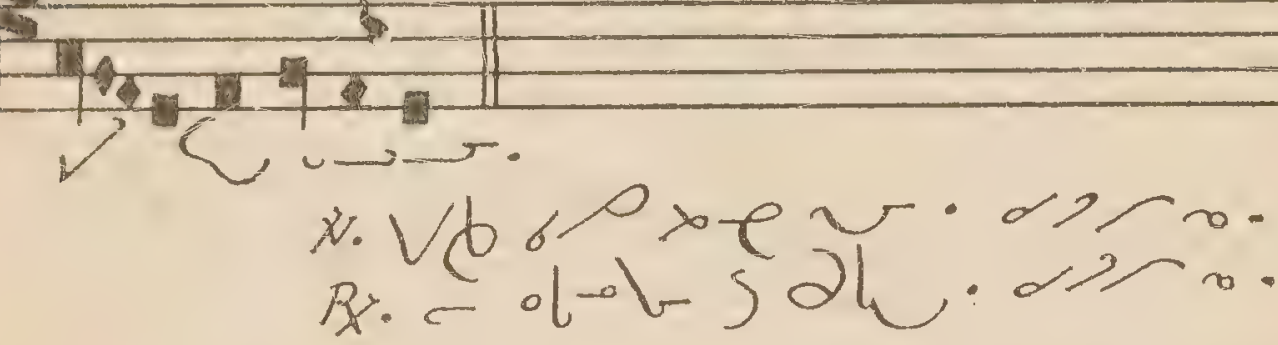

No. 42 . Adeste fideles.

ts th

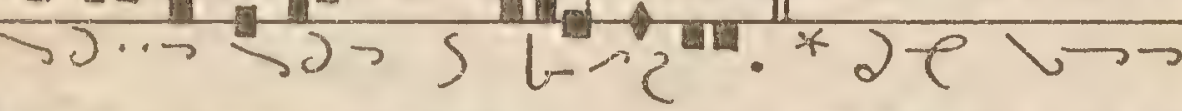

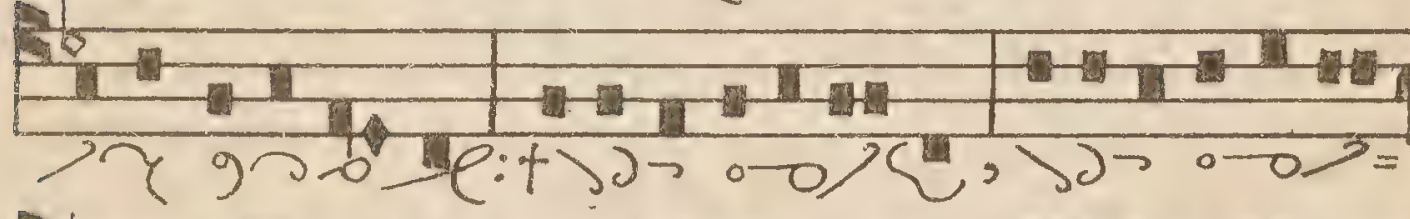

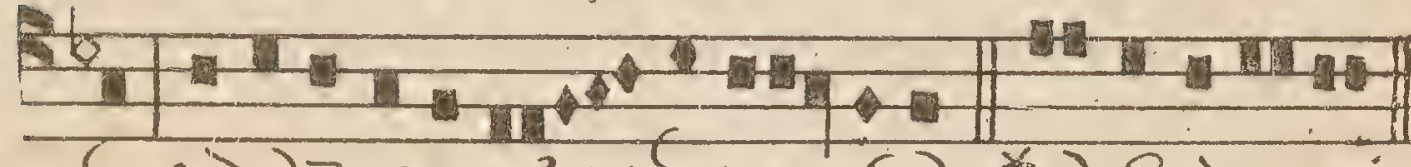

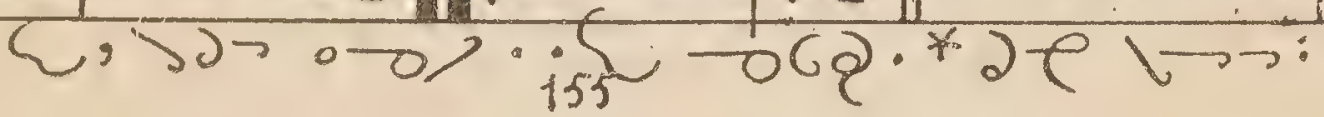


No. 43.

Attende.

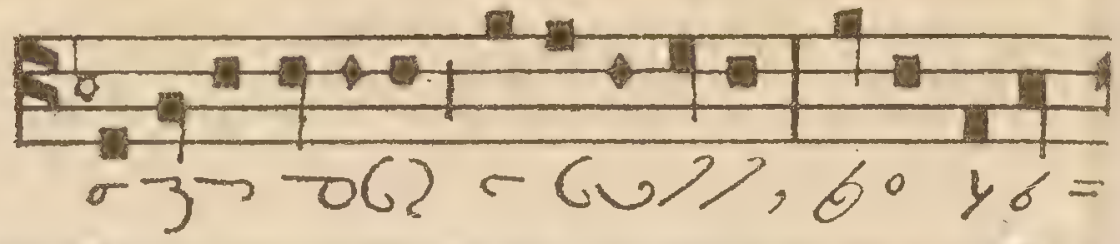

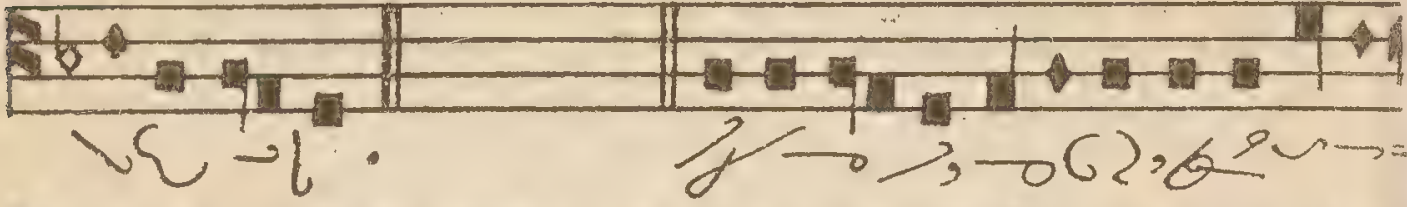

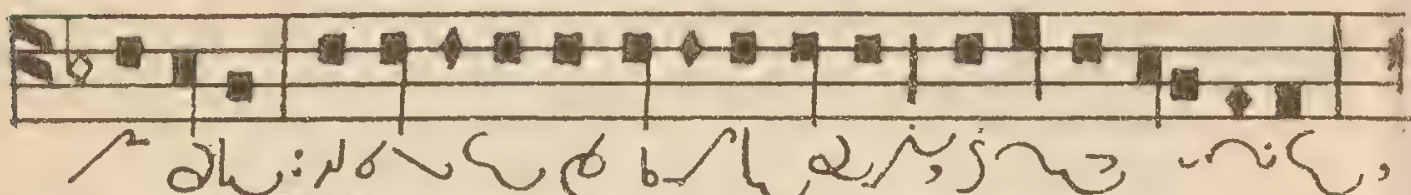
th

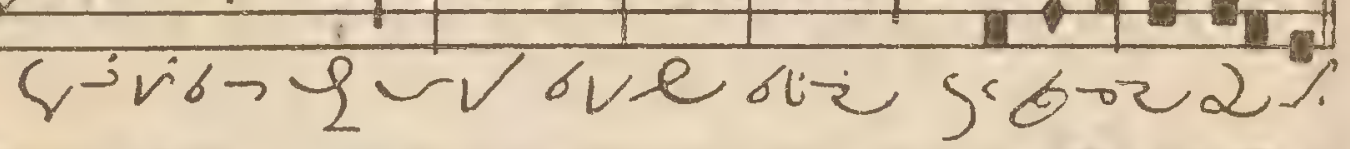

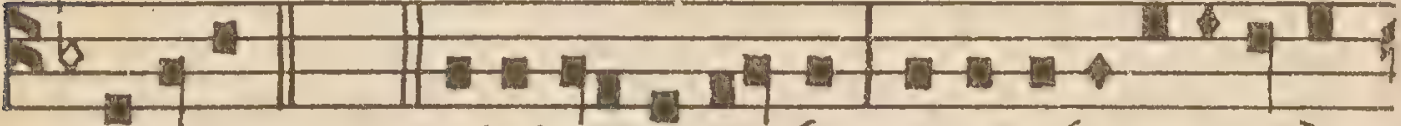

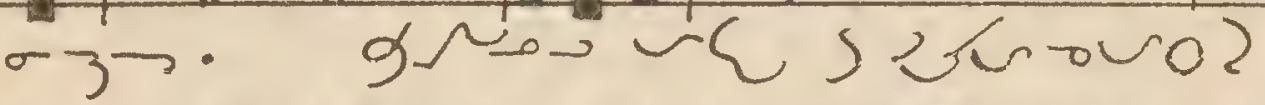

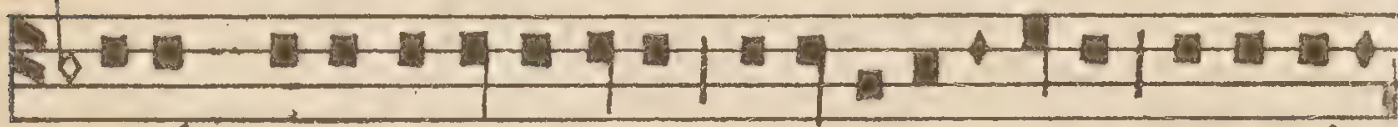

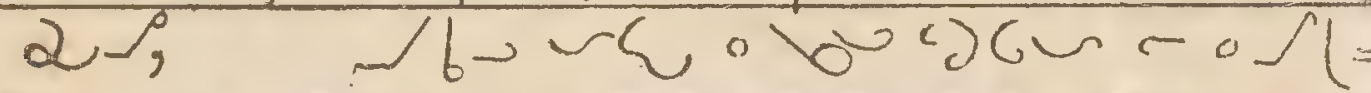

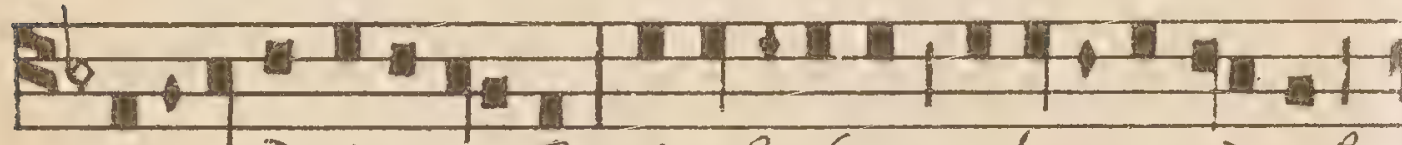

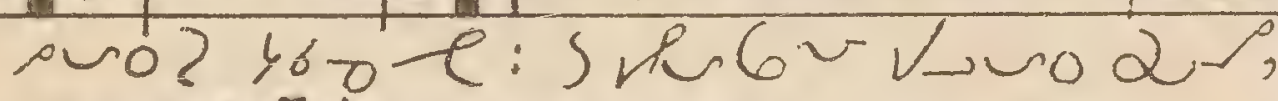

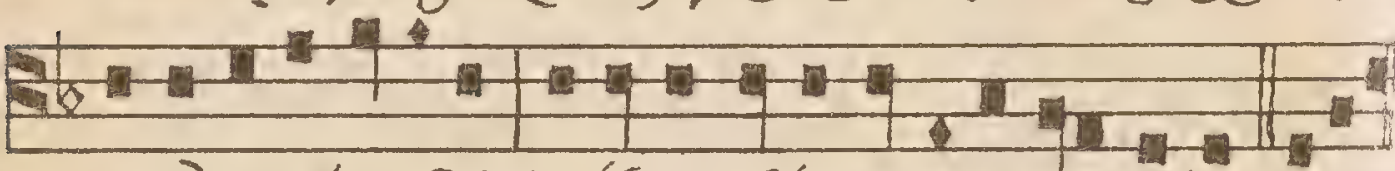

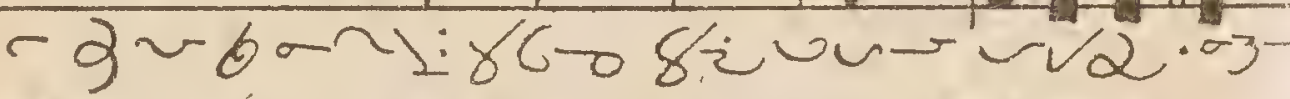

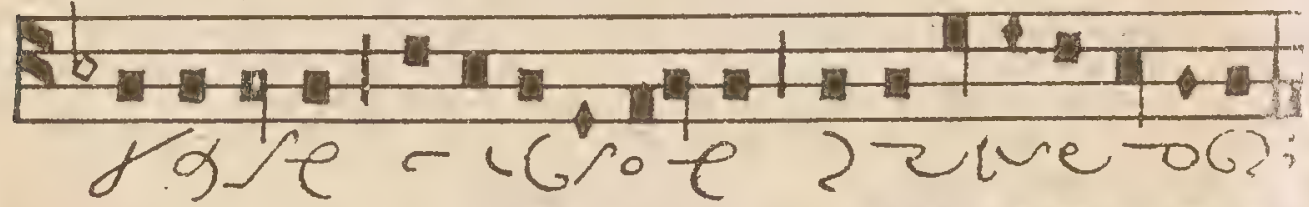

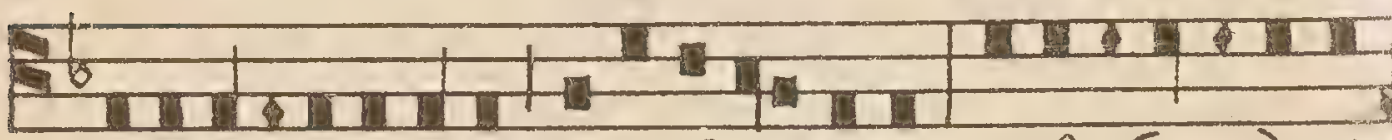

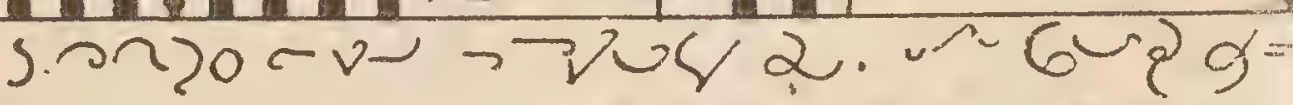
uld 
\&

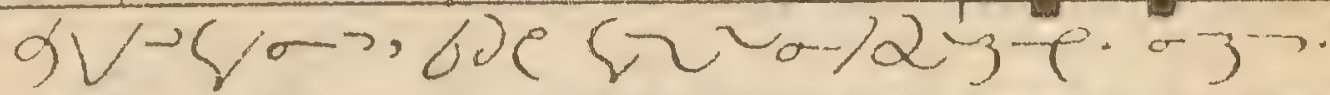

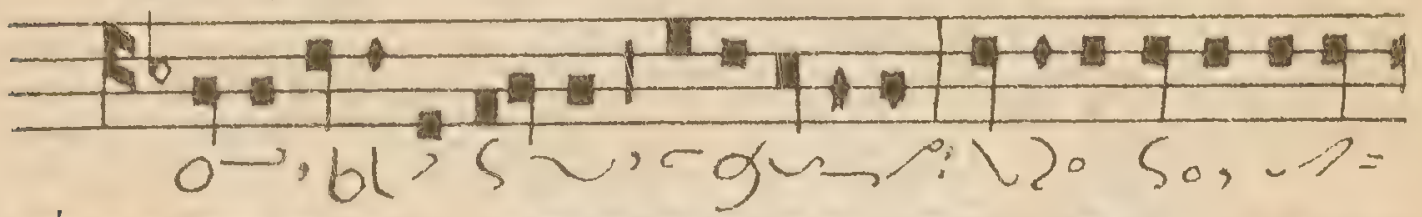

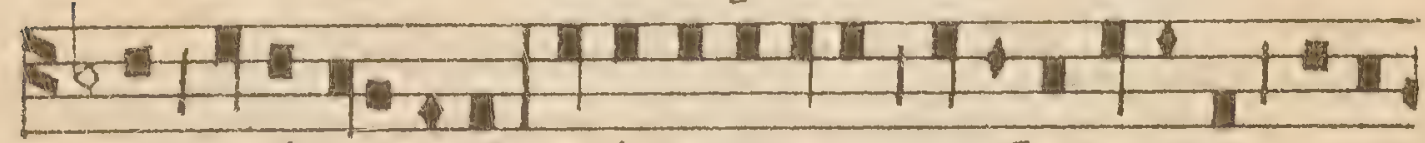

$-0\left\{\tau P, y-y^{\circ} \mathrm{0} \cdot 660 x_{0} \geq 5^{\circ}=\right.$

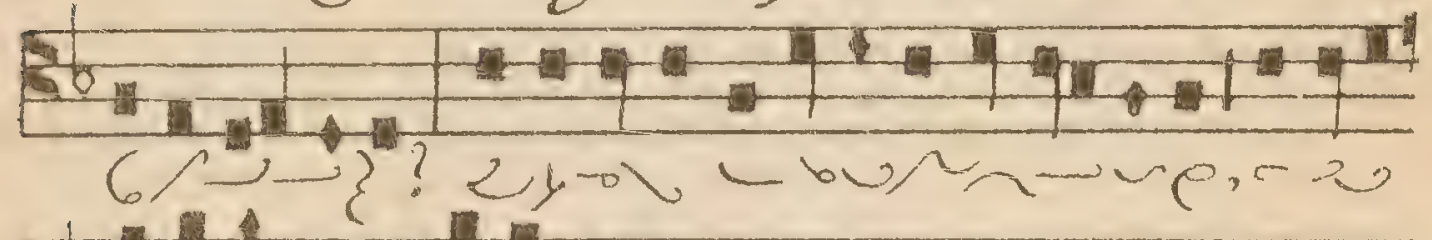

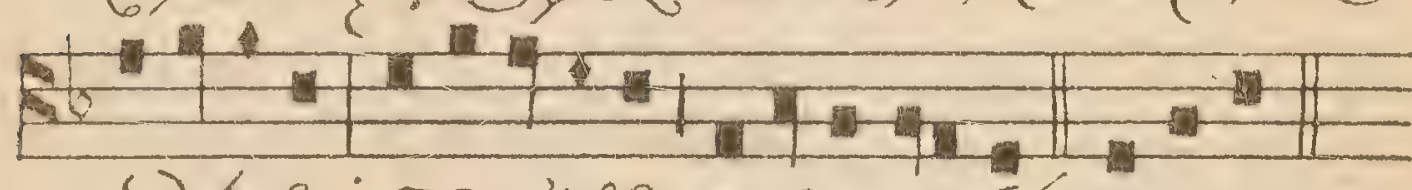

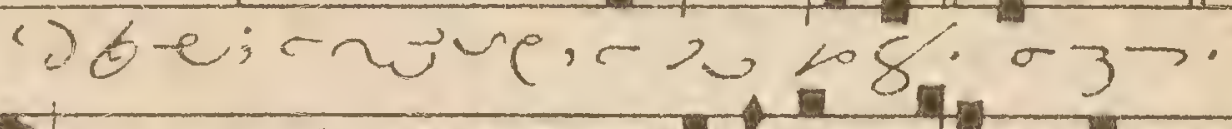

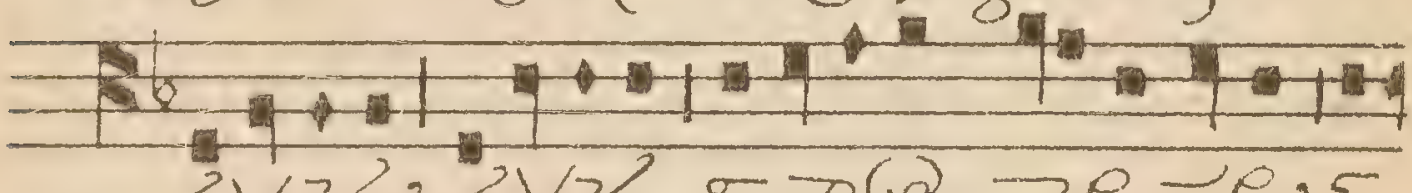

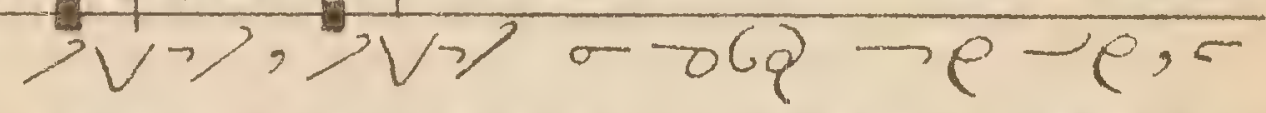

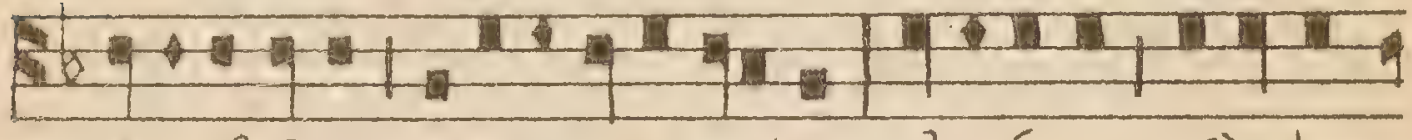

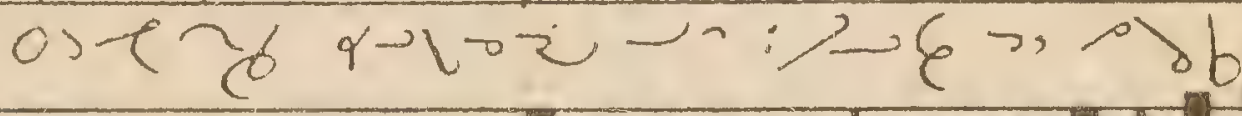

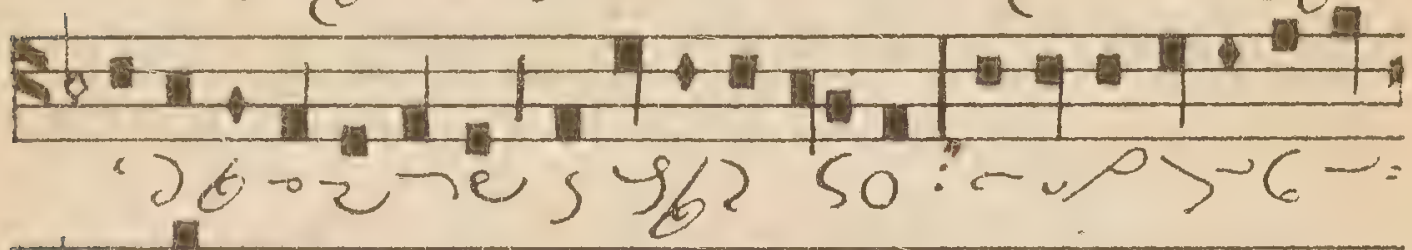
$-726-1.0315$ 


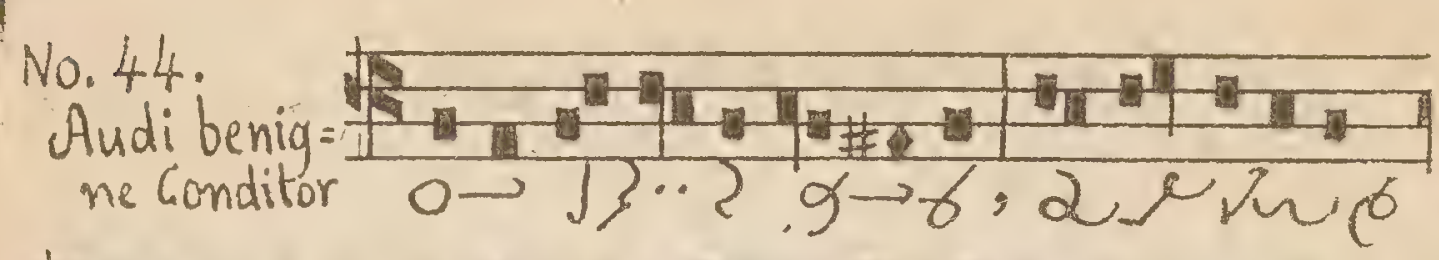

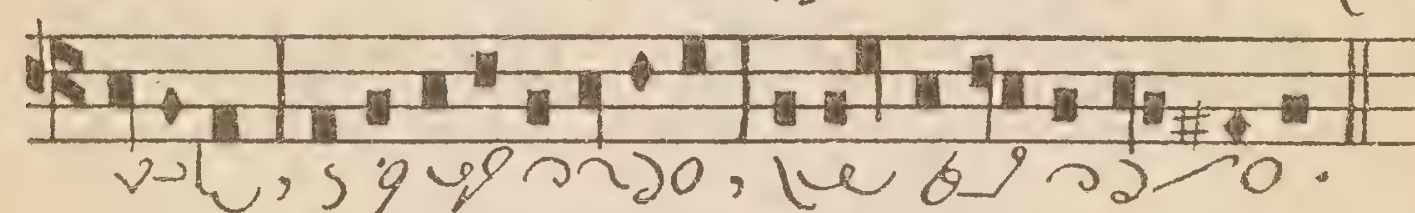

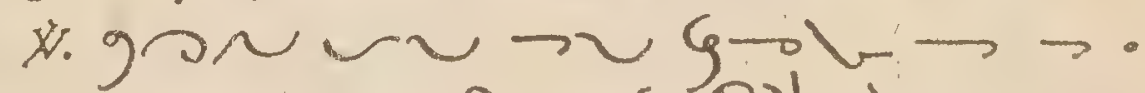
B. ᄂ. Co-2 a selulu $\sim$

No. 45. vexilla Regis.
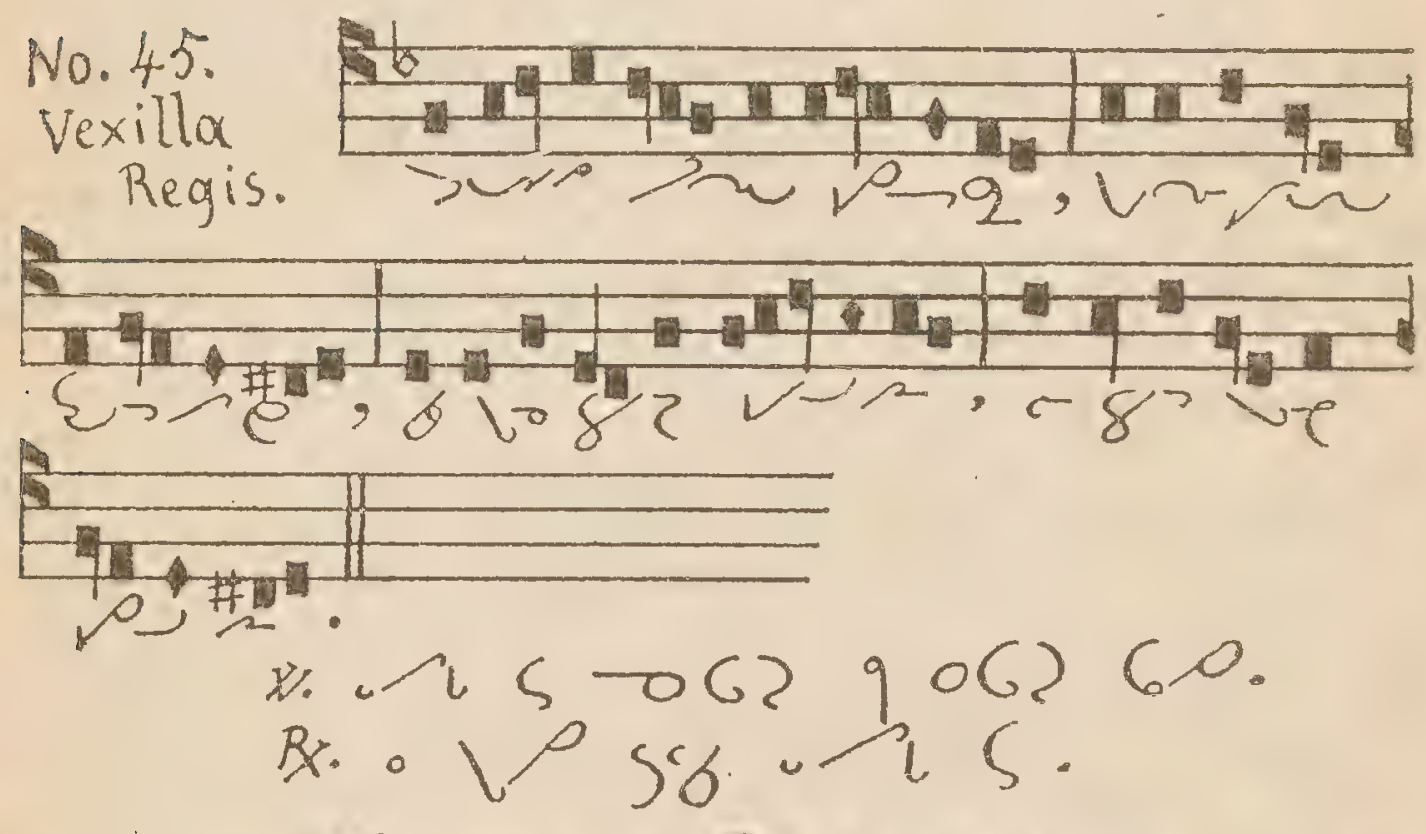

No. 46 .

Stabat mater.
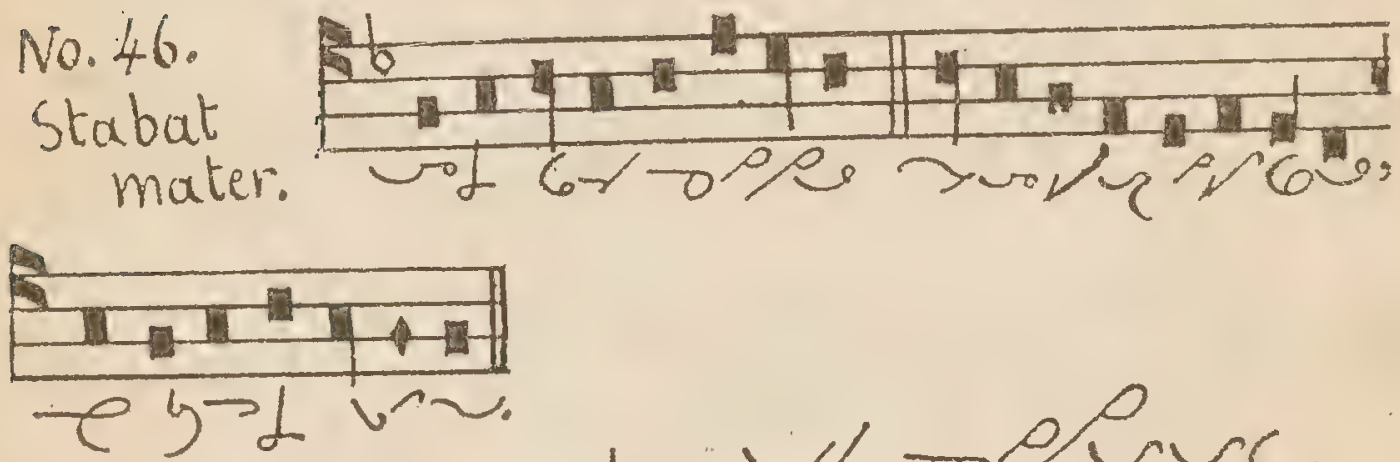

x. o s al, ve tepur.

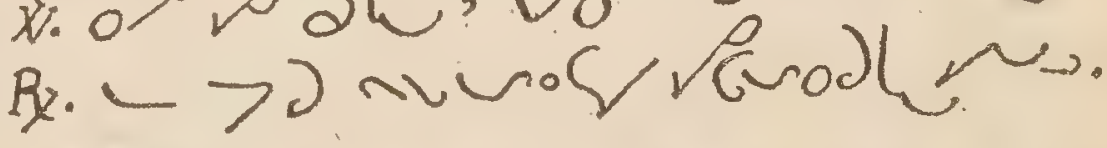

No. $4 \%$ Victima Paschali
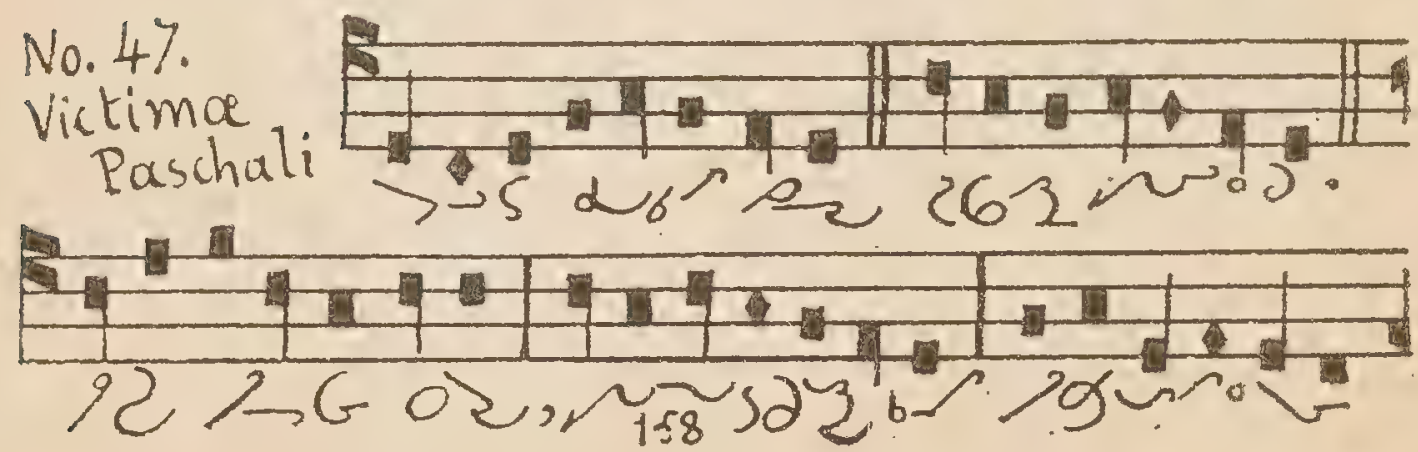
MANUAL.

$15 \mathrm{I}$
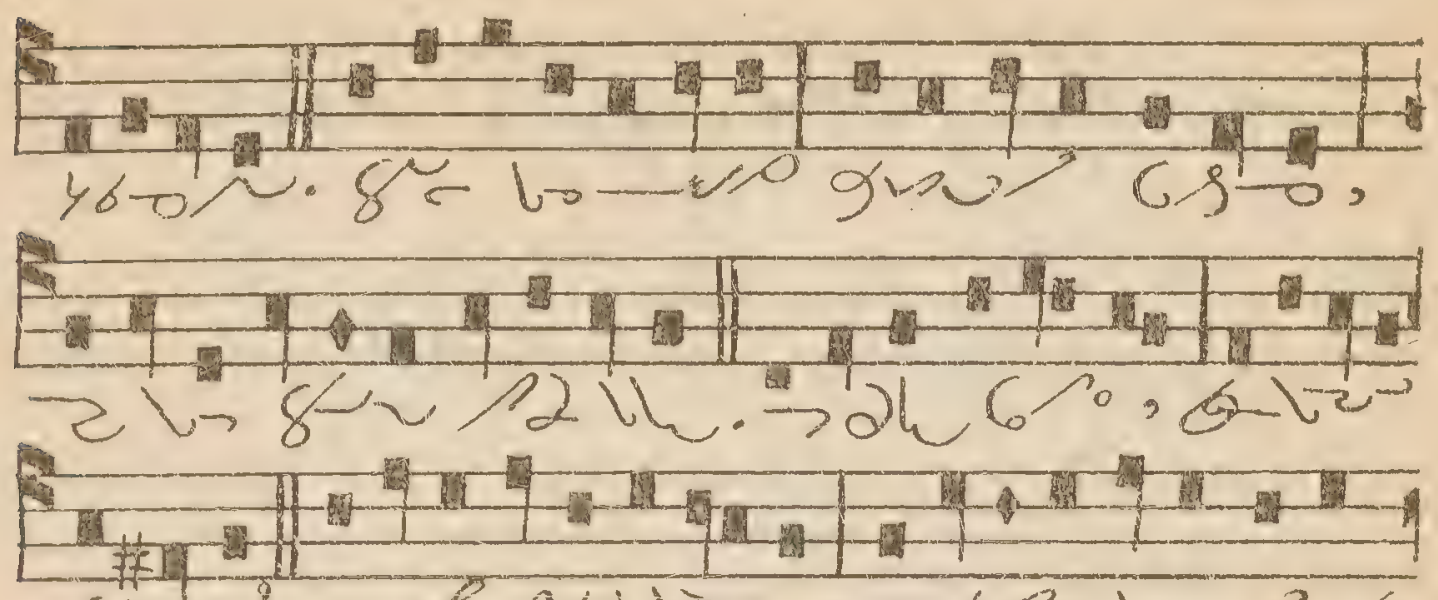

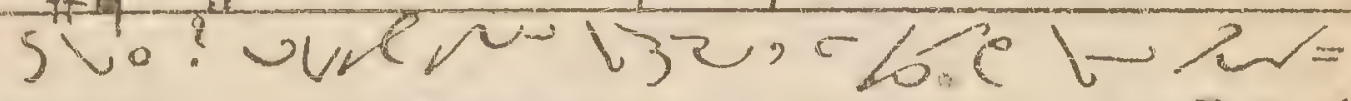

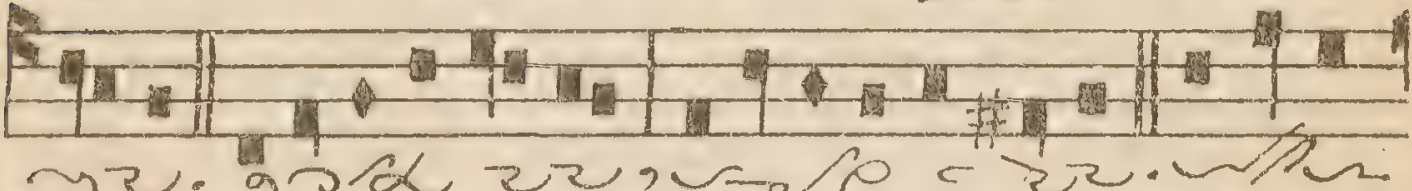

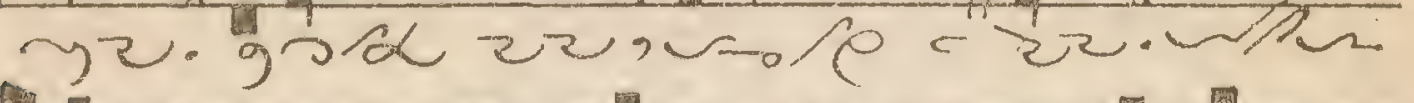
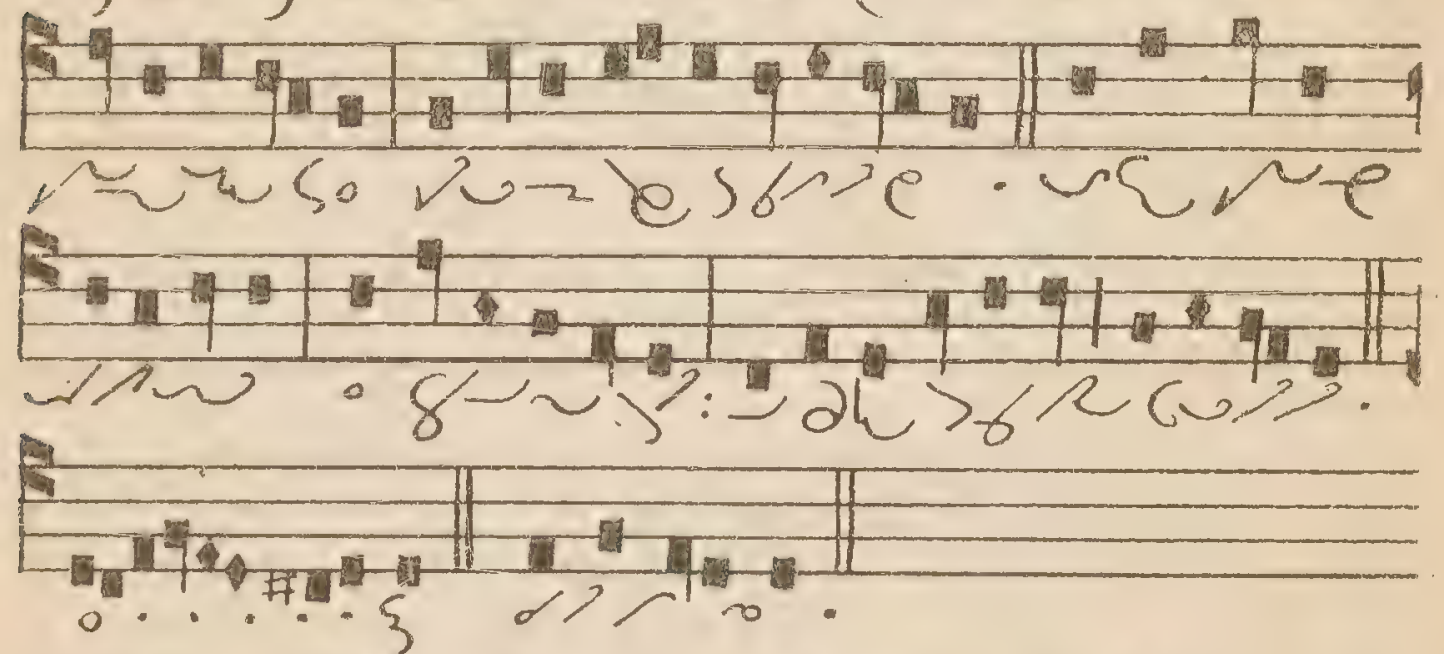

No. 48 .

ofilii et filia.
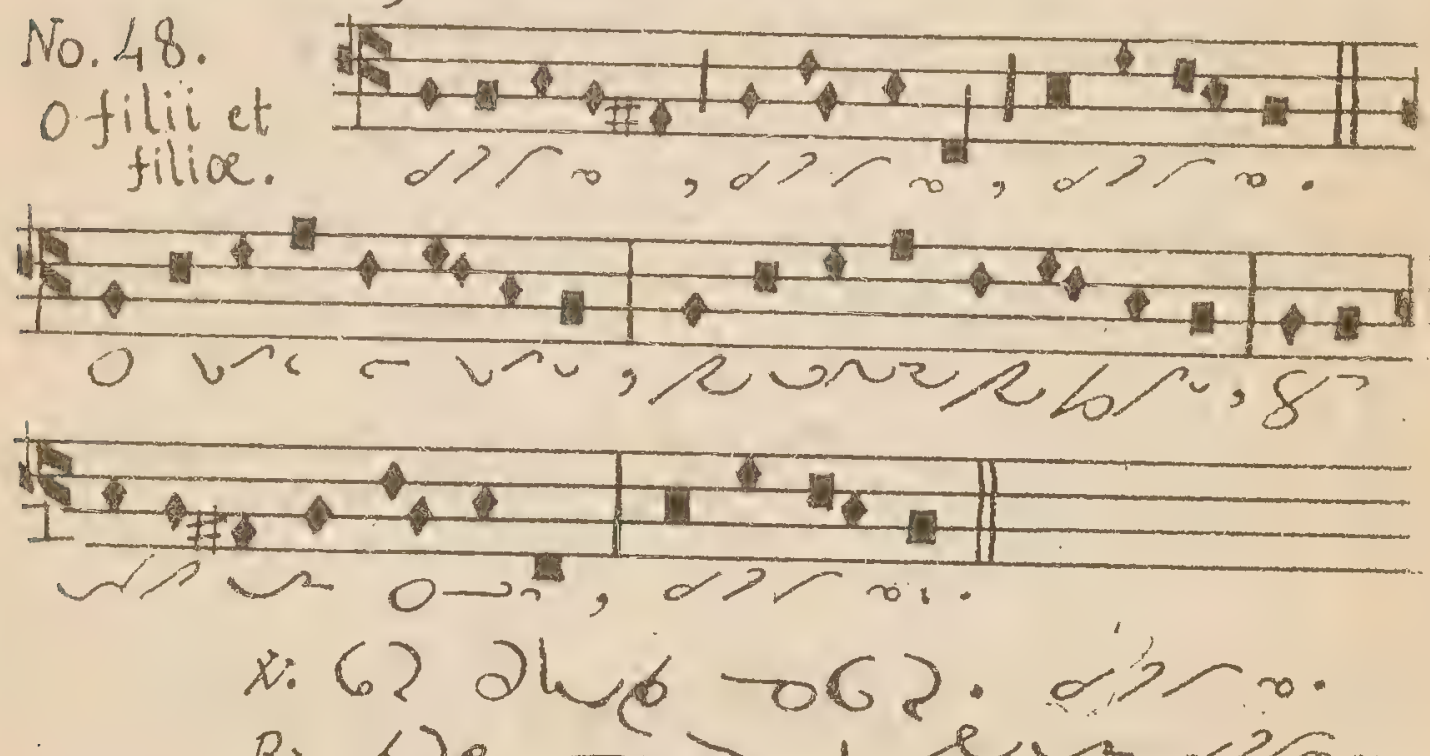

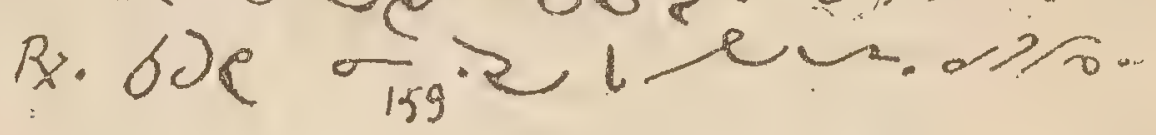




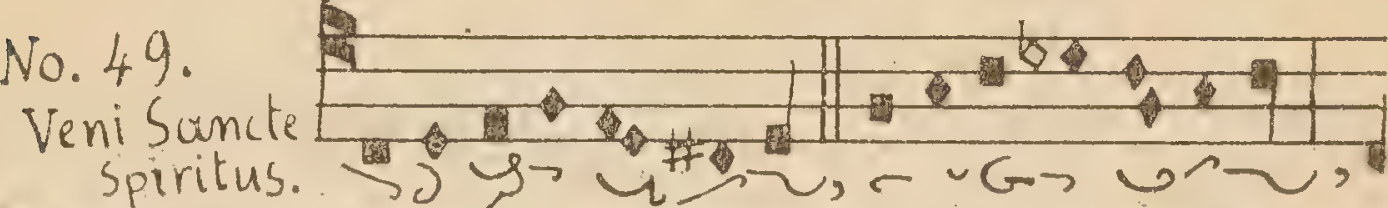

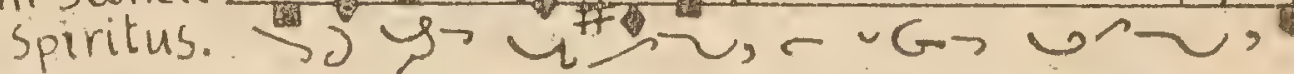

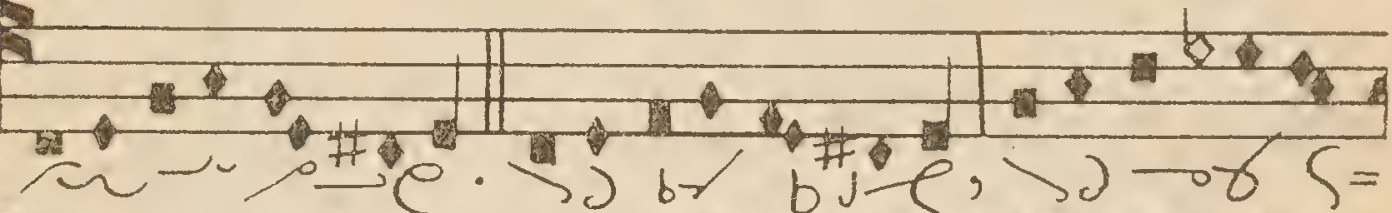

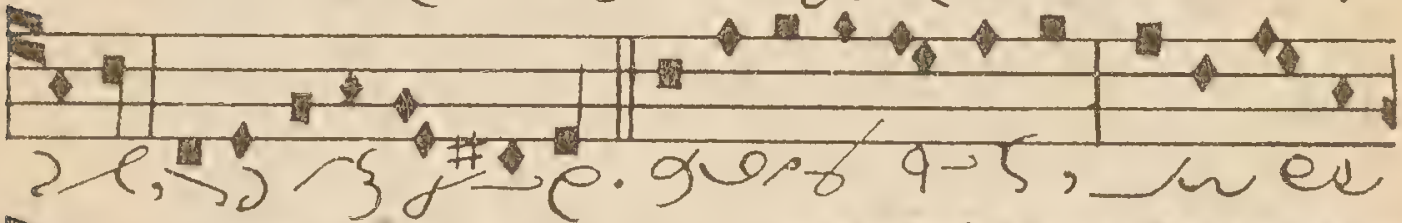

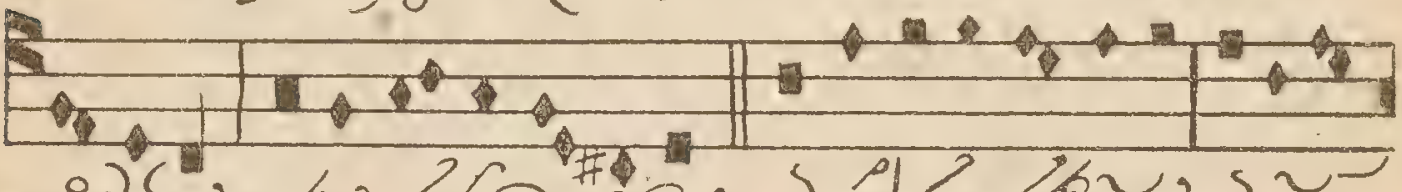

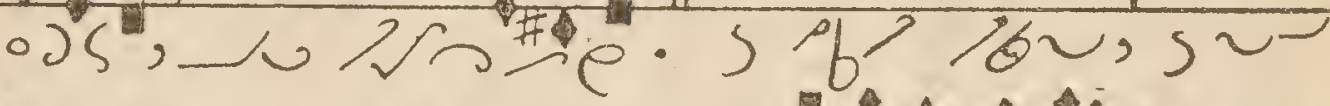
P. कa.

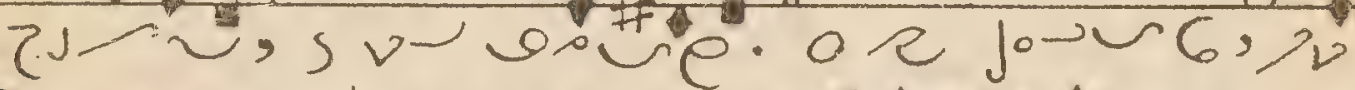

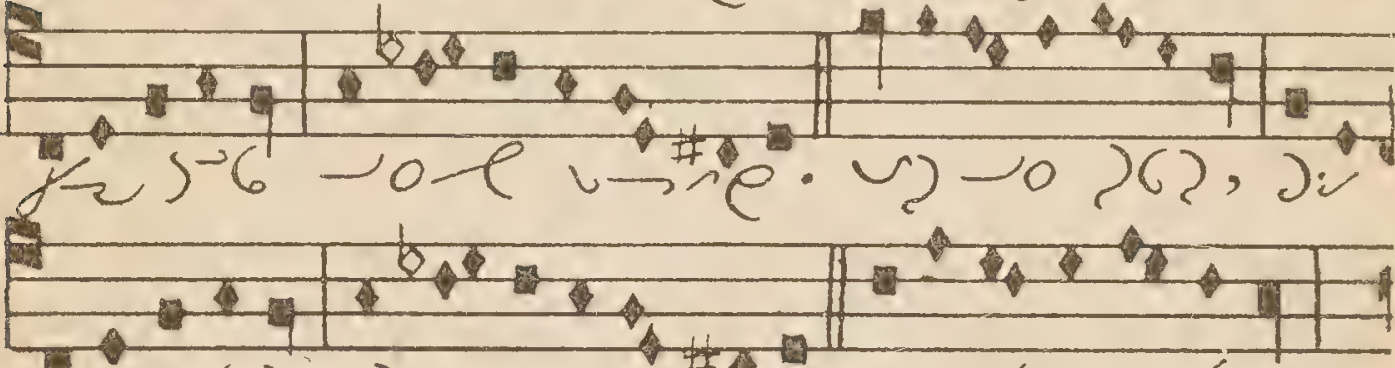

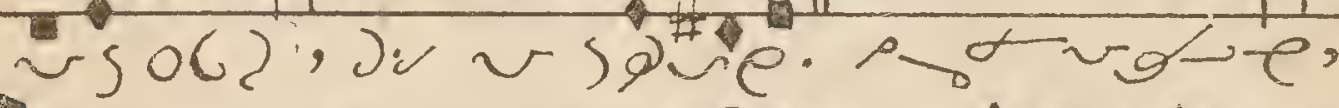

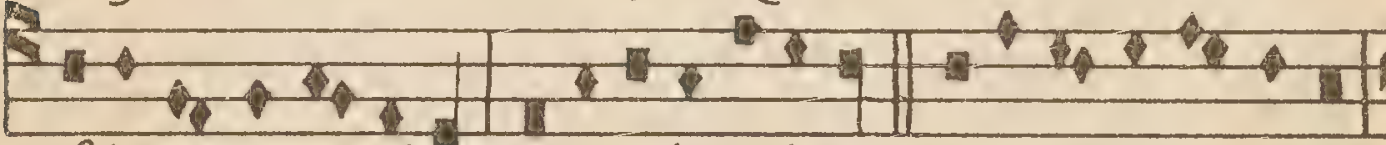

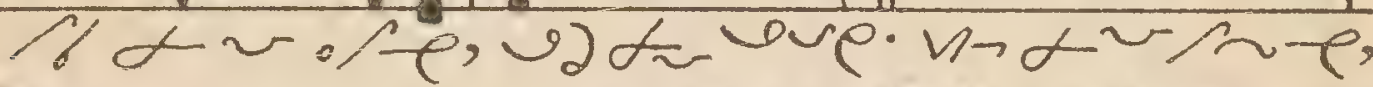

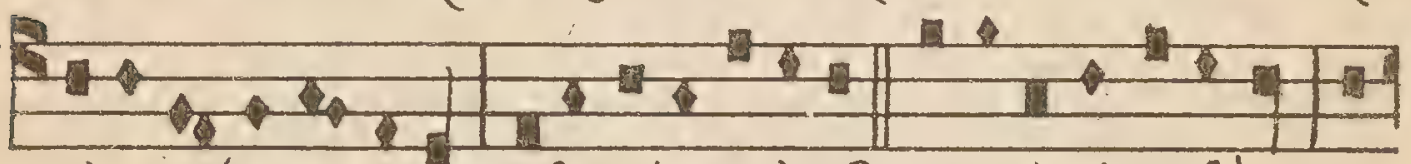

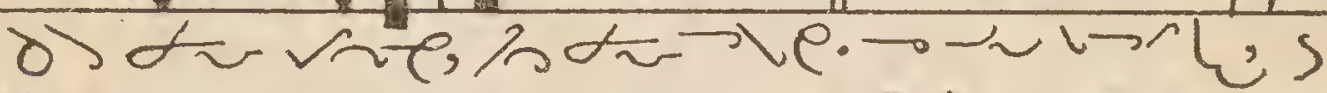

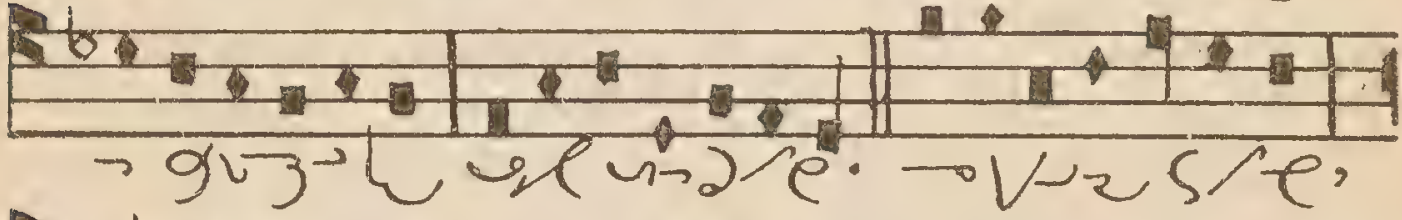

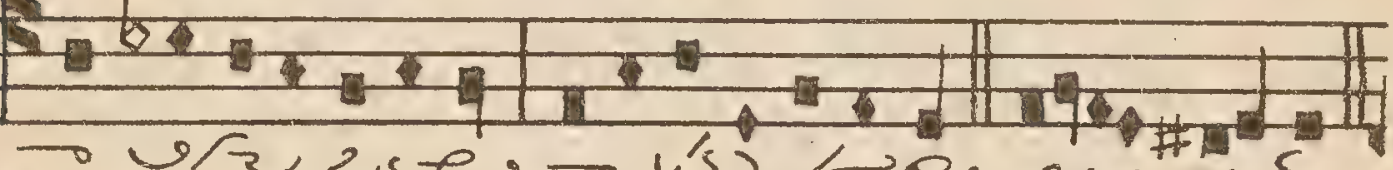

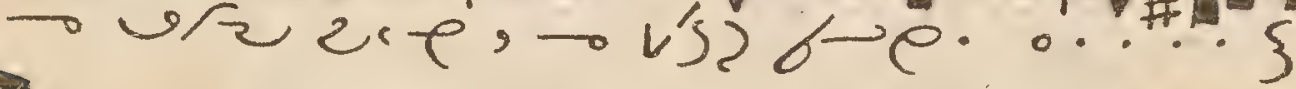

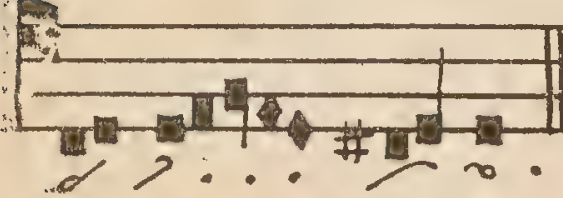

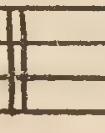




\section{Veni Creator.}
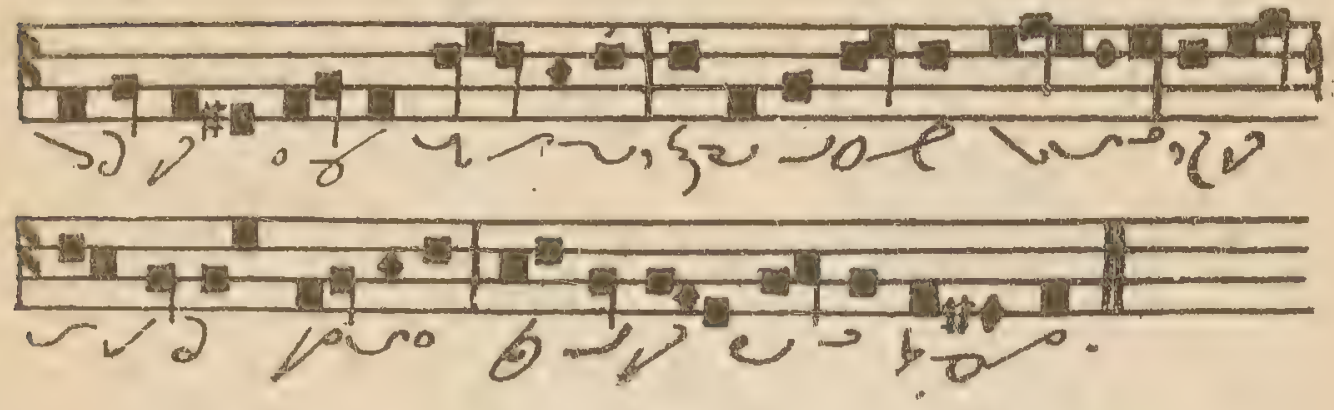

II

V.

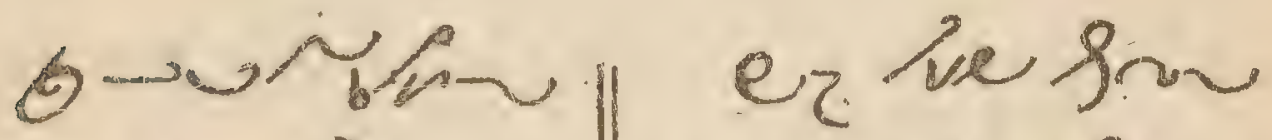

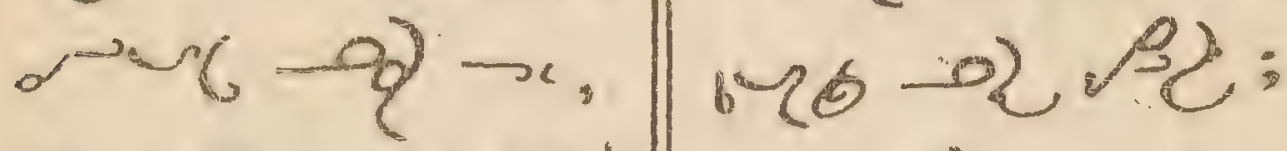

\& 612 f

- whrovo. ver e? de.

III.

Mus (2)

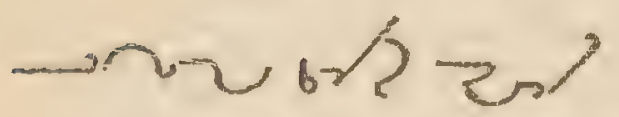

VI.

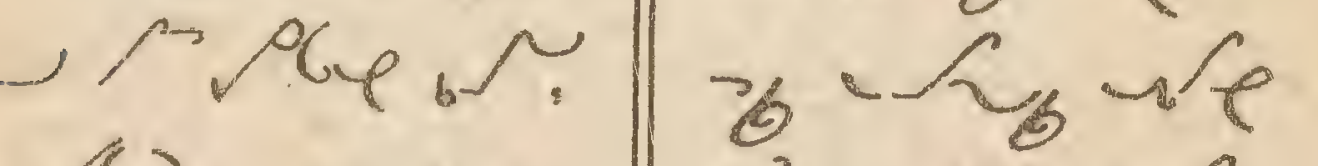

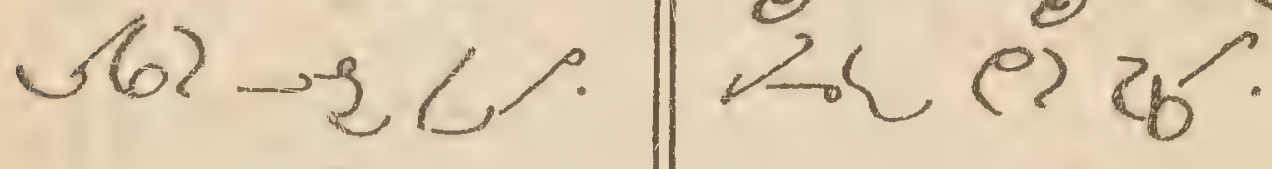

IV.

$2 y-r\{-34$

$3 g \rightarrow 6<\alpha^{2}$

VII

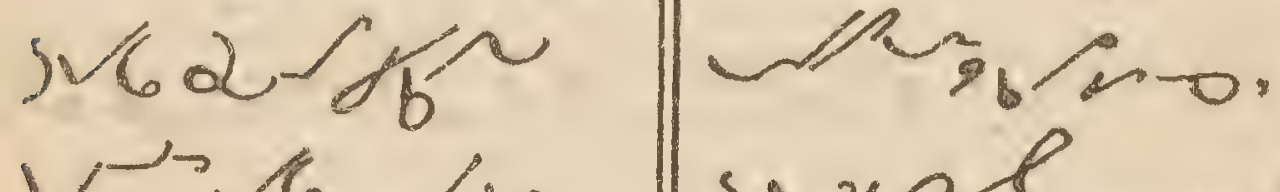

\section{व)}

$2<\approx$ क

to a for

$-10608^{2}$

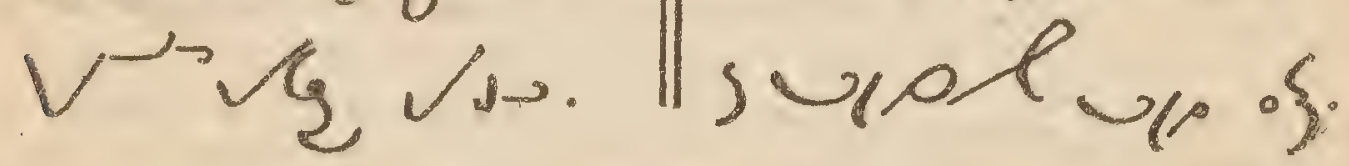


Pange lingua

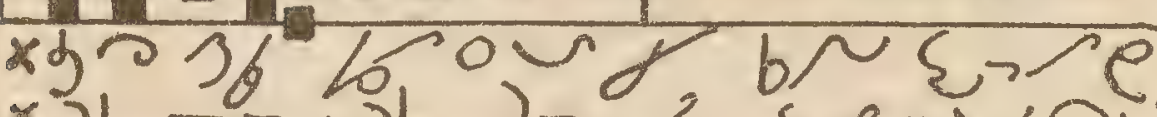

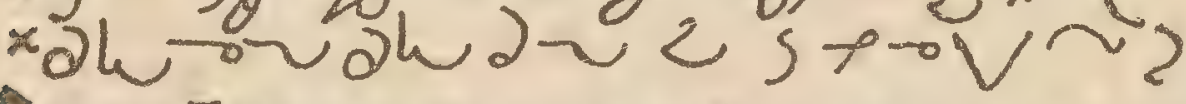

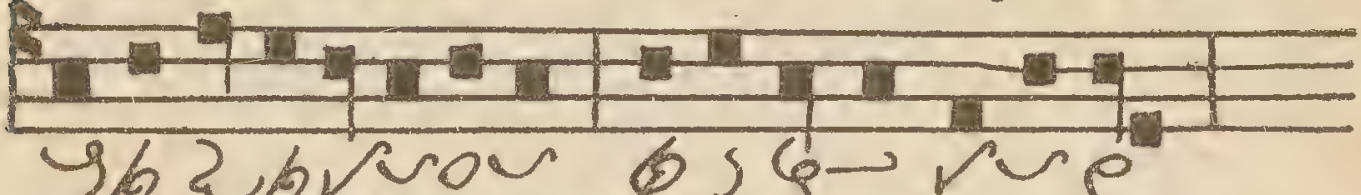
96200kon gsg rue -5 GogVur Jou UG?

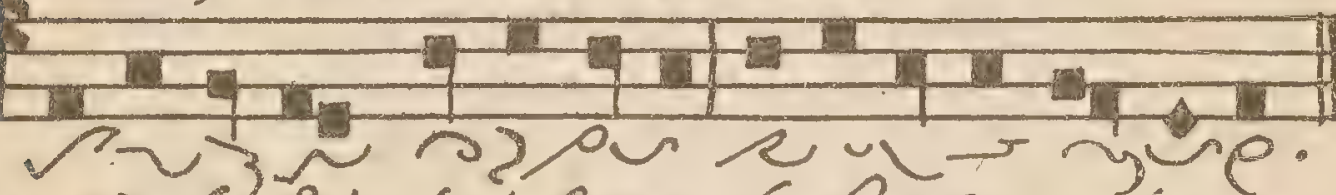
$\because 6 \%$ sopu 6 poung

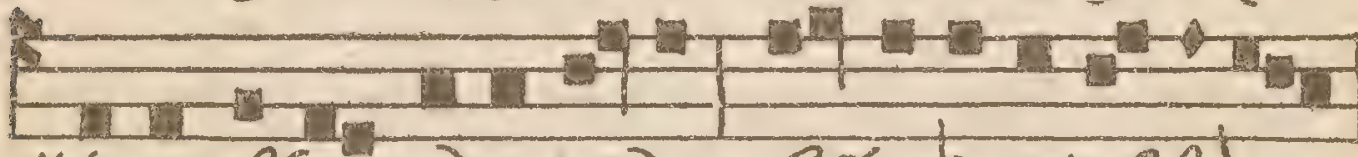

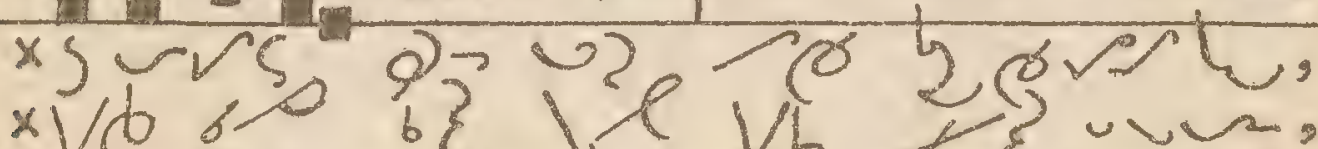

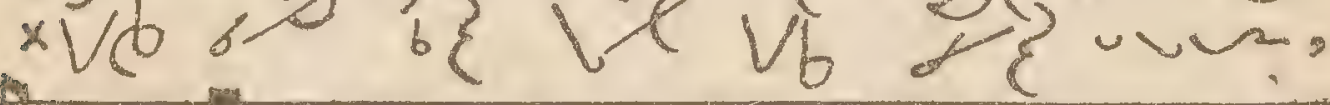

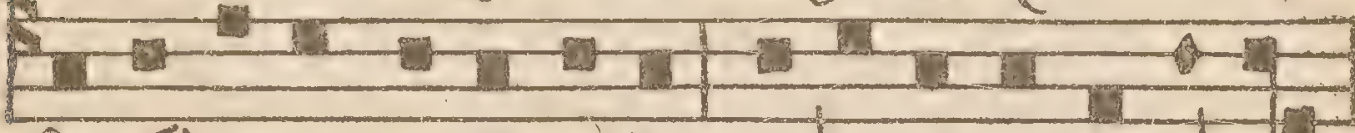

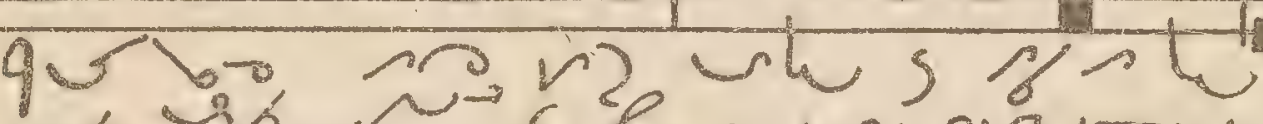

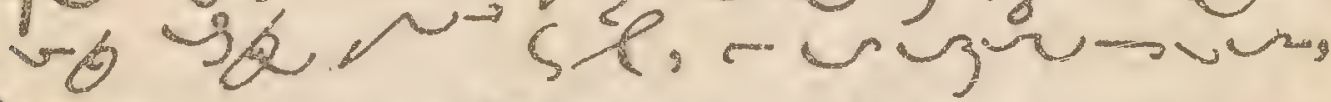

1

1010 ?

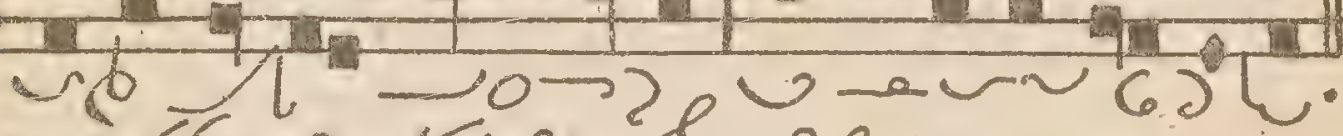
argte duol us uruvin.

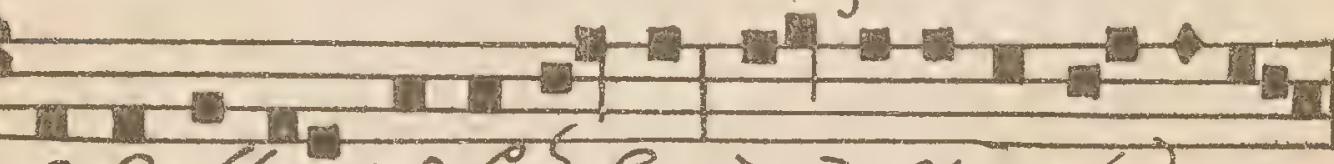

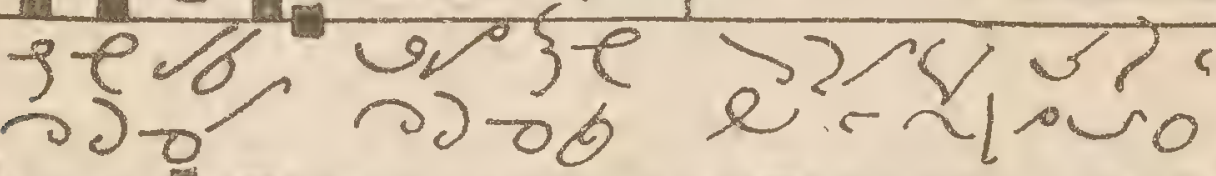

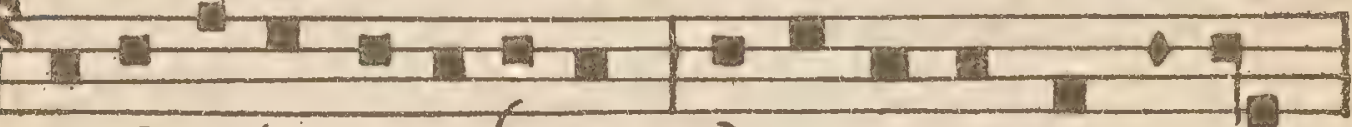
- -40 ol ir of $v \sim 66 \backsim-12>-1$

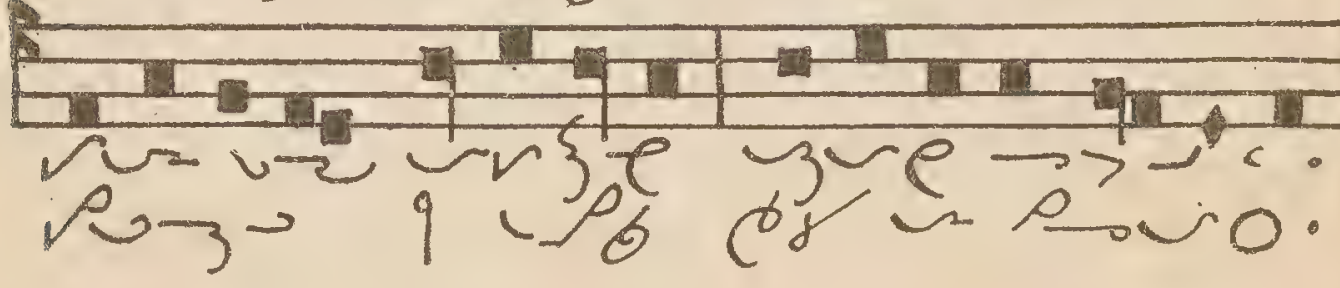




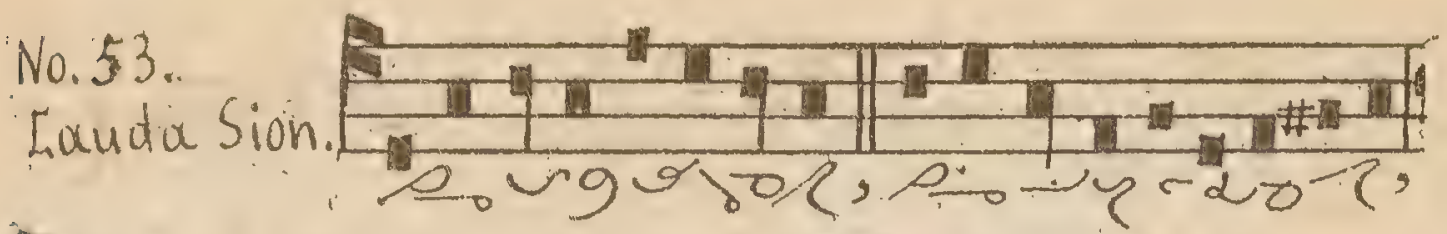

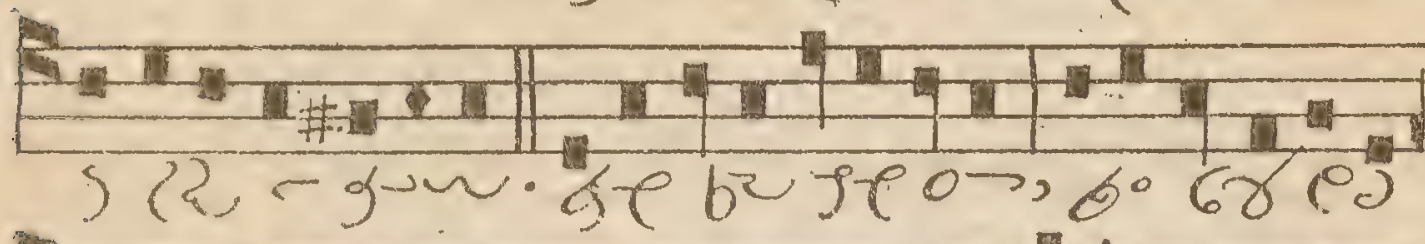

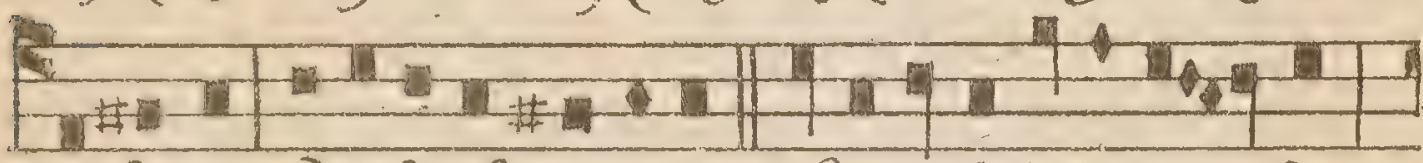

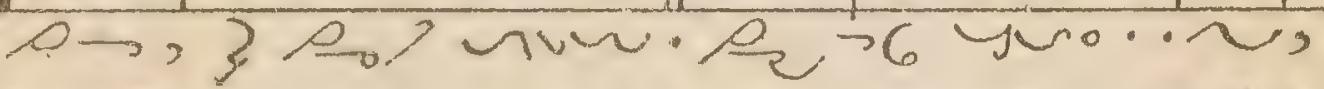

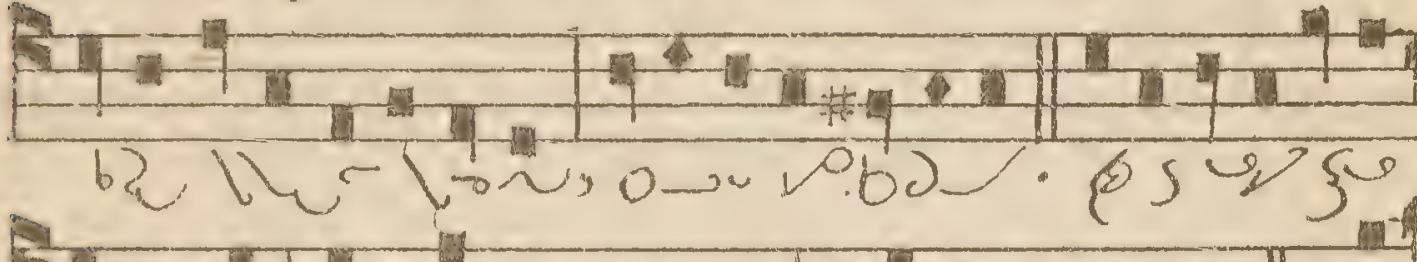

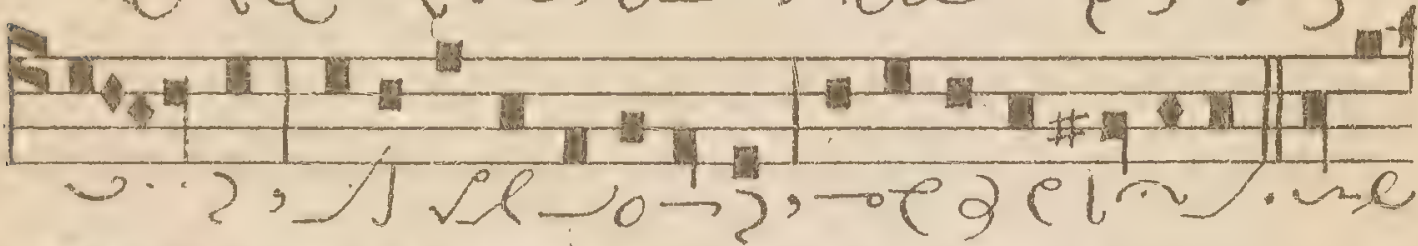

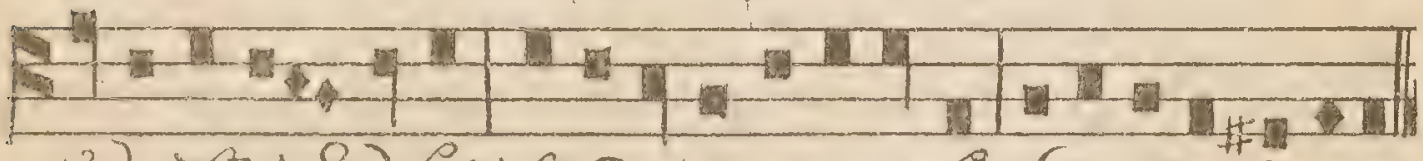

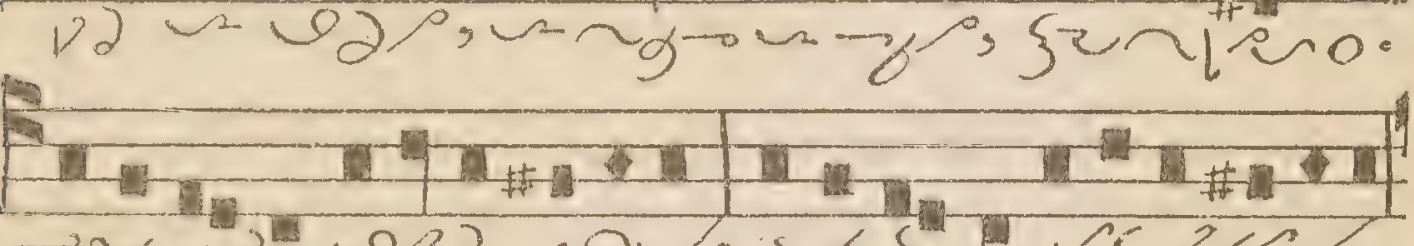

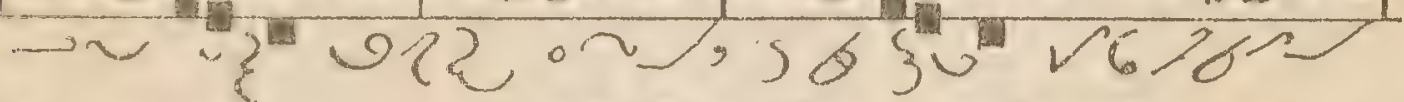

Fon

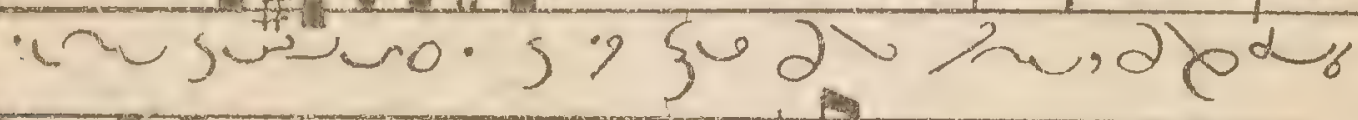
5.

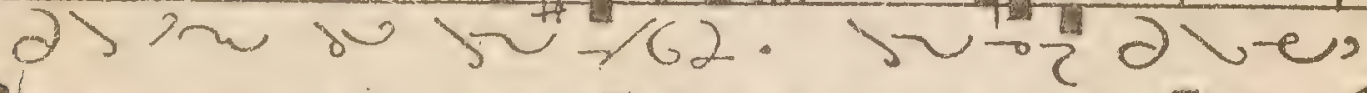

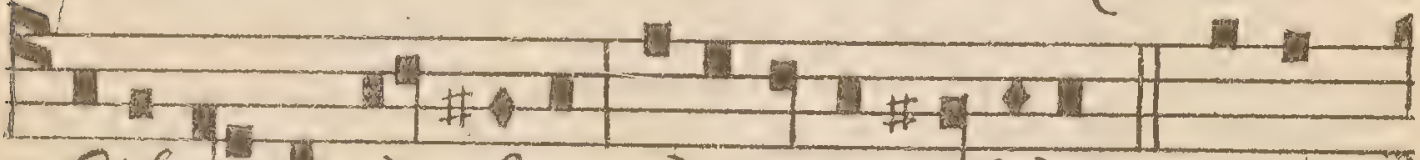

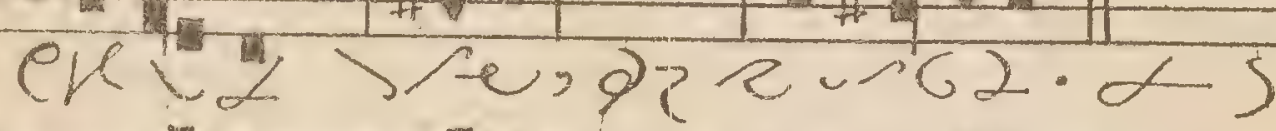

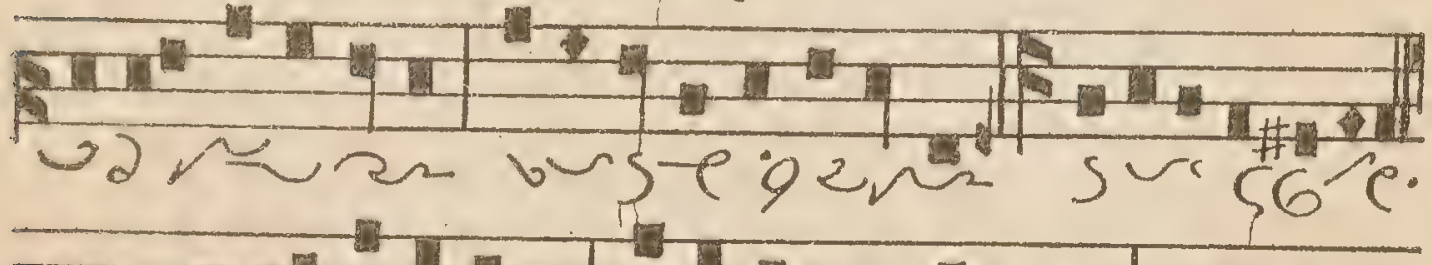

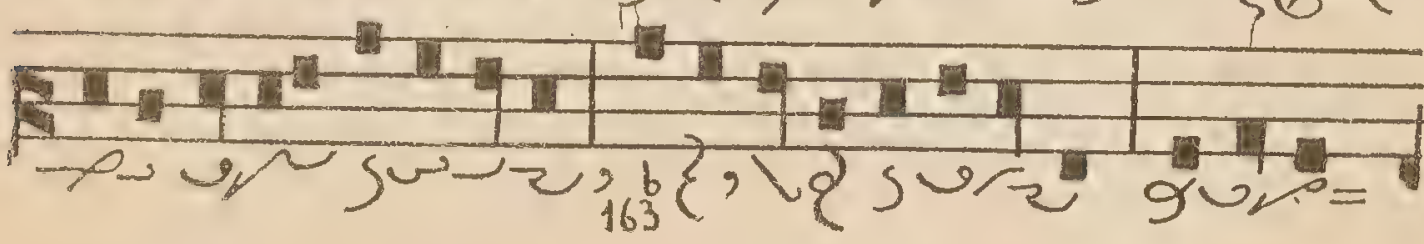




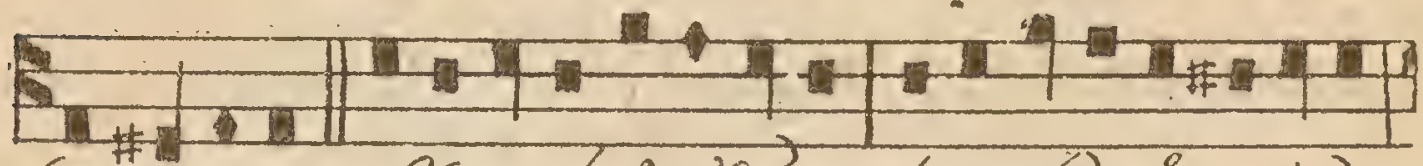

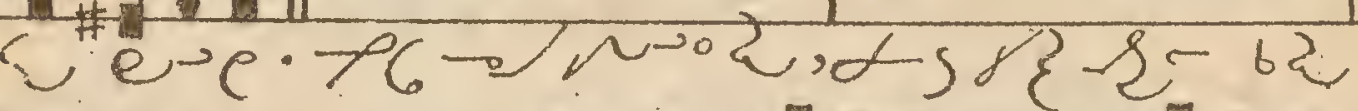
2I -

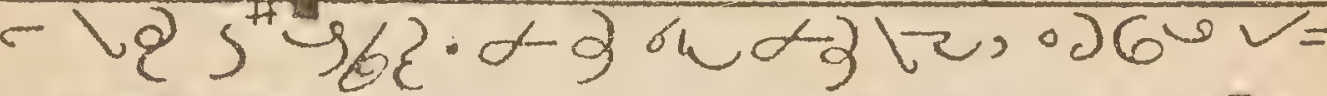

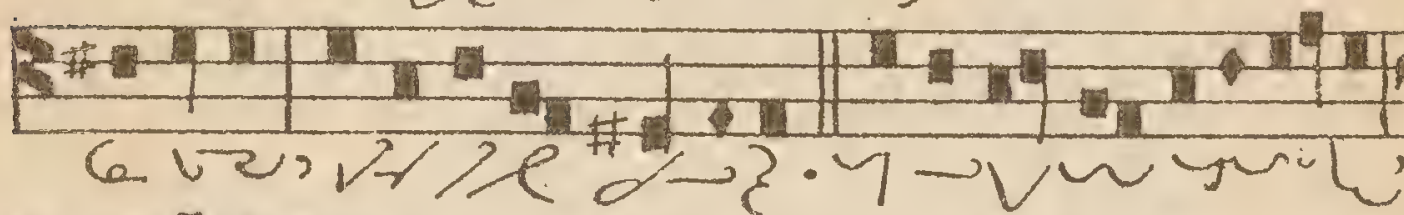
-1 - 1

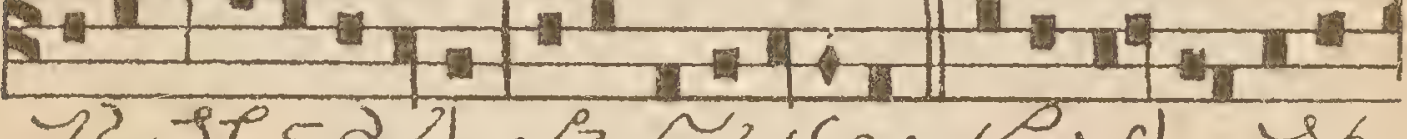

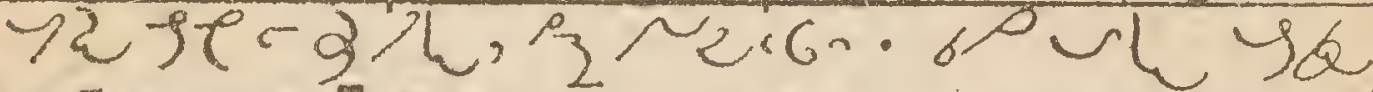

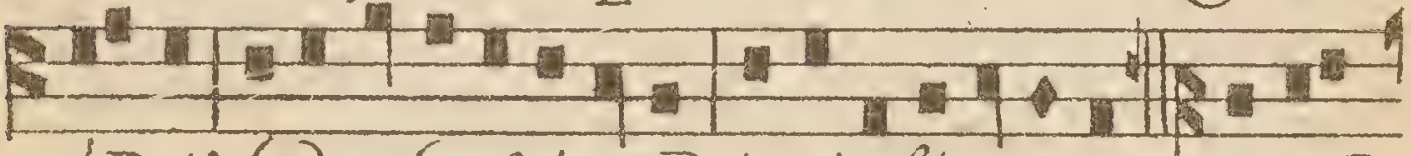

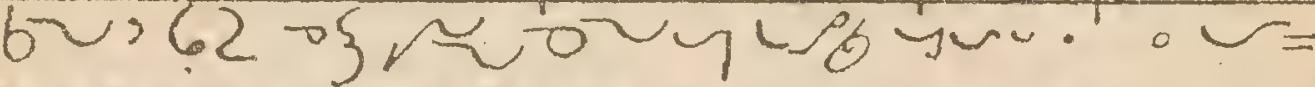

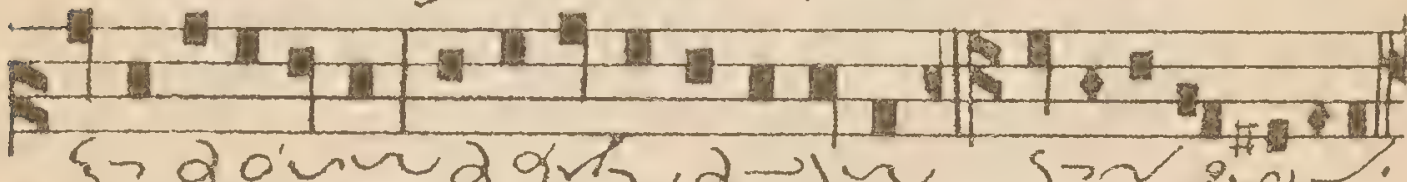

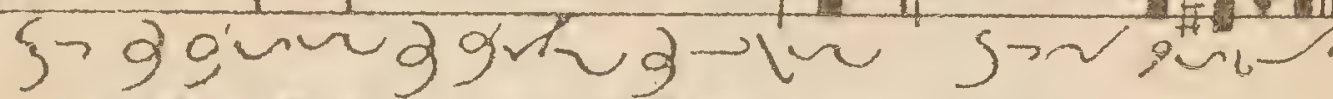

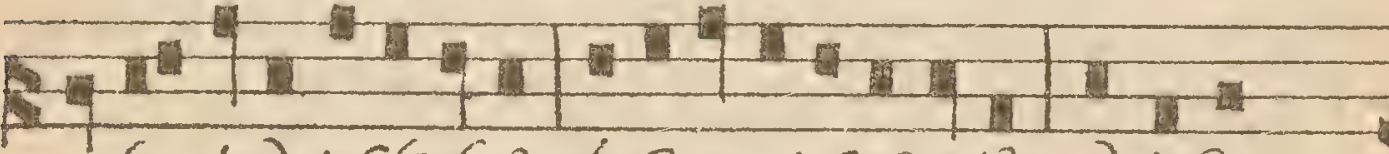

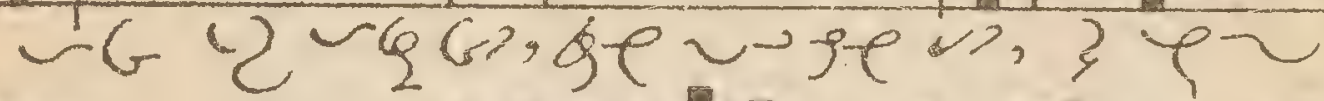

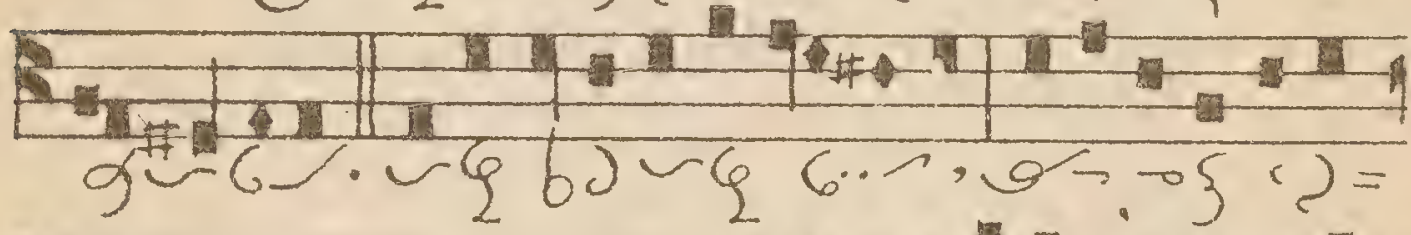

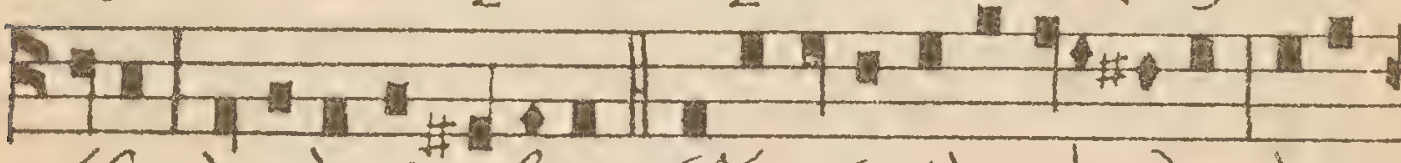

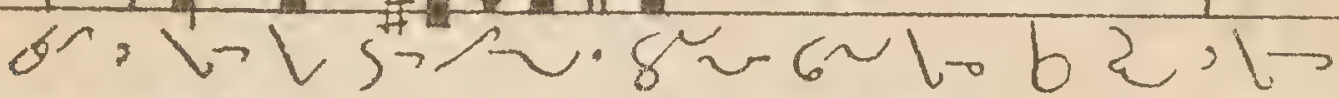

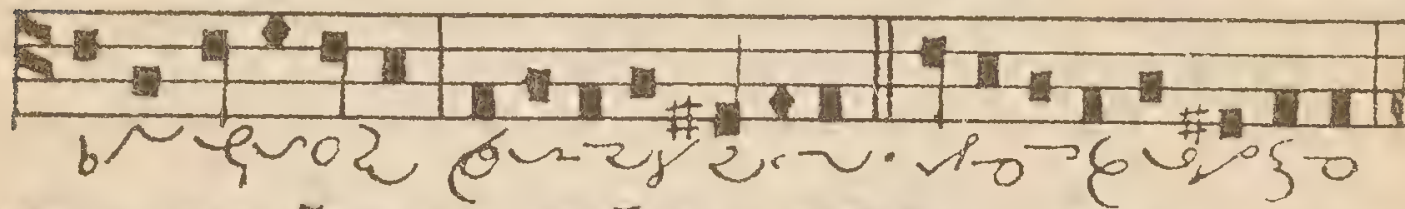

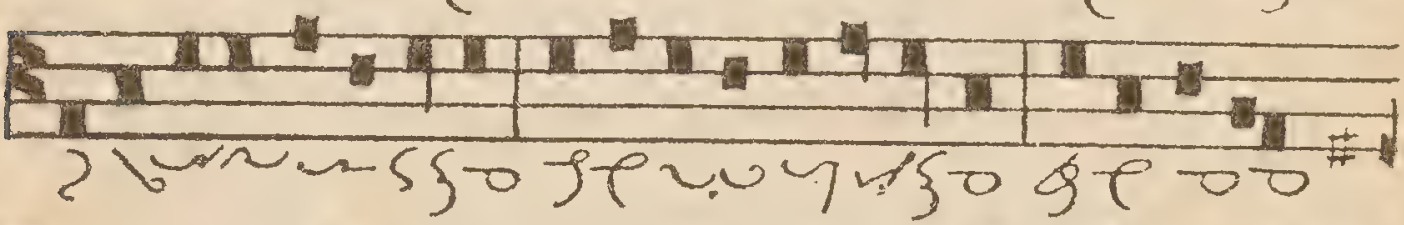

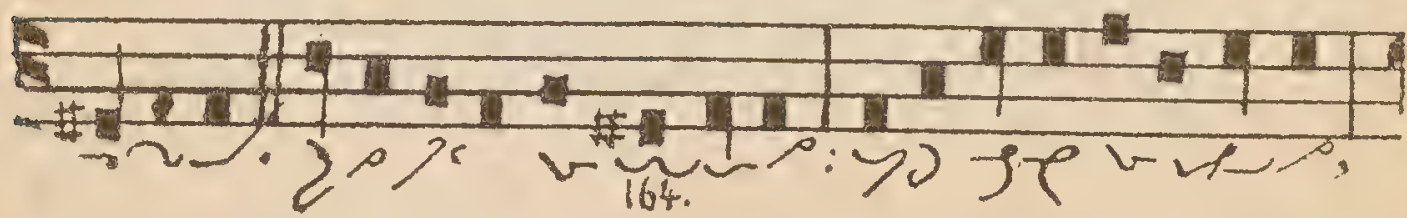




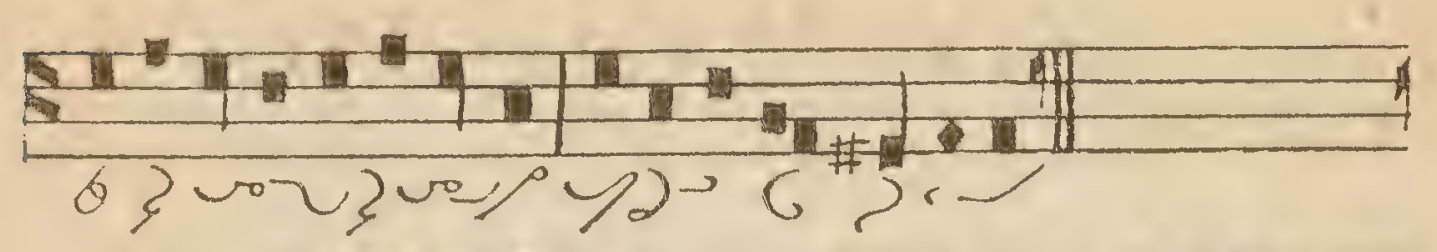

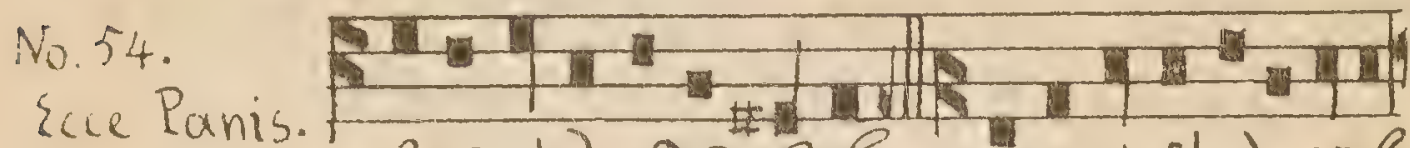

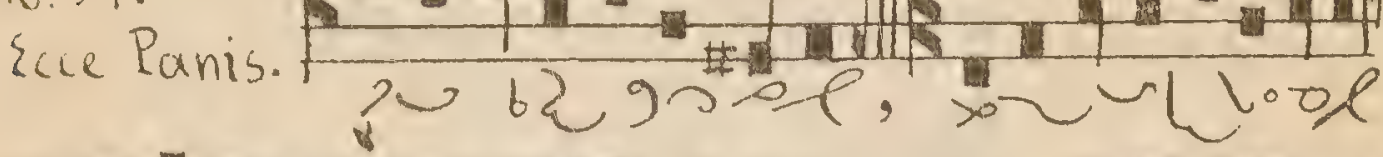

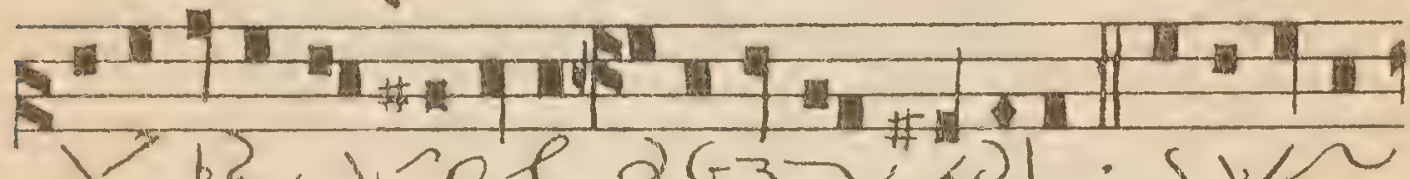

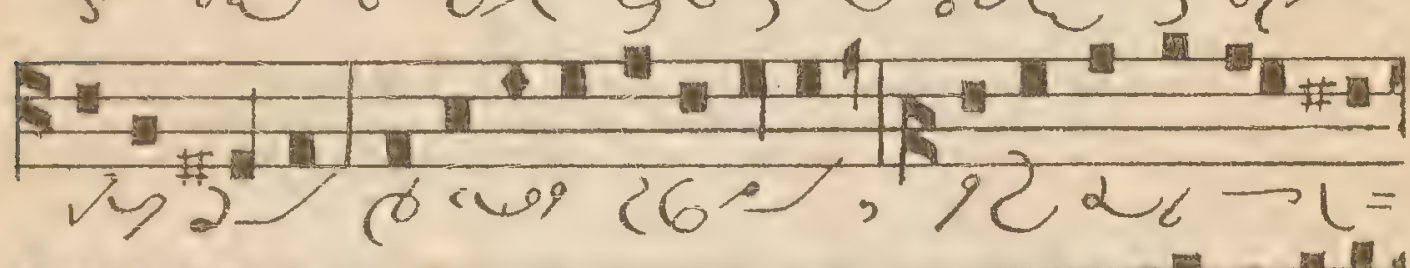

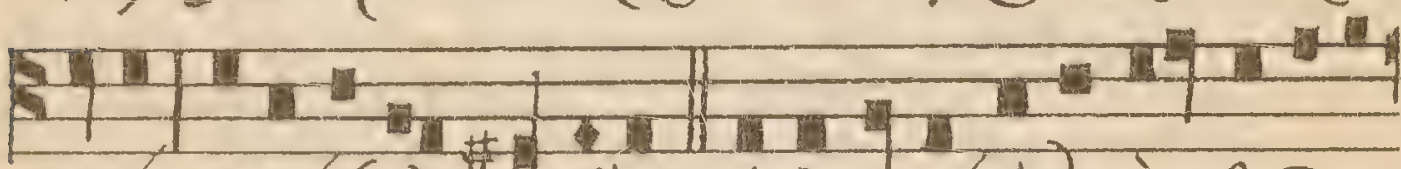

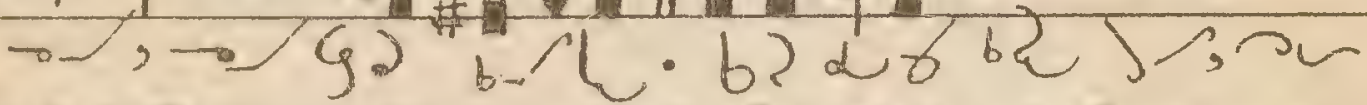

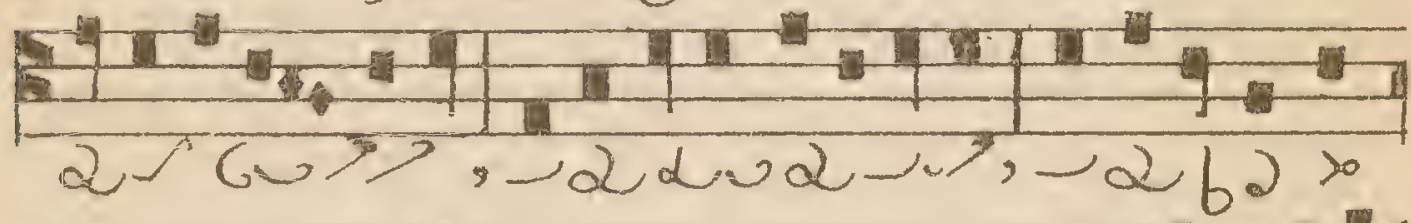

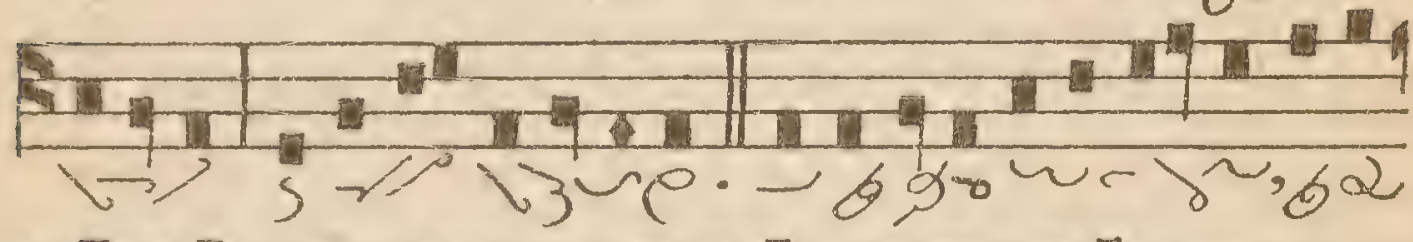

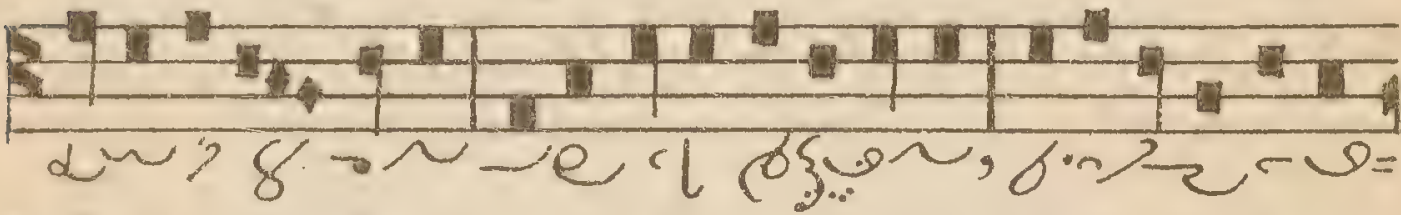

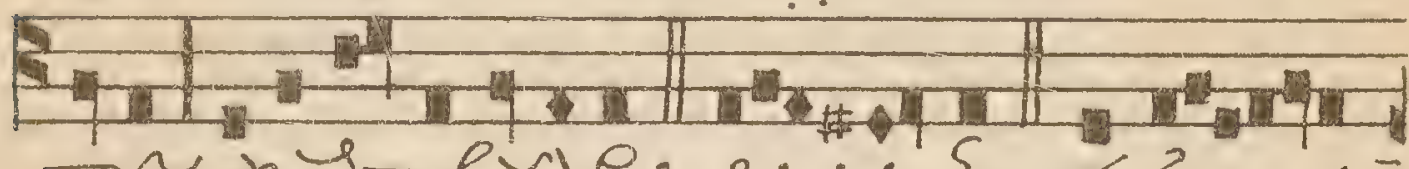
or so golve.... $\xi_{0}$, >....

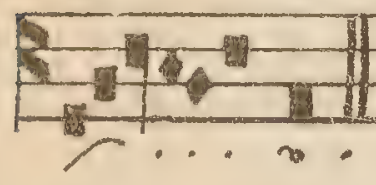

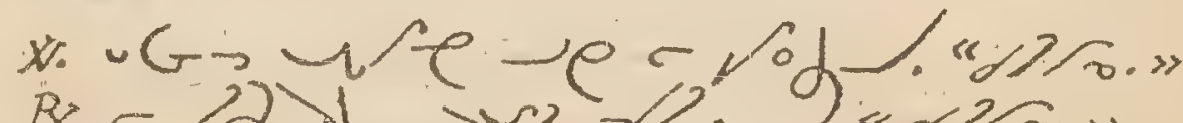

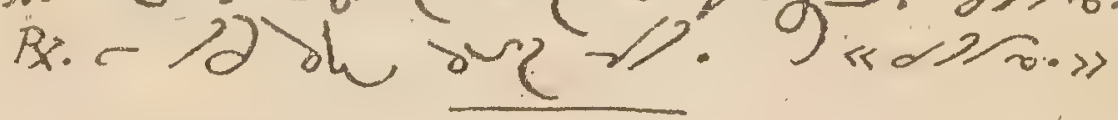

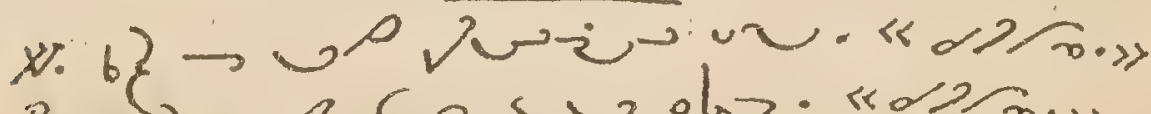

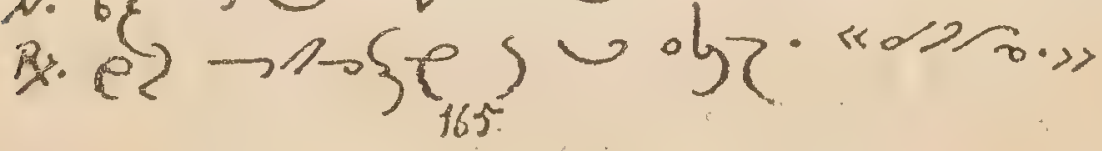


So. 55 Panis $A_{n}=$ gelicus.

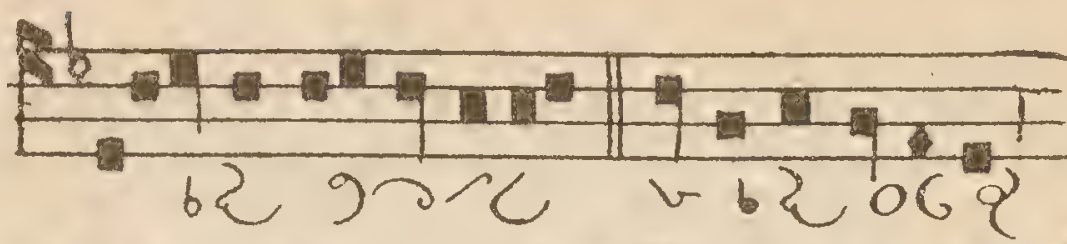

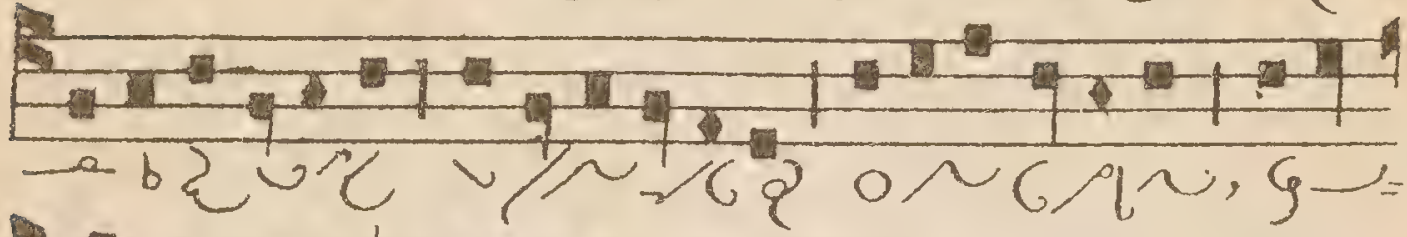

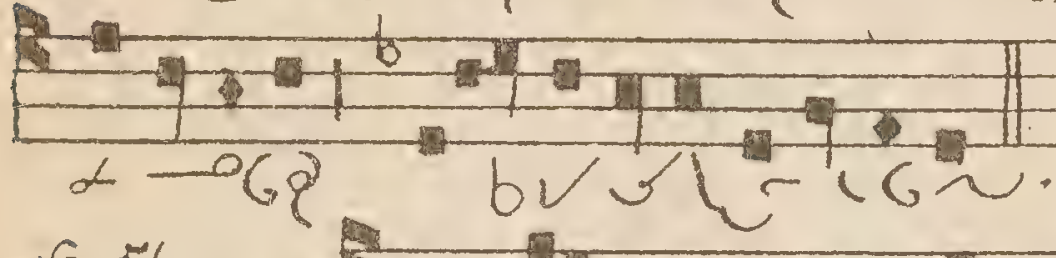

$\$ 0.56$.

Verbum - In

Supernum Ve \& $2,2, ?$

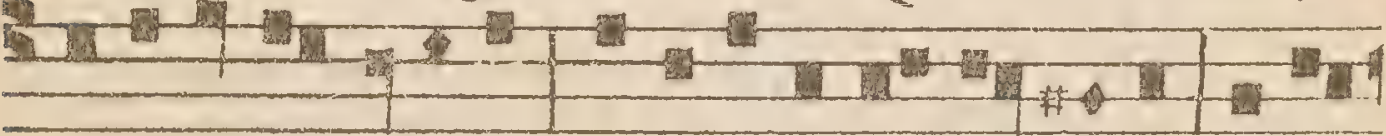

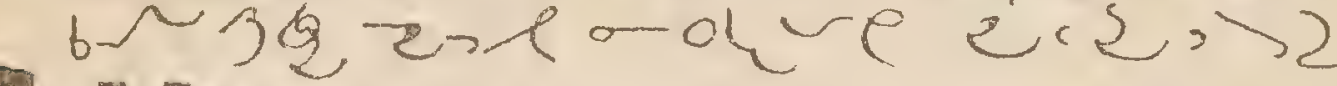

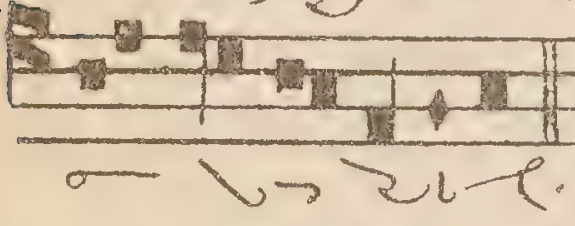

No.5\% Adoro te devote.

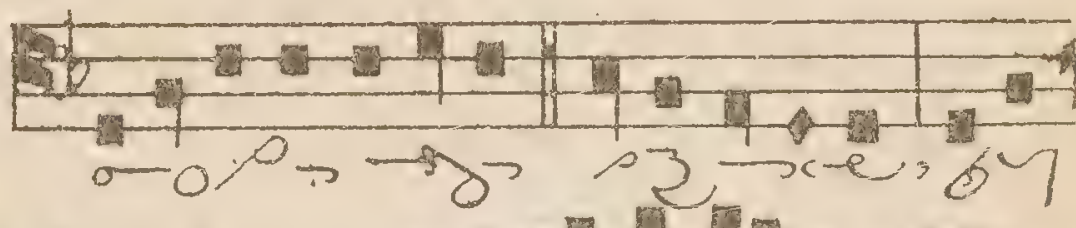

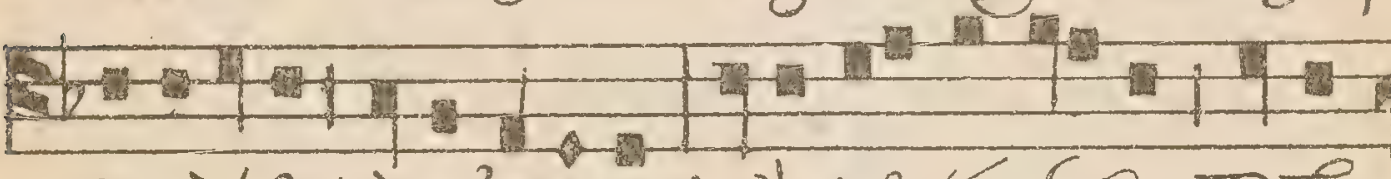

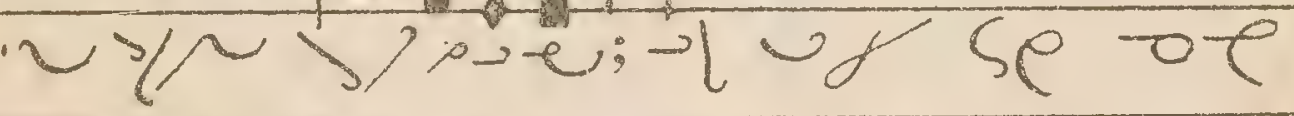

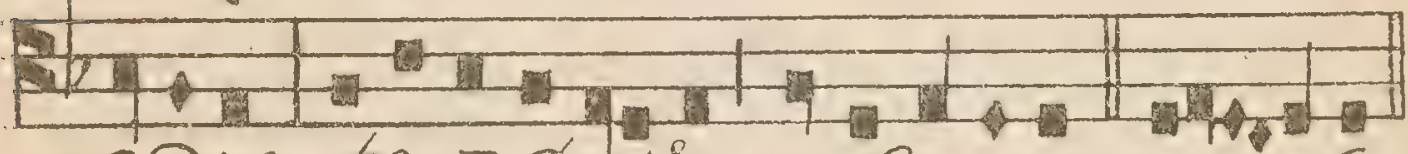
7ru $60 \rightarrow 9212$ op-sus. 0.5 .

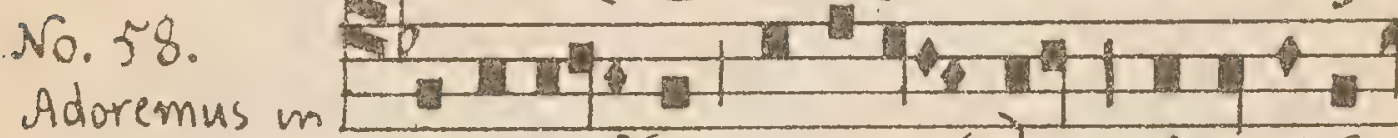

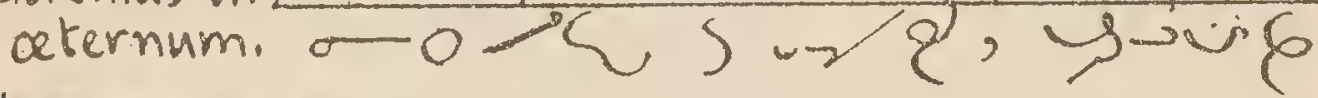

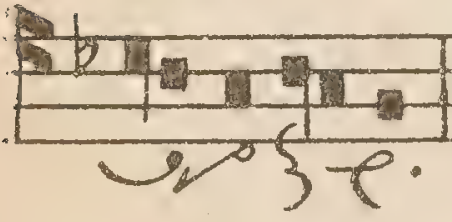


No. 59.

TeDeum:

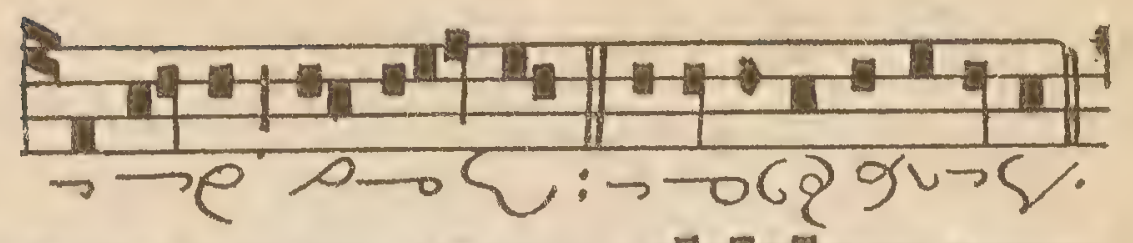

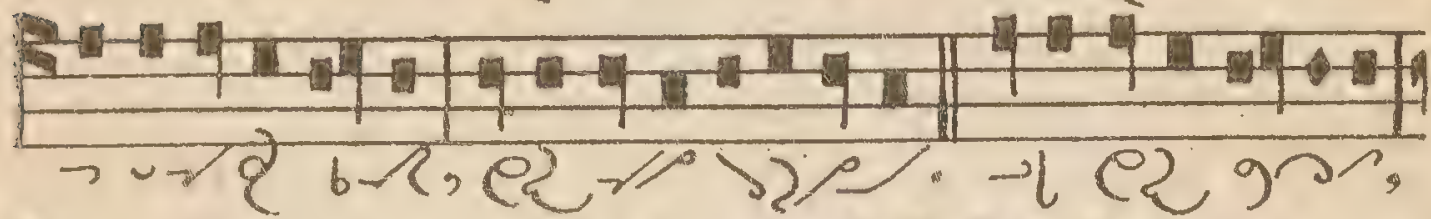

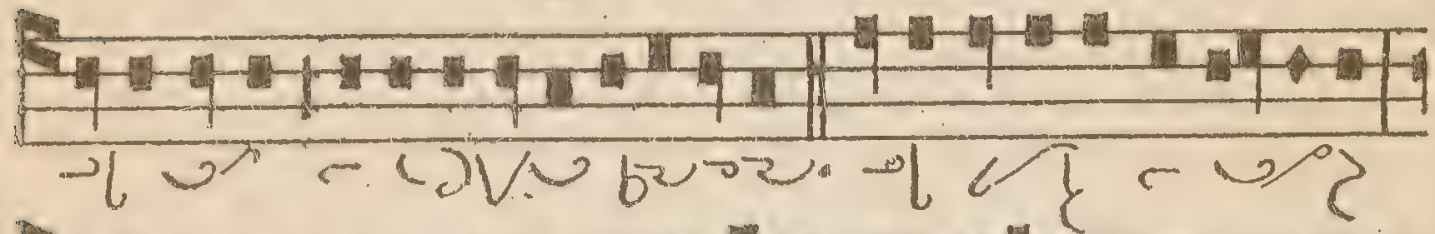

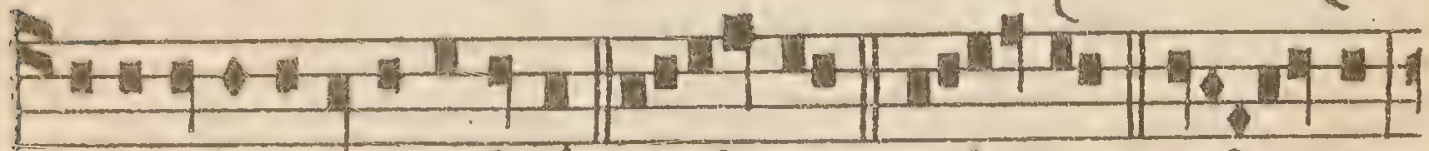

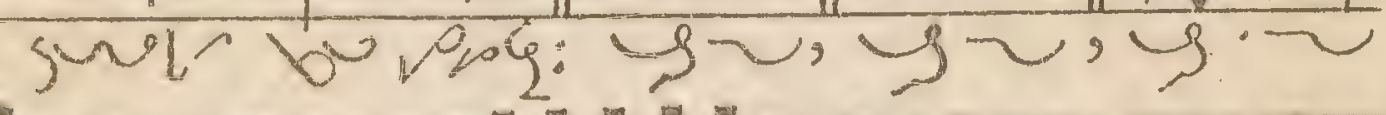
हैor a

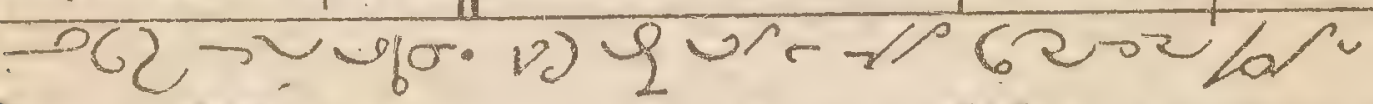
E. In

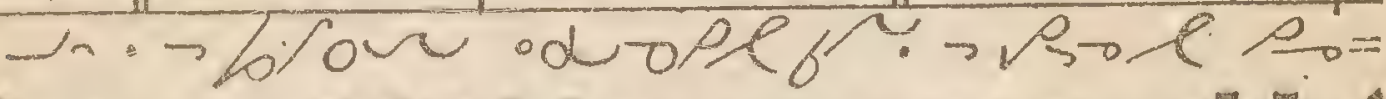

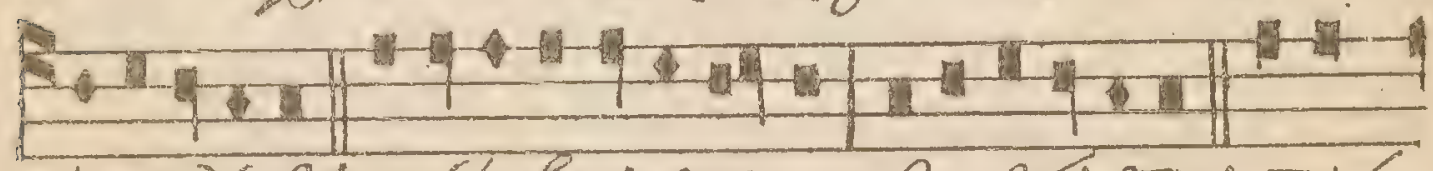

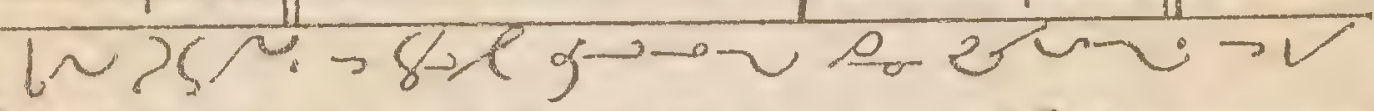

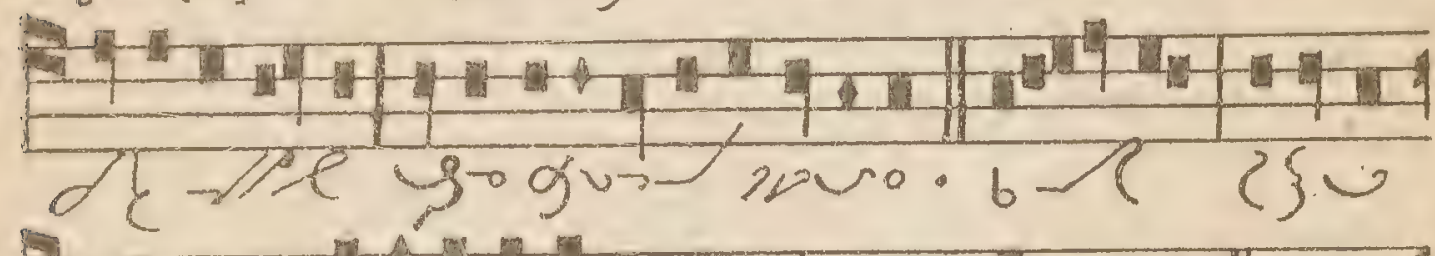

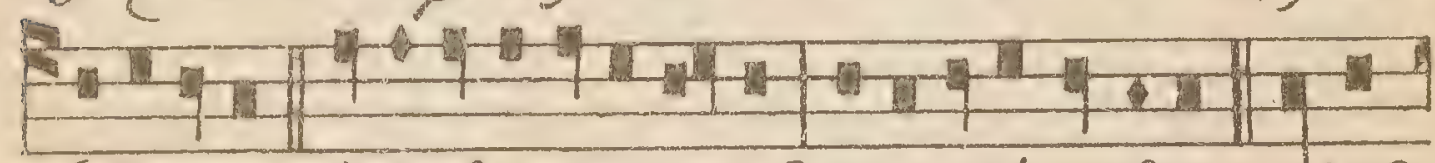

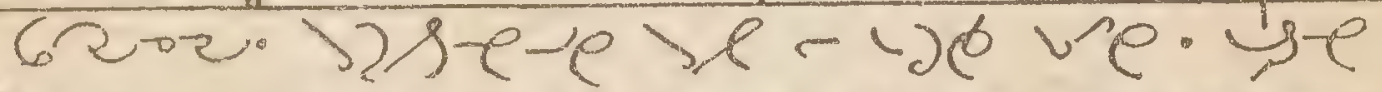

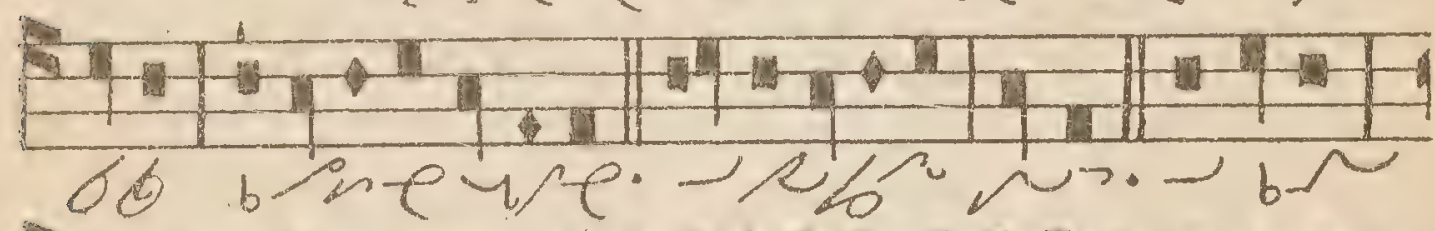

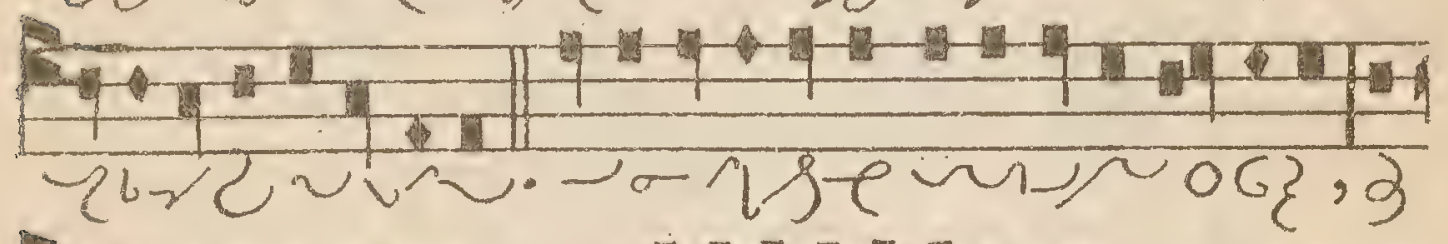

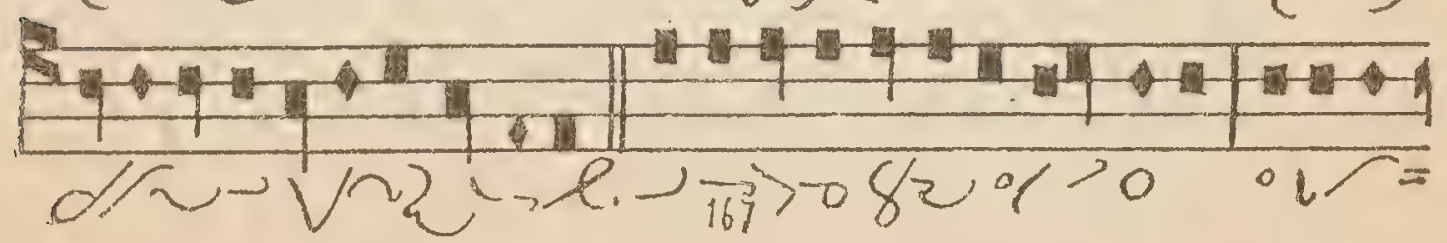




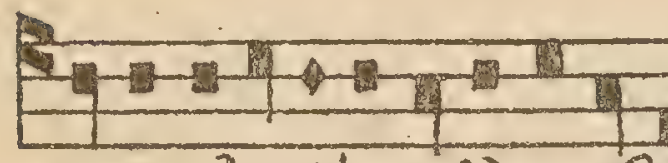

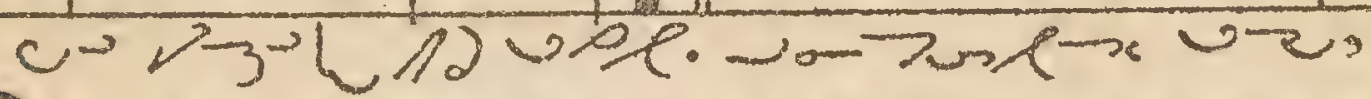

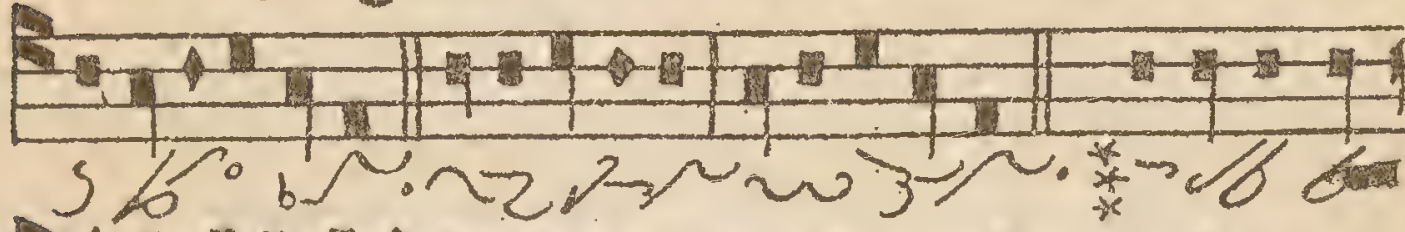

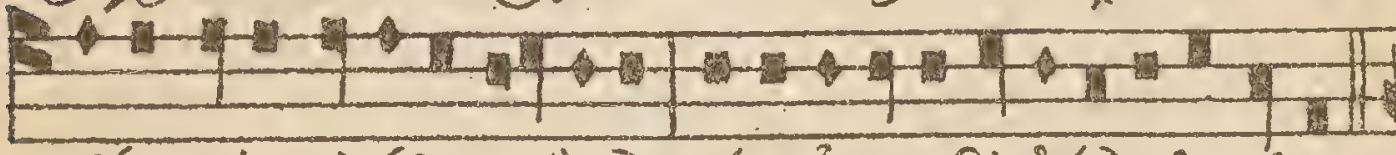

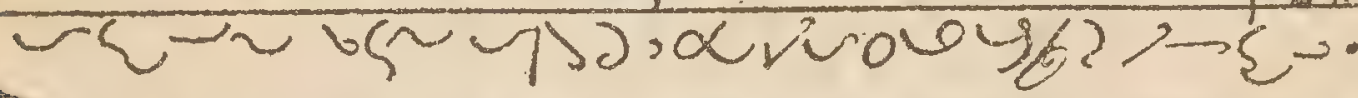

2

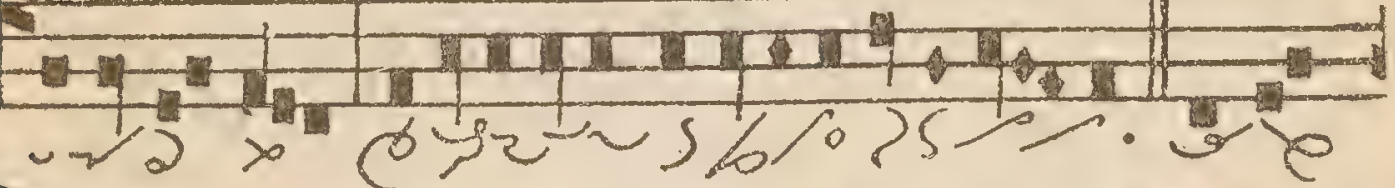

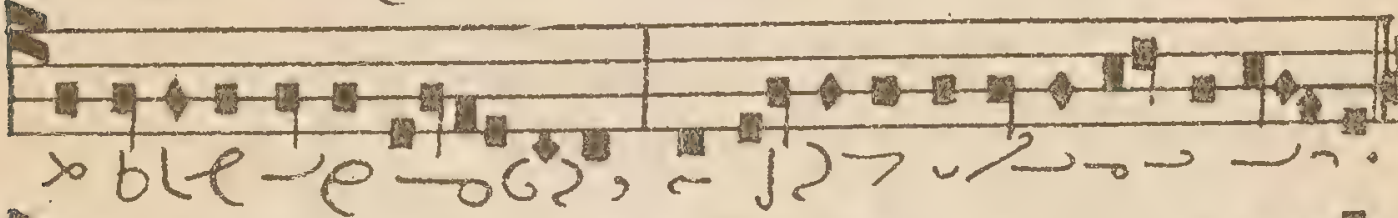
s

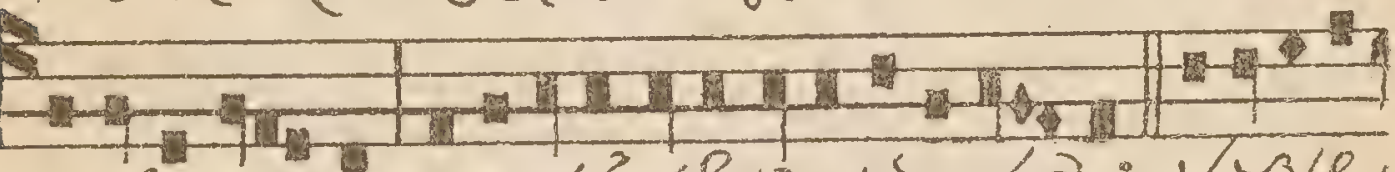

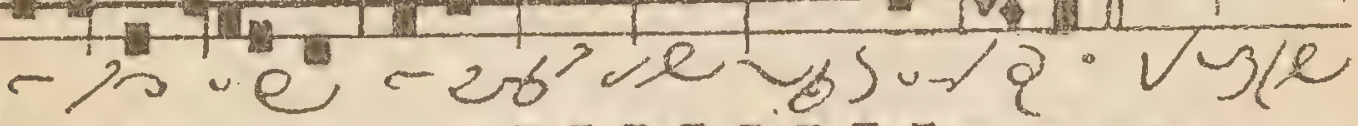

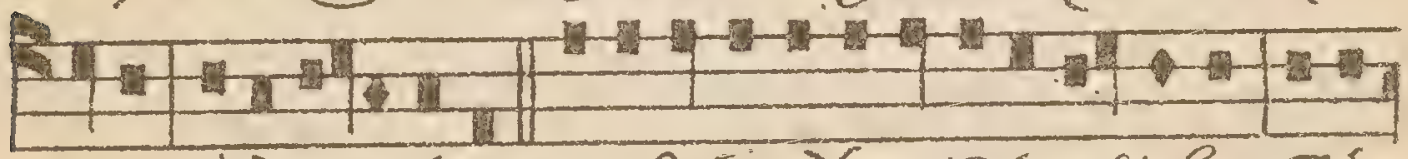

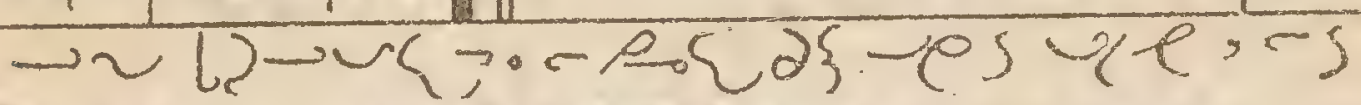
E! yoll $v(e \cup(x \cdot 7)>06) \rightarrow \sim 0 \sim 2460.2$

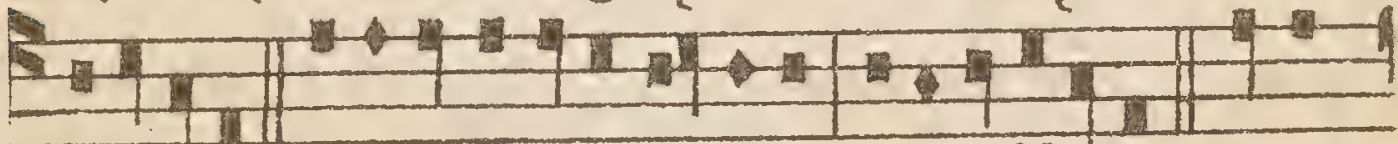
$(0 \rightarrow / \cdot G) 2 /-0, ?, G) 2 / \cdot w_{0}$

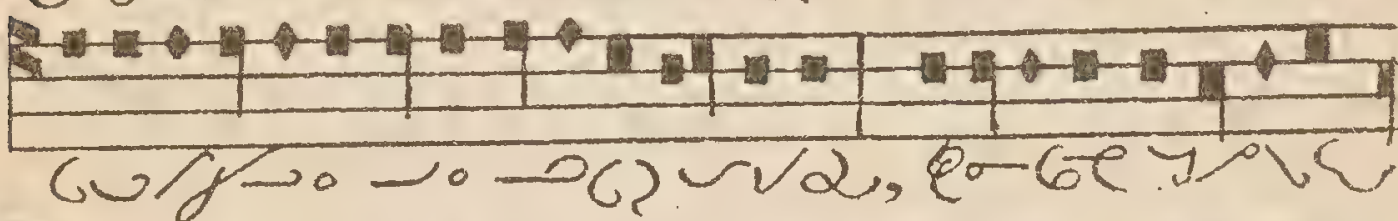
.

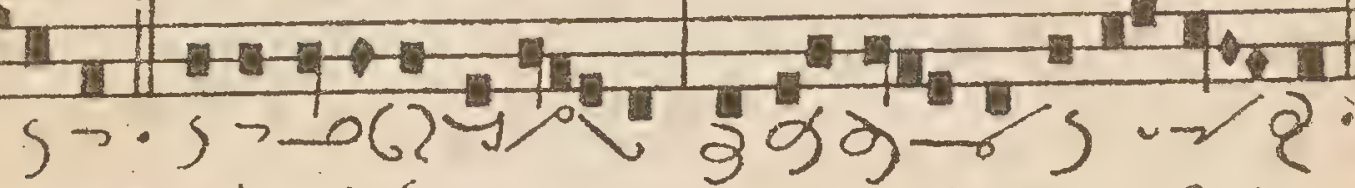
x. 12 $\rightarrow$ b 4 b-

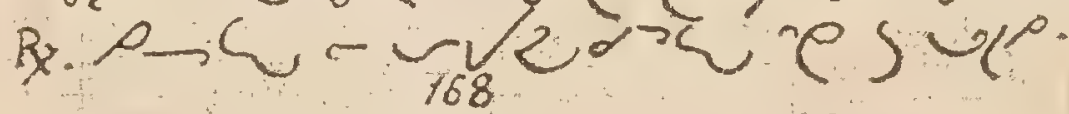




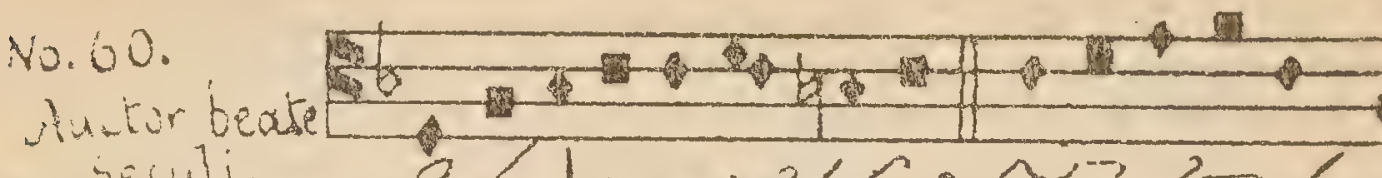
seculi. 9f los wer,

8.

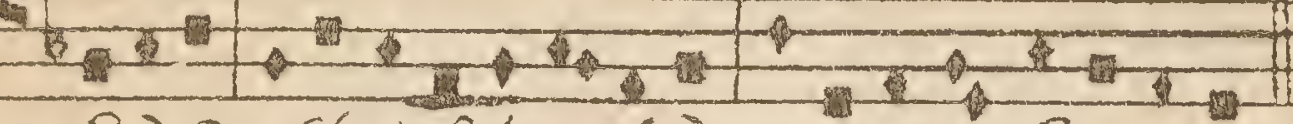

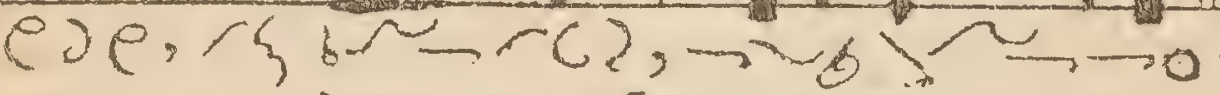

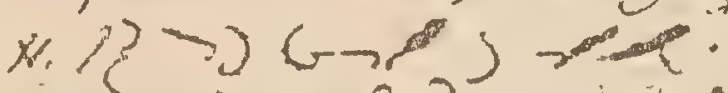

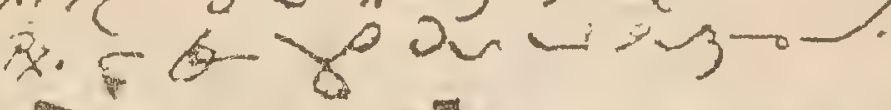

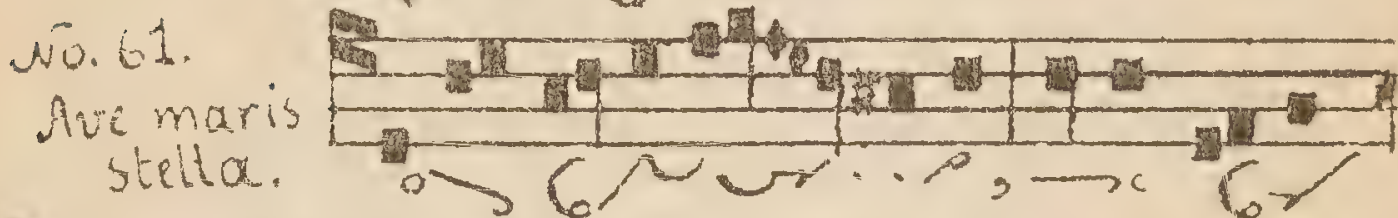

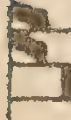

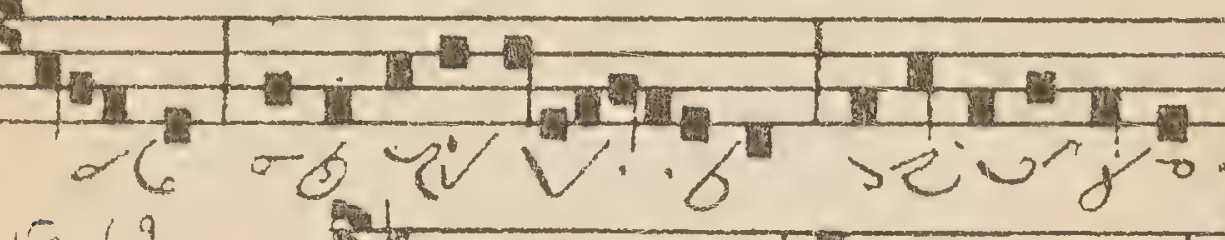

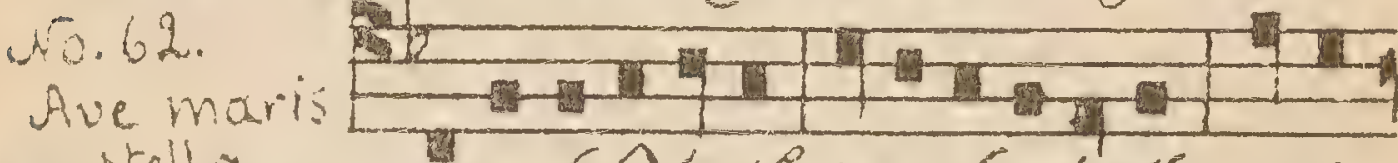
stella. of $6 x+x-6 / 26,06$

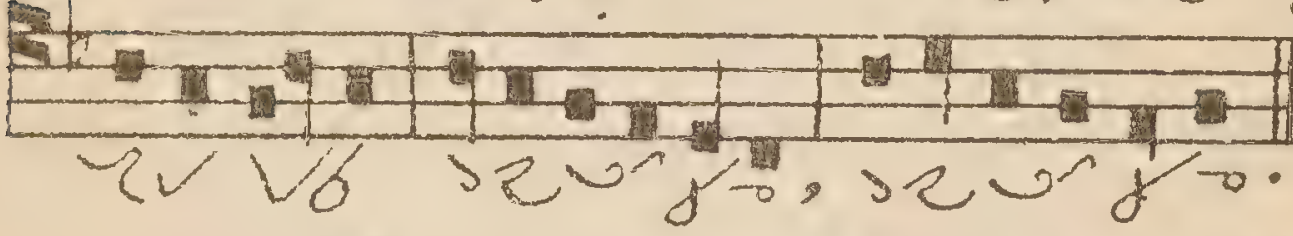
(6 


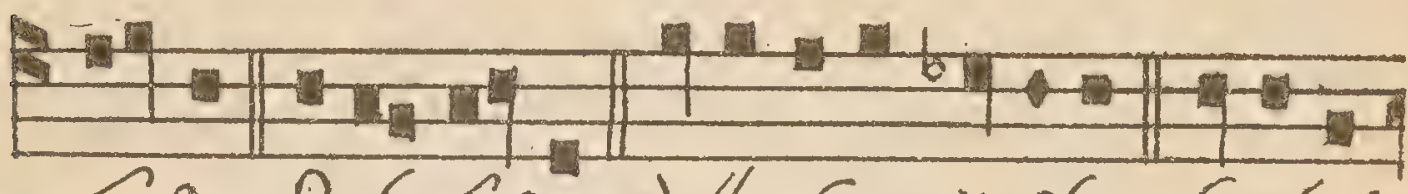

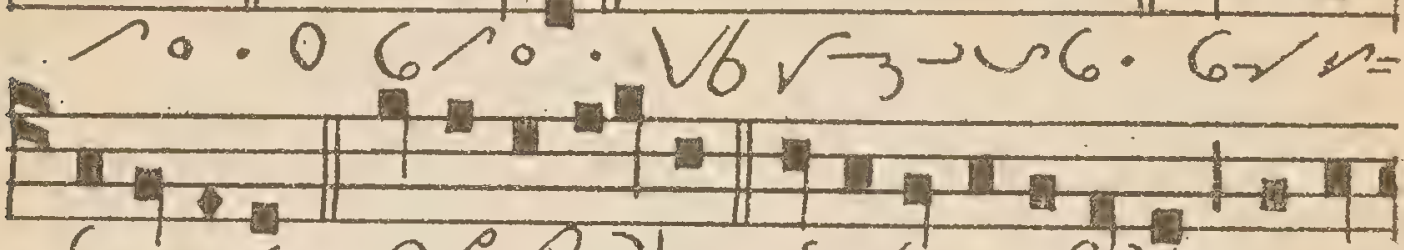

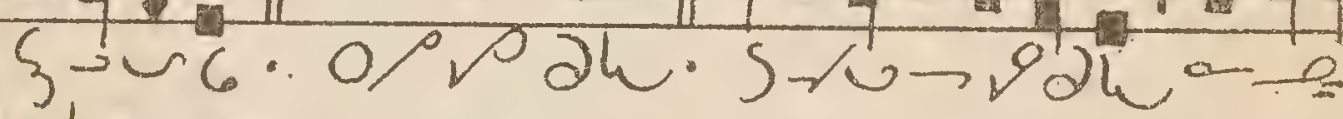
00

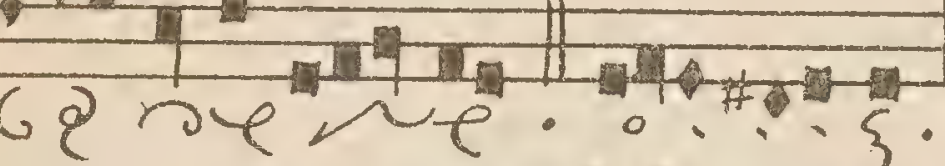
No.64.

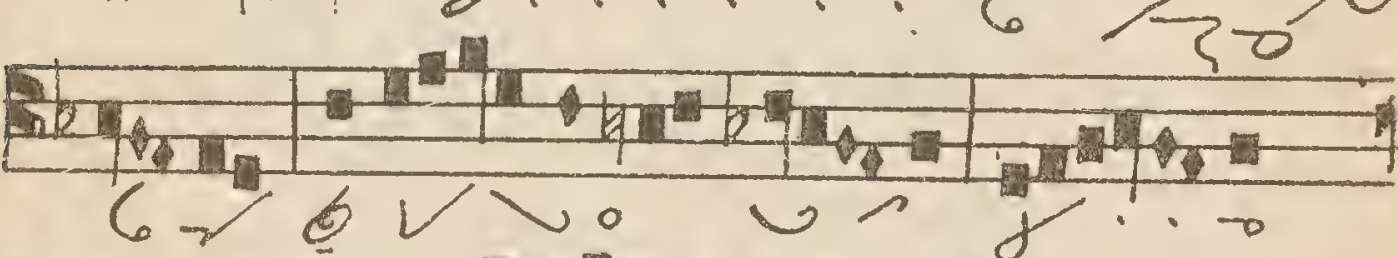
एै. 5en 7 m

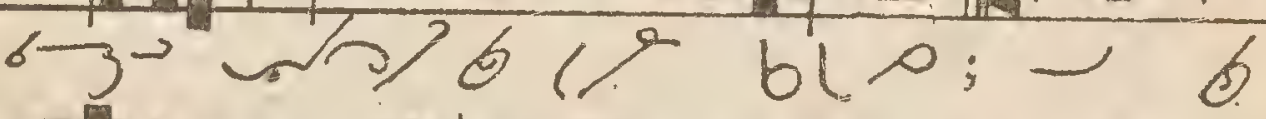

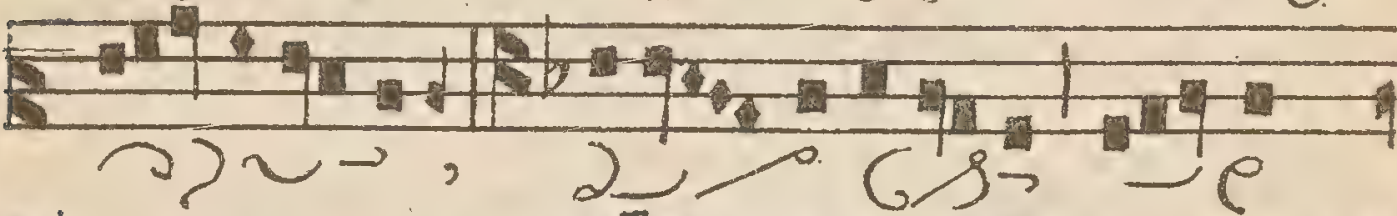

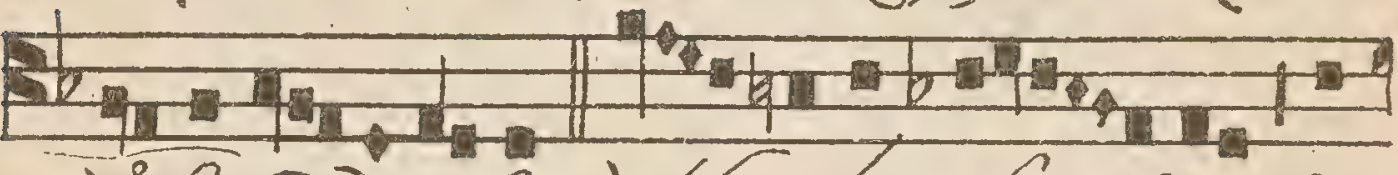

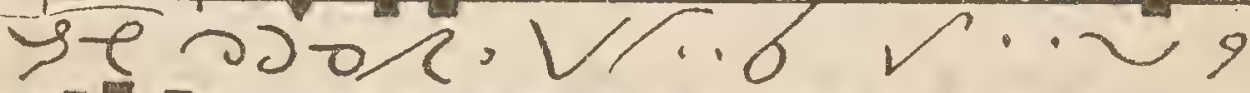

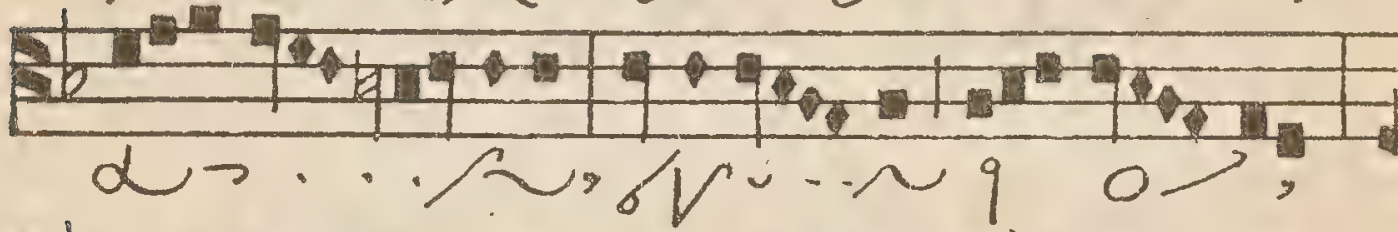

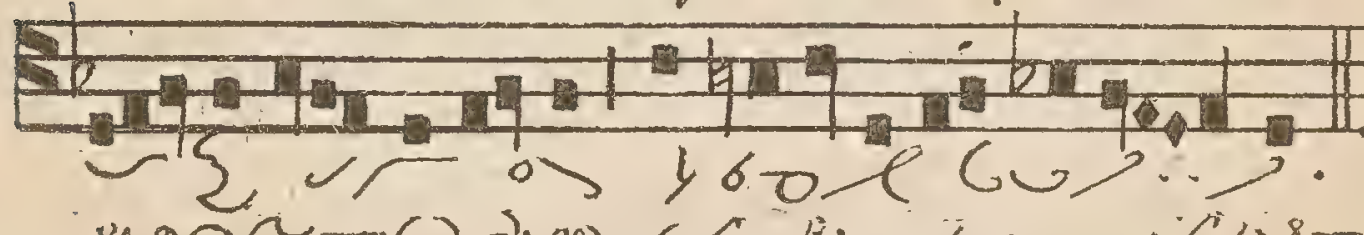

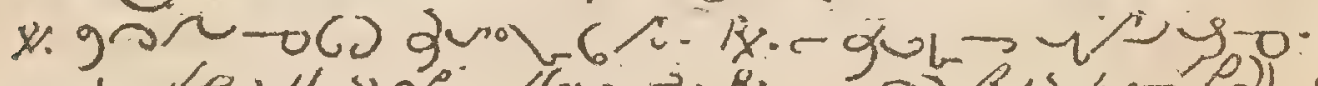

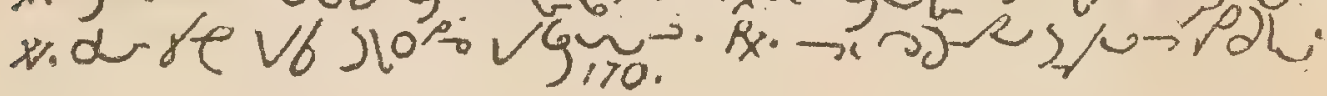




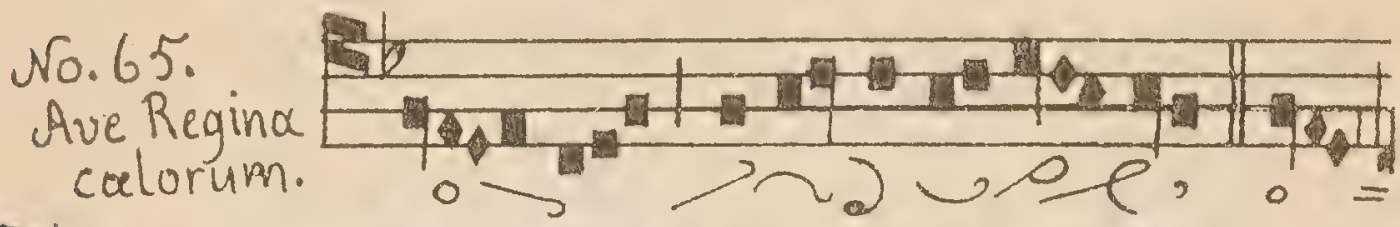
sto

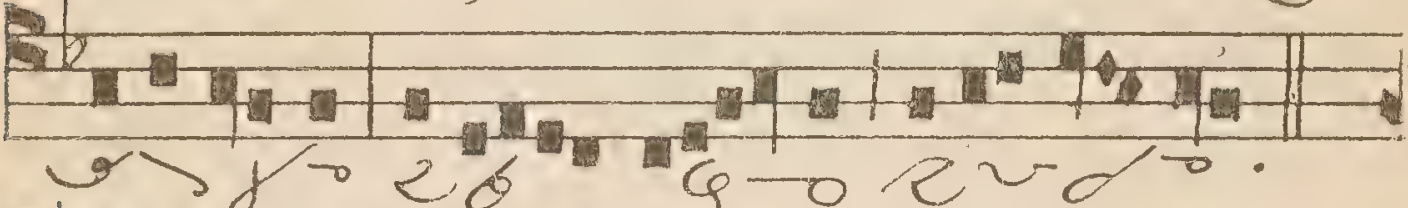

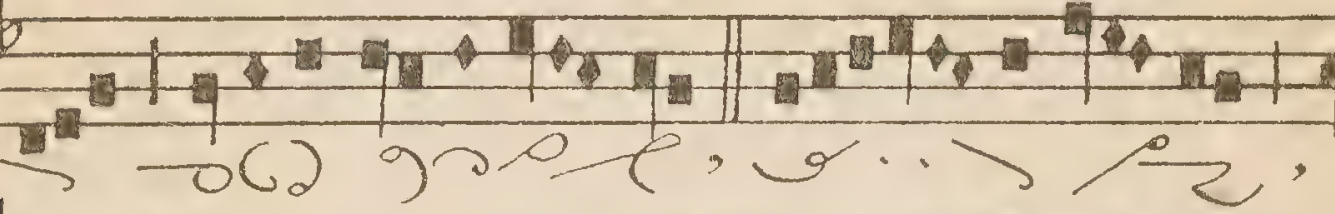

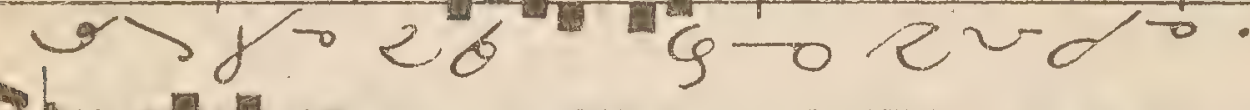

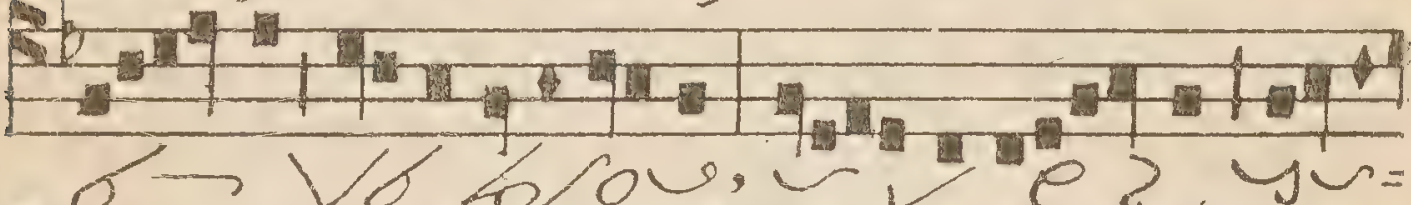

o Vo b/ow, w e z yu=

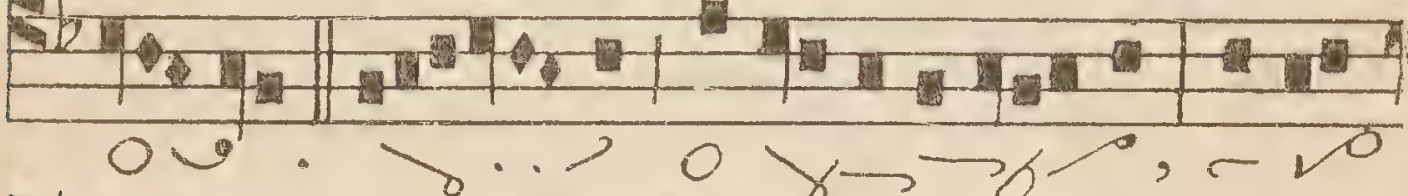

aहै

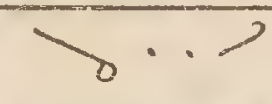
O

8

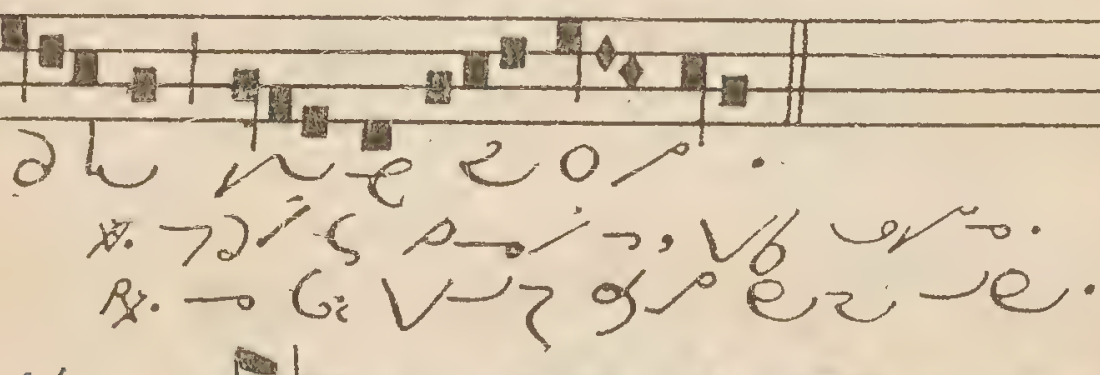

No.66.

Regina.

cali.

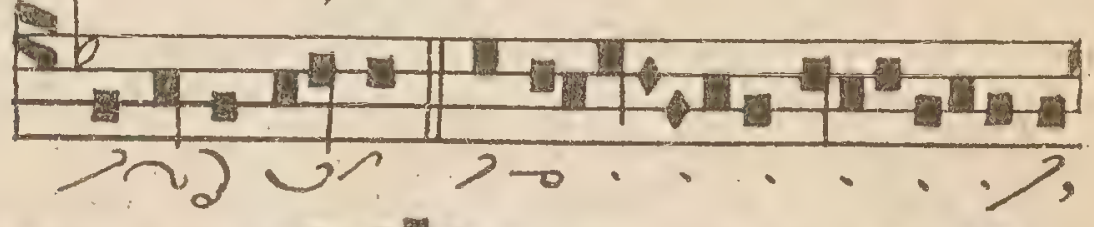

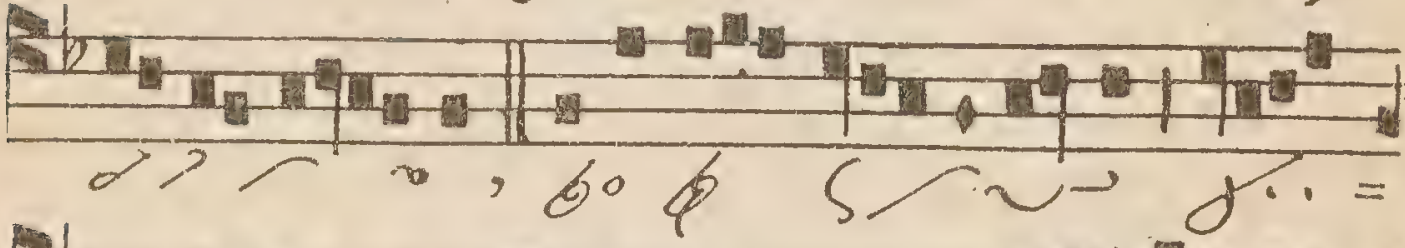

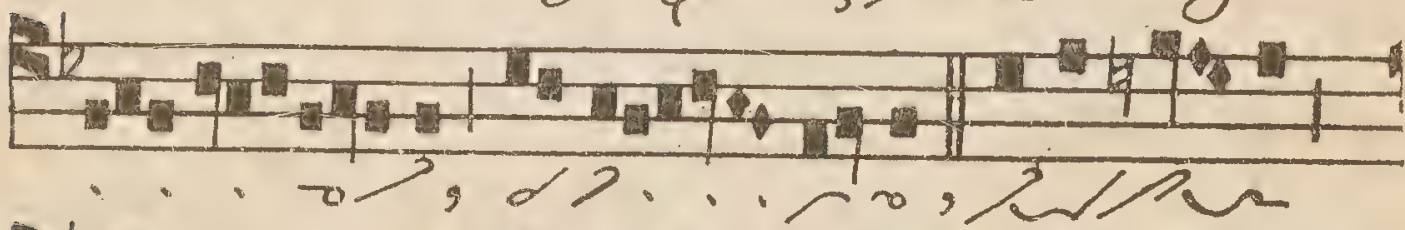

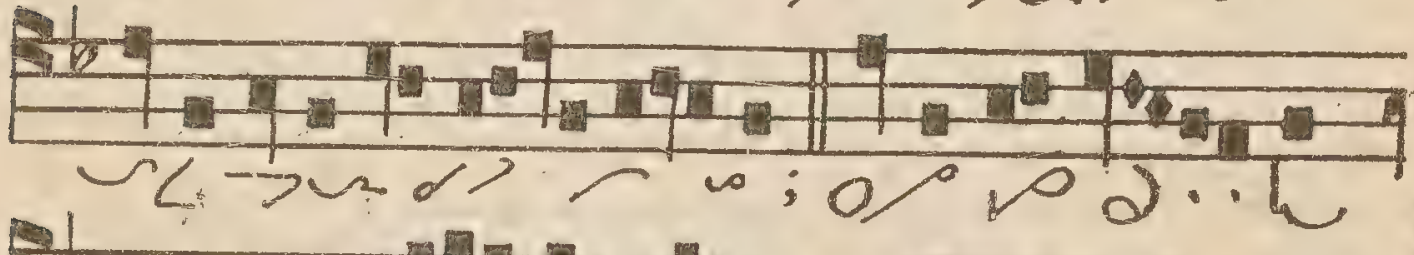

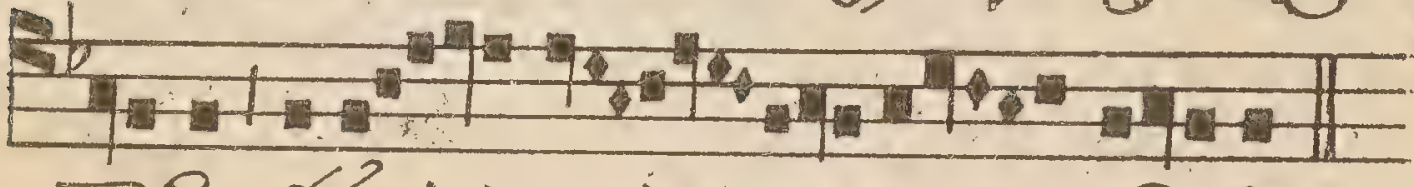


No. $6 \%$

Salve

fifgina.

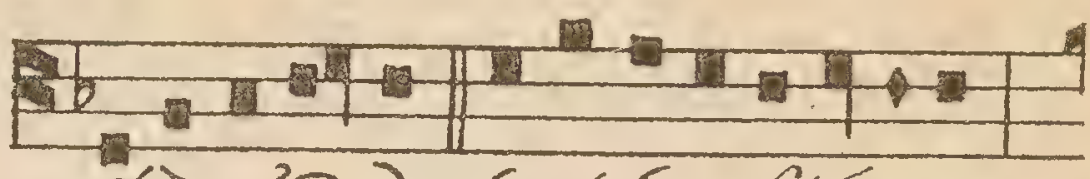

क人 ア2, 6r Guffen,

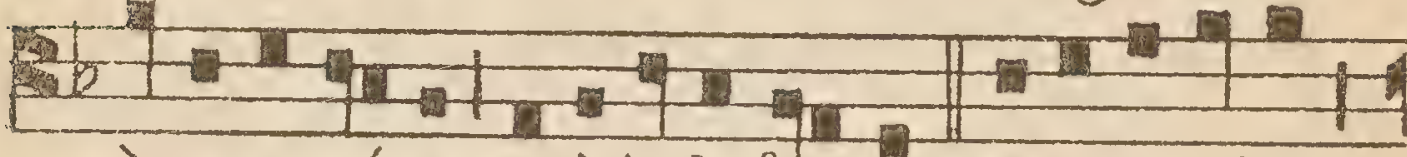

入o-60-7290

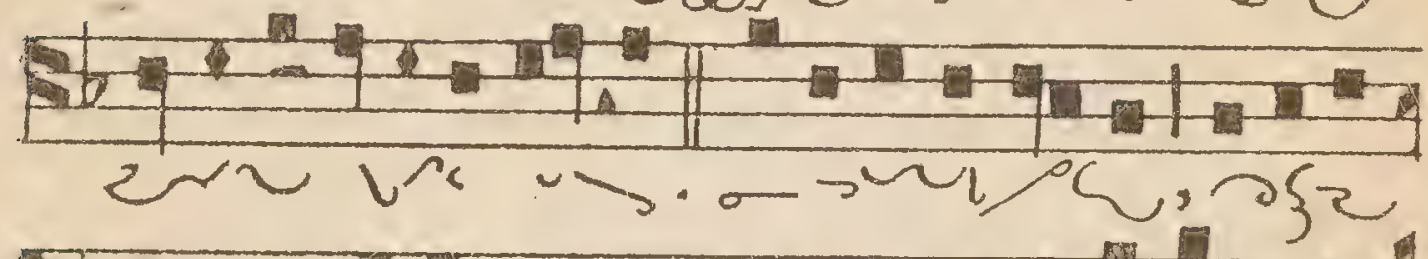

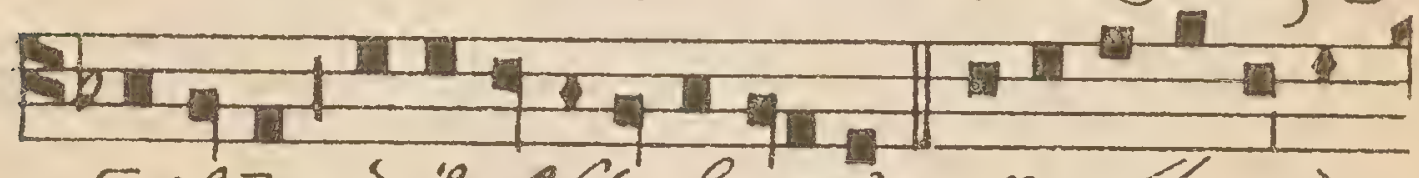

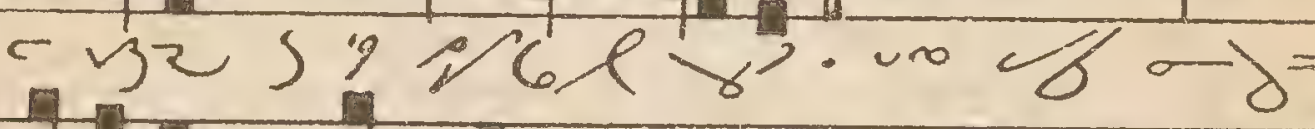
6odsare te Guffe oke

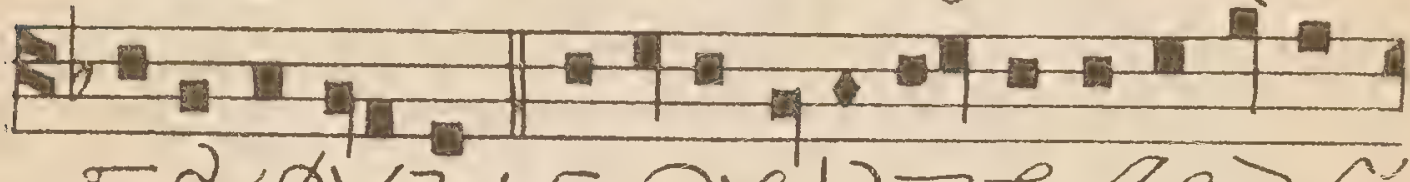

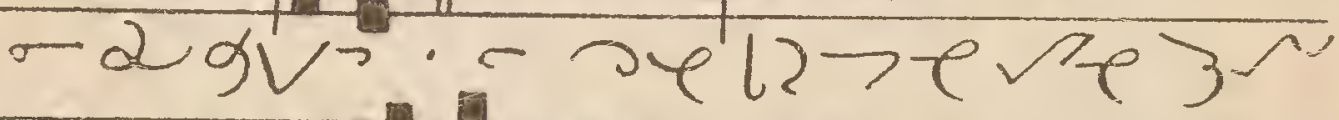

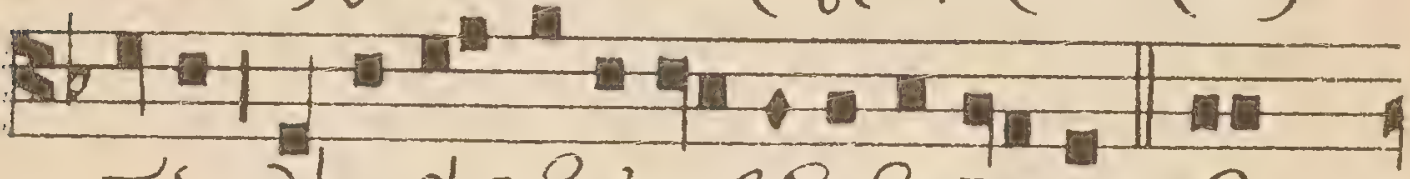

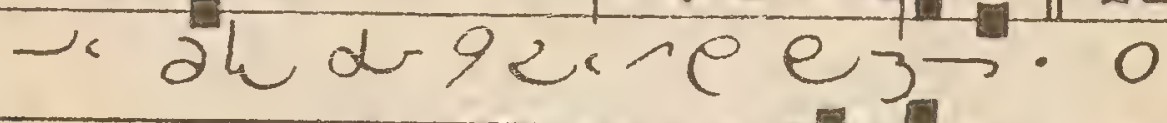
रे

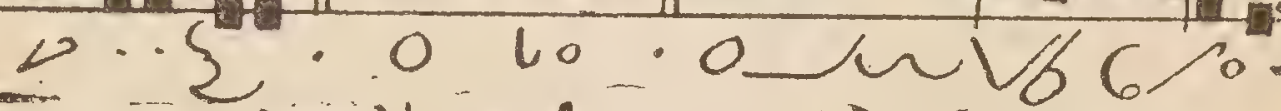
x. o p ph, yo $\rightarrow 00$ ग.

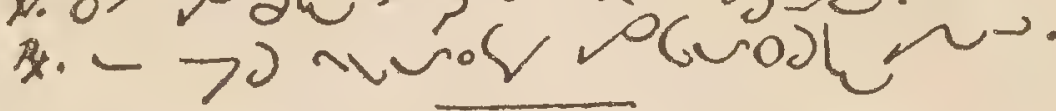
x. $6 \rightarrow-207 \sqrt{6} 60020$.

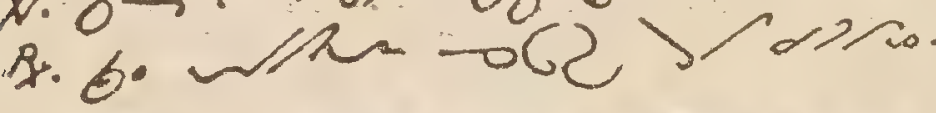

No.68. Sub tuum.

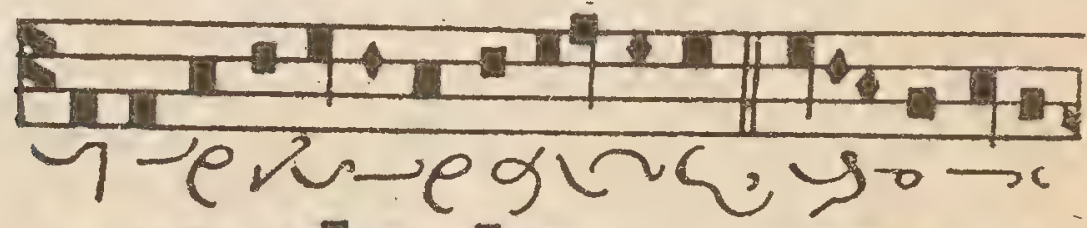

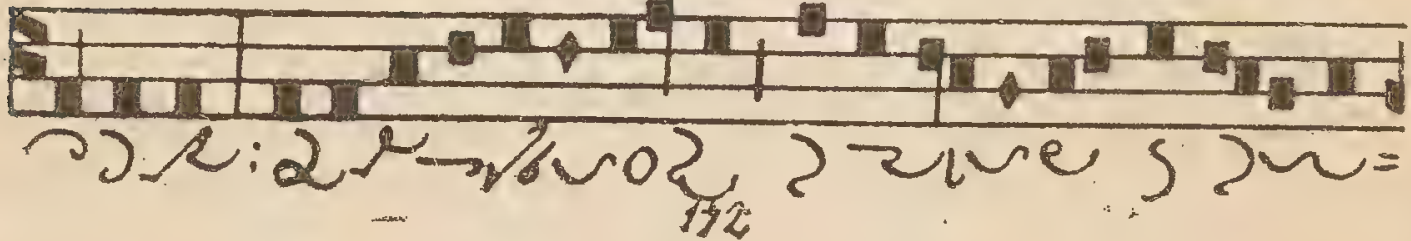




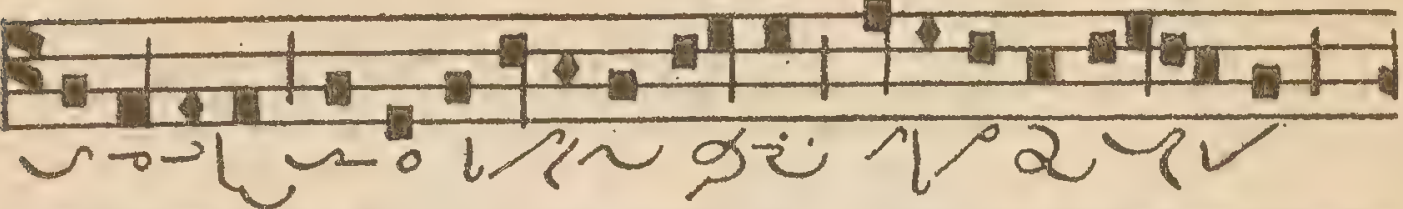

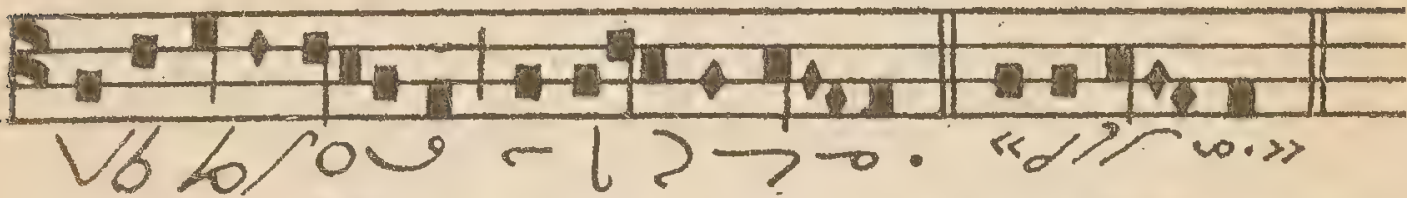

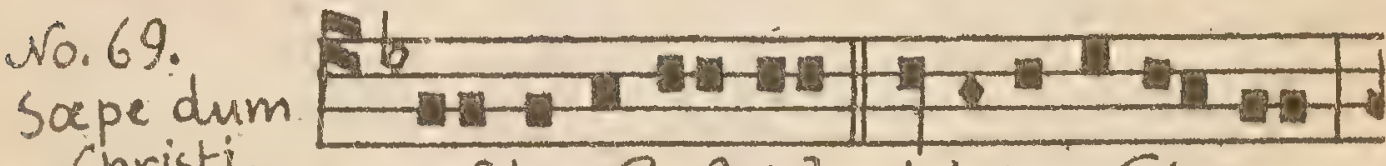

christi. Wl cru bl $\sqrt{32}$

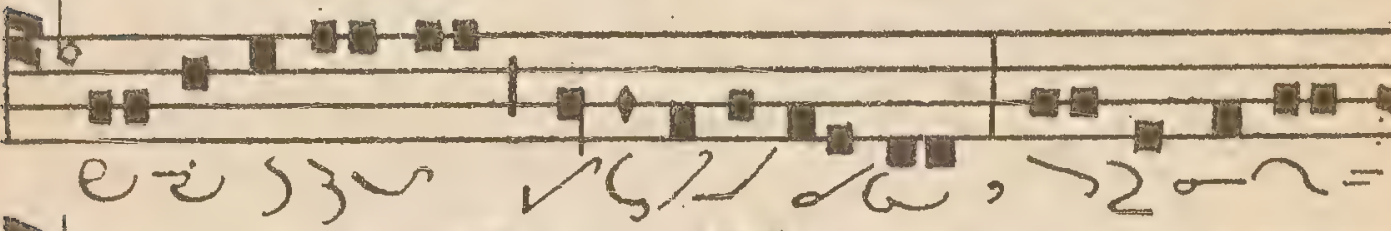

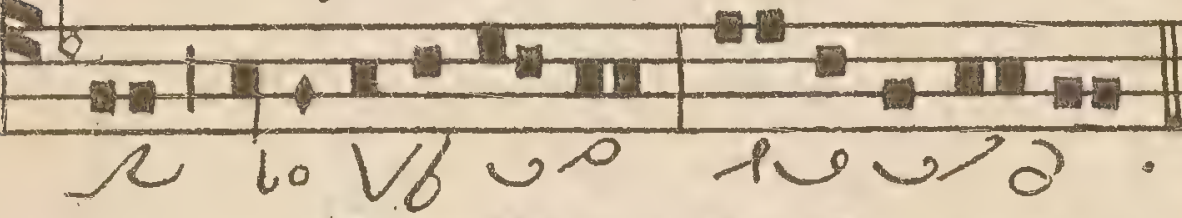

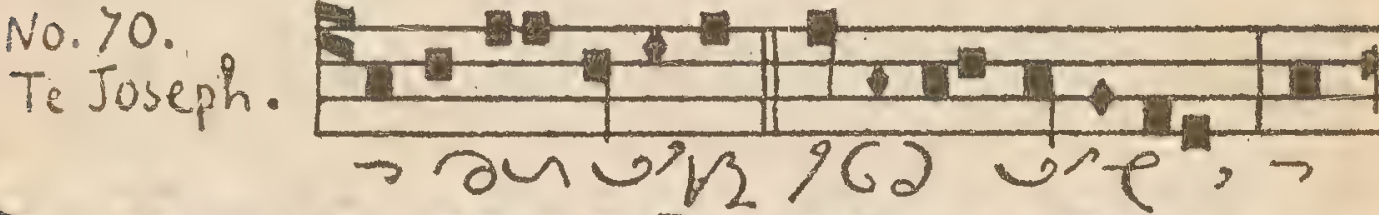

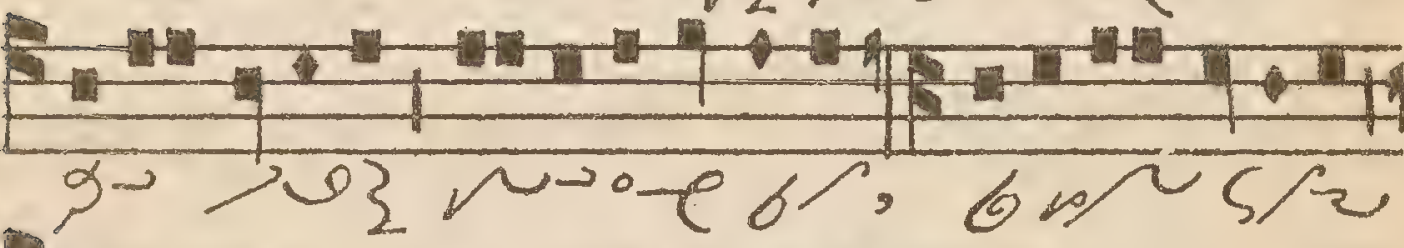

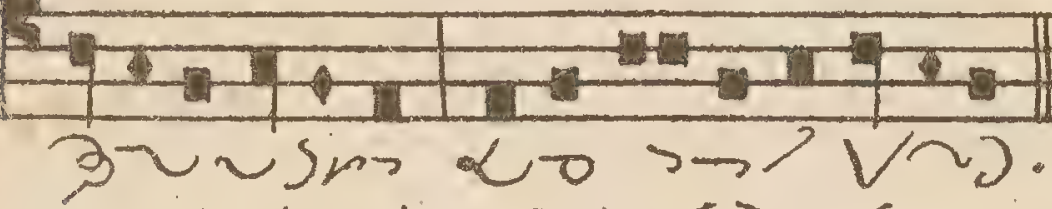

x. gu

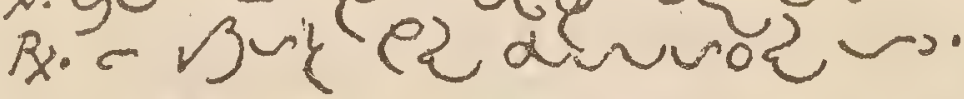

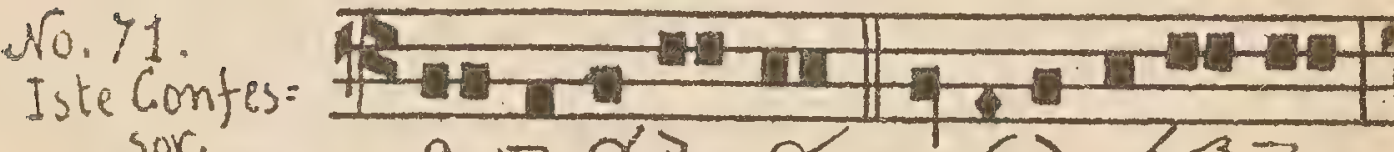
sor.

$\sim 920$ o6 632

Finc

$\$ \ln 2$ bl $v d t: 9 \rightarrow$

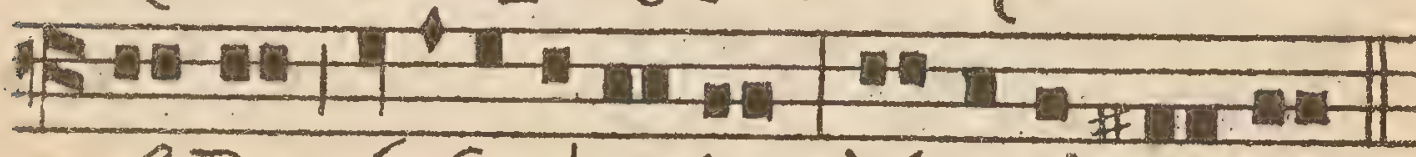

s $5 \times 10$ 
No. 72 .

Jesu Corona

Virginum.

2

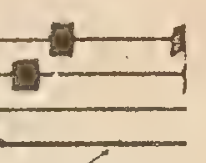

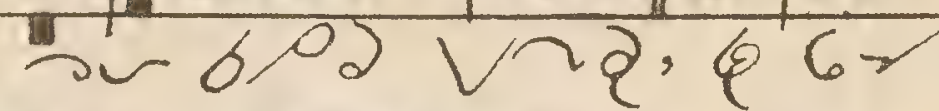

in

En m in

gub b
v\{ 9vi.

so.73.

S6 $\rightarrow \geqslant e \gamma(20 \rightarrow 6 \%$

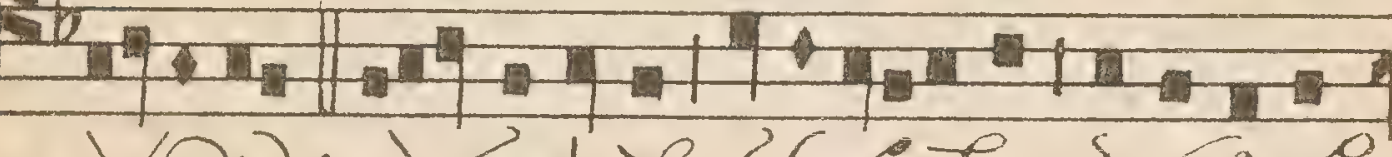
OG?

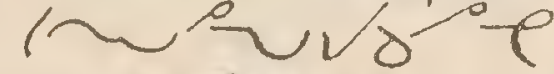
$90 \mathrm{r}$

2. $-962 \cdot \sim 0 d$ 2 0 8i $2=$

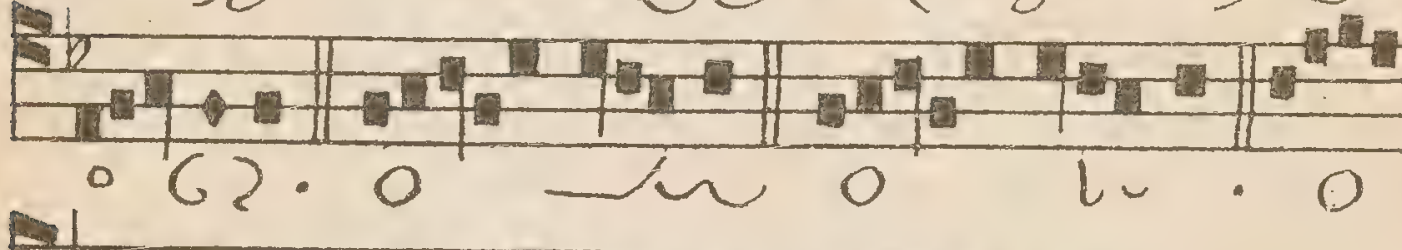
$\rightarrow 1$

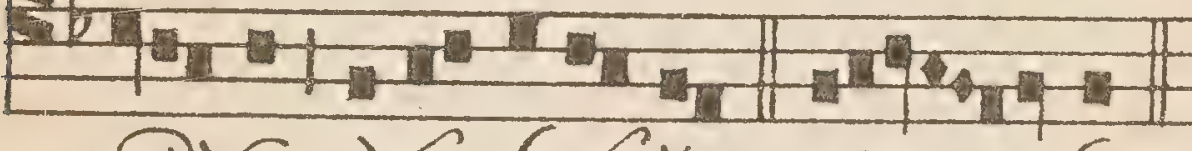

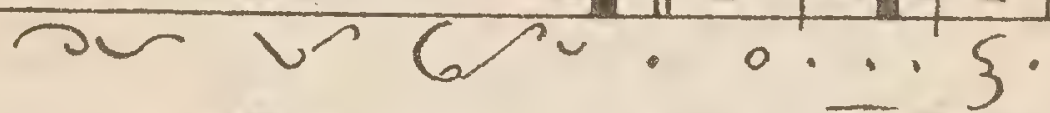

No. 74 .

Lucis

creator

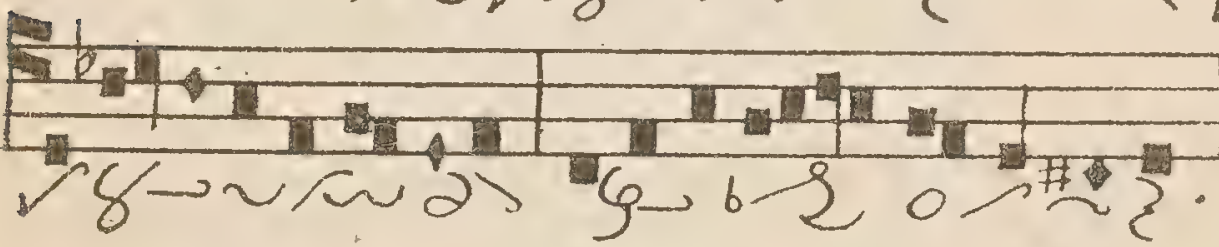

x. -

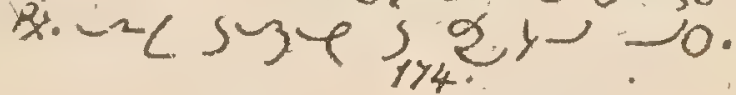




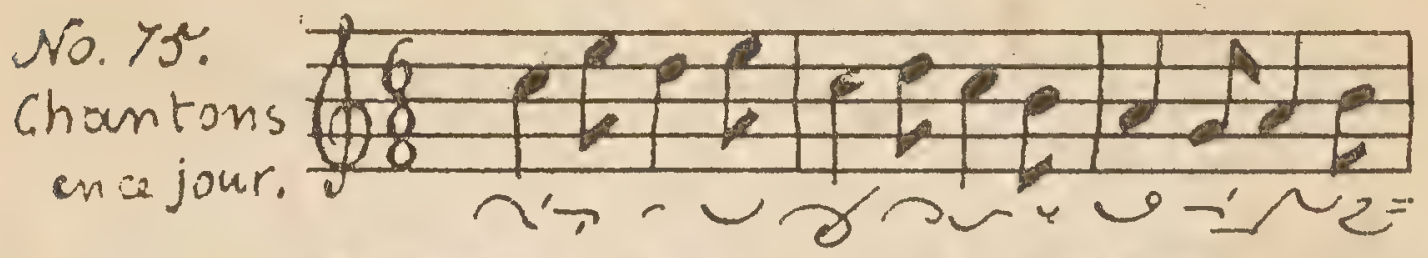

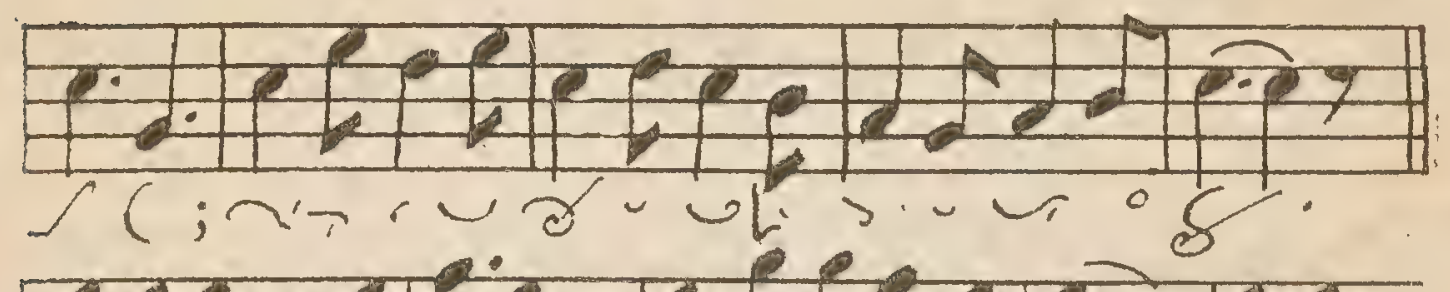
Gexe

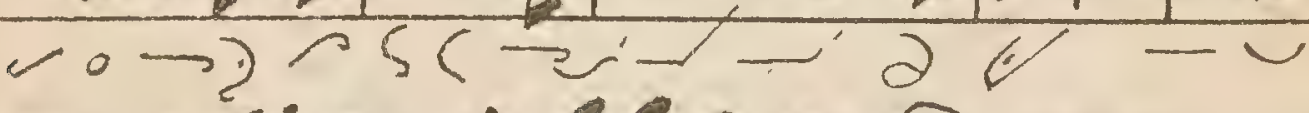
स\%

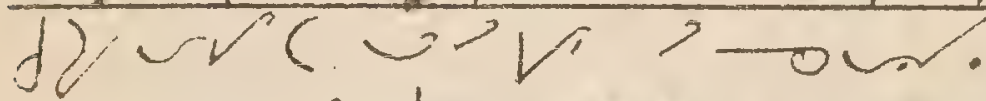

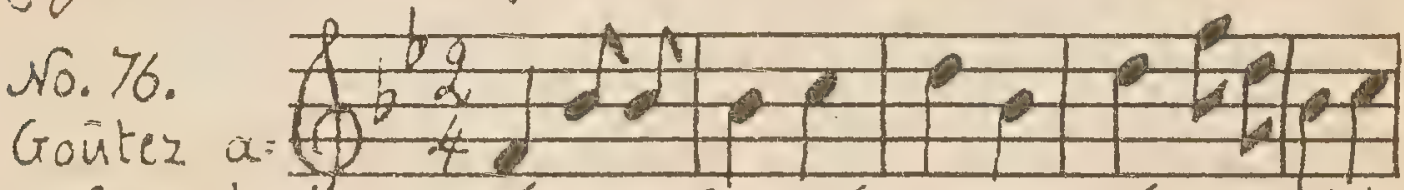
nesferventes. $\quad b \rightarrow{ }^{\circ}\left(\checkmark \backslash-6 \rightarrow b^{\prime} b=\right.$

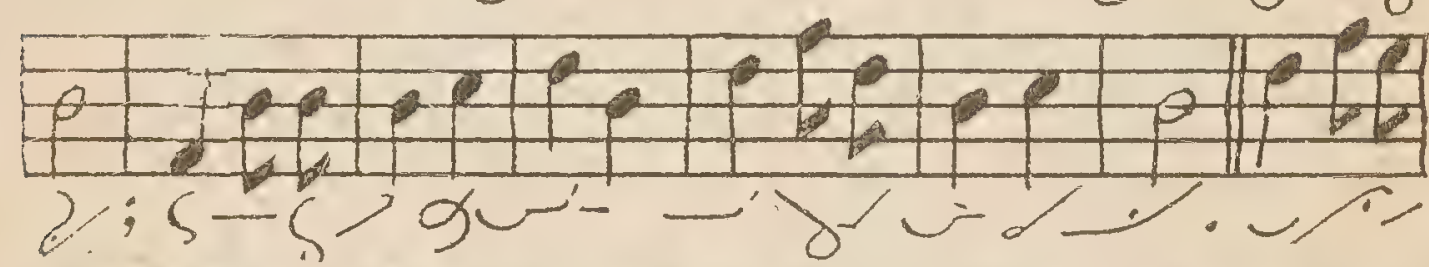

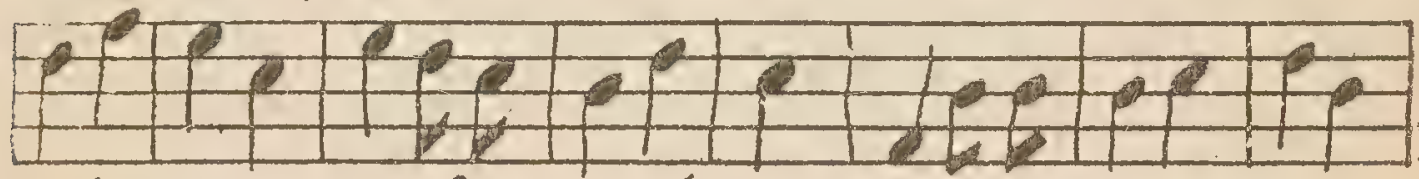
$\forall u-0 / 3$ ov - wumos u

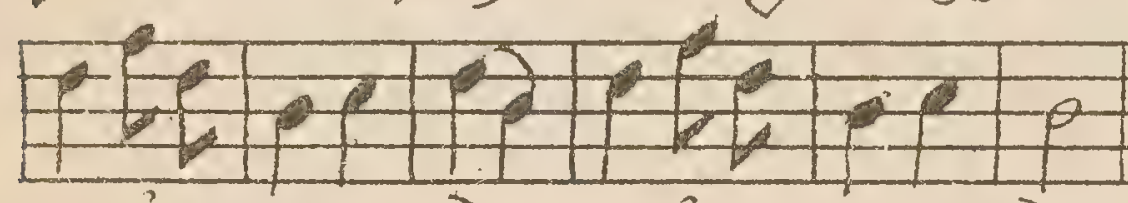

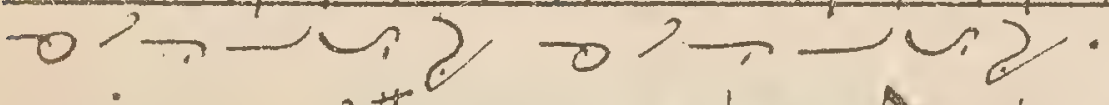

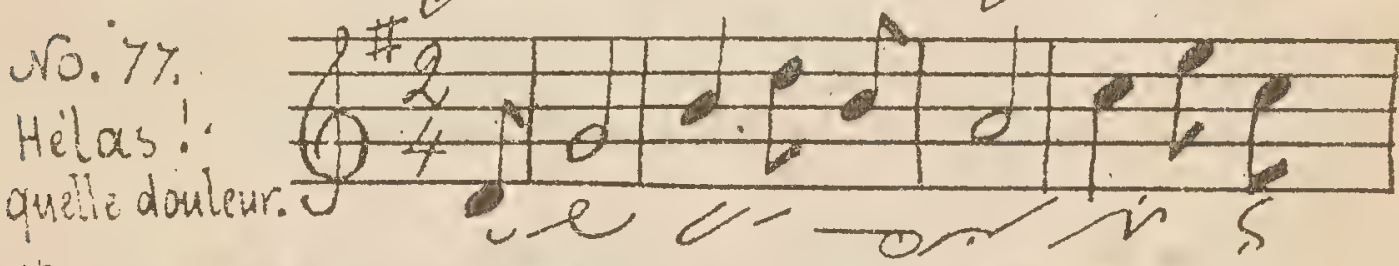

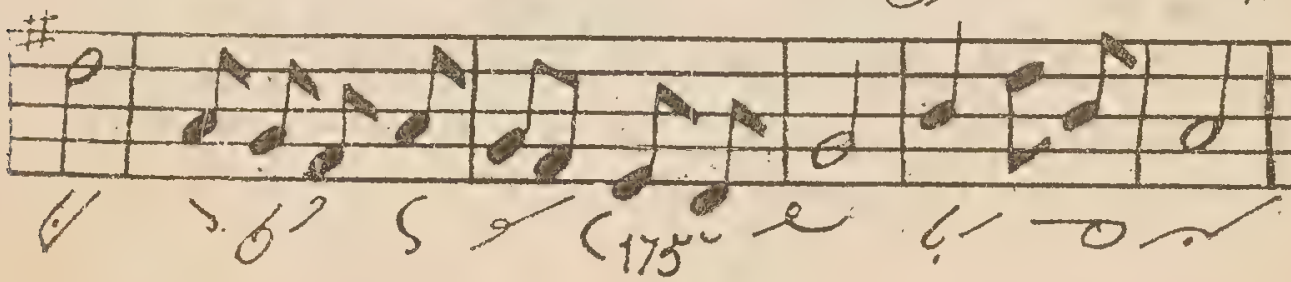




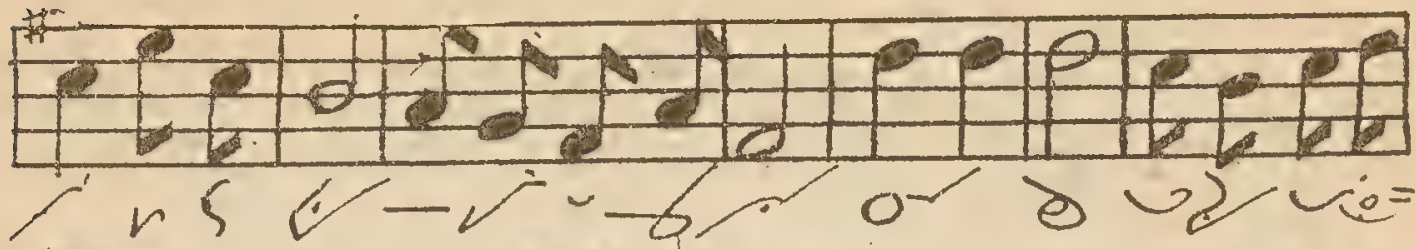

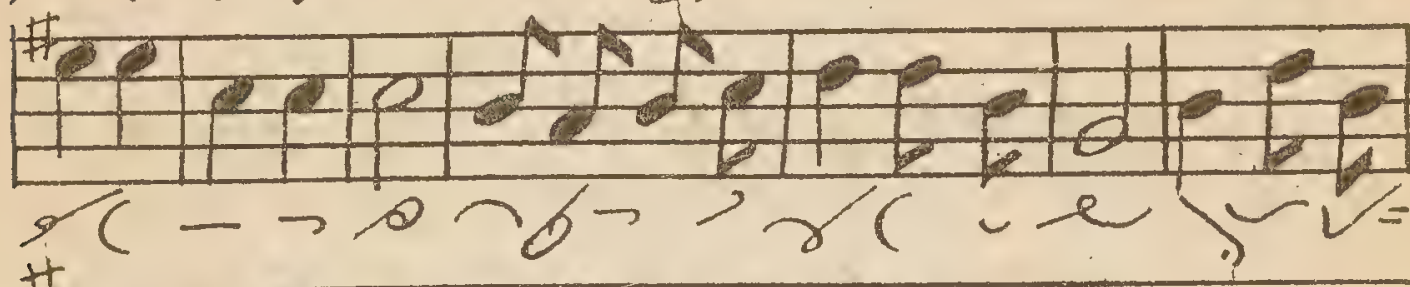

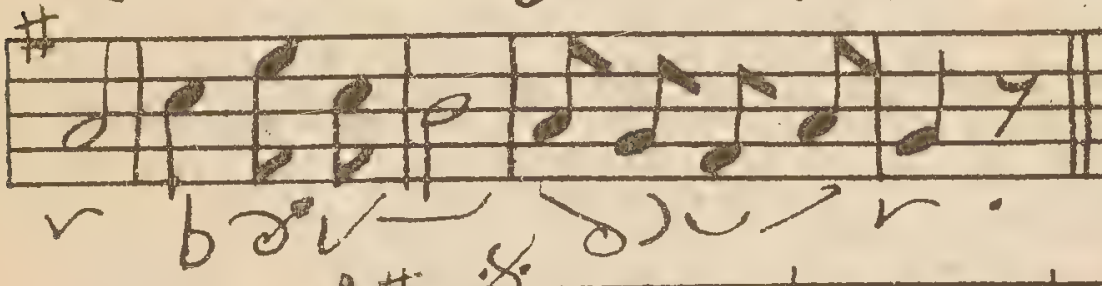

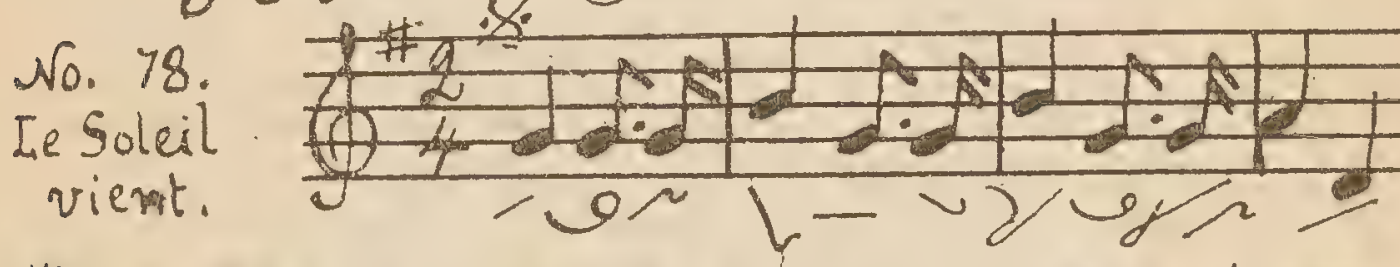

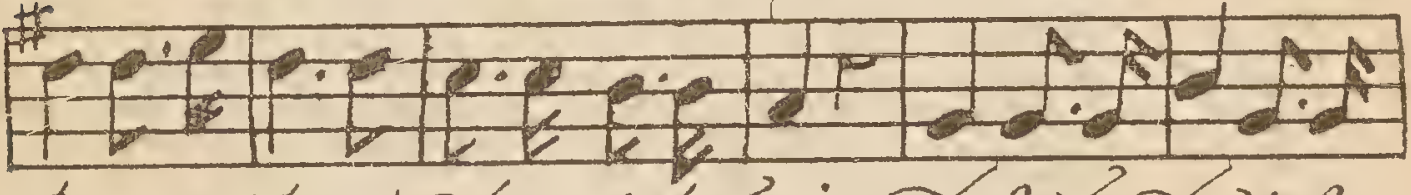

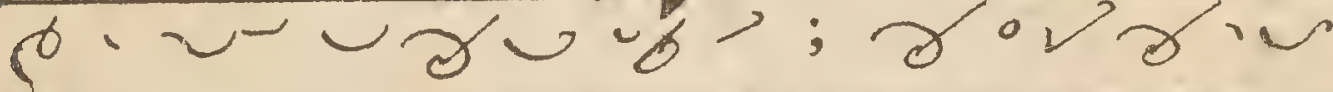

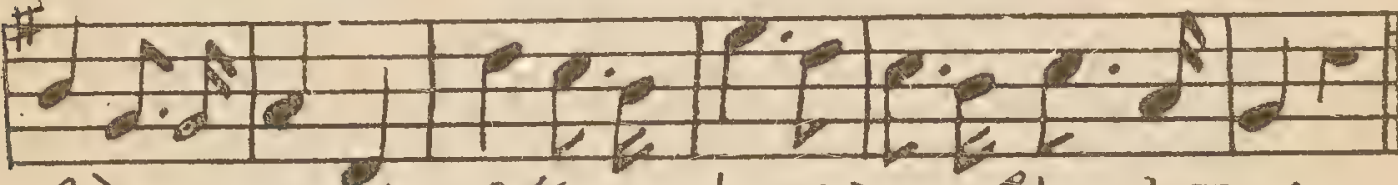

$p \searrow r ?-v_{f}<\alpha_{0}>\rho_{l} \rightarrow$

vo.79. $\frac{8}{8}$

reux pour moi.

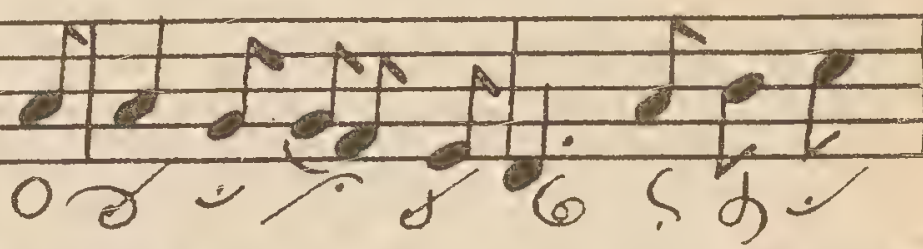

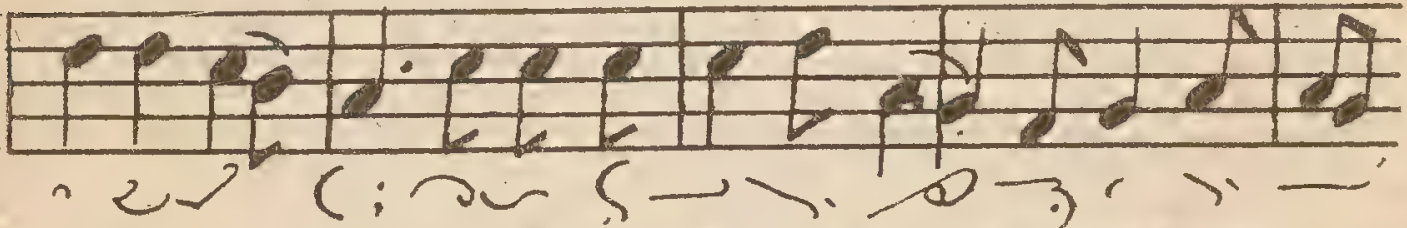

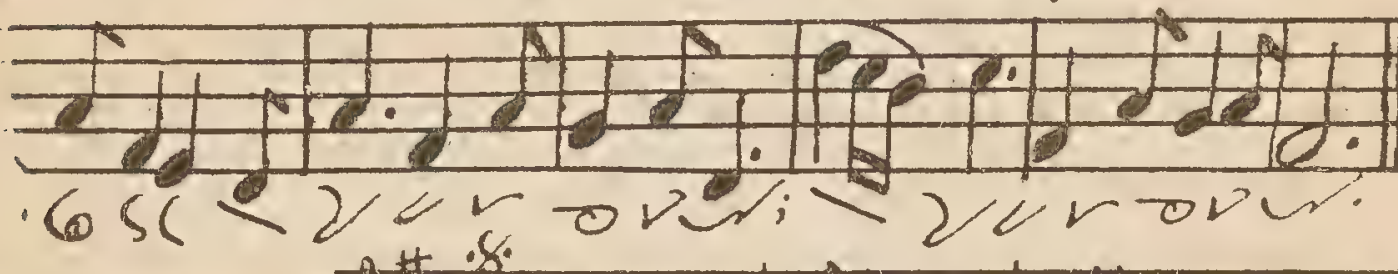

No. 80 .
venez divin
Messie.

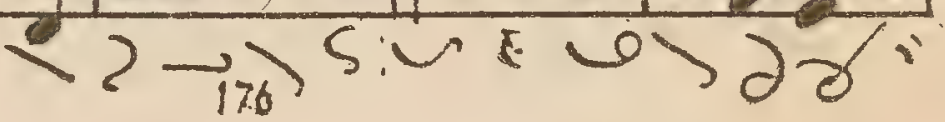




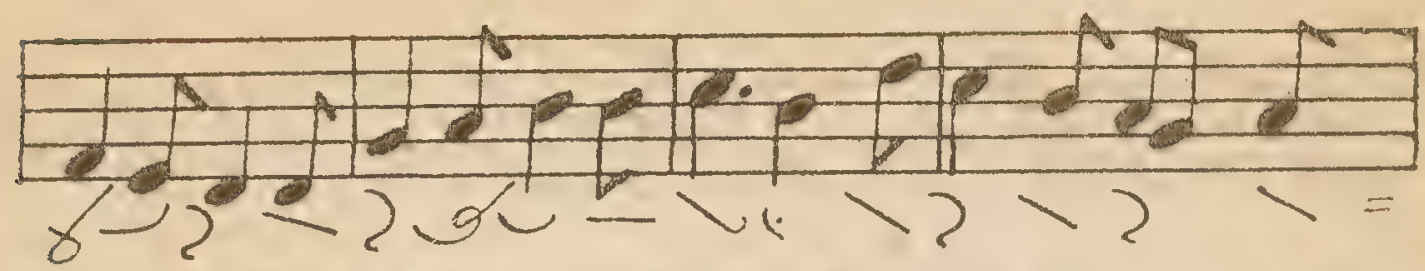

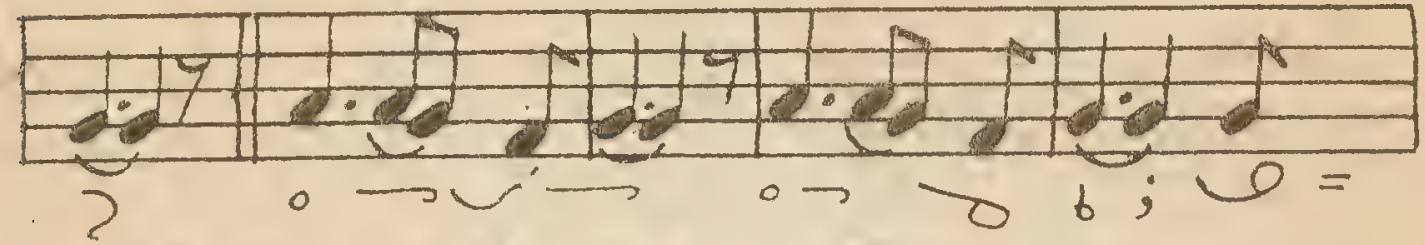

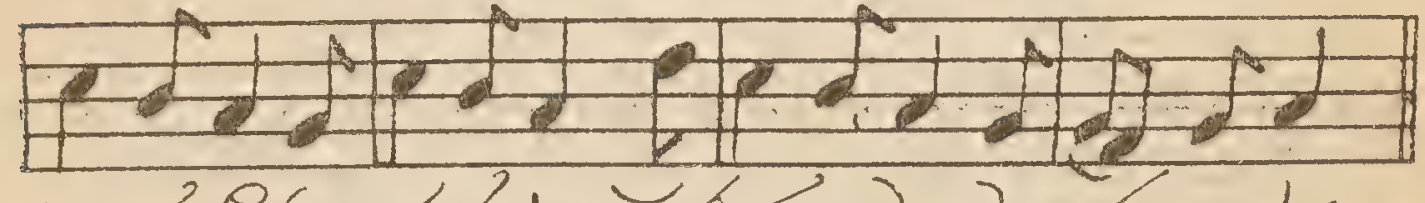

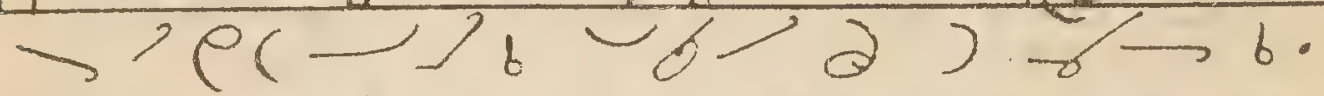

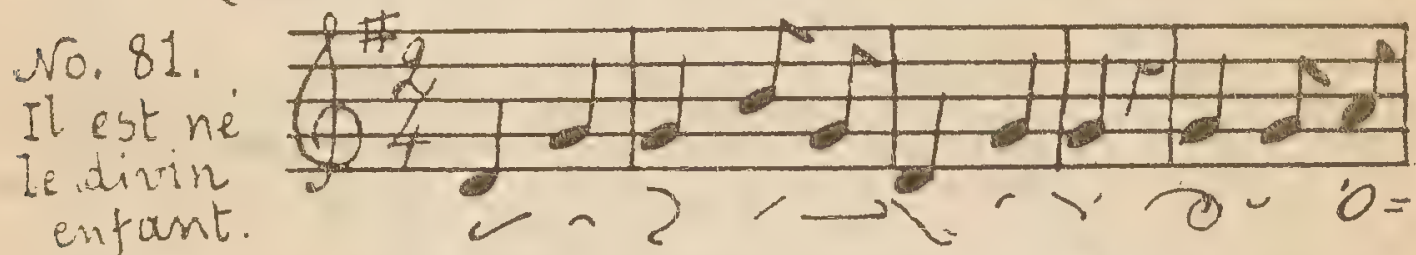

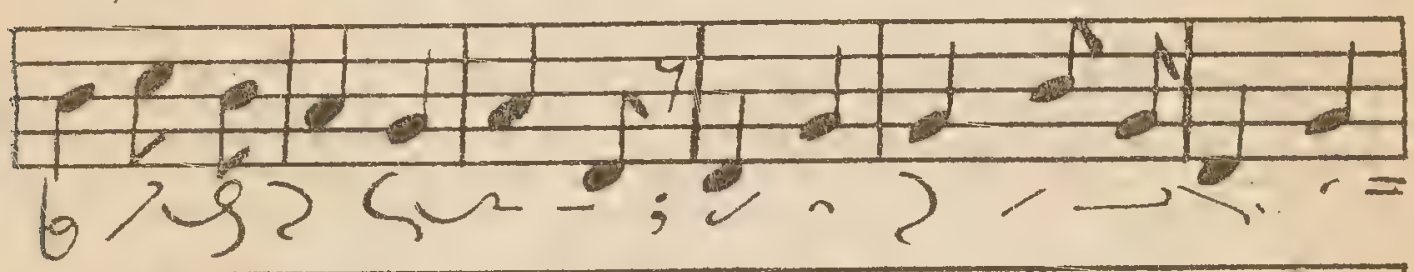

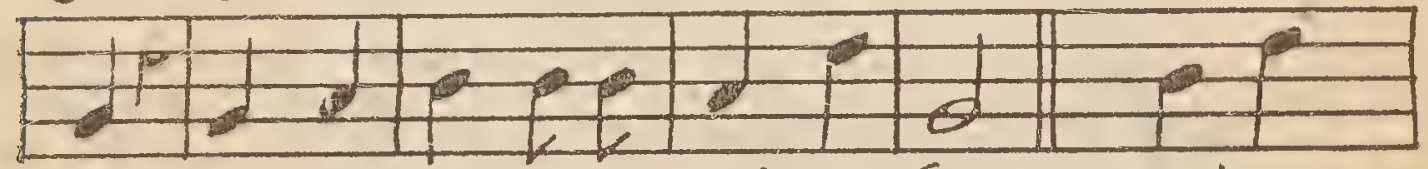

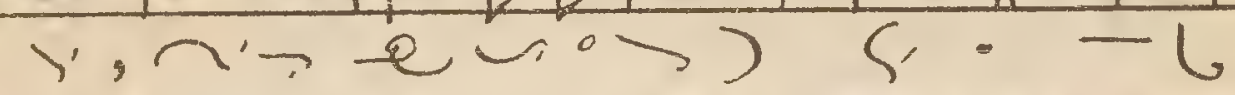
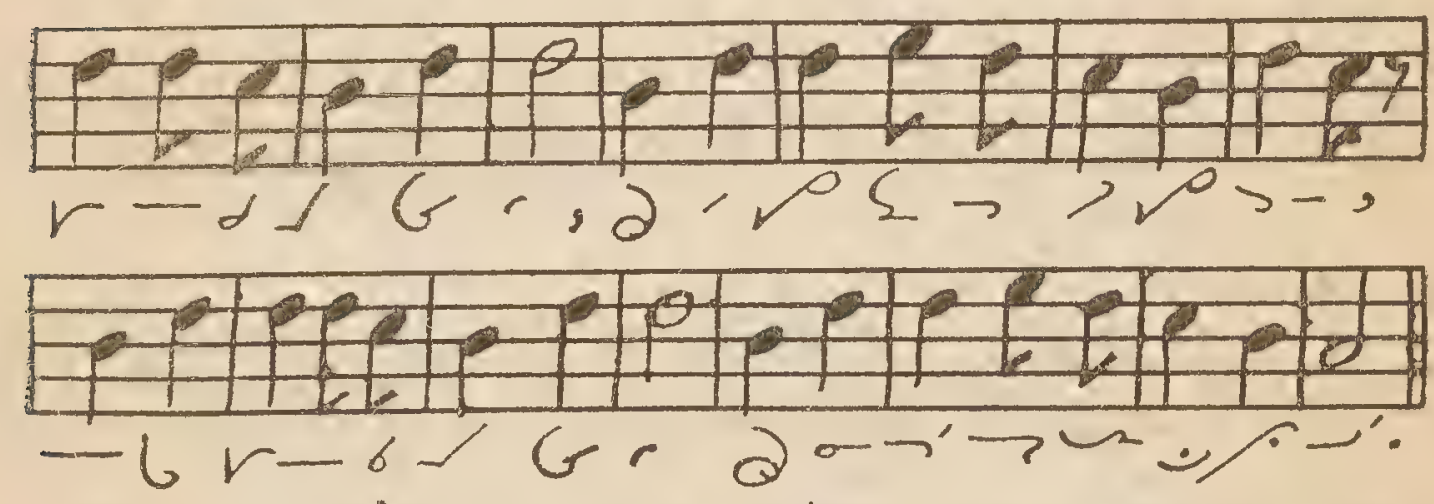
No.82.
Lefils du
Roidegloire.

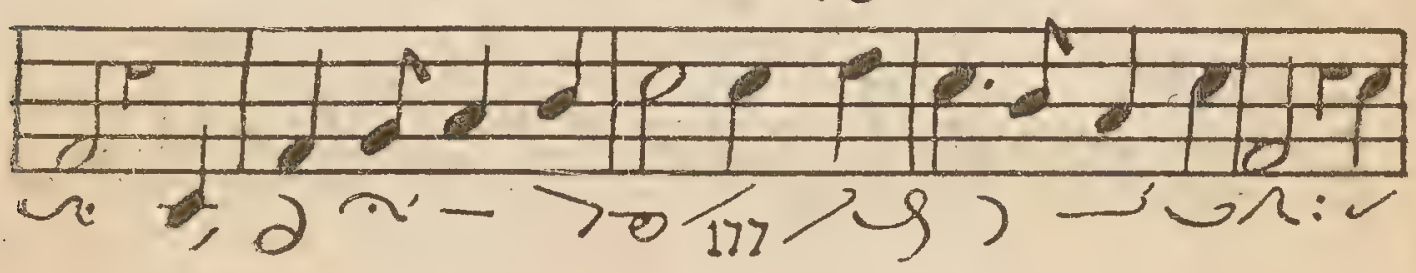




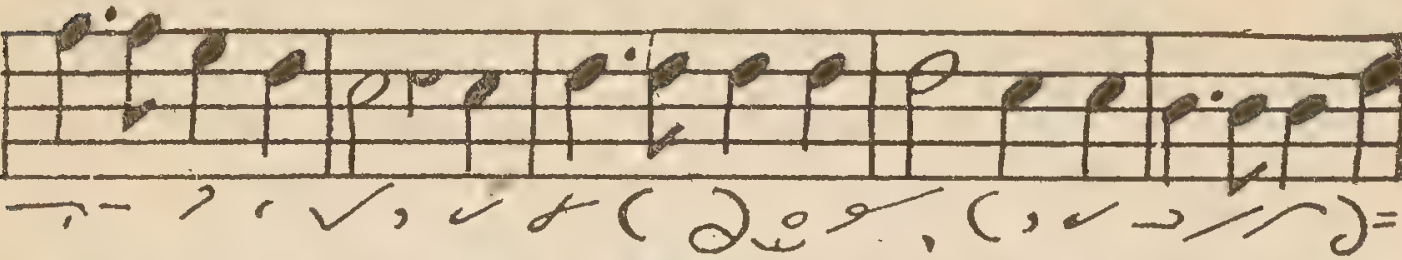
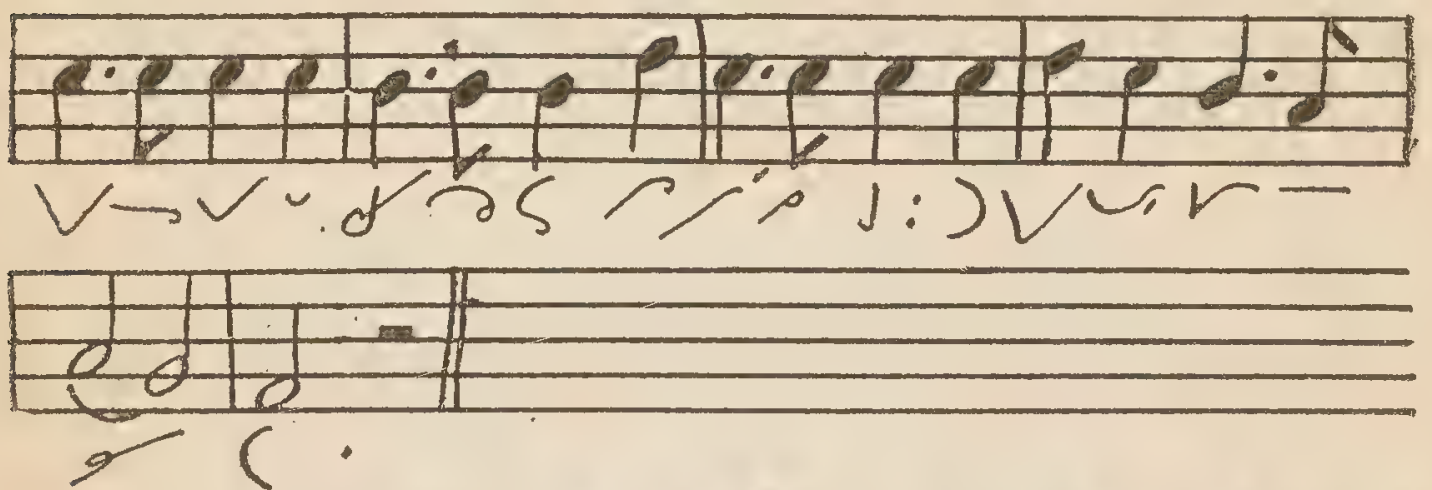

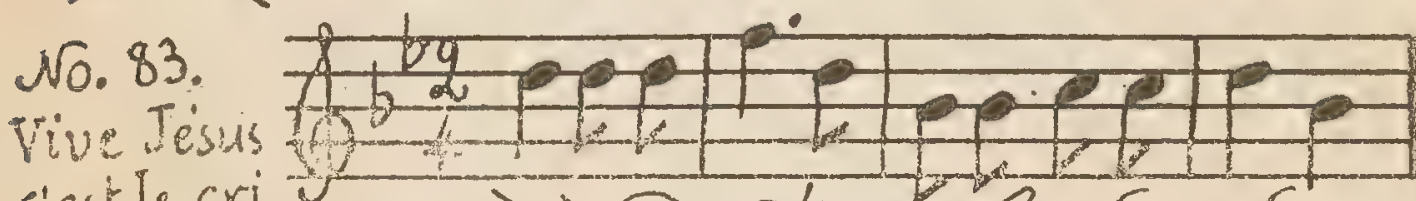
c'estlecri.u DU D.

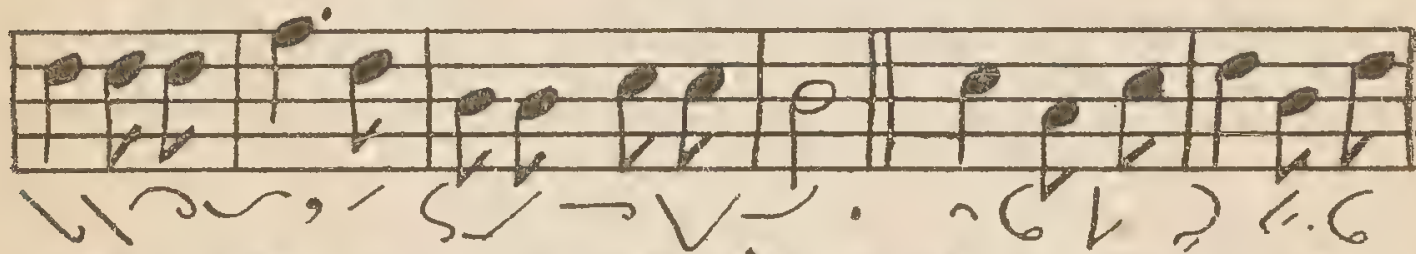

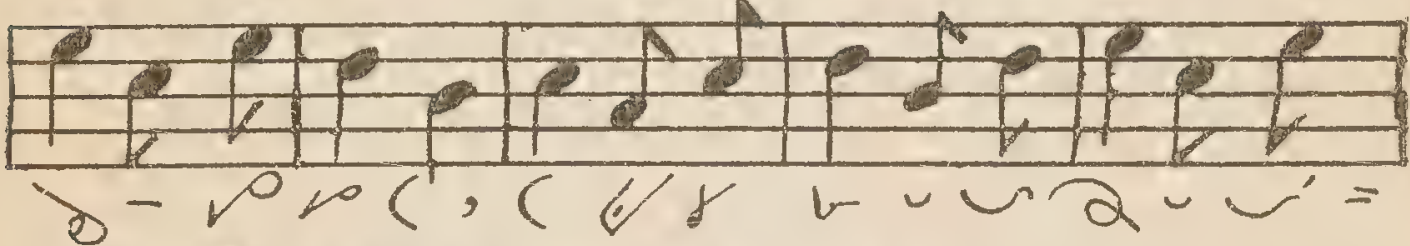

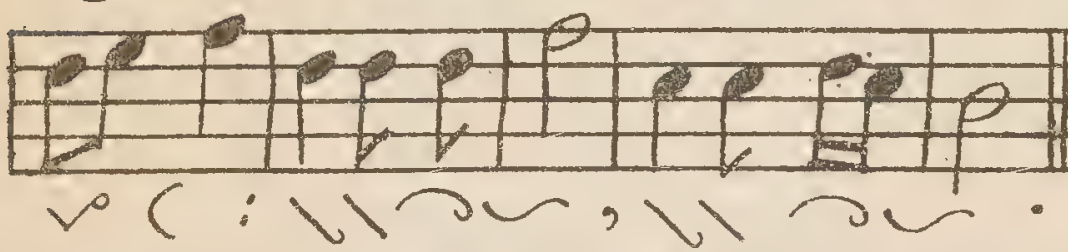

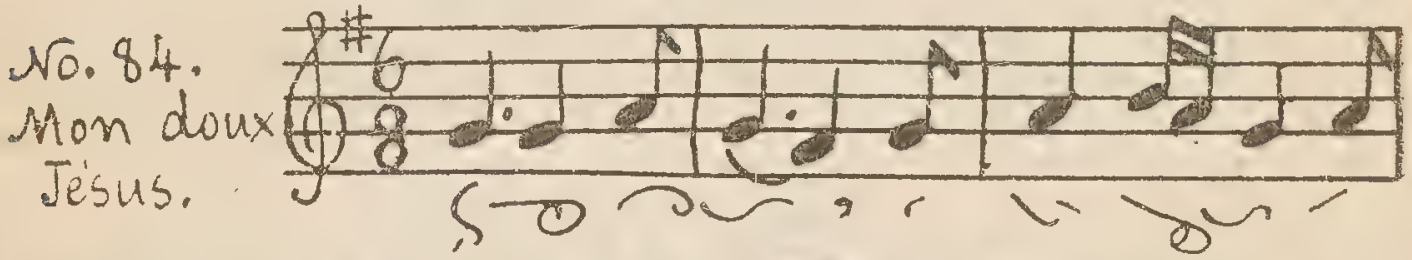

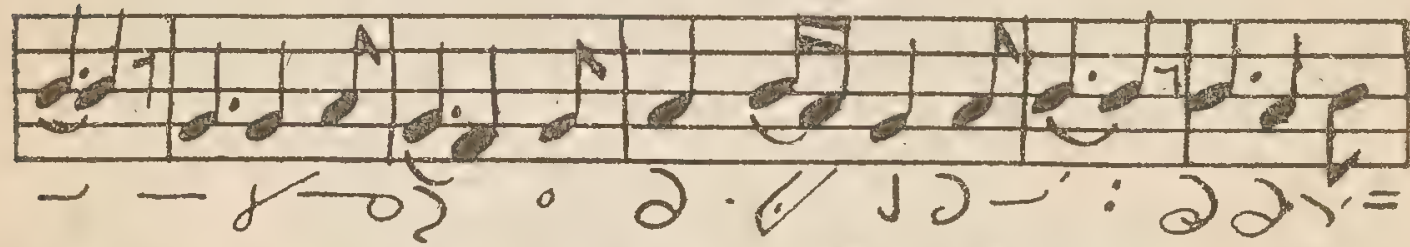

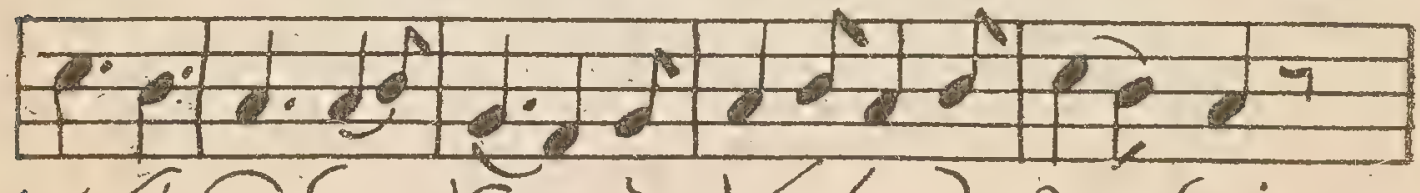
$\checkmark / \partial S$ ' 

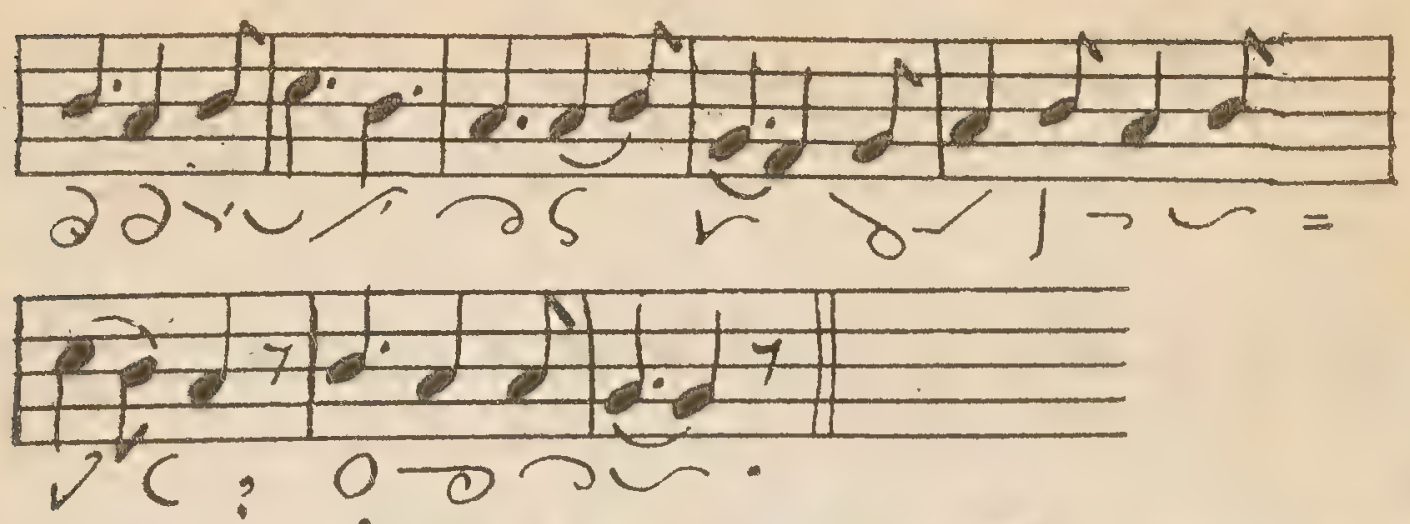

No.85.
Aufond
desbrutants
abimes.
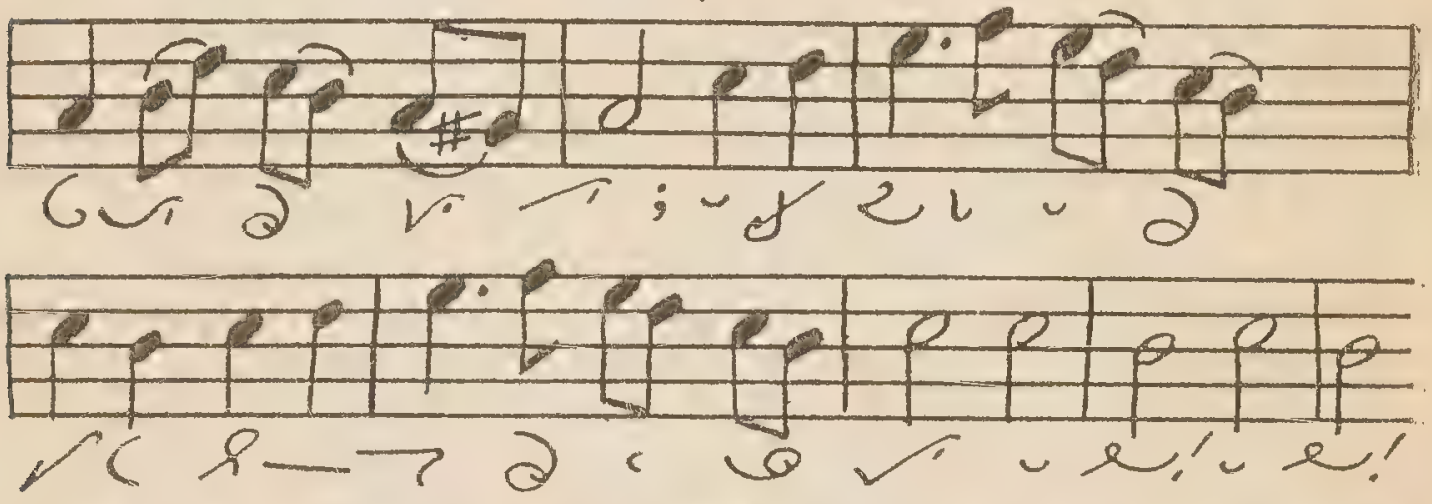

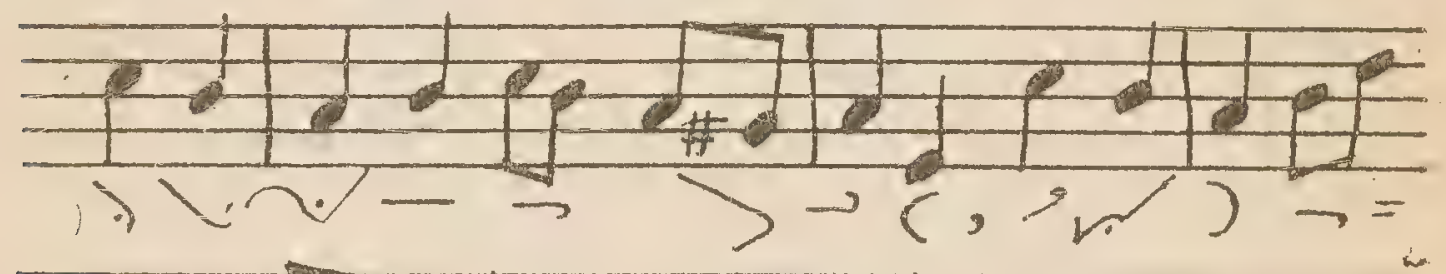

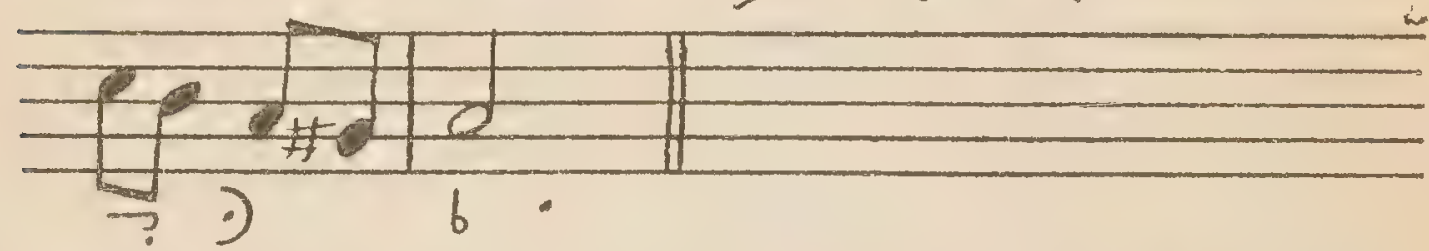

No. 86 .

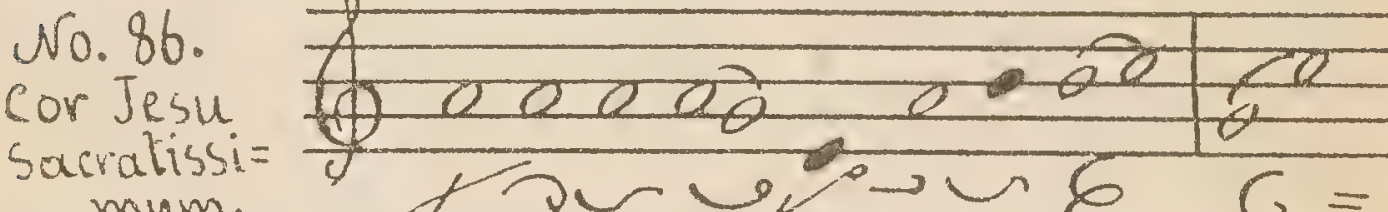

mum.

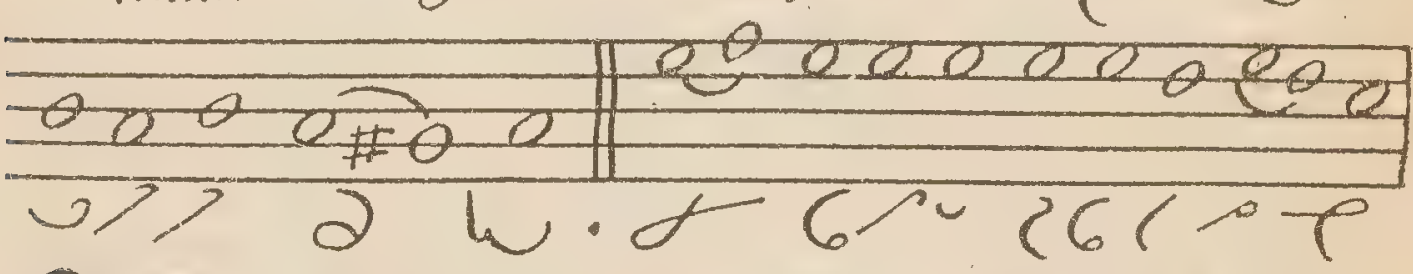

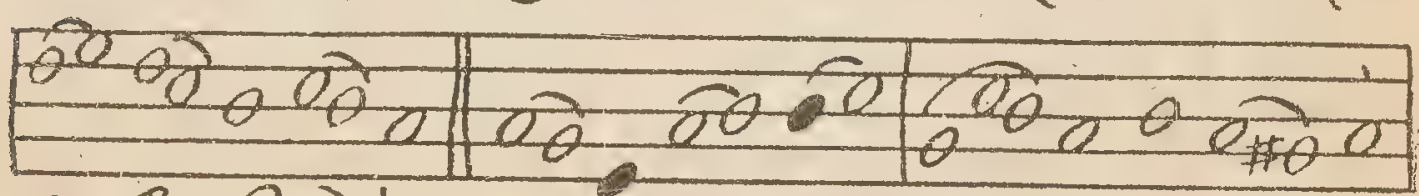

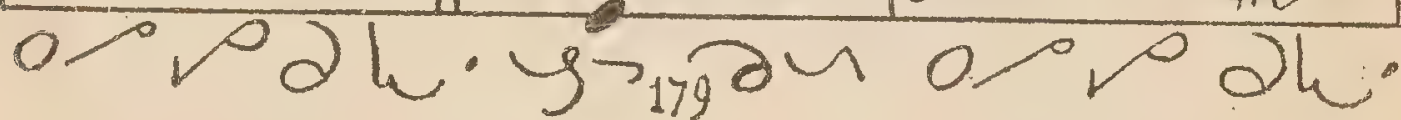


No. 87.

Un Seul Dieu

tu adorerocs.

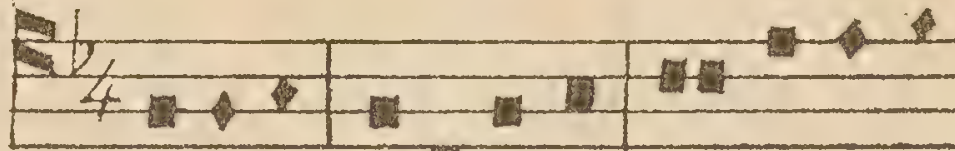

$\sim \sim 7-0 / \rho-C=$

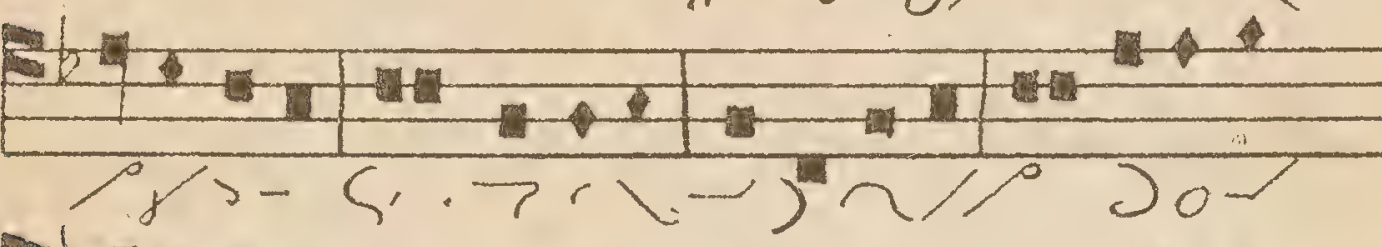

औ... $\partial-0() \zeta$

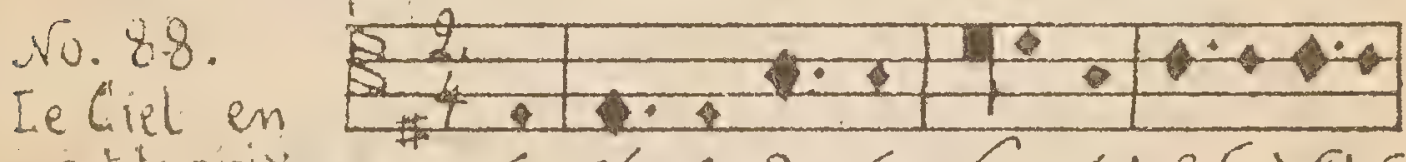

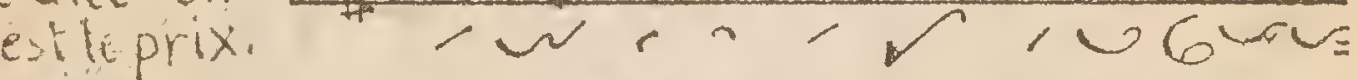

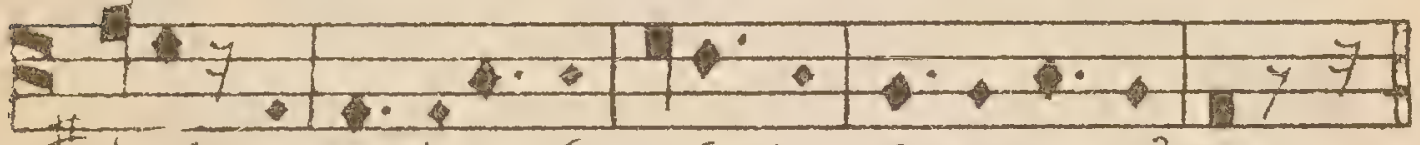

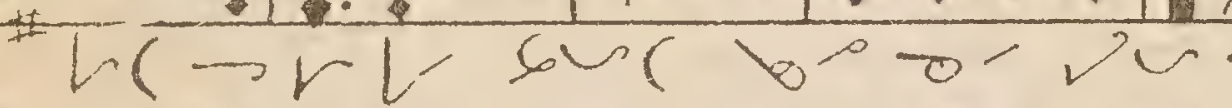

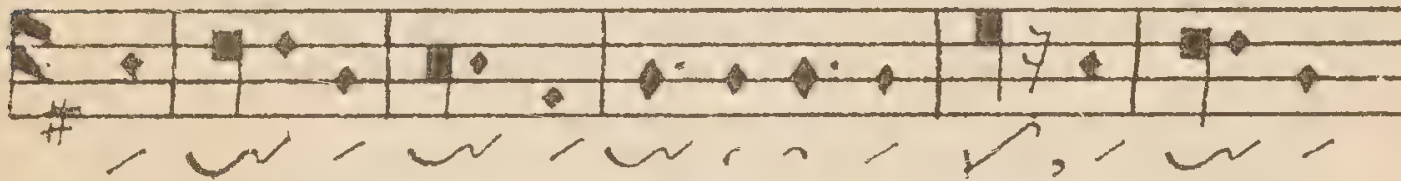

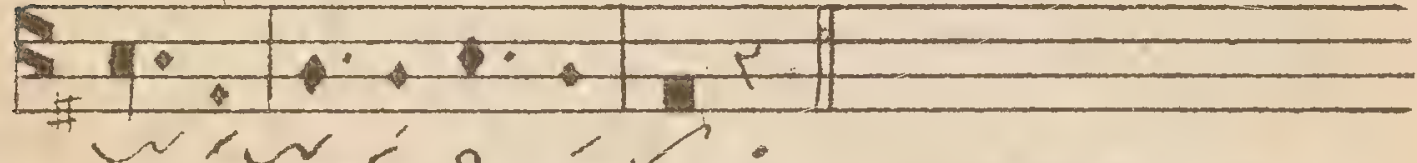

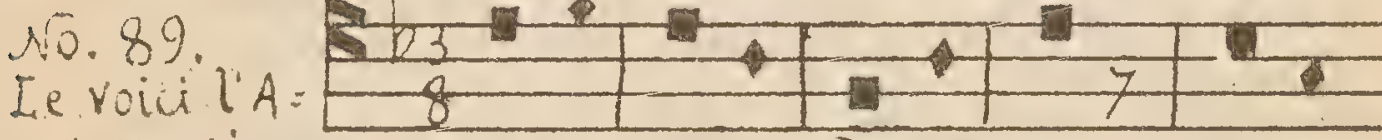

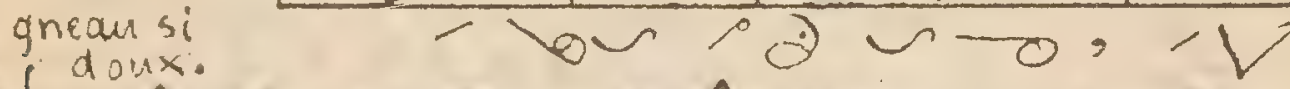

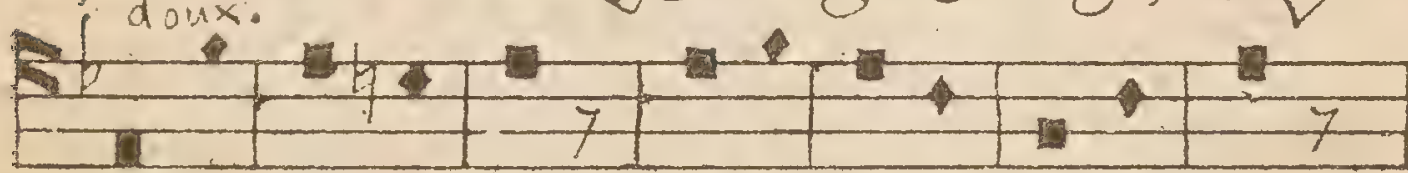

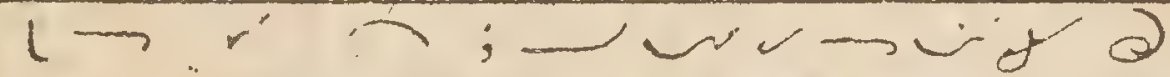

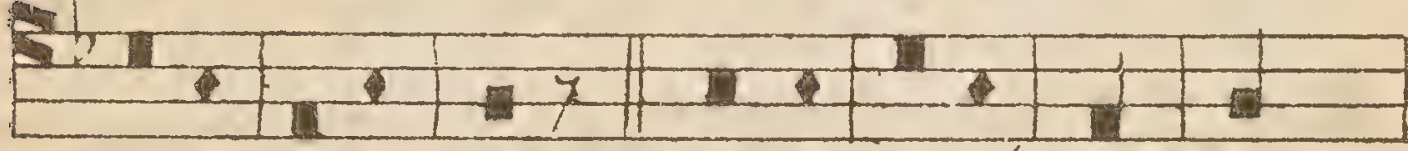
$0>>>0$
$\circlearrowleft$
ᄂ,

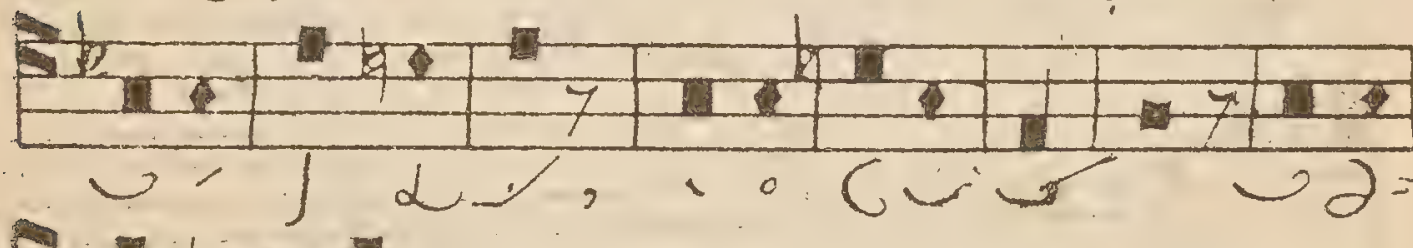

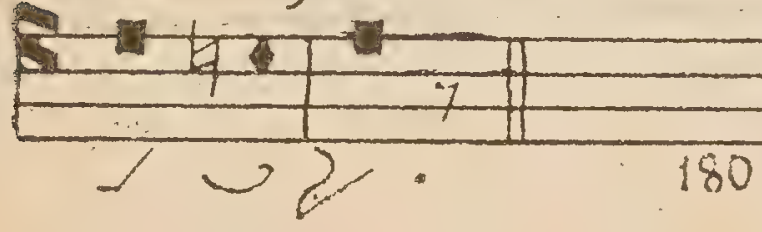


No. 90.

Vive Jesus

vive sa croix.

$\frac{1}{3}$

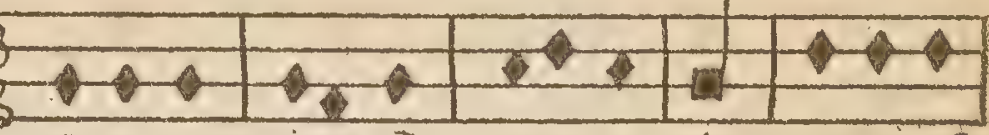
orut $D$ cos, ore $6=$

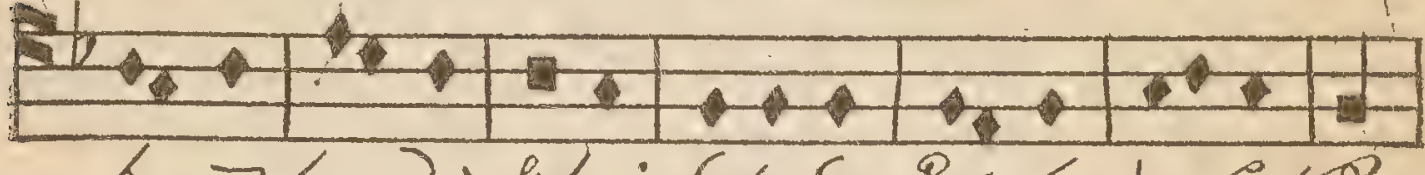

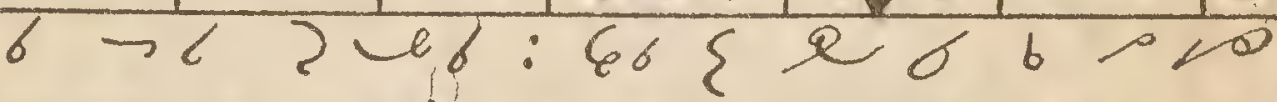

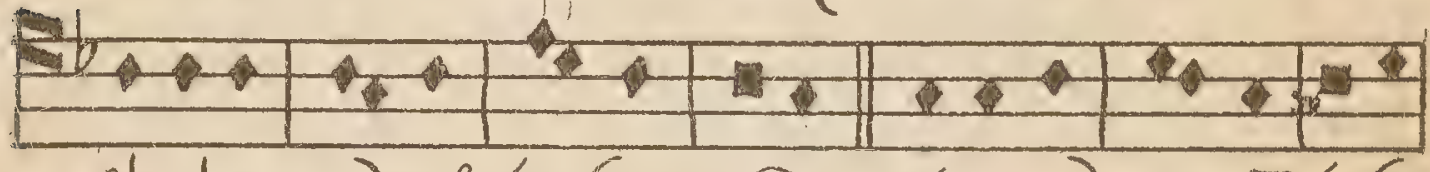

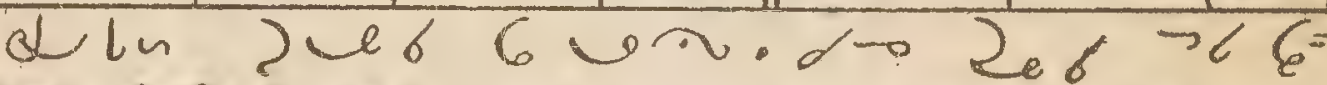

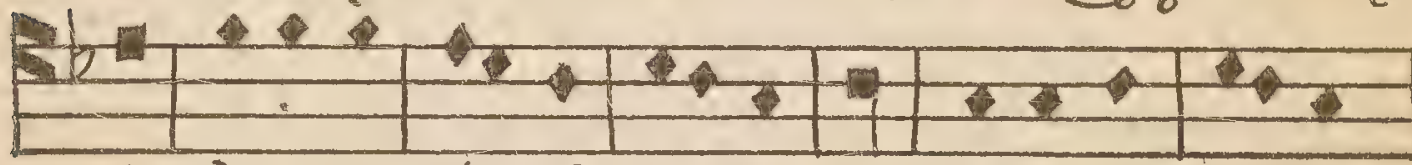

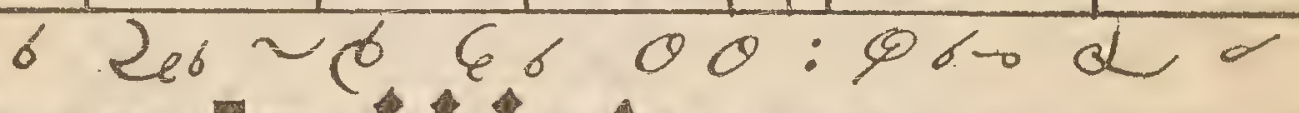

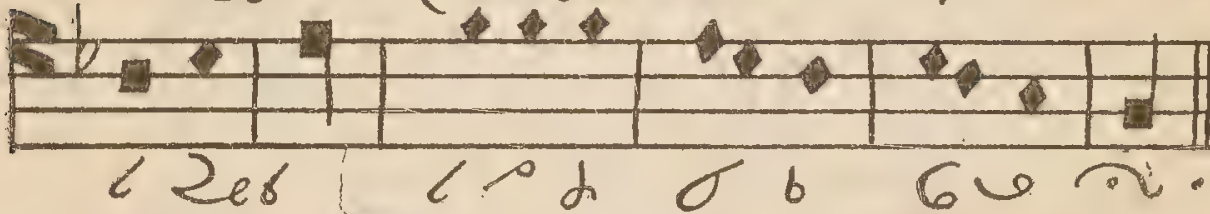
Quelle nouvelle $\begin{aligned} & \text { vey } \\ & \text { Q }\end{aligned}$

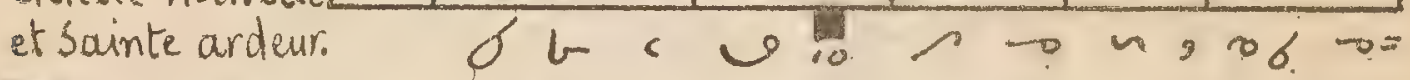
Dit

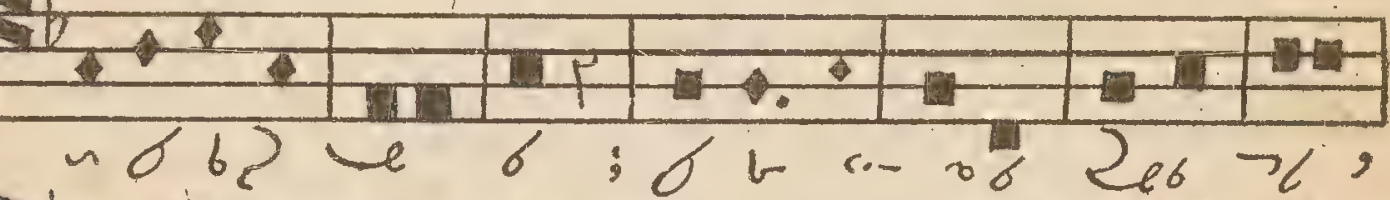

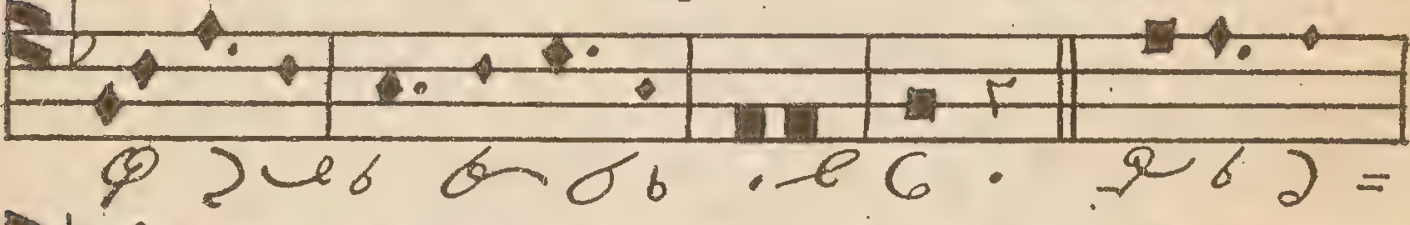
40.4.

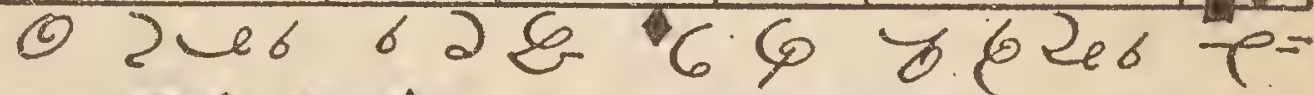

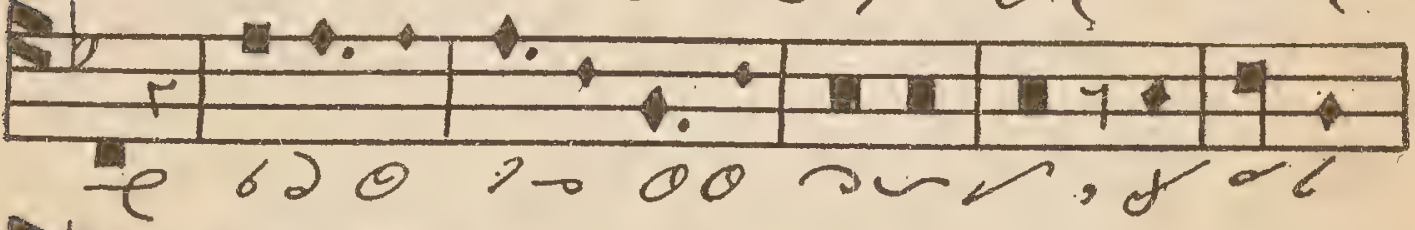
$\frac{-1.1 \%}{6966}$ 
o tloos S! Joseph. (Arr: Gelitum Josenphy

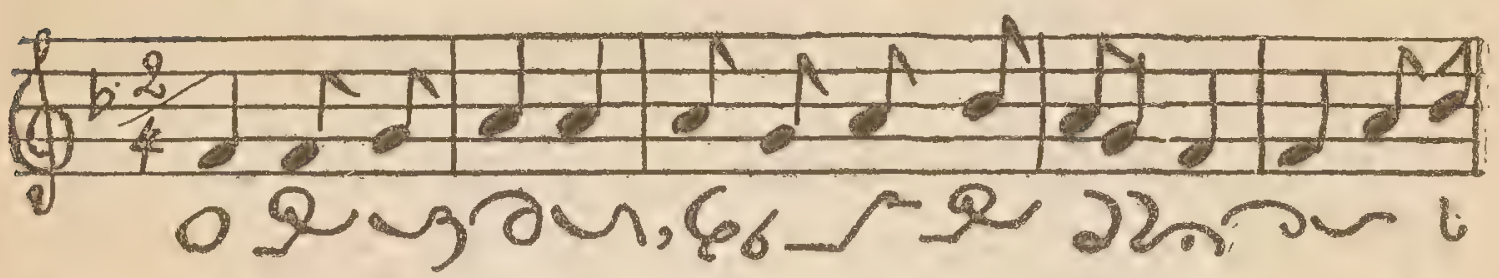

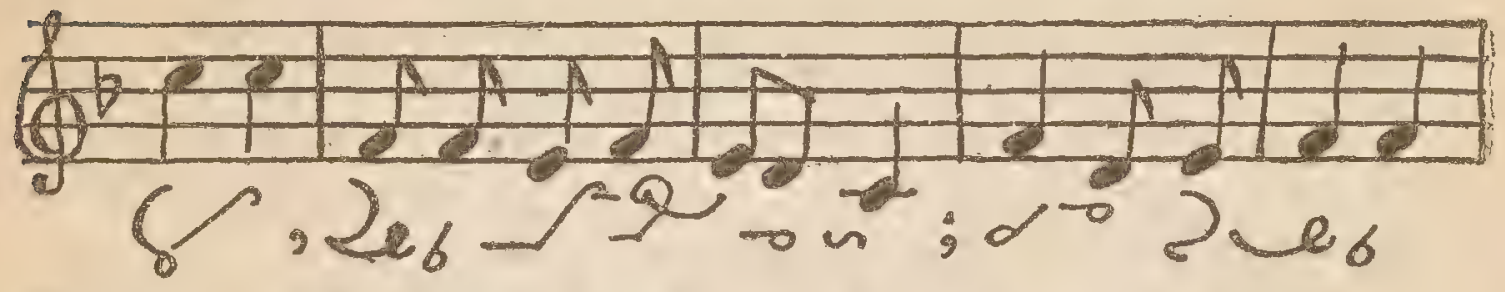

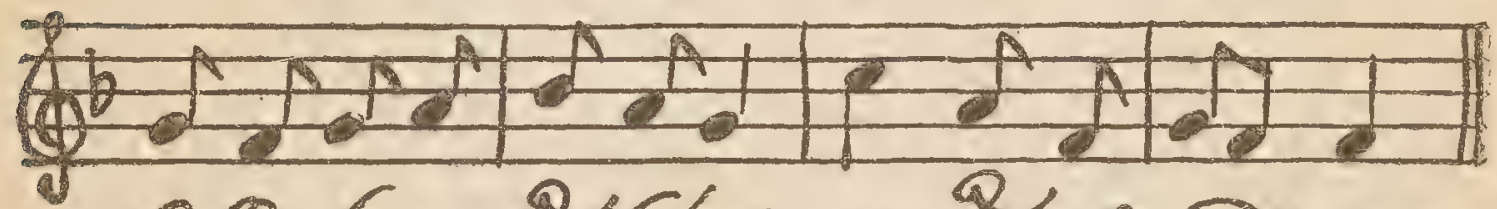
00 G edr,o ezan.

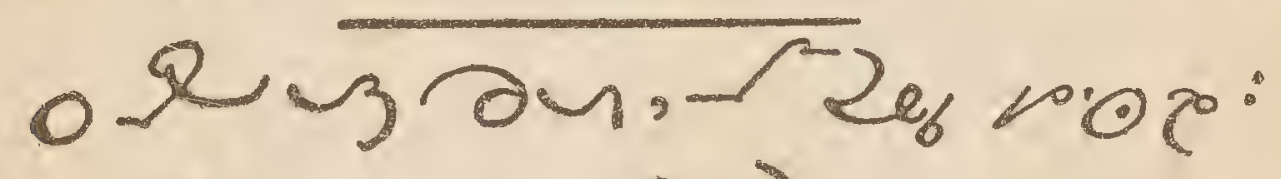
rar $\Rightarrow$ ro $\mathrm{Ke}^{\circ}$.

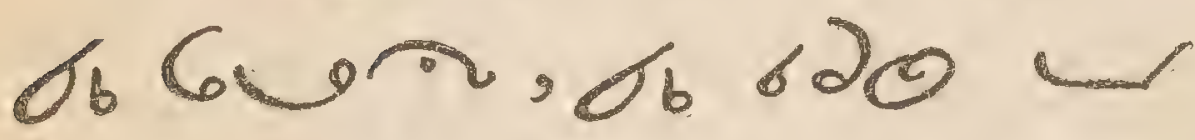

$$
\text { ol zan }
$$$$
\text { o suan. arongee. }
$$
$b^{\text {an }}$ a ee, bandee.

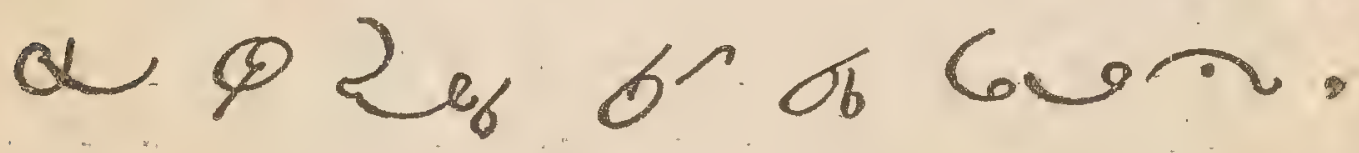

$$
\text { ol } 3 \text { on. }
$$


Tloos alta nsaitar.

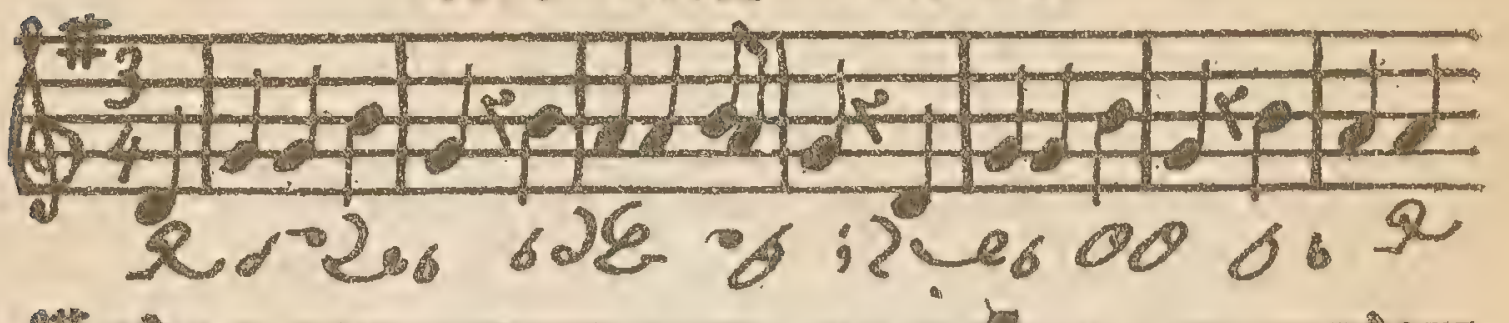

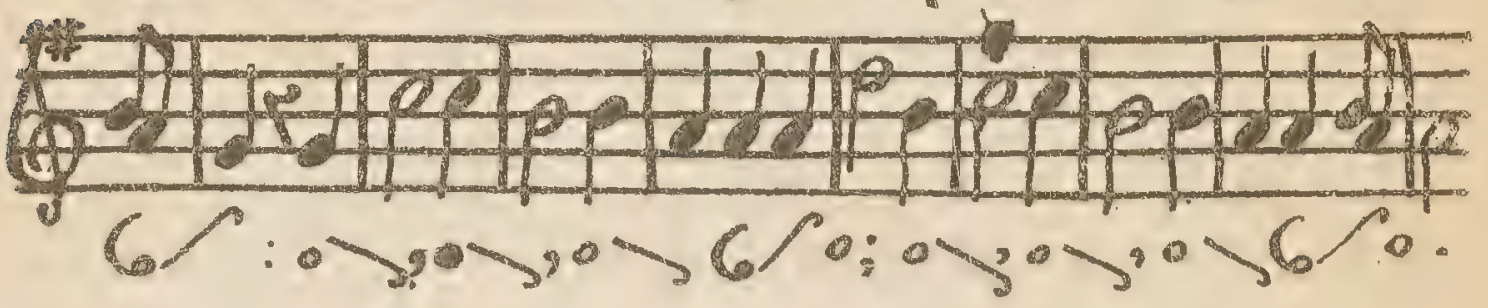

$2 \div-1-2$ - $26-9:$ रुes 00 of $260^{\circ}$. $9.03 \cdot 0 \times 6 \%$; $0>0>0<6 \%$. $-2=$

मे 686 of 2 \%e; $6, \wedge$ Ob 620 : o, $0 y, 0 y 6 \%$; a), 0 , o $6 \%$. $\operatorname{scon}^{-3-}$ $166,6 \%$ Q.0 ron व G. 68 : o>0 $006 \%$; $0.2,0>6 \%$.
625 a of 66 . al 2es 66: a $10 y 006 \%$ $0,0>0.6 \%$.

ob 200 in: o.- 8 bin ob क :

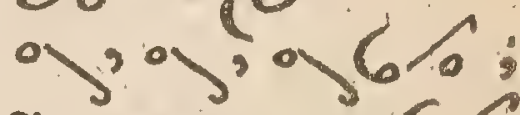
- >0, $006 \%$. $-6-$

$266 \%$ Jee 66 ; 2. 1 ? d. कु se $^{2}$ or $305.0 \times 66$; - $>0>0 \%$. 
Naitia chako wawa tloos mersi.

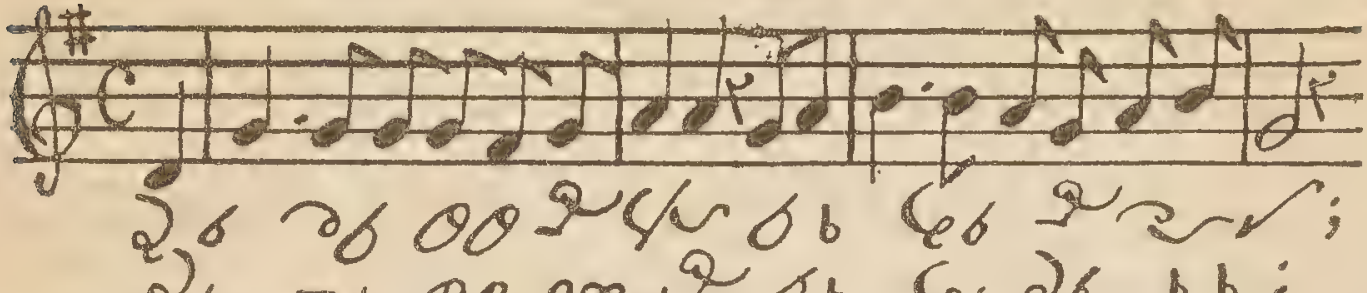

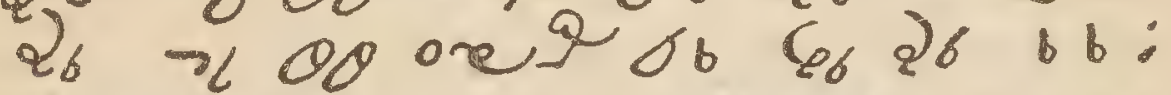

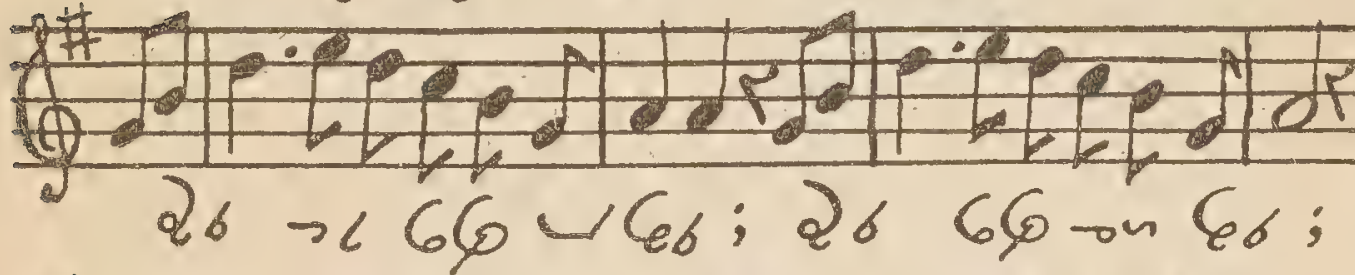

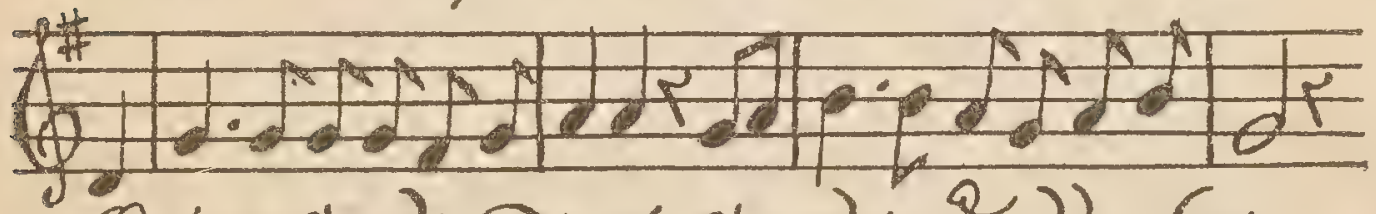

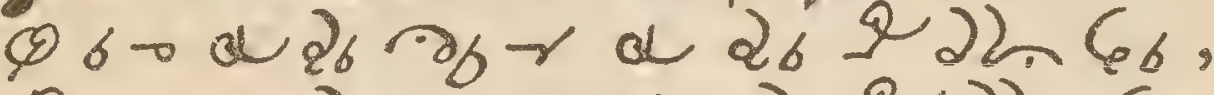

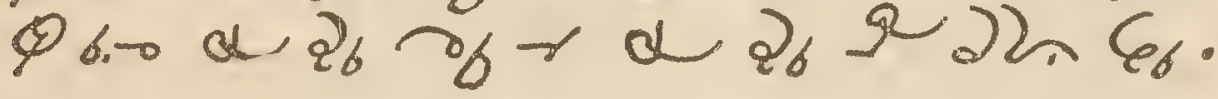

$-2-$ $\sigma_{6} \sigma_{6} Q d 2_{6}, e$

a o? 6666 a. 1 б. $>$ कृ:

r.- one ion 66 66:

Q wo Co $\sum R$

66602 d of 26 of $Q$;

666 \& 10 a d से 20

$$
6_{6}-3-
$$

bo $6666+e-\rho$

Q $d 1 \% 66$

$66.0 .0 \%$ \% :

$\sigma^{2} r_{66} G_{6}$

6606 one 10.0

ol cor ab $6.9 \%$,

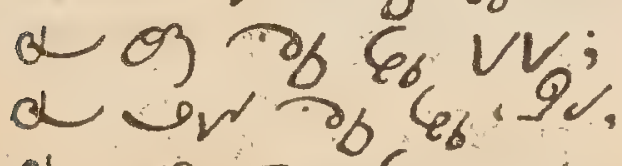

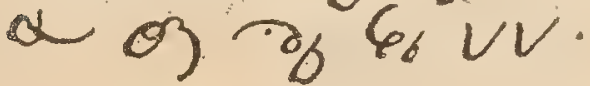

$02^{-4} \cos _{10}$ ob 0606 und 2b vie 92 है, 26 et 22n 6. 20 ${ }^{2} G_{6} G^{5}$ Ob 0.6. 60 var; Co 95 L G VN दे के क 66 ; $60<98166 \mathrm{VV}$ $122_{6} a 66$. $-5-$

$0 \times 6666 山$ a八 06 G.0 $\mathrm{C}$; ob $2 \% 8 \%$ N $\xi_{6}$ भुo शे 66. $a_{6}-a_{0}>6 c_{6}$, $q_{b} 0^{\rho} \sigma_{b} \varepsilon_{b}$ : on. - $c_{6} g x$ 66 top \& 6 ep: $\sigma_{6} \cdot-\varphi_{6} \Omega x$ 66 top \& $\mathrm{ee}$. 


\section{- Salutaris.}

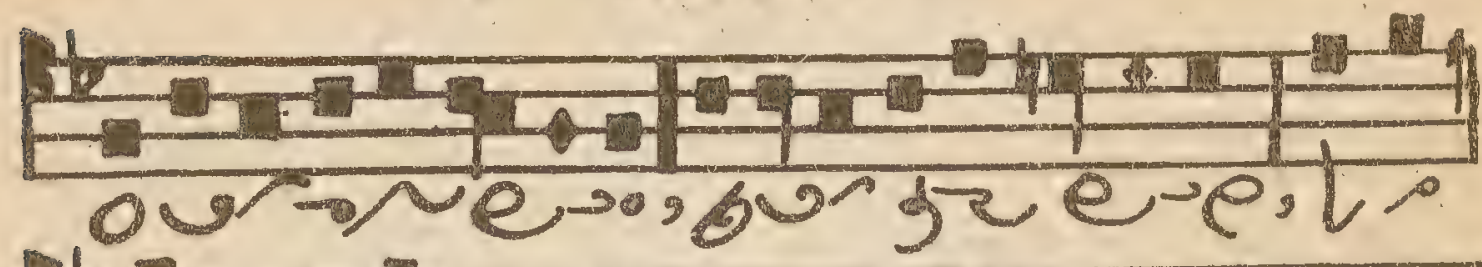

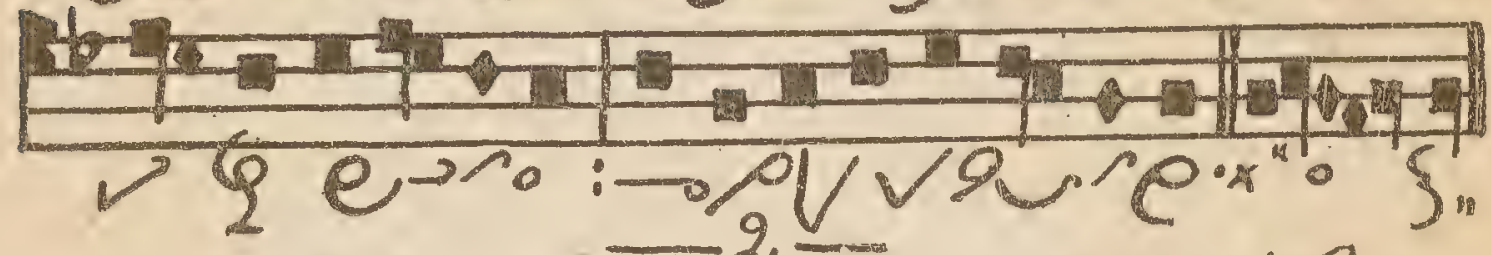

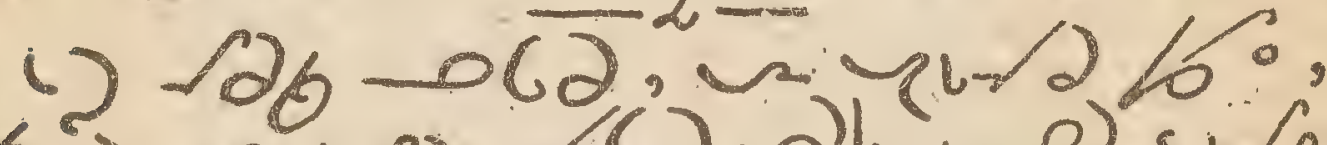

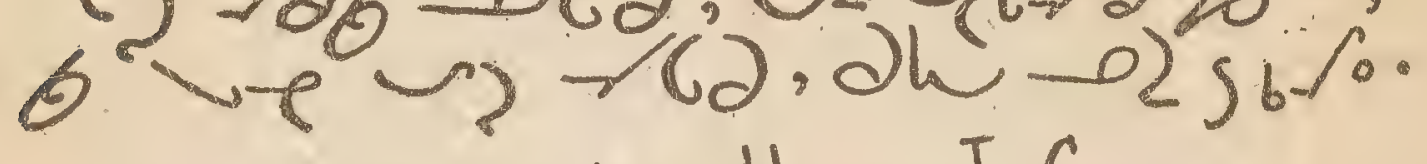
o maika Hoos J. C.

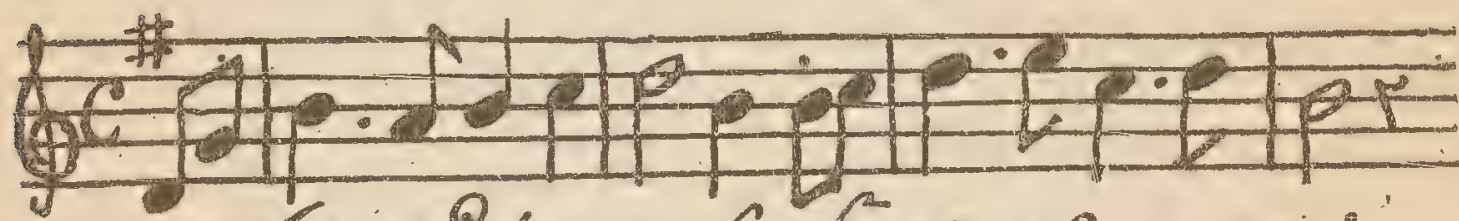

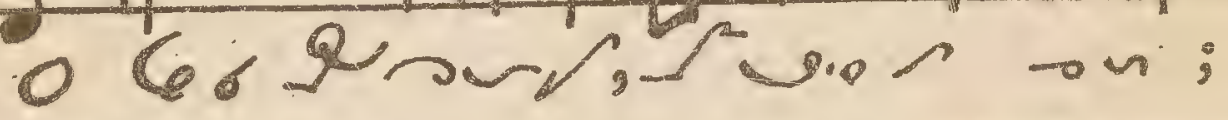

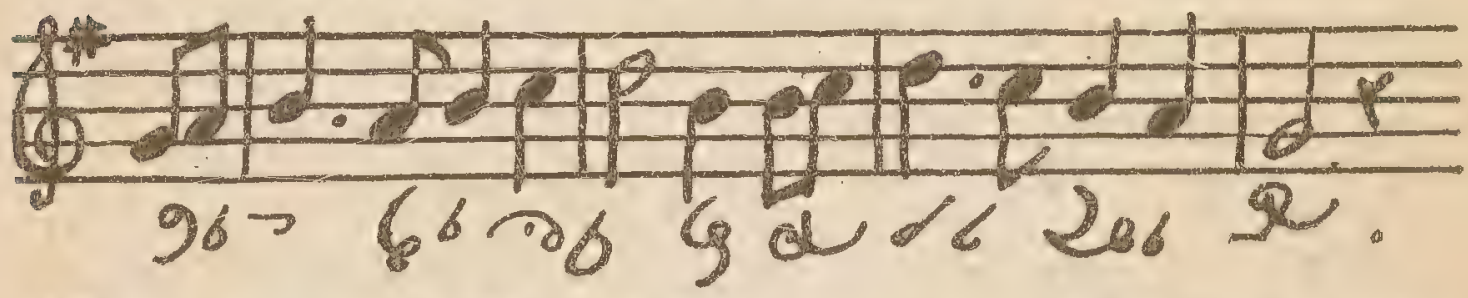

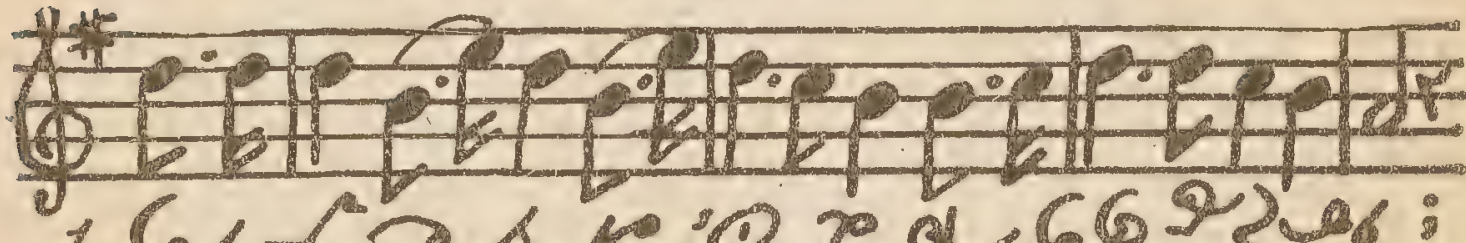

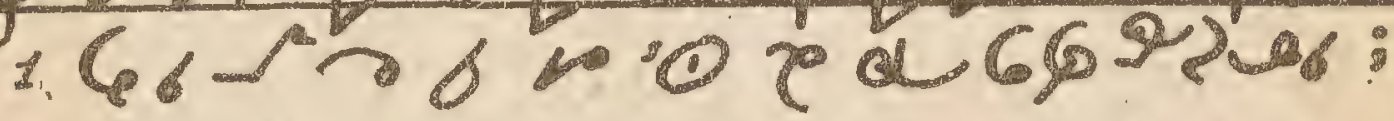

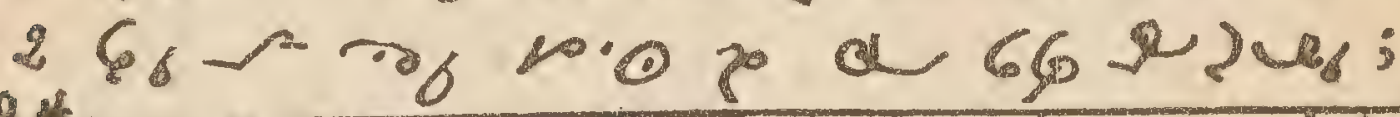

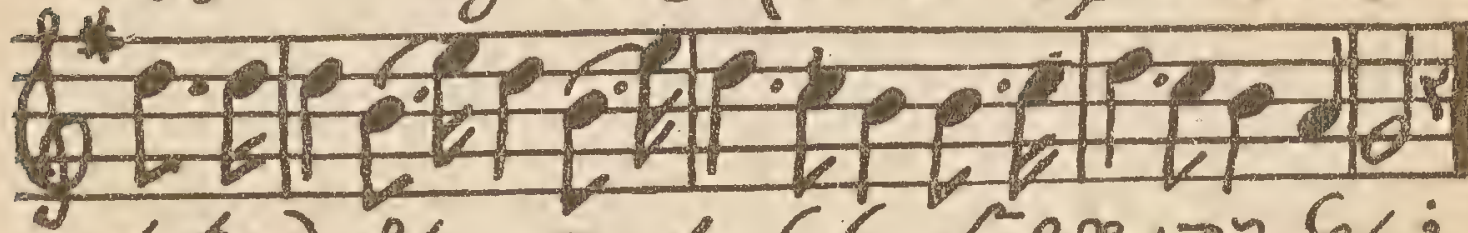
$60306>266$ poren $66^{\circ}$ 68 रुe6 $766 \%$ Nore on 66.

$96-66-2 \overline{9} 60.86$ \& 168 246:

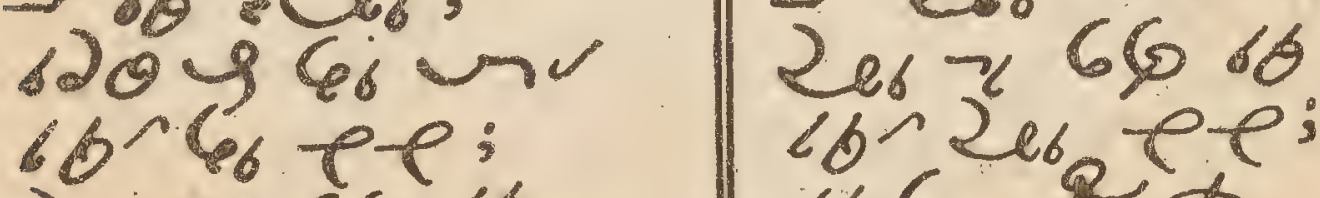
26,26060 . 


\section{Litany of the $B$. Virgin.}

en $\sim$ g.

s s

$\sim, 0 \rightarrow 2$.

$\sim \rightarrow, 20 \rightarrow 2$.

dr $\rightarrow ?$ ?

Gul gu.

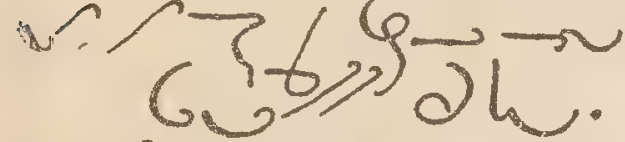

ur $y=\overrightarrow{0}$, Guश口.

$Y=\sqrt{3}-2) \rightarrow$

Guग टरू.

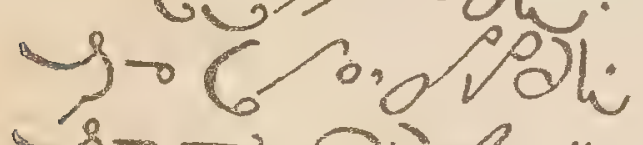

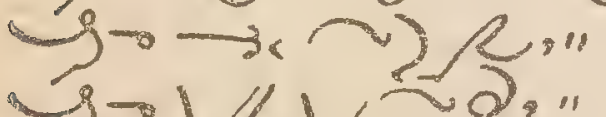

G-VEV,

6r w? pro."

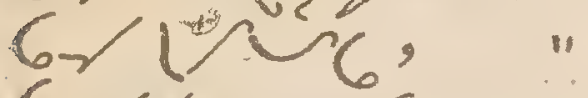

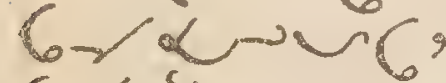

$6-1)_{0}$

$6-1) \rightarrow(\rho$,

$6-1061$,

Gror

62 "0.

62 or

V. $35 \backsim 6 . "$

Qb $1 / 8 \rightarrow "$

bु) "

Vb bz, "

名以之; "

ye حun,"

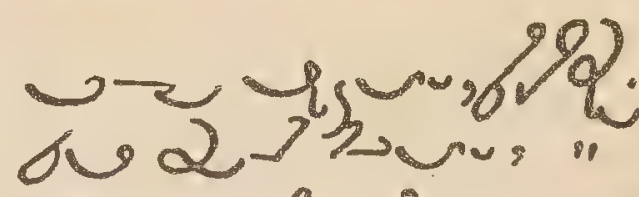
enfor" "

e o. 1 .

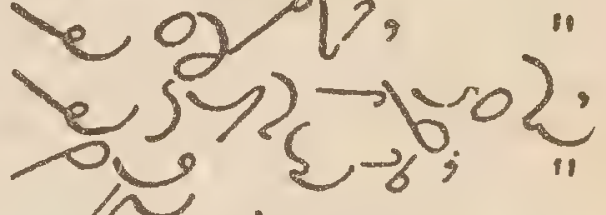

- "

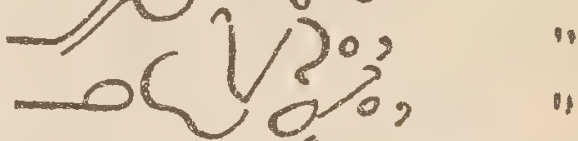

政,

(3) पर,

$\rightarrow$ (P 6 , "

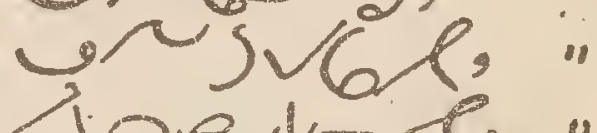
rejor," guse avole" is a diee." $>0$ b fol." 20 so el" is ou of." "D) 84. त. $92,0 h$."

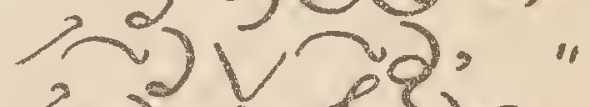
त) प्र० ej,"

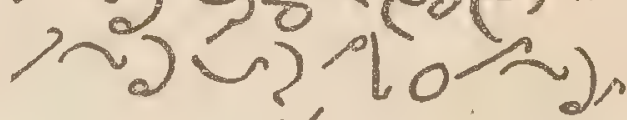
) $20,6-6$, $29 \rightarrow 502-62$ ? $9 \rightarrow 0,0 \rightarrow 2 \xi$.

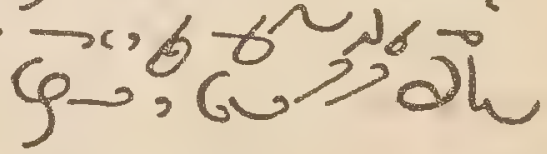

* S $10-2=\simeq 20-\alpha$.

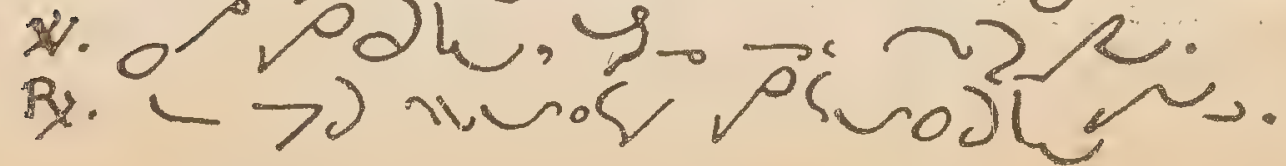




\section{Chant for the Litany.}

$-\mathrm{I}-$

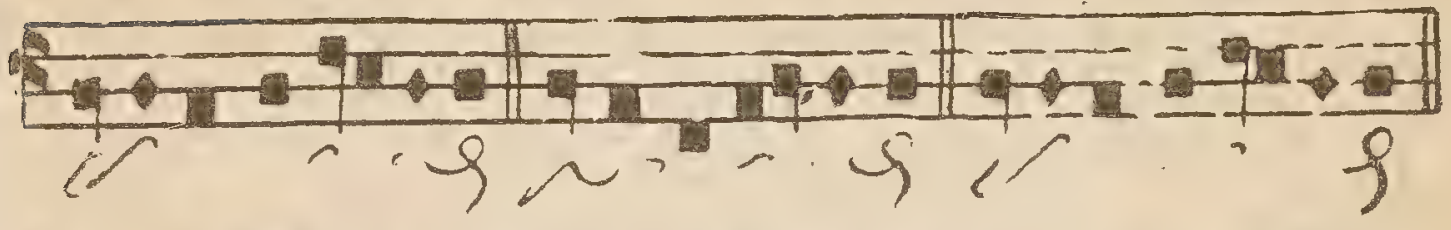

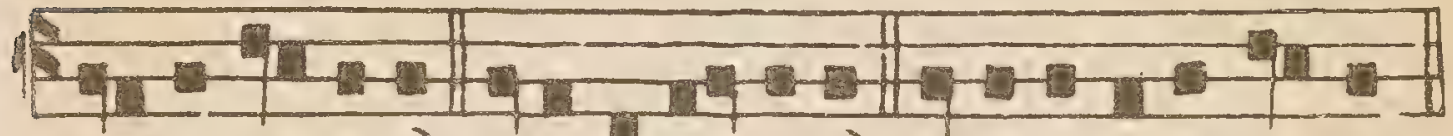

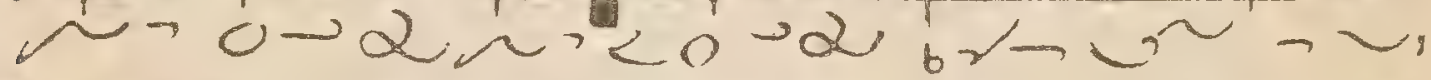

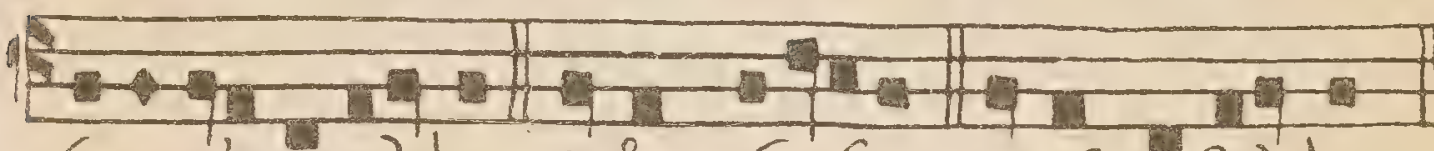
w), $2 h$ 9-6ro of

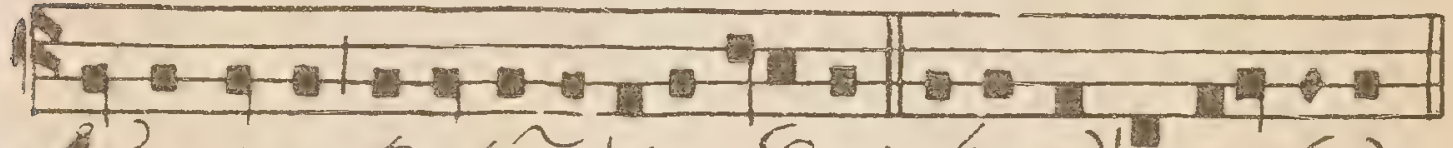

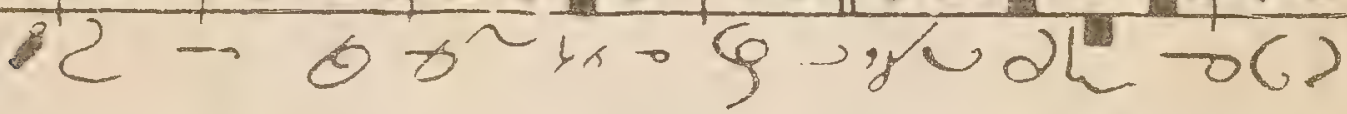
- 11

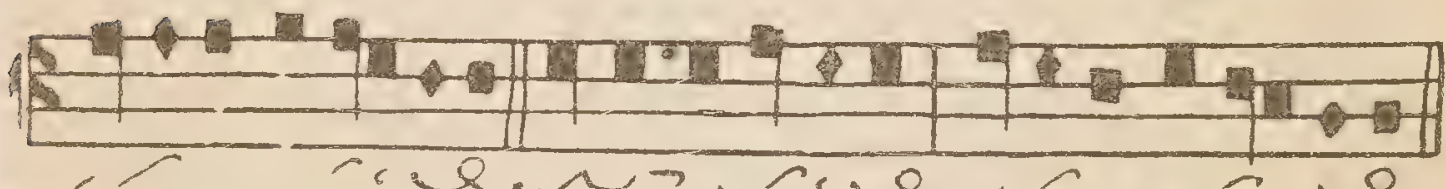

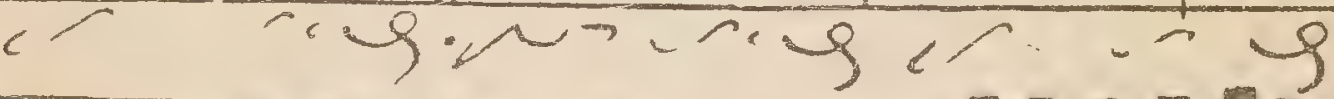
- ${ }_{11}$. $\sim 0-2 \sim \cdots<0-2$ br $=$

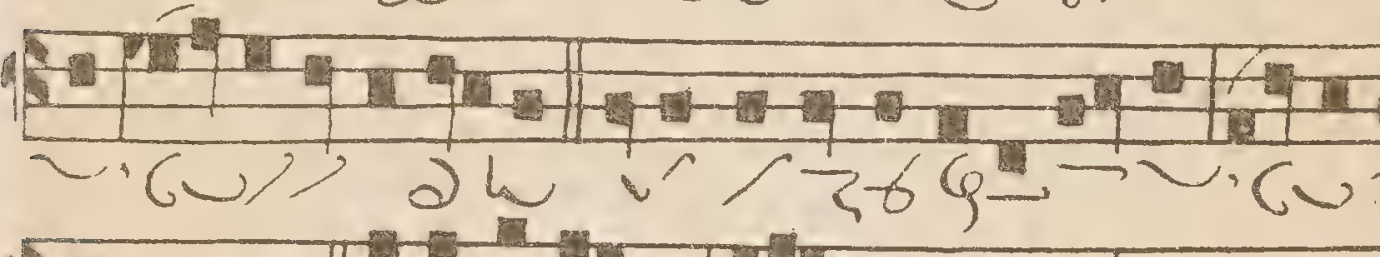
2 7म है का

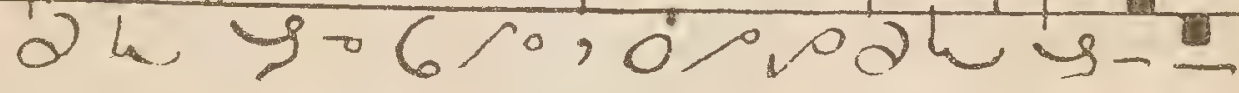
$-111-$

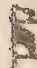

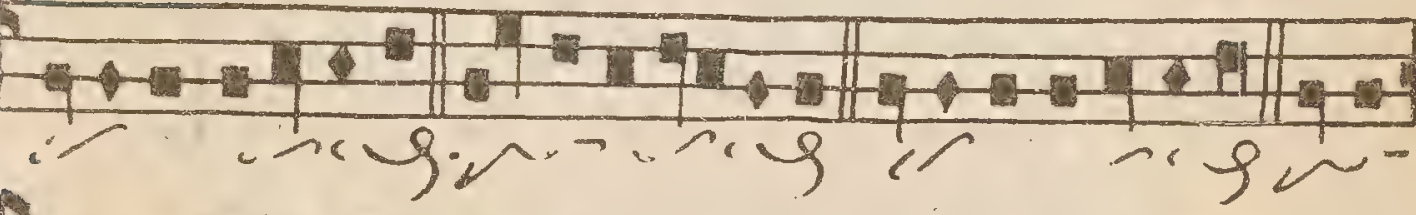

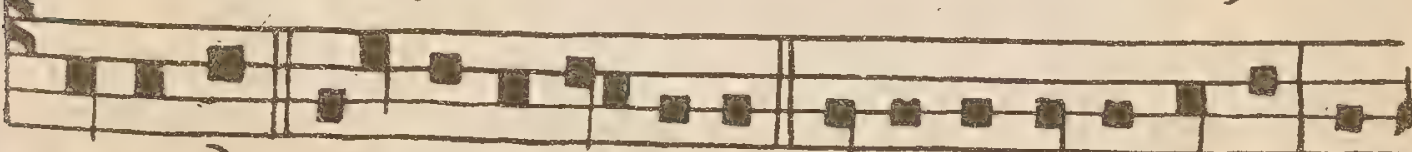
$0 \rightarrow 2 \cdot{ }^{\prime \prime 20}-2$ br $-3 \sim \sim, G=$ .

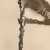




\section{Inviolata.}

38

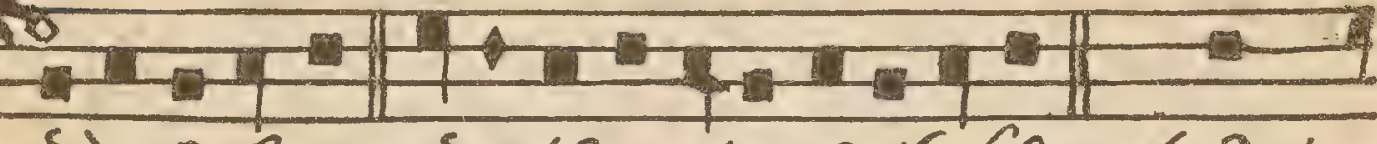
1

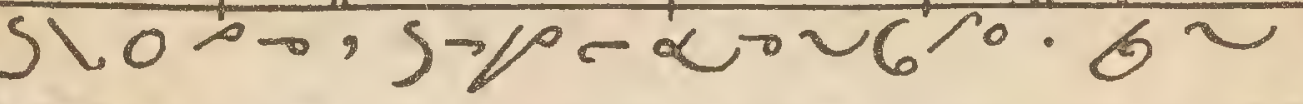
5

n.

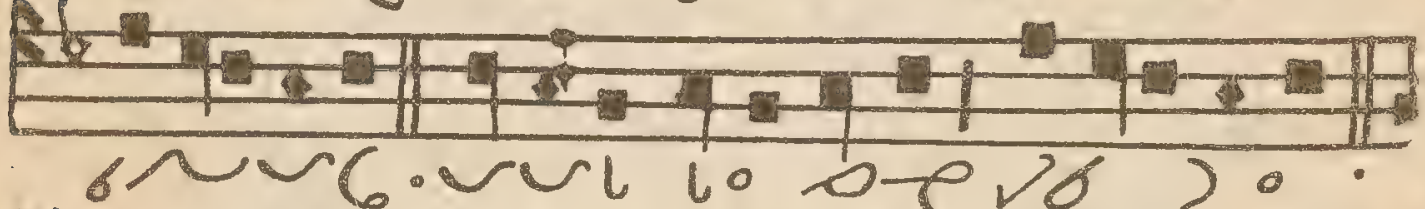

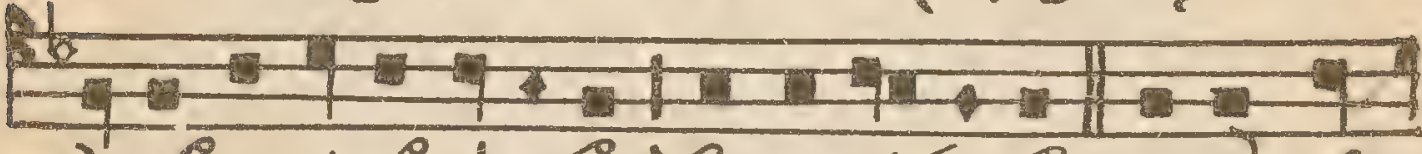

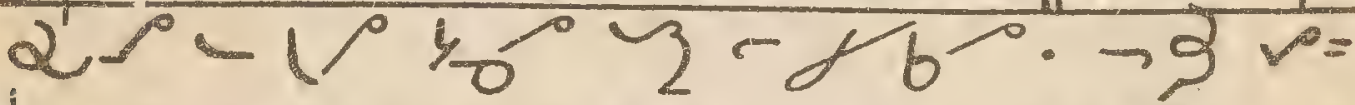
i

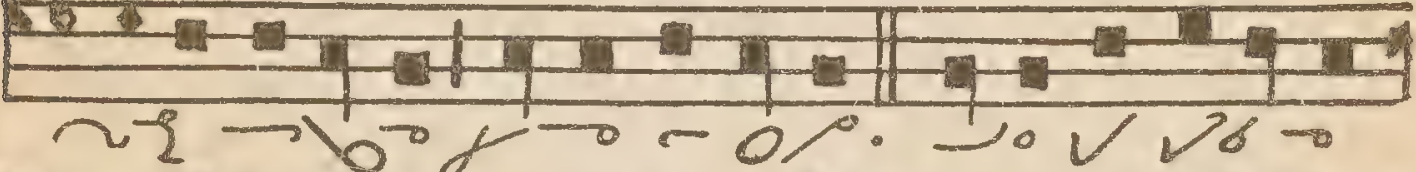
To for

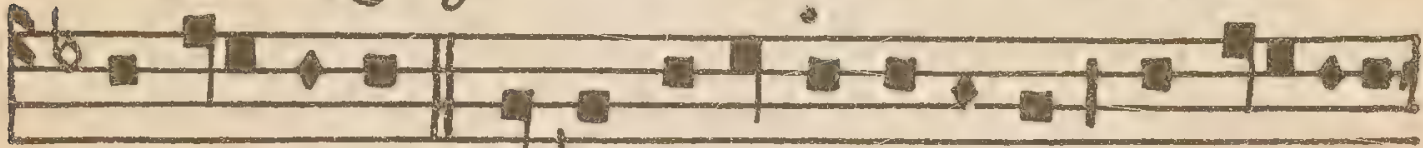

1

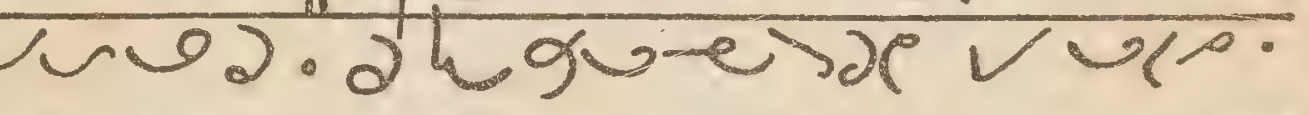

54 A

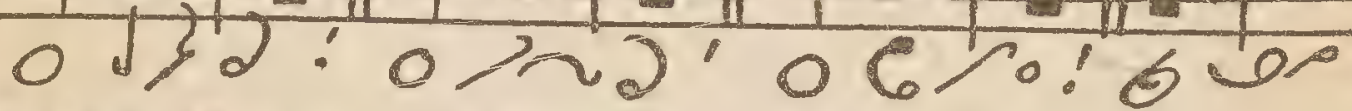

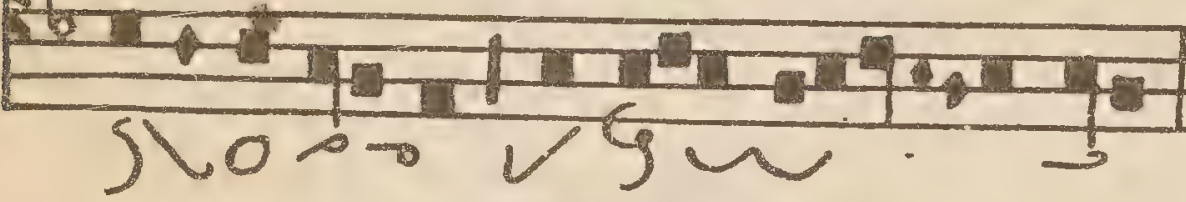
Parce, Domine

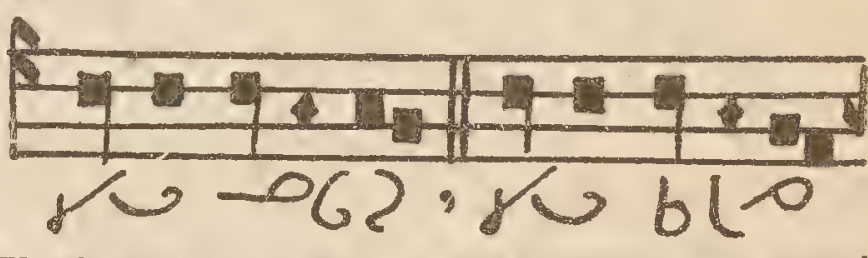

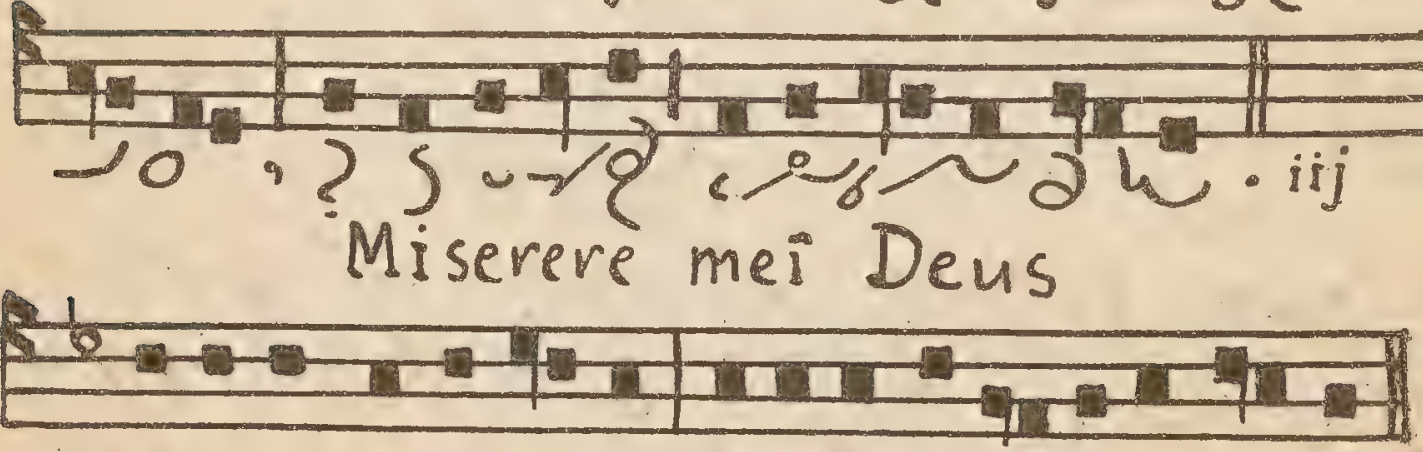


The Magnificat.

Fin

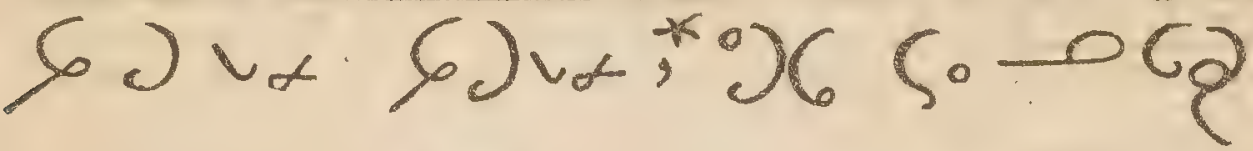

$-2 v^{-6} \cos =$

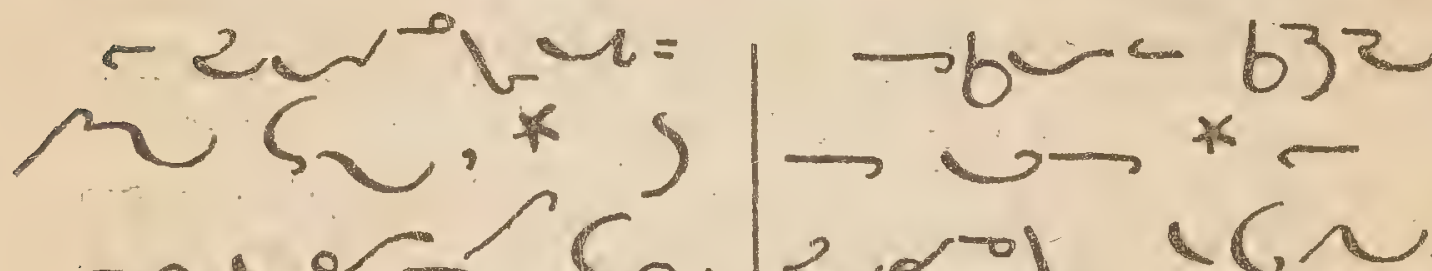

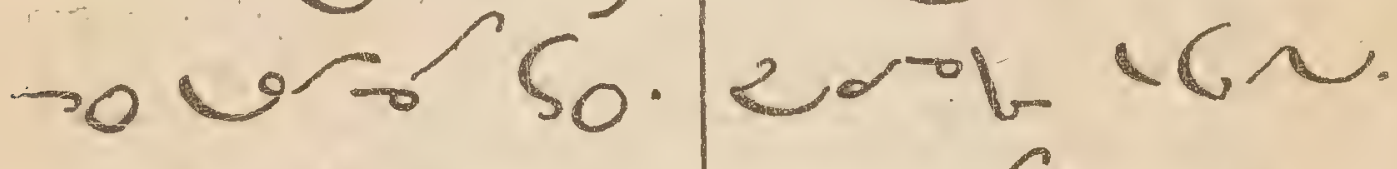

6. 1\%

Gror gun

$1 / \rho_{2}<$

$b^{2} \times-x^{2}$

: * 20

29 lates

$\rightarrow 3(2 \pi)=$

Gon so):

Uut 2

l. $\sim$ e *

G. sun Gis

Co 6 b3 $x^{*}$

$\left.-s_{0} \alpha\right\}$

$\sim$

- Guffo worsu.

$5 \infty^{\infty} x^{*}$

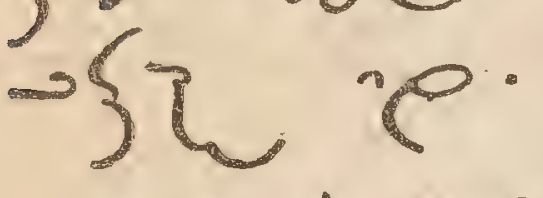

sa bjus

$180 \sim 0^{: *}$

fan CW

$f \longleftrightarrow \infty \cup$

$\sim \mathrm{P} \sim$

- $\sim 2 \varepsilon_{*}$

Vpe - w

w S w

6o bar

$10 *-\mu c^{*}$

$y_{0}$.

L $2 \Omega$

$u_{0}-0-2 / x$

ruludd

-3 up

$\{r y=2.0$. 


\section{Laudate Dominum Omnes Gentes.}

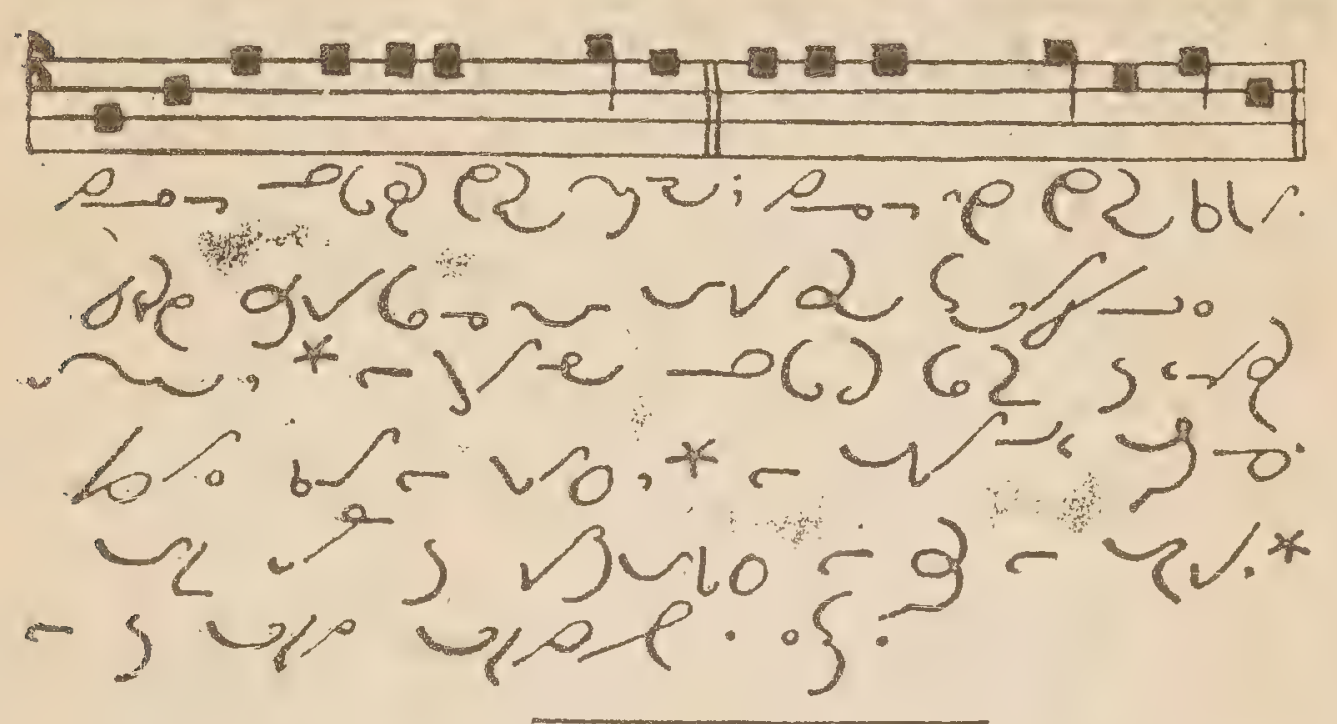

Iakwa, alta

the

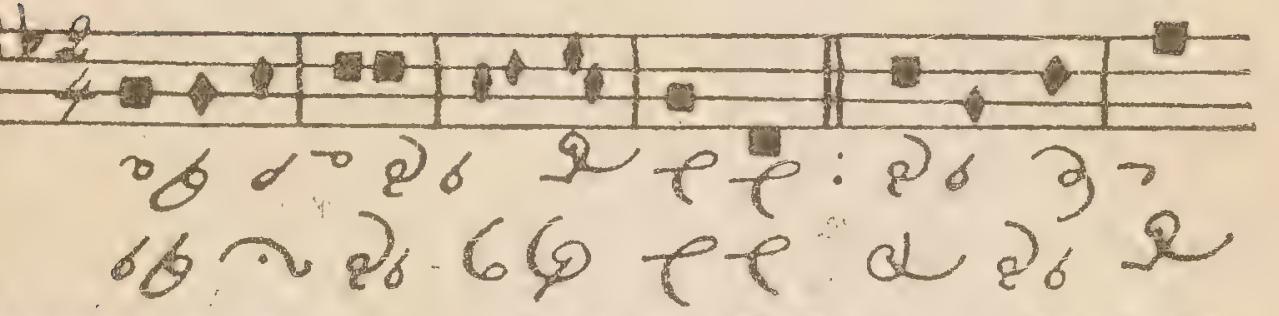

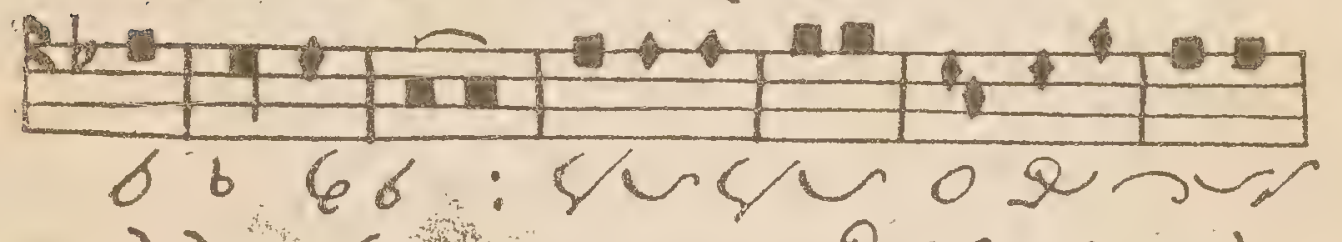

$2266 ., 066$ क $46 \sim$

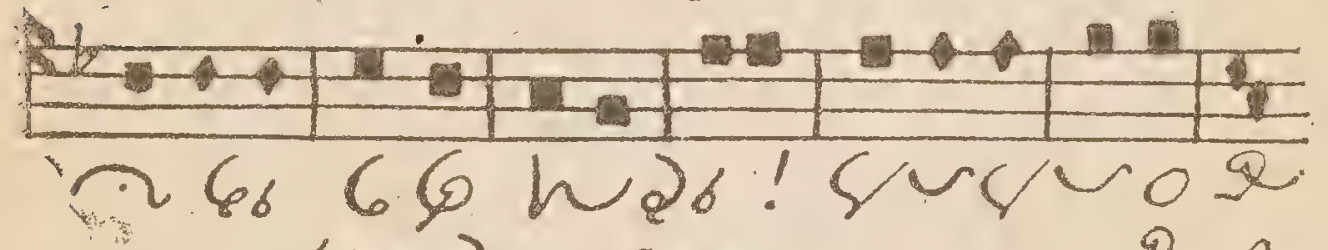

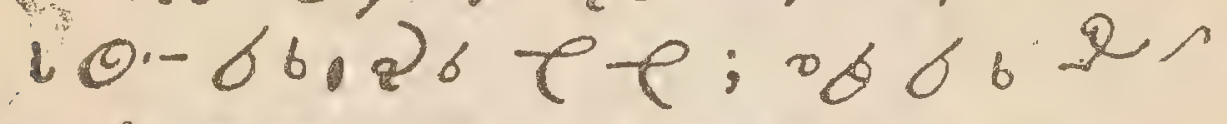

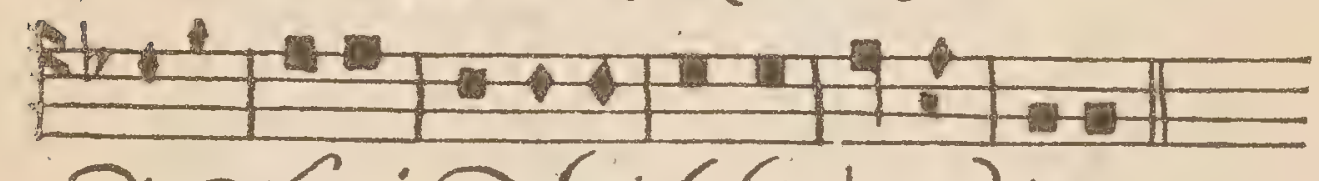

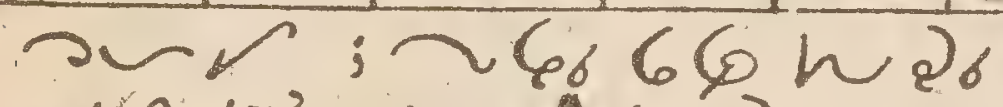

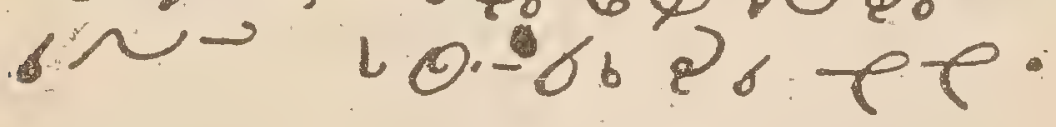




\section{Welcome to the Bishop.}

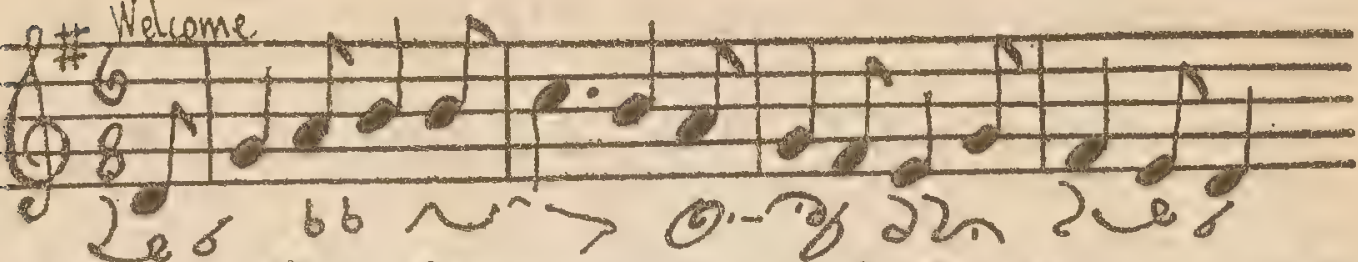

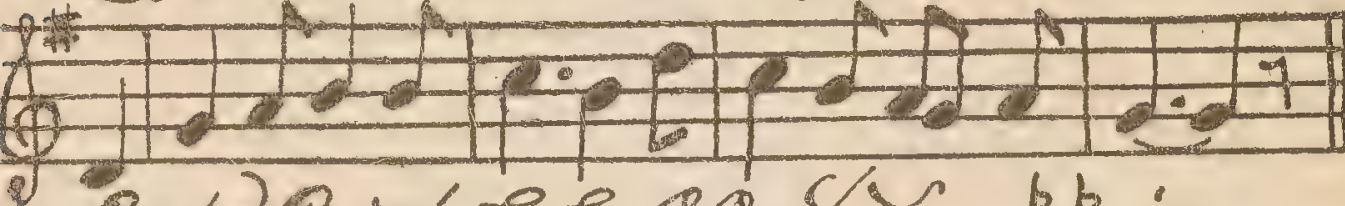

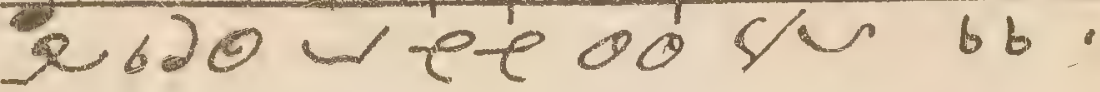

$2 e_{6} 6 \overbrace{\partial 2}^{1}$

Q. - of $2226:$

.

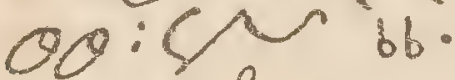
2

$2 a_{6}$ 6ь $\sim$

1026 ore $r$;

an $\varepsilon_{6} \%_{0}$

$22 \div C_{8}+22$.

$2 a b a b \longdiv { 2 }$

one $e_{8}$ ép.

$60.16_{6}-2,2$ a ser $\Rightarrow$.

$20.6 \leadsto$

nim

a 286 \%on.

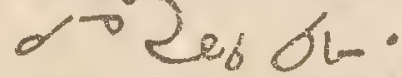

s

$2_{26}$ b6 $\sim$

66066 ee

$82066-2$. os ee $e_{6}$

206 6 $\leadsto$

6 ps $x$

600 6. S.

6 . 2620 .
206 b6

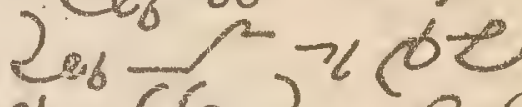

ou $66206-e$

$x$ \%. 8

रूक แ

$100 b_{6}$ क

a 60 or 20

(3) Ona.

9

2063

$66 \times 65:$

$x$ \% 00

$G 5066$. 10.

2ab bo is

ob on \& ge olo $2 u$ op $-11$

2es $60 \sim$

Les ore a ep:

$d \varphi_{6}$ व 60 ore 2 res. 12

$\mathrm{C}_{6} \mathrm{~b}^{6} \sim \mathrm{2}_{6}$

ob 0 - 2es 00 : 







\title{
Stalo Manual
}

...OR....

\section{Prayers, Hymns and the}

\section{Catechism}

..IN THE..

\section{Stalo or Lower Fraser Language}

\author{
With the Approbation of \\ RT. REV. P. DURIEU, D.D. O.M.I. \\ Bishop of New Westminster
}

KAMLOOPS, B.C, 
Stalo Morning Prayers. oiaro Prece asp to - bow 0 G, $2-5 \%$,

? Morning offering. watora, $\approx$, d $\log _{3}-a, j s_{n, 2}$ octimn to.

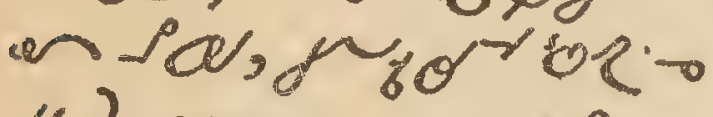

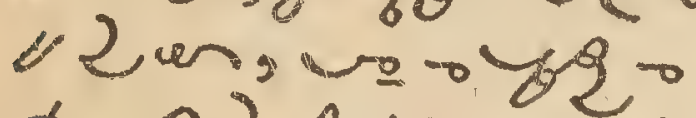
x.Q 2 injugen PQ. Uniangabm PYG, Q Q P.O \& soras —, sе Syus - 28 B.

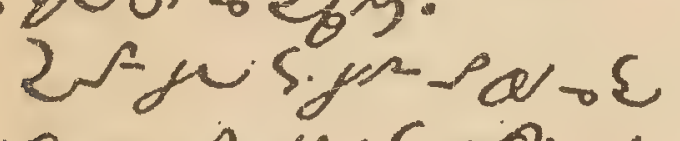

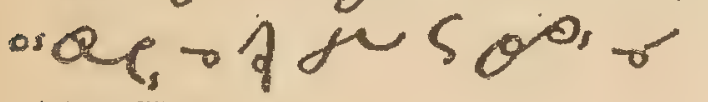
से 3 .

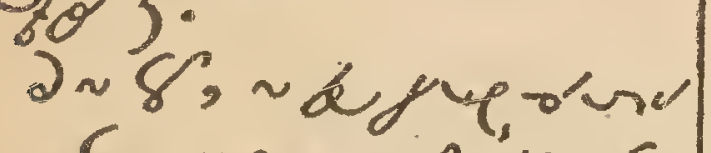
$\because \varepsilon$ os a -1946

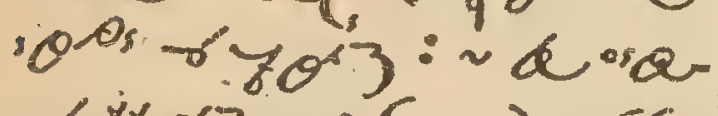
$08803-\varepsilon_{3} 20 \Omega$ mosersiogn? $\checkmark y+2$ ofe.

Pater. $\&$ Gin.

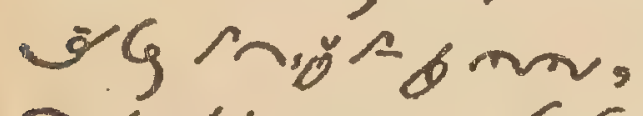
$\sim 2<$ b m $06 \varepsilon_{0}$

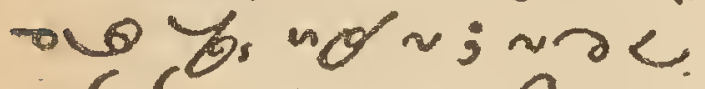

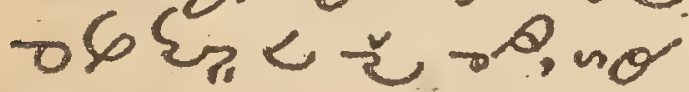

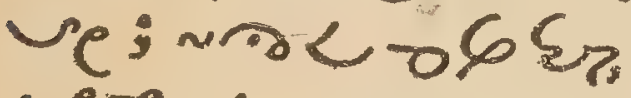
cr $6,<$ unvorio 39, 문. $\varepsilon_{0} \cos _{b_{0}}=$ W; z Survall un, vevon an a wro oy yous

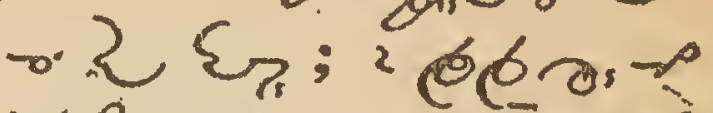

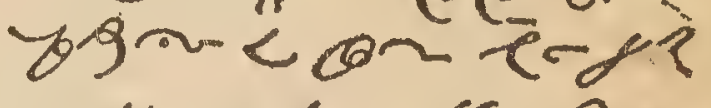

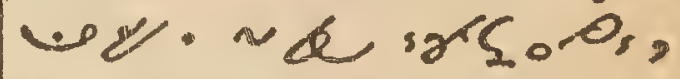
¿बr winn. जो.

Ave. o \& un. o r, setas rie, ( GQvir;opa 00 sic $2 \cdot=\sim 80$ ?

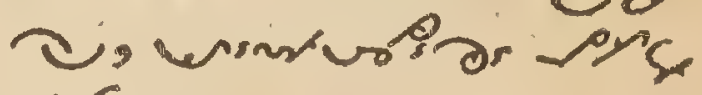

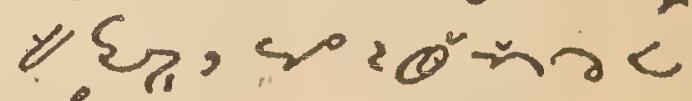
धn. $\sim$ cu. Credo. anvori $\sim 1 .=0$ surivod G, no b6:02 b6ue.

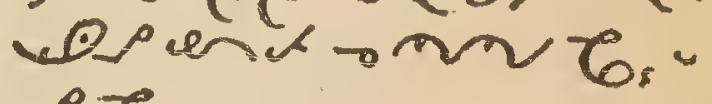
Tr;andinox - ole sin já कs spere iosw sove; owewens

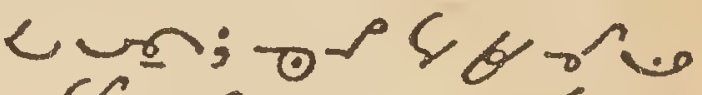

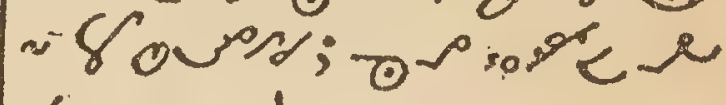
up o 2v;r-and cun, cyenci2s

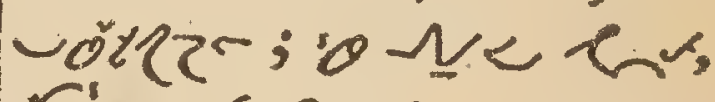
$x^{2} 65-6$ - $x^{2}$. 421 0.5द 
- a g no eb:ol 6 S जe:- $A \in<E G=$

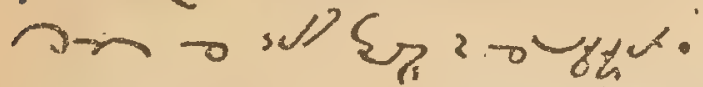
= Onudoraveris. थ -60 of $\varepsilon_{7}, 2<\mathrm{Ch}^{\circ} \mathrm{C}$

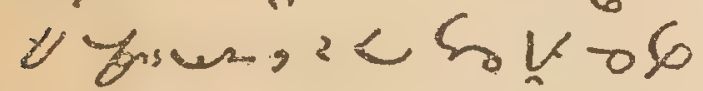

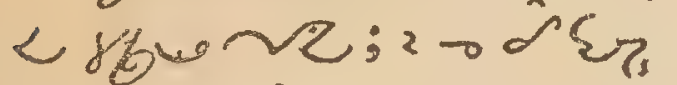

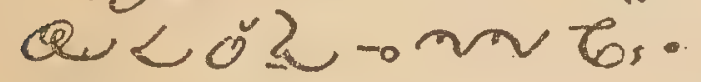
Q 2 -

confiteor. sosolepur. = scorruger sol ore "o be sol 64 ves so? ज्ञ 800 \% GuA, sol- स, so? $-y / 3 w / 3 \gamma, 02$ $66 \mathrm{~m}$ हn, '0र 0 क उre

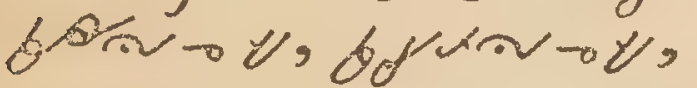

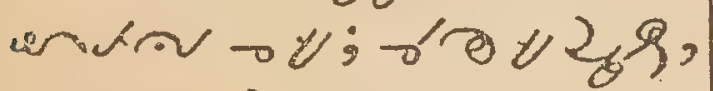
zg. - vouvise, os

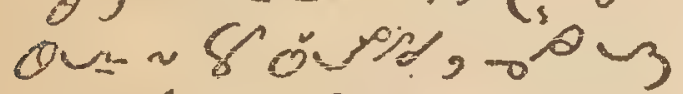
Gud, $0^{A} *,-P_{1} y_{1}$

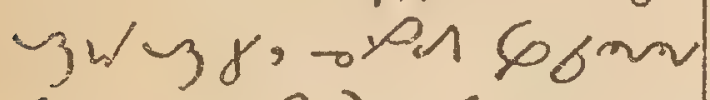

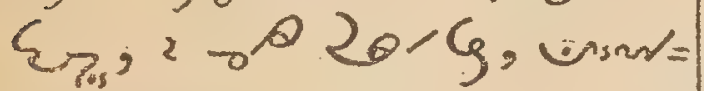

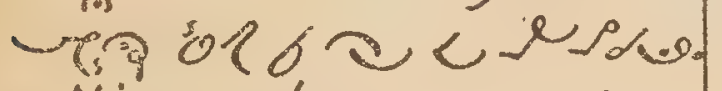
Misereatur. QS So 2 - Ano ebsolbsue. ? Surdoryun.? kuposonnto: cus. Indulgentram. o 2 . $\sim d$, or Go, , w wros,

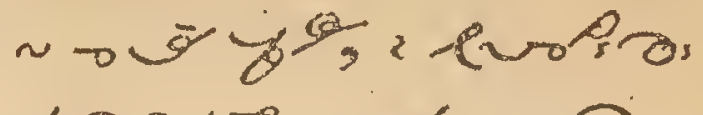
sma cos: 2 . The ten Commaniments.

$v \rightarrow$ be $\rightarrow$.

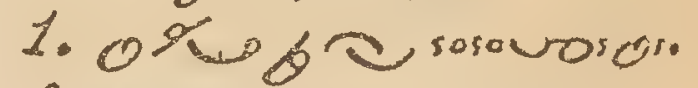

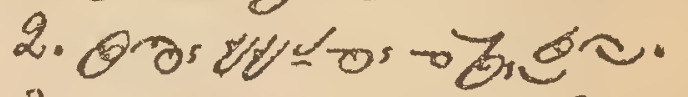
3. w d domuroson. 4. n 6 bon - $0 / 9$ ? $-6<-3$.

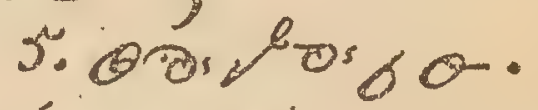
6. Oroik unt. 7. prork.

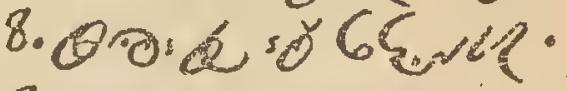
9. 0 क्र doto wer.

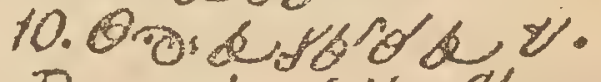
Precepts of the Churds.

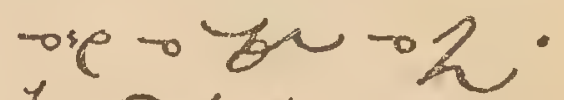

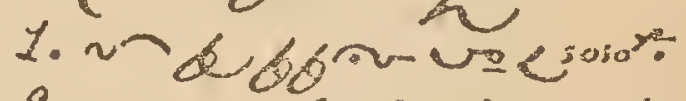

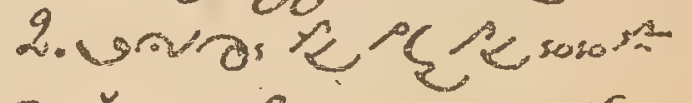

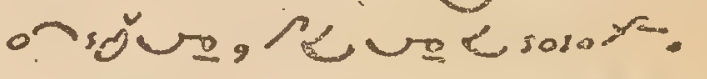
3. ofonP a so:cer. 4. 66 2prodes $\alpha \rightarrow \infty$.

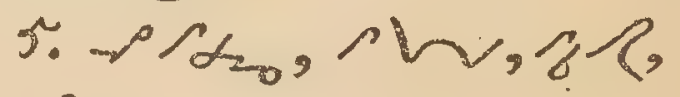
- 0 ds on ar.

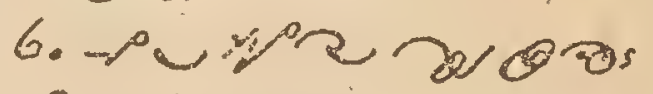
ob 020 .

seven Sacraist $x<$

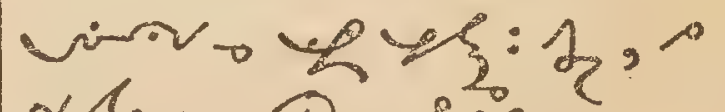
ales, $\theta$, tis, LRon. 5 , Bon. Act of faith. " Lous = rosn- to 60 , 0 
MANUAL

5

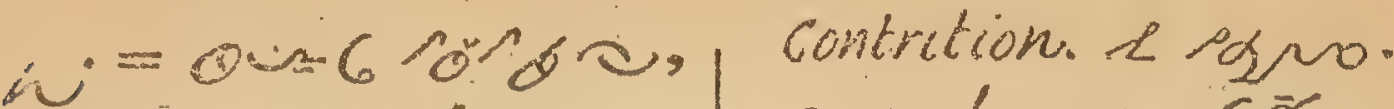

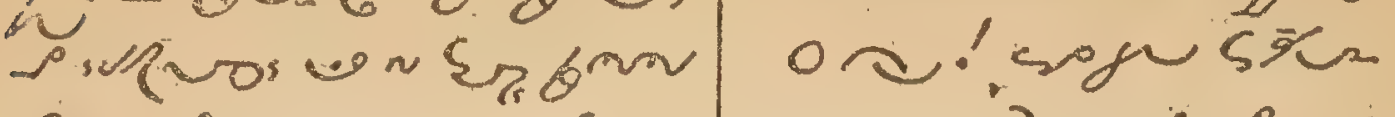

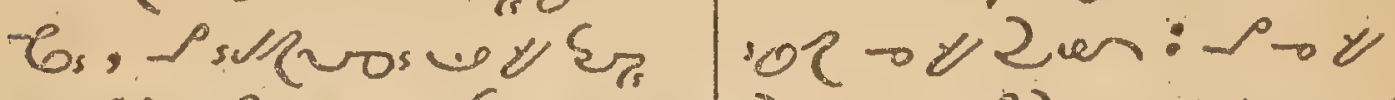

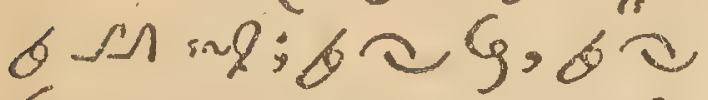

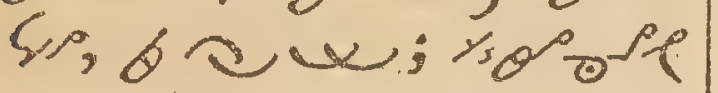

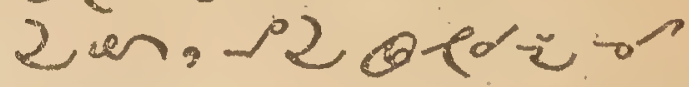

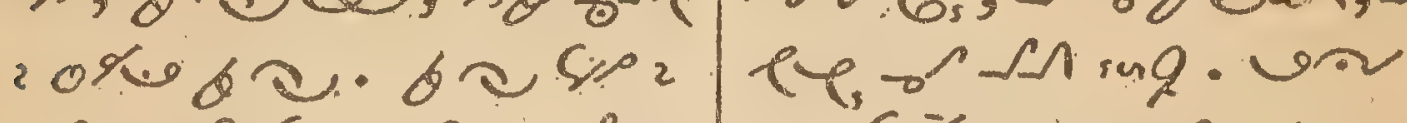

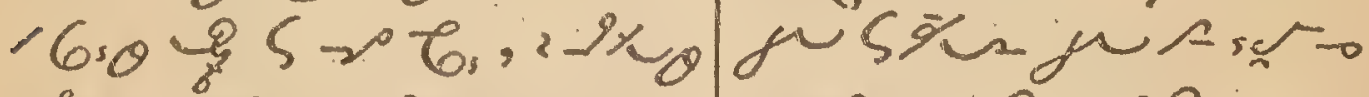

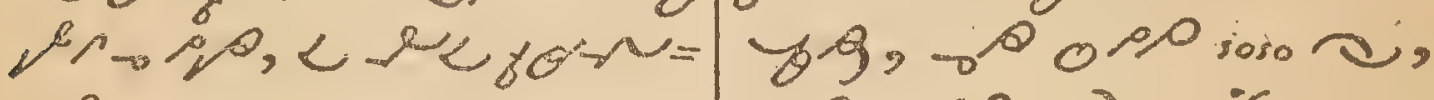

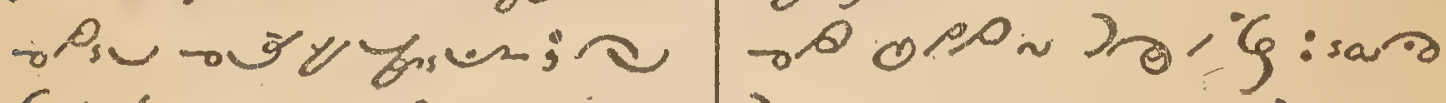

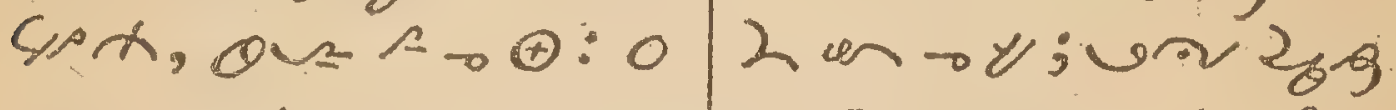

a, worgonvoro

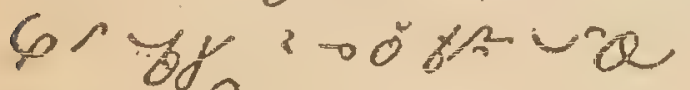
- $2: 0$ y 0 , : $<$ on $\rightarrow$ or.

Hope. i e en w o

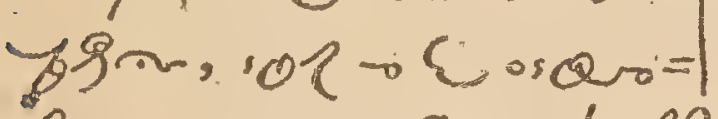

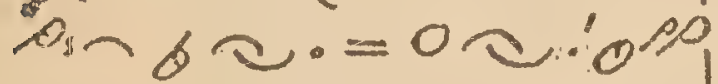

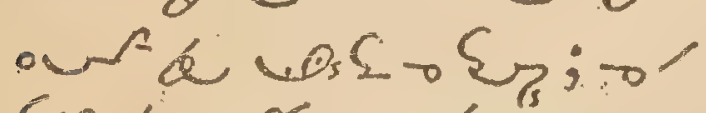

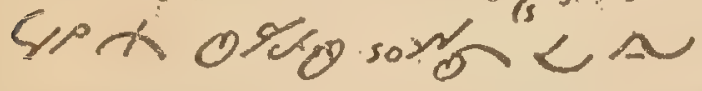

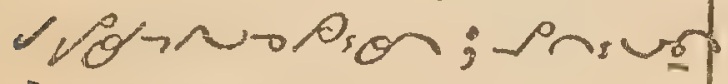

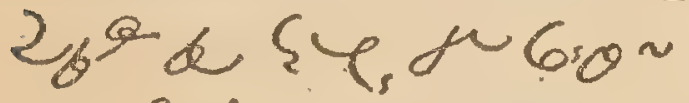
s $\sigma_{3}: \theta \omega-0$. LQ sowar OK

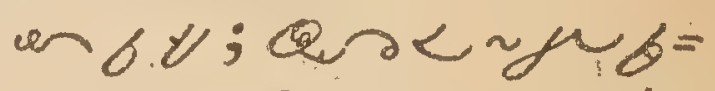
bus Q a sow? ordso= Tin $6 \%$ \% \& $6 \zeta_{5}-5$ o.

Angelus.in.

1. Nora nume $\delta_{0}=\sim$ was a upus - wernatson.008. 2. od yinara.

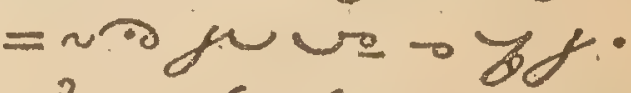

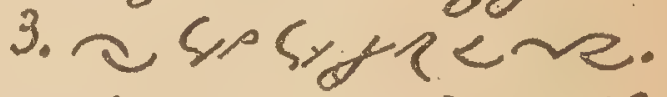

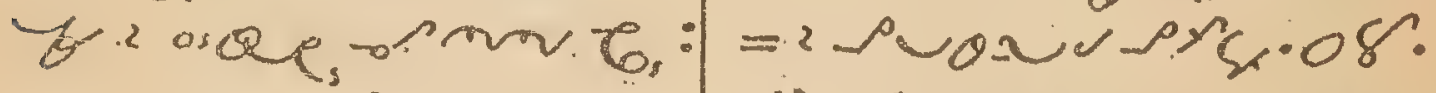

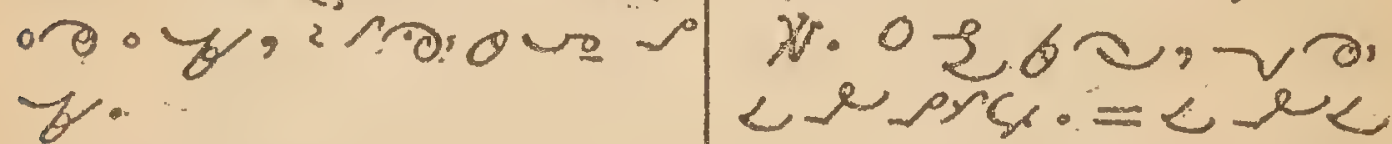
b.

Love. $~<<$ unn 0

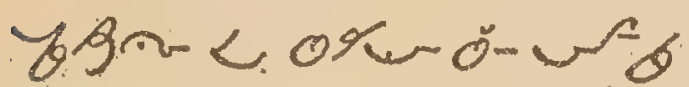

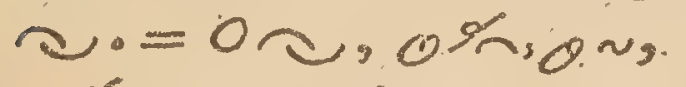
ofisurew, vew मn: $1-66$ mo so? 66

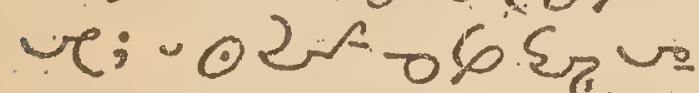
pdis; o trg pust. rop.

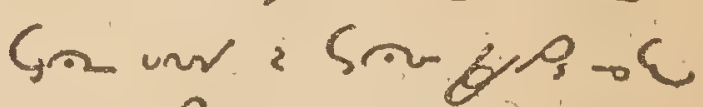
ovotos ax. Oremus. $02.5 g^{2}$ $\rightarrow$ qe sos a is y; - 0 Nonisals ex 60

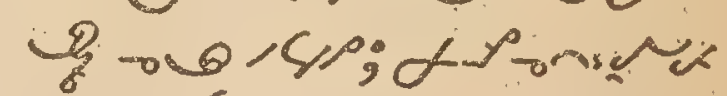

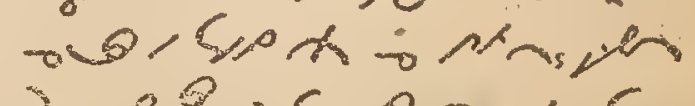

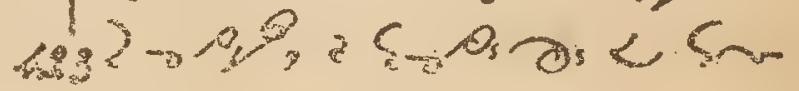




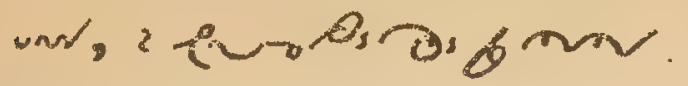
6. $~<<m$.

Subluum. o \&.

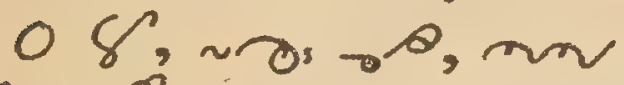
Zni; osqua a oras:

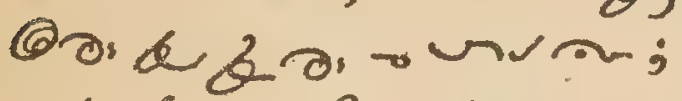

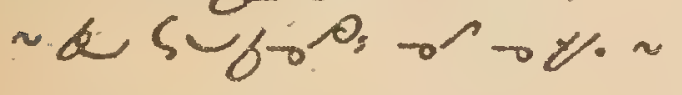
करण

Preparation for

confession.

$x \sim \operatorname{sic} 2$ voos $<$ Novin bro-1.

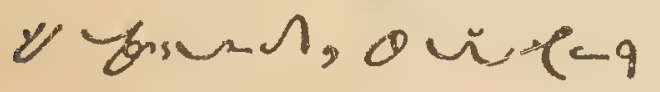
6 bif.

$x \sim \alpha y^{4}=02 \div$ c, <ed sosisto "ora a " teq a $80011+\forall$ oven 1 .

$x \sim d \operatorname{lin} 1$ \& on -redos psor onen

$=4$.

$x \sim \alpha$ und 0

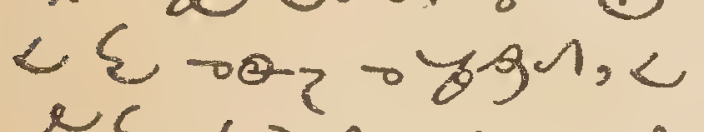

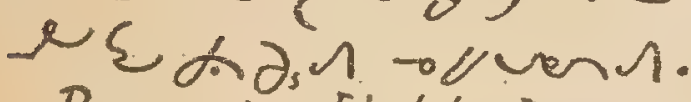
Prayerfor light. 2 e, uñ ơ $\rho_{S}<\sim_{0}$ "6 万ा:

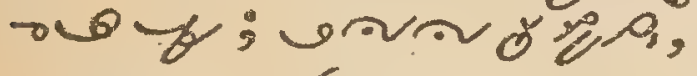
iaroro o ho. orom

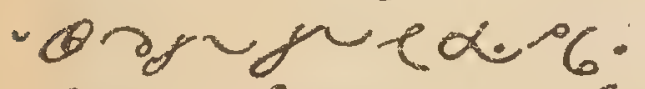
rogusing is of $($ ) bom-ons rosure argunda 6 oree no yes

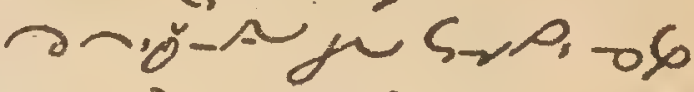
ar 2,06 a 2 o6 Vरen som.

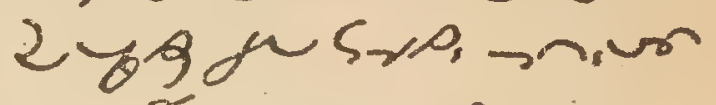

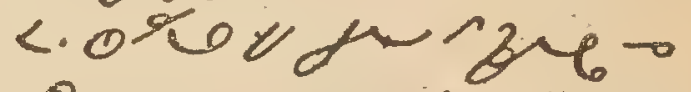
कy, जe hion. ofum rov.

$x \sim$ b fren or o $\checkmark$ sosor $\& 110$ tronsoso Gere.

$x \sim d$ tr overo Noan oN , 00\% toro, oo lohy.

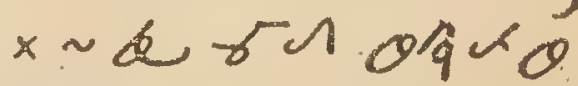
veros d Mre-ro $N^{2}$

$x$ yod vish o 10 ven $1,-3$ के $66<$ :OR = OG:O $x \sim<s e$ ara 90

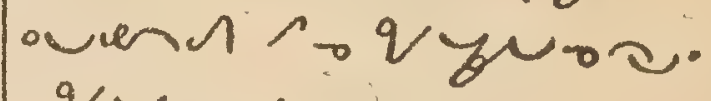
$=v$ ar 6 .

$x \cdot \sim<\operatorname{col}$ sieng ov and 0 oregr 久. = - y

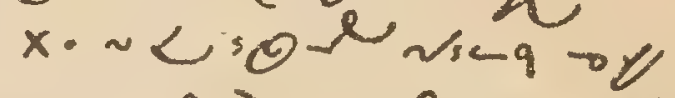
owenn? o最sue ob n.

x. L ' 0 is vend to os and 0 riga owen. 


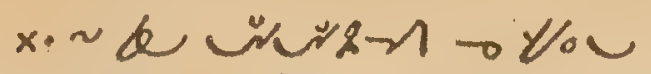

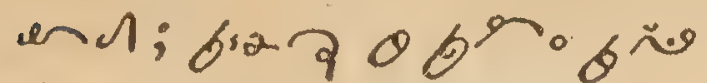
a, obn: br. G. nA.

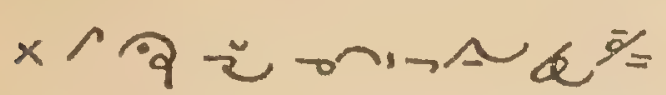
« tyowen $1<\mu$

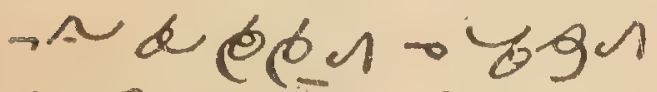

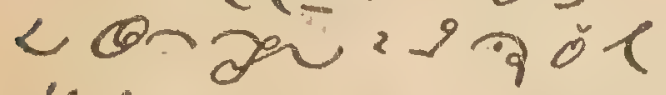
fur :Oर $0 y=y \cdot c$. $x \sim \alpha u n-12<$

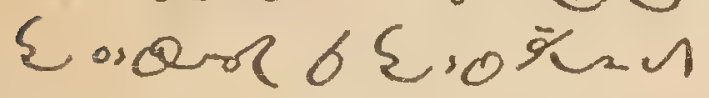
- youn $1,2<\varepsilon b=$

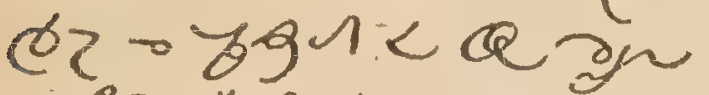
- iq ótgus .or. $\mathscr{V}$.

Prayer for Contrition.

od r d 6 osap, 65

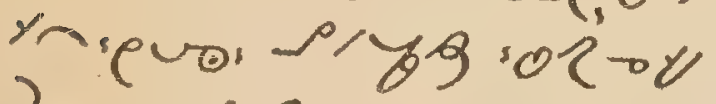
2orie; 0 2 -d 6

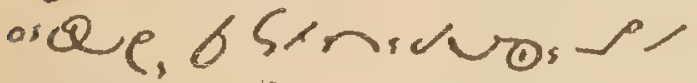
b9-V 2ons; 02 , ree 6 ose, b sombo

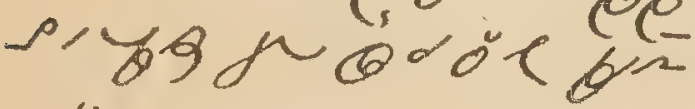
$\rightarrow \forall$.

x. OD n boses. bong ys, a rotgs - V, 'era nusyor rg.

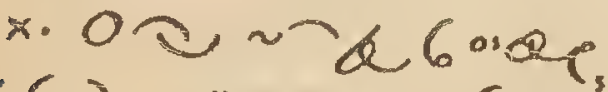
$65) \times$ manc - 0 2 a $\cos \theta$ 652 sustros o $5 \mathrm{~V}$.

*00, wa bosal. (5) osdo.p. by, ieg ᄀ.

$x$. 0 己, id cosac,

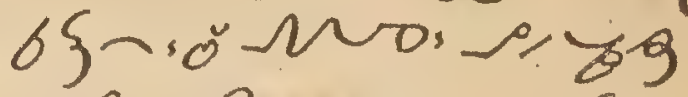
'0र 0 , or o6ई. x. यdotghso? $\rightarrow$ mar $6, \rightarrow \Omega$,

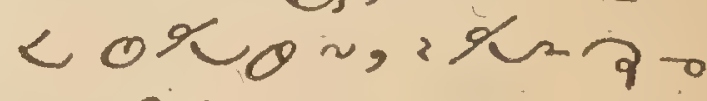
squ oyoven 1 . First consideration. $\theta=6<0,00 \sim$ m $6:$ : o $\xi_{r_{3}}$ tos ch oses or rmm 6. oर

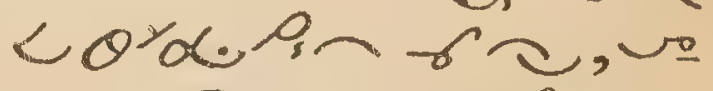

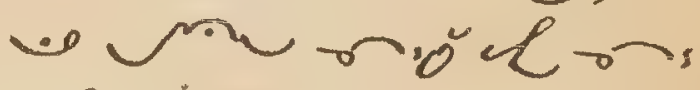
b2,

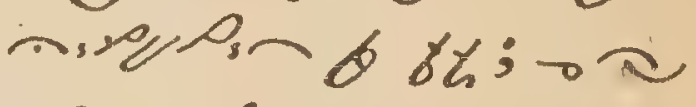
osa, or ma 6 , ond. 2 "rd. hiog, ydo hiven oथ. séc Pzg, < lany

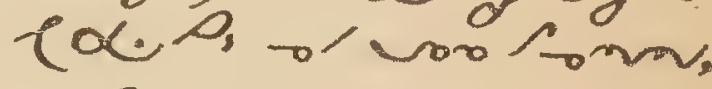
<Q y u f exp ( $2^{-9-1046-900}$ ogin, rR w on, ?

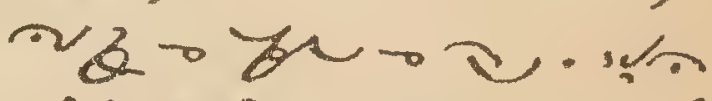

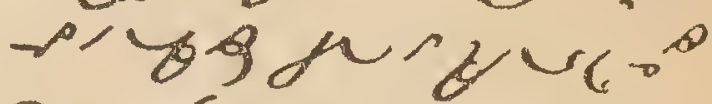

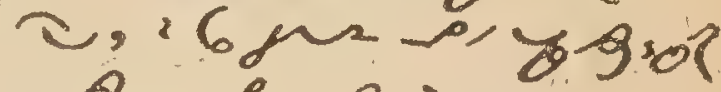

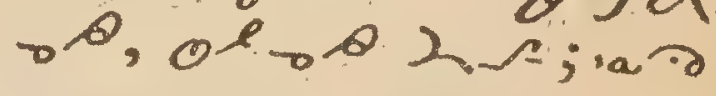
fubuc $\rightarrow 2$, saว 2 an $r y$.

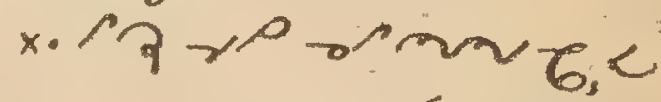




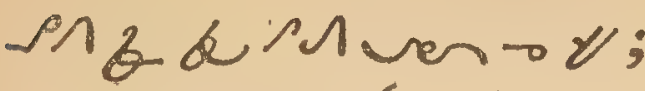

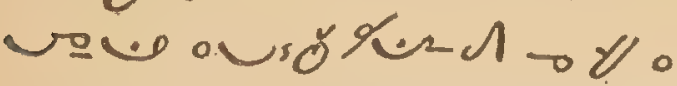

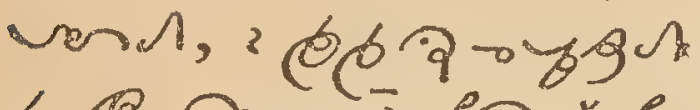
<ब \%

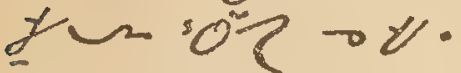

=rog.

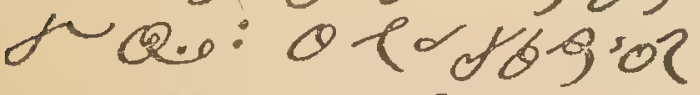

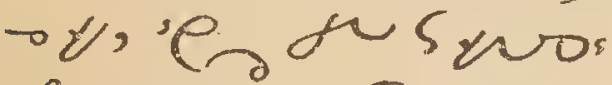

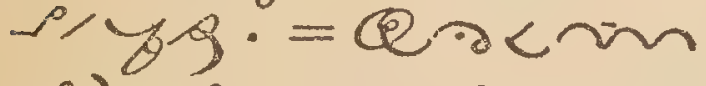

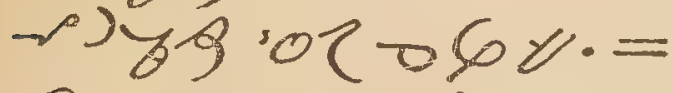
Q.

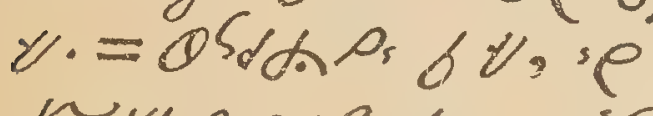

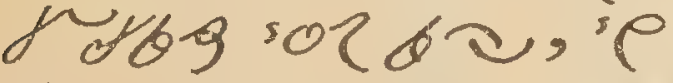

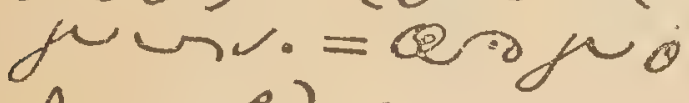
100.22890262 ०र 06 हा?

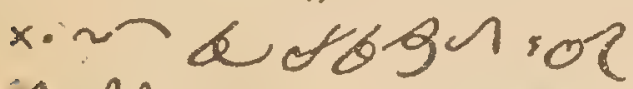

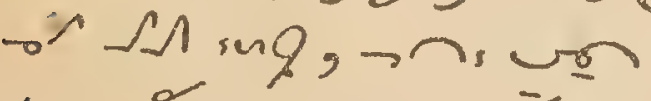

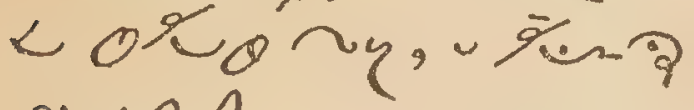
-ygh -youen. second Consideration.

Q $26<$ or $\theta$ mon

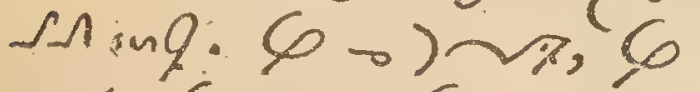
sost; 6 Q

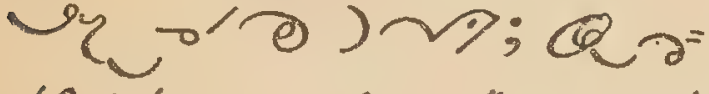
busan gases. $\left.z^{0} 0\right) \operatorname{sol}, \alpha^{2}$.

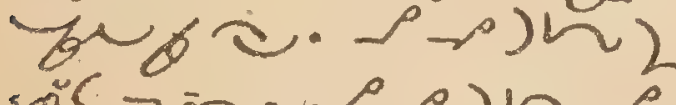
.

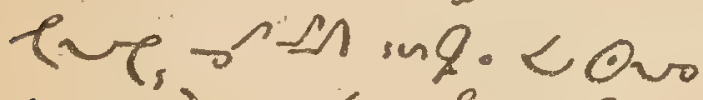
Lue h $2 \%$, of $\sin$, olndi<erive

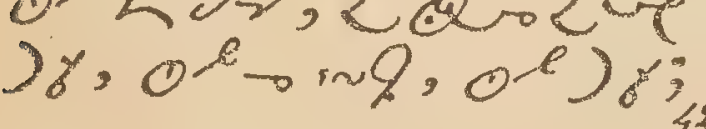

Lareverg, $o^{b}$ s $q, 0$ 그. P.o ) disf fur o Vi p. ) - dioli,g ?

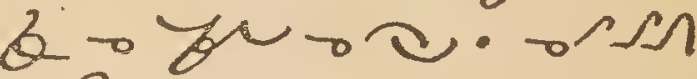
sng of se sny, soses= Sin folste

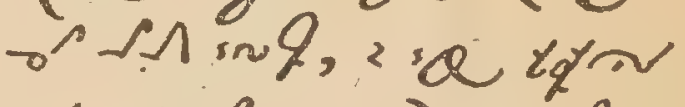
d mirs's 2 - 2 suips,s 905\%er. ) Da gu $5 \times 2+r \cos 2$ ?ar of: $-40 V 2$ en 5 bV= $7 \cdot \theta=6$ ค $960=$

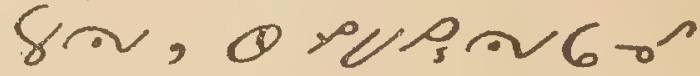
$A 1$ ing : $Q 6<\operatorname{san} \mu$ w $-y \cdot 0$. 0 च SUsy, rad; ine

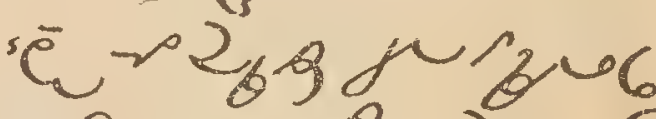
T

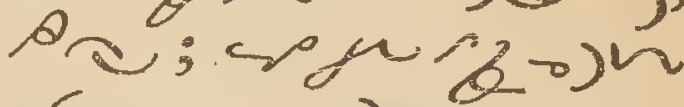
26 yer 2 \& 3 sol.

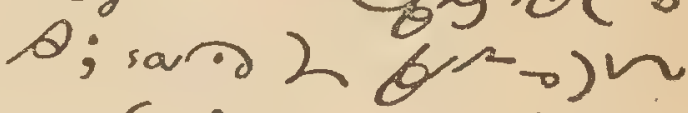
2 о6س; in suos $-A-269 ; 0 \cdot 02 \pi$

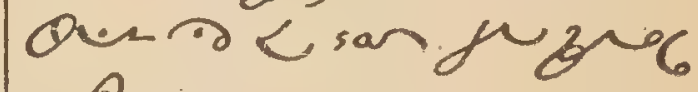
$\rightarrow \infty$.

$$
\text { x. ra d P or sing }
$$
nim prisold de ru in oע: voun.o\% un to yound, 26 ¿ ? - rqukar $8=0$
वें 
$=400+60.959$

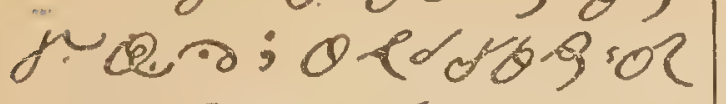

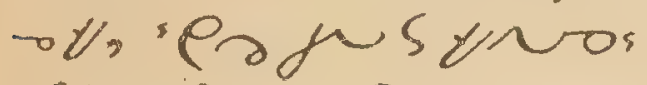

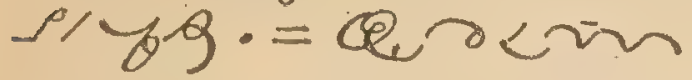
s) 89 :02 $06 \%=\alpha=$ afro sus's 'OR o $60 \mathrm{C}$.

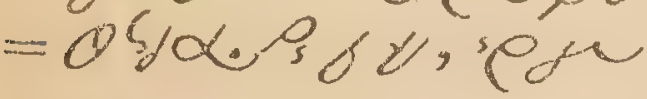
dro" $=\theta_{0}$ or gropde, sol ofo $\sum r_{1}$.

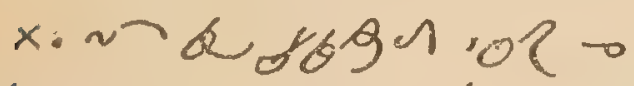
$x$ n. wर 0 \% $\theta$ s, $\bar{x}$, क व - Viownan.

Third Comsideration. Q $-6<0 \% \theta$.

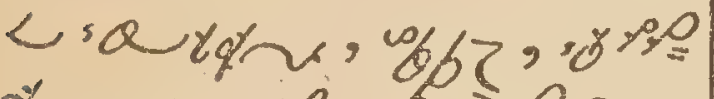
¿r, "ior, wosin, $\left.\cos / 6 e^{:-\infty}-\infty\right)^{2}$ n, Duscosi-not: dersuos ox y en one. ox, pos Pnos zan. "arró

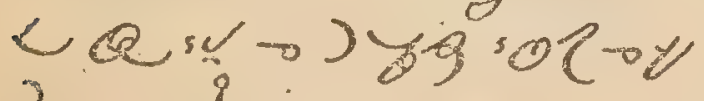
zam? : a sofun on on. ox,

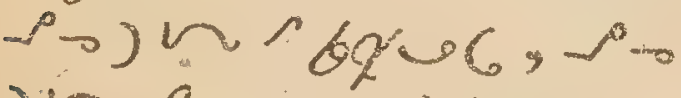

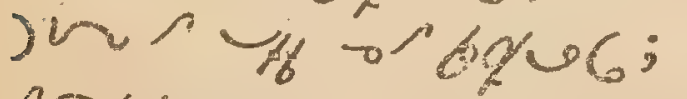
roviram ow"k八 fx; rov by aw

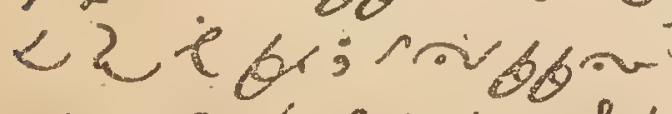

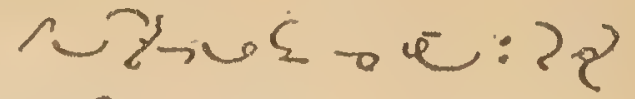
up ousue. ox,

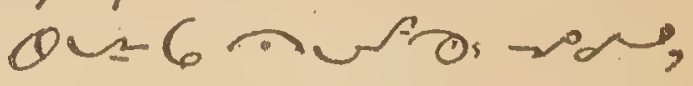
vecousos चर; $\theta=$ $=6$ od o oforg gu

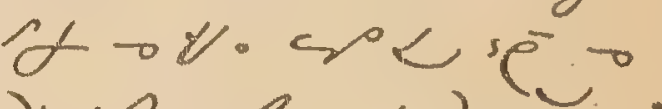

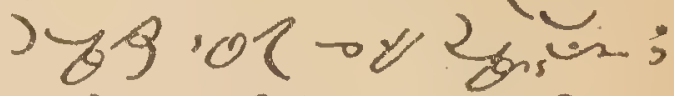

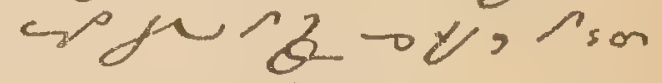
gyー几。

$0 x ! r \approx$ bo 2 , va oqA; ridorsuso - 0 en? wep:

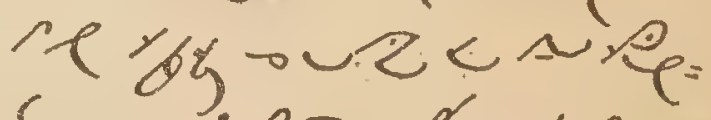

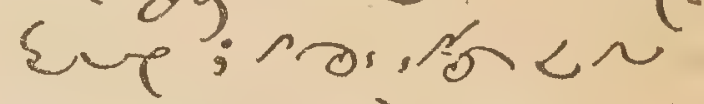

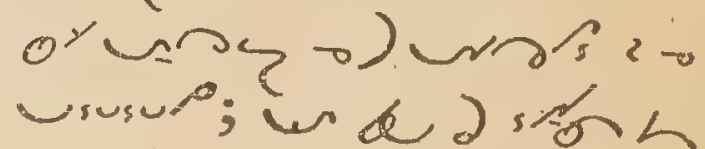
Nor al o is;sw ()) wia J in 3 b 6 ; 0 an 6 do ofry grorn;

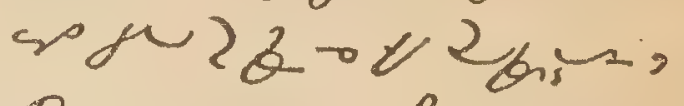
बar zren a sobr

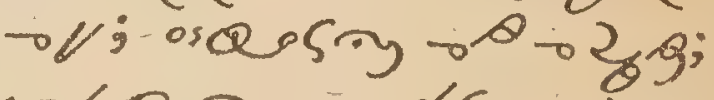
"od बतन Hoto $8696 \% \cdot 500$ mara gutere Noblo by. ote

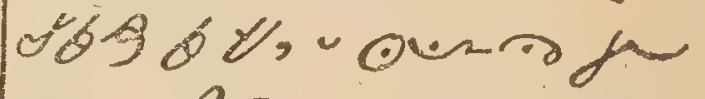
wor o, Cusagu synos org. x. Parpis num ox Lorúcs: poyo

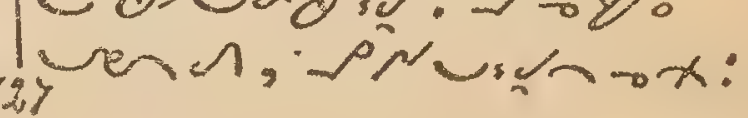


roviogras wn, 2 bo 2 , (ए) व का

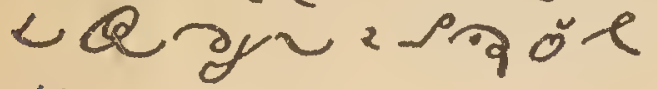
Jer sor oy.

$=$ afjotong सकां - ieragusyros -69s roforsus sol obvi $=0 \mathrm{~s} \alpha \cdot 9$ or. drg sol be, sper ns. = Q a jo hus - 269 soर 6 , '0र $06 £$.

x. difgen 'or 0 ansureolis

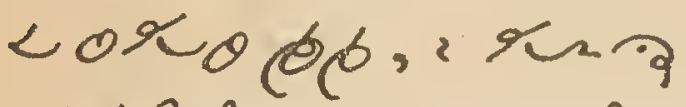
-zga ty aren 1. Fourth Consideration. 02,0 opo , op

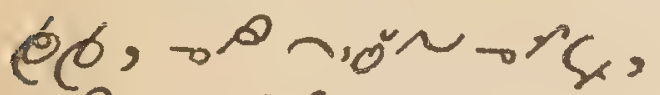

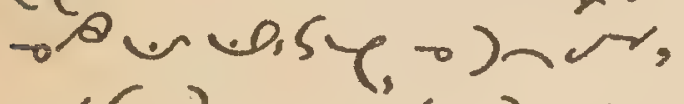

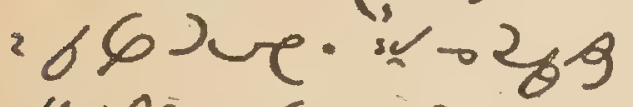

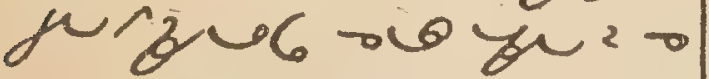
0 g. $0 \mathrm{C}$. $0 \mathrm{u}$ . Os Y

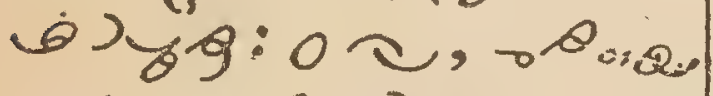

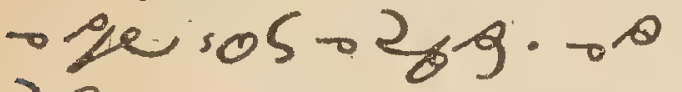
वeriom os $\sin q:$ : $\circ 2, g$ w2ien $n$, wse \%o eb. An st . bq ; $\Lambda$ sosur

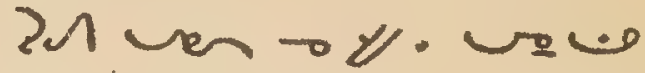
"Ǒ⿻儿口

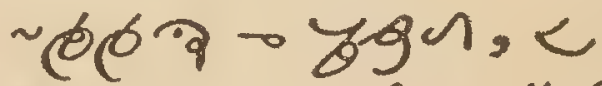
Q क्रय 2 किष ys sor $0 y$.

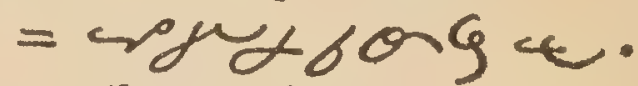
x.L eglo:

x. soso Gee nu.

Offering of the Mass. os n.

$02, b 84.0 \%$ Q02.5 , $064,62 \mathrm{c}$ 664.juog; vawi inn y.a. ode

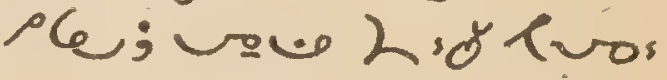

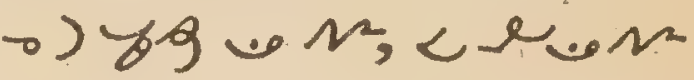

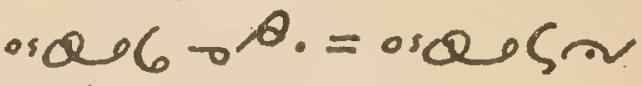

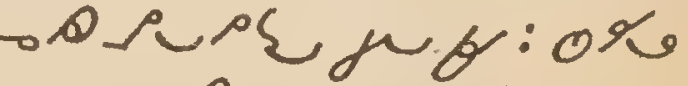

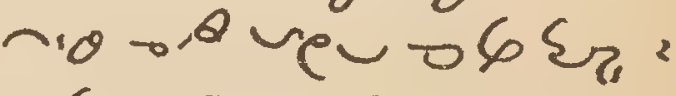
obnon; ormm $6_{s}^{2}$ -

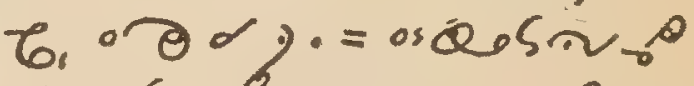
ep 0 . C \& osqe; 0 ro pe Losacs: = oses $\sin _{0} \theta$ hep ju to -oy2n.

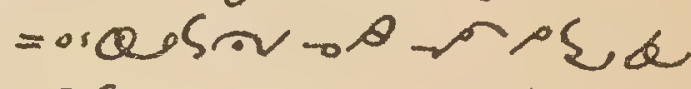

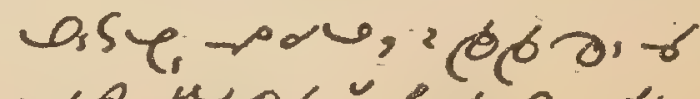

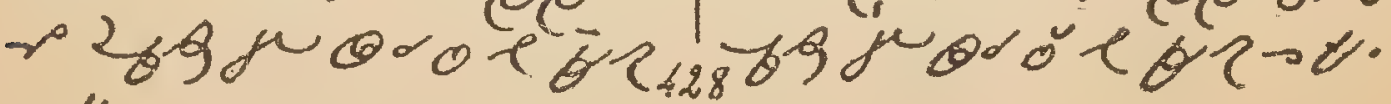
$-\mathscr{y}$ 


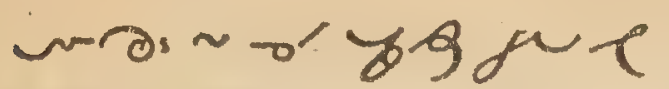 gma b.}

Before Commanion.

Faith. LQuvorn

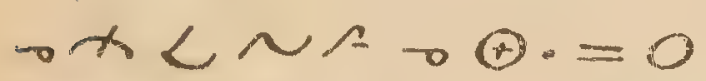
ch, an-wiru $\Lambda A_{0} \otimes,{ }_{0} \theta_{0} \curvearrowright S \rho_{,}$

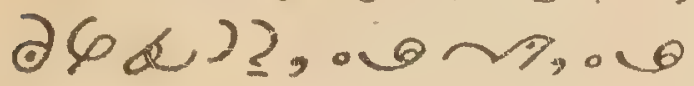
luy, o Q vio'; Qun 6

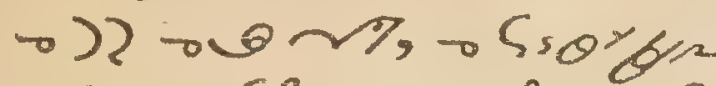
-

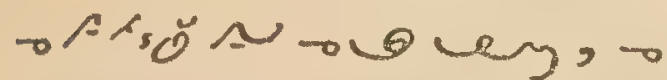

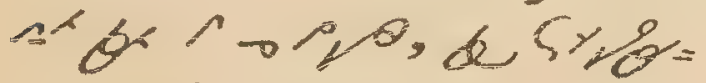
$\rightarrow$ ops orse.

Humility. L N M No. molgh .02 0 x. $=$ o dx.'oodivers

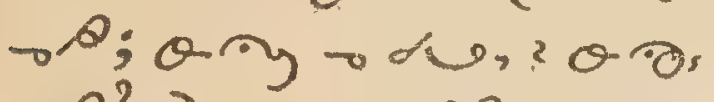
- A? do C, OPP, B A

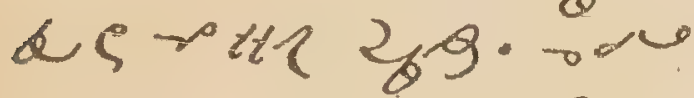
insory of g $16 \rightarrow A, 2$ Qu-6 mo: 2 sere $r, 2$ sorer, 2002 sol? ob-ox 6 6y.

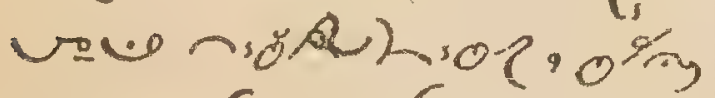
0 th: $5 \pi, 6 \varepsilon_{y}, 501$ o.epe.:

contrition. us sole - yqu soर

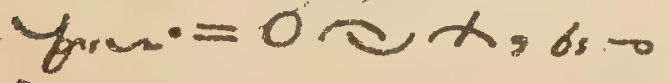
2 s.

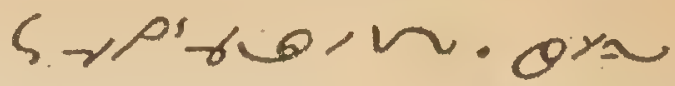

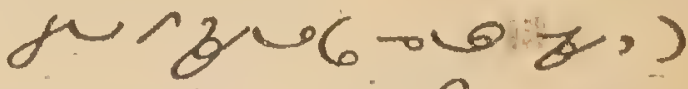
Unory.

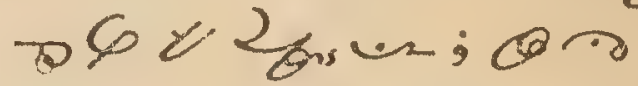
oes - inótrar V; :00o ubstagum LQ Son us as Yin by. $0 \approx x$ D.5yrar r suar Qun: QLingu

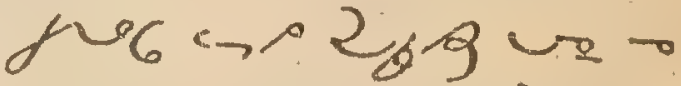

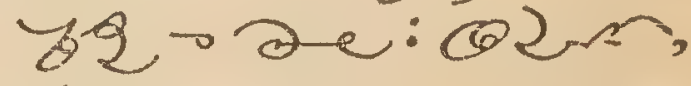

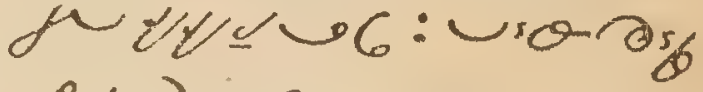
- प2 28.

Love. us end w $\sim \rightarrow x .=0$ ๖. nod cus rav, ergo ous ob;.2

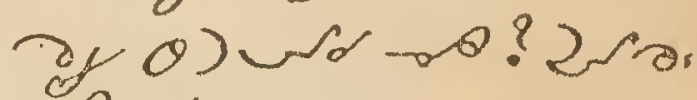

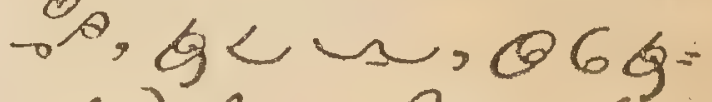

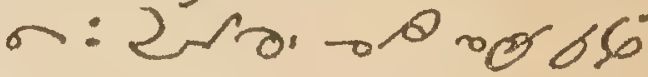
T. $20=6$ \& $x, 2$ Nai - o no blowe. Desire. n w e ooe b甶 $0 x_{0}=02 y$, ros $2 x$ sed 5 ¿x, $5=2,9 \cdot 2$ $\sin 20.00-10 \%$

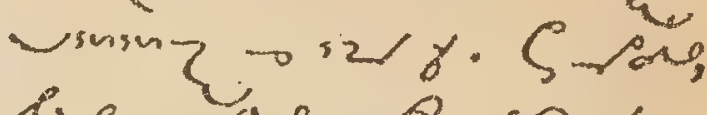
ketoraba sea $\zeta 2 x$, spol. $3 \sim 8,262) 013$

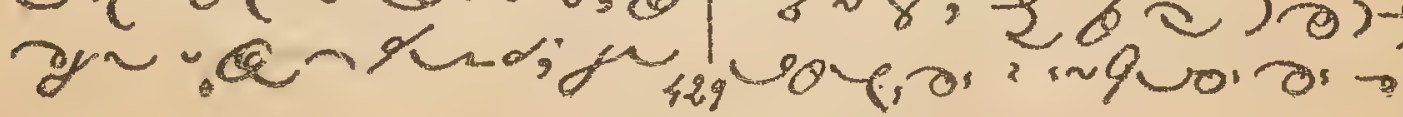


239.010 大. 52 "eac; uny te, No bis 2, 5 , inas.

\section{After Communion.}

Ox, wa son

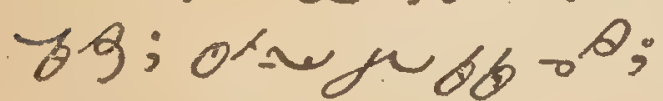
redk hon -idvioto

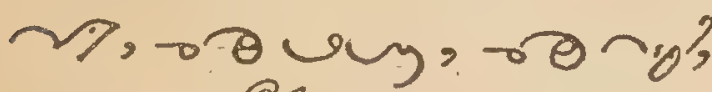

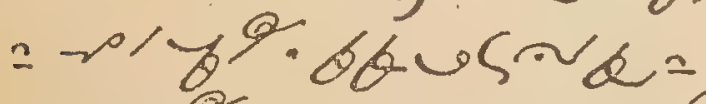
my

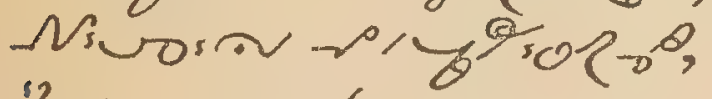

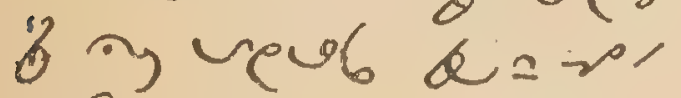

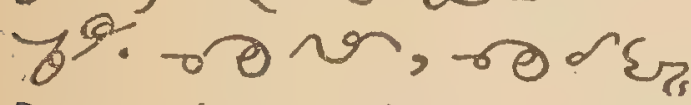
2 - nn 6 , Q < <ón $\%$ -pe: y, ore w6, DA wor, A

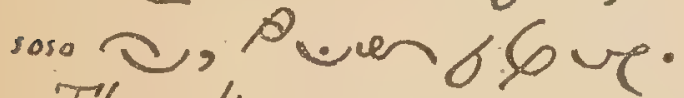
Thanks. ue unis

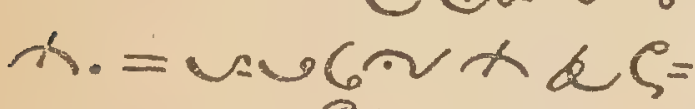

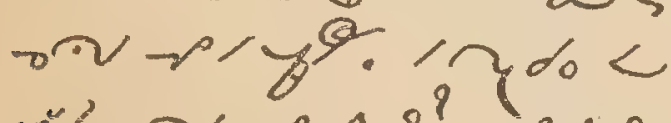
öbor ra? - per Q0h hor $\varepsilon_{\pi}$, ONnd gususi, wevoriós

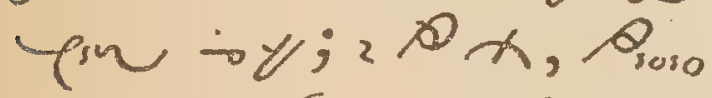

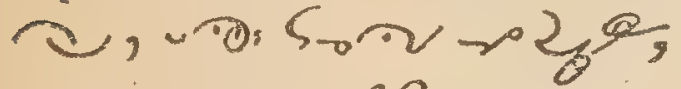
viorve; opensa w c,sy. यu, $2 x$, neincy: “o.6.0 g. Tre ropds ren

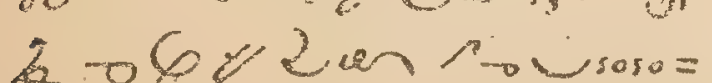

GPl ardatara

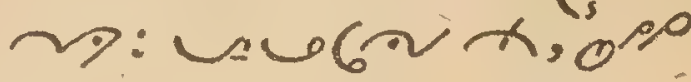
ح ح - y Petition. < $6, \sim$ r w.ol ox< umn og

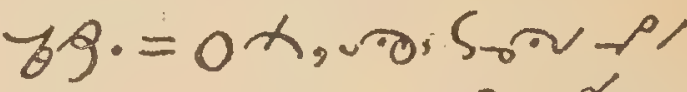

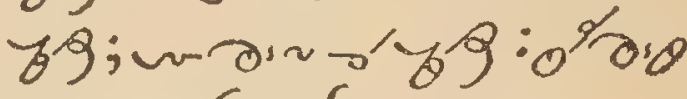

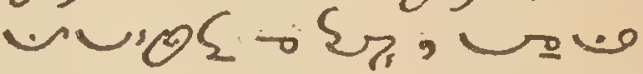
2 b8vic odety $\varepsilon_{\pi_{1}}: 6 \xi y \partial<\sim j \mu$

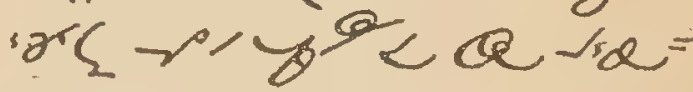

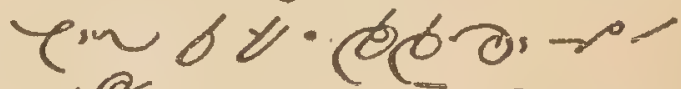
多 gus:

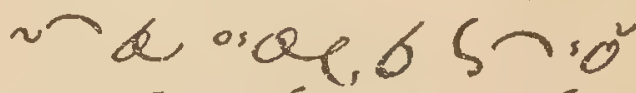
nर $06 \% \cdot \operatorname{\theta l} \alpha$. by 'eguero; \& 6 oxper.652 rs=

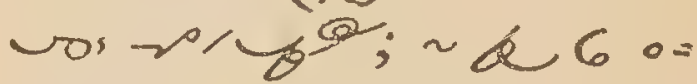
ap, 652 ebur.o? - $\tilde{\theta}$. Qf; wy

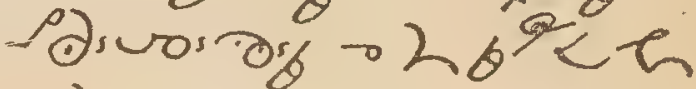
rop. on siso cuen; 0 Guor गy - WC 28 $2=$ or 2fo. Resolution. r L onvia mox niulesusi=

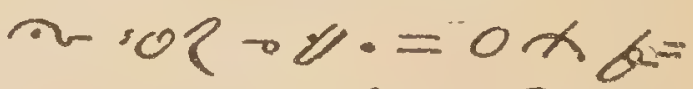

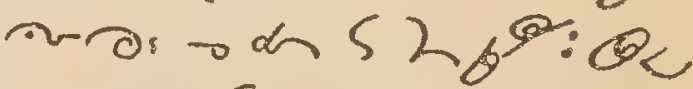
an gu $5 \%$ m oर 0.22

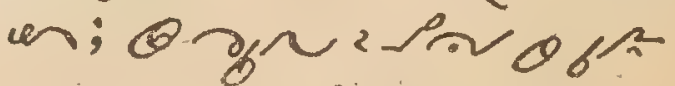
mince. Q a juosess

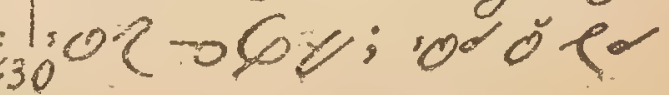


dyes sol oy, "6a gu

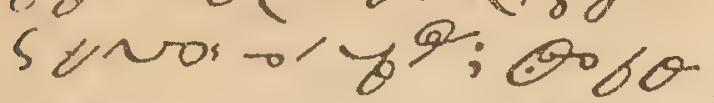

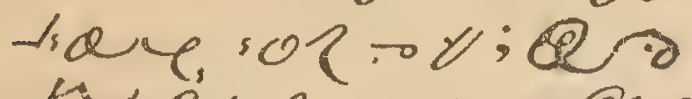
flogr on yु? $\rightarrow V ; 1000$. of $\alpha \cdot 0^{\circ} \sigma V^{\prime}$ "eragar; "eror

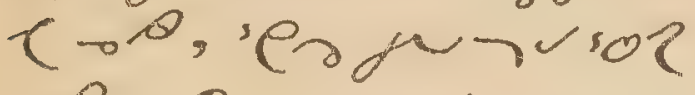

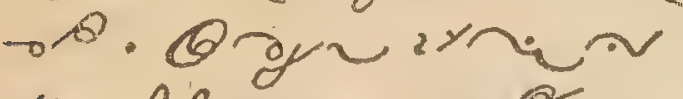

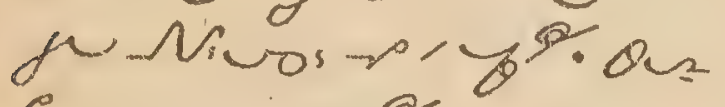
Suribg, aun

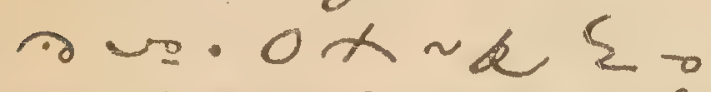
d s co 2.y.g. ol a) $6 \zeta_{Y} \sim \alpha \xi \eta$.

offering. $\sim<$ osen $\rightarrow x<$ squnim. $=0 x$ भC

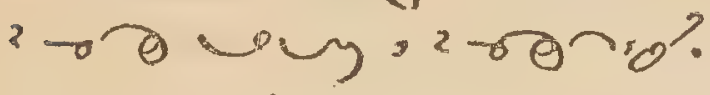
2 rendo $\mu$ u to oscil? os OST - S

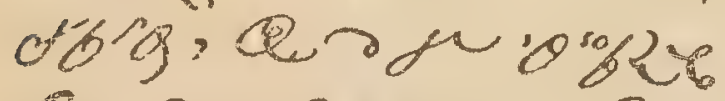
an

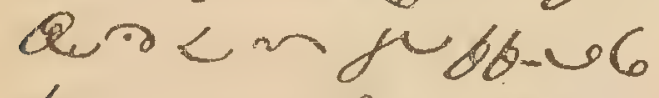
$x=-1, y-9 \cdot 0$. os -) wey ancling ber $\rightarrow \theta$; vevorama ob

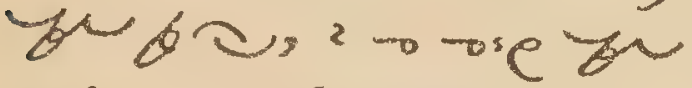
- $2=2$ en.

Intercessiom $\sim<$ bus $=$ mox owee cos $s_{0}=$ ${ }^{\circ} \cap=O X, \sim C b=$ क्ष

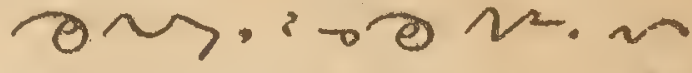

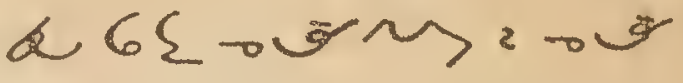

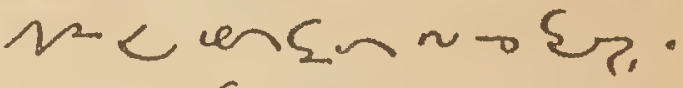

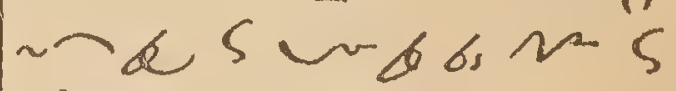

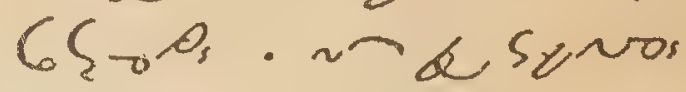

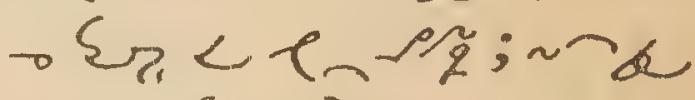
.0. uSh ing; ó this,

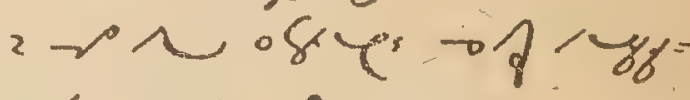
$\theta^{x} 3,<\operatorname{ern}<<$. -क $88 \sigma^{3}$.

\section{Rosary.}

Joyful.Myst.t.no \&d.

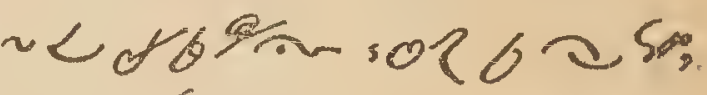

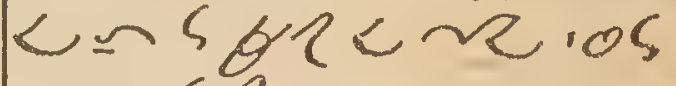
. o 8, ow unk 드.

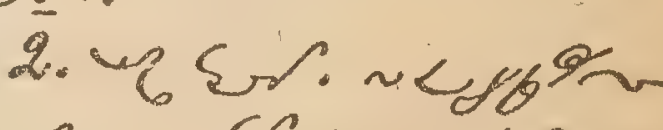

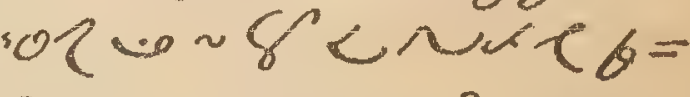
mis $4,2 x$. 3. sol $\rightarrow x<$ w

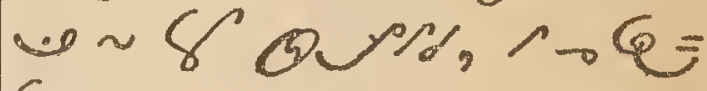
द०गा.

40 souzor $5010<469$ -Oर जo 8 < werer ore rax.orba G.

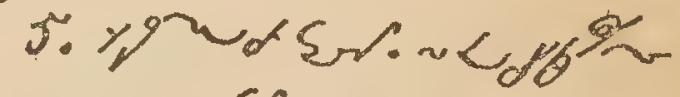
sor un \& zan,

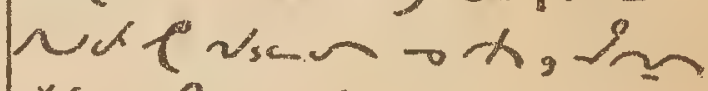
ós bipson $c$ voos. 
14

STALO

Dolorous. areser: $\sim<$ of

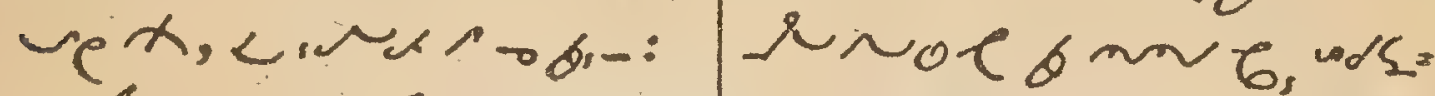

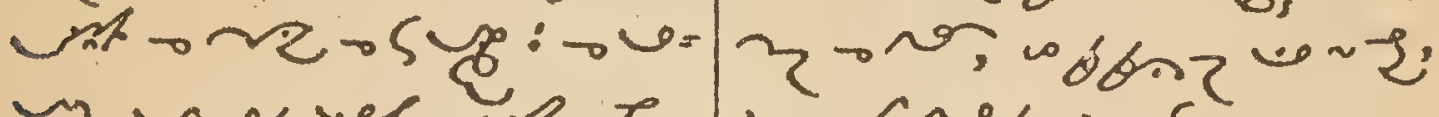
2 ung ves.

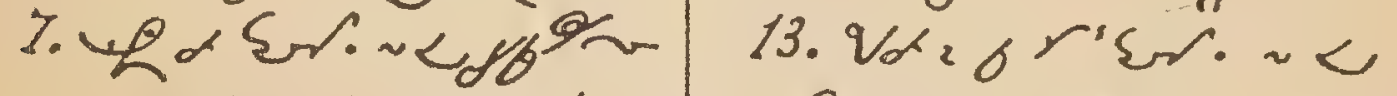

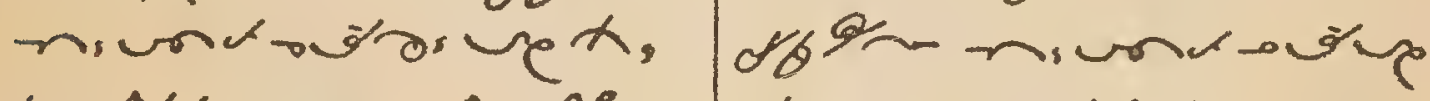

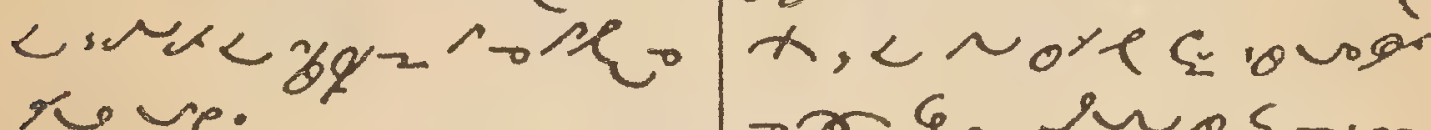
Ku.

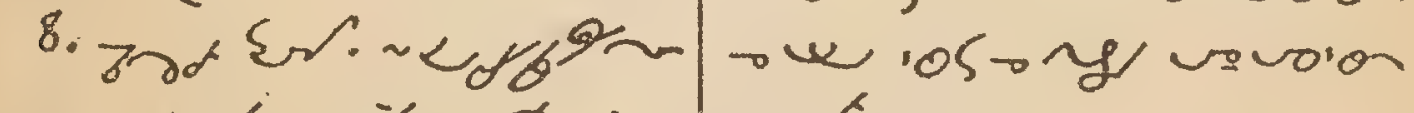

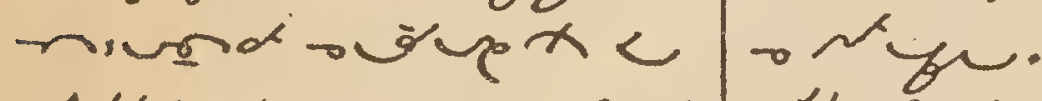

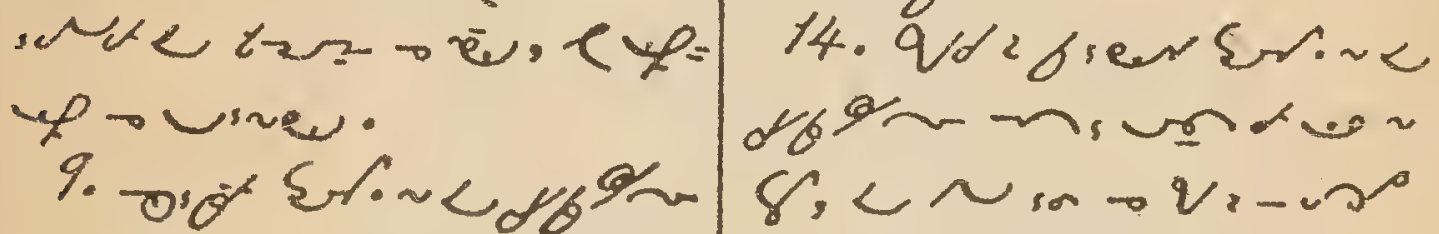

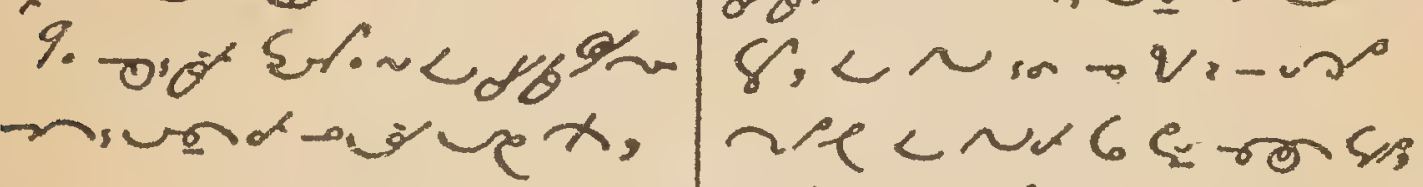

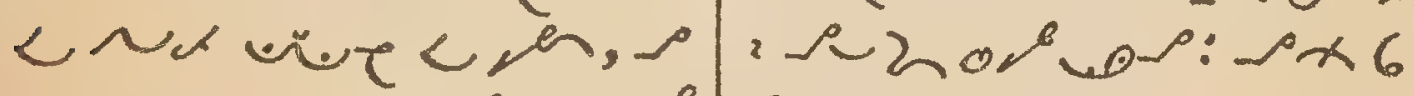

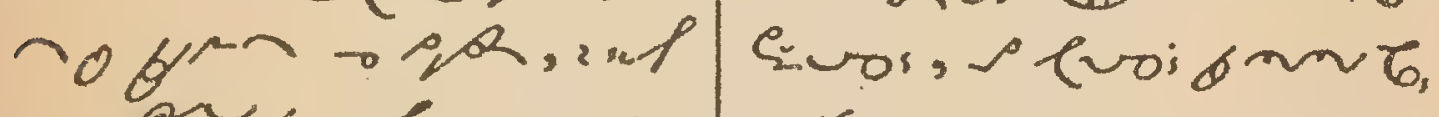

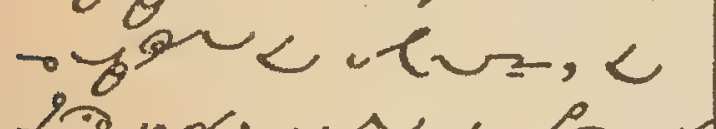
wotren.

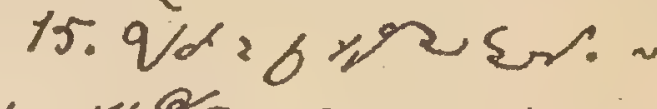

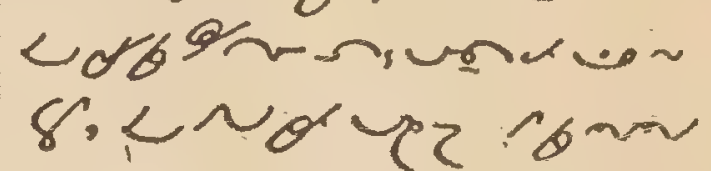

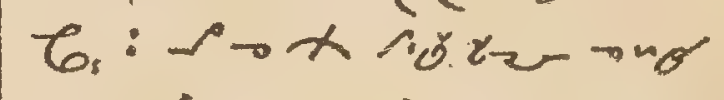

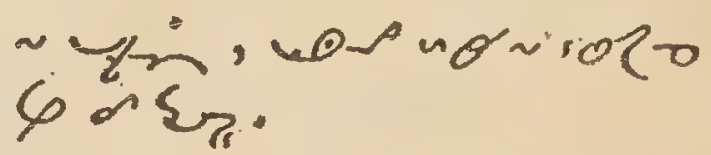

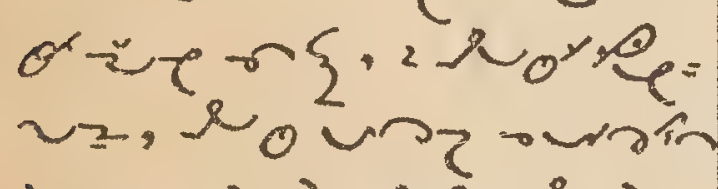

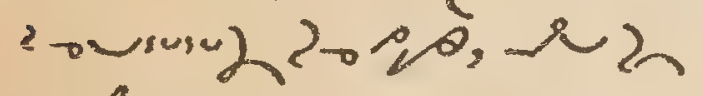
or, bone un

Via Crucis. Glorious. 11. V\& 26 ï Er. w L d

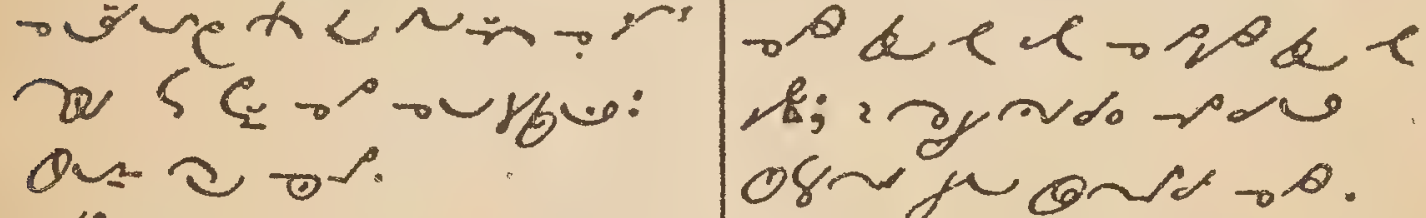
os $\mu$ on $\rightarrow$ o.

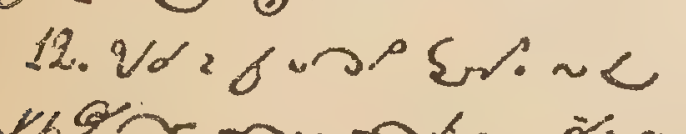
Q $=6$ or bid क्ष

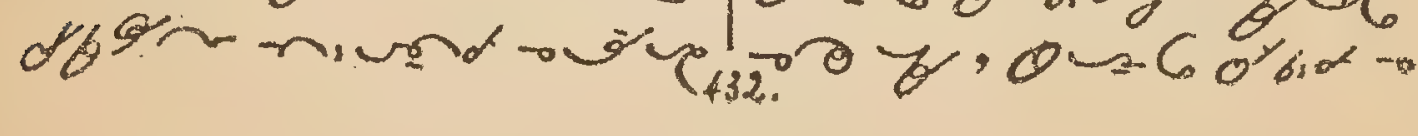


MANUAL

$-15$

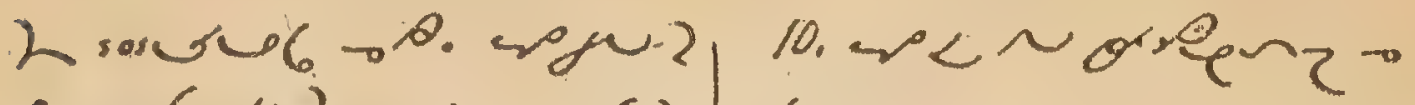
3 060 inen; $\theta=62 x$.

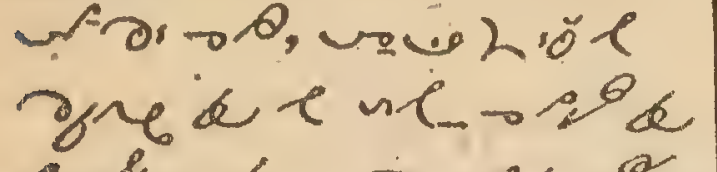

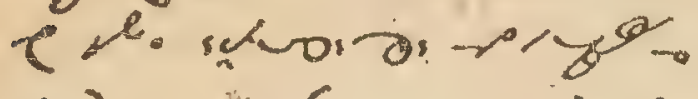

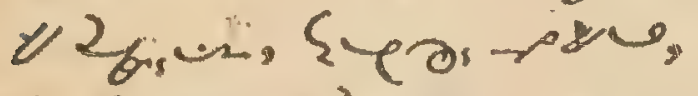
< ह p r r 2 g g < ians Sancta Mater. $48-x$.

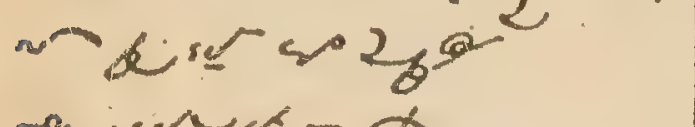
-aidus Adoramuste. eyecra Ax, 2 Aro num $=0$ ed spafel row, dererere.

At each Station. ON 2

reguns.ro, ware

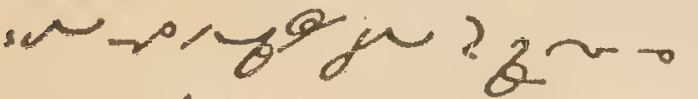
yob:; sarven. V: Syar radea siapin toviograr

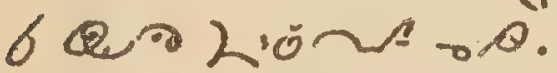

1. we Erion ax -

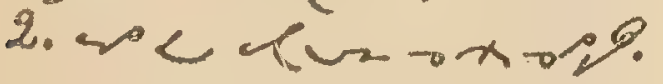

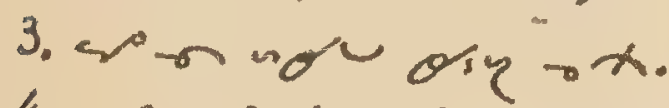

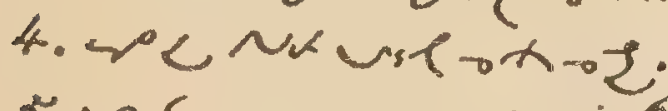

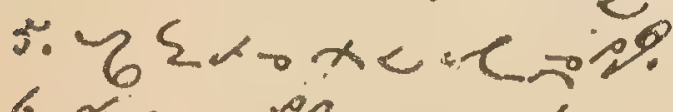

6. रु w in

\%. arazk oris ox.

8. vocisoniz ox bou a ver.

9. $x$ ondion ore $0 x$.
16. $20<\sim_{0}$ unz

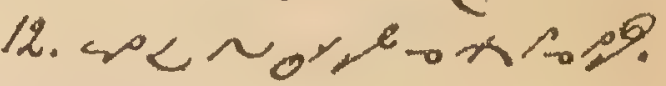

13. are $n_{0}$ se ox 6

$\rightarrow \infty$,

14. cre Noy?z $-x$.

Pateri 0 g

Ave. Os $\mathrm{s}$.

Glorix. Lo'sol- 9.02

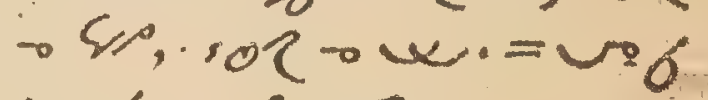

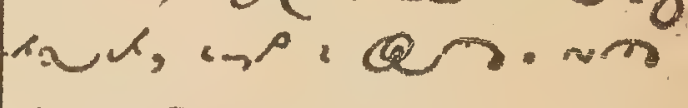
un.

Guard of Honor.

$0 x$, nd or , no 1

- tal opons हn.

Pho ré, do

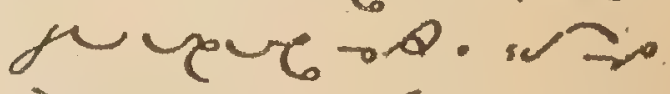

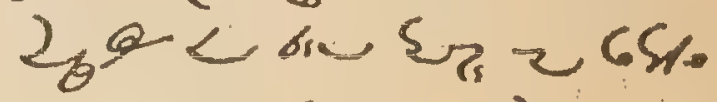

66 , o sive 6 . Lif

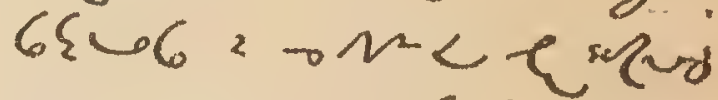

son $y$ o of st.

2u< a d bonul

CN $A \theta \cdot \angle C=0$

)'en 'egurvos n

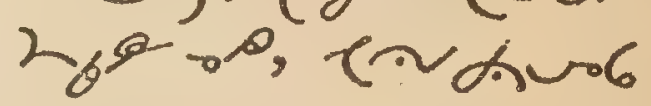

niver grofor.

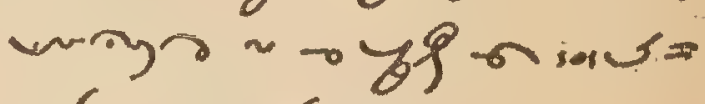

Cर क है?

Hymn. ox, 2 sacong

Q

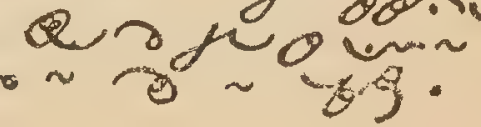




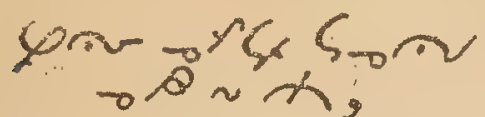

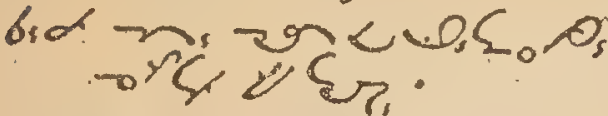

Oiner Hyinis.

1. Venez Divin Messie. $S \curvearrowright$ SP. biv volnown, sed Vosolos, $5 x^{\circ}, 50^{\circ}, 5.0$.

spu, 'e of $s$,

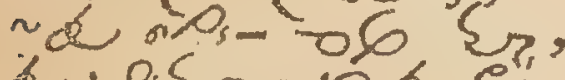
d $. L_{0_{s}}, " C \&, 5$."

2. Creator Alme. - o a sp.

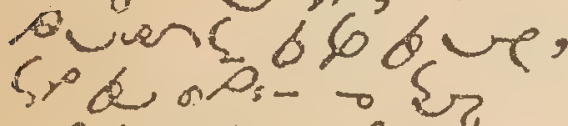
on tor to.

onf rection

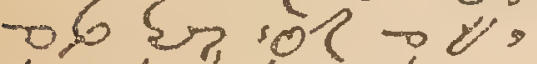
क. क्र $6, x$. a 6.0 . 49.6 .

de $100 \sim 940.6$ औ क्ष 90 र 90 ,

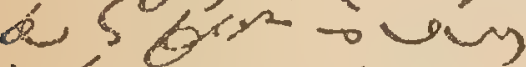

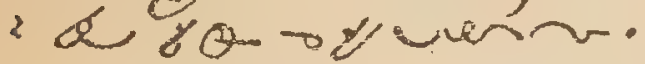
3. Adeste fideles.

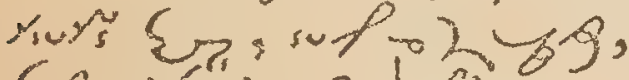
$250,26,50 \mathrm{~L}$.

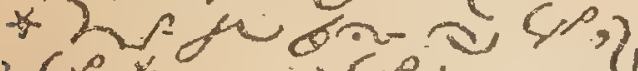

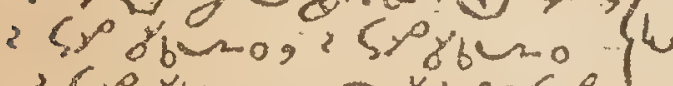

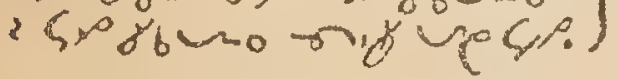
4. Les Anges dans nos.. a spo Son 6xu $v \rightarrow p+6$, n n m n

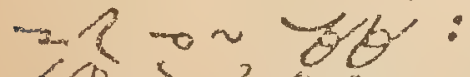

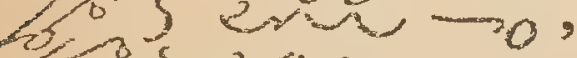

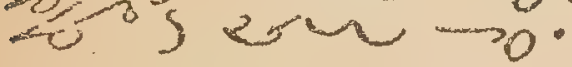

5. Andi benighe.

of $0^{3} 0 \sim \mathrm{C}$,

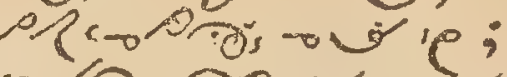
souno vare firam, حbe, wo, ors.

6. Miserere mei.

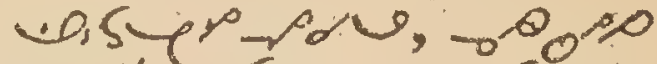
च2. 2 onsw.

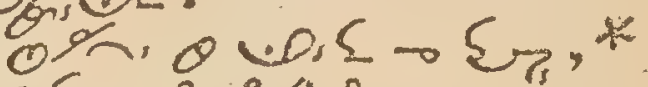

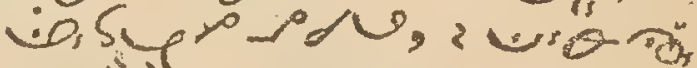
- ע゙रु,

uar $9 / 96602$ son, * w ․o un oर 0 .

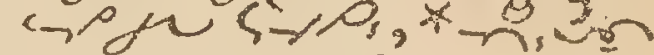

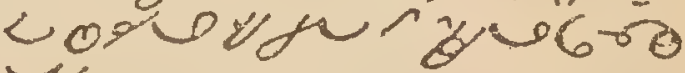
z)

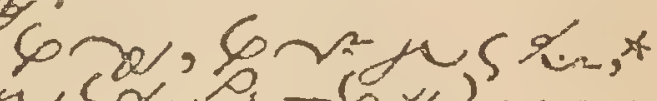
us $\alpha, 3,-5 \% 2$ s.u. opon $2,8 \sim \pi$ sos $=$ ש6, * rrven to

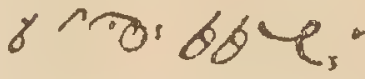

7. Mon doux Tesus.

Oर $\sim 2$ sol-oss

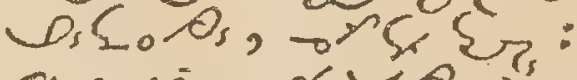

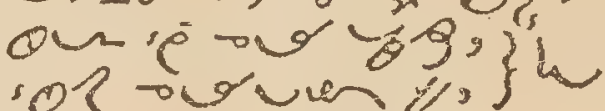

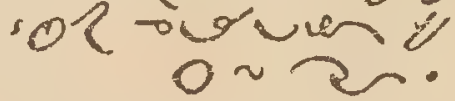

Qmobla ob

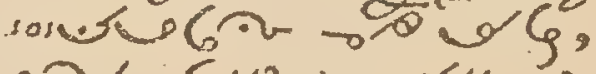

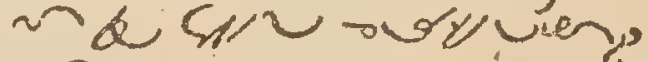
sar Uuan a ty $\mu_{u}$ $0 \sim 2 \sim$.

antargensop?

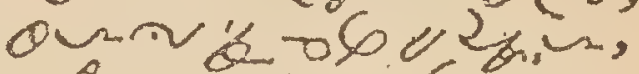

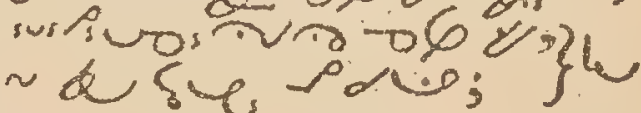

$$
0 \sim \text { ? }
$$

8. Helas, quelle douleur.

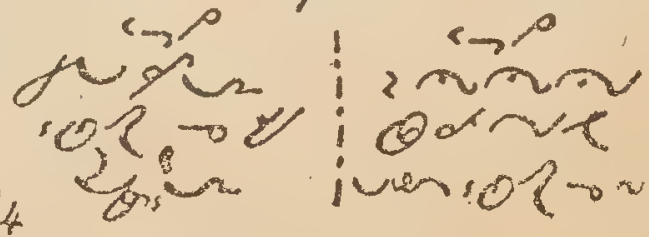


$+$

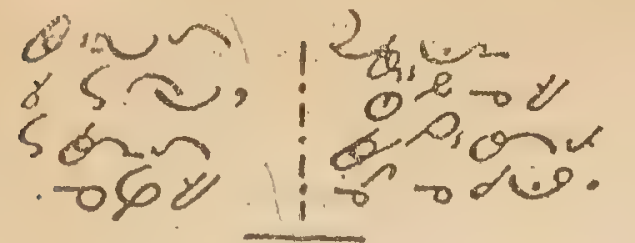

9) Au samg qu'th.

ba róch war

cNur, $x$ - sos,

cosintor rumen" uruniog, en ras.

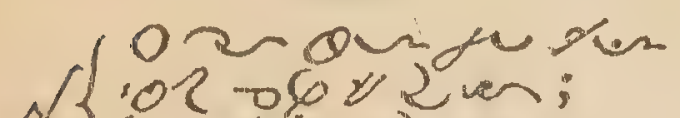
$N \begin{cases}0 \\ \mathrm{~s} 20\end{cases}$

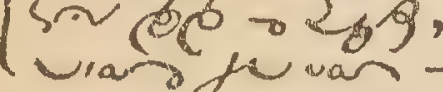

Hon $\overline{z_{0}}$ ar $\mathrm{x}$ bover to 0

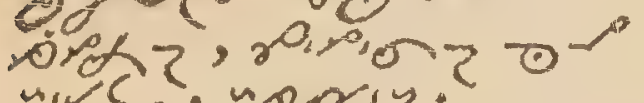

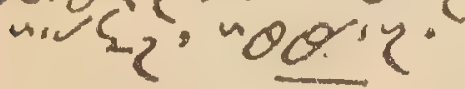

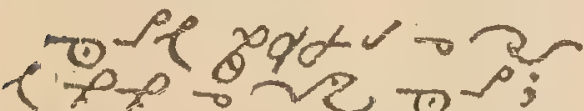

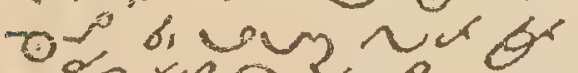
$0 \% 9 \%$

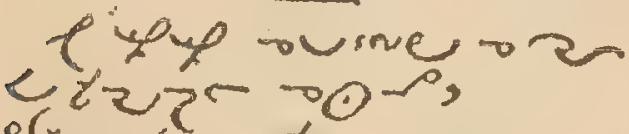
-

exoster.

Drcetentoo ortor. o.p. 100 -2 bu waro bytion or.

2-kunz ropo - ran $\alpha$ ovison: $0,0.20$ - 2 < Nutomaton.

swe.w2 ox Lpmin rops: Nog $\therefore$. e e en.

orr Qungian y.u

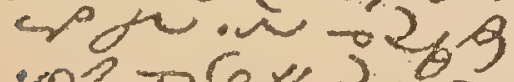
or $05 y$ suar.
10. Subne cirt.

$$
02 \pi
$$

rroife e, od,

भy\% L o

की

rod dyn-oy,

人日, ws

bs oresure to

$<$ exungon.

11. Regina Cali

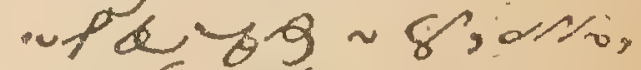
- 20 s.

$\therefore$ of to edblo

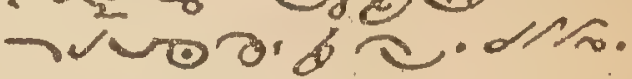

12. Veni Creator.

Ske mionm

co - 39 - 65

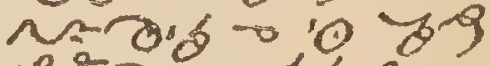

raras oce

13. Le voici l'angeace.

$n \rightarrow \sim 0 x$

, $\infty$ ( )

orma

$\sim<$ ran

14.0 Jourheureux.

$$
\begin{aligned}
& \text { vo gusuriy } \\
& \text { - T ( ) }
\end{aligned}
$$

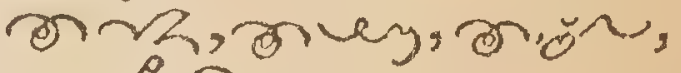

क त,

tho dunory.

ondereto,

o.

ons?

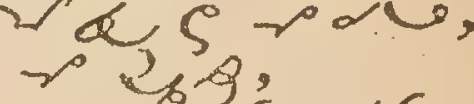

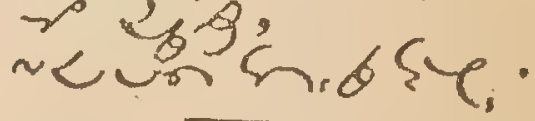

क्ष०त-

Dospesons.

"P<น

war 0 2ons. 
15. During Communion. S, $5, \pi, 52$,

16. Chantons en ce jour. $0 \sim x$

rouss re, org, du 090 2

o 2 ح-6. vis rionde लरटल रू, gebagus: .0र $-y$ umen. on $x$ orn oh 52 ctsis, crar 0 o con sus sot $\rightarrow$ $x\left\{y^{0}\right.$ o don sugusus al ay; uran 100 or m oo. 2 is osul 6 - ytr zbgo.

d कर \&min,

1\%. After communion.

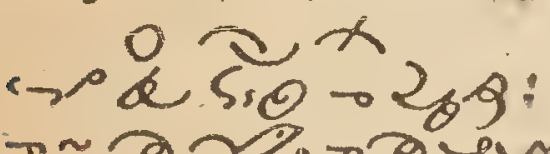

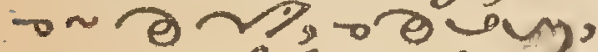
ron

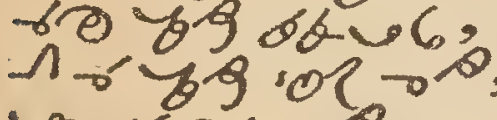
$x-62-\infty$ fing 6 . 02 .

ra o on of $\sim$ X;

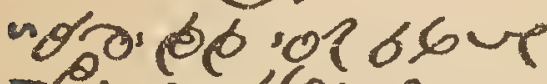
गे जen irdoros is, - dै of ह?,

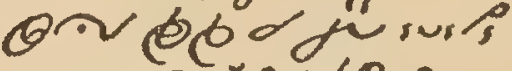
suniortiogu aly - a k rod, oum sus of च L oun a $6 C_{2}$. 18. Love for J.C.

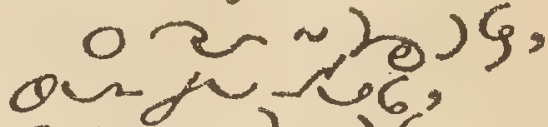
0 2 20$),$ oufu $=6$, Qny A. oxy ar: ब. Oर, 20 , sis $50 \sim \alpha$.

19. To the H. Eucharist.

ax, 2 sip, QUvan io $\theta$,

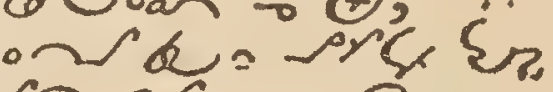
गि und $6-10 x x$, Quna भ :er, to $) 4968$. 6 , an su 8 क 269 oर $\rightarrow 0$, , 0 - 0 ;

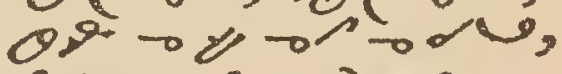
ro. $\alpha^{2}+2_{\beta}-9$, ro. nor nos.

20. Vivatin excelsis. uncrato 2 sin ncora

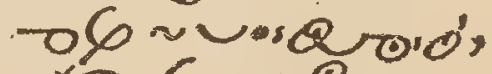
UT. S5O of $\sim 0-6$, , क. 80 , $\sim 0$ \&9 का एक , ons 436 कथ Exis. 
21. Aufond des... An oy $\rightarrow$ Shring, anc, un m n : $06 \%$ wiven,

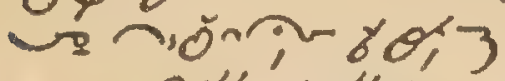
OV.OV

oom m<ow यूग $100-2$.

$0 \rightarrow$ on vorm $\sim 2$ \&o, 0 oforis Mid wus

a) S1N rion 一 2 oros.

22. Dies irae

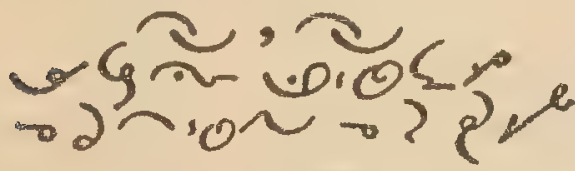

23. Pie Jesu.

0 , \& $\xi, \sim d, 0,2$. - $2 f^{\circ}$ o $-6 i=6$ ce.

24. Purs esporits, o choum Bn 2 "Qí? asty a s pi: onden w r अun 102,9 iol $\rightarrow 0$,

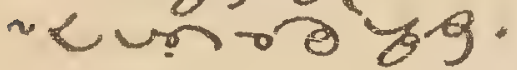

क्ष $\therefore 6.04$. 406 .

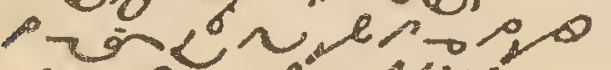

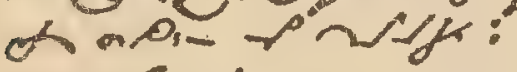
nugre ○几 $\rightarrow d_{2}$ लिभ fiom onsige if.

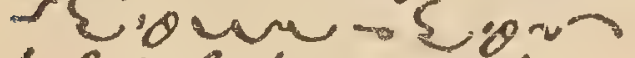
bo tomm to: ura 2

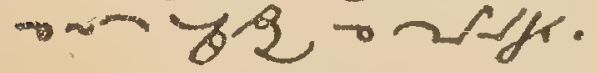
व. काan<น

क जर 10 क 0

$4 \times 6 \approx 2$

$\rightarrow \sim-8 \rightarrow 2=2-14$.

25. Panis Angelicus.

क्रि -

Dors vom, of this $x$

ode zon 'Es."

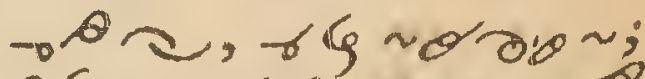

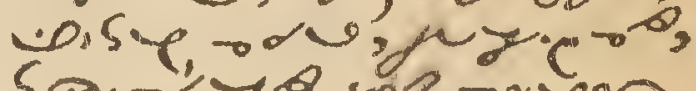

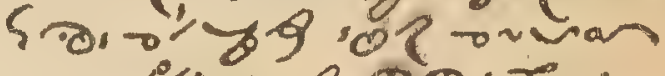
\%or in 6.

26. Welcome to the Bishops.

$\sim \sim$, 政,

ir a borm. 0 ,

-2 $5.00,00$.

$$
\begin{aligned}
& \text { जद ज9 }
\end{aligned}
$$

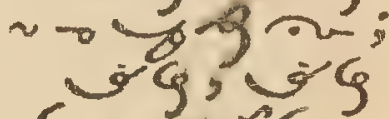

5\%, "S.

27. Chant du canot. ofio $\sim 8$.

min 09

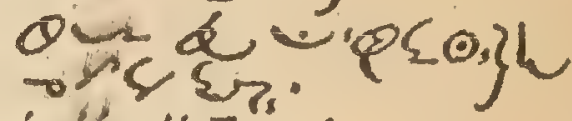

Pr. tothe H. Family. ox.'B.

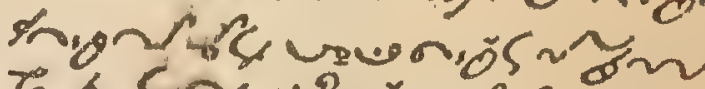

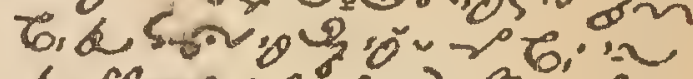

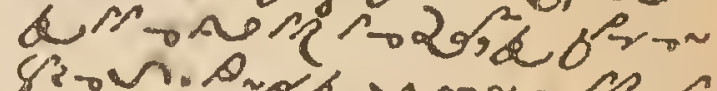
Sivv. Anoknir. 1

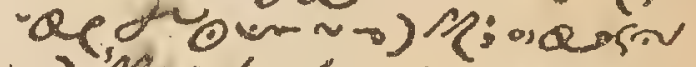
- ) " i $\sim \alpha \alpha, \sim<\sim \alpha 6 m$ 〜

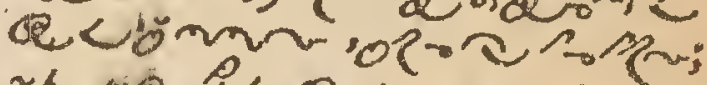

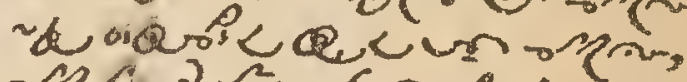
- M 20

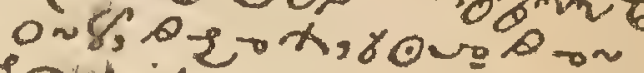

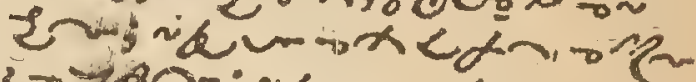

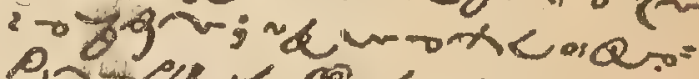

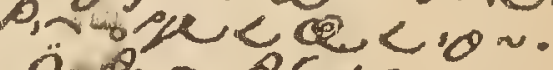

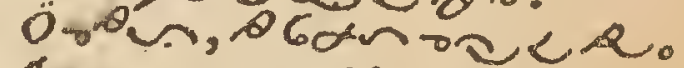

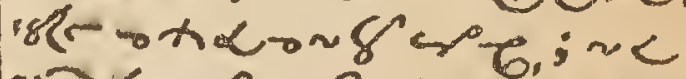

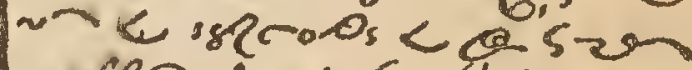

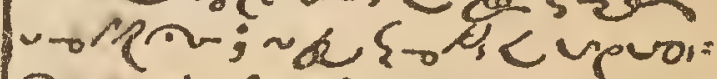

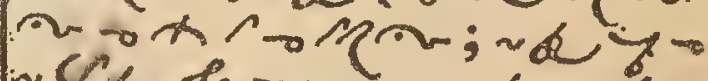


20

STALO

stalo Catechism.

I. God.

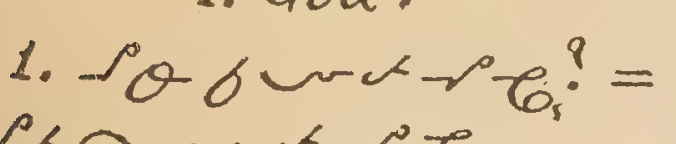

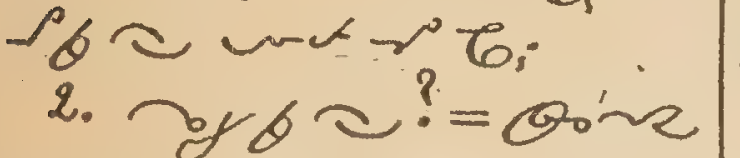

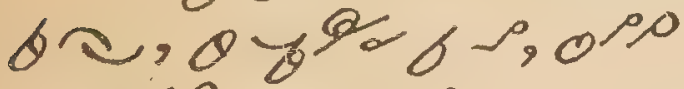

$\sim, 0$, एе $\sigma^{\circ}$.

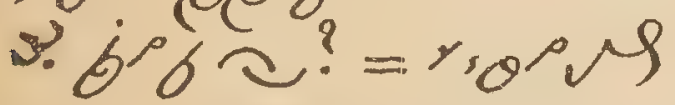

de, or bट.

4.0 b w orsors.

$b \curvearrowright ?=\approx G_{0} \approx s_{0}$,

尺 $\mu$.

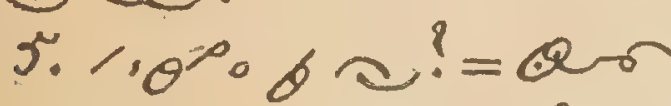

$\sigma^{2} \theta^{\circ} \theta \simeq$ : inors, oxd b

6. o- 6 worororg?

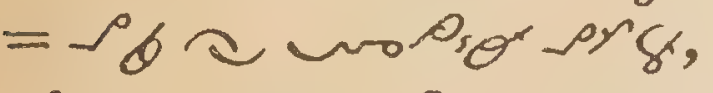

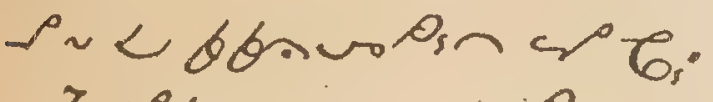

T. Pove wors o

c?= ¿

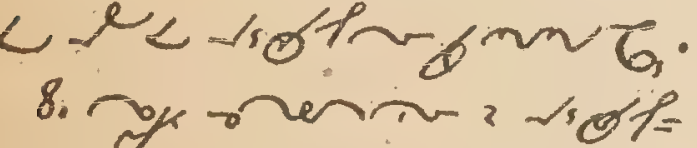

8. dy nen? " Nof

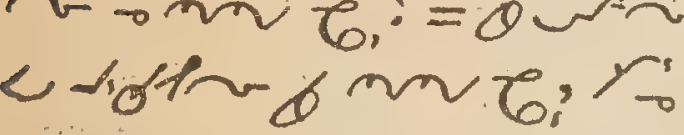

rem-ing $<$ on sur.

$m-\varepsilon$ aros $b$;

2dundin ok orooin 6 .

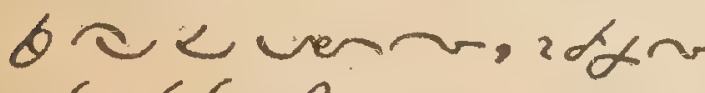

- 6 640, 6 .

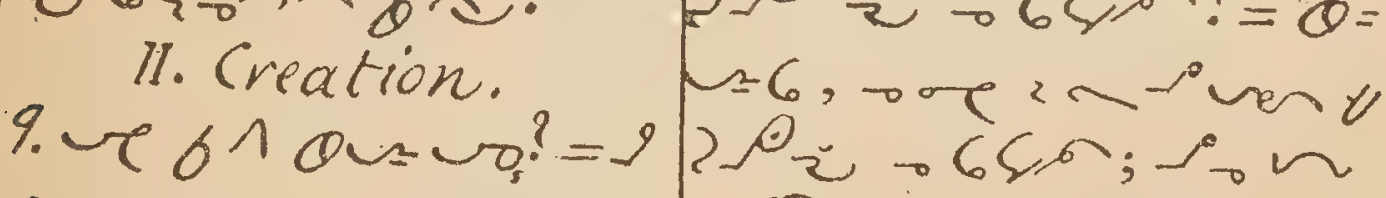

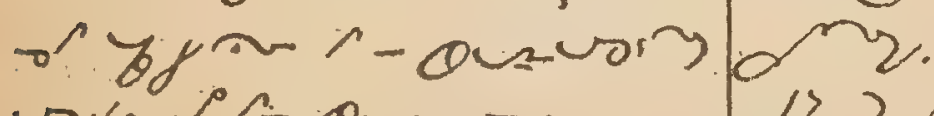

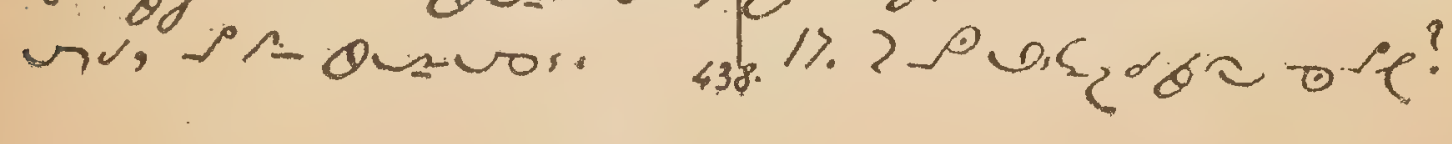

10. Ruori-onario

जu. = anur vod.

11. P<y d 1.00 .

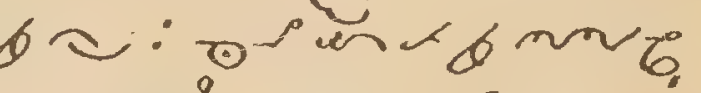

$\sim b^{?}=0=6$ b

$\rho \pi-g m a, 2=$

12, 6 .

12. 6 or $<$ inco 66 $462 ?=6 \times 2 \alpha$ - roe br vien 66 te.

13. o b ens - nog, wno um? = ¿wens nolg, wno um,

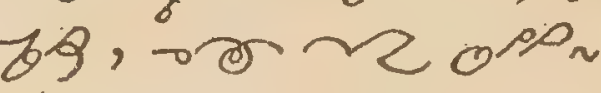

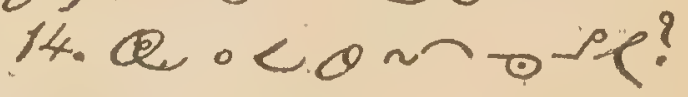
-QQuncum ore. 15. 0 .

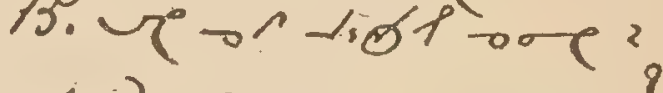

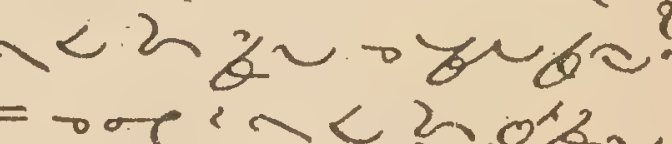
- yu-r,anos

be $54,5.6$ - 6 man 6.

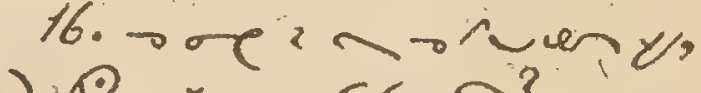
10 ₹ $064 n ?=\theta=$ $=6$, ore 2 - 10 ven To:

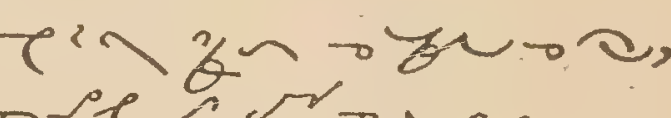
च: 
Qun $6-\sigma \sigma^{s} \backslash 2 \beta \sigma_{s}=$

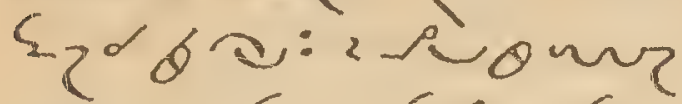
aed 2 2 sipes

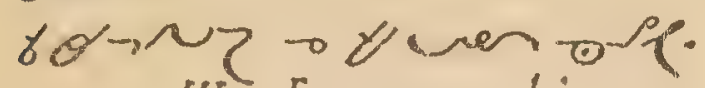
III. Incarnation.

18. breec zunen

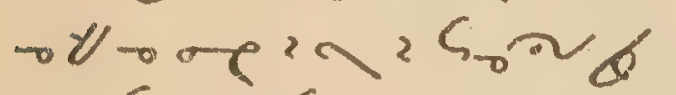

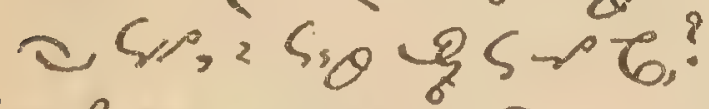
$=\rho \operatorname{sonez}<-0 y n=$ réuiventore in zomsorespes c4in wn SOUN: Wuentekrb? - 2 he res.os - 8 ars.

$19.0-y x+a s \%$

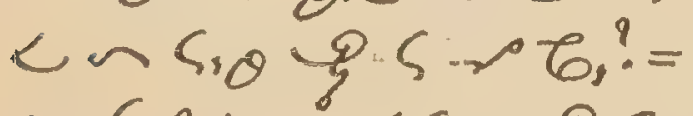
¿ sie sursiogs - b 0 , x< bru.

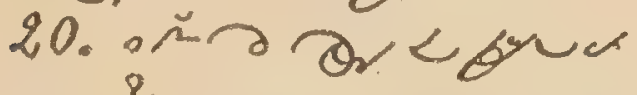

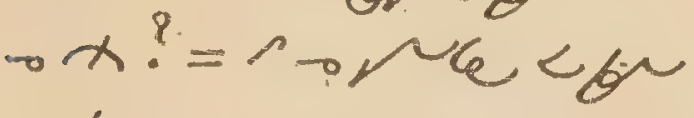
$-x$.

21. ryox? =x ous $\approx \sin \theta=\frac{9}{\sigma}, x$ obor 4 \&

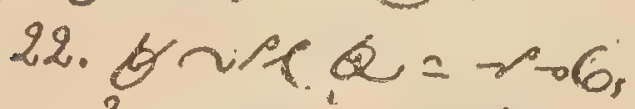

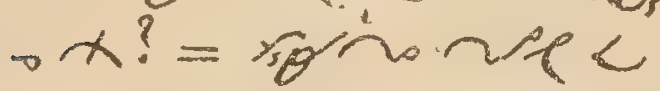
$\sim=\sim 6,0 x, 2$ OOU\% $\because<<62 \mu+\backsim 2$ ㄴ 8i: orracems

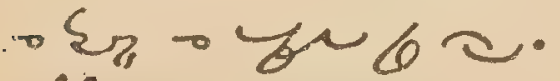

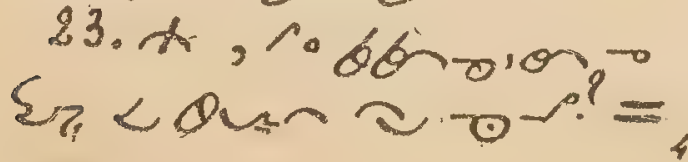

$\theta=6-12 b 6$ aros.

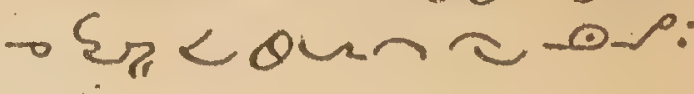
bs to soso van.

$24.062 x$ und $x$

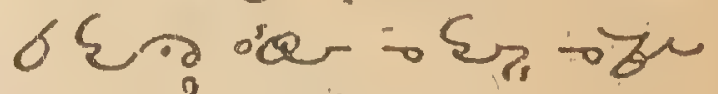
$b \approx ?=-6 y / 2-0 \leadsto$ $2 \div N,-2$ end

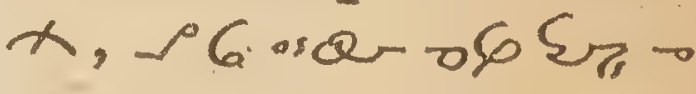
yुda.

25. $0.62000 \cdot x$ ve $-6-y^{\prime} y^{?}=$ xuma - उwe R:sk-our, noveror orys:$\Rightarrow 20 N^{2}, 2$ obuir

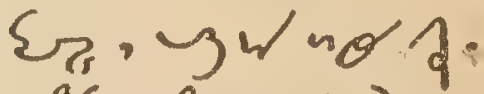
26. -8ve< 2 otum

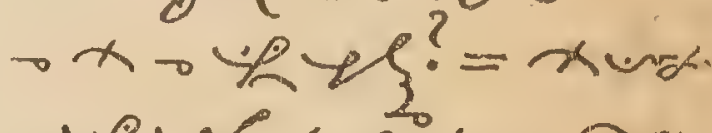

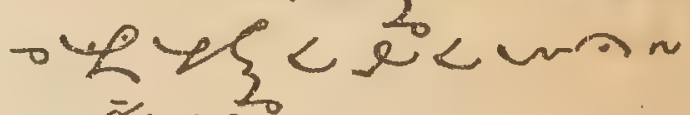
- जु 6 .

2\% 2002 iph ox? =

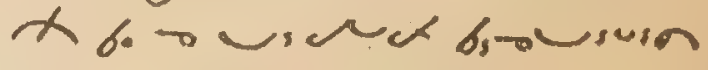
Luseropo.

28. ogue 2nighox-

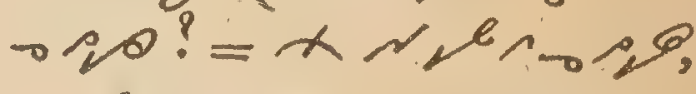
Leveránoson. จष

29. oror: $5 c_{2}+x<$

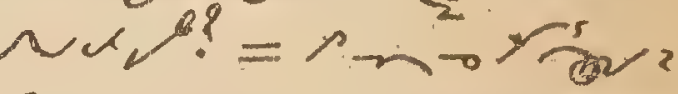

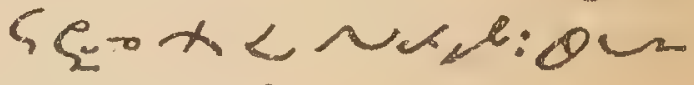
c

30. brouk $=6.6 . x$

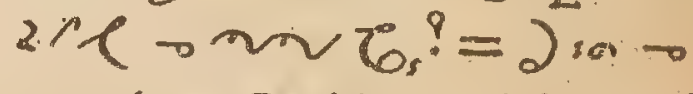

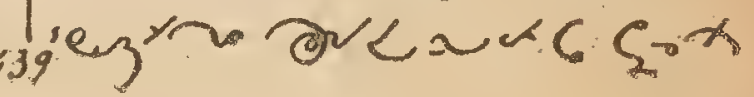




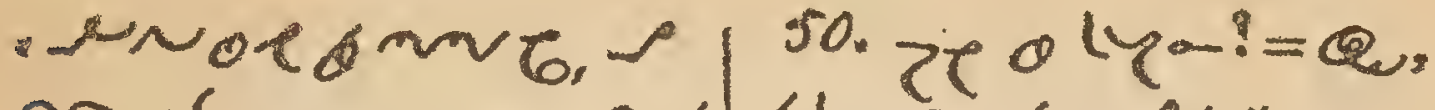

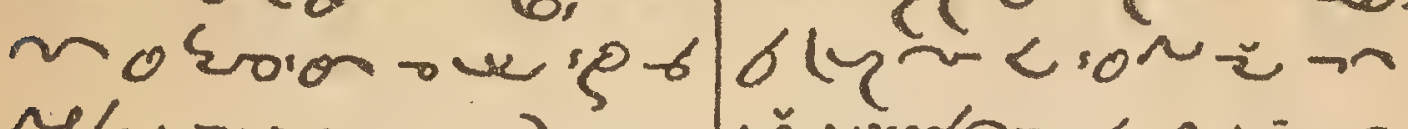

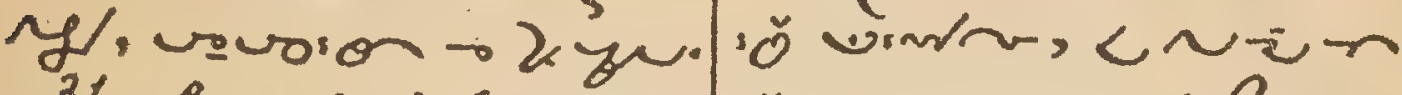

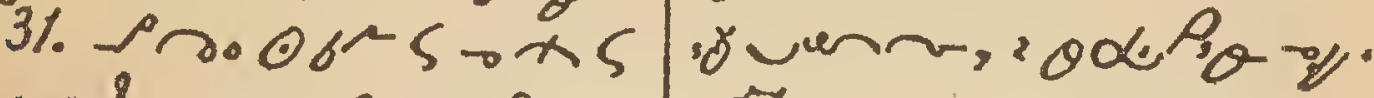
$\sim \sigma_{1}:=0 \sim 6,<2 r$

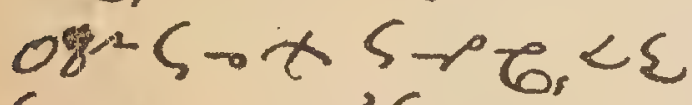

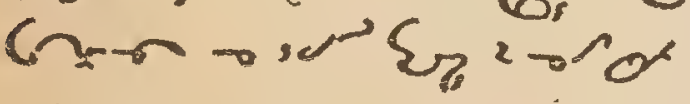
st.

32. "Qvoros.. . $\varepsilon 7,0$ ian $a<5 \approx 2$ ? $=\theta$ sou $\partial<$ shan $0 x$ - Er, ?h o ruas. mu $\sigma_{1}+\infty \varepsilon_{\pi}, 2 \varepsilon_{0}$ bin $\rightarrow \forall \varepsilon_{\pi}$. oर 0 - 11 s q.

VI. Sign of the Cross. 45. - Pure ins -1 sin.

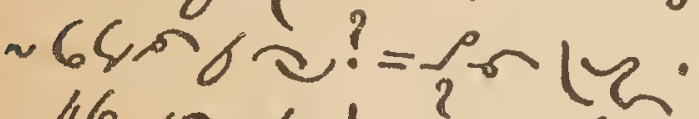
46. " 0 - d $1 y^{2}$ : = की

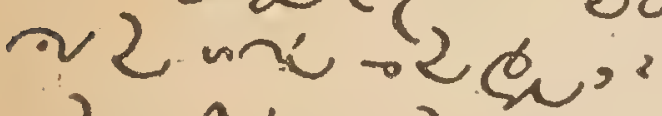
-2w几, 2 कर, ? -2m, r $20 \theta^{\prime}: 0$

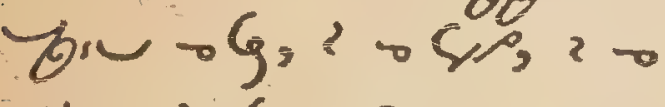
火, 2 <w. 4\%. '0 d sog d $y^{2}=\operatorname{sog} \sim \sim-3 \Omega$, 将 - x< ustros.

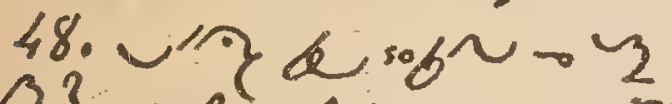

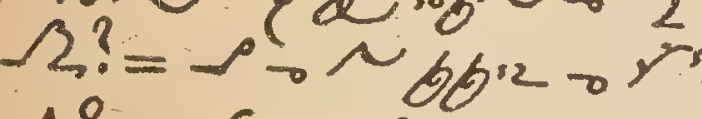
$\checkmark, \rightarrow G, \rightarrow$ Sp, ow. 49. $\partial \operatorname{yog}^{\circ} \rightarrow x$

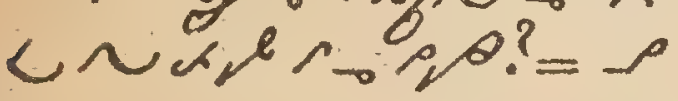

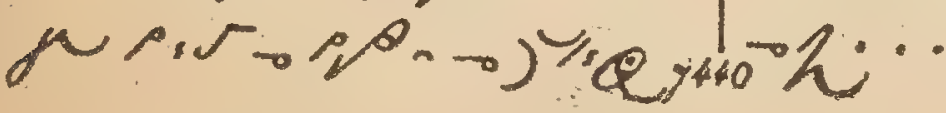

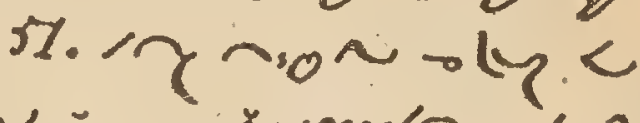
$\sim=n i \delta$ Grandm, <

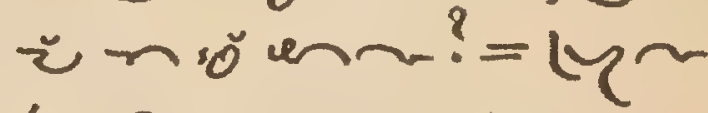
Laserar ouvaras Uen sol based < sosion d ov vint, 2 o olven.

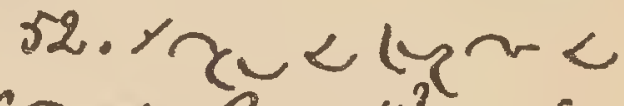

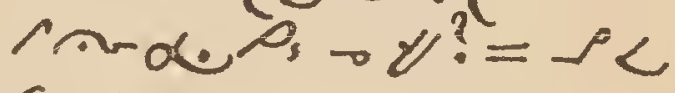

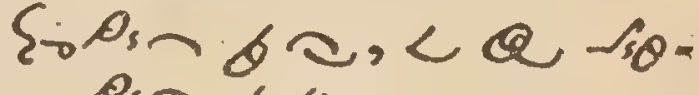
wes $8 \%$.

VII. The Commandm." 33. 的人 जoceuman? =

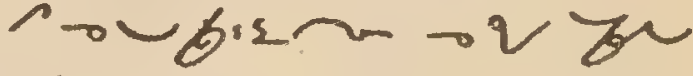
$b 2$.

$54 \cdot 2 y \sim, 2=2$ rovar -2? =6

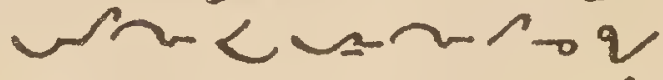
अ $\rightarrow$ ofe isg,

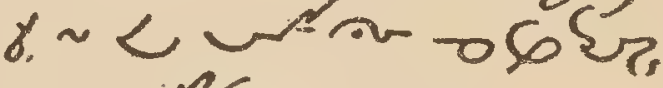
$v=-\gamma \operatorname{se}$.

25.

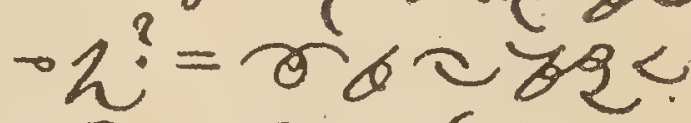
veroin obe bu 눙 56. Cundorati 
vist. Prayer.

$5 \%$ obad y unne novosn ozuba? $=0^{\circ} 06$ foron 0 C

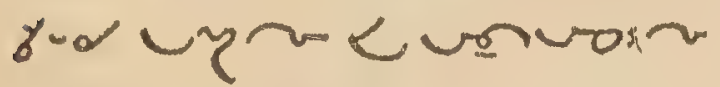
- yude.

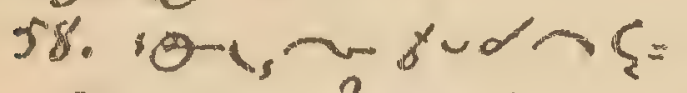

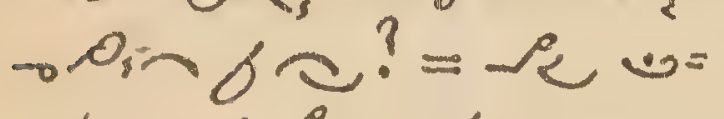
innes s roodin.

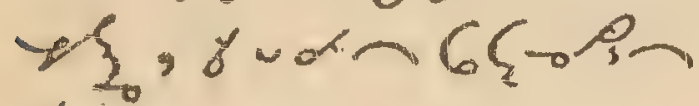
$6=$

$5 \%-9,10$ - coswing

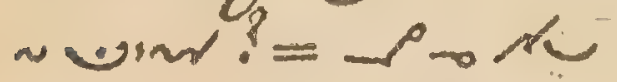

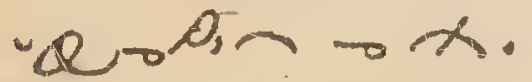

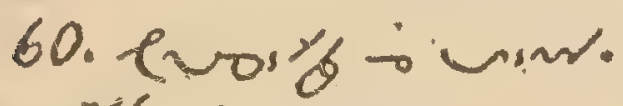
$=\pi \log$ r $2 .$.

61. Po, o ving of

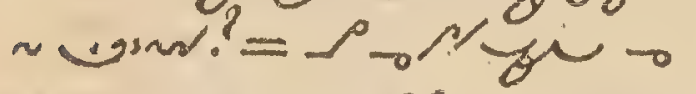
Non $102 \rightarrow \sim 8,-1-\rho x$. 62. rvory - wint = o siunger. IX. Sin.

63. जr-yven? =0 औ- - यूठव an.

64. 66" tuen? = use - Vuen: "üVareró 〜Lu solviven Prsp.

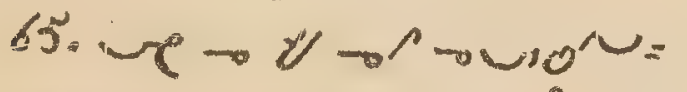

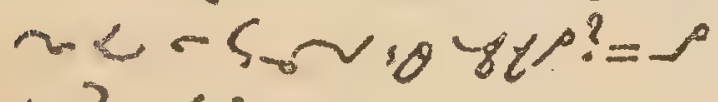

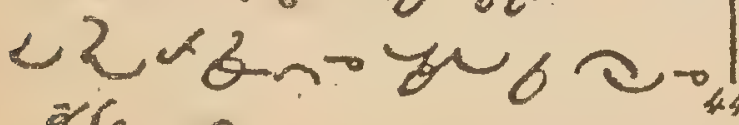
बदु

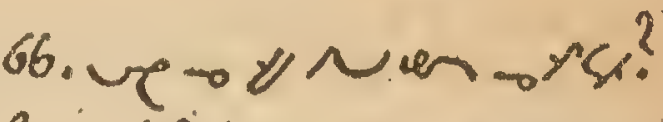

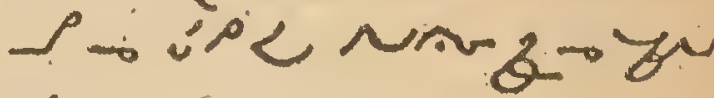
60

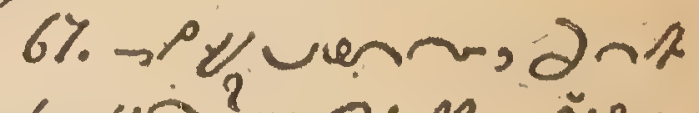
- Lyr? = $0: M-100$ - Pztr r norn,

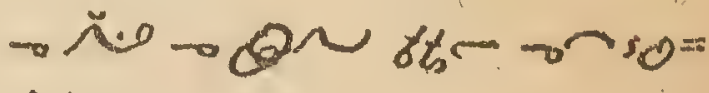
Non.

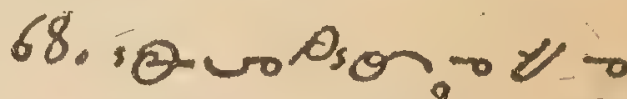

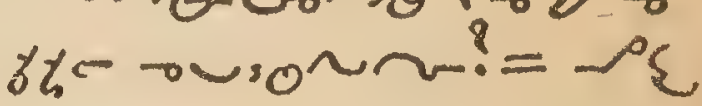
so znO. $b \wedge \operatorname{sing}$.

69. $6 \rightarrow y \rightarrow b t 50$

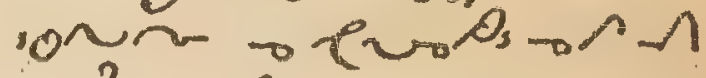

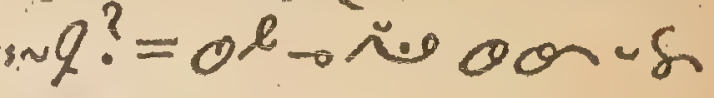
\%

70. 3 -witen on

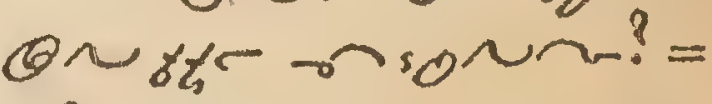

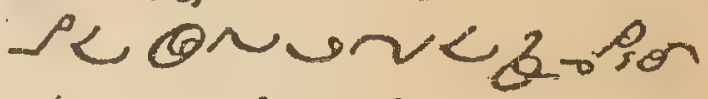

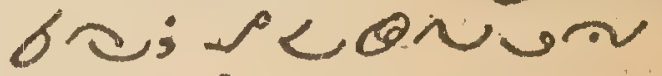
$<\varepsilon_{0} p_{\text {ion }} \mathrm{c} \boldsymbol{0}$ 71. -10 भio snibs

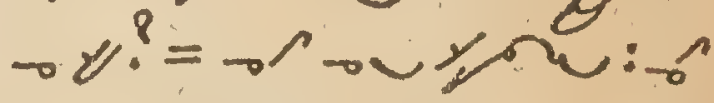
-

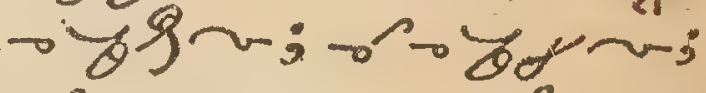
robiunnis trou

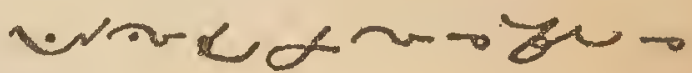
2.

Y2. Guine - Lowine - or bix. nzg, of z-9, wer, $e_{1}, " 0 y^{2}, 2 q$, wer. 


\section{Baptism.}

73. $2 \gamma^{0}$ x $u$ inn - $\mathscr{L} \varphi \xi_{0}=\rho<S$ asi- orionm.

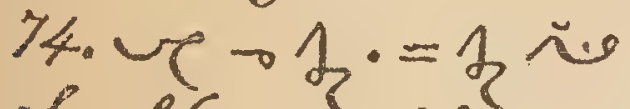
$-\xi \rho \xi_{1}+2 \rho-\Omega_{50}=$

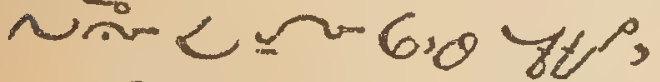

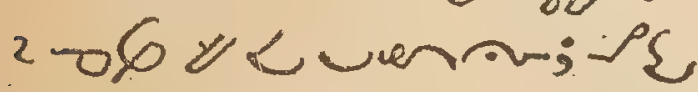
'asen son son. Lrsinga, ca sinth: < rens.

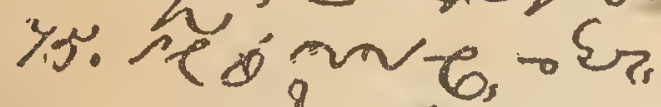
Q ison? = Q a d Lh bma bs $\Rightarrow$ ? Q vison.

$76.0^{8} 0 N^{2} 6$ of

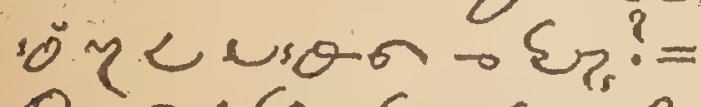
Q

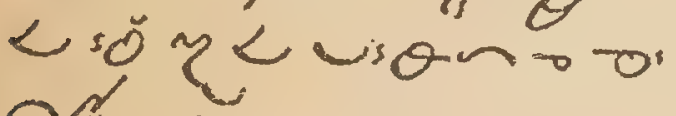
त'

7\% gy acento govion $60^{?}$ ? = bith of sol o usue,

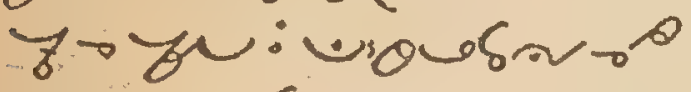
$-b_{0}-\zeta=-\operatorname{sis}=0$ 火.

78. कु - 0 जe $u=$ $\theta-c^{?}=n \theta \sim<\alpha=$

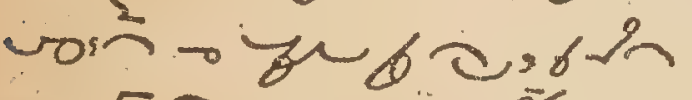
or ; squgran soर $06 \% \rightarrow 1-62$; अPUO Quvion.

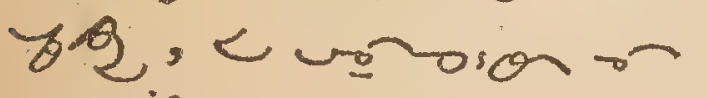
'Q⿻肀二

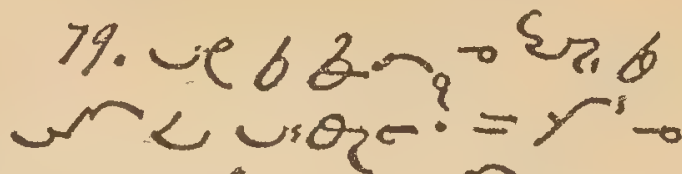

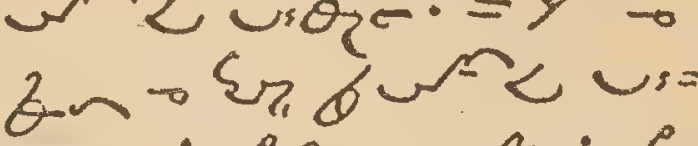
बरा ; an-arven:

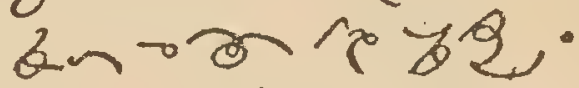
XI. Confirmation.

81. ves saler? = $\alpha$

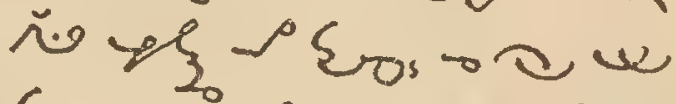
sovisg y-ar qe, <पn L susion sor

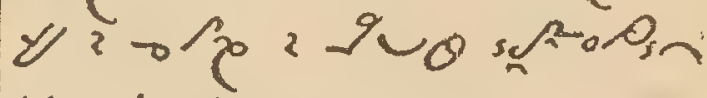

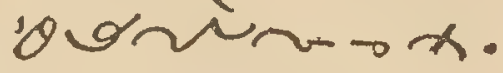

82. $\alpha+\bar{e} \cdot \alpha$ ? $=$ ol $\rightarrow \mathrm{e}^{-} \cdot \alpha$. 83. $\partial{ }^{\circ} \leadsto<\mathrm{G}^{-}$ $\alpha$ ? $=y$, ar soर ugs 0 or ,

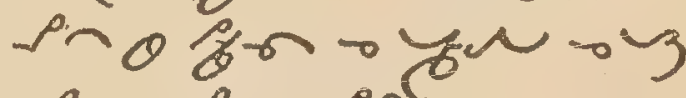
n,uspren.

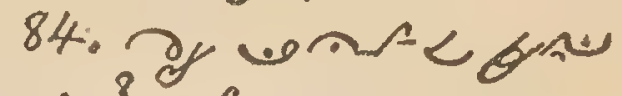

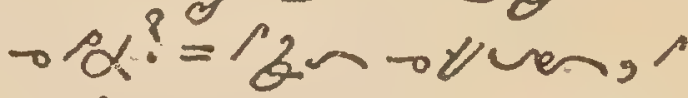
ripion evord, 1-rion nivon 6recar bon L byion ou. XII. H. Eucharist. 85. ve 0 ? $=\Theta$. rkuran 4ip $x ; 206<1$, क ri, aney, का 86. . 0 o x und o : ?

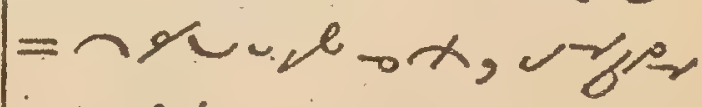
ryglogin ow, 2 4thx, ? asere ore.? 
:brop, z e Mr, p

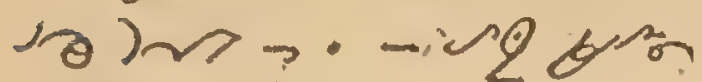

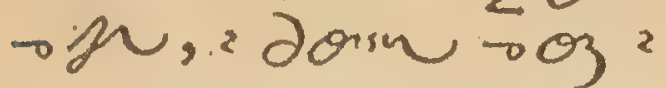
ᄀu, 2 os 0 ?

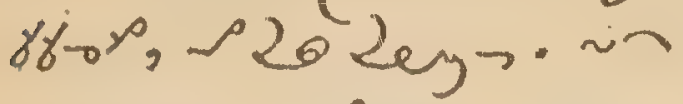
sonveros of b ugr = Y. 4 .

8\% - क्र $0 x-20$ $\lambda \sim, p 20)$ eey $\rightarrow 2$

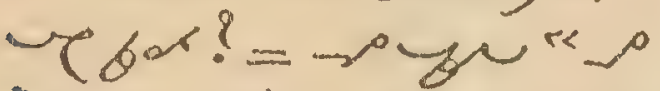

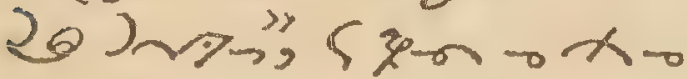

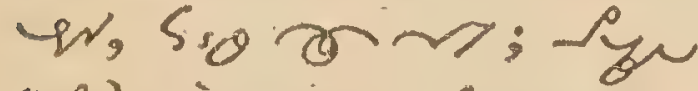

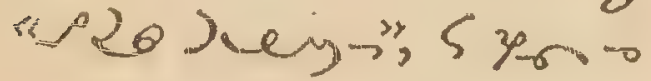
$x \rightarrow 035$. 88. L N 200 ox tox Lenc, $-03<$ on $\mathrm{U}_{2}, 0262$ Oous ter $203 \cdot=L \sim \mu_{n}$ - $x$ - ýerre, ¿S Goun - en, 0 b 2 - Not $\rightarrow$ WN $\rho \wedge ;<\sim \sim q z$ o celey, 5 orm oj, -is on warfiog. 90.<นy- $0 x$ -

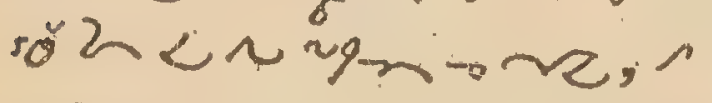
"ern or/s? Yar? $=<2$ yen ox orng. morein $\phi$ y are: Ob<几N vign or

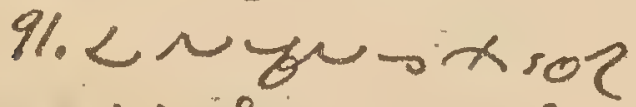

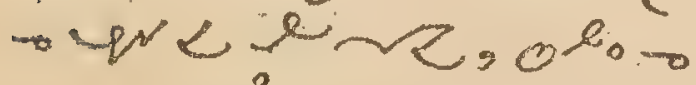

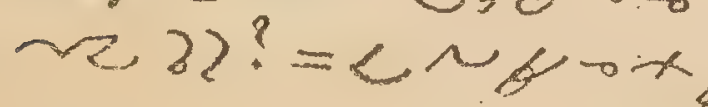

soर-4erm, Qol - व $22:-2$ ?

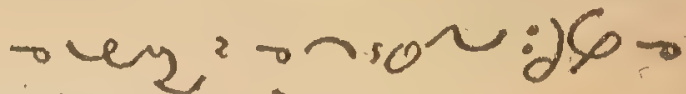
$x<\sim ?$ 92. $\% 1 x<2 \mu w$ $\sim x \rightarrow \Theta$ ? = xum - $\oplus<\mu$ som, <e somen $\rightarrow$ Nyouration und b rog, $2<$ sedrersonn. 93. 2 . "Osur $<\sim \operatorname{sose} \rightarrow$ क fogn十os ox<N

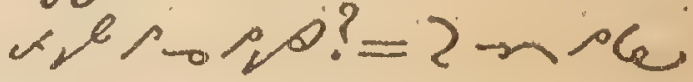
Lis'orn. L w woser

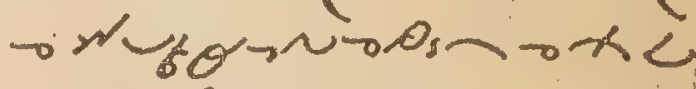
几х,

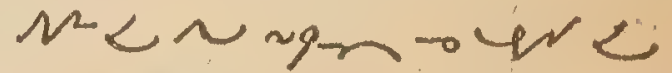
sig a me $\rightarrow x ; 2$ क

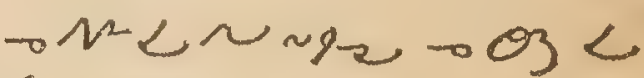

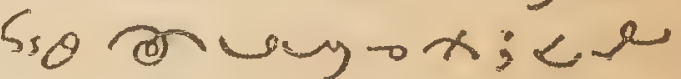
$-2-\cos \theta=-x \operatorname{son}$ Luan bus.

\%म. nog y. Wix sop $\theta 2$ ? $=0$ क 6 m कद no besor 65 ndingun purin b2; ᄂ b 2 ;

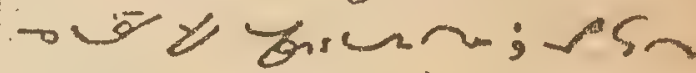
solin ope a 860 s त $\sum$ os ${ }^{\circ} D_{1}-b=$ $9 \%$ m

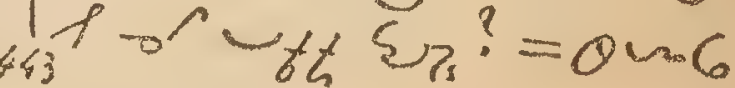


26

STALO

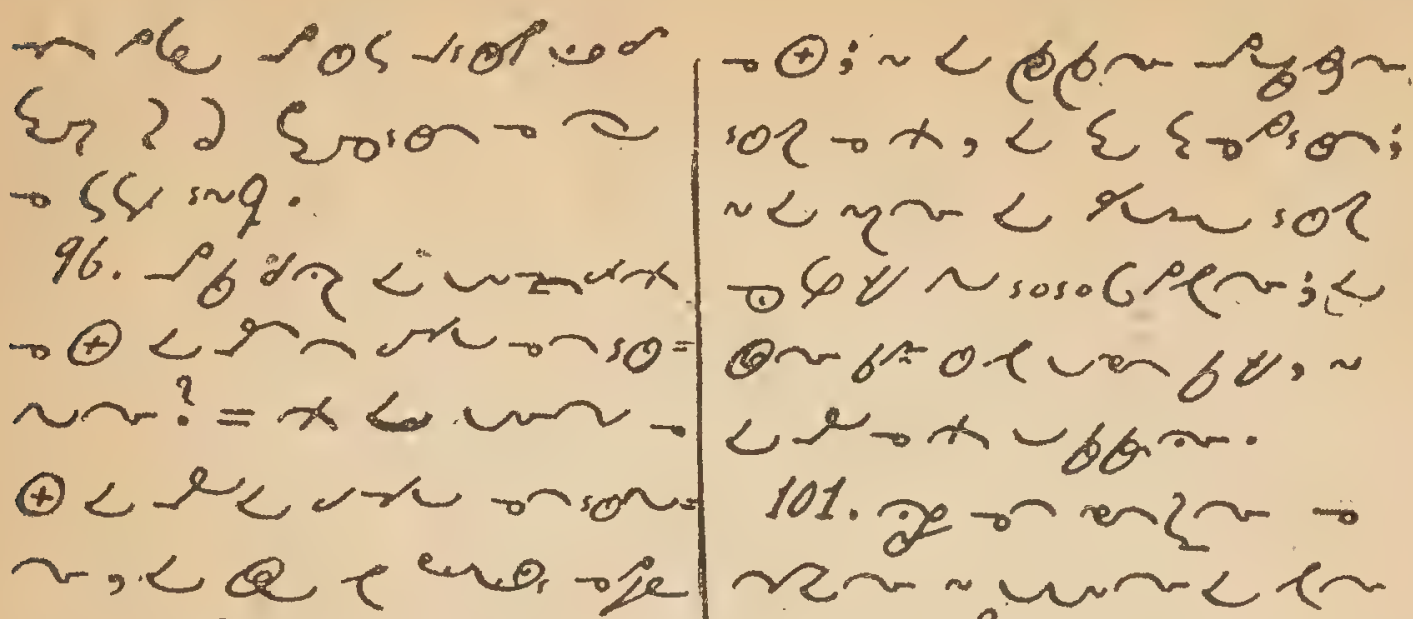

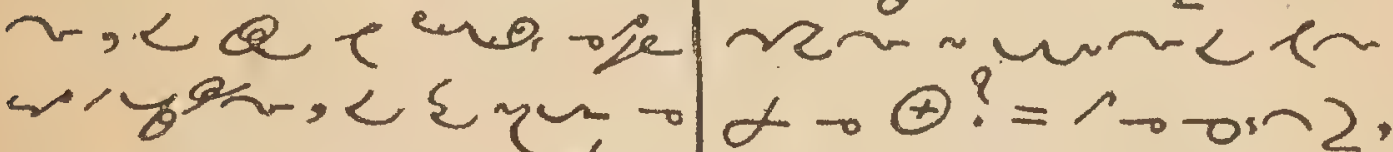
je

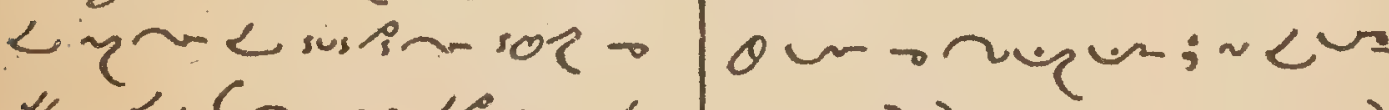

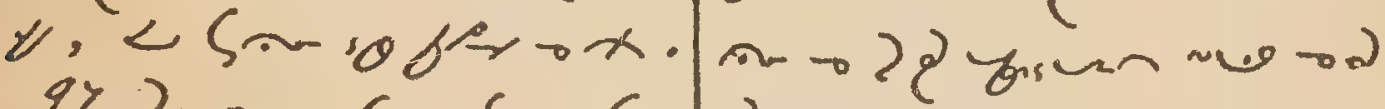
$9 \% .2000$ of $-5 \pi, 5$ 2un-

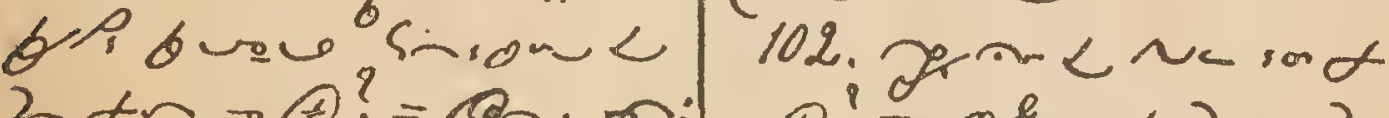

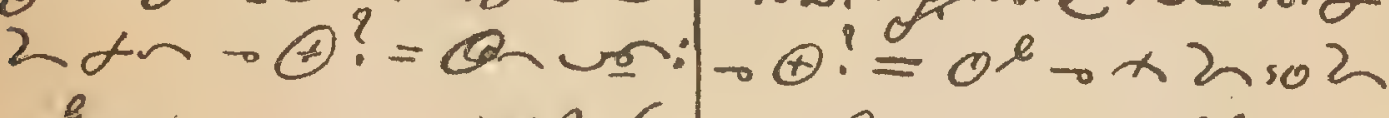

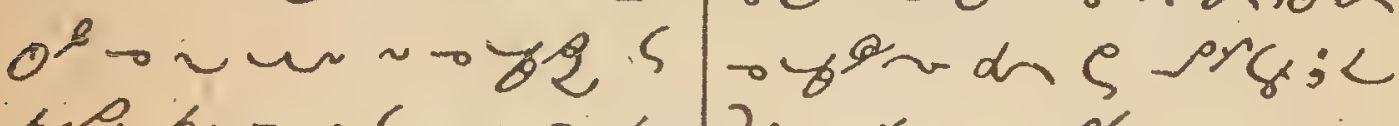

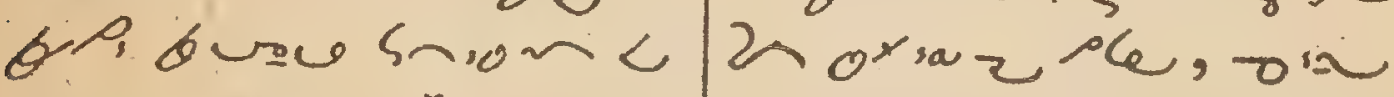

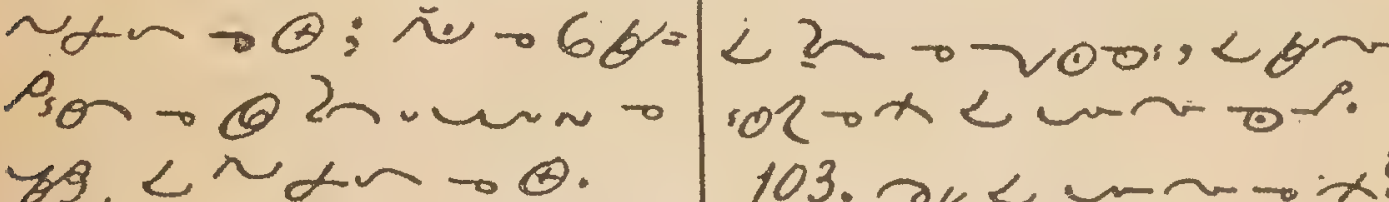

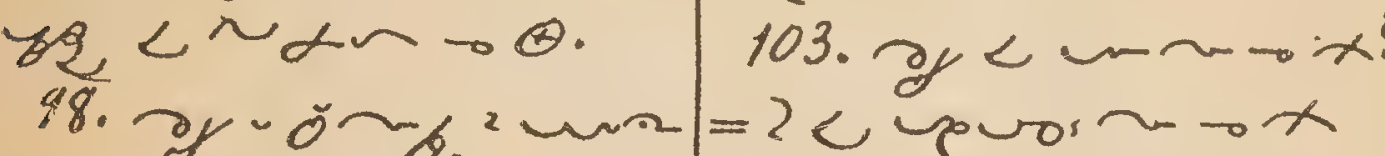

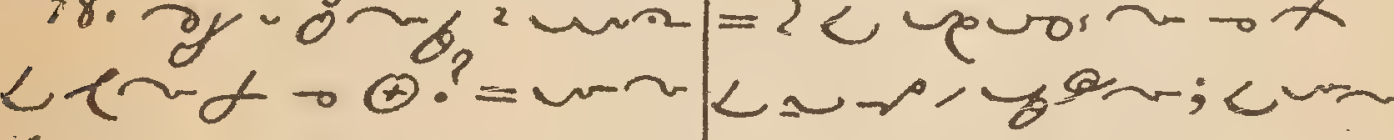

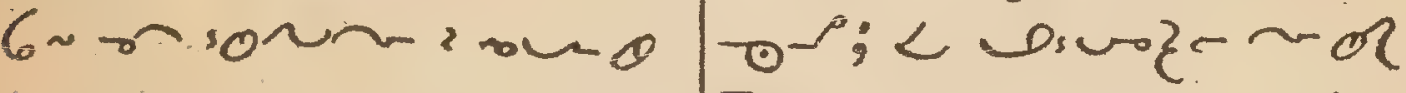

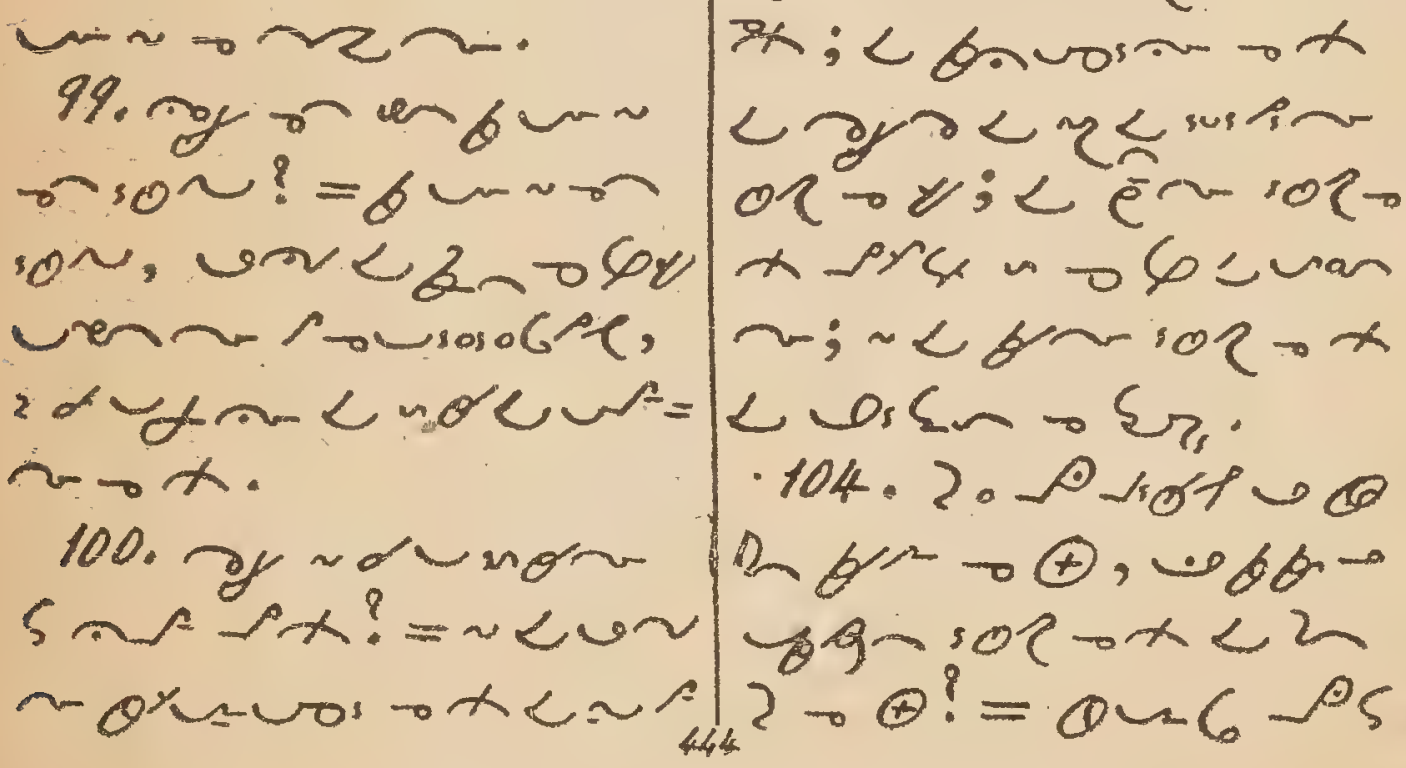


N.91 $60-268072$ $<\alpha-x<22 \%$ Q 2 : ox Q Q

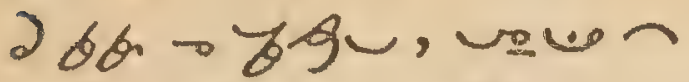
io 2 san $\rightarrow$ ge. XIII. Penance. 105. ay a p an $\mathrm{s}$. Len $\theta$ store? = gutersere oncturen: oreg, op wo, worke, tors pers. 106. ¿a a cukg? = prez, s/se voor ip vo gees Mory: " Mow 6ट<ह zon 9

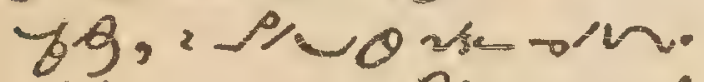
10\%. ayc otaly e rat

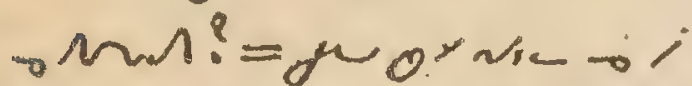
n, 294086902 yu-a, oreger -

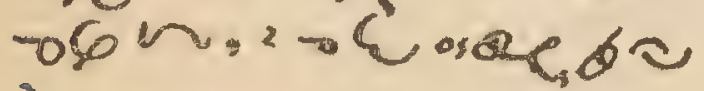
hon.

108. L N O sock 6.2 1

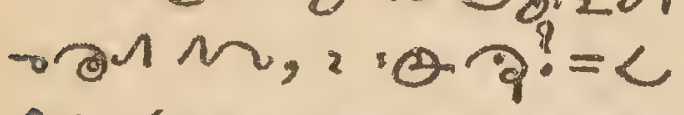
roriagubrorin, $2900 \alpha \rightarrow 102$ No, 62

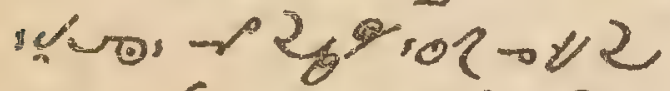

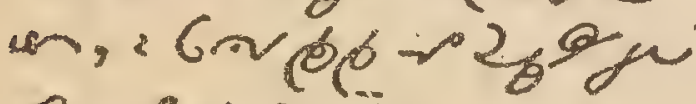
Qoler an.

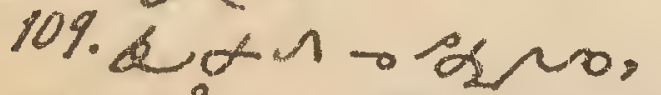

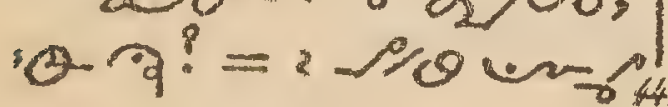

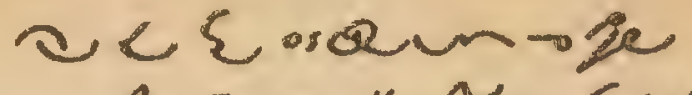
LR Q

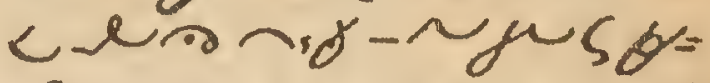
Pi d ce ta juor or br-y;: Nuobr

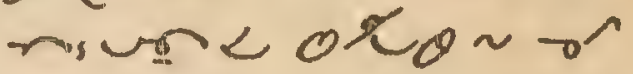
2N6; ; $9 / 40 \% b^{q}$ nive L oro ny

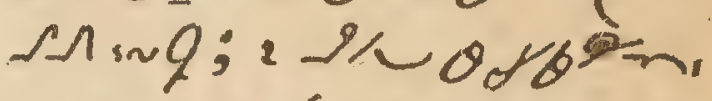

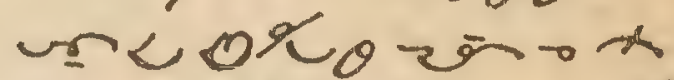

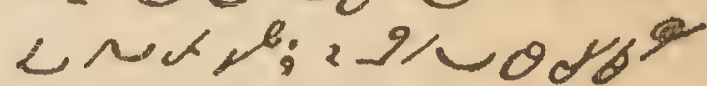
nim lor o $60 \%$ -oed-rsicr. nid-n jus biligox

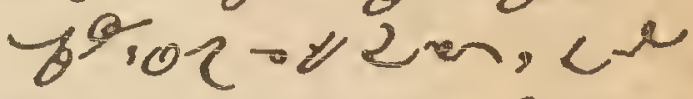
rrio-r jus bab $\theta=$

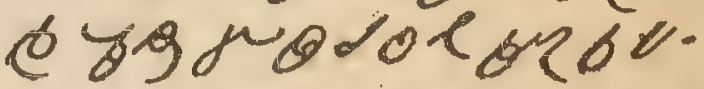
110. ro a de ensorber? $=$ rqye un ons.2 pory, a pot: ") G, oration rohy, - wan nu. soroncee

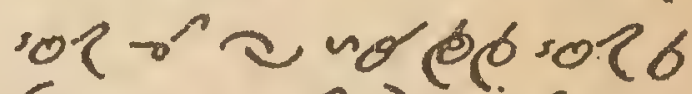

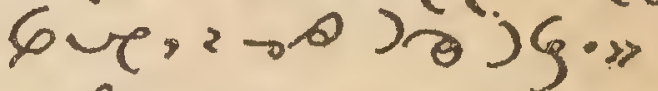
i pountrin. volo $<$ gude soso GPe; i ghom - Mroura o borat. -00-roro.:00 lo h3; 2 9 rom - Mr warkera. seonn, or odido on-s, a poin 


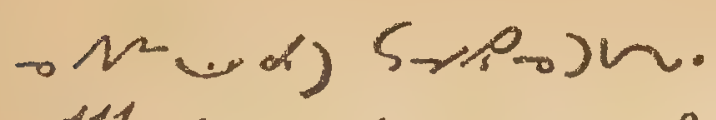
111. L worsemn - $N^{2} \Omega \Lambda$, :

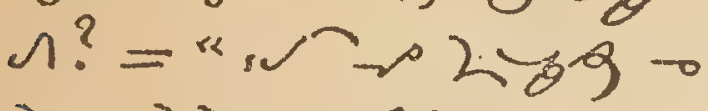
in 22 sosocer, 2.0 Ou I S SP, M 3 O c) u, 2 d z era. $2-020) 5$ a orac rorsons."s?

112. "बतa<

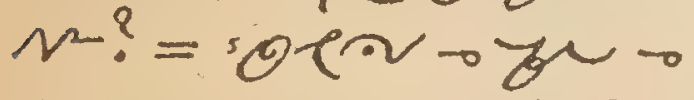

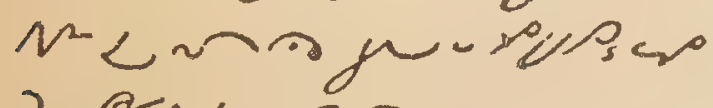
2क; - L preir, <ino gur res overob osere, $N^{2}, \theta_{0} e_{\text {. }}$ toro, a o e o hy, $\% \theta<\rho$ oq 113. L Mn ug: "T os but -89 a $ح$ ?

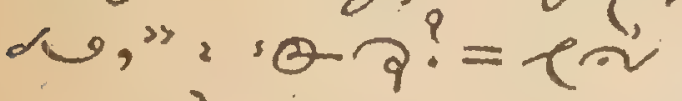

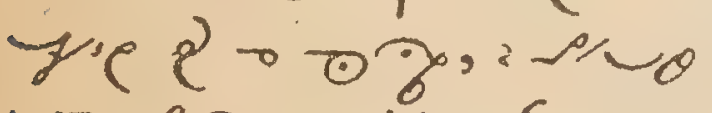

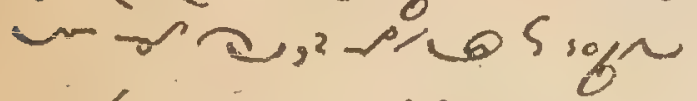

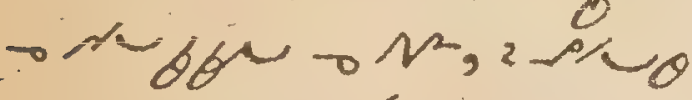
자 $2<\leqslant<\infty$ यद, 9 , prevs

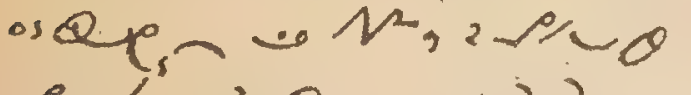

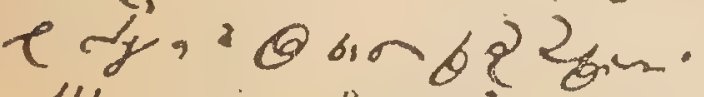

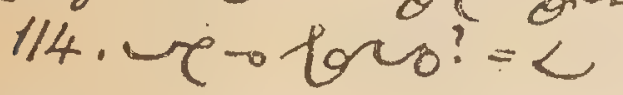
-sar $\rightarrow$ otho, ?

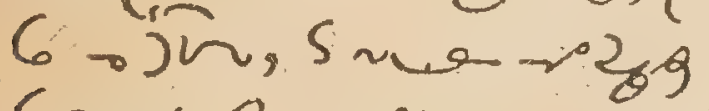
sngls we. $115 . v-h_{3} ?=<\operatorname{osq}$, $\rightarrow N^{2} \rightarrow h_{3} 6 \sim+6$ in.

116. bed an Sroon NA \& in otsonsober $r N^{2}, 2 \cdot 0 \cdot q$ ? $\mu^{2}$ Sign oun jorson

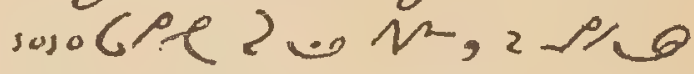
ne 0 dro or soroct. 11\%. o b en onyms

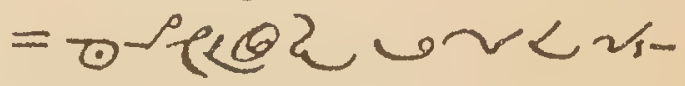
$\sim$ on, oxifur. nथ

$118 \cdot 0-06^{2}$ un on , y'? = LN กัon, ore en in rm.

119. ousogh an w nsm-ousasocr? = ose 2 bofiem<21 - Nisar robuer. LnA rorgorenun em.

120. Nunos to 2 . w yeytionr.eusule?

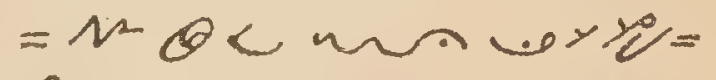
son 10 wosolex; $<\theta$ ofmorir va $z^{2}=x$. 121 - $\rightarrow$ ons: =

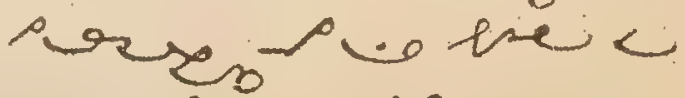


ar

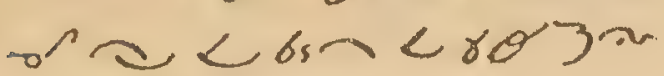
OR

122. Ps

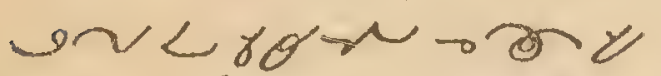
bren? = ror $<8 \theta+4$ borman, ope 2 \& SSereng; osed toss

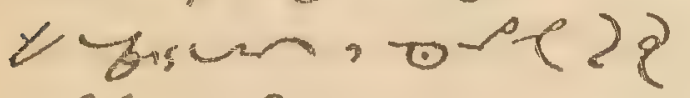
-5 sir sug.

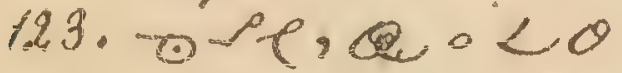
$\sim_{-} S S \sin q ?=0-C$

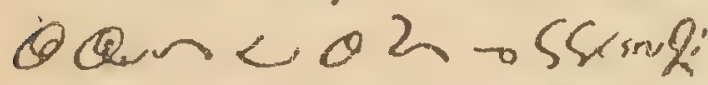
$\theta \operatorname{san} 2-30 x-$

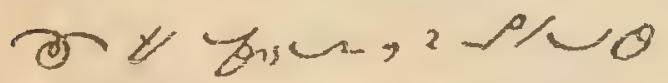
C- onm To.

XIV. Exer. Unction. $12 \% \sim r \rightarrow R 2$ ? = \& 10 of wernstox

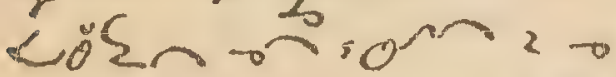

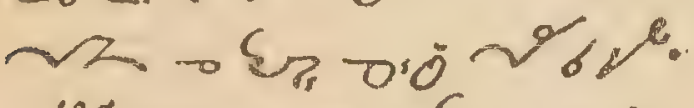

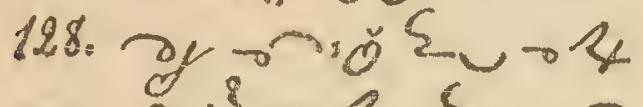

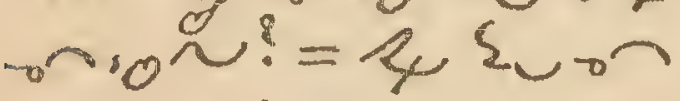
'on o 60 Q $\sim 2$ wan; \& OैC $\sec 0$ 20 6.

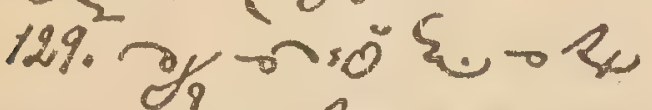
ore? $=2,0 \operatorname{san} 0^{\circ}$ or thotor ormon 6 2< 2 mson. 130. Of $\mathrm{i}$ dt on

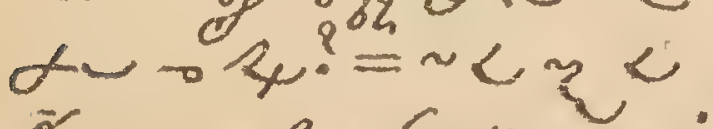

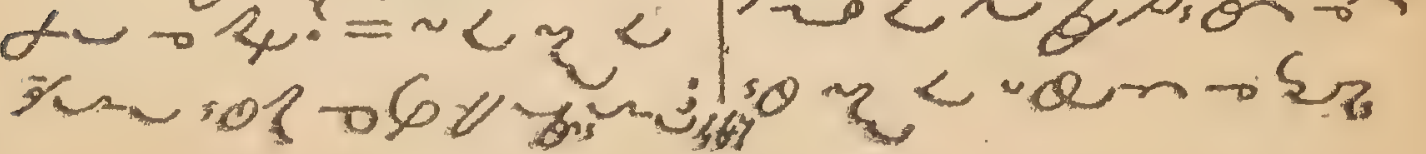

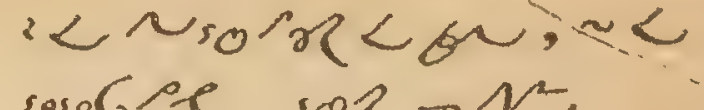
soroGec sor t $N^{2}$.

131. gf $\mathrm{N}<\sim \mathrm{C}^{-}$ R? $=$ Now ososos?

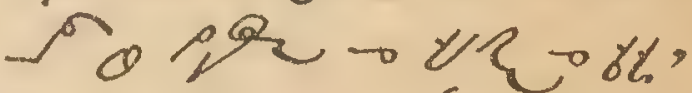
, - viurus, gnoguso? $60<3 \%$ - $06 \%$ an to $\beta \Omega$, rof, robur, roven, rovarn, r-ovink XV.H. Oraters.

132. $u+q^{-}=9$ r.

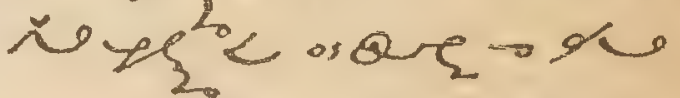

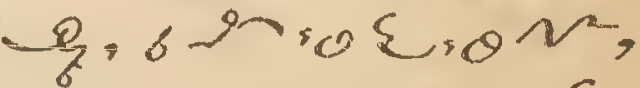

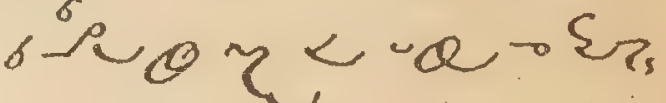
- bx - o a a $\rightarrow\{r, 0$ - 0 133. L

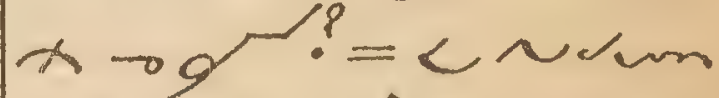
$\rightarrow x \rightarrow \oplus$ os 0 \%m of : r $2<\sim \circ Q m$

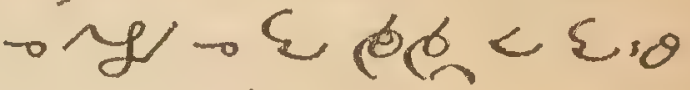
$N^{2},<\{: \theta \sim$. $134.06 e^{-}$- y $q^{2 !}$ $=0^{b} \cdot 2 \alpha \div \sim \mathrm{C}^{\circ}$ -

135.00 - $2.0 \%$

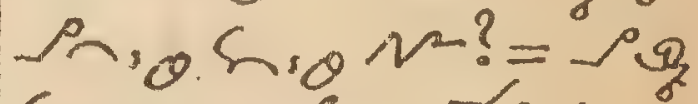

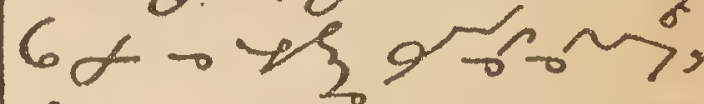

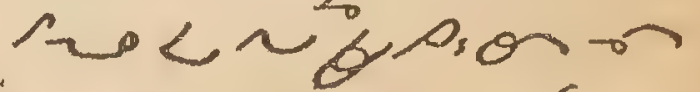




\section{XVr. Matriminy \\ 136. r. $r \mathrm{~s} ?=\mathrm{zm}$ Noy Punsto Letoriancia nsoor. \\ 13\%. - a $=18 \cos \theta-\mathrm{ye}$.}

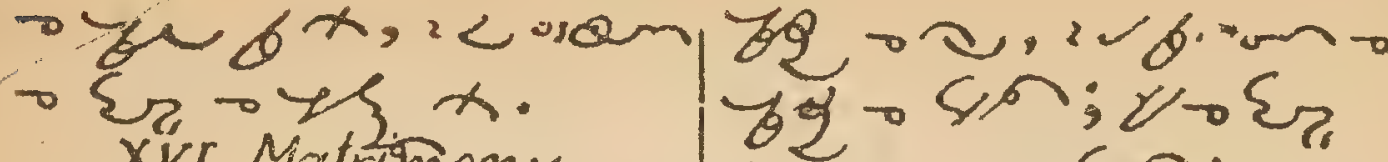

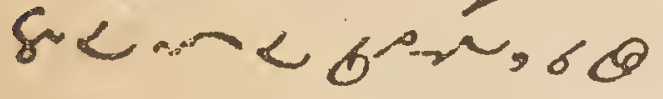

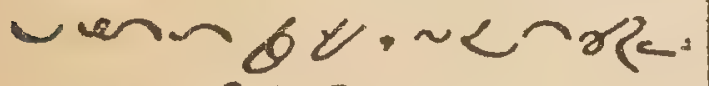

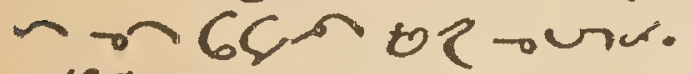
138.. - c un to $\omega<8 m$ ? = - 0 n <in, o 0 . . ob.2. जulb<ar,:2 umun on roon. 13\%. $z y-y x+20$ $\varepsilon_{7}<$ Evion or $\rightarrow$ CN? $=\rightarrow Z_{0} \varepsilon_{\text {? }}$

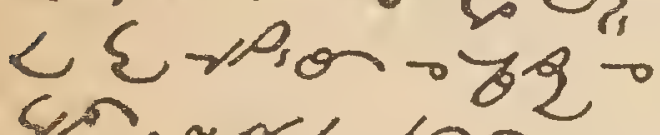
sin, no< कथ

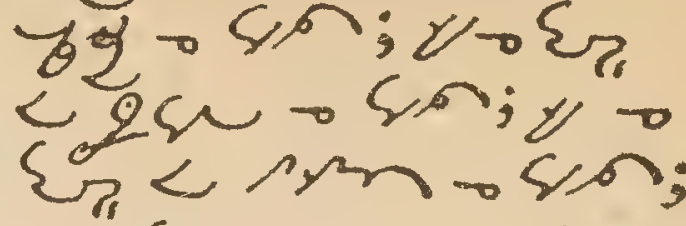
$y \rightarrow \varepsilon_{7}<$ on $\rightarrow$ s on < sin.

140. of wiven DPT <לripion<smm? = <srigon< sinn, ing Lnd sol ra; s. \& bbuar, gno< man $\sim 72,2 d-$ 几 ndi2 б八y; 2 -

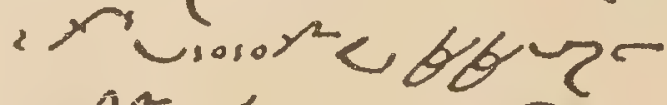

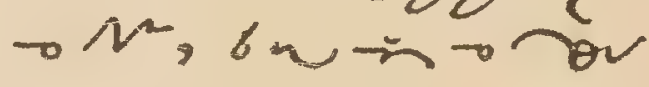
c sn.

$142.0 \sim \sim 2 \div 2$ $\angle Q, \sin <\pi<$ sin? $=046 . \mathrm{h}$

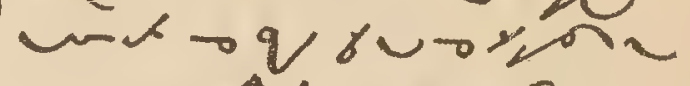

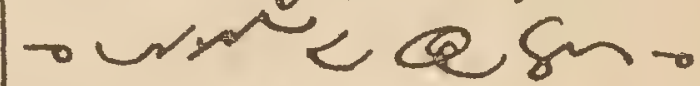
$\theta \sim 2<\sin : 0$ यदs navor ki- 
$-$ 
. 


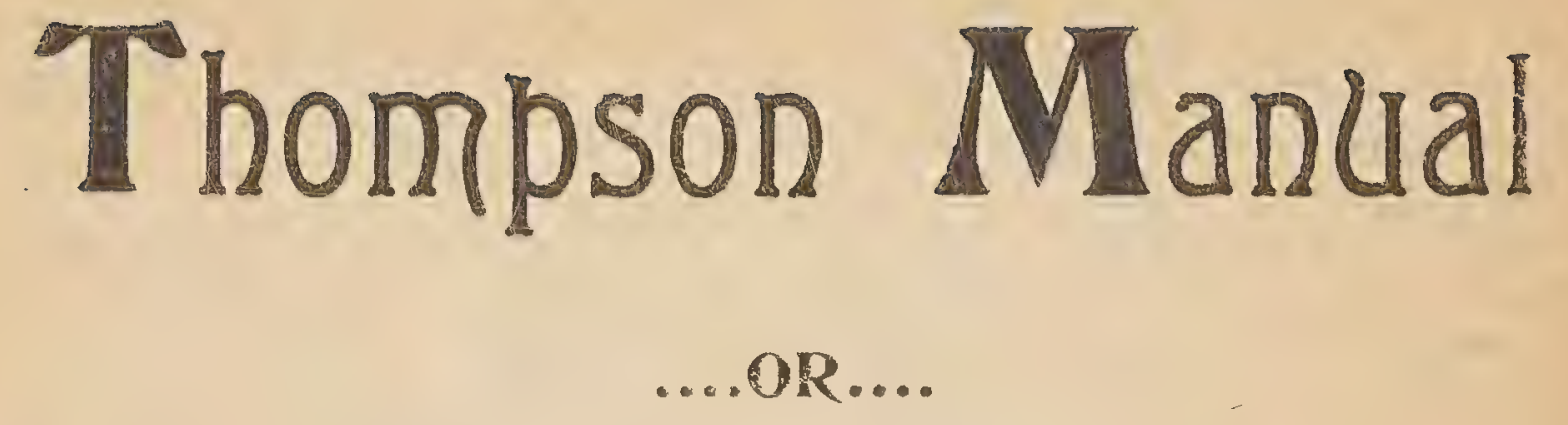

\title{
Prayers, Hymns and Catechism
}

\author{
..IN THE..
}

\section{Thompson or Ntla Kapmah Language}

\author{
With the Approbation of \\ RT. REV. P. DURIEU, D.D. O.M.I. \\ Bishop of New Westminster
}

KAMLOOPS, B.C.

1897 

Thompson Prayers.

Offering of the day.

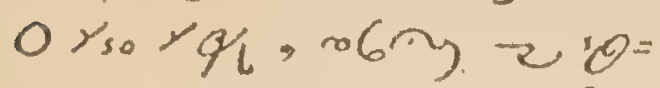
uns 6 rom so 7 wis

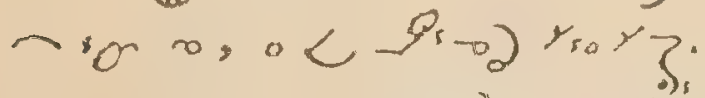

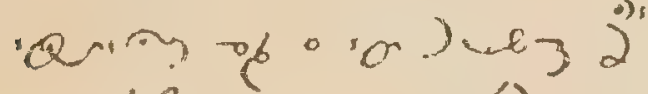

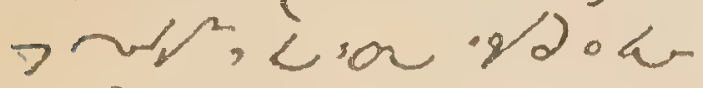
0.2 . us o 2 .

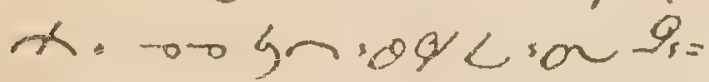

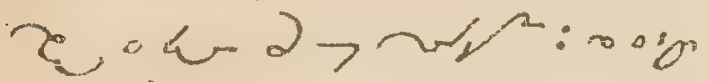

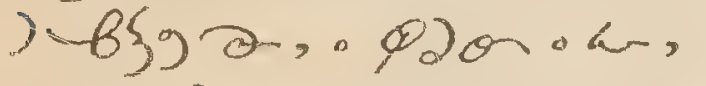

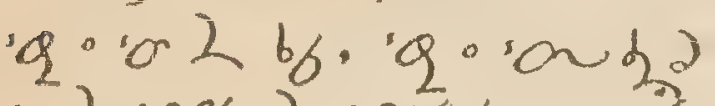
so $2.0 \%$. $2.0 \%$ gn son tse $\partial_{7}$ war, of a soso un o

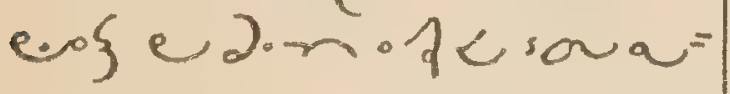
"Coum $><$.

or 8,902 . LqQ $-2, g 6,-\cos 2) \cdot \xi$ cocannegan. Pater. 38 su $\angle \cup * G$.

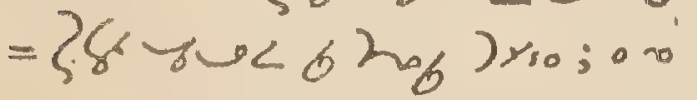

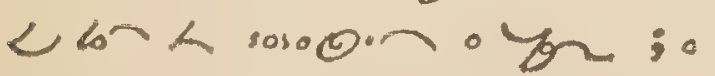
oक 9 .

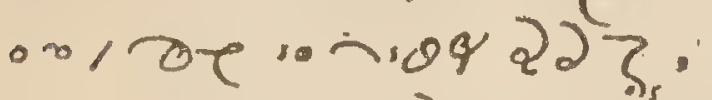

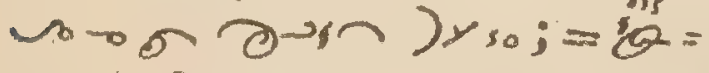

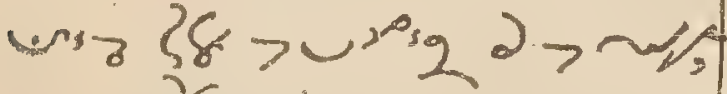

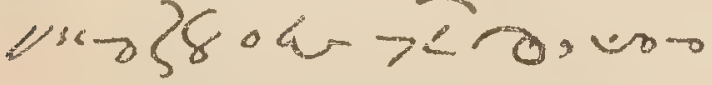
ok" $-85 ? 38<\frac{2}{2} 0<9$. on ans; y.9um? ork. an, end.
Ave. aro $8 \mathrm{C}$. गक्ष 8, bun 0 . sfe,, 0$) 0$; soolo 01

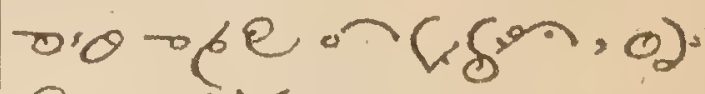
" 0 क 6 , then "6.3 ar $\because \int 8 \circ 9 \phi-296$,

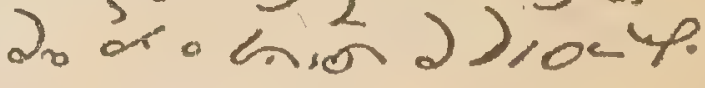
on, un.

credo. oderd $\cup$.

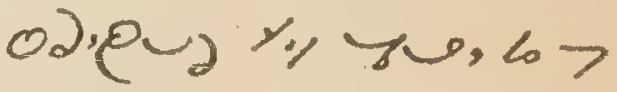
D, 3, OCon sor

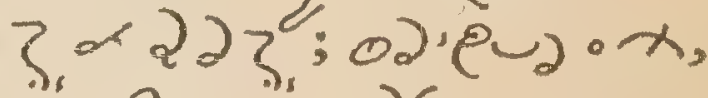

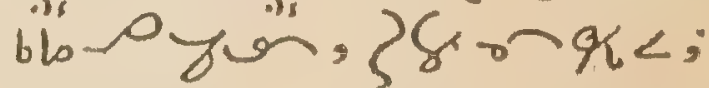
उ.बकर, pintor

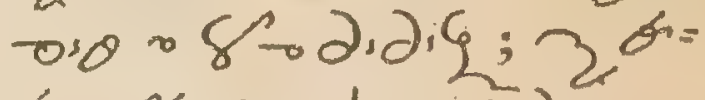

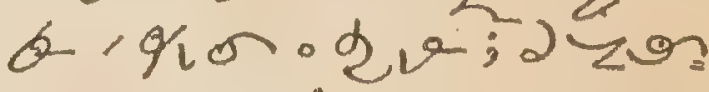
(ब) re b, " Hon, is पton; 2 on orn; 2

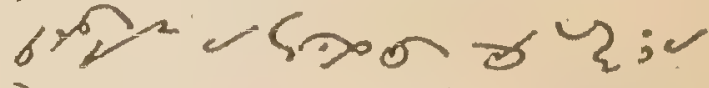
2on arst?, पcoond 几.ơn y.jylus 9a:03以0<2 $9 \xi$ - bution. =02.eva so widorobonsug;

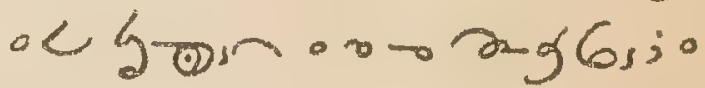
$<291 \circ a->e_{3} \circ$ , on ob:0, $-2,0<-18=$

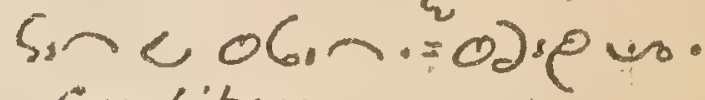
confiteor. masuge . G. = sorosyeg or\% lo

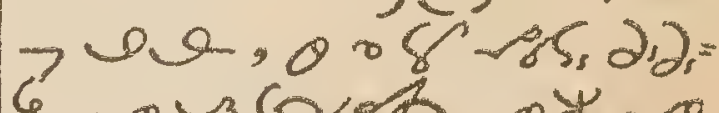

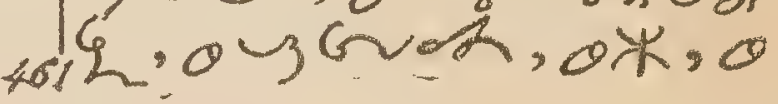


$y / 3 \sqrt{3}, 0$ - 62 - 0 $72.96,000 \rightarrow 30$,

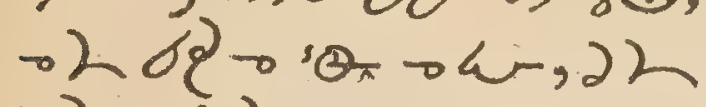

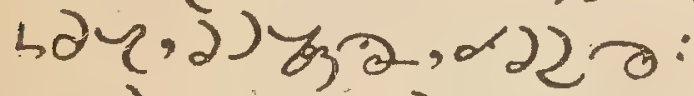

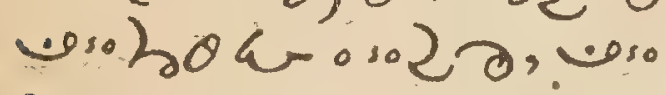
how-0s.2 2 , wrozo

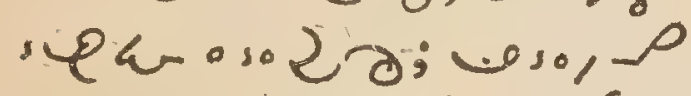

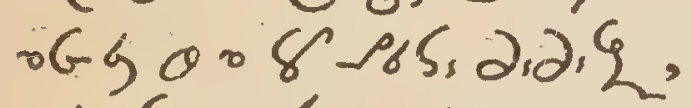
ous ch, o*, ong उस 38,0 ope, 007

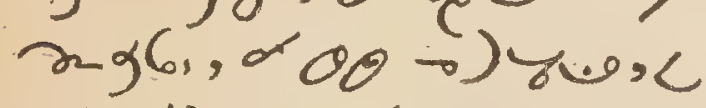
a $52 x 0^{\circ}$.

Misereatur. o $0<30$ =0

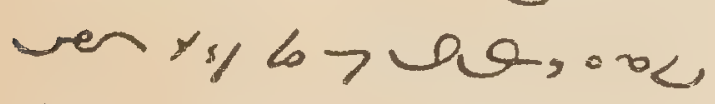
Vicin $0 k-><\partial, 0<$

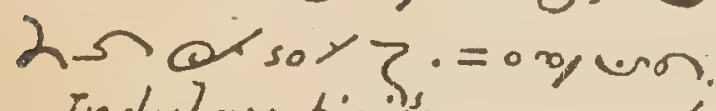
Indulgentiaim. or<sos= ven, oL Usun, o< gin

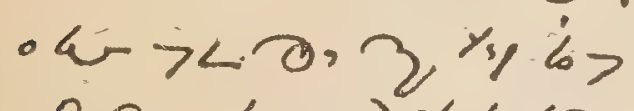
la, $6>)^{9} 9 \mathrm{dm}=$ - oun.

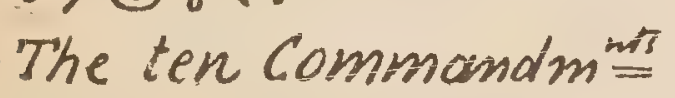
$992-73$ का $1 \%$

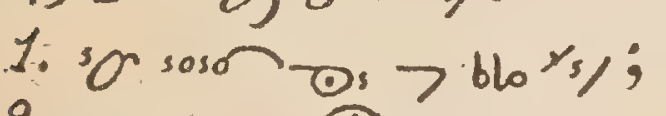

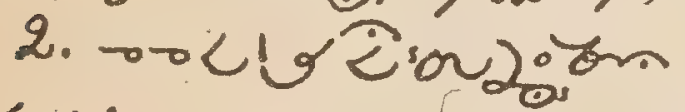
$>3 ;$

3. osanthoserson:

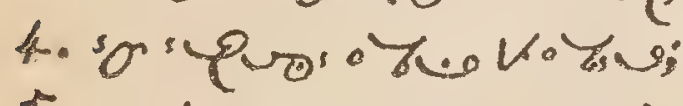

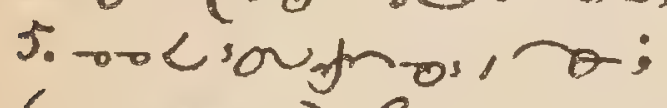
6.0060 are \%. -oba 20;

8. $\rightarrow$ क क б;

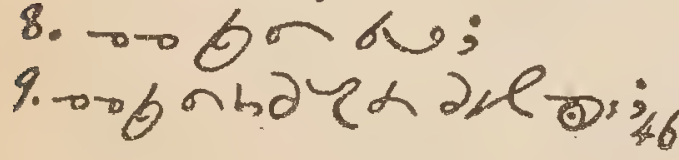

10. -0.60isy 20. Precepts of the Church. sie- bुan $\mathrm{L}$. 1. $\rightarrow$ Niter sur; 2. s.pnd d vor. कद

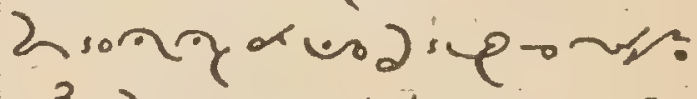

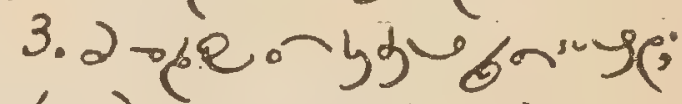
4. abe onjw, d L, 6r) गुर $\rightarrow$

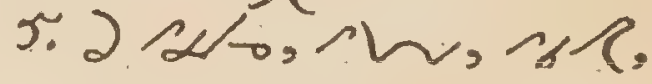
b० ) ग, ;

6. 2 टnation ${ }^{2}$ iq $>2$.

Seven sacraments. $x$

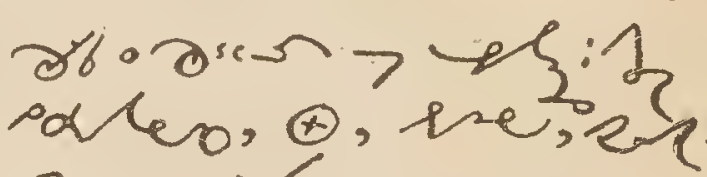
20,9,

Act of Faith. on $(0)=$ pue of a od, on. $=02.0$ os $\%, 3.2$ re - o $>$ 2ag6, or so 5 ; 2 - 2 ce. $\alpha>2.96 \%$ or $\Lambda \%_{0} \beta_{1} ; y$. "

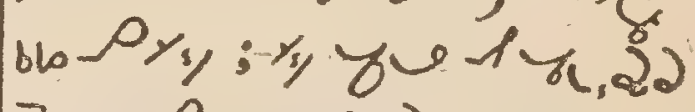
T. . Pin tha Len = (a) L suevosnoun

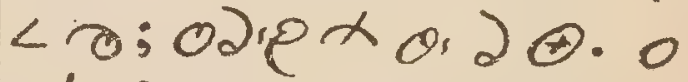

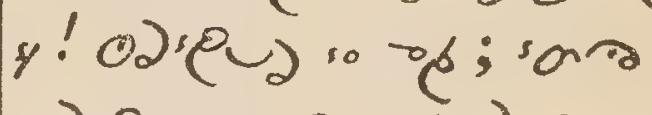
ode 2 - 6 . $100 \cdot$ वर्रe $2,-85,0$ 
MANUAL

3

contrition. L.of Co.

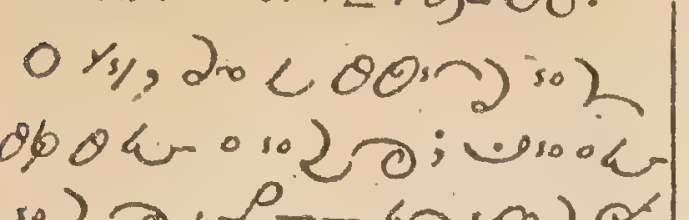

- soरे, porgara

sorz; wsookmsos

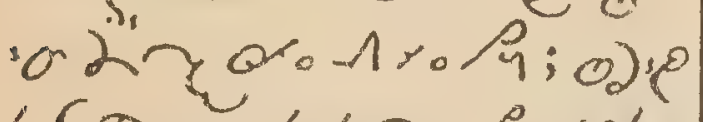

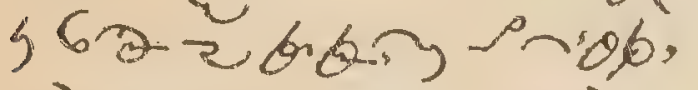

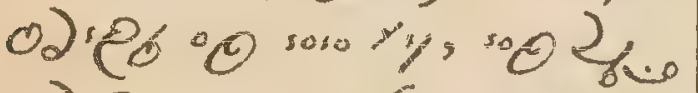

o).

u; 200< (19-10) :0

$2,06<-6$ - 50 som

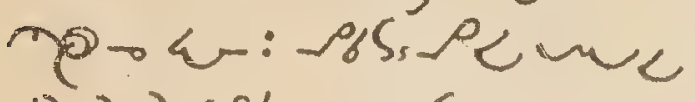

करे 2:06<-6 o

LैP.

Angelus. I. vo $y \circ 6$.

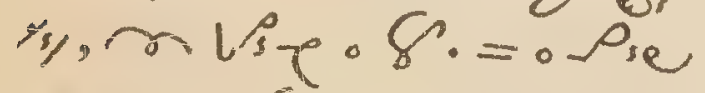

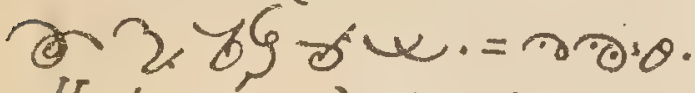

II. $6,25-300$ ars. $=00$

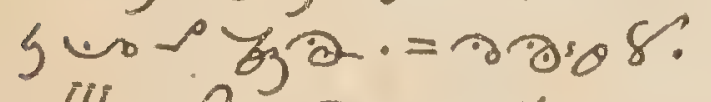

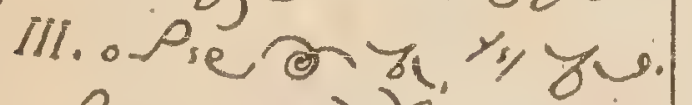

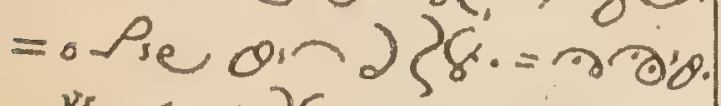

X. $6538,0.00$ y.

$y_{y}=0<$ un o c nork,

1 P.obgr alis.

oremus. 0 \%s, $\theta$ rom so

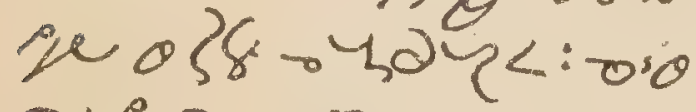

risonerome

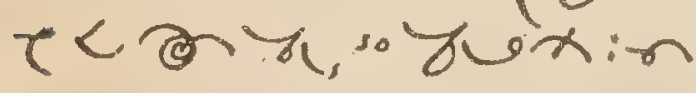

sone bro. 2, der

गpgore 5 n, $0<06,02$

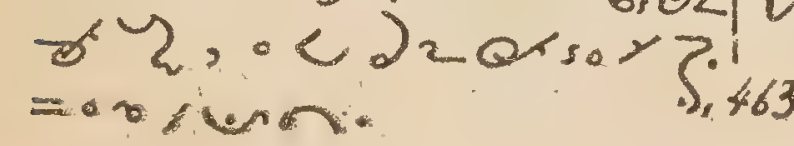

Gloria Patri. fo $\sigma^{\circ}=$ w, roon, unc zer, ot Pbs, $00<$ lin a $<$

Sa $:\}^{\circ}$

Sub (uum. os )s ofs. rog< or $0<$ sa 0. of 5 , 0 soo o tis an $y_{3}$;

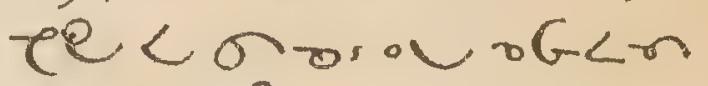
$\phi\left\{<; \rho_{0}\langle q-q)\right.$

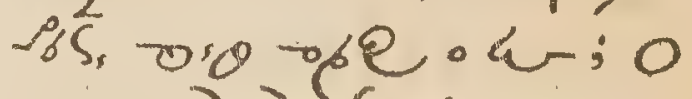

${ }^{30} 0-\partial . \partial, \zeta \omega>0<>$ QQ ) Y so 3 .

Before meals:" o \%, woo $38 \mathrm{u}$ so >usige sakn: - so $\cup$ oG $\angle$ o $0 \backsim v$ $\sim q l<x$.

After meals. $0 " \mathrm{l}\rangle$

- lo va, qa 6.2

ope o re sola gi; ;

gra $\sigma_{s}$ so zer o 2 pig.

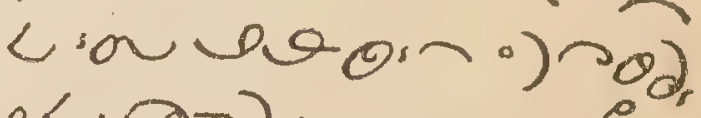
$0\langle\partial-\partial>$ oros. 好公.

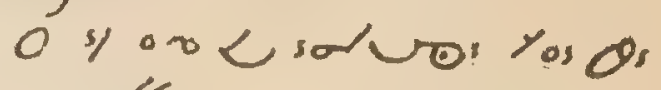

2)' $/ \xi,<$, 2 < orio

$\times 3$.

Form of Baptism.

N... ory $^{\circ} \mathrm{O}$ )

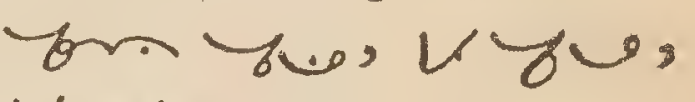


Morning Prayers.

*27ongovzo, Vu. 00, un.

veni sancte. ONw, $0.0038,0<6 u n$.

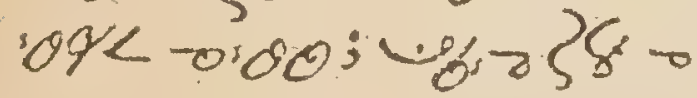
rogk, oc ancene $r s \%$

Adoration. onessic oxy, oc sorove $3=06$ $>\infty, 1>>242 \sqrt{2}, 169$

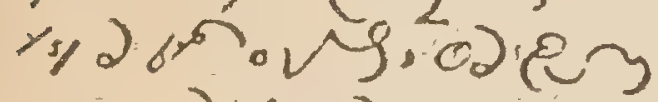

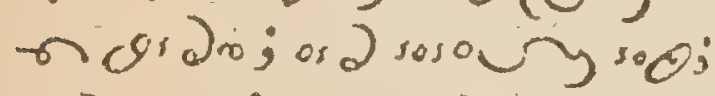
62n-1002 50208600: - $\partial$ बrरु - be cus.

Thanksgiving. one ree $x_{1 /}$ oर $\operatorname{sen} 3 \alpha<0$; $=0$ 人 $\pi, 0,2 \times 6=2 \%=$

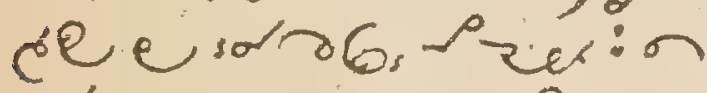

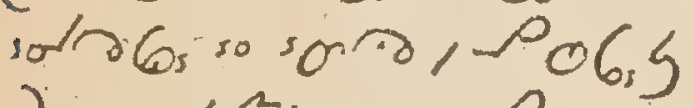

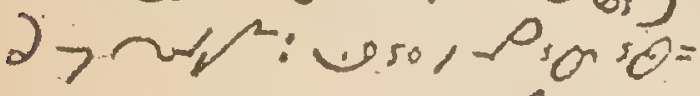
wing op: $>$ witio of 2

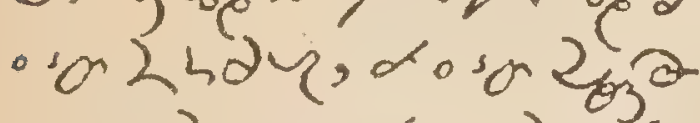

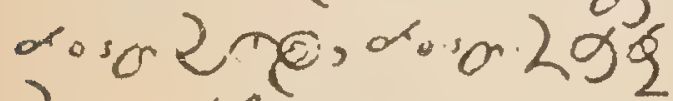

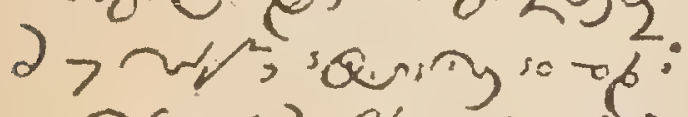
ग6, $02 \%$, ०र गुर usengocengy-p os. $2>$ wh.

Resolution oredoe.

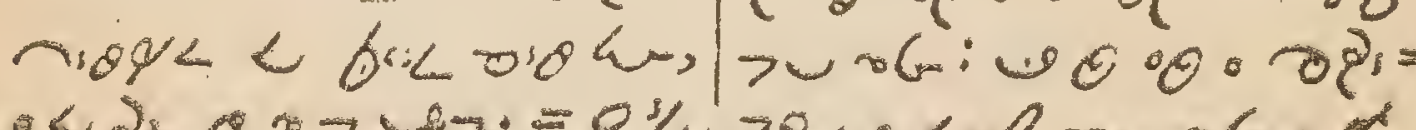

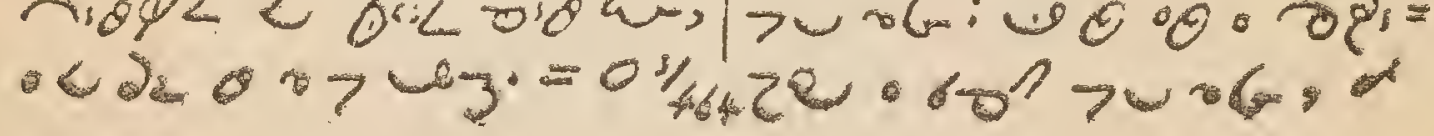

fox, as.2:095

(a) uns) $0.2>$ w/s

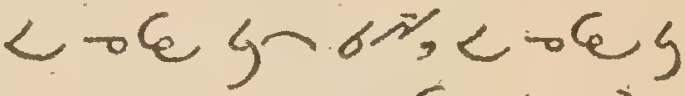

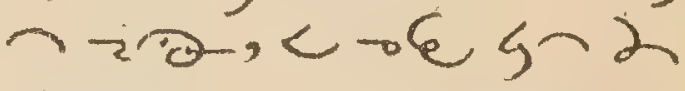

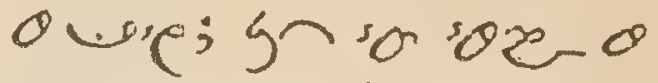
r, og son jggdmo 29): Ua.0. $8=$ \$areb or

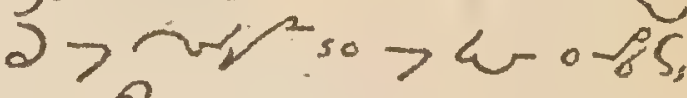
- Pre: la ex.20\%

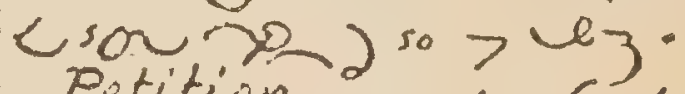
Petition. : $0<\cdot G<$ ary<son gen: $=02$ 96,9 . won. 299 :

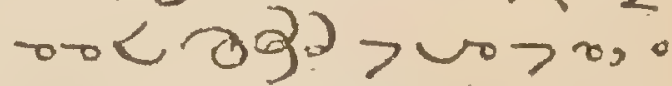
reन0: 0 - $02 \%:-1$ ० 56.05 so bu o obe anog so 20 ofe. $0>\mathrm{U}_{3}{ }^{\circ} \mathrm{C}$ sou 1 - 2 so 2060 o 6 $2 \sin 29 \theta 2>$ wद ion dis e 220 an. 20.

Pater. 38 youmag. Ave. rrosog. credo. 0Jigu. Seven Sacrix bee or. Act of Faith. ore ode $\left.\boldsymbol{r}=0,1,0.0 \cdot-n_{0}\right)=$

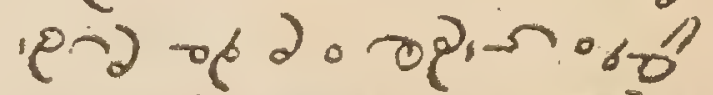

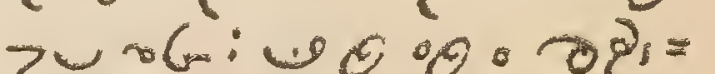


Pbs por osin solzo. Hope. a c arueso r.ogl = os/gen - $x$ yon 2 is, $1,0=$ a. 250\%<soubsen 6. - L- oso 2 or ocsor

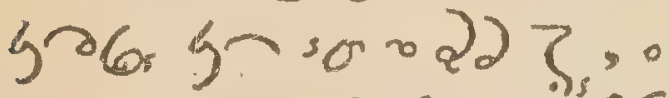

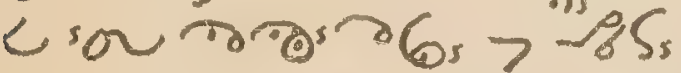
) ysor $3 ; 00$ r lim

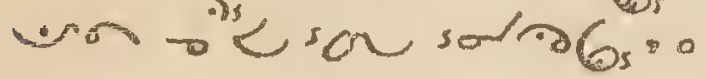
$<-6$ - $<$ un $<$ के 10 $10 \%$.

Love. oncienc: = $0 "$ iens:0 on ope - Liog, n bolo ofe vo, oOrapridse=

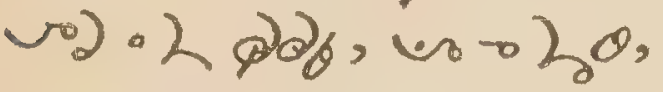
riens so.

Contrition. o o b b

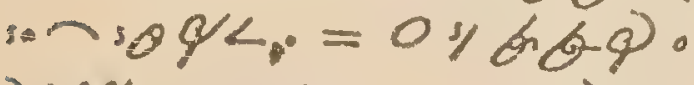
25040 - p62 0 w. 5020 . 0 - 5 का s. $2504<$ -

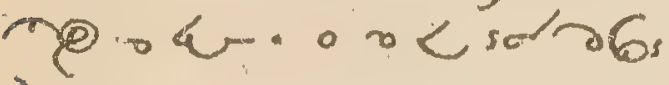
20.

Invocation... $0.8,20$ in yos 8$\}$

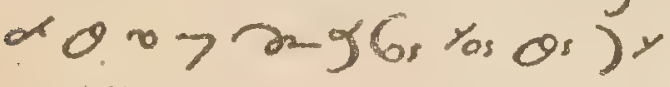
so 2 र.

To the B.Virgin. Or \&, tuan ys d bolys:

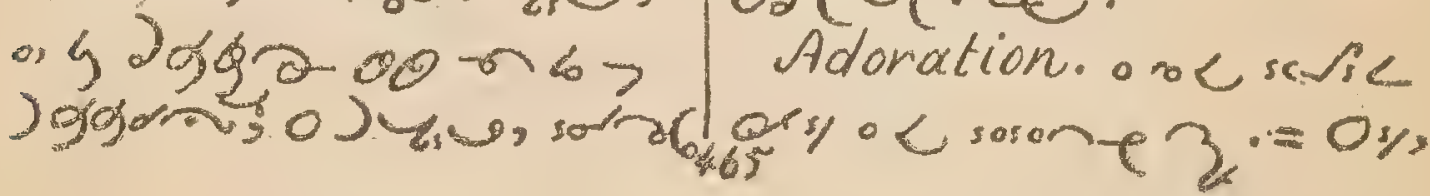

$-2098,60 \sin 602$ $100-263$ - 0 - suso, $2>$ wis

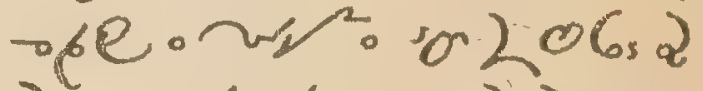
$2\}_{1}$ do hion $2 \partial b$ $\varphi:$

To the Guardian Angel. 0 so $\rightarrow 0$ on 0 ) $x=$ sor 6 , ar soy?, 000

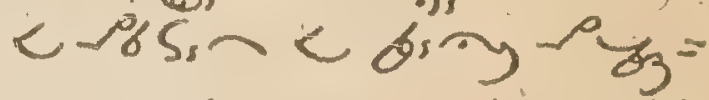

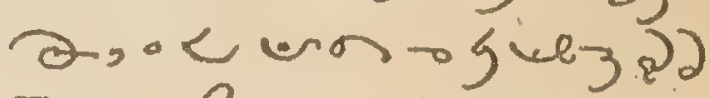
3, , proc won o e

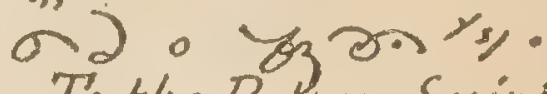
1 $\left.-0 x>22 g \sigma_{s}\right)$ >so

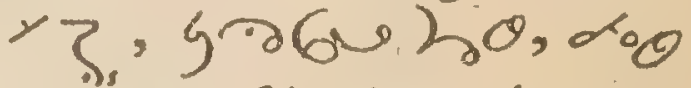

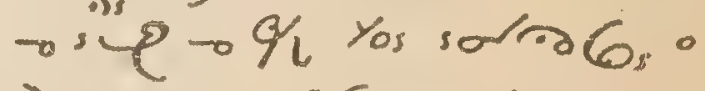
)ช, $8\{\sim 6 \partial>$ vis - $6 \sim_{5} \sim 6$ ors, $<$ sou soso $=$

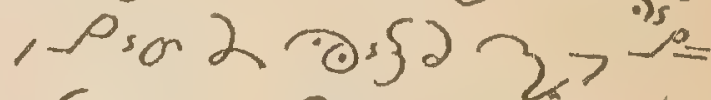

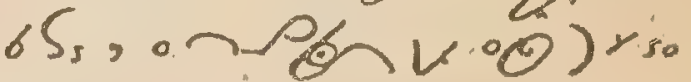
yz. o v/un. Angelus. Non. subtuum. os Jides. Might Prayers. io Jym tro, w. Veni Sancte. Oy wor Acts of Faith, etc.. ore odeve.c. Adoration. one salic

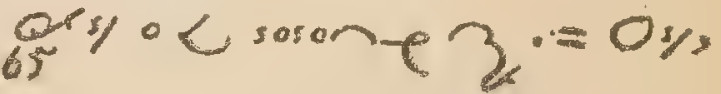
To the Patron Saint. o

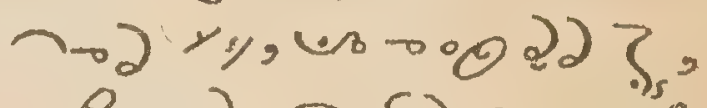




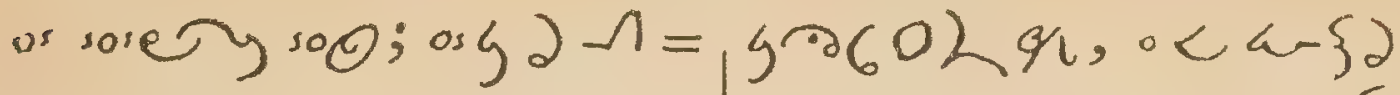

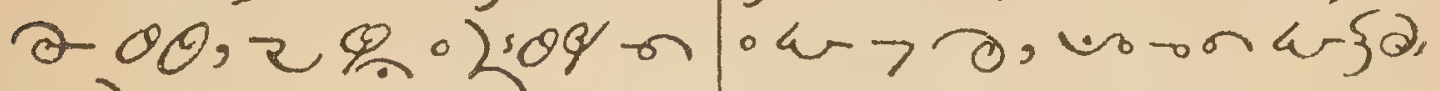

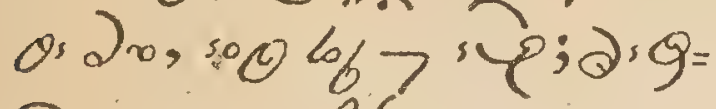
ग. j; a : 20800, n แ> 9 d o. 0 .

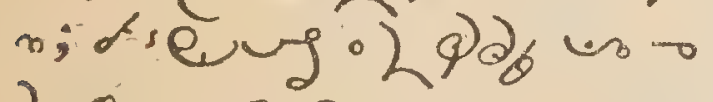
ho z seng 500 .

Thanksgiving. one $\mathrm{GC}$ ys a operesolven. = osgd or $(2,029 \%$ obe esalabs?

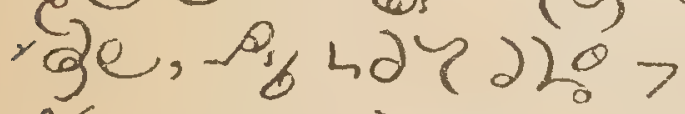

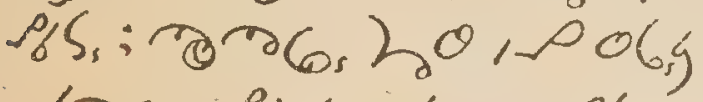

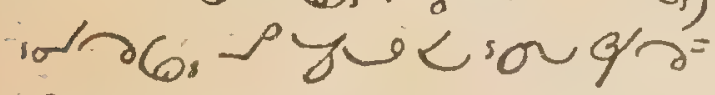
$6 ;$; soe 2 r $\pi 6 ; 0 ; 0$, ope of prex. vob2002\%, 0 eदेग of गe sol 6 6.' jल6, $12-007296_{s}$

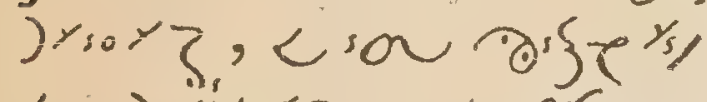
6>) ğgorn, z 28 s न दुरा -

Prayer for light. one sue ${ }^{1 / y<}<$ son gen ${ }^{\circ}<0=$ eoint०u-

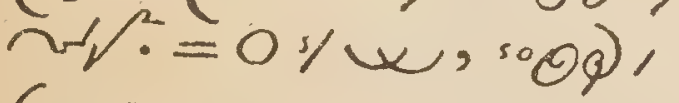
cose on so\% ope.0 $>$ 2. $96 ;$; esg o so $20 \theta$

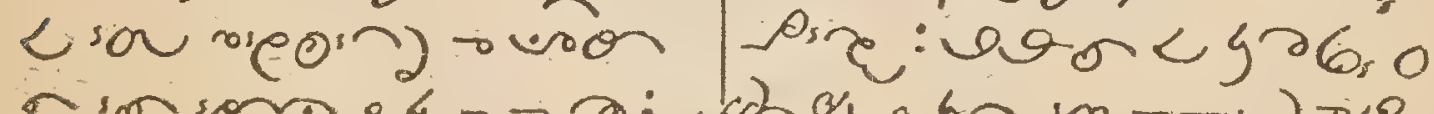

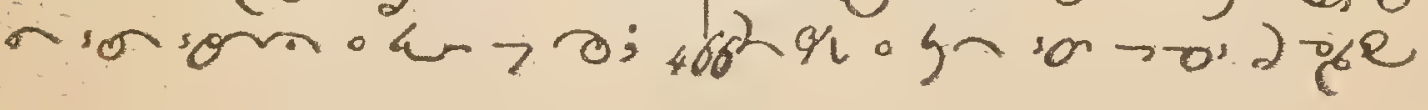

on pos as?.

Examen. onesye。 $u->\left\langle\partial \partial>-v^{2} .\right.$. contrition. b2>029l - voonevere च

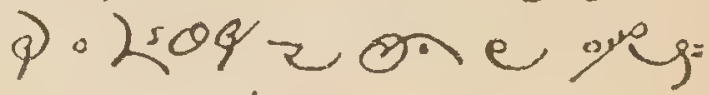

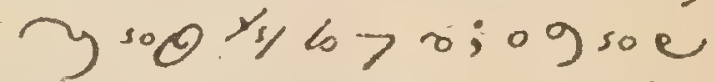

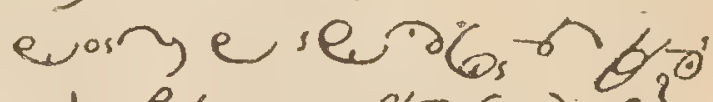

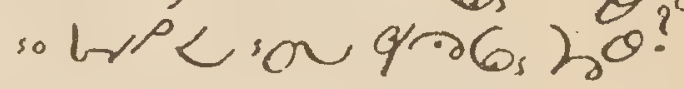
odre $q, \log >) 13$, wo

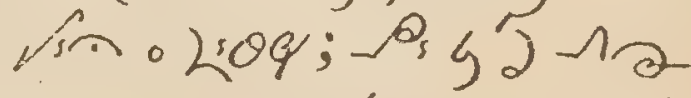
00,0 <isolato, oL $q$

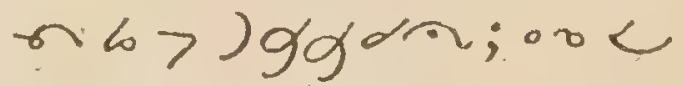
पiक $6,1,0.030)$ o.go ex pen, ably,

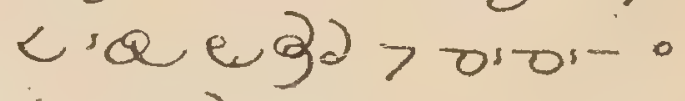
¿- oso 20 .

Firm Purpose. or $<Q=$

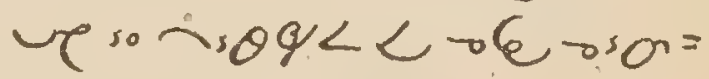
कर

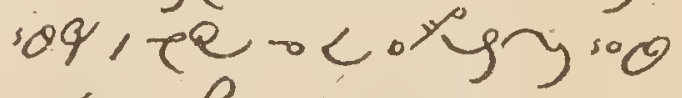

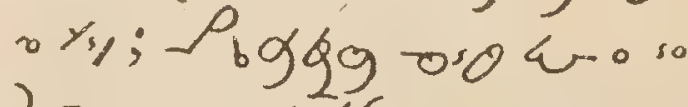
2 o. sor a gfing ovion

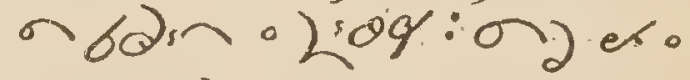

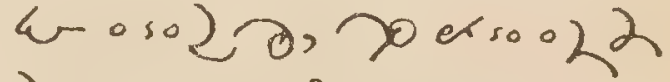

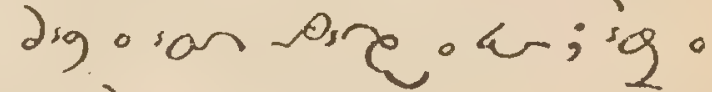

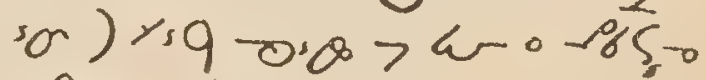
os 
- "n

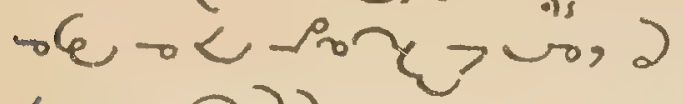

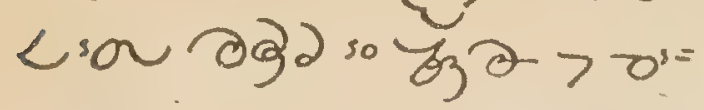
$0^{:-}$.

Confiteor. sososugq ${ }^{\circ} \mathrm{G}$.

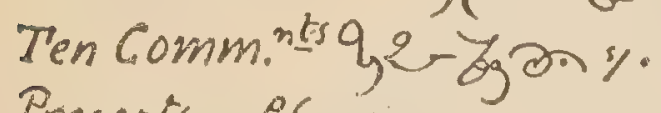

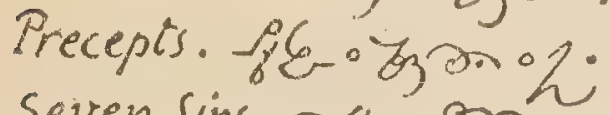
seven sins: ar yomo ob. e. an $>0$ : so 7 sog: :

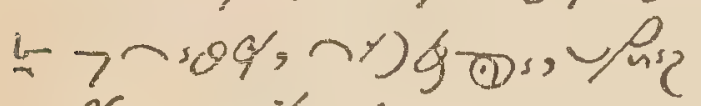
YS 3 , oin, Nor.

Night offering. $=00<$ Sq2 $<$ ars, कro wo 80

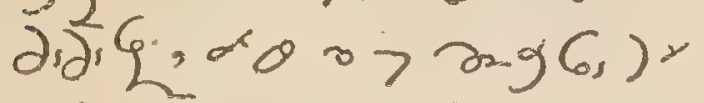
sox $\zeta_{i}=$ wo-o $02 \%$, oso ( o ) $)^{5} \partial>\sim$, 。<

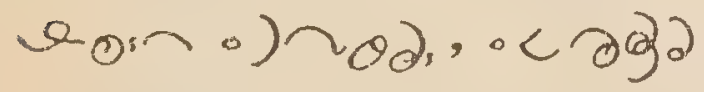
s.

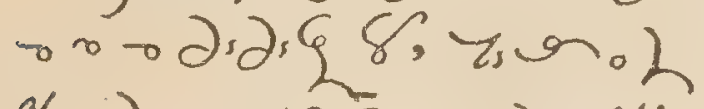

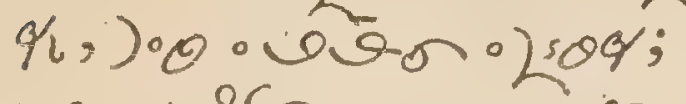
so $\theta+8\{26,-0-7$ in,

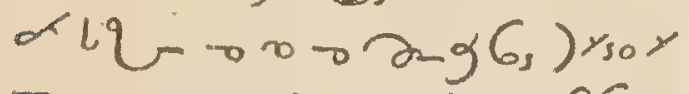
$3, \times 6 n 600 \%,-8 \xi n=$ 6้ $\partial>n-2$,

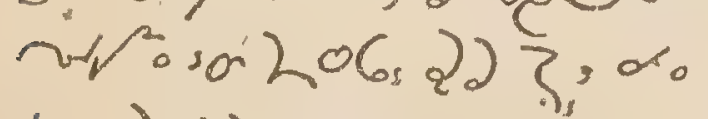

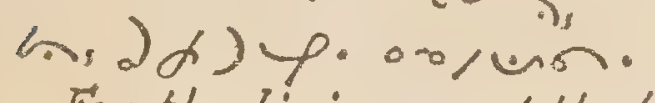
For the living and the dedd. or L gque on $9 b<$, 。 06 a

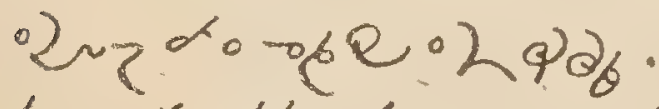
Sor tosgh, yos om on, yos

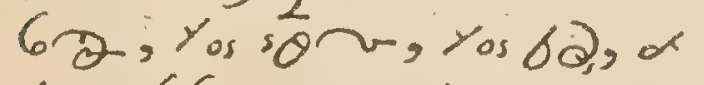
ros $p 6$ G. o o 2 os ros ros

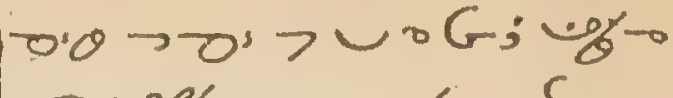
- neg 0 - $0<$. 256 ,

$0 \% 6>0<) g^{d m,}$

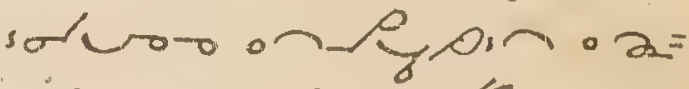
(6) ros os 2$)$ s. $\}$; o. 》o, on bn, d. $0.80, \mathrm{cot} 0$ ) 9 )

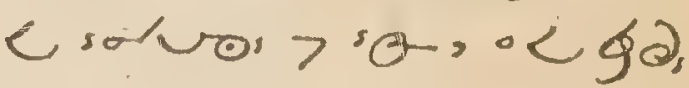

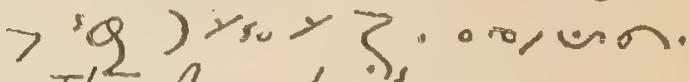
The Angelus. on.

subluum. os jools.

Morning offering. Q/a 6 , "0 2 , $2,8 \xi 2,2>$

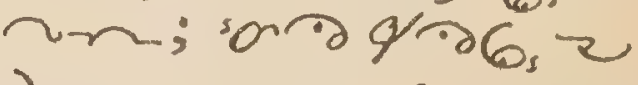
$2 \curvearrowleft \sigma_{s}$ so wdr. squs

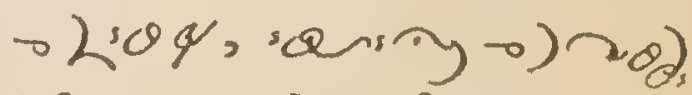

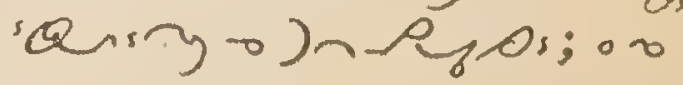
< $90^{\circ}(0,0): 0 \%$

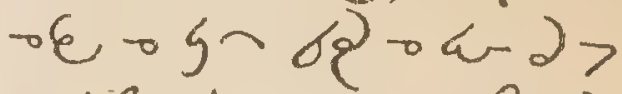

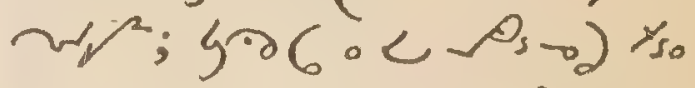

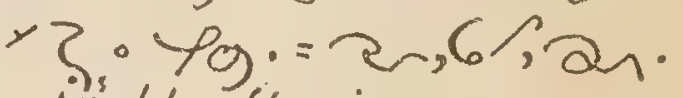
Night offering. $0 \mathrm{ys}$ so $\mathrm{O}_{\mathrm{O}}$

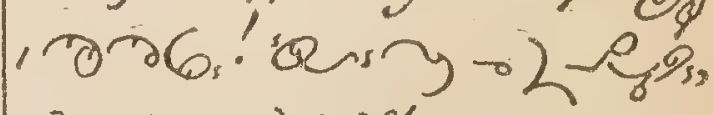
Qus? o L sod, sQus?

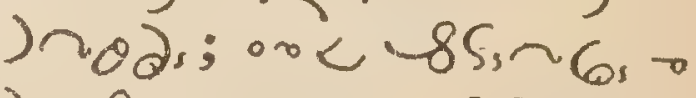

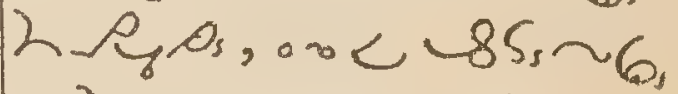
- 2.00, ore $85,26_{s}$ -) nods, < u ga f, - <-e o 5 Y $2 k$; S ( $){ }^{\circ} 0>>99$. on 
Preparation for Confession.

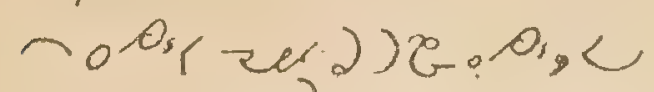
ce $0<$ ógor

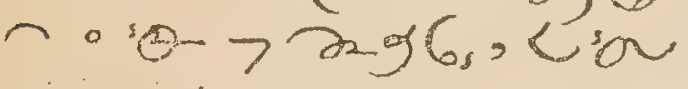
ब) oup or $09 \%$ Q $\alpha>9$ o....

shov, eson e o-p - Ln>920,0<sonsu $+\mathrm{s}<\mathrm{on} \operatorname{siceg} . .$. bow, e ne bis on $\sigma\left\{\right.$ arolo $\sigma^{2}>2 y$. -Govoroy, 20\%= $9 / 9,<$ on $30 \%<$ in

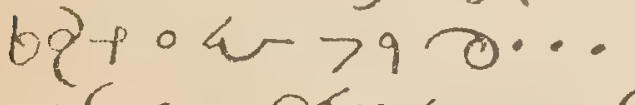
oGoworyeson Gs= is on:og/q....

Prayer for light.

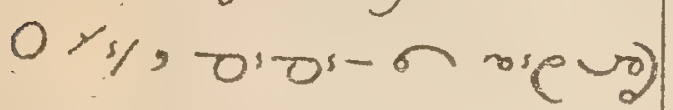
r none one ring "ब)

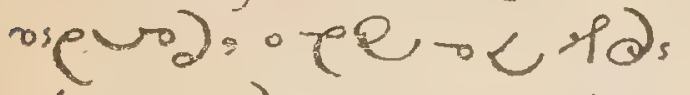
- L a so 20, to<un o<prsog; do L s ('on'os obe o h-

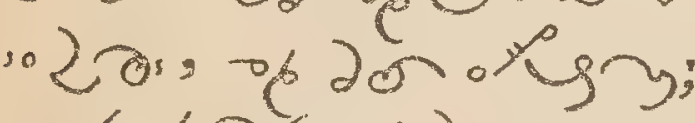

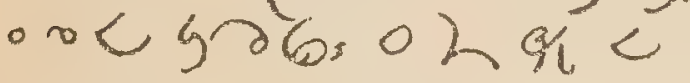

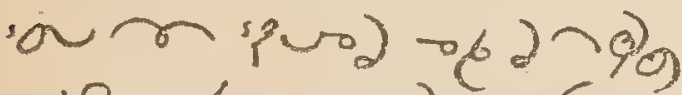

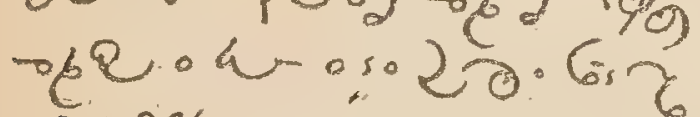

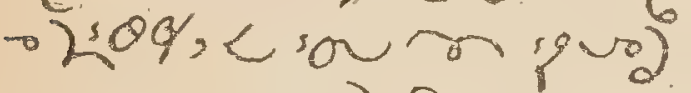
-von 0 of 4 z

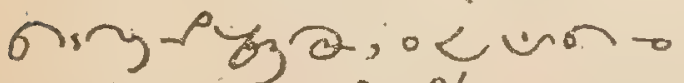

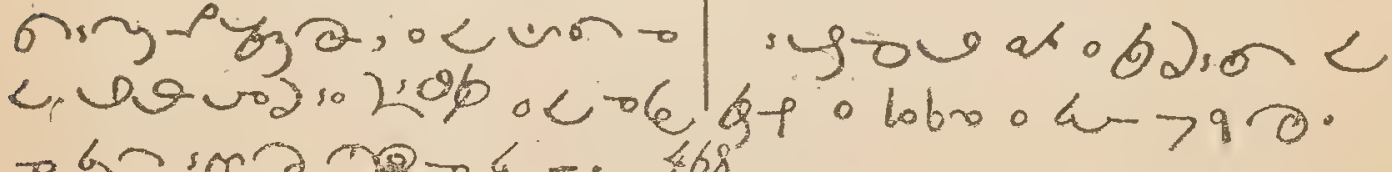
- grsorarate 468

$$
\text { =rsion... }
$$

yणu०620>6=

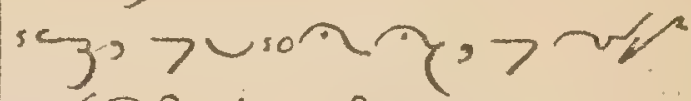
का -f fow

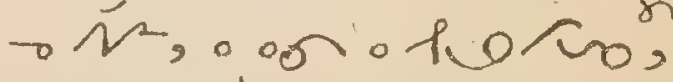
. of $\circ h_{3} . .$. ifowowon< dis $\rightarrow$ nsane, Dontor, o tR...

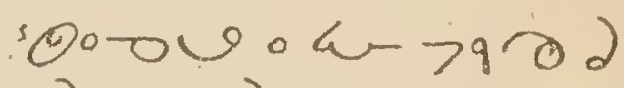
2य( $9,2 n \operatorname{seg} / 9, \partial>$

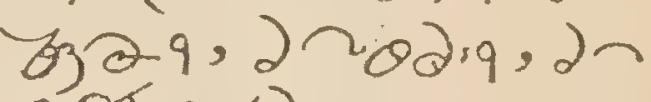

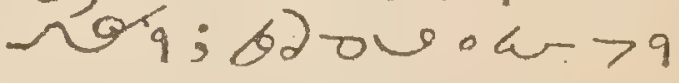
o 2 ob 20.9 क <sseq...

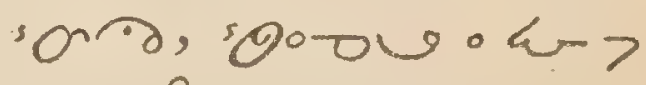

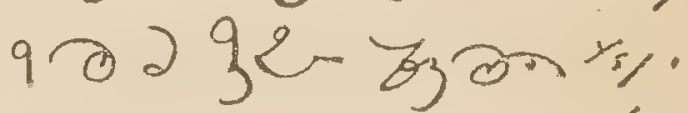
$={ }^{\circ}$ sosaves $>$ blo $Y_{s}$. "On'a, so-owoun

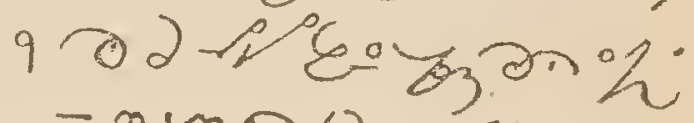
=nongtos 4... "Ord sooton? $10 \partial$ वर पर 30 कर $0<->0$

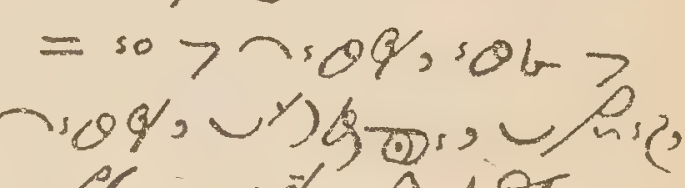
भ $53,6 \pi$, حeir.

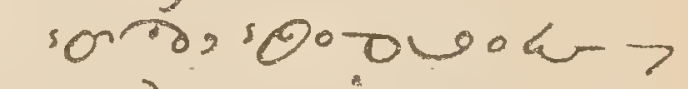
id juon cmeves yst... 
on ow ver abe o $4>90 / 900<\rho=$

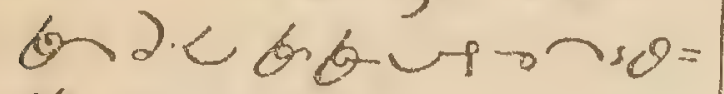
$99 .$.

Orotow a onsogac son 6 क o a s o o chano $289 / 9<$

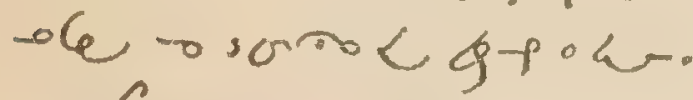
sक्sov....

row oys, son

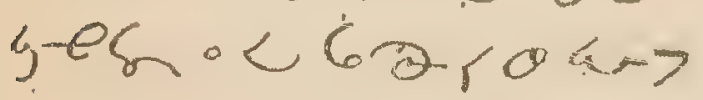
iro, olverpong. g/a $<$ - of $\rightarrow$ som : an....

o (row ors $<$ son $)_{3}=$ os to s 10 .

Prayer for Contrition.

$=0.4,0$ s sess G. (OOn) solsog - so $20 ; 0 \$$ n. 2. $\omega$ - so 2क;:

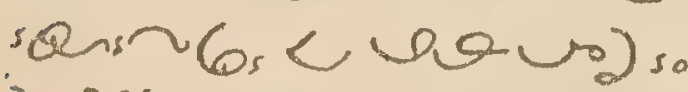
$2804<-6$ - 52 sor: mo. 04 .

$=0$ o

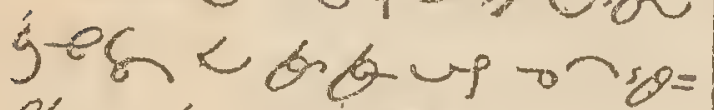
$99 a-7.2$.

0 s.

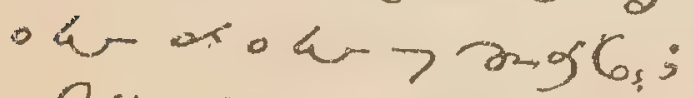

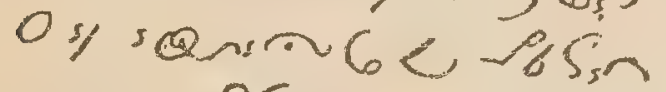
gra 859 a $<$ कर

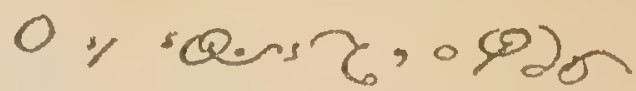
, an, o<s?, $5>$ r.q $00, \cos 526$.

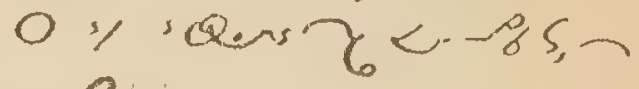
$(-8$ क्षin 2 : 0 s, scens $6<$ - Bs, . (n). so 250000 , o o ope o ang6,

ore sa) za arror?. - inon a ods mose

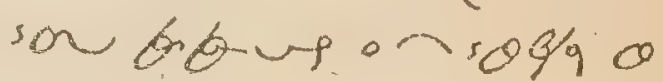
L->9 as o son

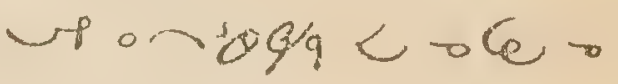
son < g- of First Consideralion. odip o yso Y $S_{i} ; 0$ :

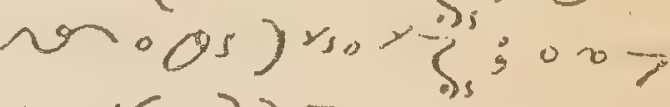
$20 \cos 22\}_{s} \cdot \operatorname{Ton} y=$

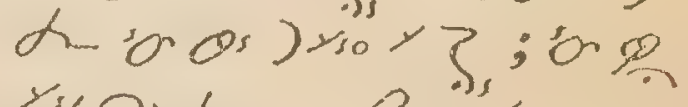
ry got, is orden mor - Y, dust उor $\rightarrow 0$ orpur $<$. Uबis ys son scensy ysy

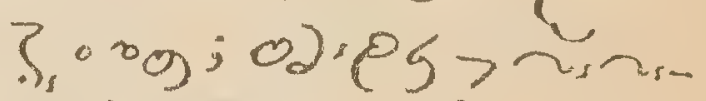

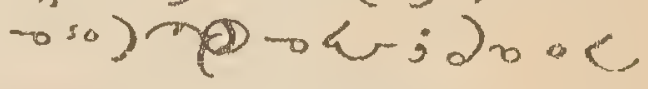
cos 0 : 209206

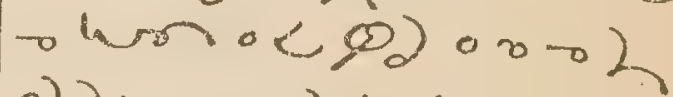

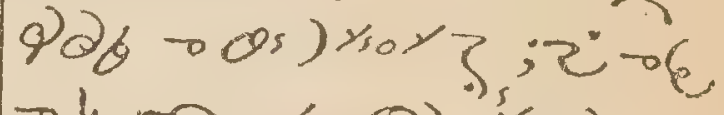

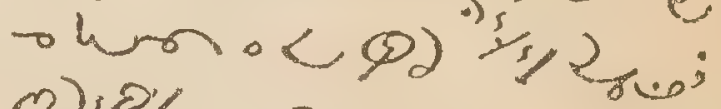
0) $P$ 'S $>$ या - 201)

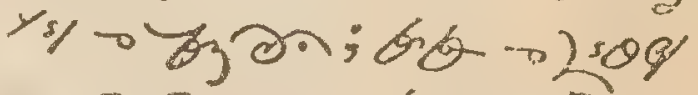

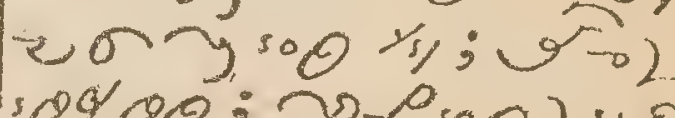
$40900 ; 301002=0$; 


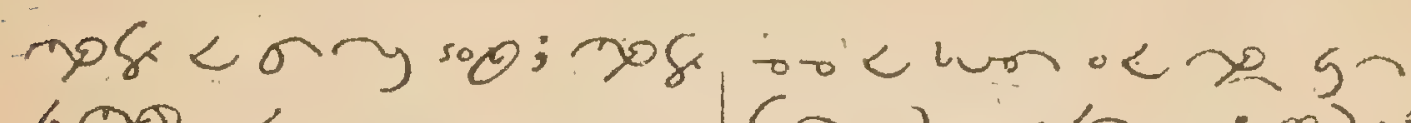

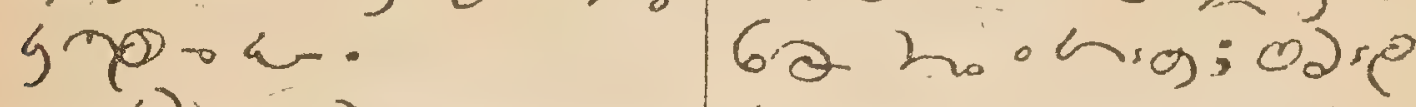

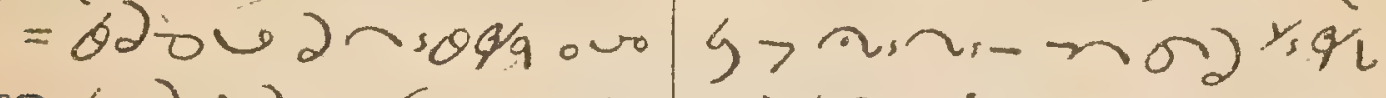

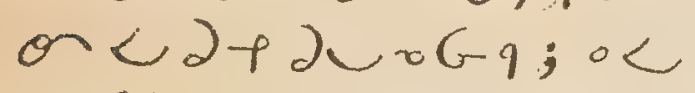
$\omega-\xi+0 \omega>90,0<$

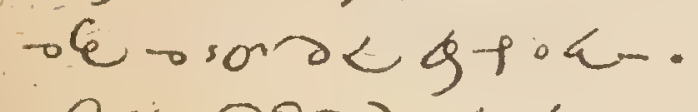
$0 \mathrm{~s}, \mathrm{n}^{\circ} \mathrm{d}$ a 0 k - so 20, sogallate

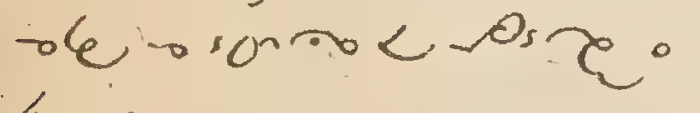
a.

is e bofdoch dok $>2.96 .5$

85 , a : 0 - $852 \circ 2: 09$, $\angle \rightarrow 6-5$ sond 20 ar;

Qeason 8 (1)

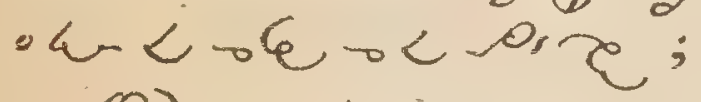
- 9 don.ari, 'qu.so $3 \times 900<$ son jar $^{\circ}$; $85_{5}$ o son $1 \mathrm{nol}$ ) $\operatorname{so\theta } \theta 0, \alpha \theta$ ote $\cdot \partial_{2}=$ $96, ;$

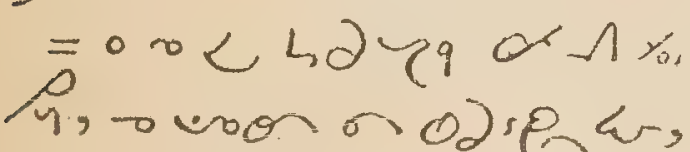
uson bbup on:09/9 oin> $>9,0<$ sa $v$. ayp sonsolq <-

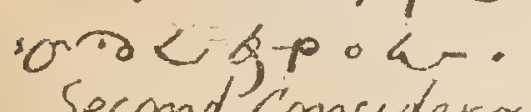

Second Consideration.

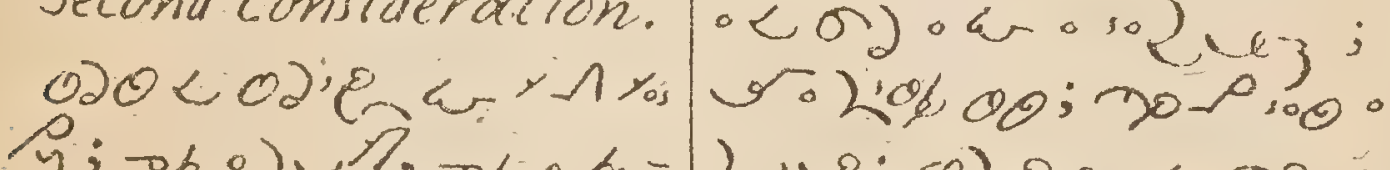

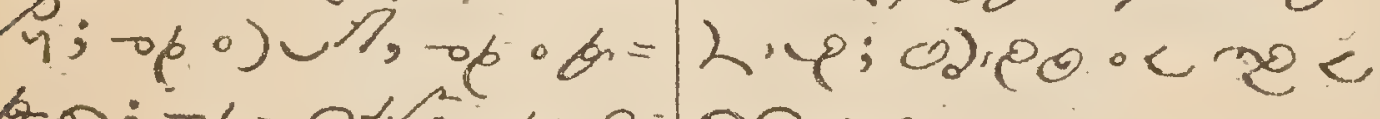

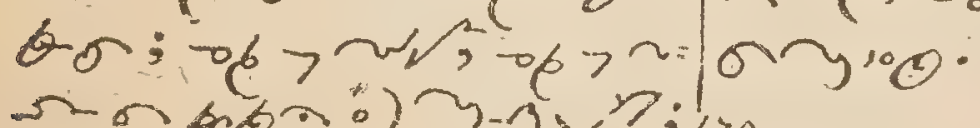

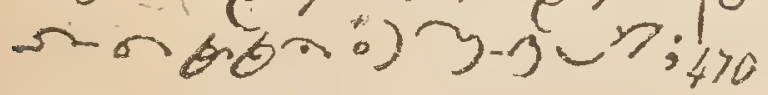


$=62$ ow $2 r i c 9 / 9$ vion

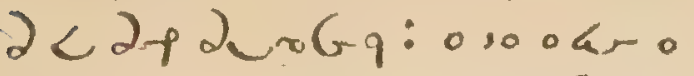

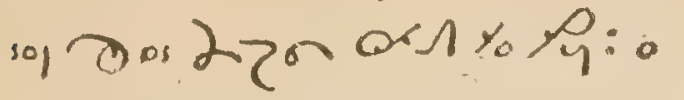
oc ongnt on.9990a - sol o, o< havions

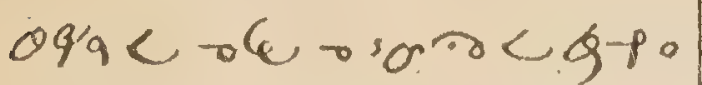
u.

$=0 \mathrm{~s}$, pord a.k.

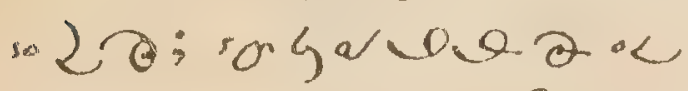
-kanden sine.

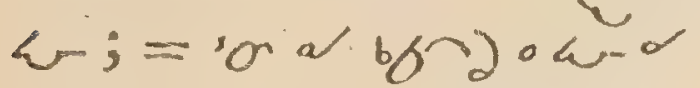
- $u r>2 m g C_{1} ;=$ Sor a o

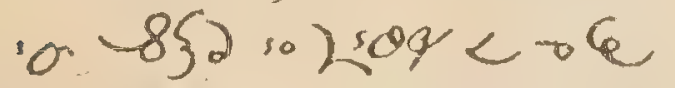

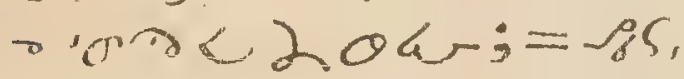

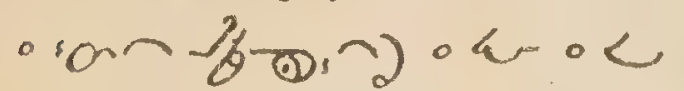
- - oर

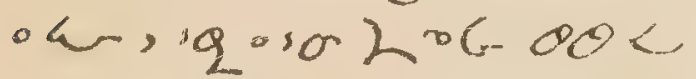
a sa6,; = 98 s, os a

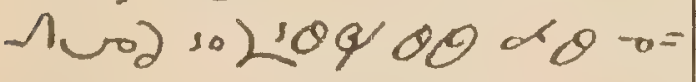
te. 20 g6.

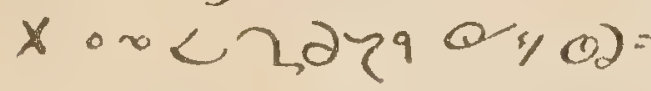
ip ods a , cia

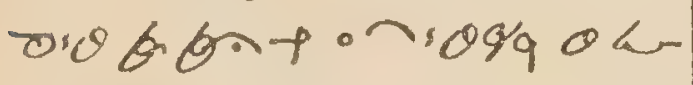
790 .

Third Consideration.

0 \% ode o odp la, soc soln $6,0,06,500$ sola 6.2 rig a obe. L.

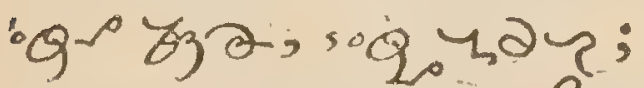

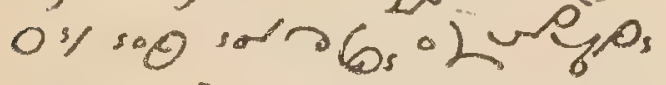

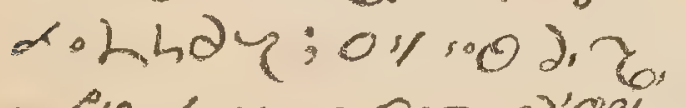
- Perion rom clog',
"0 26, orsor?, bf

Y 1 y aroso $2 \partial$, opese end ..) ing $<$ -

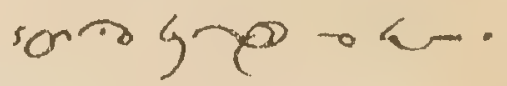

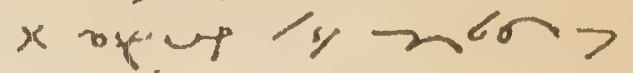
o, 1 , 0.9.;

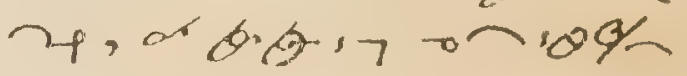
0, $6 \partial-9$ a $4>920,0<$ Den poriog/a < ve, - अ $2<$ \& x 70 orgok; ss,

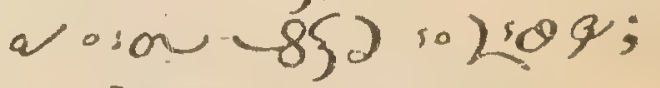
$=.2$ on 0$) \log 0$ ar, iq. on d 2 ; $=185$. sa bol so zog oso obe.4; = -85, osa

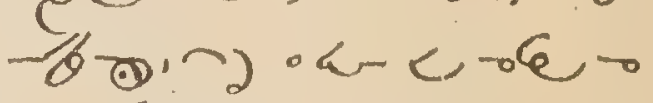
<.oire; = o p)on. $m, s \circ \operatorname{son}) \cup(\mathrm{ars}$,

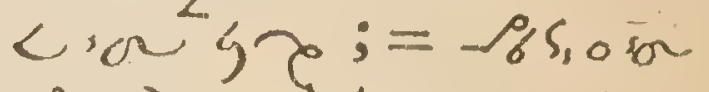
hara) so liog ars aro ofe o Dzg Gs. $x$ or a < $4,2 y 9 x$ -unor a $9 \&$ / $2^{\circ}$

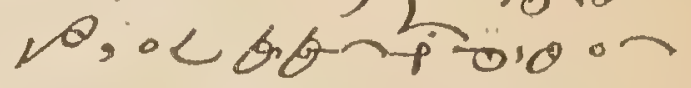
sq9 $\&$ ar $>9$. 0 . = Fourth consideration.

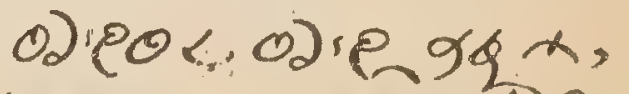
uno, zer, igr?: n, nufze, भiszon

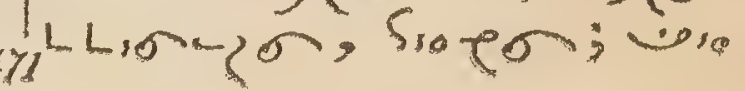


- w. - solo osdzen; 200 son $x$ - so) 20 -4; 0 大; w wo on10र तथ $1906 ; 68)_{6}, 9$

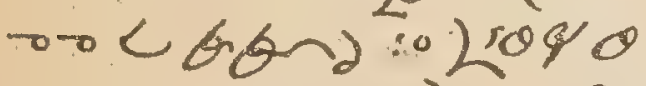
unsoरे; 69$), \rho_{0}$ <205 20.? 0 \%, co so a osoldo 3 m; egry smy 1290 ; ging preboriegry

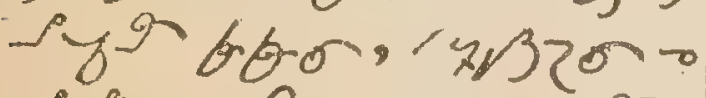
i-f, o on squms zon.

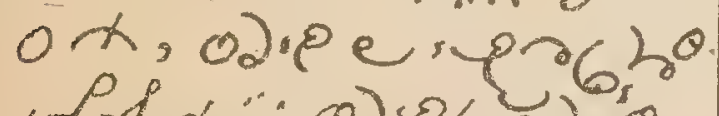
- $1 ; \alpha ; 0$; nor -2 L $b q^{\circ}+\varepsilon_{-}: \partial_{0}<$

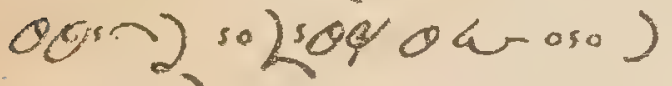
ez; 2 r.o< 0 2 ou.

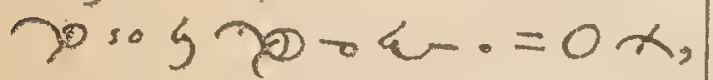
cosn rwon a g g) po: pewe revol

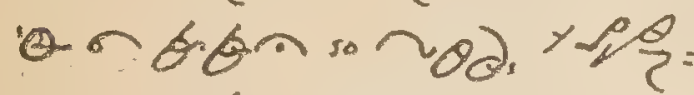

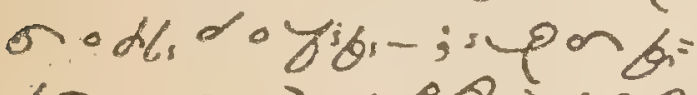

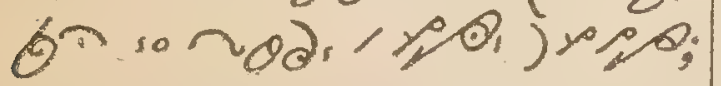

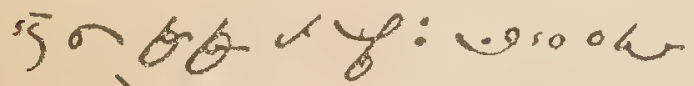
o sa $200 \mathrm{dr} ; 0$ ) 350 honin, 2 ga so 4 .

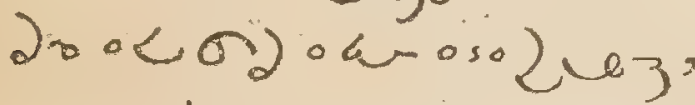

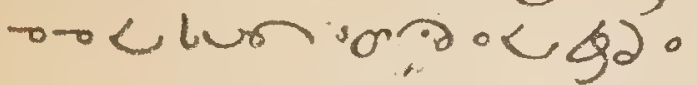
U; 'Qn'? 00 -209';

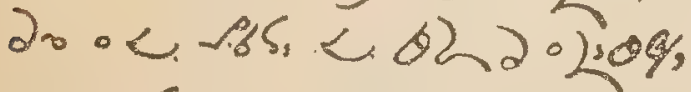

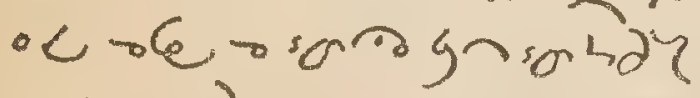
ou. 2a $y^{2}$ by, o osgo

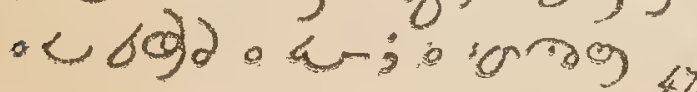

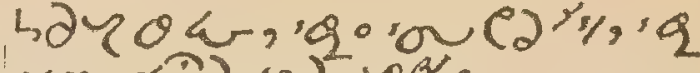
oroñ so Li, $0 \%$.

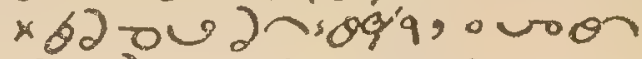

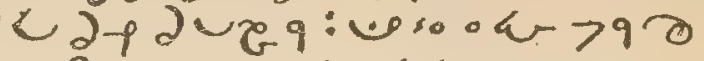

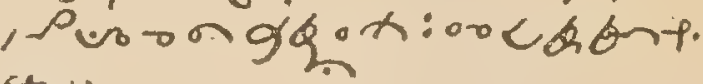
4 . sog severc

Betore Communion. T. Adoration. o v $<0$ 's? rox mas $) \theta=0$ s/ $x, 0$ (e) $n \operatorname{\theta s} \alpha$, soc. s/ Tu; of 2 o osos,

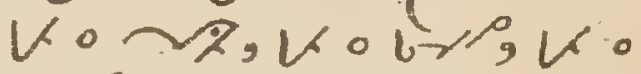
जyo: 0)हु non o a, o so n 68 oso - $\delta$ - $\partial . \partial . \xi$; so s) $x^{\circ}$

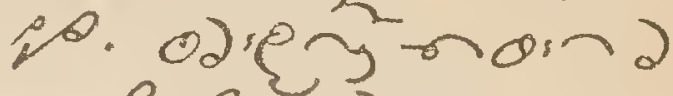
s. Wras: odsen n Qind so urs for ) re?

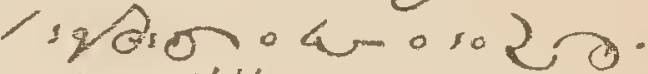
Humility. ore ne -0. $\theta$ or $\theta x .=0$ s $x$ 2400 ef: 20510 10.06 A $22 \mathrm{sog}_{3}, 0=$ $6>1,6,60$ soso; 206 $\xi>9 Q$, los $>$ Uoo; 20 so so $20 \rightarrow a-$ os 92 vese, os 52 b....

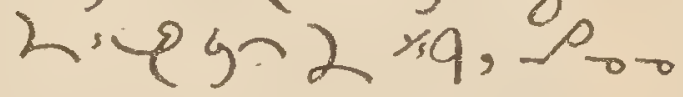
<oig as 99; 0 20.8 'ordar qri? wso, pos No 00, vi, $\rho_{2} 5$ 00 - $299: 00<5 \%$ $2 \%, 0046,0<, 99$ 
Contrition. on< $6 \partial<$ $\theta$ an $>0 .=0$ son $\rightarrow$

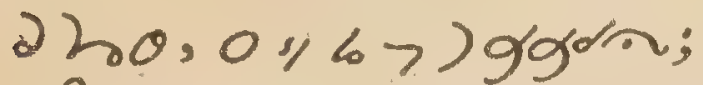
Pos bु 90.9. L09 nsene - gras.

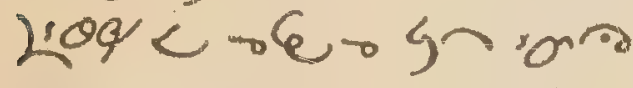
7o - an. oresald on $9 /$, numse

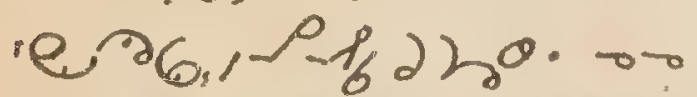
soxpe ofy d2s0\% w - ae...ver. - - ros roigrye; व.m.

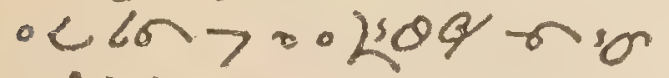
1230.

Love. ore conese= $r \circ x .=0 y x, 69$ e iev 6,30 - 6025,20 - ter orenso? <lunocecseryro

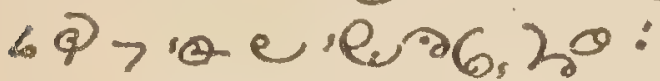
wro pionlens

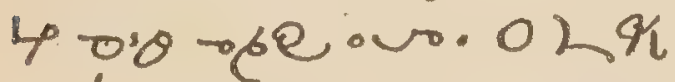

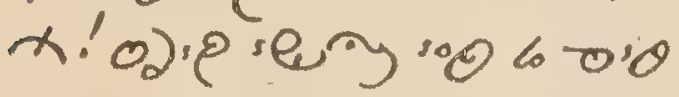
rebeno.

Desire. ancentor

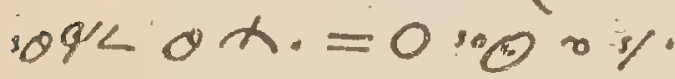
- g $\rho_{\text {so }}$ - $1220 ; \mathrm{urg}$

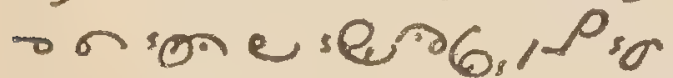
60. L L:OQ 00. 0.0 0 2. 0 sty. os lyry.

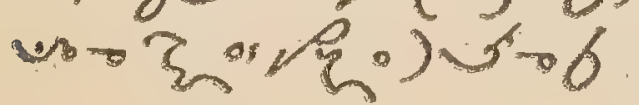

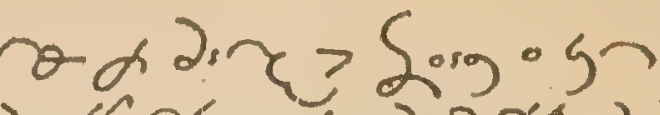

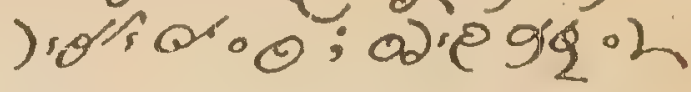
sor oce - g 200. - 8 , पi, on 201 yo, uga, furo. Li, 04 ox.

ard en:

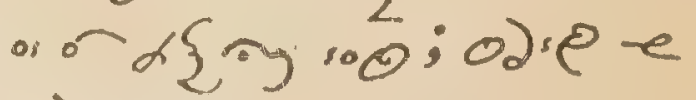
- L509 00; 0.0 r. "qon $\alpha$.

After Communion.

- o< saine ox. =0 qi $b>\langle\varphi, l\rangle$ saso, Ory z at 2 Liog: os a row?

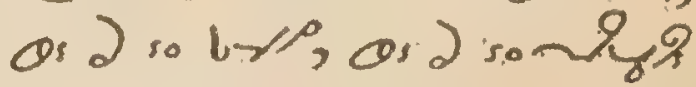
0.102 20. or P. 50 .

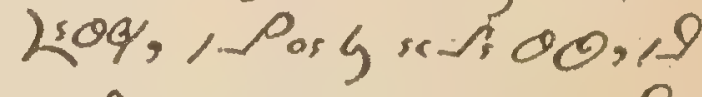
or hod so lsog OQy, Dos

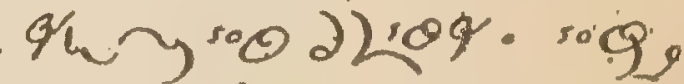
No, $\left.d_{0}>\partial_{2} g \cdot G_{3}\right) Y$

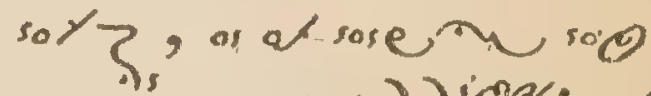
- os zuar 22 iog; os $y=$ nond yalca iore 긍 ०) $(56,7$ '010, 100\%) $\partial$ re de. wo. hove: o re eupon. = osoris 2 rog, os แ> गوव 70 ; Tig so esa ?!. 473 . 300 , or $x e-<$ 
. यु, 500 म - u? ug so L'0\% oL $\%$, yurosolsog; 1220. $24,6 \Omega_{2} \partial 250 \%$ ox, -) $9 / 367012$ mo on, 0100 ro $8^{\circ}, 5$ row so $\mathrm{L} 0 \%$, (a)

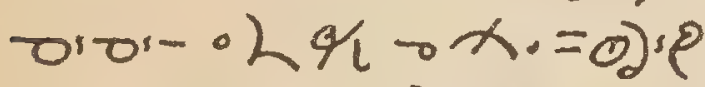
- eगy soo;

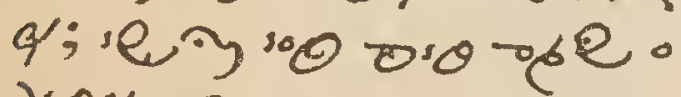

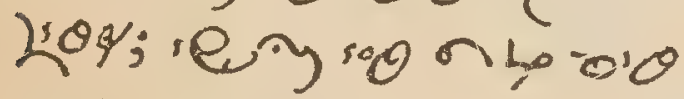
ofevisengromb

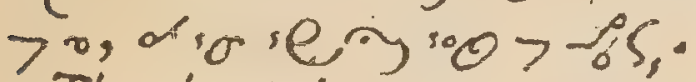
Thanksgiving. o oc ofe - $x .=02 \%$, oigd - rinja reen nokn?

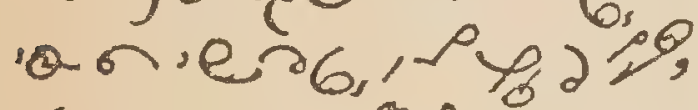
or val wh $>$ whos os 2.6 o 00 obe. $100 \%$,

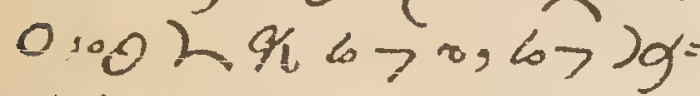
gor : -ociun ocin

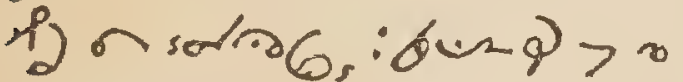
- Lieg orcos, Pas ra zar. 0.2 nin.

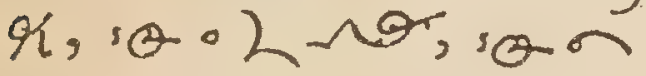

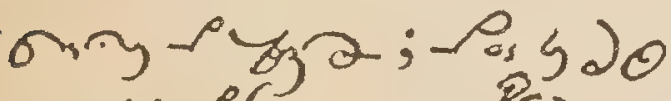

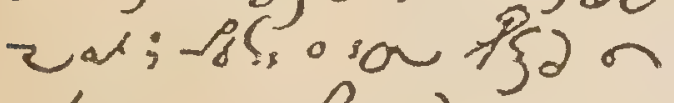

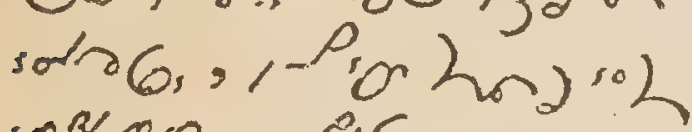
$\operatorname{sog} \theta>-85$.

Petition: o o o g a $o x .=0,62,0,010$

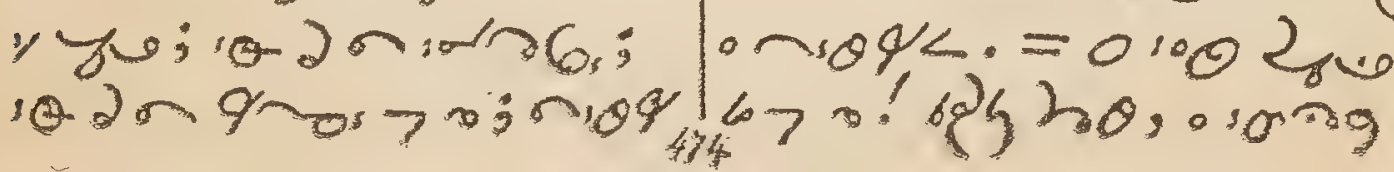

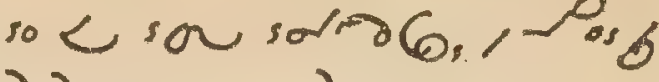
अ - voon. L $9 \%$, «0ro be a tos $50 \%, n=$ $\rightarrow 0) \cap 0$ ). $0<$ ara $22002 \pi, 0<-85$ y in 06, 0.00 solac oर $q_{1}, d 0 n<$ salver op ०र $\varphi_{9} ;$ yor yos

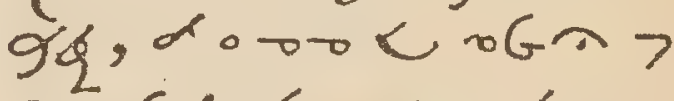
296 ; dore solvo. $\circ 9(>296, ; 0,2)$ 125 . Offering. or $<\cdot 0, p 3=$ $\partial<\theta x .=2 \theta \cdot \theta=$ $26,0100 \mathrm{yll} 6>) 99^{\circ}=$ n; -b2 ho, < -85, gn ic 06, 0100: कo $2 . \mathrm{cm}$

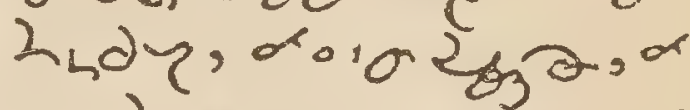

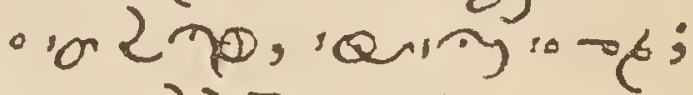
- og 22 ?, - Vag, गदेन >

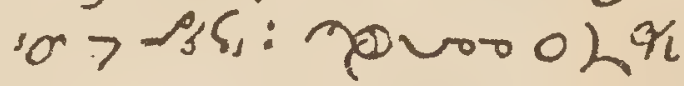
u.Liog, k. Lhal, -) तods: zun ton

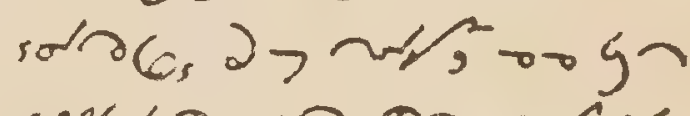

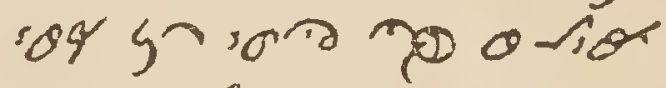
O:00 L Yy Q2? Resclution. on eque Resclution. 集

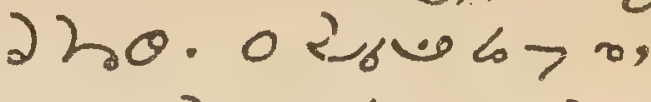


is $0.00^{\text {? }}$ or zat ode a o so 20 ;

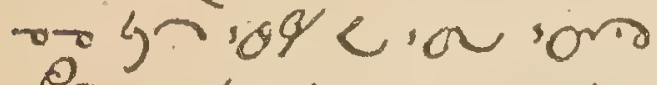

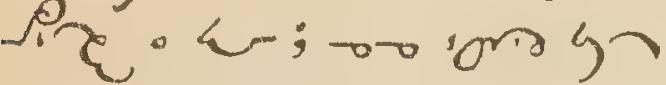
o hoy 0 \&; 0 क 20 vico) $109 ;-0<100$ " भou

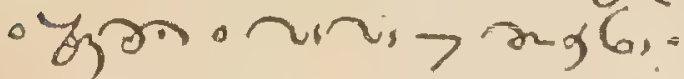

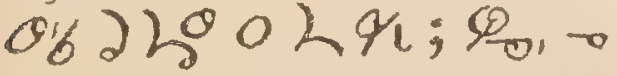
won el 20 10 Lay;

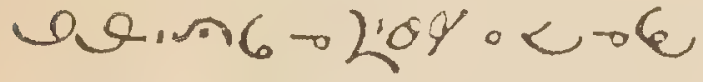

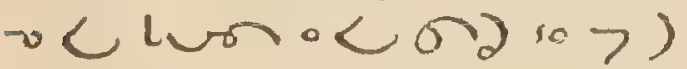
$\log \partial-00 \partial>$ w.

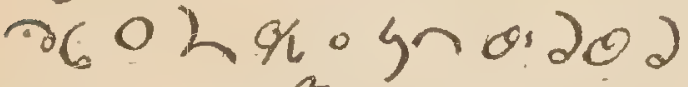

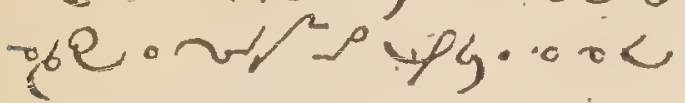
ins.

offering of the Mass.

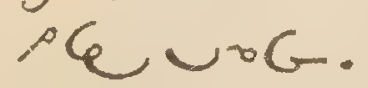

0 ; te, or (b) 6 , -von". 29\&; on b) 6 , won émes:

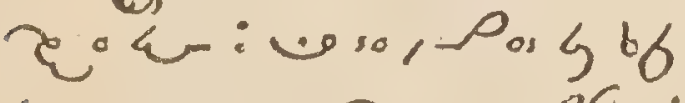

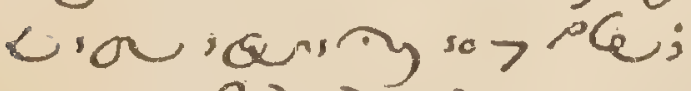
Us so, 10 2 2 so Log on:

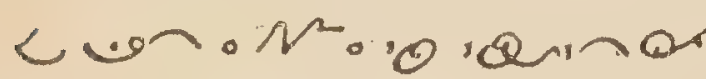
- O. ians yoo so ple. Liar sosery soo on 9

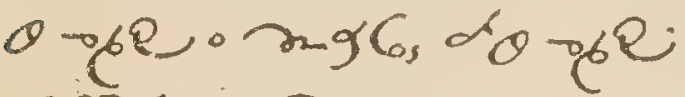

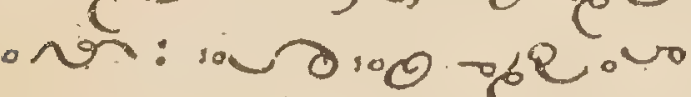

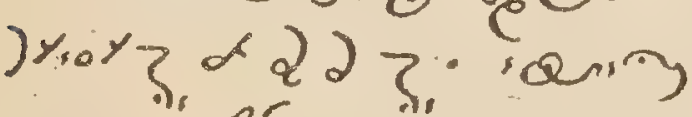

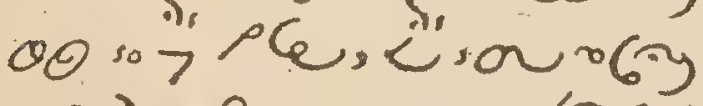

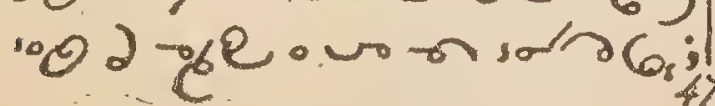

iens 00 so $>P G<$

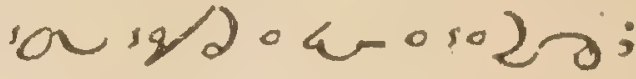
'anity 00 so $>$.6 $<$ rou sais $6,20,4$ ia

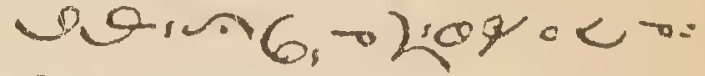
द o mak $300>0.100 \cdot 572$ arsoy?

\section{The Rosary.}

Joyful Myst. 1.0 o $<u_{3}=$ 2-र< $26>\varepsilon, 10$ inon.

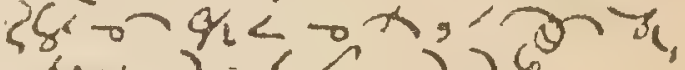
$3=2.6 \% 2.2 .2$.

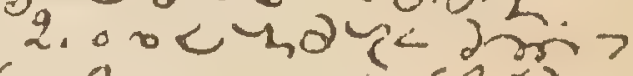
En's won a 8,12 ion दू. $3 \sim y, y_{3}$. 3.0 $0^{2}<43 y<260$ Ertion. 34 or $9 i<x$ - का 2 ज. - $2, b^{2}$

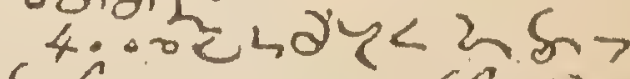

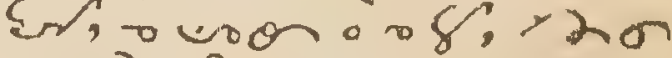
ar. kop, 'ap oxars yen.

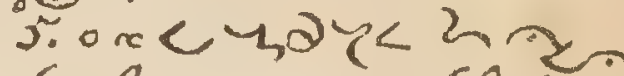
7 sor won o. 4 dis 3

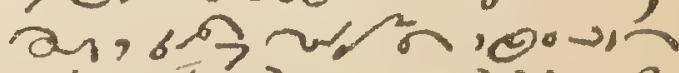
ox, x bqus on d) cas.

Dolorous. 6.0. 2 पे $2<$

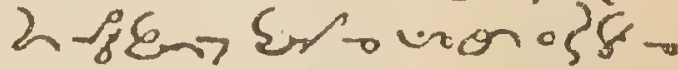

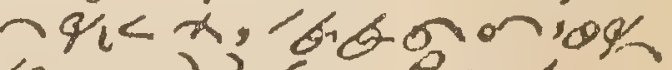

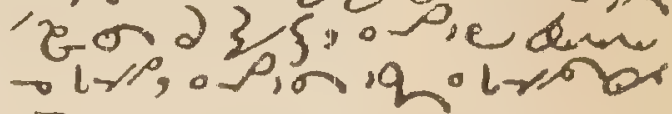
? 3 i:

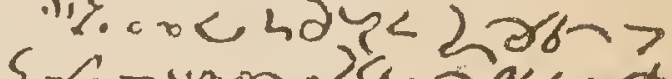

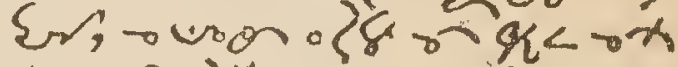
'zre )\% 次。它.

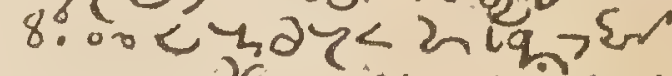

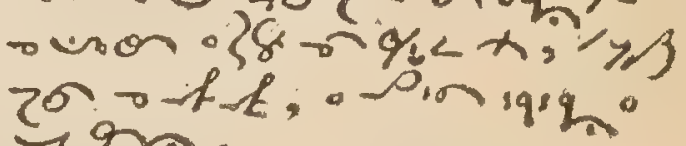




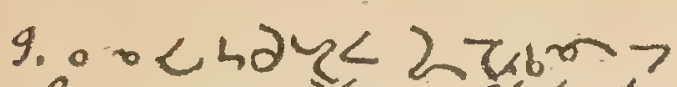

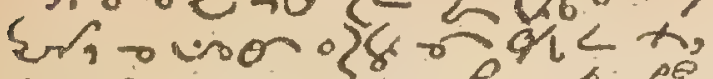

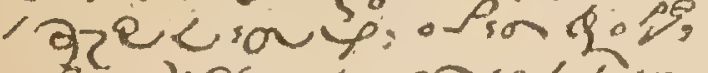
- Binlign, uen ro la cion

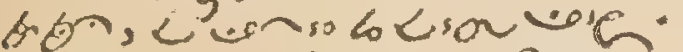

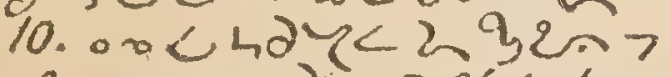

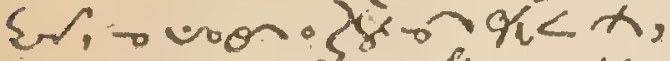
जi 0 \%

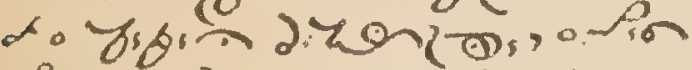
R.c

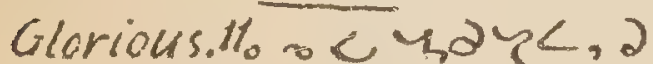

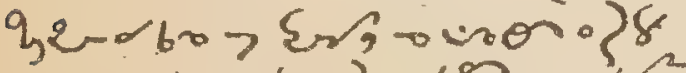
$091<0 x, 2,6 \%$,

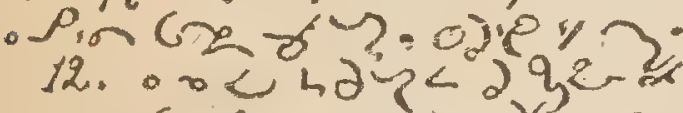

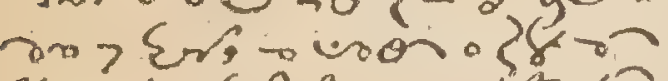
$\%<x, 64,2>0,0$

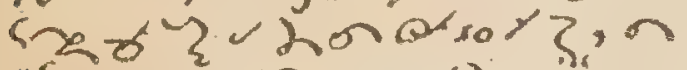

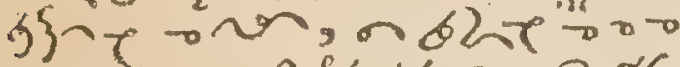
Y.,

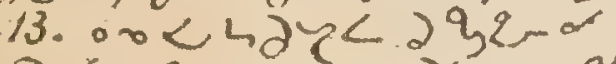

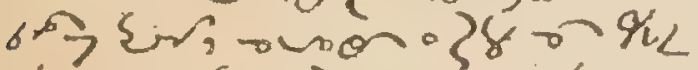

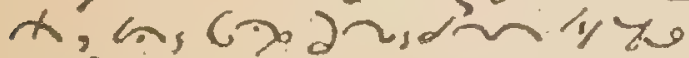

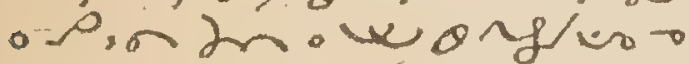
tyon.

$34.00<42 य<2$ 3 2-a

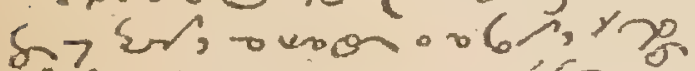

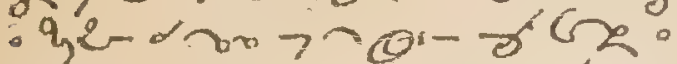
x.

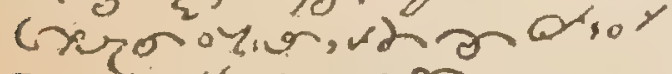
उ, gु

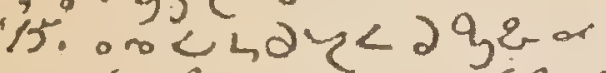

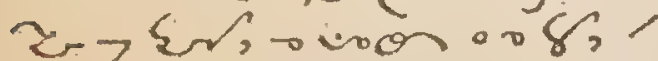

$q_{1}$, (on) Y Y

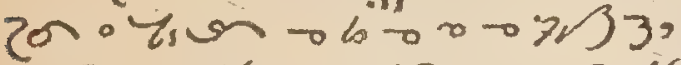

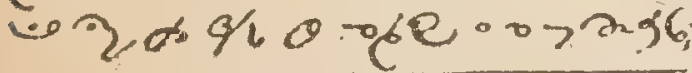

\section{Via Crucis.}

ocruxave. 0 roo sus opo, $0 .<2$ s. fi o : 0 ;

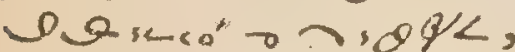
$\psi_{\text {siz }}: \omega_{\bar{p}}><0$ Preparatory Prayer. $019 / 3$. x. os 5 र

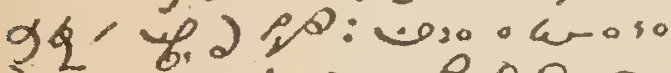
之o. dn: Mis, plat 6din. h. - Pie lavaj so Logl ore - yo sar mo t L. $\angle \rho g \sim 0 .=0 \% 20 x$ Intentian. orous orobs

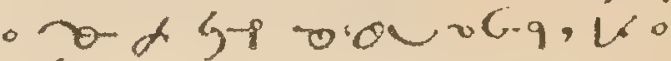
obs w o son.

sancta Mater.

$$
\begin{aligned}
& \text { - 8, tisun" } \\
& \text { rota } 220 \text { Liog } \\
& \text { 几́ba. }
\end{aligned}
$$

I. station. $=$ Adormus. $=$ os soren-100 $\mathrm{O}$, o Pa $\sim \sim=00$ - $\%$. 38 - पe 2 क

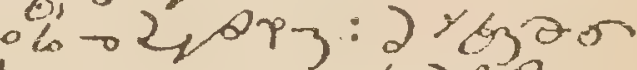
- 2v<ior 2020 .

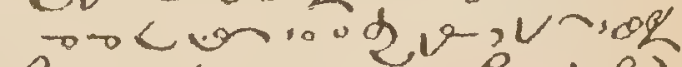

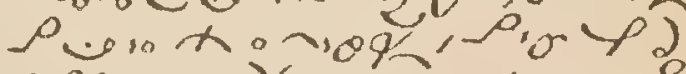

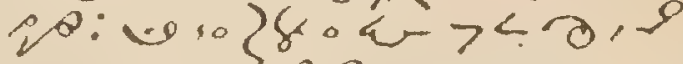
stoxaga.nime

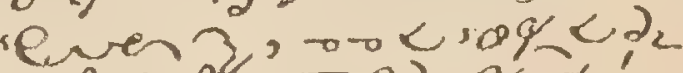
an $1 \% p_{y}$. =02 $q x$.

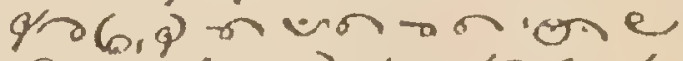

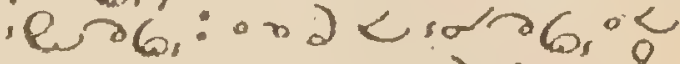
un $\rightarrow<n$ -

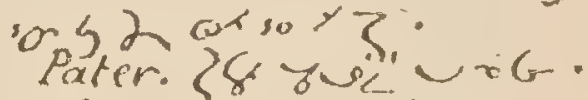
Ave. 7008 un.

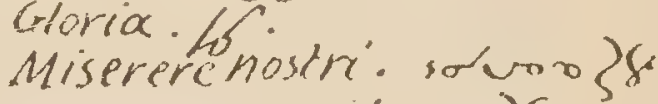
$H_{L}=O q_{L}$, solvo 38.

Fidelium. O \%, onc sol=

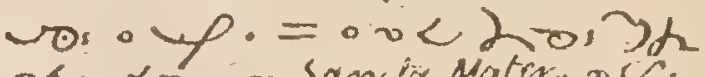
कs, $y_{3}=$ Sancix Mater. of

II. Station. osioner $\sim$..

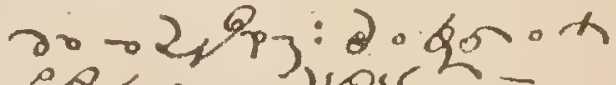
-9a<ia Jips =

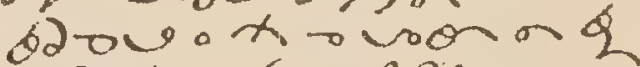

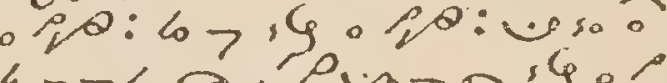

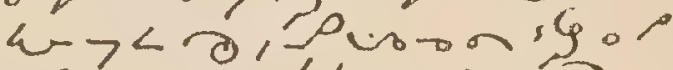
$\theta_{0}=0 \times 15 a^{\prime} z_{0} 0<0$ - द्य 0.0 a $01020 ; \alpha 00<$ $536,0<\beta_{1} S_{1}<$ ion $\left.\Omega_{0}\right)$

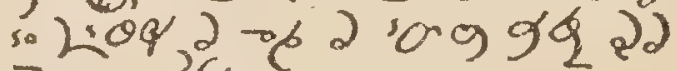

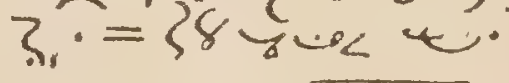

Jil. Station. os soic $2 . . . .=$

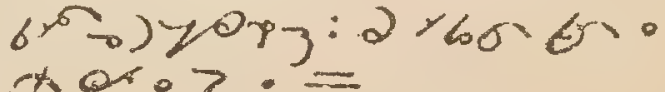
476 or: $3_{0}^{\circ}=$ 
bolow o $x$ : lon bhs

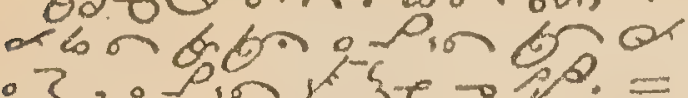

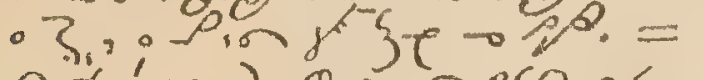

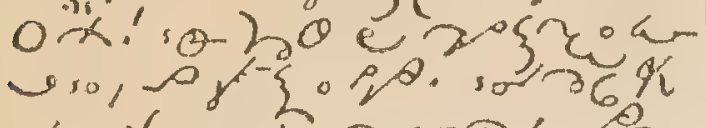
- $<-6 \rightarrow$ ton rome-? or. $=$ दू ye 4.

IV. station. os soien.e. S- oyonz: 2yyo. $x \div y^{\circ}=62000$

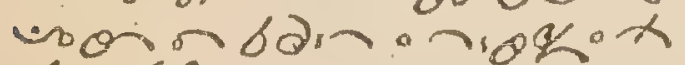
क. s. $z<0,9+0,990223=$

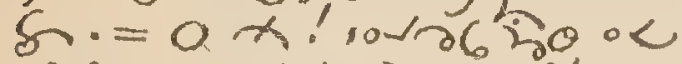
sh, Lion कlond ro lieb o

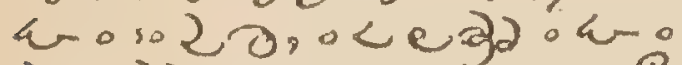

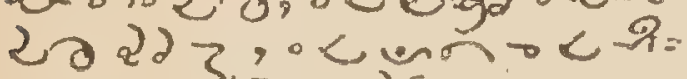

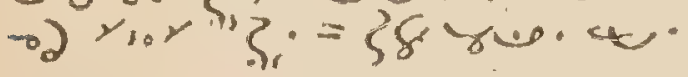

V.station or soren..4.

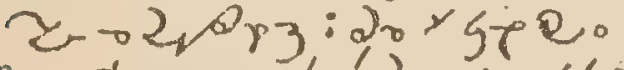
$20 x .=c \alpha ! 20 y / y=$ Noc).05\}a. $20: 00$ on "One prear. ore

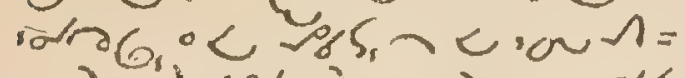
ग. Q $2 \zeta_{1}=\zeta \delta$ z

WS. Station. or soren. 4

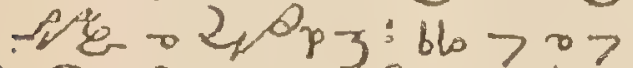

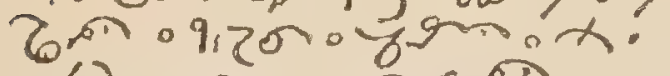

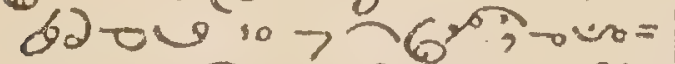
one sone seunar

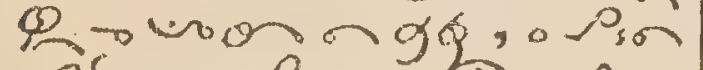

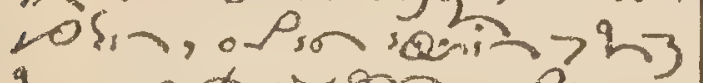

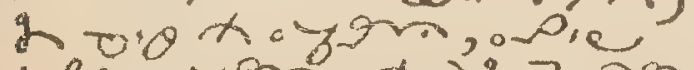

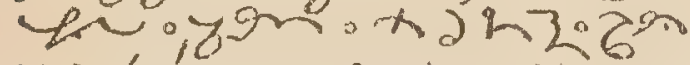
$=0 x .00<9, \dot{0} 0<-7$.

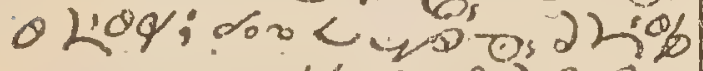

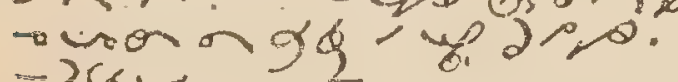
$=38 \times$ s.4.

VIT. Station. os soserom. of o 2asp 2 ind of on $\left.x_{0}\right\}_{1}=6200^{\circ}$ mon cbor. ox, ina on on or:?: wso \s. दा $7<0$ / cog. $x_{0}=0 x \cdot \operatorname{org} 2$ wie TIA teriokmanzo . $3620,5262,0<1$

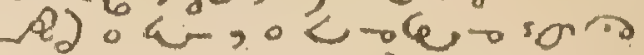
$5700 a-=38$ - $=3$. VIIT.Station. os sore an. $12-2^{2}-p_{3}: 2$ os 2

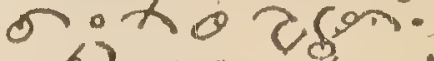

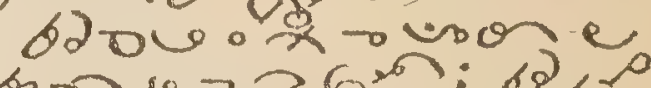

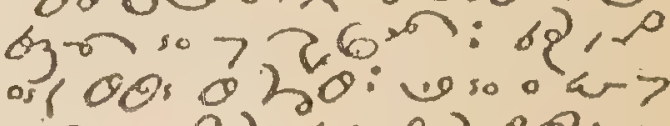
q $0(-\infty) 5 y 2$ p o< dos 40 a $>90$. $0 x !$ sal 6 ha

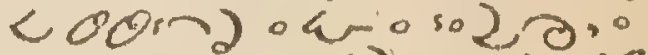

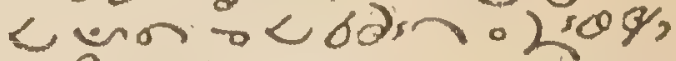
- 0 -o hon sona s

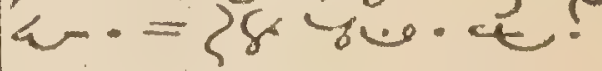

IX. Station. os sosen.

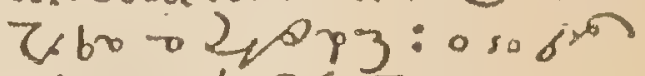
eb? oxoros: के o 2 , bn, som,

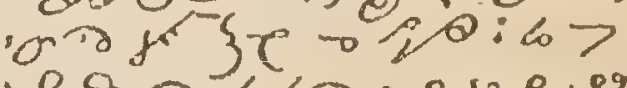
as on bin, o so en

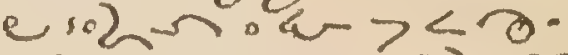
$0 x$ osos-aro $280<$ -6 - लra 570 - a :

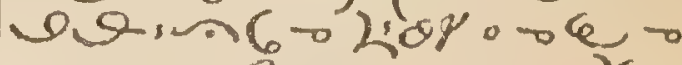

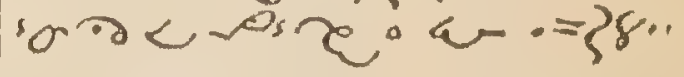

X. Station. os wesen.

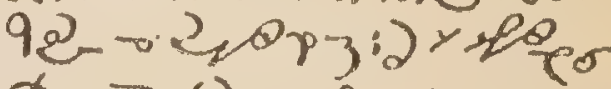
- $x=60$ - 0 - uson

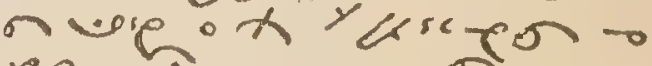
roin: : son ig of te

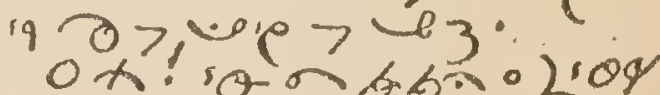

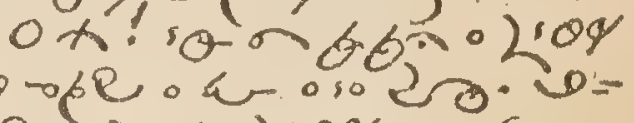

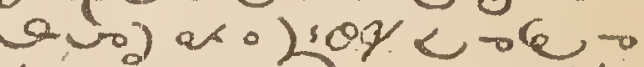

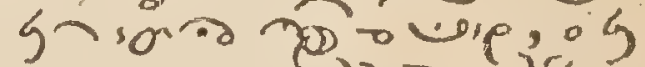
in or an $3:=3$ six.

XI. Station. onsen. $92-\infty 60 \div 2,0_{0} 3: 2$ 。 sore ox al.

6) ow unon abon.

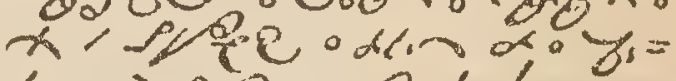

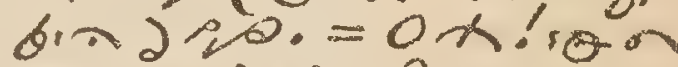
3006,20 wa us on 


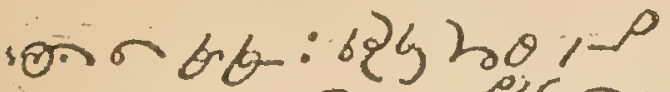

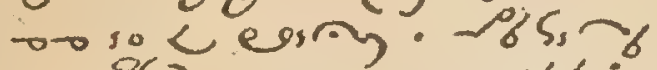

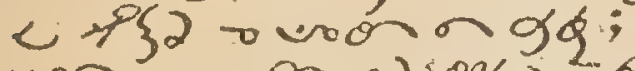
vor ow on 0 iog jas

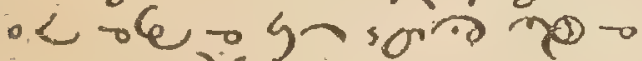
an. = 38 yo.

\section{XIS. Station. os soren.}

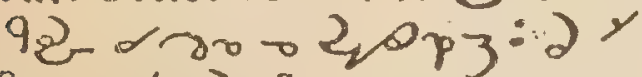
Po . x d io.

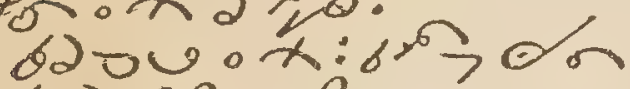

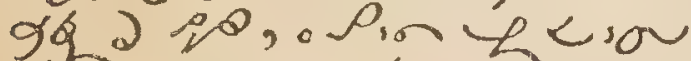
gen $=0 x$, o. an

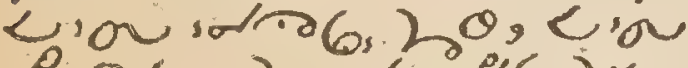

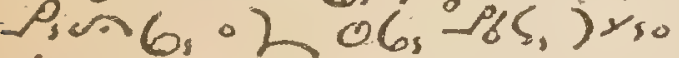
$\checkmark 3$ : uso $=0$ o at 70 - 29

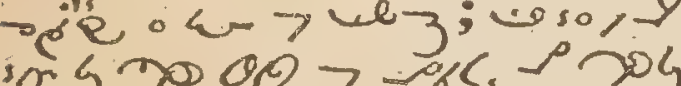

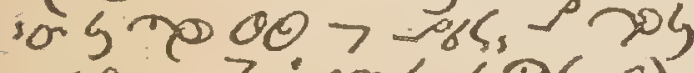

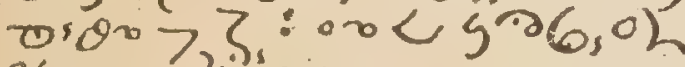
$\sigma_{l}=38$ \%

Xill. Station os sose $\rightarrow$. 92 $\alpha 8^{\circ} \div 2$, op $3: 2 r$ See ox o, pa.

odow ruoneven eton onigh o 8 \%

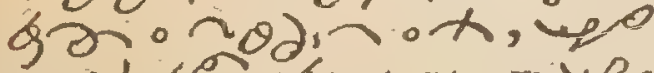

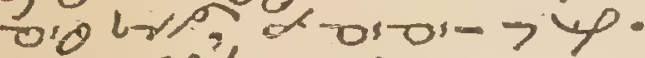
o r 8\%. so ho odnén ०x. 526$)_{2}^{\circ}$ ०ट तe

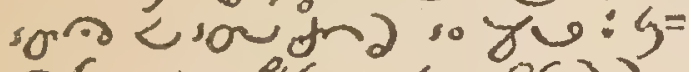

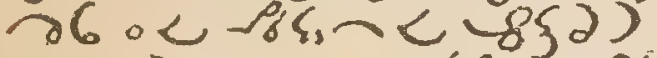

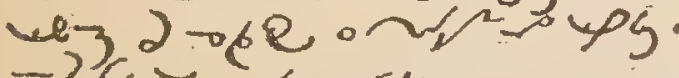
$=38$ \%

XIV.Station. os sosen. $92-\alpha 5-20^{4} 3: 2 y$ yifo $x:=62$ - 0 won yapror. - $x$ वे $\operatorname{tin}>$ ? $0 x \cdot 9 a 6, x^{2}$. y $2=$

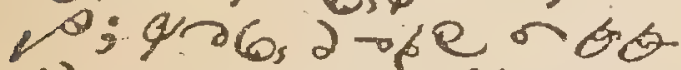
'म $-125.0425)$ mर

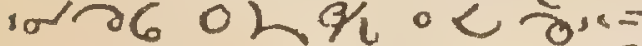
$\mathrm{y}>102109,0<-85$,

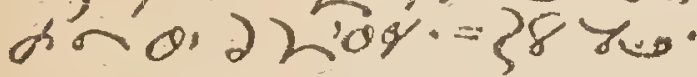

or idaver.

$102 / 0^{\circ}$
Hymms in Thompson.

1. Venez Divin Messie.

ong $\theta$ \%?, $9 \cdot 0$ र क $98<$, बio भion

$0,0,0,0,0,0$

aigging

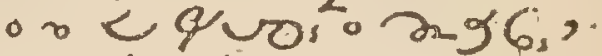

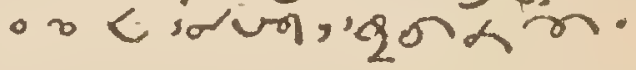

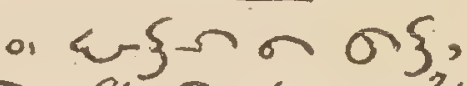
- sog zenar aid o 0 (s

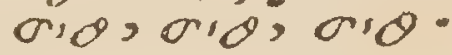

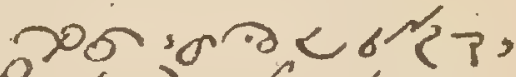

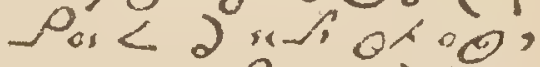
$\left.q_{1}, \infty_{10}\right) q_{3}<$.

2. Creator alme.

$0100 \%$ yu, yo oee rowar, aig quoro 2.96, \% 0,942 के ? ?.

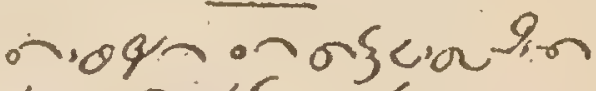
obu a mig6, ol, o: $\theta$, exm ox,

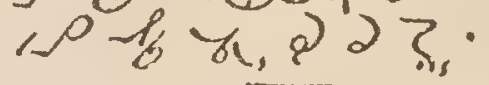

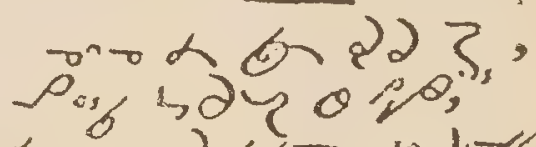

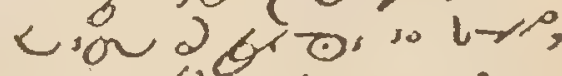

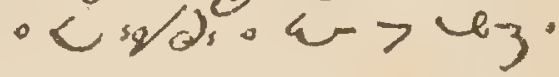

3. Adeste fideles.

onow, ang b. केण <.og'a, ong, onouls? *bolर न gh on: orovio sasure,

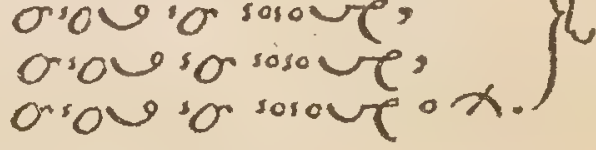

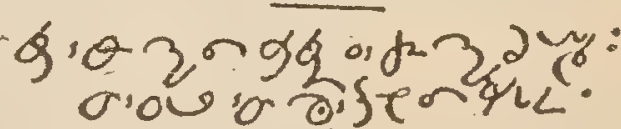

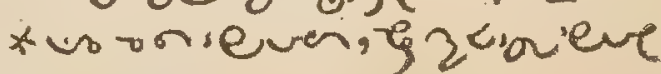
48 - onower. 
4. Audibenigne.

0 sog lo vo $y_{3}$,

grarchozion,

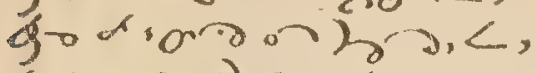

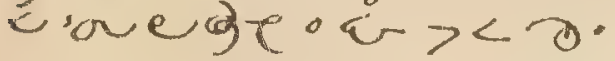

5 Miserere mei Deus.

: al $620,02.800 \%, *$,

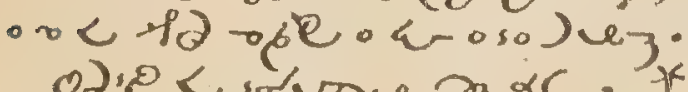
Od.हर

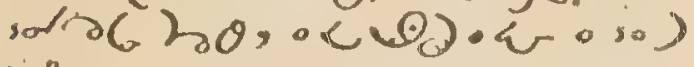
e 3 .

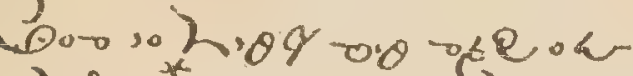

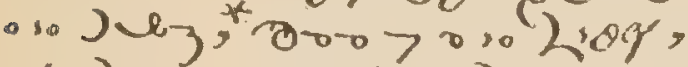

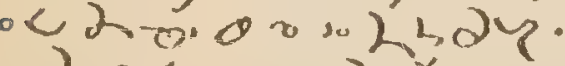

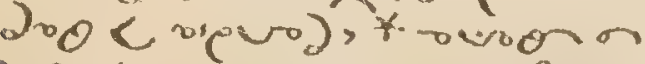

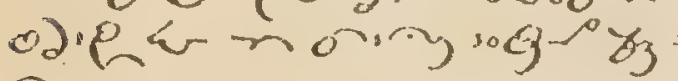
2.

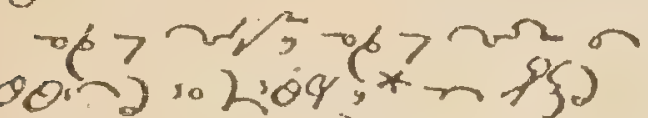

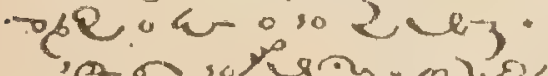

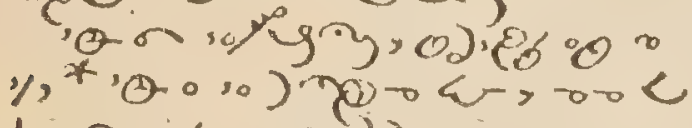
hin o< syga.

6. Mon doux Jesus.

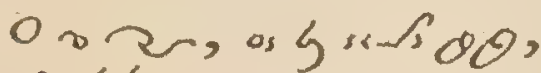
209 2oroons jor $\}$ ope o i o)

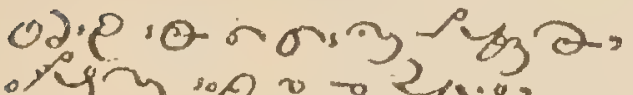

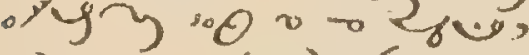

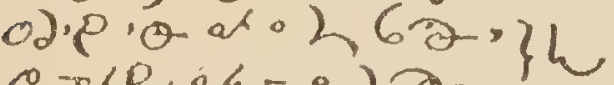
0 0 ○ 2 .

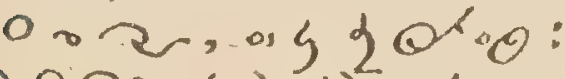

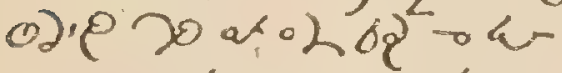

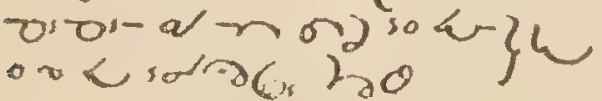
or 2n.

7. Jini Peihe' dis prion enfana or 5 Tok.

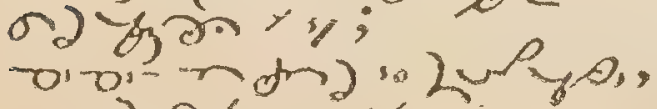

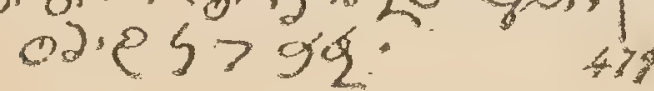

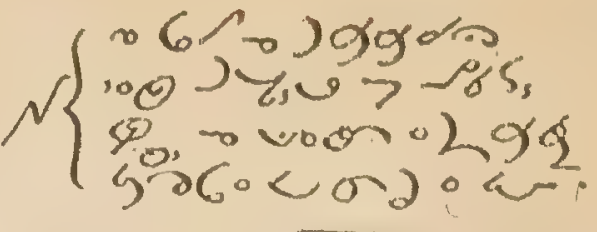

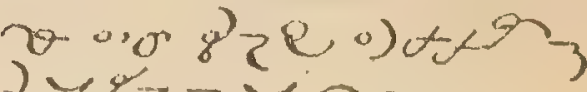

$$
\begin{aligned}
& \text { व) } \\
& \text { ciovoding } \\
& \text { 2.八.-5 2p> we, } \\
& \text { - d lorion }
\end{aligned}
$$

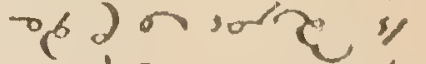

$$
\begin{aligned}
& \text { Lio o.o for and } \\
& \text { «, o-jee; la } \\
& \text { varmigojon }
\end{aligned}
$$

8. He'las, quelle doulcur.

2! ! je,

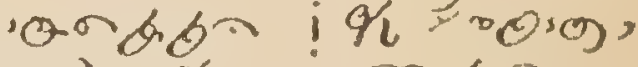
-10 Liog! i mbity oh 2 .

orab i token... . 10 L9\%, i 0.500 , $10-102$ b $6 . i$ a 2659. 9. Fins dernicies.

aco $x, \alpha$, $6: 2 \cdot c$ b6-20) 3 , प्र०० 56.

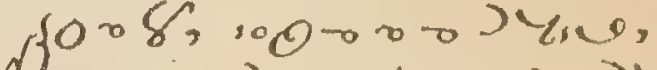

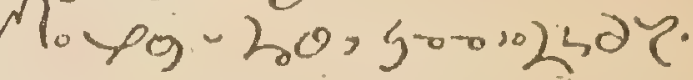
- 100 ) $43,0.92$ b 630 , bथ $6 x, x y^{2}$ wor.

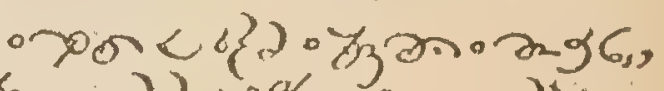
6ु- 2 Li, 10. To se Joseph.

orn, soondbre

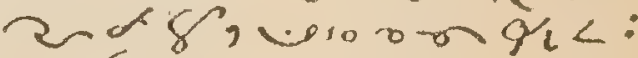

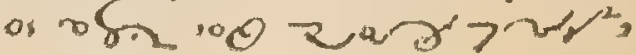
on $\sim$. 
orn, od, व\% न ok

On $\sim$, , 500 so 2.09,

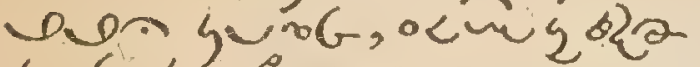
<-égrivon:-ses or $\sim$.

11. o crux ave.

0 sog soso $>$ Po, o. 62 "ns ar. 0 : Gicro or sog<, Viciso on $><$.

12. Au sairig qu'un... groba bo - Lon lo pa, - d6a040100. so so 10 y.

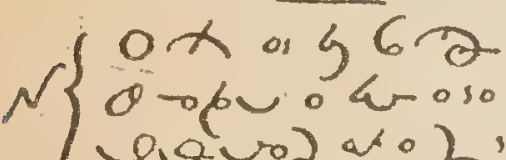
बर्बin a. र $10 \%$ > a so "2 70 - $u$. gos $20: 0 ?$ ᄀi⿻ 2,0$) 663$ (6) 23027520 . o 2 a hionce. ginion $203 \rightarrow 2$

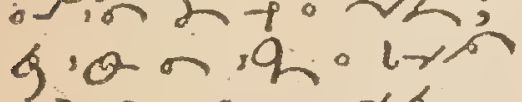

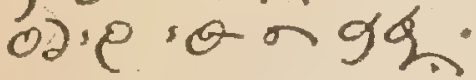

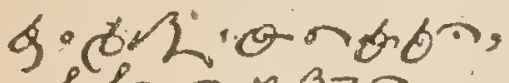
n-1 1 - 2133

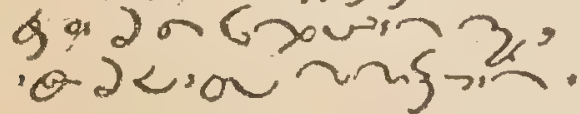
5 os $\log \delta$ - 0 , c) बen 99 क 2 r 20. क्षon $23 \%$

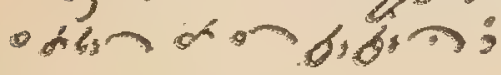

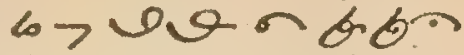

<iden.ario.

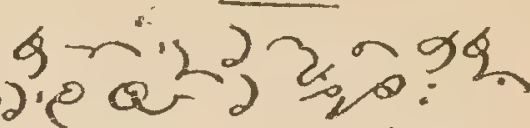
6> पan bon - Psar hor.

ox. 0.0. - a $\infty$

- 22 \& a > - 3 ; 0.0. 85. O теن: fo. Regina cali.

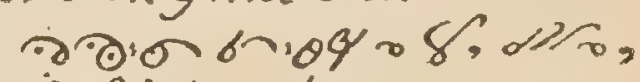
sog- yw os Go z a of In,

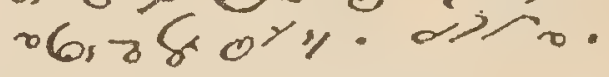

14. Le voici l'Agneau... $\mathrm{N}, \mathrm{\theta}, 200 \mathrm{0}$ कr so. ró?

15. Adoronstous.

' 0 susuc $200 \Theta$,

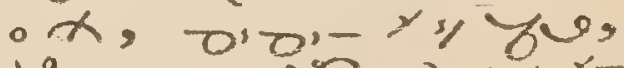

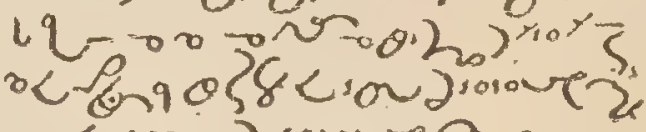
uar 2 rosove 2.

16. Before communion. oder o.

ग $0 \rightarrow \Theta$, $x y$, vow?

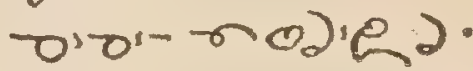

2055200 , $20059 \%$, se soso $>3$

न.ंस 20 人',

$$
\begin{aligned}
& \text { or and } 35 \text {. } 5 . \\
& \text { arlera, }
\end{aligned}
$$

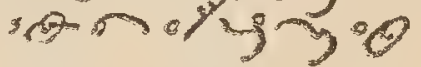




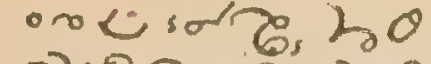
व): रूandoर भi几 60 \& 0 \%.

1\%. During Communion. ons, 2, $0.0 \%$, $0.0,0.0000$ L:0\%,

ris?"gonar.

18. Chantons ence jour.

$$
\begin{aligned}
& \text { O o } x \text {. } \\
& \text { 20 m } 2 \text { isud 10 1.09 } \\
& \text { ode } \\
& 5220 \times 10 \times 3 \\
& \text { w, } \\
& \text { " } 2<1.0021 \% \\
& \text { - joinga. } \\
& \text { - L व } \\
& 0 \text { o } x
\end{aligned}
$$

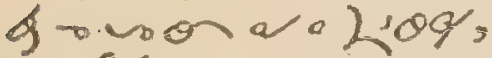

$$
\begin{aligned}
& \text { "ssiringa }
\end{aligned}
$$

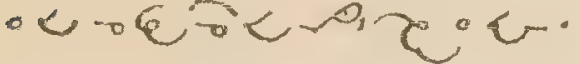

19. After communion.

$$
\text { " fux, }
$$

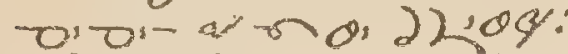
ord o por o laps.

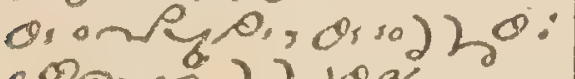
- ory of a Liog, Avod: hiog 00: on 10022109 , oded'

icura. खि" .०ळ 100 o s010 $Y_{\%}$ lova ośn - or are pun micumar कo ana:

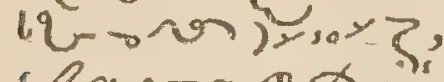

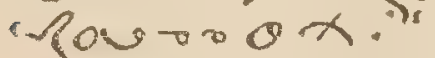

20. Panis Angelicus.

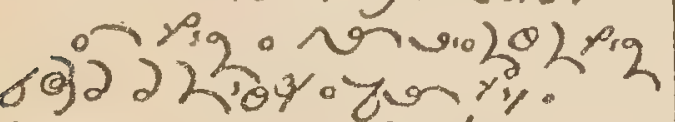

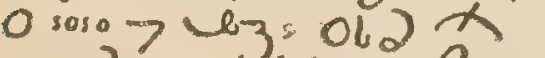
hog $5>\rho_{4}$

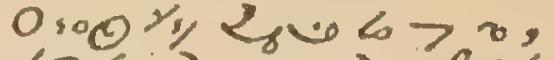
. 500 $102104007 U_{3}$ "र ior 2 or soy?;

21. Pitie mon Dicu.

0 solq, 02. $5>99$ ब०. 208 - का इ?

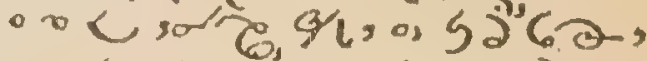
or (usungr:anoso) 0 .

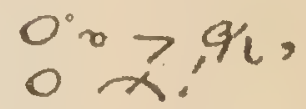

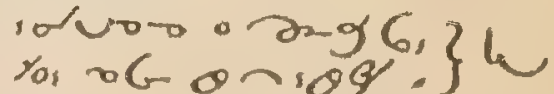

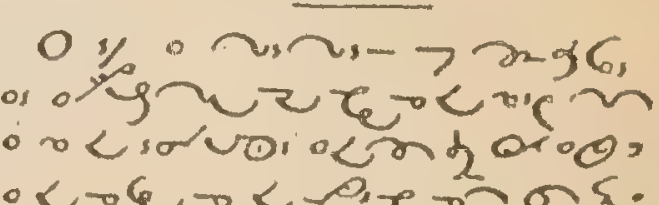

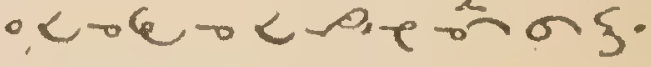

$0 \%,+0<$ wonoly

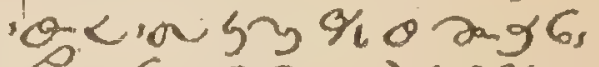
Yun 00 : hiog, -

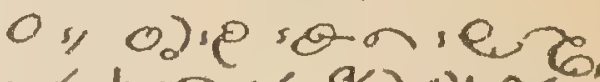
oर

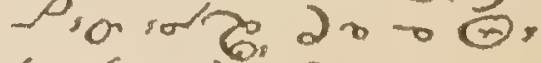

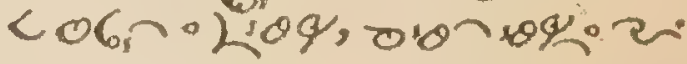
22. Tesuis Chre'tien. or 5 ra, as alsog; os $50 G$ OY so $Y \mathcal{G}$. -od piscounof or $5 \%$, os 5 及.

23. Umis aux concerts.

$$
\begin{aligned}
& \text { - corgstor on, } \\
& \text { us ty a } x \text {, } \\
& \text { ज. } 38 \text { - thu<. }
\end{aligned}
$$

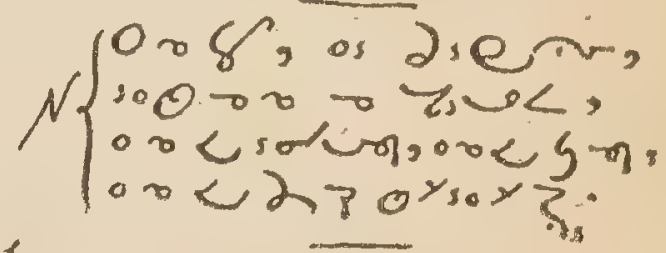


- 10 then a 8

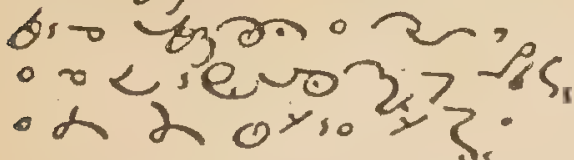

- bicon:ora 2.ow 0 \% 8 ;

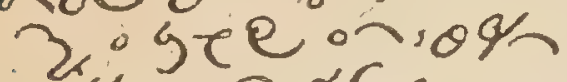

- of $7256^{\circ}$

24. Fundixmontal truths. OD:e- $1 / 6>n, b>0=$

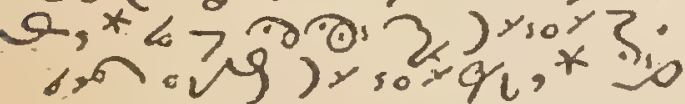
blo $40, q_{L}$.

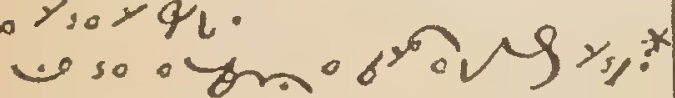

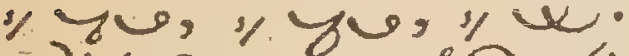

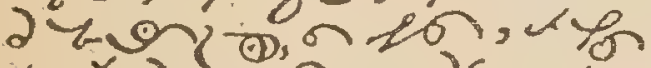

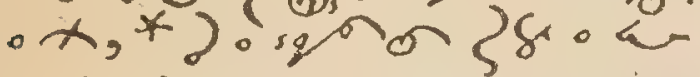
$7<\operatorname{li}_{3}$

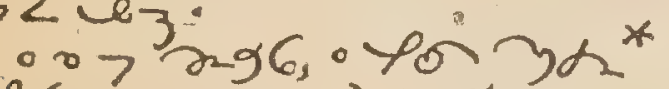

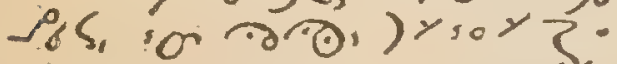

or. $4-7$, 96, , ison ryo, 85, on 6 a ) $19 \times$ 14

25. Propagation of the Faith.

LI $\rightarrow>$ ang $\sigma_{5}$

- 9 dर कीजि?

oग: $<>96$

रद 525

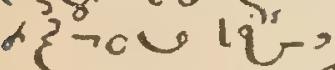

- $00.0,109<$,

मदou कn inter

gq $>$ m 96 .

26. Welcome to the Bishop.

$r \leadsto$, w o is,

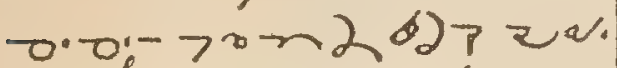

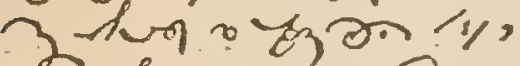

risur 0 - $\rightarrow$,

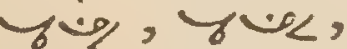

गे, $2,09<$,

$3 \times 2, y+\infty<$

0.00858

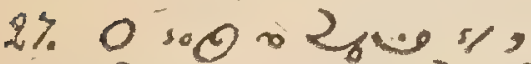

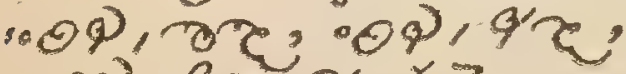

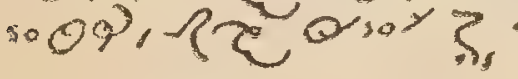

Visit to the B. Sacrament. 0 x. od.er 0.20 .

Ox! 0.6े बर:a yदs og 00

ox" \% बe ; $\theta$ ) $\theta$ u, 6as rar, onjor zas

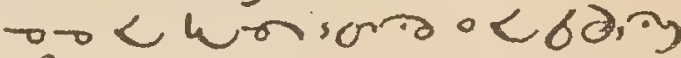
pory.

oxiseng 10 .0 The.

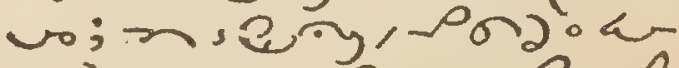

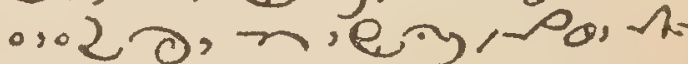
Ro. so, hiog.

ox.'m zony pros 2

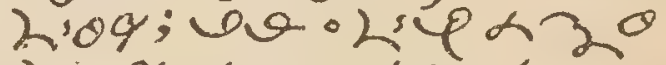
hiog, $<$, in 920,540 , $<2,0) \times 10 \times ?$

Guard of Honor. Enroffment. Ox! log $>0$, lo a No' ro n.s9: sue, evo: ast.

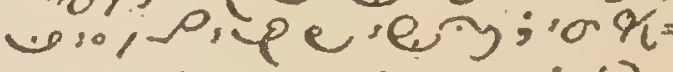

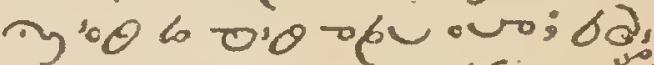

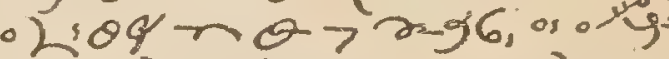

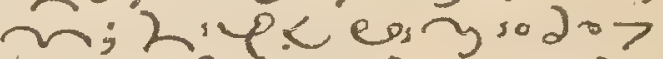
$e_{3}, 1,0, e^{2}, 2020=$

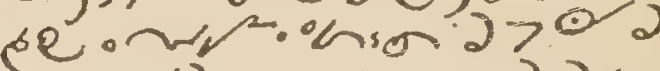

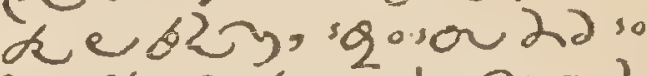
hisqo, (an boing - h

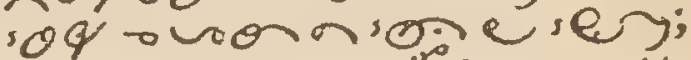
- Liares on orgnogk $\rightarrow 2 n g 6,$. offering of the hour. ox!

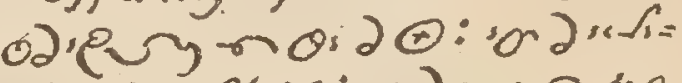
(2) $10.0900 ;$ os 2 sose $3 \% 0$. os 2 pry inis.

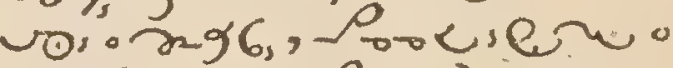

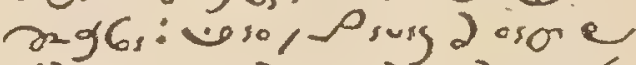

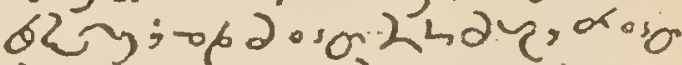

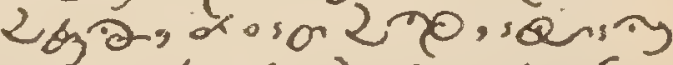
so 0 b. $9 \pi 6029 / 0<-6$ - L9i20 a oर susg $2<$

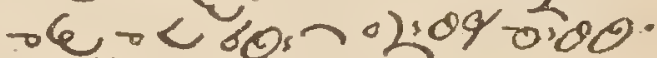
offering. $0, /$, s ing

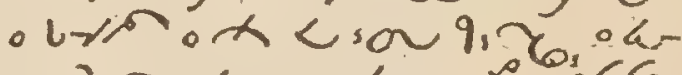
$01020,<$. 50.69

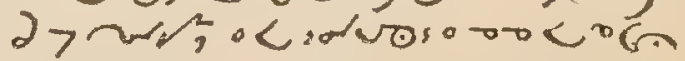
73960

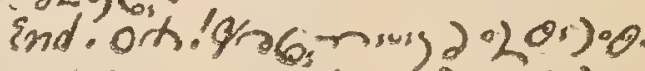

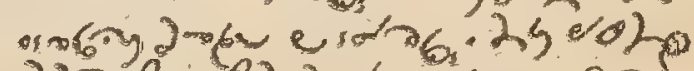
Q2 $3,0,0010600<04003$ $482507-85$. 
Gatechism in Thiompson. I. God.

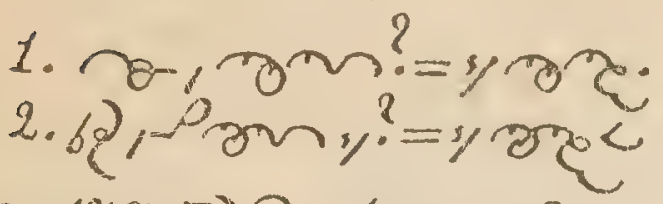
on wevol 2, chouleng)

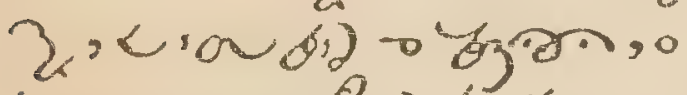

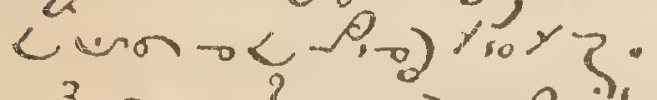

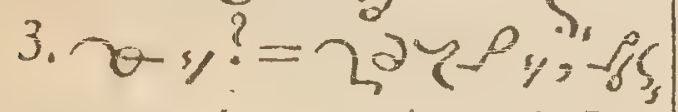
nos $n, b>0$, b va

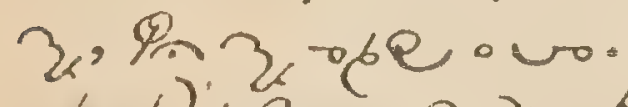

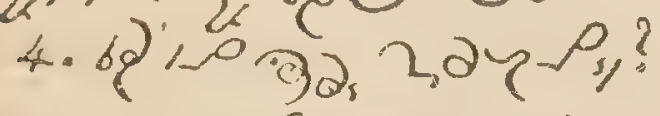

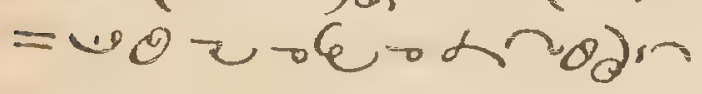
$\%$

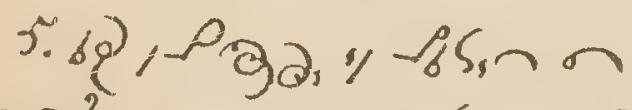
on? $=\log 26-9$ ary, a z-6olun - <八.

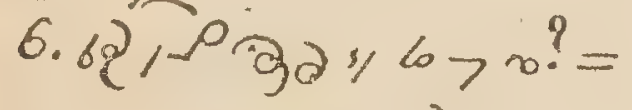

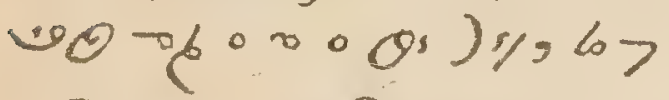

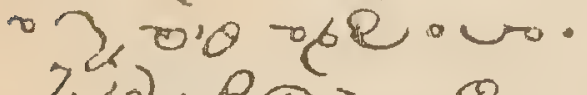

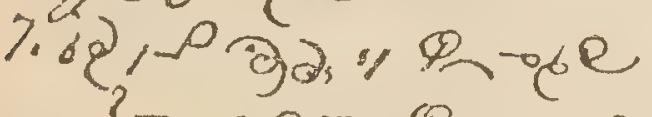

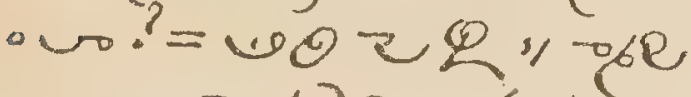

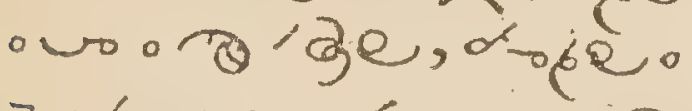

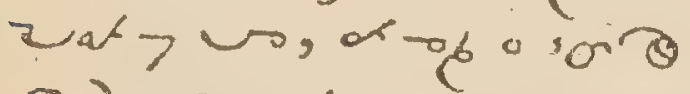

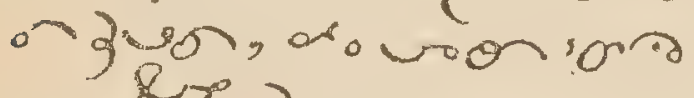
e ke aniog<.

8. $2 \operatorname{sg} / \cos y ?=32$ ot 2 oro 7r. Trinity.

9. Y<יव. y? =-0: blo $\rho^{2}$.

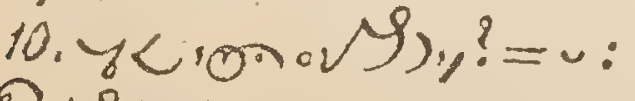

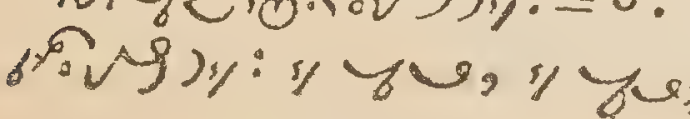

"W: a so on $\partial \mathrm{C}_{2}$ 及.

11. woso $2-\beta ?=2$

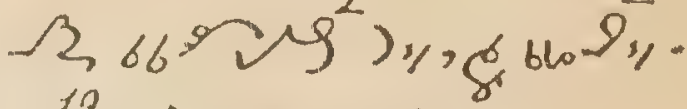

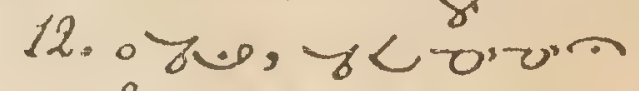

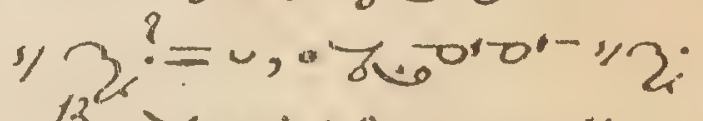

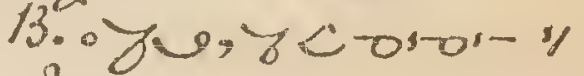

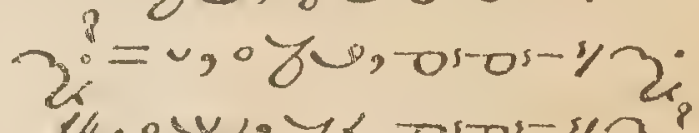

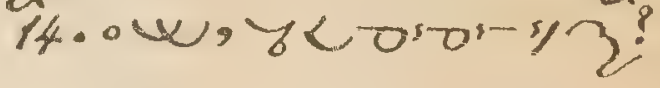
=u, aर, or-or-s? 2 .

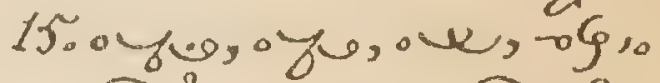

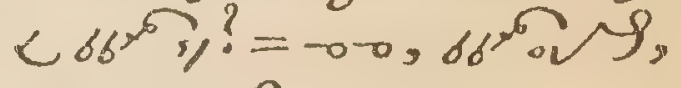
$68.610 \rho_{3}$.

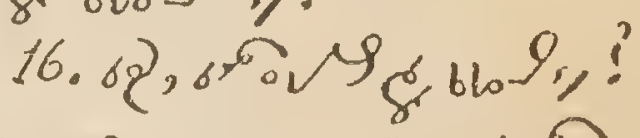
$=00$ z mun. M a ofe our.

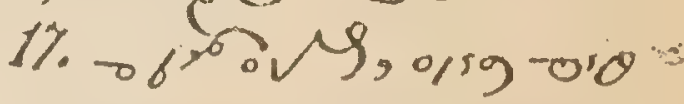
- la>r, olotof $\}, 0$ lo Q? = = o, po, Pu= woo b 18. o b. ov 9, z< tion - blo oid blo? = 02se fior - br ore bla. III. Jesus Christ. 19. - G< बr, $0.06 \%$ \% $9 .=02,5$, ,

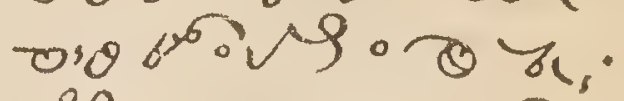
20. \% s9 0.0 6.0.' $\therefore$ का ? = s 21. 6 र" y

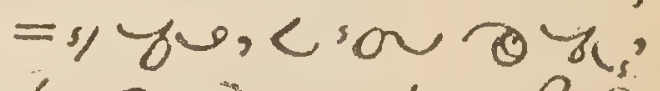

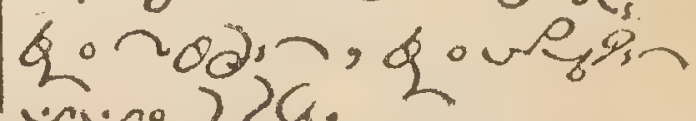




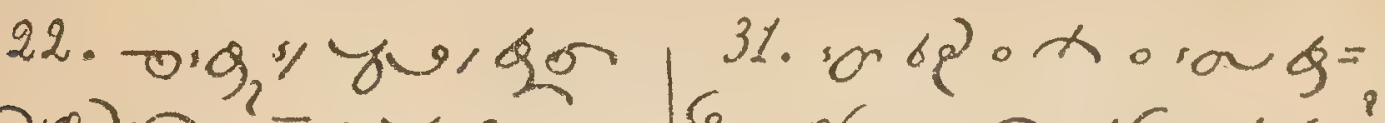

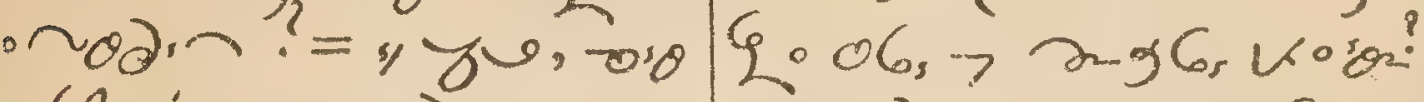

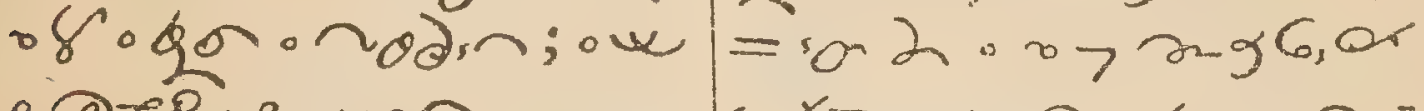
- dee una.

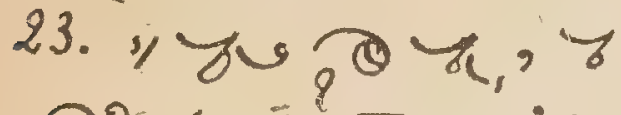
<风<

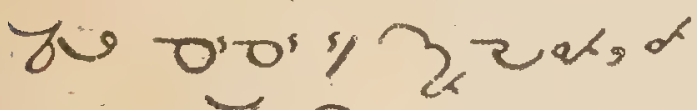
oid. $\pi, 3$.

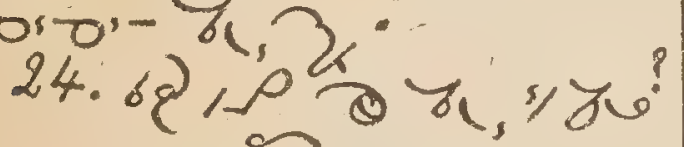
<ar sol:e $><$ ? - L sar 2 orsoy?

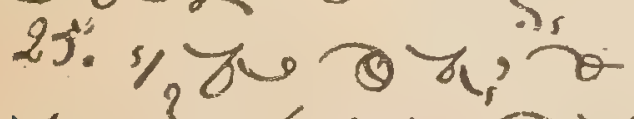

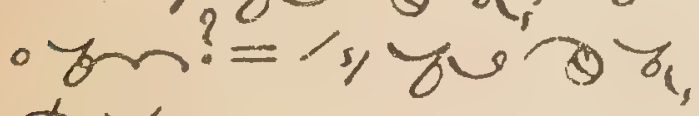
xtom.

26. $b$ - ox? = x, , $10 \%$ \% ब द,

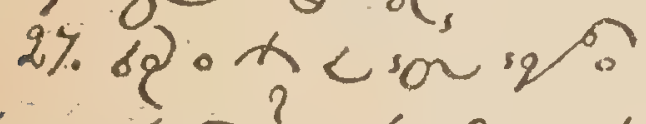

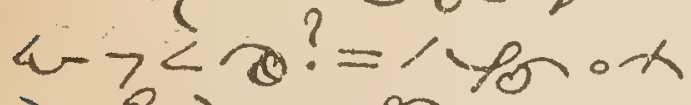

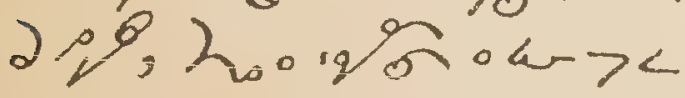
$\theta$.

28. $-9<62 \cdot x-6$

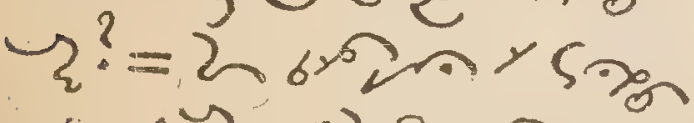

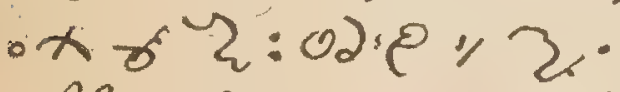
29. y con ox कर है,

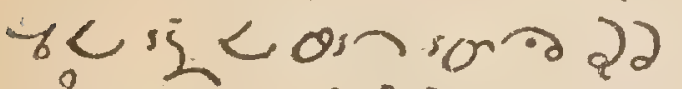

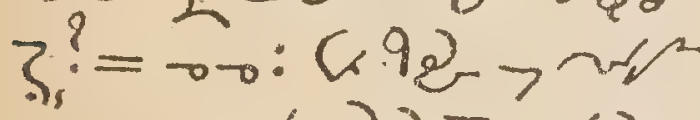
es, os ox Q2 $3, \times 2=$ on orsoys:

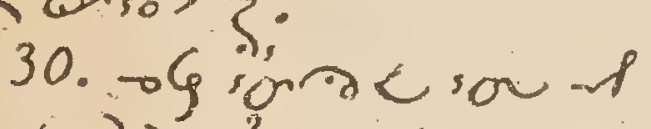

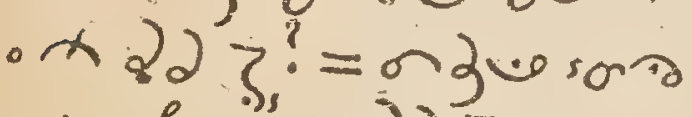

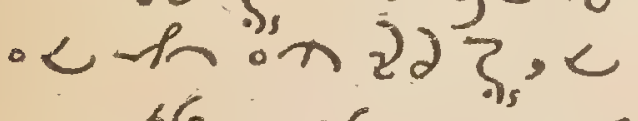

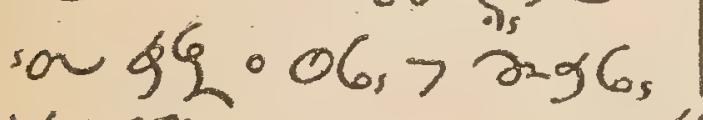

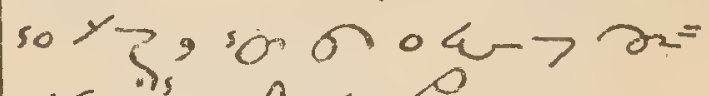
$96,3)-1 \%$ 年.

\section{The Charch.}

32. 20-28se o. $2=$

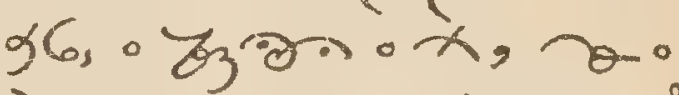

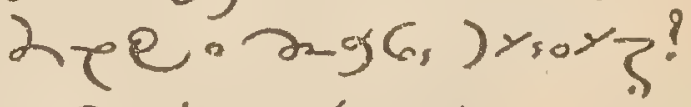

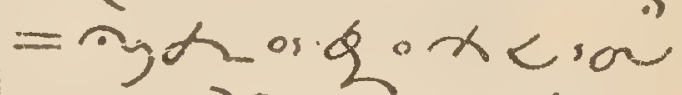
on。) हु.0s, dy

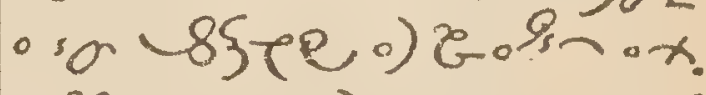
33. Uoso o) 飞.o.

$=0,10>2096$ bo - -sog人 cion dign.

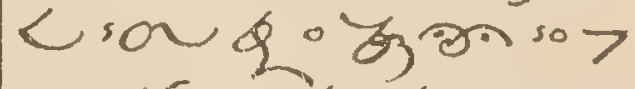

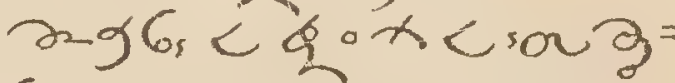
S. 2 g6 60 bु को $50<$

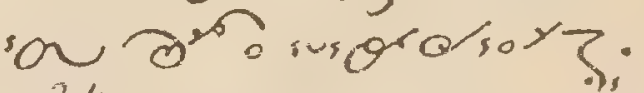
34. حo so $>2 x g 6_{5}$

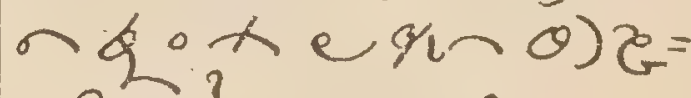
or $\theta_{1}$ ? = coso , $v$ 。 $\leadsto, k \circ M$.

35. $x$ ? ) e⿻一亅八

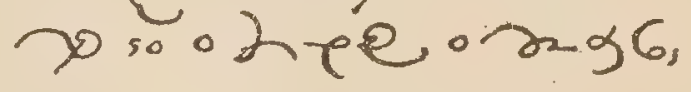
or so $\times 3$;

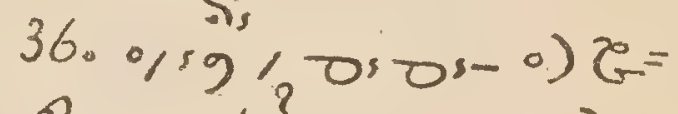

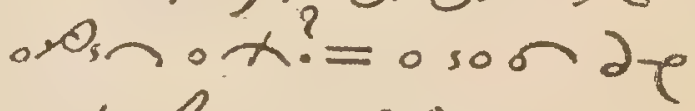

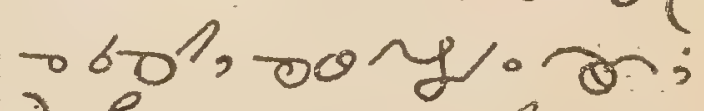
2 P.oson and K. sor. $4843 \%$ 20.0. 
ox? = A s soo क人, $\rightarrow<\theta^{2} ;$; so oरम

38. o) 6 ops o $x,-\gamma$

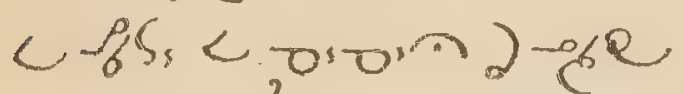
as $\partial g_{1} \Omega$ ? = $\times$ 徆品 " ogn:" <-

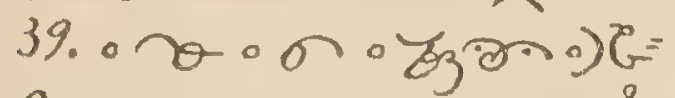

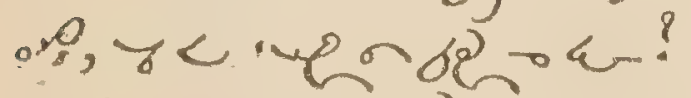
$=0.1$ :

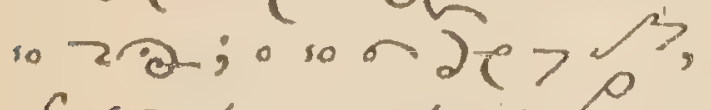
fion $\xi>$ "ust, of का wor.

\section{V.The Creed.}

40. $00<680<220$ s.

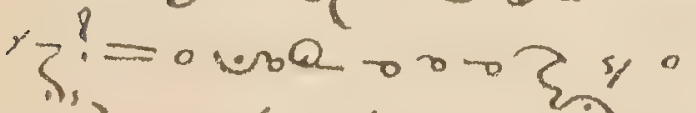
¿is's or so Y Si;

传. - ro wo, algr pe

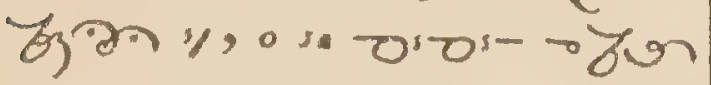
"

42. $219 \circ 62$ - o mon

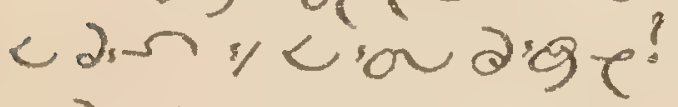
- 2 o). $2{ }^{2} \mathrm{G}$.

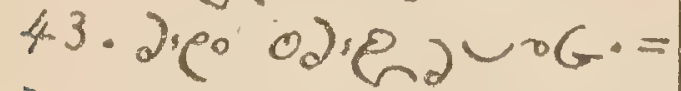
0)

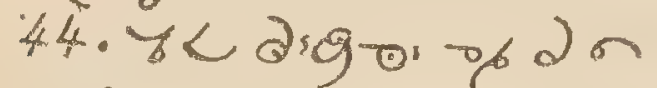

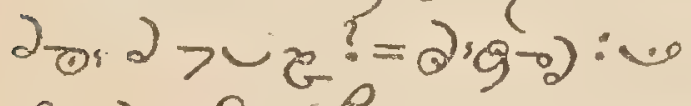
$0.20,0 \rho^{\circ}$ ars $\zeta s$ $<\sin (\theta)$ o
VI. The Sign of the Ciross. 45\% p vo, meonec

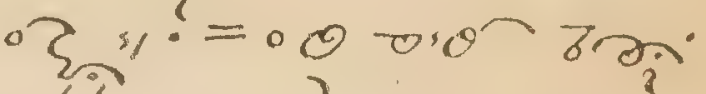
46.0068 . 00 \%

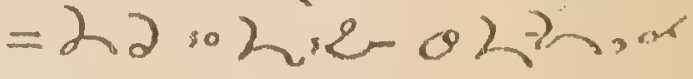
Q) $16, \alpha$, R,,$\infty$

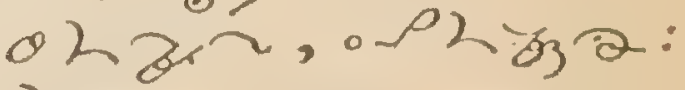
व) 火. o

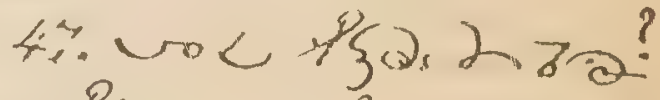

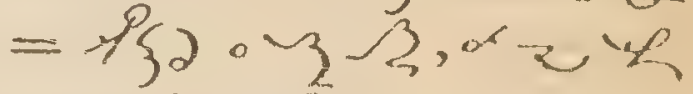
- x 2 के.

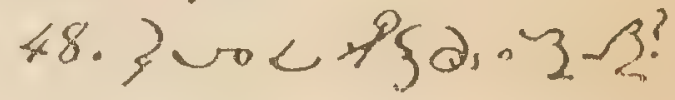

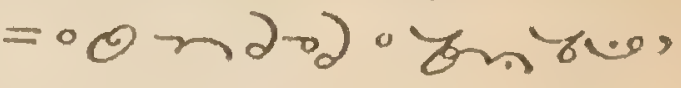
以ु), k.

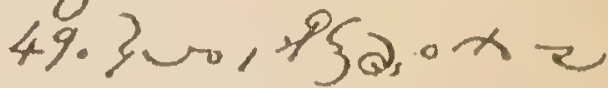

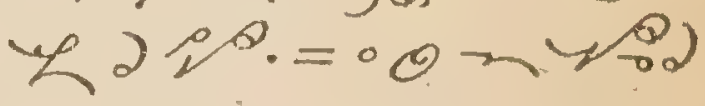
- Zon (ब, 2 h, वर 32.

so. Lu $x-2$ ? = fs - son- a, ona mo, d - biker< senok.

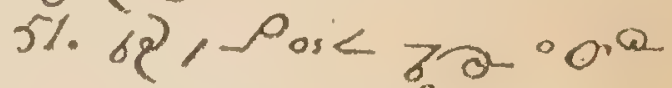
b, , s,a mo? = cson

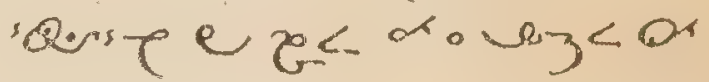
" Cin g-on $0<$ ond $<$ duc, or a $w_{3}<$. $52.62, \rho_{05}<720$ b6 ce०am? = c savgen

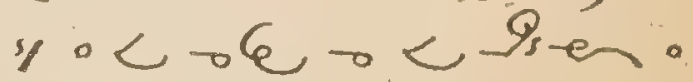
a.

VII. The Commandments. 48553 . Jig beton on 


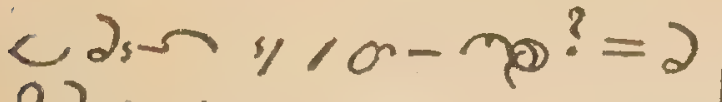

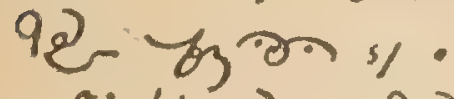

$53.6 i s .20092 .0730$ $\%=$ son sorovor $>$ tho $\%$

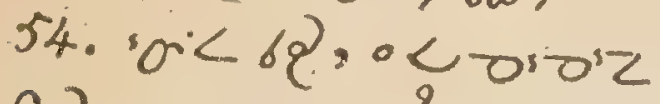

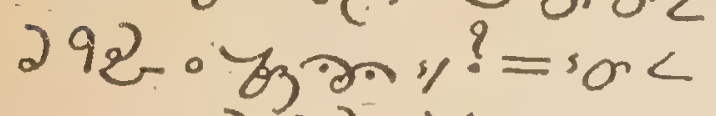

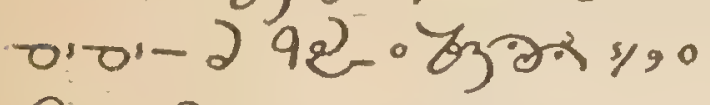
luve" do ope orso= qu, doreverepe: pag< uniea<.

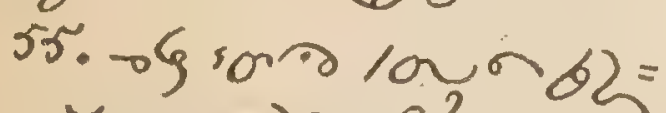
(०) 10\% so "< 《u<son

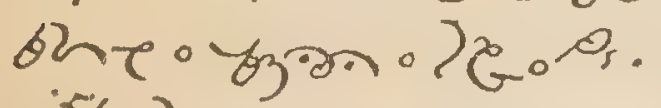
- 56.2000 = ros ónor 4 . VIII. Prayer.

$5 \%$ oce ocie, z<

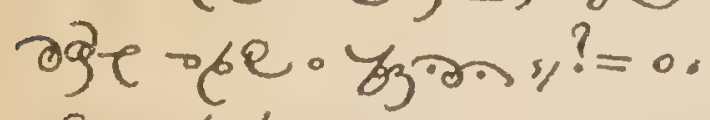
re-<gen" ro< $\partial=$ ar< 0 व अुक्र"

$58.00<690<$ gen $\$$ ?

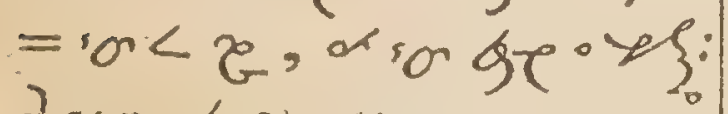
2o, o gen $\%$

5\%. \% ope ou? : so >

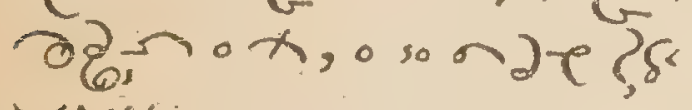

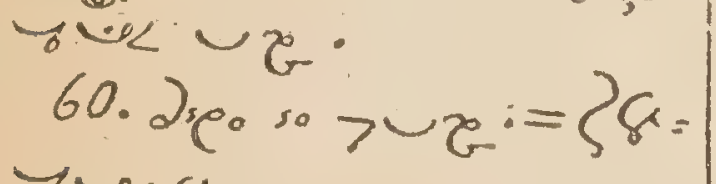
61.4.

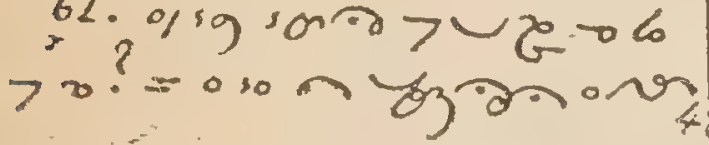

ब. 8 ᄂ

62. 200 so >

गुण 8. 4 .

IX. Sin.

.63. us so oan? = are"

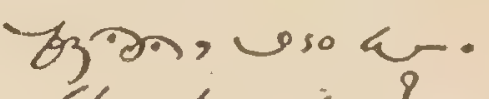

$64 \cdot g_{1} \cdot a-?=200$ a-: broos. 2 -sog< <

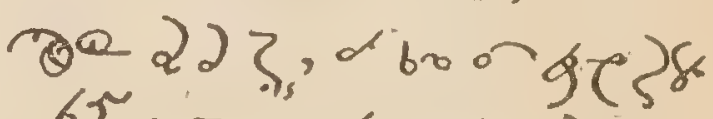

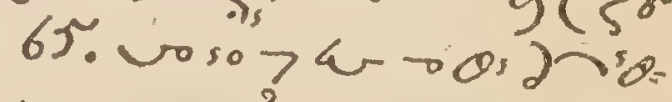

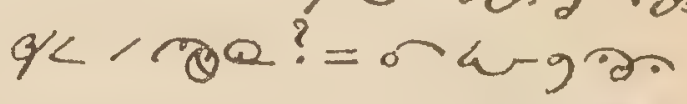
oredownory=

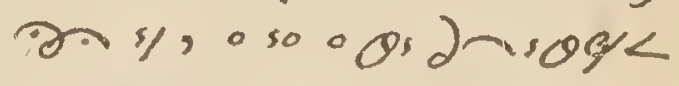
1 to.

66. vo so o a nonge pry? = oso a $38 . \operatorname{yg} 20 \%$

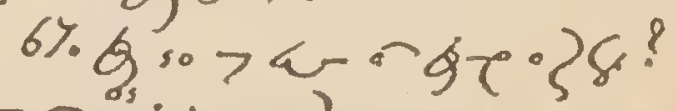

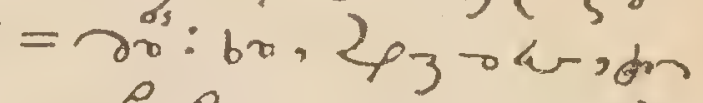

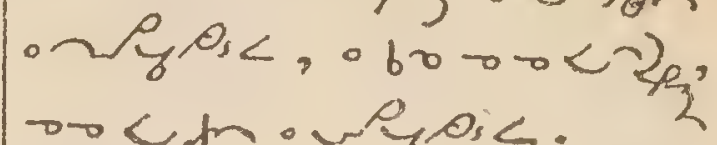
oredn...

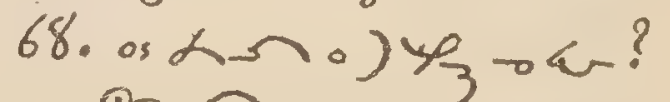
$=0,8)=1, \alpha 2,0$ $\Lambda y_{0} \rho_{4}$.

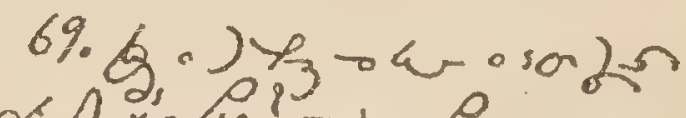

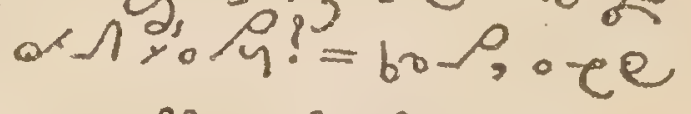
- $<$ in $\rho$ ye.

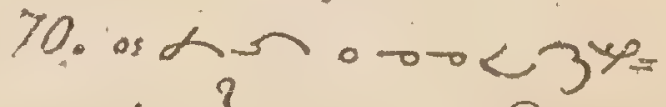

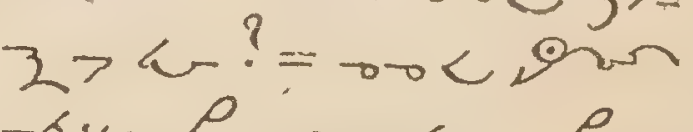
कर <yon<yen y" 


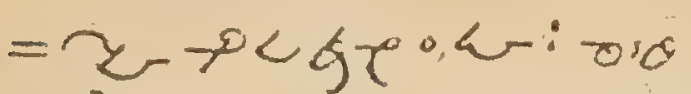

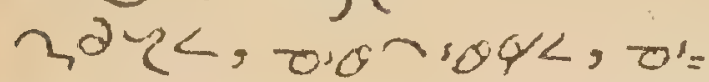

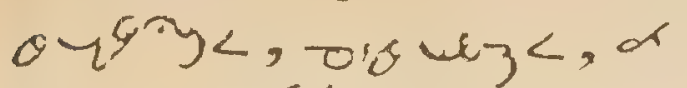
oron $\Omega$ ore.

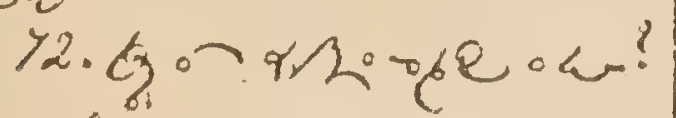

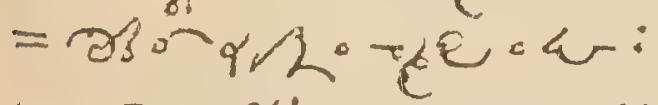

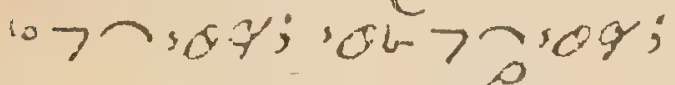

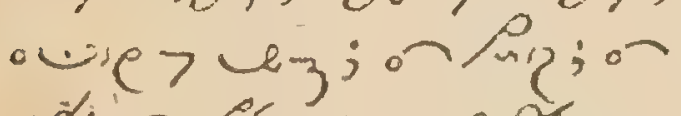
oriorsisn nor.

X. Baptism.

73. 68 ox, 0 an or

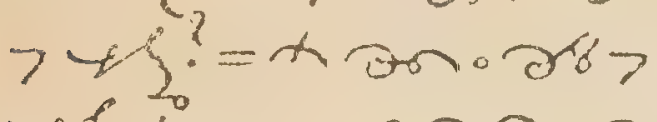

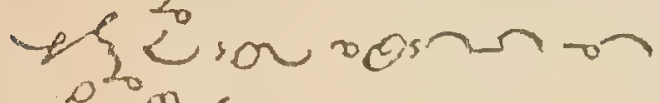
weres

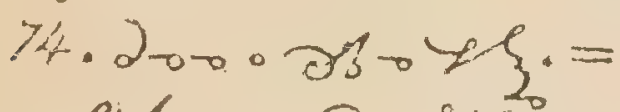
h, sheo, Q, the, चह

r. us so $z^{\circ}=1$ bro $\rightarrow$ thas in $0<-0$, os तog< - तुQ, od sond of

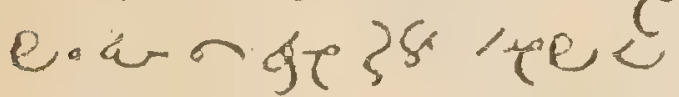

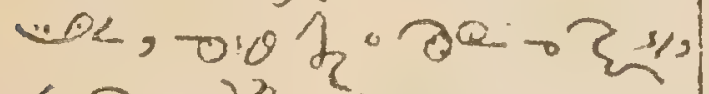
$\left.\therefore \cdot z_{0}\right) z_{0}=0$.

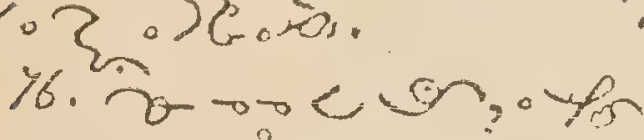

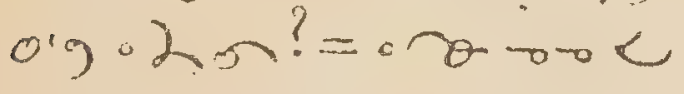

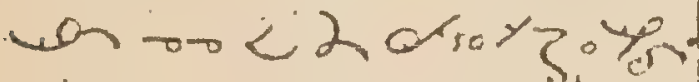
पy. च< $\sigma \cdot 2,02-9 c_{1} ?=00<$ 2. $\operatorname{mos}^{2} \mathrm{cos} \cdot 10$

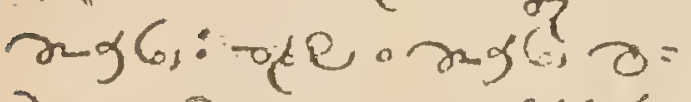

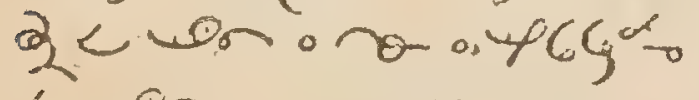
en.

48. os 6 og a son जि, $R_{x} ?=2 x^{2}-2$ of 2 .

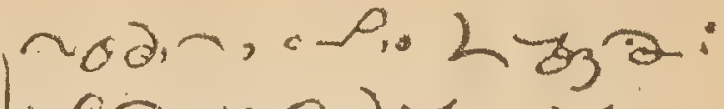
winy so 0 ther. Vye, vw.

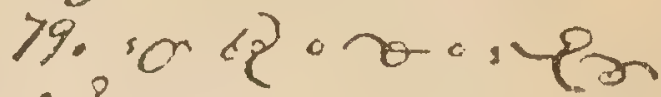

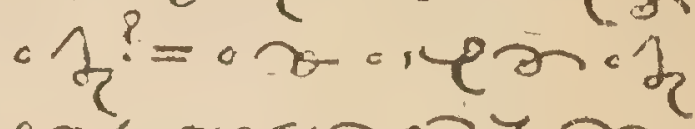
orercesn a byan.

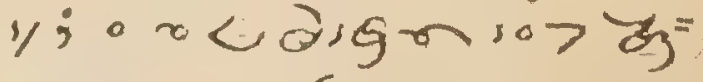
क;

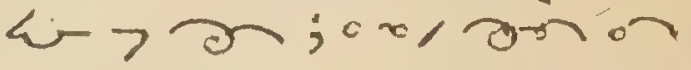

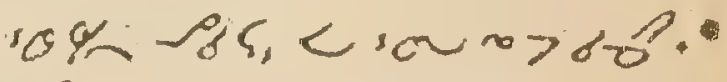
80. ve on. 0 -

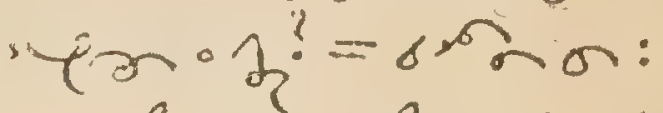

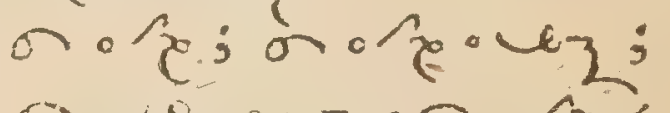
or ope जo a o 'an o ragl. $X T$. Confirmation.

81. v so acter? = Go, to se 7 yk os sw - es kin, in recin on aqu;

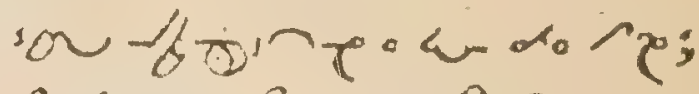

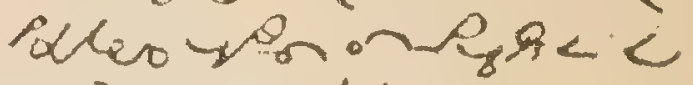

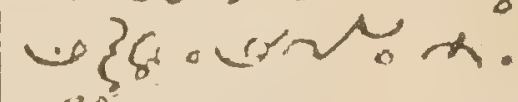
82. Do oratcon.21

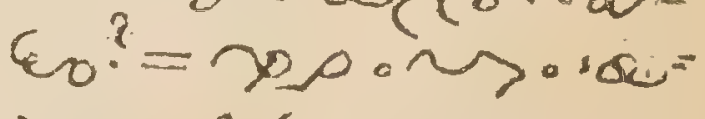
cee $2 k_{00}$ ang 83. or $n$ on $\leadsto \cdot 2$. n. o. $\left.240^{?}=\mathrm{G},\right)_{30}=$

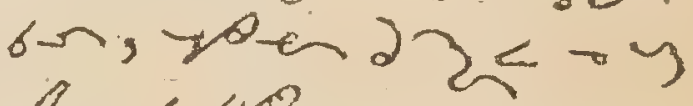
$R$, or whon.

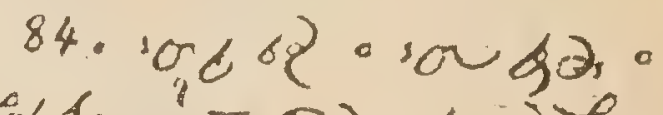

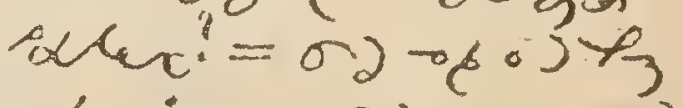

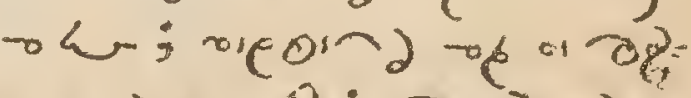
(8) c) 200,$0 ; 0-2 \cdot 2=$ 


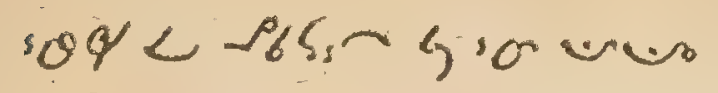

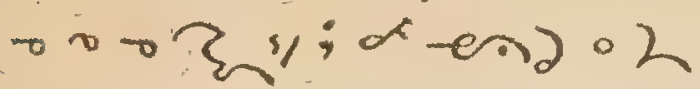
sog, < on hinsw. XII.H. Sucharest.

$85.78 \circ \Theta ?=0 . b_{0}$ so $>$ Y, 0 , 0:- 2 - $1 / 2=$ (x, of $2.200, k_{0}-2$

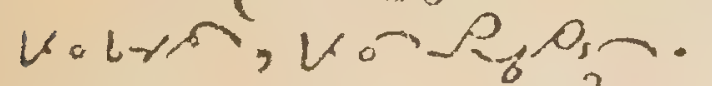
86. 20.0re: 0 ? = x, o मryno, 1:Q6, Y.

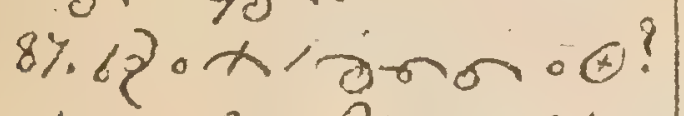
$=10 \%$ Prom ingoversinco Pin rro, opin aro Y, o pin yon: go

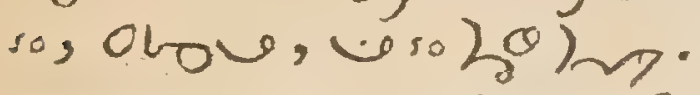
con yon a gr, in ) $\mathrm{k}$ og, o sink

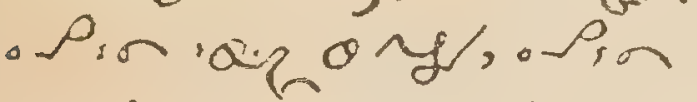
ge: gowro, obou, Us 20 Jurivor vo

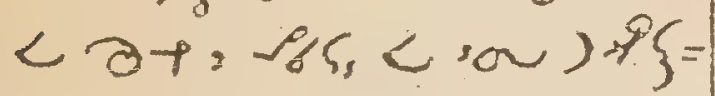
ก.

- 88. y bुanox, wo 20 Li, ws ho jur, - pin dg? = o k3as

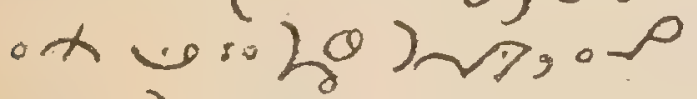

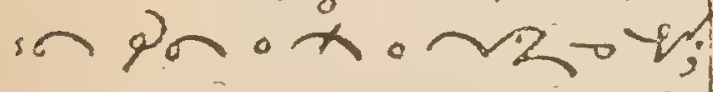

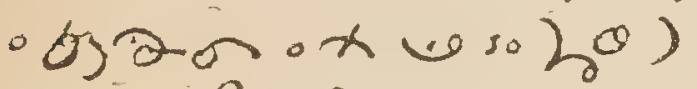
l-f, a pro $2 \alpha \circ x \cdot 1-=$ nog.

89. a $700<24^{\circ}$ प्र 2 rax ax og 2 inen 48 ox; y soracseon

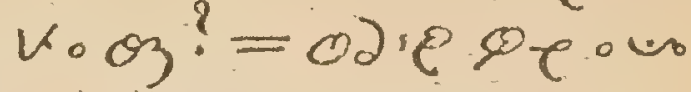

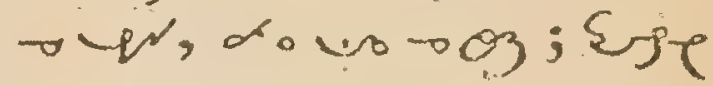
- vo - wo ontojib ) $10 \mathrm{~g}<$, $x$ eve $<-6=$

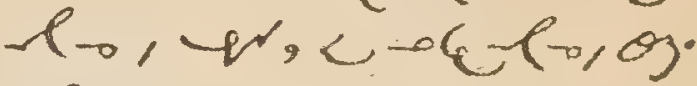
90. w, -

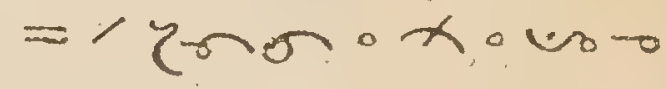

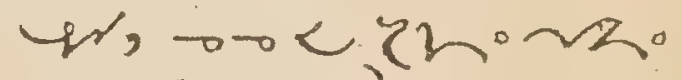
x: Appokon, d re - Yion.

91. - b3anoxo. of is so 20 ) orrox.0. 2 us yr: $\rightarrow: x$ of $\partial \circ 0$ on dusyrink, जs $\rho^{2} \theta_{1}$. $92.68 \circ x_{1} \rho$ का० 0 ?

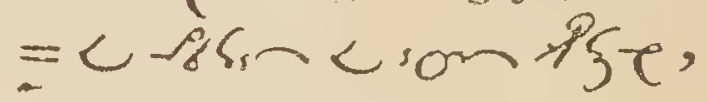
o cuntoemigh nama< - fon 2 \%?, - Lsan alin rar. Sork.

93. Jig val gon:-er

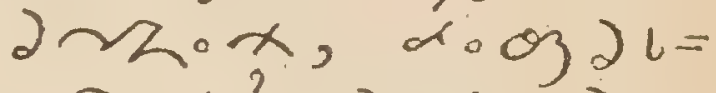
का०ג? = 2 K, d。

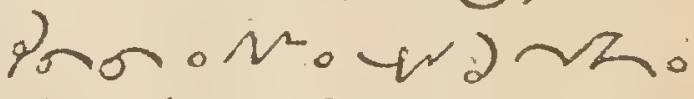
x, d.03 2 urdox: $x \cdot 2$ icon $10>0$.

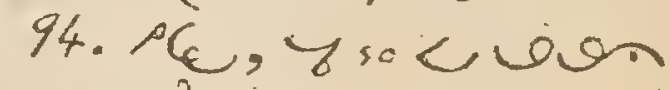

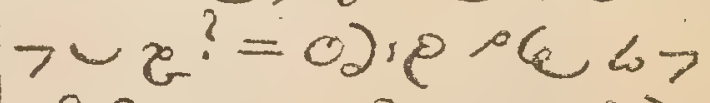

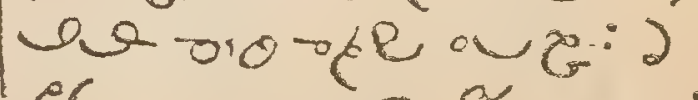




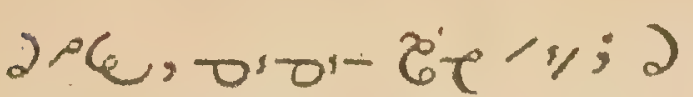
Pदe oro-eor and

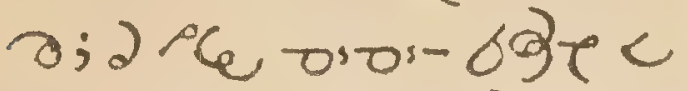
a gen $1,0<\partial_{1}$. हैu⿰ 一⿻上丨

95. A6, yso < yon. $\varphi^{?}=0$ a 5 per orom

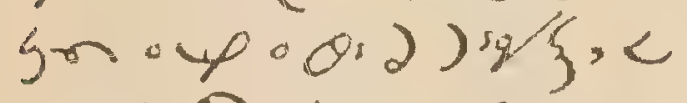
a is on.

$96.67,10320 x 2 n$

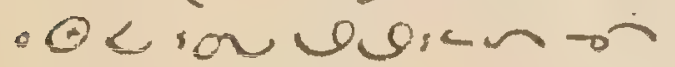
wir. $0_{1}<$ ? $=0$ O n niag

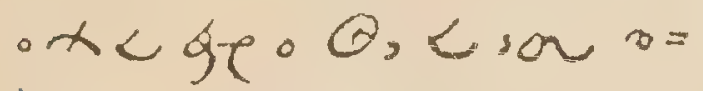
on, oc a naro

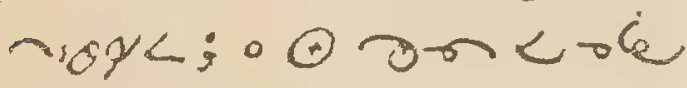
- C 2 . सe 2niog<;

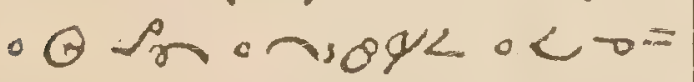

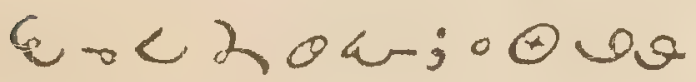
onn onsogk ross

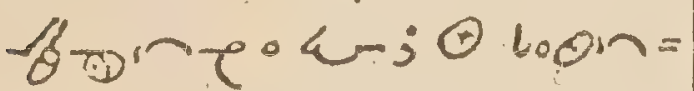
$\therefore x$.

9\%. y<un we the

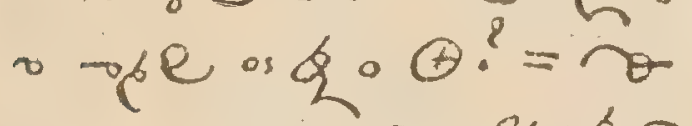

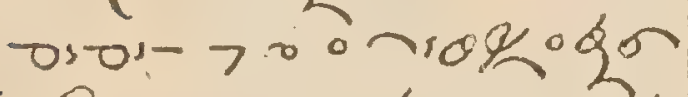

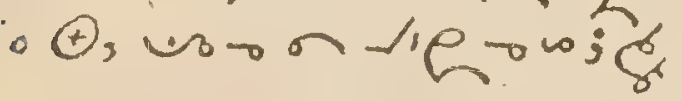

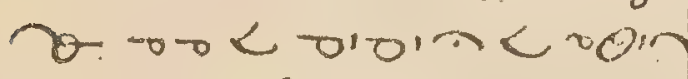
- isog $P$ Q

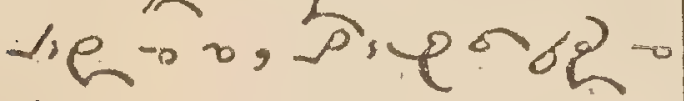
w.

98. $50<68 \circ<9 e^{\circ} \theta$ $=0$ e 2 ge. 0 , on

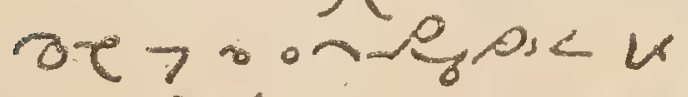
- vol<.
99. sonterns $R_{1}<$ ? $=$ on $<$ se $<4$.

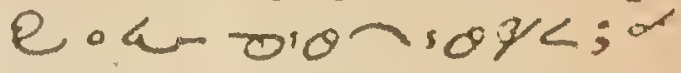
da. 'criever. 100. $00<8$ oc ierr ox $>$ 0.0. ? = o. - ian aigt axdor? dब; 10 lavenik usaljen oxis: cra o ofe.ar $>$ ojoc waverrop/ <-a romege wid son ene oropk ox.

101. on cre? on $\sin 9 T^{\circ} \cdot \theta ?=\rightarrow 0 \alpha<$

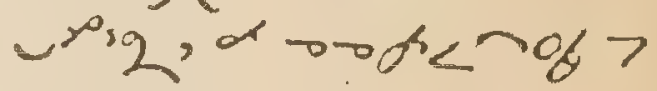

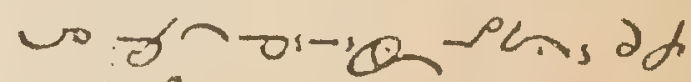

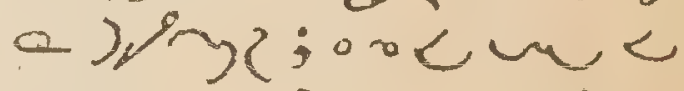

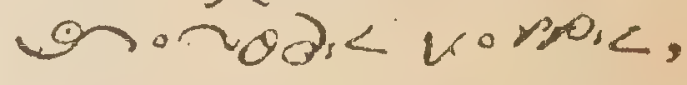
- Luns< a a.ad चा $0.90 \%$. 102. $0<<8$ कर 70 < ग) o以n 0 - $20.0 \%<$;

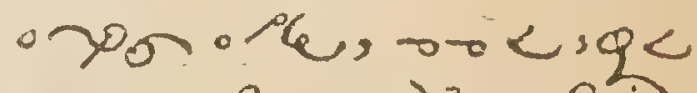
sor $b-(0,0) b_{0}, \theta_{3} ;$ riok d Arse rox.

103. inc eq an 60 - $x ?=\sin x+z)$ qर; 006 - $2 ; 0$ int $48<\operatorname{sen} \theta-6 e^{2} \cdot 0>e_{3}$ 
sobitex awon wan

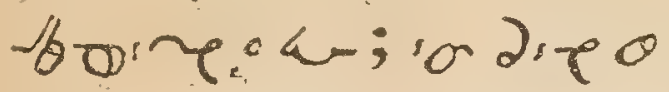
$\left.x_{0}, 0 y<V_{0} n_{0}\right)_{s}<$, - de ovez; sobro $x<-9 s_{1}<$ son bse.

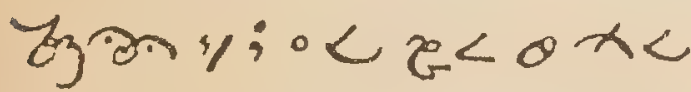
m yon te of $2 b<$. 104. $y<$ m. eston

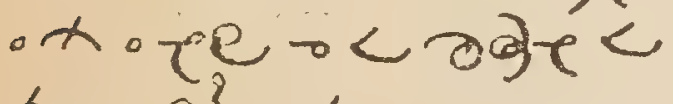
sc: 0 ? $=4>0 \cdot-2=$ con, o e $0<0$. evgr:日: $x$ in $100<$ zerpo, Gsolpgen ox, wosols qnds aso o ye. Xlll. Penance.

105. cs 18 os a gs. rद lee? = os os o पk the $2.20:-2 \xi$, espo, serose, loro. suceno

106. cs $($ be oson 2$\}$ ? - $0925,0 \rho_{0} \alpha$ Liog-

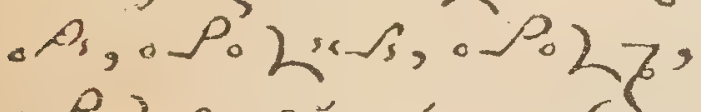

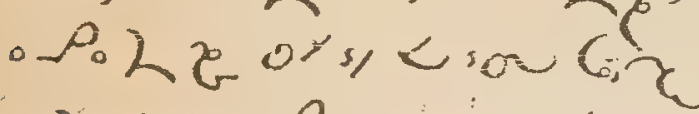

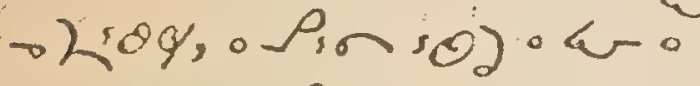

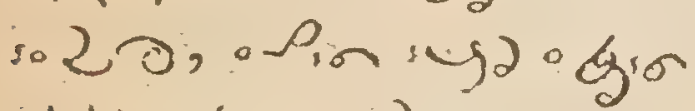
- letsok- so 22 .

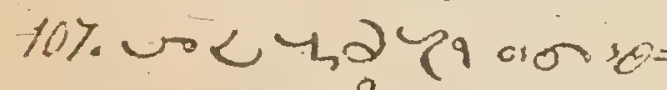
भ० $\Leftrightarrow>10 ?=0,0102$ - k- o so 20 , o pso his -92. - tan क

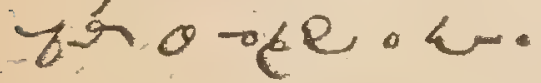

108. arpon<sytok

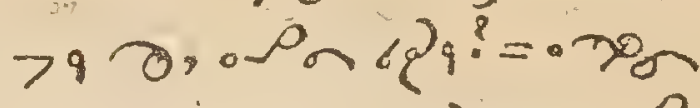

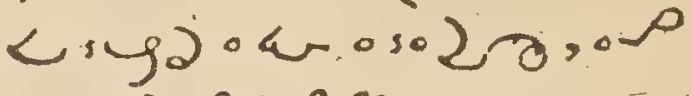
in gे करि.

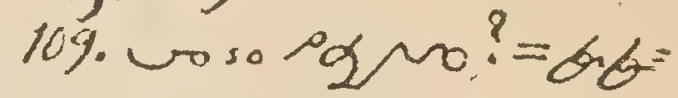
3 कin a a na $10.0 \%<$ - so igro.

110. der lq. songt. odv? = aGS ars <

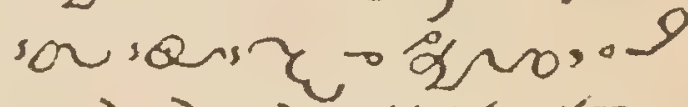
in 2 a so jog ars $\}_{s}$, - Pin ga ruon a ojic

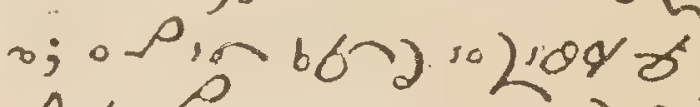
$\Lambda$ \% 4 , owon and

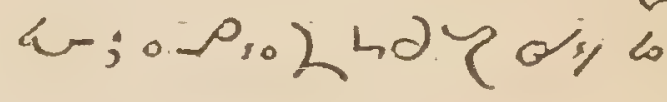
70 l he, thone we 8$\}$, t ison

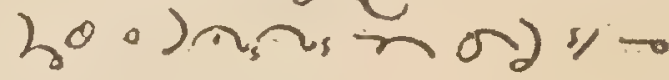

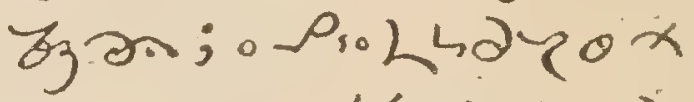
-ron a 9 , pond peson sqon: 4- so 2o; a pro blon so)

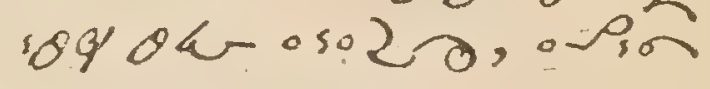

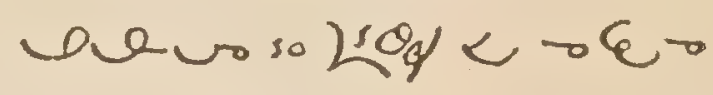
Lר

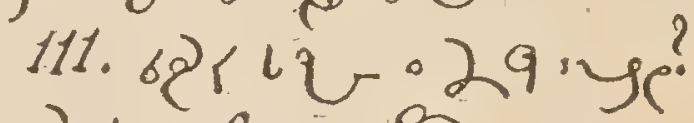

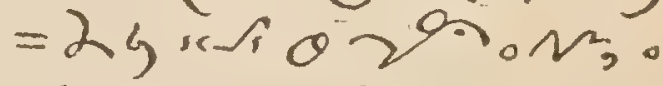
$\rho_{50} L_{\mathrm{C}}, \rho_{s 0} L_{63} \partial: 2=$

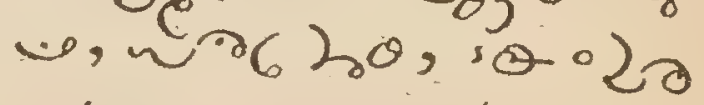
okn. sososuye 5 oys 6

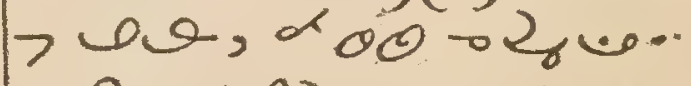

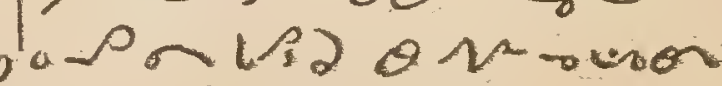




\section{MANUAL}

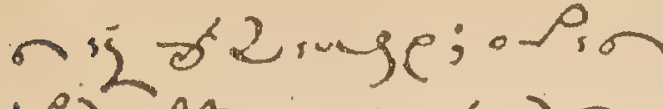

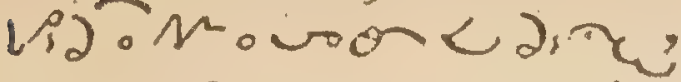

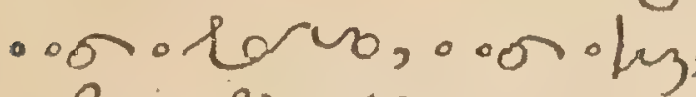
- Prol Lij opravone

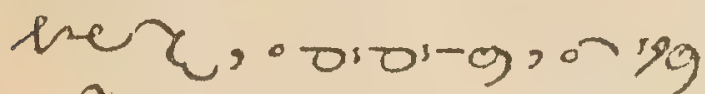
- Psn sya ok osozo. $112.3 \sigma<-6329 \cdot 70$ Cuser? = be o 1040 w.osh hige, do ore

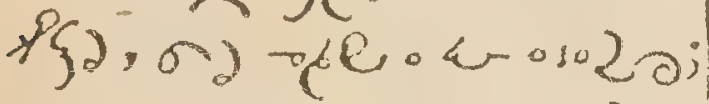

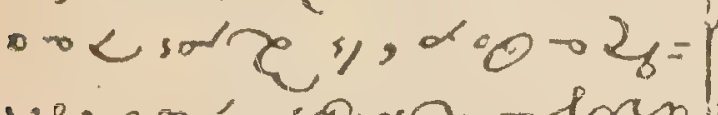
$\because, 00<$ sourncotor nisgon.

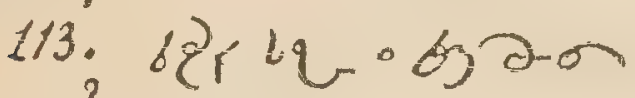
- $N^{?}=\alpha\left\{2 \circ b 3 D^{2} \cdot N\right.$,

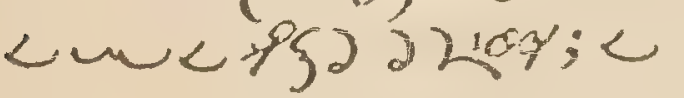
werorevo ovoone $A$ vericusersid

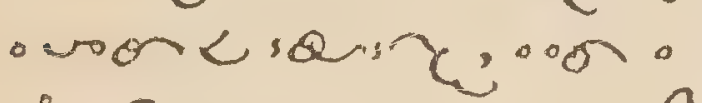
tero,orohy; 9

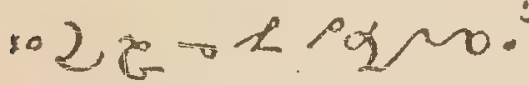

113 bis. ost \&8, odiza

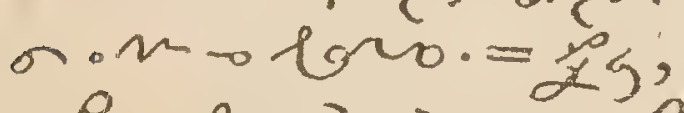
- Sn mod solrog,.

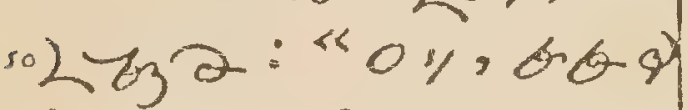
- Lior o ope ou oso ग.00 nll 70 \% म Gevol soljog c ok.

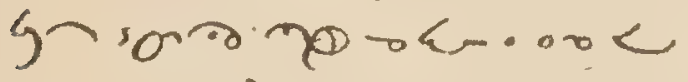
solno $6,20 . " 1$

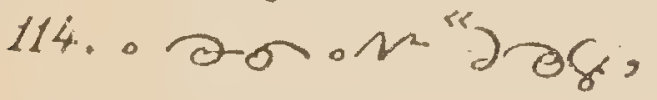

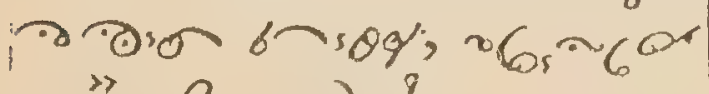

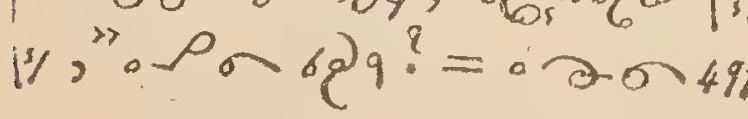

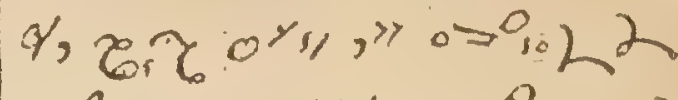
sats ox on 686 , ontion 20

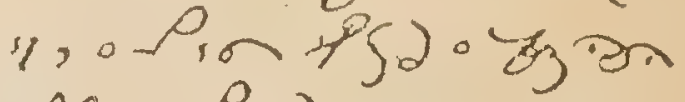

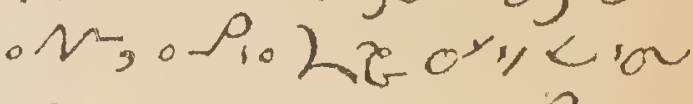

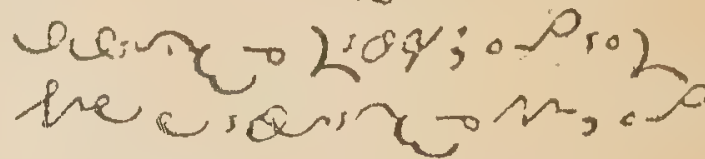

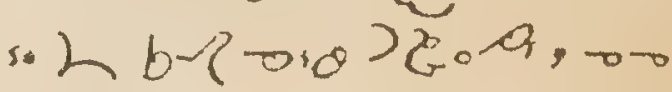
<sqund ma. 115. Wiso 600 ? = ron. r olono, o 16n hoc oso 20,09

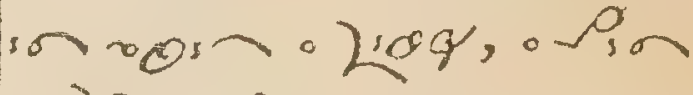
6030 . ie.

116. wo so ohy? =02s=

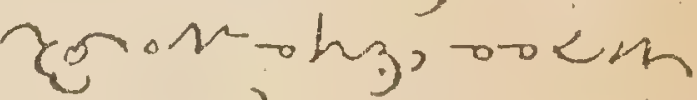
o 2 - . so 2 .

$11 \% .68+19,0 \mathrm{Ce}-0<$

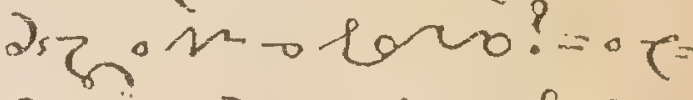

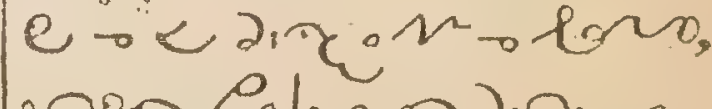
- Pond oh on dr.

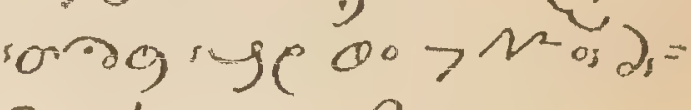
zihz, rpporear o $4-200$ - 2 is; irspe os $>$ r, os nvij

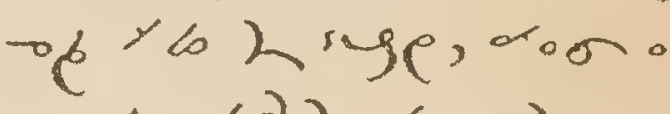

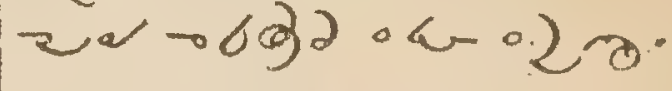
$1 / 8.68+12-0.1750$ $b_{n} \rightarrow L_{0}>902$ s9eq? =

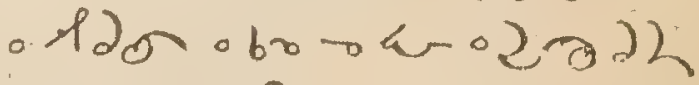

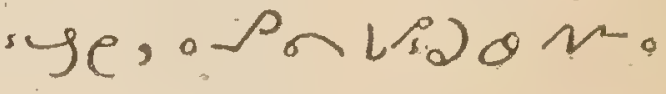
urdon se. 


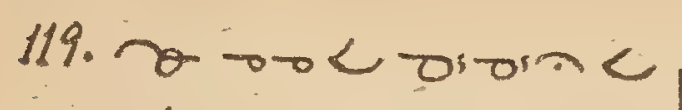
ion o h > $e_{2}$, पर oron<syc ? = or <oitin<son.k> a, ore orous - $>$ a, कर्वा esug, \&, seogo Li: $\sin ^{2} \mathrm{x}$-h y y 120. De-retastise

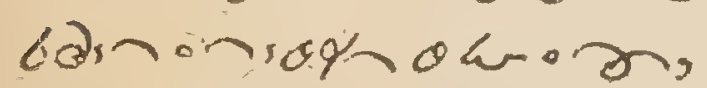
कर प्रान< nem 20, -

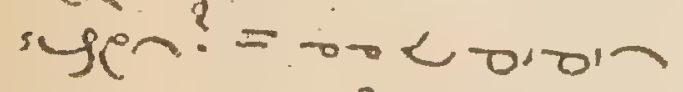
L"yen: Pestom त $\mathrm{c}$ - 4 .

14\% on a k enoboa disg, n- ow Nonsye, y<orom

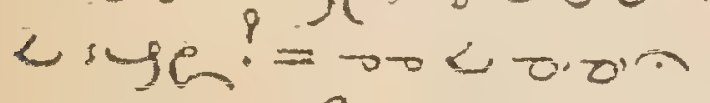
usg, Purnsun bQ $\rightarrow<$.

122. प<Llin.Mnsy - ang $g G_{1}$ ? $=$-o< < ine lin. Maro a sye。 ong $C_{3} ; \rho_{6} \zeta_{1} \rho_{n}, e_{2}$ or sequax.

133. voso Pese? = $s=$

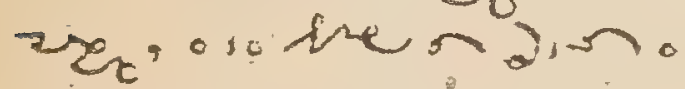
minse, souke $a y \cdot \omega><3$.

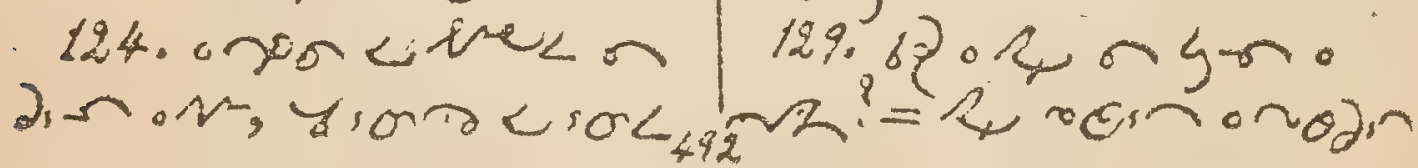

sor<egoriore $<$ oinceroc $><$ or 0 or luno res or.

$125 \cdot \operatorname{sen} 88 \cdot 2 \operatorname{sen} 6,0$

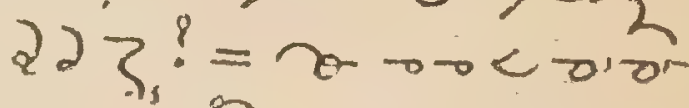

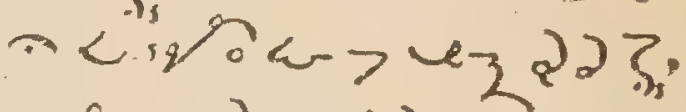
cyor son ar Jig. $126.7<\rho 5 i n<i a$ sin $)$ ) 125 ? $=00$ :

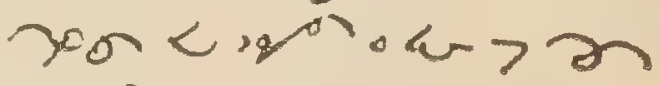
- < 2 sor so $>3$. 126 bis: pwe are. or ${ }^{\circ}<-><0^{\circ}=$ oion

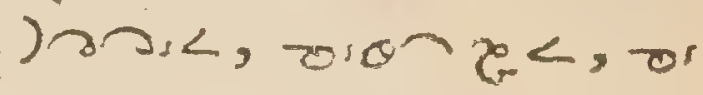
on werogl, oro oke $x>0: 00=$ C बCE $\partial\}_{i}$

Yur. Extreme Unchion. 12\% wrok2 202 broso $>$ y sa yon.0. noarm : P. G. 128.68 .2 or 5 क 0 Sên? = स gron yon as yey, d wein

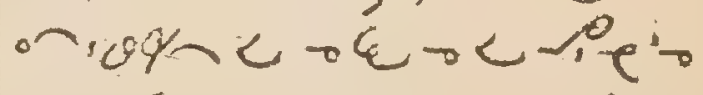
กon 5 .

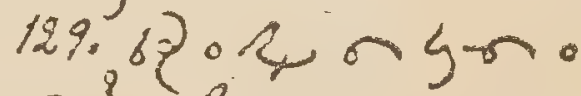
$<><$ ig o $<->e_{3}$ 
- 90 \% -

130. '0 68. o ostor

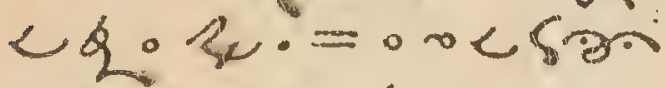
okn ondereng.

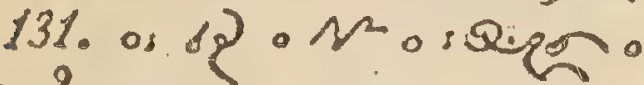

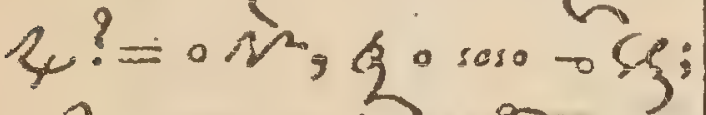
yon 0.00 o) $46 G, v_{0} \rho^{2}, v_{0} Y_{h}=$ 2, wo vorn, wodkn, katgrn; o Prom: oclim ys rober.o (o) ojoth, (onf), of $\mathrm{Ll}_{2} 3$, + 0 290, कo dls, do dibs-. XV.H. Orders.

132. $\sim$ sog? = of bos. $>$ y. कn oxe (2) $>$, e a $02 \delta$ - 2ngG, of

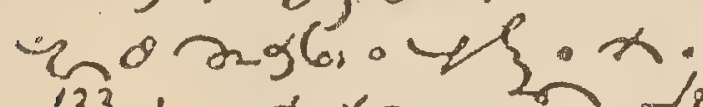
133. wrxyon कof! $=x$ rorono $\theta, 2 \alpha \cdot \alpha$ arr. of: 2orkeno

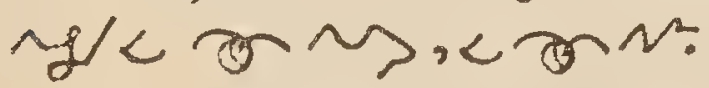

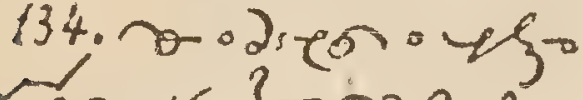

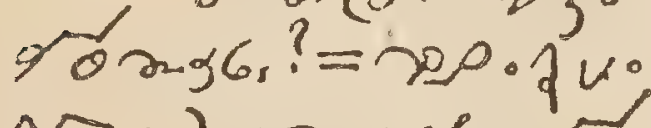

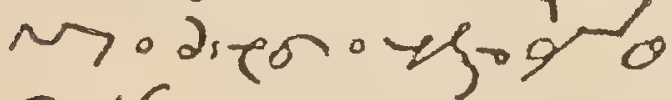
ang $C_{5}$

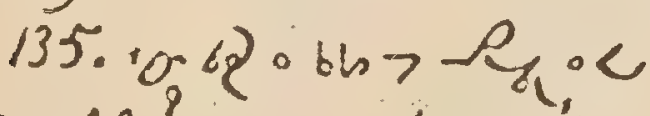
on $M$ ? = ox

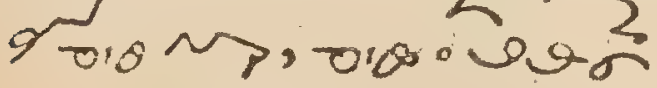

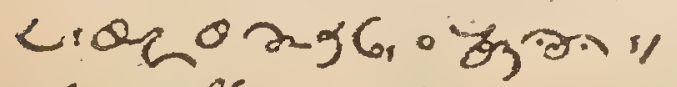
ar: yspox.
XVI.Matriminy.

636. woro $1 \mathrm{sm}$ ? = r $\mathrm{sm}$ bo $13>2$ 2. $13 \%$ monsenor

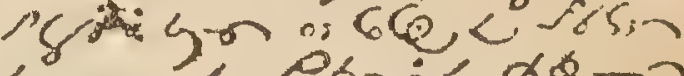

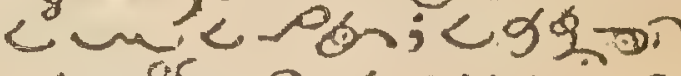
o<-85. $2<$ rea. ¿zer a sevons.

138. '0 62 20. 대 n ro? = o r okar "Lisa be, no oc ma< 5 ;

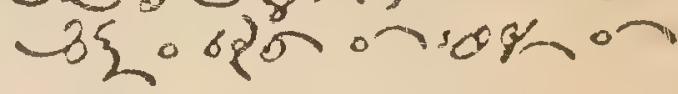

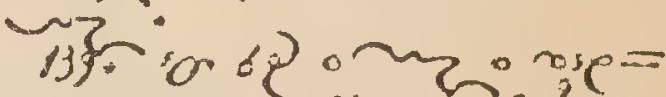
orno 0 .

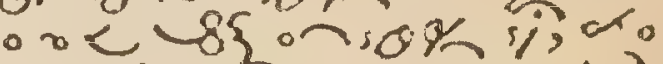

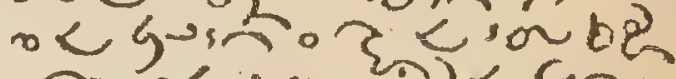
10-o<m inde sin. 140. 18. $10 \%$ " की nz. $=-0<$ cogn on en Escian sin; $\rightarrow 0<$.0\%

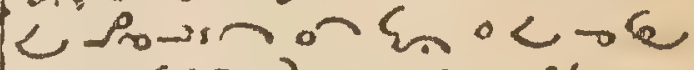
- 8 in 30 - $10 \%$ m

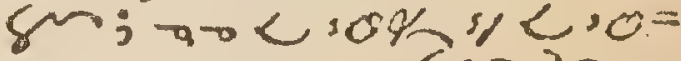

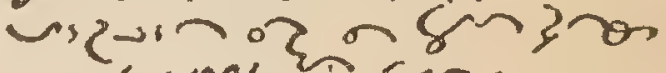
का दा

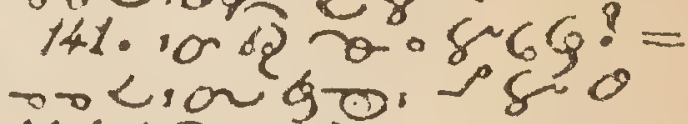
wa; \& $>20 \gamma$

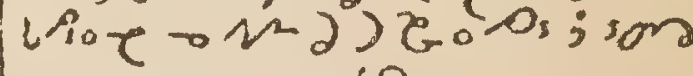
in morn ake ourvo $260^{\circ}$

$142.09<950000$ $<$ व 2 gin ho $\rightarrow 0<$ ordine gm.

0गे:920z-) $20 \cdot D_{1}>3963<-60$ <

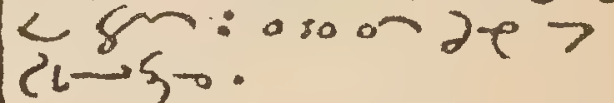


$\therefore$

. 


\section{Lillooet}

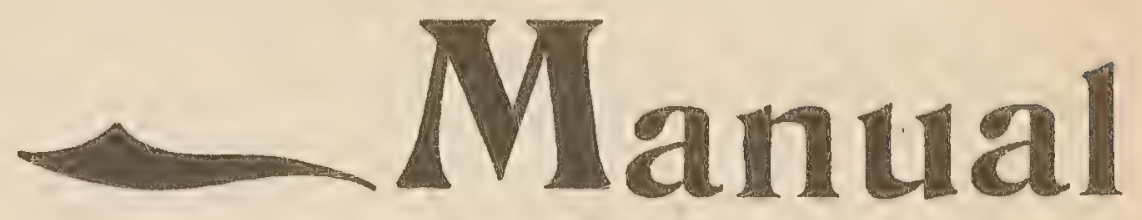

....OR....

Prayers, Hymns and the

\section{Catechism}

..IN THE.。

Lillooet of Stlafliemoh Language

With the Approbation of

RT, REV. P. DURIEU, O.M.I.

Bishop of New Westminster

KAMLOOPS, B, C. 
Lillooet Prayers. Morning Prayers.

offering of the day.

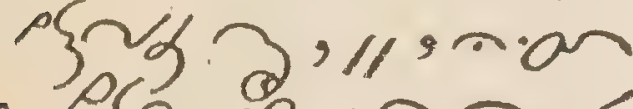
a PQosina do प, 5 2imcos, 29

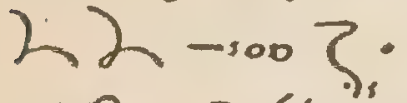

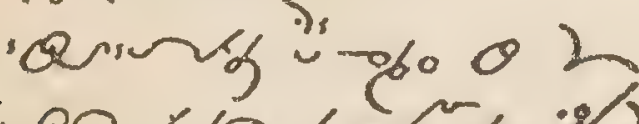

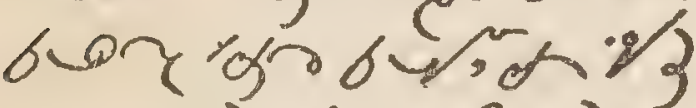
- 100 Liz $\therefore x$.

a 620 sol $\$ 620$

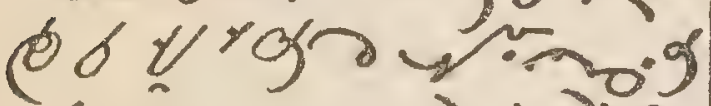
er coneusn 2 hayos

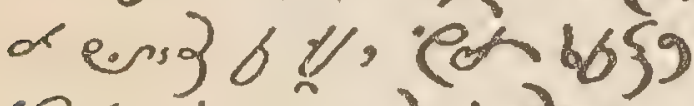

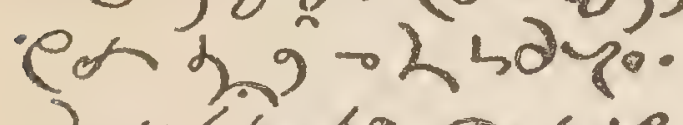
2 , 2 g9, mo b riv obo on यsm

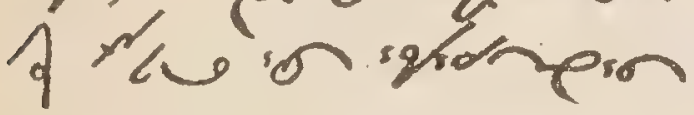
11.

$08,6 / g 2,-2,50$ sw on usm 3 ; "an' $\alpha \rightarrow$ gi, Nh"

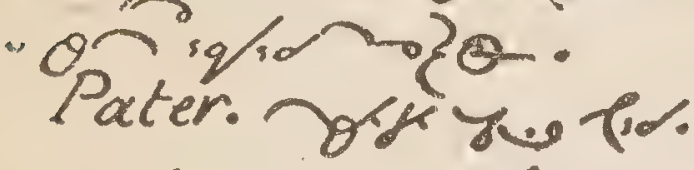
Dotryer, a'bsoo: 6.760 osols onousm $=$ का अo jman

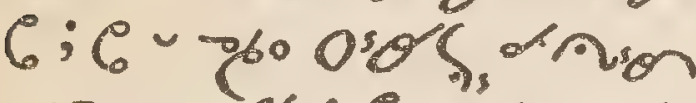

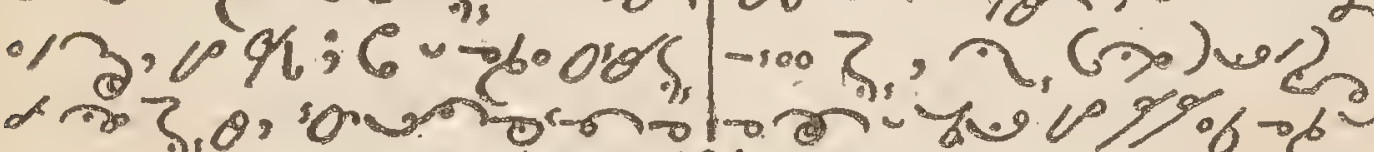

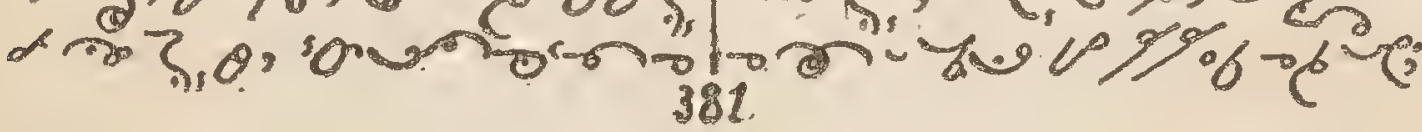

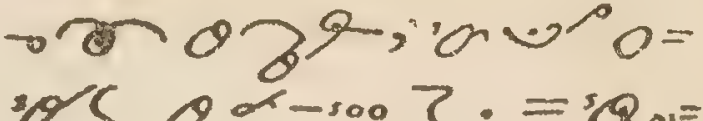

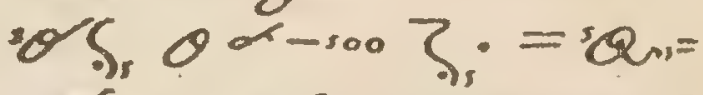
\%

6. Hor o dyet bor. ovezuofo n

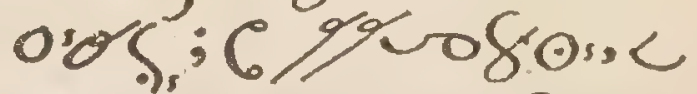

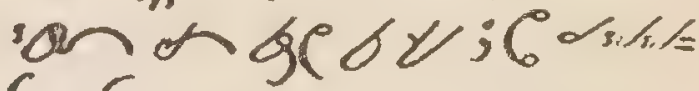
jogrost son breig 9 . 6 -ysn.

Ave. of $\mathrm{s}$-jo. $=0$ 8. y ye, "od 3 ; ब1 106 of 29 m. कc को $=6,8, y^{\circ}$ on 11, of

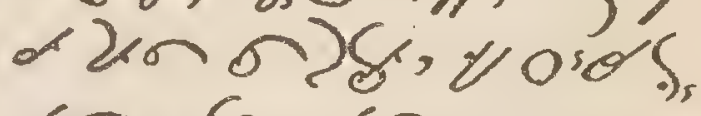
diri, Godion Yqu. 6 'sín. credo. 02.03\% $=0 D^{\circ} 0$ के श्र०6 or, 206r 06 soo $\sigma_{0}-\omega_{0} \gamma^{\circ}$

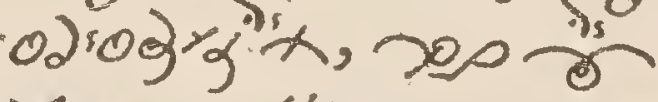
ton, अfforizm 0, $\pi, 0$, so 8 , w sctso; 2 solso $N_{0}$ so 8 y

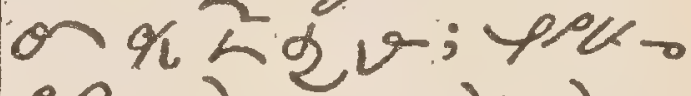

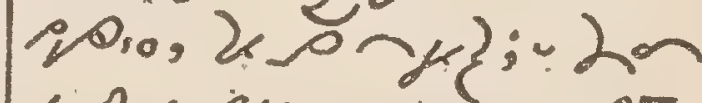

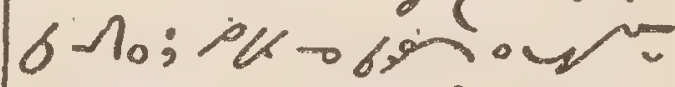

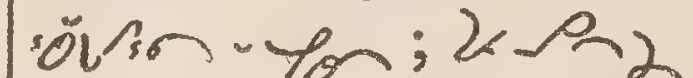
वा 7 प 
rewnon, isione - 6000 $0^{\circ}, \sigma_{0}-\bar{b}=$ $02.09 \%$, w, Go-Cso

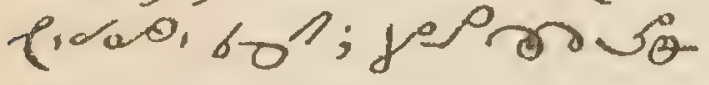

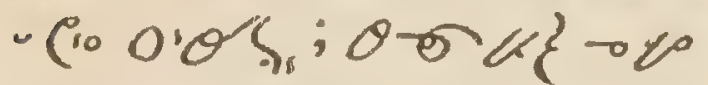

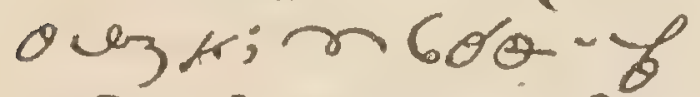

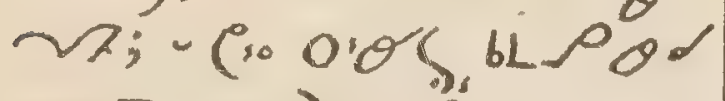
arno $3 \cdot O_{\text {d }}$,

confifiteor. osoǵgrgso

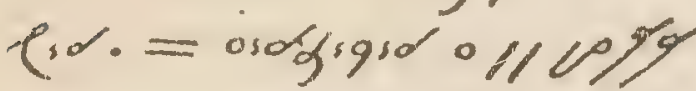
- b o c, \% 0. 68 แL prag; of $2, \%, \pi, \pi / 3$

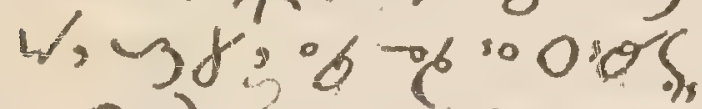

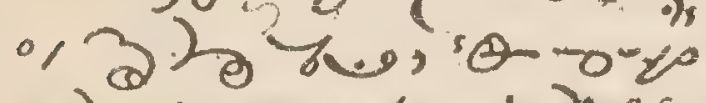

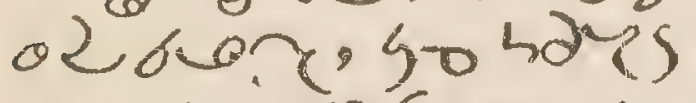
- 50.5069$\}-20,50$ bor $25,5 \mathrm{~kW}$ ए

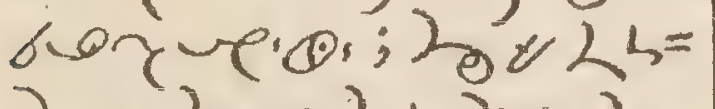

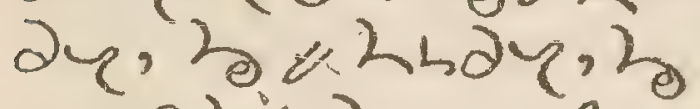
$y$ is Lisdy, .5:

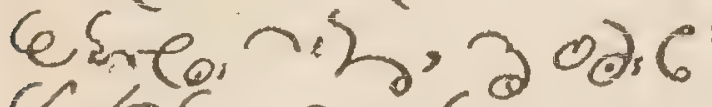

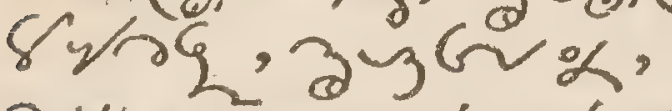
उ*, ब1 $\mathrm{y} / \mathrm{z}_{\mathrm{W}}$ $\gamma$, at of so $0,055_{1} \% 3$

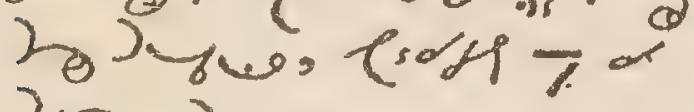
20020

Misereatur. $e$ sog. 11,3 vo of ow? Pincos rót von con. 2022 s. $3: 6 /$ sin.
Indulgentium. 611

onc log.of ob ve. ernogonds, savis =

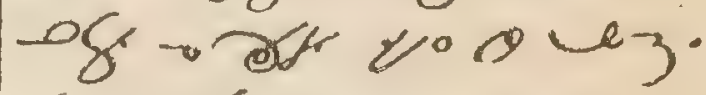
. 6 - sin.

Mandata Dei. C $^{x}$ $1 /$.

1. $20-1 / 10$ osoros. 2. "a br oัk19 znmall. 3.6 br on ens in.

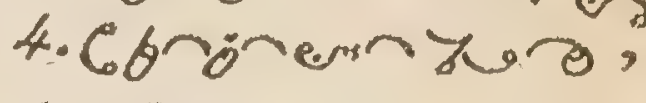
tivere.

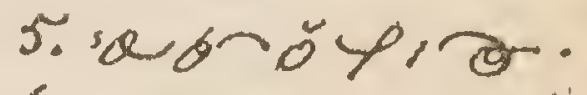
6. 'a ón carns 7 sa or 0 .

$8 . \sec 60 \%$

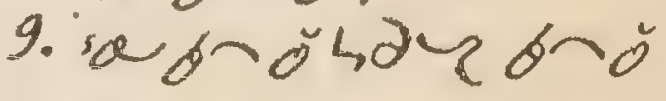
os 7 б.

10. "a bro"say bro" Q)

Precepla Lcclesia. - S of joos.

1. Cobonamen ro (so Nos ain. 2. No.6. \& i- $10 \mathrm{C}$ 6 ob vin. 0 . $6.06 \%$.

3. 6 . o ososg.s.

k. b-o niong, mion

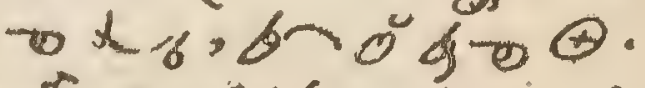

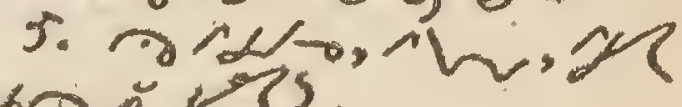
382 br o. 
6. '\% ratersa b 0 S 6 . .

Septem Sacramenta. xद次然:

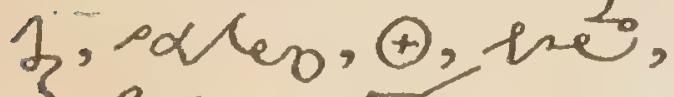

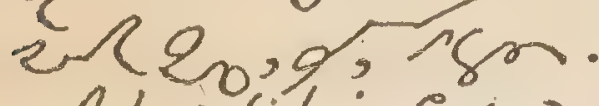
Actusfidei. 6 y $02509=$

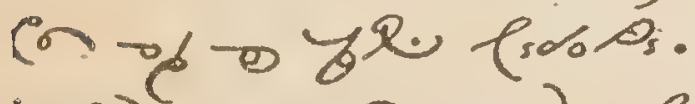
$=02, \theta 11,2002$ - soo $\}_{s}$ - (so osols, $2^{\text {son }}$

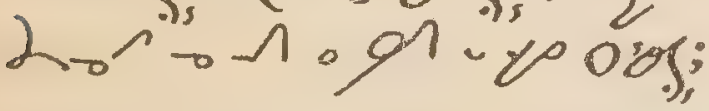
"yos"ly ysi"

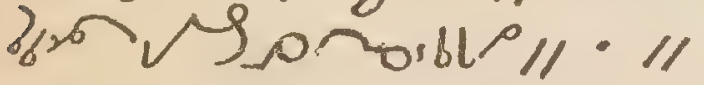

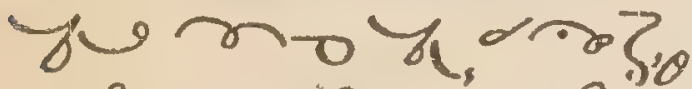
- Por o cll a Pso,

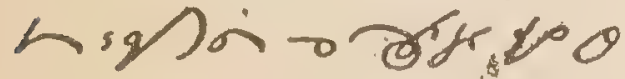

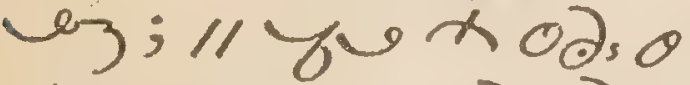
ब- $\Theta$. $01102509 \%$

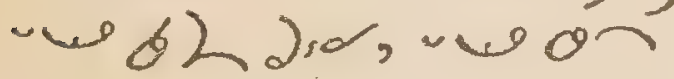
Qsduofen apsoli; Q) asdrso $P$ la

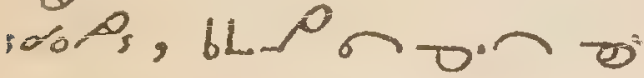
\% Spei. Cysyena uayto on sarised il. =. 011 an bs osol $\xi$

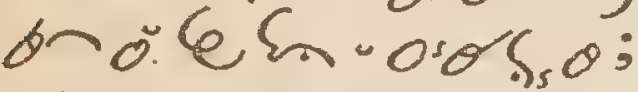

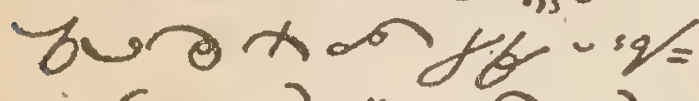

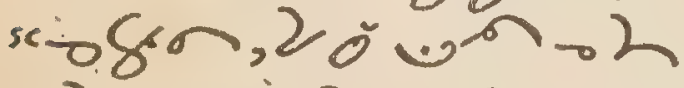

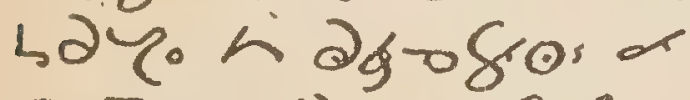
कर, $0.62 \mathrm{mco}$

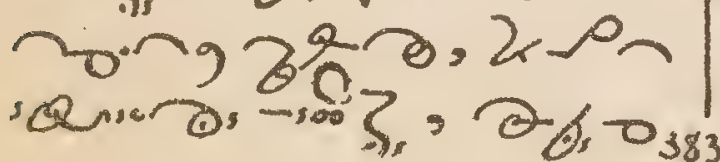

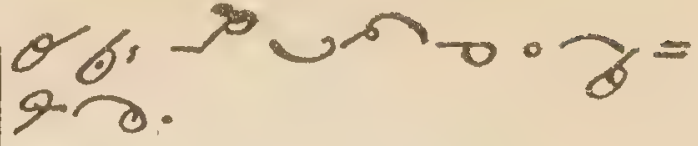

Caritatis. 6 ron sol $\zeta 11 .=011$ - 6 . $6, " 20,0$ salk, जos एurgorolsy vol obue; org sork urobo o. lidy lo sorkकl. न2.

Contritionis. $L$ equo. $=0116 \xi \sigma_{g} \rightarrow 200$ 26 ar; 2 - 2002 bar on an moo? 2. 2.1.9. vog o 6दa -bury. - $2=$ रे०, 3 so $11,3 \mathrm{cl}$ 2...

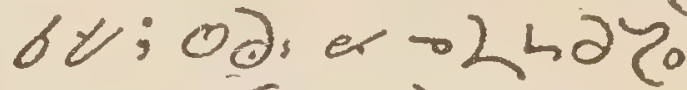
$4 \operatorname{san} 6.62060$ V; 6L g《e onoum -2hyy० hion b秋人 पद्वि:

Salve Regina. I $M$. $=068,300 \%$ \%

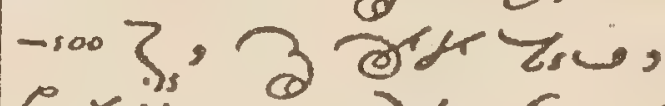
6 rus 0 agrof,

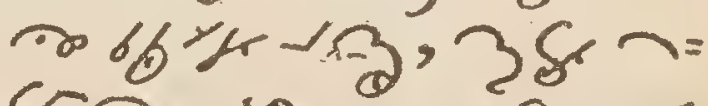

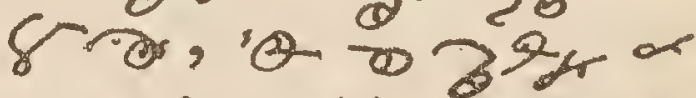
अ, $x_{3}, 96=3,0$ कर 
engr coresgofos. 068 , g्रक bn og

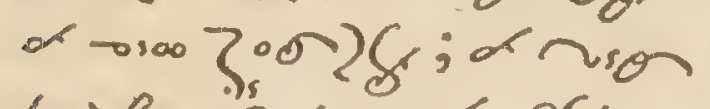
h tor, 6 ' eurd wogor \%or orare. Angelus. in rid.

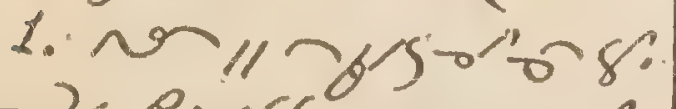

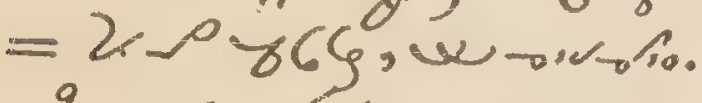
2. 工 $2_{0} \cap 011 .=$ 662 जा को

3. Jun m $=25 \rho$ म p. O truen II fidos or यन क $2 \mathrm{~g} .=5 \mathrm{~g}$ cos, का पर कर susiogin.

oremus. 011 bir का oje or oryo may; \% n m a

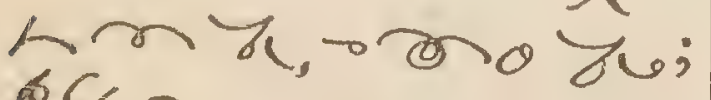

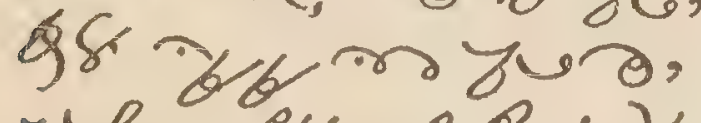

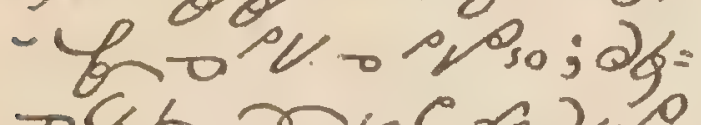
ofh ror 60 s 20

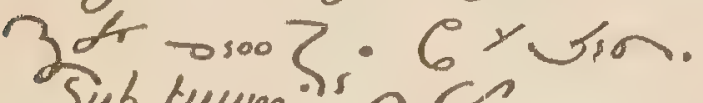
Sub tuum: 08 . or, 6ros a ton II, 2y sos: $2: e-85=$ vogbs ciang 98 . 2 ss of bs 0 y.

Night Prayers. Adoration. 011 ser $=$

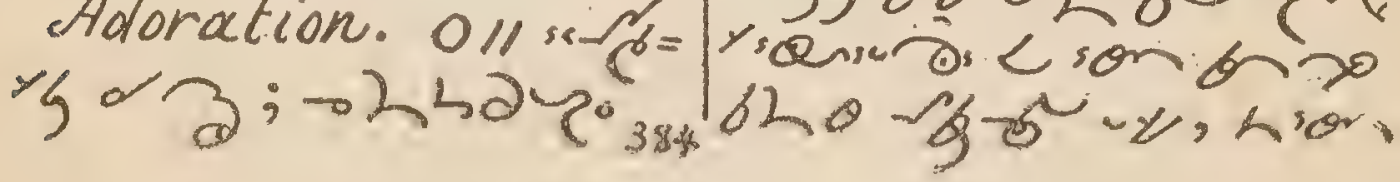

eusgnon की ब1m;

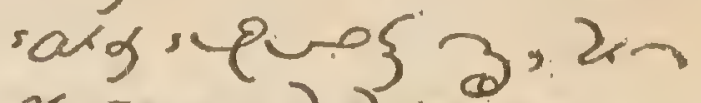

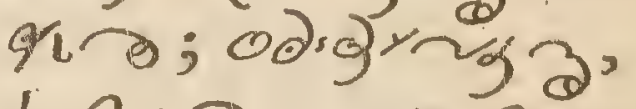

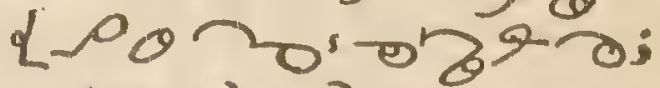

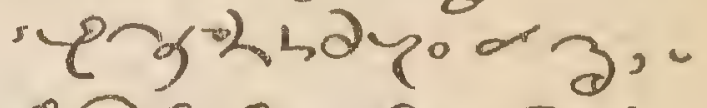
o jor olsaritis

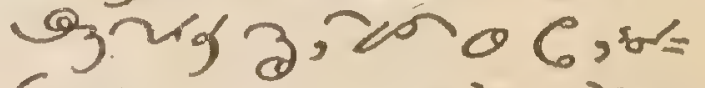
frg 3 of $\alpha$ - bar L6 $\theta$ dg 6206 ; sorfy - bo oifs win 2 ,

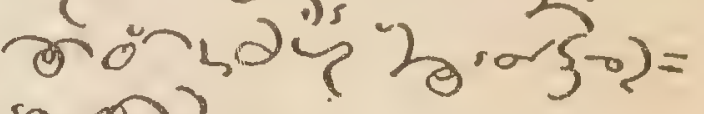
s 02.

Thanks. 6 ×

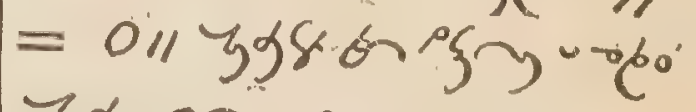
उ9 onsans. sarg o r bेयद b;

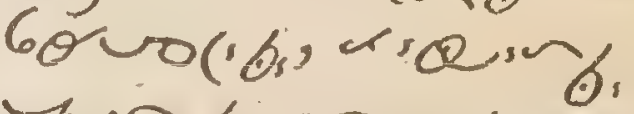
zor Gue havosit.

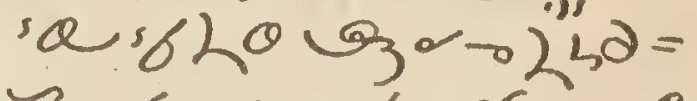
6 aren b s $y \cdot 6$

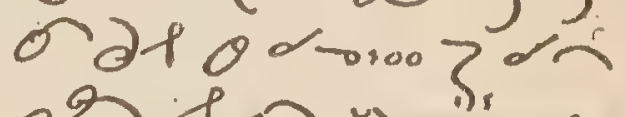
ol if on ynis ara o $\} 2 T$.

Praver for fight. $6 \mathrm{6} \%$ Th . og ofo. = = II.

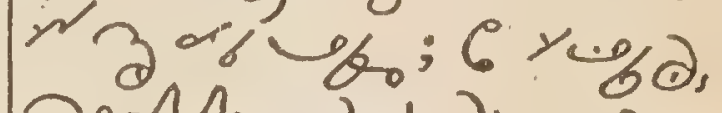

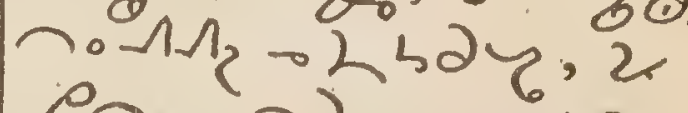
ening 93966 ,

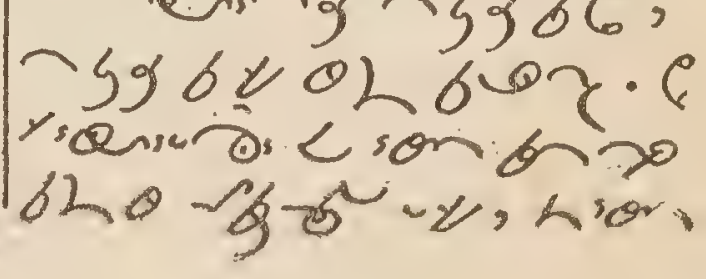
o. ba 0 sanin to 
ongg bhosbobor be:

Examen. Cy'ssand gon biv on borad бr.

- viro siney arz, ongore, nerin 39 ter hor. or dif - Lha to - 20 onic $=$

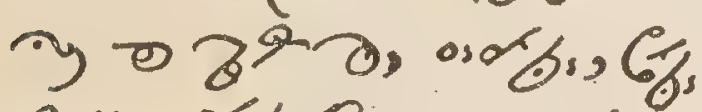
OII, Org Q070idn c-o: pospy ong

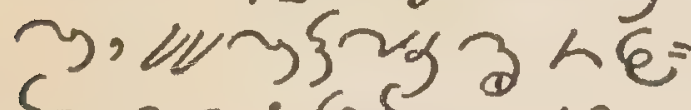
kero; cen pisur $\therefore 6 x$ b $99-2 b)=$ To 6holorsute o herz.

Firm Purpose. Cogre

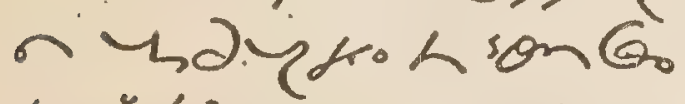
$10526 \mathrm{~V}=0 \% \mathrm{Ha}$

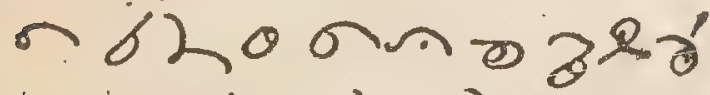

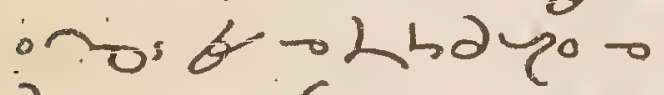
20 a o jy a; 22 64; or serbl LOL - So; oysieb Lloun Diana 62166honens -2hay<iong. 人ion 264,62 L co-g- $-p, 62 \alpha$ erobs a on $5=-192$ नryisen on $208 \mathrm{~s}$ oryong on 0 略. 385

Pater. roysoria Ave. of 1 po. Gredo. 02. क्रु Night offering. OII ssiniz 5.62 m ulgonn us) ज. 068,03 ) 5 No, ks 996.09

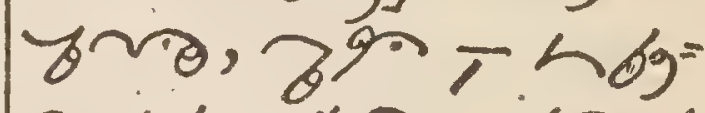
o b V gro 6 ma, rion b) $\varphi .6 \times$ sin. Preparation for confession. Leader. = dororgo Ap - $\therefore$ - propio, QPos. $0.05,0$ <2an $60108=$ sodor dforon. zariotos id is olezts dinol. Gga vllowizt. $\times 6$-scrot, $\angle$ sog 1

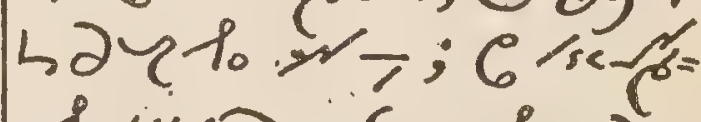
ot $80-\cos 10,2-$ on iv dat ov ougt. $\times(6 y)+12(2,5$ bún cyplónas Lsank tow edor. $x>12208 . .$. Prayerfortight. oII

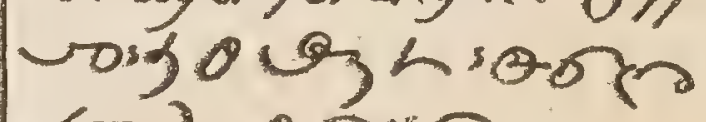
art hoon.. 


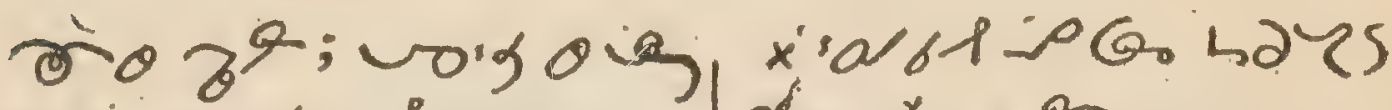
- idi $\rightarrow$ to , s o th? hoxeign; Links 30 - 60 ) $h$

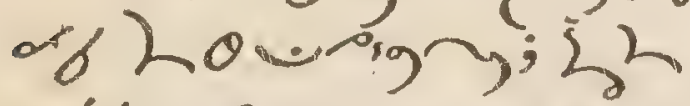

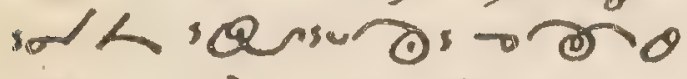
je, $202 \mathrm{e}_{3}, 0.605$ (s, $\rightarrow,-60 \% \theta 2 b=$ or. $94-2426$

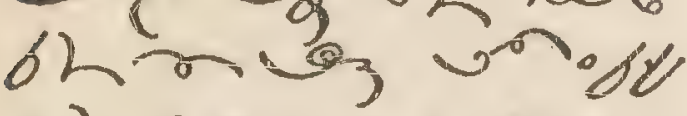
-horing aro उद; wos bfy 6ho blर -थ. $\times 6$ × ojo $111<-\xi_{i}=$

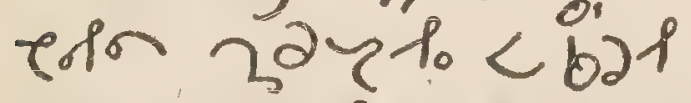
- to ovis $\times 6$ ergtiong

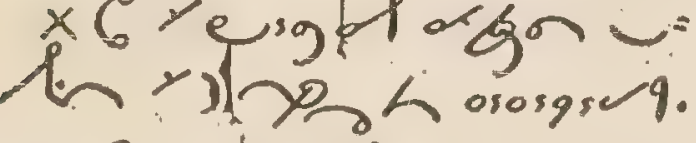
$\times 6$ y'igot arver 人iquspotarin,

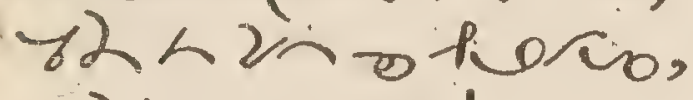
पih 20 hz.

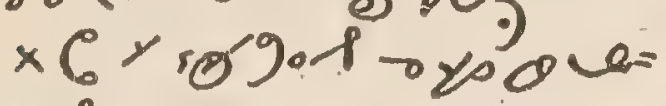

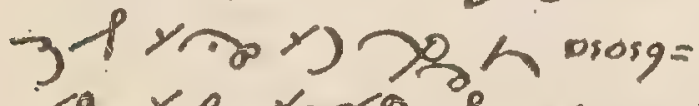
iA Ys rsogot - 20 ezt 5610 2, d तन 0 ogat 64 $\rho$ ค, $P$ Q o o r ros sol

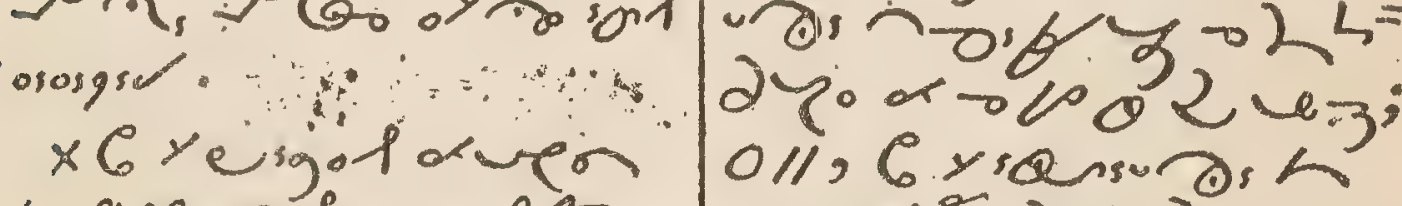

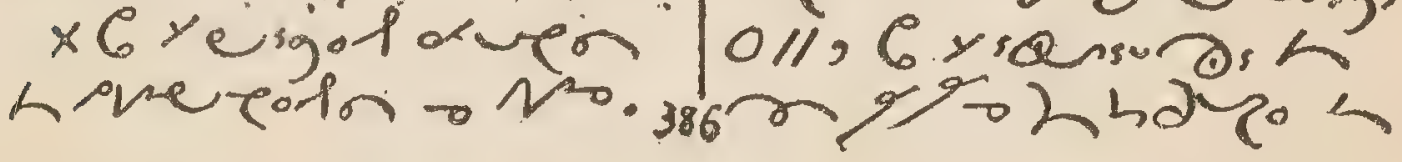

=6brónens k. $x$ ald $P$ Gaid rep Q

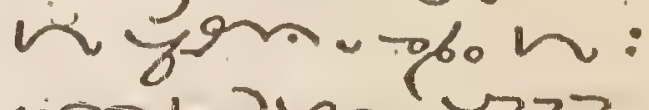
sombays, Thरेग, or ? $63.683,06 N^{2}, 061$. $\times 6$ ×. lon "ion Durain= -89n"l"abort. $\times 6$ Ysg.t agora 6 100 $640 \mathrm{Cl}$ -

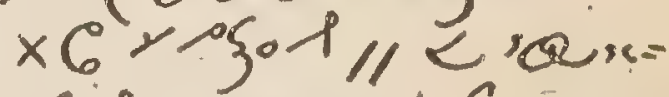
-6Pro of

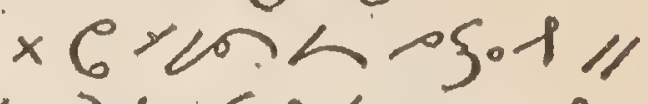
人 2 का 662.

Prayerfor Contrition. Oll 6 'Yanirosnos

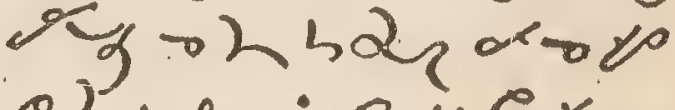
oL ${ }^{3} ; 0 / 16$ isas खा 2 र or $01002 v_{-3}$; 


\section{LILLOOET}

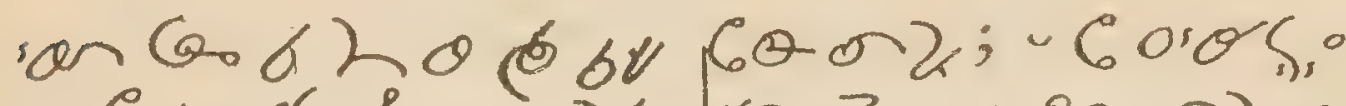

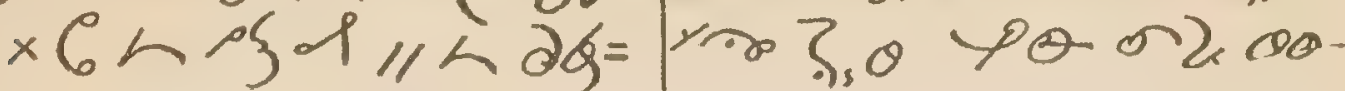
- $89 n h$ b volo d 1 y or ret.

"

$\times 0 \| 6$ "iqundar on $93 \mathrm{ke}$ 々 मेद

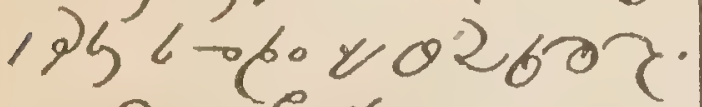

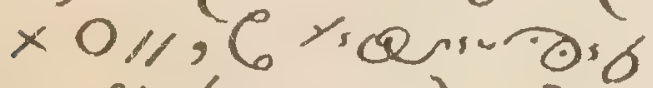
2Lion b siqu by.

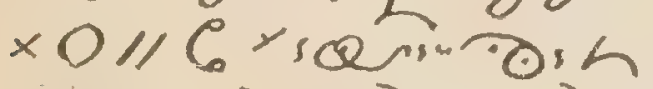
bु $30-252 y .25$ opo ע0260?.

$\left.\times O / 1, C^{Y}, Q n-2\right)^{5}$ < $\log 3 x+25 \partial\}$

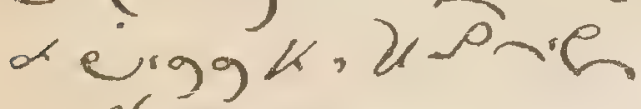
us 3 .

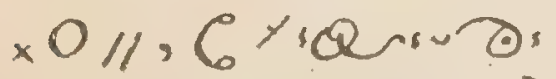

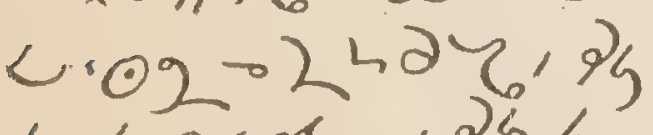

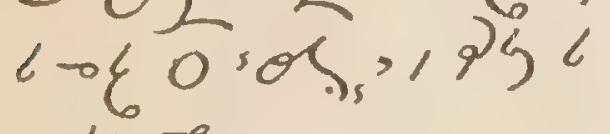
orice

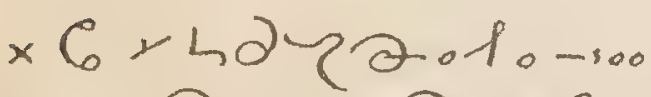
3 . no rara 6 . 'First Consideration.

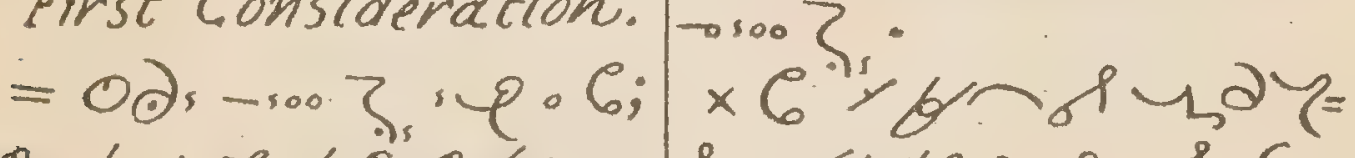

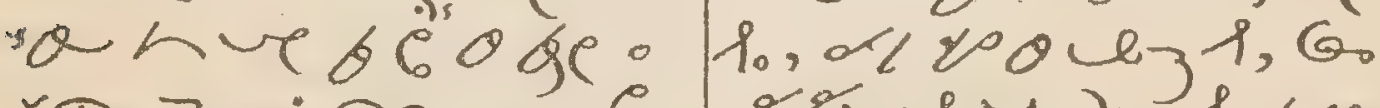

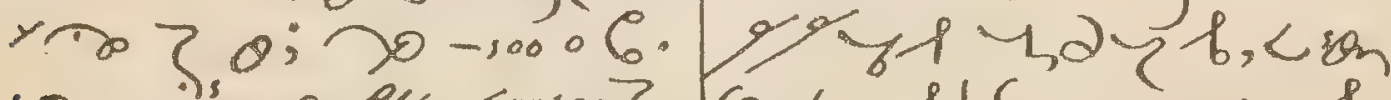

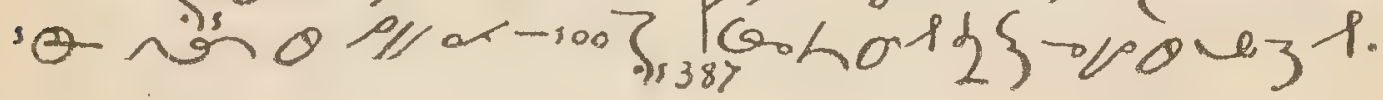




\section{MANUAL}

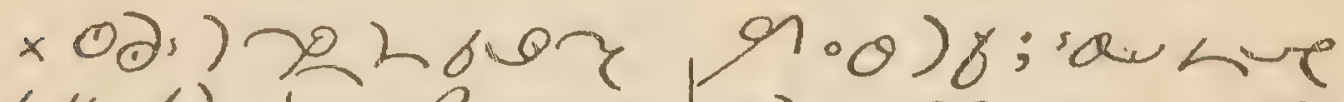

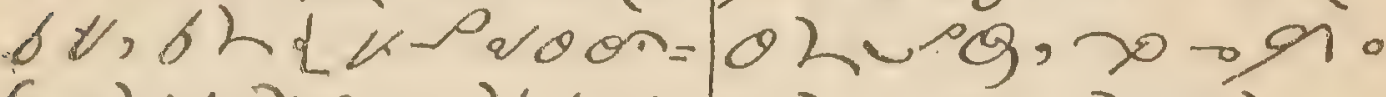

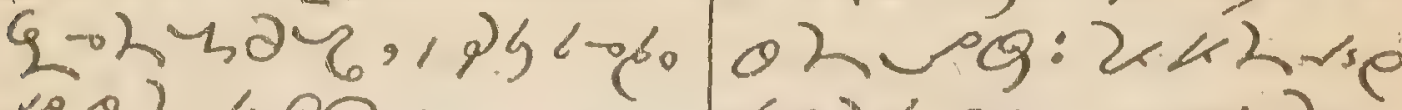
एक्ट 6 亿.

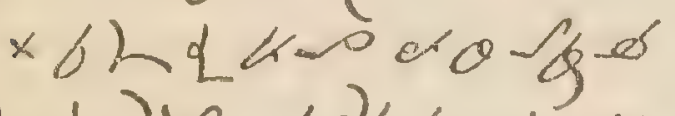

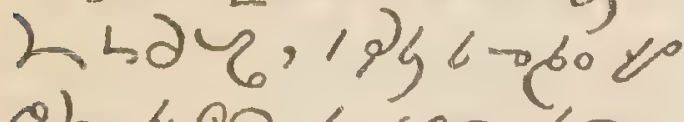
oh borcson br 69m oy.

$\times 62$ d $k$ erob

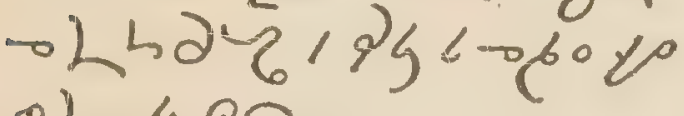
oh अवr.

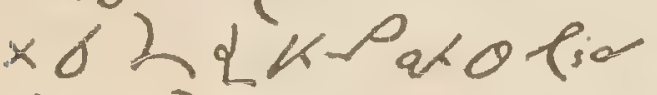
-hbаप, *orkeng

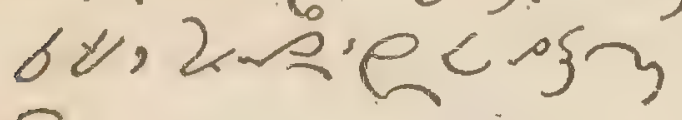
3

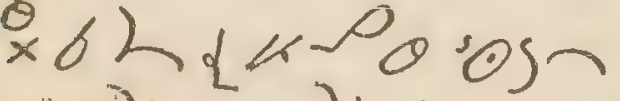

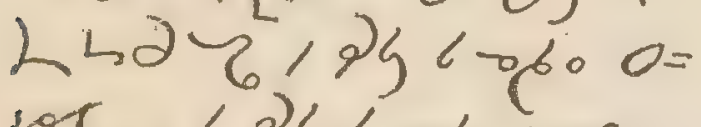

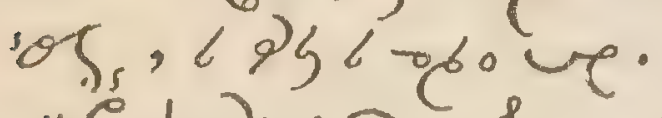

× chaya-torn - ar voniras oy. Second Consideration.

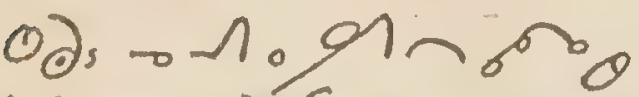

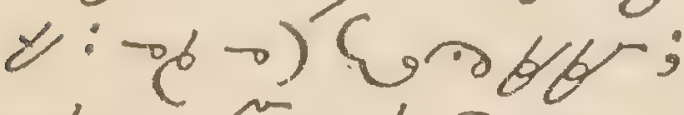
ob us, of nman

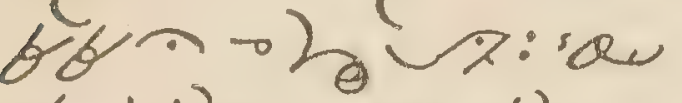

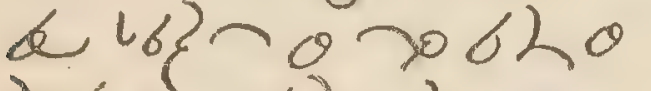
)'dson; bqor hom $=$

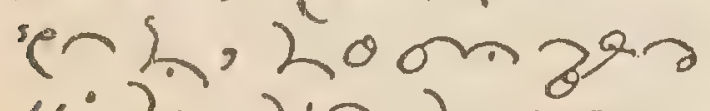
$11 ; 20) \backsim 20 \mathrm{~m}$ gq; 2 o) n 2esn - 10 श; sa hueo hб, $0001.02 \%$; ojobr ovo; 2x 2ase or ong $3^{9}=$

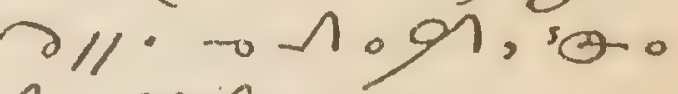
rolferon 2,0 - isgn. dVo)

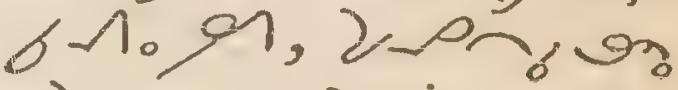
- 260,2 का लो

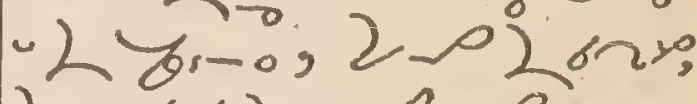
2 ion 6 hors pad उ9 6h0 os $: 0 y=$ ज्ञा.

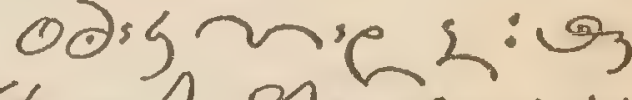

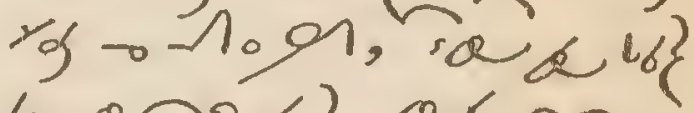
hoporobur - ע; 011,6 -ras es esos on 5 ;0 m y an 12 bа - to nim a ano 39, 36.20. उा1; y

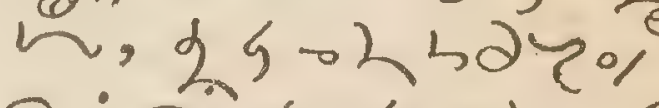
る; 20 人 $990 h, b_{0}$ oboun; hy यद2 $60 \% 3.203$ कर: नin 3 .

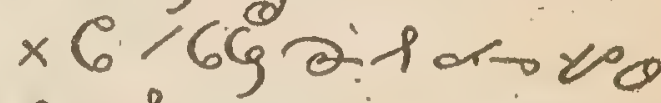
ez- 2 a

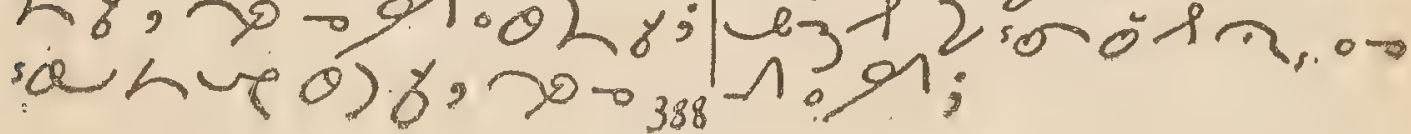


$\times 6,8$ at 2y ¿powit, Qo gers 1, dy b, <ian Go al 2$\} \rightarrow 20 e_{3}$. $\times 0$ \%) 2 6or 6

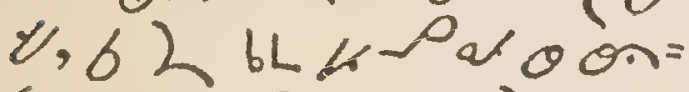
$\{02, \partial\}, 196$

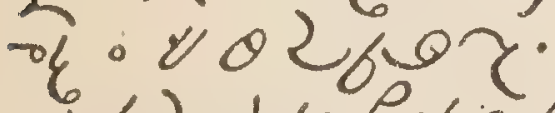
× 62 Lस $40-\beta$

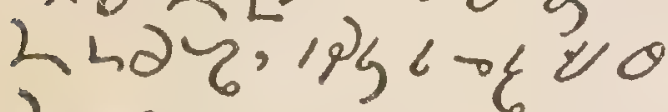
$2_{6}$ or $<$ ian 6 - $89=$ mb. $\times 6$ L $14 \rho_{a} \theta$ b

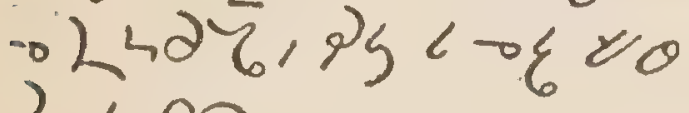
एकिर. * 2 Lksar ofid

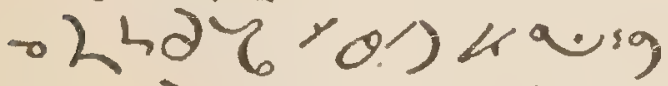

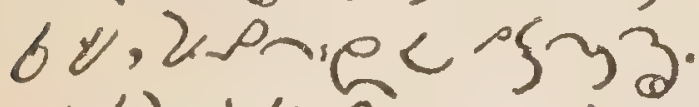
× bLसक०ण2

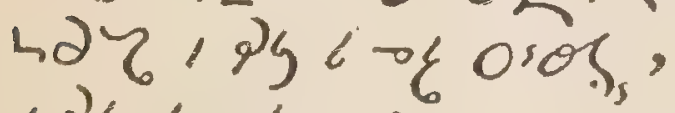

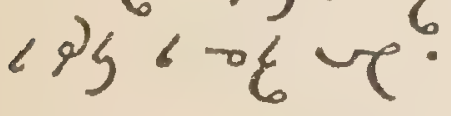

x Grhaydol。x un. angl.

Third Consideration.

$=0$ as $609 \& x$.

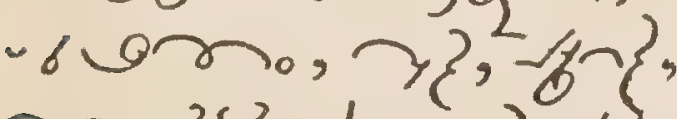
muरsर, hiकर

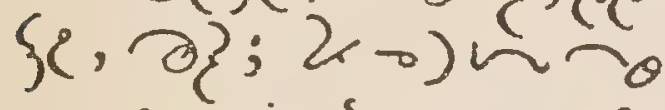

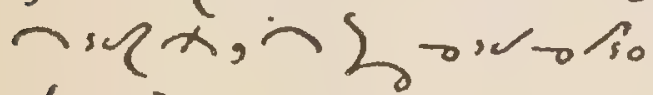
x-2060र部; ox $200 \mathrm{n}$ 万om of ; b8or रion 389
《ध ग3? ogs र sen sorabor-io? ox $2-0) \sim y=$

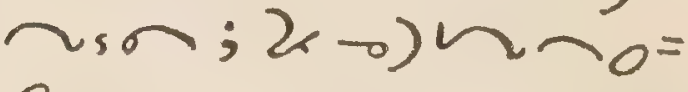

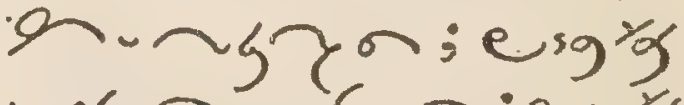

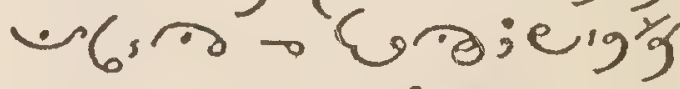
yon wrojesgy पin? non bt:

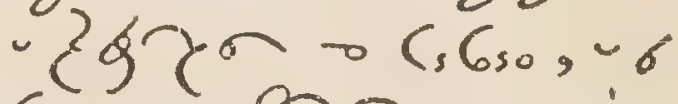
Gion $y$, 0 .

ox:0.6.6. on sor

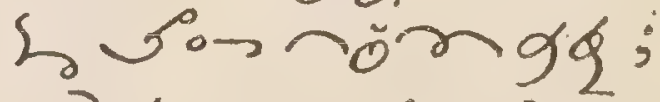

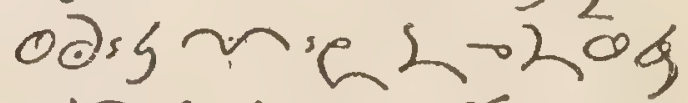

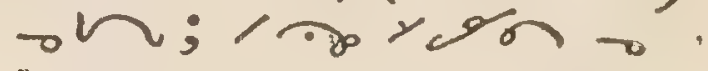

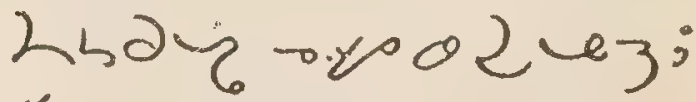

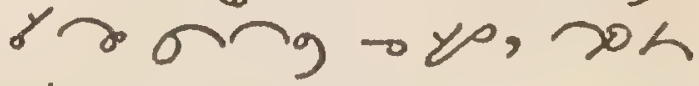
49 -

ox! esgìg ono

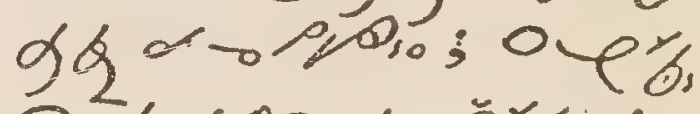
る人ion 6 ork: wh Cona xiss=

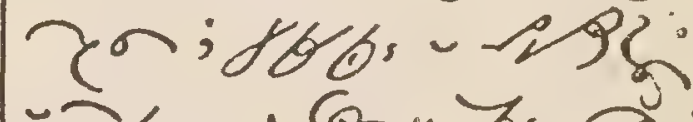

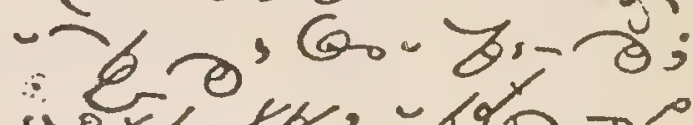
एर $86,-69 \%$ - 10

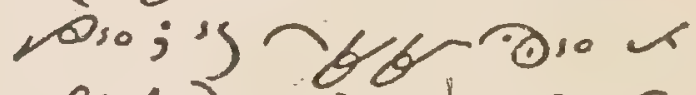
$\varphi_{6}: 2<-0 \sim 2$ ros $=$

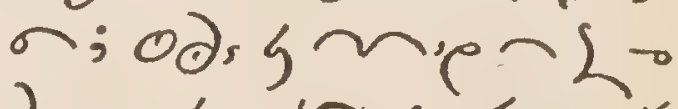
toogriar न3 - 2002 - 3 ; sack hर म ब Q '0

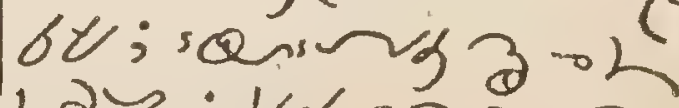
मे 6 ; the of enr? 
-hbayouseng. oho haे b\%; حso $2 \times 669 \times 2966$ ?

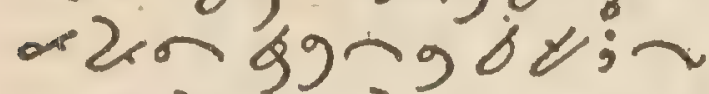
or 25 रू a ros, 2-prse 15911,2 pisena andar.

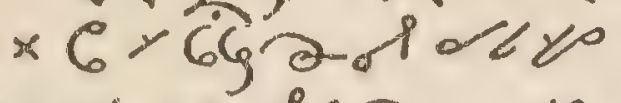
or bre: 2500 rortok:xi - Ppro.

$\times 6 / 6$ of $22 y b$

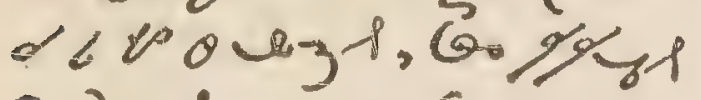
$2 \partial y b<\operatorname{son} b_{0}<$ in $=$ $19\}$ D 20 lezh.

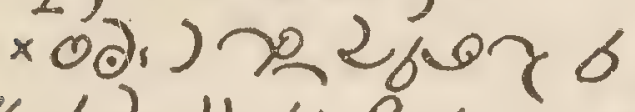
$\%, 62$ bLस a aro on =

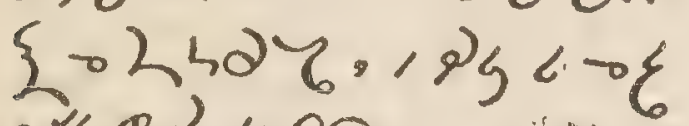

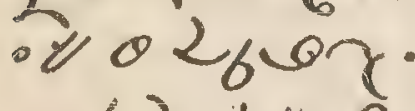

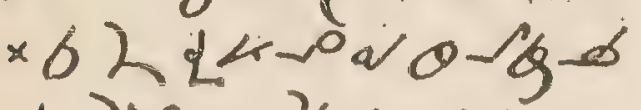

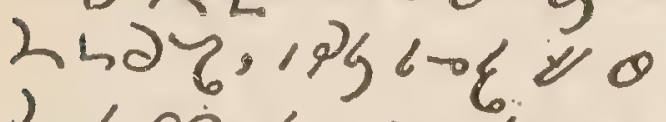
$260 \gamma<\sin 8-89=$ nov $\times 6$ L

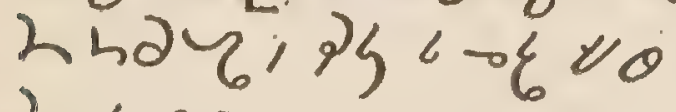
युor. $\times 6$ LL alator Lha $y^{*} \theta^{\prime}$ ) keng 6 \%, 2 $\times 621400 \mathrm{OL}$

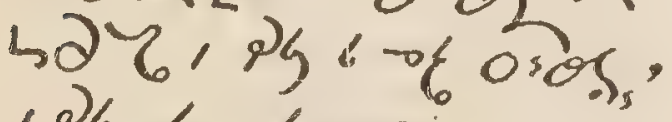

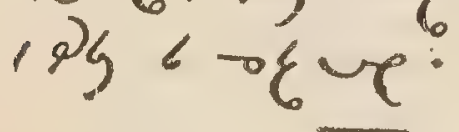

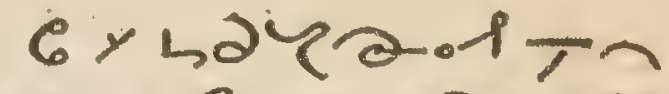
f.obor nogp. Fourth Cionsideration. 0113 की aryo. 3द $\varepsilon-6,628.6$ ofue; क\& - रमेद -रo ain - खीo अव. (No haप: oll Ge corron 6o. - ha Ly y:0 "Ian byera Lhay, 2 - 2 ro,

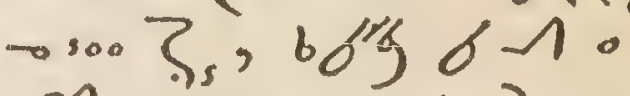

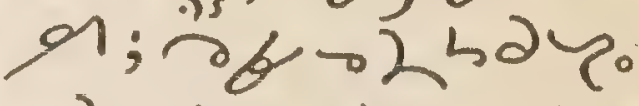
- 2 or 5 , drmos प्र०20 62006\%. $\times 6-662 \sigma^{\circ} \circ$. निOC, नrol्रF;

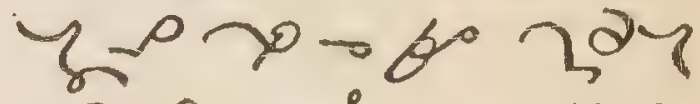
oricusstll to orert. $\times 618$ of 2ayb

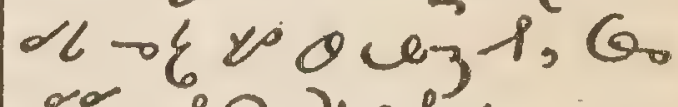
roit 2ayberon $\left.Q_{0}<\operatorname{col} 2 \xi-20\right\}_{3}$.

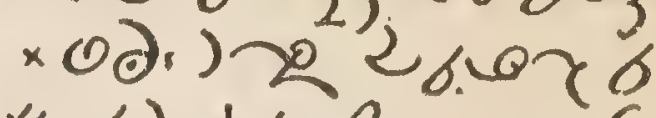
V. 62 LLPat $\theta$ on

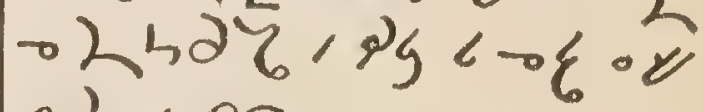
- टुक्र.

$\times 6 h$ Lus aro-gar Lha

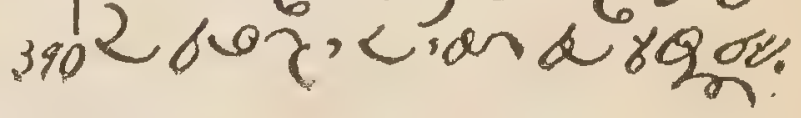


$\times 6224 \rho_{e} \alpha$ ob

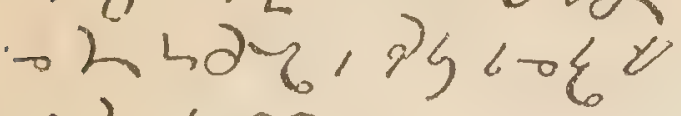
0260 .

$\times 62$ Lis a o pid - Lhay Yo') ueisg б'v, 2- 0 ' 32 . $\times 6^{0} 22490.02$ 2 मे $2,9 y<-6$

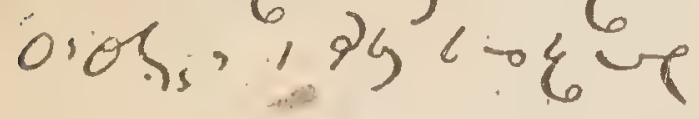

$x$ ososgsog $\cap p s 0$.

$\times$ L 2 ro.

Before Communion.

Faith. 6rodigcon u人

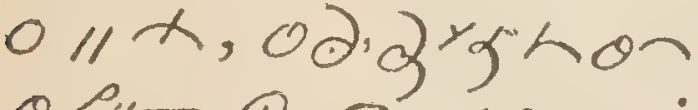

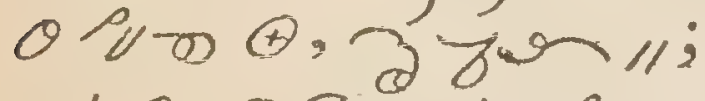
ot mo, urpo? - 60 o; orrmo, mo brters ocso s? No - ton o M. polo; drotrito,

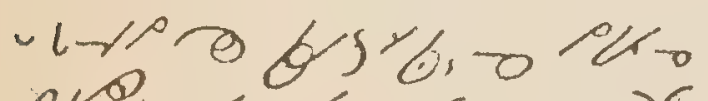
poso ris se a gro,onds. Humility. 6 yojen $3^{2} y_{0} \neq 0$.

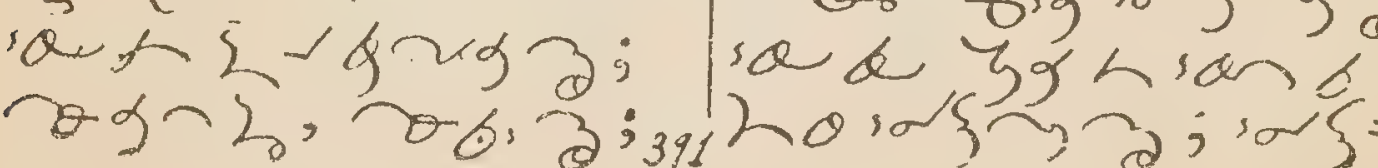

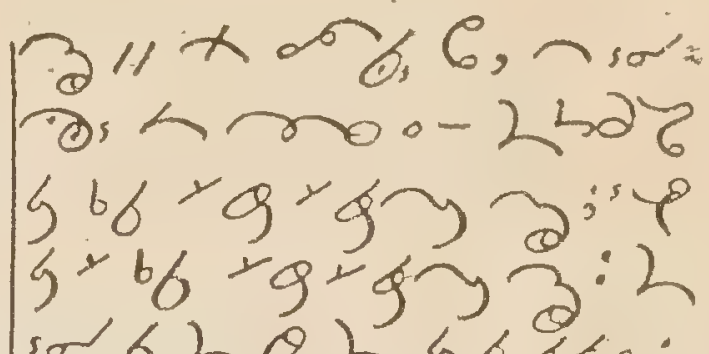
sa 62025680 ; - o-sididotos, cor $2 x$, sor a b 39620 os प्रक 92 तs. 6 शूर, द. 90 e. contrition. $6 \times 69=$

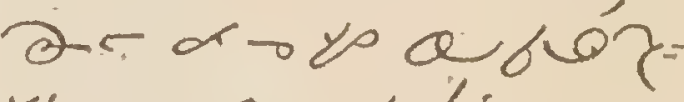
ror. $=011 x !$ ? 20.69\% divoL bor:'ia d दुर son 6h0692, :2 m०ब3:)

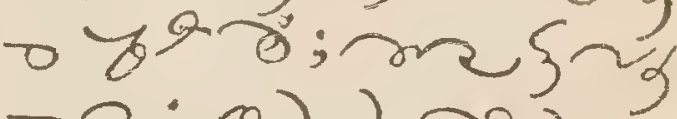
- 3 ;0d 2 22 6बर 64, sad 35 6ho: Go6ro vi: 0 र

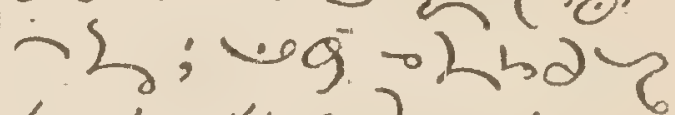
- obo \& Q 2ez; ía $620 \operatorname{son} 5620 \mathrm{Nug}=$ nove. $6 x=0 \| x$ एका

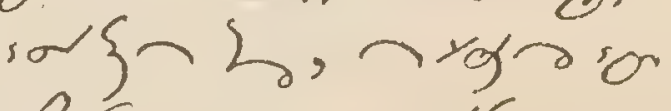
$\rho G_{0}$ O'g so's? sa d 39 人ian 6 


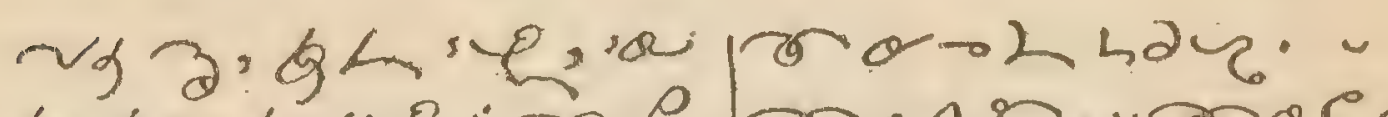

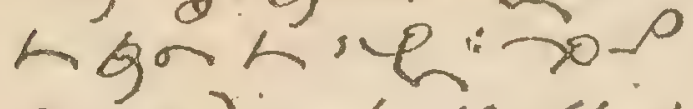

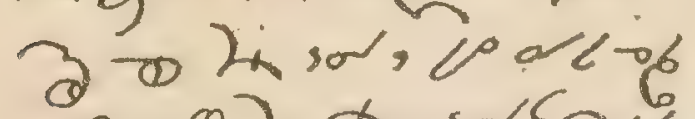

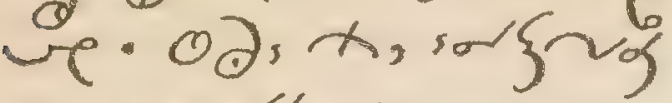
3.

Desire. $6 /$ e $\xi(a)$ $x_{0}=0 \| x !$ 'so's

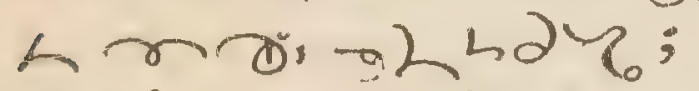
$\left.\left.6 / \cos ^{2} \rightarrow\right)_{0}\right)^{\circ}=$

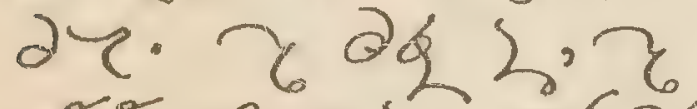
प्रcein $250 \%$

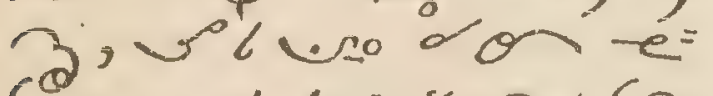

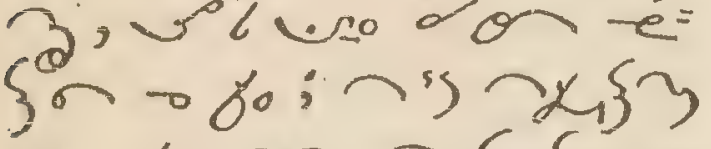
ə, 人 r. 万人.

068, tin 1120 24, $2 \%-2: 60$ oin का मूरे. yx, seb कू "रूमा $2,0 \partial=$

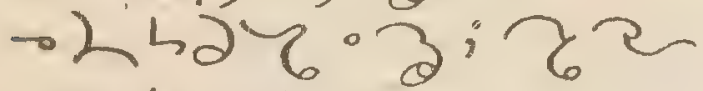
ser ơm.

After Communion. Adoration. Ox, M.

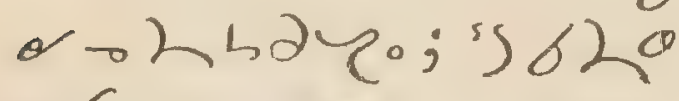
e\{r; dow wex

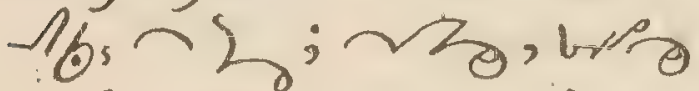

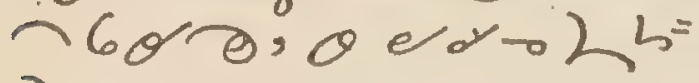

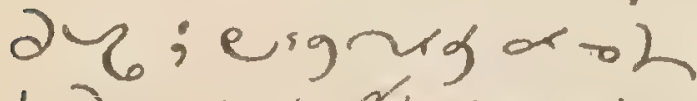

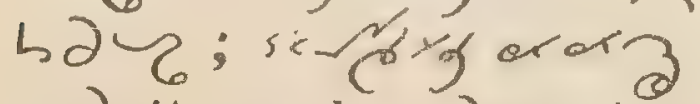

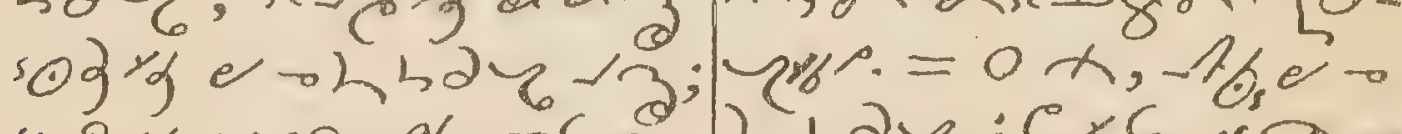

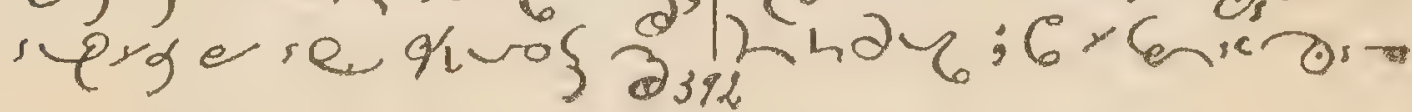

sot, oor troo 3, 60

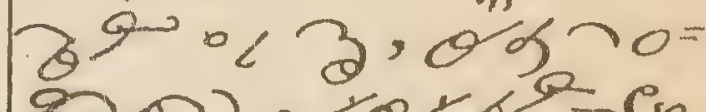

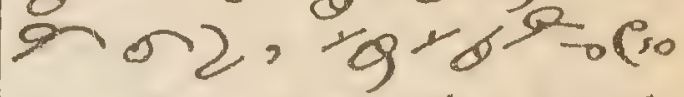
व. $\operatorname{coc} x, 050 \%$

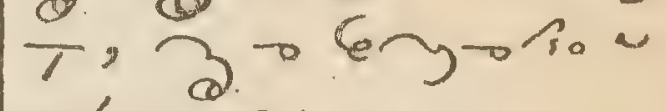
$\rightarrow$ o. $v^{\circ}$

Thanks. 6ry-gron, $x \cdot=q$ res $\sigma: 3 x$ Mbs er b ha co, od ŏmns, piano $20 \mathrm{~s} 60^{\circ}$ s, so 6

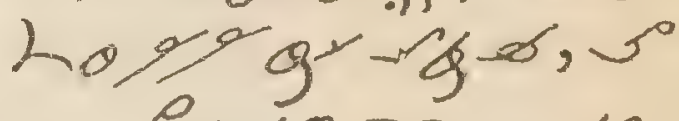
$\rightarrow$ Pogr r o vo, यक a dx, osorys vo. $\rho$ - ये मे. Obs $\partial \xi-z y^{\circ} 2 L=$

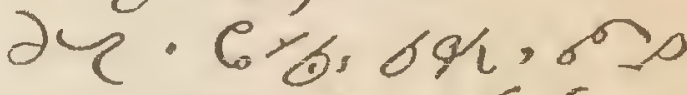
n sorpor be $\left.e_{1} ; 03 x-0_{0}^{\circ}\right)^{6}$ ) En $060 . ;$ 200

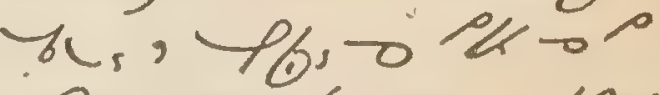
Dso; 万iglsina - Vea

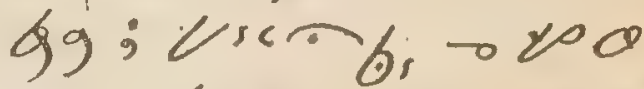
$490^{\circ}$ or osolgsto jor rion

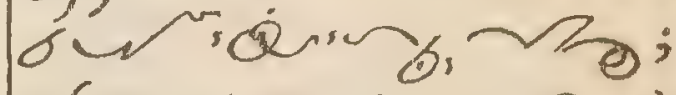
ofरng $8 x, 0602$ hy o or $\partial$. Petition. Q y UUก $x, \alpha \varepsilon x-8 \sigma 2 \partial=$ 
242,$6 ; 26,06$ in - oso s, $\theta$, जor - $56^{9}$

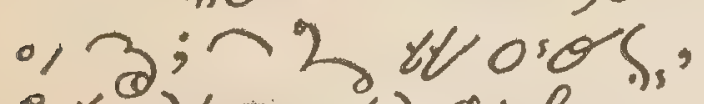

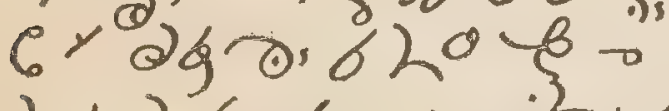

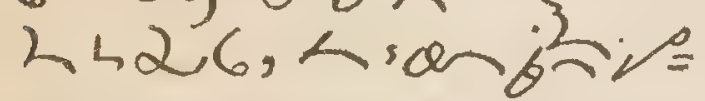

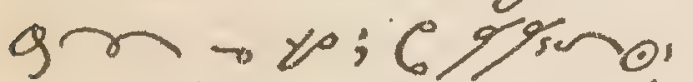
$\rightarrow$ L 26,620 gen

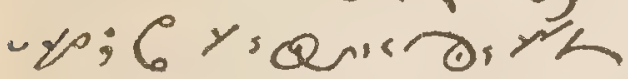
b 6$\} 9-502 ; 0 \gamma e$ igg 6\%:एकर $202 ; 6$ yaurios roho.iog

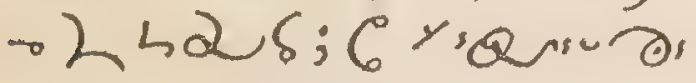
6ho griss o s esoith

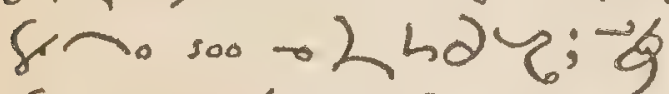
$8-0$;i $2426 ;-I=$

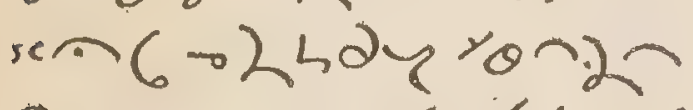

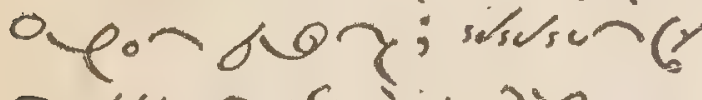
roll oig Lisar.

Resolution. Grender rit. 3 gor oygsh

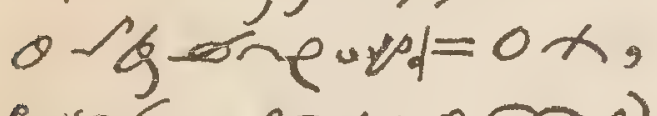

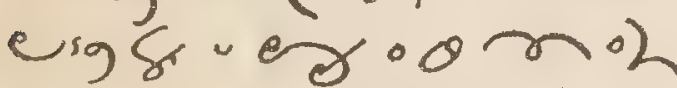
hat:

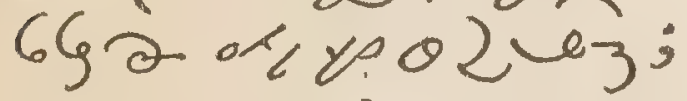
a 639620 eegn

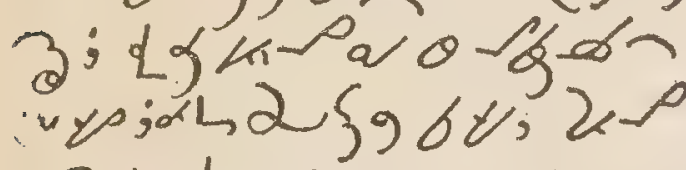
"e八2, sal 0 ;

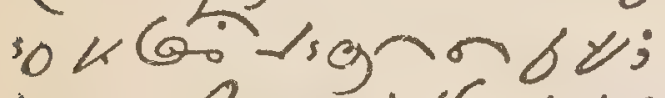

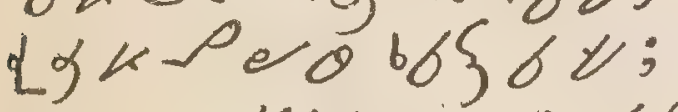
a enggy

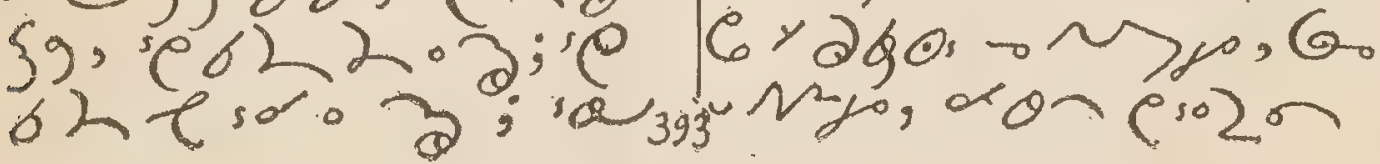

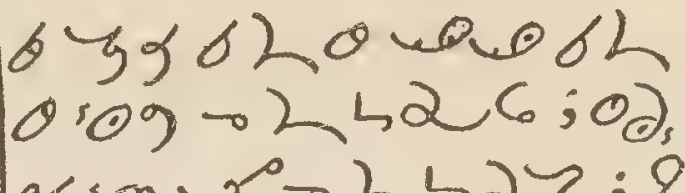

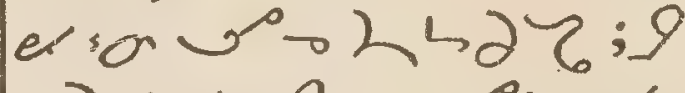
ods s p.on siox 6 \%gor en - hidY: Do-pregar मng g?

offering. 6 or $Q$ sol $=$ er, $x$ ren,san $\rho e=0 \pi$, "Qnis

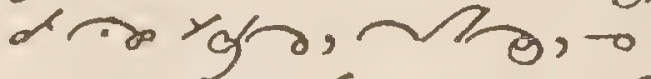

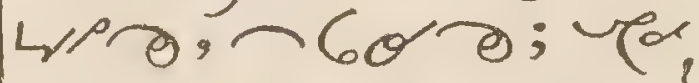

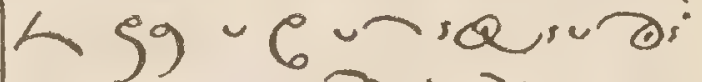

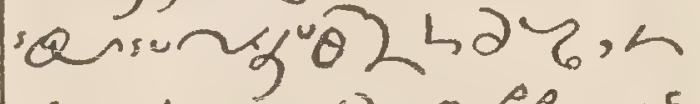
son 6 - 62 olines oेs of squm $0 \mathrm{~L}^{2}$ Lo ks araronin $=$ es, hor oo, o L)

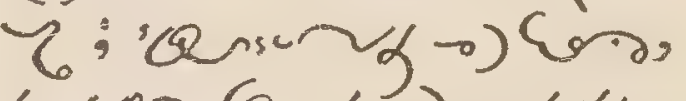
hion (a./in) by. sas (sq\}) hon or

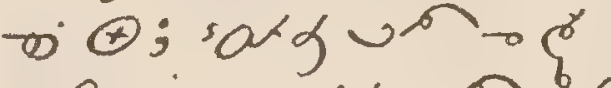

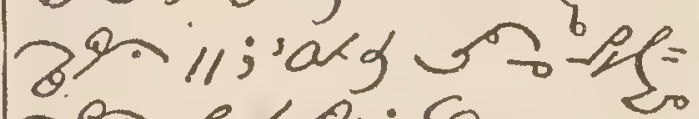

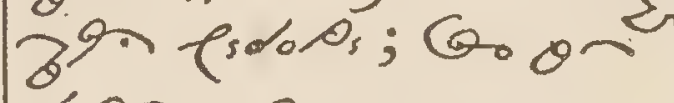
ororeso. Intercession. $6 \gamma^{2}=$ $x$ ron $/ \operatorname{san} e s+e=$ $n=0 x, 6$ b 08 uns o ono esdols, ब, 几 0 \% . . 
Tos $\theta$ Sosings N

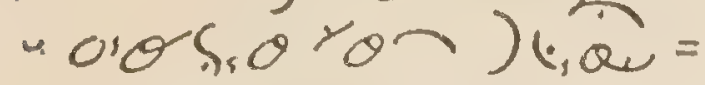

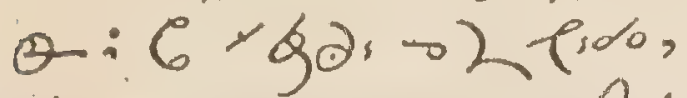

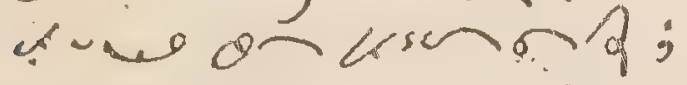
बas- $6-82990$ od 0 को का एर "k> W-o nigidge.

offering of the Mass.

$$
\text { of nets. }
$$

$0 / 1)$. btowbia, wounth $62,0.4,0$ m. 6.620 Ne; vos y bo gn o senis B ar. जo 22 of $242 y_{0}$

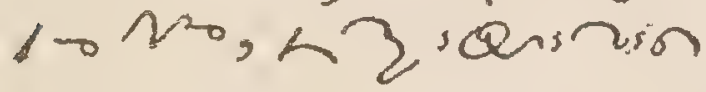
Q.

Quinug $3 \cdot$ P. bh $6^{2}, 2 \rho 3-\pi$ de osol, de obon,

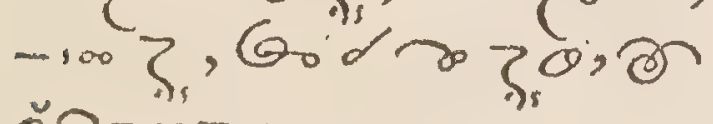
oัค

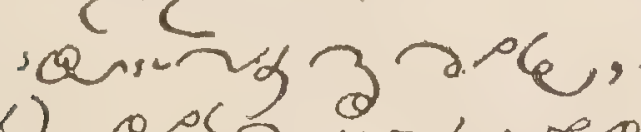
b)o of 2 , soweo

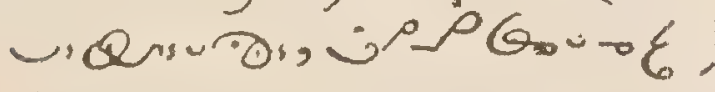

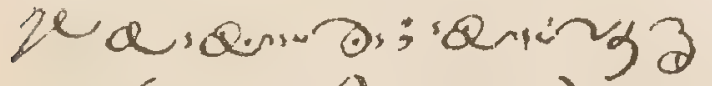

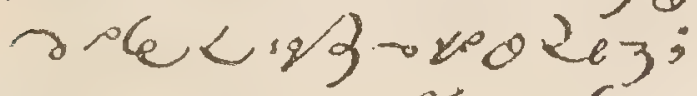
-añy a ${ }^{\circ} \mathrm{C}<6=$

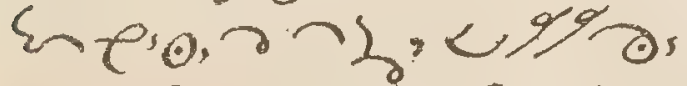
$-2, \partial y<\sin 6.020$

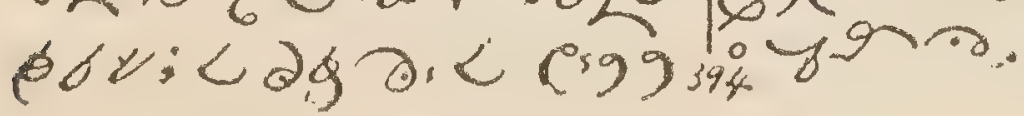
$872 \xi$. ए人bar, 6h2。 $\rightarrow+\infty\}^{\circ}$

The Rosary.

doyful Mysteries. 1. is

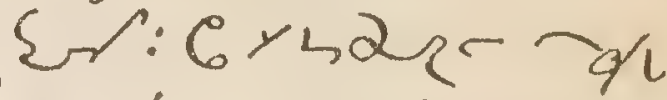
yox, mo k, o,

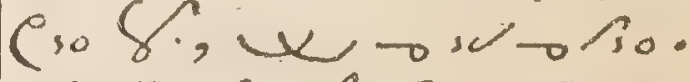

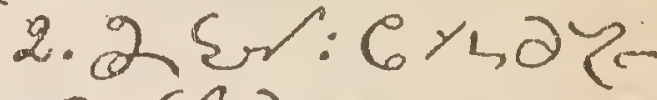
- Csob, deson 2 भt.un $x$.

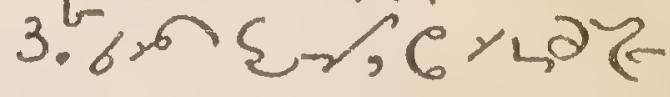
Oryox, ridsdo

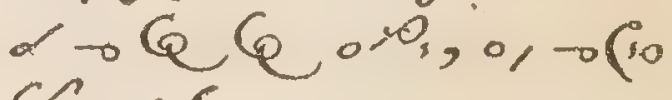

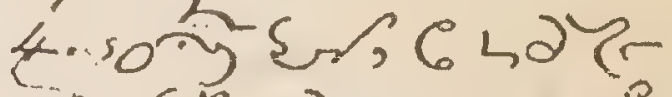
- esa $8,-2 \sim$ - ridas, sagen yurs 11 tun-

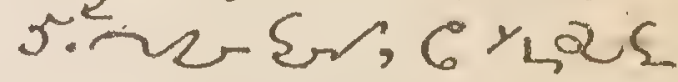
$\rightarrow$ eso 8 duzan

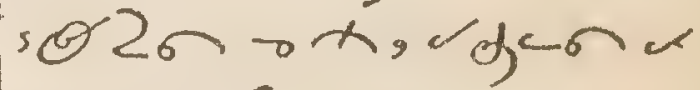
- Pidopio.

Dolorous. 6... PeEs?

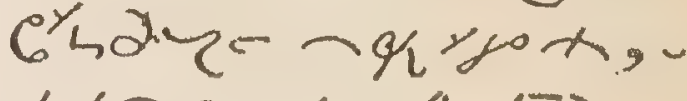
bb八a d 1sd 2 , vira kn $3,0^{\circ}$

\%. ors Ed, chat quepot bynon 0 पर 0 - n

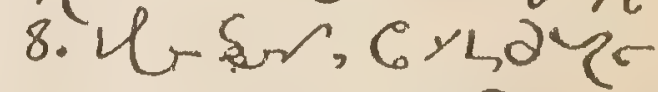

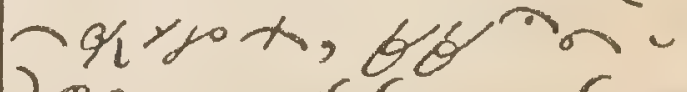

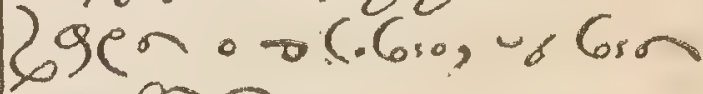




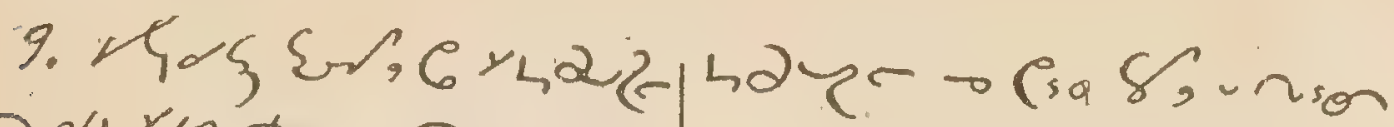

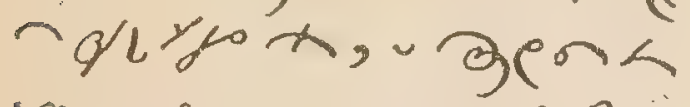
ar h h o os,

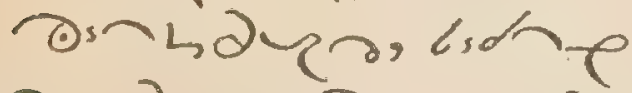

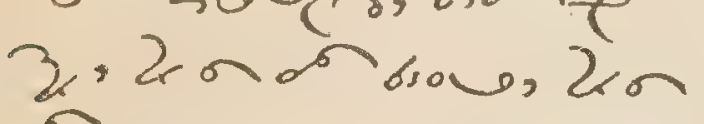
onee.

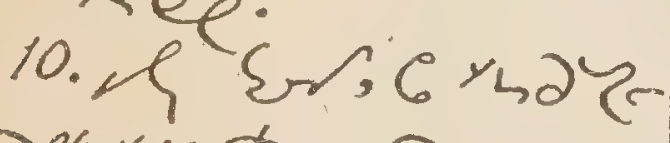

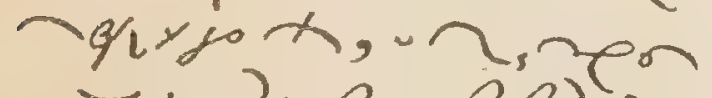
$-86,2 \rho-9 \rho), 2$ bद - $692, Q^{\circ}$

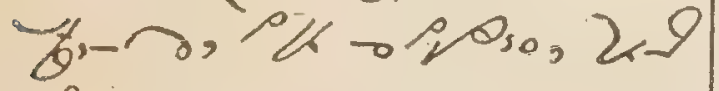

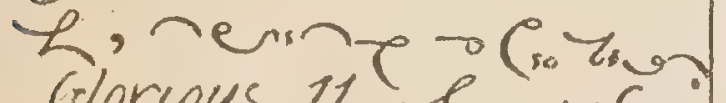
Glorious. 11. H ops:

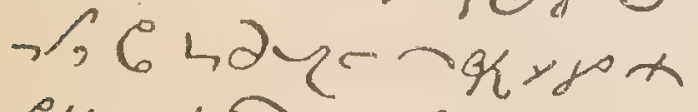
एक - Ton, ods 11 2.

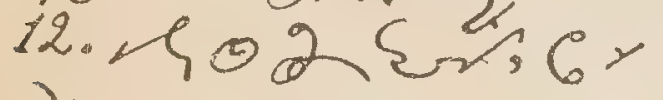

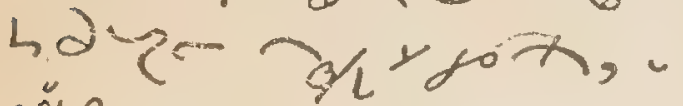

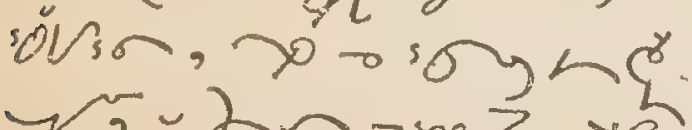

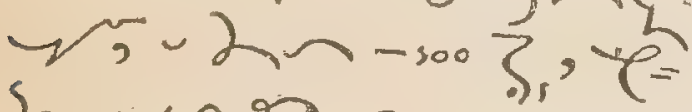
semor, nenineo

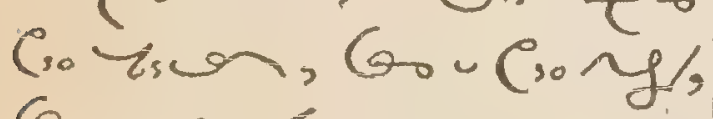
Go $-\cos \theta \cdot \theta$ :

13. hor singy hoze حqugor, $(\infty)$ बदिका ren, Mn, we

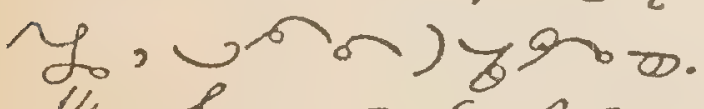

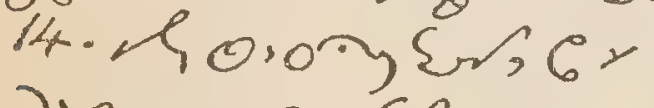

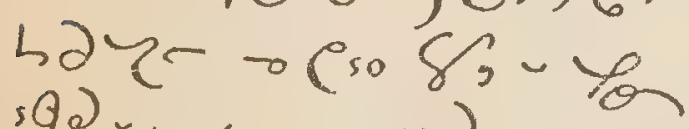

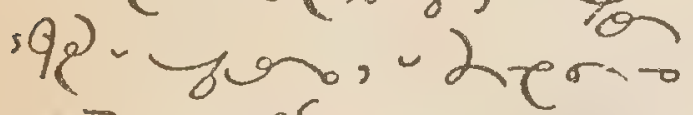

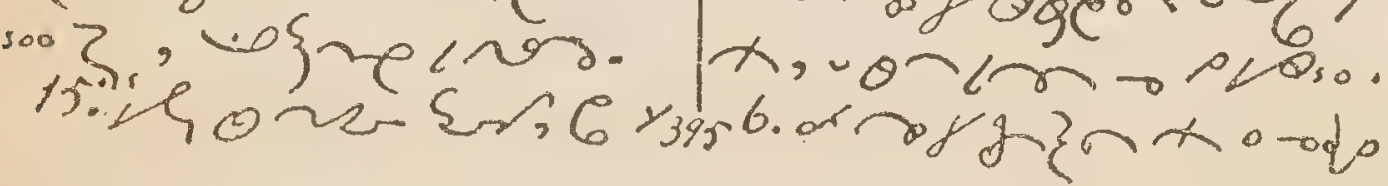

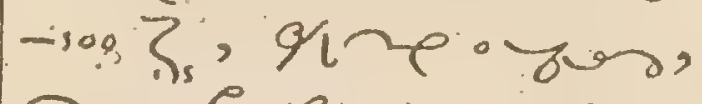
$306 \%<+0000$ so $\sin ^{\circ}$

Via Crucis.

Preparatory Prayer.

$0 x, g^{2}=$ er $2=$

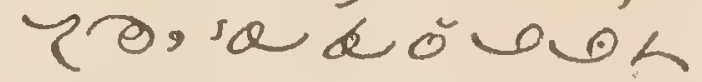
$\operatorname{san} \theta \varphi d-90$ soin san osticog;25 ) iso; $\theta \sin -24 d=$

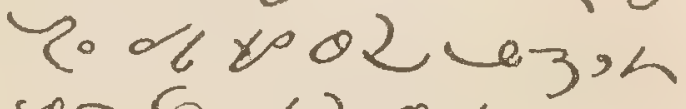
$\sin 62006 \%$; Oें 02 vez.

Stabat Mater.

o 8 xisen

ming $\alpha 5426$ - O八 6 sol 2 . Adoremuste. sc $\mathrm{C}^{\mathrm{y}} \mathrm{y}$

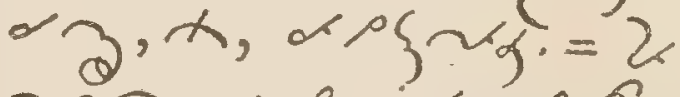

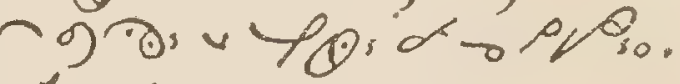

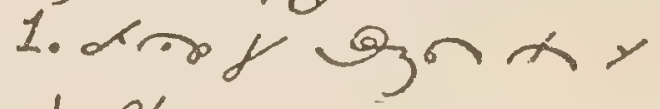
- de 94 nan y. 2.d rode us Tent $\rightarrow$ PQso.

3. ar कo \& con gु, $x$. $4 . d x y)$ yon xim.

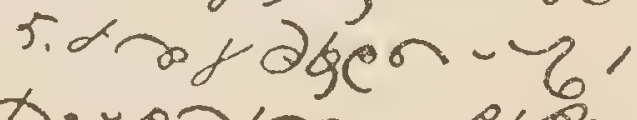


$6 \sim 9$

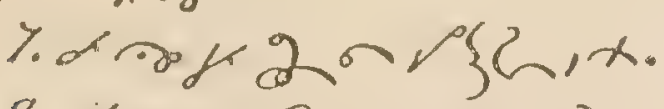
8. 2 rofersen $\times 2,2=$ पथ - )

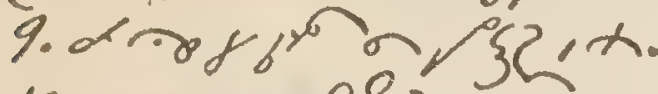

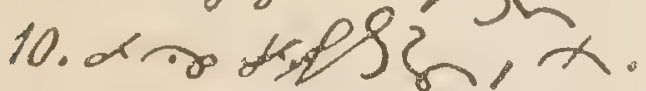
11. a ry $\alpha$ to $\theta_{s o}$

12. disfy Yonixdo Pso.

13. dक्षuln, x呰 Coso.

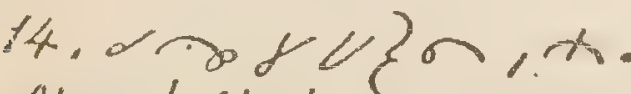
At each station: Ox,

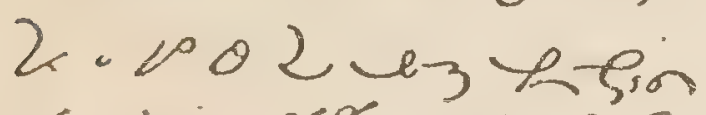

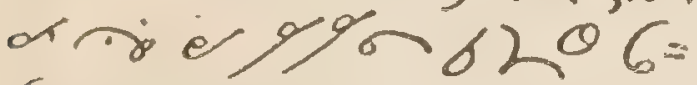
दo व 402 a

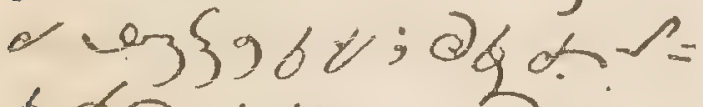
\&-63 $9 \mathrm{~V}$.

Hymns.

1. Venez, divin Messie. 26/ gु, \&. $46 \% 086028$. $\sim 6, \sim 6, \sim 6$.

2. Adeste fideles.

- $60,-r^{2} 0$. क्र०८म 6 , 260, 260 की जiर: dease " yon, 260.03p. $\sim 60: 09^{\circ}, \theta$,

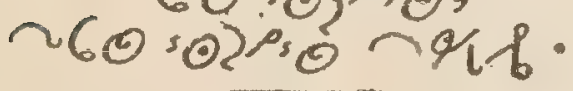

3. Aus sang qu'un...

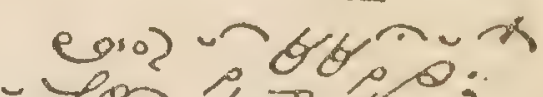

tor :- o o

यद्य 69 o to vis $x$.

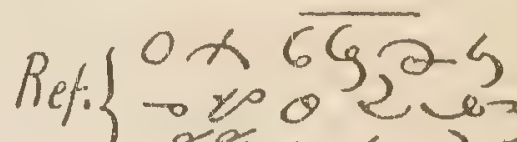

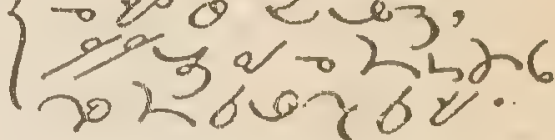
जक्न $x$.

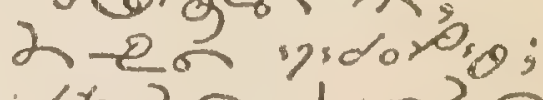
- $25 n$, sion $2 \sigma$ क्न. परून.

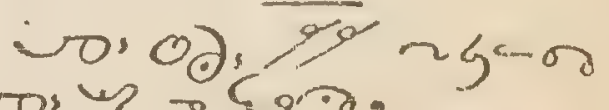

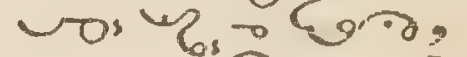
'O U L for ?o? tos galdid.

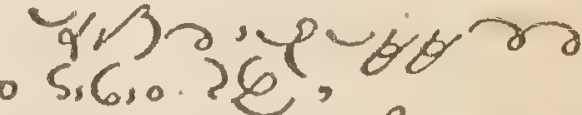
moron $\rightarrow$ sinen एक करुषन. ign ing ops, - La inia op. enger yy 2 ren.? - Lon sereve.

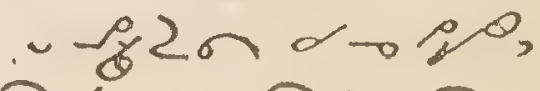

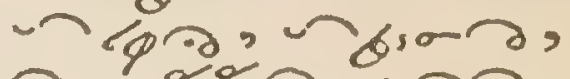

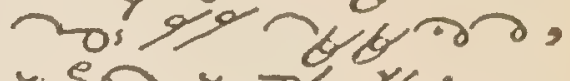
- Sू - वहै.

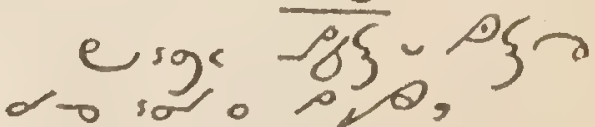
clorabra, ition sesar.

$0 x$ ogsan

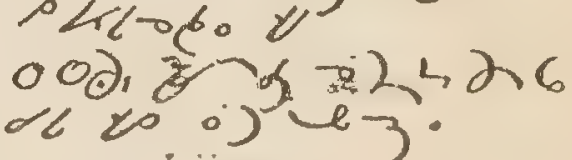


4. Miserere mei Deus. ek-ens, उ 106 人o?.

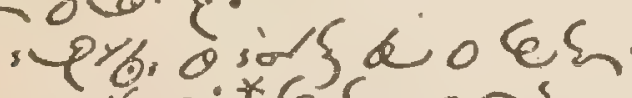

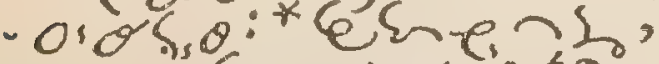

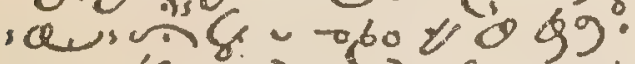

जós - h $42,6<-60$ 4099 , *on 6 . $426,6, \pi 6=2426$ 0 - Cso.

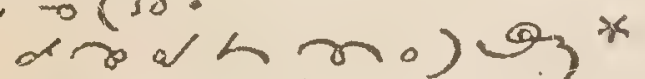
Lon.y tho oins $\rightarrow$ का $\theta$ \&

op $206 \mathrm{~g} 2$ + yqe's op. 4099 .

5. Mon doux Jesus.

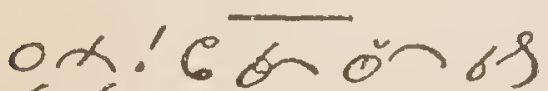

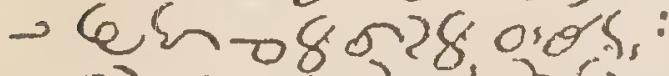
$000.042 .0 \%$ 62 .

\% eprez z rexy.

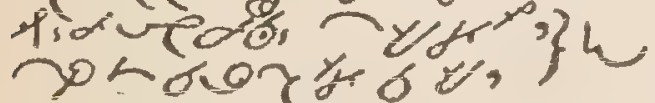
crin.

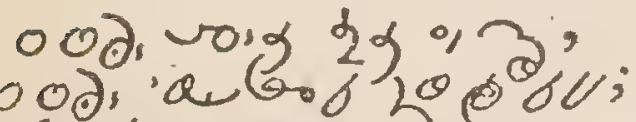

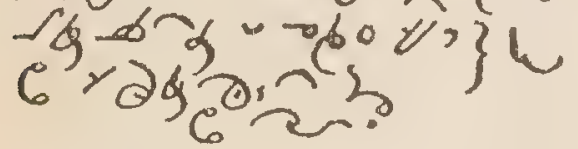

6. Helas, quelle douleur.

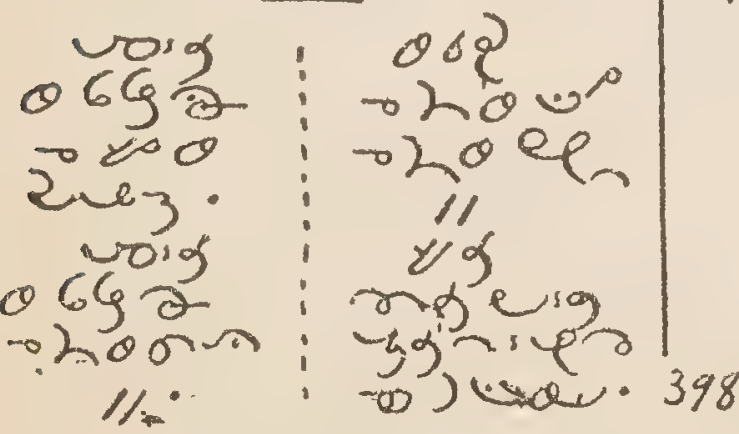

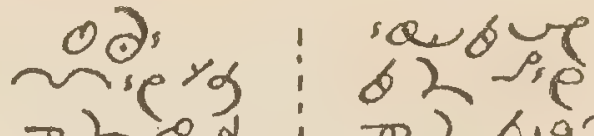

$\begin{array}{l:l}220 \alpha & 0260\}\end{array}$

०े.

n'e 10 \%, 1002

-2015: 52200

7. o Filii et filiax.

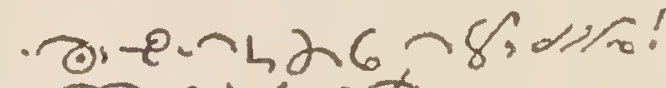

- $\theta$ कु $x$

sols gro w's है,

8. Venicireator.

\section{ति, W}

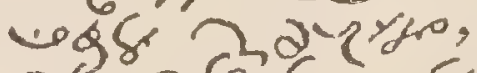
re.e cén.og of०

ح $2608 \mathrm{~g}$ ह6 की 20 \% - cso Dod yas. 9. Le voici l'Agnexer.

$$
\begin{aligned}
& \text { ore. } \infty x \text {, } \\
& \text { ros } y=100 \text {, } \\
& \text { "Pry soofs. } \\
& 3062 \\
& \text { sपy sols } \\
& 6 \text { yry orifin. }
\end{aligned}
$$

10. O Jourheureux.

voig $02.09 \%$

0, (ब)

Q.0, wo, $x$

चु, war, द⿱

$$
\text { "1 } 2 \text {, }
$$

vorg odsogys. 


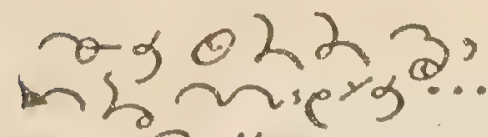

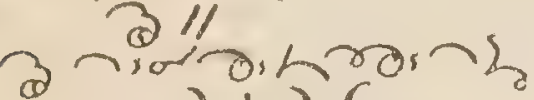
0,02426

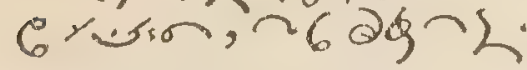

$30 \overline{8} 8$

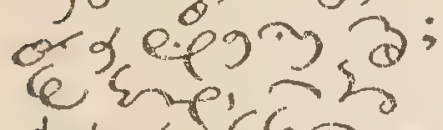
Lo a 69 क.

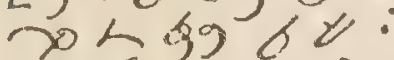
बogs $\rightarrow-0$. 2 $2 "$

11. Venez, Tesus.

$262,26 / 1$, $\sim 6, \sim 6$ के 02426 : 几 य,'

12. Chantons encejour.

$6 \times$ का का 20,

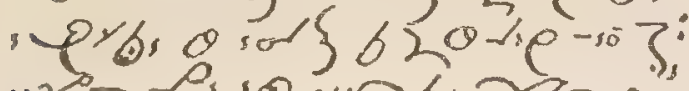

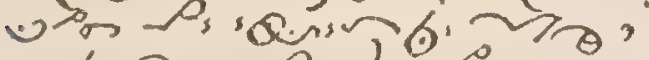

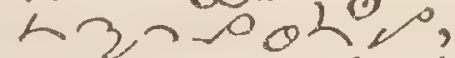

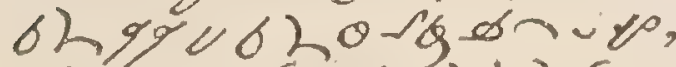
$6 \times$ esg guaro 2426

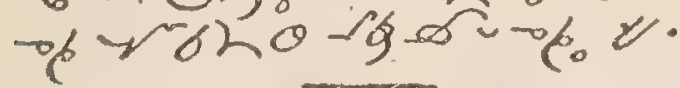

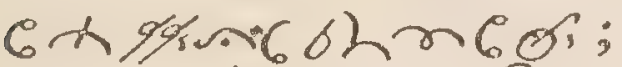
क उ2 $98020-19620$, जo por

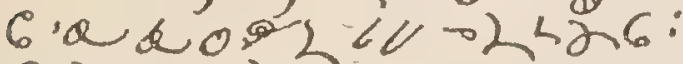

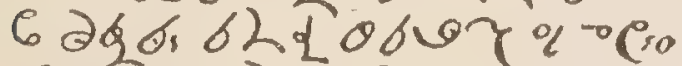
$r \rightarrow 40306$-soo ?r 13. After Communion.

$$
1 \bar{x}
$$

196 er oy 0 h h 26 ; - $\theta$ r $\theta$, Q. $60 \theta, \theta \alpha$ 万. eignin -2426 , soरrig at w - pros a $09 \overbrace{0}$ को 53 a cio?

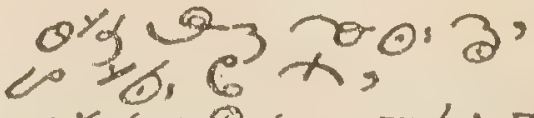

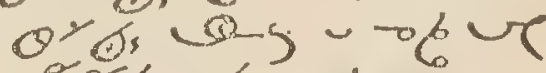

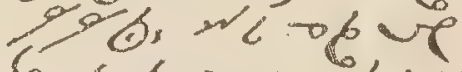
ens. o. 0.6

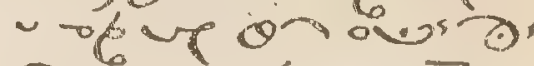

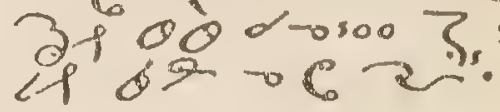

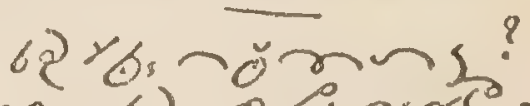
"a oho ioro's,

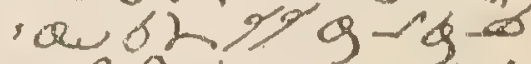
wowign -

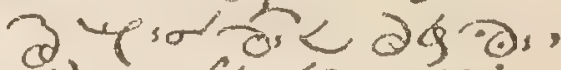

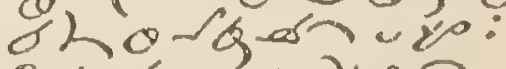
6 resion zay a $6 \times 2 \%$ के

14. Holy Eucharist.

6 rogs 20, 2ун $1 /$ كु

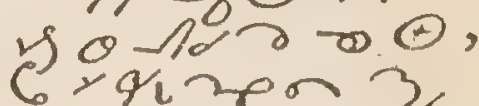

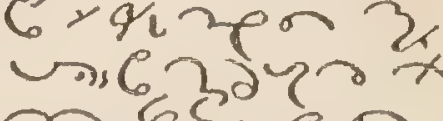
mesten. $96-20 \times-8 \oplus$

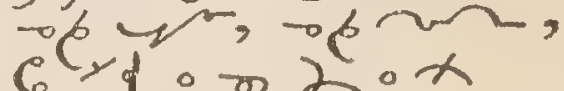
cis क $20 x$

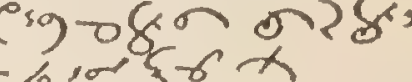
कर 2rion tigon osuo?. 15. Panis Angelicus.

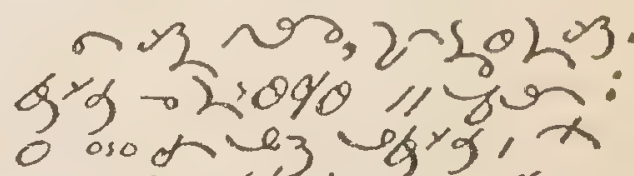
न 594510505.

031128006

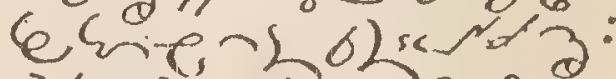

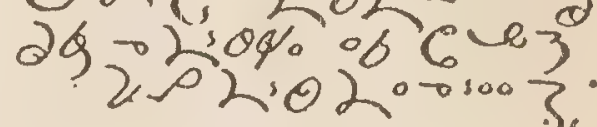
16. Guard of Ronor.

$$
6-6 \text { ? }
$$

399 ong ovin $x$ 
Gिखें

संखिया

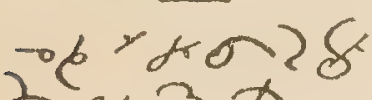

do, $\rightarrow$

of 9 क्ष.

$\%$ T so DsOAO.

ryxorsogro

o a nia oje

व न

a -8 -

c 29080.

$\%$ c so Diggro.

2 का. 2926 of b 6 - 26.

1). Maria mater.

o 8 rog tus

6h-cesn 8 o,

$\sin 642038$

29086, $56 \%$ म.

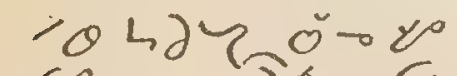

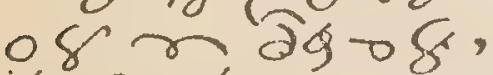
Lan ge ougy

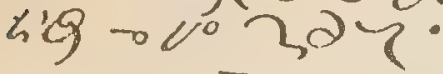

$$
\text { ge ovons }
$$

नrzla"

oriaboro

62 - 611 . of.

18. Dies iras.

$$
11
$$

ogf r. $\rightarrow e \varepsilon s$

$\rightarrow$ poc 60 \%.

19. Aufond des...

$$
\alpha \text { कि }
$$

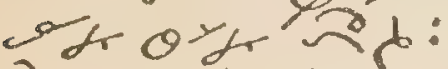

2 o $200 a_{3} \mathrm{y}$

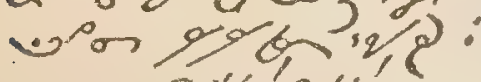

$$
\text { OV.OV. }
$$

havy a d ifide

पर 0 (Q) of $\partial 1$ 2क्षण.

- eso 6 -

6 "Q igrd inogic

$$
\text { 29Q? LQ⿱ }
$$

6 ot iensiog

$208 \times 6$ soo?.

20. Holy Eucharest.

खुरा "ुw,

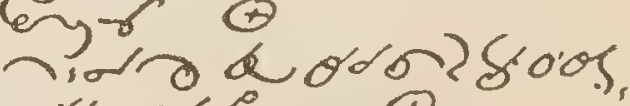

$0 \%, 01 \%, 0$

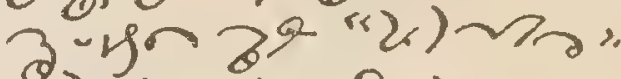

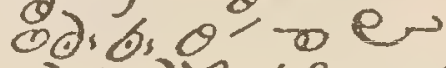

- रेव 6 6 envers?

obibiokiol 3

व. $\rightarrow 24.2 \%$ क. $0,3 \% \%, 2$

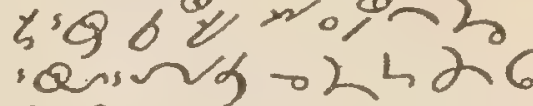

heigor a 3 .

21. To the Holy Ghost.

ow mos.

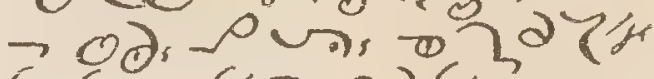

घर

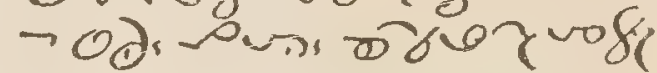

"so $3^{9}$

7 a dasing 35

ood.

Prayertosroseph.

Prayertos!Joseph. On,

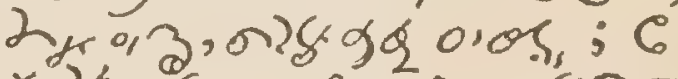

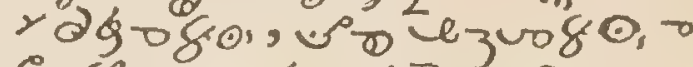

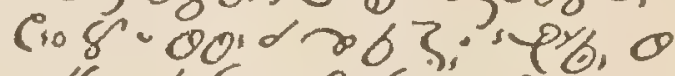

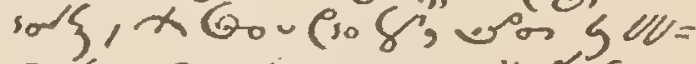

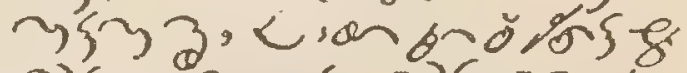

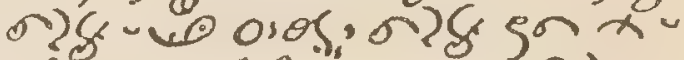

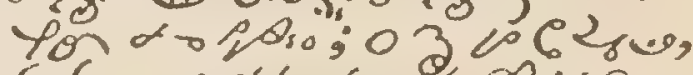

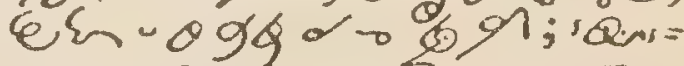

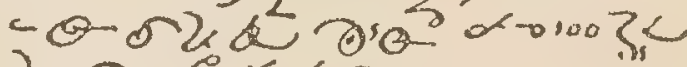
For the living and the dead. cyeser. 60 o'ot $a$.

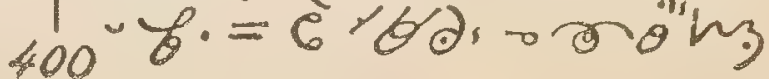




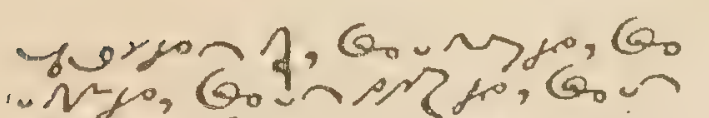

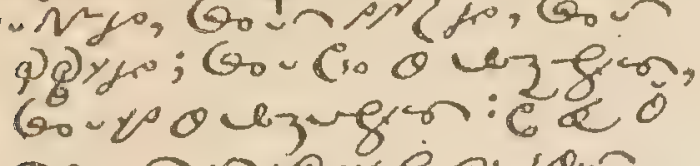
veniniangigin

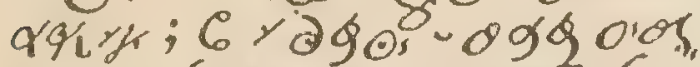
$G_{0}-\theta G_{0}, G_{0} \cdot \theta d y, G_{0}$

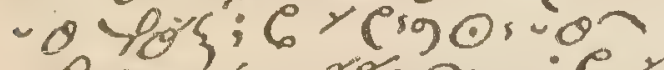

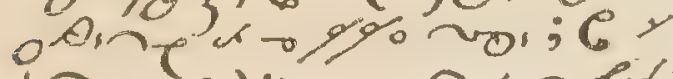

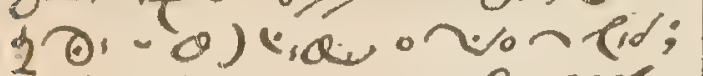
cymogor o vo voros. $011,31062,2,6=$

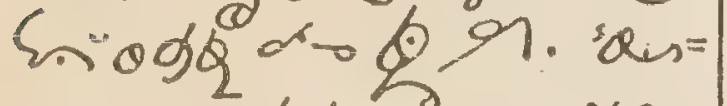

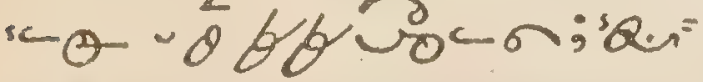

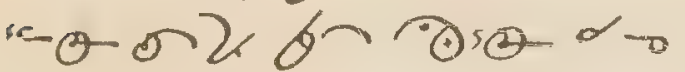
soo 3, 2 . 2.6 sin.

\section{Gatechism}

in Lillooet Language. I. God.

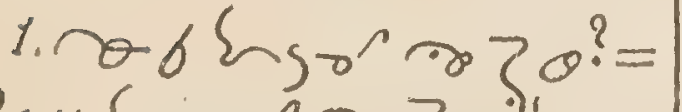

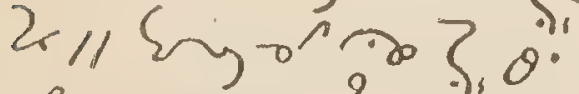

2. $\theta$ पुg 11 ? = "r MII

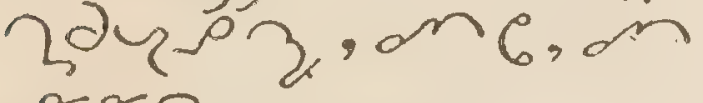
3.62

3. 8 ¿ $_{11} ?=6$ " $29_{11}$.

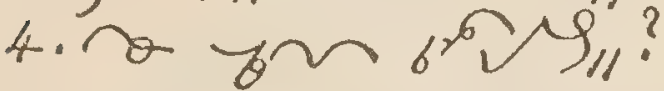

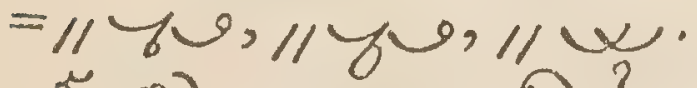
5. Od.

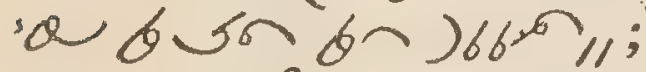

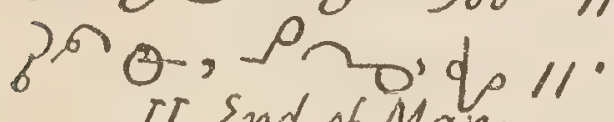
IT. End of Man."

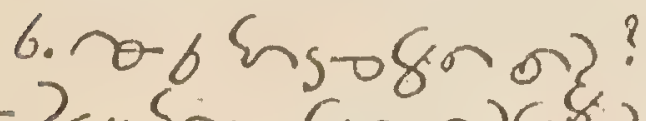

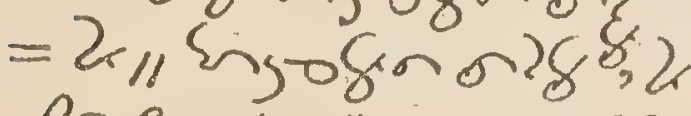
pกรำ uscog $=$ an 6 .

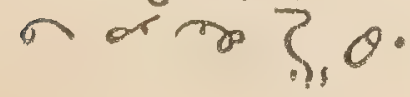
401 14.20 - so 9 ye 602 ?

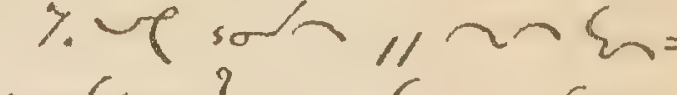
solfon? = " Engogon 4 san tige -roo ?.

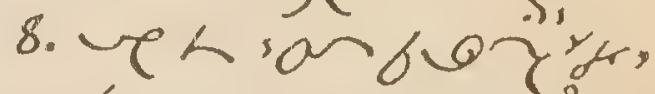
<少人 $-190-300\} ?$

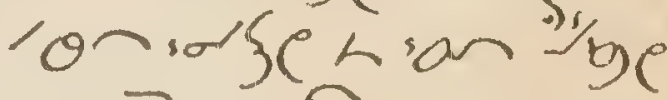

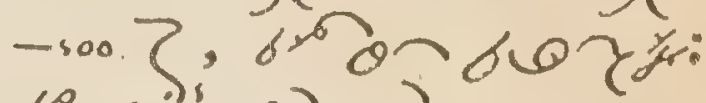
$0,02.096$. asolugril1, 2. we osansoga "or bark; ¿ge on 290 fin": 9.64211 O 10210 ? $\left.=2.02^{2}\right)(1002.09=$

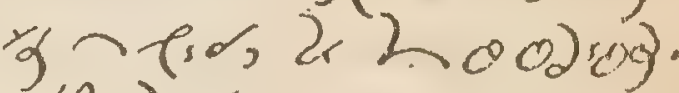
10. 2.00 a y o 0 0):0= 93 4 ..

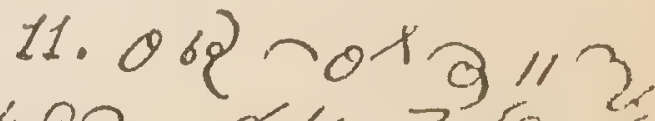

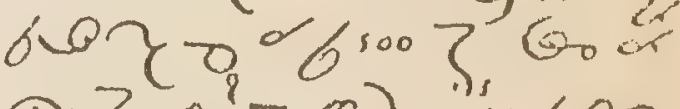
$\partial\}, 0$ : $0 \partial$ " "ll 60$\}$. 0\% Iifcreation.

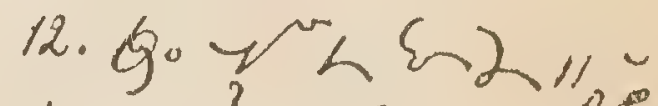
of . ve? = nभन oris ovigar bogue

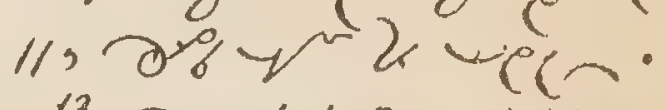
13. 20 b bor b 0 \%, $6 \mathrm{p} \sim 9 m ?=116$ कर b以 k, blongm, moydy.r.mon 


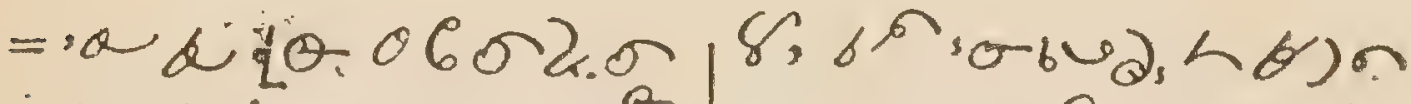
न्रा:

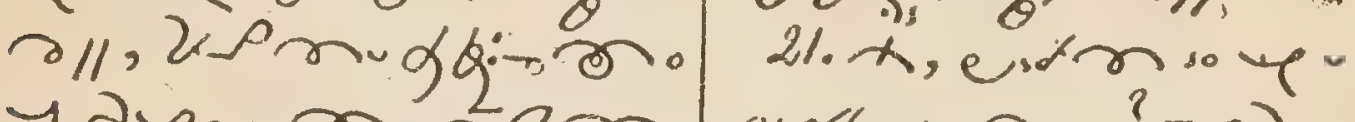
मेय, खा०

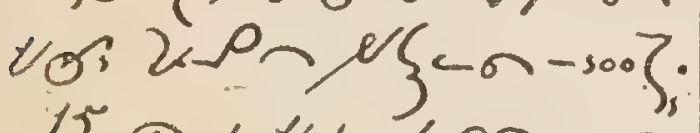

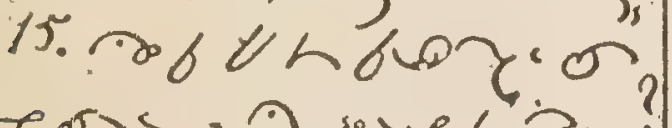

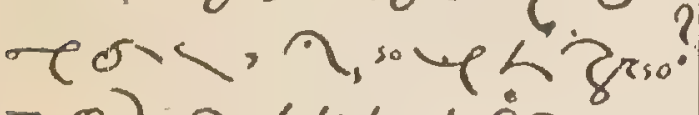
= 0का

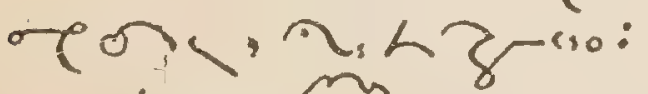
soind ne.

\section{Jesus Christ.}

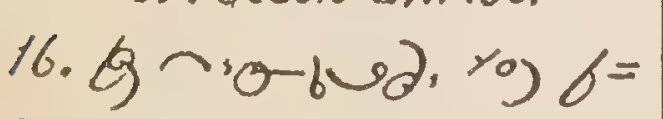

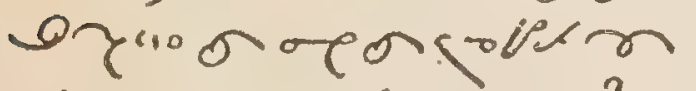
a $\|$ on a क ? $0^{*}=$

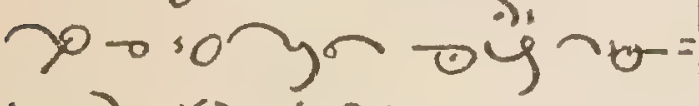

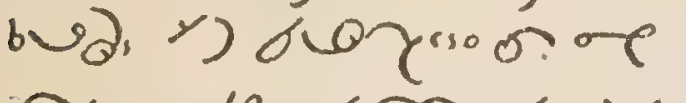
कर on के 3,0 .

1\%. $-\infty\}_{3}$, ? = Ar八 ton ma, $\infty$

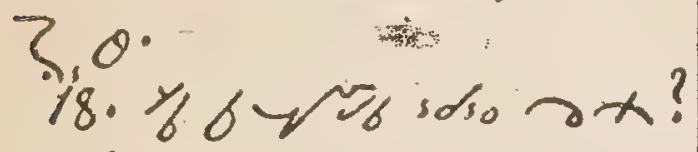

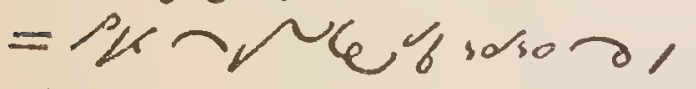
大.

19. $396 x ?=x 02$.

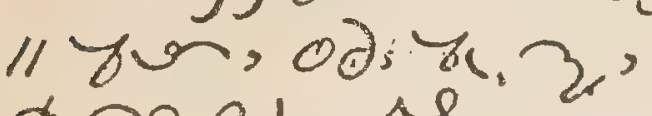
xpodo y."Iron.

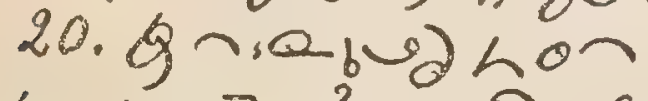

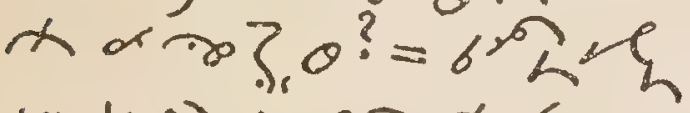

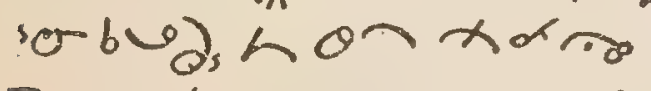

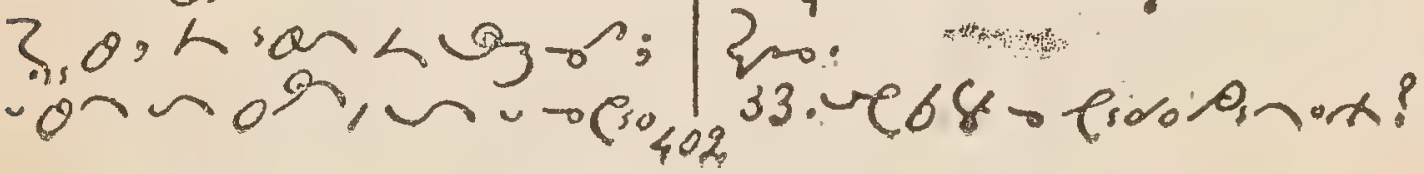




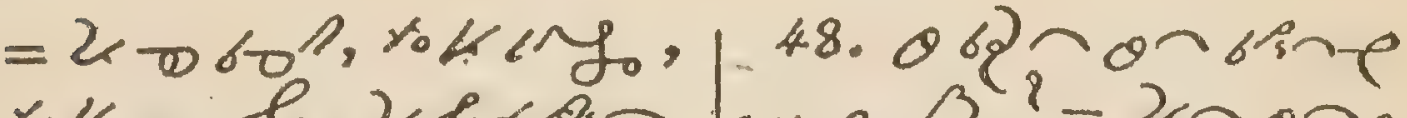

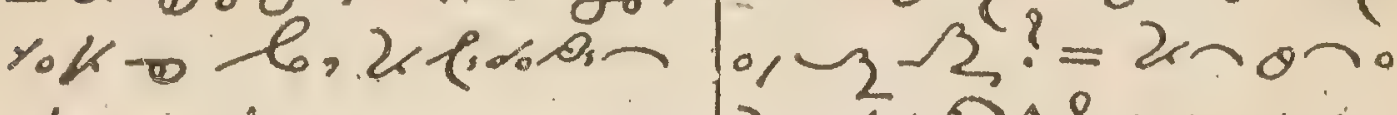
x.

34. no-6\% kidop $x ?=2 x+g \alpha$ podos, $\rho$ sa b

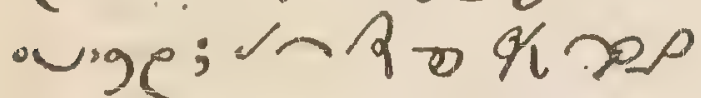
eise.

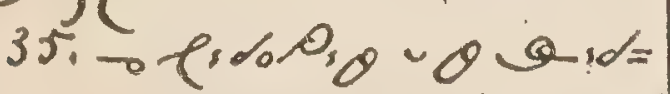

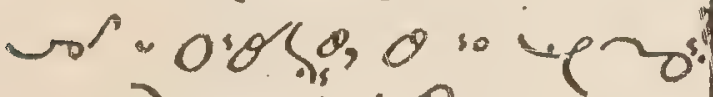
$=0 \partial_{s} \rightarrow$ esdotio ros.

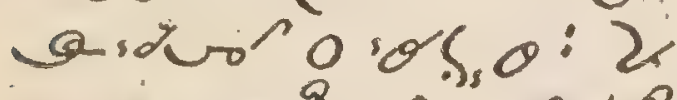
IN

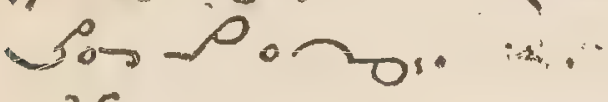
36. 76 gुn by के

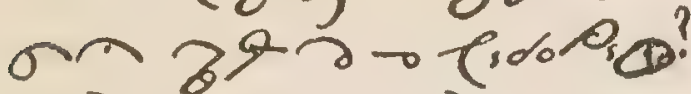
$=2 \div$ sod 02 रूn

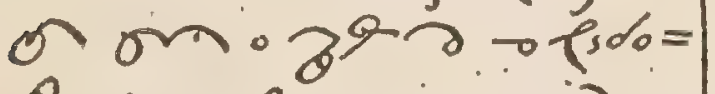
pis: Mrisiv od -o. ot.

$V T$. Sign of the Cross.

45. Anue, azen $\because 81^{11} ?=2$ nó 14.250.

$46 \cdot 0 \times(4$ of $(0-)+1=$

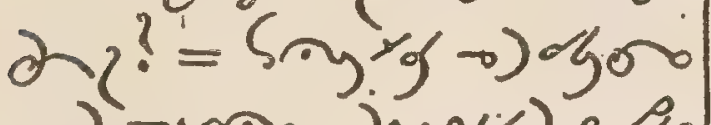
-) Tion, jus - g. ) (4) $-9 \circ 8,2-0) \frac{0}{2}$

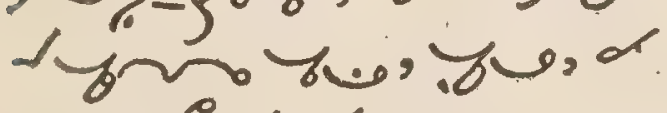
火. 6 \%sn.

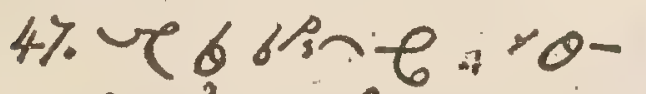

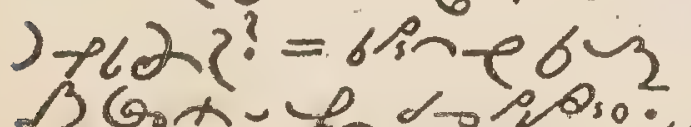

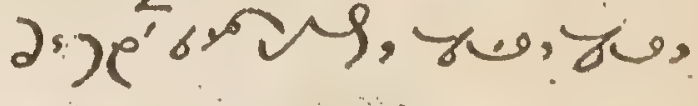
49. o 6q on is - Pon d r P i, ? = खnoy. Paga.

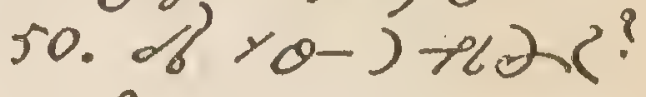

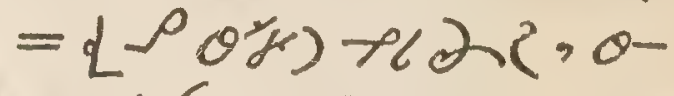

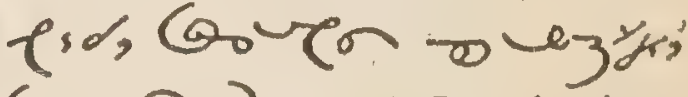
co nayser 6 y. $51.068=0 \%)+2(x$ - $\theta$ pid, Q - - Ta 6

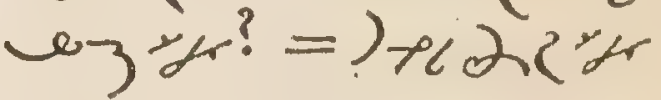

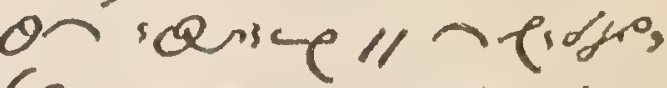

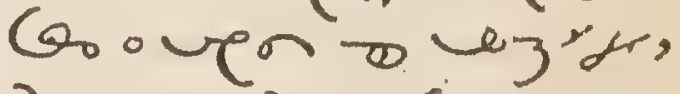
रू d बक्षण 116 yfro 0 s.

52. 0 68 0 o

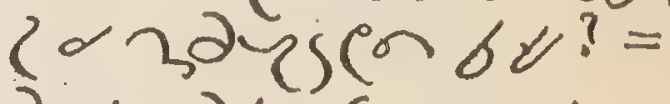
एद 2 gण on b-1ig-gin 64. VII. The Commandments.

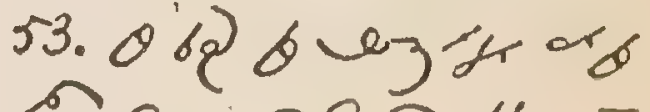

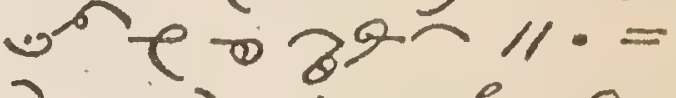

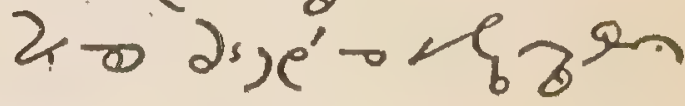
11. 53 bis. $2100-\mathrm{C}^{2 n}$

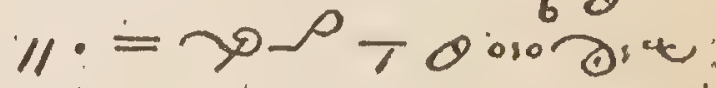

$54 \cdot 0) 6 /$ h of $60=$ ne- n $^{2} 11 !=\alpha^{\circ}$ ine 0 H 3 in,

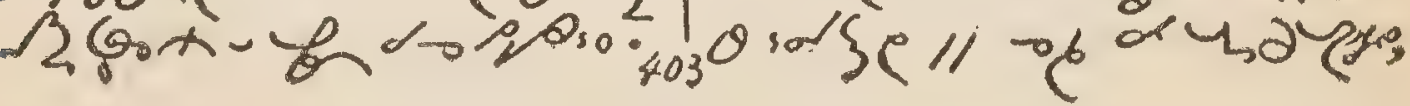




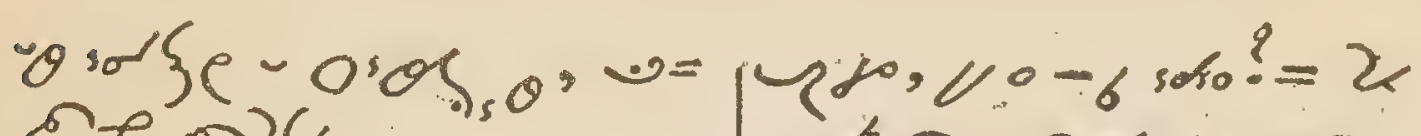
ne on 26 .

$55.0 \mathrm{~g} Q \mathrm{Q}$ ine

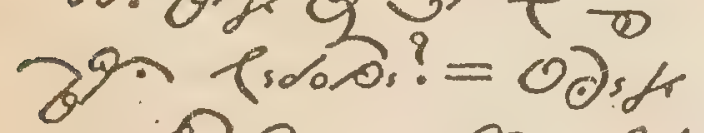

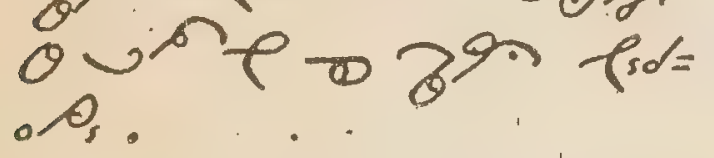

56. $2 \mathrm{~s} d \theta-z^{9} \cdot \rho \mathrm{s}=$ - $A^{\circ}=6$ bo gun - Gur cer.

VIIT. Prayer.

58. 062 \& e 3 \%

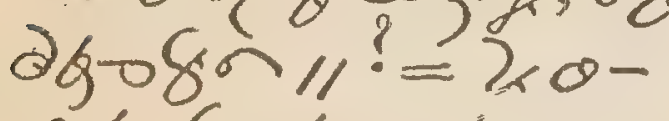
esd, corlonge o - $\left.59 q_{0}\right)$ 59.0106 ol $060 \mathrm{r}$
pso? $=20$ on $3, d=$ vofin boy $9 x$. 60. 2.dog rofy y

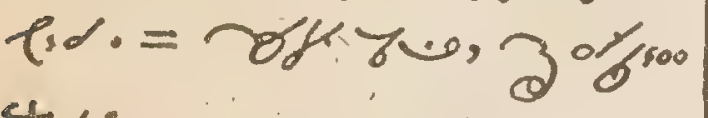
4.

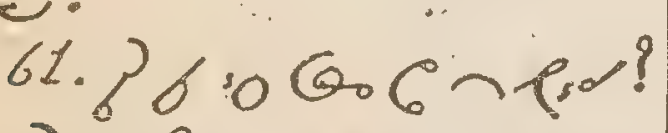
$=2 \times 3^{9} \cdot$ od no, (so. 8 s y 62.2 .006408 nesd. =orifyer. IX. Sin.

62. $4-6 y ?=2 y$

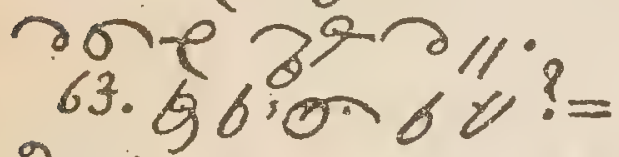

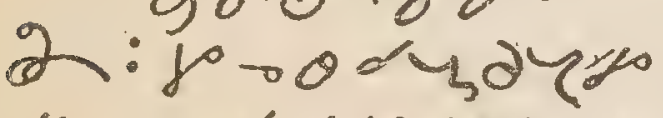
$V 0-6$ sdso; fo rofize Q 64 . 64. जe 6 th orn $2=$ 如

- Gono rojyen

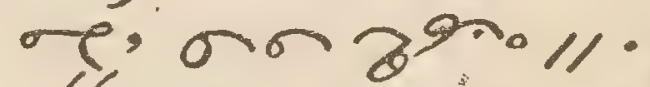
66. ve by Que jor? - 2 varezar 110

6\%. 9 Һs 640 lez 8 ? $=2: 100$ Thrsso o nofron Go rom so, thon. 68. v bעbRora $60 \% 0 .=2$ on onedi 11, ryofin, 2.ogno 100 . 69.6 \% rn 6ofo on sand $\sigma \wedge .91 ?=\gamma \cdot \rho_{0}$, hule rsondy प, 2 sind ofin-1 70. vello is< R= or $\operatorname{cogh}^{\circ}$ ? $=2$ sa.

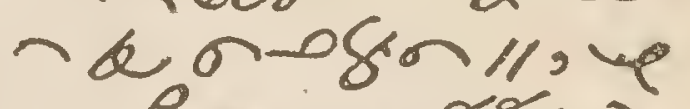

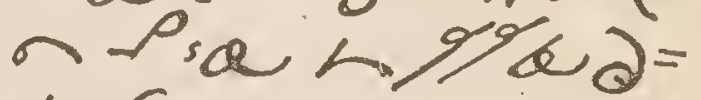
sogon.

\%. ०४, gen o $4:=$

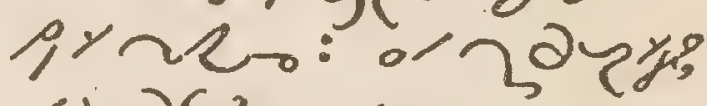

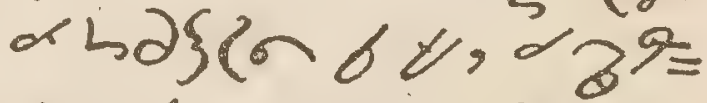

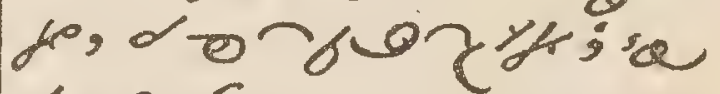
6 lise Dansof: nII of bory 


\section{Baptism.}

73. 0.62 on $62 x$ o\% $y \xi$ ? $=x$ ह 2 .

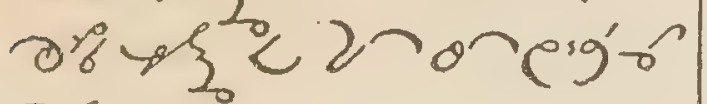
C6080 :

Y4. $2 \mathrm{sg} \sim 64 \xi ?=$ 及 $^{\circ}$

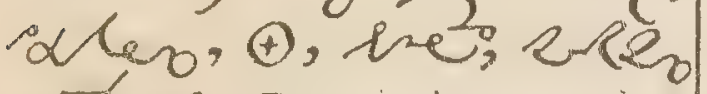
4, zm.

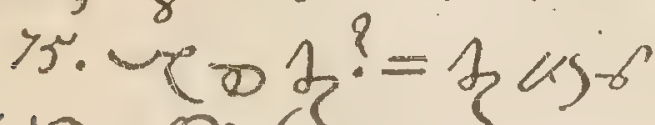

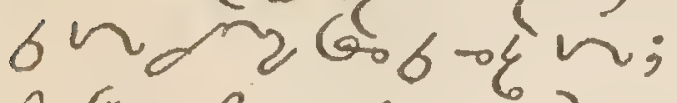

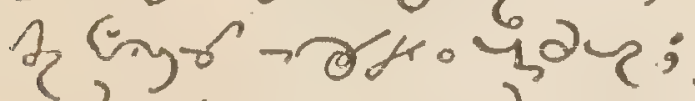
<द⿱ $\operatorname{arn} 11,<$ द ton pios, $<\}$

\%. - $0.05,0$, se 60

- 0,00 Q $20-100$ ?

= का दुव के 2-100

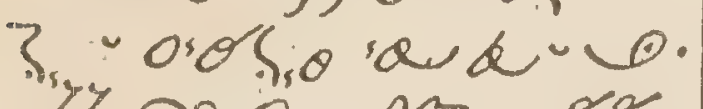
sy. no Q ugr 1 - orol, $\theta^{?}=\operatorname{sa}$

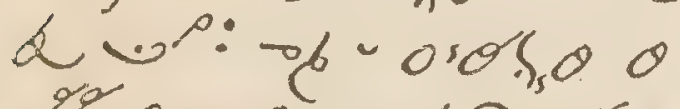

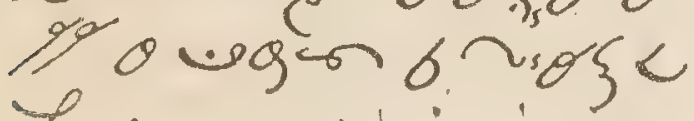
h.

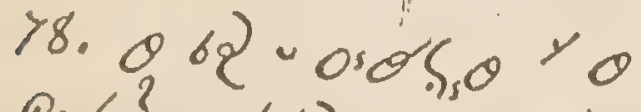

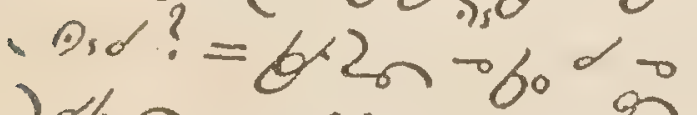
)dyon, wegan,

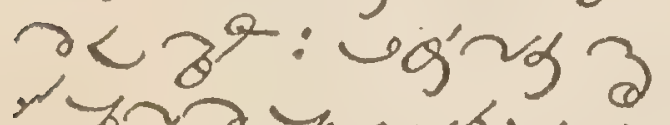
א.

79. Y9 b e 2 or $5=$

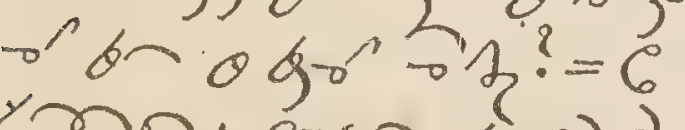

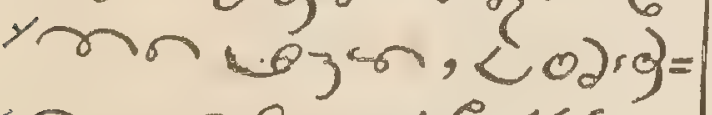

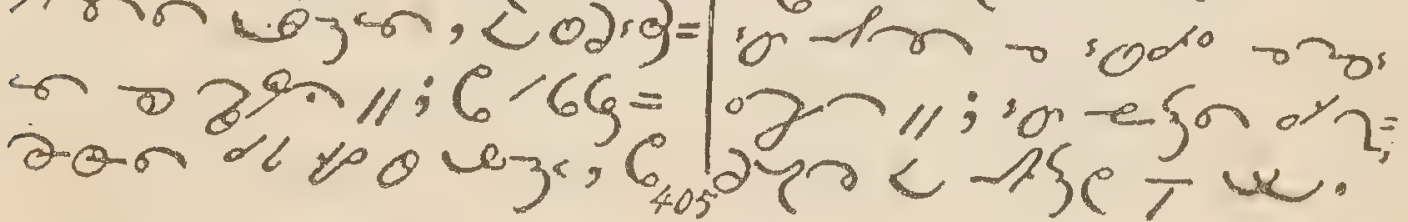

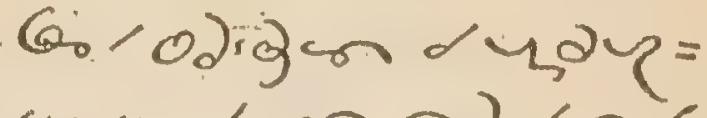
so u of on apsd 万? s\%o $\rho_{s}$.

80.veroreron

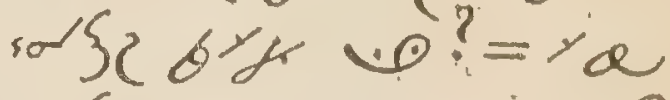

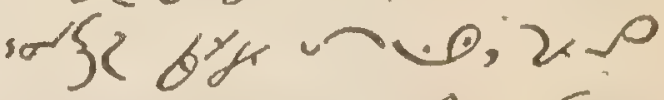
ron -onbr, 6ra कृ?

X11. Confirmation.

81. - क्यk? =

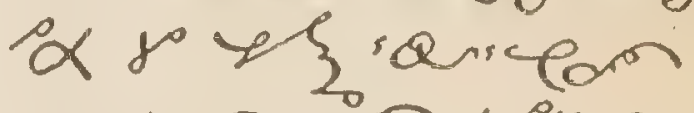

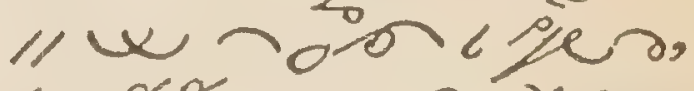

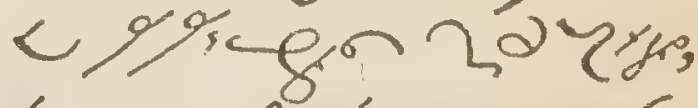

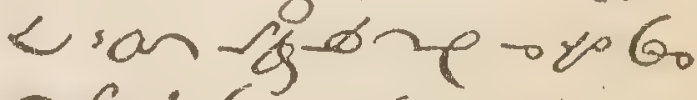
crichogn $<$ ary $x$.

82. bo'anga d

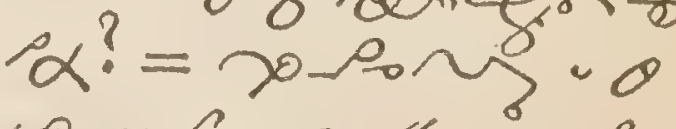

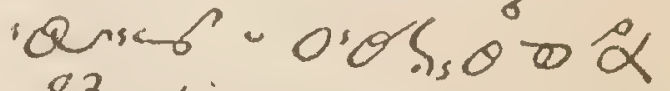
83. Lim-3y 'ansign क क $\alpha$ ? = 'Gin, ) pos'sogn, saga Jdakro "VD

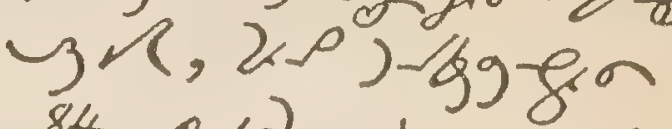
84.068 o do 0.05. "a: se gon क $\alpha$ ? "

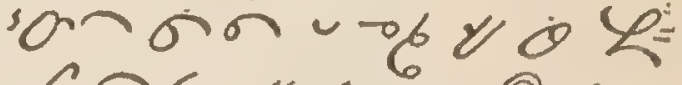

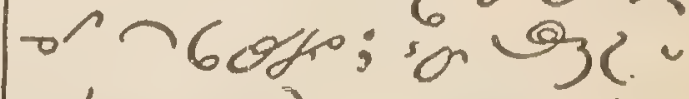
- 6 o dpsar pidoli. "n shon sodo rzos

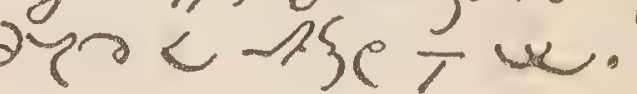




\section{XIIT, H. Fucharist.}

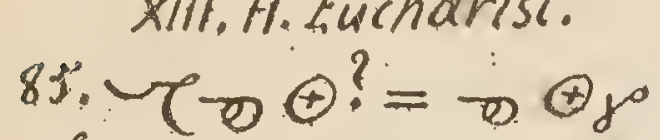

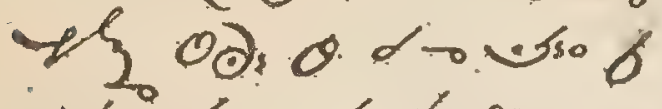
पर्व d wiogos 11 . bux, do m.; Lipr, 6 d 2 .

86. 3o urgon b ang? $\rightarrow ?=2 x$,

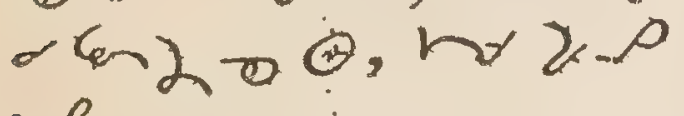
h.

87. $398<2$.

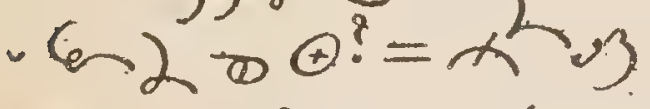

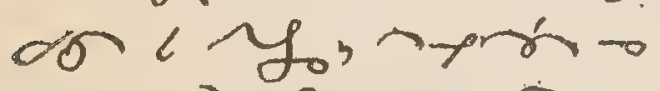

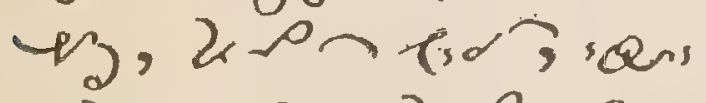

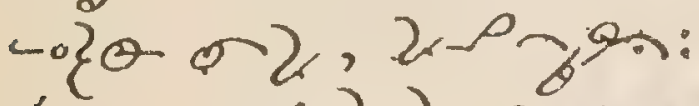
कू, प; 2. यें

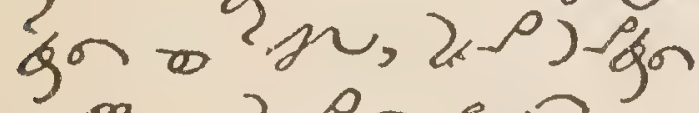

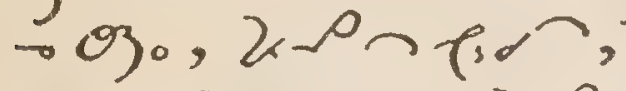
ariso on, $2, \rho^{2}=$

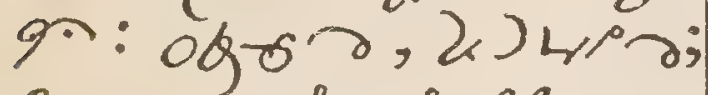
6 'vn.t bु sक. $88.6^{9}, x^{2}$ ). 2) vira, $20 \mathrm{Q}$,

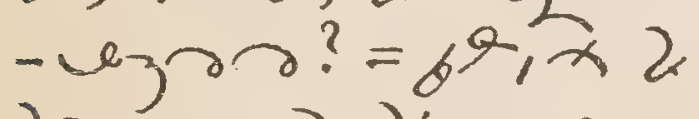

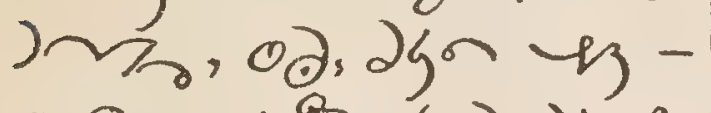
m; $\left.6^{9}, \times 2\right)$ 以is

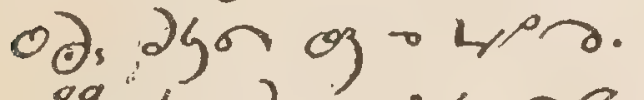

89."x-gon er-ma,

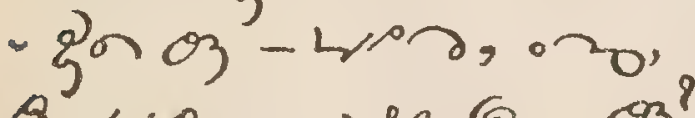

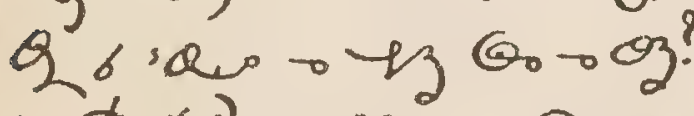

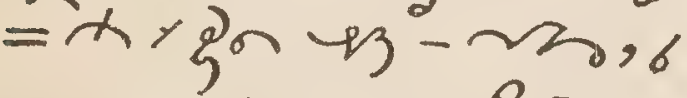

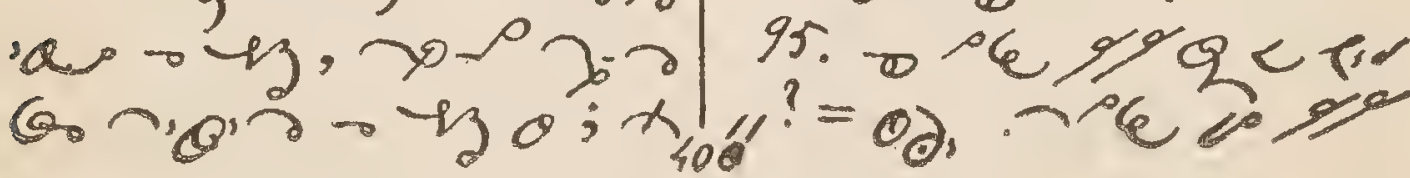

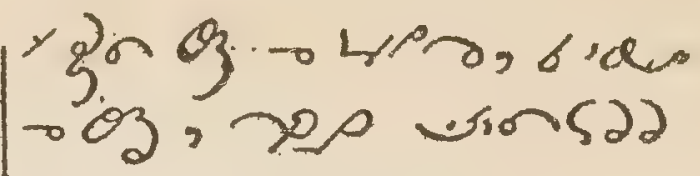
6030.

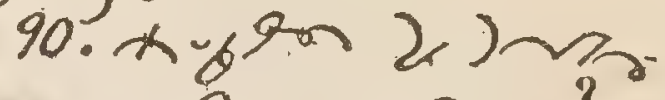

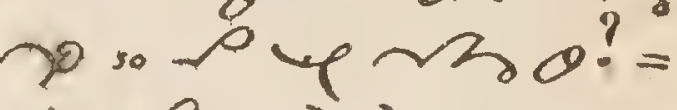
$x-\cos ^{2} \mathrm{x}$ ) d八 Mo;: $G_{0} \theta$ - vira, $G_{0} \frown$

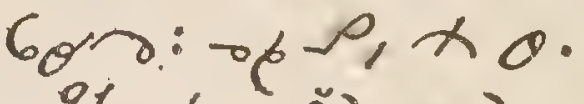

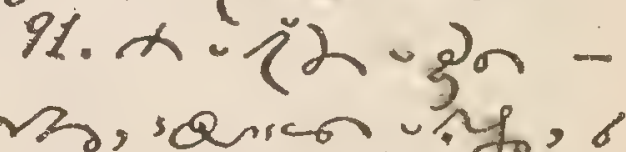
अ, squn usy, 6 ž y

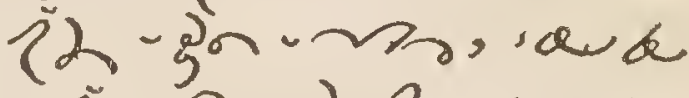

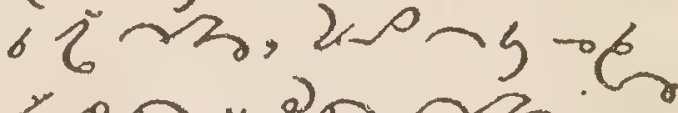

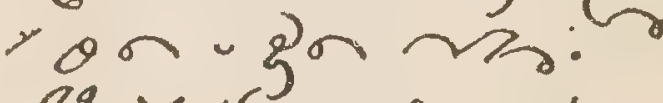
$42.398-c_{3} x$.

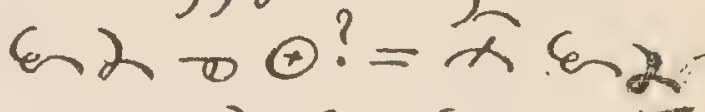

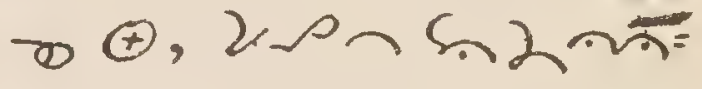

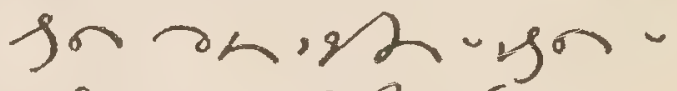
Ton d t.P. $\theta_{10}, \theta_{0}<$ 3 6ofio.

93. of 6.ann $x$ प्रव

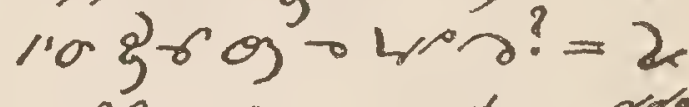
- mosent ad

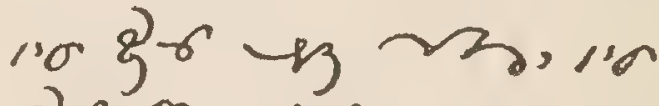

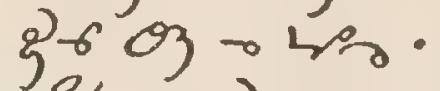

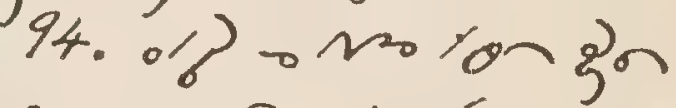

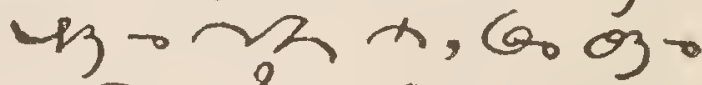
wh $x$ ? = P क ' nion a pasco. 95. कर श्रश्र<रा 
$d 6-6 \cup r d: 20$ ro oq $\stackrel{\text { - }}{11}$; 2 aroi $\theta$

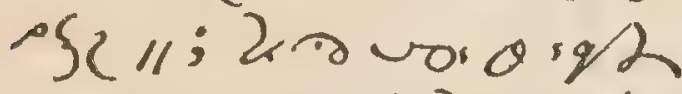

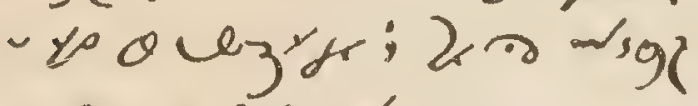
- 0 oped T.

95bis. olo' 9 ce of

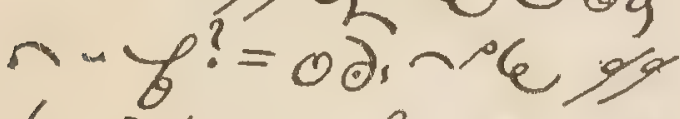
br agon-b. cio osol,

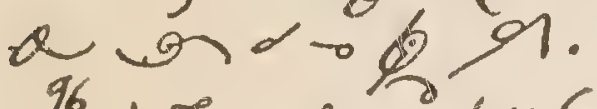

96. $v-4 z-x-6 x$ - $\Theta<20 \operatorname{con} 2$ $\sim 60$ ge० $?=x 62 \%$ $\theta<2 n \sin 26 \%$ ro, cian d 1 br ग'or on yell, 2 mb\% yon $308 \%$

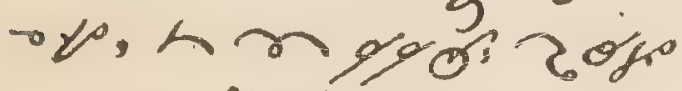

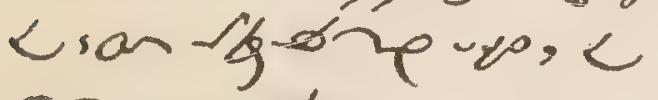
ryes $x$.

9\%. $\theta g-6$ क $\Theta, \alpha i g=$

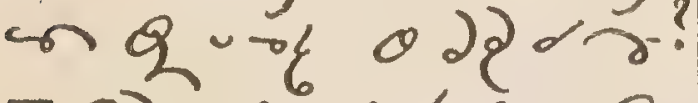

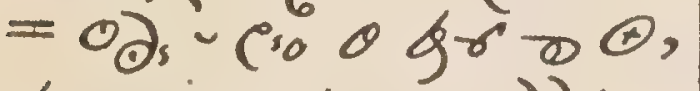
"gan - व 6 व गेगें " v o gr, $00+200$ Nigun.

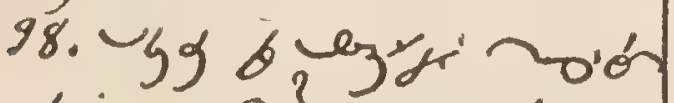
oge $\oplus$ ? ? = דón $\theta$ ge क $\oplus, 2 \rho \cap \cos ($ 6ofso, 6. $>$ jo.

99. 39 b unzter on

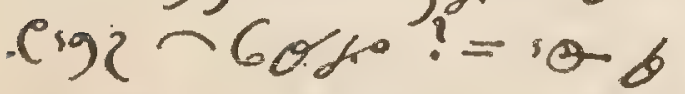
yor ra $x<$ on ar $\Theta$.

100. Jy \& rods:

$x<\theta 0$ क क $\theta !=0$ D) $30, x<$ क o d

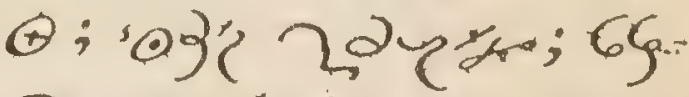

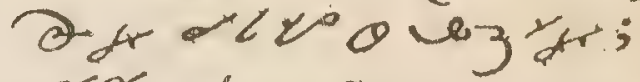

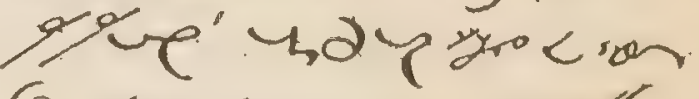

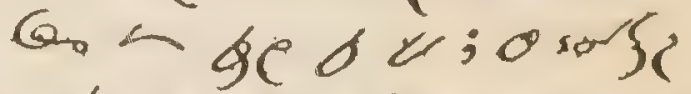

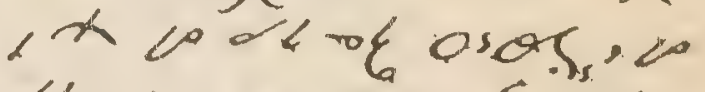

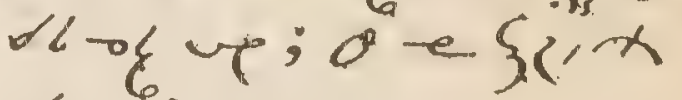

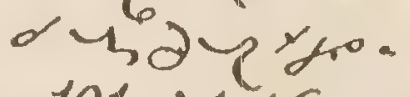

101. 398 - vezr? or dN.eugc $\theta$ ? = "a d Ut b.uge, "ar by of y nion zidor:

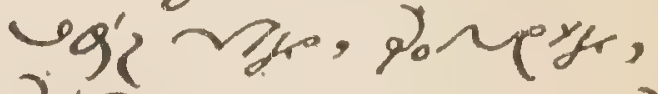

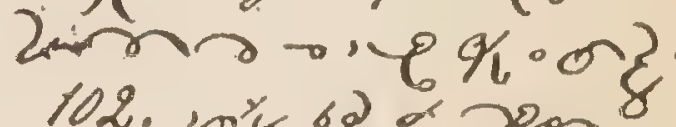

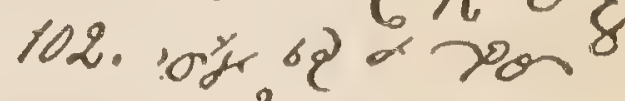

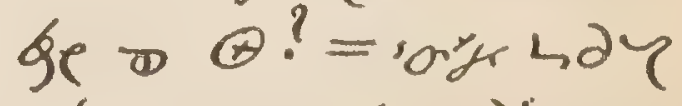

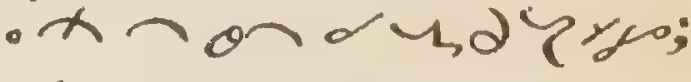
-pon m kas s " sotos $\theta, \operatorname{soc} \alpha \rho \wedge<$ in tr, th.

103. inyer of yen e?, $x$ ? = resine 2

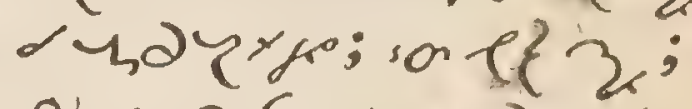
- évn vi man agrofon $d x$ of cyor;iere sderxiygn gigf on -gónpu;irerarr

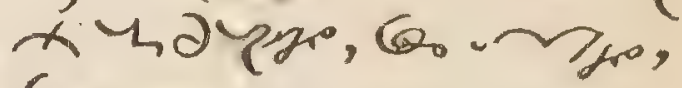
Q. - a boryo;

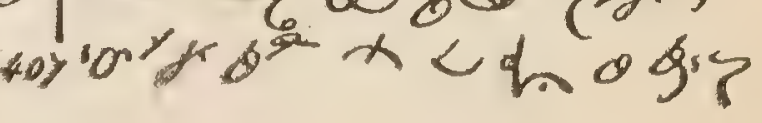


Ty

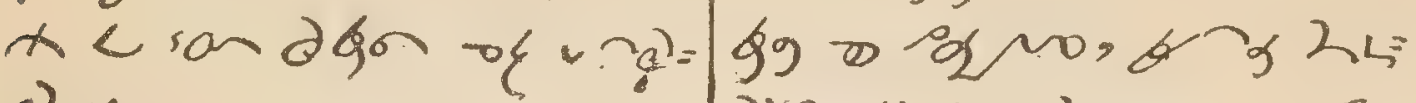
Qb 2 po.

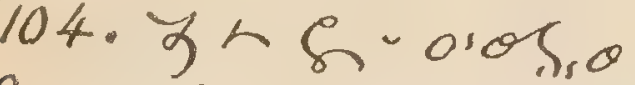
in

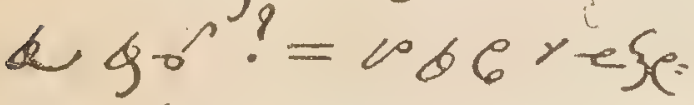

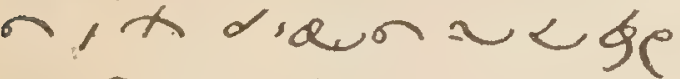
(ब; $\theta$ बisin,

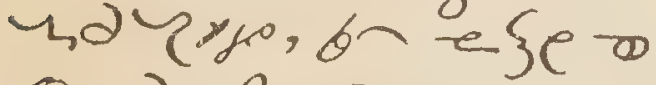

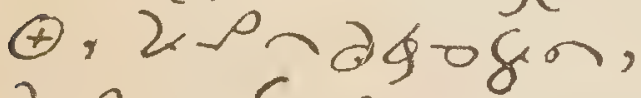
2ar of ofon bine. XIV. Penance.

105. oxp yo 3- 3 , hmot of o isme?

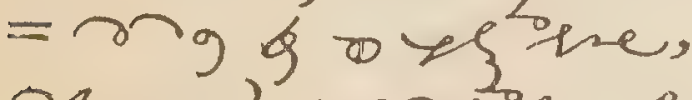
या कर 2 or: aro, ososgr, torro,

\section{ers.}

106. oyg yo \%क्र $\xi^{?}=2$, र $2 \rho 2 \alpha$ $\rightarrow$ (rdops; 20 hind,

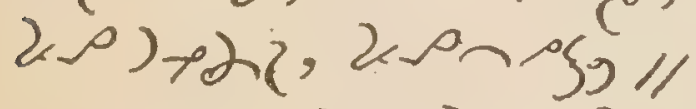

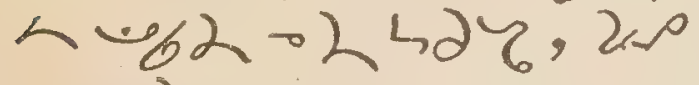
(.0.)

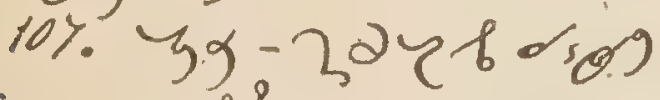
s. $2 \frac{1}{6}$ ? $=\alpha$ s) 0 m $2-24 \partial y \alpha \rightarrow k$

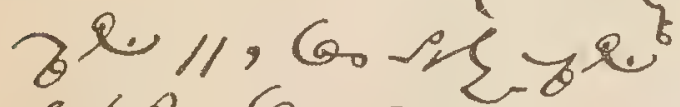

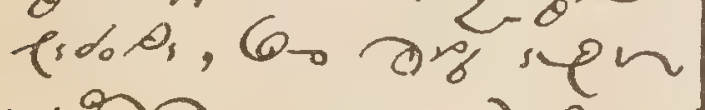

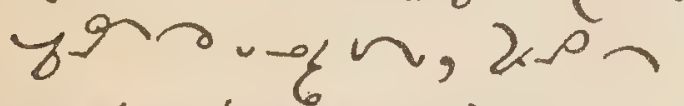
s959' $\left.\alpha 996 \gamma^{\circ}\right)$. 108. dion rigsat $\rightarrow$

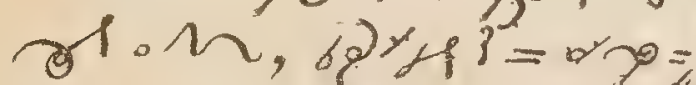

2द 20026,6 , g, $9024 \partial\}<\operatorname{son} \theta_{0}$ 6ho66\%.

109. 68 yp yot o o o

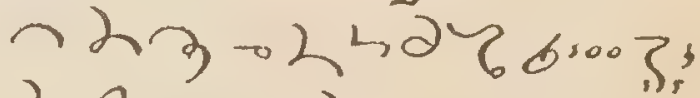

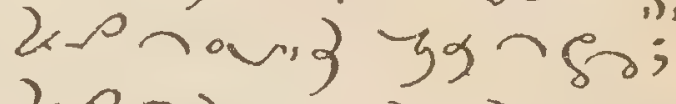
यक $23-24246-1$ on, wa مourga $39<$ עत; $2 \rho-23-242\}$

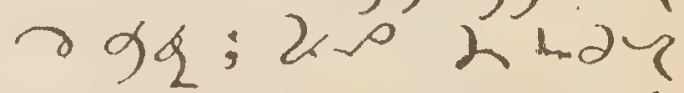
hon $\theta \mathrm{c},<$ and o,

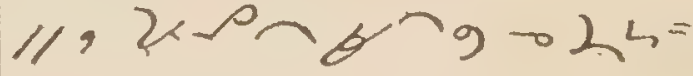
गु न

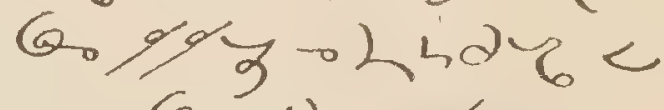

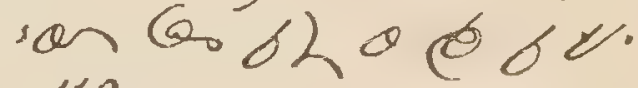
110. 11939 \%org. vos o ososgid ? = $29^{\text {oso }}=$

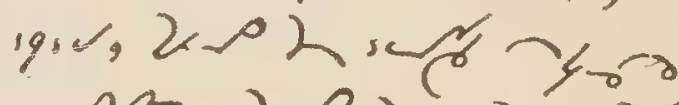
- No, 20) 10 hर 28 ) 9 : 2u: :Qin कoh?, क 2or n; onoysgst oll lo def of of r,

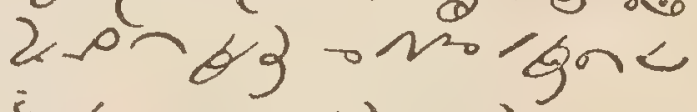
Leion 6 ha 2 osogred

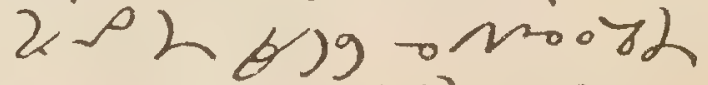
LiQ.

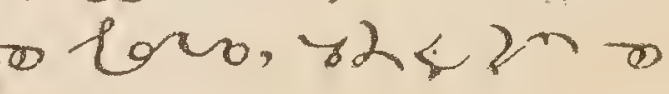

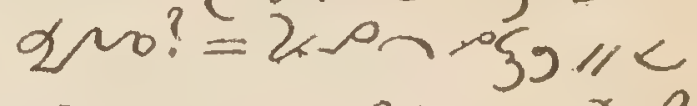
serira o a ro. x. $x, 2$ oregs Yy 
$h_{3}, 2 \rho+63+N^{2}$ her, y2 6) $\theta$ ror

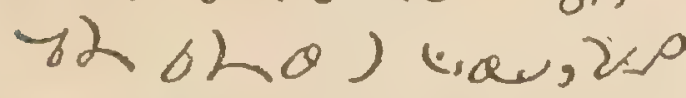
of 3 - No - of. n. 111. a भon (b) - nb; Jo b so Gry = 91? = 6 - 24.2 6 . in o) ososgst, (son we

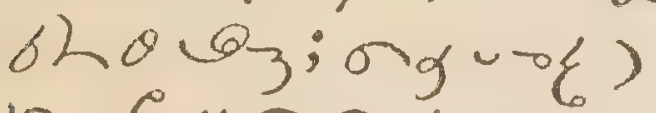

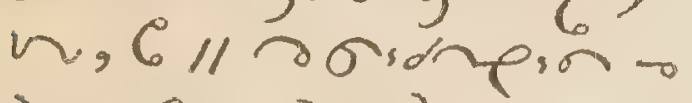
in, 632 2 2ussen=

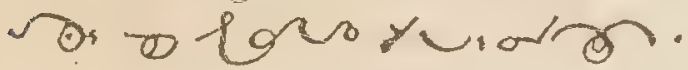

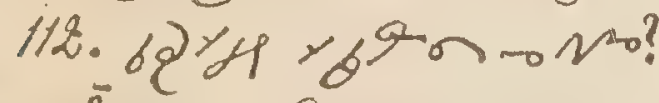

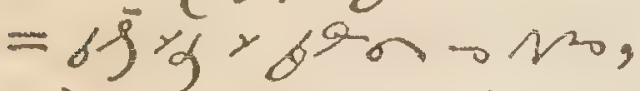
6 hभ, $024,2 y$

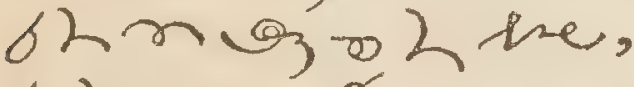
o hmajosksa. mor $x^{2}, y^{2}<$ $100,-12<20$ कh

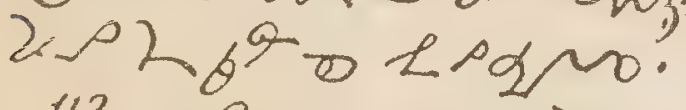
$113 . y^{2}-120,26$."

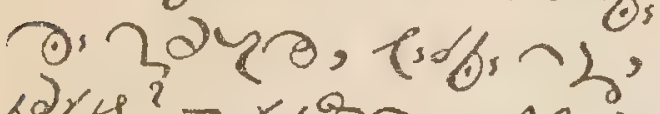

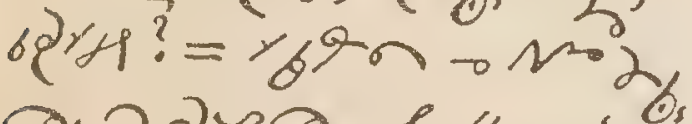

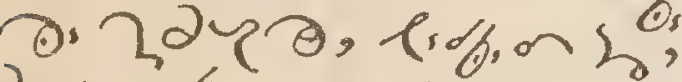

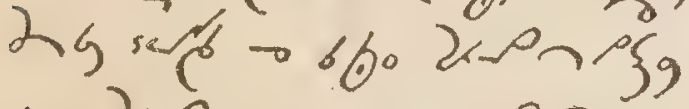

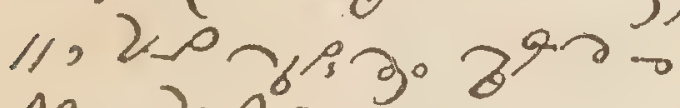
m, we es, $11<$ megran $-\mathrm{h}$ रेद, wh the usum $\rightarrow$ m, 2. 2 Lers, upisan b) ieo भा.

114. (T) tor? =s

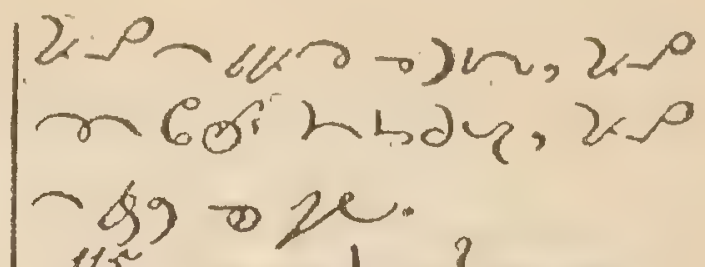
115. us ohy? = sans: m 0 mo wh, sa \& $6(490) n$.

116 . ar $700<$ ososget 9 ,

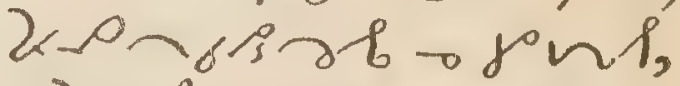
borp? = or poon bh ososgs $\alpha \rho \cap$ si 0 f) $\sim, 2$ g Gorosger

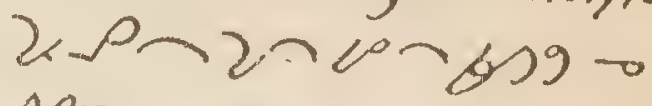
No.

11\% 206ror, sen rm? = करra

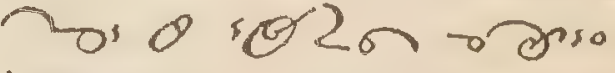
n, कर nem? 118. م-6.0 G.6w? "ynym? = का ia de a a buron

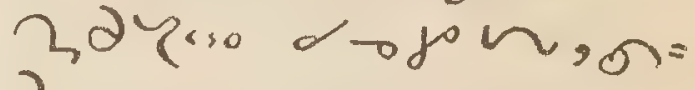

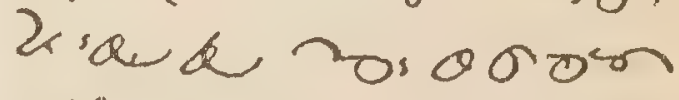

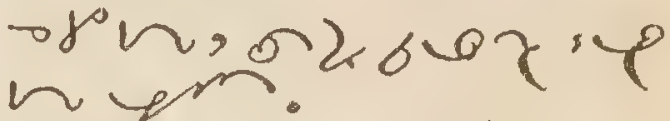
119. of so k. blor b"Pn yon on oso

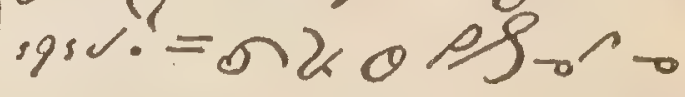
wo lez ron ososgre" or 2 o Koo ron ososg = son on bor sen Yh.

120.0 so 2 25n.

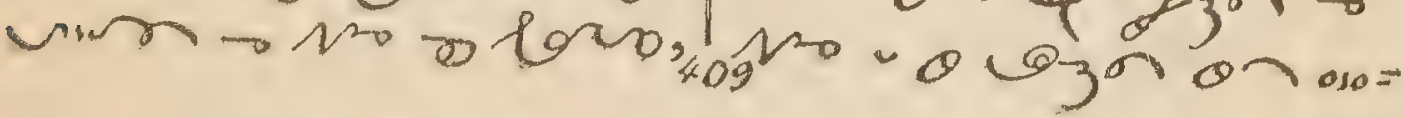




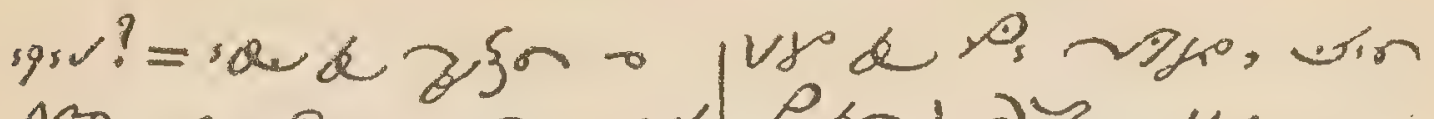

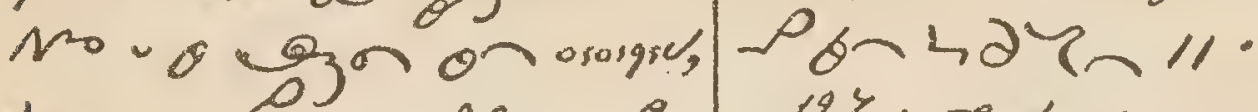
Longh $\rightarrow$ No, का 2. $x$.

121. บ क मिए? = renso, 2 whe lise

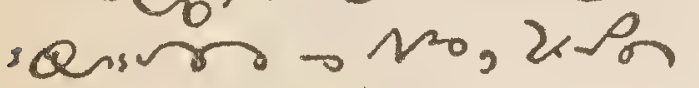

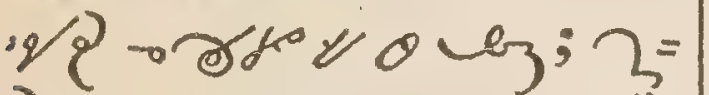
से व

122. उब 0 - 2 sat ros of ig to किin $=0.2 \sec$ d ros on ig or o aro n, on 2

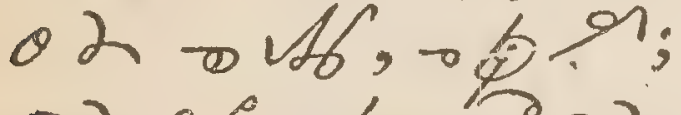

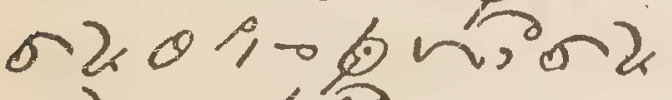
0 म

123. \&Q so sue of o

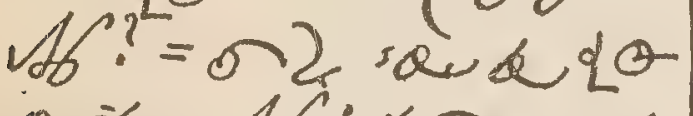

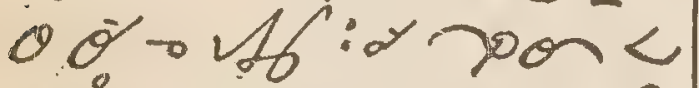
sisn - osin, 20 ก 65003 .

XIV. Zxxt.inction.

124. ToR $R Q_{0}$ ? =

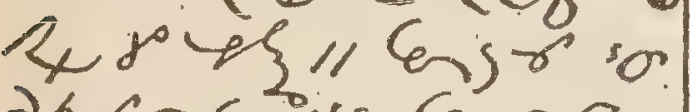
2द्gr $6 \%$ \% Inion 2 gr $p$.

125.06 39 से\%

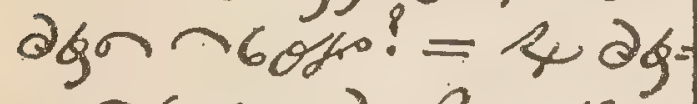
n $60 \mathrm{ge}, 2 \mathrm{ph}$ oss nhaygro, cion ian

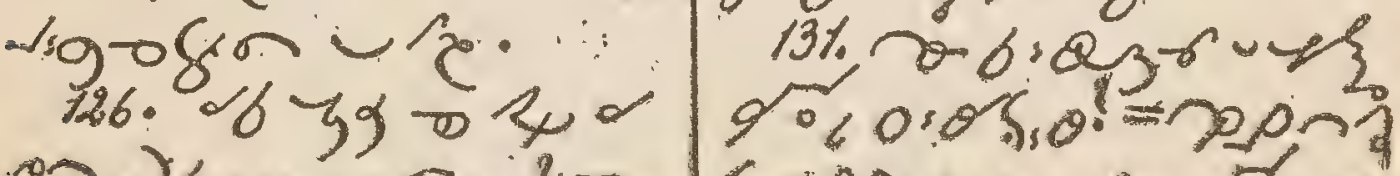

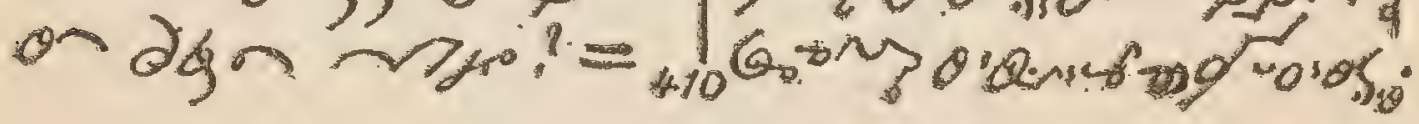




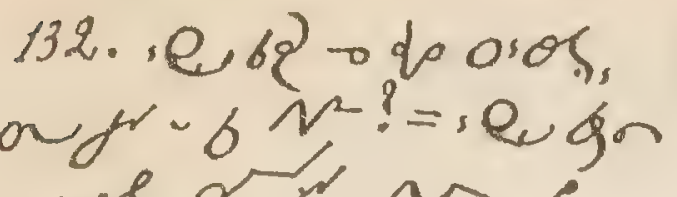
का of $x_{0} \sim, p$

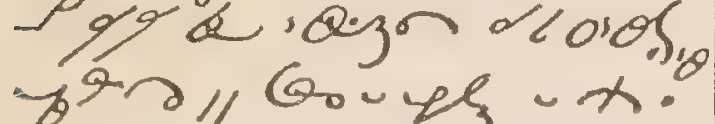
XVI. Matrimoñy.

133. जक 8 m? = 8 m

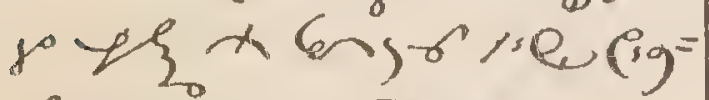

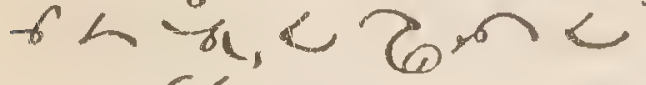
an 68 .

134. जe \% का. जन क क , $\mathrm{m}$ ? = -

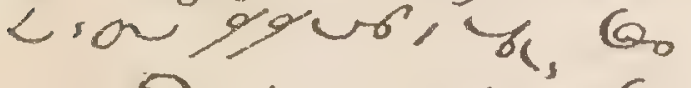
18 , <ion on $45=$ do," "a 6 ह бo-,

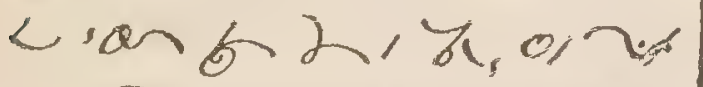

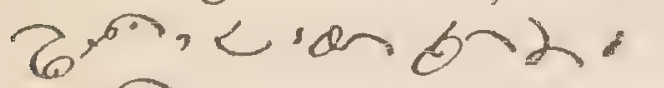

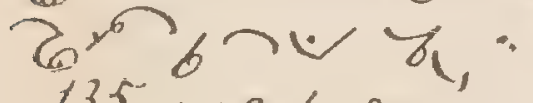
135.vebez'o. - 6 - $\delta^{2}:=6$ > दुon 11

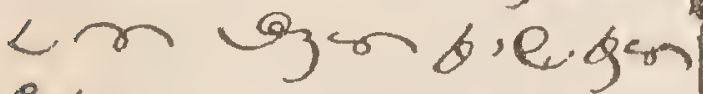
"revrz 39न 202 "11; $6 \times a+20-39-2{ }^{2}$ inert.0.

136. 0 \& $e_{2} \sim \operatorname{sic}_{10}$ ?

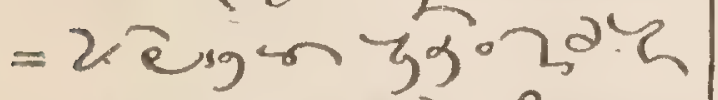

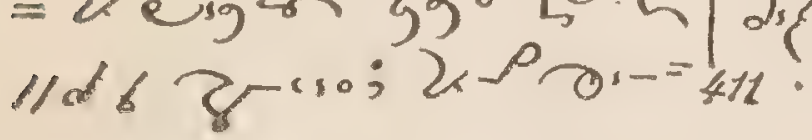

20 $39205-980$. 13\% - (6) ise ns $=$

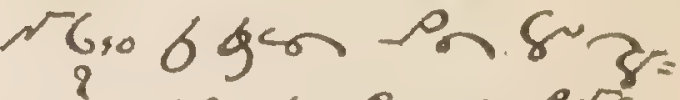
"s? = s cor se.

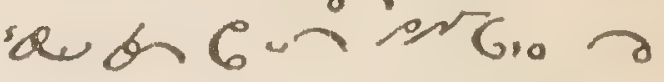

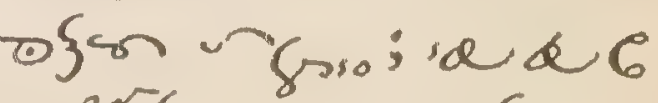
-erkso rian o sun 8.: 'a dion

138. ve be en ost $\xi-1: a$ su? $=6 \times 66\}=$

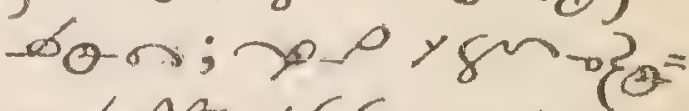

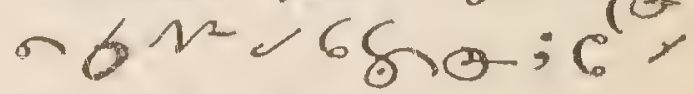
ma gun fido. Gou of bance 2

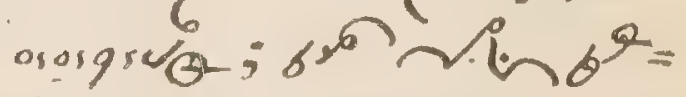

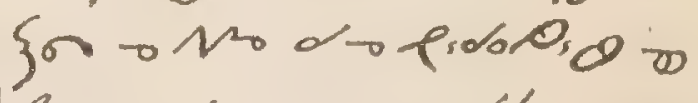
za, rigna dón by reenor 800 .

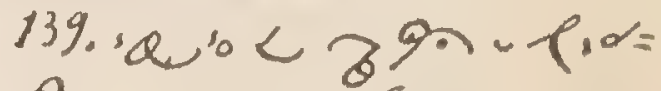
sician a san, oo 6"aud on"a 8"? $=0 \partial$, bo ح2-0.3:

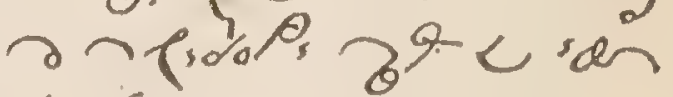

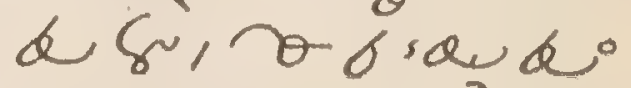
Do" "an $8: 2>0$ $\left.2)_{3}\right\}^{\prime} 6<-\left\{0_{0}\right.$. 


\title{
Okanagan Manual
} ...OR...

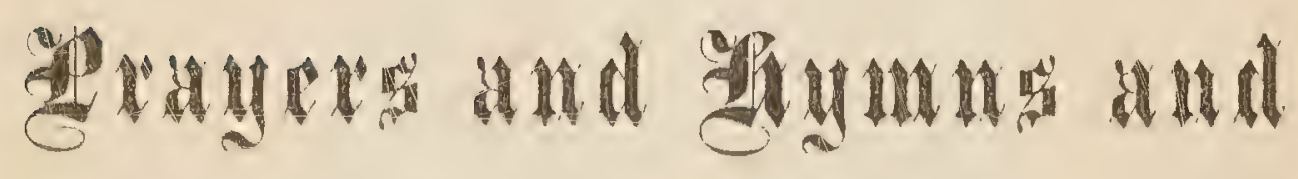

\section{Cutedrigu}

\author{
..IN THE... \\ OKANAGAN LANGUAGE
}

WITH THE APPROBATION OF

Rt. Rev. P. Durieu, O.M.I.

Bishop of New Westminster

KAMLOOPS, B. C.

1897 

okanagan Prayers.

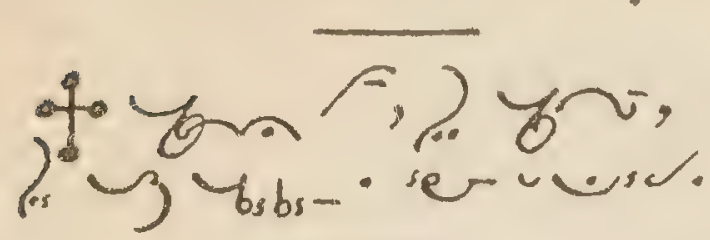
offering of the diay. on, 2 sy, ow

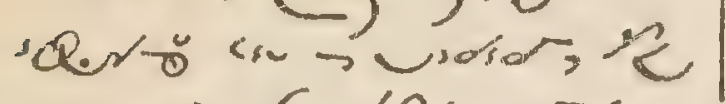
seros, 5 sigs $\rightarrow k_{\text {r. }}$ surry ra ue of Didid, re get-jes

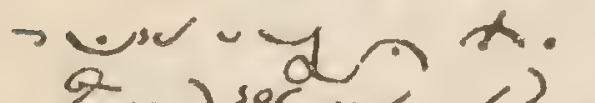
$\left.\left.Q^{2}\right) \operatorname{sog}-<b\right\}>\alpha$ $\rightarrow \rightarrow \operatorname{sos} ; \gamma-\gamma<-10$ - $2, y$, $190-\alpha+10$ sons ( ) ing, ions?

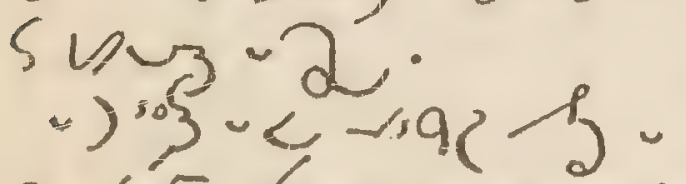
רidst, वa u - )aco-gom. foresoyolays. 0 en 8.66 2. "arn? cinosis rean. L) गuar.

Pater. $\circ, \forall \cup \longrightarrow=$

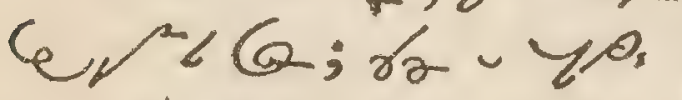

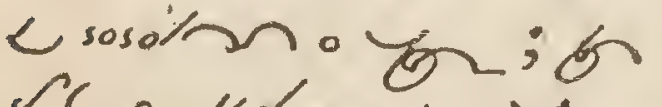
S S.o. Uda - U U

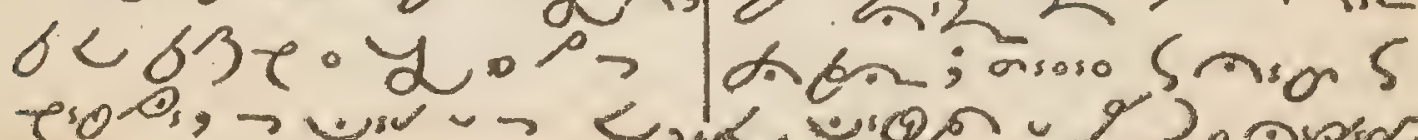

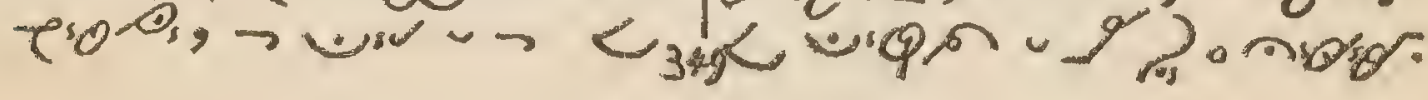

saris as bisis $\delta \cdot \sigma \cdot \alpha+g^{2}, 1$ Unu-

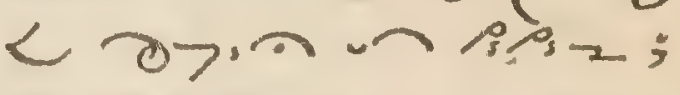
6 - $2=\sin$, q $6<c^{2 / 39}-\alpha-$;

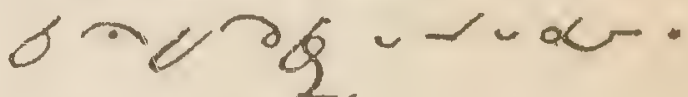
ser-ust.

Ave. 2, 8, a - pe, $5,2,2$ bie- $-20-2-6\}$ o) ser

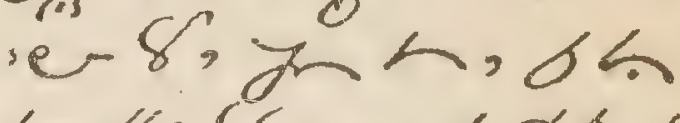
$b_{x}-V_{0} \cdot \int_{2}, 6906$ $\rightarrow$ Yoi, $12, \sum_{0,5}$ buz $<<2$. ser vis.

credo. 0 sod $\{<>$

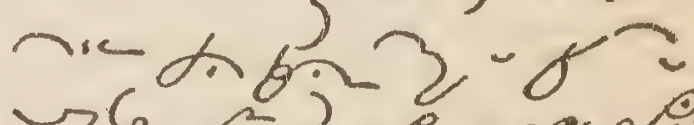
ग) ग) $02 \xi x, 72-5=$ $\sim, S \varepsilon_{2}=\mathrm{CSP}_{2}=2$

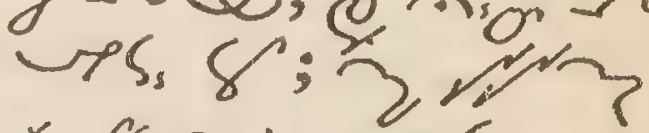

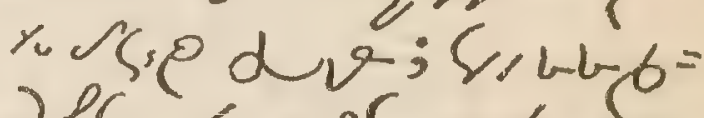

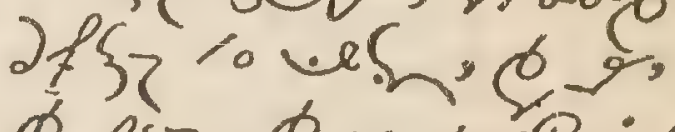

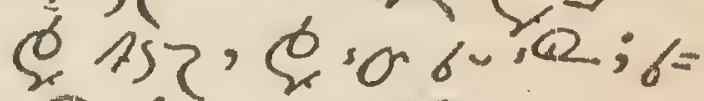

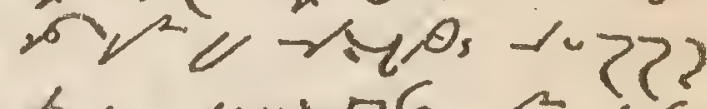
b'ovurens 26 少小上 
$=23 \cdot 02\}-2 z_{3, b}-$,

7. - ver 60 - 03,5

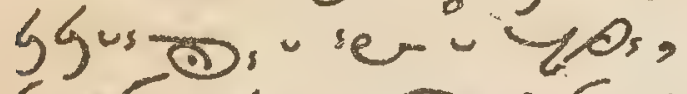

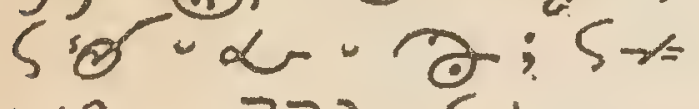
प्र० - 222, 5y>,

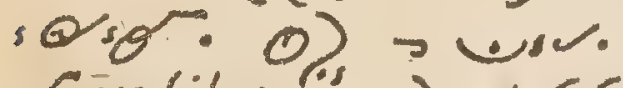
Confiteor." 2 S SSE uh nimponit - see- $83>-4 S_{1}, 04$

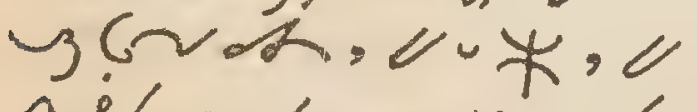
I/ $3 w \cdot 38006$ urevu yor, ? 2 bu) $\sigma^{-\alpha}, \alpha$,

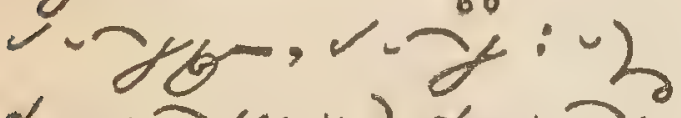
w.

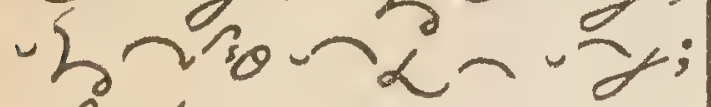

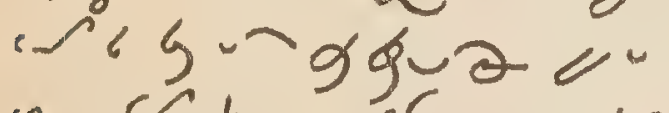

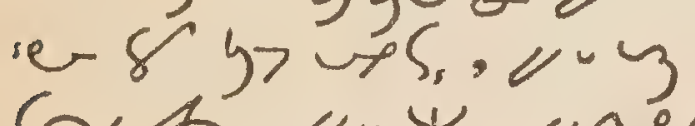
GA, vu*, Ung

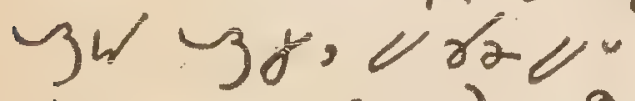

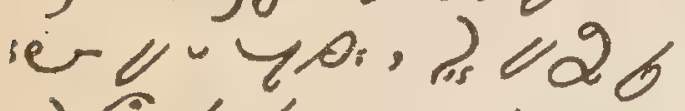
-) 6 bi-v. Misereatur. ien $6<$ 393 - 2 - $\alpha=$

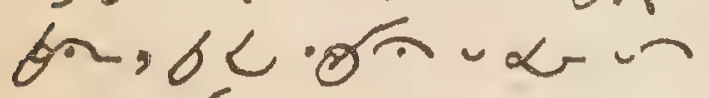
$\gamma^{2}, 56$ an $u v u=$ Es. =ierusu. Indulgentiam. .e bc 3925. 56.0\%, $562=-\alpha-y^{2}$,

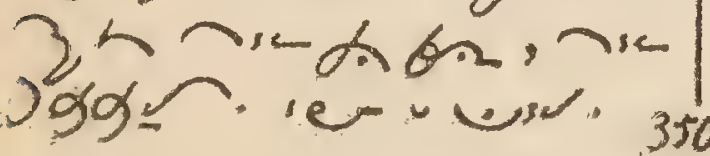

Ten Commandments.

9<- < - J6न.

1. 0 < sore $>2$ 人.

$2.906 \cdot<e<$

- कान.

3. sen-cey-yr.

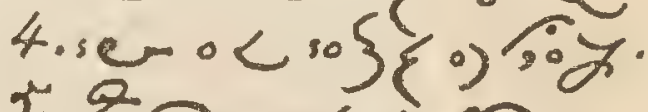

5.900 .

$6.9 .(1)(2$.

1. . $<20^{\circ}$.

8. $9 .<8100$

$9.90<675 \circ<28$

$10.7 .<$ แ2 $0<25$

Precepts of the Church.

2.tec do in.

usdid 5 went yn

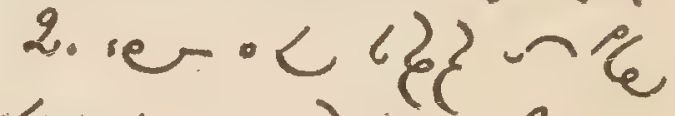

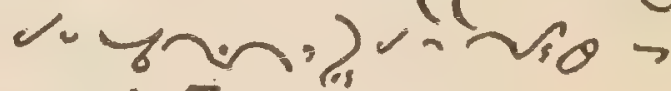

vidid.

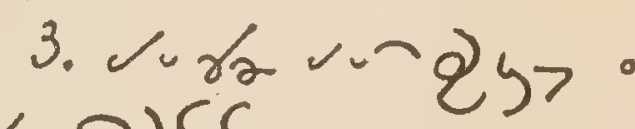

(几) $\{$.

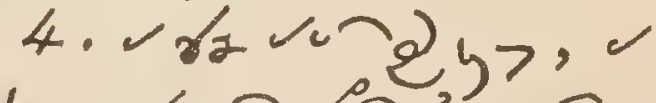

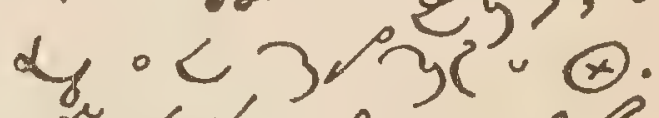

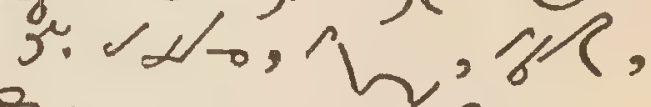

शर

6.unzerpocos

- 2 .

seven Sacraments.

xo:ar nus

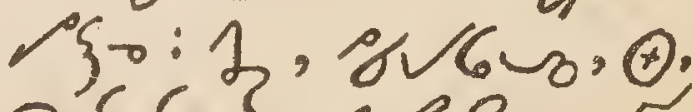

उर ह?, यर20.0 
Act of Faith.

a $\left.\}^{\circ} 02\right\} z=0$ 人, -ro

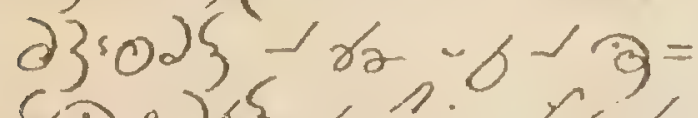

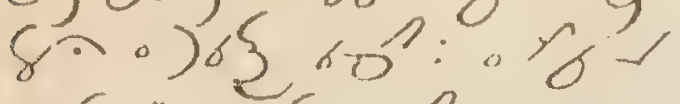
3800) $6\left\{\begin{array}{l}0 \\ 0\end{array}\right.$

Hope.ser sos. $\left.\mathcal{L}^{-2}=0 \wedge \therefore 6\right) 2$ $x, y \%$ whe $\phi$

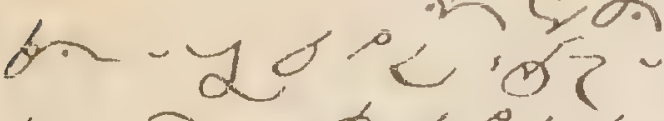

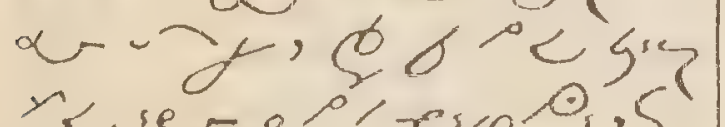

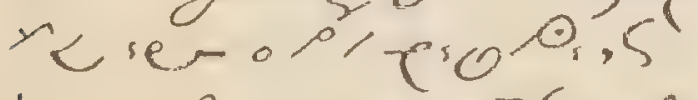
$3>6$ ? म $\because-6$ ए ग) $759 \circ<427$. Love. er < sogs?

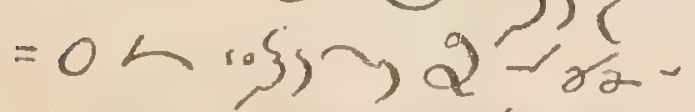

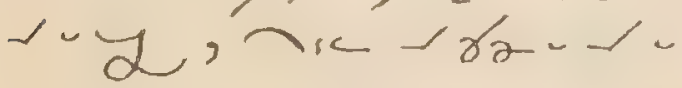

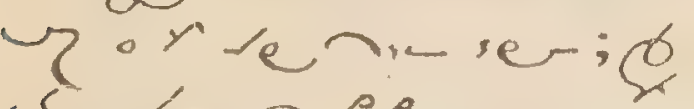

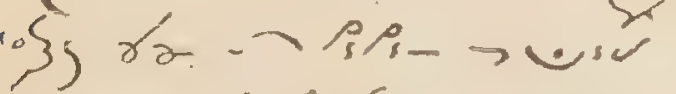

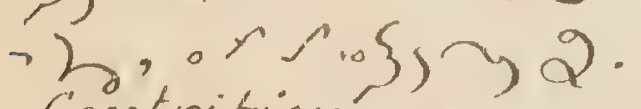
contrition. ere $\kappa_{2}-\partial_{2}=0 \wedge{ }^{\prime}$

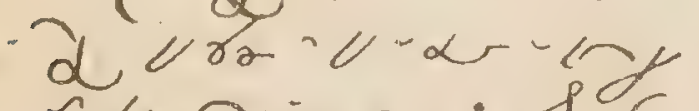
- N de ric en; 15

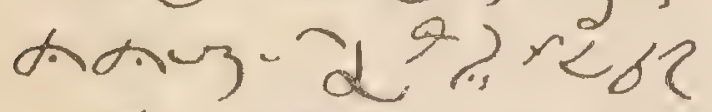

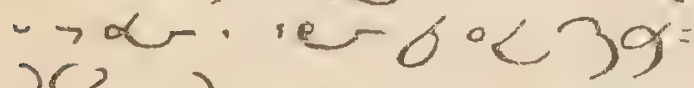
उदर - 3 . Angelus. 1. N 1 . ऑ( $2-4 \cdot+2.8$. $2.692-25=\sigma^{\prime} 0-351$

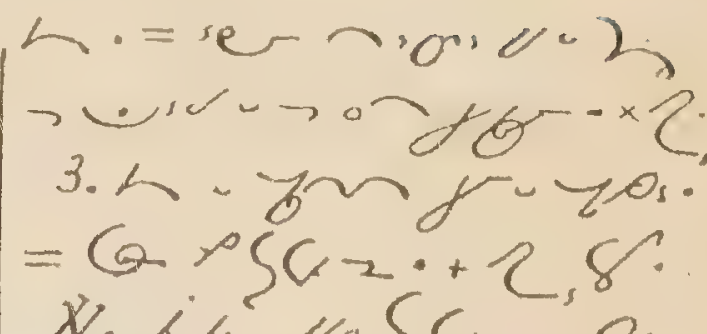
X. Jor-voSkz, Ore

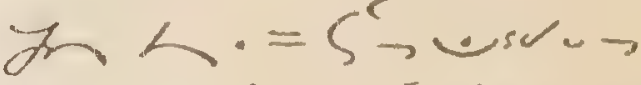
L serdiz, 5992 - 6 $5 \sin x$.

oremus. Oh! en

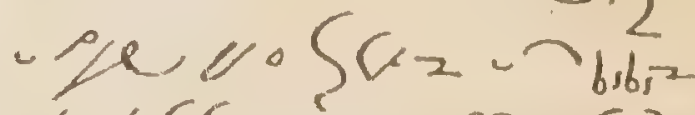

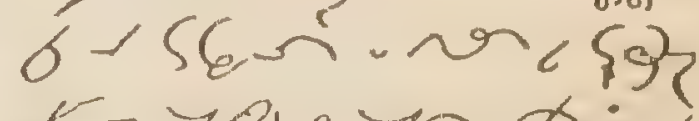
$\delta \rightarrow 4010 x ;$ जल =

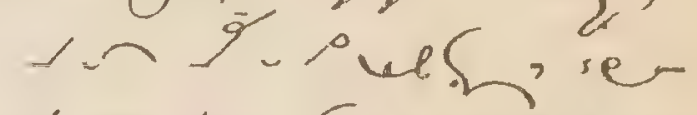
6 a gie 58 rados 472,56 andury = Eरr. ser - uer. Gloria Patri: to - 5 ,

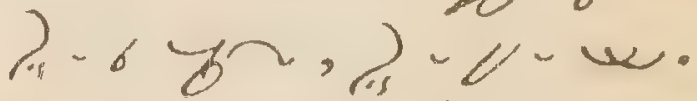
$=-4$ unsas

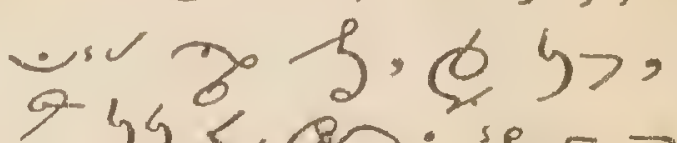
sub tuum. o 82 iser bั 5an 2 ; ie 9

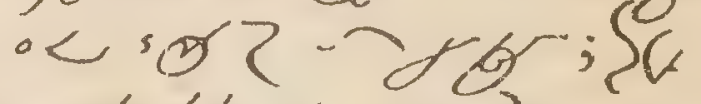
$=69960$. 6) - $=$ करे? पद $\sigma-20\}-\alpha-10$ $8, y>-15,0), m$ 
Morning Prayers.

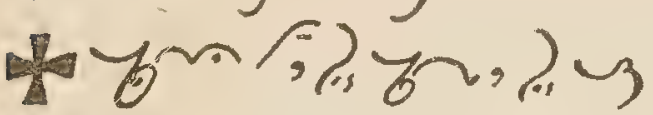

robr-. serscuss.

Veni sancte. O.hw, novoS $\mathrm{C}_{2}, \mathrm{~S}_{6}-2 \mathrm{2}=$ $-12 ; 6-07-2 z, 5 \approx$ - Adoration. "). Uu C $\log 222 \cdot=0$ nuser, an iso 3 h.

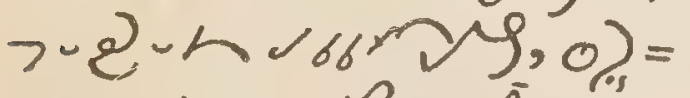
ritevidR。弚;

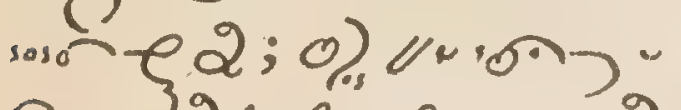

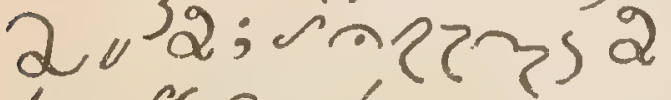
de ship var vuc. Thanksgiving. se- $<$

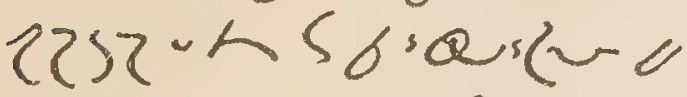
$2 \cdot=060)$ s.e. 1

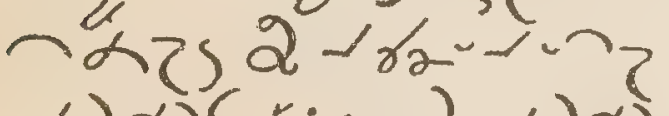

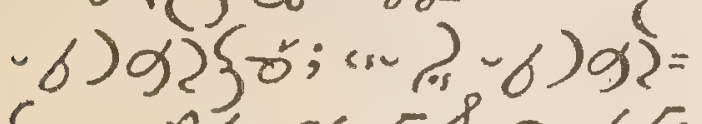

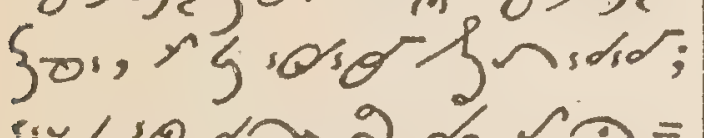
"worng 2 ra $=$

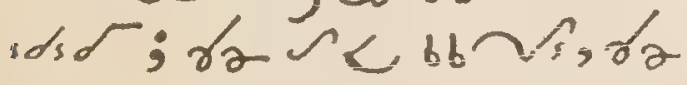

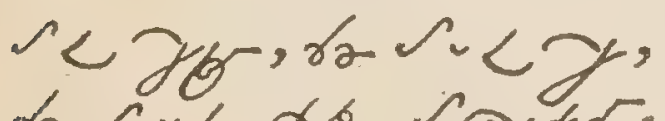
ras $<$ gq, nises, sarny wra. 6 ags 06) rs.e $58033-6.0$

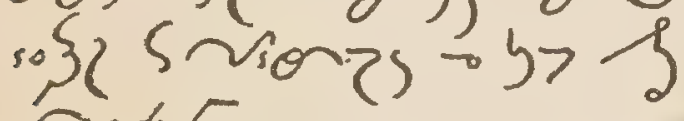
sidid.

Petition. .er- $<63$ $2=56$ b $20-\alpha=5$

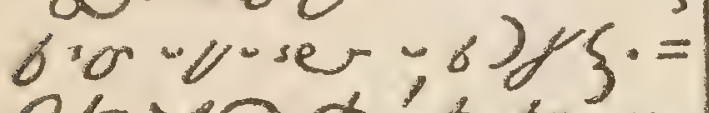
oxyาx:'大on 352

2 火usdo; 9re $R$, ? एव) - U; resons? use-, 5y Jgg v unPi-; SAronMU- $590<-19$

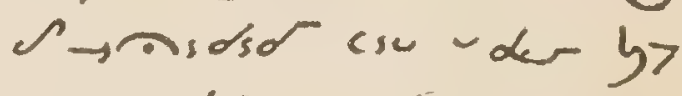
-6-1in: hon-2

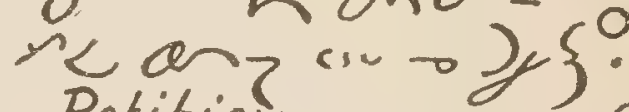
Petition. se-C sos - 0 b gam.=0人?

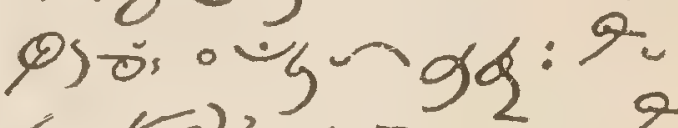
$4092-475$ 8.5.5:700:0 6.06) -5ip; se-6

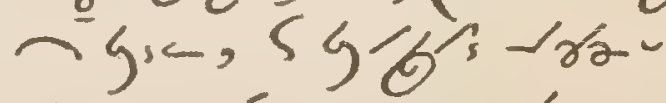

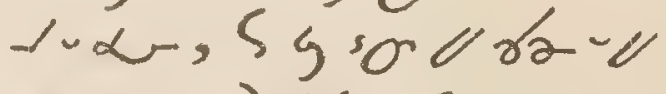
se- - ) $8 \xi$, 5 vion - $20 x-4<-3$

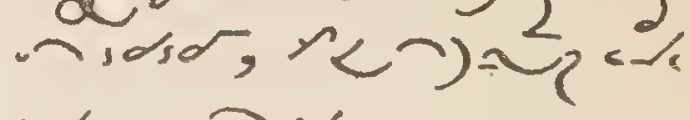

Pater. Ave. 2, 8 credo. ग3 502$\}$. seven Sacr. $x$ brar Act of Faith. ser- 2$\} 02\}$ ? Hope.ser-co- $\mathrm{N}^{-2} 2 \mathrm{2}$ Love. se-cosss?. contrition.se $=2$ Invocation. ser $6<$ sgan vure- 8 vos, 


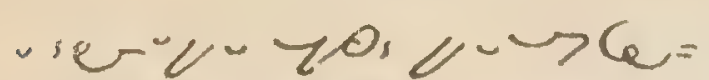
$\mathscr{L}^{2}=$ To the B. Virgin. 0 sers, yn ? - y, M

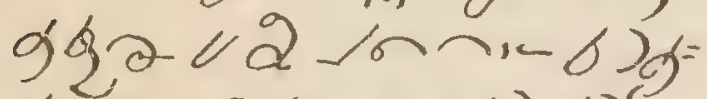
कर; 0 « $\$$, 2रe 9 क; - Usoson, $\mathrm{b} \sim \operatorname{sos} \mathrm{s} d \mathrm{da}$

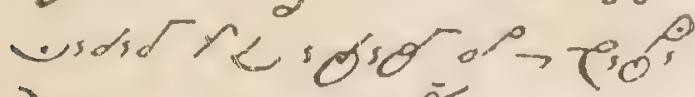
P, nc.

To the Cr. Angel. 2 ser

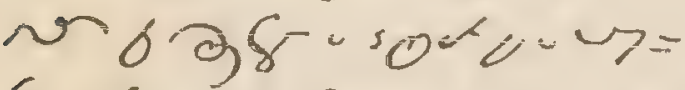
दr, on 5 y ofo, $S \rightarrow$ was $>-4$ के ${ }^{\circ} \rightarrow \mathrm{T}^{\circ}, \mathrm{S} 955$ - L'or.

Tothe Patron Saints. OSC

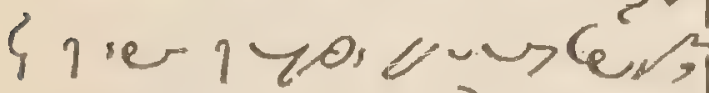

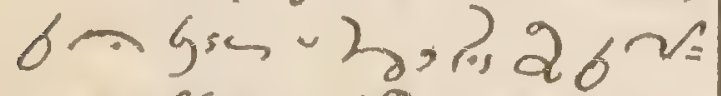

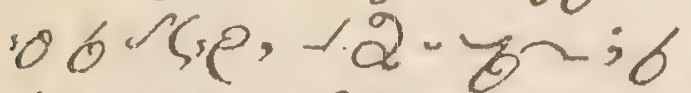
bs wet -2 of $\rightarrow r^{\circ}, 5_{5}$ $\because \cos ^{2}<\mathrm{Cl}_{2}{ }^{\circ}$

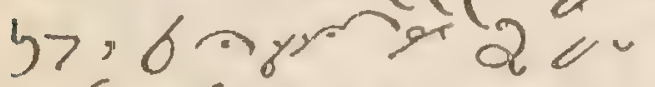
verserserd. Angelus. on

Sub luum. os $\alpha=U 2, b<-p$, 02 serb两人;98 - L बकर थ $6=, 140=$

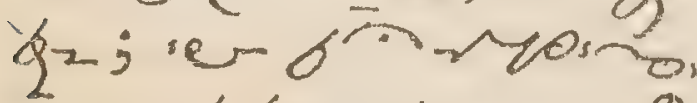
$\therefore b>1-1 \alpha+\alpha, 02$ जis., nse ser, mse thboum urs?
Night Prayers.

of ber ; a Acts of Faith. ser w, 23: 02$\}_{2}$.

Adoration. ser $6<$

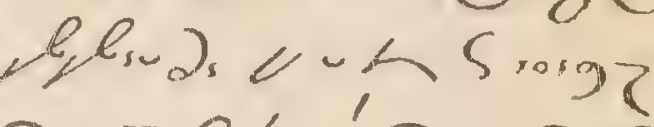
2. $=0$ h' 2; 3 usmo2, -9s - a - we

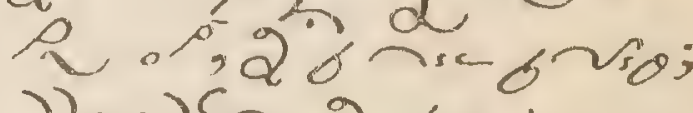
a)'or\}บ 2 1 y> r.

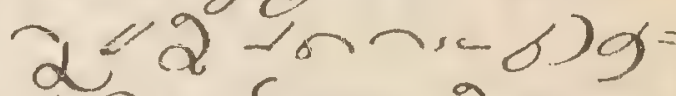
कर; 10,3572 工 -raser, 6 so so ra - isp

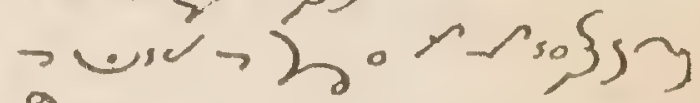
2.

Thanksgiving. se $<$ $232-5$ - $20-14$ b of, of ) $15 \mathrm{se}, \mathrm{dr}$

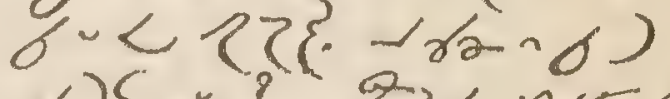

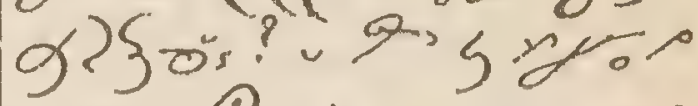
$\rightarrow e^{s} \rho_{s}, y>6$ $4-2 ; 26{ }^{2} 6^{x}$

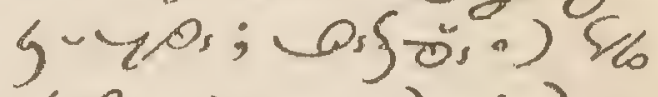

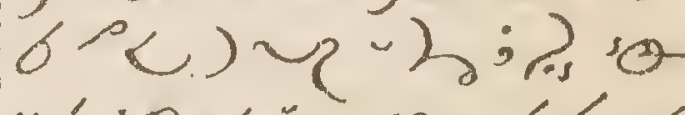

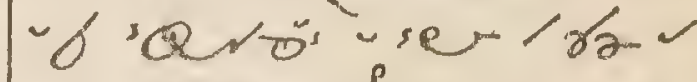
- idse b $\$ .2<2$

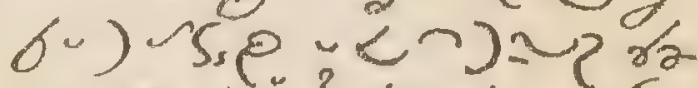

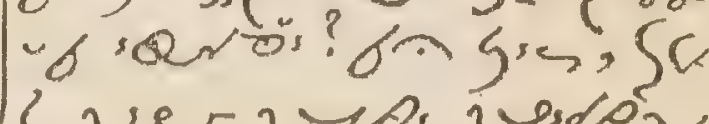

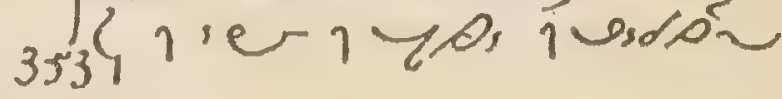


ưver $<2\}$ -

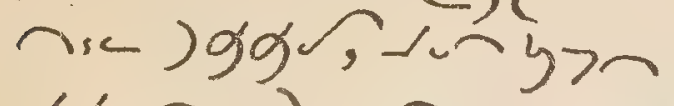

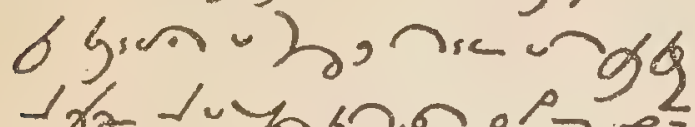

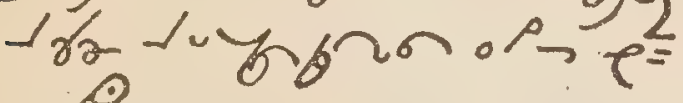
o Ps.

Prayer for light.se crsors un bugm

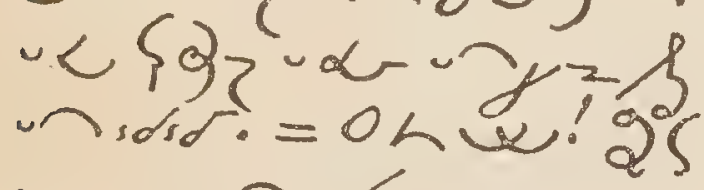
yूă 2 कa use-



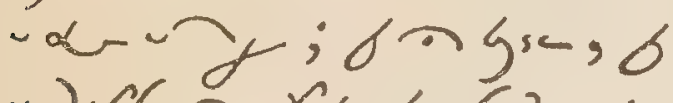

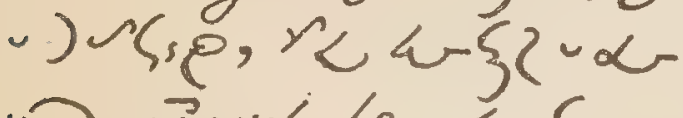
gride aris-s

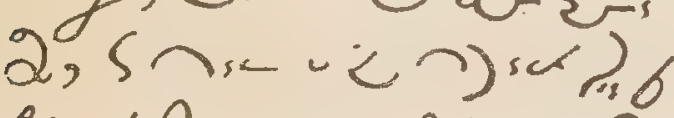

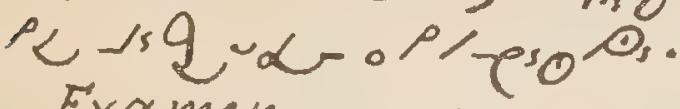
Examen. se- < yz"

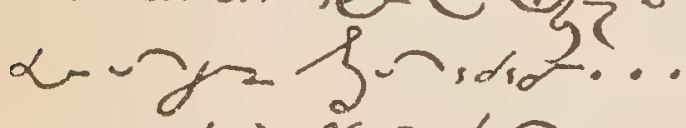

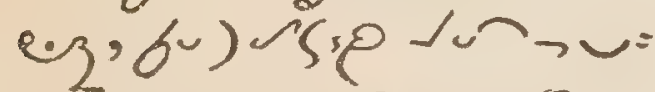
sut ven 2 - -9 s. ) $4-9 \cdot 5-2 \pi-0) \delta\}$

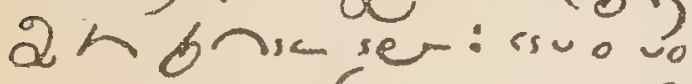
)

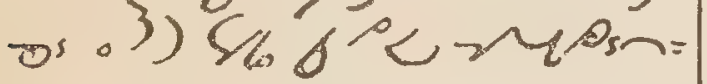
$2-5.0,5)(2,60)$

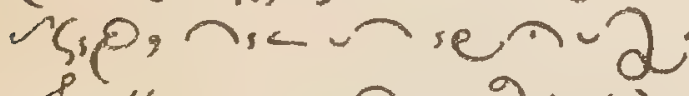
busp $202 ; 6)$ gरी o ) rise 6)9g $56 \mathrm{~g}-\cdot 35 \mathrm{~s}$ - 0 a 1 क $599 \%$ Firmpurpose. se- ze

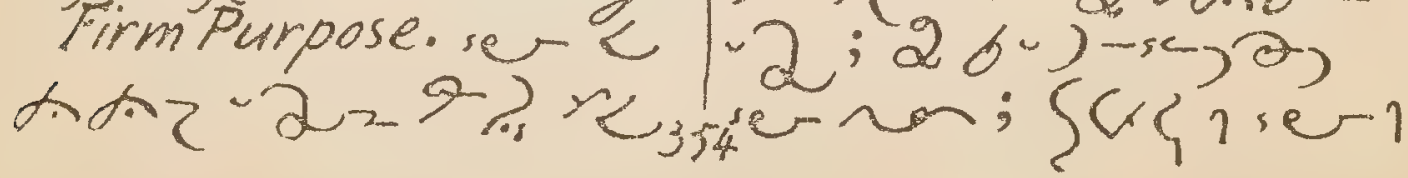

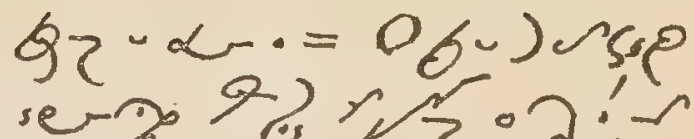
se 3 का 202 ) $4096996 ; \csc 65$ ab ósencous ヘ -

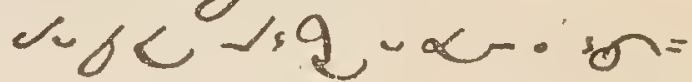

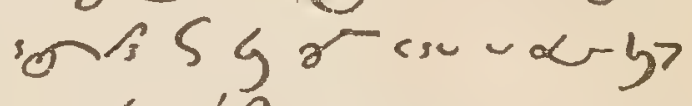

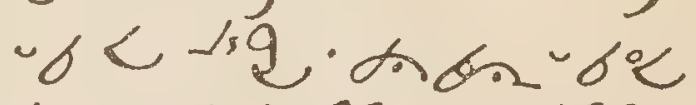

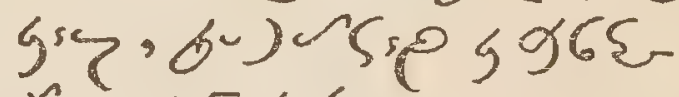

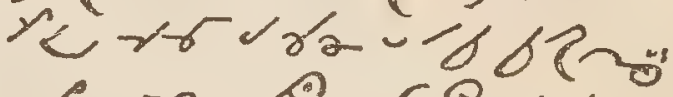
- $\rightarrow$ Tsol, sque in -7 regal. to ra of confiteor. S I $\{$. Ten Comm. - कु 人 nyn. six Precepts. If 250 . Capital Sins. my 6ring - o) ang: ᄂ aja, rror 2 , ws voni, $13,5 \mathrm{~s}$, $\rightarrow ?$ S.

Night offering. ser 6 L9Q2 os Uih, Use

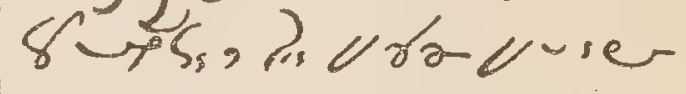
Uu ros un $\rightarrow$ ed $=$

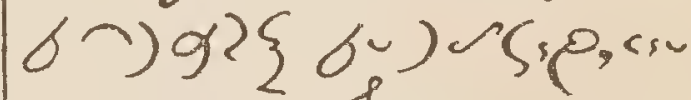

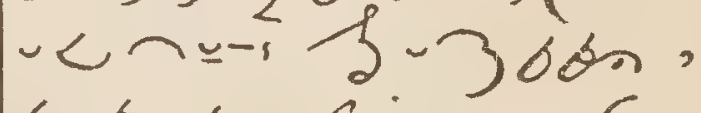

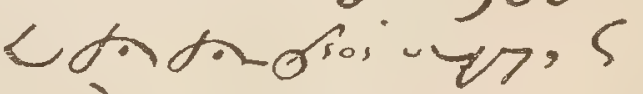
(2) - ver - for. 2

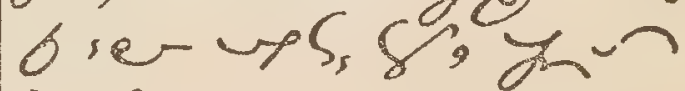
) तspz, 26 ar 


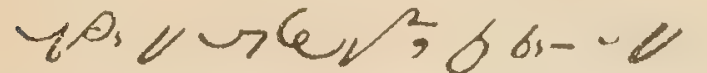

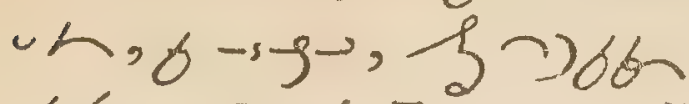

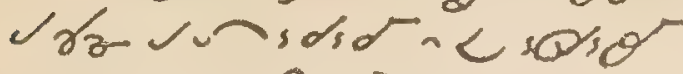
or esols, mes ry $\alpha$ g. sersusu.

For the living and the dead.

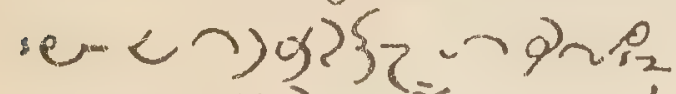

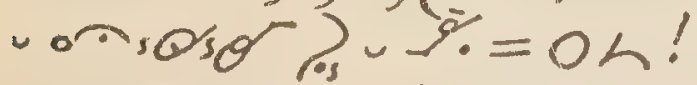

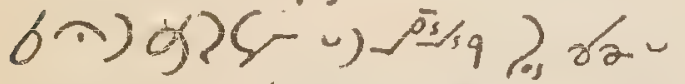

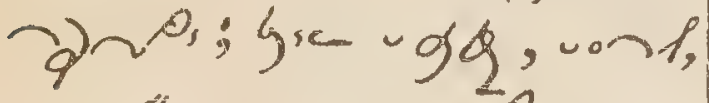
ט

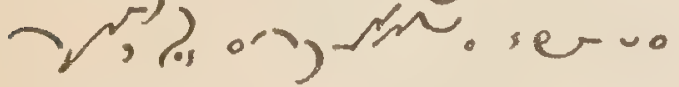

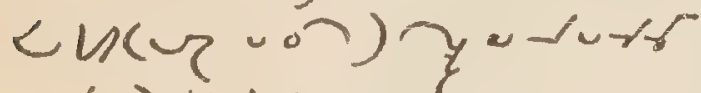
uo) 6s: ger unbiav F.0 -2 - yo.

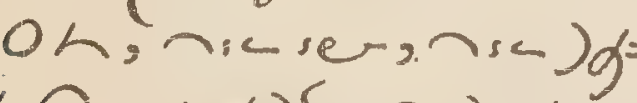

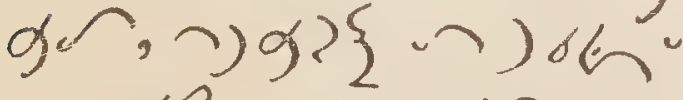
owsendas gno;

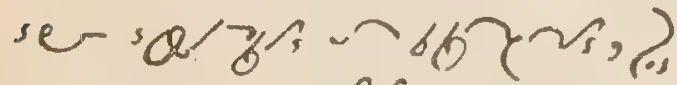

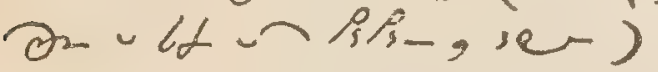

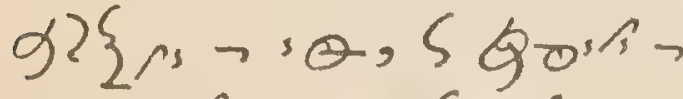
mon't vurberser $\rightarrow$ cis.

Angelus. Nor. Subtuum. - o di-.

Morning Offering. o\% 0 \% $60 \%$, $6-2, \rightarrow, 12066$; 1. 690, 6-1usard isus $\rightarrow$ ids

sarn 2 , sarny

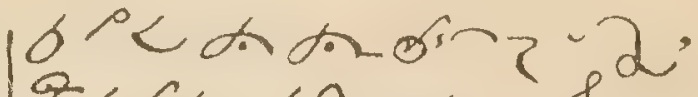

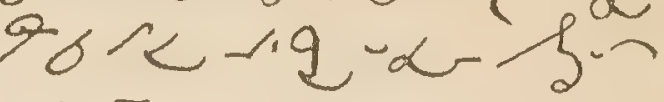

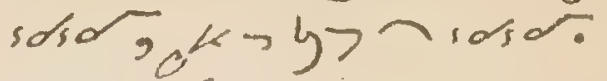
gyts g jou wever: 2. 8 . 2 . right offering. on! $26630 \cdot-2$;

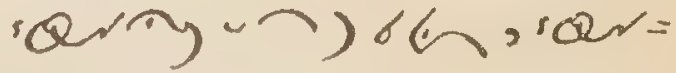
भ. 2 , 'arny-u;

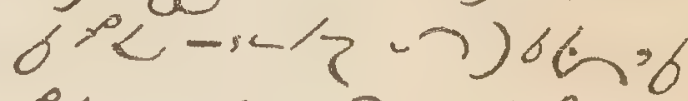
长-2. 2 -sch Ir $90-\alpha+59$ v-2 \% s. p. eviun Before meals, on 5 , boc serob 2 uno - ceréry an;

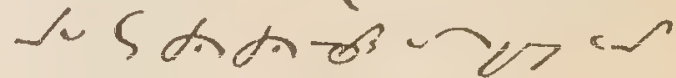
5 S 3e volog. After meals. O人一n ser, ancono b\%: ravuerdugaros 2. 6 ofor bus

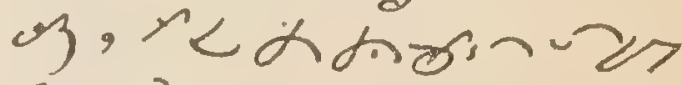
$5935 \mathrm{cos} \theta$

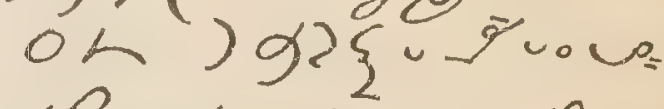
sol -36 on, re roin's revan Uu $\rightarrow$ ce 2

Form of Baplism. N... ugri, 2 a - ys, sarn 364 is w. 
Preparation for Confession. $\times 1) 0$, bu'sus,

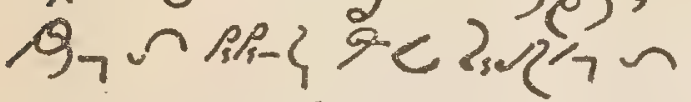
by)

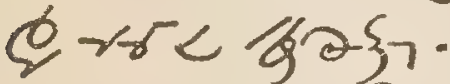

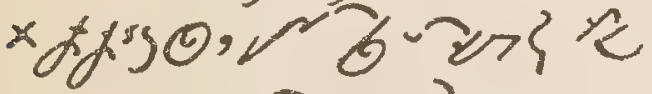

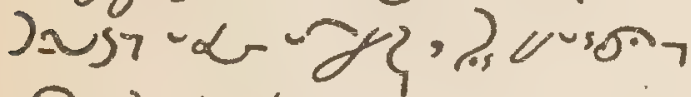
-2दण्र.

$x>70$, < y for . SOS 5 9< 0 R-2 $20-2$

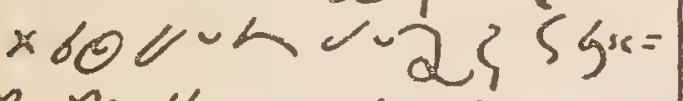

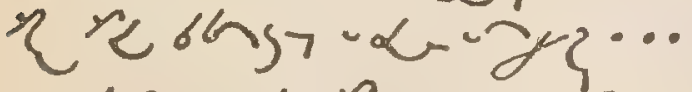

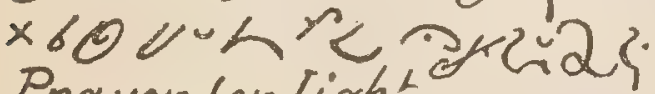
Prayerfor light. oर, -16 几 $\varepsilon_{3}$ orgu

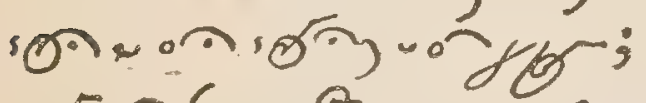

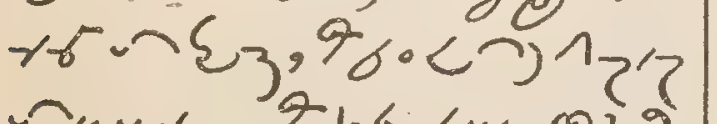
भु-

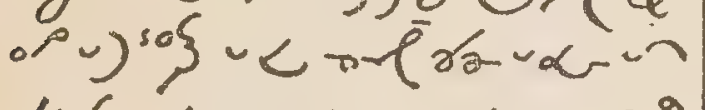
$8,61-260-1 / 2\}\}$ शे.

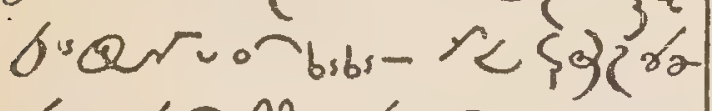

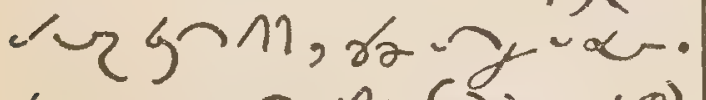

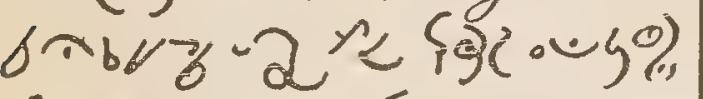

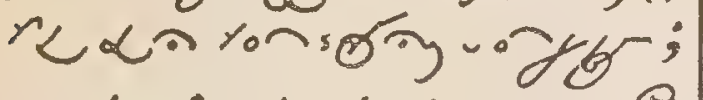

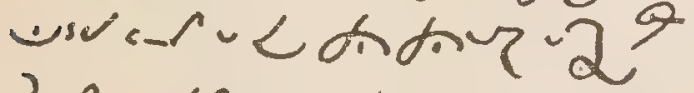
?. त $62 \backsim \alpha$.

$\times \theta^{a}$.

xüg ) $\{\varepsilon-\}$.

x) $99 \xi-\tau$ - Q en $Q_{E}$, 火ronor, 次hn. x) $99 \xi-4$ - Qur? $8 \dot{2}$

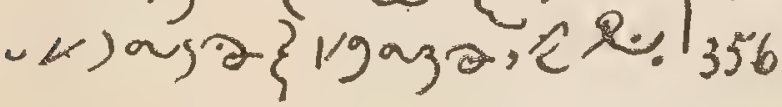

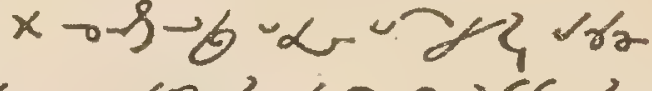

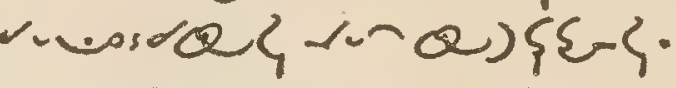
$x+-9-6,2-2<-24$ - $86 b^{\circ}=0<$ sore 4. $x-9-6-\alpha-2, z-\varepsilon$

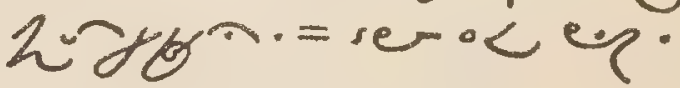
$x \rightarrow 9-6 \alpha-2 \zeta-$ या

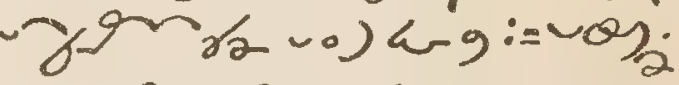

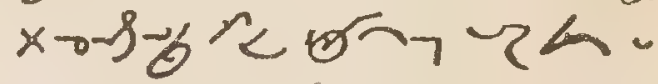
anर $<<5$.

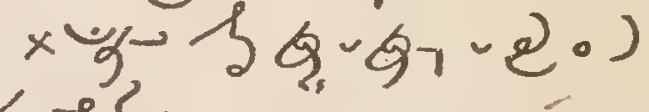
<-g?.

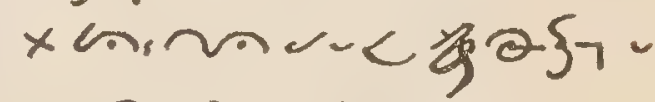
$\alpha-2 \gamma\} \cdot \alpha$ diso.

$\times 60$ Uuh, r gen

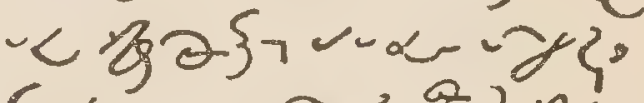

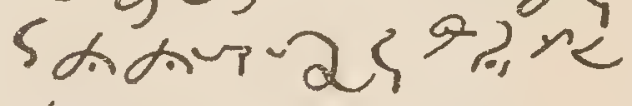
Q7 - .

Prayer for ciontrition. sen 60 bu 0 b serin ig sen b०< sarz - ) y g.

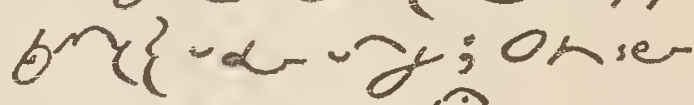
bousas si-2. u-

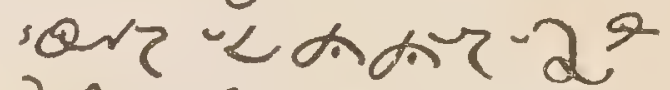
?. $\times<62 \rightarrow \alpha-$. x O4, serbesar? <-se-2 rou แnsuv L, fonsons sus. x OK, sen boc sor? $y>x-1-2-12$. 


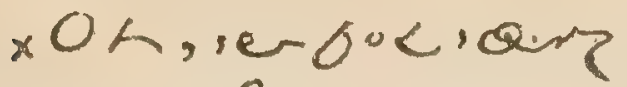
प>-<-2 $2-\alpha$.

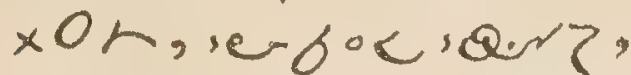

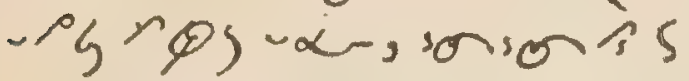

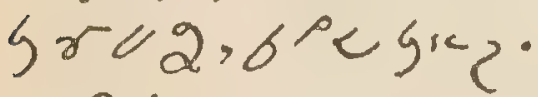

$\times 0<$,serbociaer revior? a 2 , ?.णra unyor.

x ren < or cruek, or rea, of -dz $2-\alpha-j\}$.

First Consideralion.

o) servurters, ${ }^{2} \theta-$ onn.usd y uE,

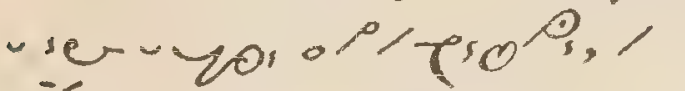
I/s, $5<$ cos is wuter,

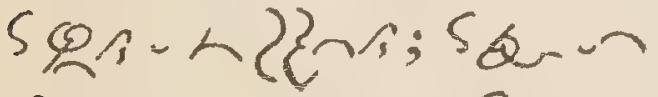

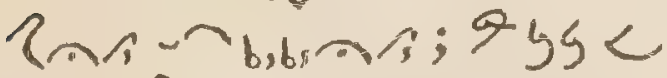

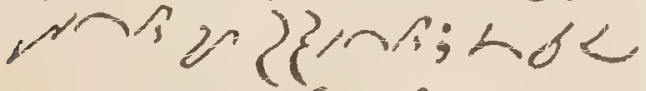
Qannargher:

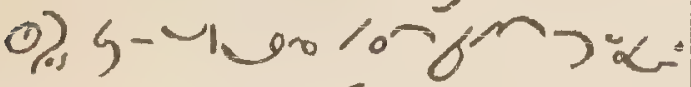
ग5y

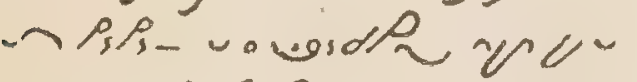
$\rightarrow 6,6949<9(-)$ ) $\%(0)+2$, lon,

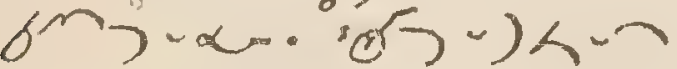

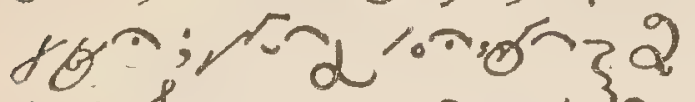
$r ; 1,4.2<2 ; 5,2$.

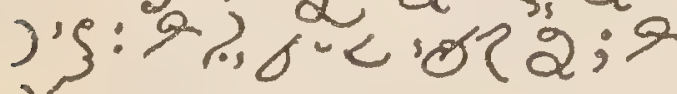
r. $-<0<\alpha$.

x० $\mathcal{E}_{p}$ morguren जदp: var

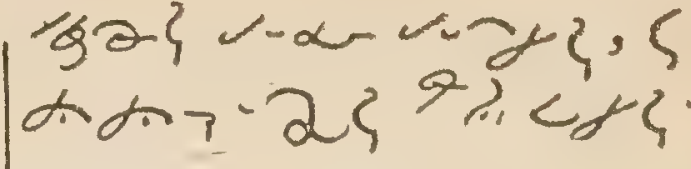
$\rightarrow \alpha \sim$

$\times a \sim<\sigma z-\alpha-\alpha<s$

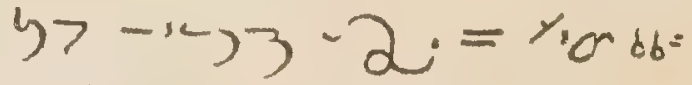
2i-2-" $\alpha$, inion $\mathrm{Sn}_{3} ;=y>$ ( ) $\times$ $2 r \gamma+\cdots-\alpha-5>5$ $-Q)^{-\alpha} ;=\gamma \cos \omega$

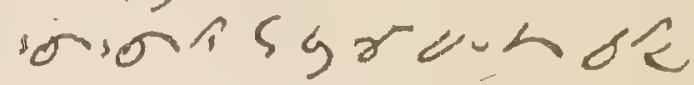

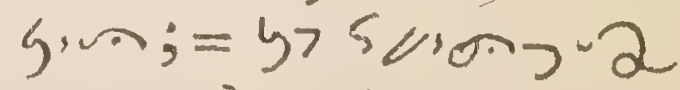

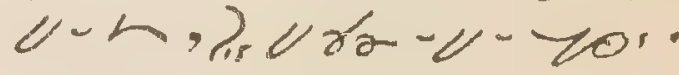

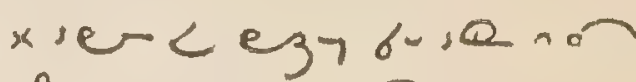

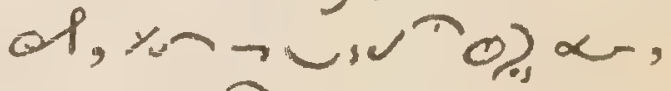
-rs r 72 .

Second Consideration.

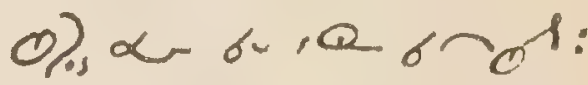

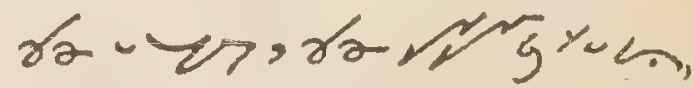
2) ra widis ra 366 . गfinen 29 ysen

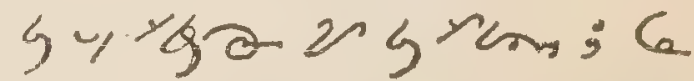
$4-3-400605$ )h Jft?; «- $\alpha$

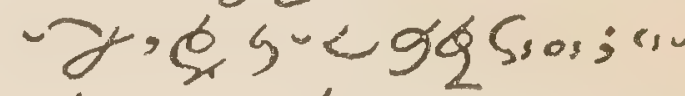
- de bor sonar . Q 6 8. 9-72 « 2 ,

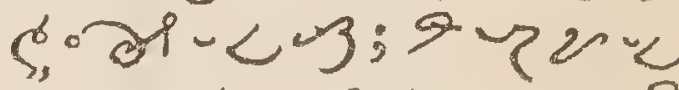
ivis:al-cحiu;

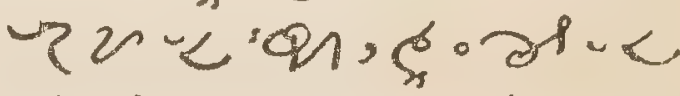
sQh; अu < <-ia \%om

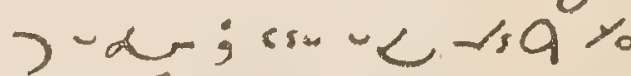

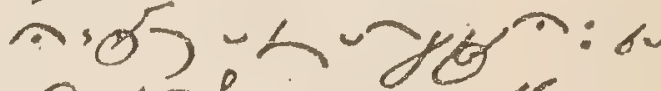

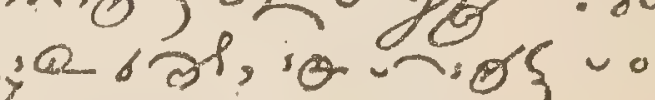


12

OKANAGAN

usp, fr bm, on biaror users, io

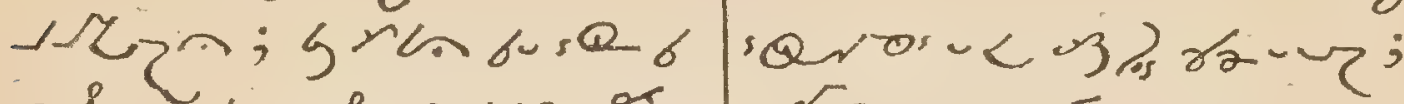

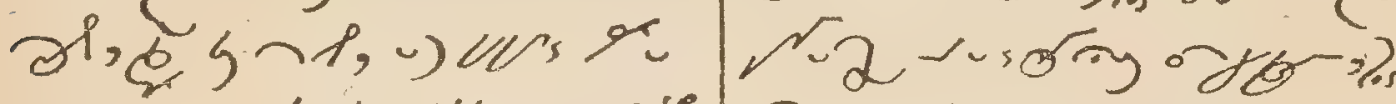

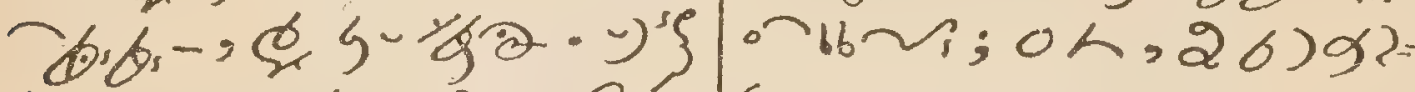

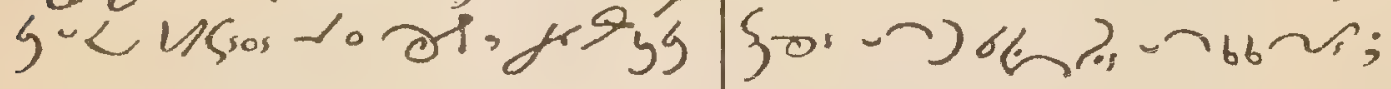

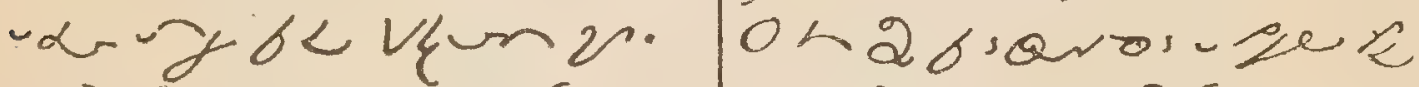

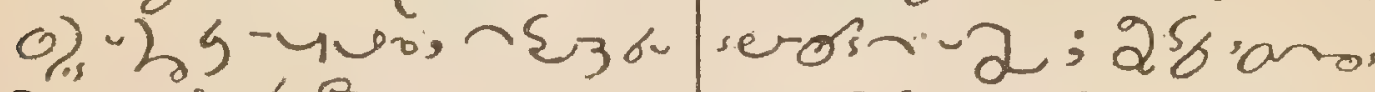

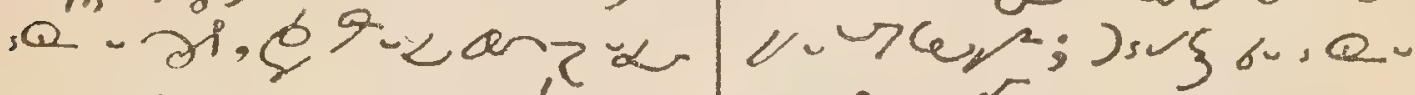

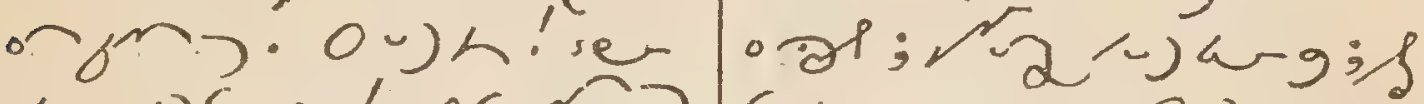
$6392\{-5 ! 05,-2$ s

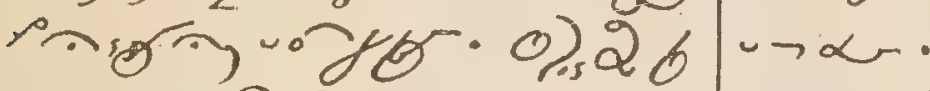

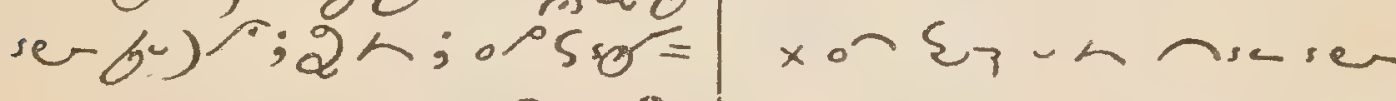

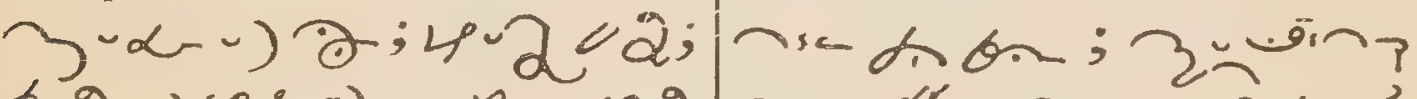

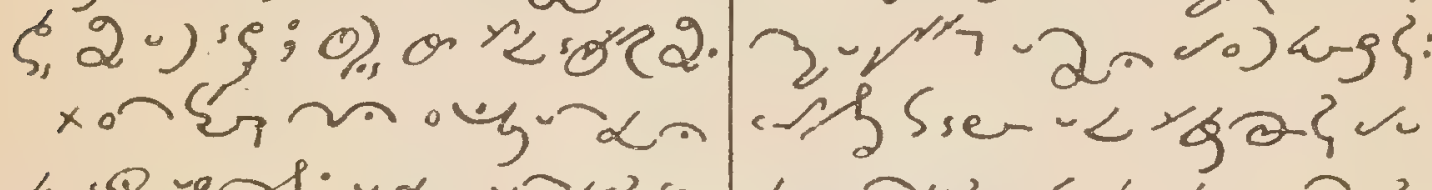

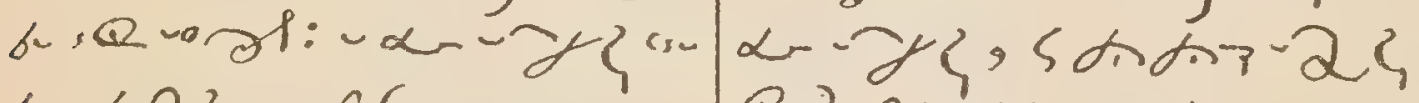

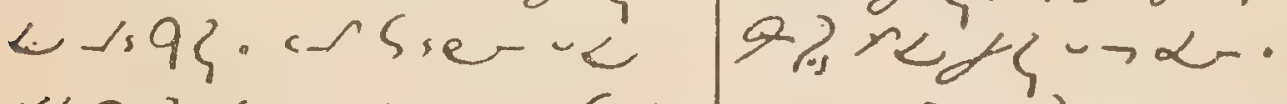

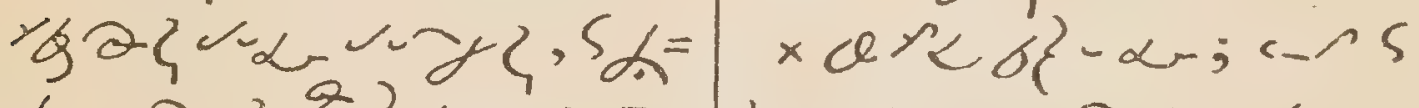

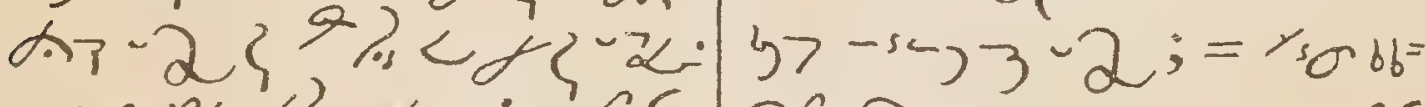

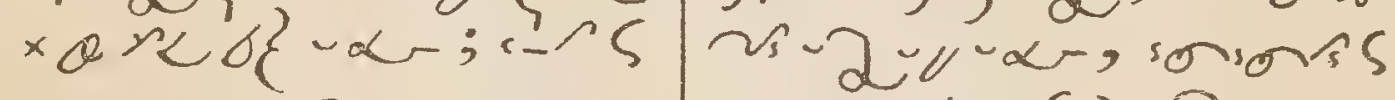

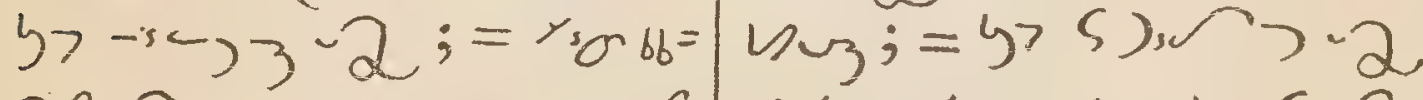

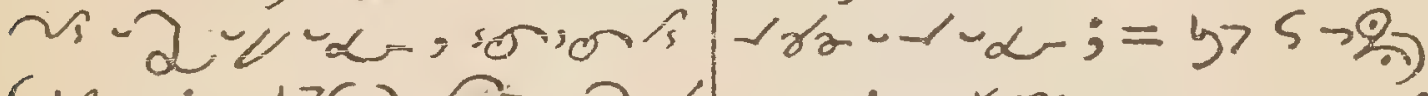

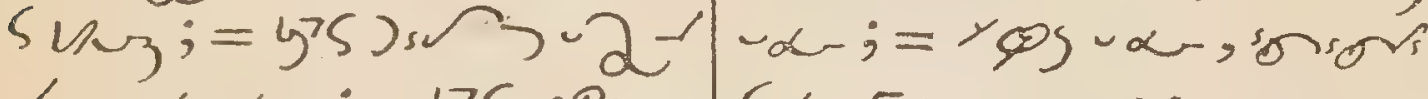

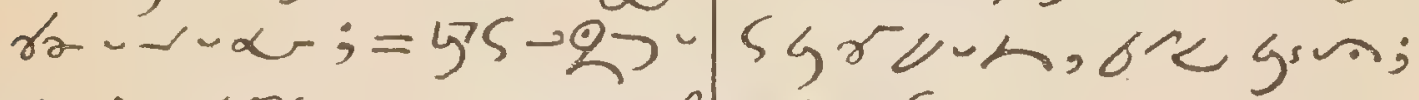
$\alpha-;=y \operatorname{sg}-\alpha$, , s.

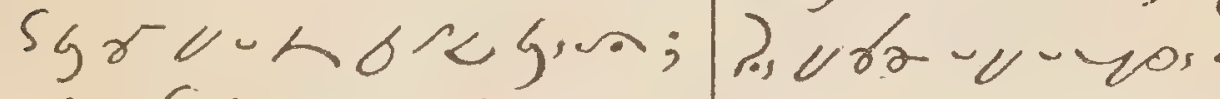

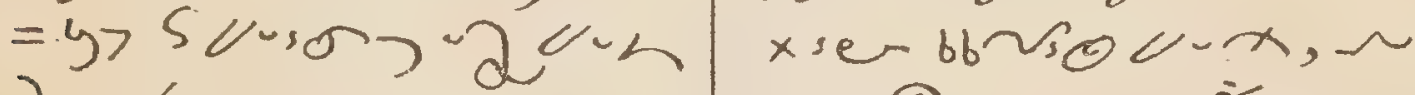

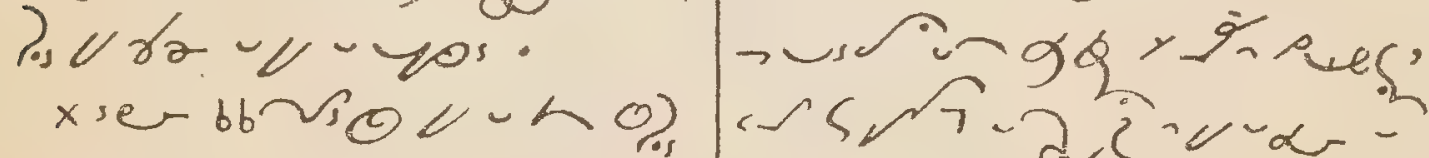

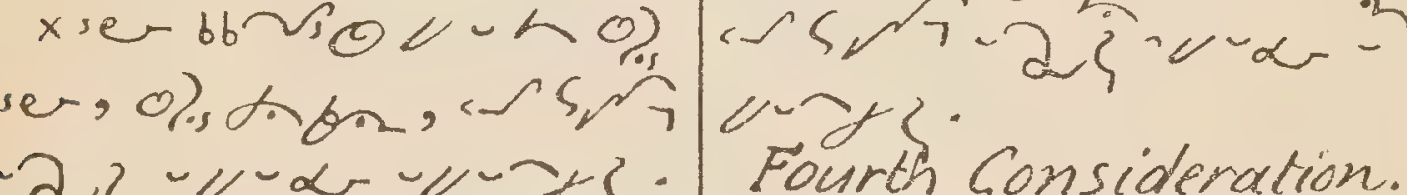
Third Consideration. : Fourth Consideration.

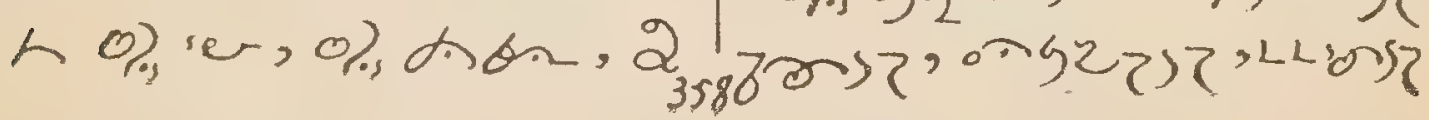




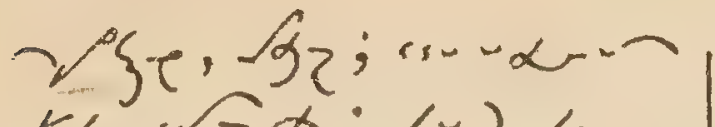
fin $\hat{r}^{x} ; 1-2<$

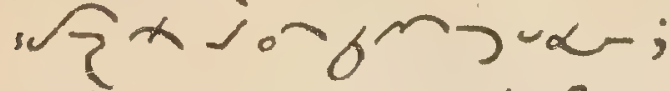

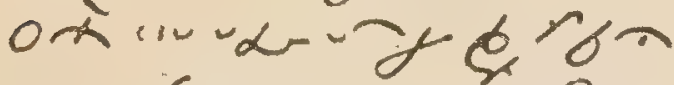

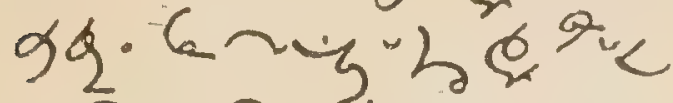

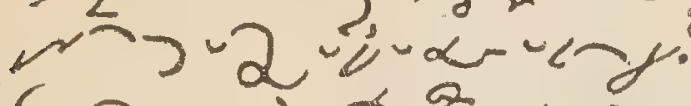

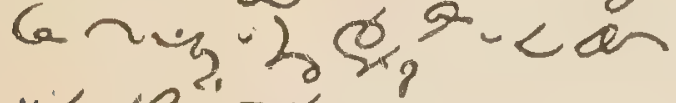

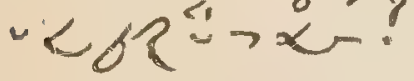

ox surargor unen;wemy or

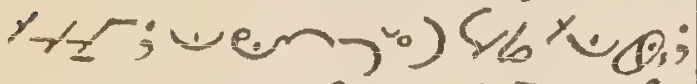

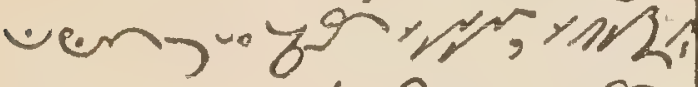

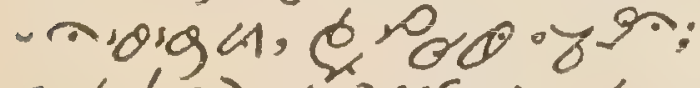
$0 x(0), 60)^{\operatorname{sog}}-3$.

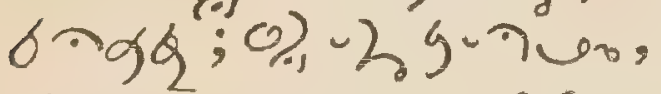

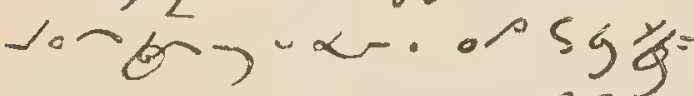
क-vuk-ugiors angu-x-g; orteb? $u \alpha \alpha$.

oxung gangé ees : 6 un $^{2} 20060 \%$;

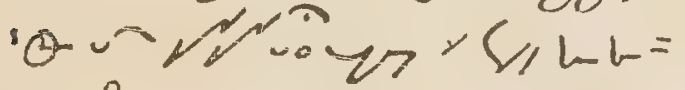

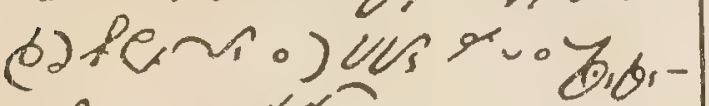
sio - r r voy wh

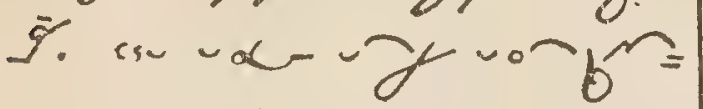

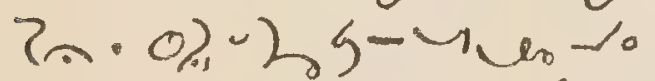

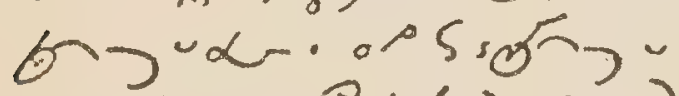

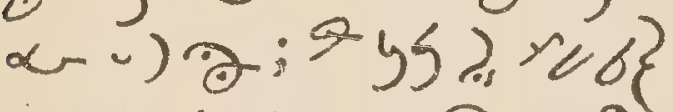
- - ;

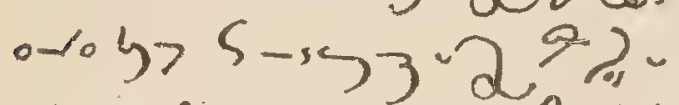

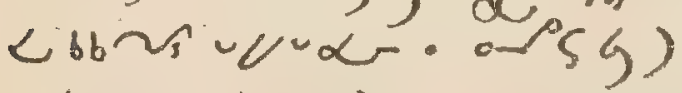

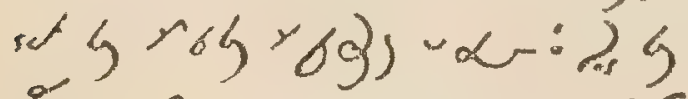

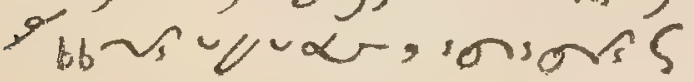

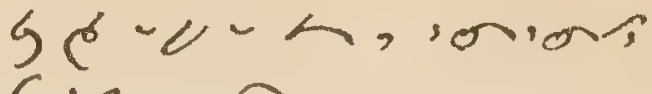
Sमs.

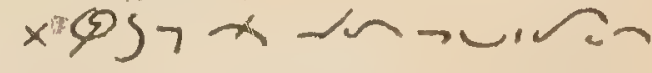

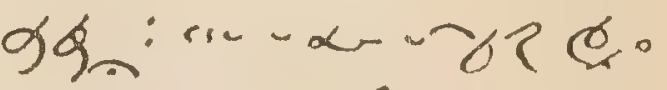
oyg $x$ : a sience

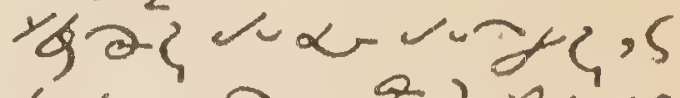

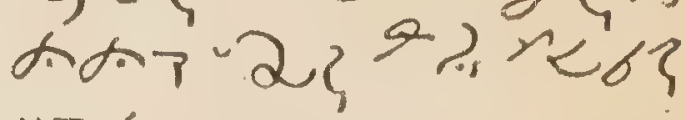
$\checkmark \rightarrow \alpha-$.

$\times a r b\} \sim \alpha ;<15$ $y>-s, 3-2 ;=x_{s} \sigma 6=$ si $2 \times 4$, inions Sun $3=5=5$ ) s $\alpha-\gamma \alpha-\alpha-;=y>$ $\left.s-Q_{n}\right)-\alpha-;=\gamma \cos \omega$ sonis 5 \& ‘ gin; = y> Skus

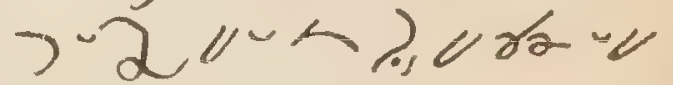
vos.

confiteor. $\})\{$. Act of Contrition. Q9 on!.PSG rga vu

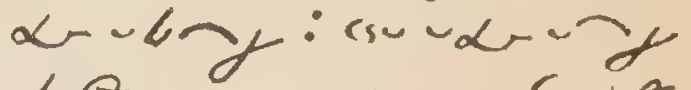
b9-2'onumis; "surб : 6 का;0) $35 \%$

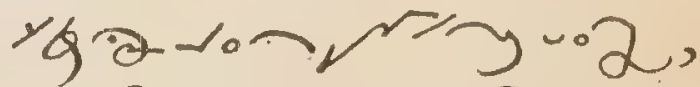
0), 26 soso 2,260$)$. o), brem-ancop-

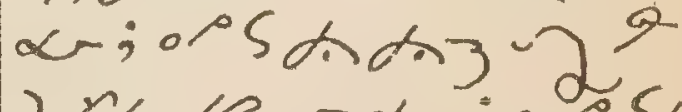

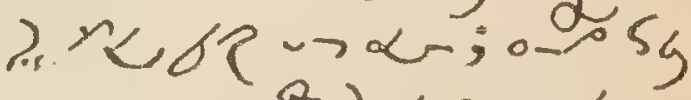

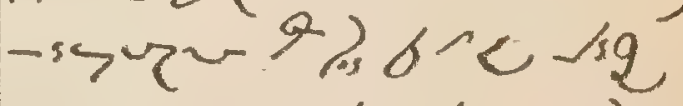
$-\alpha-$ seriong:c 22 . 
Advice before Confession. wor to Sumi xe

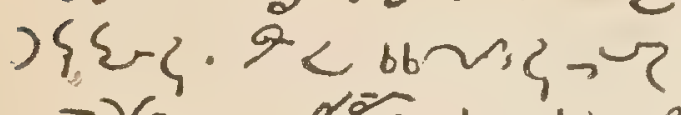

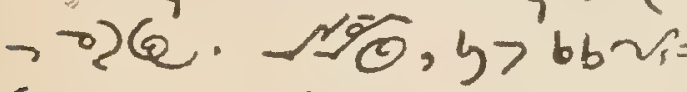

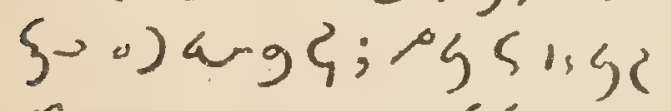

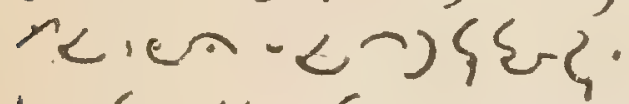

y> $5, \alpha \partial\{7-\alpha-\mathcal{\alpha} ;$

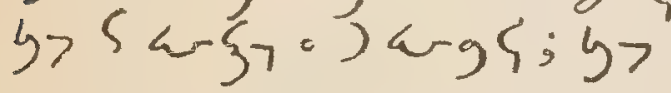

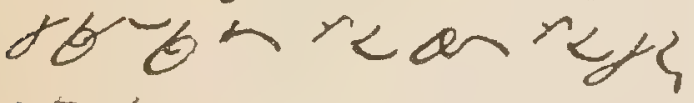
$\rightarrow \alpha-$

After contessicn.

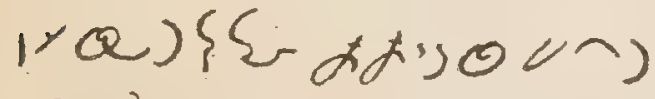
ㄱㄴ? $23-\wedge-v=$

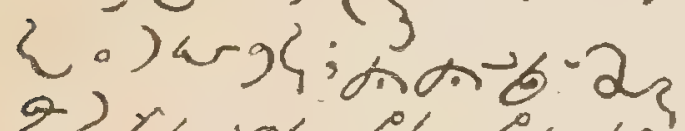

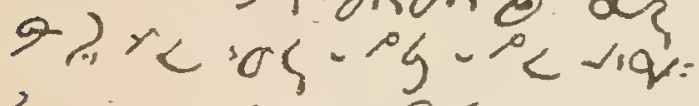
ㄴ.

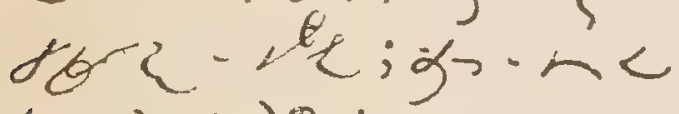
ज“द; ) अ

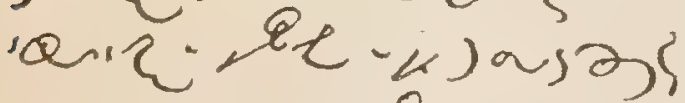

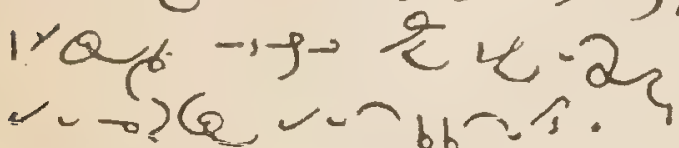

offering of the Mass.

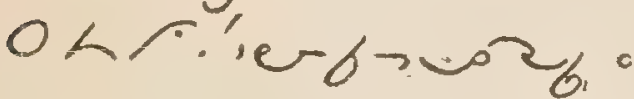

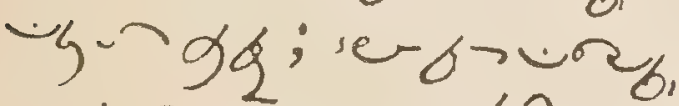

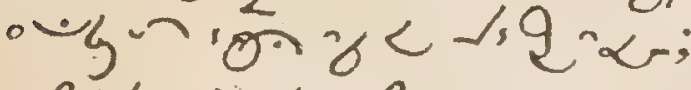

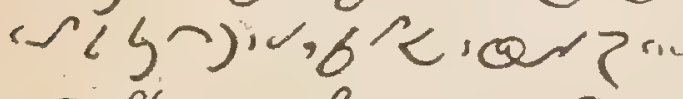
rele, sions-2\% - At, - Rtucian.

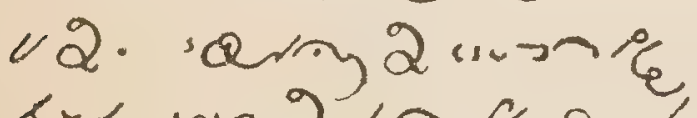

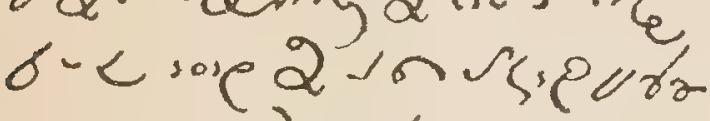

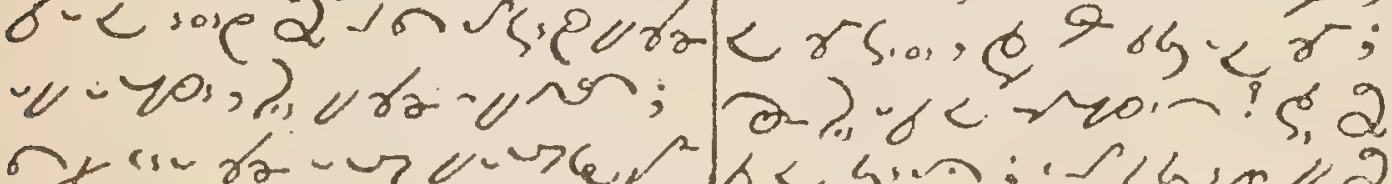

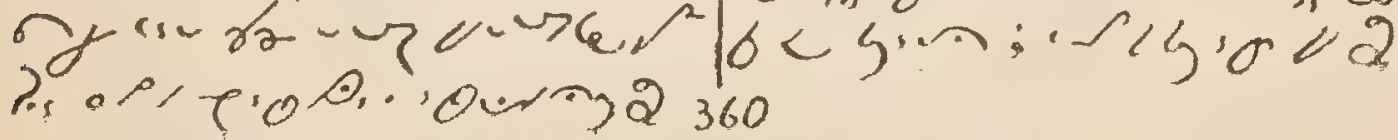

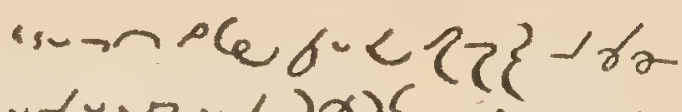

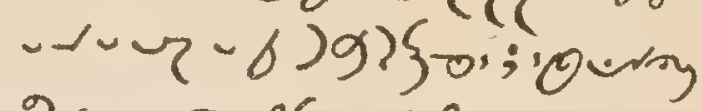
2 sun 96 b6e sort $x_{2}$

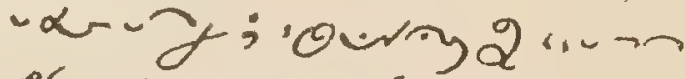

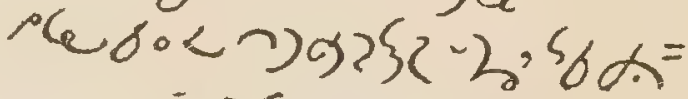
$<\leftrightharpoons \dot{9} 2\} 2-256 \alpha=$

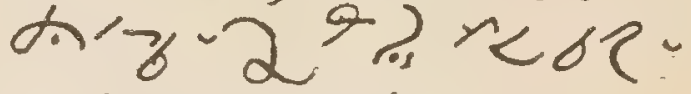
$7 \alpha$. ser $\sigma \circ<$ ser $2-255$ on unks:

\section{Before Communion.}

I. Adoration. sere d) $=$

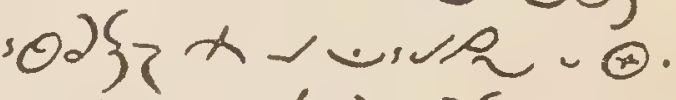

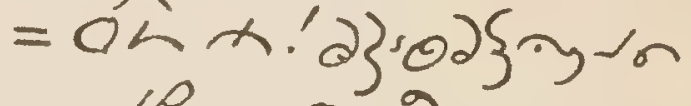

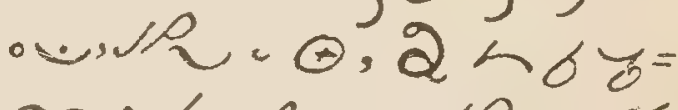
mita 4 wast , o - था, $x_{0}$ ) $(\mathrm{r}, \mathrm{r}$. S6; DJ'Od\} NUNR

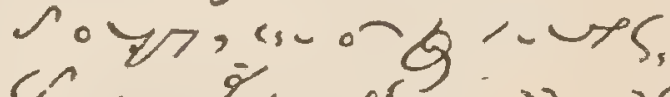

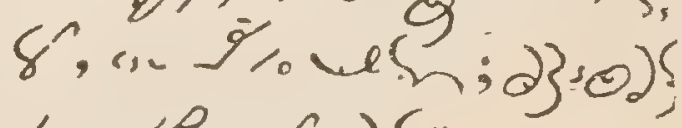
rup $\Omega_{0}$ )

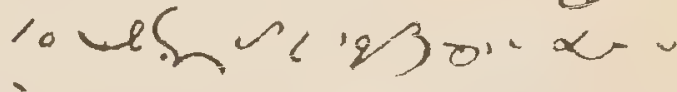
) 2 .

IJ. Humility. ser < m, $2=0-x=0_{2}$

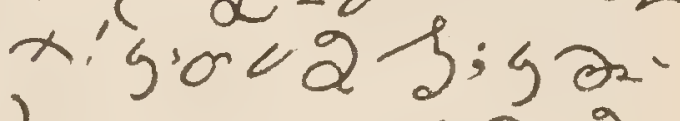

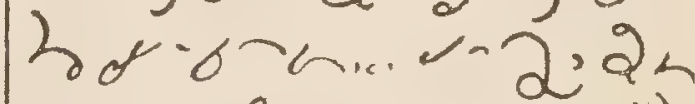

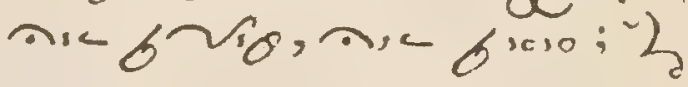
ग.

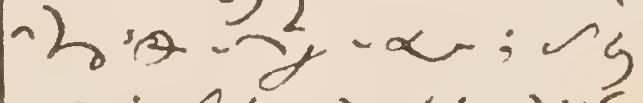

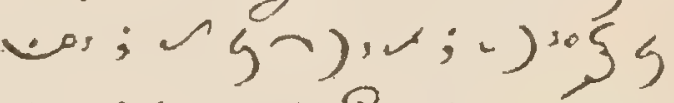

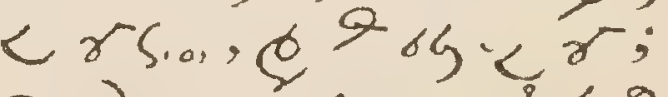
$\alpha-y<-201$
$b<5 \cdot 5 ; 2$ 
\lrcorner $09\{: \cdot e-6 \cap) g\}\{$ 6) ) sie, ser biagse, conirition

Contrition..e- oc

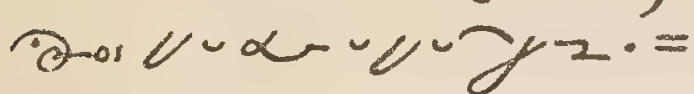
"L6mans vण2, on

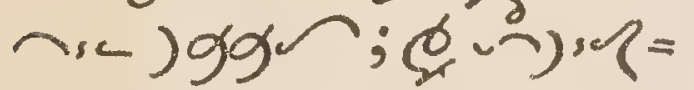
3- -

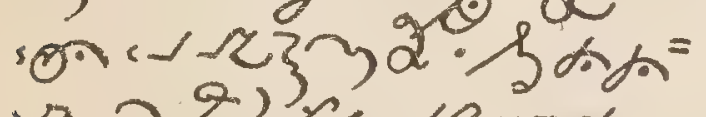

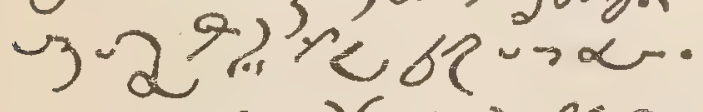
se- $\left.6^{-1}\right) 92\left\{6^{0}\right)$ ase,

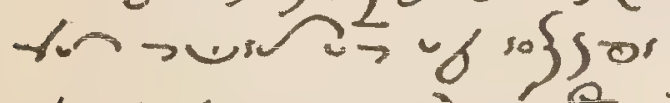
trón

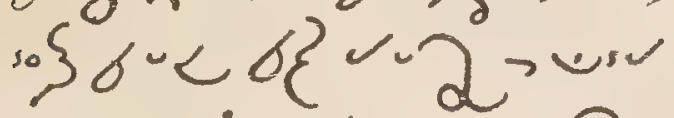
- De: a b uor 2

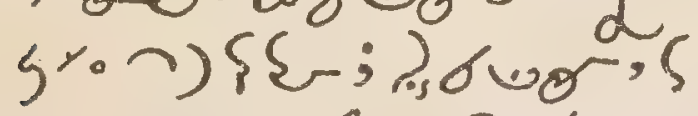
nicesergin $22^{-1}$ an u.

Love. erancesoss? x.= = nx.'nd ) $9 x$ rofyng 2 ?

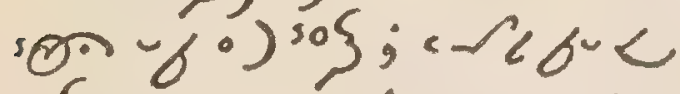

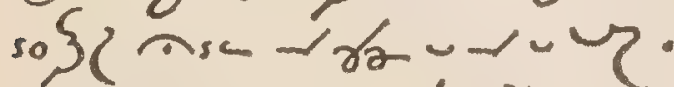
०6u (s.pt! of bu

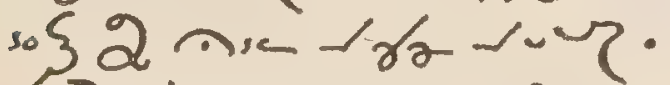
Desire. se-c 0s-jz $x_{0}=026$ se- 4.1

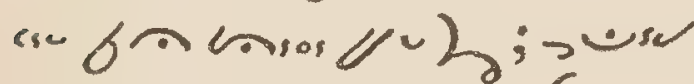

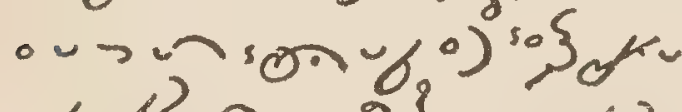

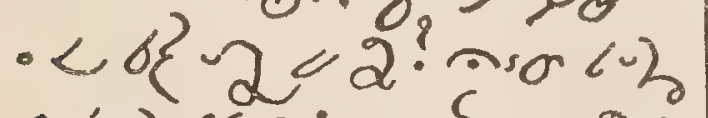

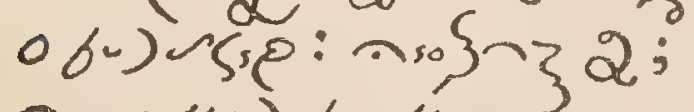
moservatón. 361

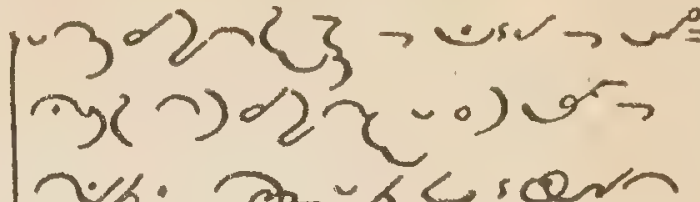
\%. $2-6<$ son 270n 55 a 02 . se- 8, y $42 \pi-5$ $-7,6$ y 2 - $60 \mathrm{r}$ $2<x$.

Sion, 2a, riononsos - Ason; verun $9-202 .-10 ;$ तsonenser Fenson.

\section{Atter Commanion.} Adoration. ser $<$ so $=$

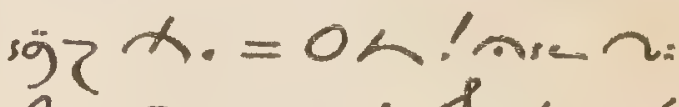

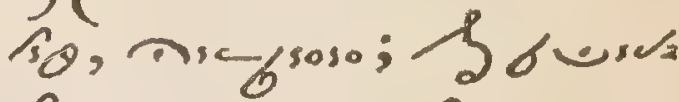
era; wus no 27 urst -0) Glo, wive

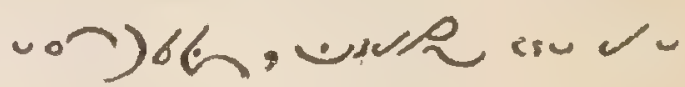
3. कात2 2 ,

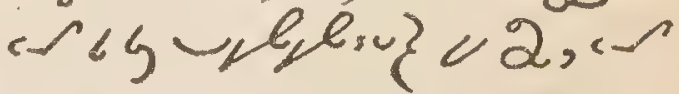
«Uजo $-2<2 ; \pi$ in sspars 2 - 2.

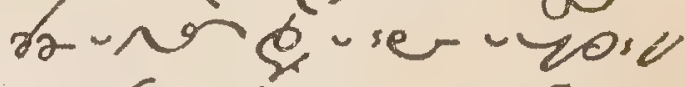
-7e prosg 2 a

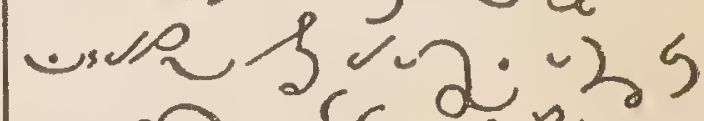

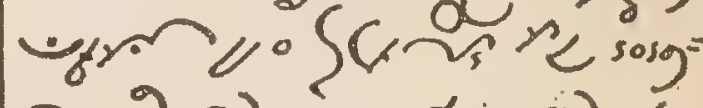
-20\%, ssoro; 2 6 'C 2 бa- 7 . Love. sen< solstex. = su ousp una 人 nie Jggr: ol oben senigor -2 grisoss? 
2ns - 1ra-1un? 6 oj- 2, 0.60) rs.e.6

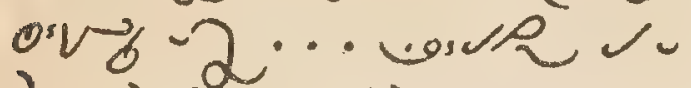
3.) $5,5,69) \cdots 2 x$. Josorg nicien. OSk 'enon, 02 e. 8.6 ar-2द, 5 sogs $>-26$ -) नsect.

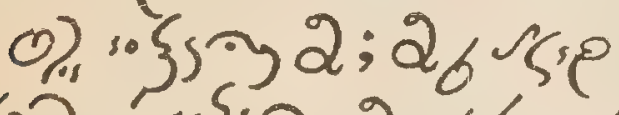
- $2,503502-120-1$

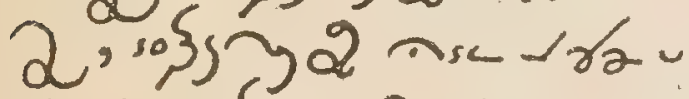

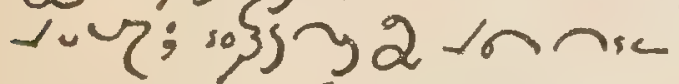

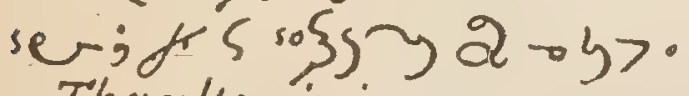
Thanksgiving. ser $C$

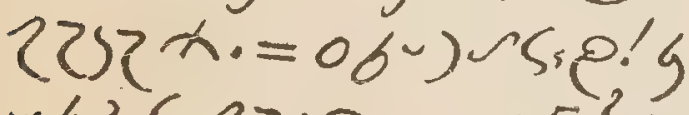
" $525(25)-\pi)^{\circ}: 5$

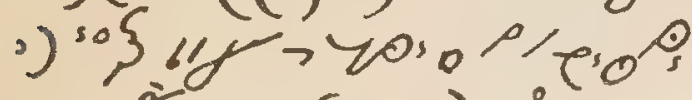

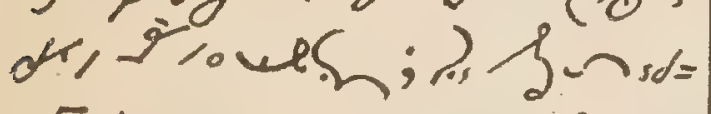
so on,,$-2 \cdot \cdots i$ रा? $2+x_{2}^{2}-2 \cdot 2$ 6u) riencier, ase Jga 9y 9 -

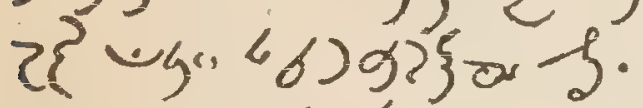

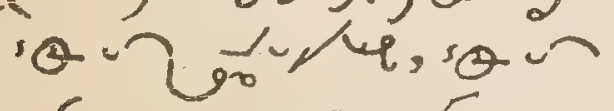

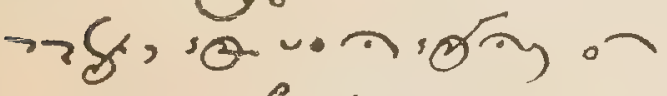

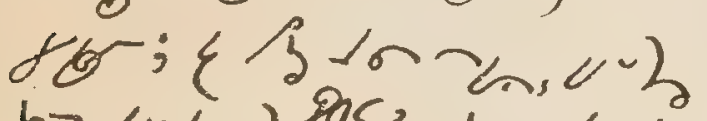

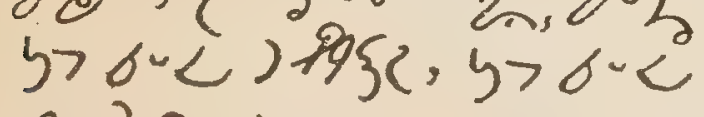

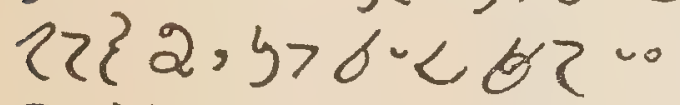

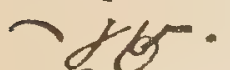

Petition.

$\cos v-x .=6 \cos 2=$ 人)

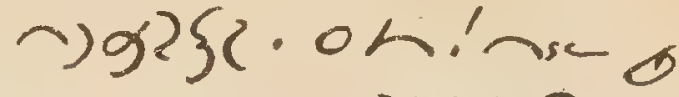

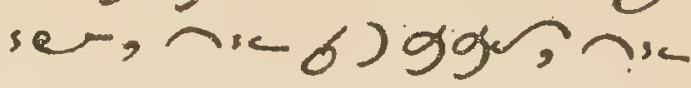
6 io, ez -3 - $9 \varepsilon^{\circ}$ 68 arser, ob - 0 , 83 - $065-$ s010; 63 re 8 m 02,59 55-< V6? 2 .

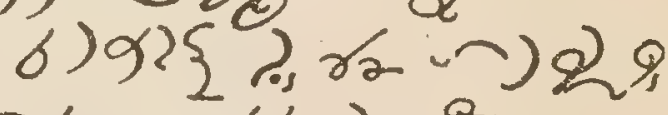

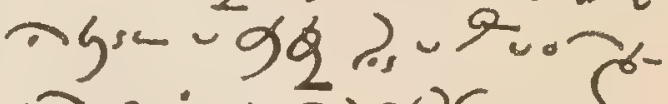
- wois of $925-9 \Phi$. Yorvo eoses $36^{2}$; offering.sen beson:=

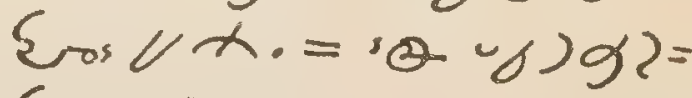
\}o, on $\rightarrow$ se 6 ) g9? - पyu 2 -) so $\{$ - Qusve ob> 2. > un b-

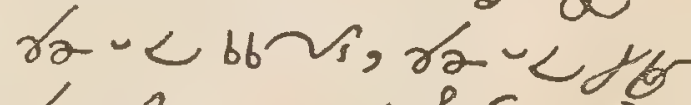

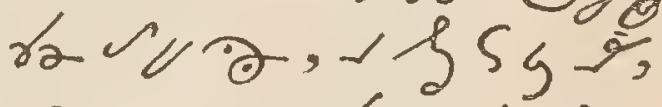
sarry rura. Inser

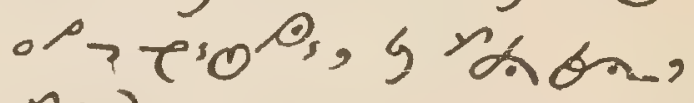

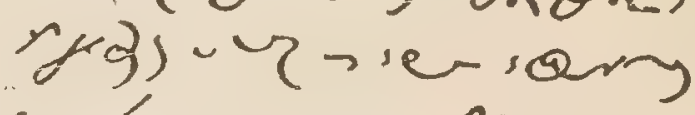
su ga. serachson ve

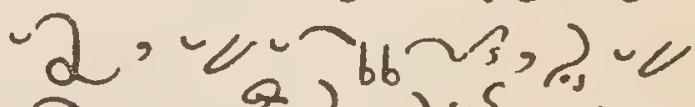

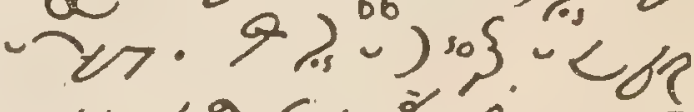

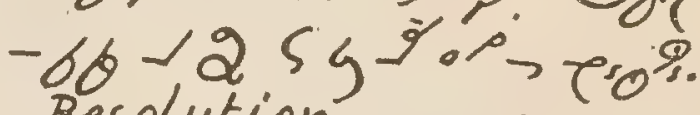
Resolution. ser $<A \alpha=$ $7-22=0260)$; in ben, पybems 12 ? 575 万 $20-\alpha=2$. 9.)

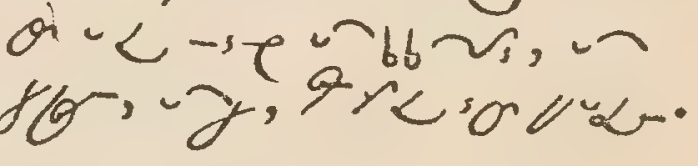


MANUAL

17

are 2 , ore ans, Dolorous. 6. sen de bs=

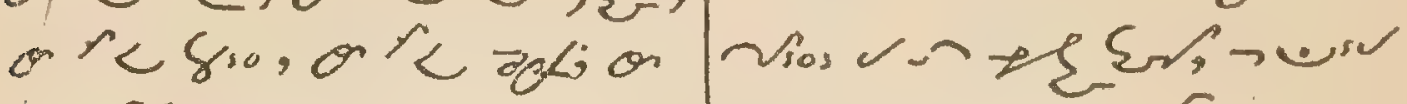

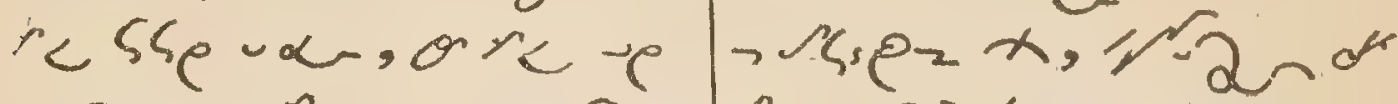

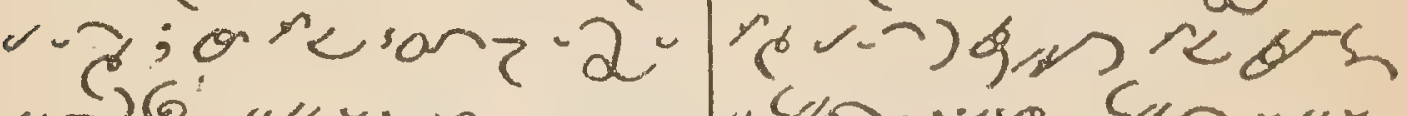

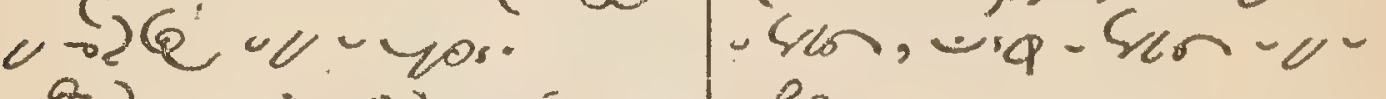

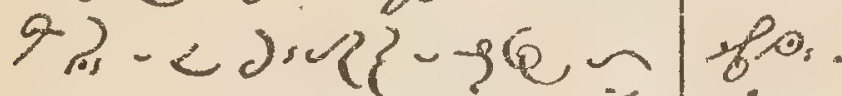

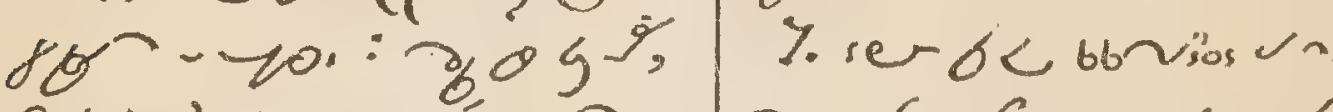

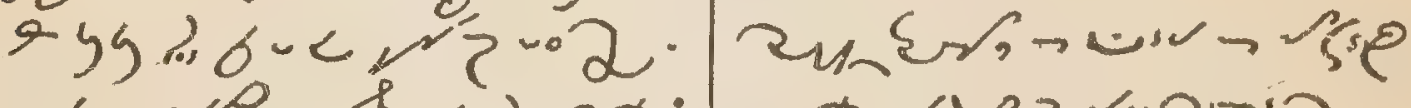

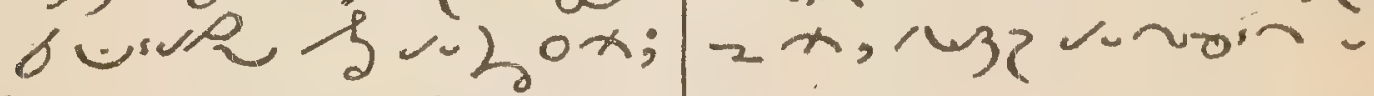

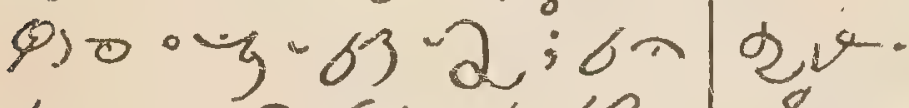

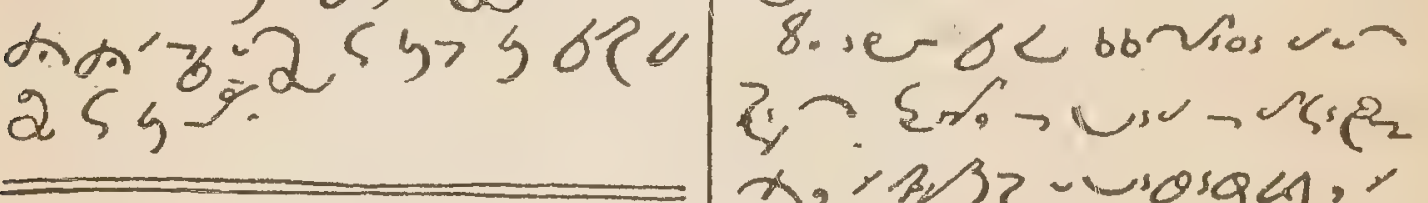

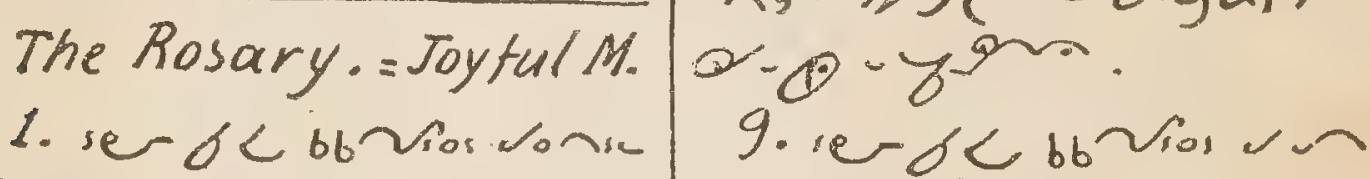

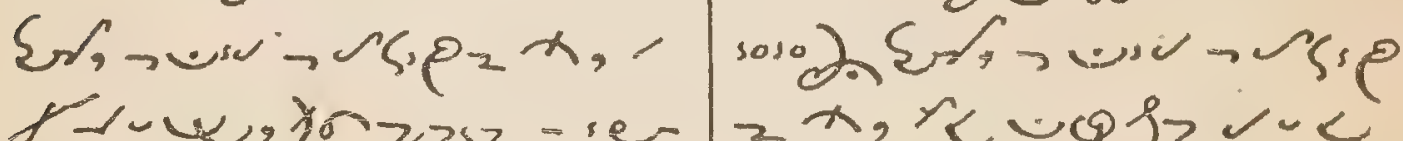

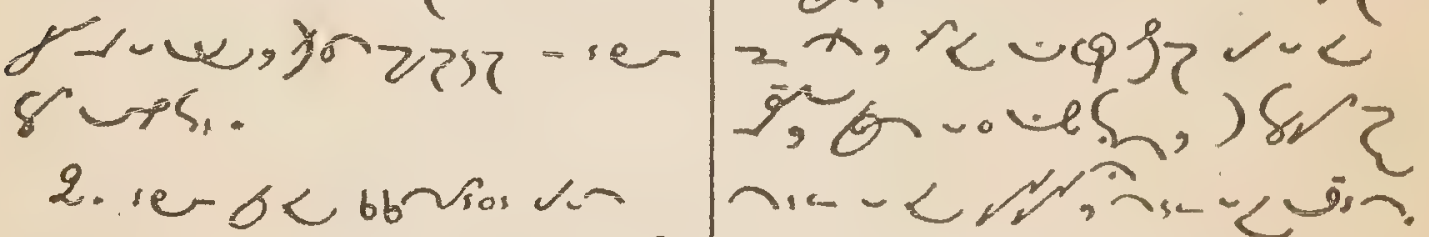

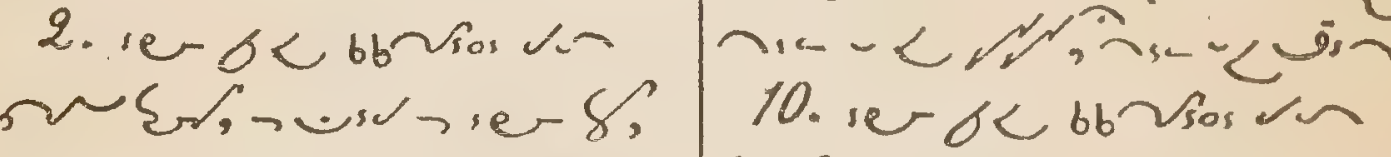

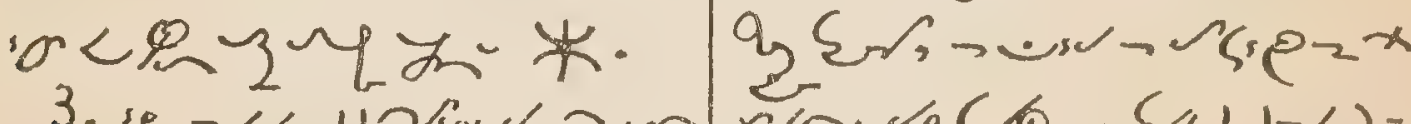

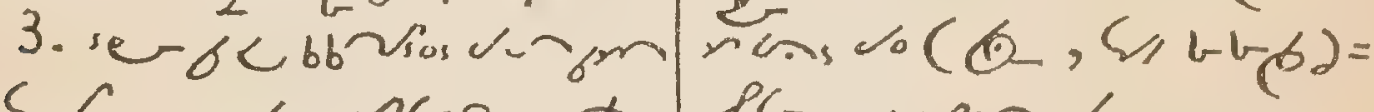

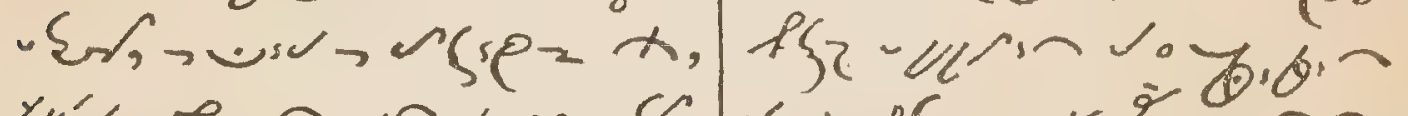

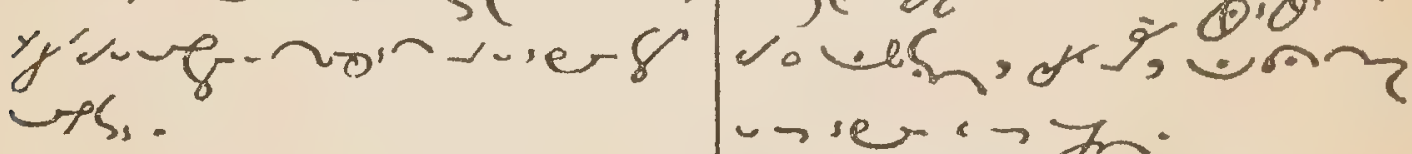

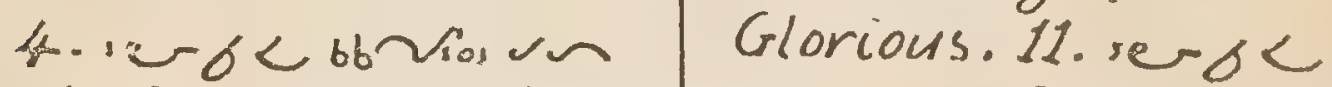

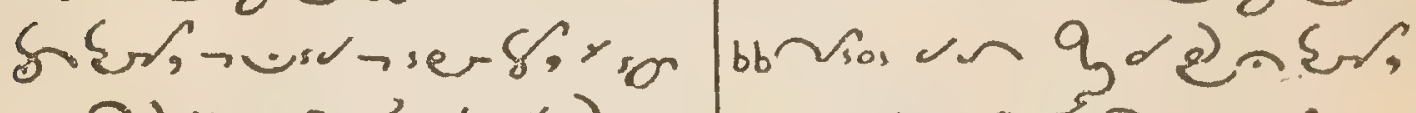

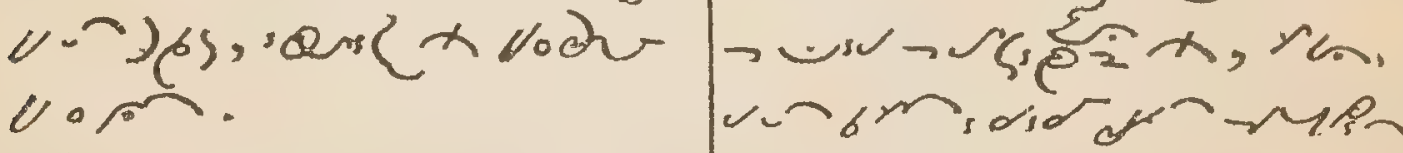

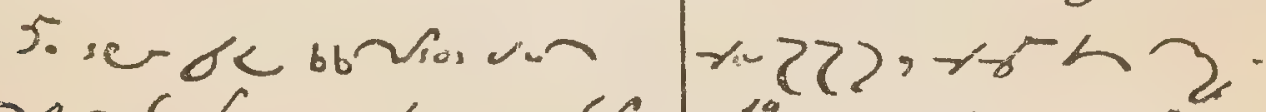

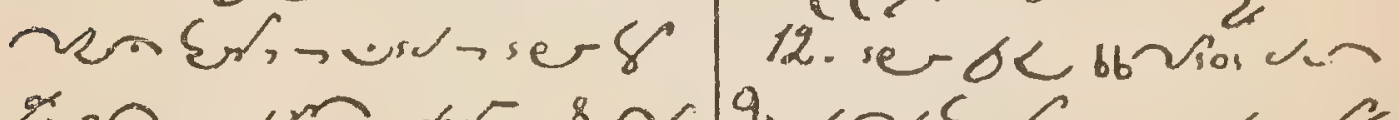
\% 
18

OKANAGAN

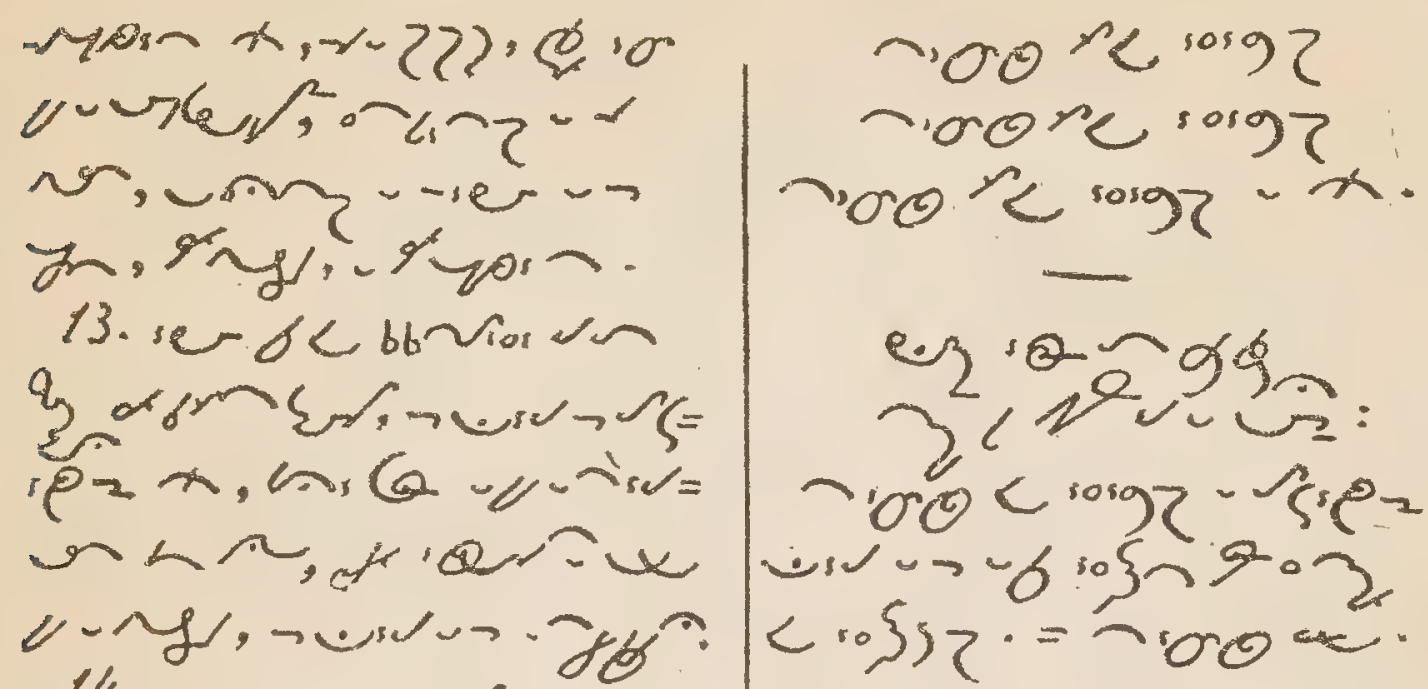

14. 1er 60 662 siov

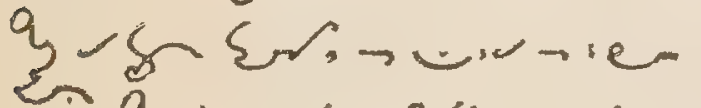

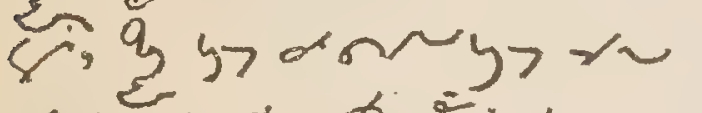

nion-

mos u yatorasu

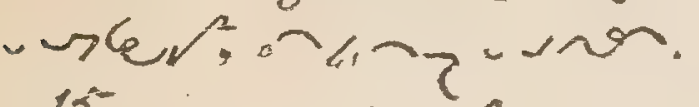

15.

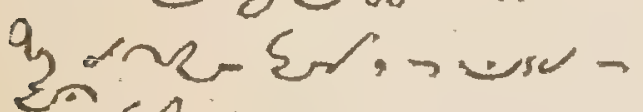

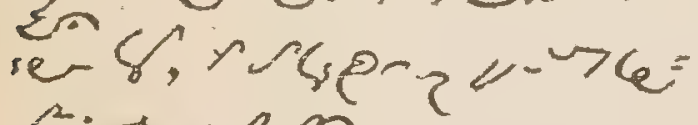

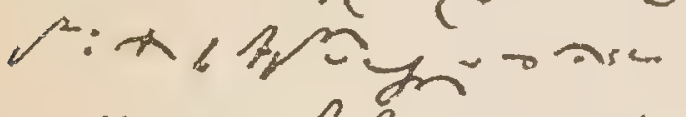

nier ats 3,2 -

siondravery

so.

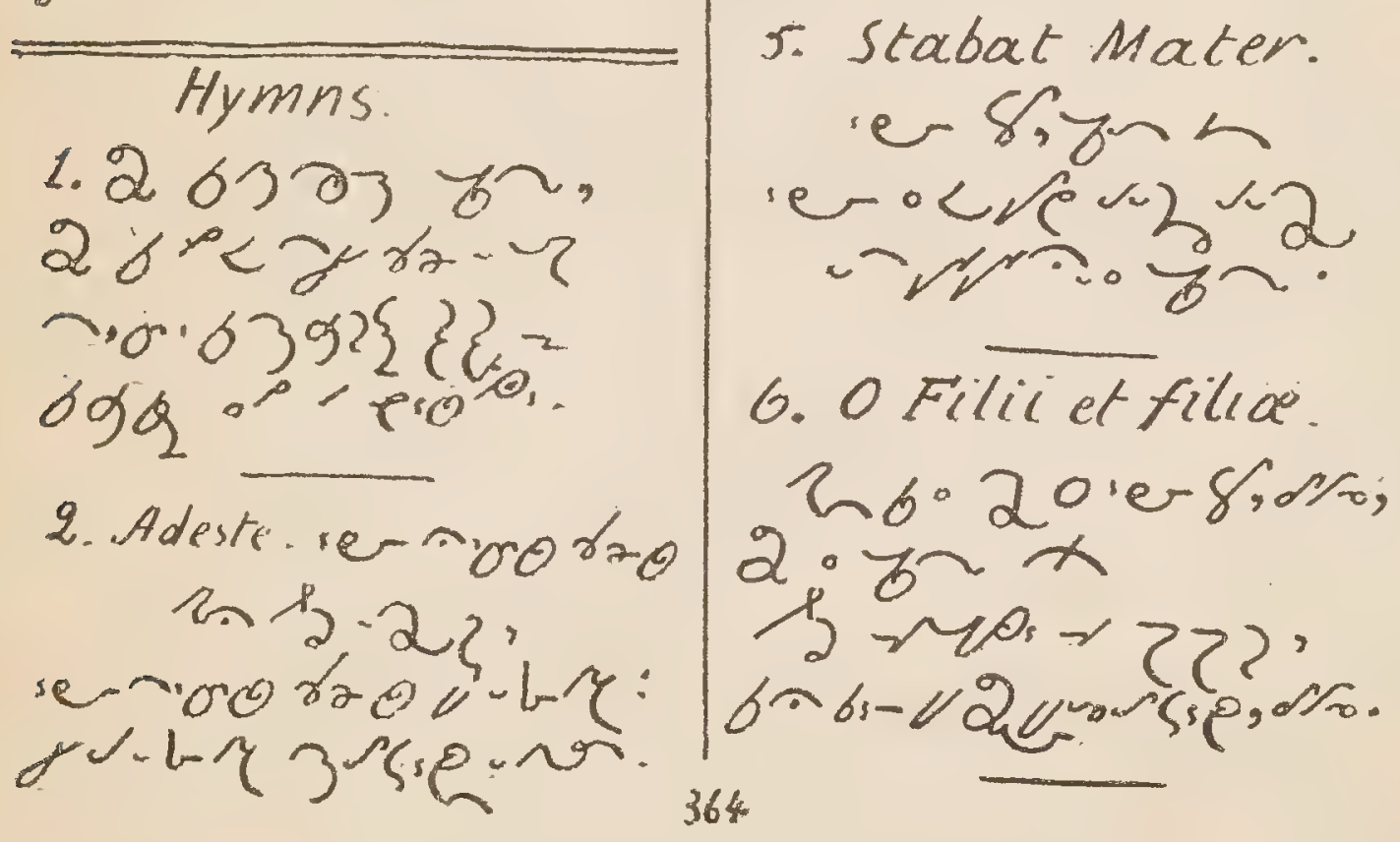

3. Audi benigne.

02 re-6303

borif = grotron=

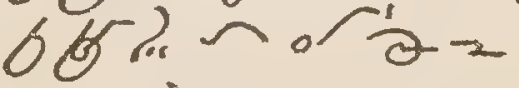

(C) ) (52.) argz.

4. Vexilla Regis.

026 ror over.

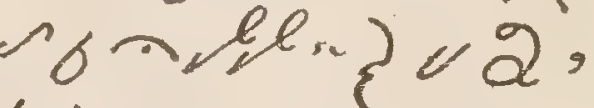

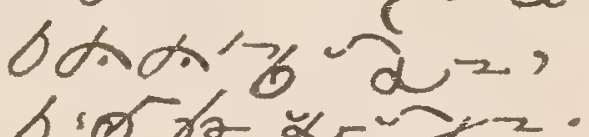

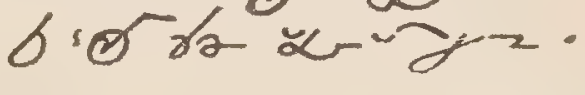

5. Stabat Mater.

ien 8 ign 쓰.

6. O Filic et filla.

26.20iersids;

$2 \cdot 6$ - $x$

मे भis 272 .

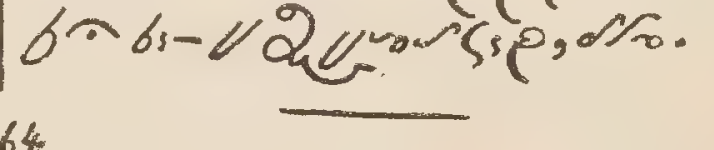


1. Passion Hymn.

$\mathrm{ez}^{2}$ Nir $\alpha$ ror 2 T. wesn, $56, \frac{d \sigma \alpha}{6} \alpha$ - rey $x$.

Retrain. Oxarson

ne: of $62 \cdot \rightarrow \alpha$;

$\alpha r+\alpha-2$

पवे - 0 -

2. ez $3-\Omega^{3} \rightarrow 2$. 3 ;

son.

: 4 LLions?

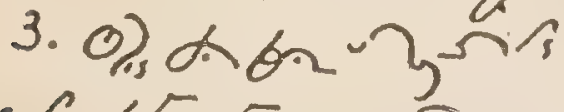

chror -

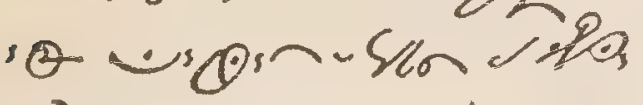

0.

4. r.trug

yn- erosgen

ez- rewl Gars

(ब) नूदs?

$5.6 h 2^{2} \cdot 5 \cdot$. es us sia d o d D)

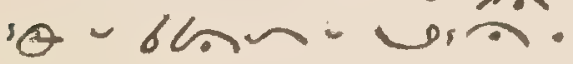

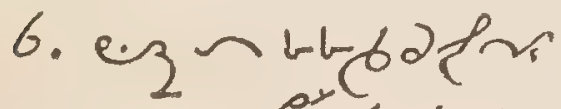
unin ackin

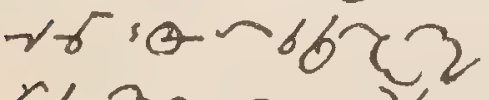

r< $3=0 \times \mathrm{k}^{2} \mathrm{~g}=$.

\%. ez.z⿰冫欠

n-sin-ies:

o) 4-6.

भun का "و औ०. 365
8. Miserere mei.

6 ग92 $-200,2$

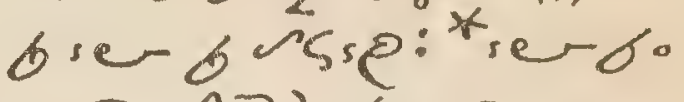

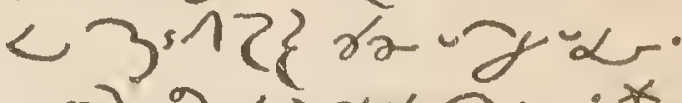

0), 26) g9 几: * $6392\{-2,56$. ra- $\alpha-2$.

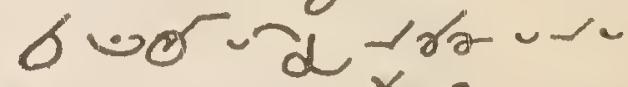
$\alpha-\cdots, y$ *ceser orn 2,5 son vuser רแ2s.

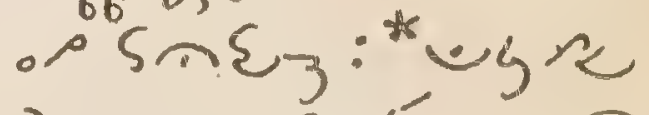

o)

Stg.

ra viosatro-366

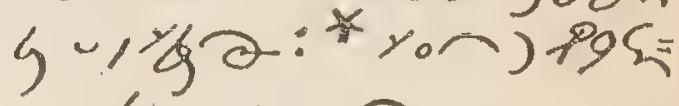
万ra-

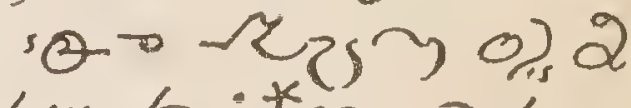

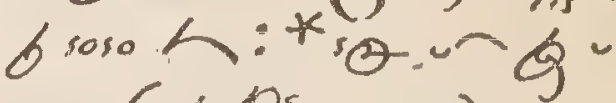
$\left.\left.\alpha-\varsigma_{6} \phi\right)_{0},-1\right)^{\circ}$

9. OBon Jesus. Ox:bez $z^{y_{2}}$, $6392\}, 696,6>201$ \%., 0 - 6 - $2=$ warverz oser 2 \}

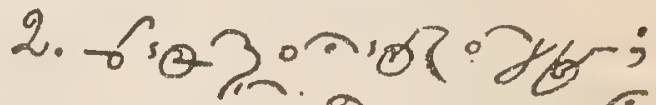
sonurn 2 brerb; $\left.\begin{array}{c}b+6, \alpha-2 \\ u \alpha-\alpha z \\ \text { osen }\end{array}\right\}$ 
20

OKANAGAN

3. \$r-6 402 ,

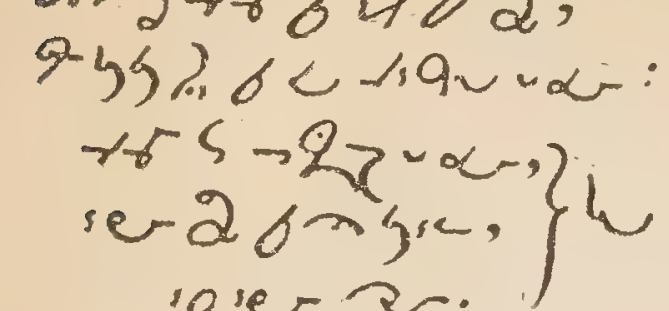

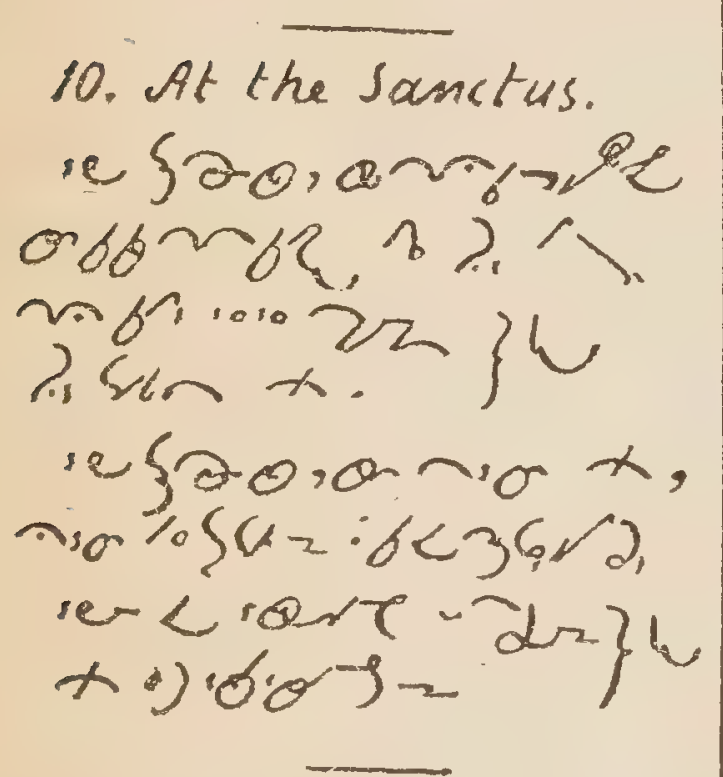

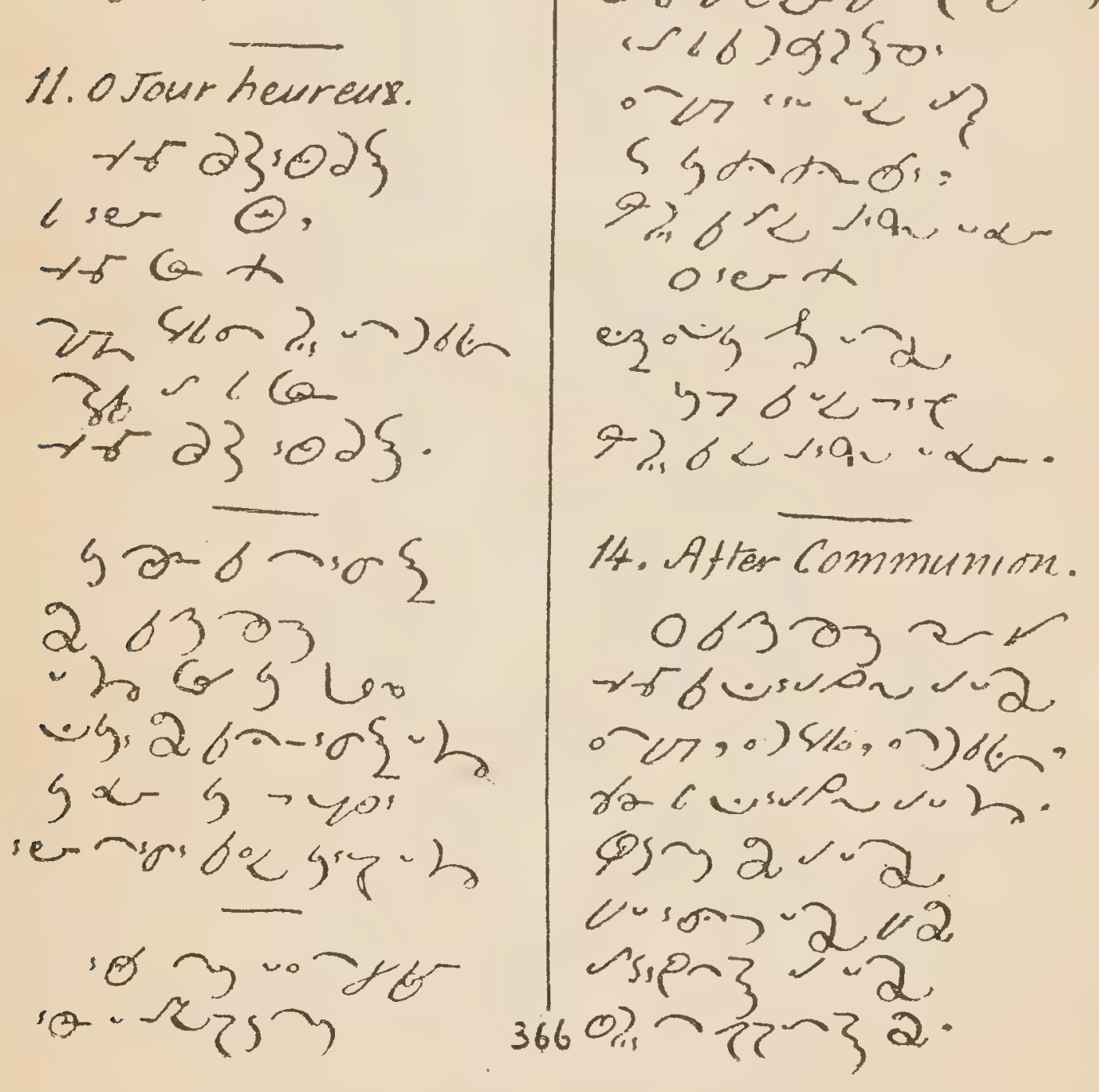

s- $0092\{-2$ 0) d. क्य. सar - a 6.5 - x 2

12. Ame Tesu.

ר.'A, ר.o.h

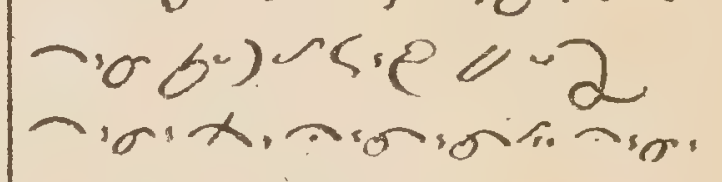

13. Chantons encepor. 0 en $x$.

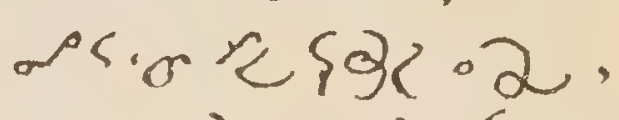

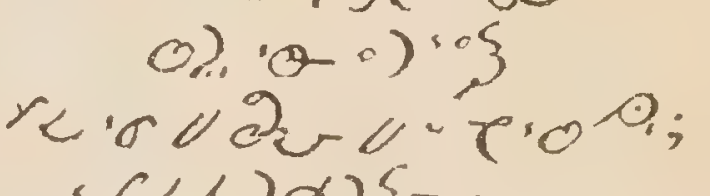

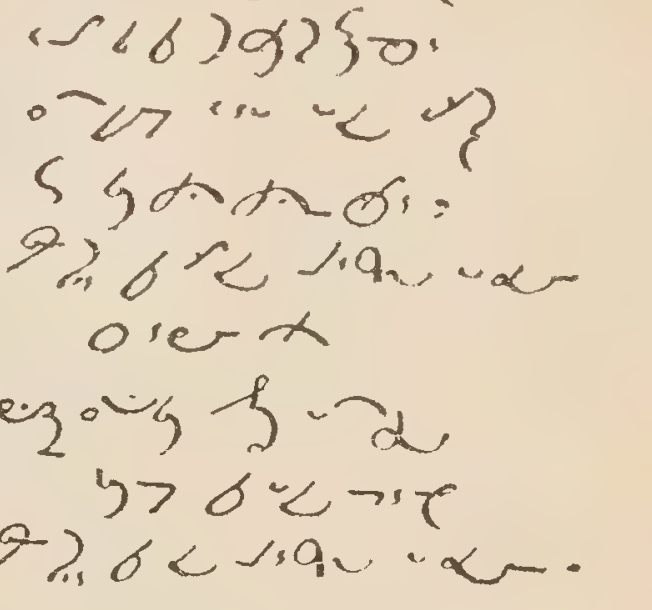

14. After Communion.

063232 -56 usuon 122 raccuspus.

$\sin 2-2$ us. $45,-2<2$ $36607,17-32$. 
MANUAL,

21

15. Panis Angelicus.

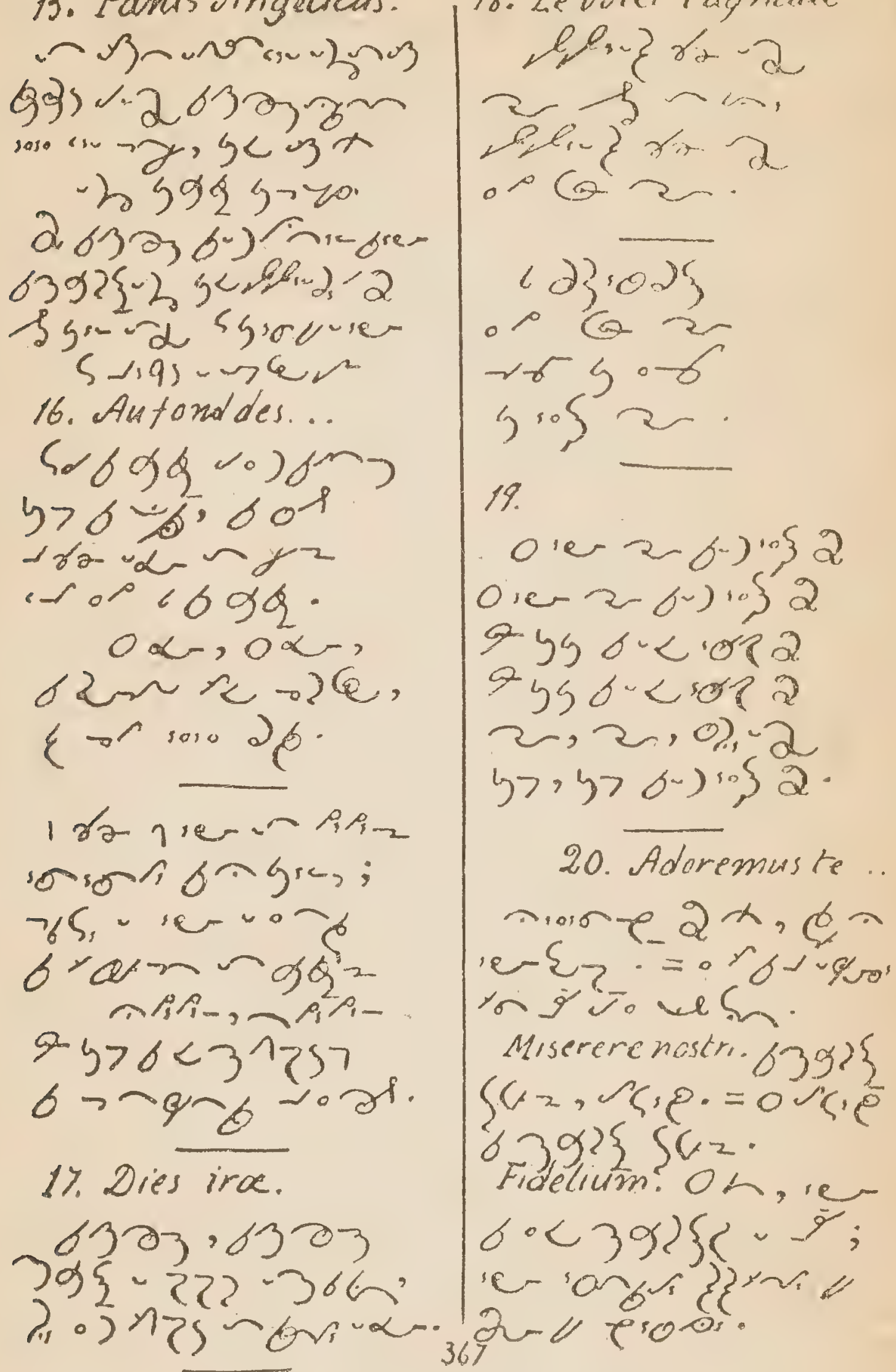


Catechism in Oranagan. r. God.

1. 2 - 6 队力? $=\angle-66^{m}$.

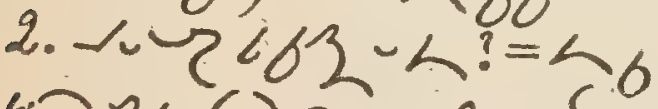

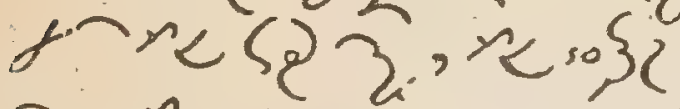

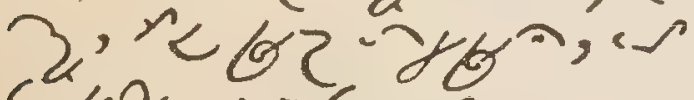
$5,159,56$,

3. $\theta-<?=\alpha, \zeta_{1} \sim_{s}=$ b- $2, y>c$ usde, ac aron, nicierz, ems 3 \% -

4. 127 ? 30 < - 万.

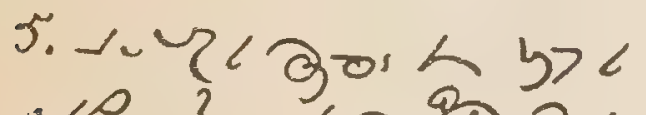
نup?= -人, l, war zon.

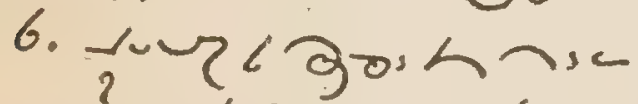

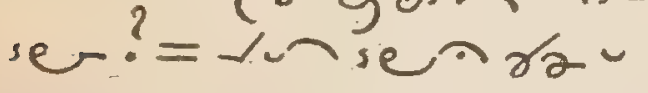
Tuuh, Amonien

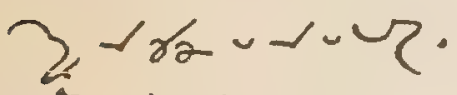

\% 1 प्रा क-s hem ran? $?^{?}=1$ 果人

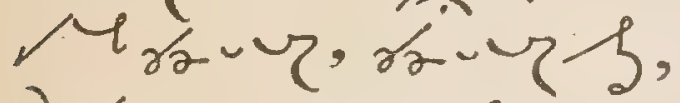

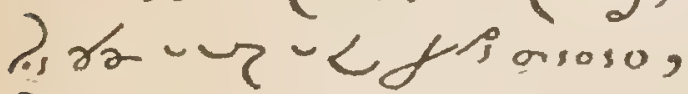
7) 120002

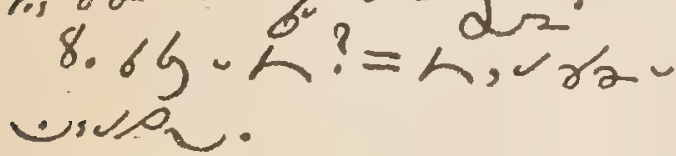

IJ. Trinity.

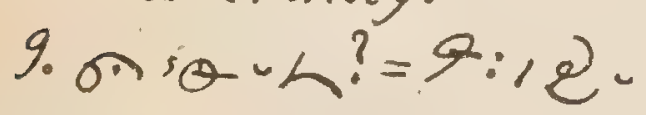
几.

10.0.0. Y UU人? =

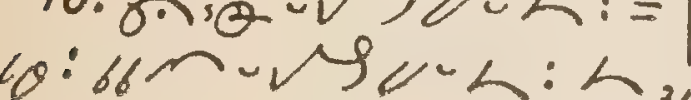

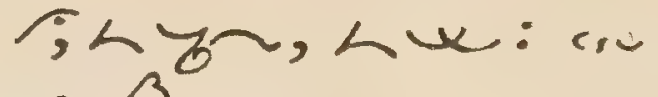
$\xi \beta$.

11. -7 《 $-2-3 ?=-2$

及, टर人,

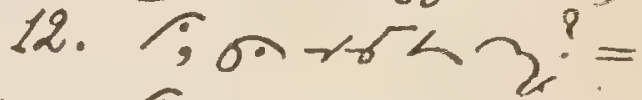

lo, S-

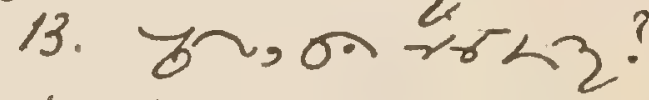

$=10$, bु, $16<2$.

14. W, न मर<2? = 10, ,

15. ; z -8\% 乌.2-人.

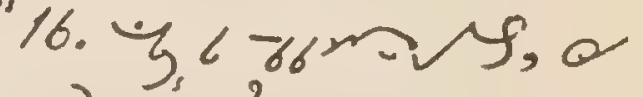

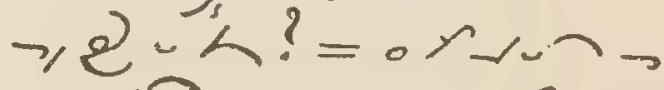

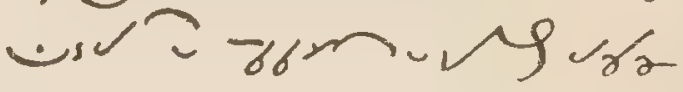
$\therefore$.

11. ᄀ-68 ma 19,0 oges - गserer,ogaroria -A A

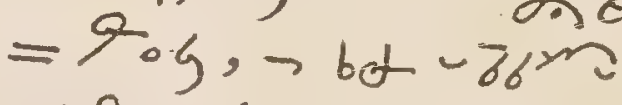

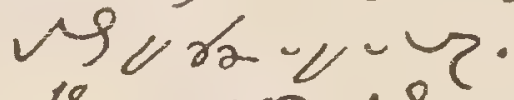
18. - -86m- 99 , का 66 . 1 है- $-1,2 ?=060$ : हरन, III. Jesus Christ.

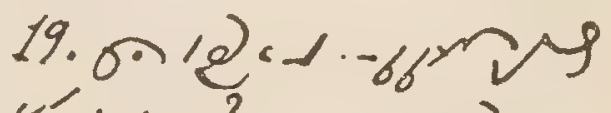
8 r $20, ?=0,12 u$

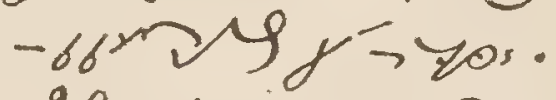
20.0g0

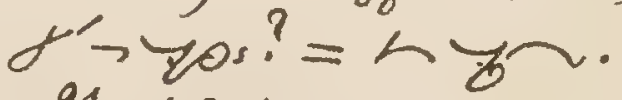
21. 3र人 पुर 


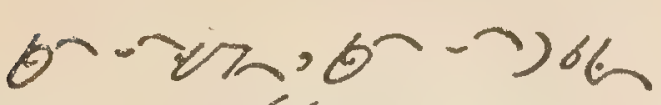
कus 0562 .

22. -105 人 万人:

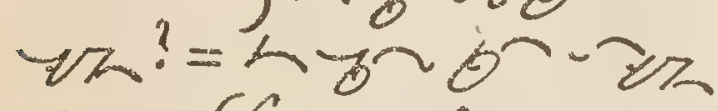
rued 8 , we re

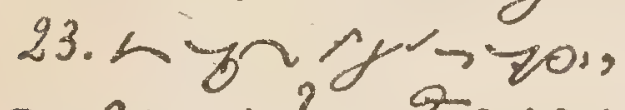
の $40<?=9: \angle 反 2$

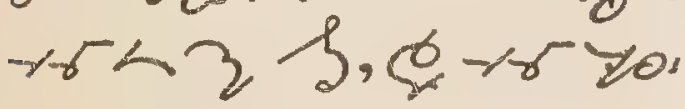
2.

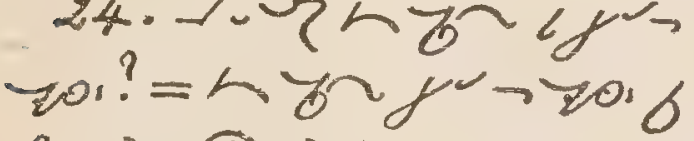

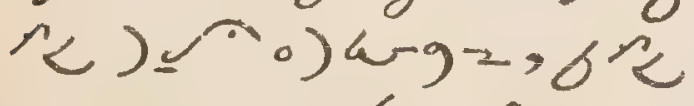
saryureas.

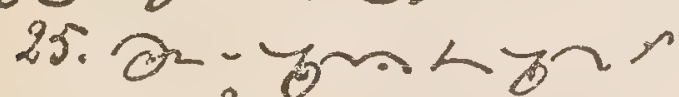

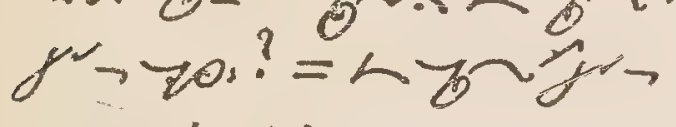

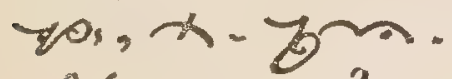

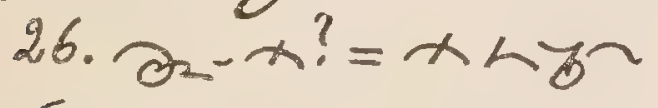
$\gamma=20$.

$2 \%-y,(6)(x \circ)$

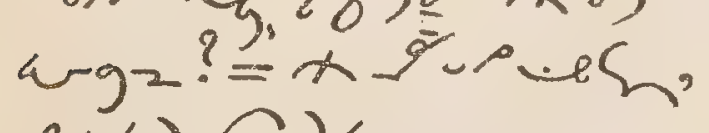
( (b) So) argz.

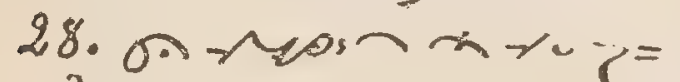

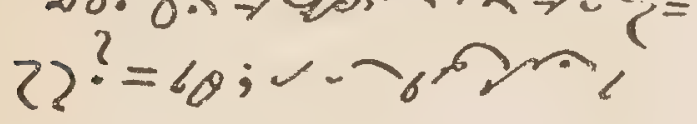
reso $x-2022^{\circ}$ 29. xम-105-2र?

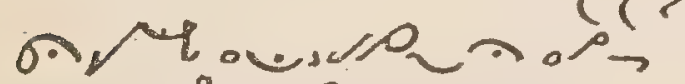
$r_{0} 0, ?=9,4: \mathrm{Cr}^{2}$ ?

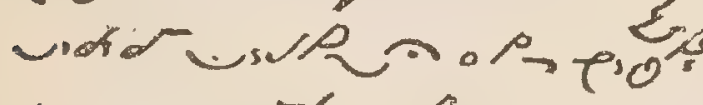
"onvurtert.

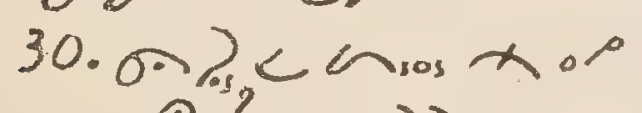
-

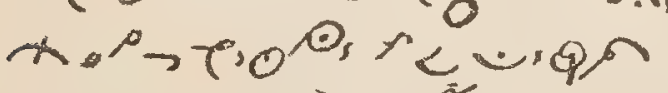

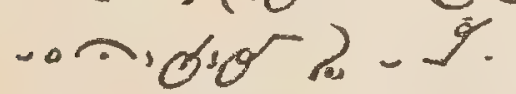

$31.52 \times 22 \mathrm{x}$ 火心 $=$ की - a 22 - ian-ser-zo u- $\rightarrow$ (e) 22 न.

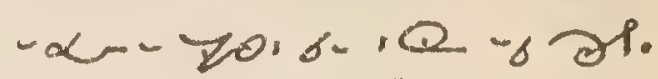
IV. The Church.

$32 .>$ an $51382-1$ sor-x, $>\partial_{2}<0_{2}$ - Pos un verar?=i.

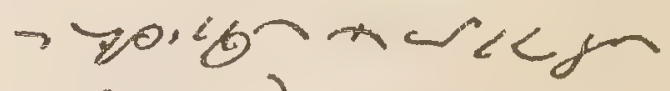

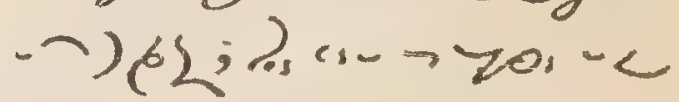
33. - $3(6-6)$. … $\theta-20$ - $72-2$. (ब3.0र D. 《 > 20, - en $x$ re $3=$ 反-201-26-1 cian-sorventer: 34. 2n - > 20 - -6x

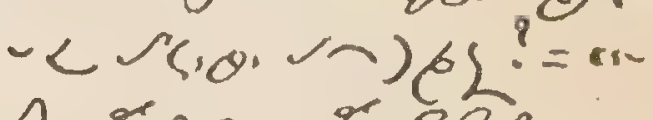
f, YM, Yere. 35. $x, 0 \cdot \theta-y=1$ $3, ?=9:-2-5,02$

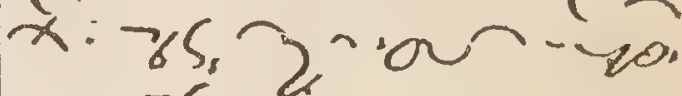
v-76,

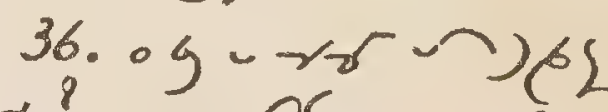
$x ?=\pi 0 \xi 7$ - 0 की $-y /, l_{C} C_{0} \cdot \mathrm{Ung}^{-}$ 37. $\left.\partial 2-u_{2}-0\right)(\underline{2}$ $x ?=x-\ln , \xi^{2} 0$

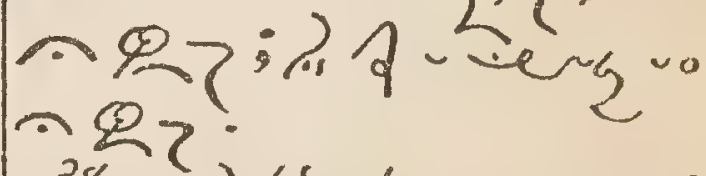
$36938.0)(2 x, 0.9726$ 
1 \% $-\sigma^{\prime} 98^{?}=10,-0$ ) $62 x$, y> 26 dx. - 38 -201: 人we $-198 \mathrm{r}$ जाn.

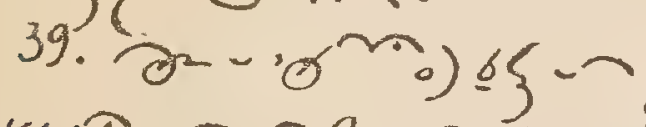

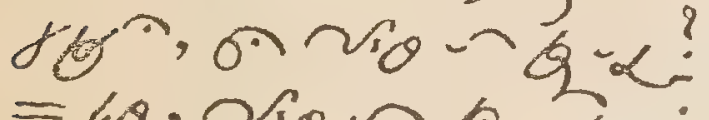
$=\angle \theta, r_{0} \sim Q-\alpha$. -r רaja; -ohibb-ior, on of - or.

\section{The Creed.}

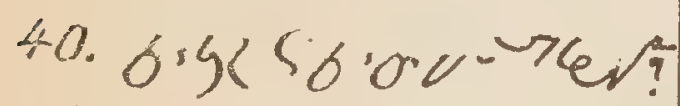
= oc mis...er.

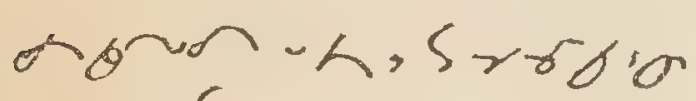
unc $\boldsymbol{r}^{2}$.

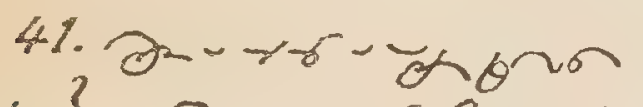

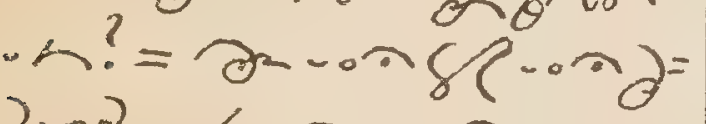

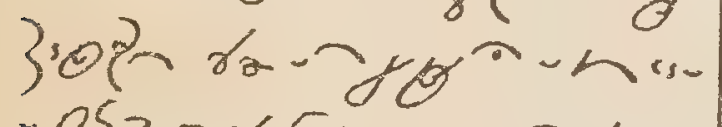

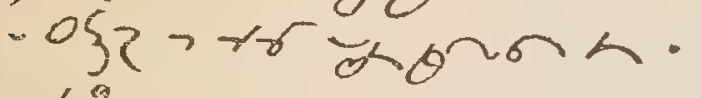

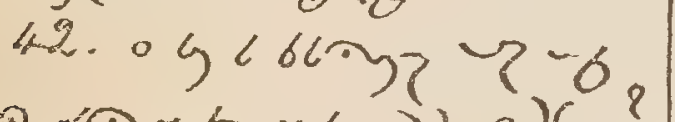

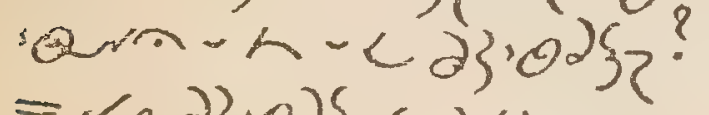
$=\nu .0\} .02\{-0,6)$. 43. $0\{-2\} \cdot 02\} 00$. $=23,02 \xi-2,0$. $44 . \sigma 2\}_{3} \theta \alpha$ - कर八。

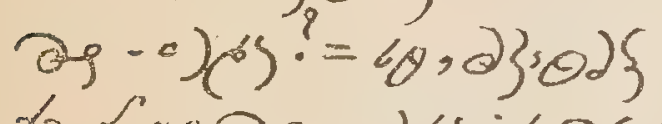

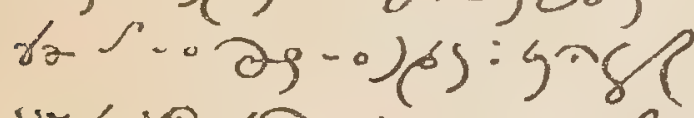
"6:arn-n mons-

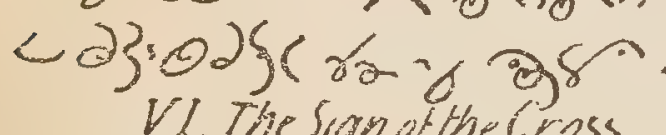
VI. The sign of the Cross. $45 .-17$ romun.

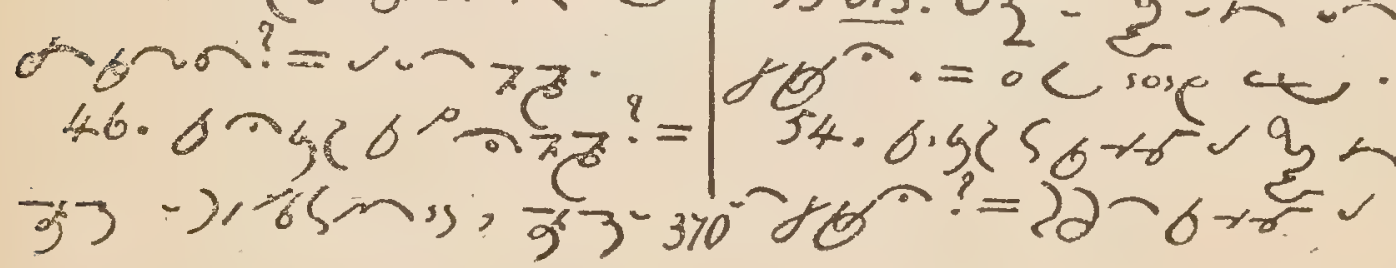

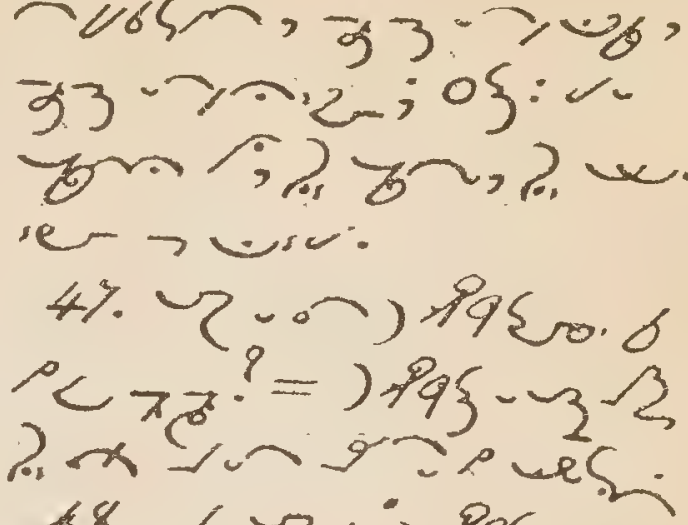

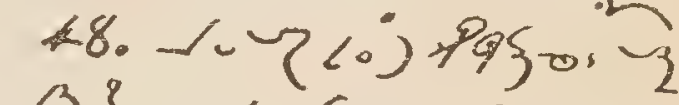
$\mathcal{R}^{?}=-\operatorname{og} \Lambda$; $\sigma^{2}$, 世.

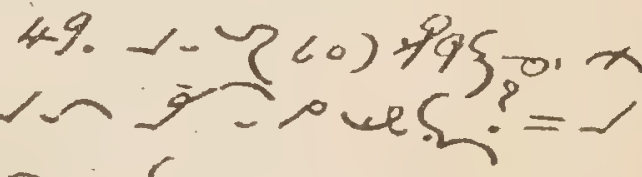

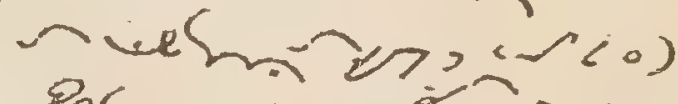
Pas xin gres.

50.4556 a $?$ ? $=57$

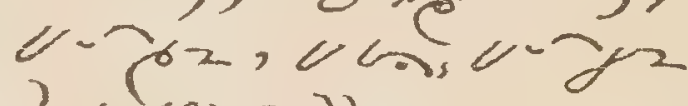

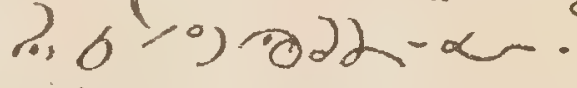

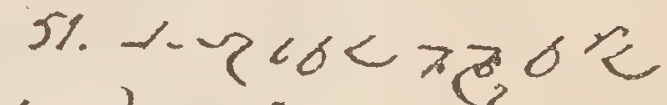
or $>$ d o rigre oerc- $b_{2}, p_{0}-y^{2}$ von, ore gin $5 \sigma^{2}=$ $6<-b_{2}, \lambda_{1}-\gamma^{2}$. 52. $1-7.0$ a $300^{\infty}$

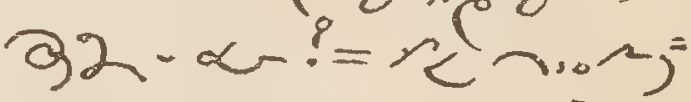
2-4 6 regingos 1 q $-\alpha$.

VII. The Commandmonts. 55. Jog Sounsz - $6.0=$ nin-n-c gz? =八 - $29-q$ q -20 53 bis. o\{

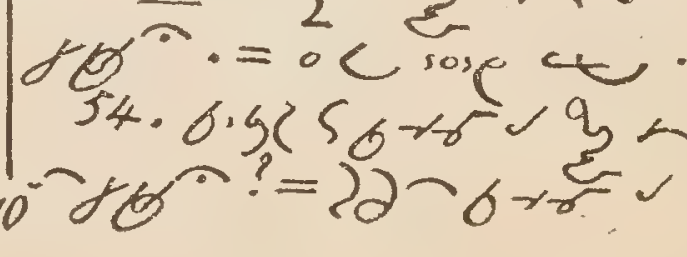




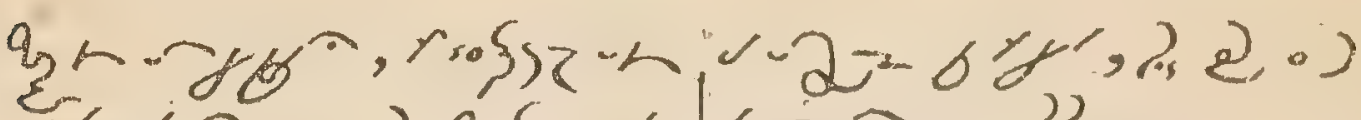

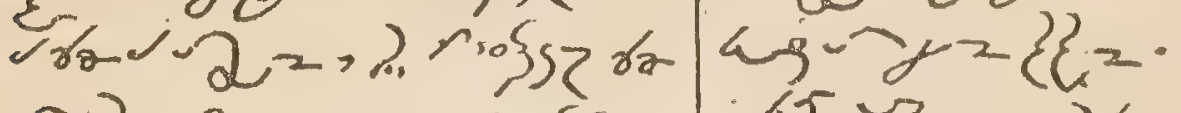

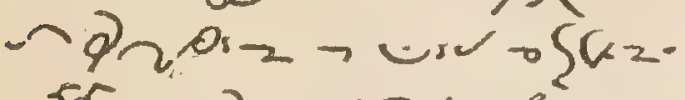

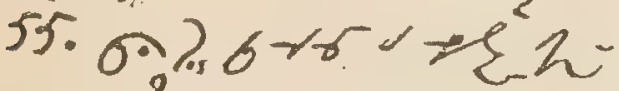
$2 b^{?}=6,2-r^{2}=$

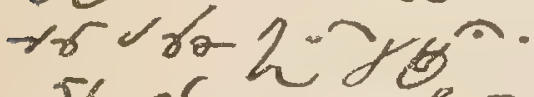
$56.0\{2-2 \varepsilon=86$. vis. Prayer.

5). 6"हक्ष

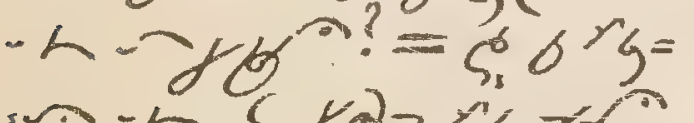

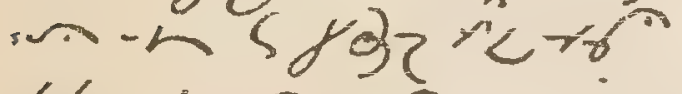

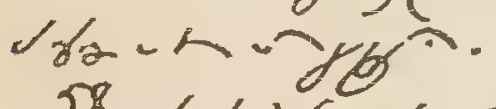

$58.6 \cdot 5<56$ g.⿻一㇉丶

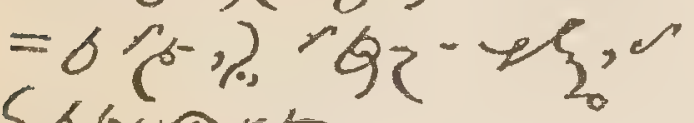
Sogsin. $59.050)(6)$ - o $05-1$ ra. $\left.\operatorname{so}^{\circ}\right) \mathrm{cs} ?=\csc 6 \mathrm{a}^{\circ}$ x, ज्ञ

60. $e 2$ an 0 - (6) $=0 / 2$ U. $\rightarrow A^{2} \angle Q . a$.

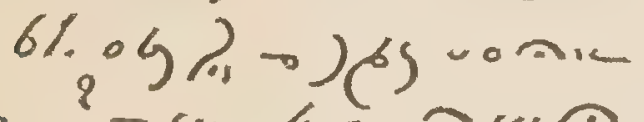

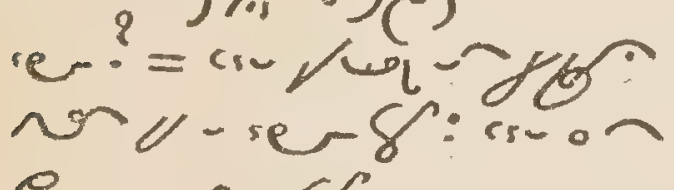
ere $e^{2}=8$.

62. 02 … $63 .=$ 2. 8 ix. Sin

63. 20 ) $\mathrm{chg}^{\text {? }}=10 \overline{\mathrm{c}}$.

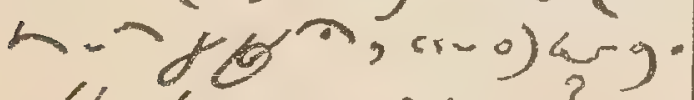
64. Goc (-0) 4 - ? ? = जorio) ang: $20 \log _{37}$

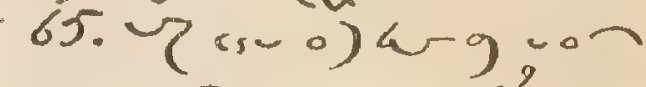
ag $-2=6^{\prime} \mathrm{g}^{\prime} \cdot ?=$

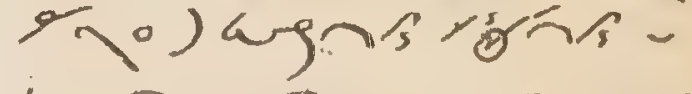
人$-2=6 \% y^{\prime}$.

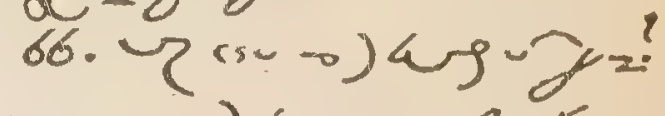

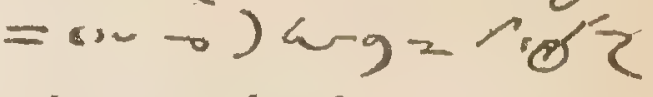

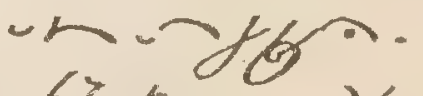
6\% $90(0)$ ang - $2 y^{2}$ ?

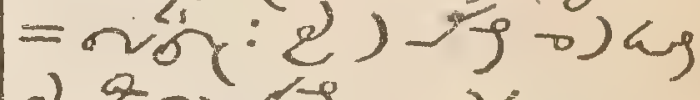
ᄅ9ก

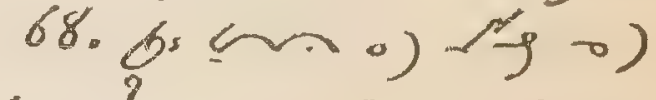
an? = 6 mat-a, 6 :ane 28061. 69.0 , 0) (1, o) ans. bcionv-nio 6 d? $^{\text {? }}$ $=2-, a \mathrm{nos}^{2} \mathrm{~b}^{-}$. 70 . bs m - 9 -) $a-9 ?=96 \mathrm{mon}$

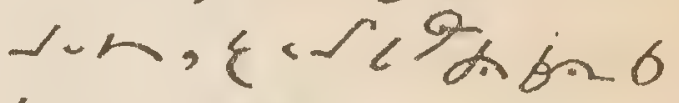
gin-

प\%

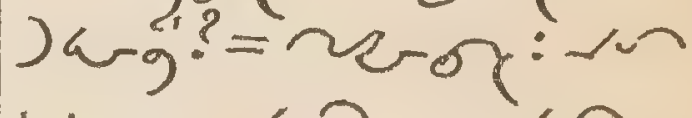
biblz, a $-2,2,-1$

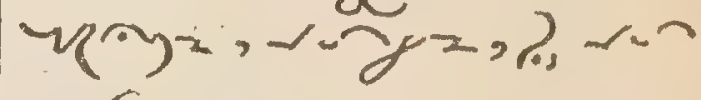
$>26 x^{2}$. $72 . Q 2$, eary $20-0)$ ag? = munz ra - o) ang: (a, ar, varg a-, wis, yes, ¿द, sp, >3द. 


\section{Baptism. us ? = Ayrm () 662 . \\ 4 . C2} ake, 0 , he, रह g.

75. $4-3 !=32-$

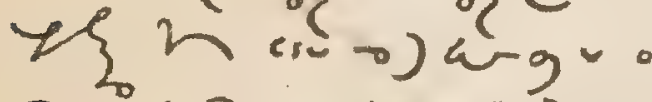

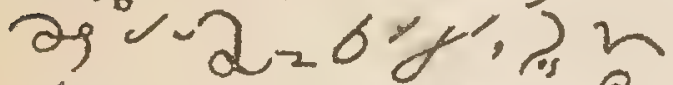

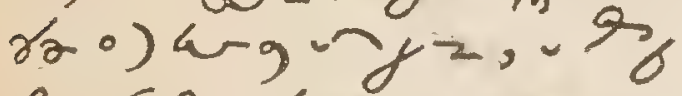

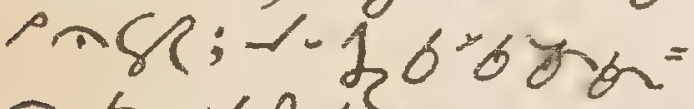

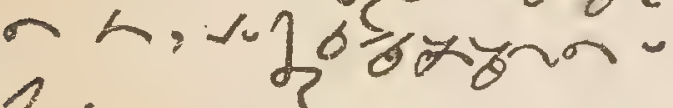
h

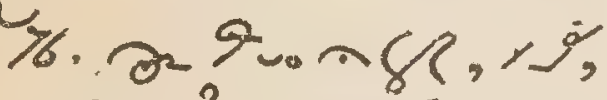
bS S:on? = का शrom \%, $9<$ an 4 ?

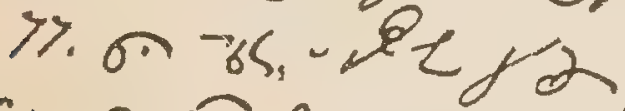

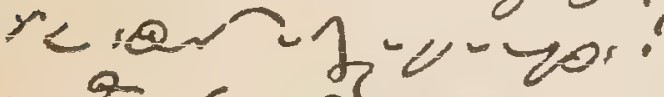

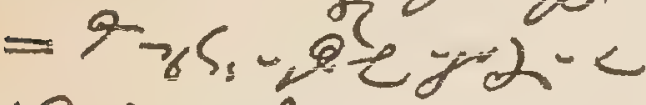

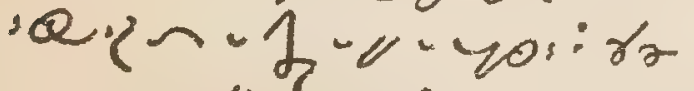
ros ge hn wo.

क.

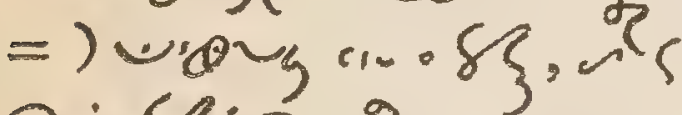

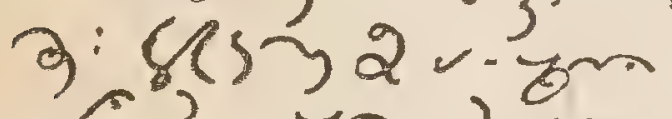
?? क्रे w. T\%. con $-y^{\prime}=22 \alpha \theta$ - 6 br r 52 以 860 ; 22 $23.0 \mathrm{Q}$ - $\cdots$

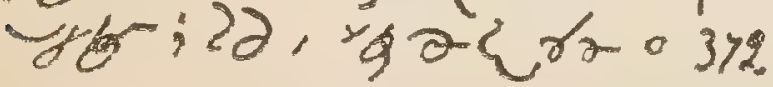

Jang-; 22 on-2 b> re-was fien ton.

$80.7-0^{m} \mathrm{an}^{n}$

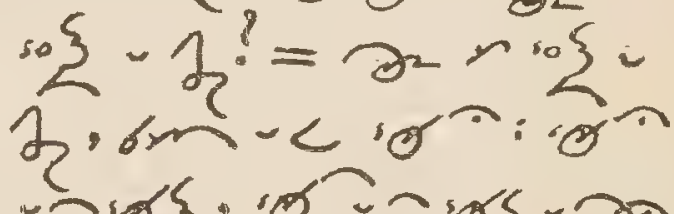

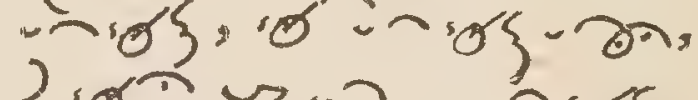
ग. - Licensin y-yor. XI.Confirmation 81. 2 - Pakeo ? = o :

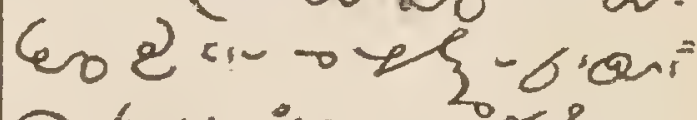
人从wivmorlen, o<

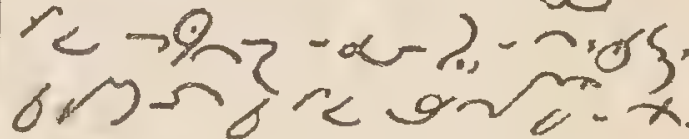

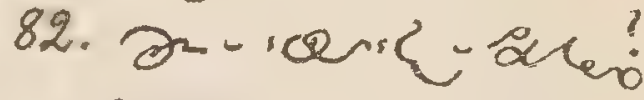

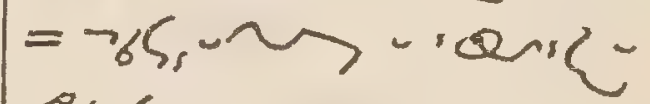
areouryo.

$83.64 \cdots n 6^{210}=$ wn. Paleo:=66r,

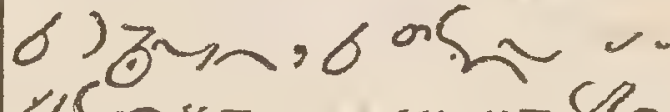

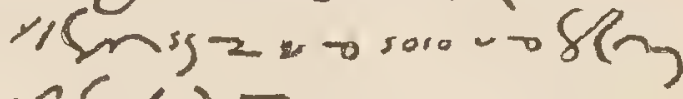
(56) 可。

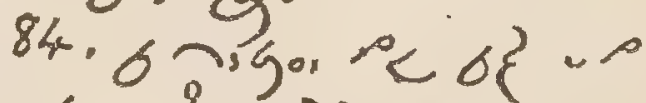

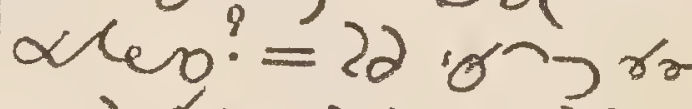
-0) - 9 - >ang; 3 (19) 33 gara, acusien Wonnun; la $9=$

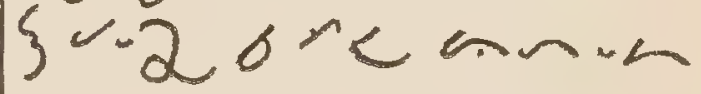


XII. H. Eucharist.

85. $\checkmark \vee \oplus ?=\Theta 2 \cdot \varphi s$ jo Mus ravise vz, hen ר)6

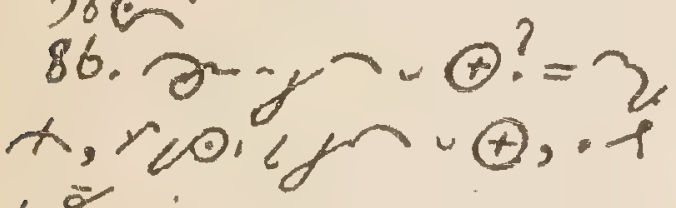
19.

8). "5i大r

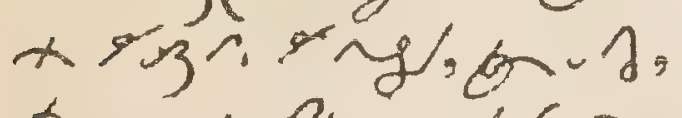
er, eson, ex.

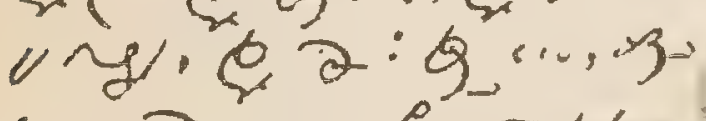

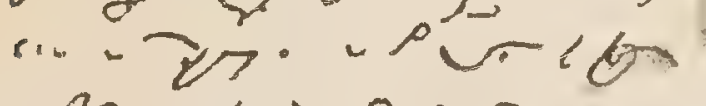

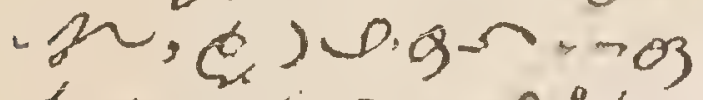
be, es

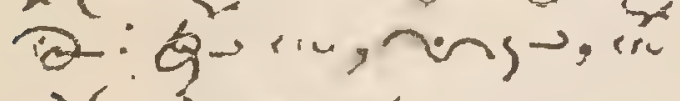

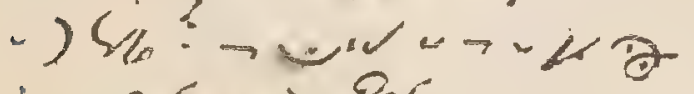

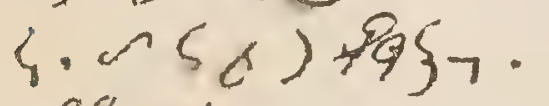

8\%. $x$ refer:....27 … $) C_{L}, \varphi \cdot 5 c^{?}=$

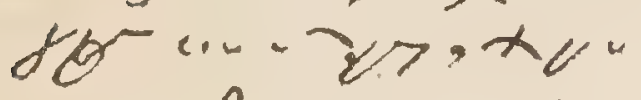

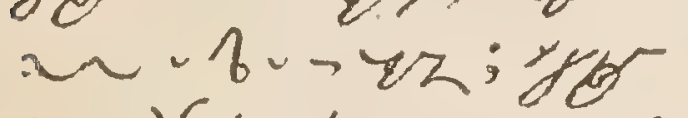
(i.) Sh, xuvevos $u \rightarrow$ sen.

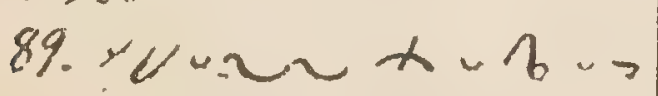

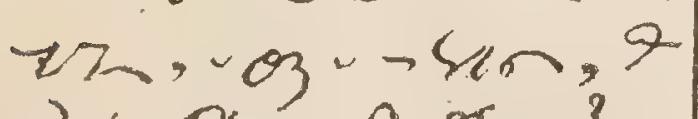

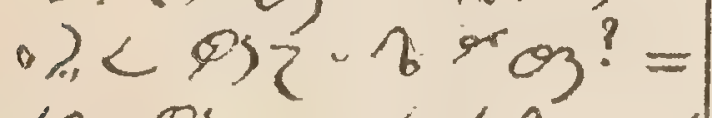

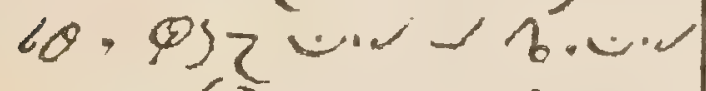
- $9 ;$; 5 रे श. 2,903 .

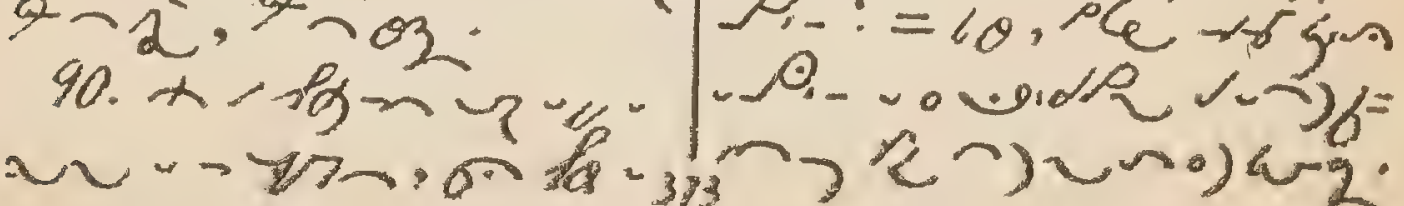

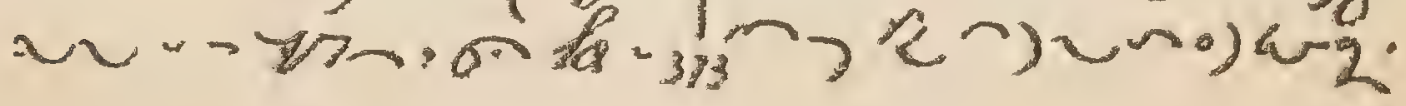


96. ny, 690 xy 8n-06r(xn-1) 6h=? = csedis $x$. Cथल

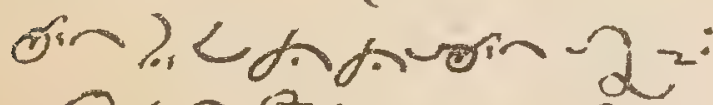
- 0 gn $9<$ whye $12 z ;-\theta \sqrt{k} n$ dretar y-a;

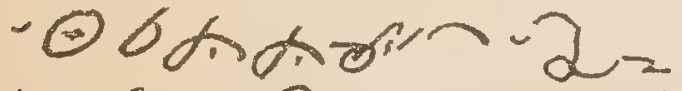

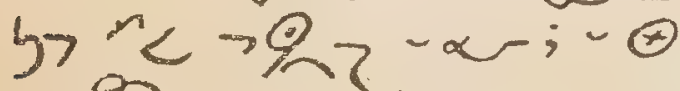
$690 \%$.

$97 . \sim \Theta \curvearrowleft \neg$ टकान人。

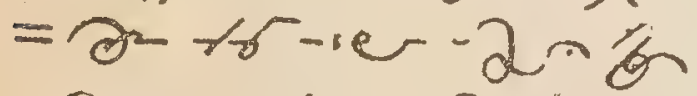
$-\Theta$, שיduren; $\partial^{2}{ }^{2}$ reroin

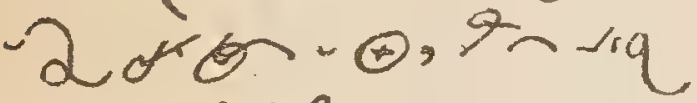
- ien, \& ${ }^{2}$. 98. 95 S b she Sien-c ) $\left.{ }^{\circ}\right)^{2}=:=6-2 \sigma$

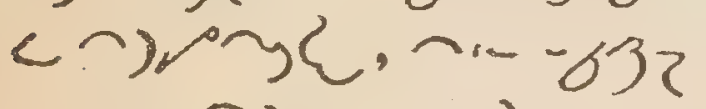
- ser - 96 -

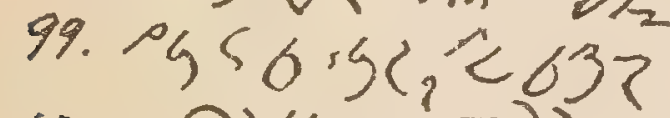

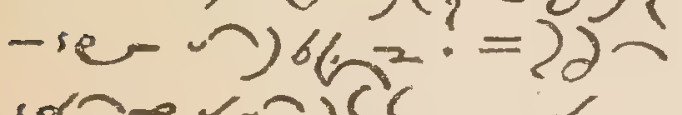
ल (

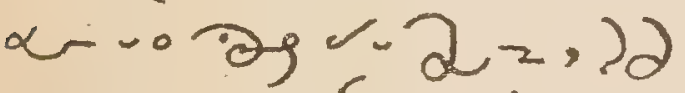

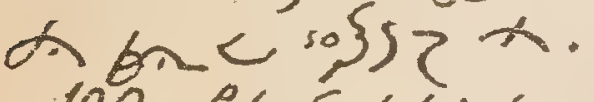
100. $955,6.52<-16-$ <" $\left\{5_{2} x ?=2 \partial \mathrm{kgn}-\right.$

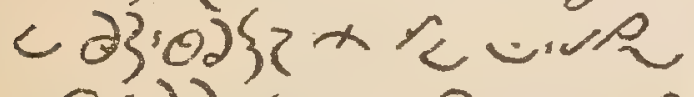
$-\Theta ; 2 \partial \alpha+2-2=\sigma^{\prime}$

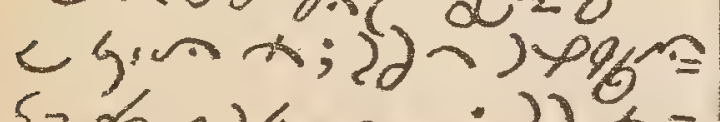

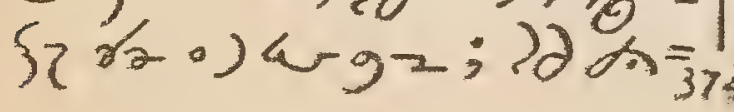

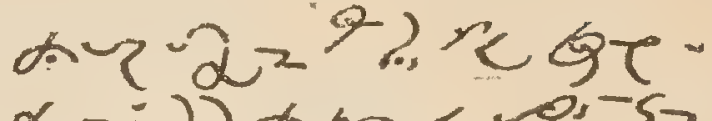
L; ; $2 \mathrm{arac} 0 \mathrm{~s}$

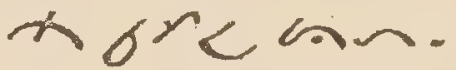

101.055 osg rg 32 . ser- $2 y=$ ? = $6 \mathrm{C}$ 2, $590<2$ 的 $-2-(n)>70$-g 5 क.

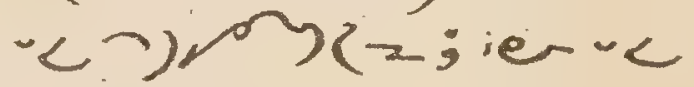

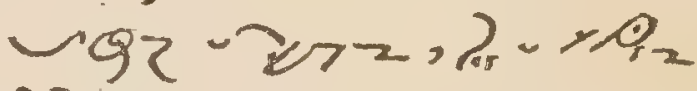
$2 \partial \rightarrow$ wथ -

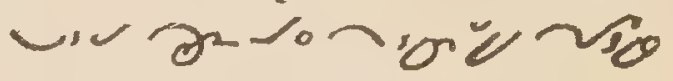
u. rsie.

102. $0550.52010=$ लेख? = सra) नोर bs. $\sigma<66 \sim \operatorname{sos} v-x$

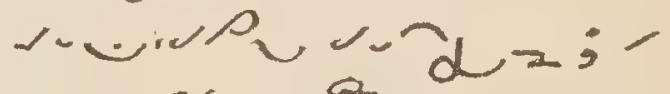
on Ple, Yroniono

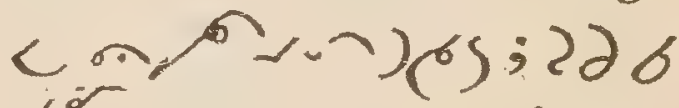

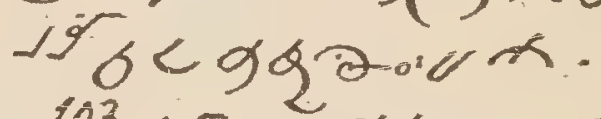
103. 乙-< $98 \partial=$ ax? $=2 \partial \operatorname{cic}^{2}-2{ }^{\circ}$

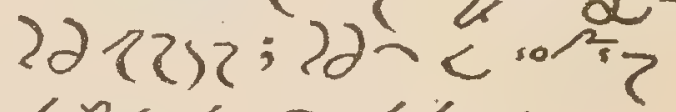
6re gin drasen

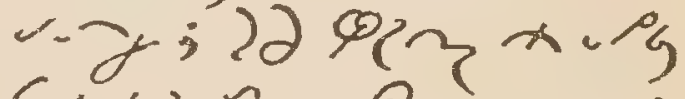
50 . $2<-9<-\alpha$;

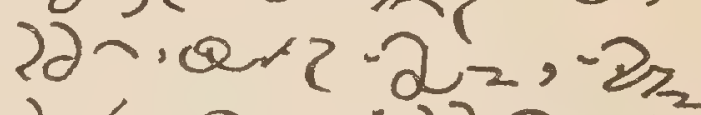

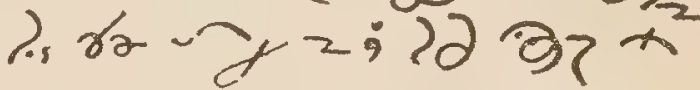
प> -

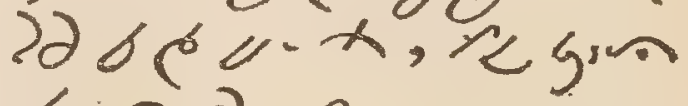
ชa-ר की 104 . Oner por $\xi_{2}$.

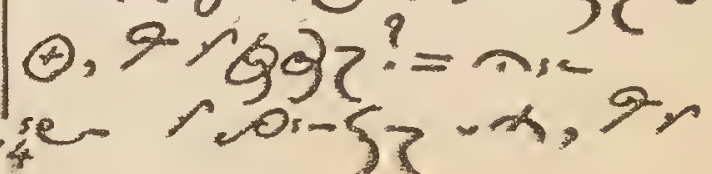


492-0: 大 $0<2$ $-2=-1-P_{3}-\xi_{2}-\otimes ;$ s6byon $x$, inion?

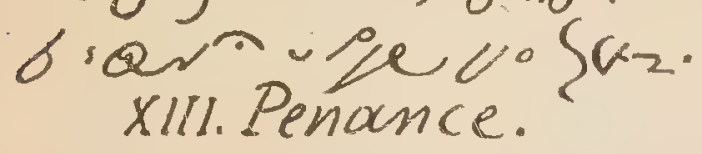

105. g一gर G०m)\{हn? $=22-y^{\circ}: 1^{\circ}-93-1$ ang; $20-5$ y

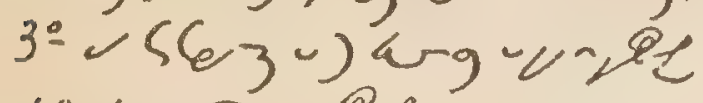
4:6.0 क. (e) ang;

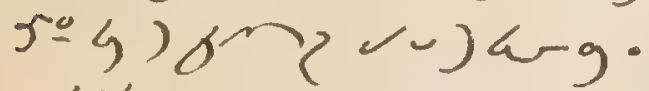
106.6 ? 52 Porno

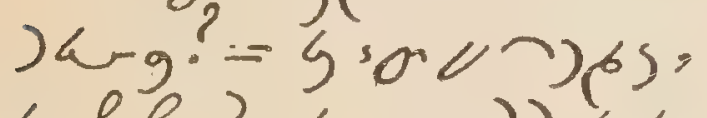

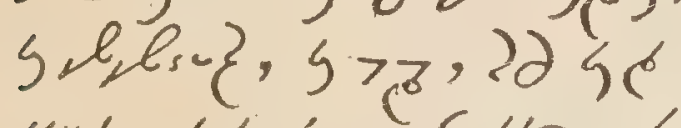
vun oc gan souns ra $\left.-2-\alpha-; \sim s_{0-1}\right)-1$ ang, $5 \mathrm{~S}_{3}$ 马ुहैu) arg.

10\%, 7 न แ

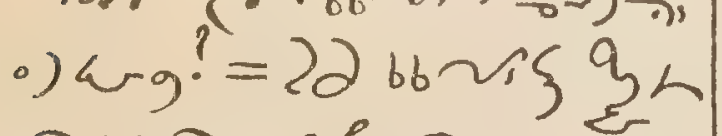

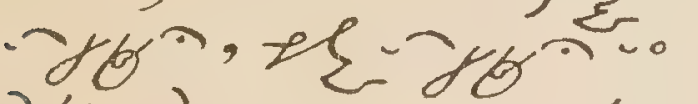

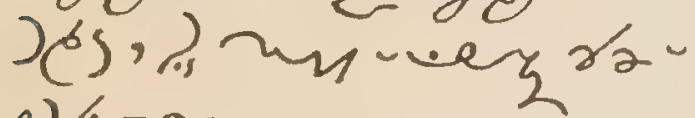
o) 4 -

108.6 ? $52 \sigma^{\circ} \mathrm{C} / \mathrm{g}$

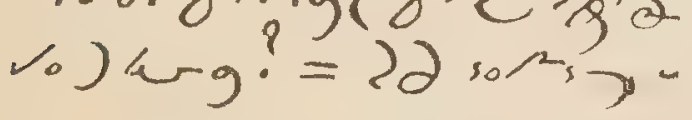
人 b<ian-c -2 " $10 \mathrm{arg},-<\alpha \alpha=$ $2-2,9$, मе कर - $\alpha$; $=2 \partial a_{0},-b_{b, 6}-\alpha^{-}$

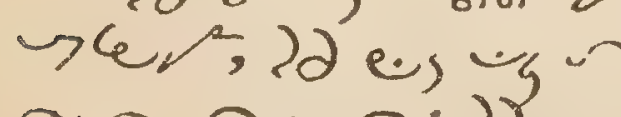

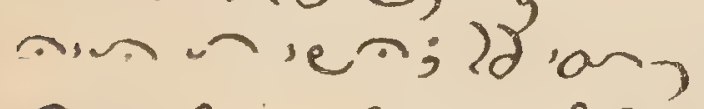

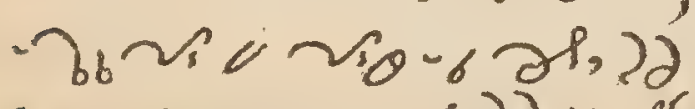

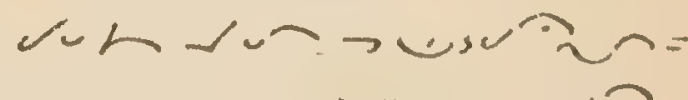
unser, 10sur (0) - 0 :

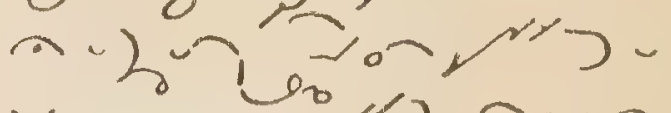

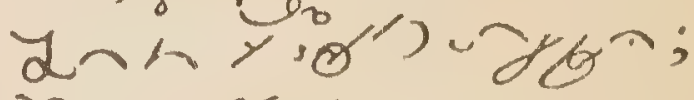

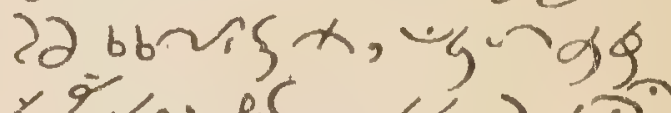
y 90 us $6<$ ) -) arg; - $54{ }^{2}-2$

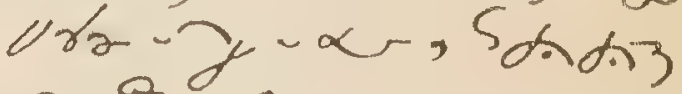
2 ??

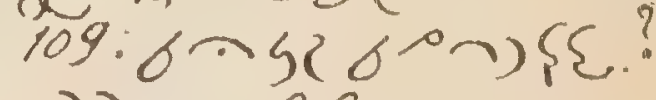
$=225$ selise - nz

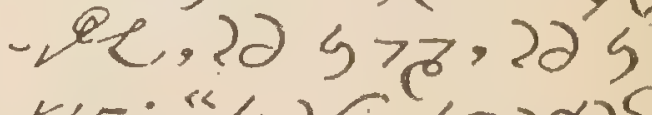

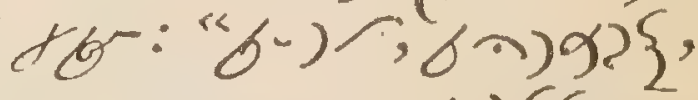

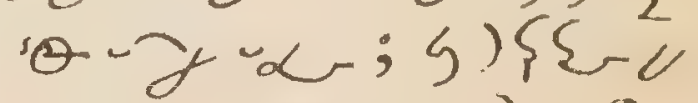

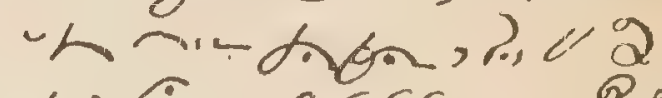
b) : 15563 - 8

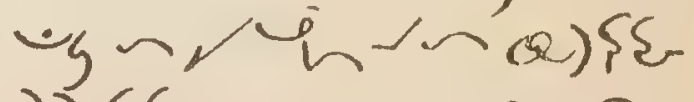

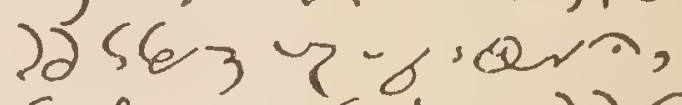
celoro, huz; das $=$ E37.6.arm" UK)

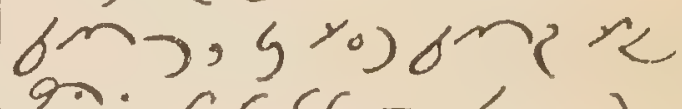
ga; $\sim 5(4) 3$ tru angar o) की. $110 .>Q$ (E) $\rightarrow, 0) \mathrm{ang}$ ule, $2 \circ 26$ ? =

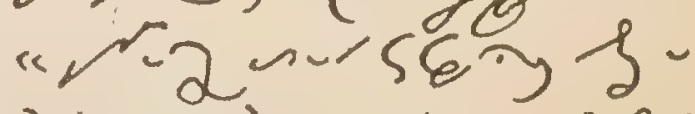
) ang; , ..0) a-g o) 1? 5; 28.007 (a) ang; ser $6<-) g 35$ 26-) f bicer-loro

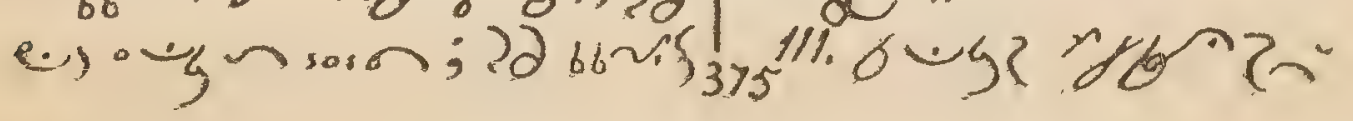




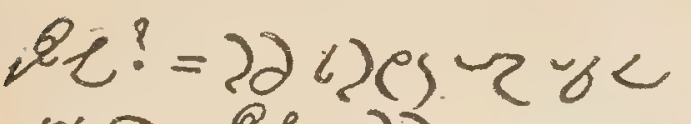

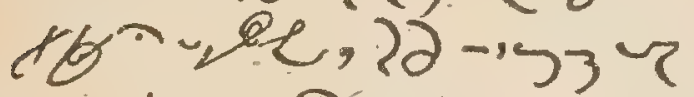
$2 \partial-33-2-6<-29 x$ c. Coro, $6 h_{3}$. $112.7 \%$ के ren \&e toro? = 2250 $6,5(, 22$ urson, 2,22

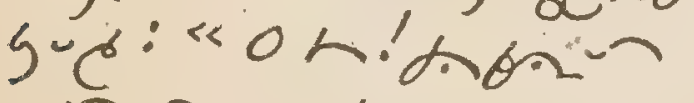
N-2 vra-valag ordf गses; 55 त. 3 - 2 \%

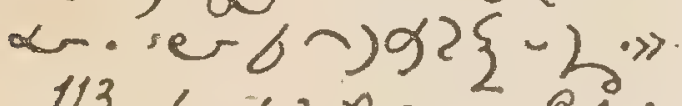

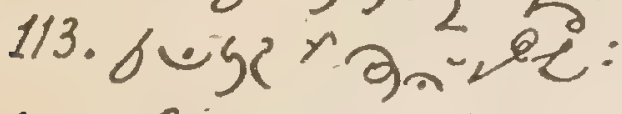
"क., $1 \%$ के $2,66-0$

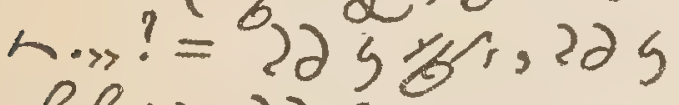
eless, रa र25-人 6-10 $3925,22 \alpha+3-29$

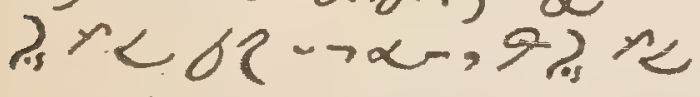

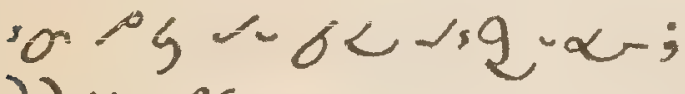
22 แn

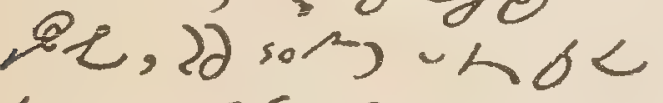

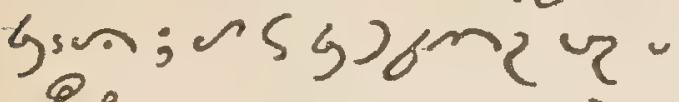

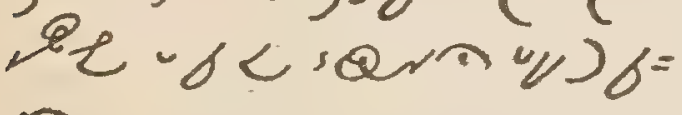
n.

4h. 7 - 6010? =6" arr.let-torro.

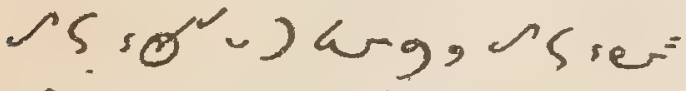
or $-2,-56$ bs 115. T-hi? = orar

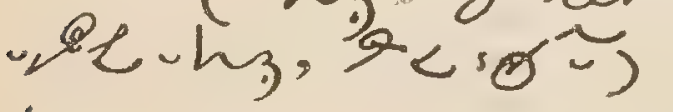
ang.

$116.2265529 \times 5$ sor

b- - $42-2-4$

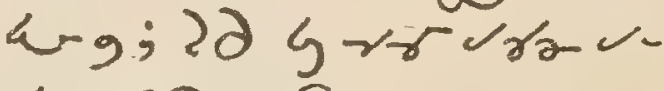

$638^{\circ} .06$ re 1.92 - reco.

117.22 b.5र $2,9 \times$

'as - ie lons? =

gorara uletero

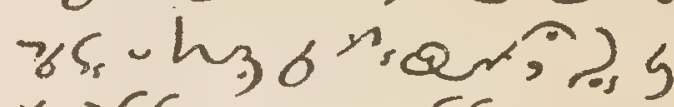

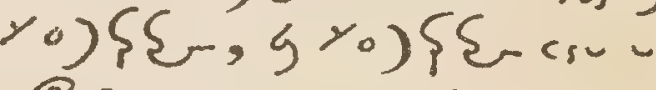

Qe-biarmihz os,

(b) ang $5 \varepsilon_{3} ; 5 \%$

\{द, - 66 -

र6з ra-ri.)an,

?.

118. 22 65 $5(\%)$ म1250

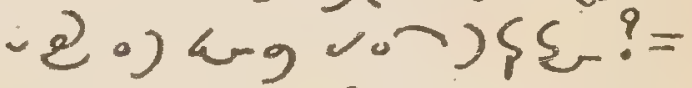
×0) तरा - 2 - Ja-g

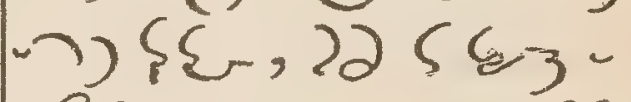
R vound us. 119. 2 . 78 - 9 क -0) kng, on 160 ) $\left\{\varepsilon \cap ?=\partial\right.$ ? $^{2}-\gamma$ -Sn 0) kng, श्रr un -0)ang, g/r

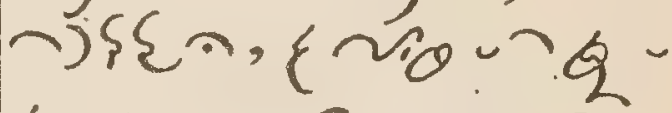
Lusw on er em. $120.29+6-1 \cdot \hat{2}$ (0) $\operatorname{lng}, 9+6$ andin

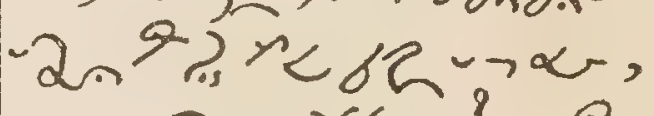

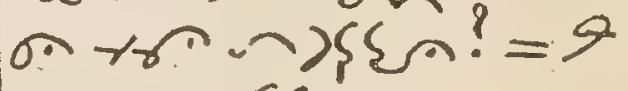

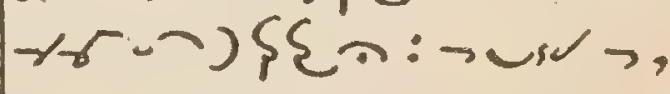
Vio 0 jo) ang.

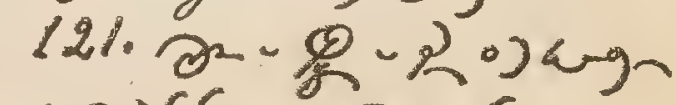

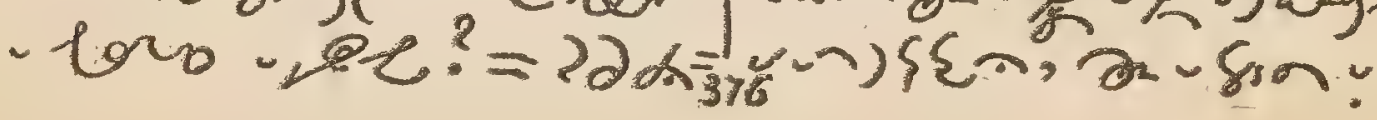




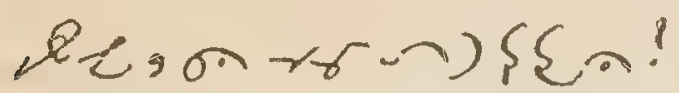

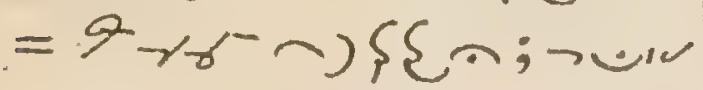
$\rightarrow, \sim_{\theta} \simeq$ gn ua.

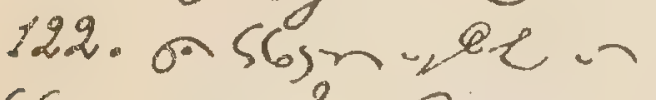

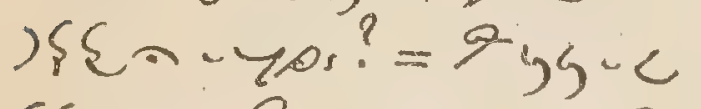

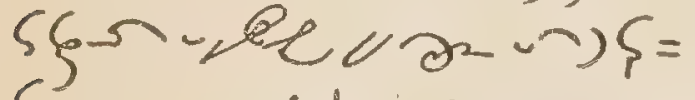
\{n-yos: $y>$ in 9 , c.suryx. 123.4 pises? =

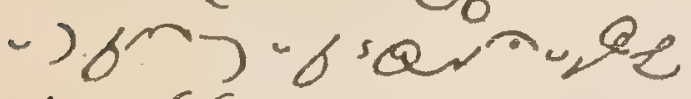

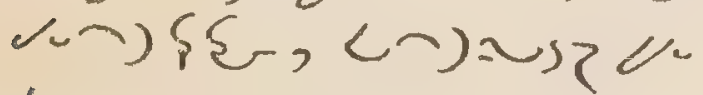
人。) 4 - 2 .

$124 \cdot \operatorname{ros} 64-1 \operatorname{sic}^{2}$ б) m $^{\circ}$ ? = व (songren )

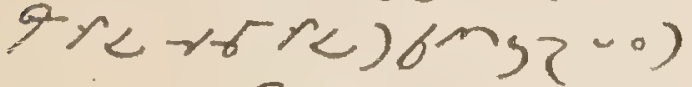
angz, 9 ys s seros. $2 z$.

125. rstr-yor $2 x$ on re inn ro ourgo $\rho, p_{0} \rho_{s} !=\partial^{2} \gamma_{-\gamma}$ ) 'o $P, 22 \times \bar{s}(\sigma 6) \delta=$ ר.

126. O. $5>6$ west $36 \mathrm{~m}$ ? = $9: 22 \mathrm{~s}, \mathrm{n}$ (J) onms of ang $\mathrm{s}, 5$ sourer.

126 bis. $(2 \%)$ fm $)^{2}$ -) argz? = baisa $=6 k$, c) 92$\} e-90 ;$; is rawe

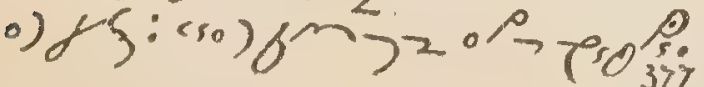

XIV. Ext. Unction.

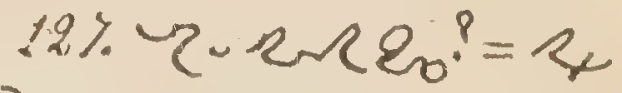

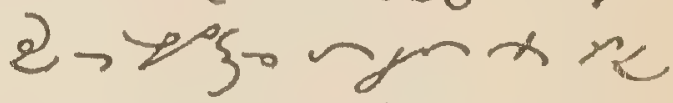

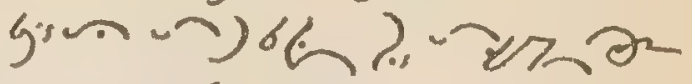
"c N/Sion.

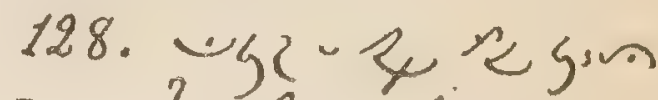
- 666 ? $=4$ r. $366-<-$ Ssos, ?os d. man $2 \times-$ iqs निलड.

129. "थर = द serosn

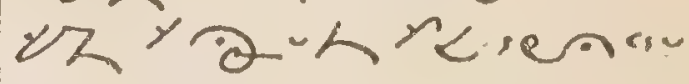
(1) $6 \mathrm{~m}$

130. ल5 sige 20' so

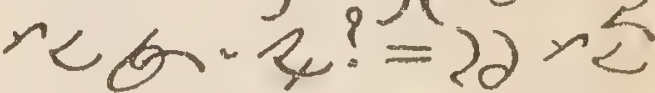
\%क( ㄱ) $\$ \subseteq$.

131. . p s 5 sg . ec r.⿻口丿 bn-rosotrhj;

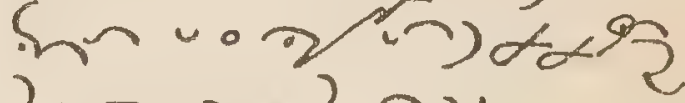

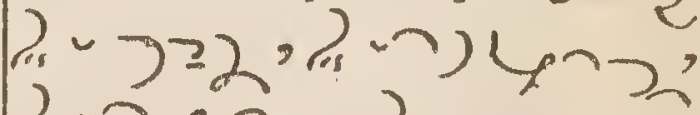
?. $22-2, ?$. ros orbin, be 6 : ser

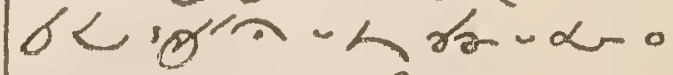

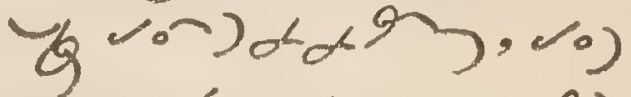

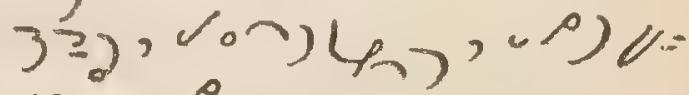
ess, w Tosbs-: XV. H. Orders.

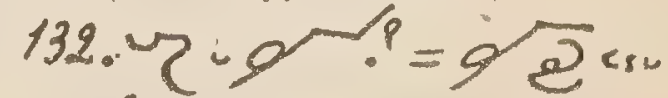

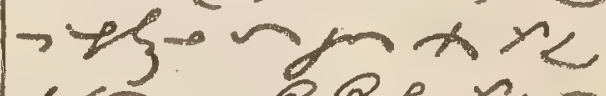
for - e, 298 
- vos- JE- arin - U-uor-yon $x$.

$133.59 x$ con? ? $=x$ rgn $\theta$, sugen r电的, r

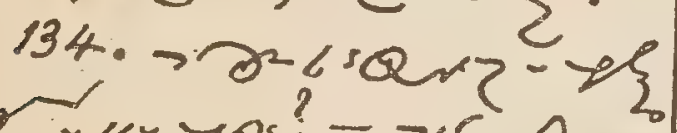
orvur? = $2 \mathrm{~S}_{s}$ की,

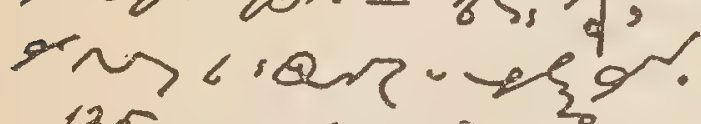
135., nigos, 2 -

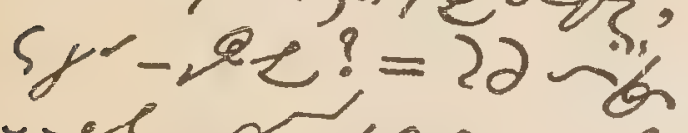

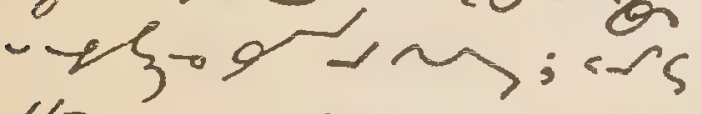
6n - caton<3= sin-20.5 86 -

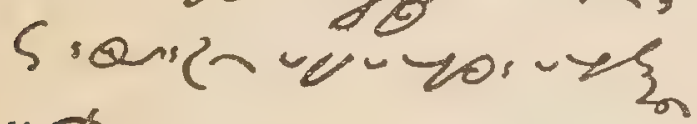
$-x$.

\section{XVI.Matrimony.}

136. $-2-8 p ?=$ isn

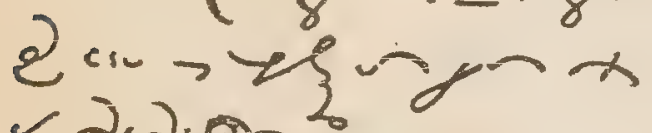
$\checkmark \partial \cdot \partial \cdot$ की

$13 \%$ - - - qu-zan? in $\sin -\partial \cdot \partial$ ब $y>$ cserurym; ) $2\left\{Q_{1} ;\right.$;

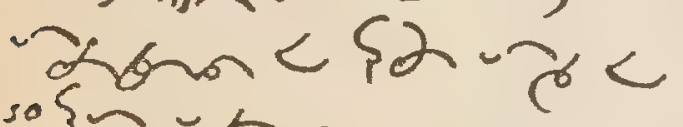

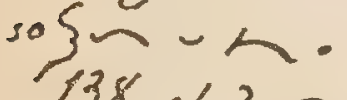
$\left.138.552 \partial^{2}-50\right\}-\cdots$

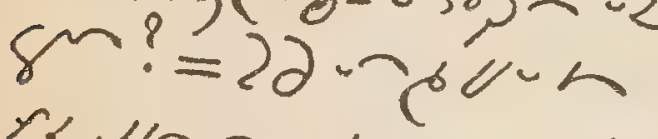
resinn-ciesur

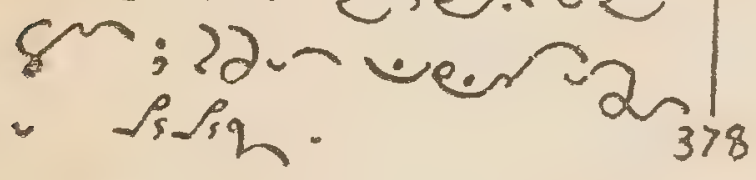

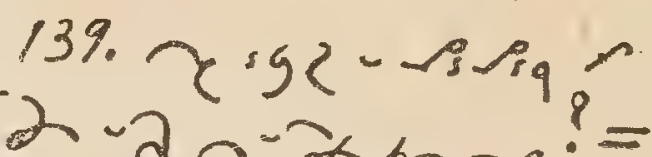

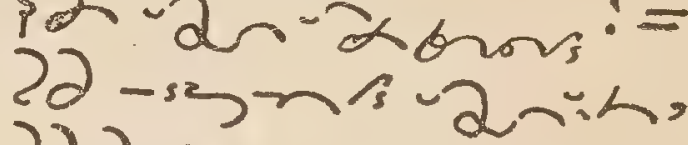

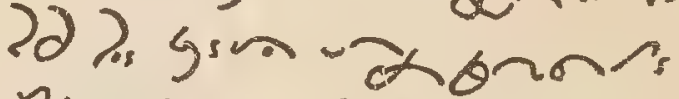
rosin kenegn. 140. T-2. 一人净ig

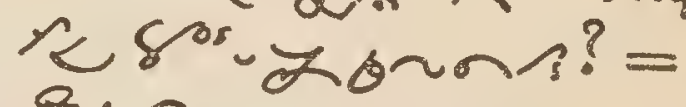

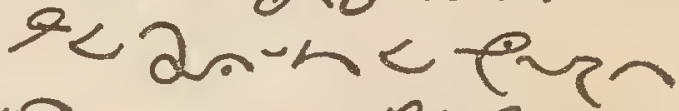

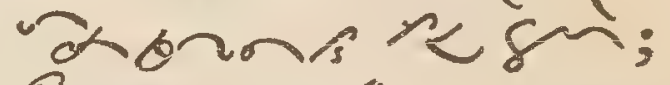

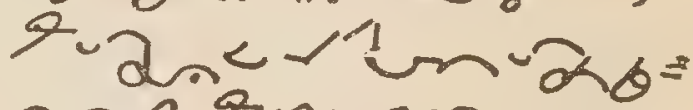
nisesnoan

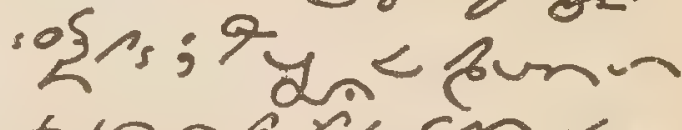
कonns re gnvar 9 r.o, 2 s.

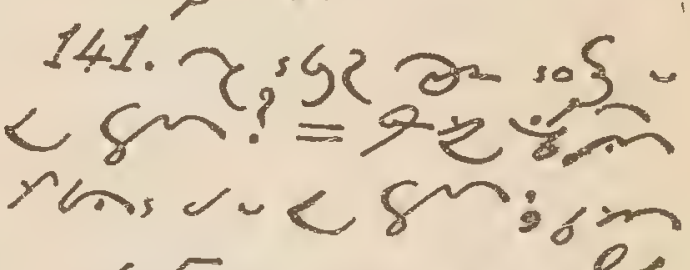
indor < 80 -

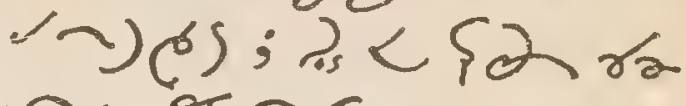
142 क 66 र. 142. 0 < $\cos 91$. ) वरroำsin? $=10: 94 \pi-n$

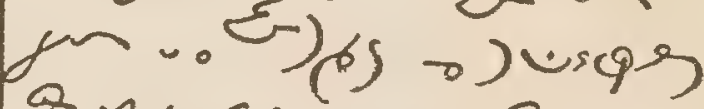
aresn $\partial^{2}{ }^{2}<$ romes sin: isects in $\{$. 




\section{Ibushwap SIDanual}

OR

moravers, Ibemns and

\section{Catechísm,}

IIn sbusbwap.

Ilkamloops, JB. C.

1896. 
IMPRIMATUR.

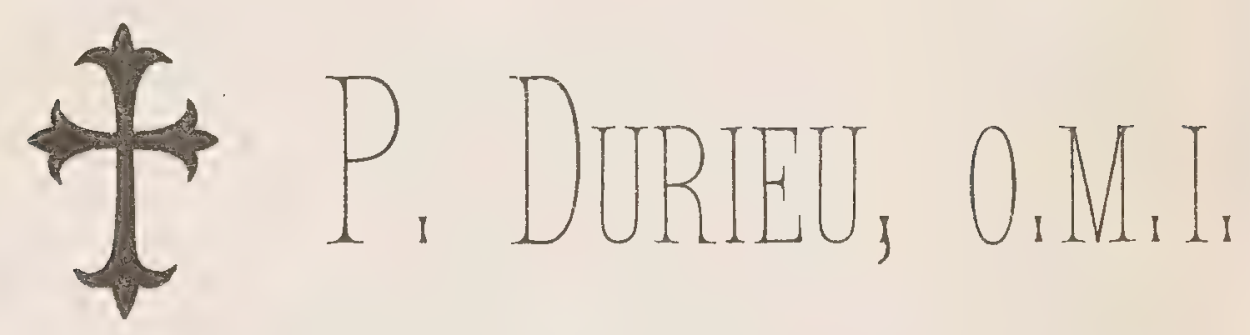

September 29, 1806. 


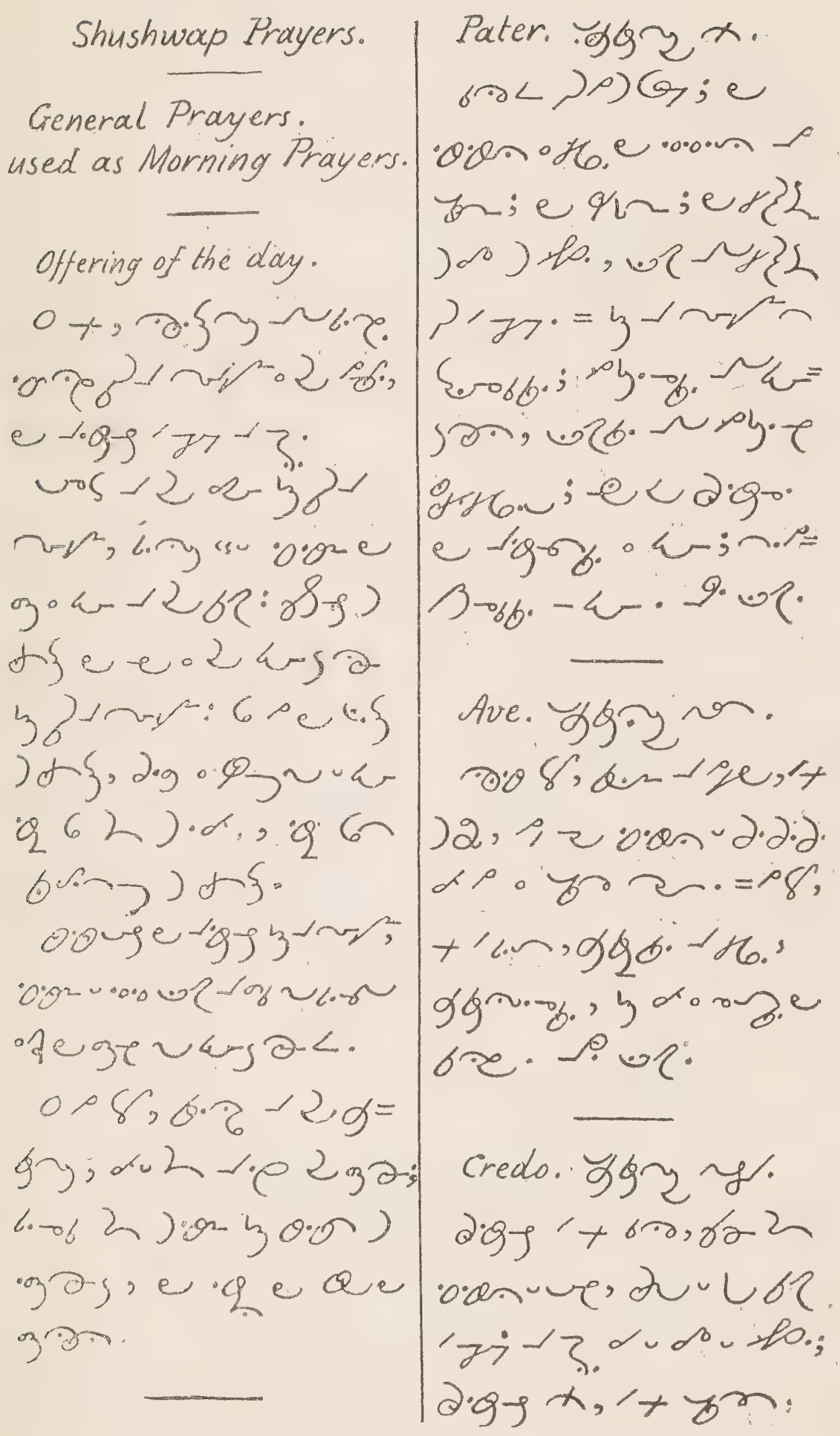




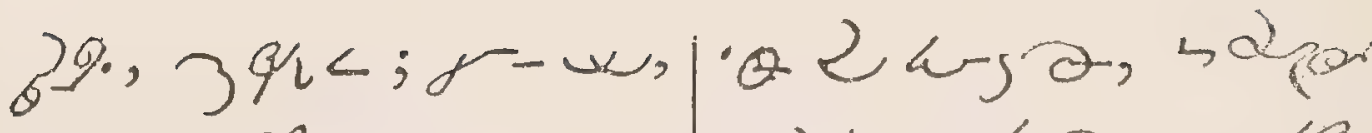
l.- 534, ang,

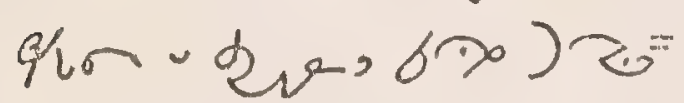
ब., 개 $\xi_{2}, 2-10$

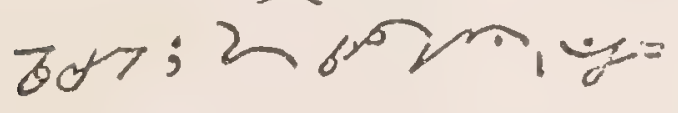
6.0.0; Hols >'27; $7620.70 \alpha$ '大

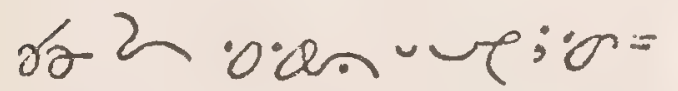
$\tau^{6} \mathrm{~A} \partial q, 6 \bar{q}\{$ $106 . \alpha \prime(\cdot 02 \cdot=2.95$ '十w, dupd bo' . 9593 , de खेव

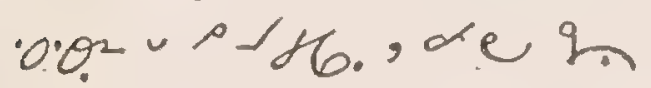
$-\cos 2, \alpha 6 \log _{0}=$ o. 0.0 0 म6. - su 6

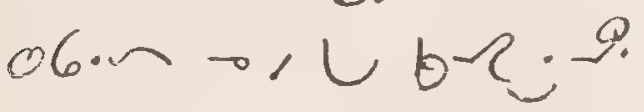
. 2 .

confiteor. ygun 2 $\left.\rho_{\text {.g. } \partial \cdot}=\operatorname{sig} \partial-g\right)+$

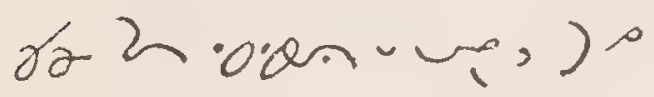
$\sin , \cos (2), 3$ moter, ) y $38,000 \cdot 046.100$

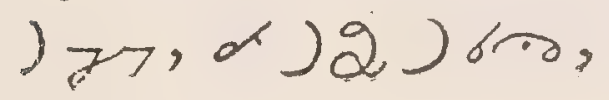
ok-: 2a L L., 200 $2_{6 .}, 2$ ho $22_{6.5}$ bogjogisur do, ) येde=j

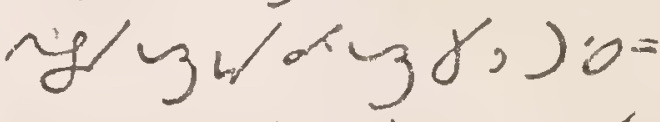
10.86. 10. J78, ax

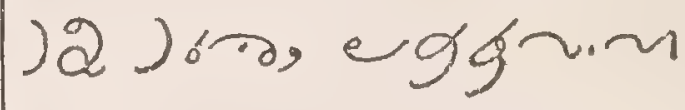
J $7>19 l$. Misereatur. pe g g = urd 7 ga 20 an ve, sedvand and <op, ded a ) $77-3.9 \cdot$. Indulgentiam. se gqurud e V.u,

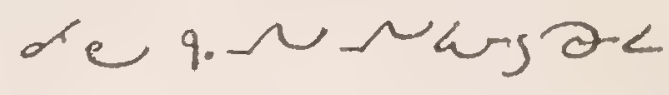

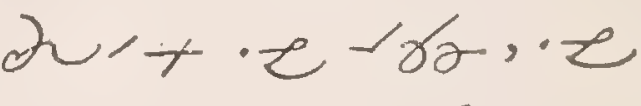

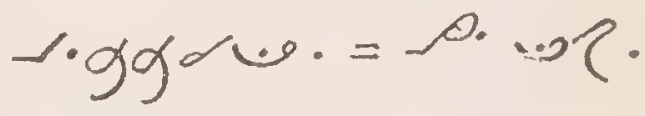

The ren Commandments

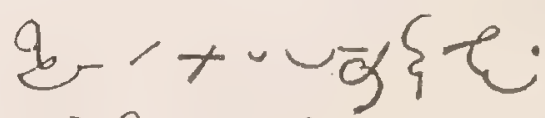
$1.3901+600000$ 2. ecken-.tom. 
MANUAL.

7

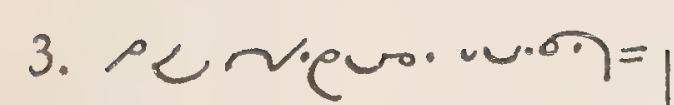

4.

. 6.0 .

5. e⿻一

6. $e x \partial r$.

rexdet.

8. ex. bz-

9. ec hayj.e

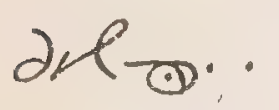

10. $\left.e<\alpha_{2}\right) \cdot i$

$\partial \times l$.

Precepts of the Church. $-(-16).(-3 ;+b a$,

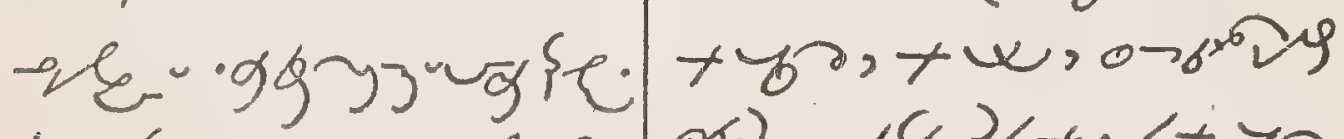

1.. . ${ }_{0}$

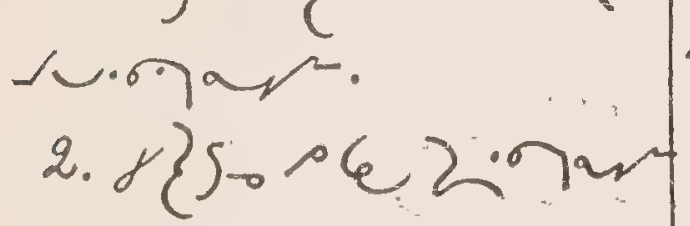

3.

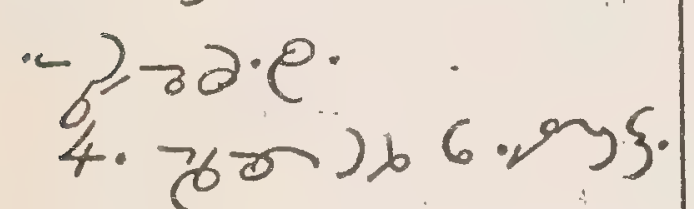

- $\Theta$.

5.) Jol, ) h, ) 8 ?
6.) $\sim-\alpha^{2}-\alpha$ un.

Seven Sacraments.

ove, ares,

rदि.

Actus fidei. e 2.97 - $0.0 \mathrm{a}: 3(\cdot \sim \cdot 96) 3$. - 46.$) 27-13.200$. $2,-16.30)$ s. 200 )>जब. e a. unas= $\partial<; 02 \cdot 00 . x) \Theta$. ot.'zalgy us o.on;

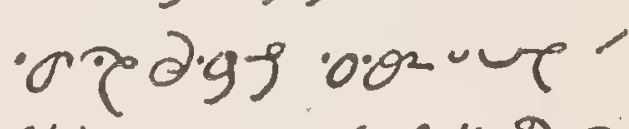

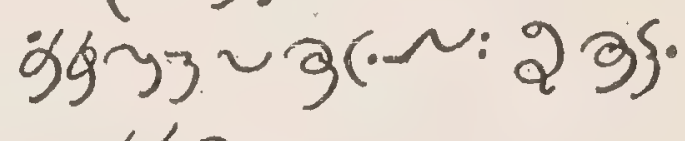
.9633, 76 ᄂ ס.

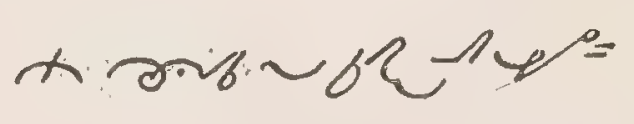

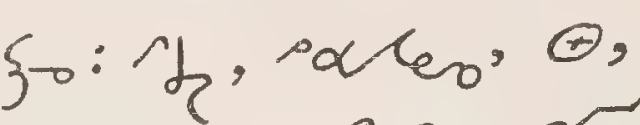
$=02.00 .1+22 \cdot 272$ ord, $68.2+1+60$ e 32. 


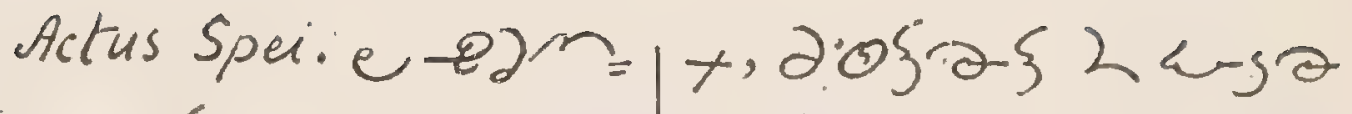

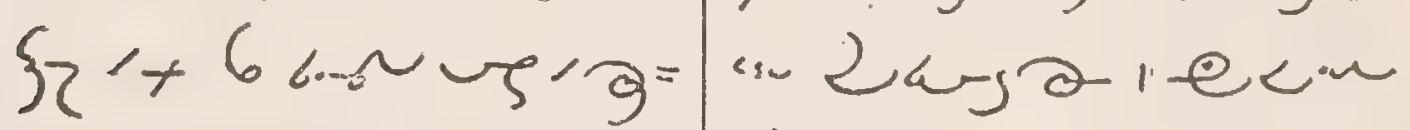
$\sim$ cur $=0+, \circ$ 几.gogol, so yox pondoruqe a.ro. Naja, -is 100.) 小5, चुव

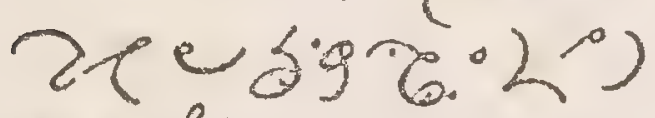
o) so. el. $6.27 \%$ ? 100

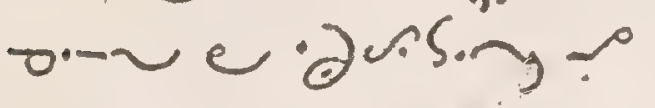

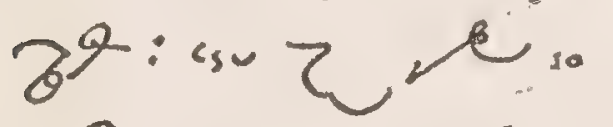
39; v5: $3^{9}, ?$ su 700 so.

Caritatis.e oas

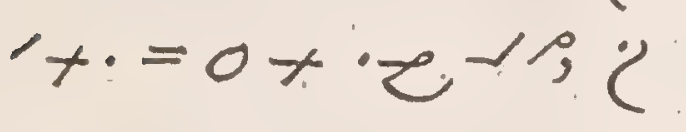
$\sim 0^{\circ} a \cdot 0^{\circ} \cdot \mathrm{y}=$

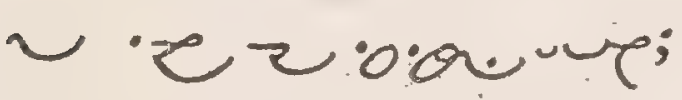
are ocarg.0.0. 86. पर $1+62-) 89$.

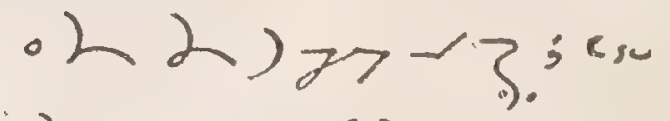

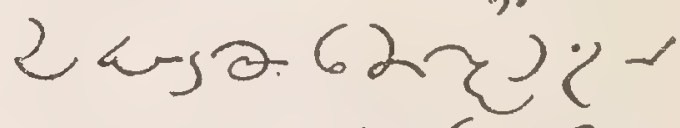
$\bar{\sigma}: 0 \cdot 0 \cdot \partial \cdot \partial \hat{\partial}\}$

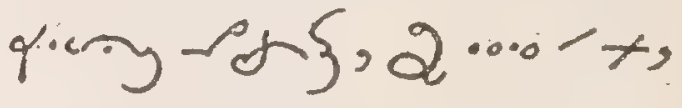
2.र-10)6m:60

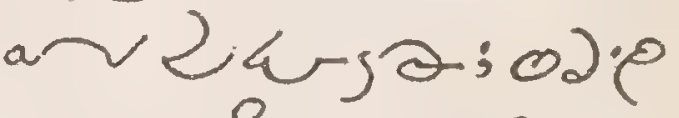

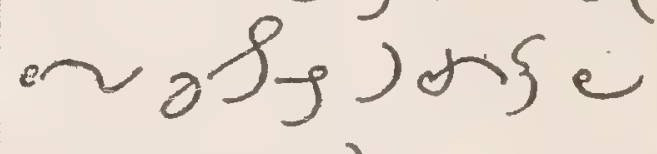
च बr.रद्यु; 6 कबत 62 l. बa ee y'ge d'g?. in. s. 996.0.

The Angelus. rn: L.un- + ice -8 . $=2,0 . \xi \sim-6 r$

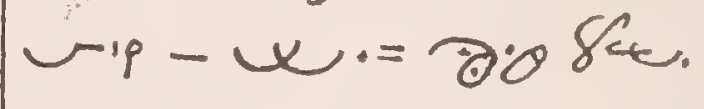
II. hovuag)t.

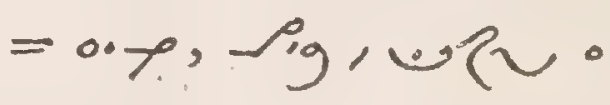
$b^{9}=8 \cdot 08 \cdot 4$. III. $1+$ brg- $-16:=$ (a) $\alpha_{\alpha} \cdot=\theta \cdot \theta$ contritionis. e $\partial \cdot 0\}=+9 g \pi \cdot 0 \cdot d \alpha, \rho$

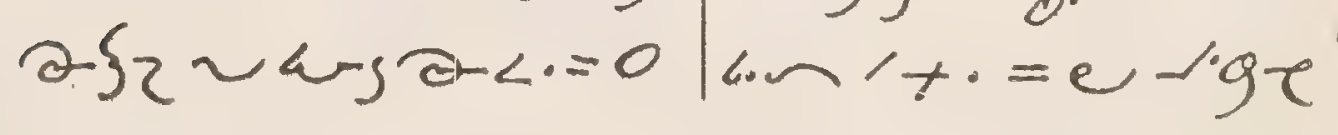


MANUAL.

9

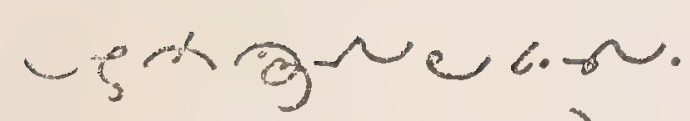

oremus. $0+8$ \% 8 .

ngon: vaven

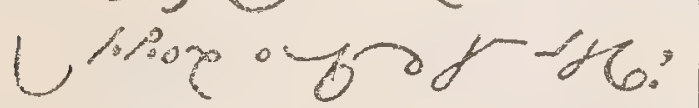

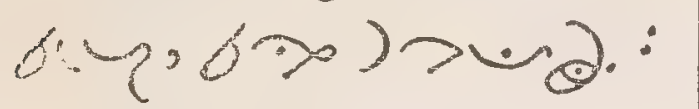

su br. o.

cदgr, 2त)

Giloria Patri.

Giloria Patri. Lo Jom,

d) fo, a) $\mathrm{x} \cdot=$

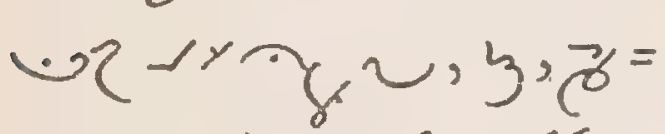

क,

$\rho \cdot$.

sub tuum. o8, 2

cunctros 1

.20.6. $) 2$, ex>

\{. $-1992, \alpha \cdot 2 r$

919-6r75; $102<$

- y.guc geou.

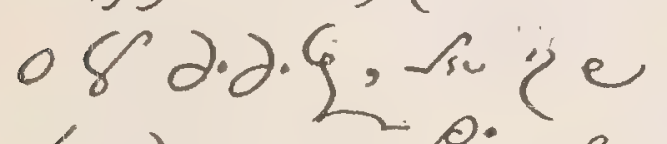

मे) यो. 90 .
Before meals.

$$
\begin{aligned}
& 6 \text { ग, } 2 \text { คक } 6 \text { : } \\
& 6 \text { ค न (6.-) गे.; } \\
& 6 \text { - ef-6 Lu, } \\
& \text { su } 6 \text { бa-jo, su } 6
\end{aligned}
$$

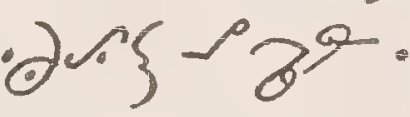

After meals.

$0+z^{\prime}-r^{\prime}-1$

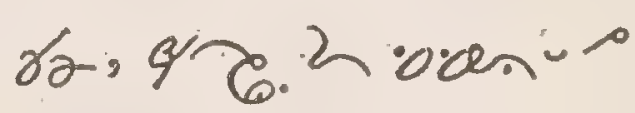
น.

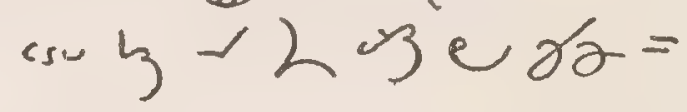

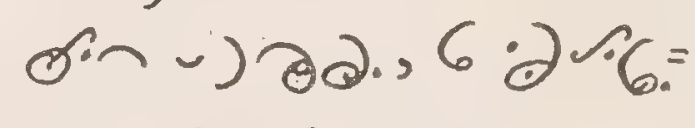

ry $z^{q}$.

$0+96)_{0}<0^{\circ}$

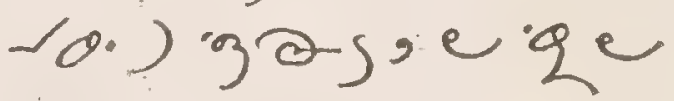
2.) $27-3$.

Form of Baptism.

N... uog? 2 2

fin rsid, 
Morning Prayers.

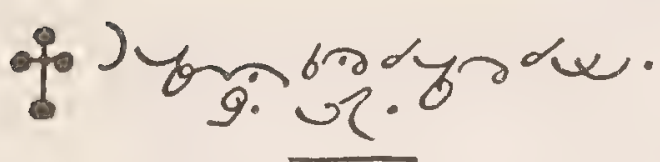

Venisancte. $0+\psi$,

3ว) $\alpha^{2}$. , e burg. o小 $\{-2 \cdot \% \cdot \% \cdot d=$ \{. च Adoration. po.s.es) te a.ove 2 . $=0.2$

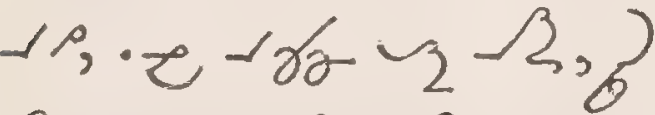
$9 \cdot 1+) \gamma^{\circ}(9, \partial \cdot 9 ?$

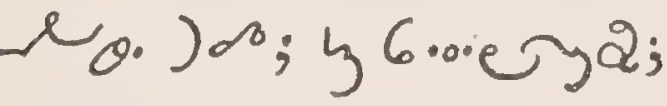

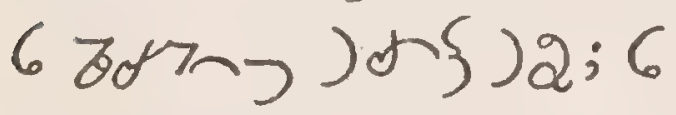

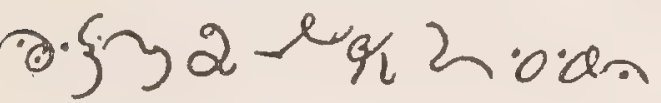
u.

Thanksgiving. Pe okt t tore b. $32<\alpha .=$ 6 hol, ogy n:0.0n

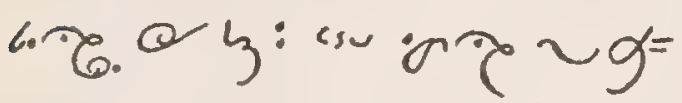
\{.76.0 206.3 3 $\Omega^{2} ;-\{1$ l. $\mathrm{S} \sim 0.0 \mathrm{~s}\}$

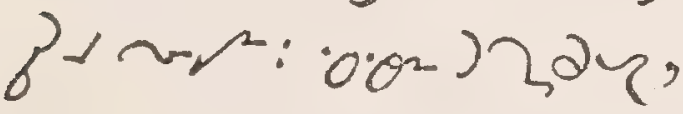
:00 ) $8^{2}, 00226 ?$ $3 . \sigma^{2} n r^{2}, 6$ congu

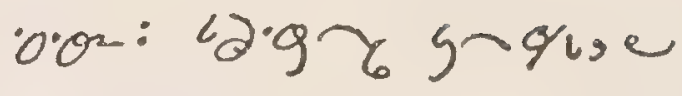
. 0 r 22 2.on 2 a $3 b^{2} \sim 2$.

Resolution. "e 63T od $\xi<e$ bof ore $2<$ ) p Nor. $\left.=0+\sigma^{2} \times, \sigma^{-}-\right)$ 小 $\{2 \omega 2-2\}$ क rar,eroर $r$, er. 2 acsos. e-e.2 $68.2 \cdots$, .2 $202-19,02 \cdot g=$

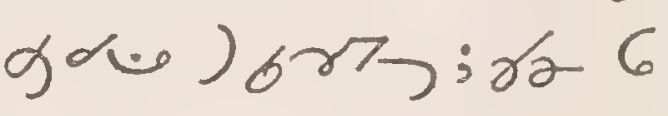
2 แ. 2 , e e e f $=$

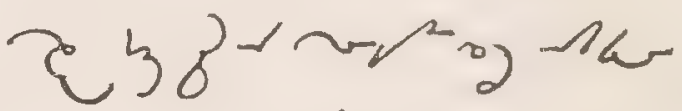

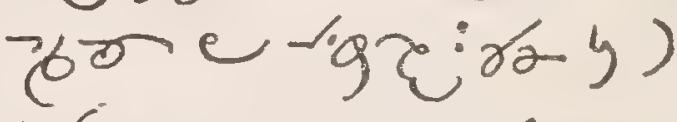

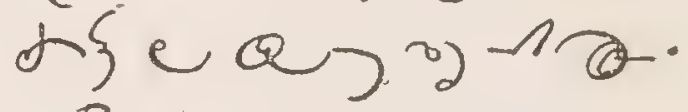
Petition. se $0.0 \%$ e

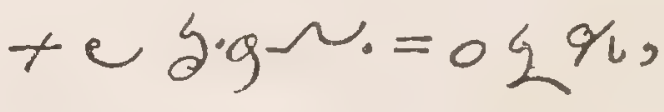
Q. $\cdot 9 ! \cdot 2 \sim-29 q:$ ocu.rar joe eg.9\%: $e<\%$. -29gryogng;

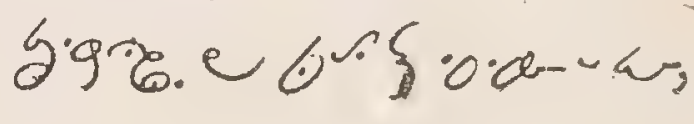


MANUAL.

II

.h. 2):0.0n

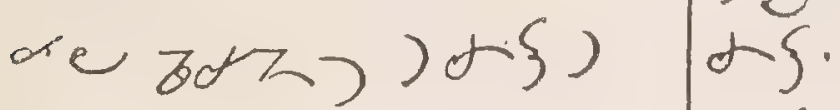

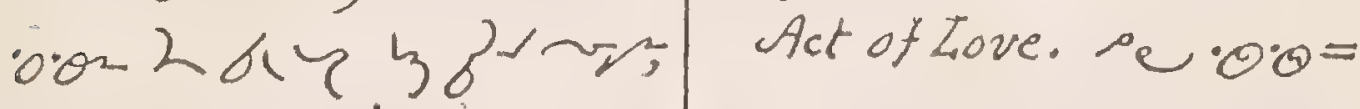

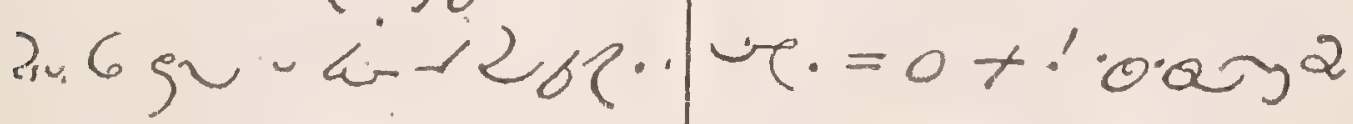
乙: $\operatorname{con} u$ J

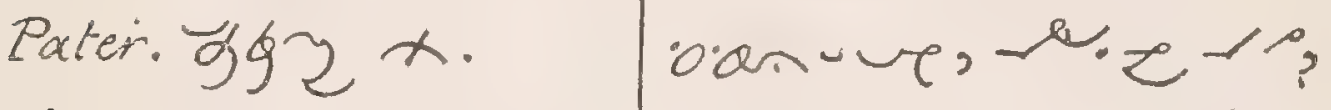
Ave. पुgz wo. diong.0.0 I bris,

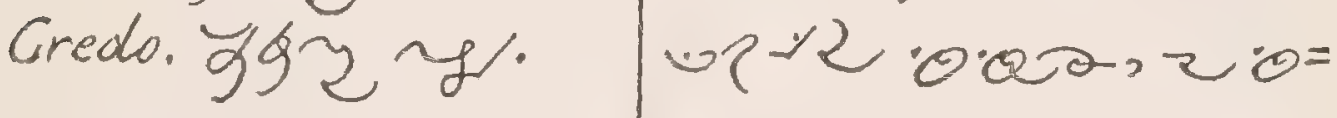
Seven Sacraments. $x$ eq 2.

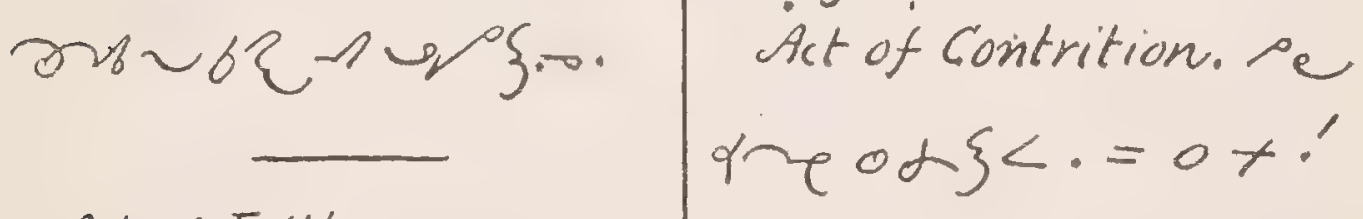

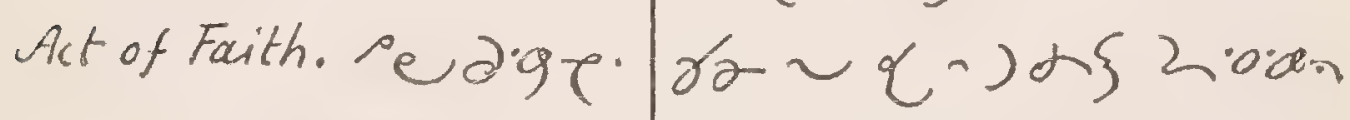

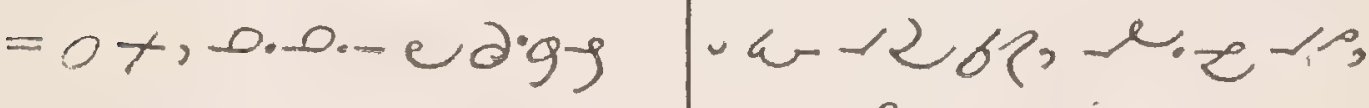

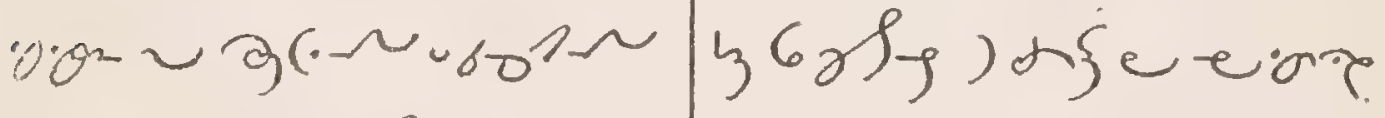

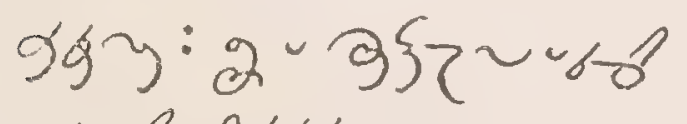

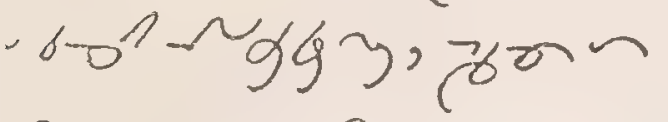

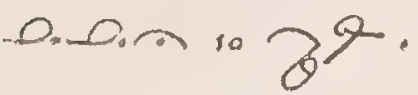

Art of Hope. Heroin

end $\}<.=0+, g \sim$ 0261 th. pogge\%.

Invocation to the B. Virgin. $\operatorname{seg}(\alpha<\sin \alpha$ 大 (

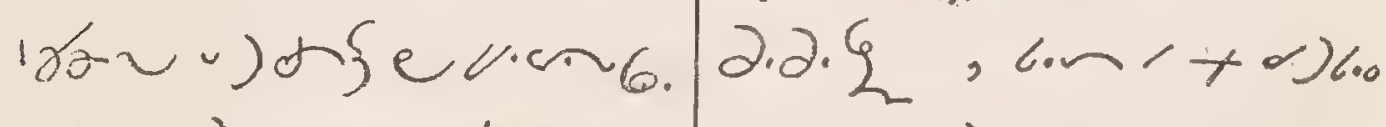

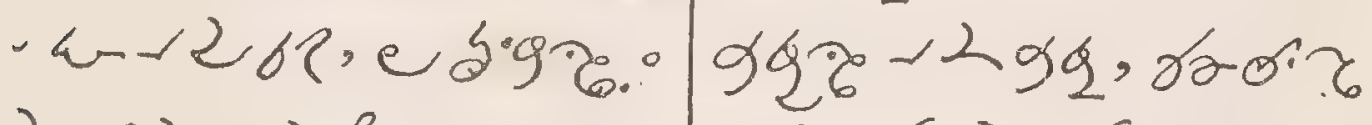

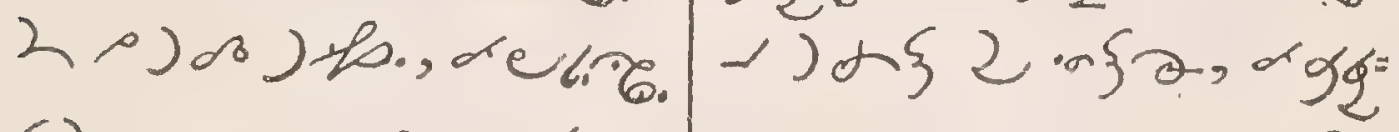

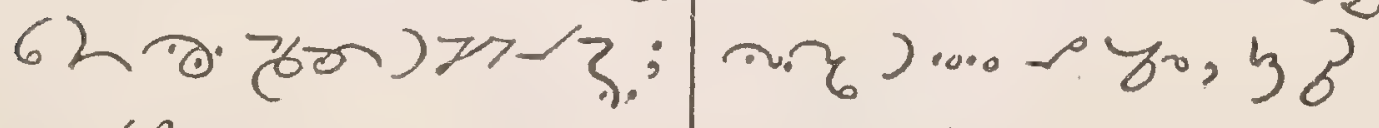

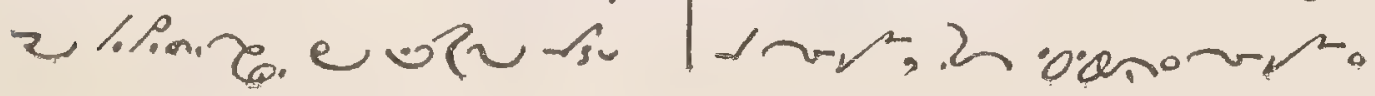




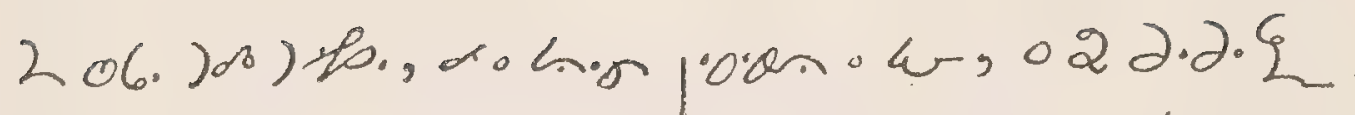
12600.

To the Guardian Angel.

$02 \rho$ in $3\left(\tau^{-1}\right.$

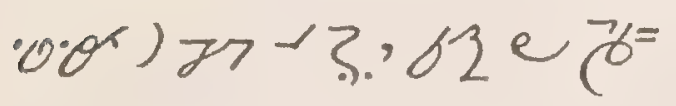
one c.S.T.

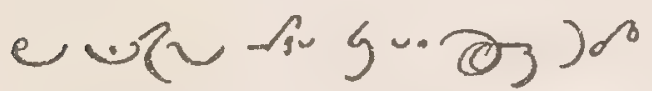
) so, e e y.ge $26=$ $y+x^{2}$.

To the Patron Saints.

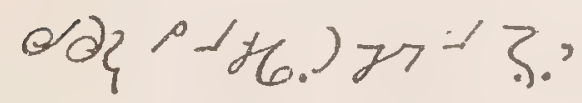

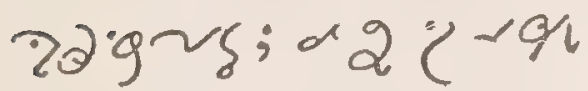

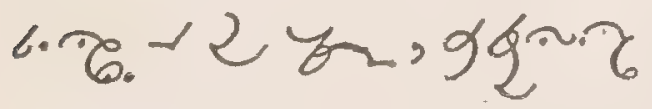
)t,e 0.00g't जर 2.) $100,622.2$ pon) 2 (6) p. .र.

Angelus. Non.

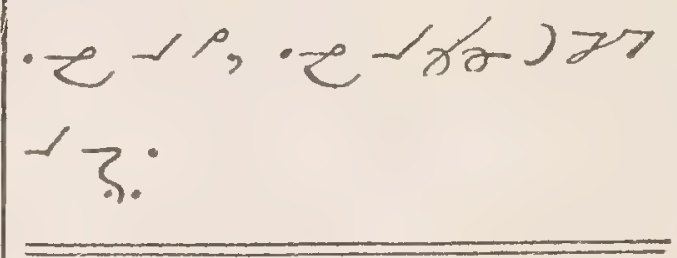
Night Prayer. of 2zmos,dyond Veni Sancte. $O+W$ Act of Faith. Pe age... of hiope. erasor... of Tove. Pe oar... of Contrition. e $\alpha-c$.

Adoration. po.s.a) te rove $\lambda \cdot=0.7$

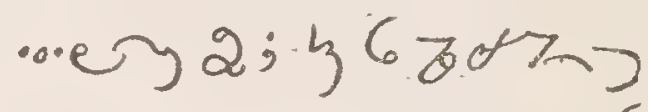
) 小2, 乙织加 $1 \sigma 0 \cdot) \cdot 2 \cdot 2 \cdot 2$;

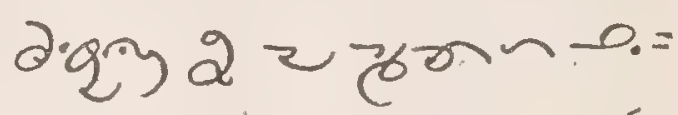
-

Sib tuum. o. g.- $\sim$ ) 2 - 2 - liggole, $-\alpha\} 6 \cdot) 2$ e (. $\{\cdot 6 \cdot$, $00322.000 \cdot)$ $02 \rho 1$ lon 1 t: e

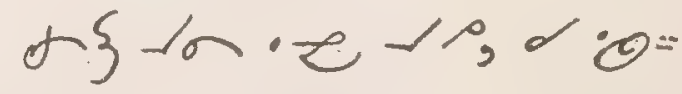

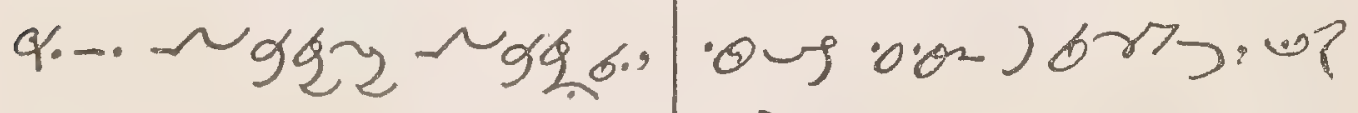

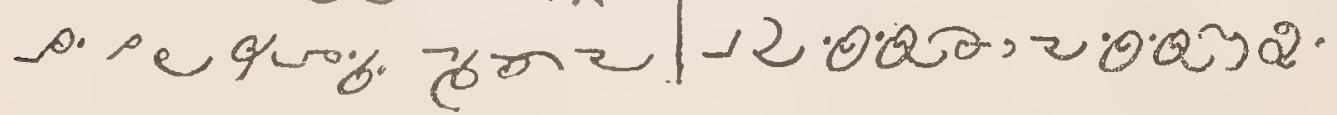


Thanksgiving. se phi for $\sim h-\xi \cdot 2$, de e

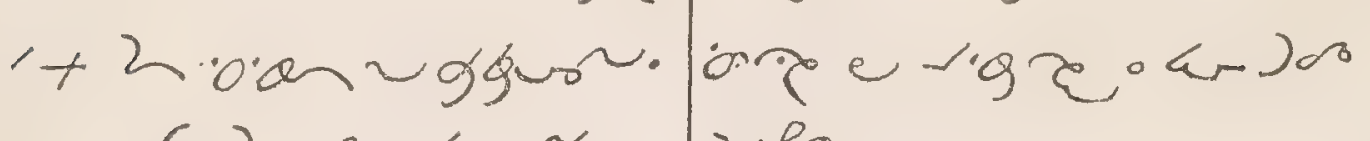
$=\cos 2 \alpha-g-96$. e.0.0.0 e ogn $20^{\circ}=$ an $\sim 9 q z_{6}$ ? "eq y

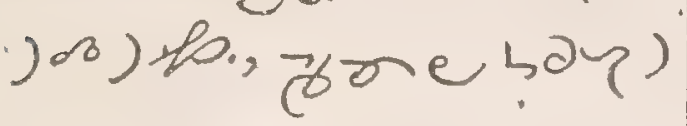
250;2637.0.6.5;

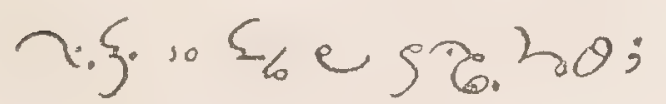

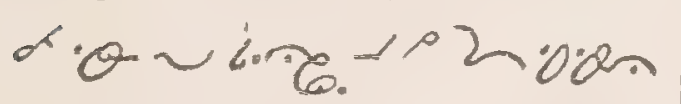

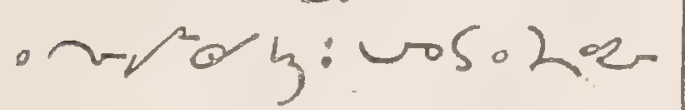

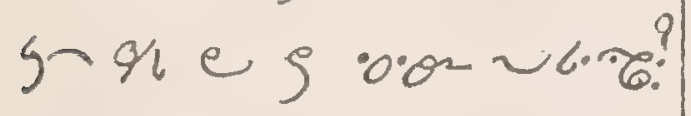

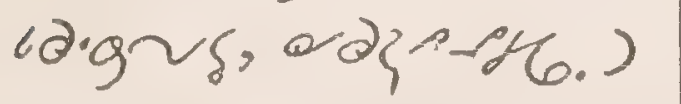

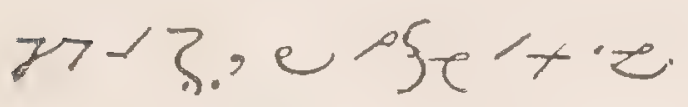

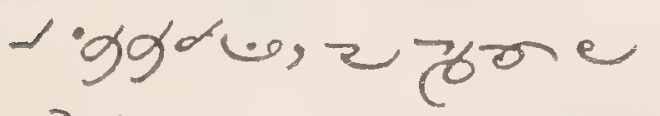

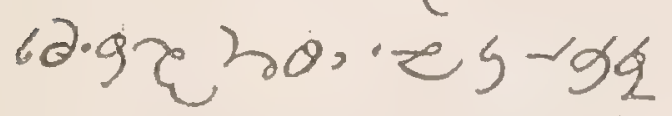
z"oven? ) obles. Prayerfortight. se -.0\%erteca.g enedine vara< $36 \div \sim 2:=0+\omega$,

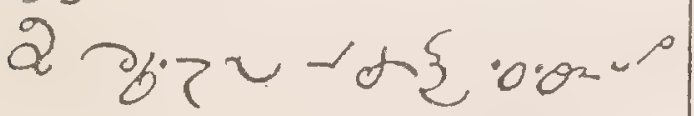
16.: \%. उo- je

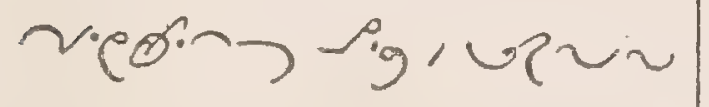

Examen. se ge $\simeq$ as $2<36 \sim \sim 2$.

Contrite Prayer. egor

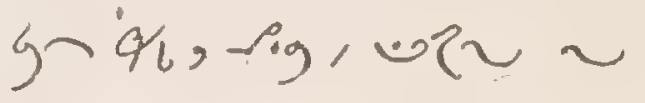

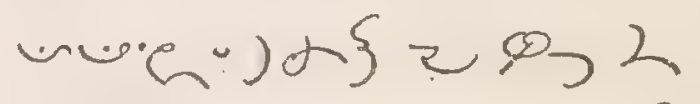
as \%力 2 t.e $-10: 3$ su

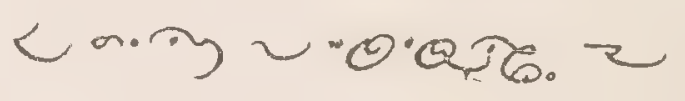
ح.j. so $\varepsilon_{10}$ e 50.20 ? 0).

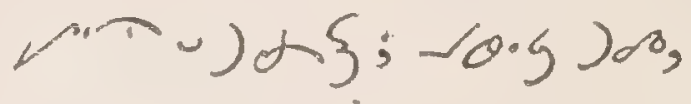
$3075) 2.96255$ qu的.

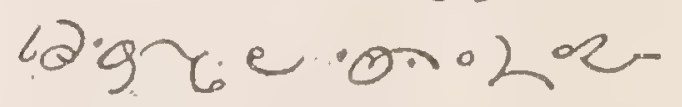
tho b bog, e 9 1 D.D. - o a 1262 . Firm Purpose. se da = oin-oj<etar egr $a \cdot=$ ogh $q$,

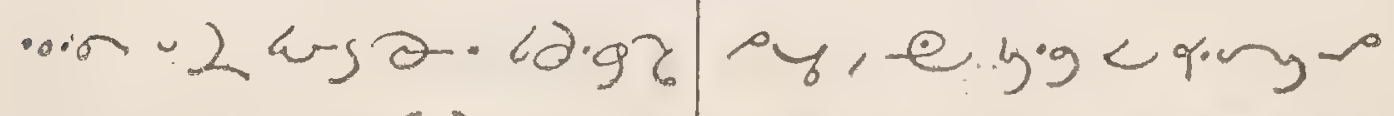

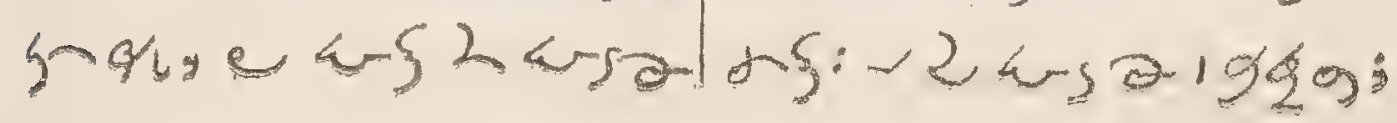




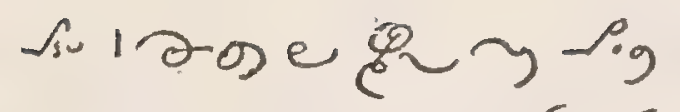

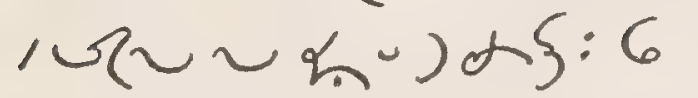
20ई $3-4-1260 ;$ 206222909.2

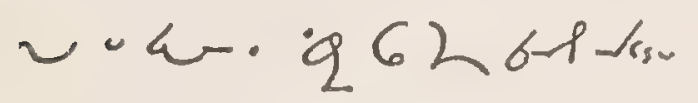

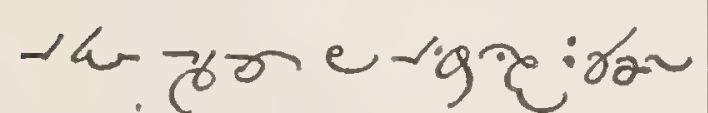
ᄂ $296.519\left(6 \varepsilon_{y}\right)=$ 2.5० $20.0 .200 \mathrm{n}$ vo, (0.)क) Ho, d ereshrue

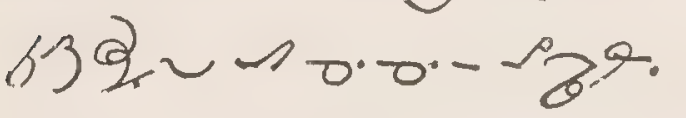

Confiteor. 9592 I.g 2. Ten Commandm. Q The Precepts. ork-'gg 33. Seven Capital Sins. Sorf

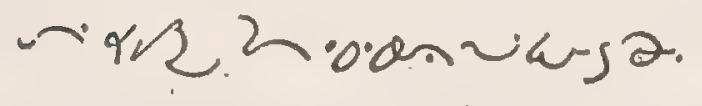
UQQJa, U.0. voo,$\dot{\sigma}$, , eror, Y $(8)$, n. not.

Nightoffering. ègq () $+0,0(4-2.2 .\{, 0) 0$

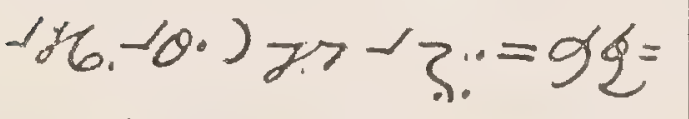

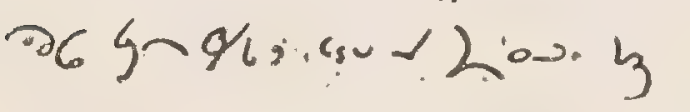

1) vn, erarnu) कa.se 63Q up $3^{9}=2 \rho-2.2 \cdot \xi 8$

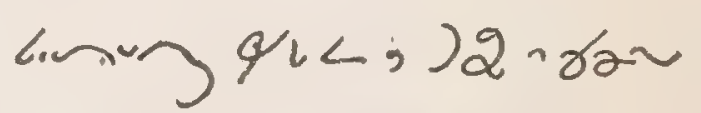

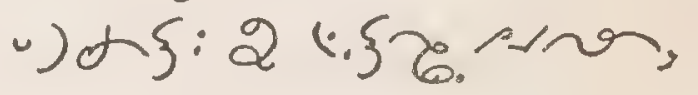

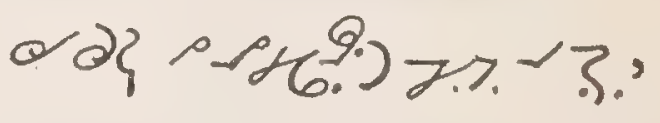

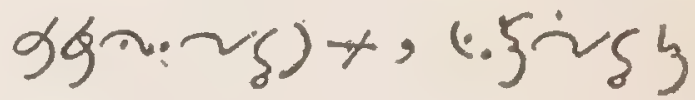
bun, 200 .

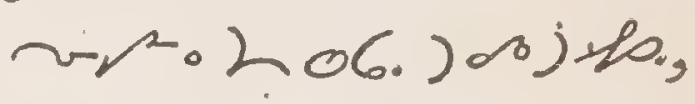
d.on.2 ba. 9. एर?

For the living and the dead. se gof ve ofrlyc

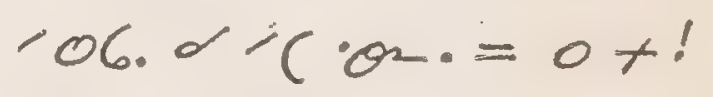
gón " L-..pe dion

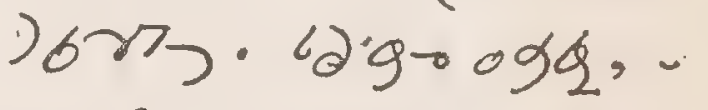

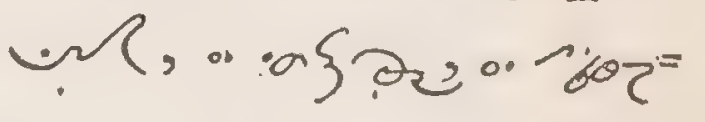

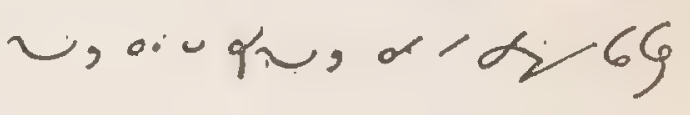
serviso. angan?

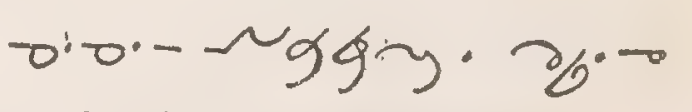

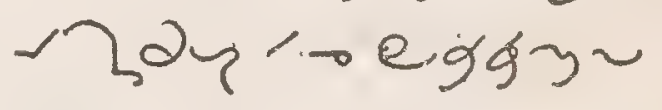
176.

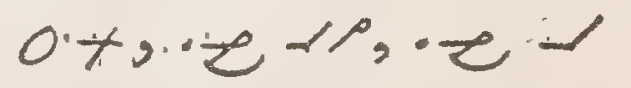

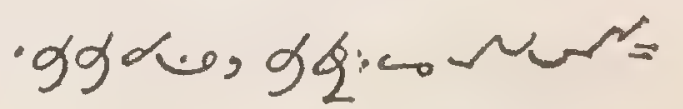
y.u o.j.jas. pe 


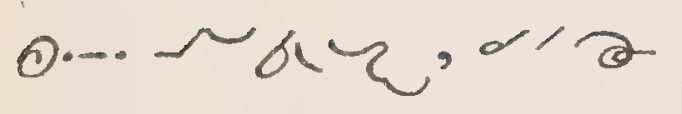
(4d) brys, se, gg= w. A. de g. 1 Q) $27-$ ?: 9. एर.

Angelus. $\sim$.

Sub tuum. O 8 .

\section{Morning Offering.}

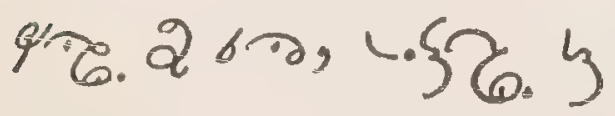

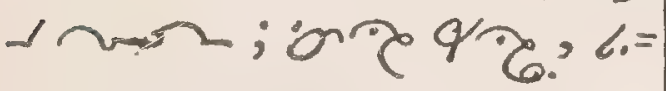
2.3.

uy - ) d

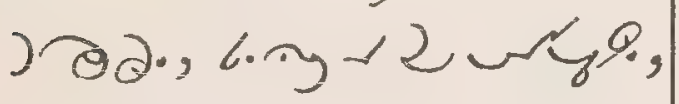

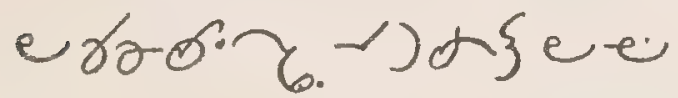

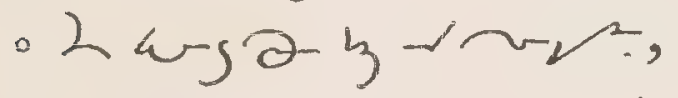
- oxog 6 25) 2, 8,2 .

right offering.

0 , 2638.200. biog 2 wryo, lons)

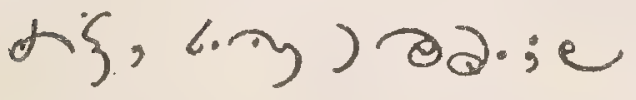

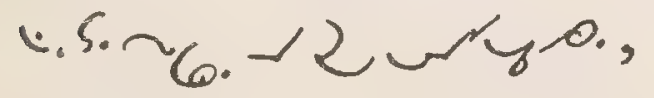
ec.s.r. 1 ) d $\xi$,e

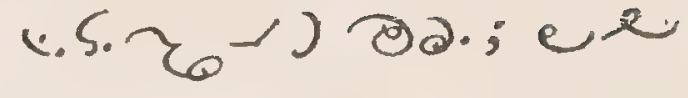
- Lv, de e。य 6x) Jar; a 6.5. $12 \cdot 03 \sim .9 .0 ?$

The Memorare. Ifo,

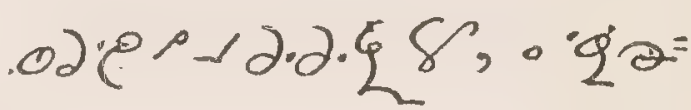

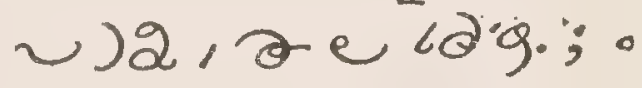

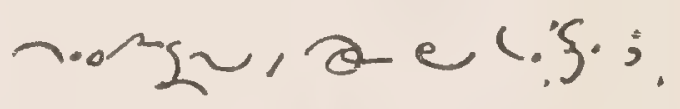

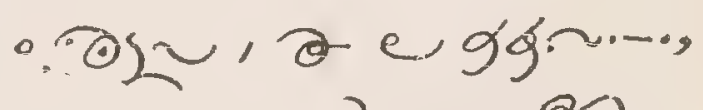
- y.g (ákneg); री 3 d.d.G.e 2 :0.

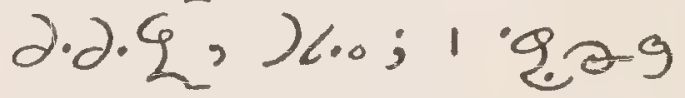
) $\left.2,1) \rho g_{g}\right) \propto \ll \alpha$, $1 \cdot \sqrt{9} 27.6 .0 ; 0$

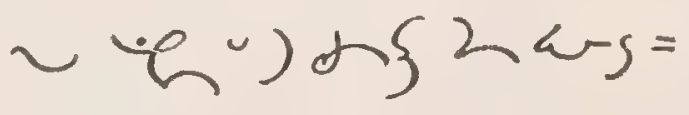
2; 0 l. $1+$. e क). 2 gq ग, 9. pe रु. 2小\}, se gq= 26. 
Preparcation for Confession.

Leader. ح $0^{0 .}(\xi) \cdot g=$ gryze e e lo.gor

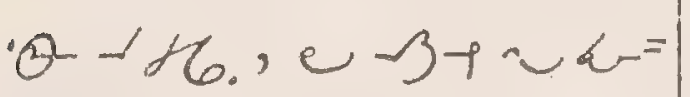
sक्र, de d- 2 ) $0\} 2\}$.

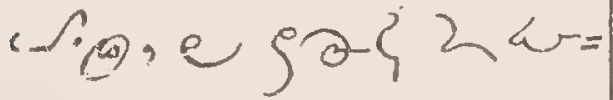
sol, en.or. ryo-?.

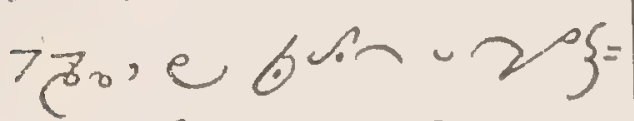

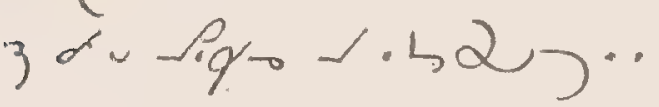

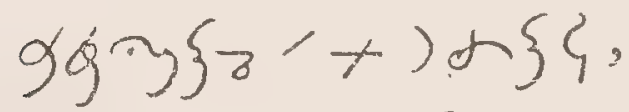

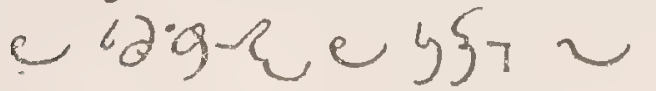
usal..... 9.935? $1+e \frown 0=$ a. $\{$ d $\}$.

Prayer for light. Alltogether "6 y bro, Nieug lig

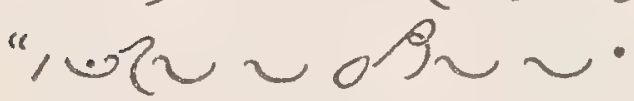

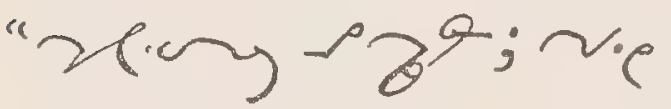
"us, o-e e py.z.' "2 arga, ocule "Lfगeq. Jos "e $390^{\circ}$. 2 ars?,

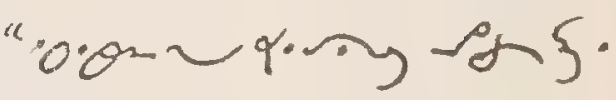
") $00.04 g$ e $l .2 \%$

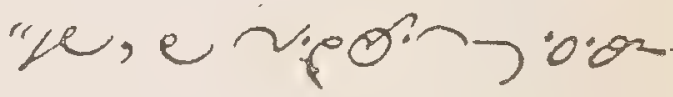
") Y., $00 \mathrm{n}$ र $60 \mathrm{z}$, "0.0n ou- 2 бर. $2=$

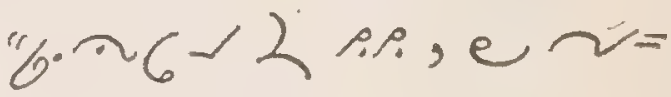

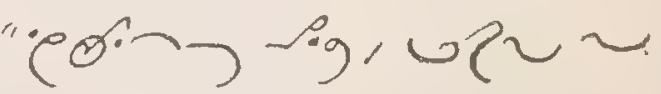
"Leeseryo "Ba. su 6 ).or o

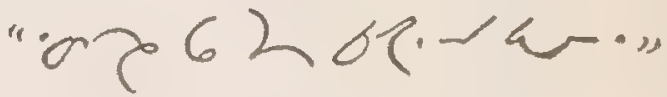

Leader. PV. ...... gro \&. 16.9 ,

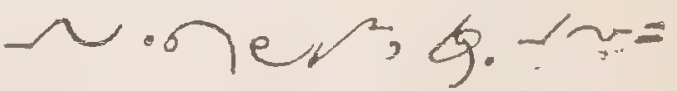

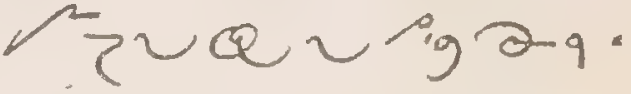
... 4.25\% vos $6=$ कर $\mathscr{\ell}, 0-10$ co,oron.

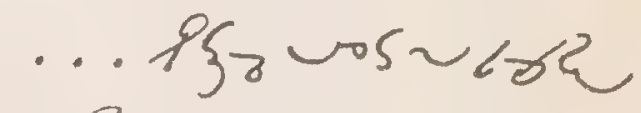
- Q e jal, ja $\sim, \circ e$.

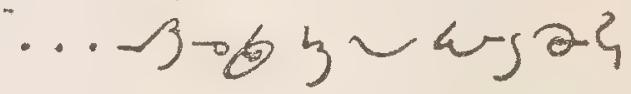
2 $\left.: P(\zeta,) \propto\{\},) \cdot 6 \theta^{g}\right\}$

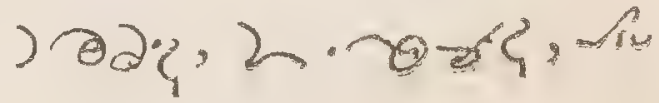




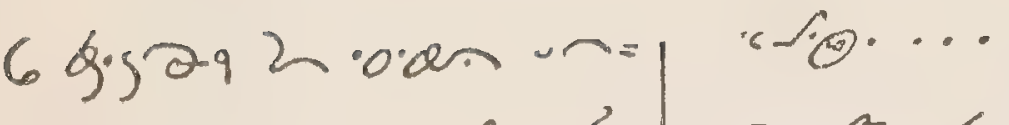

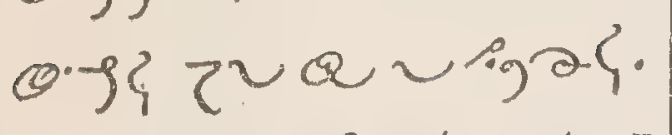

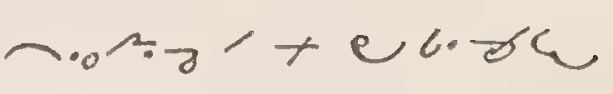

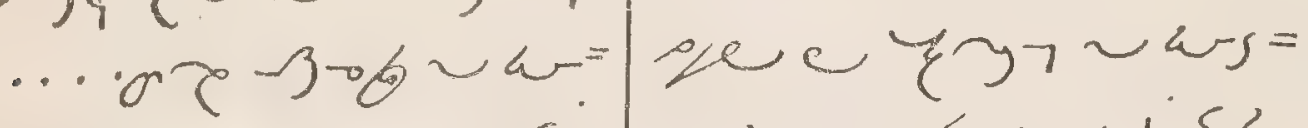

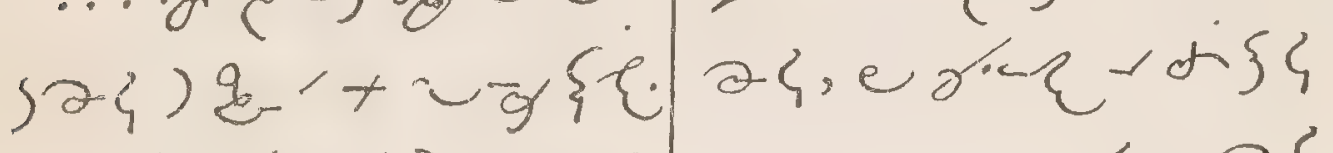
All together: "39+6 ee orpe argal. "o.0uo., q ...."

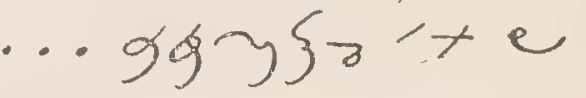

Lieader. ar $3-30$ $\log \partial\}$ ) $3 \cdot \bar{g}\{c$.

All together:" e. $\}$ ro -? "Ansts a ..."

Leader. 02 -3-自己

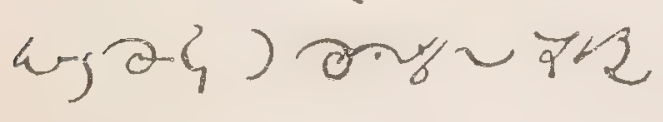
vargo.

All together: "บQQ, "b, CoOr, a.:." Leader. irro-3-o $\arg \partial 2$ 20 … 3 3 3 \&. $\sim 978$ $\sim \cos 2 . .$.

C.de eg 几ro. Prayer for Contrition. Ali together: " $O+, 6 .=$ "m6e К-)小ई-2k= इ2;0t, $4.0_{6} e$ फ ")小 2 ars 2 ; 0 t

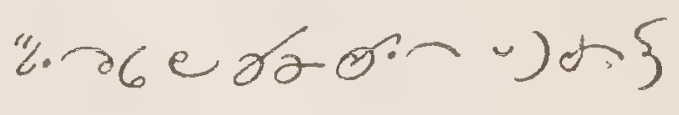

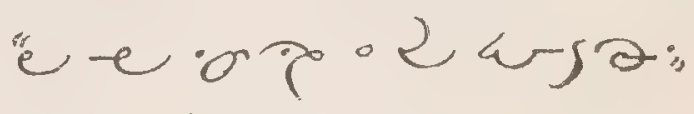
Leader.... ot, 4.06 e).d दuargadok $-16 . .$.

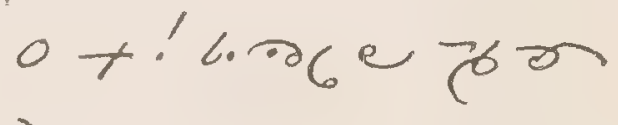
- 2 ḱ a e ee - 9 r.a... $0+4.26,0 \log 2 \pi$

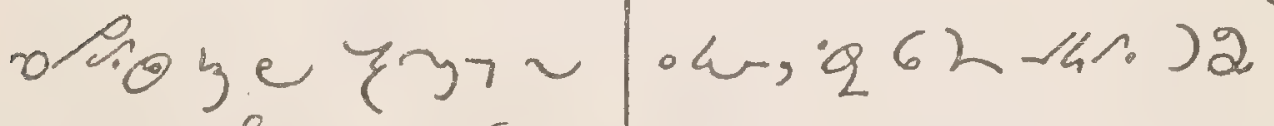

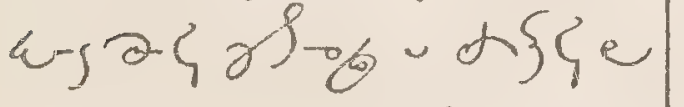
e $9986 . .$. e areensy $\operatorname{sig} \partial \ldots$ $0+4.26 e$ रक urg. un... 
$07,4.26$ e 76

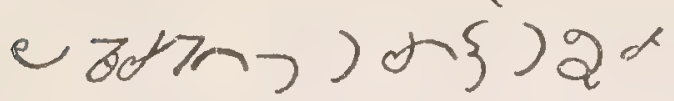
20.0n o म6....

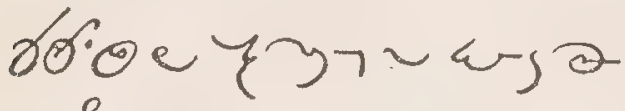

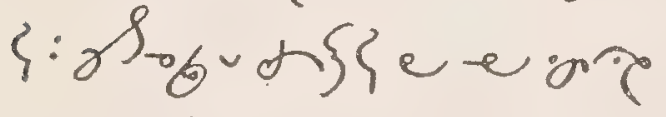
curiganagak.

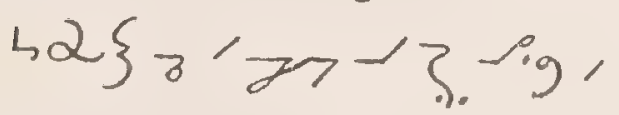
uथ $u$.

First Consideration.

All together. "Od:e ve "y>-z;-uece)

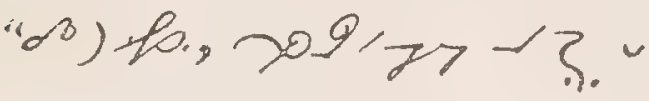

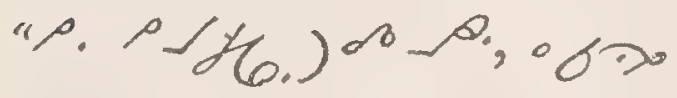

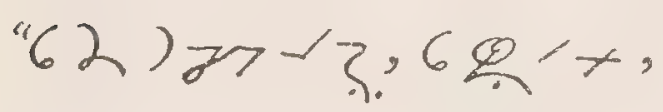

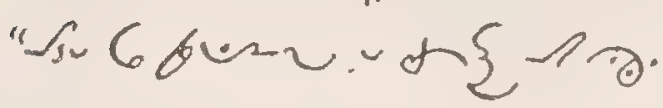
"八; oc vipuneien, "ocrieune h, oc "rpunete: 6

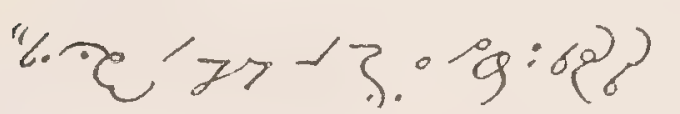
"10.00a-gag;02.0)

"प.2100. $689-2 a-5$

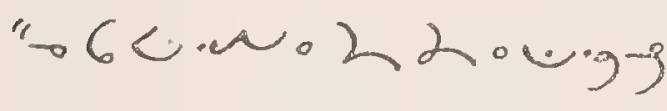
"(z) 了, ; -6en。 2

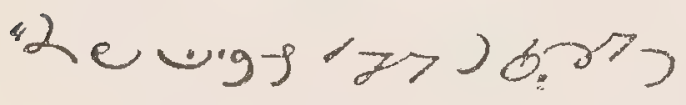
"ocenolderengs
"

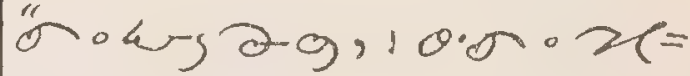
"c) $\sim+18$ " द ") "3 6 vN "26.0"

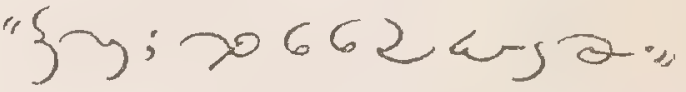
Leader. $g_{3}$ ) $\{\xi<w$

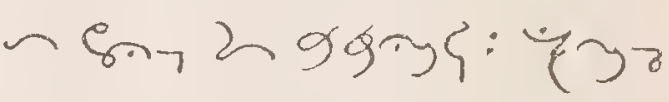
Uूलक tererjensal. $\cdots 0+6 Q>32$ 4ya, 6ra a se e "रु e

6) $\cdot 5 \cdot a-206-46$. 6rone (.5) d se erded ) a n...

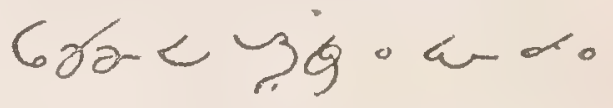
ento...

ong a, 262

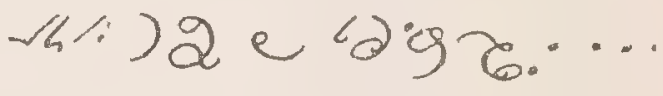

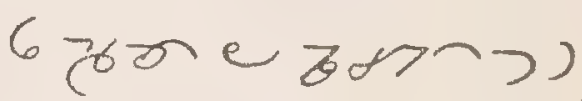

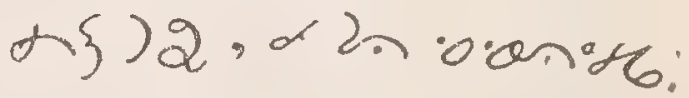
...xóne

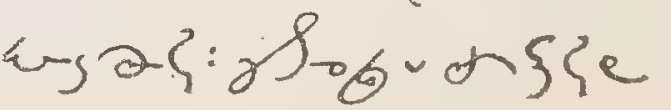


MANUAL.

T)

ere.ope urogr,

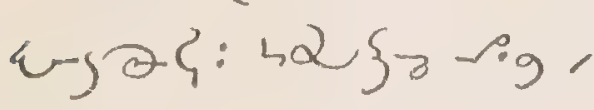

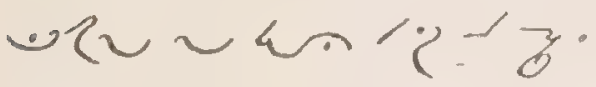

Second Consideration.

All together. "OD. $\mathrm{C}$

"

"(ootr; ) n f r )

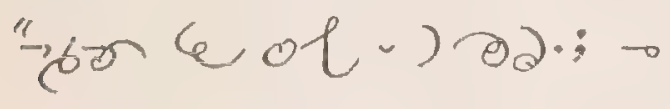

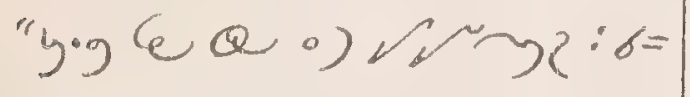

"(र) 2 -6.) z $2(0 y)$

"बक? "s. 2 as

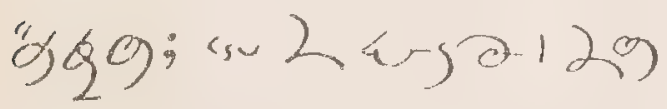

") $(-3 ;-6)(-3,>0$

"z62-3;-6) U,

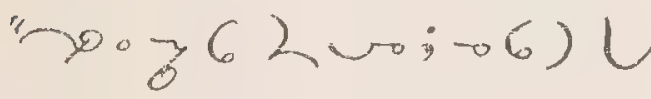

"y $3, \infty \circ 0^{2}(6) d>$ : su

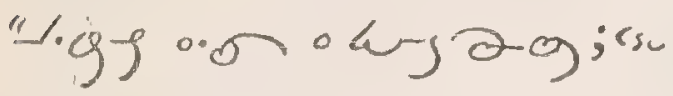

"ligg :0 o $2(\mathrm{cu})>x-$

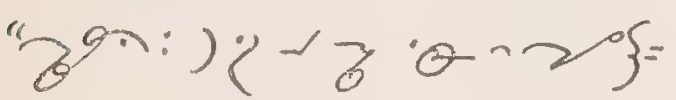

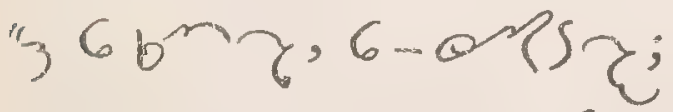

"‥ 9$)(1-b, 6 \sim b ?$

4) $16,626 . \tau^{2} 286$;

"lsu 6 (com $\sim: 60^{\circ}=$

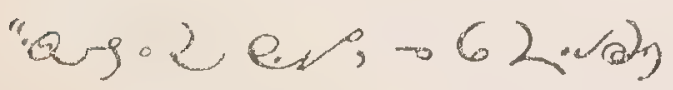

"ortegr.

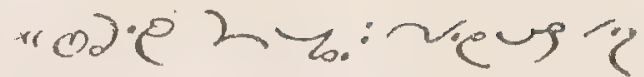

"z 4 -

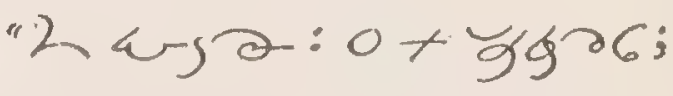

"द

"म 8 ", 2) $60,2-x$;

"३2ईटk-ga;

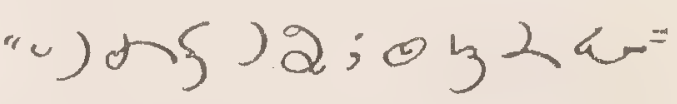

"अव, 2 san 2 ars;

"2万)小5) 2; 2026

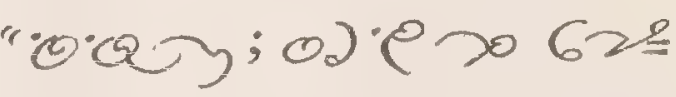

" $\{3$. "

Leader. 9 y J 5 res

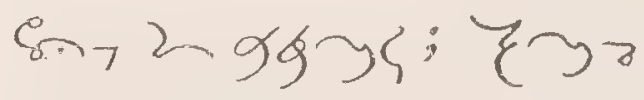

$\sim a r, a \xi, 0.0 .-63=$

orterpregt

u. ...

$0+6 a>32 \mathrm{~L}=$ sa, 6ratage - ree ligr.a...

6).d joud a a

6....

6. उक्ष e(.5) d fe e.

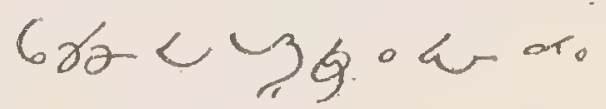




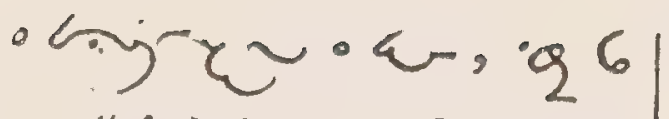
2 - 2 e 4.96.

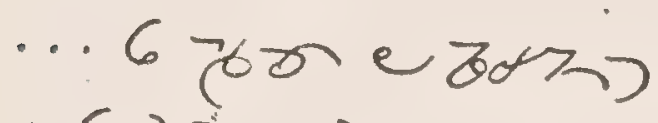

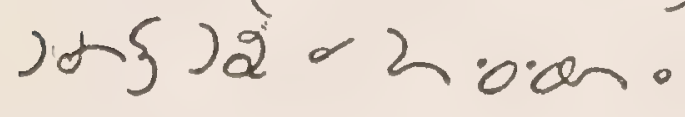
t6....

bo.oezry 2

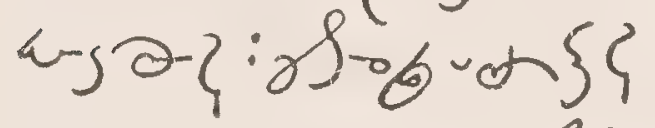
et.oreevist

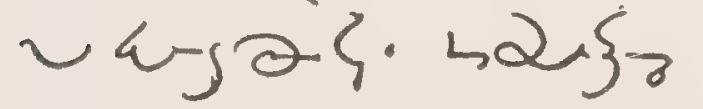

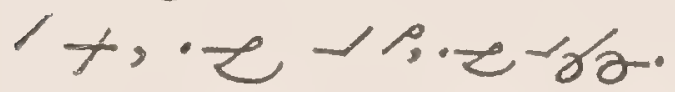
Third. Consideration.

All together: "o 0 . "s, e ". 206., 2 ".

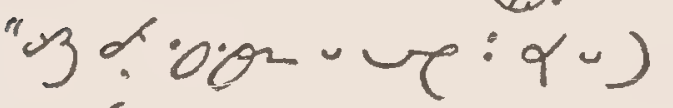

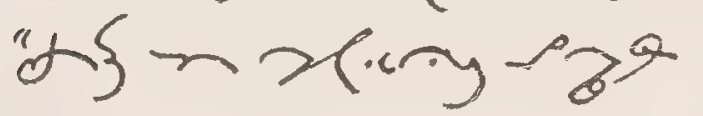

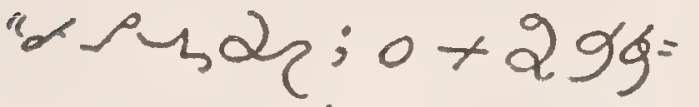
"6. 2 Nyo. or $2_{L}=$ "2u;0+2 l. 2. "x ho Lha

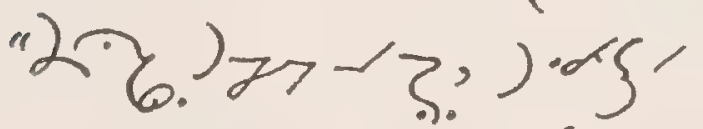

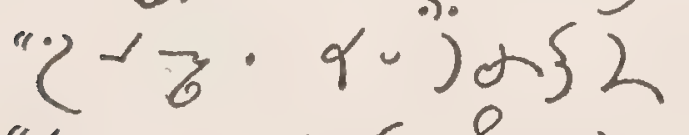
"a, a; 3689 )

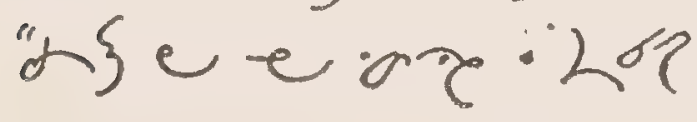
" 4 .".
Leader. Neup $>$ - $-\mu, \tau-\gamma \alpha$ :

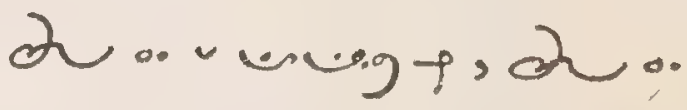
- q.4 \}. Sine ye $\partial \cdot 0 \xi=$

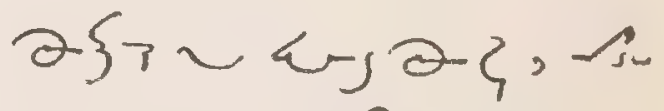
ie zerstasse e 42 areegru e.

... $0+6 Q 3^{2}$ as $\partial, 6 \gamma \partial \partial y e$ e are grou. $6) \cdot d \xi \cdot 4$ dok. $-\pi 6 . .$.

6 бक्र (. . ) 小 er are e $\alpha a$. ..C $6 \gamma<3 \%$. andok- $16 .$. - hyz: Lr, 96 $2-14.12$ e 596. ... 6 бర e उofs $\rightarrow$ ) 1 ) $2, d 2$ .0.0m : 6 . 
6roeryt

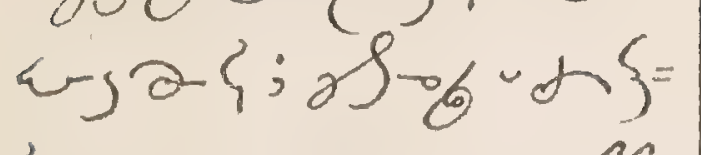
दee rocuss $\xi+\operatorname{\omega g} \partial\}$ : haY Ja

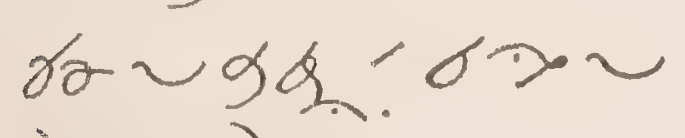
) $\omega$ ब.

Fourth Consideration. All together." odex "rongl: 20 , "3r, - is $2,-0$ " "Y, h.0 sर, 952, "S"s?: 2 arga, बsu "1.2य x; ho。

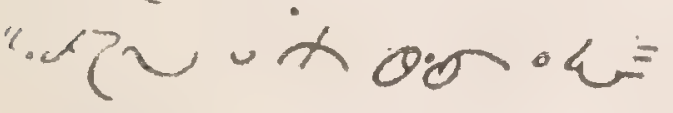
"s2क." "Ox! 1 hasa 1 "g\$o.; bq be e

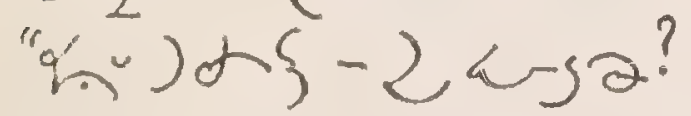
"ol te eq. 2 "L-ga:?

"ox! teara

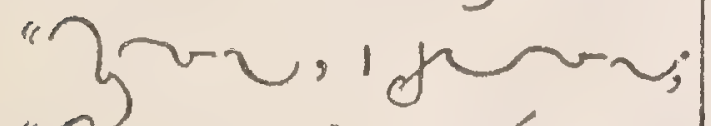

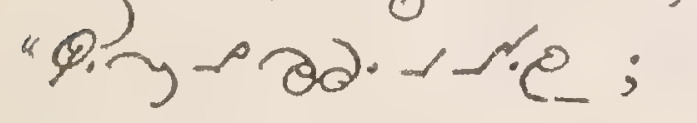

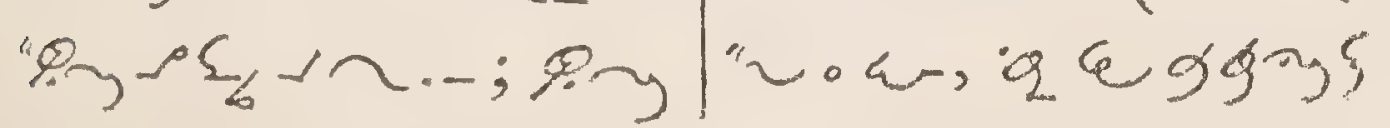

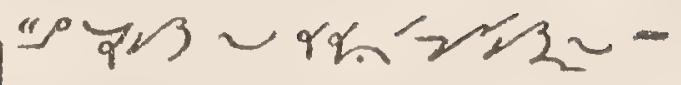
"Ton, ح (\$y). "0x! 02.5च.0.6.

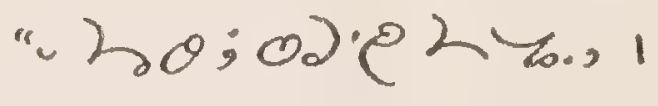

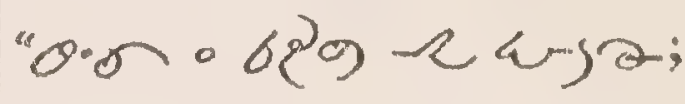

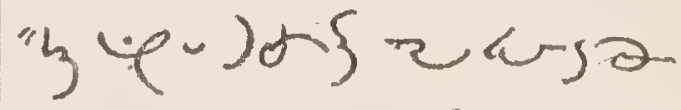
"( $9 \cdot$ - $320 \xi 04$. "2. ce Larga.

"ox.'界, eg( ) > = "जब, e wure tor" "界, wignear? "vogion a rusor. "Y8-; ;

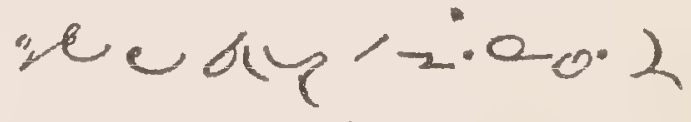

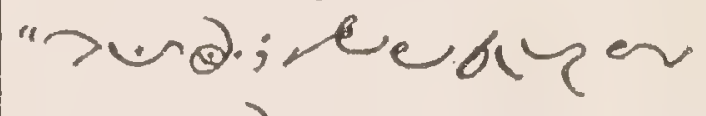
"broo: Langa cun.

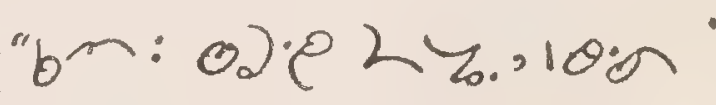
"arga; 20 ow; "口马"

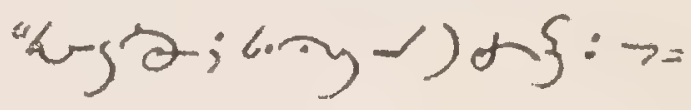

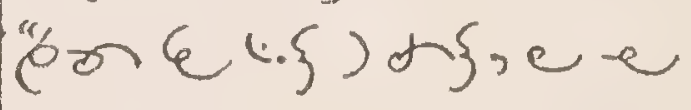

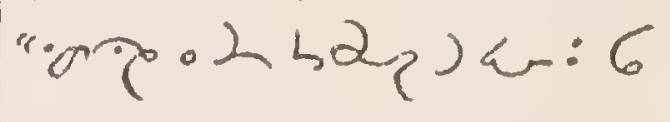
"). $15 \cdot 2230.0 \sim 2 \%=$

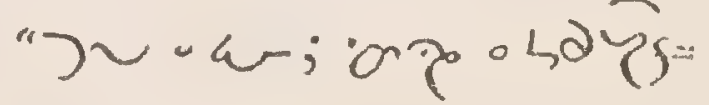




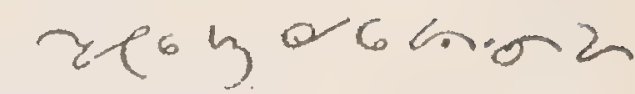
sigap.e<Lג\}r $\pi$ ए $\log$.

Nक9., 万ण Cᄂ=

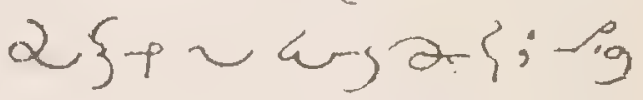
- Kele igo

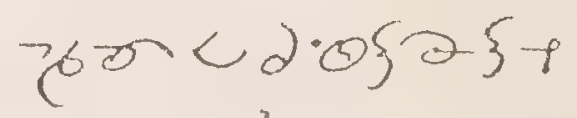

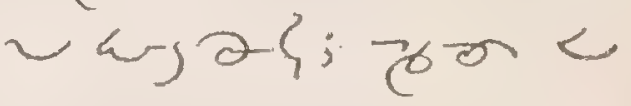
z) 2 an a 260<63+1 Q $\sim \arg$ क Ko....

6 torec.5 d se - .00022$) 4 .$. 6rate 30 ordo $n-16 \cdot \cdots$

- hyze 2 सा. ) 2 , e $\$ 90 . .$. (76 एवसे)

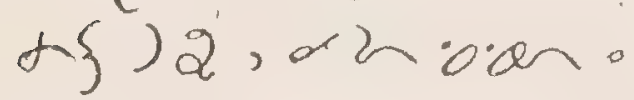
Ho.

The Confiteor. 鸟) iga. Act of iontrition. Yygue

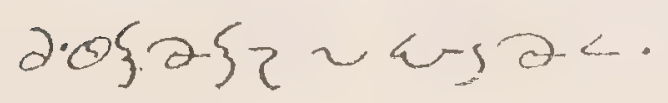

After Contession. - Q U P.sal, "R

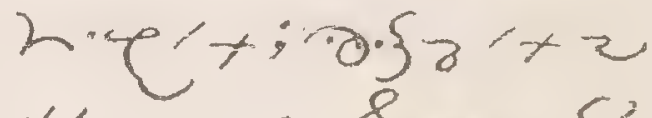

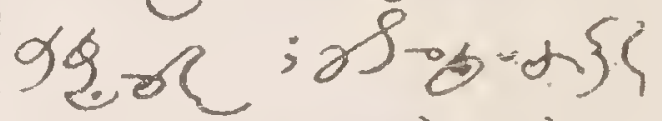
e-e iree dis - 19 कर wS

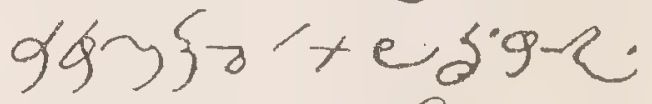

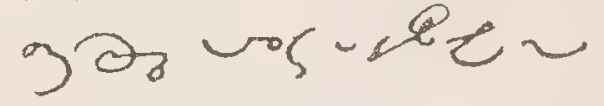
"मर gquद, c.5rete

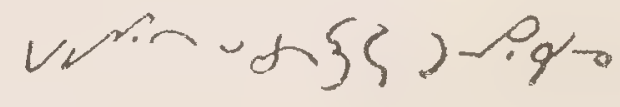
$\leadsto L \alpha$ ? 
MANUAL.

23

Prayers for Communion.

Betore Communion.

Fith.e $0.9(x-9)$

$$
\begin{aligned}
& 12,9) 2-965:-9= \\
& \text { oed } 962.0 .90 e
\end{aligned}
$$

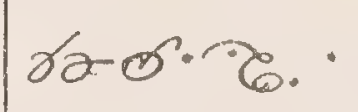

$\Theta .=0+x ! 2 \cdot 9-9 \mu$

contrition.e $\partial \cdot 0 \xi=$

afe $\sim$ as $a<$.

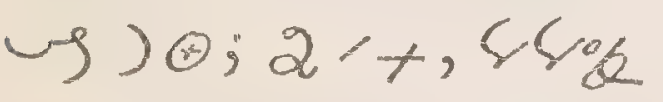

$0+x \cdot \gamma \sim(h-)$

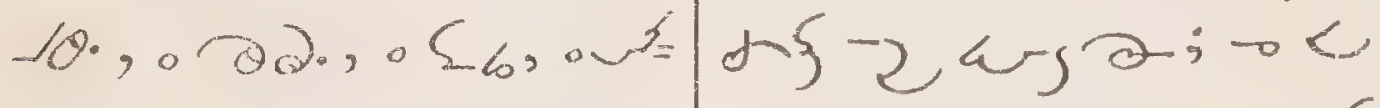

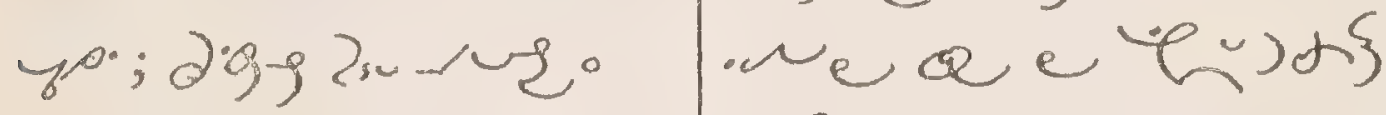

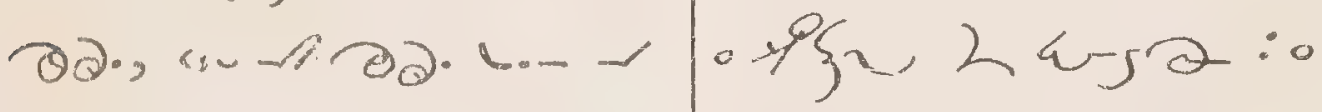

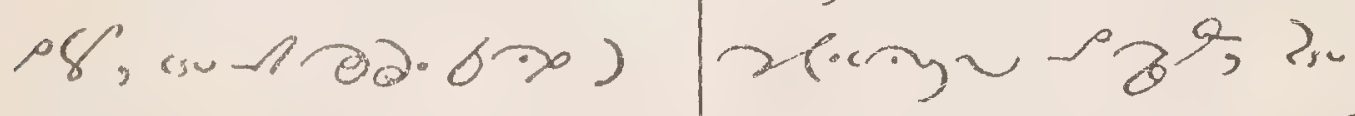

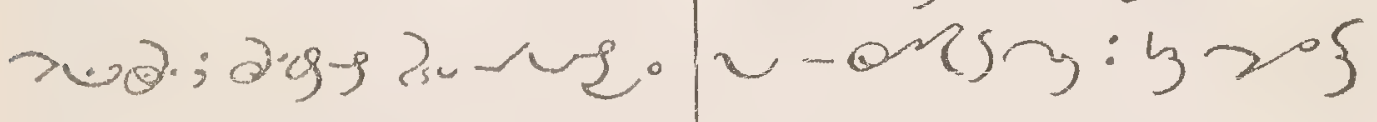

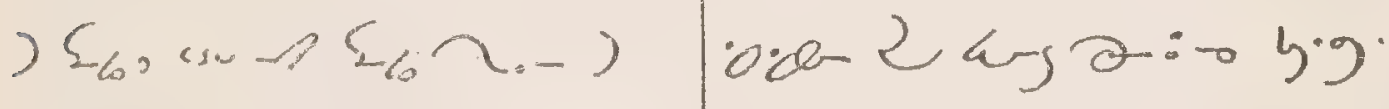

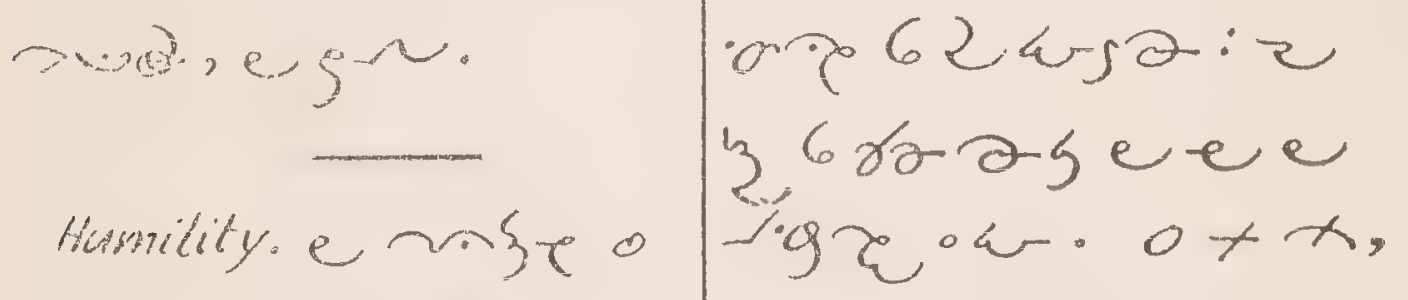

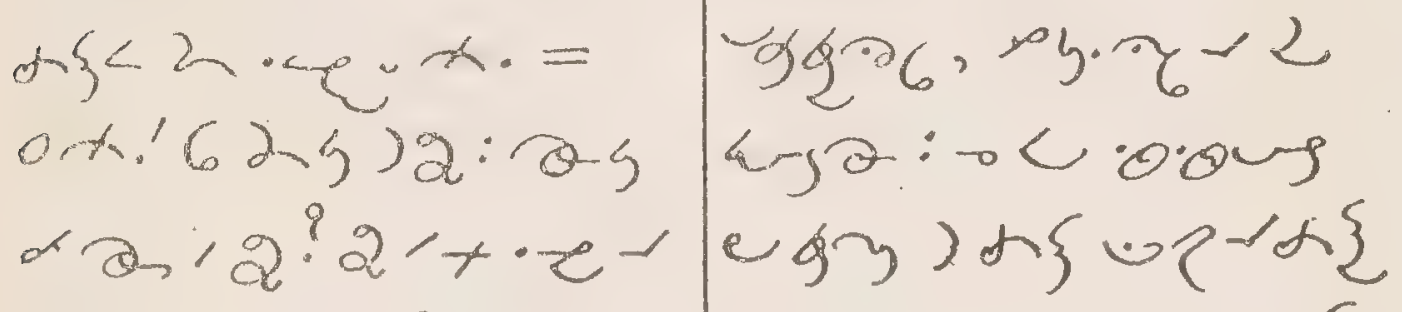

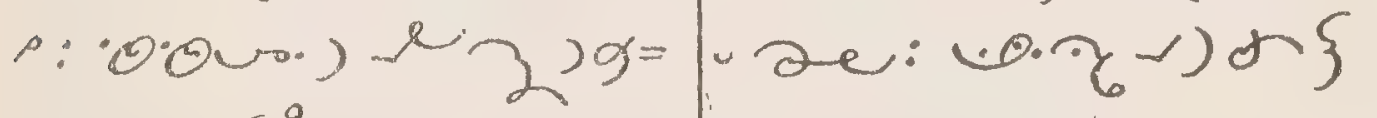

q-) d s? ho uneg - 2 arga.

- L2.

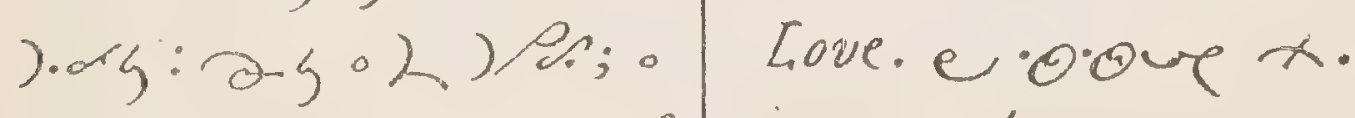

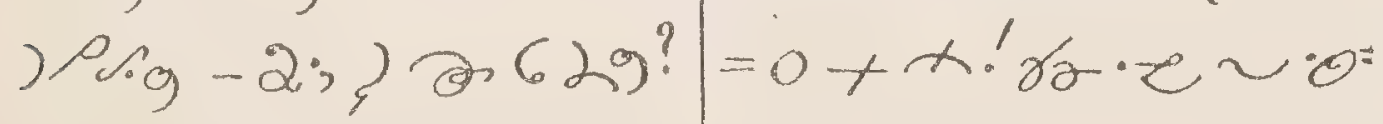

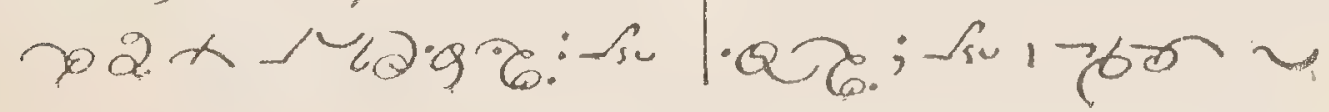




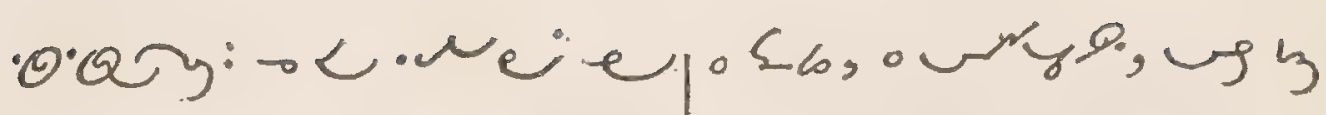

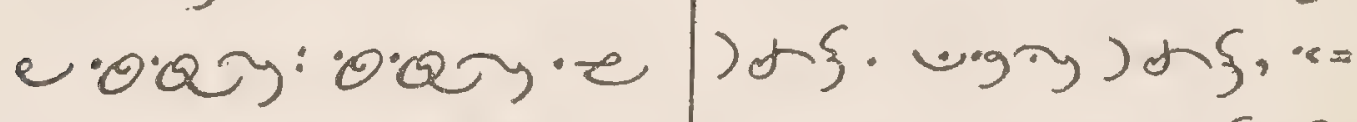

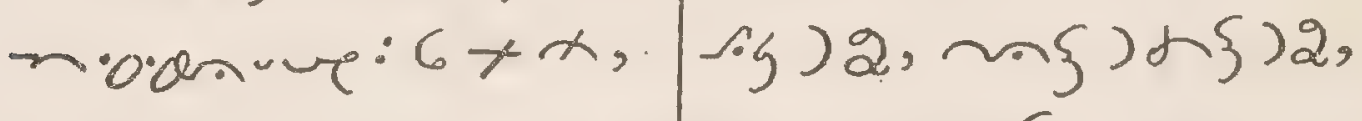

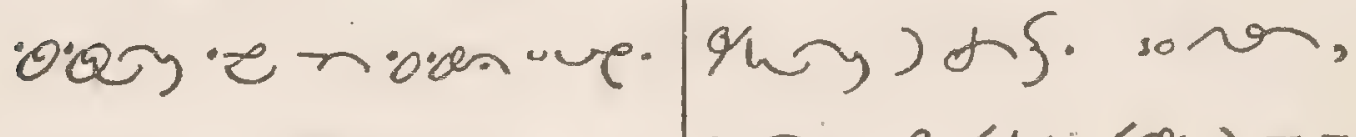
so $8-1 \% 6.10)$. Desire. e-rye $x .=-1\}$ : pe o. $\{: 00 y$

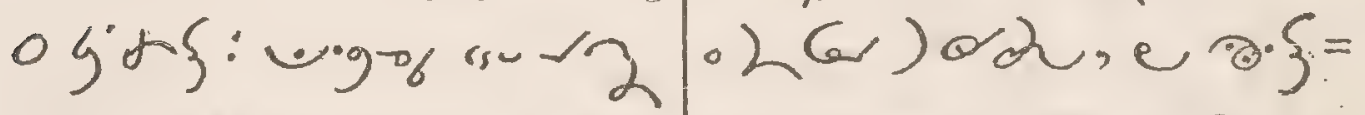

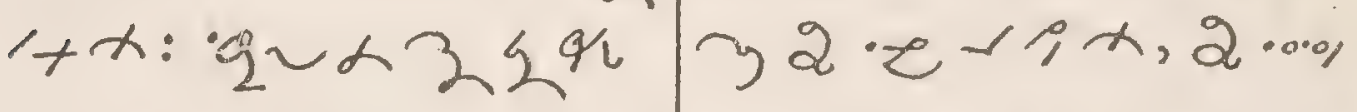

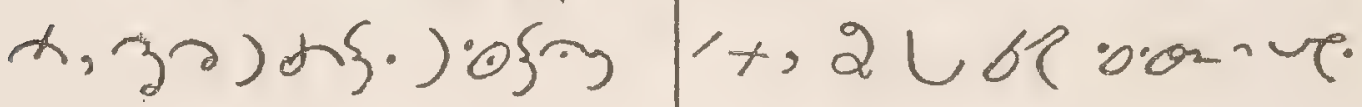
०र八u)

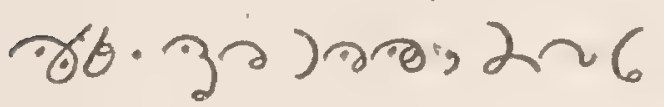
2): $Q$ ᄂ 2 '十x, उə ) $\partial 2$

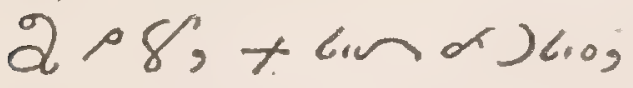
कृ ' ג: З2 - very, od.ev

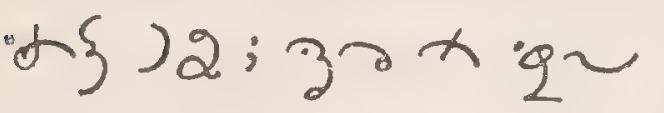
$\alpha 2$.

After Communion.

Thanks. es $\rho$ r $x$. qृ. $x-2, \xi)$ 小

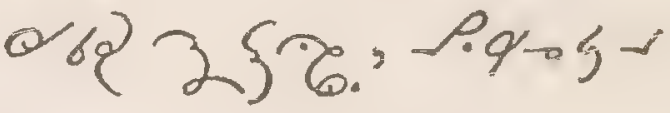
म6.5:-ura< ?9 ) an, sullgre: 2 -e-1, x, 2..0, 2,

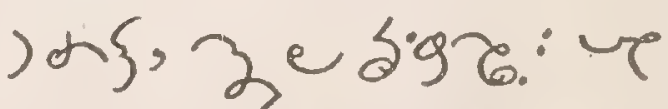
w ) 小\}; $2 p-1 q, ; e$ gو

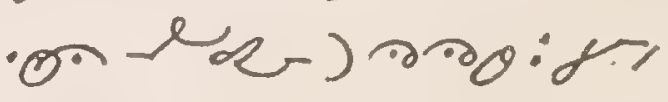
- $16 ., 6 \infty, 1>00 ., e$ a. 6 - एक Adoration. = 0 x: 2,12 ija 2 iga: 3

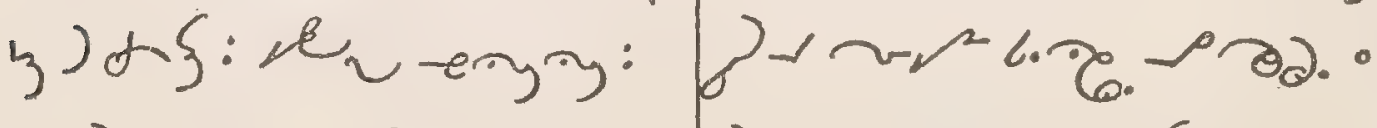

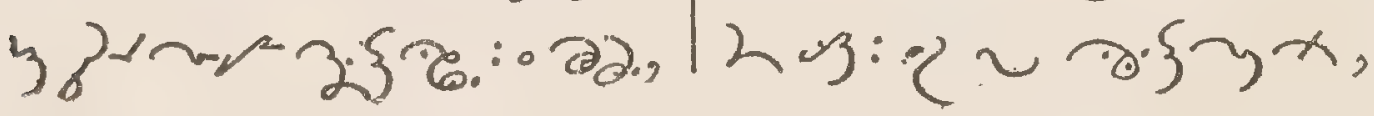


MANUAL.

25

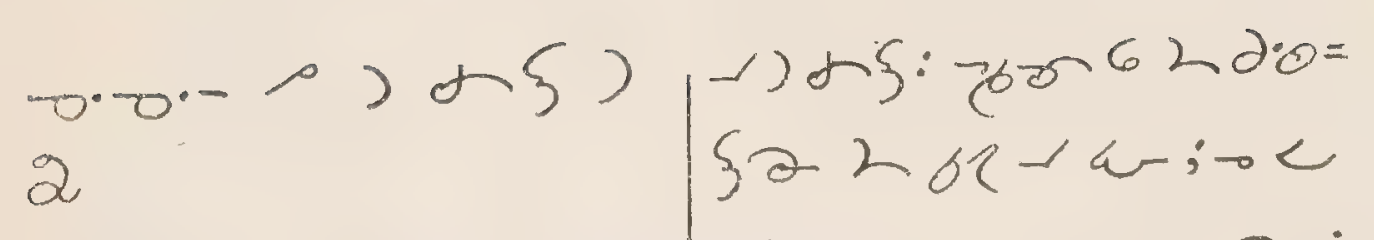
woreungr:

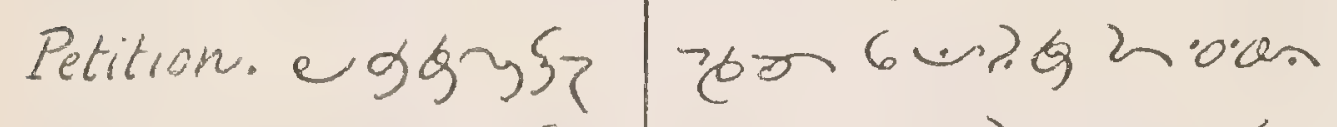

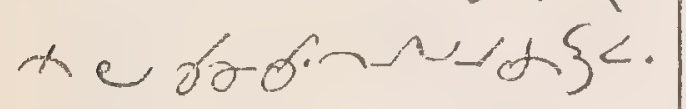
- ar: o holes $=0 \times ! 2,3)+\xi:$ qE Vß马)小s:or दe for) $\alpha \xi: 2$ <.w, $\alpha$ areen

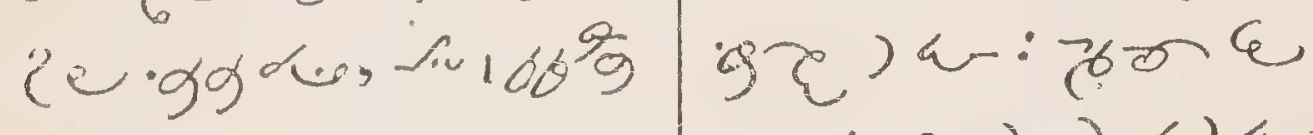

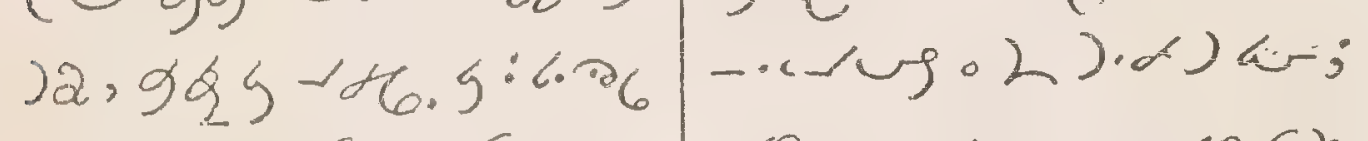

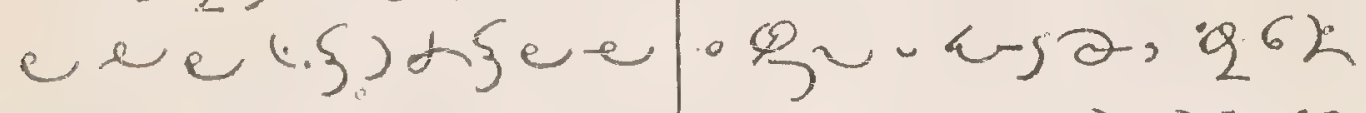

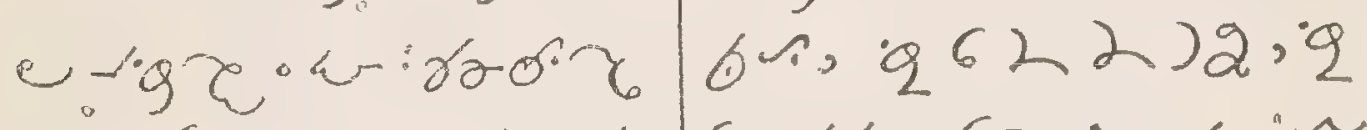

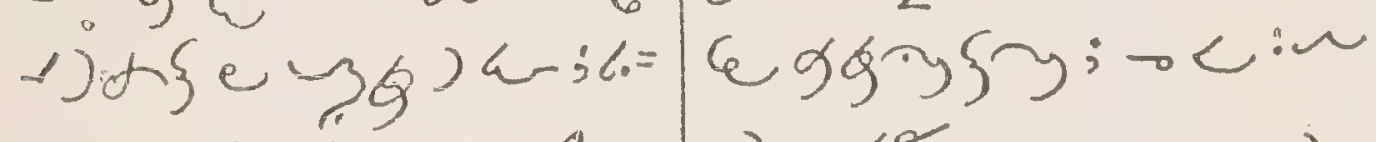

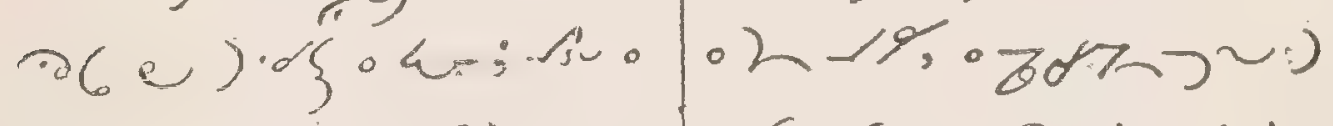

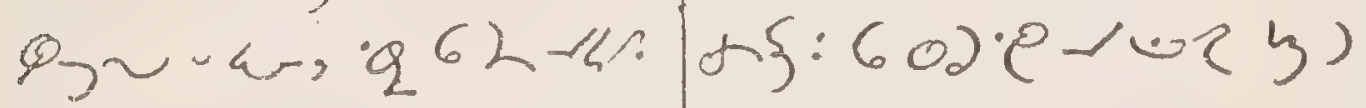

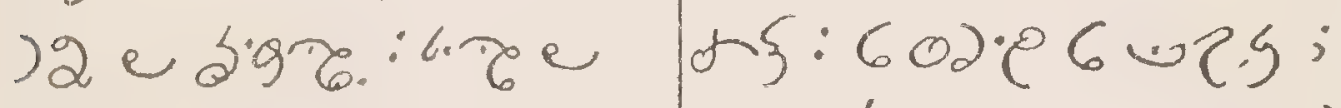

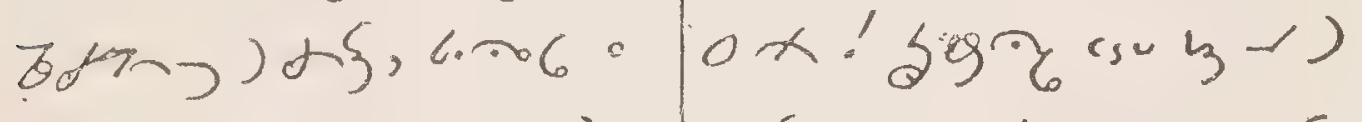

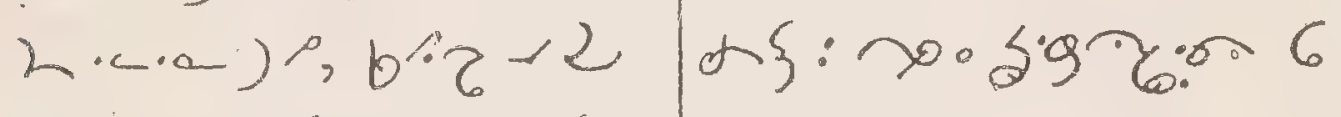

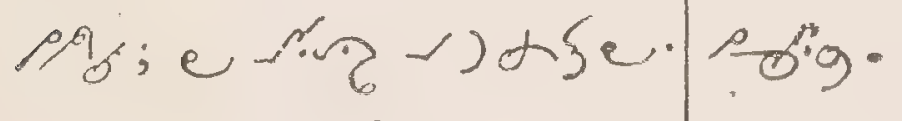

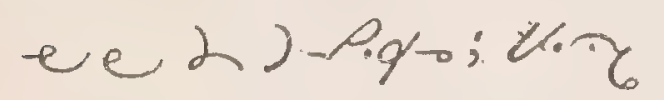

th: $00-6 \cdot-30.00$ - Nogje.

offering.e (.e) $九$ vos: $\sim 9 c^{\circ}=0 x ! 3$

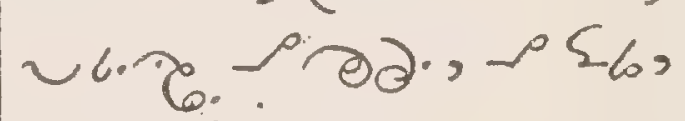

Resolution. e 36$) x$

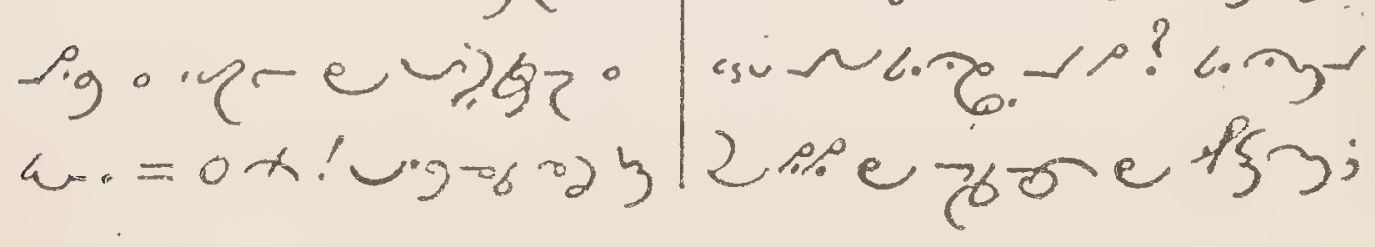


$\therefore x y-)$ oh,$e .76$ o

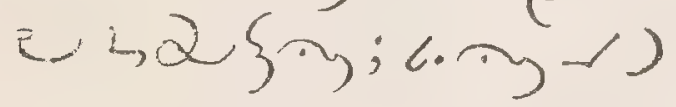

Do,eter 2)

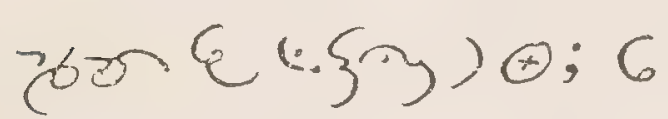
<. . $\{-q,+\cup \bar{q}\{=$

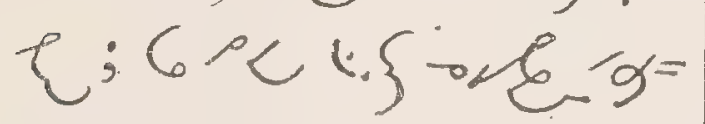

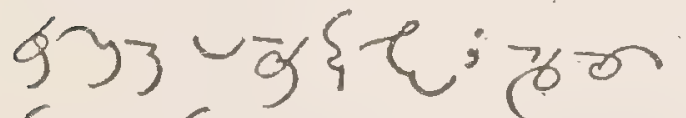
द $.5 \cup 5 \sim 6.202$ 2 .

Intercession.e 3() xuse oreve ggur $=.0 x \cdot \operatorname{s.g} 0$

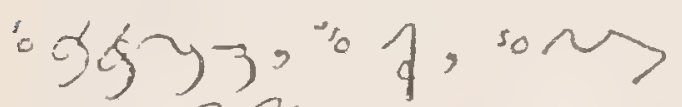

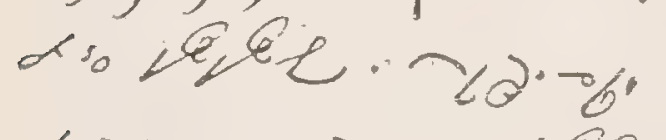
IN? $20 \cdot 6 \cdot-18$ e क० म6; 200 Sel, e jqo.; $=$ $3 \% / 12-16 ; 99^{\circ}=$ o. 几):an:0.0n) bथ $-\frac{z}{b} ; b \cdot \sigma_{0} \sim \sigma=$

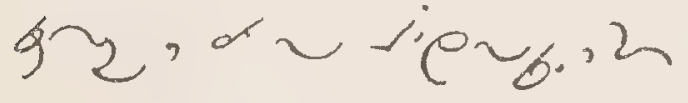
. $5 x$ ar. offering of the Mass.

$$
\text { ot, wiegsig, }
$$

wer h $90 \%$, ug s.g, UR Ler, revg rig, erv-h to.; su 1$) \cdot d g e d .9$ ग- लद, - 12,20 ) $\{$ )

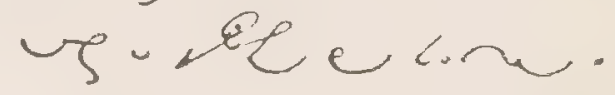

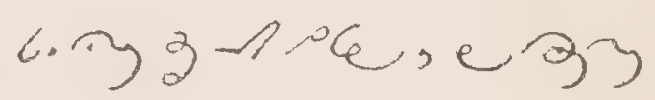
70209 h 20 on $\%$.

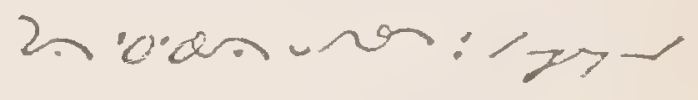
\}. a $\alpha-\infty, \infty \alpha_{3}$ "s. l.ry -16 ery "अ?. 2 . 0 an. an 4. - Le $\sim 6$.

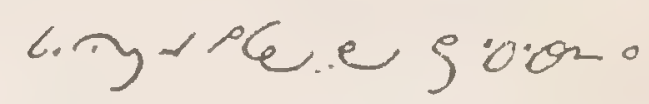
undor; lon 10

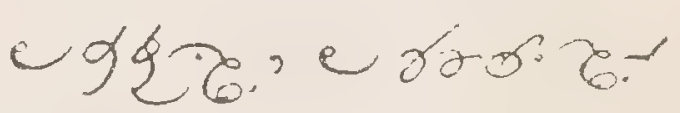
) $5 e-e_{0} 202$. $68+4 \cdot e$ en. )小 
The Rosary.

Joyful Mysteries.

1. . मेयन- ).

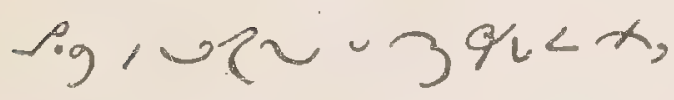

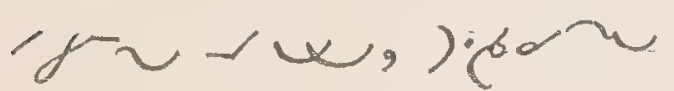
$.08-2.2 . q$

2. Po haye un sei Ero rig, we ups:

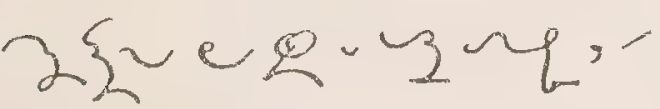
的㕲。

$\overline{2 y-) \sigma^{2}} \approx \varepsilon=$

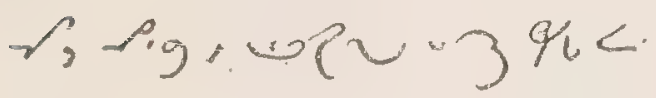
ג, r... J) edo. $108+2.2 .{ }_{0}$.

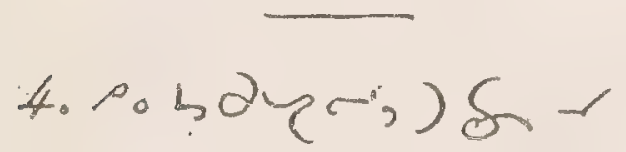
Er signow.

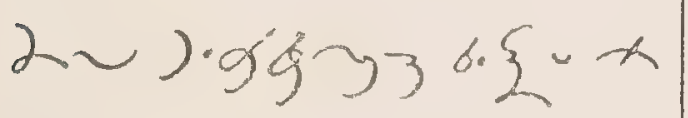
) $x^{\circ} \sim$.
5 , ob

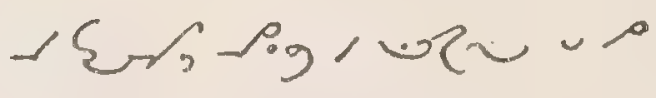
$8 \mathrm{~s}$ उan, of

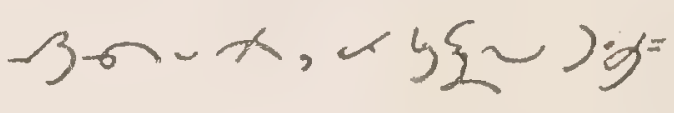
צบ3

Sorrowful Mysteries.

6. $\left.042 y^{5}\right)=6$

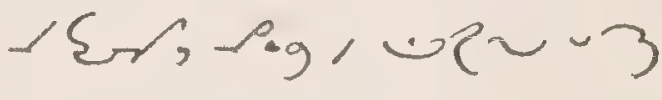
$q<x, \pi$, w

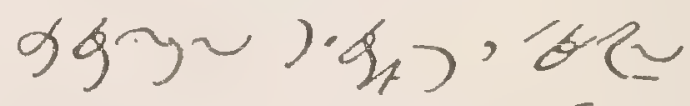

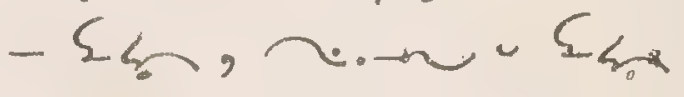
) 20.

Trsobay-) angEr, siglupu 3 ke x, $=20$.

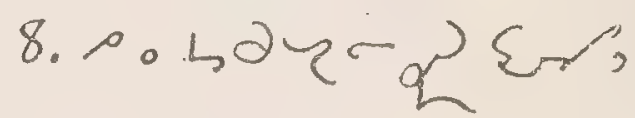
sig, एव - $3 q^{a}<x$,

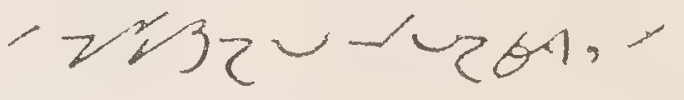

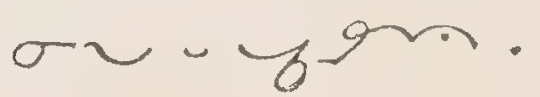




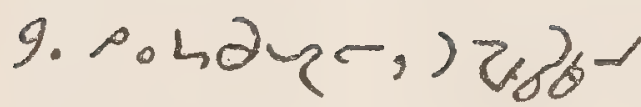

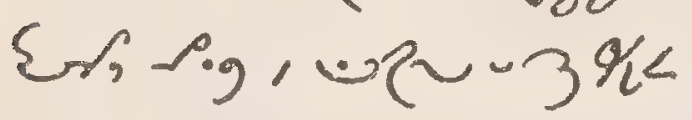
x, 一 - g\{kचeb?, 'Q - >0Q. $\{, z \varepsilon q$. wnic.

10. s.bayr, lig

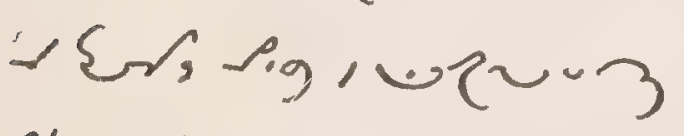

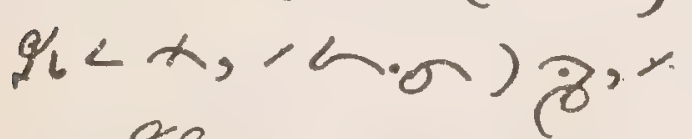
voriszu-vond

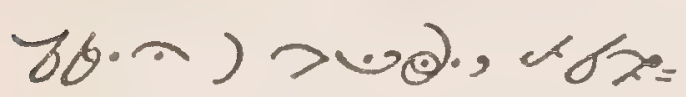

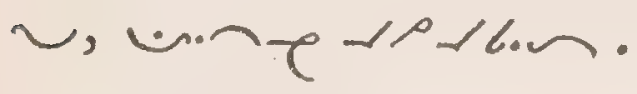

Glorious Mysteries:

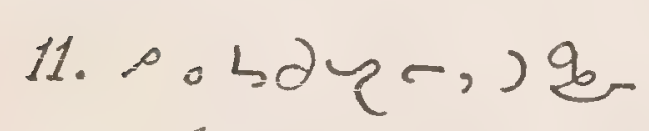

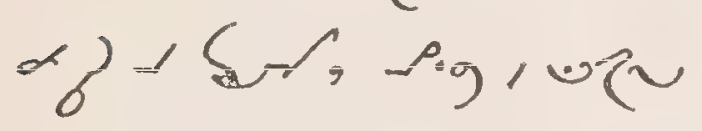
$-391<x,-\operatorname{coc}^{2} 2$

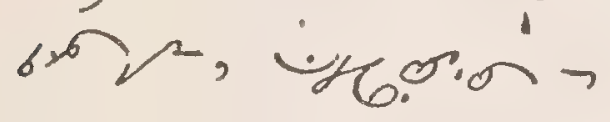
ro, ole +2 .

12. p. hay , Jq

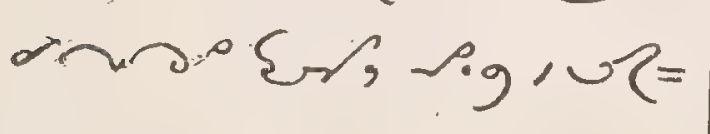
$\sim 39 t<x, 4 g_{1}$

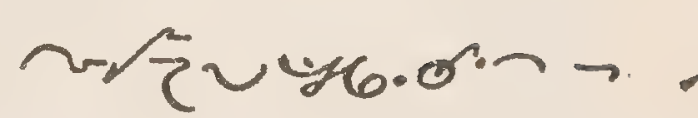

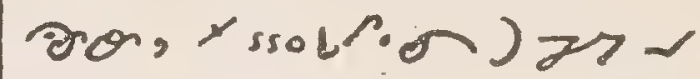
3. unene dastons,

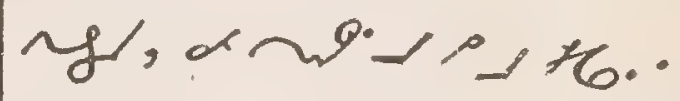

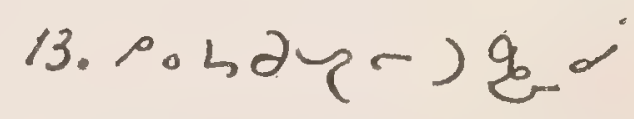

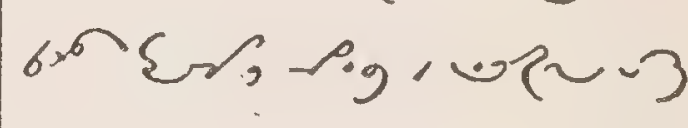
$q(<x, \text { 的) } 2>-\}_{0}$, 6Gu20.70人 tom - Linu-w) y, ur-an.

14. Pobay-, )ger - Er, -ig, upu us,

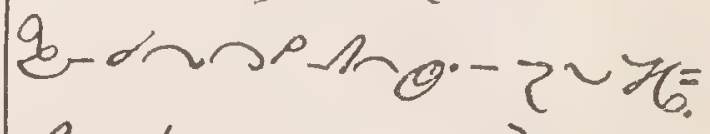
onx, «bxu:dx

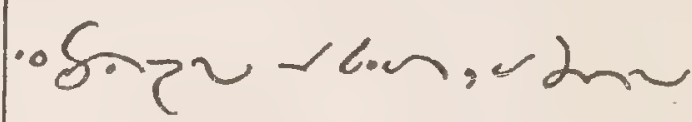
)yetz? ssol.

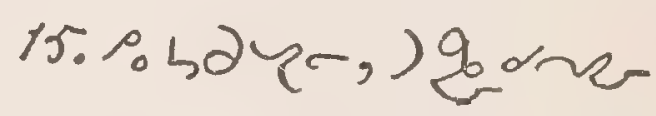

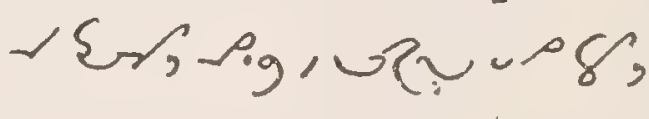
- ब1 2 2 ) $21-3 ; 2$ xus

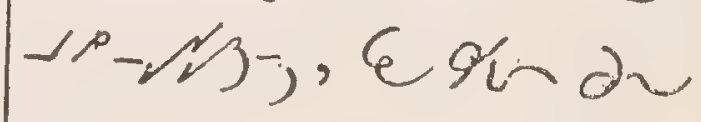
12.003 s s 46. 
Via Crucis.

- crux, Ave.

0 2.01 1 -

$36 \cdot x) 2$;

o'cob-d

V०क. Na-san.

Preparatory Prayer. ox. ص.ص.- 〜 $0^{\circ} \alpha=$ 6.1巴 (人). e-95.50 >0. Irop.

$25 \cdot 2+2$ - ig, जथe (y - 600.)

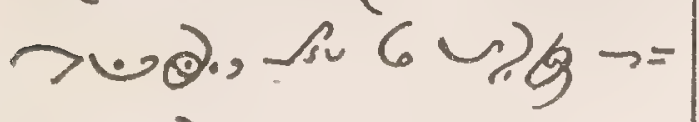

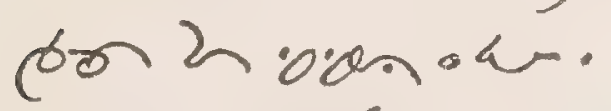
«? eqLa-sa. Act of Contr. e $\partial \cdot 0\}=$ $\partial\} \tau \sim \operatorname{sic} \partial<\ldots$ Intention. LQ $\left\{\xi_{7}\right.$ 。 os o 997 च 9393.1 $6 . \alpha-(6) \ldots$
Sancto Mater:

$$
\text { s 6, lin } x \text {, }
$$

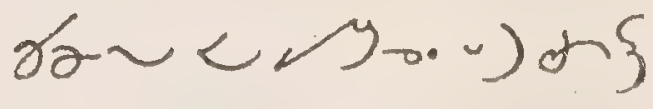

थ

\section{Station.}

Adoramus te. "s. Sy

) $2 \alpha, \rho\} \gamma \cdot=2$

96. - 60 (e)

consideration.) $s x$

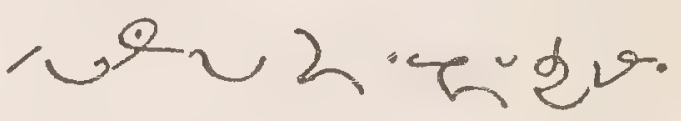

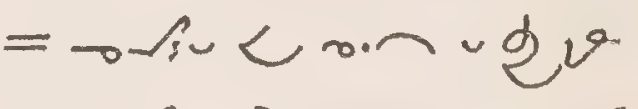

<o $\{$; d x wh

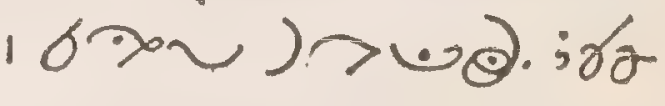

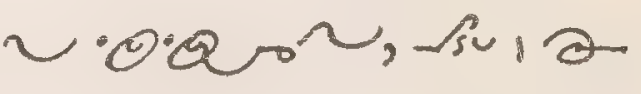

reerb.

o 291,en.6.-12

uga, - su 1670.$)$

गब; उ

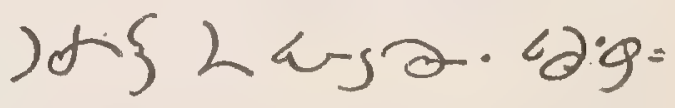

revire logr

- a....

oph!

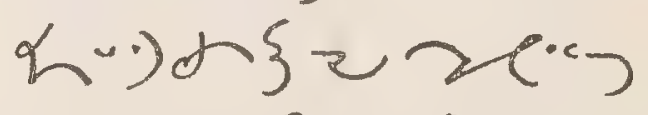

$1+\alpha$ ze; 69 दe 
30

SHUSHWAP

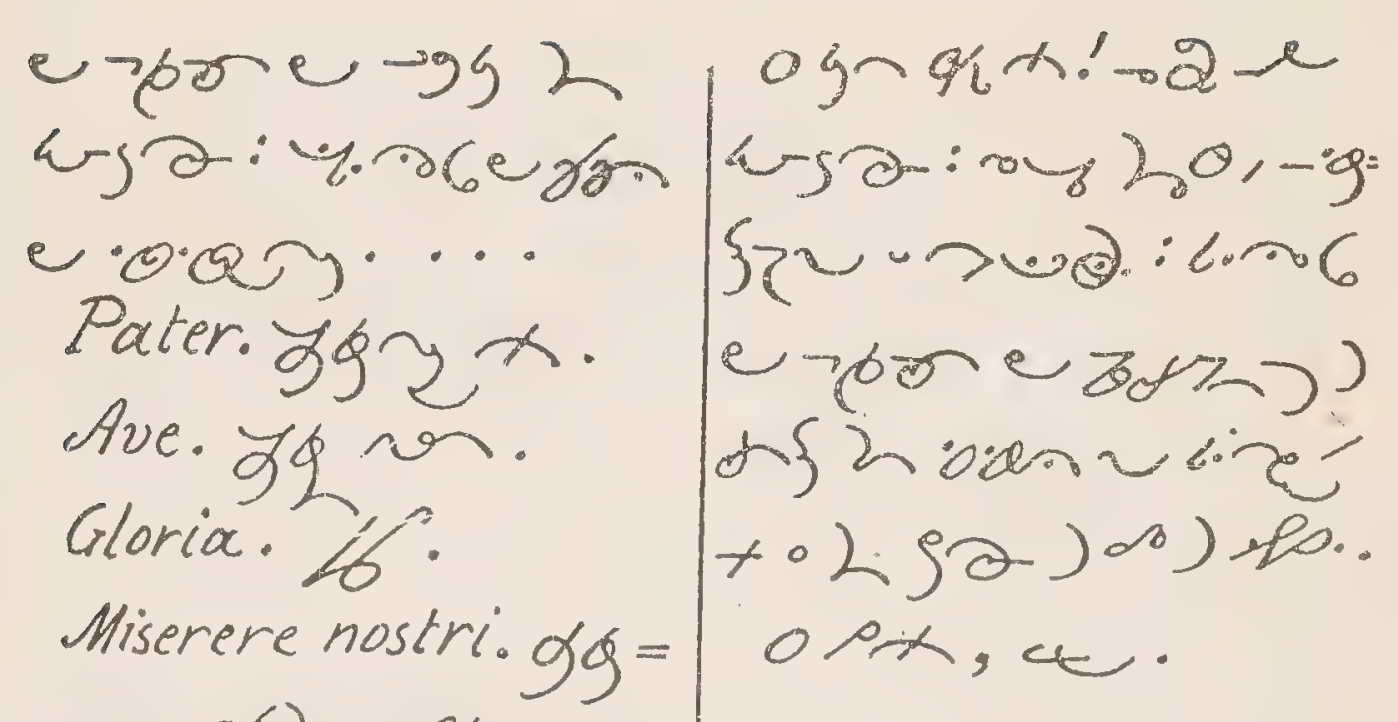

rob. $\alpha_{Q} \sim, g \cdot=0$

q, gqurg. Dâ.

III. Station.

Fidelium. $0+\rho_{e}$

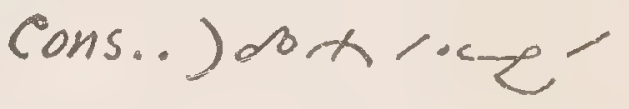

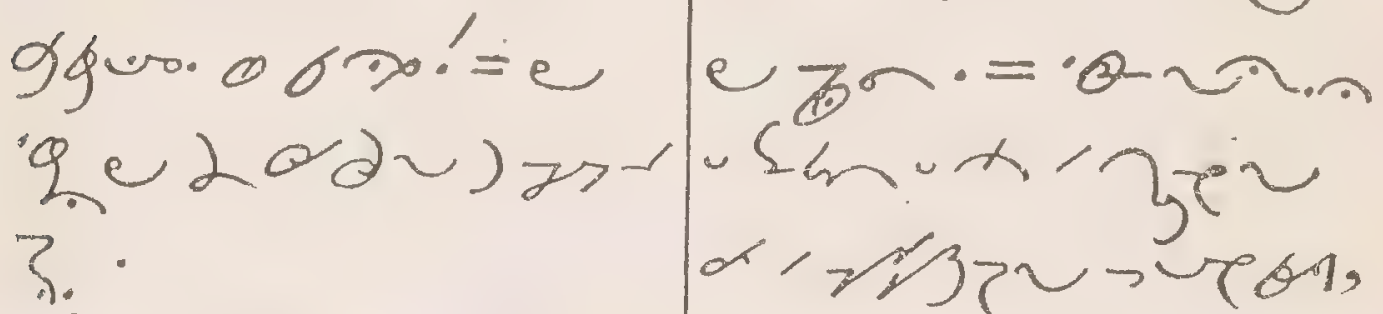

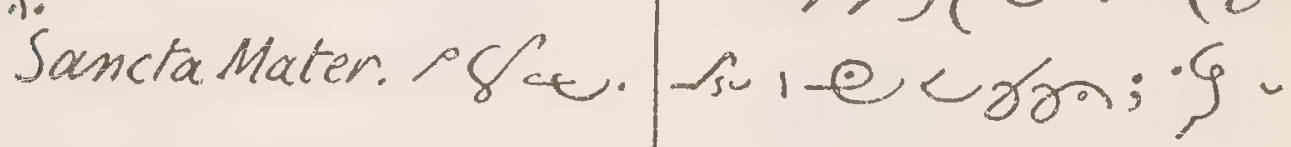

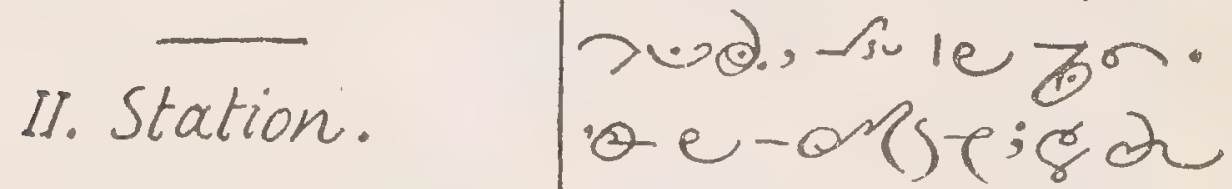

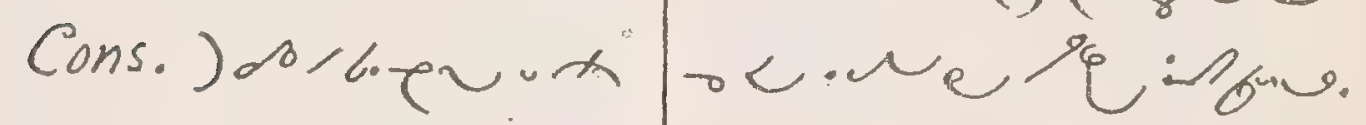

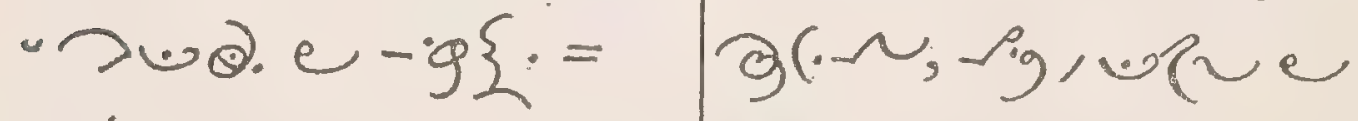

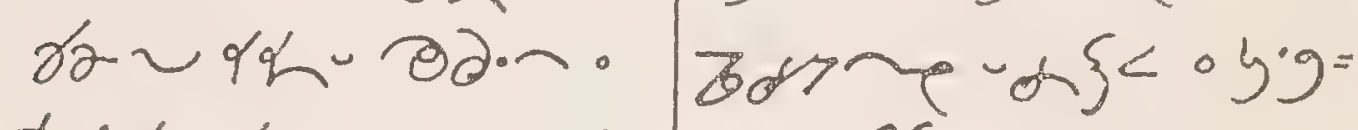

从;

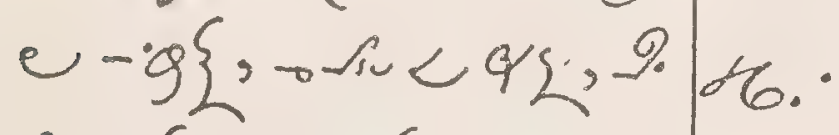

Pu

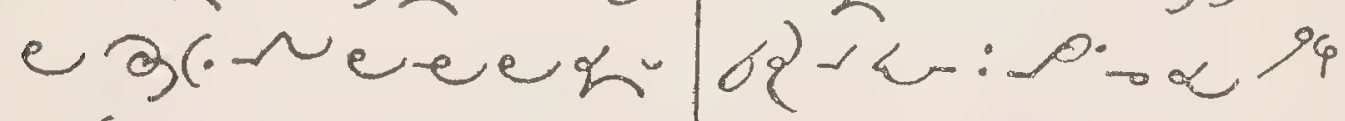

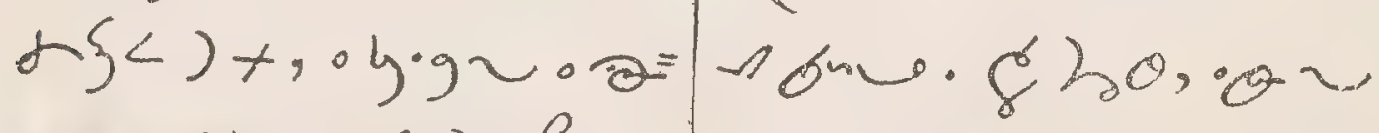

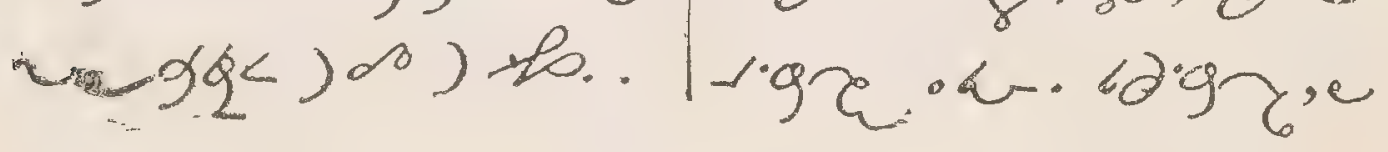


MANUAL.

$3^{\mathrm{I}}$

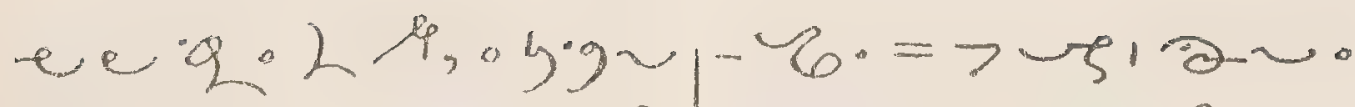

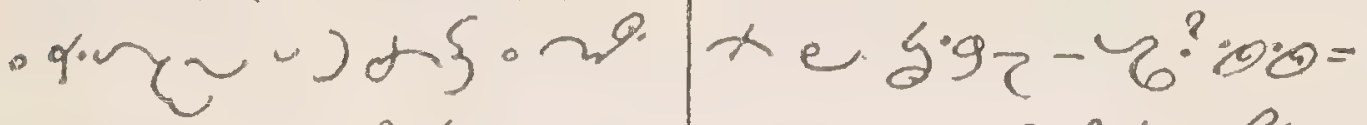

$-H_{0} \cdot=0 \rho x, c .$.

IV. Station.

Cons. Jos $x-52 \sim$

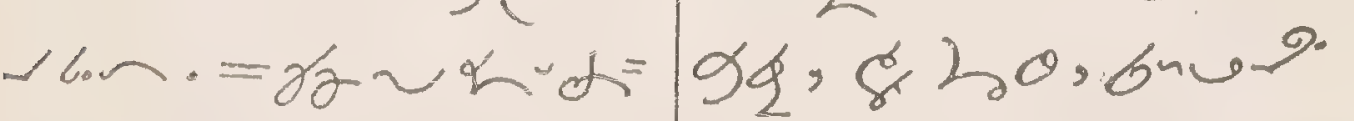

\{.

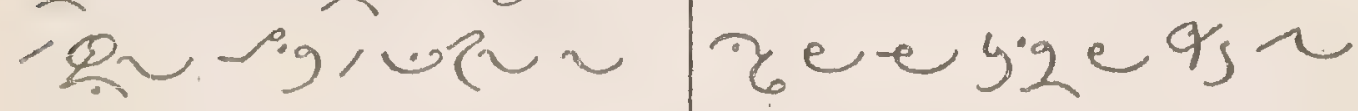

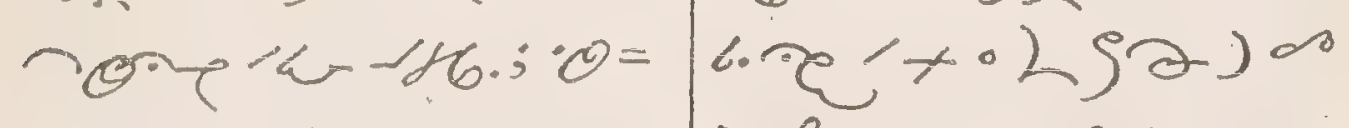

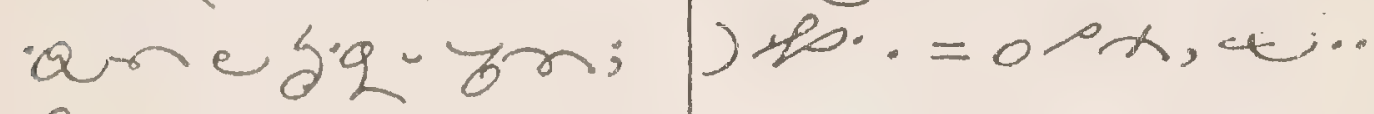

grieun, oeefre

- jge we $2<$ ) 7

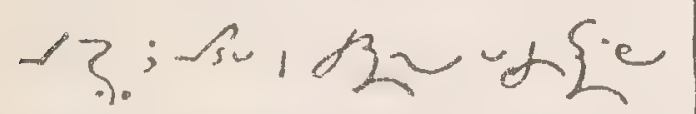

squ romean

$\arg 2<$

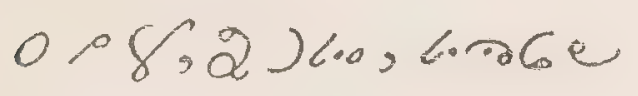

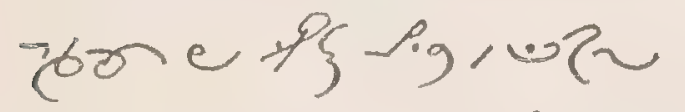

$\sim 9 q$.

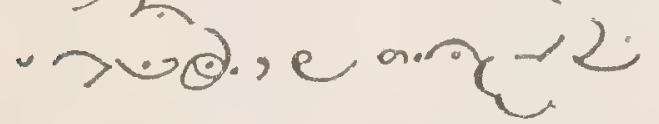

ura:e é y:00

eligrear. =0 P.

V. Station.

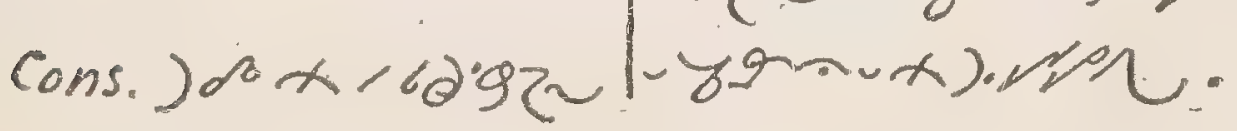

VI. Station.

Cons.) os $x-9.192 \sim$

$\partial_{b}-\rho-\partial \cdot \partial \cdot=\log \theta$

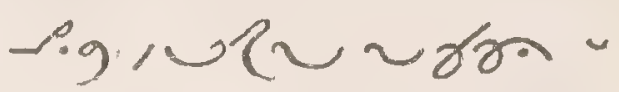

小\{z-d.2.;穴的

) ح. 2.

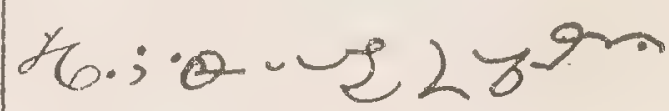

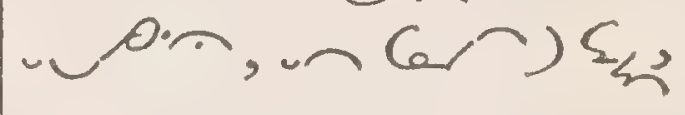

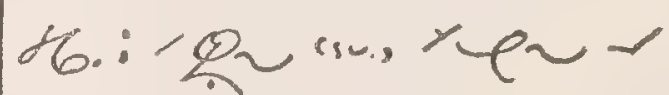

(2);

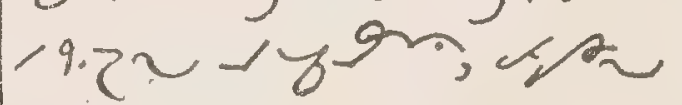

) so., 2 hos? 


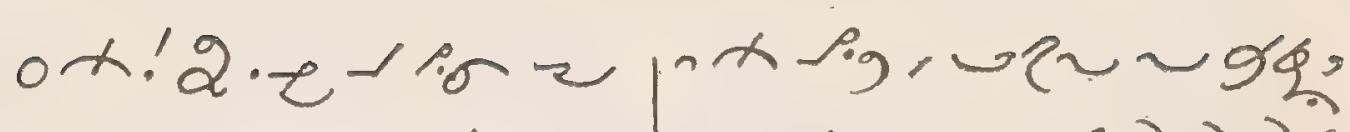
.0.

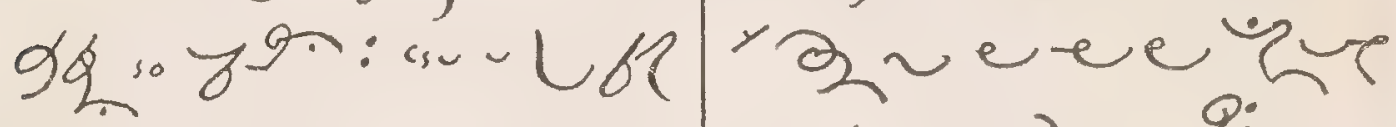
$\sim 4-5 \theta<\cdot 4.26 e$ e.joe biong 2 ayo. $=0$ oxw..

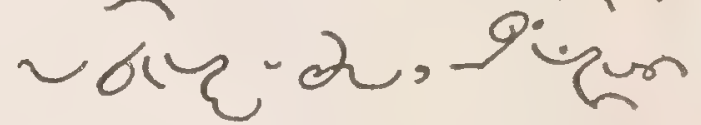

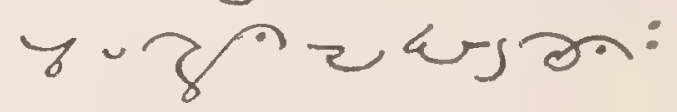

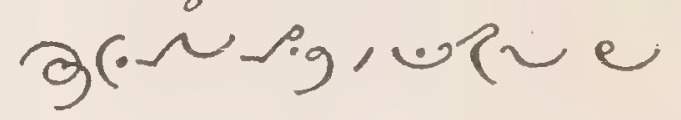

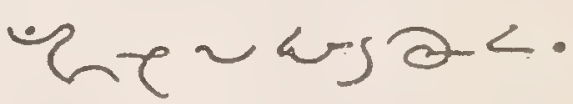

VIs. Station. O\{qu, $\alpha u j \xi \sim$ Cons. Job $x$ r por

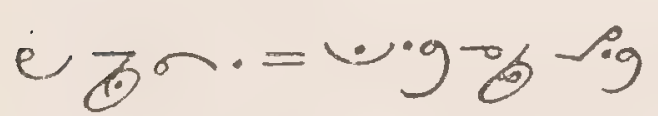

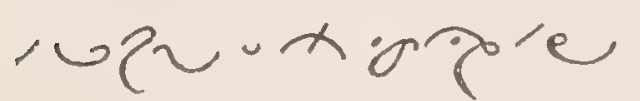

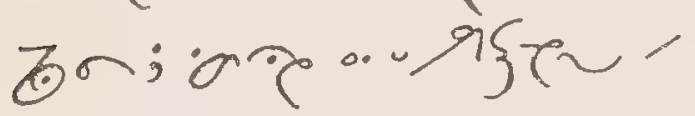
$4-26 \cdot 9 \cdot \sim e r=$

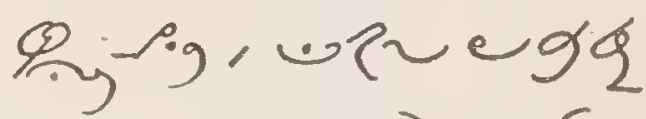
(6) )>.बे; $\rho_{0} \sim \alpha$ ) eqJ 2arso:

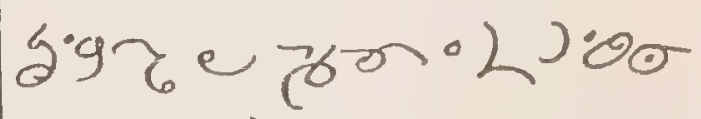
- ard hol, de e y.2.re lorén. jol s., are cy.ze \%ั山 $20^{\circ}-16$. ox!'o eque a. 6.2 as $2: 5 \circ 9=$ reegreeser or. = os $x, 4 .$.

\section{Station.}

Cons.) dox gon

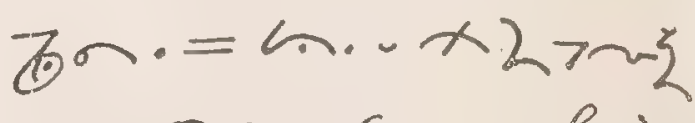

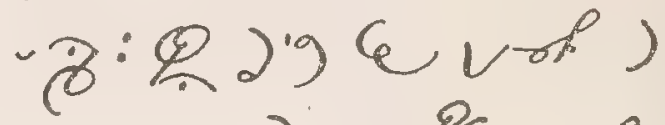

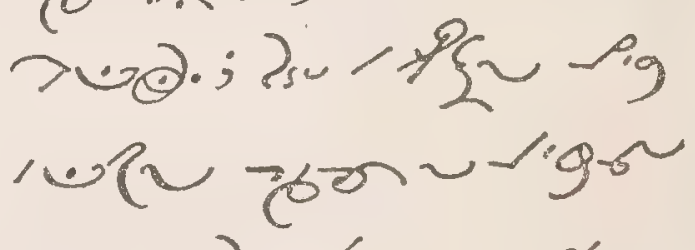
WIS. Station.

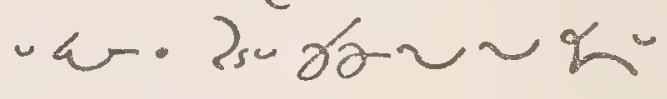

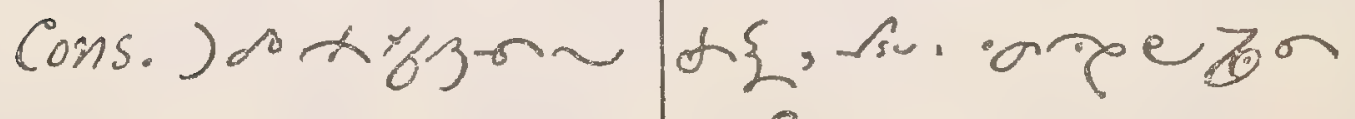

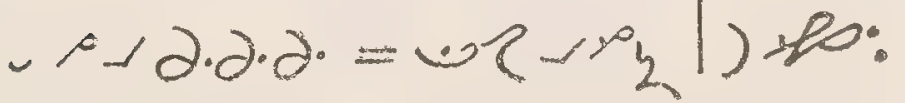


of $\%$, giree

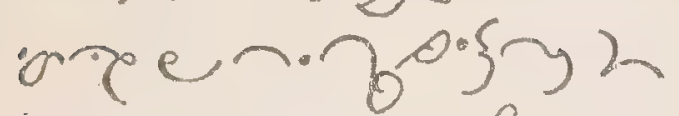
(usa joo ) Po.

\section{Station.}

Cons. ) d $x$-oge $x=$ $U=42\} z-i g, 02=$ $\sim \sim \gamma \gamma \sim q \%, x,-$

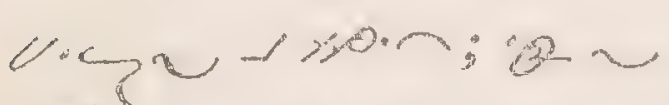
"C-

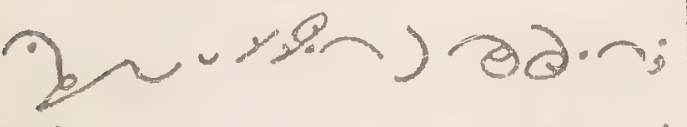

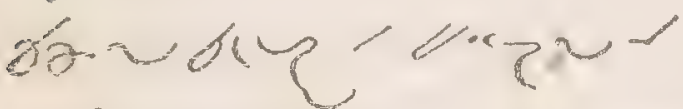

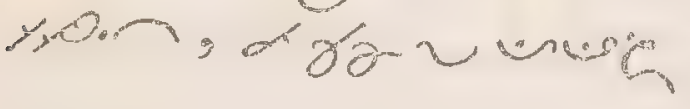
然 ig, . . R य

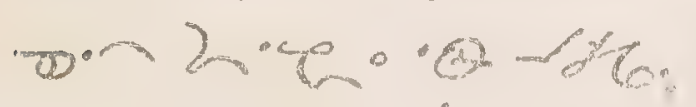
og-gitido.c

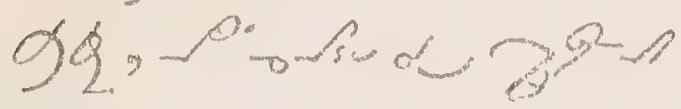
ong $\log$, w

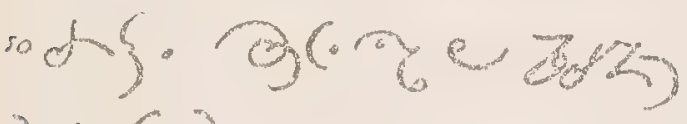

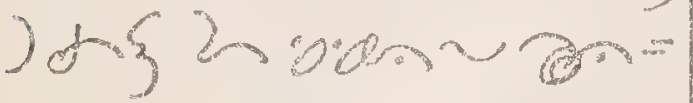

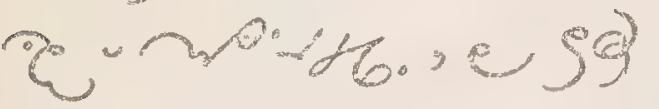
-4n-20?.

X. Station.

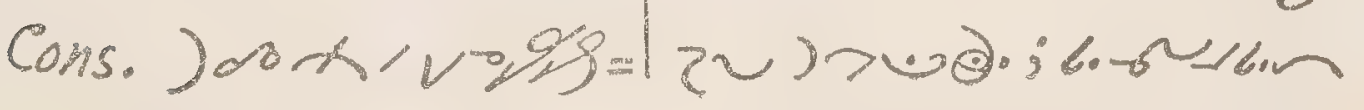

$\left.\mathrm{r}^{2}\right)>\omega 0 \cdot=\operatorname{lng} \mathrm{\theta}$

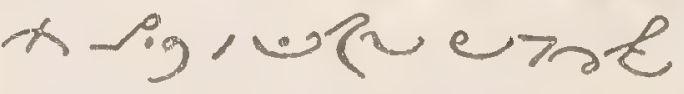
) बब: : บ - कo ) vorod 2

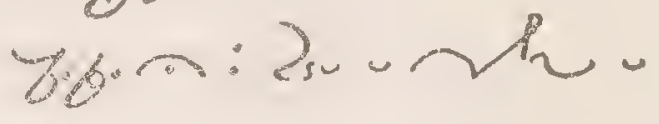
un: 3su - >oga -8y;

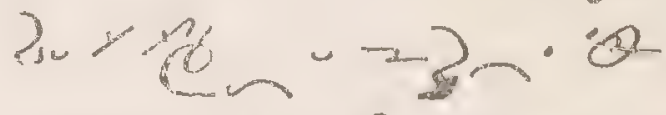

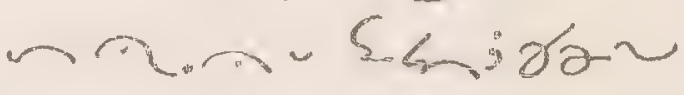

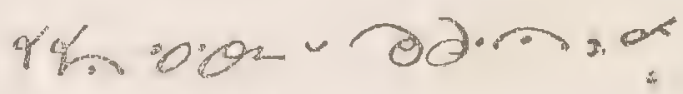
ror $\sim 0$ <. $\sim a-g a<$ ? $\rightarrow 9 l_{3}$ rest-sesere

Xil. Station.

cons.) dox - boou ) $>\cdots \cdot=\log _{0}$

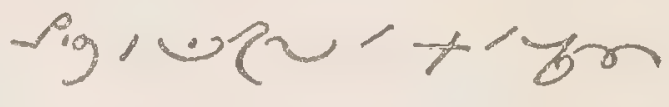
e б $-925-26.90099$. av) bav o os o of:
$05) 607,093$ ore regroar? 


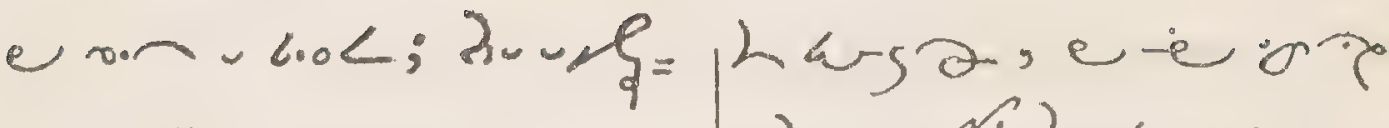
$\sim-2$.

o 5)

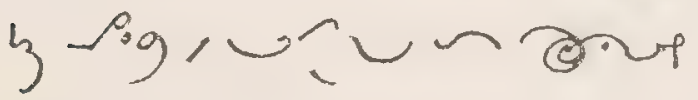
1 $00 \rightarrow \therefore-9$ l

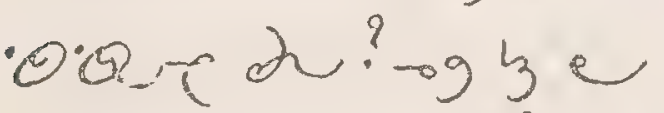
QRe $=$ asoc? ? zerstuske te aroe gruan?

XIIS. Station.

Cons.) dox-uste $-2 \cdot x \% \cdot=\log 0$.

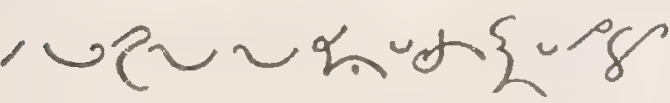

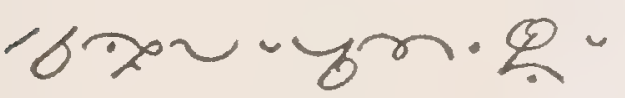

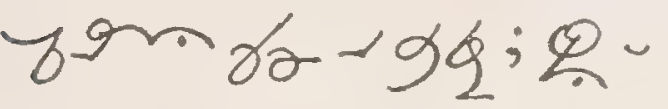
Ufiri dub.r on $w_{b} \rightarrow \sigma^{6} ; \Phi+\alpha\{>0$ inopre Dog. $x$ - Vipues $\operatorname{sig} u$

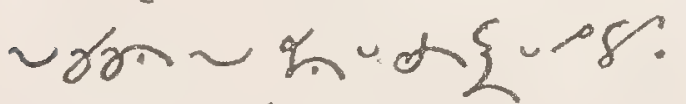
o 98 ' su - 2o 26 ?

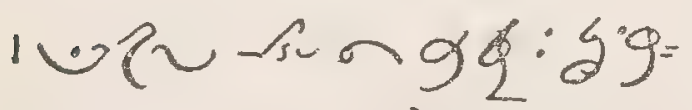
zerone $\cos \alpha\}$ - 2 virkara.

XIV. Station.

Cons.) $00 x \% \operatorname{mon}\{=$ $\left.v_{0}=0 \xi q_{L} !\right) \alpha \partial \mathrm{d}$ e. $202.196 \sigma_{6}$

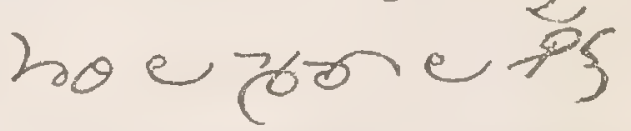

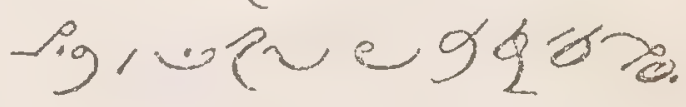
>.ब. e चक्ष ग.

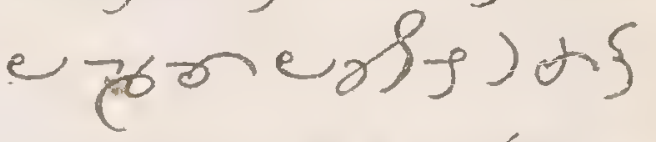
e-e aroe ligk

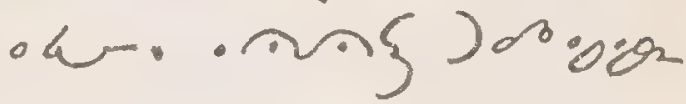
La-gक; उक 62

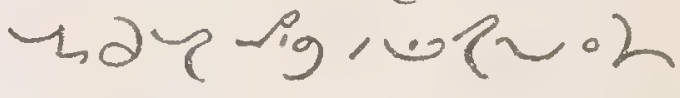
2) $\$ 0 ., 068,25,0$. $625) 75-3$.

Condusory Prayer. 02.8 is Q 2 a.s. 2; 6 Jok $0.02 \cdot a r ;$ 670.2kata eraengrear 


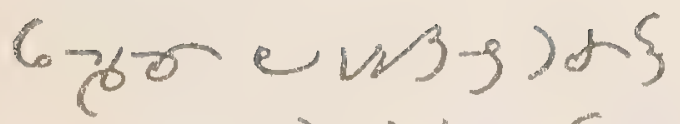

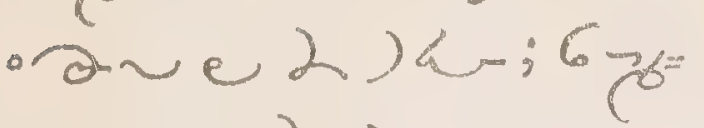

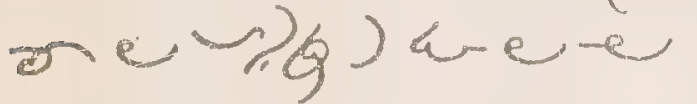
e-igre. w. $262-4$ ) x quegyjegace

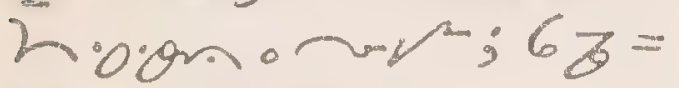

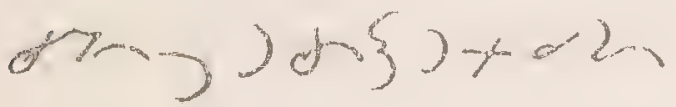
cas. 16 .

Hymns.

1. Christmas.

" $t$ : 向-íg.".

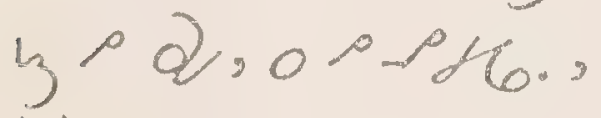
bre gersu;

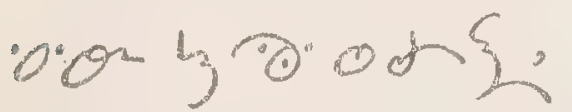
- vinz< s ron: lo, $320 \mathrm{cos}$ to'sen o. jt.

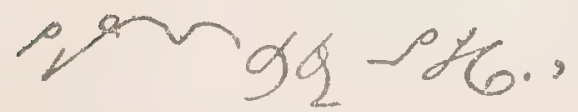
-eun aquar

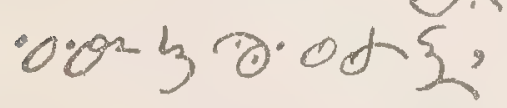
eun.ere g6sa. losento."4.
2. Same cir.

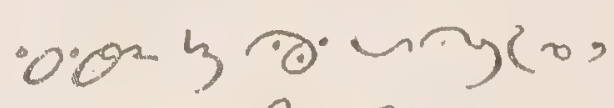
ung of curon: $1+y^{0} x$

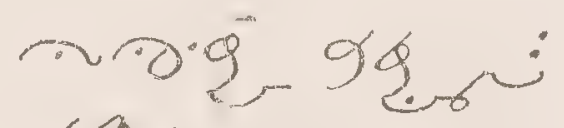
los an a. tols emo.

3. Adeste fideles.

$$
\text { I }
$$

ר.98:0.0noders 八.gr, न d)

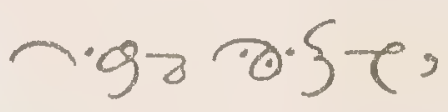

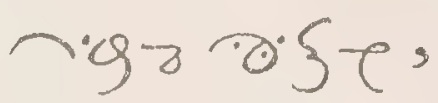
-9gatese-3 $91<$. iT.

tod $2 \sim 96,0 \sim 2 z_{2} u$ s.

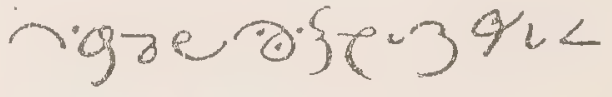
ta $\sim 00,-96000 \%$

$$
\text { ᄀ. } 9 \mathrm{~g}, \mathrm{\sigma} \cdot\} \mathrm{C} \text {, }
$$$$
\text { ᄀ. } 90, \partial \gamma^{\prime},
$$

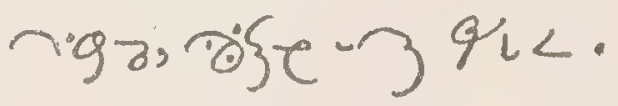


$3^{6}$

SHUSHWAP

4. Venez, Divin.

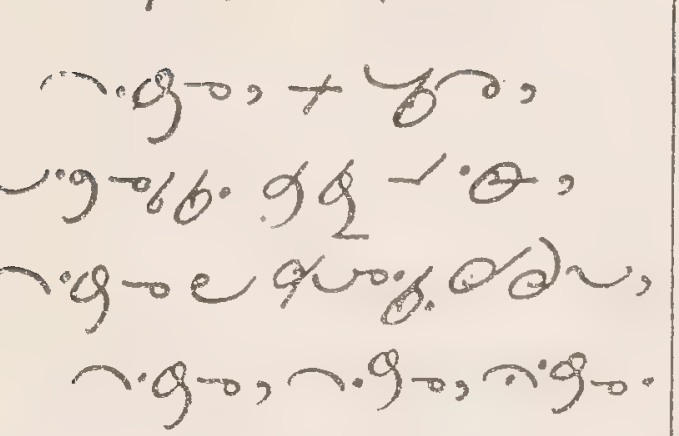

5. Creator Alme.

$02+y 0$,

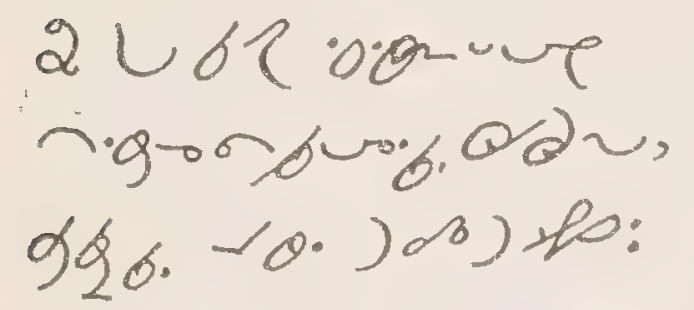

od.en...n nym,

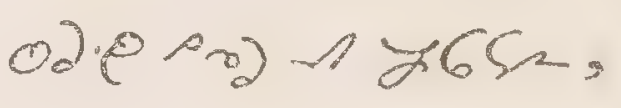

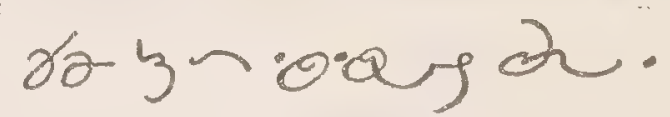

3.

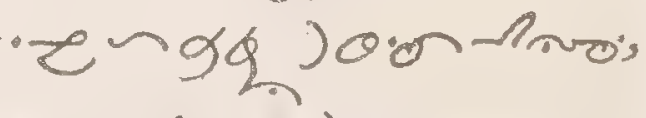

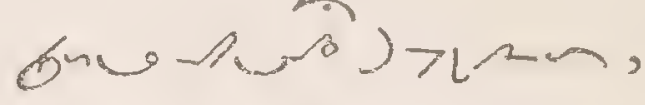
- engq jom ano.

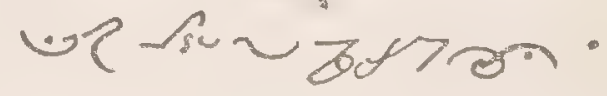
4.

ace sul (2) Jos) Do.; sol.c co. d .

6. Il est ne', le Divin. एक

T. Audi, Benigne Cond:.

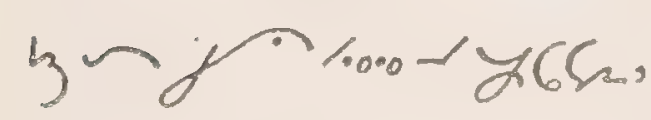
० $<-1, \ldots$, D०Q h $\{0.02 \cdot \% 6$; yof $\mathrm{y} / 0.0 \mathrm{~g} 6 \mathrm{C}_{2}$,

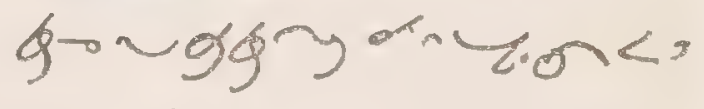
क्ष $3 \cap 32<$, e S9T $\sim 4-50<$.

s $b^{\circ-\delta e g n a i n, ~}$

8. Stabat. Mater.

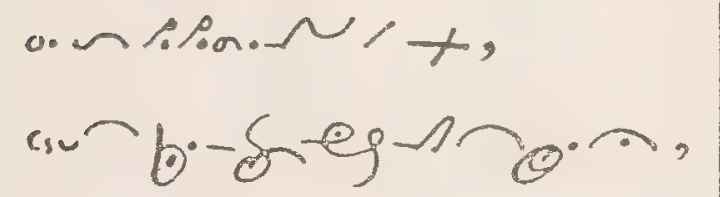
Q. 62 mas:

$20\{r d y 64$.

2. a. con, qus,

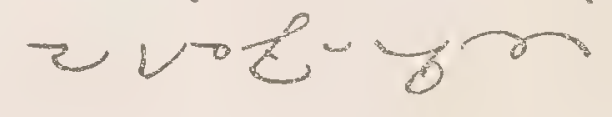
020 p 1965,

- 
MANUAL.

37

9. Passion.

1.

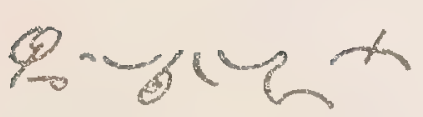

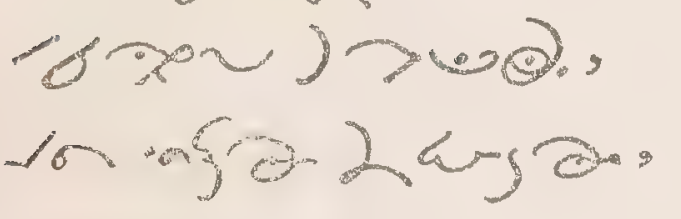

s buc

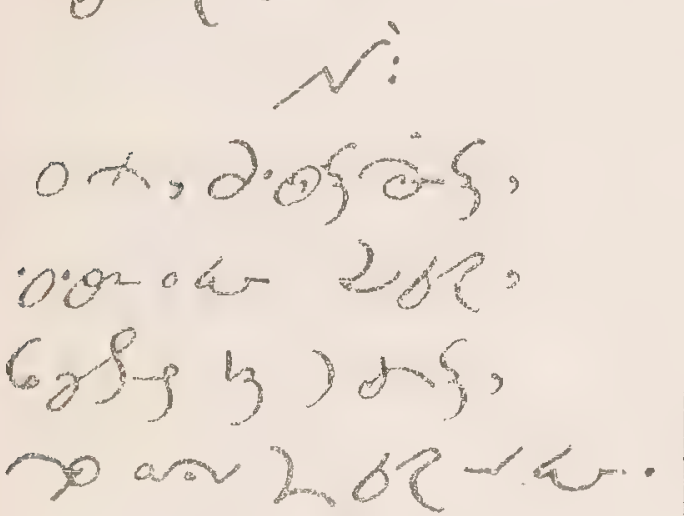

Q

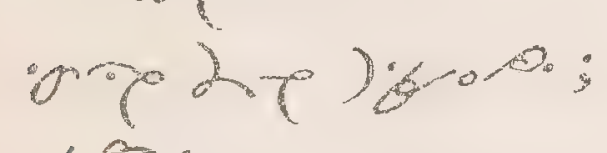

195? Lons?

457 क 430.

3.

Q9

0.-

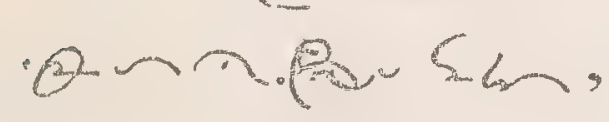

olevou.

4.

里秋“面之。

"ग्ड?
$>$ fore o q $>G_{2}$

ste-onst

5.

$-0.55-8,>-\infty$

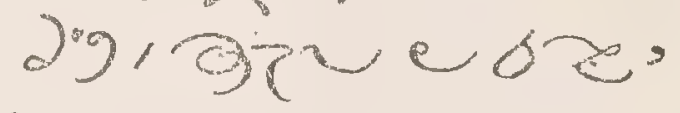

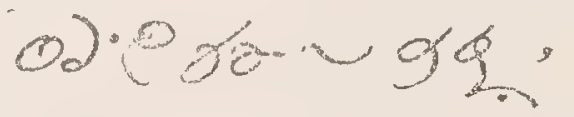

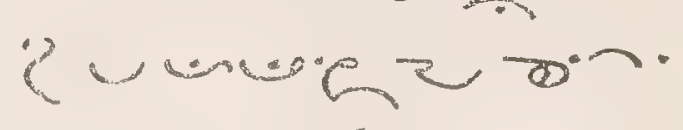

6.

Poryog?

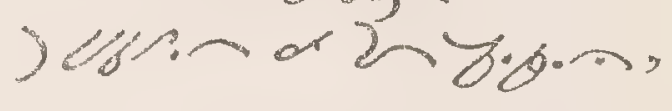

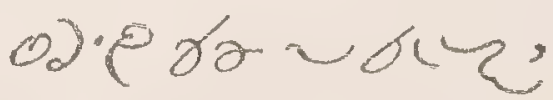

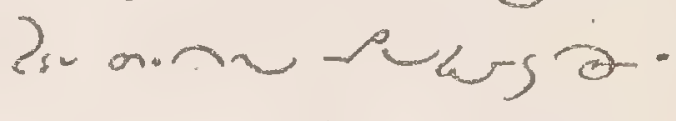

\%.

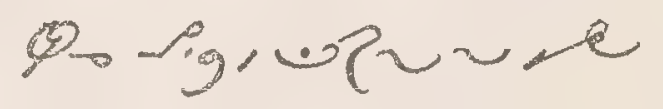
$\sim 2.00$ )

ode end?

ral 2 .

10. Regina Coeli.

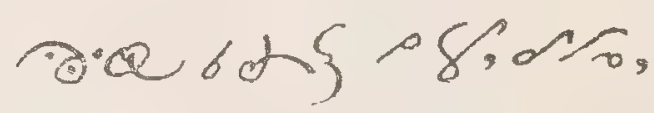

वेक्र

yn

gुo.o.) 
11. Unis aux concerts...

$$
\begin{aligned}
& 1 . \\
& \text { antorsing, } \\
& \text { ar }\{e \text { e. } \\
& \text { d } \\
& \alpha 04.0<\alpha \partial \cup \text {. } \\
& \text { r: } \\
& \text { o } 9 \text { S. } \\
& \text { 3०a } \\
& \text { 2.e-1P } 1.0<\text {; } \\
& \text { Powos? } \\
& \text { 万.9.6." }
\end{aligned}
$$$$
\text { - } 2.17 \pi+3 .
$$$$
2 .
$$$$
\text { su } 63 \text { rou. } 8 \text {, }
$$$$
60006 \text { क } x \text {, }
$$

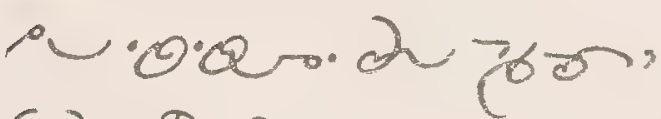$$
62>2)>7 \text { ? }
$$$$
3 .
$$

- qLu - o $\}\}$, 9क्ण. 108 :

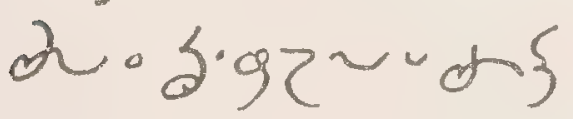
sion- $92-16$.
12. Purgatory.

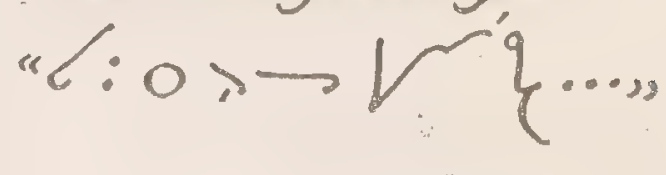

L.

0.6. 2 6no o.. ए o.0.02h. 2 b2 L,

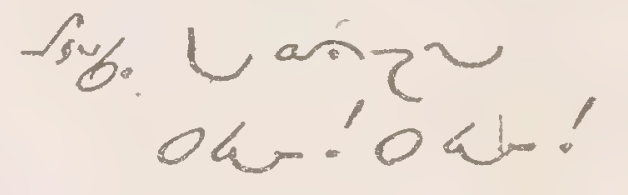
उद6. 2 fogn,

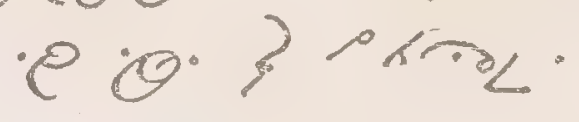

\section{2.}

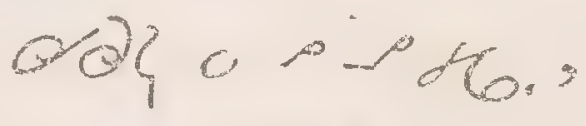
92. <4.97;

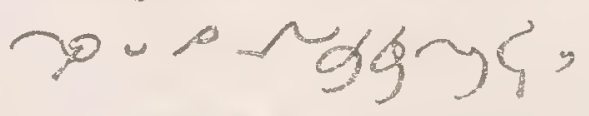
00.230 ql'ib. - $596<-9 y+$

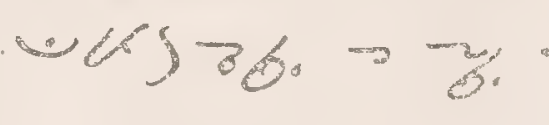

13. To the Angels.

$$
1 .
$$

Qd\}opin, र' xyo.9: 
$82 \cdot 86 . \sim 969$ ?,

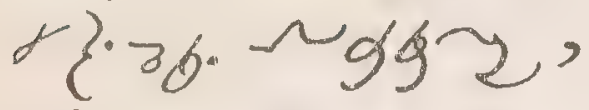

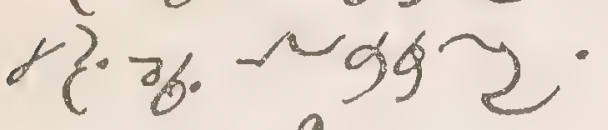
2.

$\tau^{2} \cdot 83-2<+$

- y०9 26$\}-6$

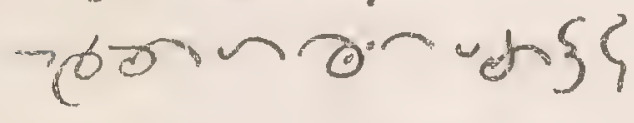

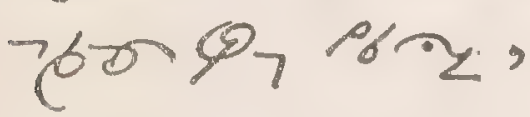

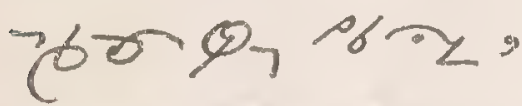

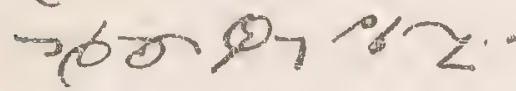
3.

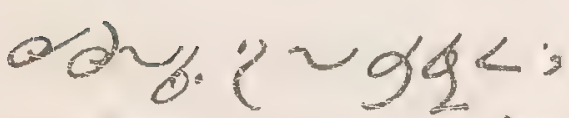

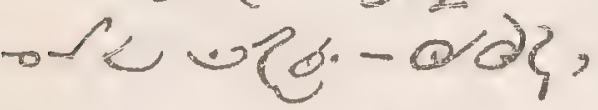

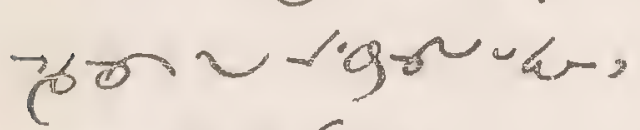

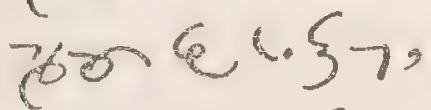

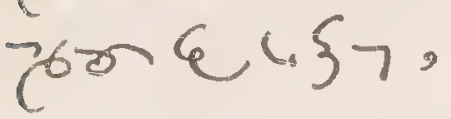
तुक $6 . \xi_{7}$.

14. Miserere mei.

$1.962 \overline{20} 00.00$

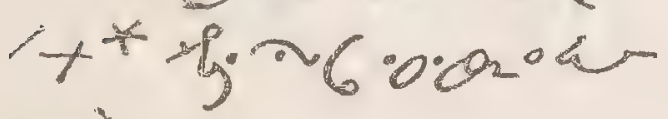
1262 .

2. 2 99

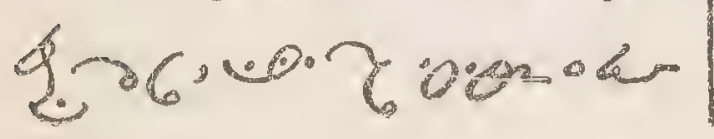

$$
\text { . }
$$

$-262 \cdot \ldots$ 3. .0. $\zeta^{-1}$ ) d 5002 . k- $1262^{*} 9 \cdot \tau^{-1}$

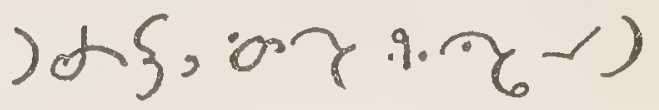
小\}.

$4 \cdot y^{2}$.eo. $3^{x / i g}$ 世2nanese . 5. (कб) (n.

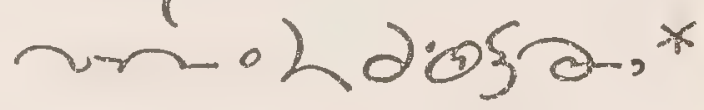

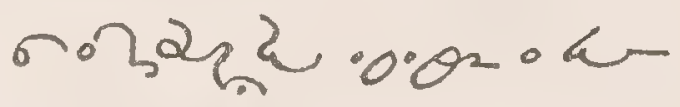
- 262 .

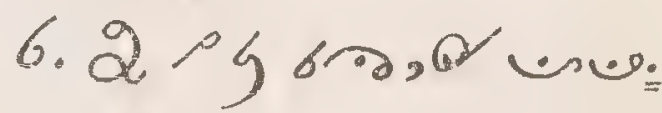
sy*angy, ) "c.ugag.

15. Contrition.

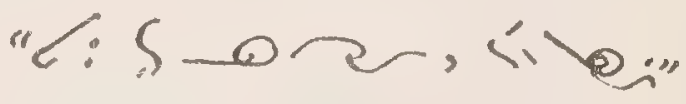
$-1$

$0 x . \log 60^{\prime}$, sy. o. -. - क. $) 2$ : -0. $\partial 0\{\partial\}$ र $\}$ - onarga<, ope. 
2.

02.00 a.ry-ars, enugry 2 gra, - by mo 62 ks 7or 62 La a h ope.

0.0 y 3 . - Low grpe-ngres

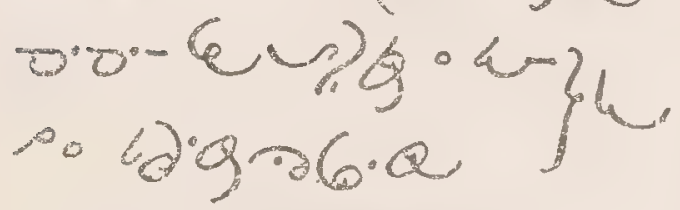
oren.

16. Real Presence. "ci - ed \& - - .

$\therefore 0.0 \%$.

Dos $\theta$,

$200-27-?$

?uary.

$2 \times 5$ मक

Euar.

¿u35y:

se rouno.
1\%. Before Communion. "Tio of of $6 . "$

1.

$0.0-c \partial 97$

- s ra,

o.o.n

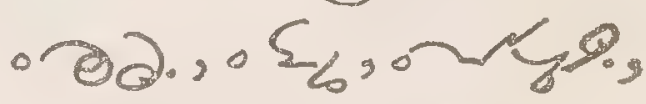

sko-1

o.o.e aigg. 2.

$205 \sim 25 \%$.'

2 ' 7 - $q$,

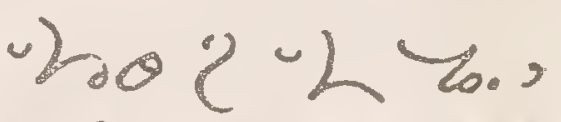
op

$$
4-5-8 \text { : }
$$

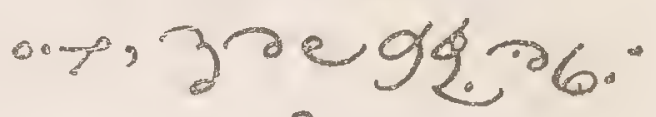
3.

oratyara,

¿थungry;

मे० $12 \log 2$

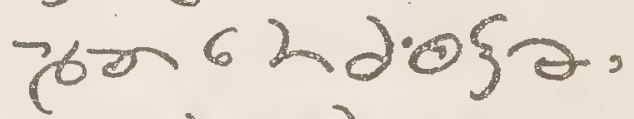

- 0 L 68 -

. $600-2<$. 
MANUAL.

$4 I^{\prime}$

18. During Communion.

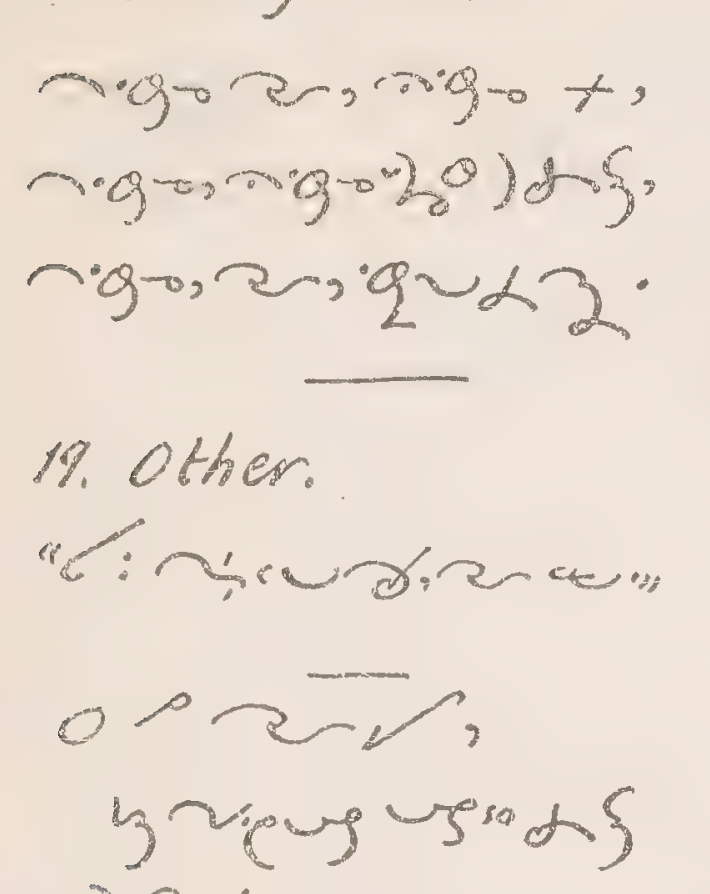

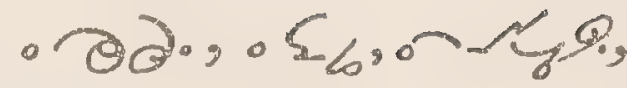

$$
\begin{aligned}
& \text { 00.-3凶2 小\}. } \\
& \text { थ गु मे, } \\
& \text { "s.t }) 2,94 \text {, }
\end{aligned}
$$

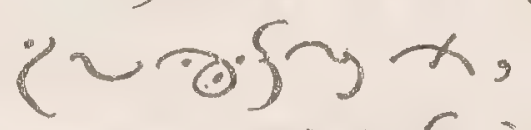

$$
\begin{aligned}
& \text {-0.- } \\
& 2 .
\end{aligned}
$$

reug Do. 2,

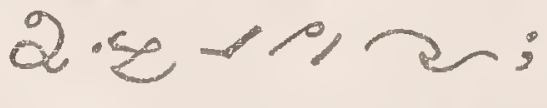

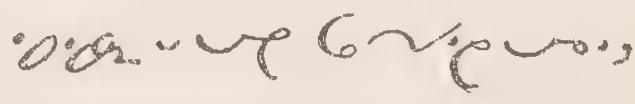
surl, 2 Lioden QLbT:anue, oonve C. 5 . vin? दोy,

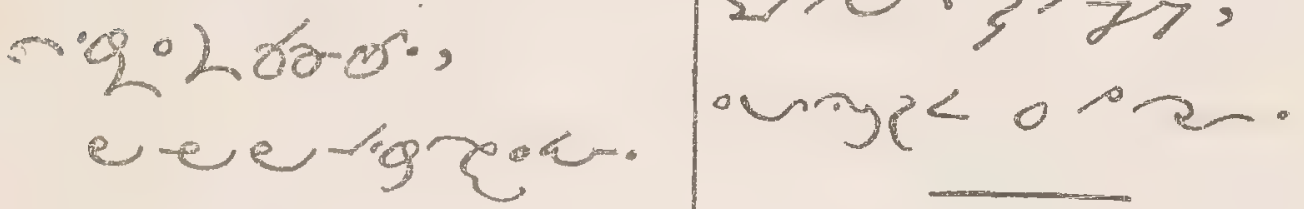

oprd,

2\% Tove \& Desire.

con 621.00

ereesigeor.

30. After Communion.

-1 -

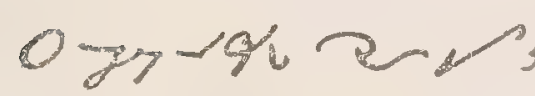

$0.0 \cdot 3-9.2)+5:$

ore, opg कr, 602 . "Wary, 602.0 . o.a 60D.6 U.0.9. eny $) \theta$, तण द.व. 
22. Panis Angelicus.

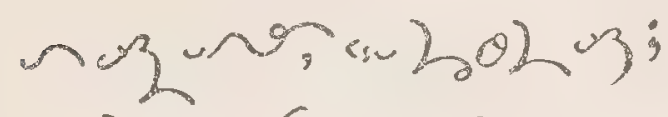

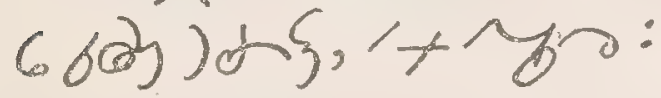
$0.00 \Omega 60.02$, hoo, $985-96$.

02 t, )6的:-10, 992620,2 - 12

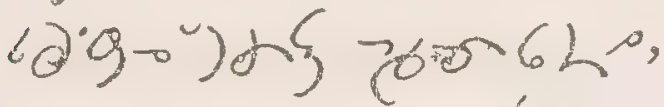

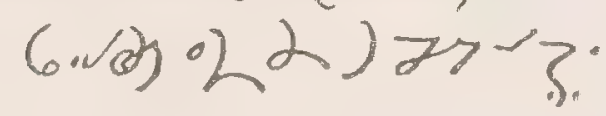

23. Sorrowfor Sin.

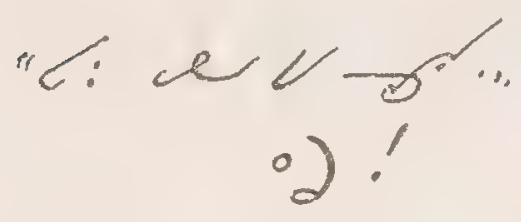

Q $\sim \gamma \%$

no)

).0.02 2 ansas.

$$
\text { - }) \text { ! }
$$

(6)二 $\%$

2o ).ot $\xi$.

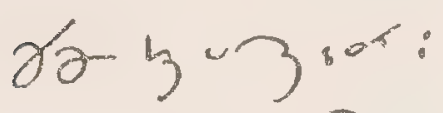

$$
\text { ponn, }
$$

9 -क्ण.

$$
\text { 2 6bor? }
$$

$$
\begin{aligned}
& -2 ! \\
& \text { - Larga } \\
& \therefore \text { - } \\
& 92.062
\end{aligned}
$$

24. Dies iroe.

$3 y-9 i,>\not>-q i$, -gger $x)$ - ( o क ,

ग) $\partial \partial \cdot 2)$ ज'

\section{Heaven.}

0 ( $27-3.30)\}$.

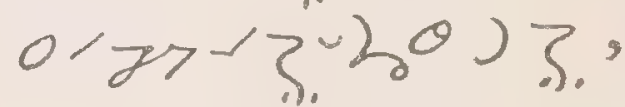

0 ' $27-46.20)^{-14}$

- 20 o ge 2,

$-2001.08$

$-20) 6.06 \%$

26i same.

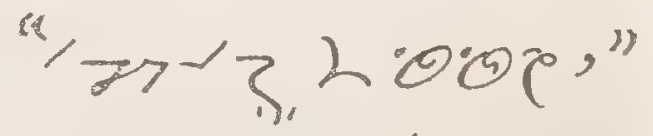

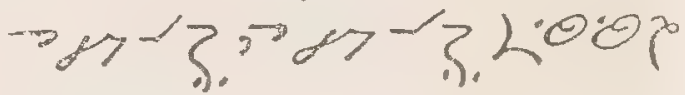

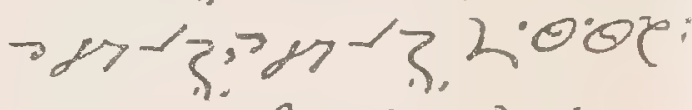
अ-3:

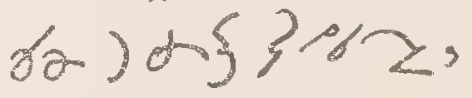

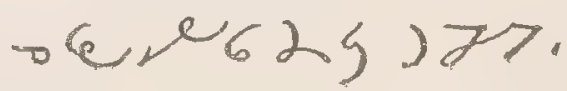


MANUAL。

43

To the Holy Family.

ox! ban.ovir.

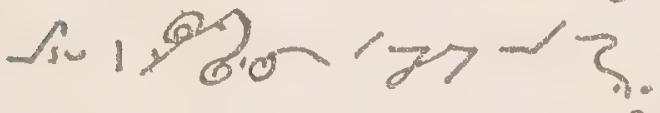

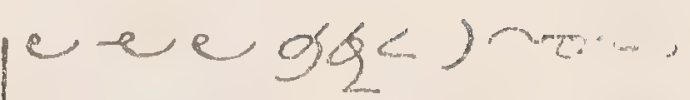

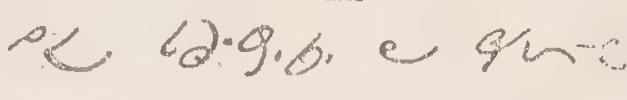

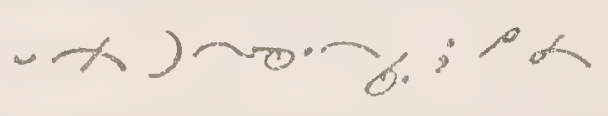

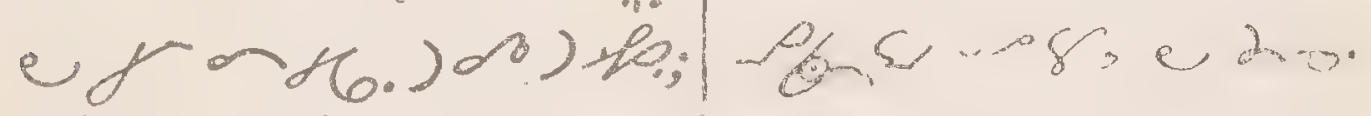

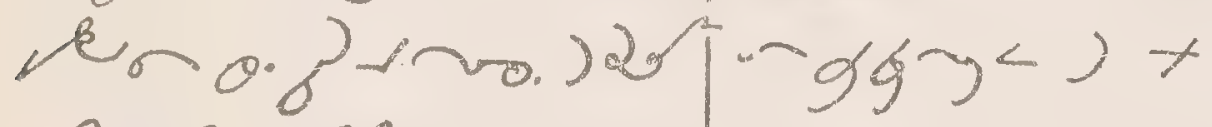

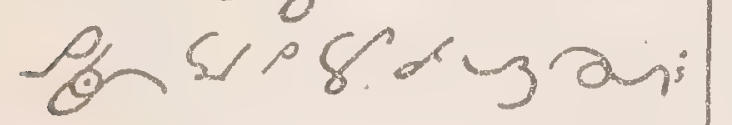

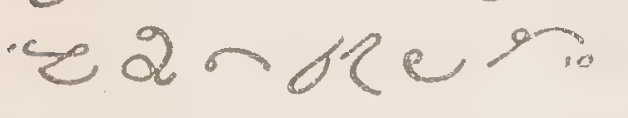

Guard of Honor.

ro.e 36.603

ox!. שe e p.ee

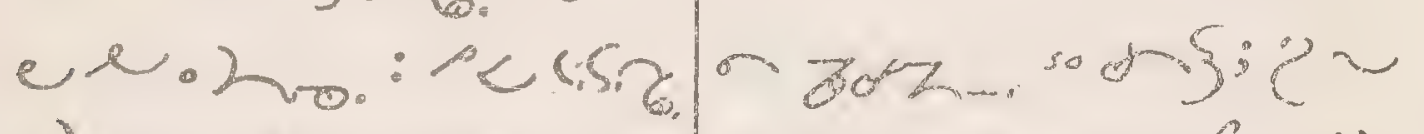
1) )

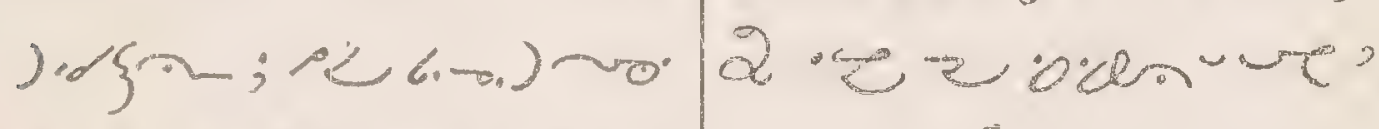

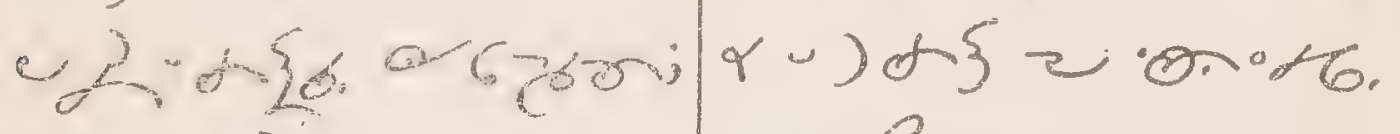

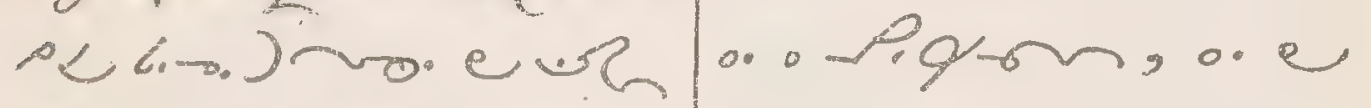

pro doterare ungu dage

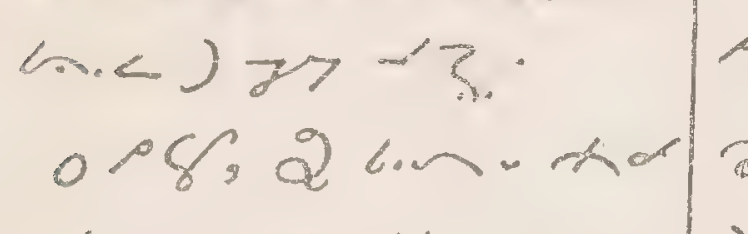

s. तु $\mu \xi,-s$

sogerecks

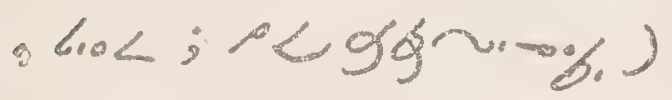

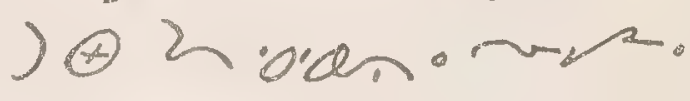

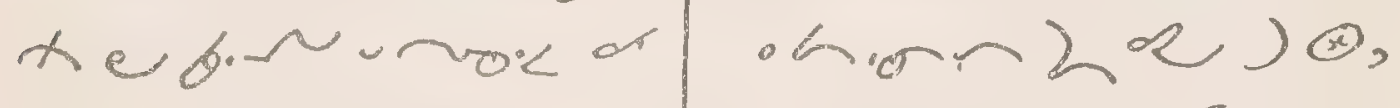

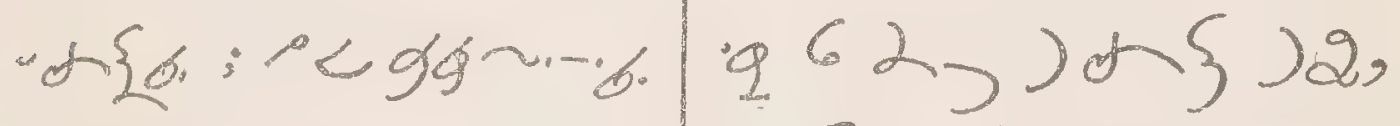

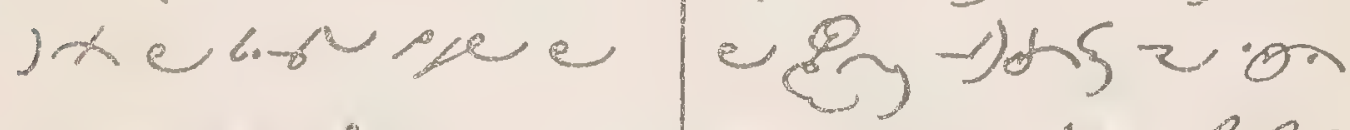

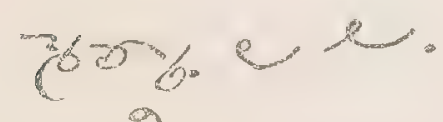

$02-321 \cdot 2 \cdot 62^{2}$

حoas;

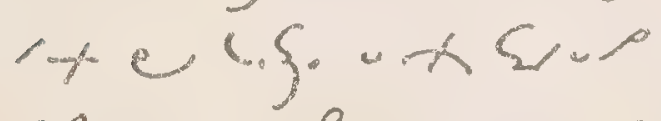

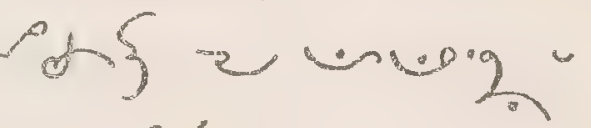

$8)$ os) fos; oc (.5.)

(2) 16. 
Hour of Guard .x. Offering. ox.'ंgn, 50.) ब. उ6

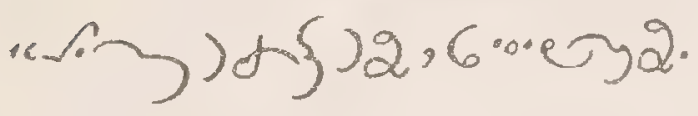

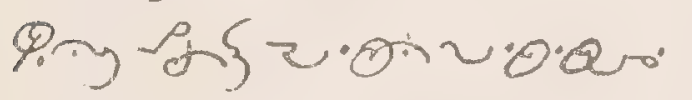
- 86,90 . a suciou

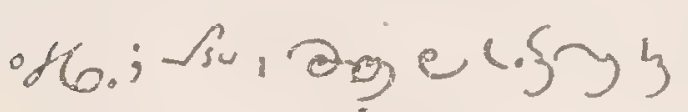
$30-02 \cdot 76 \cdot 000252=$

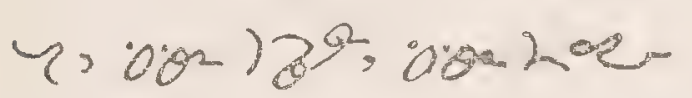
$33-.02 \cdot 36$, , 50 esu

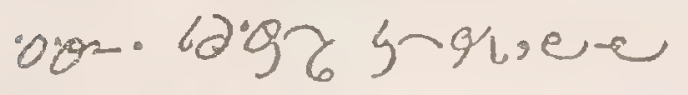
e.grakarere buin-jor $\{-2$.

ox'bry - Sh ox, e \% 6:ar-12 62: $13 \% 9$. 'or6g,are ggun.r. $186.0<.992$.

\section{End of Hour of Gnard.}

ox! g/ze. n z .0 2 - b6r. 20.32 el.5y. .0. $\{2.000-9$ 里安。

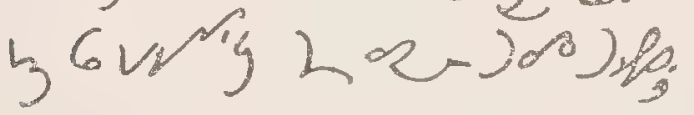

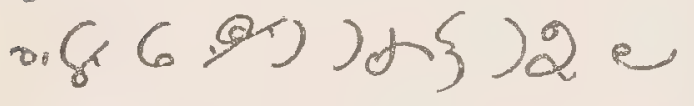
bore.ary.
Visit to the B. Sacrint.

ox:OD.E pus )

ox!ysoe बे.gृ. म ल.

ox!'年的少. Ia, ). $\xi,-b .9$ are $\varepsilon q=$ .ry of

ox'ouy ce nasur; zods SIQjuhasa,

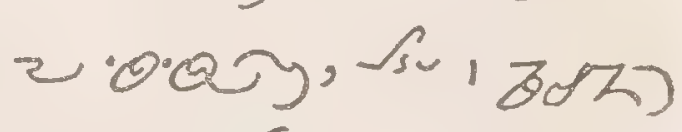
$\sim \operatorname{s}$.

ox! 6f? P

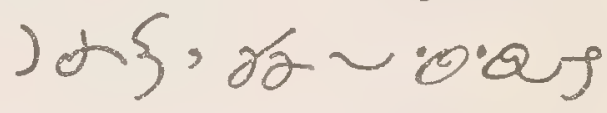

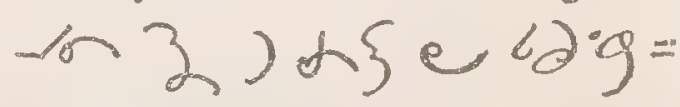
6.' h o e rog $\rightarrow-\zeta$. 
Catechism

in Shushwap.

\section{God.}

2. $2013 ?=+83 c$ 2. $7416 \mathrm{a} 4$ ? $=$ tomeneorn? a.e e.ow d a

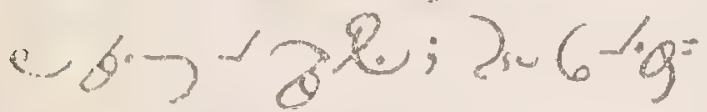
sर․

$3, b, x^{3}=2 y^{9}$

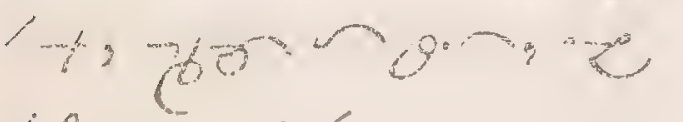

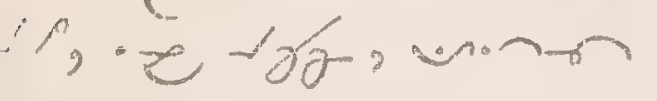
de on ver.

$4 \cdot 7-5192 \cdot 5 \cdot 2=$ dze $y^{\prime} ?=\ldots \mathrm{e}-\mathrm{e}$ 八 बिए।

$r>s^{19200} x$

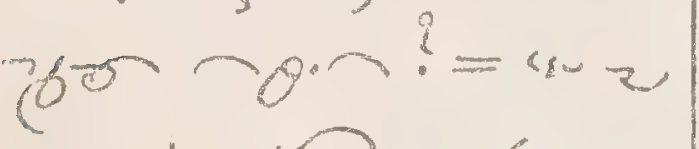
e, Uf tod ae 儿Q.

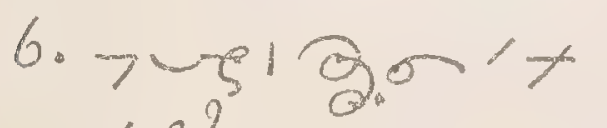
ए

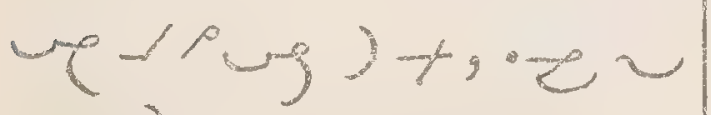
act.0.0nue.
7. >ง 192.0$)^{1}$ 둥. $0.0 e^{?}=$

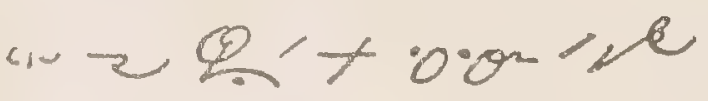
$10,20.0 \mathrm{n}-\mathrm{T}$,

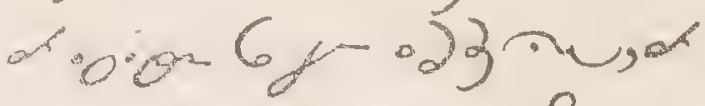

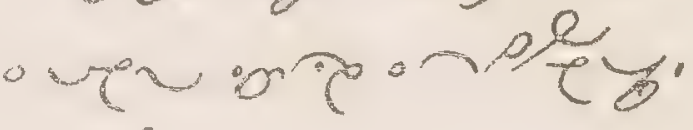
) 2 .

8. $) \cdot 0 \cdot 0 \cdot x^{?}=$ = .0.0n J 0.05 .

II. Trinity.

$978<0.4$ ? $=$ $0<\cdot 0 ; 29 \cdot<$. 10. $48<\cdot 0 \cdot(9) x$ $=6: z^{\circ}(g)+: t$ bid, 7 bo, tw: su aser 2 . 11. $-3-3-2 ?=29$. $(-)$ ) 12. ( $80,0.0 \cdot-)$ tal?

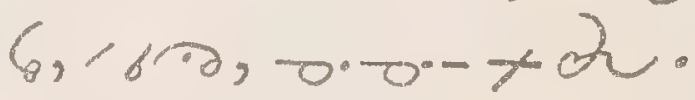
13. - 万人, -0.0.-) +a? $=6, h \partial,-d \cdot 0 \cdot-x d$. $14 .(x, 0.0 \cdot-)+\lambda$ ? $=6, \omega, 0 \cdot 0 \cdot-\alpha$.

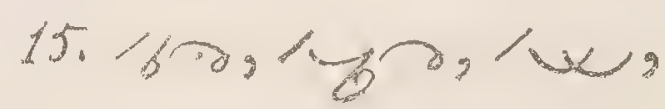




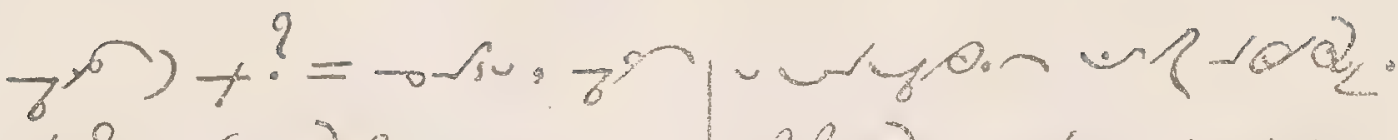
N, 68, $2 \mu_{0}, \ldots$

$16.62,7,7,9,683$

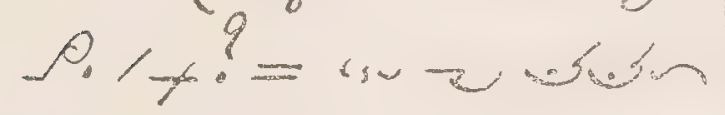

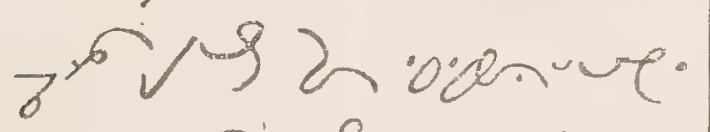

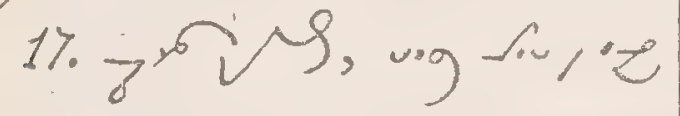
$-19,4 \cdot 9-\infty, 10.6, \cdots 09$ $1 \cdot$ -

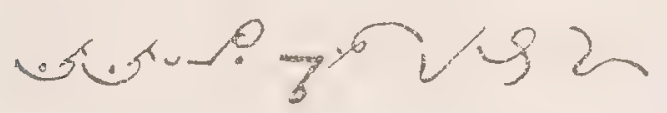
o.enure.

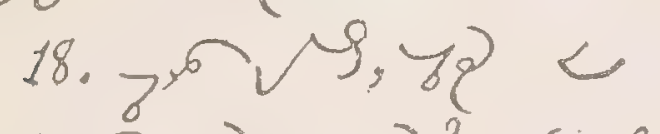
$\sin , 8>-\infty)=6,-6$ b c c o 111. Jesus Christ.

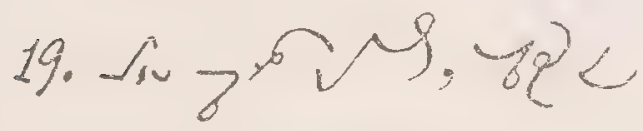

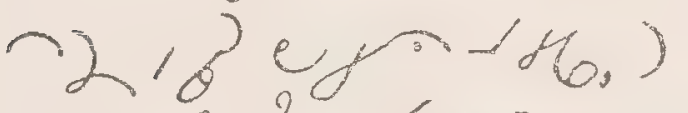
s) $9 . ?=6,375$ M 2 e $(n-4)$ s).8.

20. $\operatorname{vog} z^{\circ} \sim 9$, -2eef $-160^{?}=1+$ 万人。

2\%. od $1+$ bo, 'g-v $160^{2}=1+$ bre, en

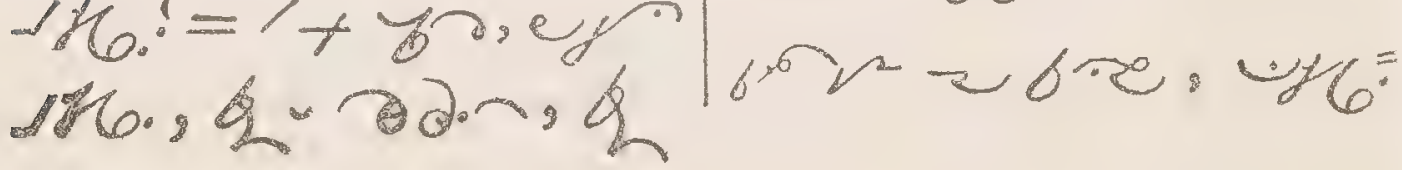

6. - w.

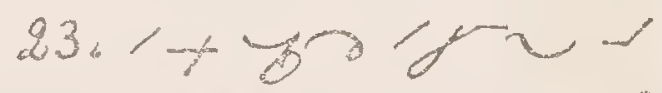
H. $=-$ sin, $1+x$ ys +2 3 , d 6 "s 2 . $24 \cdot 745+18^{2}$ $-60^{\circ}=e$ n. $1 / \mu$ sol, ore $\alpha \sim)_{71}$ $\checkmark$.

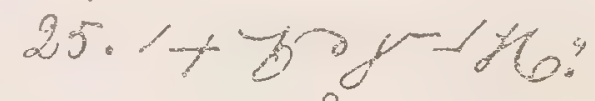
an, ton? = $1+30$ f-16.9 x. bm.

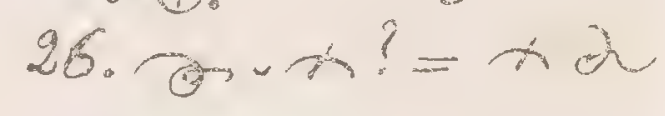
$1+60 y^{-16}$.

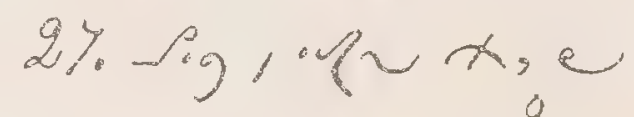
n. $\sim \alpha-5 \partial<$ ? =2 be > a.n.x-Uargo 0 28. $72<76.5 \circ$ $x \rightarrow \infty \operatorname{son}^{2}=6,2$ 
बin.

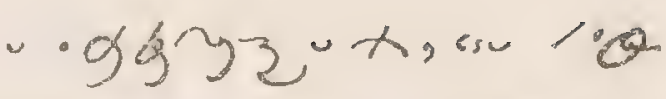

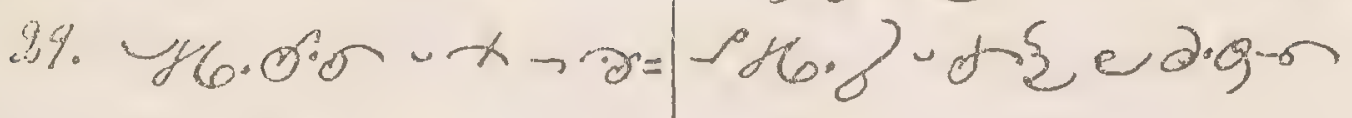

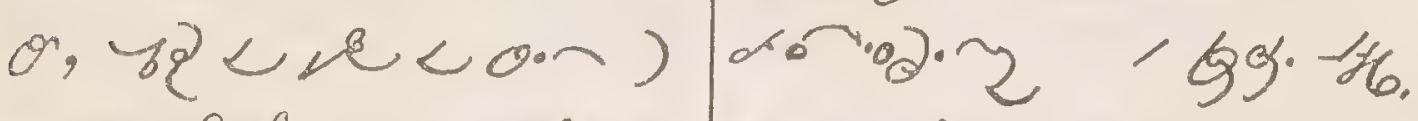

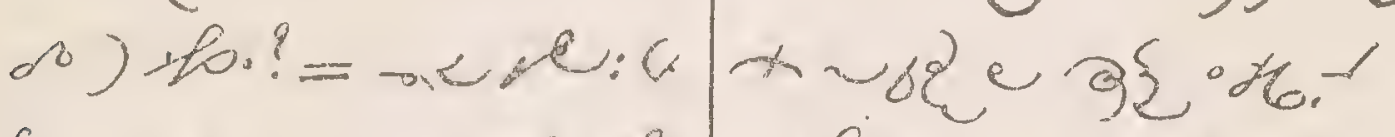

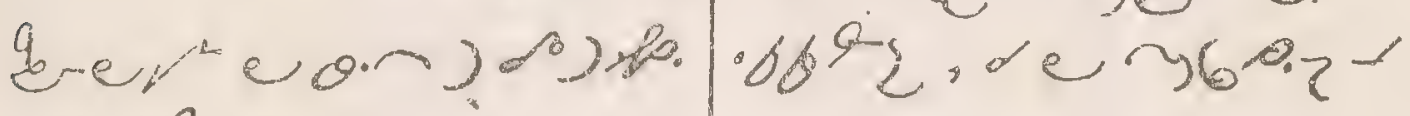

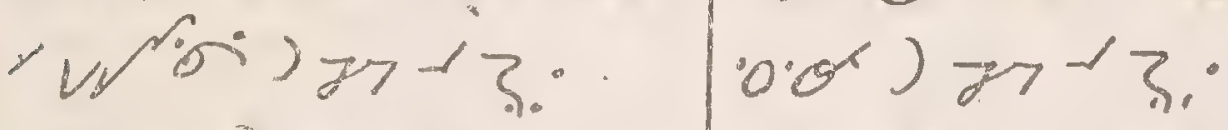

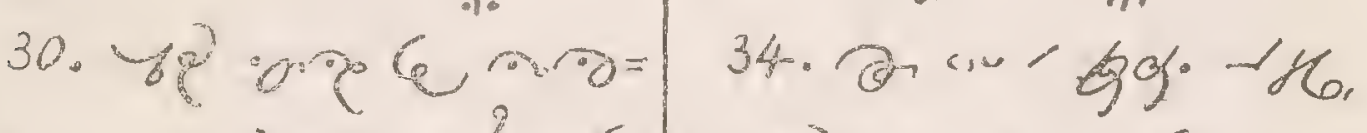

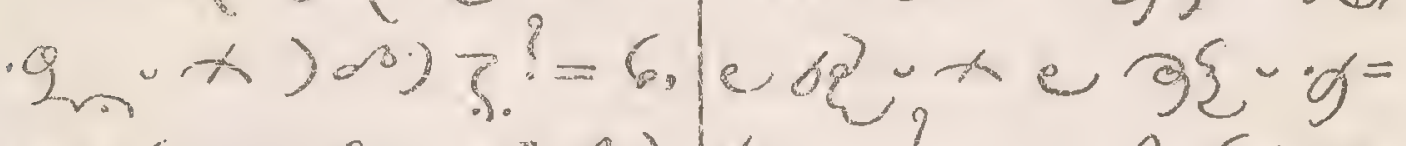

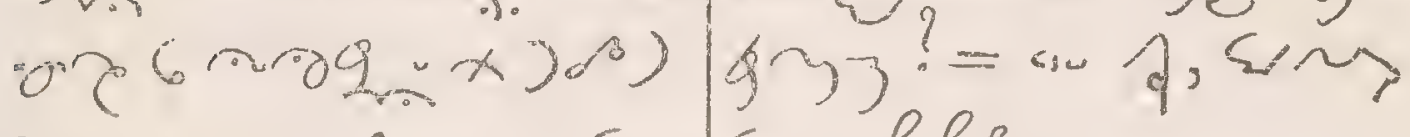

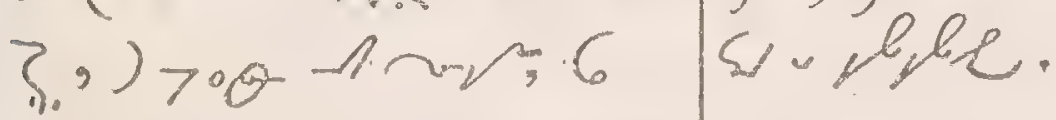

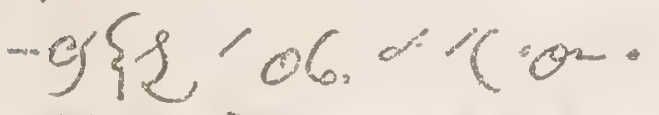

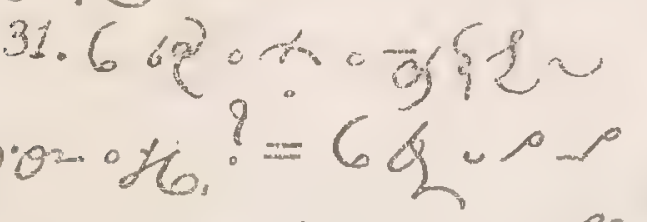
6.) $87-13.6 \geq$ $-4 .+16$.$) '$

JV. The Church.

32. o, oute ( ) to. $3^{90}-x$, ar $3(2)$ (2) $79-3 !=$ बे।

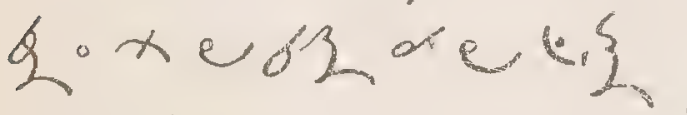

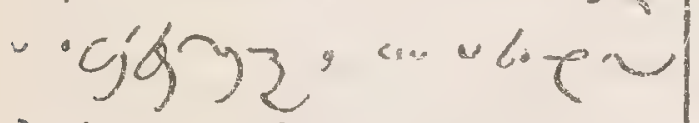

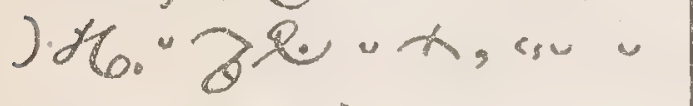

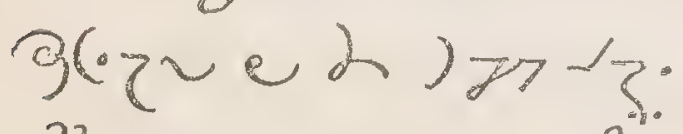
33. vs ‥999 $4 \times$ ? $=$

$35 . x$ - $82<62-1$

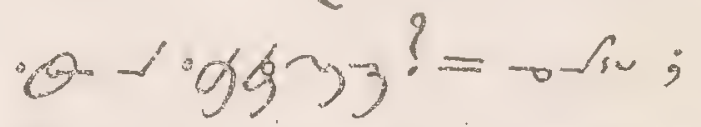
3.0.9692 $\cdots ; \%$ . $2.2 x^{2} \cdot 26.77$ 13. 36. $\log x-1.9973$ ? $=\cap \cos 2-160^{\prime \prime}, 5$

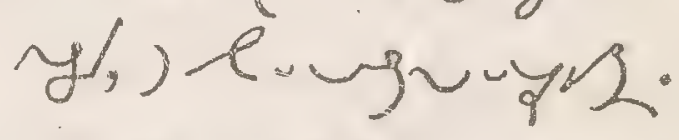

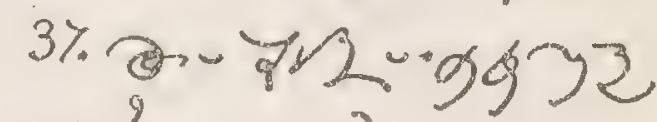
$\cdot x ?=x, \alpha \cup \frac{92}{2}$ -.0ggry3'-4Qpe;

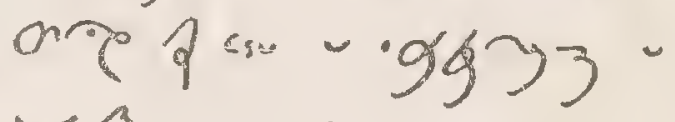
虹的。

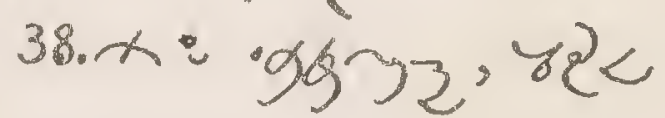

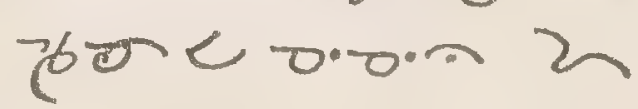




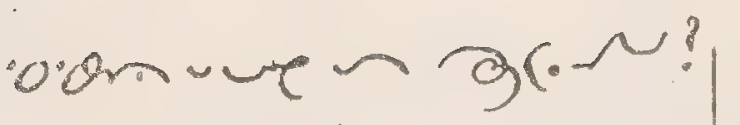
6.ben $0.0 \cdot 2 \cdot g=$

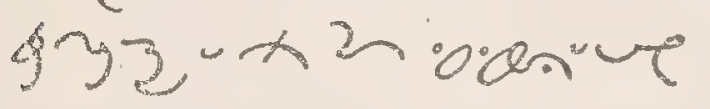

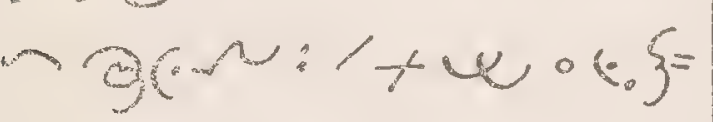
zuene.

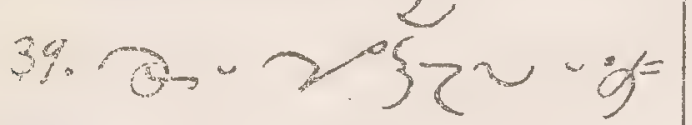
$\left.\sqrt{3})^{-1.962}, 2\right) \cdots$ beaga? $=6 ?$ $2 c^{2}-4-5 \alpha, 00-$ Naquan .

The Creed.

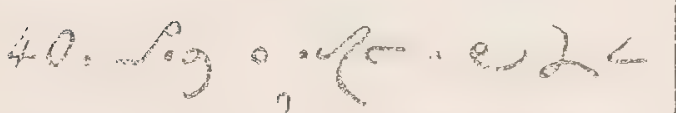

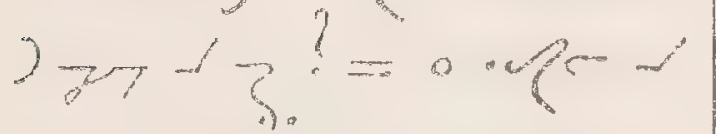

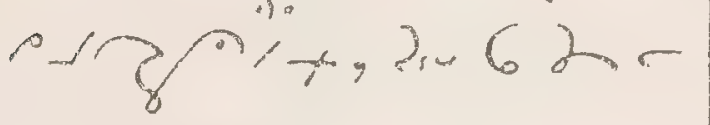
$287 \sqrt{3}$.

41. $\partial " 1+0 \cdot 0 \cdot-1$ ? ? $=\frown 45 e^{-14} \cdot 0^{\circ}$ - 2 .g-

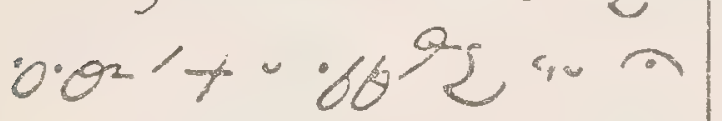

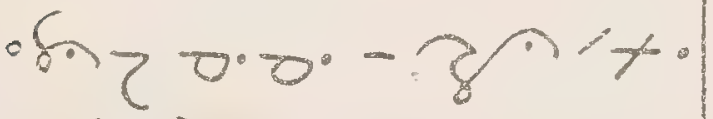

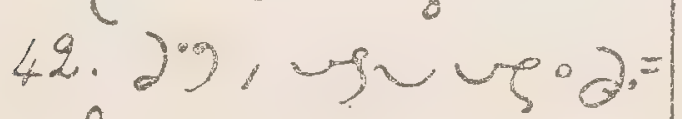
$\left.9 r^{?}=-\operatorname{lom}\right) \operatorname{gog} z-g /$
$43 \cdot 082 \cdot 0-952=$

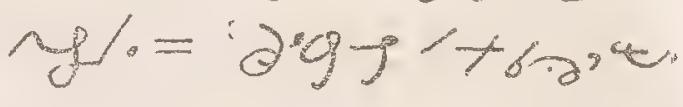

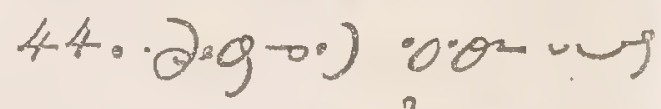
$1.962 \cdot 1 \mathrm{~g}:=6.2 .99$

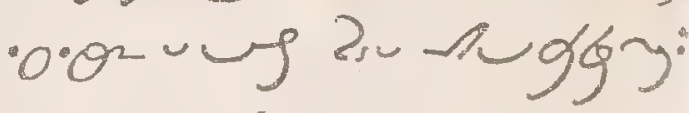
दeg, $2001+4$ lixe

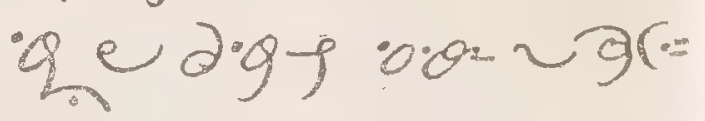
$\sim$.

VI. The Sign of the Cross.

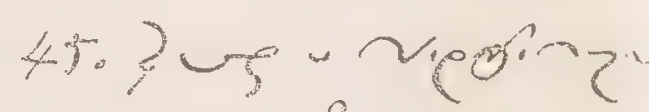
$4+80 ?=273$ 46. $-190.010 .780^{\circ}$ $=3+9)(5-9,59)>5 h$

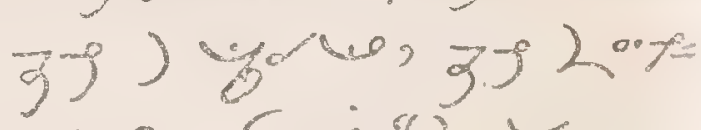

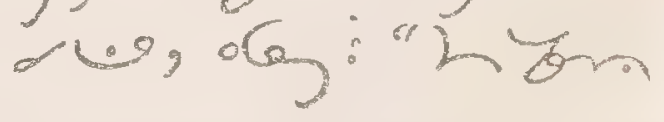

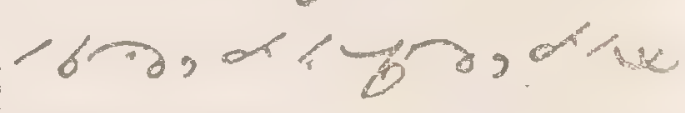
s.

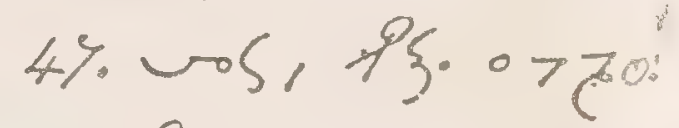
$=695 \xi-2 \beta \alpha \Lambda$ 6r-x)>48. 3005, भ कु० - 2 $\mathcal{2} ?=2 \cdot 6,1+6 \mathrm{~m}$ afo, a w. 49.3w5, 1950 ( ) ( ) > ब.? 
MANUAL.

49

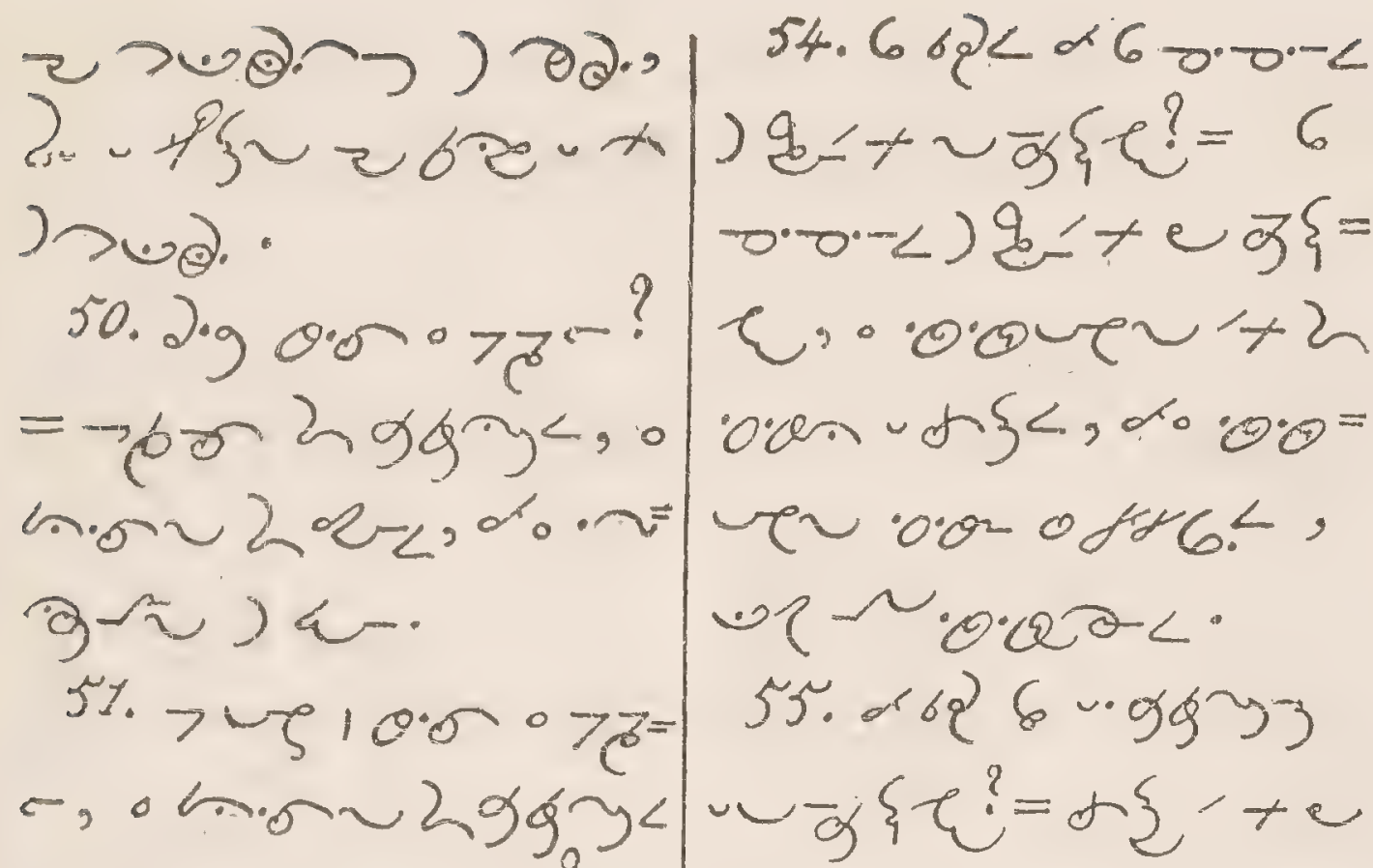

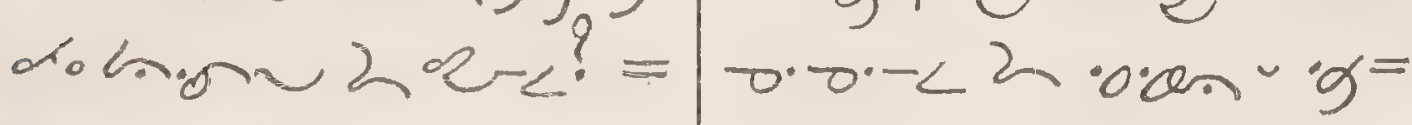

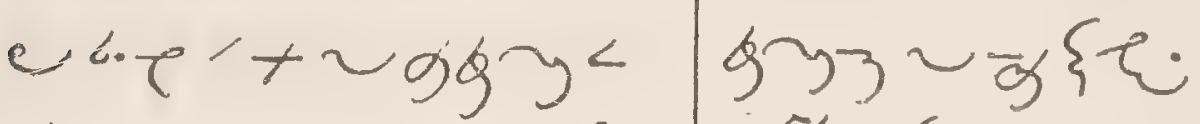

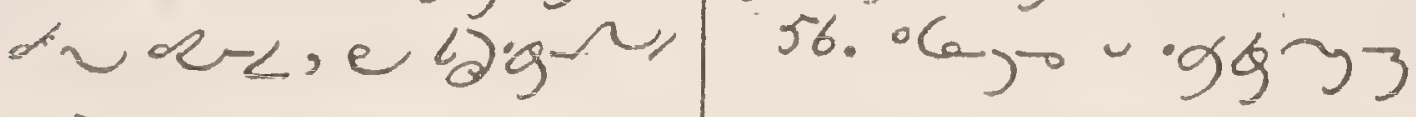

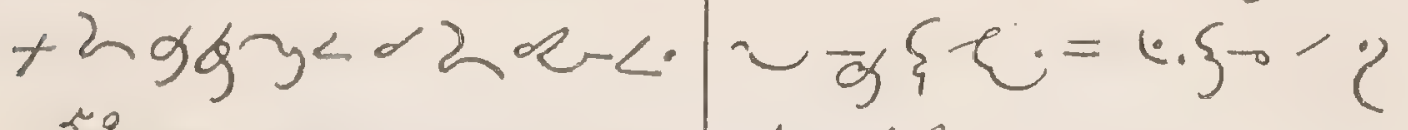

52. > vos $10^{\circ} 0078=1$ vis, w..

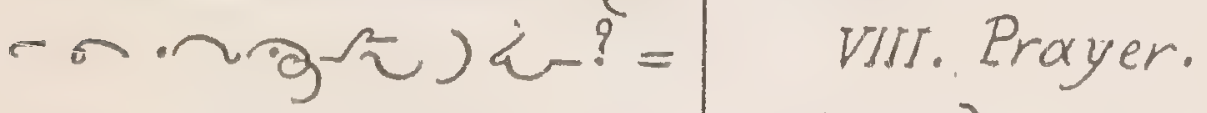

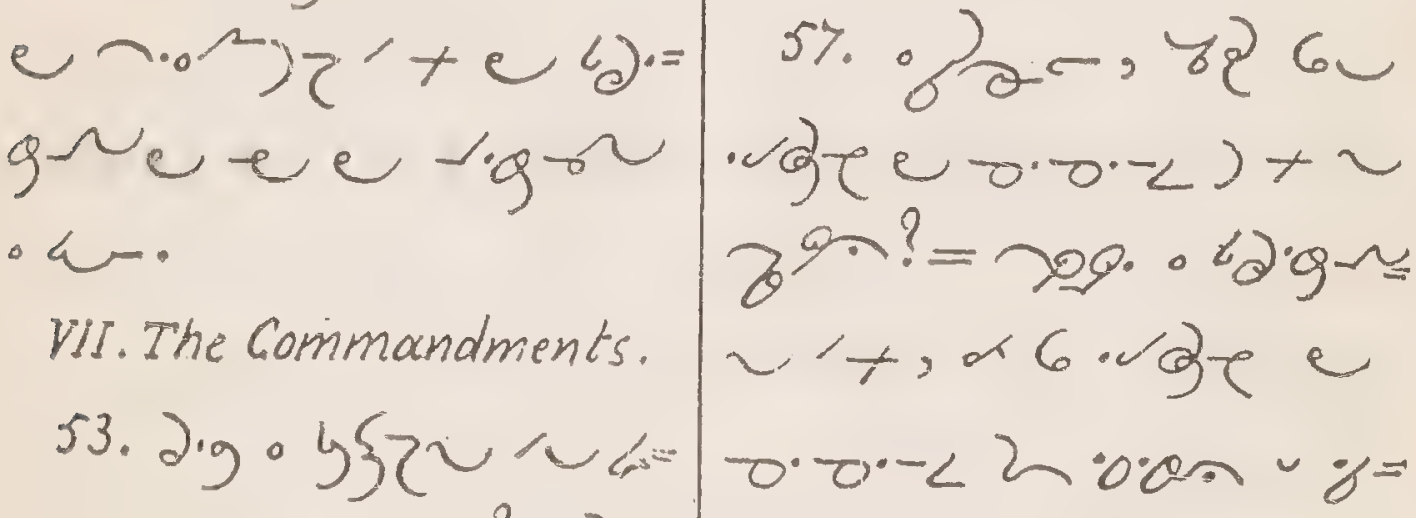

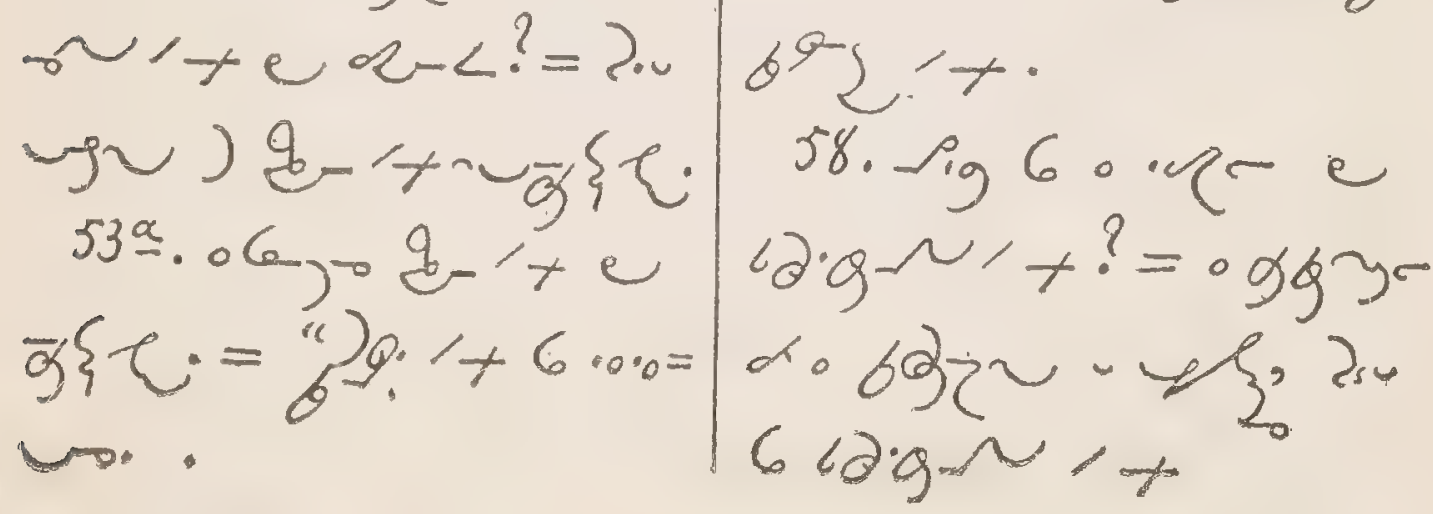




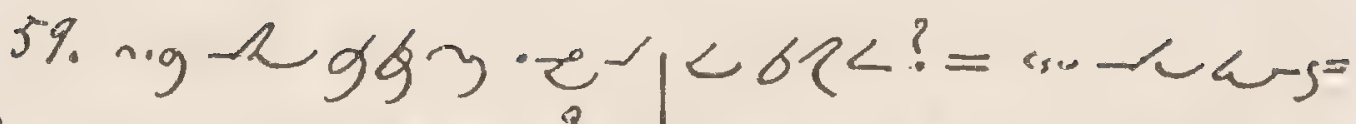

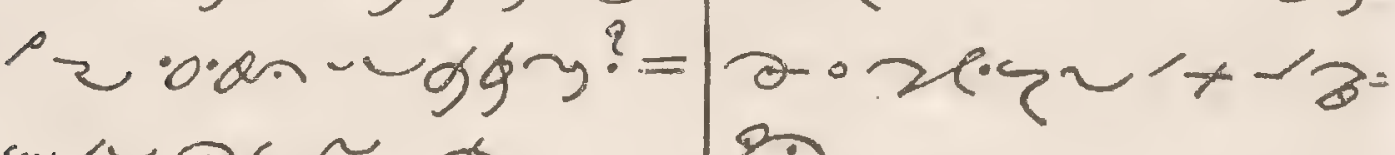

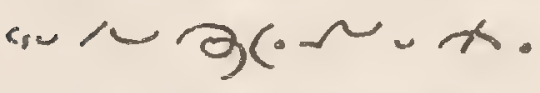

60. 06,0 , hoggry. $=6 \partial \angle P A G 4$.

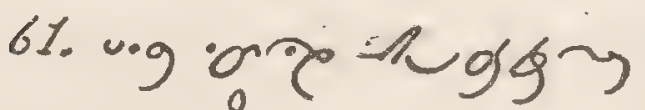

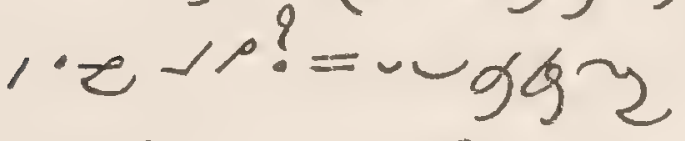

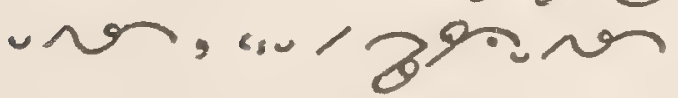
) 98 b.n. .

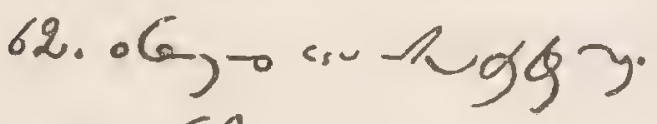
$=8.08$, a

JX. Sin. कर.

6\%. 6.0 rucursa

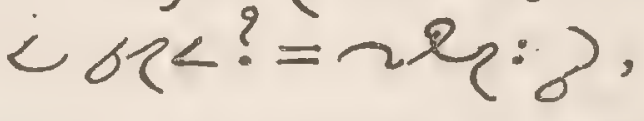
d, «h-so, d

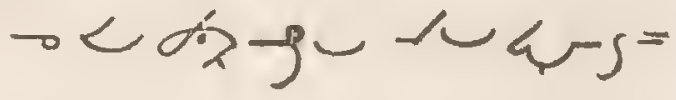
2.

68. น⿻. $\cos \partial ?=\sigma_{s} \sim$ $+, 2 \sim) \cdot 2+\frac{1}{6}$ 69. $9 \cdot 1 \operatorname{sis} \wedge \mathrm{L}=$

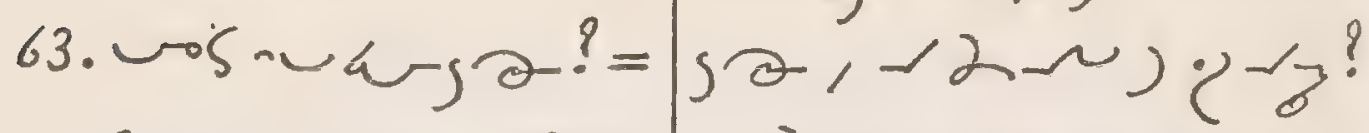

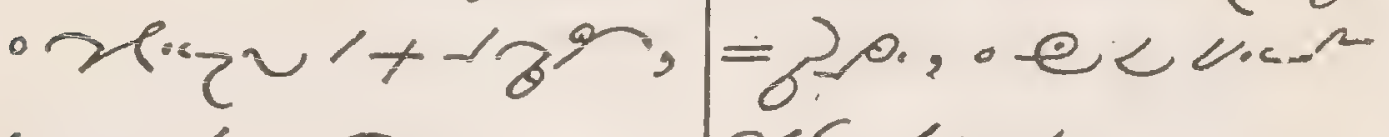
su- urso.

64. g.aruarsa?

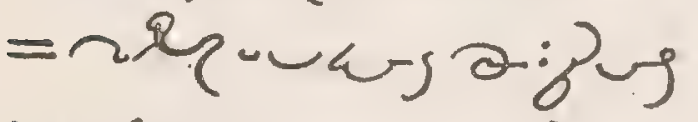
)小 $\{<-\ln 2, \alpha) \cup$ $62<$.

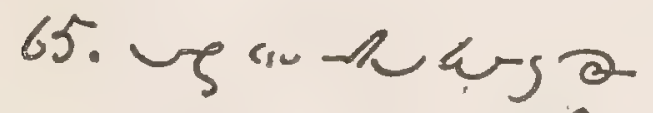

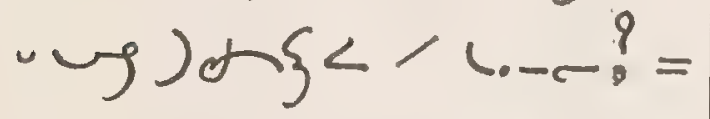

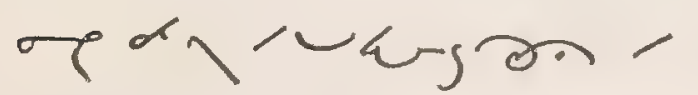
2loñ, $1+3^{9}$, rug ) o ' 'gra. a $6.620<$. 70. $\sim \sim<\omega$ $\sim \operatorname{cs} \partial ?=\multimap<f^{r}=$ usक $\rightarrow$, r. $8 \csc \alpha$ ara 1-ectaic la.g $\sim 1$. 71. g.oreqeu ard? = reror: ᄂP.: $<,-\infty \xi<, \rightarrow y=$ की $<<, \rightarrow$, $<$, d

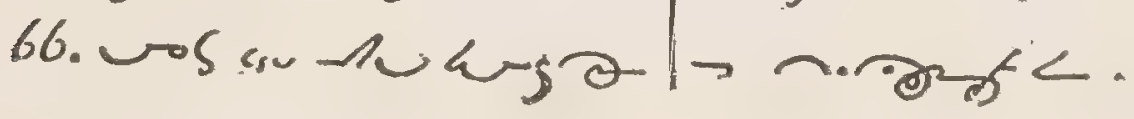




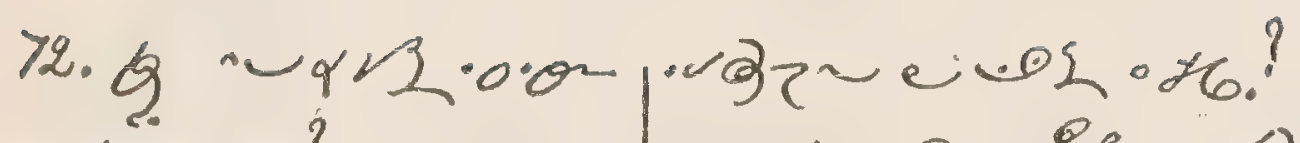

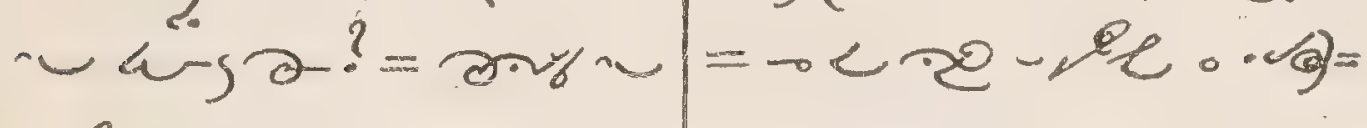
ar $0.0 \mathrm{nu}$ us 2 : wara, vor?

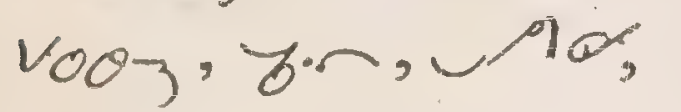
${ }^{\circ} 83$, - ans. x. Baptism.

73. >nosuxibz

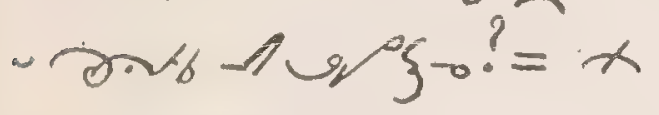
bz-arsergoe exphyor.

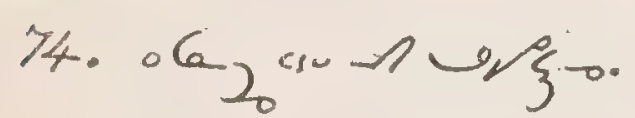
$=$ z, $\alpha \mathrm{ten}_{0}, \oplus$, ive

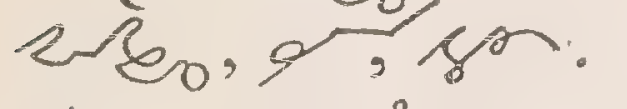
75. $5 \cdot 3 ?^{\circ}=2 ?$ uand us od $\xi<$ 'fa; वr qू-ars= क- म. 2 (2.) $x$ 12 .6. 9953 .

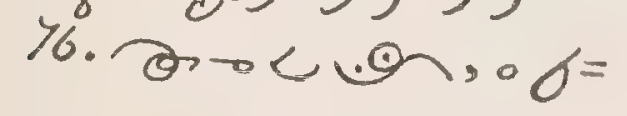
Du, $\rho \circ 62 \sim$ ? = के

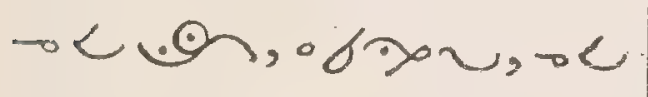

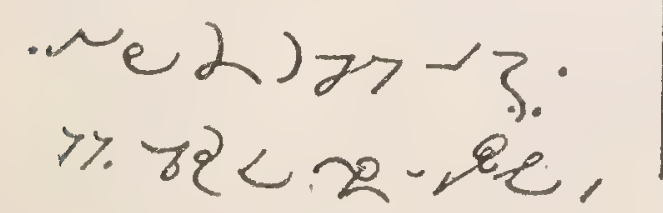

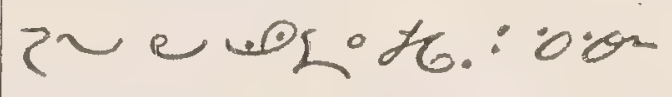

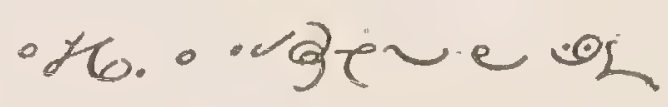
78. $.9 \cdot .2 \sim \circ .05=$ $\sim ?=6$ ح. $\{-86,2$ 外, 2-6669: ogry 2 2 tom. $160, \alpha$, bor.d 凶.

79. - ig..pय. के ore en? $=6 \gamma$ r Lonzerget

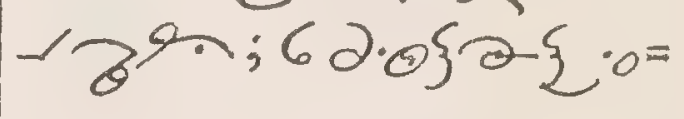

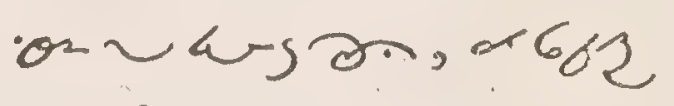

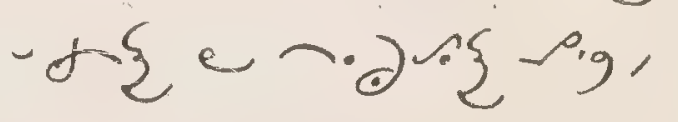
.र - $8^{9}-994=$ $32.0-\pi$. 80. $05,-2$ सु - oouni e on?= o.0.ounenan,

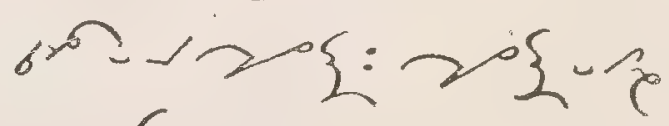
थर्... 
$625 \operatorname{mos}\} \pi$ $16 .\{>16$.

$X I$. Confirmation.

81. wos sakes? = areof yp ror

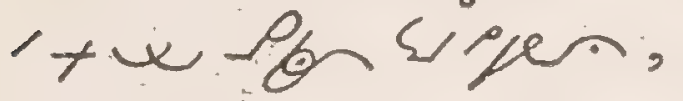

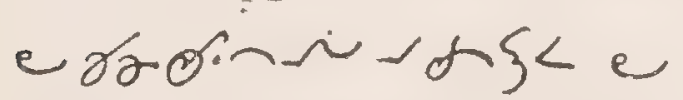

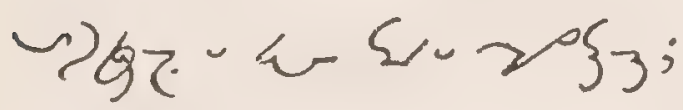
)ruegr.

82. ब. 6. $\{2 \sim-\rho g=$ $\left.\mathrm{cos}^{2}=2, \sim>6.\right\}=$

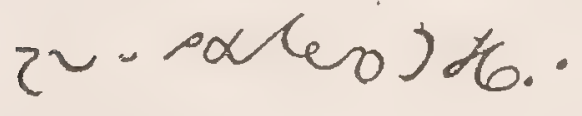

83. $\sim \sim \sim \sim \circ \%$..0 $=$ $\sim$ - pakio? =gor.=

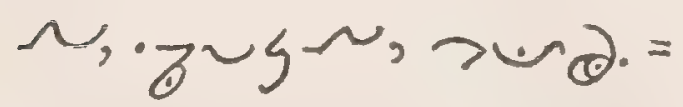

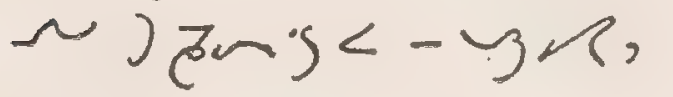
2. $6 \cdot-1 / 4 \sim$.

84.6 bo $-2 \sim e \cdot \frac{9}{9}=$

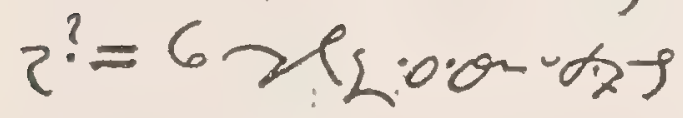
nu-so, 6 ipe.

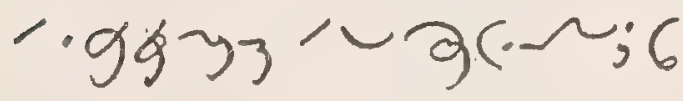

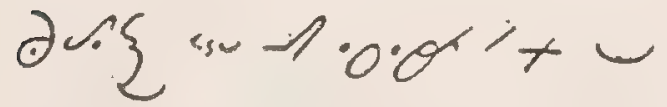

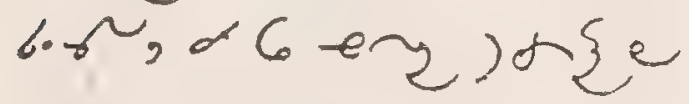
$\log r+k$.
XII. Holy Eucharist.

85. -S - $\Theta ?=\Theta, \sigma$ "x - y $1+60 x ;$ sso 1

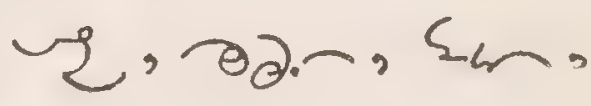
wro.

86. $\cos L ?-\Theta$ ? $=$

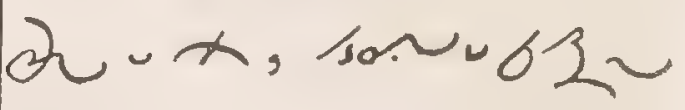

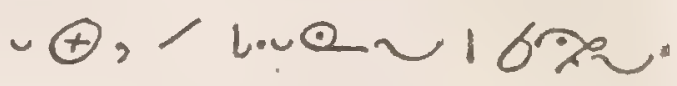
8\%. $\operatorname{sg}, \mathcal{\Omega \sim} \times$, $62 \sim 0 ?=\cdots y)$ -

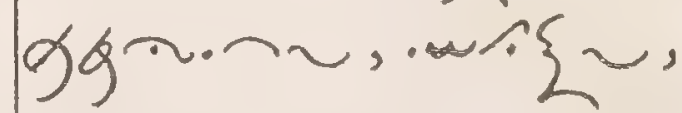

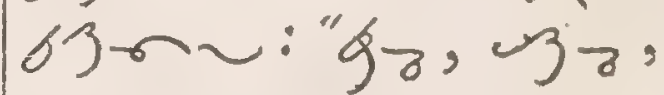

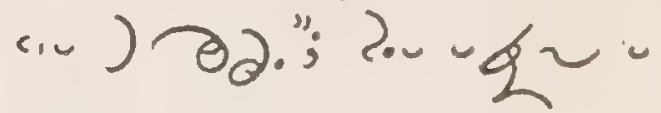
qu, $, 2 \Omega \sim-\infty, 9 g=$

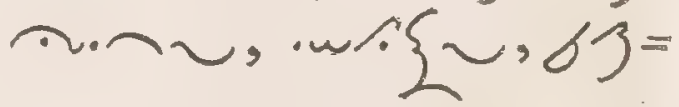
r几:" $\log ^{2}$ g, su

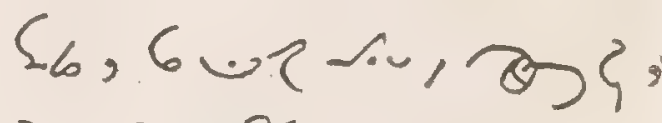

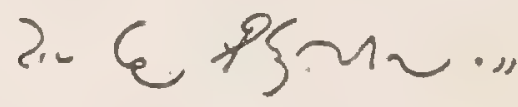
$\left.87 \times 166^{2} \sim \cdots\right)$

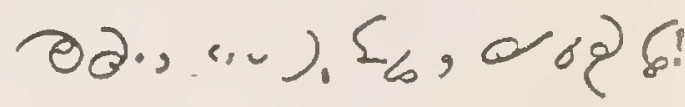
$=\left(b b^{2} \simeq \sin \right)\left(\theta_{\partial} \cdot, x\right.$ $2(2-1) \theta_{0} \cdot 2 ; 16$ 舟 (iv) $\Sigma_{L}, \times P L \sim$ 
- og) S.n.

$\arg 2<(6 x) 2=$

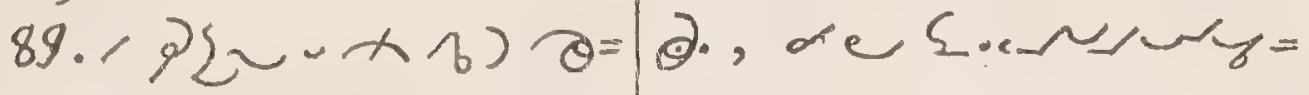
Q.人, . og) (n, , , ) $6<\varphi_{0}$ \& $4 \cdot \theta_{3} ?=$ Ge und b, or g: $69+$ ०र-11, o? OOj;

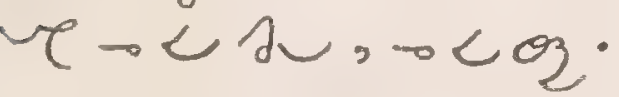
90. x> lighu-C D) $)$ बिन, प्रे K. $\partial \partial \cdot \sim-x ?=x$

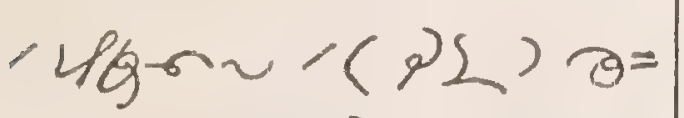

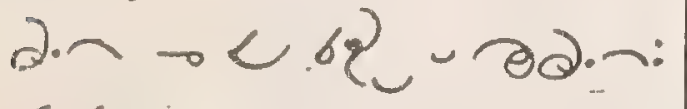

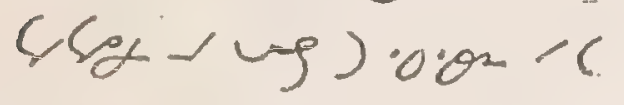
199-.

91. $.66^{9}(-x) 1$

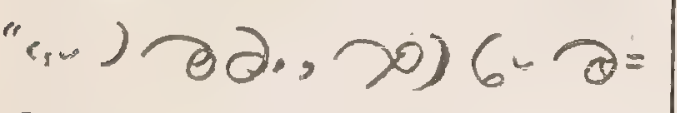

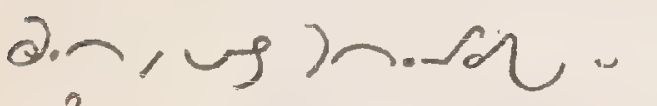
3 ? = - $\mathrm{Ain} ; x \sin \alpha$

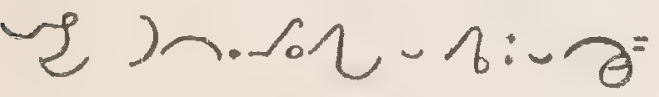
din, c En, whr. 92. > cos xis

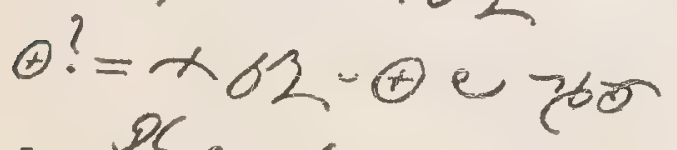
e Pfe, de pone $\theta .<$.

93. $2 \cdot 9 y, 2 y 2 \sim-1$ ) बे.न- $x, \alpha \cdot 03)$

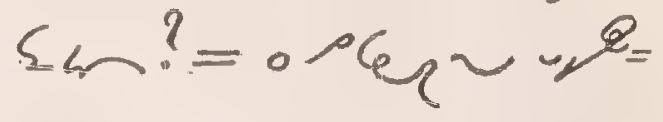

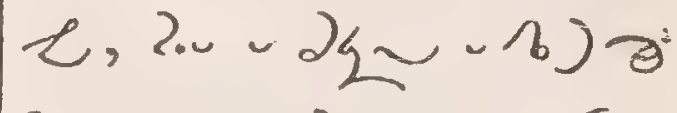
d. - . x, $\partial \xi \cdot 03) \leq$ - x: b.e $1 x$ en א, एक 94. $\rho(x, \gamma) \sim$ gqa? =

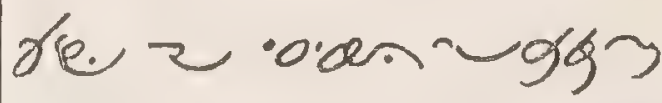
) ค६, 0.0.-

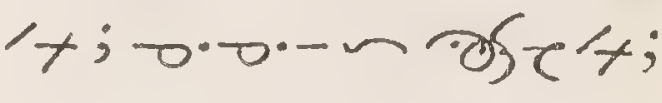
0.0.〜 $3 e^{\sim k s}=$ o<; 0.0.- eld.g $\sim 1+$, de

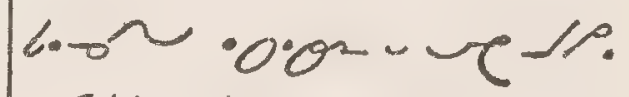

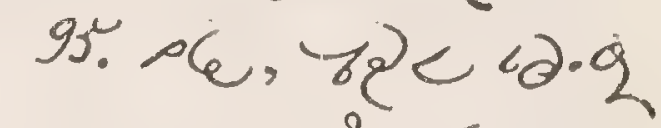
( $\operatorname{con}-1 / 60^{?}=6,16$ ca. ars, en $2-4 \cos =$ 3..

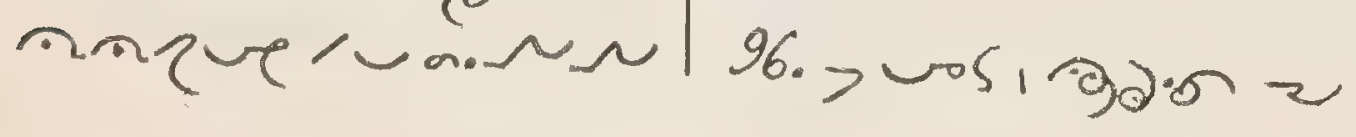


54

SHUSHWAP

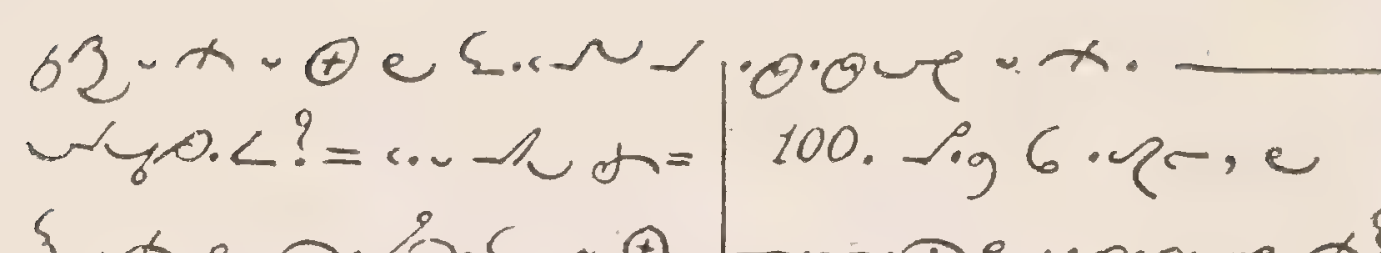

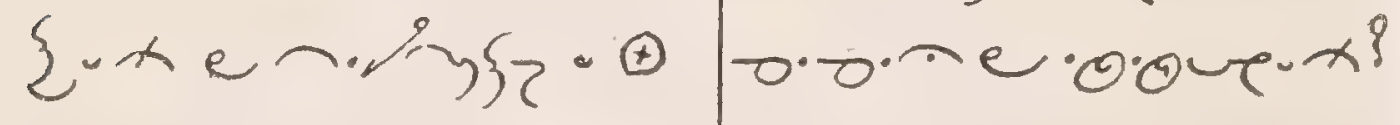

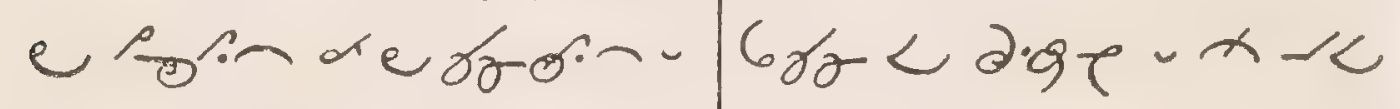

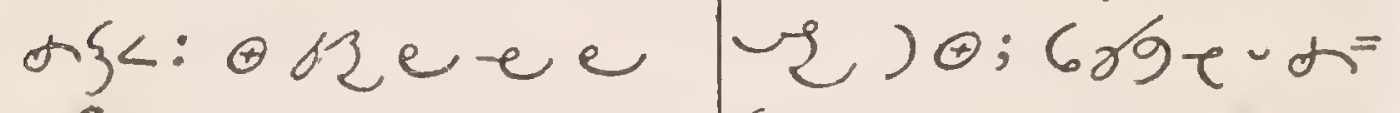

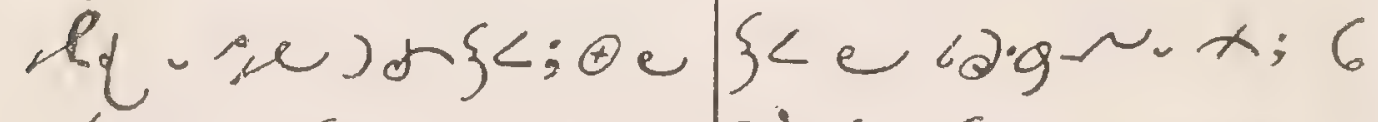

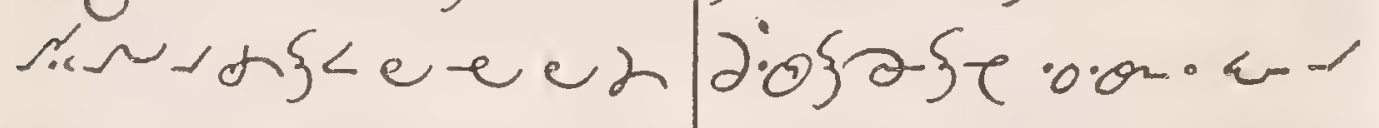

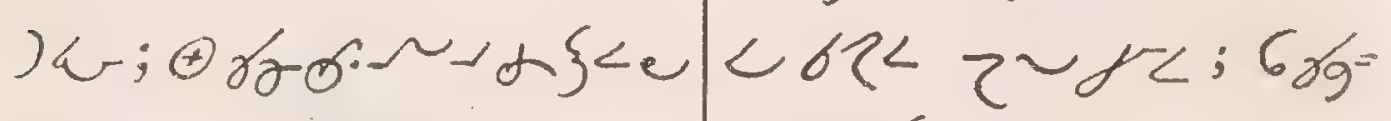

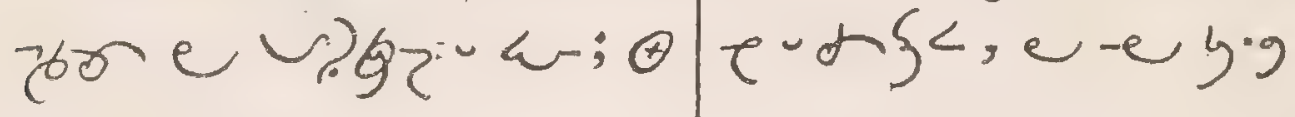

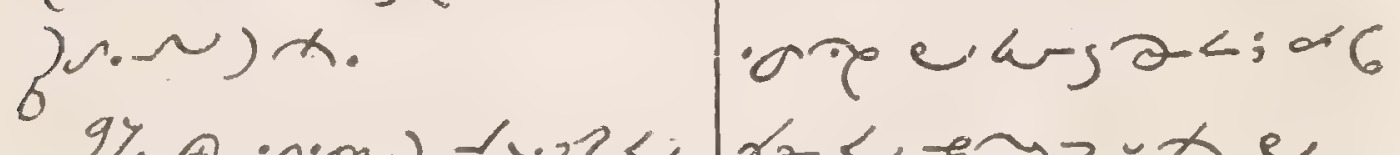

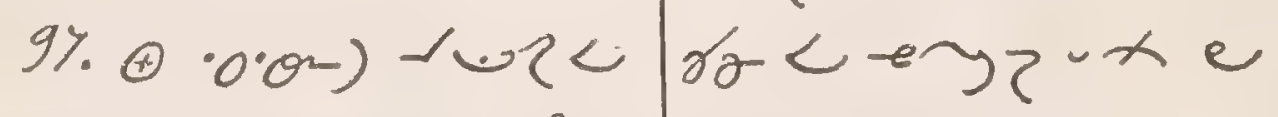

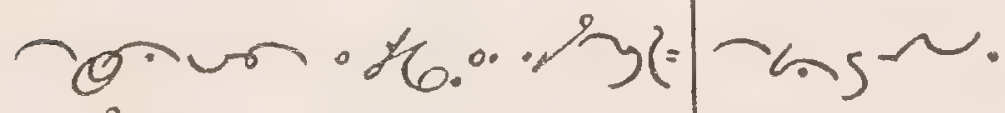

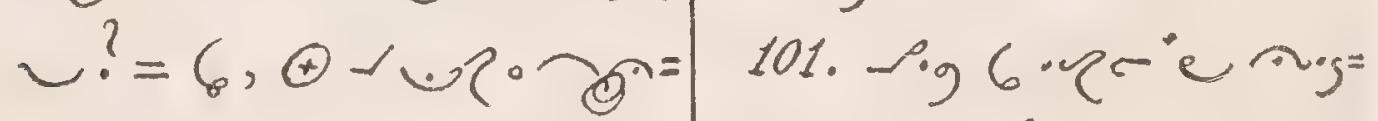

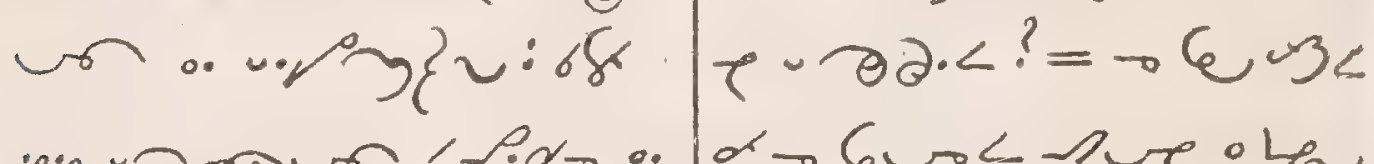

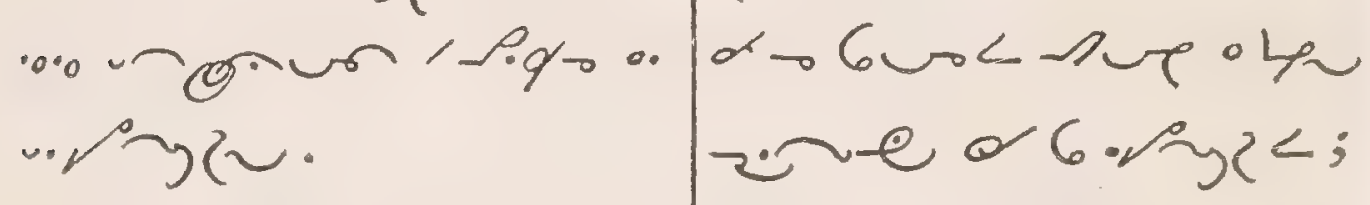

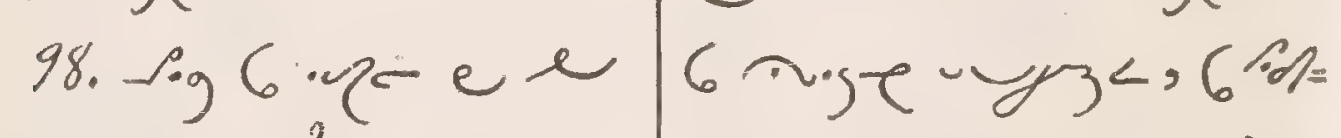

e.

ᄀ. $93<<, 6 \cdot z<\curvearrowright=\sim) \cdot-19$.

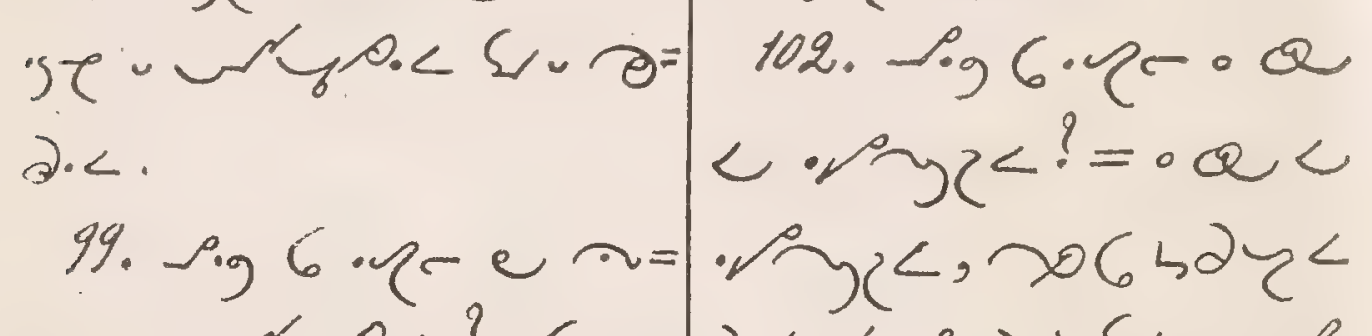

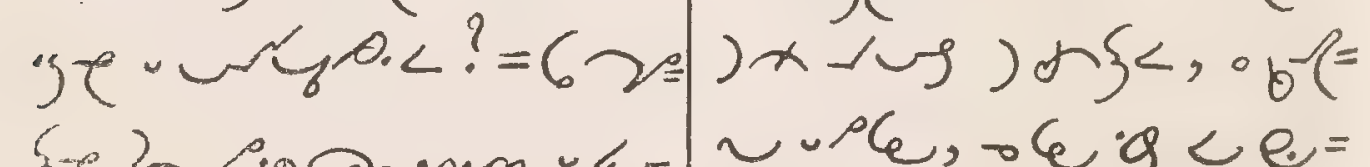

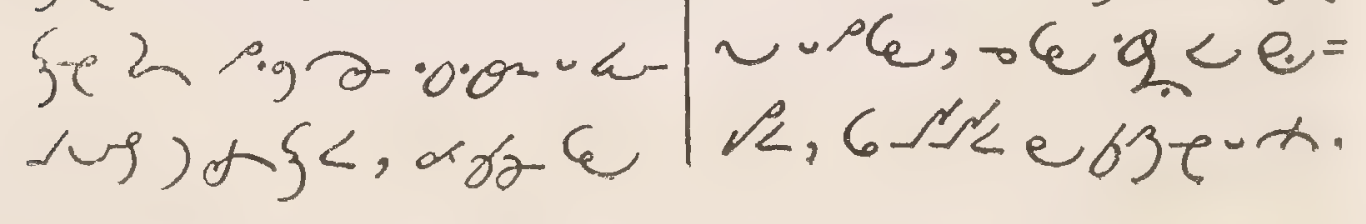


103. $056<63 c^{u x ?} \cdot \operatorname{ong} 9 \cdot \sim 9 \delta 12 \mathrm{k}=$

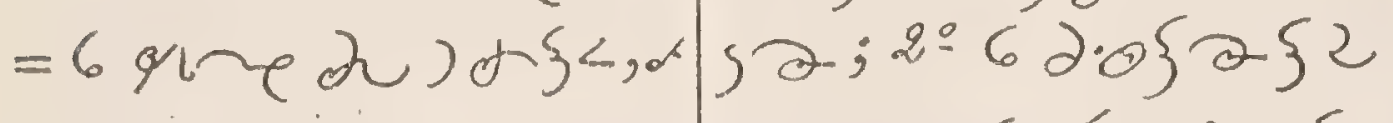

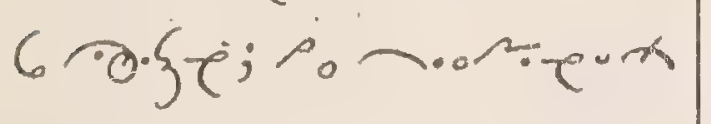

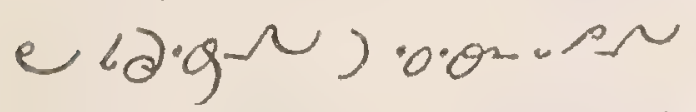
र-; 6 qरe ox-igg 6 .

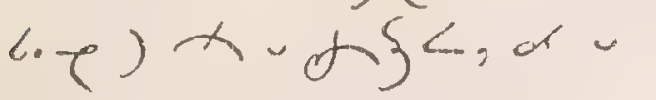
Dे.<, , o.0. $\sim 20$

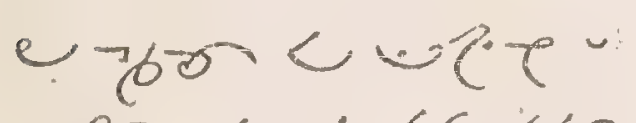

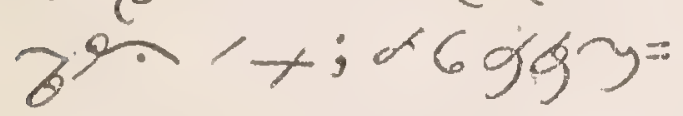

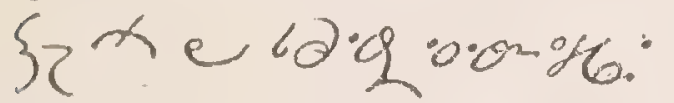
104. $2 . e \cdot \cos \simeq$

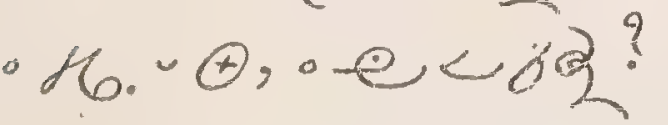

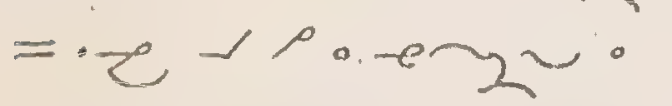
tox.ec.se ᄀ.pr: weered 小 $\{x-2-2$, or lage, or b. -1.0 tope. XIII. Sacrnt of Penance. 105. $\rho$ ig $6 \cdot \Omega$ o g7u

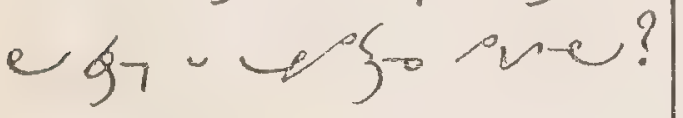
=。gequepo se, 262 k:

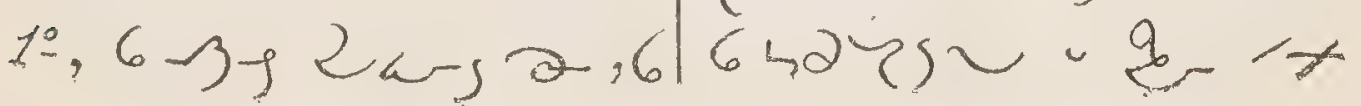


$-g\{t, d$ orle -

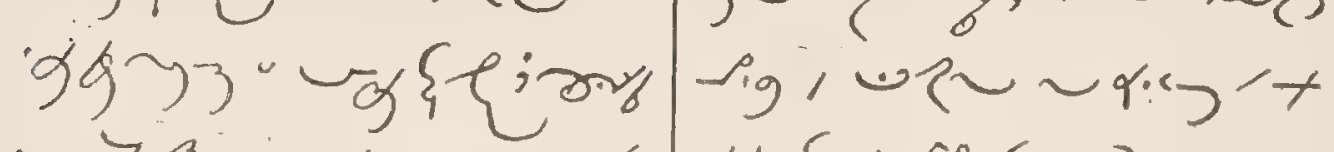

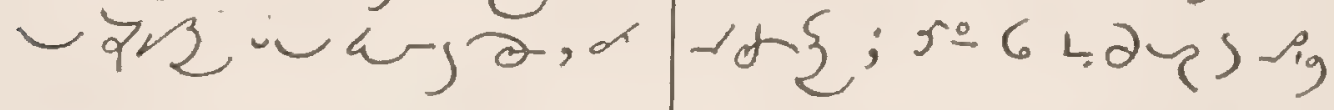
-

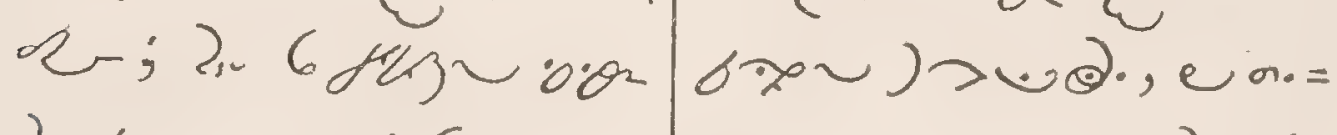
$2 \arg \partial, \alpha 6 \cdot 9 \circ \sim \sim \cos \partial ; 36$

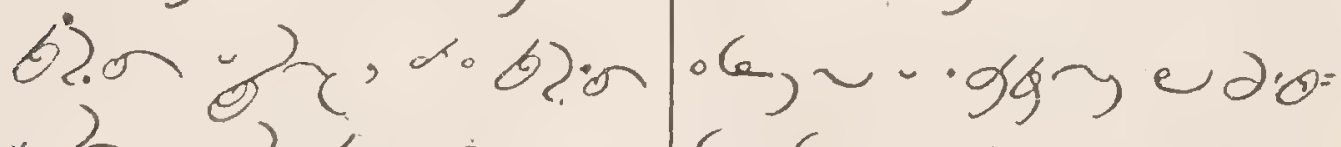

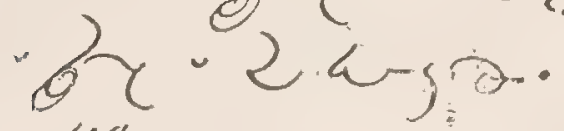
108. 0 Q 1372 $\log _{3} \partial, \log \cdot{ }^{\circ} 6$ ? $?=0 a \cdot 32$ $\operatorname{lig} \partial, 2.02 .0\} \partial\}$ $\sim 2 \arg 2,6 \gamma 8-9$ ohferero.2 aro.

log. $\operatorname{sig} 6.2$ द o. - e $\partial \cdot 0\} \partial-\xi_{7} \sim \varepsilon=$ $J \partial \zeta ?=\circ \partial \sim e \partial \cdot \theta=$ \}क्रयद, 小\}e- or $02 k=$ sक, 1069 gr $51+e$

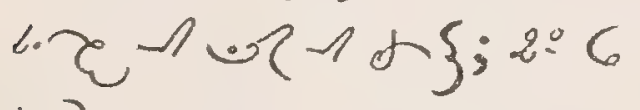

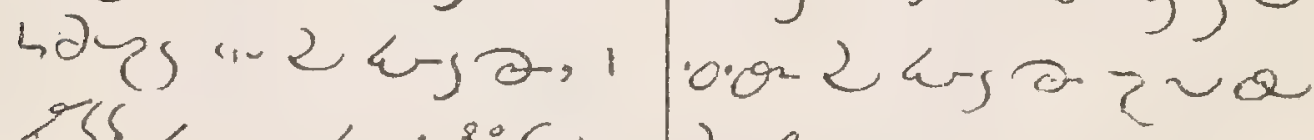
गुरुम

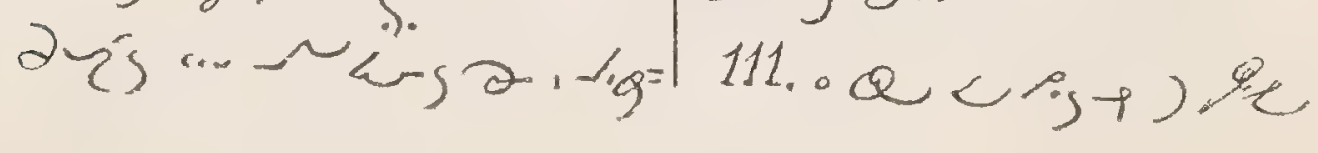




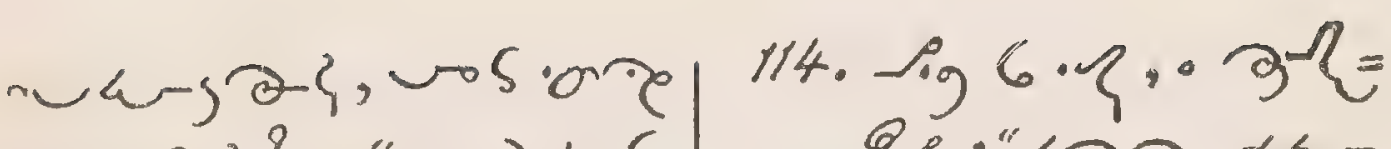

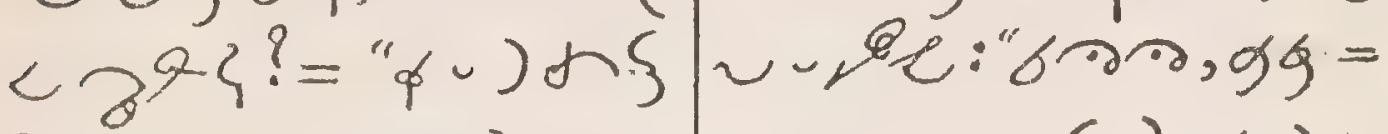

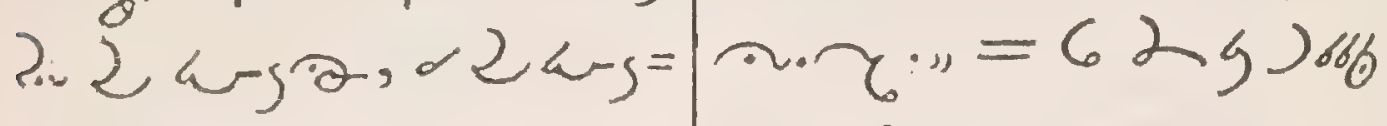

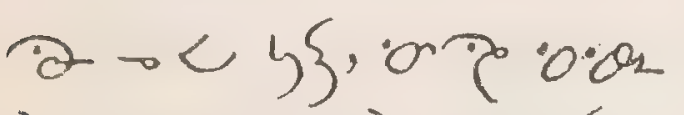
$2 \sin 22,0$

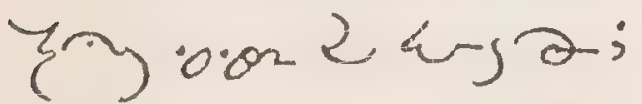
$993\} 1+e g g c^{\alpha}$

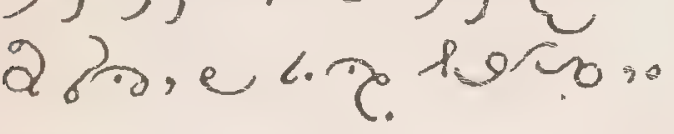
oguron.

112. $-9.96 .4,0630^{\circ}=$

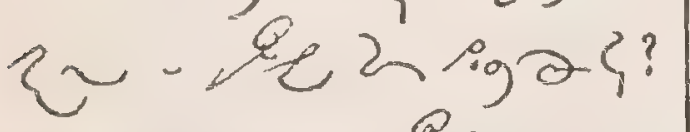

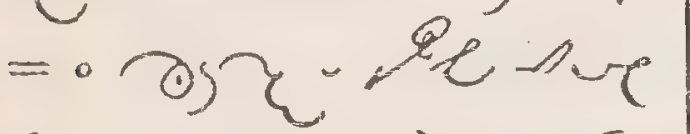

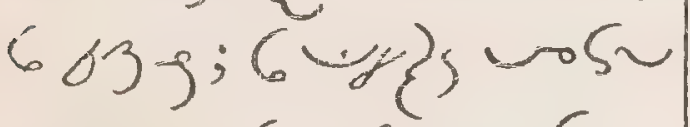

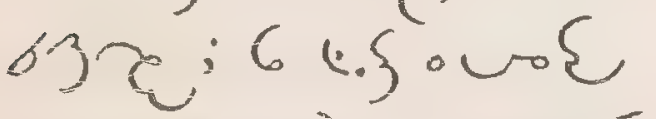
यद्धरुक, 6 veng o.gue $4=$

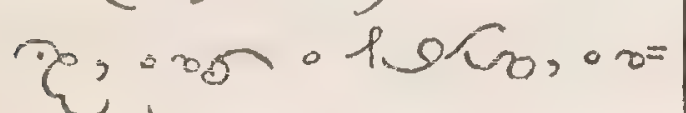
on.hy. 113. vos $1 \frown 03 \xi$,

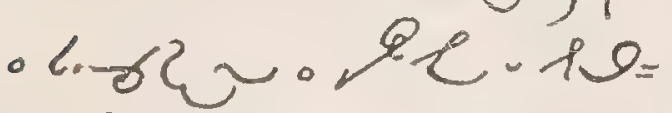
$c_{0} ?=6 \cdot 2 \cdot 325,6 \circ G$

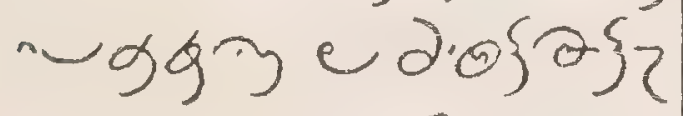
vasa<; 6 चरा 七6зद. om. $6 \cdot \log , 60.51+2$ gor (2, 60 99 ) h\}e : : 2 d.g $\sim \xi \sim-\arg 2 ; 6$

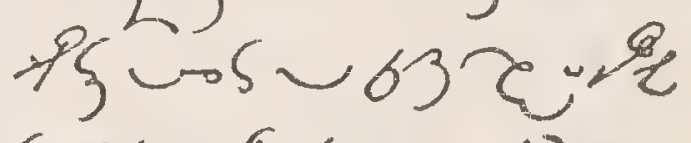
6وg $9 \xi 1+e$ o. $9=$ द2. $\xi-1)\} ; 6$

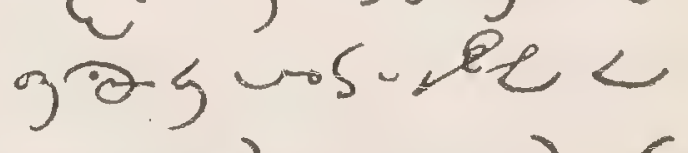
4 दरवक, 2.- 6

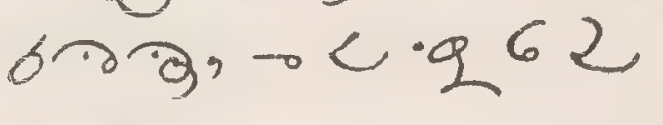
m. 115. wos - Ros? A. U b '. बQ Qee revolo

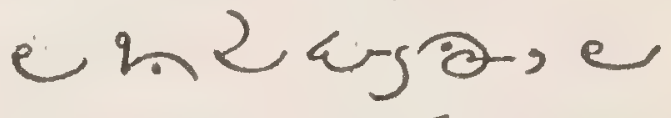
कor -) म vr.jure. $116 .(\cdot 0)$ ) P R $0=$ (o) $\cdot 0 \cdot 04 \sim \ldots$ ig $2=$

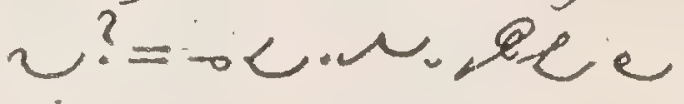
in 10 (n) ).0.0n

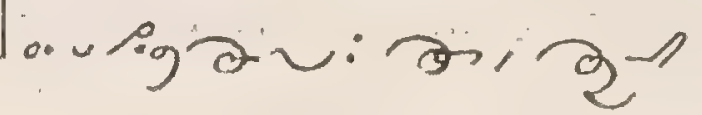




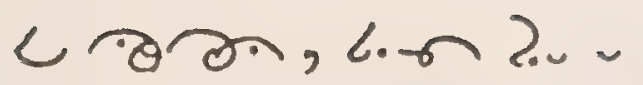
रण 0 ; की $20<$ के力, oc. be - bethofo; pp. - hy $\sim$. $11 \% .6$ o - Be. 6 rom $^{\circ}=$ S. hy $\sim$. ecurble $c_{0}, 6 \gamma<\gamma_{2}$ जक; 0.0 .6 .29

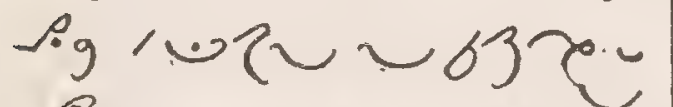
Qest.g r - hl $\checkmark$.

118. क. 29 ( $\sim-18$ unga 2 iga, चर< टर ठद 2 us $\partial ?=6, \% \cup 8 \varepsilon$ $\Omega \operatorname{lng}$; sofo $\simeq$ igd, .0.0n ka $\sim$ ig $=$ 的, 此; 血? gaน, ou. Len

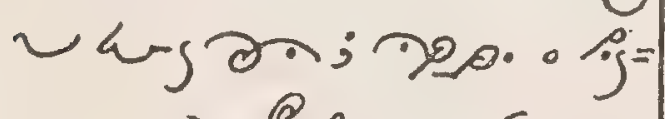

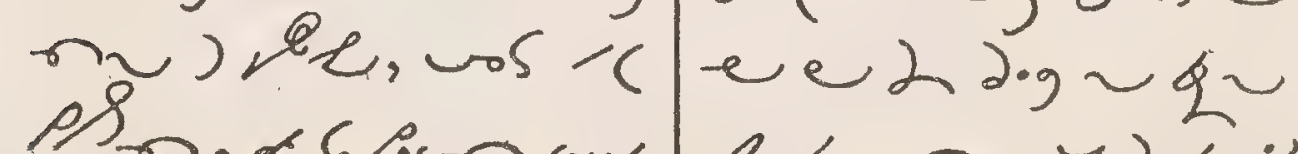
6). Son $\cos \alpha$ ars; $; 0$ ते ign :0on re

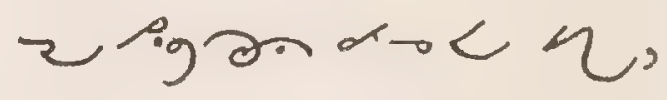
$200.0 .2 \sim, 6 \% \kappa=$ of ) $t$.

119. के a. क्य $e$ ßrचarga,e

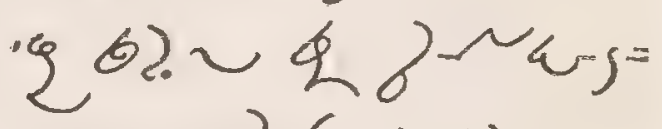
म, पर $6<2<$ $62-2 \arg 2 ?=6, \%$ $\sim \Delta Q-2 \cos 2,2$

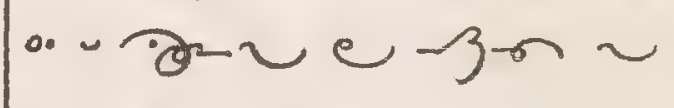
agan,e.z, क? $\sim$

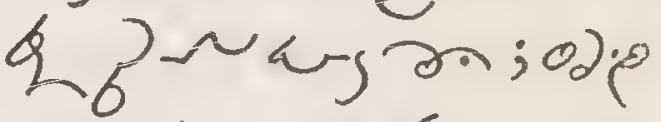
-onz y yoive

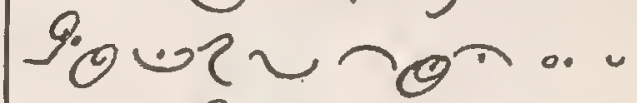

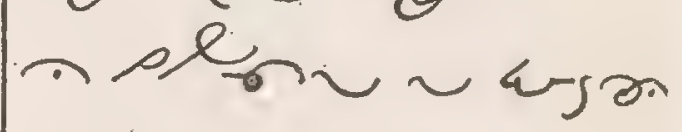
टi.gra. 120. $\partial$, ... $\partial 2 \sim e$

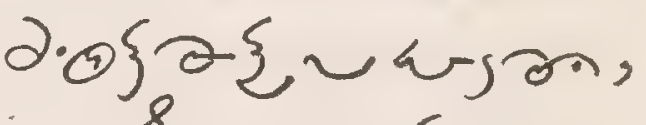
e orpe usan,e

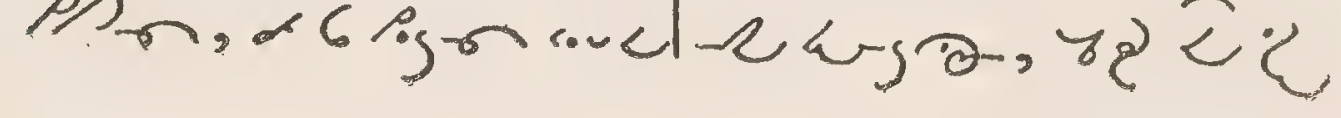


MANUAL.

59

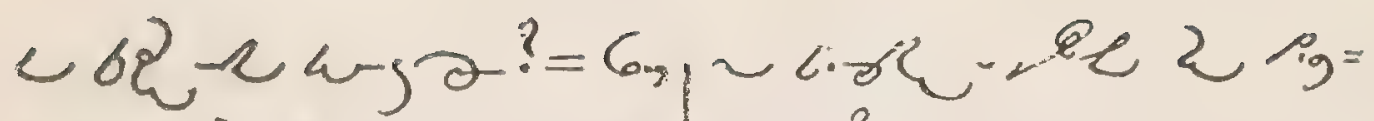

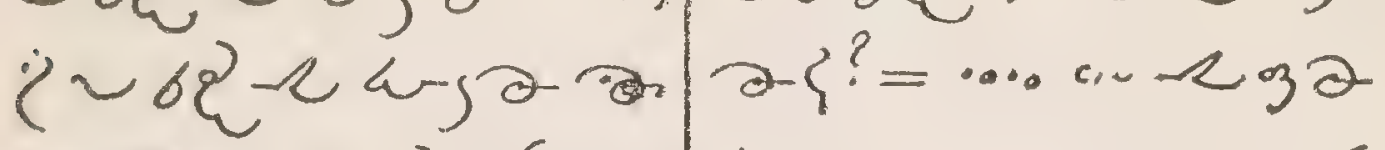

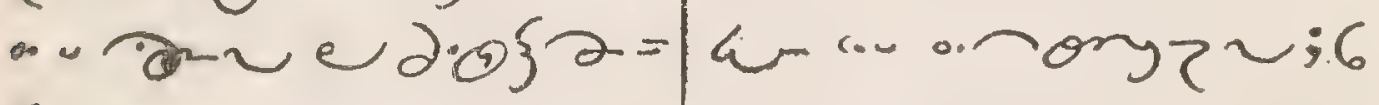

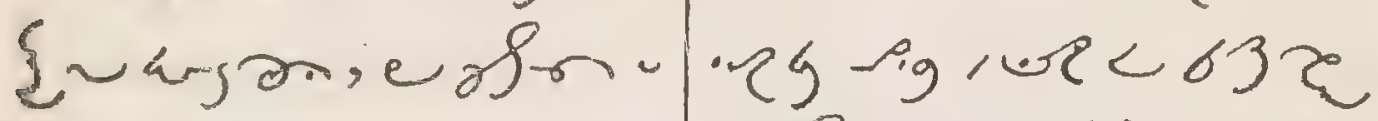

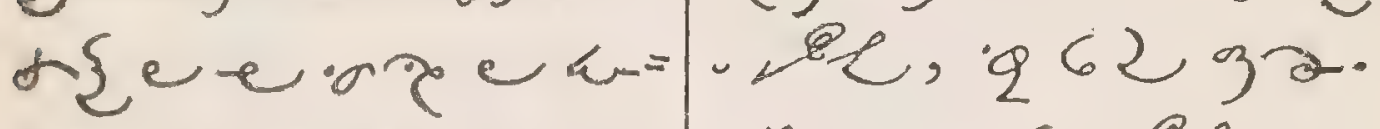

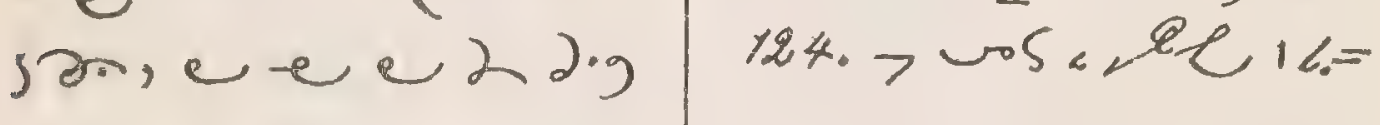

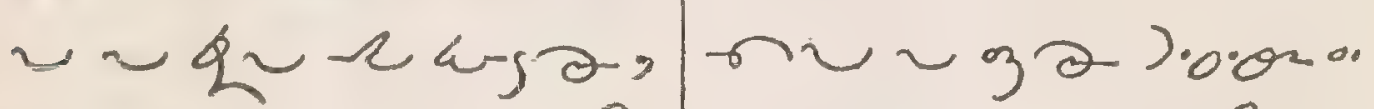

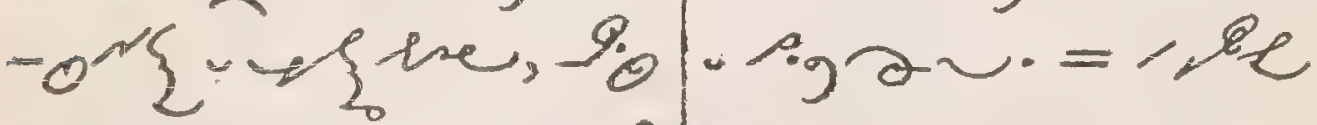
चर R-nulson 2 e ge cunger iga. $+2 \arg 2<$;

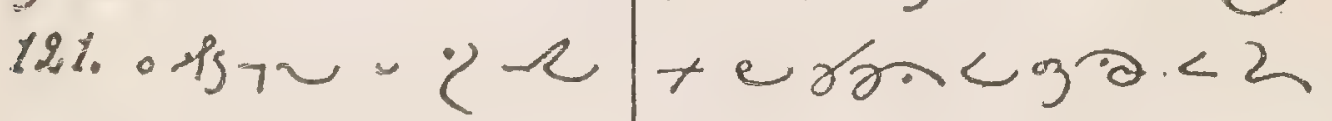

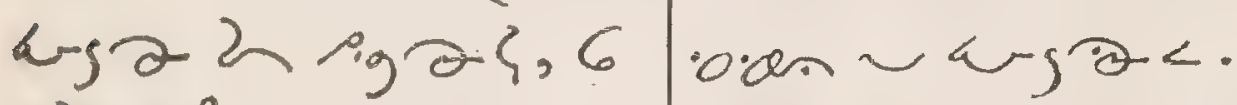

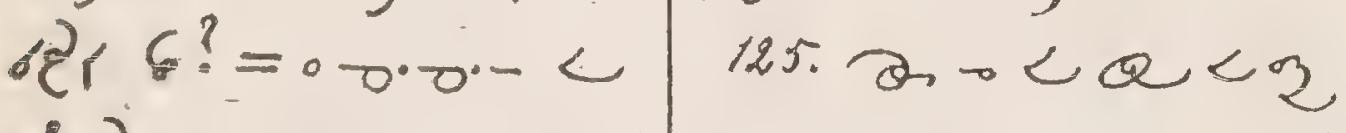

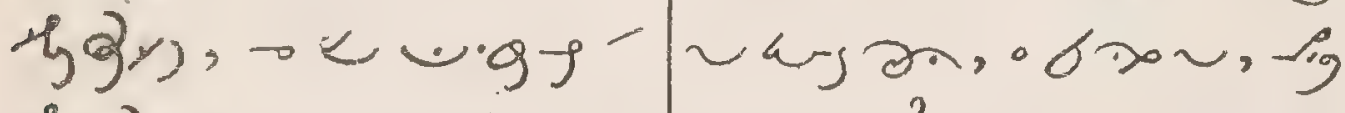

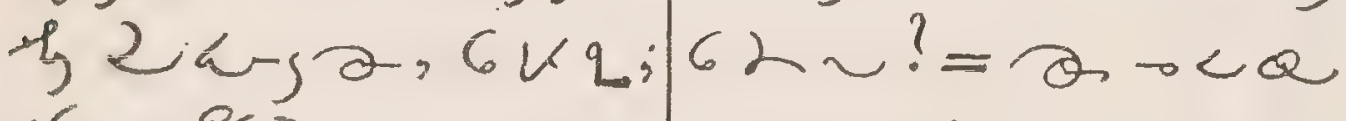

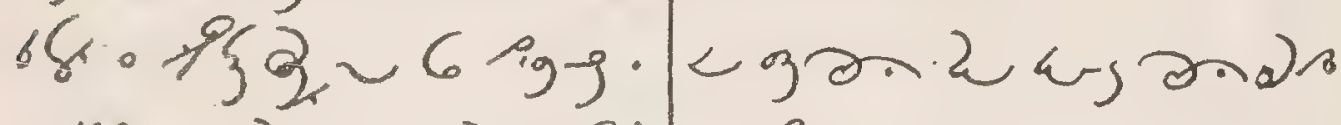

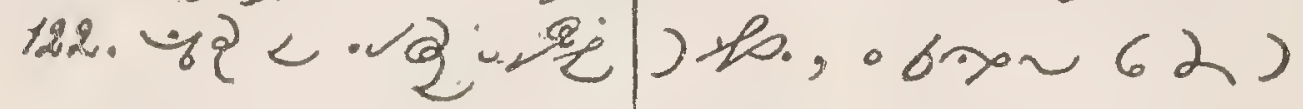

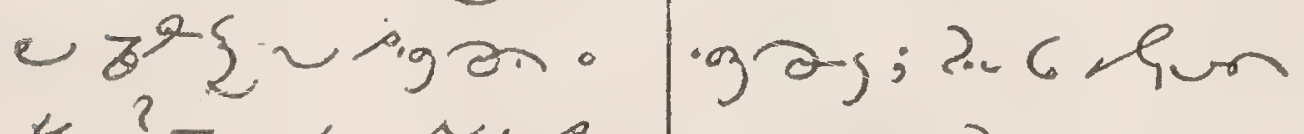

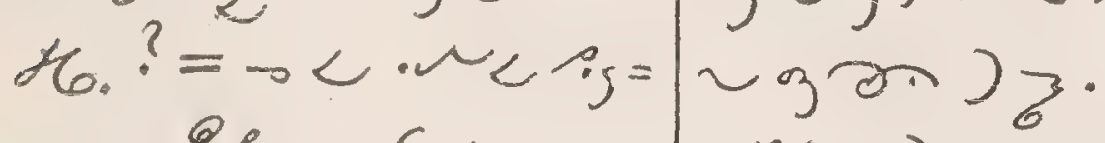

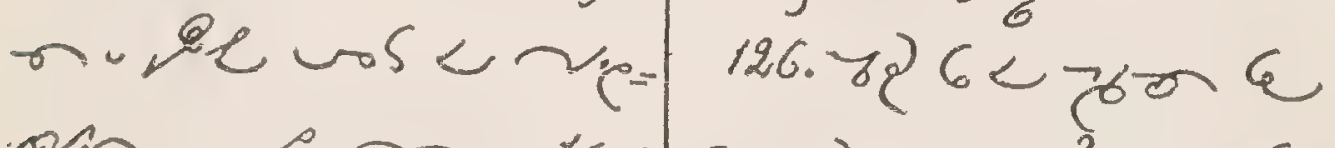

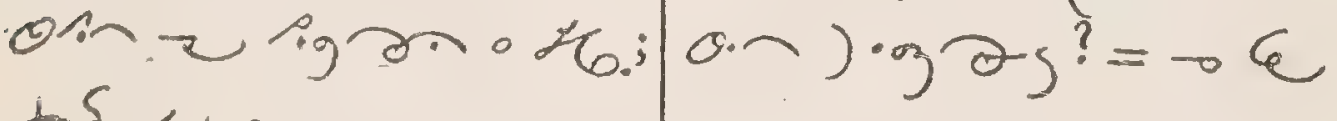

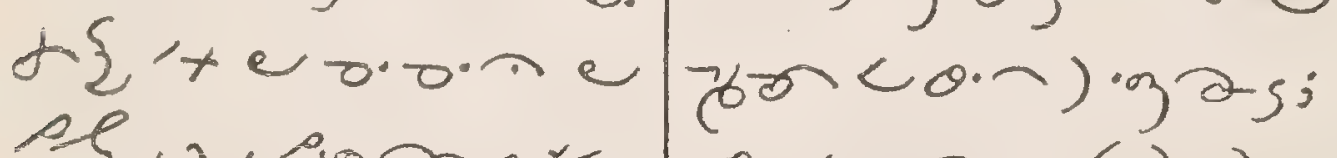

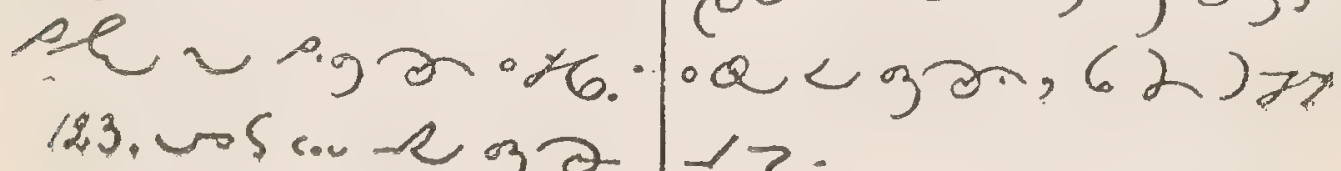
$143.05 \cos 20$ og $213:$ 


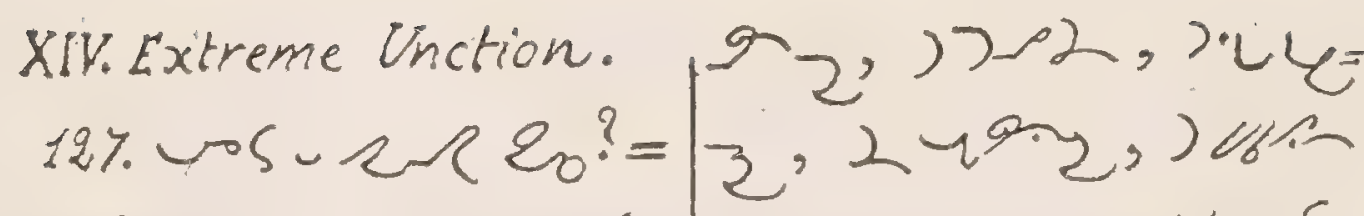
N $\left.\varepsilon_{0}\right) \cdots-1 \cup \rho \xi_{0}$

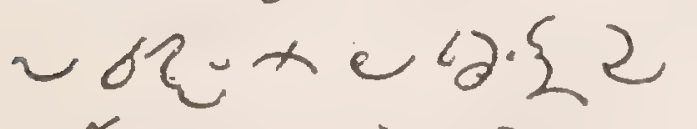

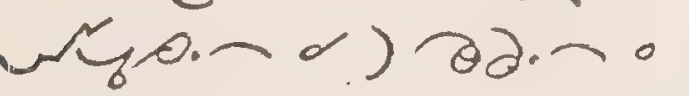
म6. वरe 128.0.6\} - $2-22_{0}$ ᄂ

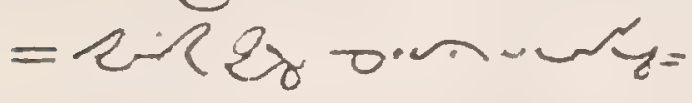
Din woe ob,d toin. okee 'ige 20$\}_{3}$. 129.062 o (a) $\{$ )

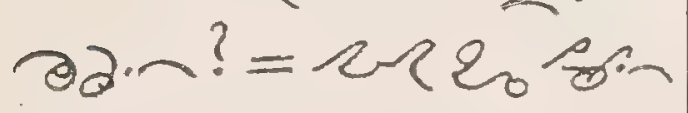

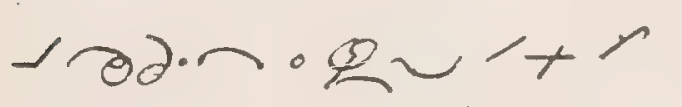

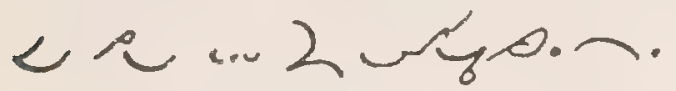
130. Sig 6.0 us u. c бr, rरeq - uR e ? = 6ran ट ग0\}

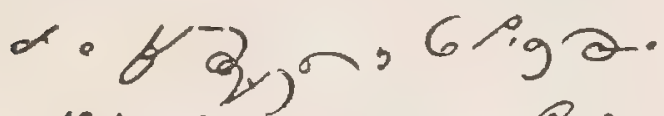
13\%. A.g, .

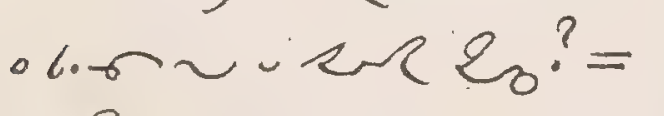

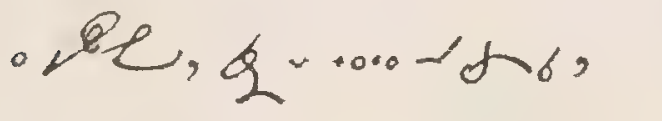

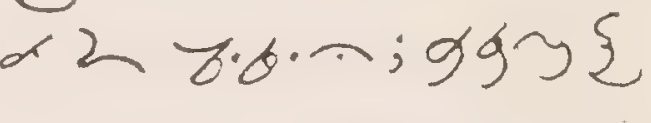
$1+e 9 \cdot 2 \cdot 0.00 \mathrm{ar}$ - $6 \varepsilon+\alpha^{2}-\alpha^{2} z$. - 2 , - (Lez,

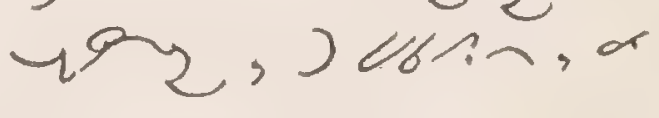
$=7.8 . \cdots$. XV. Holy Orders. 132. w5 9? =9 “- $1 \xi \sim 6 Z$, e f 1 \&e,era.

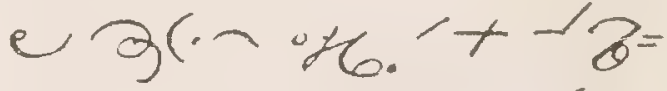
x, derde 6. म6. - $133.5 .9 \times-82 \mathrm{C}$ f $?=x-82=\Theta$, $2.0-62-0 ; ; 2$. ".ou)yede Qe, efre 己"

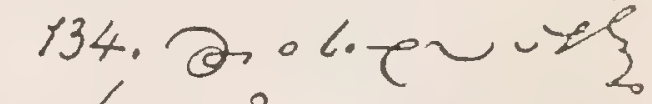
$\sigma^{2} 2 \% 0^{\circ}=20.025$

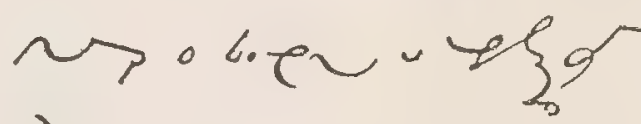
$3 \%$.

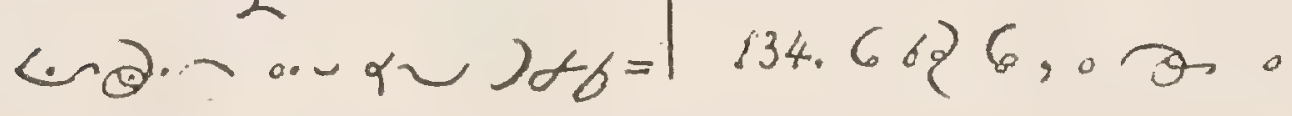


MANUAL.

$6 r$

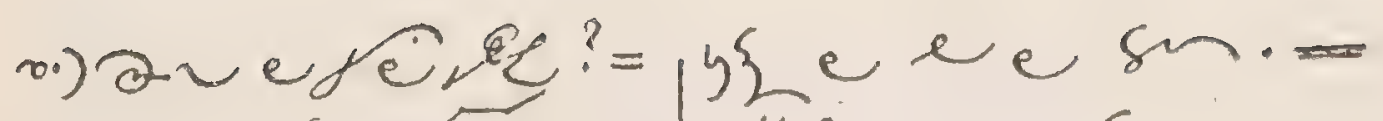

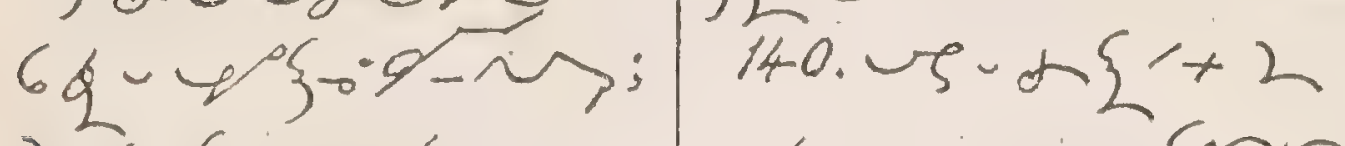

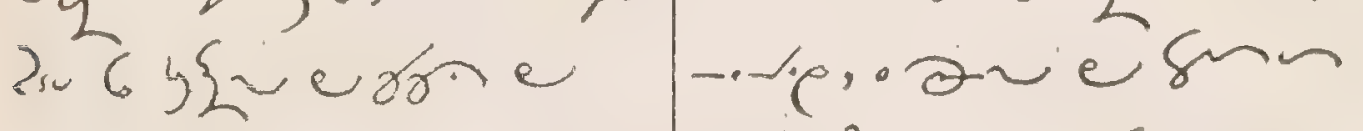

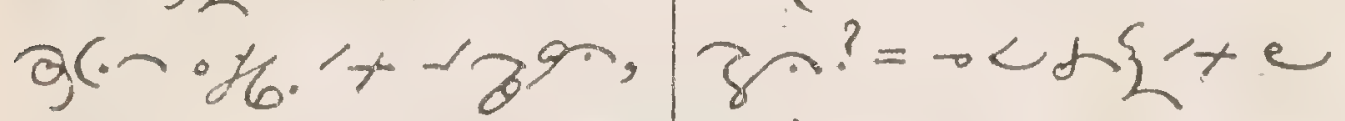

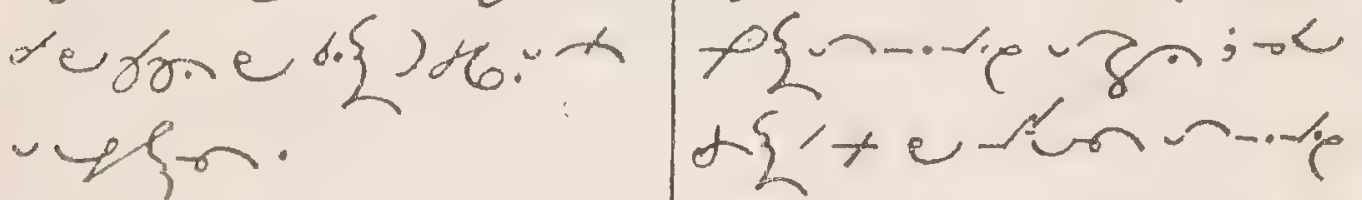
XVI. Matrimony.

136. vos $18 \mathrm{sm}$ ? = $\mathrm{sm}$

o"

) 66 .

13). vos - quen-rgon?

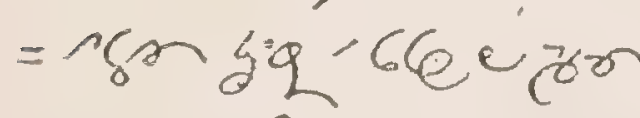

ere ponisong

- ree 98.0.

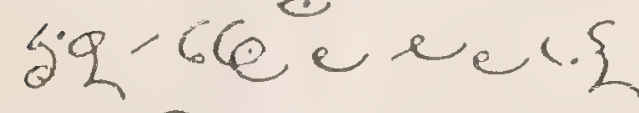

- Pereora, de

o:日r

138. 6 oq o 0 . 0.an

esm? $=69(9)$ ) $x$

eblece rat

6 un ot

139.6 of $\sim-100$.

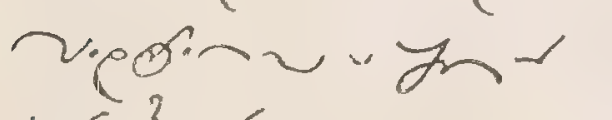

小 $\{?=6 \cup \cdots \cdots 1+1$

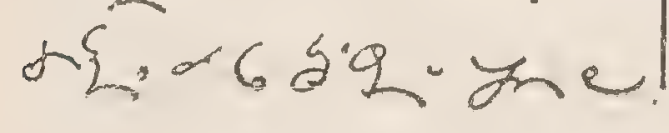

-zacec sn?

- $v e \sin ; 0<\xi$

te ous $\{$-..."

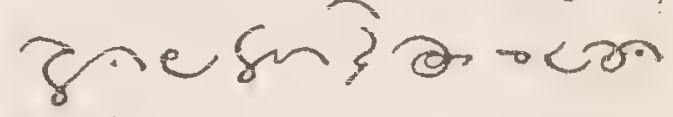

esin.

$14 \% .608 .2002$

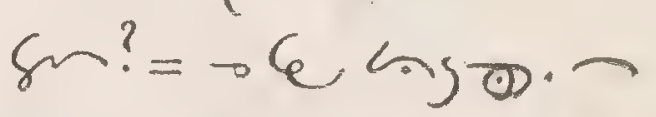

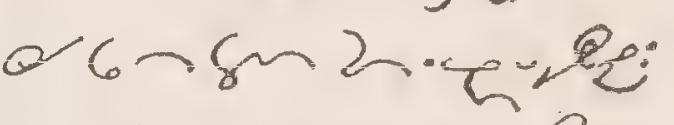

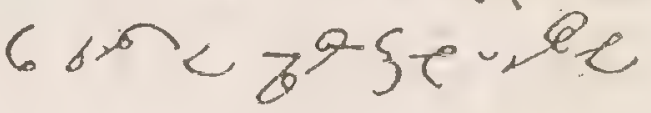

).967y3; por.cos =

$\sim$ :0.0m 9 \%

$260^{\circ}$

142. $78<9\}<-99=$

yzeresinas

"zesm? = $6, q$, d

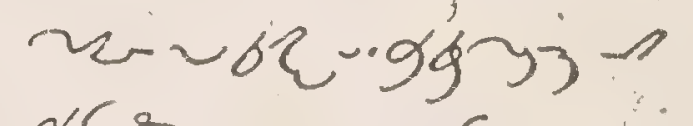

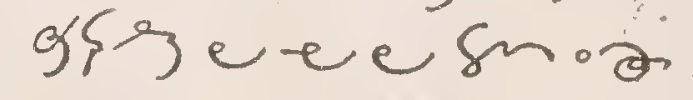

oco.2e, gn:esun

ese $\{\rightarrow\}-$ 
Supplement.

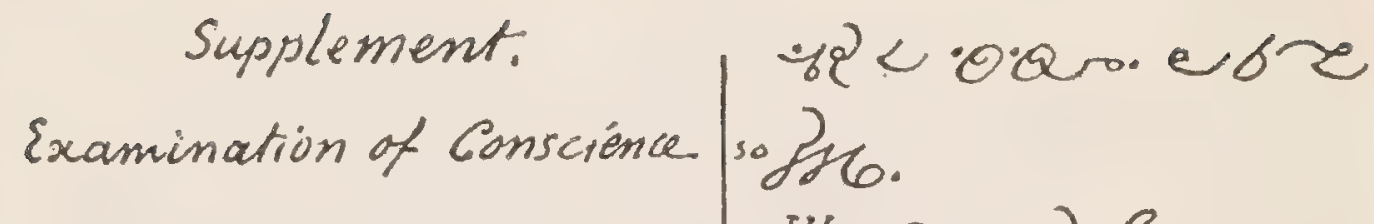

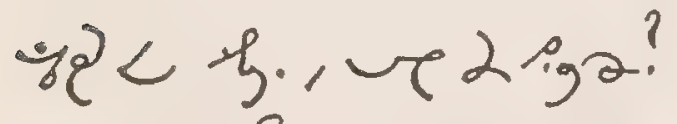

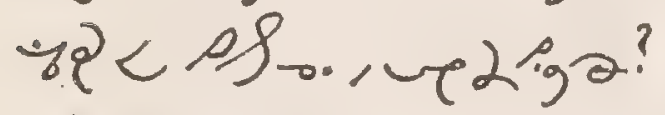

I. $39.1+610000$

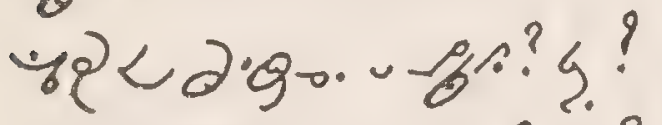
पर(श).099य? है?

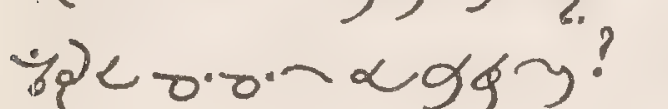

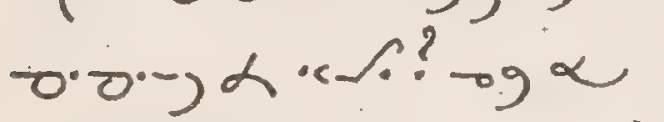

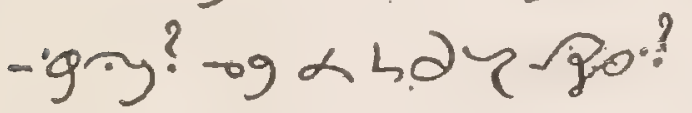
II. e<afi- 't-13n: 0 , ar. ? 9.8 III. Pe vievoundor. 0.) bro.2 nas? $010-0.2 \cdot n$ sh? $^{\circ}$ ? IV.Pel.j.orakol.o. o) infigun-..'s ba,

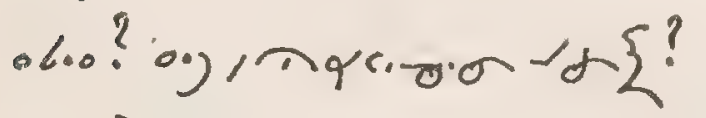

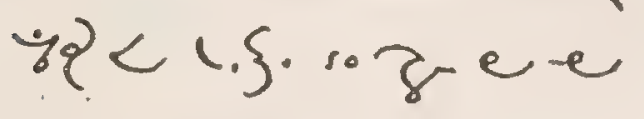
VI. exdre.

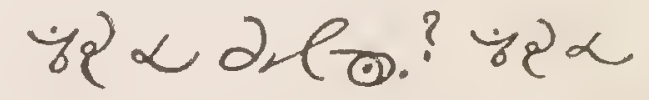
ars s su- 16 ? 2द? उ?

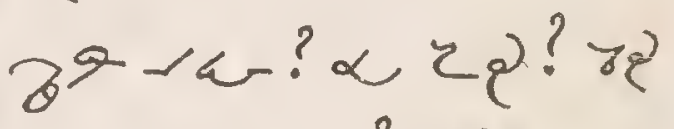

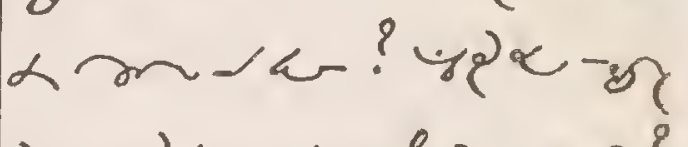
ja, k-1ut, 2-0-8? Q.?

vir. eadre.

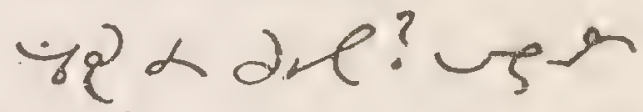

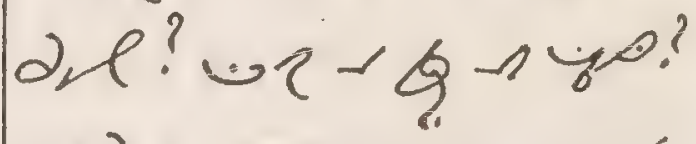

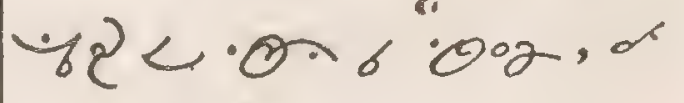
-<ま:?

vir. e八 b Q.

IX. ec hass a dre.

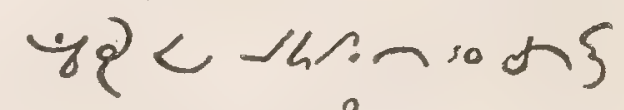

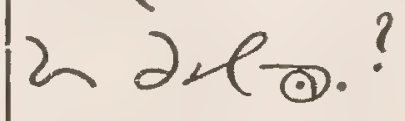
$x \cdot e<3 \alpha \xi \cdot \mu \alpha l$. V.ectra, n.

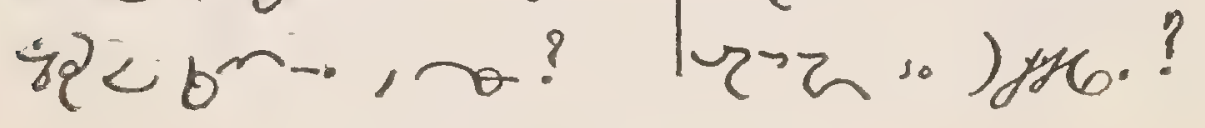




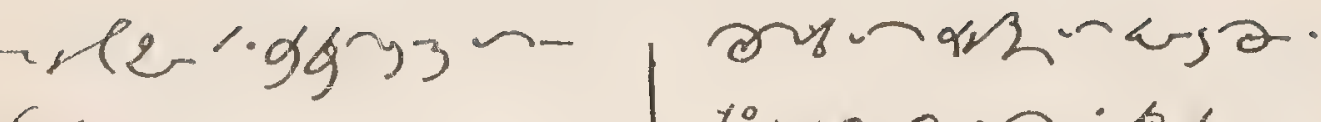
ose.

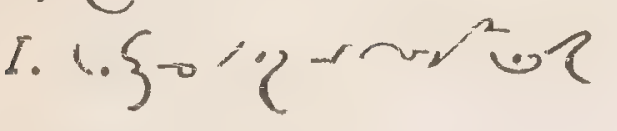

r.nes

$11.825 \rightarrow 0$ Liner orpo) (

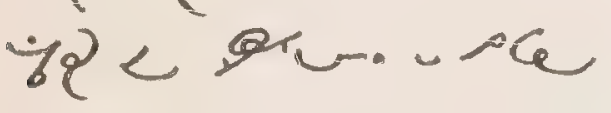

h.nes? - 6Gr? Q ? ?

III. $\cdot-2 r a \cdot e$.

皱<6-2 320

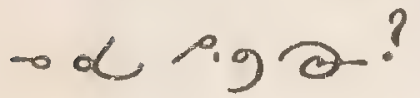

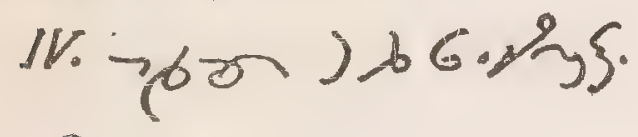
$\sim \Theta$.

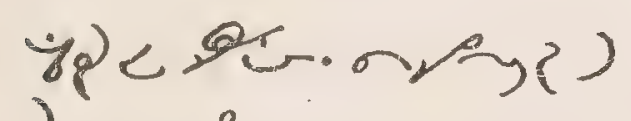

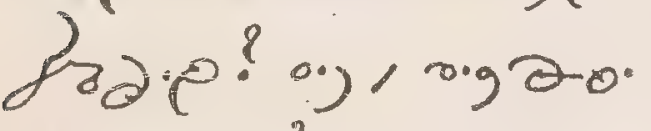

e.gn?

V. ) $d-1,1 \sim, 3$, - उळ.

g. eर, उa?

r. ) varester

on w.

goznus areat.

1ㄴaQ) $\partial:$ Q

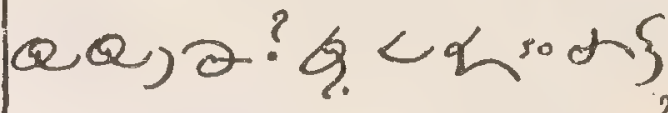
- granine-nue?

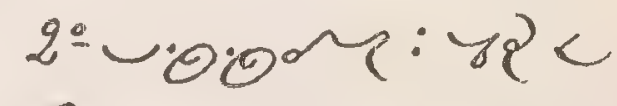
. $\operatorname{soch}\{\alpha$ y 0,0 < lo.9. $9 q, 099>3$, s rokn?

$3 \div \check{t}_{0}$.

4ำ :

346

5다: \& $\alpha$, ip

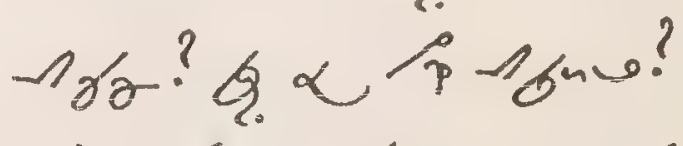

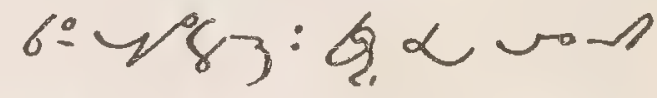

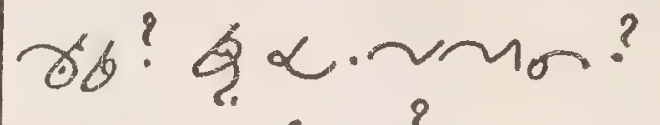

Q人.

Y. Or of: \&人. on

2..6., 29, 20

or?:0, $10.0 \cdot \log _{0}$,

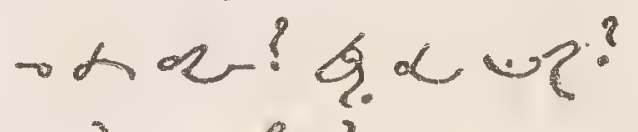

उq< e ? 

Skwamish

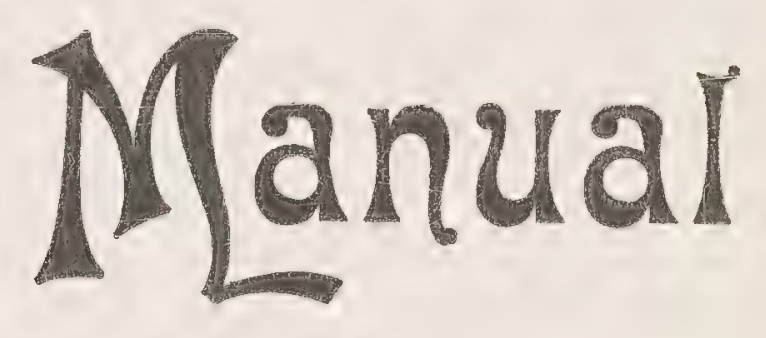




\title{
Skltamigh gthamal
}

\author{
OR
}

Prancts, finmus

\section{and Cattelisim}

In Skwantuln

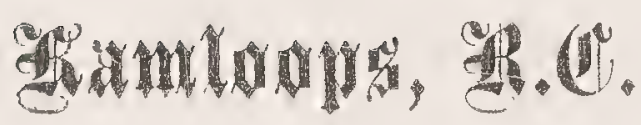

1896 

Sk'homish Morning Prayers.

In nomine Patris.

$-2.9, \cdots$ $\left\{,-\infty \psi, \cdot \alpha^{2}\right.$ \& $\rightarrow$.

Offering of the Day.

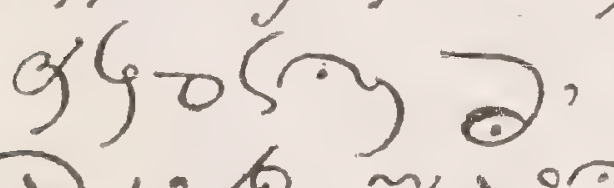

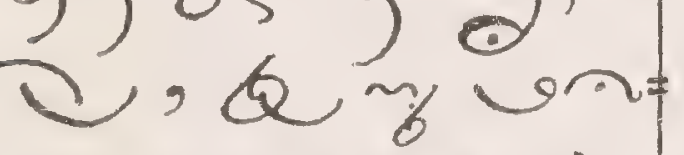
D: $7 \theta$ 卉, 6 25 son, 220 on ofols 2 ल4 ta $62030 \%$ $02_{b j}, C_{0}=$ $82=x$.

of 2re or $-02+x=-0)$ t. - 30.30 $2_{0.2}-269,7$ ondis ot te e

b2 ifक्य, e, 62 talary - 2 , 9 .

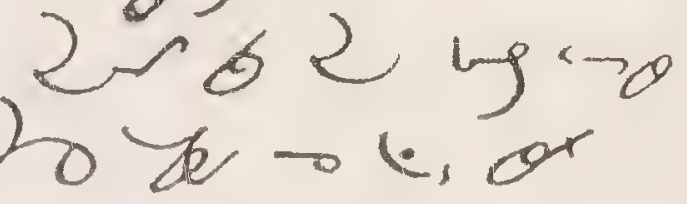

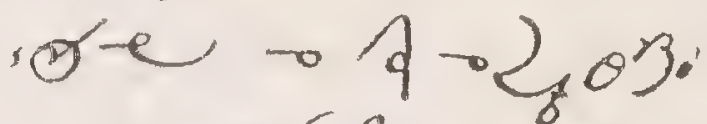

0 ax 8 , sor 6 फल - 296 . oorse of?

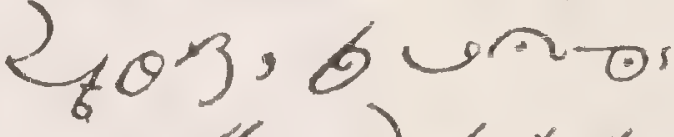

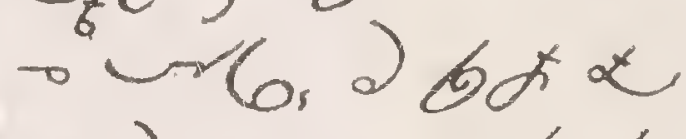

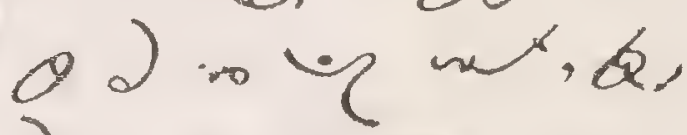
and o.er 0

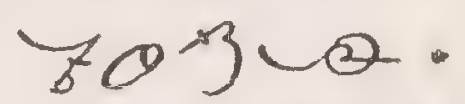

Pater. gry gुG. org do ori, sal a bate ot. w6, - 2,09

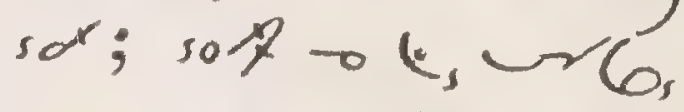
x unser proge; 518 - $4,46,>0$ $3+36-10$ - $9,-\theta \rightarrow k$.

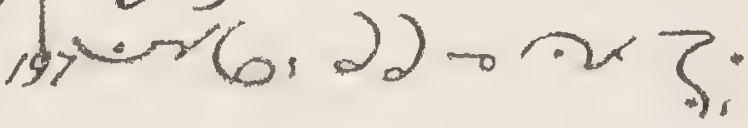




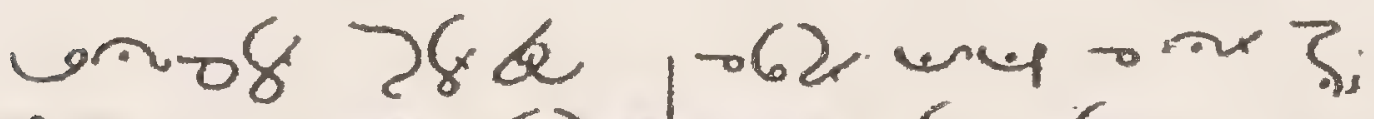

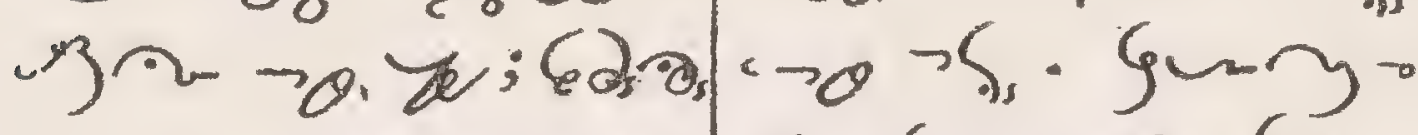
-t o uner, $\rightarrow=x, y$, a e $\xi$,

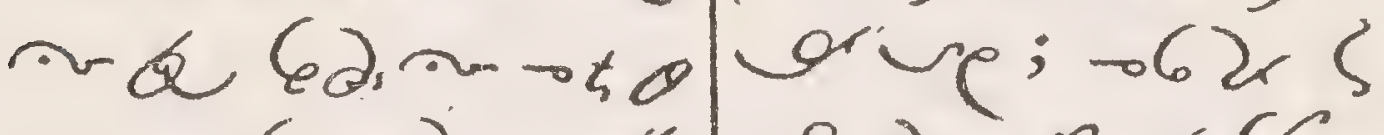

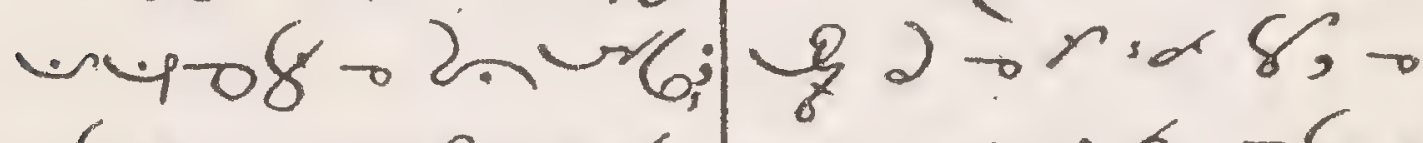

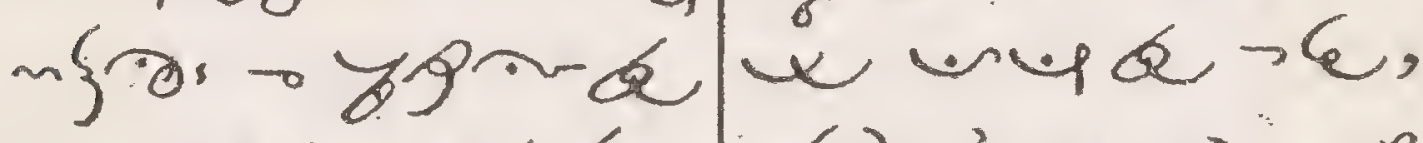

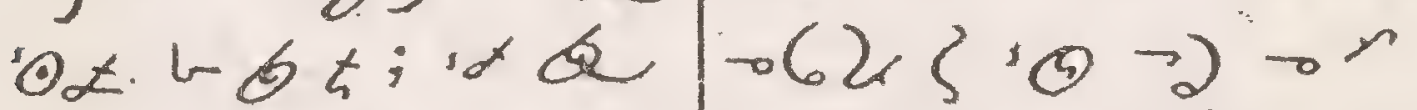

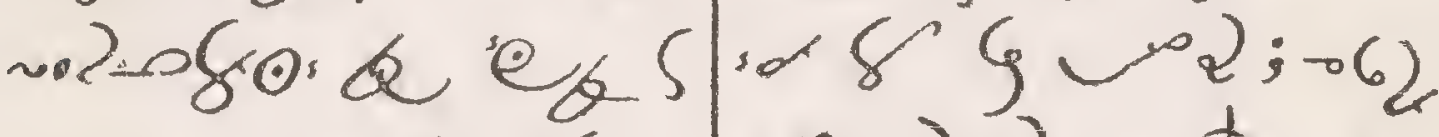

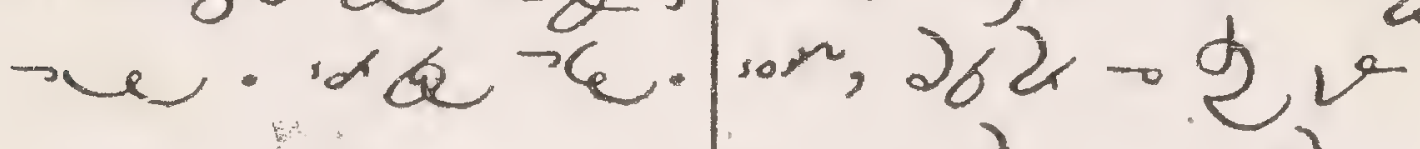

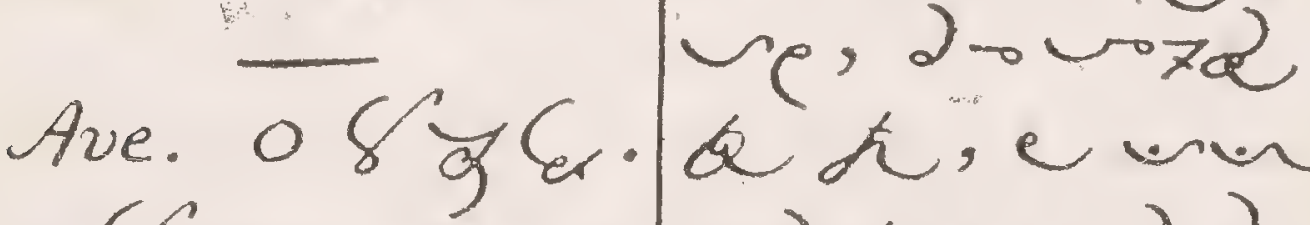

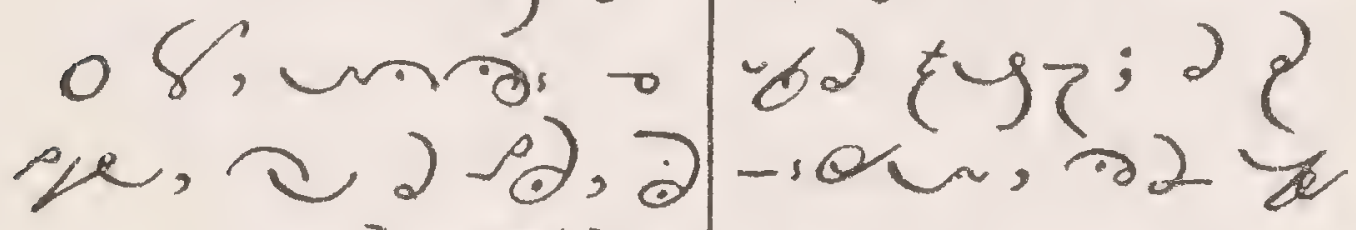

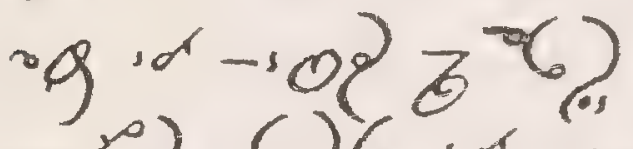
$-8,63\} \%$ $\{2$.

. 8 , थ2 2

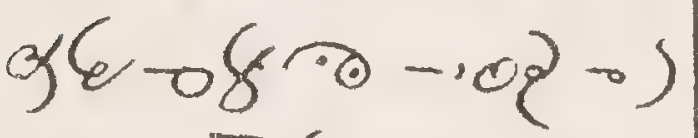
$\curvearrowright, 28 \% \sim 6$, $\rightarrow \theta, \cos \theta \theta$ $\sin \cdot \operatorname{sac}^{-1} \cdot$

-65 a $20-2+30$ $-6 \xi \rightarrow 2 x-h_{r}$, $6262 \rightarrow$ win - ¿ g, og el -08 व 6 ? $-2-53 x$ $-2, \omega_{0}-2$ bot:

bing ow, Credo. Gun g96. od g600 60"

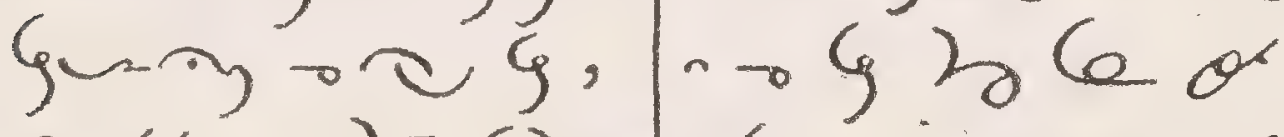

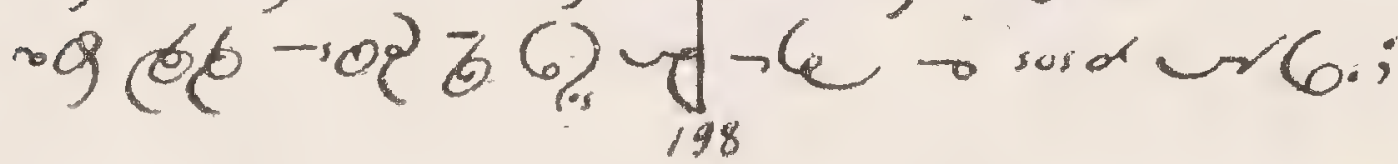




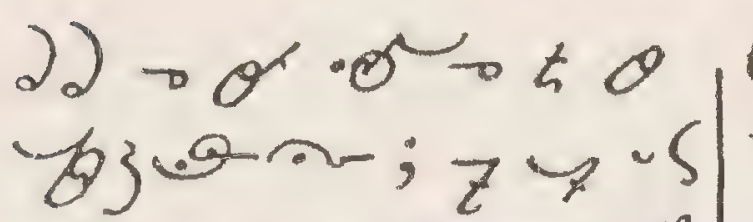

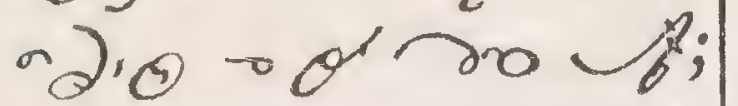
w rod unb 9 , Q $\partial-2 z_{1} \cdot \alpha^{2}$ पe.

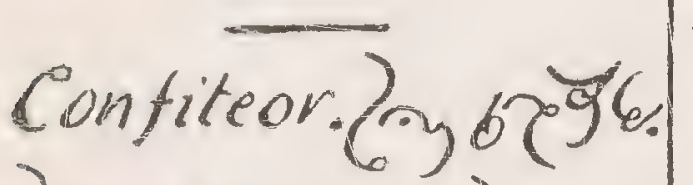
ने कर -so? I $900-102$ a 6) ve, -iिए -

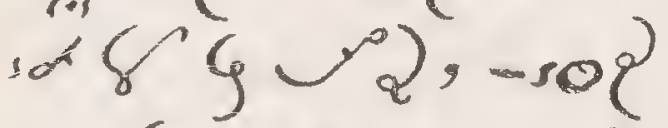
पूर 2, -10? *, -10 $y$ g/y पर, -, op o $\approx$ $\left.w_{0}, \cdots\right\}, x_{i}$ - to 0 2

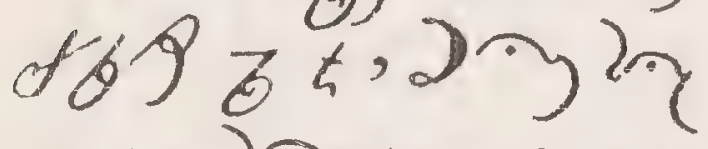

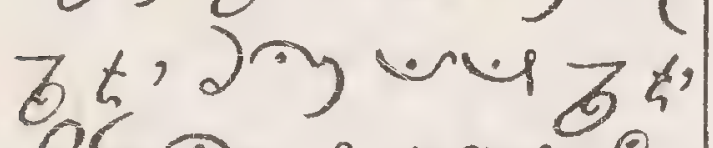
Oद⿵ जिए. - 20t 2029 \% . $2022,9,-20$ ing 20 2. $8:-6$ ४⿻
র〜2, 2*, $\partial^{x} y / y^{2} y$ $8,2 p \pi \sim 6$ ट 's. of, $2 \approx \operatorname{cog}$ ep -s0र व 6 ? पर $6201040-63=$ and id b ? $801+2\} ;$ a te.

Indulgentiam. sor e ungo80., ×639.0\%,639

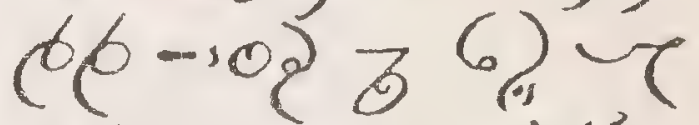
$\left.\theta)^{2} \rightarrow t \theta 6\right\}=$ an. ad

The ten Commandm. g-y $y=2$. . : a gior. तongro. 2. 0 . $19+200$. 3. $\operatorname{sen} \alpha 2 \cdot 20_{1} x^{\sin \alpha 2}$. व\}, $9 \Leftrightarrow$ व

Misereatur. 5 ungs 


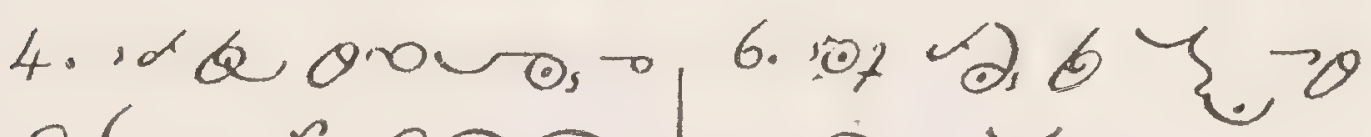

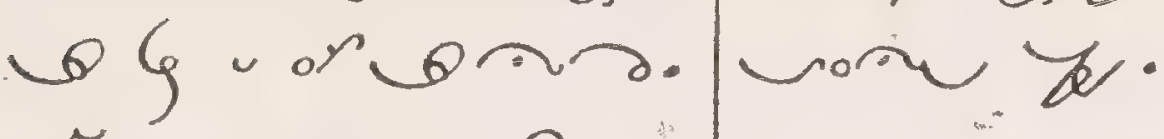

5. $0,6,60+Q$

6. '0t, 03 .

7. of $9+(-2) 6$

2.

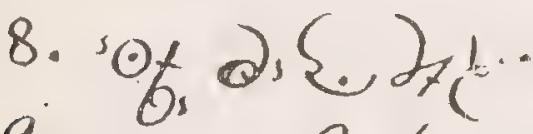

9. $0.0 \%$ of 9 \& $\sim=$

3)

10. 30 of 9 क क.

Precepts of the Church.

of $220960 \%$

1. sd b tot क 6

-6 a sas 2 .

2. 皮家 2 -

se, is, sosat , o (.) $\rightarrow 6-6 \cup \cos 2$.

3. $\rightarrow(, 4,2)$

कू.

$4 \cdot \rightarrow(-1,2)>$

$\alpha_{6} \partial n \rightarrow \Theta$.

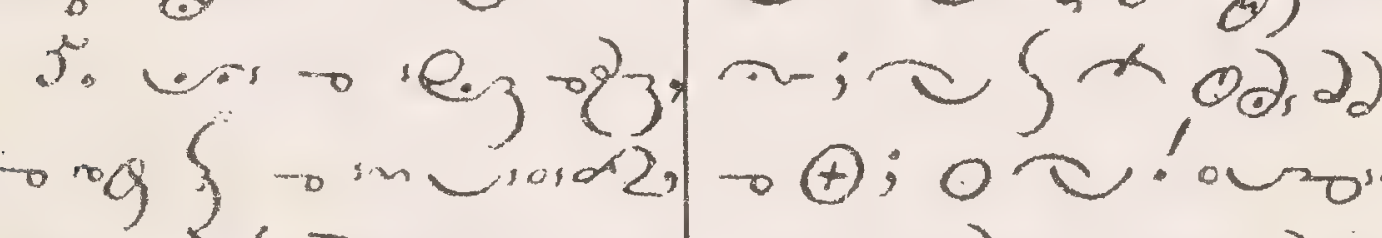

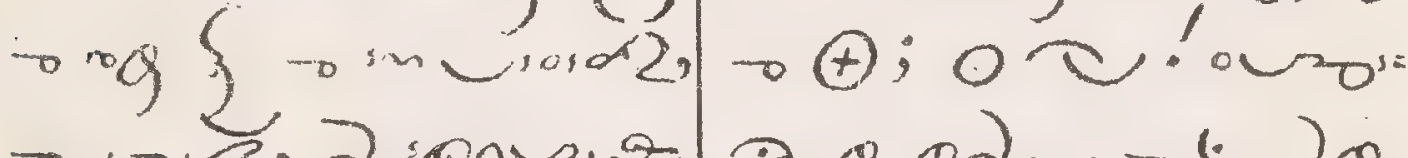

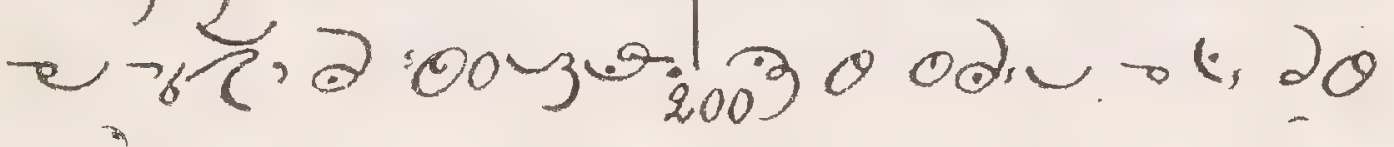

Seven Sacraments.

x女 bysergon

ar $g=r_{0}, g=$

Gu, $\oplus$, plse,

2nko, 9, Fon.

Act of Faith. 'o b

od, ot, Yz

- 960 or.

Oे, 2d = ट, 2

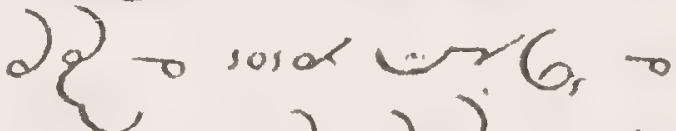

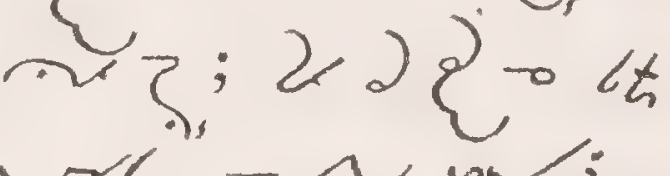

$\sim 6,8 \sim \mathrm{m}$

¿ 9,2$\}, 2$

w; ग2

or

$\int 5-85=03,2$

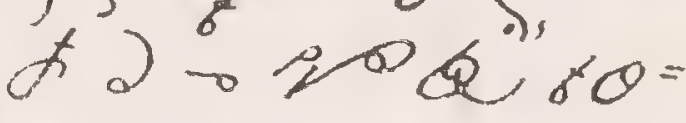

$e-0^{\circ} \sec \theta=$ - $; \approx\{x 02,2)$ 
2 ir $\rightarrow \sim \xi u s_{s}$ Act of contrition.

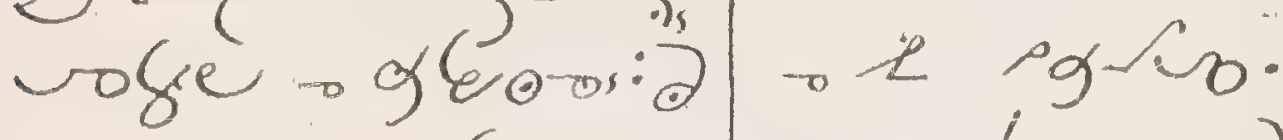

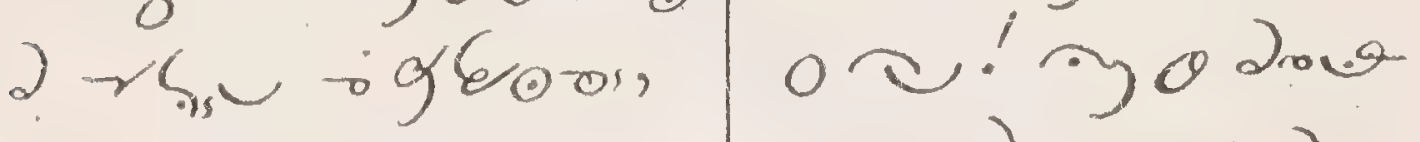

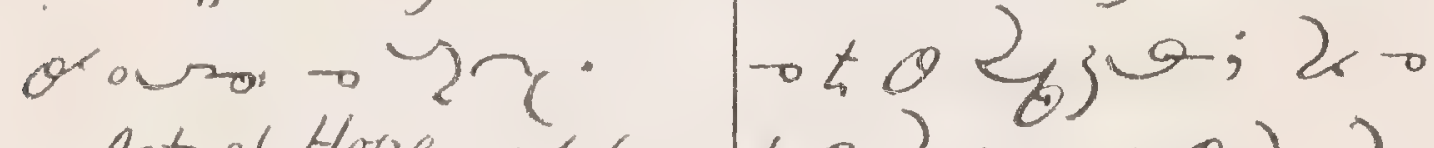
Act of Hope. io a t $\& 2$ ive 0 ) $D$ r)

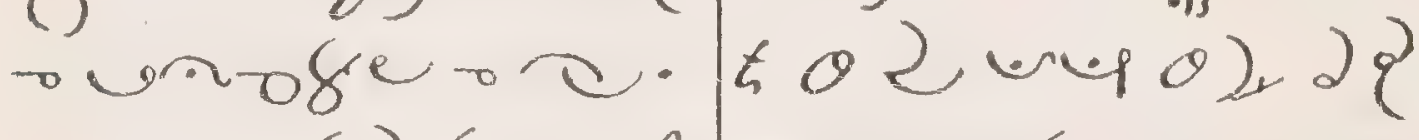

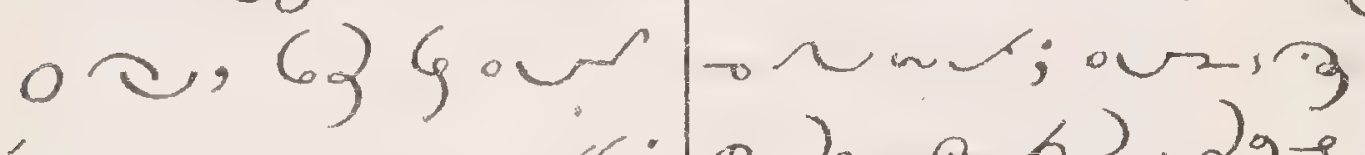

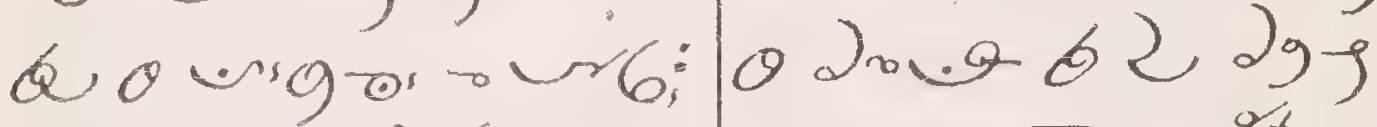

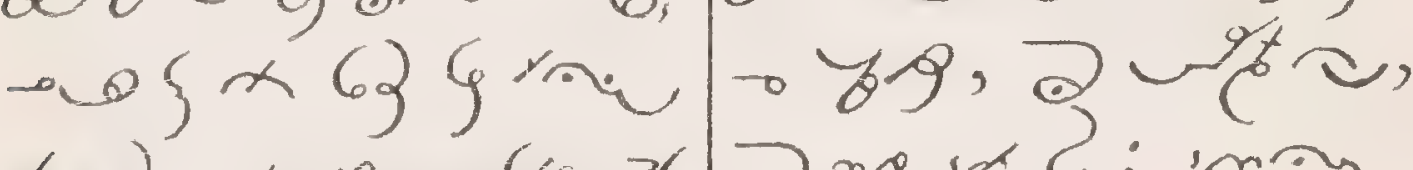

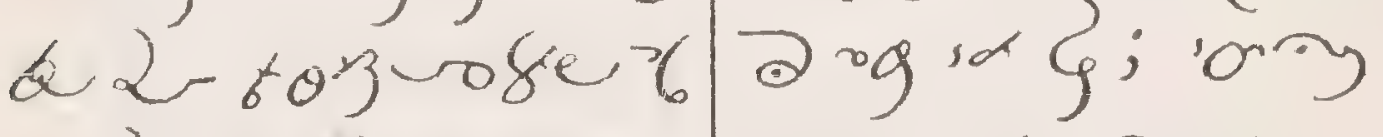

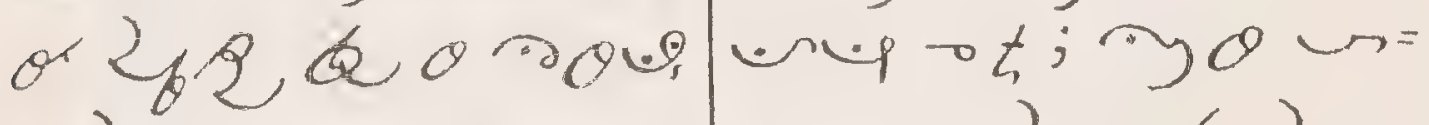

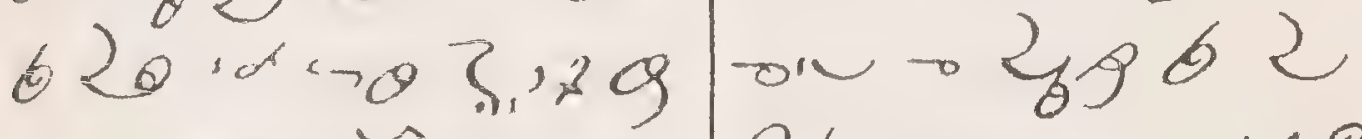
omie o yry,

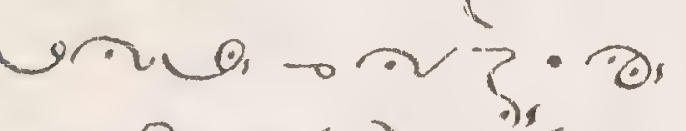
- 2 or 290 $\rightarrow C \rightarrow Y$.

Act of Love. id \&

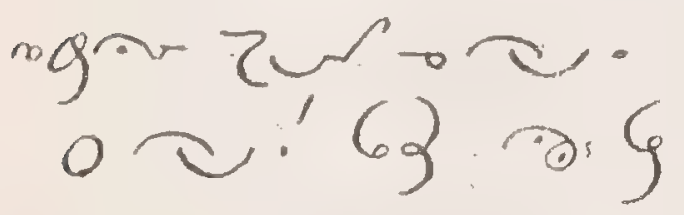

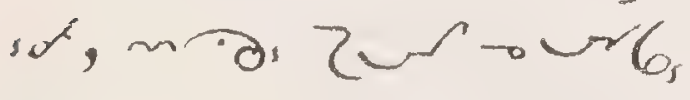

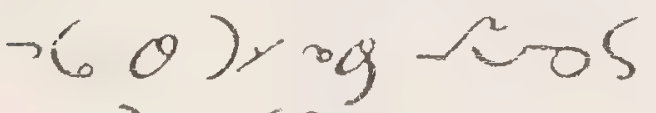
$-0006)(2,0)$

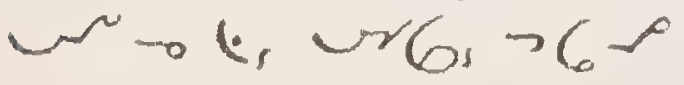

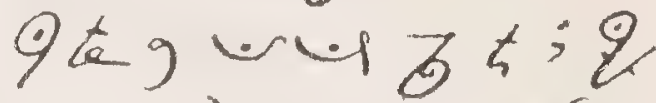
so 20 b6. DC Q $\frac{9}{2}$ o id b row 2 . 2 .

The Angelus. ㅇ. 1. e. nons in or sod $8 \%=0 \%$ cecoug, ow

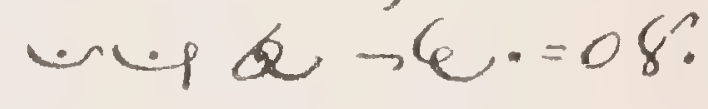
2. $-2, y e,+2$.

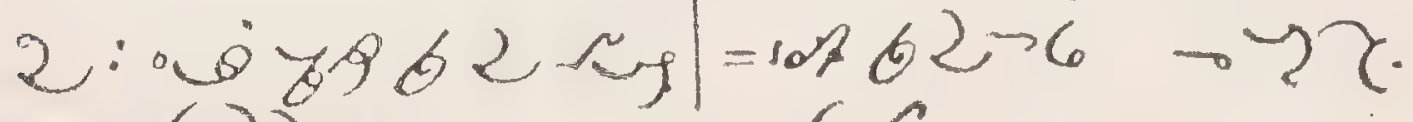
$\because 62 \%$. $20106 \%$ 
3. $\{\circ \approx s-\mathcal{*}$.

$\left.=-\sigma_{-1}-\rho\right) \delta$.

क. -2$) 8 \cdot=$ 边

Night Prayers.

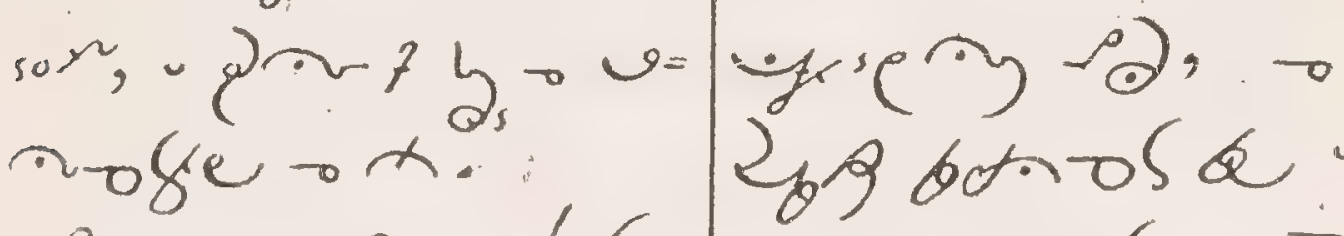

aremus. $0 \curvearrowright$ ! $S$ $6^{2}+a \mathrm{ger}-105$ arjo: on 5 ' of a s g o o s; ny 0 or 2 sor. $x$, $-9 \times 2 \infty 2+\rho$ - 4 : 00086 a Son son. 2 - 0

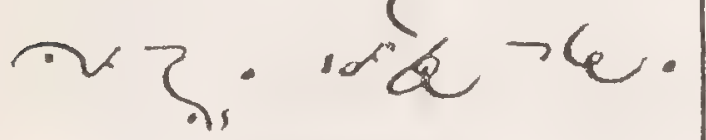

Sub tuum. o 8.
04, , d 2.2 ก ०尺, 2) e of

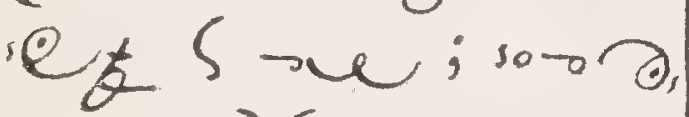
$\left.-t^{-2}\right) \mathrm{s}$. “oinuosis a द re, ods vosin

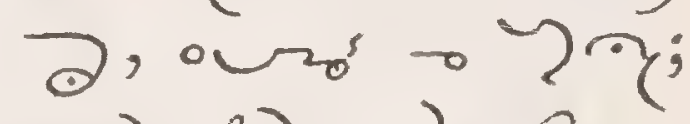
o $2 \rho-2^{\circ} \theta$ $\theta$ g or un

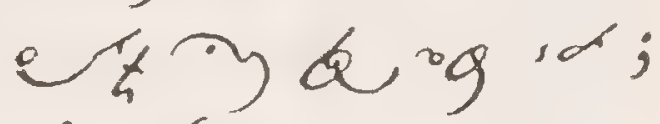
sosing 20 . 2,9 \& 63 g sol $20+4,6 s \rightarrow 6$

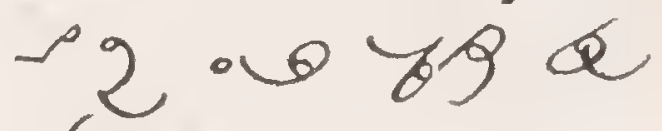
$\rightarrow$.

Thanksgiving. sd a gya 03 390522 - $4, \tau(0) r^{\prime 2} \theta$. तब 92060

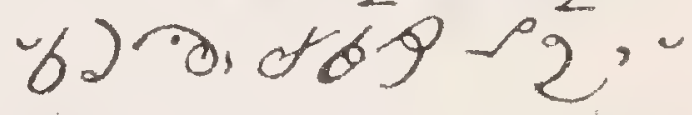
202 620 व, $1 ; 0$ 


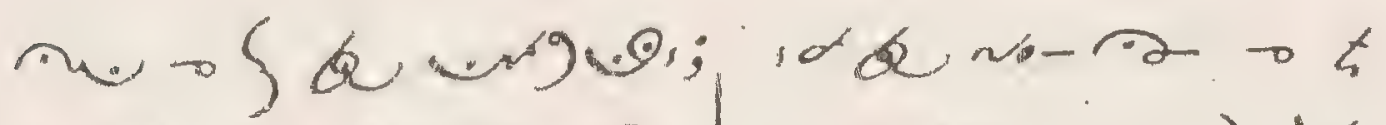

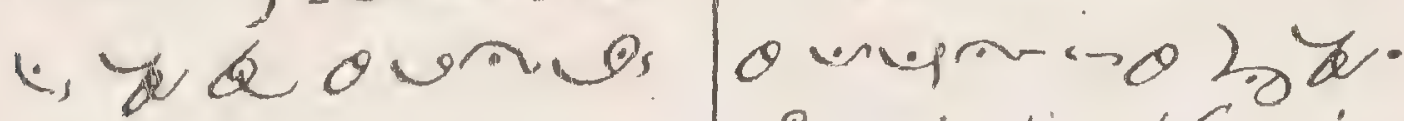
- sd -2 i. 26 ;

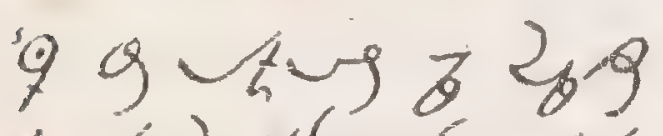
6 iar 29905 . sid $2 p 22 \rightarrow \pi s_{1}, \sigma_{3}$

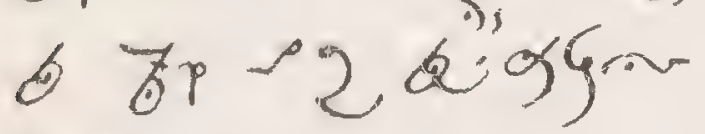
-2.

Prayerfor light. sot a ger . 0 :

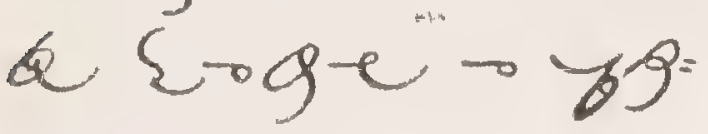

$0 \curvearrowright !-2 \rho \sigma$ 25 ए; ; $a \div=$ $90-1$ 26. $9=$ गक क्रव -90 a $62 \xi t,-97$ a 63 , wat ot

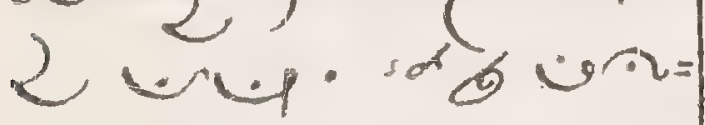

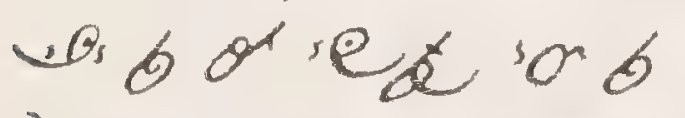
Lo bus ot , k $c_{1} \rightarrow 9 \partial C^{-6}-b_{3}$ un of

Examination of Conscience.

Contrition. $\rightarrow 0$.

Zfoc sog 20 , y-iy $5 \sin 62 d$ क्वे's ot 2 un

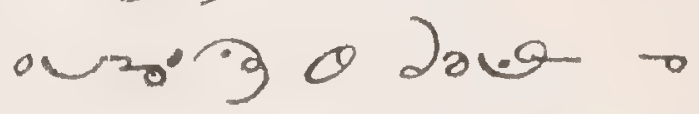
o) 0 sag tyr;

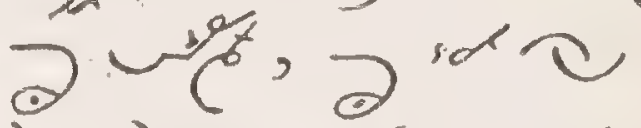
ध० $23: \varepsilon-\varepsilon+\operatorname{soc}$

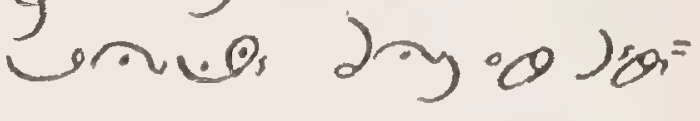

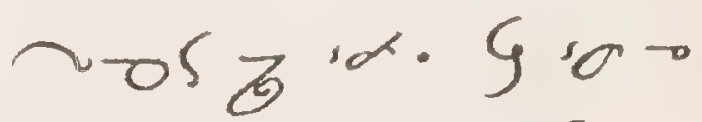
402 Jon of. unam a la usgy 0 , wa

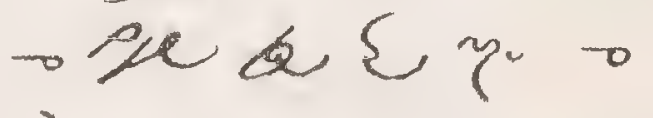
यक日 se的 620 sos.

Firm Purpose. soce y)- 3 - 2 a

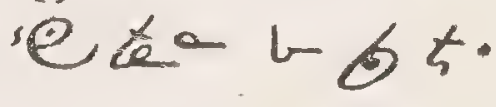
otag 'a $\rightarrow ?$ $203^{\circ}=0 \% 28$ 


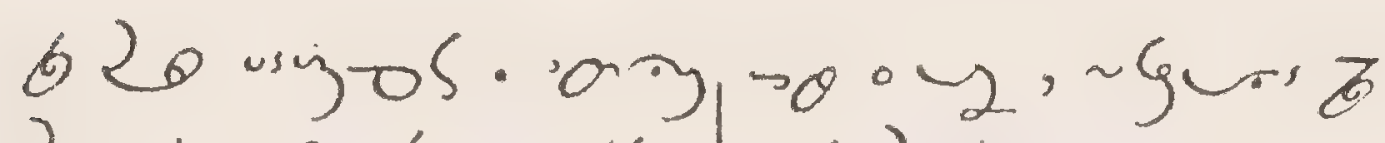

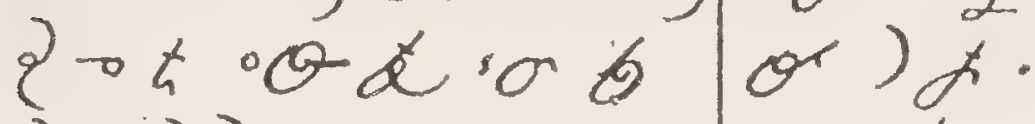

$202 \div$ sd. ox son Angelus. $v$. $6206 ;-\operatorname{lin} 3$.

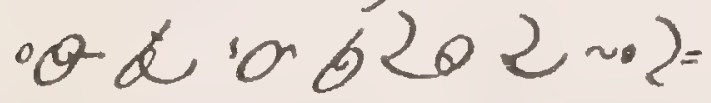
$9 \rightarrow 29$ d e光? 西

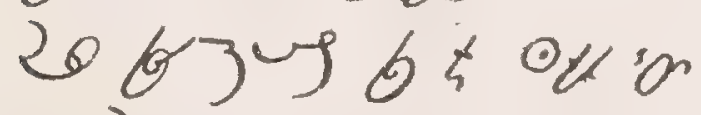
b 20 b.

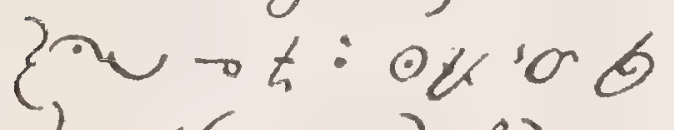

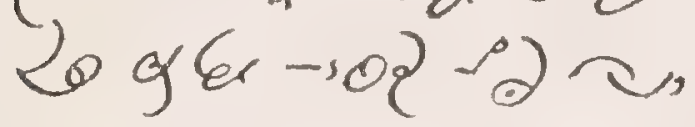
Q Dous - 0 '2.

Pater. Gig oुg Ave. O 6 gुb credo. Gungुg. confiter. 3 कु 6096 .

Night offering. $0 \propto$ sot a bygus, b2<a b2s $3+$ a de 62 -6y - 72 .

0 so $\delta$, 2) Jon $\therefore 2.62 n y \rightarrow 2$,

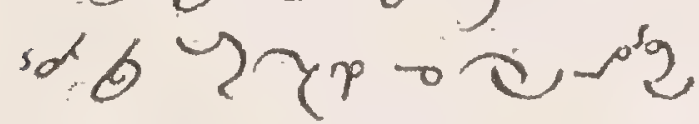

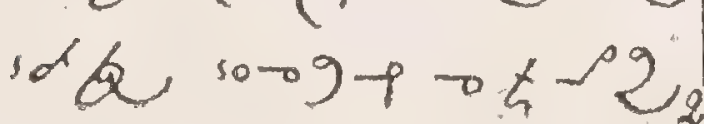

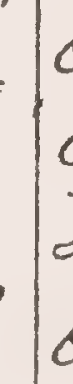

b soog $9-p$ - vop a sed bog a 的的, ban

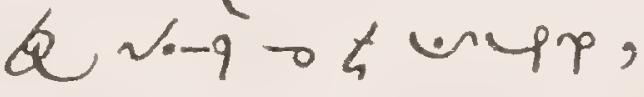

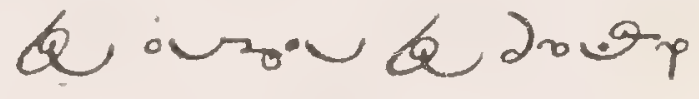
$x$ id 6 -yop 2 a $9+-\operatorname{cop}_{1}$ \& $603=$ $p \rightarrow$ t unp \& $\sim=$ $\mu-69 p-02-$ x.7 5y 2 \& $10=$

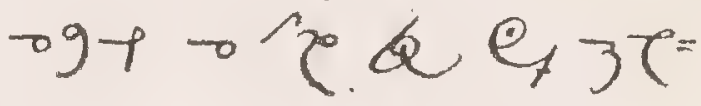
ve mup. by. $=-2 \cdot\} \sim-\xi$ $\sim \psi \cdot \alpha \alpha+\varepsilon$. x. so \& GEr . 乞 他-9e-8g= 


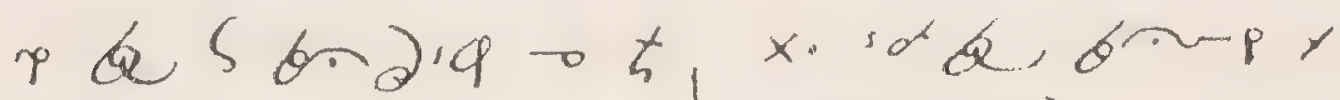
unep.

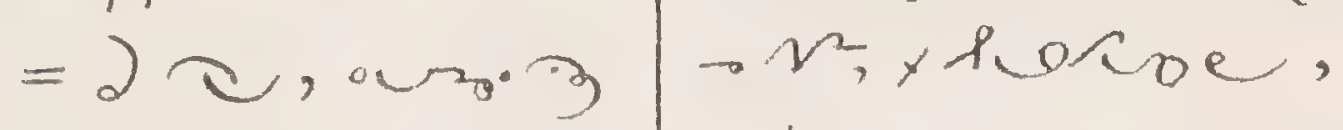

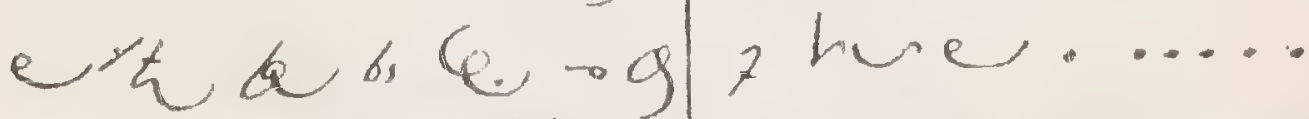

× )

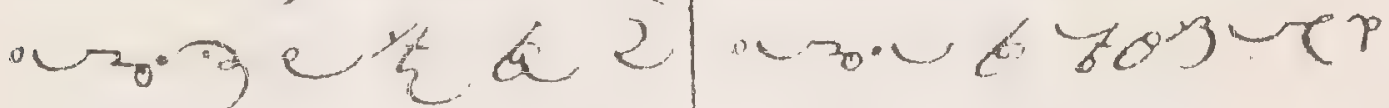

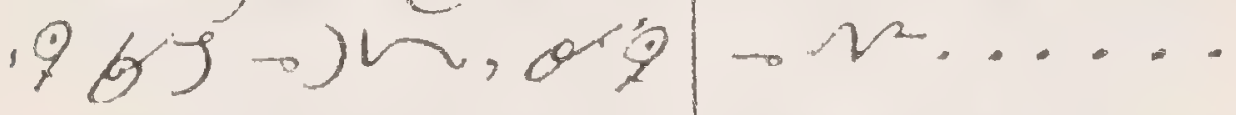

$-9 \Rightarrow k+8) d x$

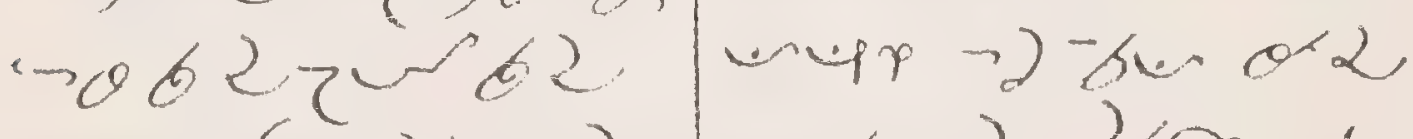

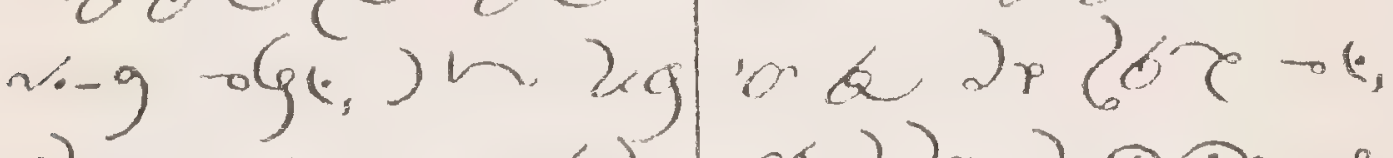

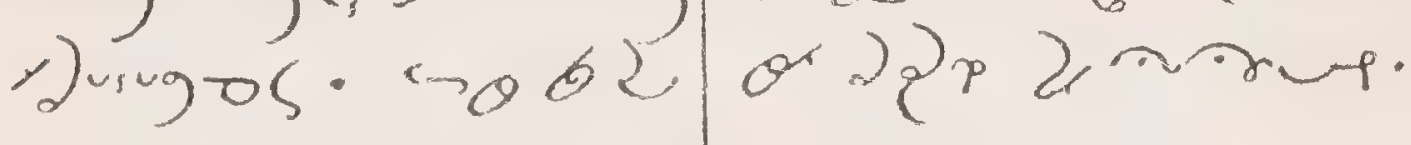

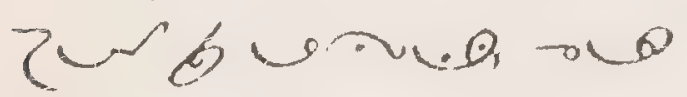

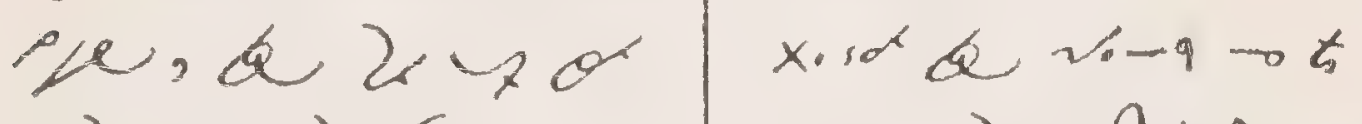

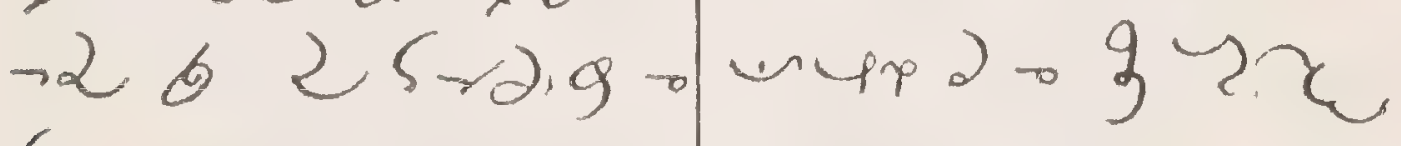
Ge, $9, \theta y, \rightarrow$

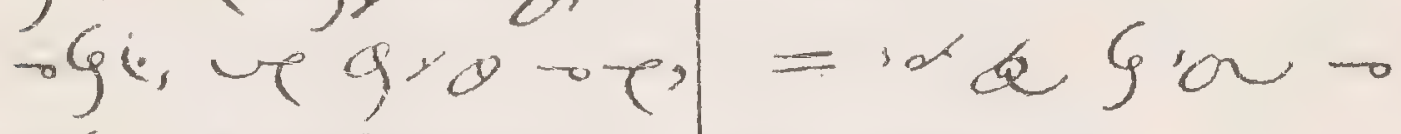
$\rightarrow 90,502$.n. $-96-2,9,62$ Sr 2,9 \& 62 \% 9.2.

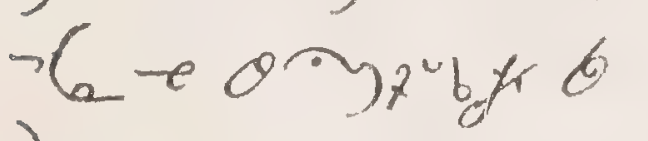
2 .net 0 . $x$. sot $\alpha$ bring $\alpha$ 16 biond $2 x$.

$=\operatorname{sat} 60$ का 6 - 6 ह $6 \operatorname{sos} 2$, , $x$. id 2 2. $-9 \rightarrow 6$ enep 20 of

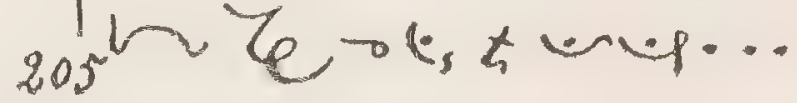


14

SK W AMISH

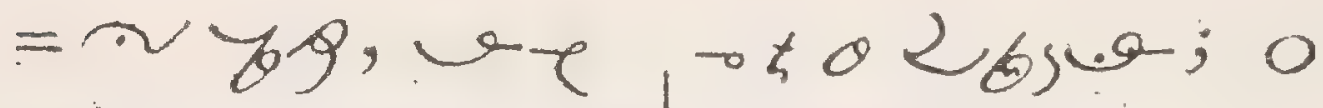

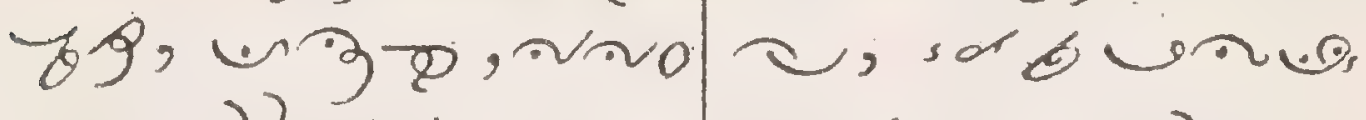

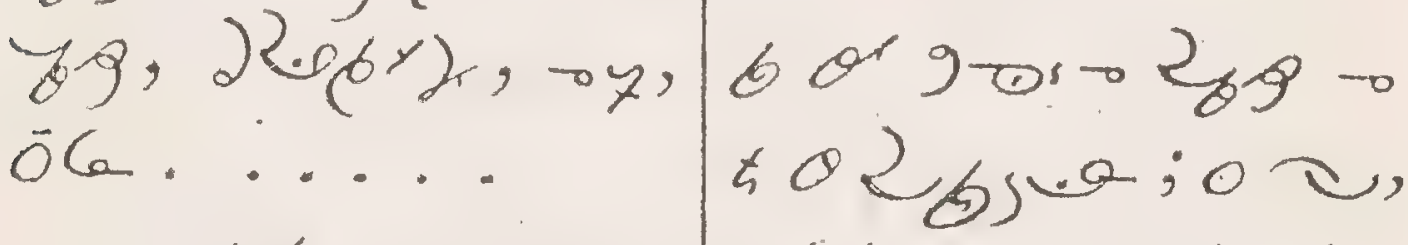

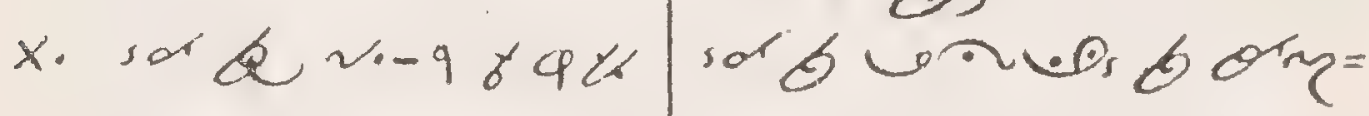

durerupuer sos-28962\%

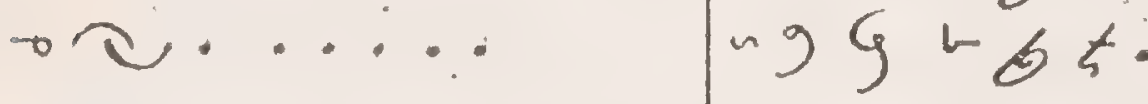

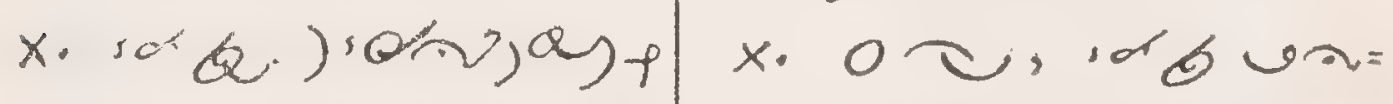

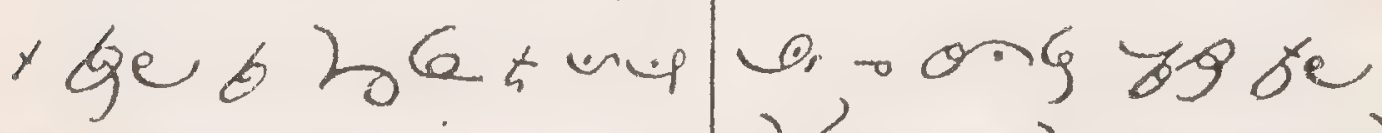

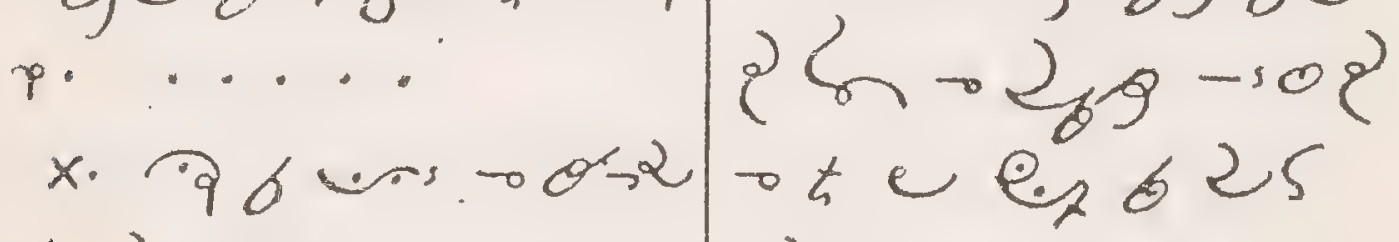

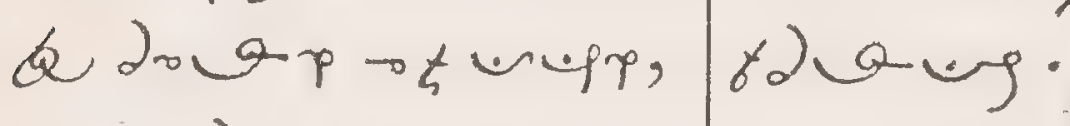

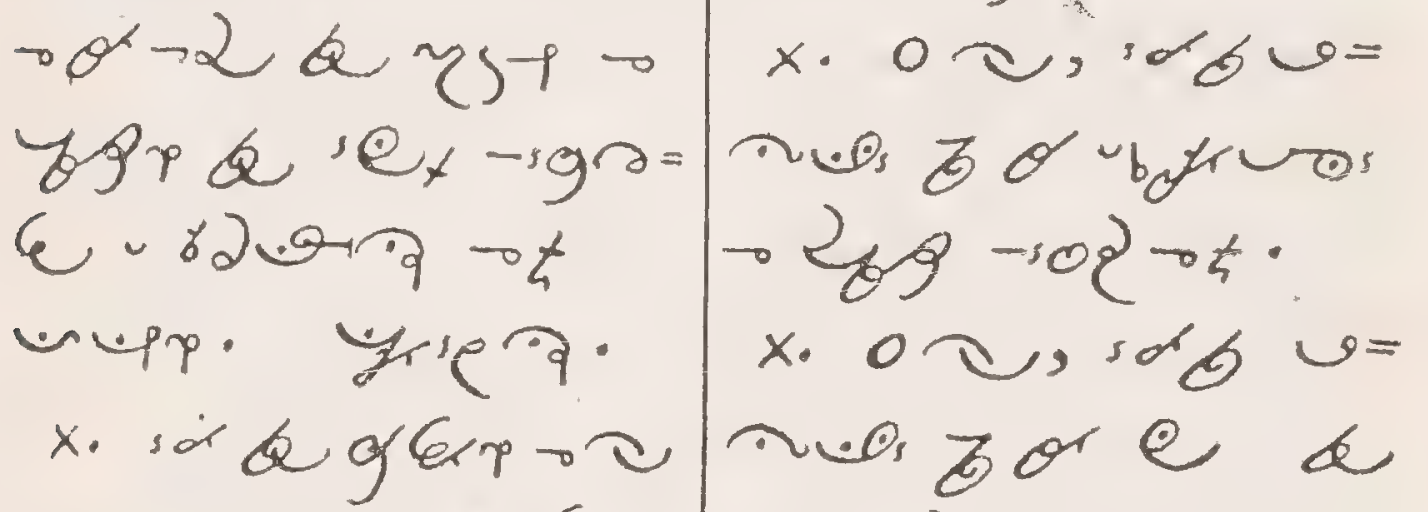

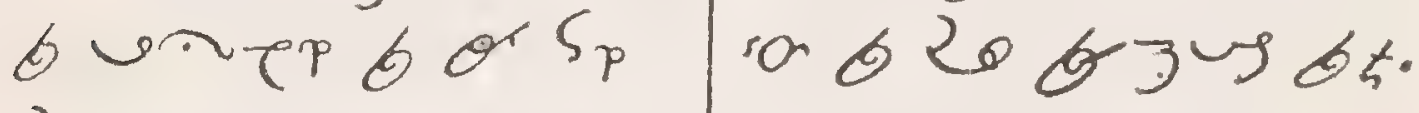

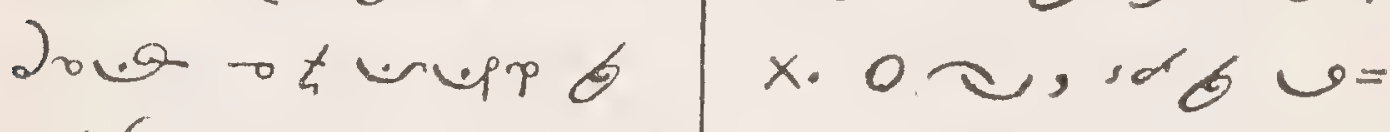

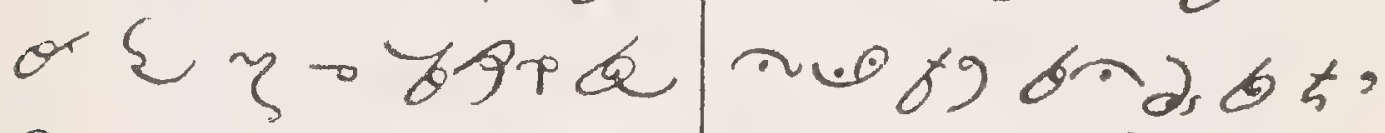

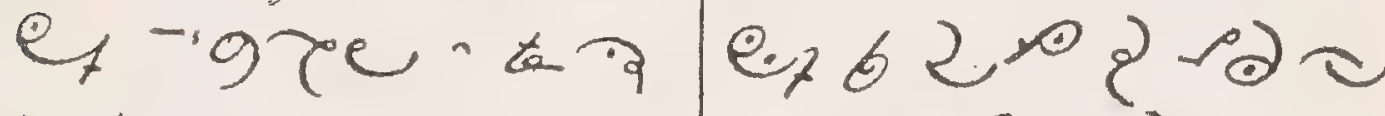

to 6 .

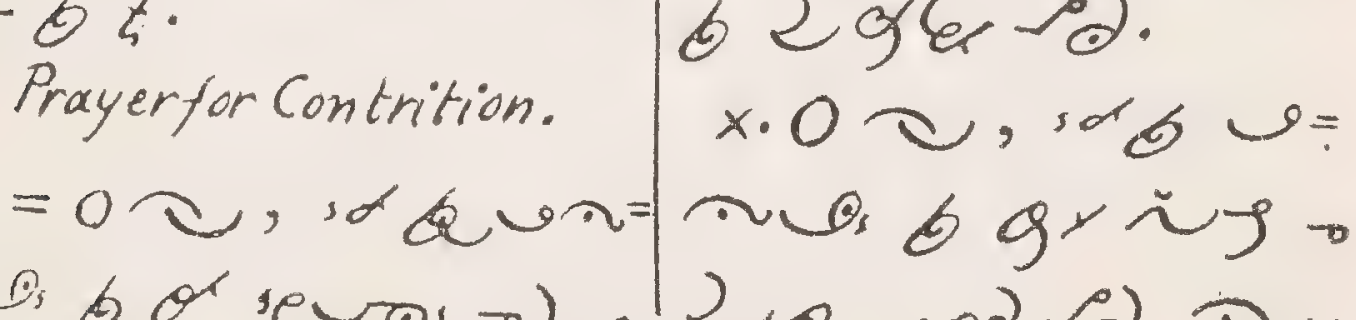

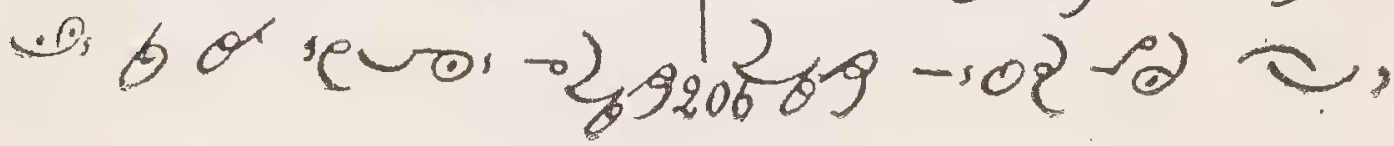


$-302-4.465$

First Cionsideration.

$x$ sor a otgop o

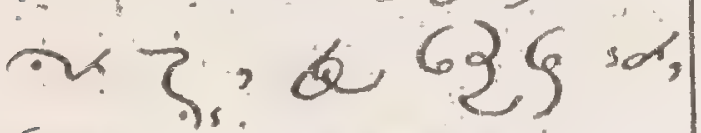

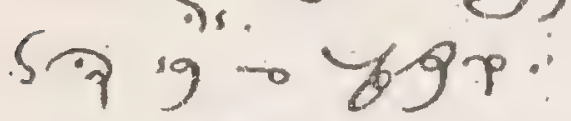

$=020 \% \approx\}^{k}$

$62 \zeta$ sd:- sos

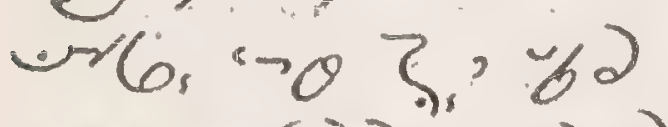
$6 d^{t},-6024 \% 2$ ?

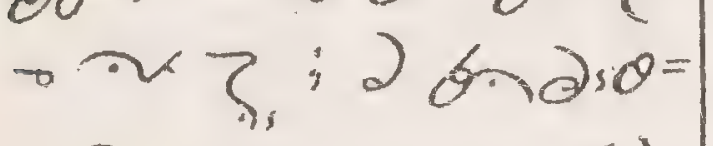

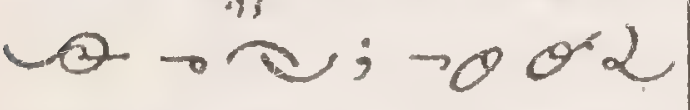
的始a un - $990.622 \%$

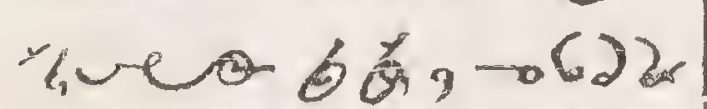
कि "थeo-but

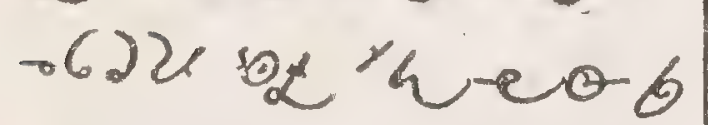
f: 00 une थ $\}_{s}$ forg: 9 youp- $\Omega$, - उत $9 \times 0$ un 0 th $\rightarrow 062$ seig -2.

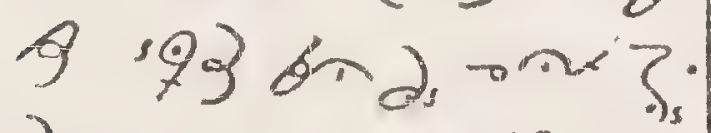

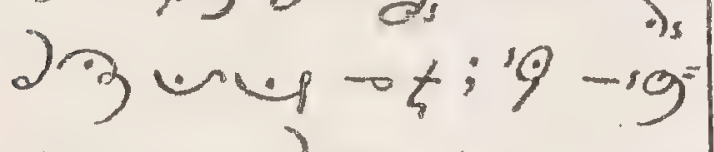

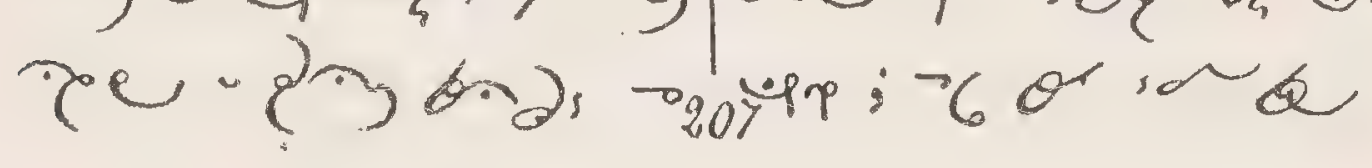

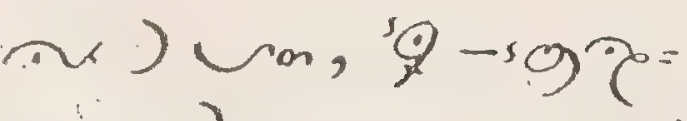

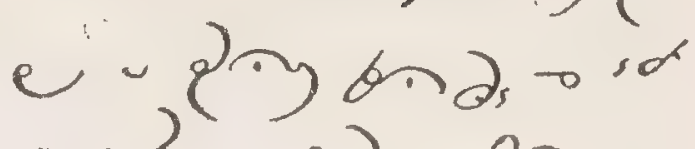
$\operatorname{tr} \xi \cdot 0 \partial, \mathrm{ery}$.

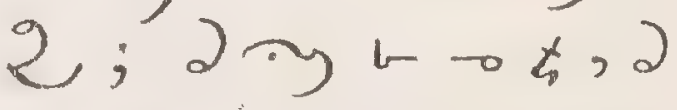
गु

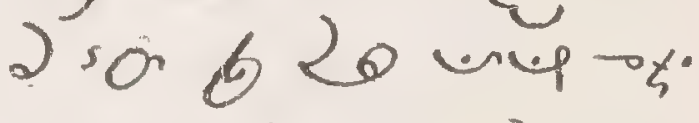
$\theta \cdot-29620 \cdot$ os 2 ¿ 5620 $-2,9-2, g$ ard वरा; व;06 sacos $\partial ; 2$; 6 2 ‥ $-5 \cdot \theta 3$ zeon -28962 9) $16 \%$ क $6 ; 03$ 7 मक $+89-1032$ '25 36 . 'थ :0 है $209 \varepsilon-9 \cdot d\}$ 5 गेers -2 .

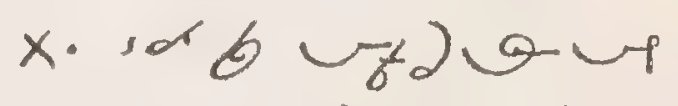

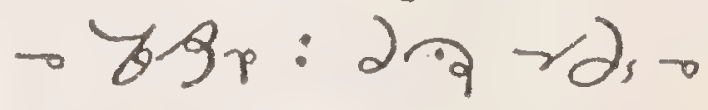
य了, \& 629 a

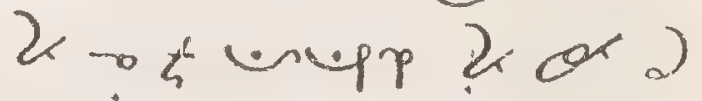

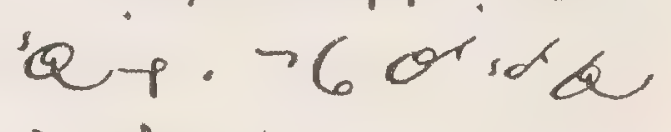
$20 a_{p}-102-t \omega=$ (⿻)丨 
16

SK WAMISH

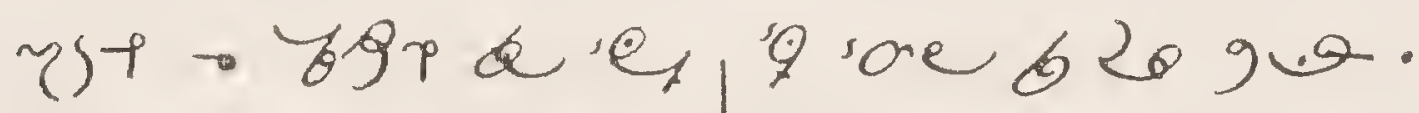

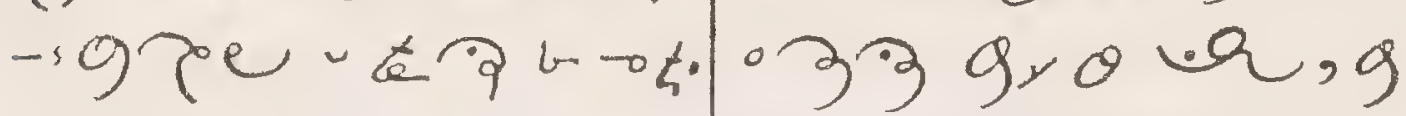

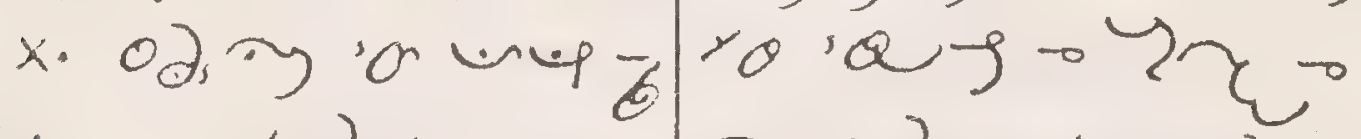

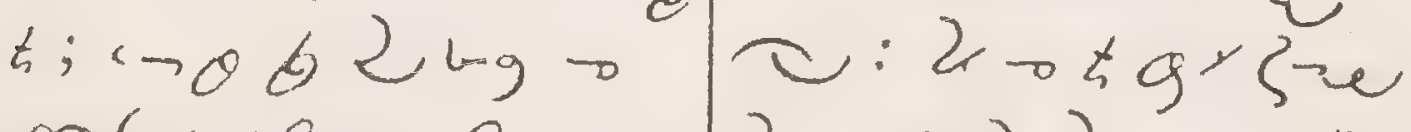

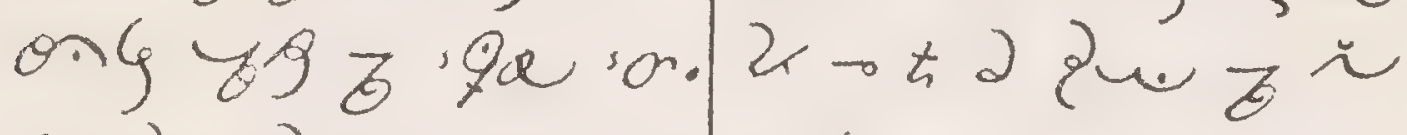

of ? -2 - 9 -t,e wh.

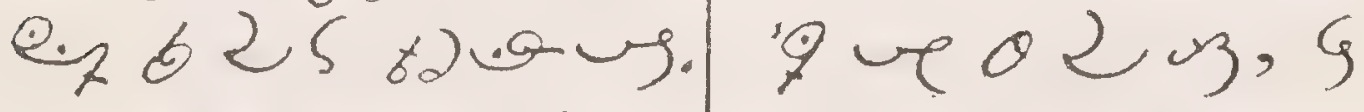

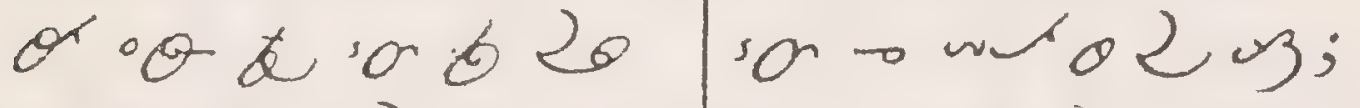

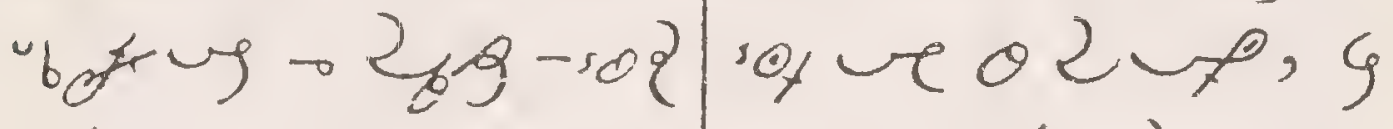

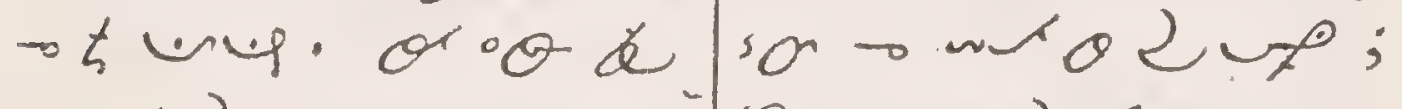

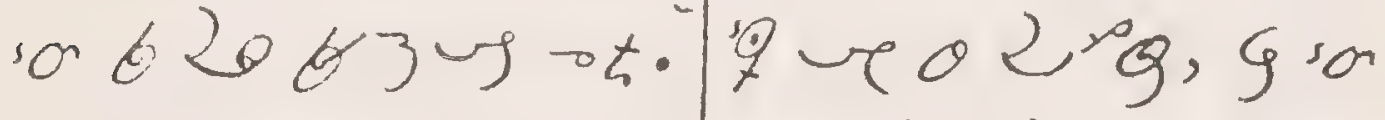

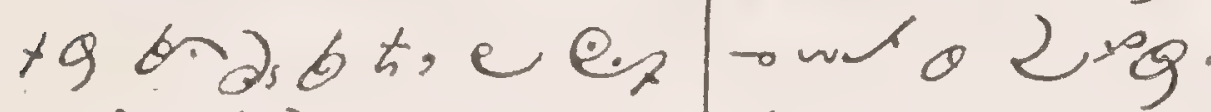

b $2 \rho, a \rightarrow a, b \quad x-2023, b 2$

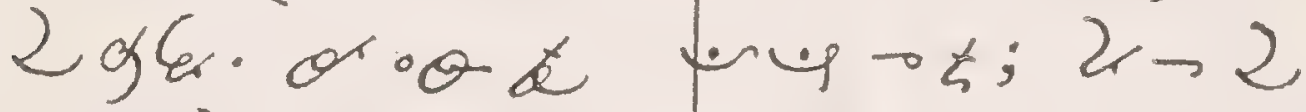

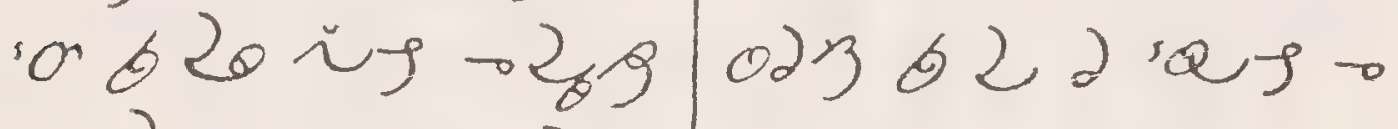

$-102.0,-102 \div$

चr 2 .

is erbs.

$\theta \sim$ un of $\rightarrow$ Co 0

brofio ungof;

Second Consideration. 3 was, 3

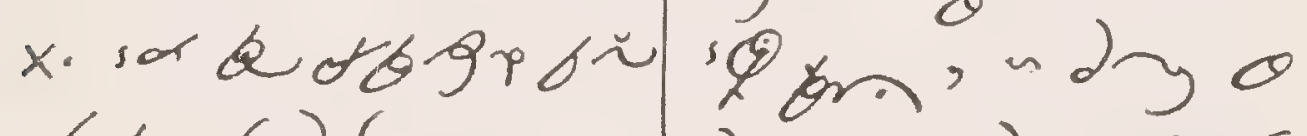

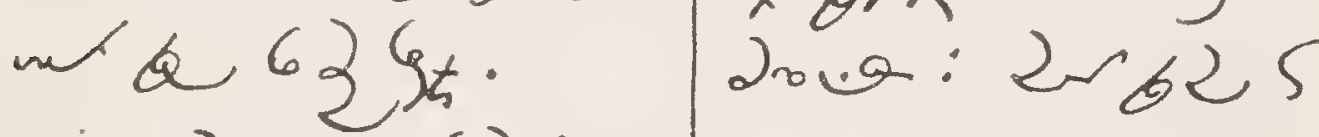

$=020$ \& 639 a $e_{7}$ and, or 9

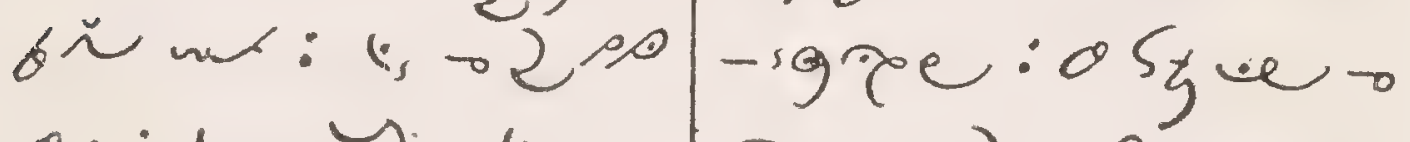

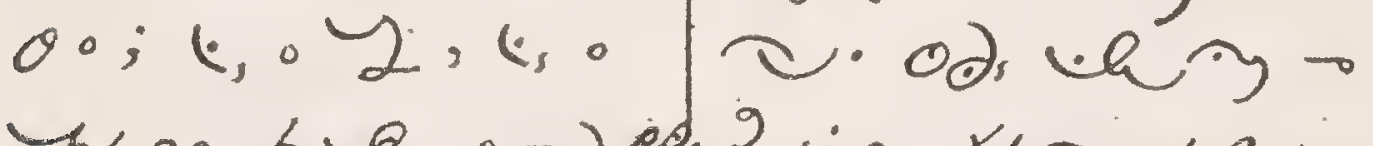

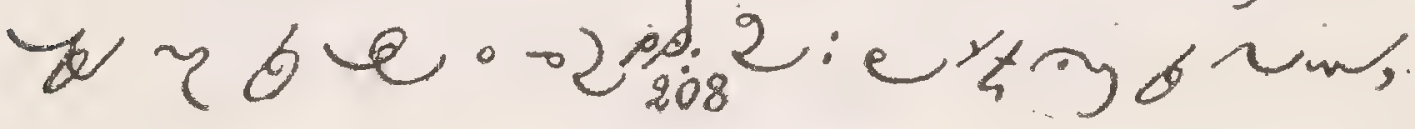




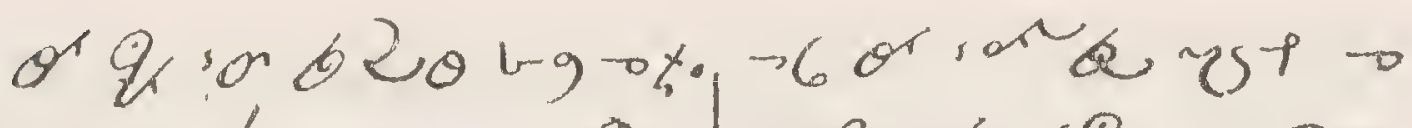

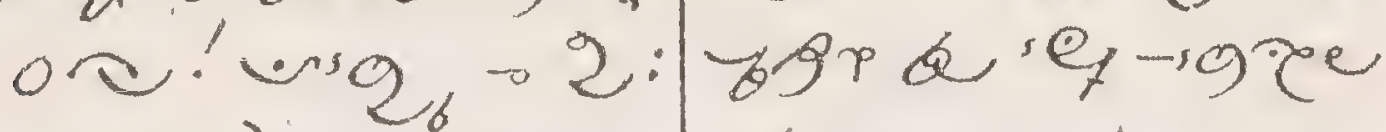

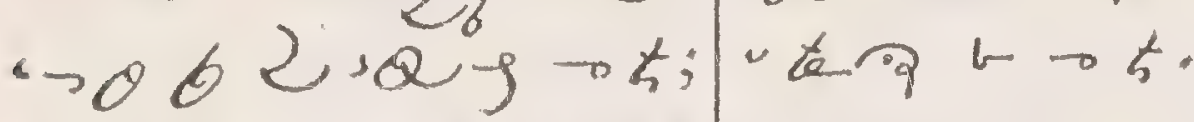
$\zeta \sigma^{2.9}-2,9-2$.

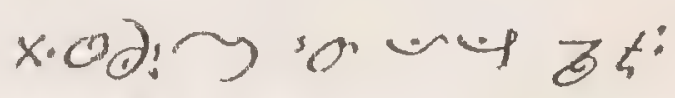

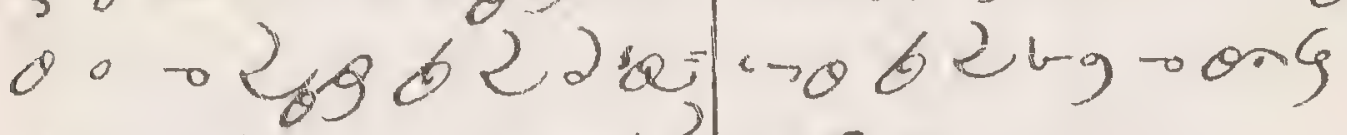

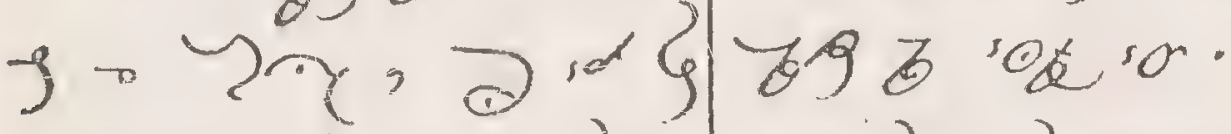
$\partial 2 \cdot \partial \operatorname{soc} 6$ $\lg \rightarrow t, v-$ G $362-2 \rightarrow \sum_{3} \cdot 9: 9$

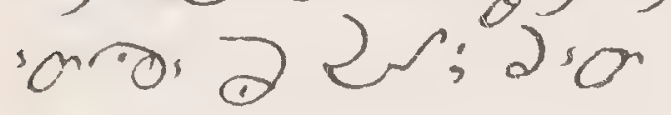
6 2osats. so 620 in 6 . $\theta$ मे 2 en-2y 62 79 क 69 西:

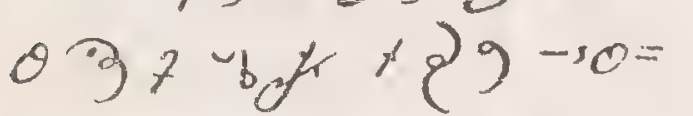
3e-y, $964 \cdot 2$ 'on 200 ger.

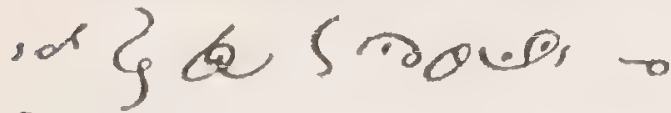
र.

x. sor b - 82.94

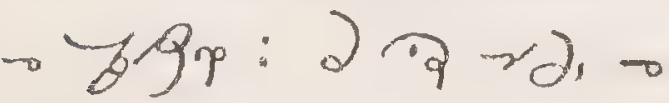
$\sim_{\text {mat }}$ a $, 62, \xi t, 2$ 2ody bor un $\theta_{7} 2 \rightarrow 2_{6} 9 \rightarrow 4, c$

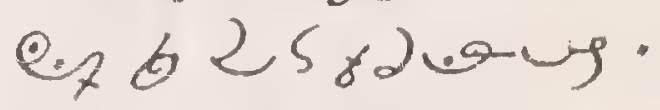
or. "b for to 2, क-10? - 5 unep. or ${ }^{\circ} 0$ ह 10 b 20 $63-9 \rightarrow 5,6960=$ a) $b$ t, e e.2 62

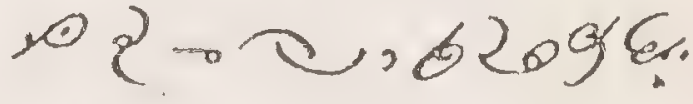
or $\theta$ b son हर 20 几フ十2,9-50? ¿, $-\operatorname{sog}$ - 16 ,

Third Consideration.

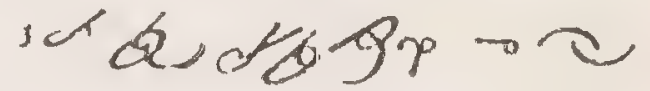
6. 63,9 , or, b 43 Gr.

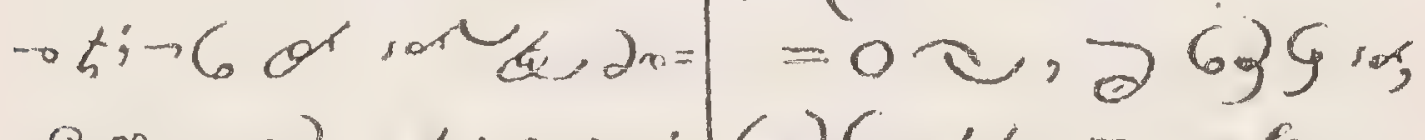
. 
2. वसंख्य

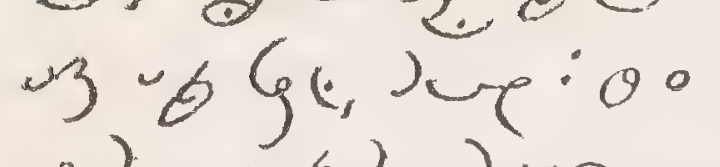
-26962 don

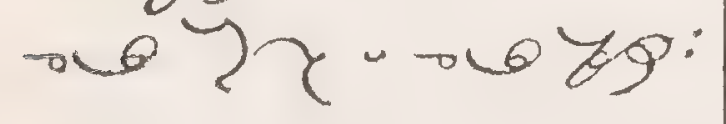
oc, 2 wig. 9

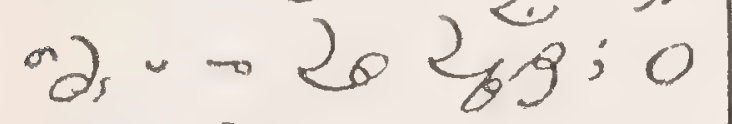
c. 2 ne-ke

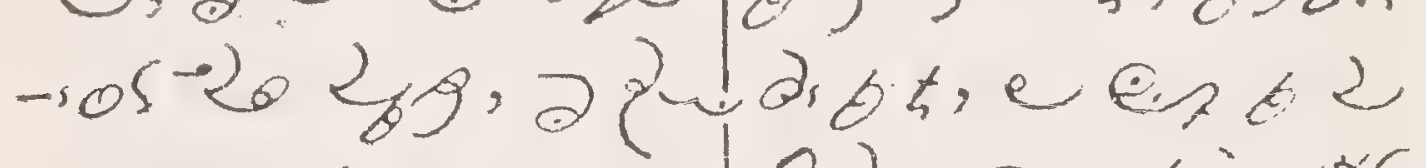
$\rightarrow \sim]_{5}: 0.9-6,4$ zi n'; 00029 -0 2un hing $-60-289629$ 2) $565^{\circ}$ x. $2 \div 2,2,-\approx$

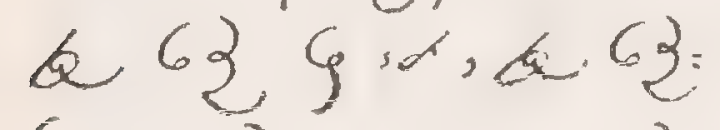
Gr: 2 o ung t, 2

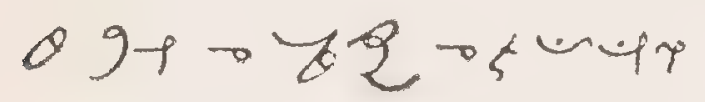
-6 or iar le derer

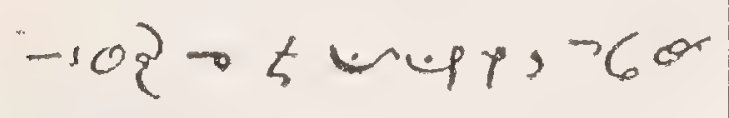

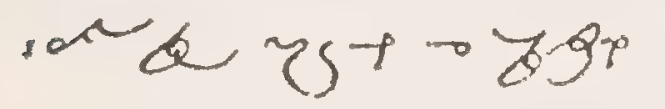
bertgre-ta $1-t . .$.

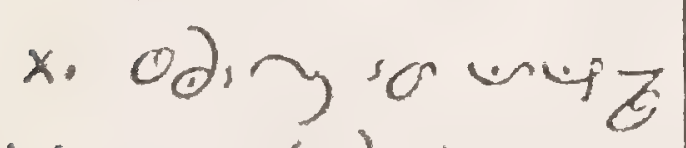

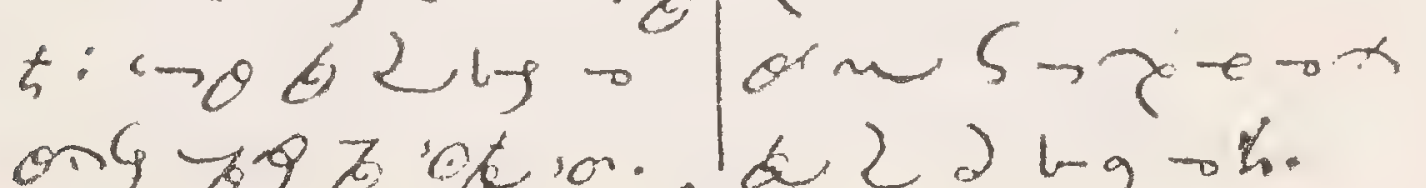

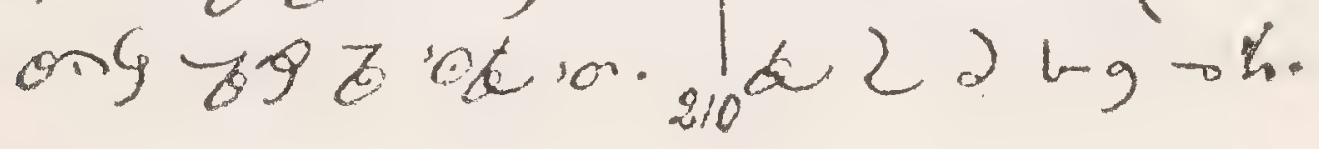

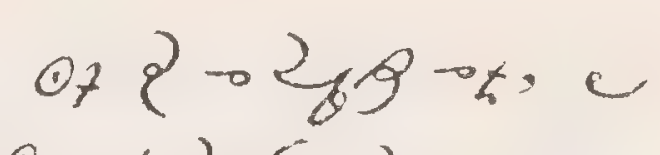
$e_{7} b 25 x$ anes or actot 20 : $=$ Hug - 2 कु -t w山. or oc tor 2

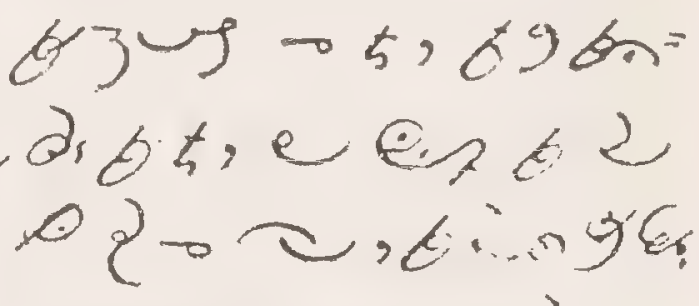
orodice चु- 2 कुC, $-502-4,46_{i}$

Fourth Consideration. $x$. wa ctogip $-9 x$ \&629-u.

$$
=02.6 \text { a } 629
$$

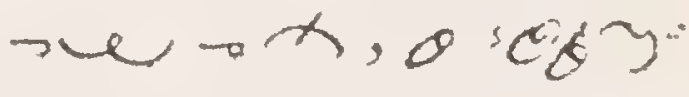

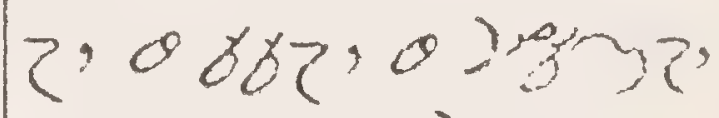

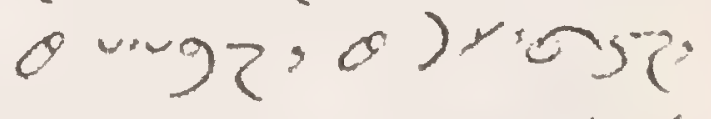

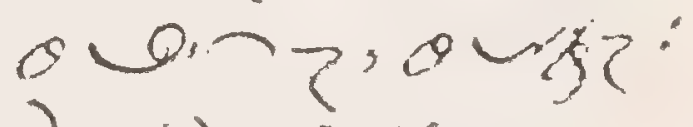

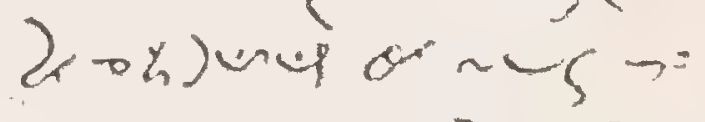
$x+-x ; 2 x-2$ 


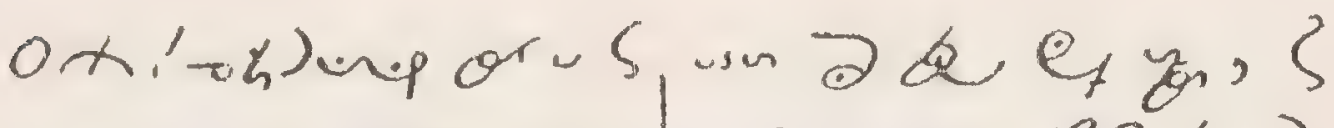
su; or. 3 y or ify ow or

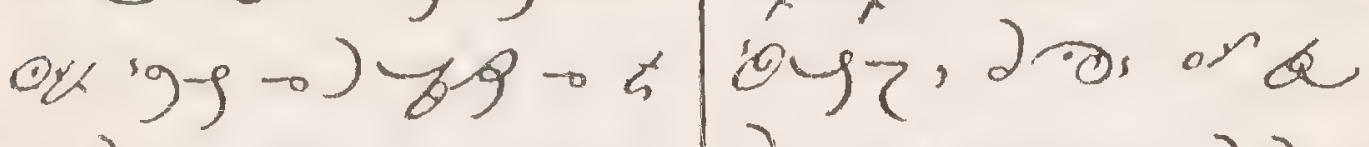
o 26ja; or 3 के $9 \times 0$ son bur. $\rightarrow t$.

$0 x ! 2 x \rightarrow \ln 2$ $86.05,2-0) 22$ ef $28605: 2$

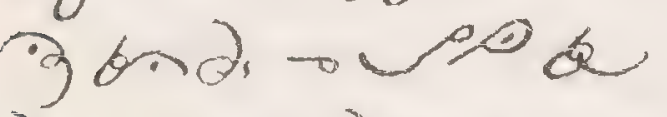
$2 f f ; 2+y$ or 2 .

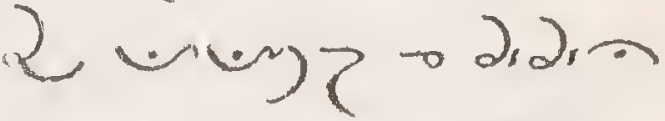

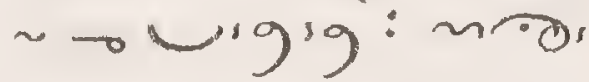

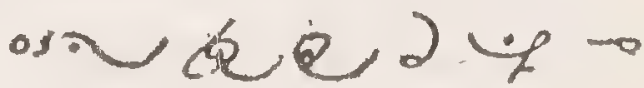
多; J s दo os -bora. $2 x-5$ J f00 5:02, el?

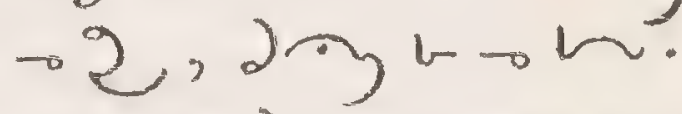
$\rightarrow \theta b 2$. $\rightarrow$ -ais, b 2,2 br In, ond $\rightarrow$ Te, 20., $\sigma_{0}$ ) <g? $\rightarrow$ ? द. ox! केण zus. $2,-6$ ots u; Oे th

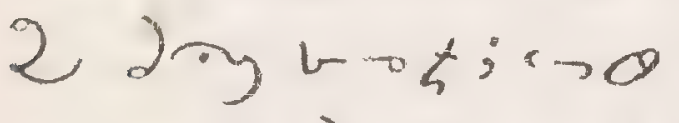
反 $202,9-50$ 2es: $\rightarrow 002$

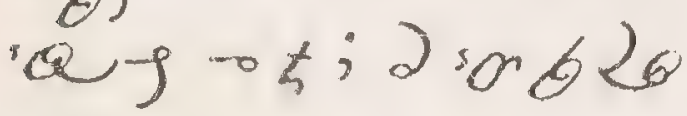
$\log \rightarrow \frac{h}{h} \cdot$ ox! 20y bia 250

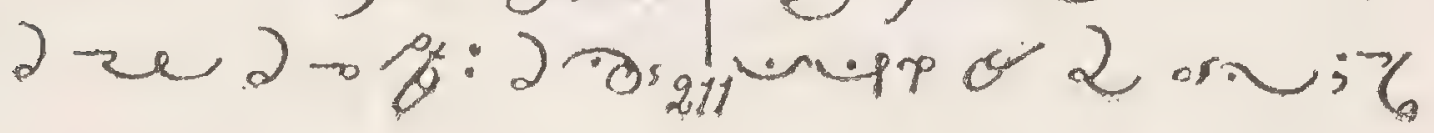
2 and $92 c^{2}$ $6516 t \cdot \omega \pi 0^{=}$ Sod 202,$9 ; 0=$ jo oor 1 ze 9 2. $2 y+92,990 \%-9$ $b_{t}: 037$ bots 89 $-1036 . y, 364$

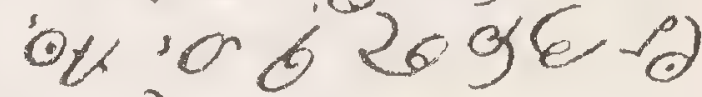
is द \& 5 movo 0 2.

x. $2 x_{p}-2, x<$ $63, \xi$ or : $20 x$

, 
ors $a$ Jones-io? $\rightarrow$ t usp; $\rightarrow 6$ or $1 d$

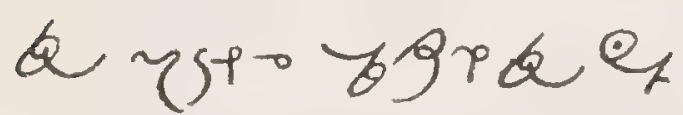
-s9e ka b ot. x. odrin son un t; $\rightarrow \theta 62 \operatorname{lng}$.

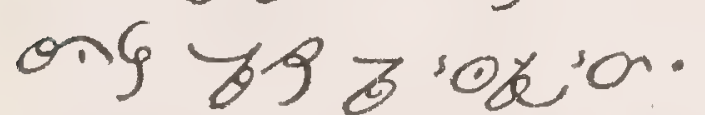
o, 20$)$ - $9 \div 5, e$ e. 625 to.aug. or. $\theta$ as $\operatorname{son} 20$ by - 4 i.

or ${ }^{\circ}$ d ' bjus to t 69 onds bt,e es 62 so $\rightarrow$ at or 0 औ'करण $\sim \rho-2 \beta-10 \%$ $2,-108+\left(., 46^{\circ}\right.$

Act of Contrition. - Losora. अवर

\section{offering} of the Mass. of $y^{6}$.

'eक\%); $266-62 t$

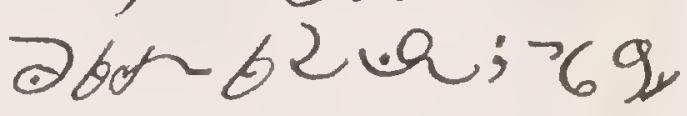
2 bet b) unos?

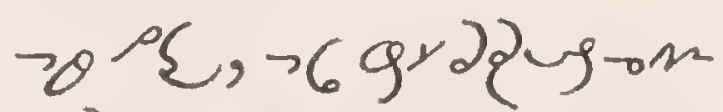

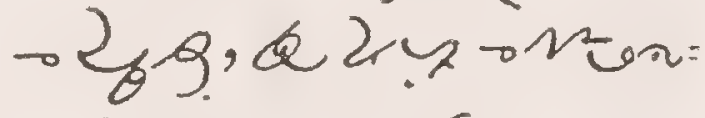
osa. un ostaga

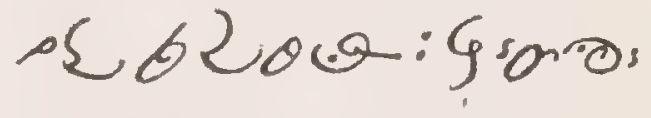

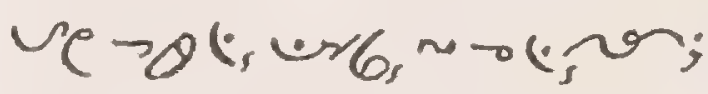

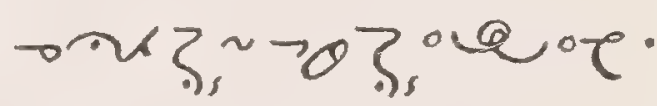
जnofng a 20 म 62

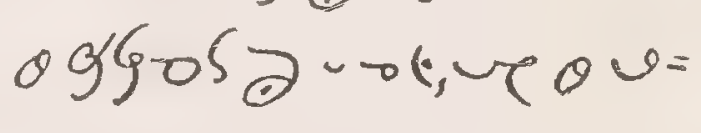
थ⿻日s, vor, reavne. unosy aroos

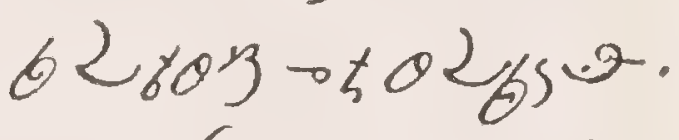
unotng 20 os a o nigus $-2, \sim 0$

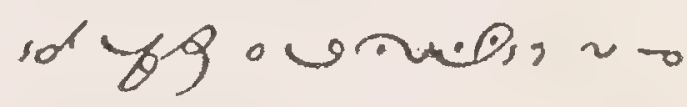
r.9 ons, a ents $\mathrm{q} \sim 2$, $12 \partial y+2 \cdot ? \cdot$ 
MANUAL

21

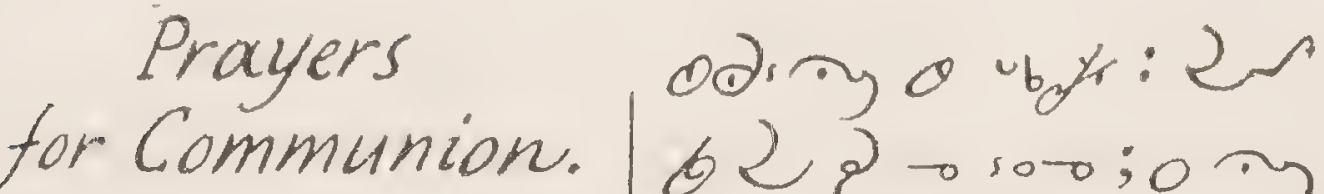

Ber Cann

Before Communion. Y 2 ? 9 -sagee 62

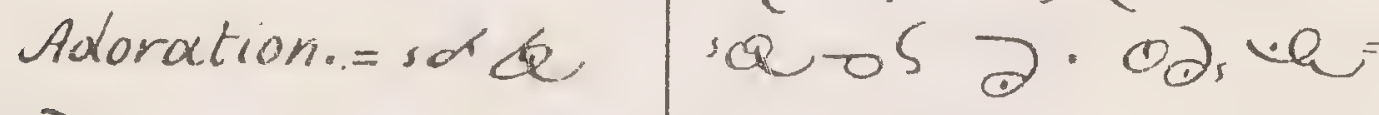

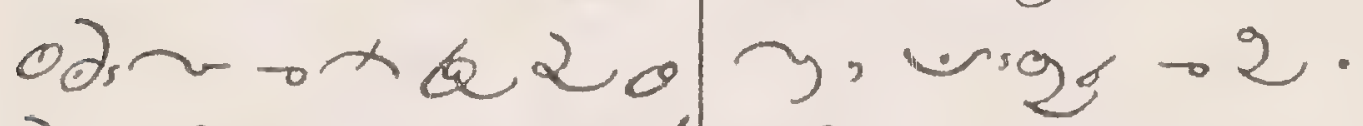

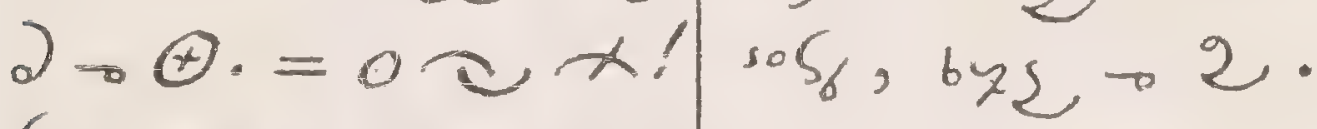

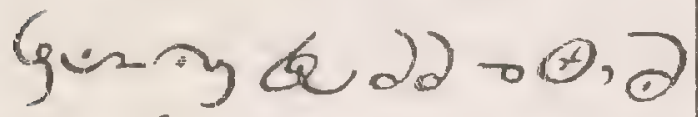

$\simeq \xi, c, \partial, \partial u, D$ Contrition. id a

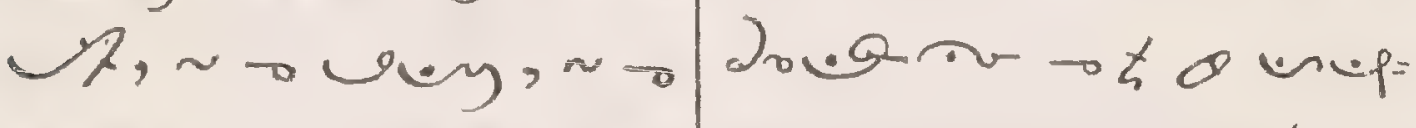

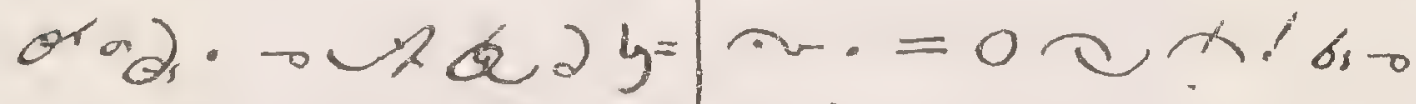

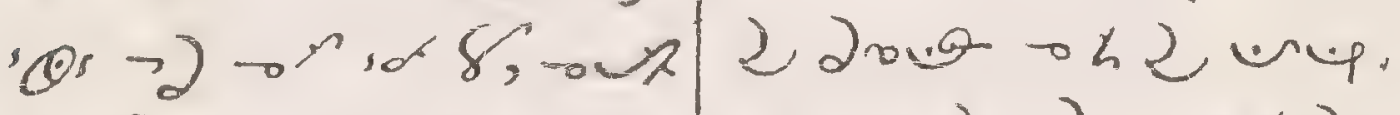

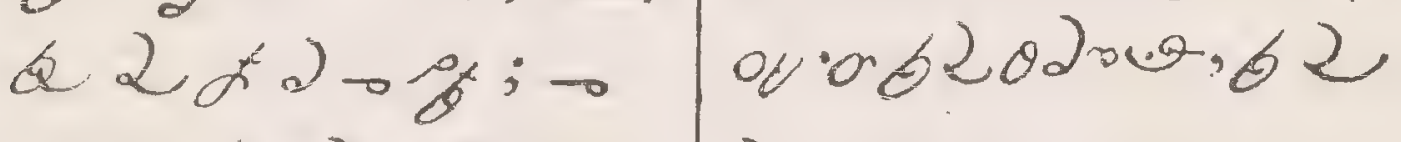

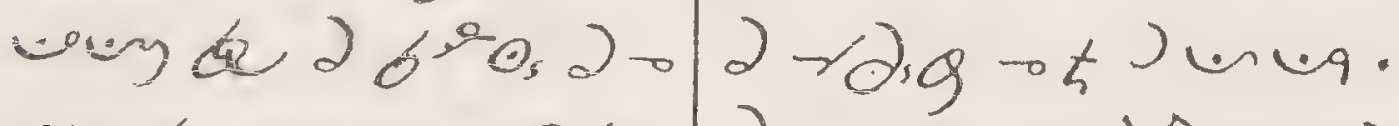

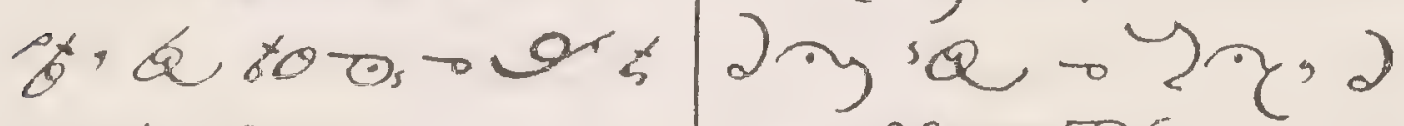
obuar. y pevos 2.0

- द०-2, 9,02,

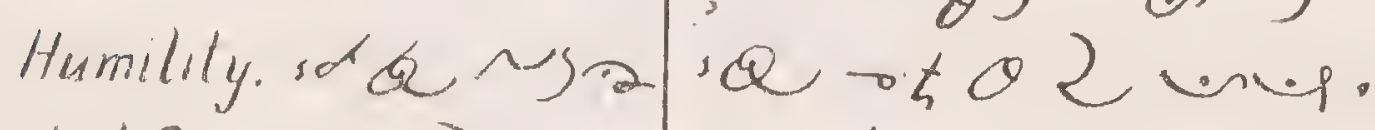

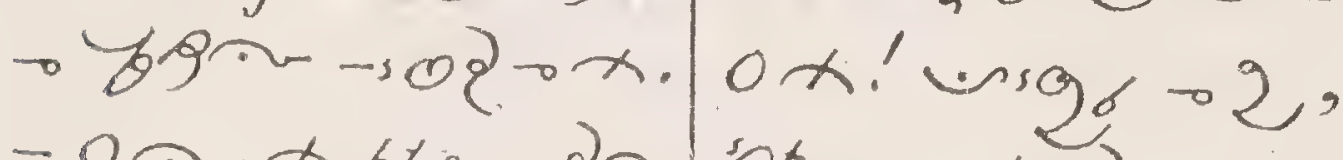

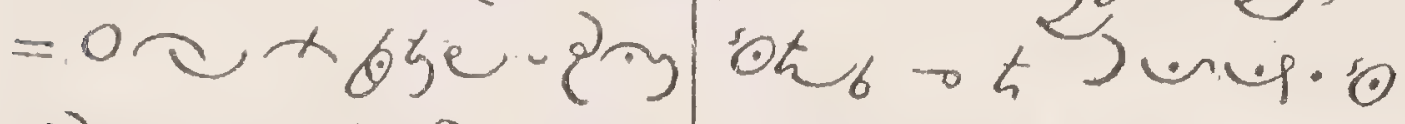

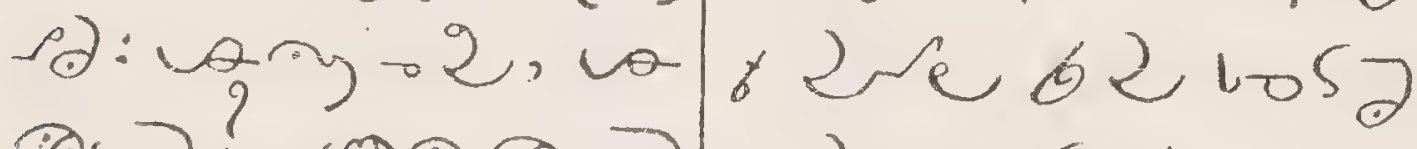

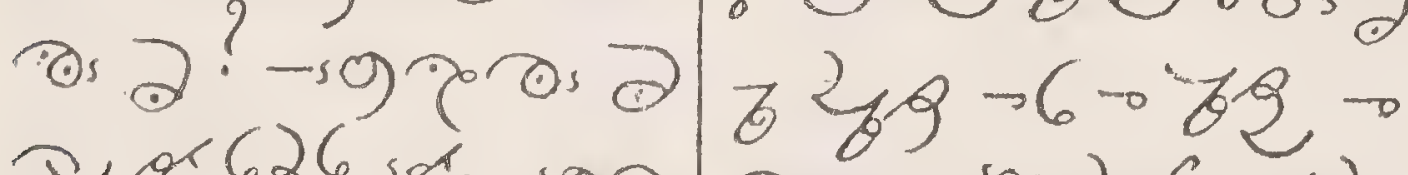

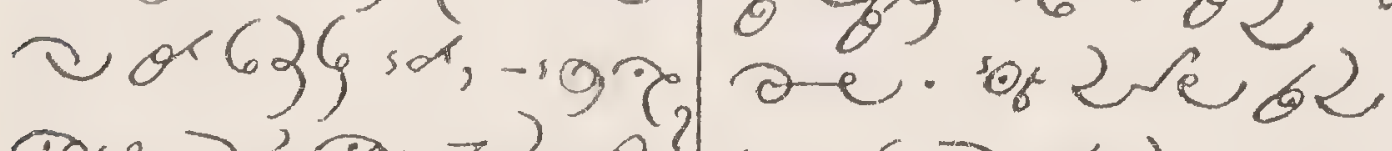

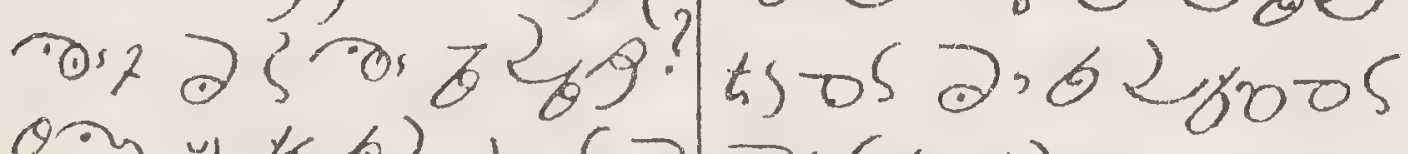

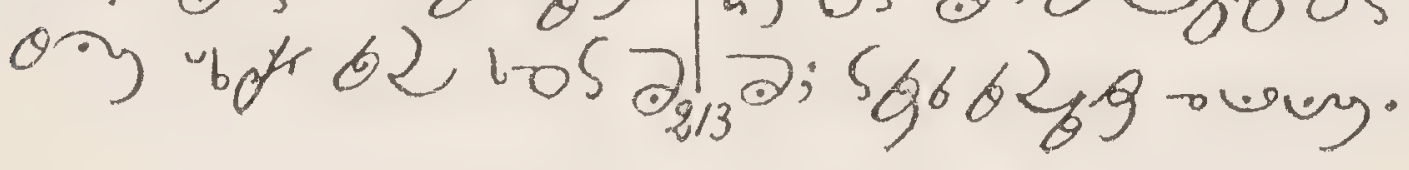


Love. $=$ so $a$ og

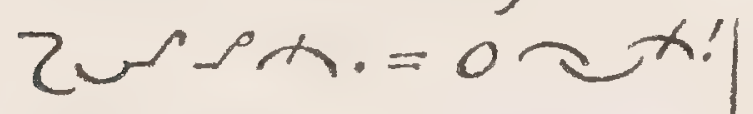

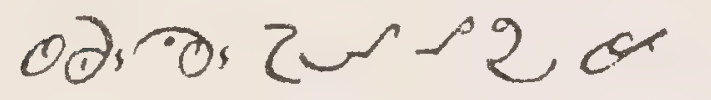

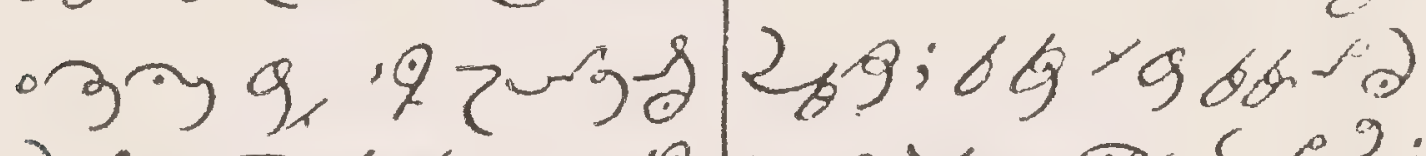
2n, $2 \operatorname{gan}, 9$.

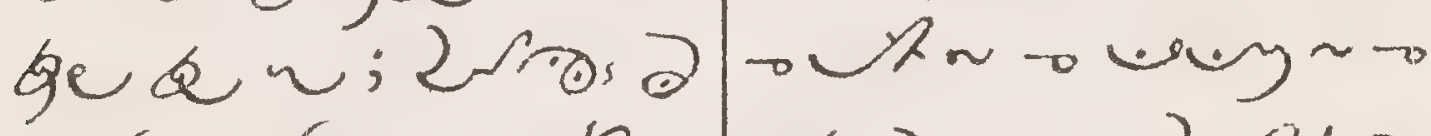

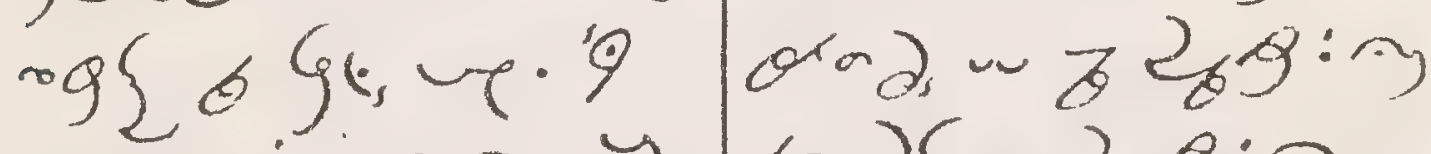

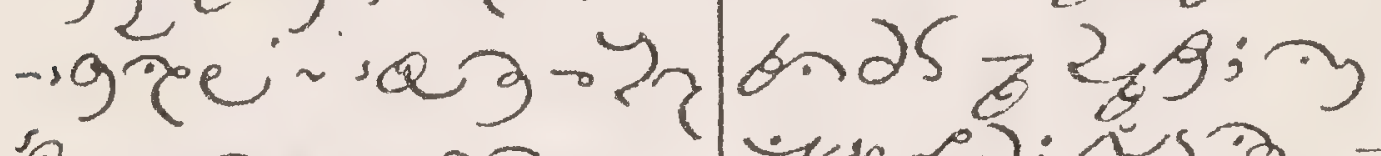
क- 9 re -93 s.9.

Desire. 'o a en $x$ bbar. $=20$ 28, $\rightarrow 0 k \sim m$ ox.eer 2 .

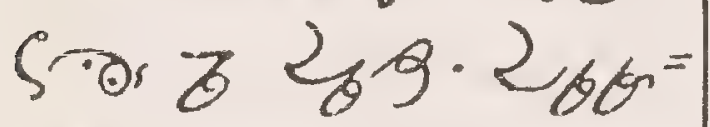

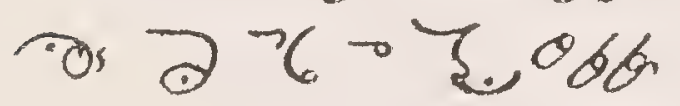
b- $-2,5620$ শ凹

osd 2) ^ว, कoy 2, bua. S 1 . $2_{6} 9 \cdot s_{6} 2, b^{2} b z_{3} \beta$

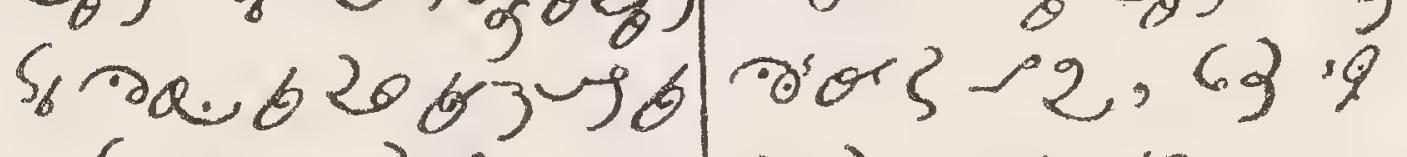

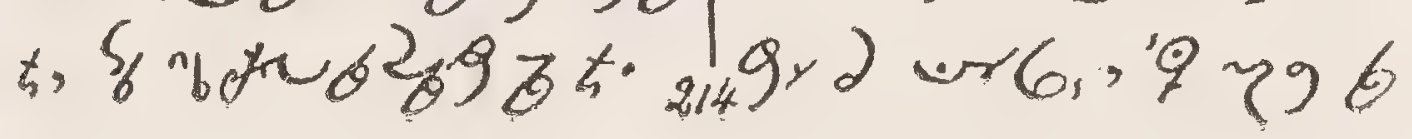


MANUAL

$2 ; 3$

$\left.20636^{\circ}\right)$ 的

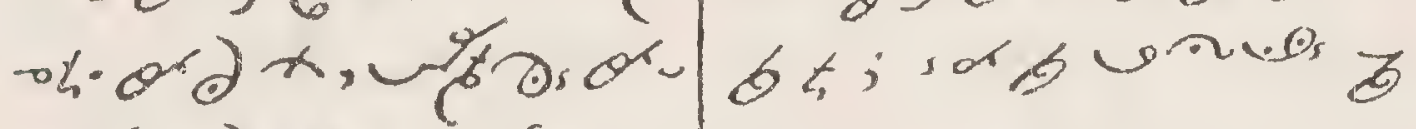

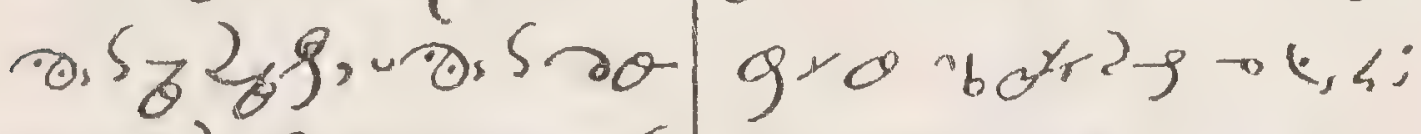

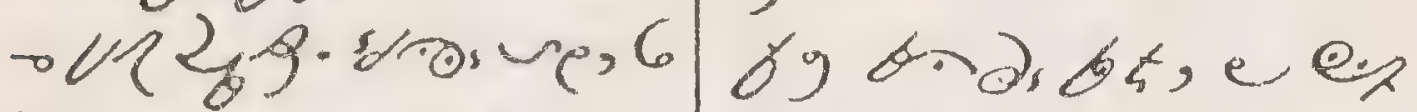

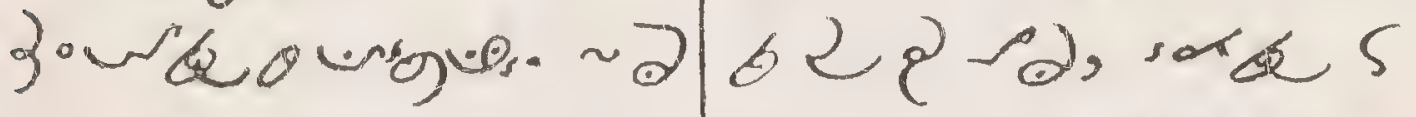

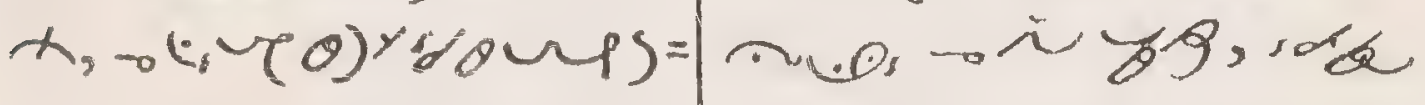

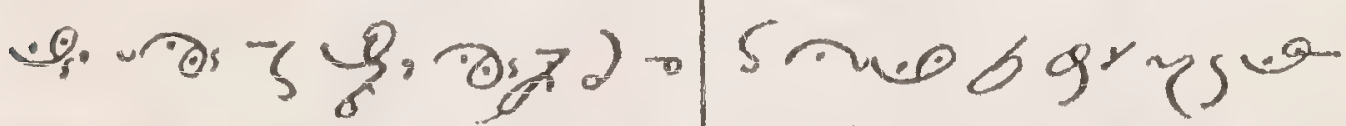

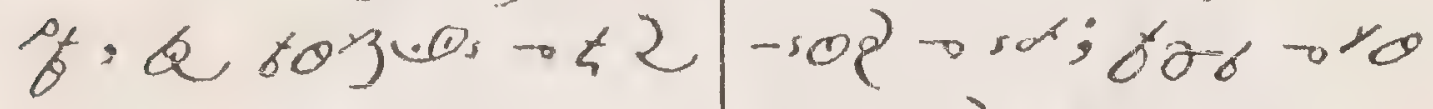

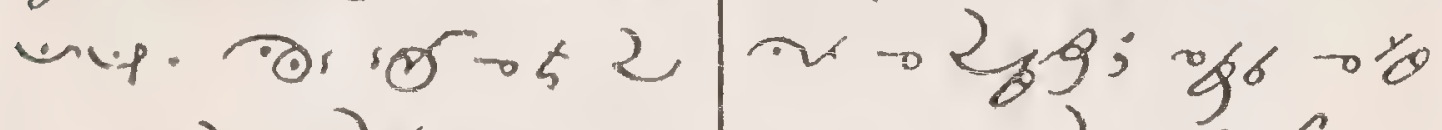
जथ मेकर;-0 च-2, - 289;-36-

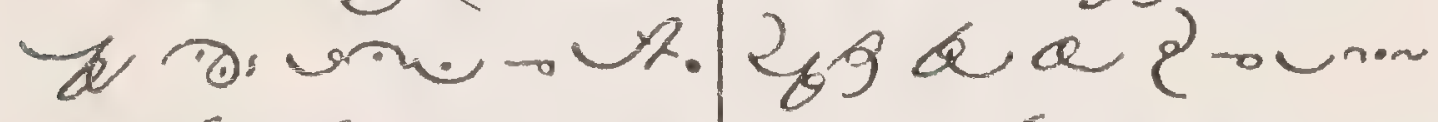

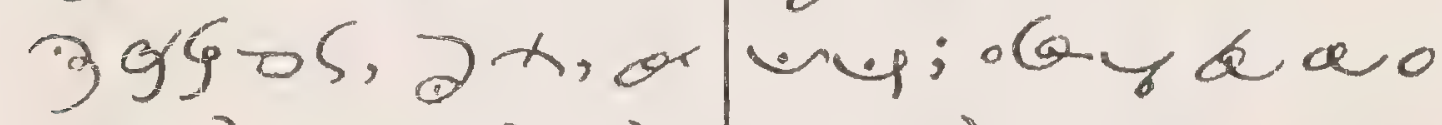

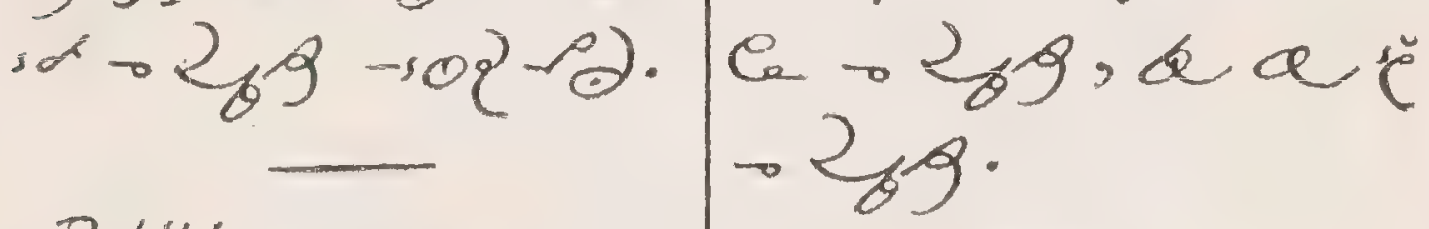
Petition. 10k uness= Resolution. id d b. e-

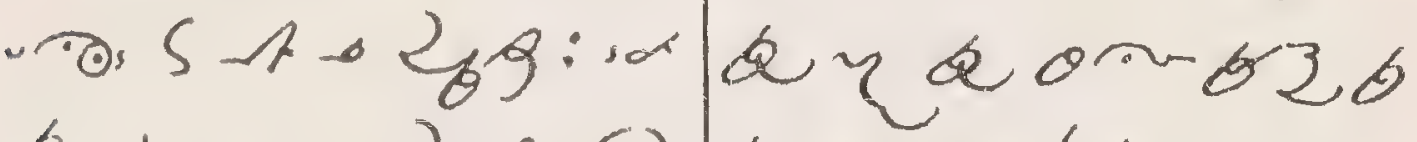

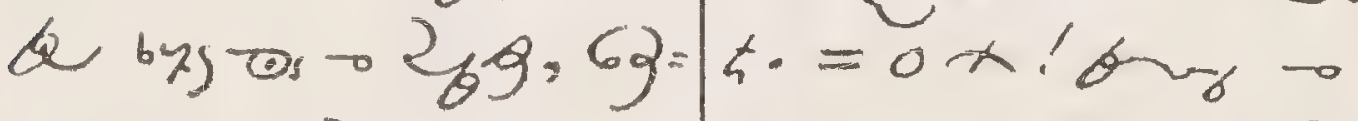

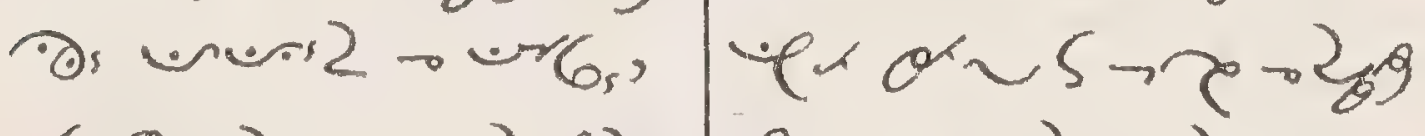
-69 r 2 -

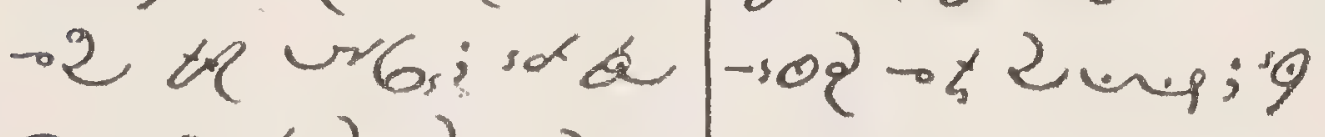

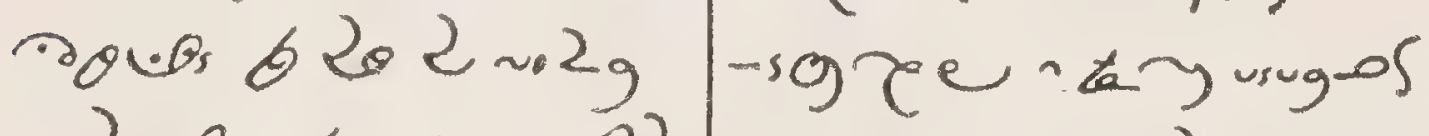

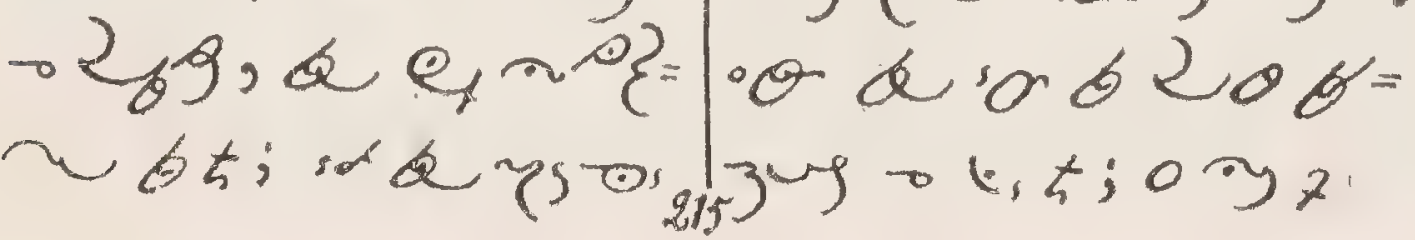




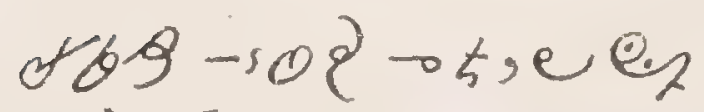

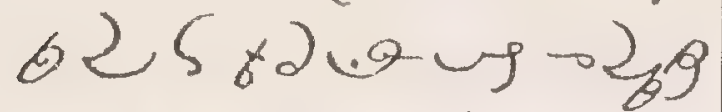
qu te $\approx 0\}-s 0$ ?

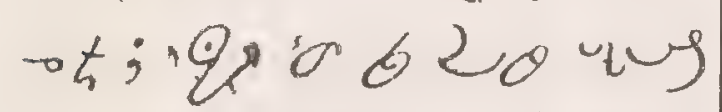

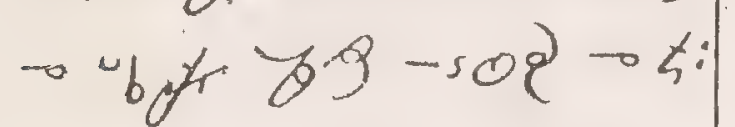

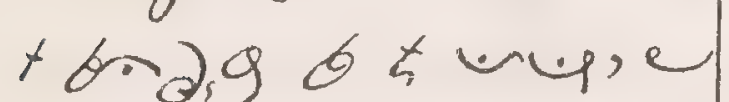
estरrogase es

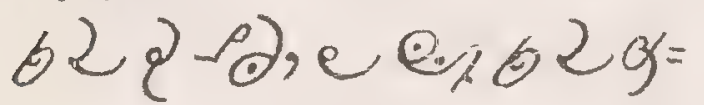
द-sop P):"q-igpe

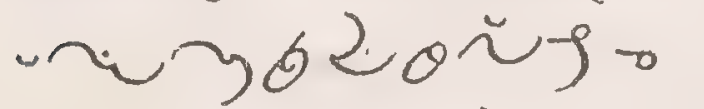
2, $9 ; 0 \partial \zeta_{3}-2,9,0$ $0 \partial \ln ^{2}$ द. 0 人.

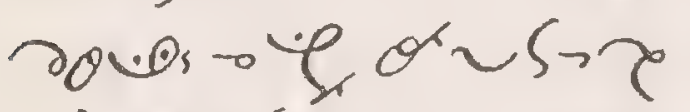
- 2 g : G'o d o $2=$ $0,0,0)$ ysd.

offering. ad o unr.

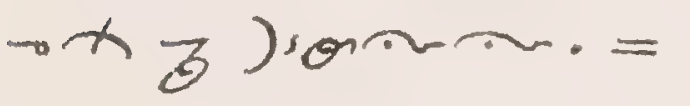
oxi'no wh ro, vine wh, wary $\sim \sigma^{\prime a} \partial_{1} ;$ or-igrz 62 ) son os ond ande! unoting

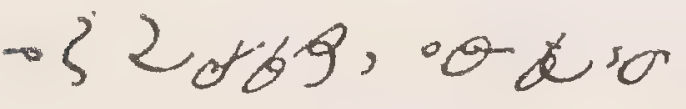
b2 0 sonsmus.
वरगे पn-का 2द⿱ sor b2, obot os

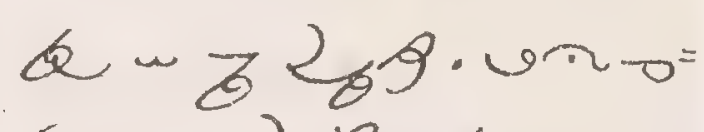
sing - 2n, de e $92 \sigma^{5 \cdot 0 \pi y} 70005$ द 2D- ब. Gबr $x-6-9,2 x$

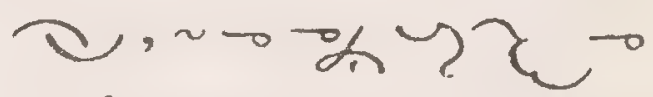

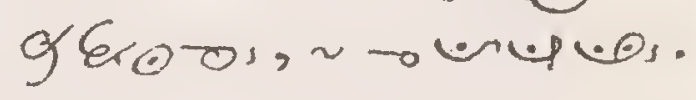
Intercession. sor a rivax 6 ungo" gre. $=0 x i^{\prime} \operatorname{sot} \alpha$

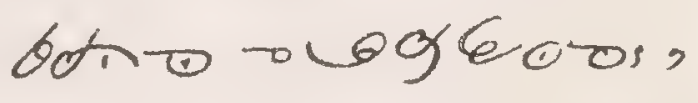

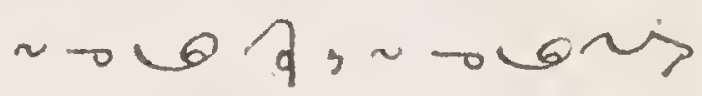

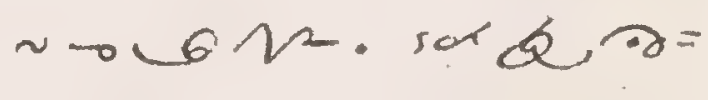
orsurs, grmadbase" a -iver is id a $S=$

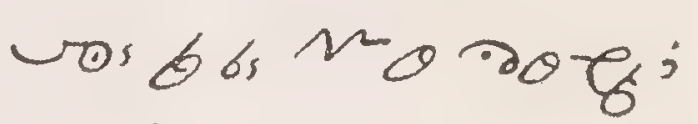
sa \& t averer ven

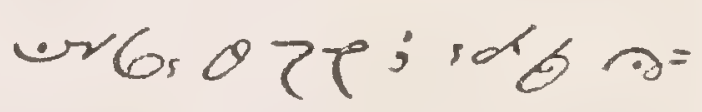

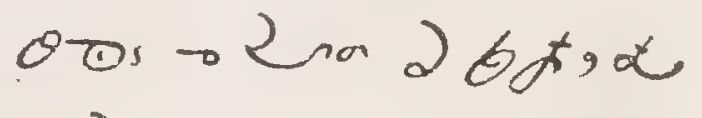

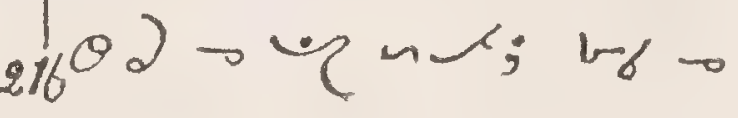




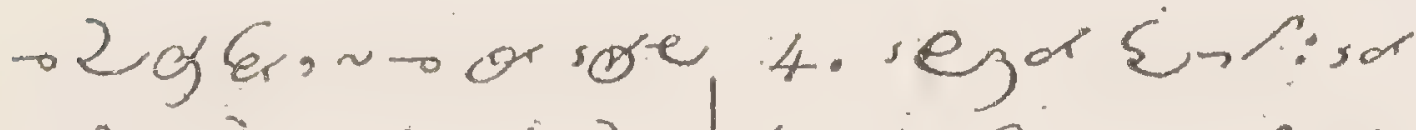

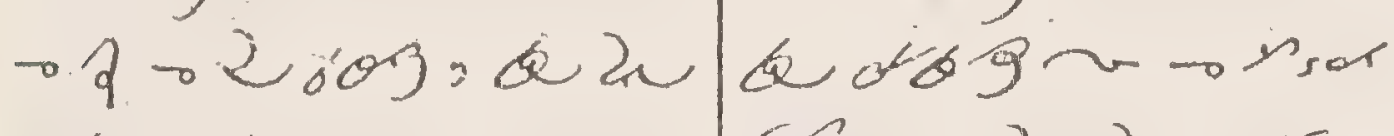

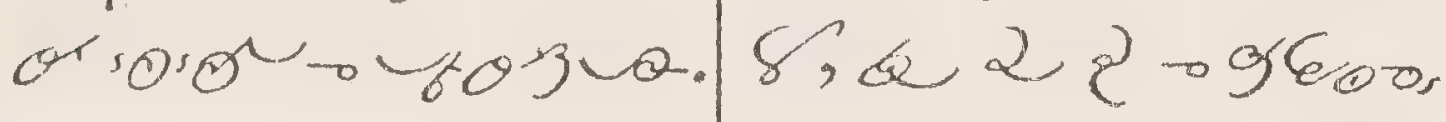
Q.

The Rosary.

I. Joyful Mysteries.

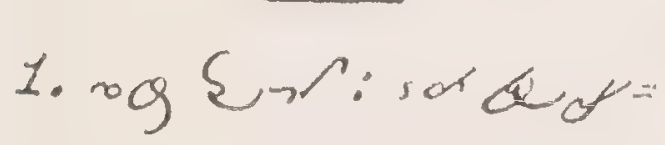

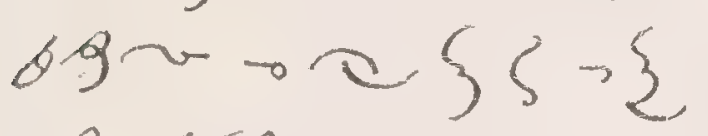
or sot 8 o T. unandat?

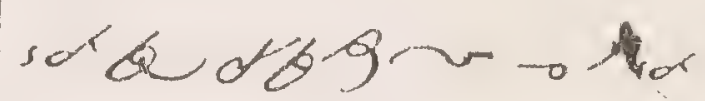
ร, \& $2,8 \sim 72$

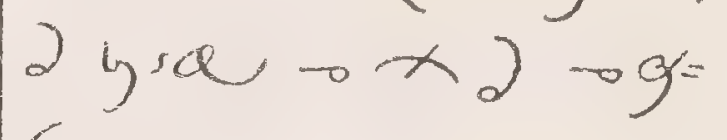
Erotos.

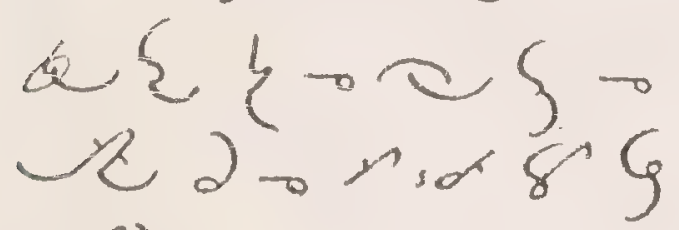
i).

2.

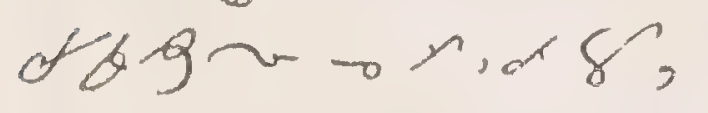
\& 2 \& bue 3 r, त2,

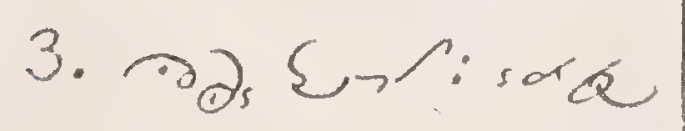
ofgr arex,

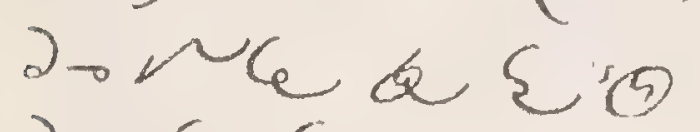
D. Q e o os.

II. Dolorous Mysterícs.

6. oxd Ent: sd a disn- orvex 《2. 20$)$ प; - 次文然。 wy, m कर $-\cos +\zeta_{s}$.

7. ound Lᄀ ? sor botgin - o $x,-90$ o or 2 or दर $28622 \rightarrow c$. 21\%) 


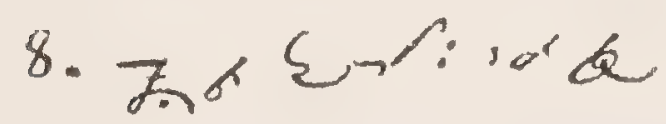
otbar o. or

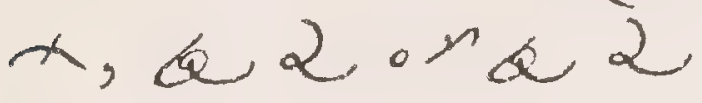

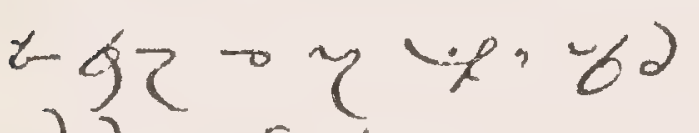

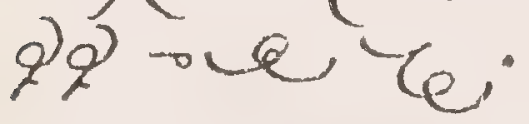

9. Wor L. Pis a ct 6 - o - or

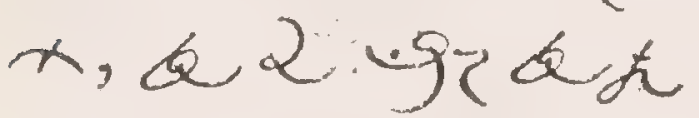
we $\rightarrow$ क wers o

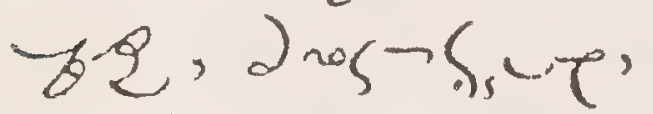

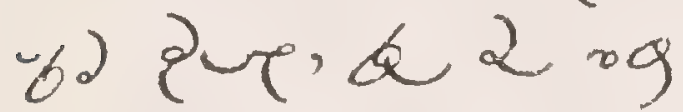
- $\%$, 2 og no.

10. Ior Sor: sor ce detion one $x$, d exs $2 x$

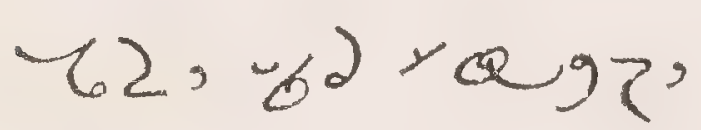
-62 घ-37 025 $\sim \rightarrow$ ig' $2, \partial \rightarrow$ - bd d, boine $\rightarrow$ sot त2.
Gloricus Mysteries.

11. $g \alpha \sim \rightarrow 2, \ldots$

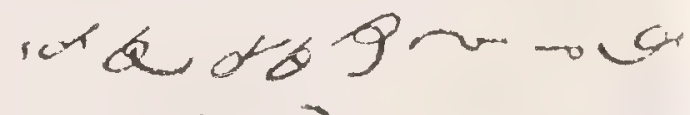
-

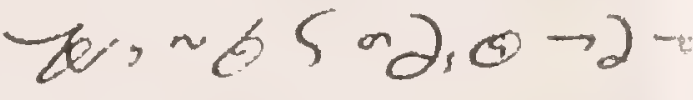
or: 0 D. 062 . 12. gor $\sim 2$ \&

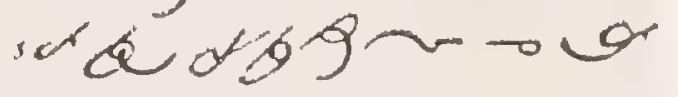

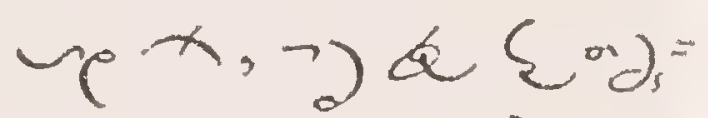
$\theta \rightarrow-20, \partial=$ $-\operatorname{leg}^{\alpha} a \sim 0 \partial$ $\& \rightarrow \approx\}_{s}, \sim m ?$ $\rightarrow$ Non, botec orsot 2及, $\sim 2, \sim 0$ v6r.

13. gor $\rightarrow 22 \sum n$.

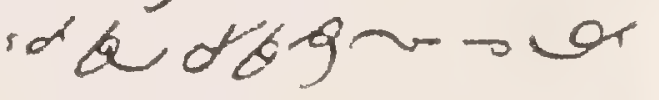
rex, \&. $2 \cdot 2$

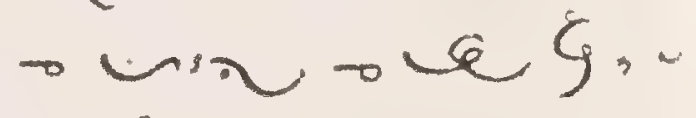
6 Sut ox g/, $2 G \rightarrow k-$ 218 ? 


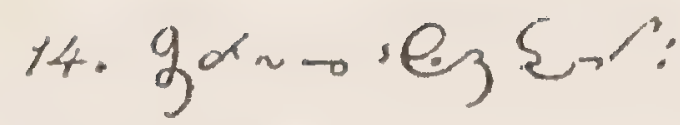

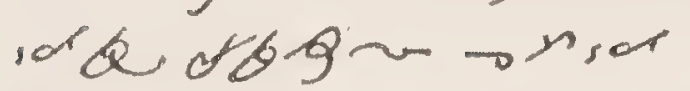
S, a $2+\sin \rightarrow S_{s}$, -bare-

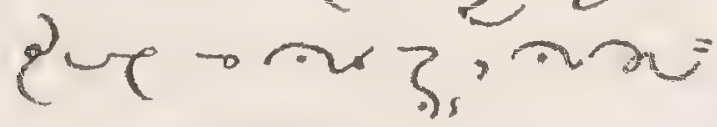
rore.

15. $9 d x-$ uru

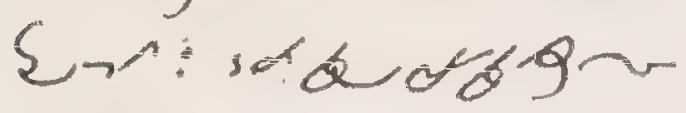
orsal 8; \& $\{$ u Sर $20 x\} ; 20$ x a thoog sod or 4209 sor -rop - Ges soso werlos.

Via Crucis. Preparatory Prayer.

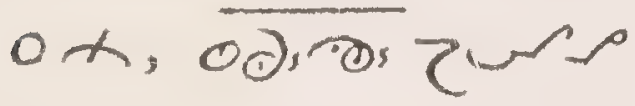
र: चर वर्व

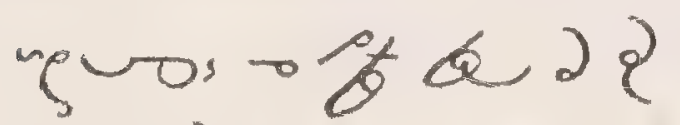
- or $2 \alpha \cdot \cdot 33-2$

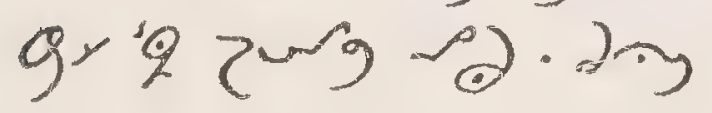

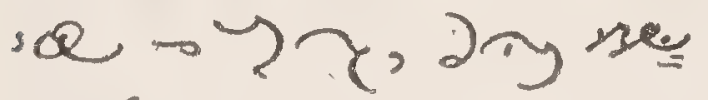
$\cos 2 \cdot \cot 2$

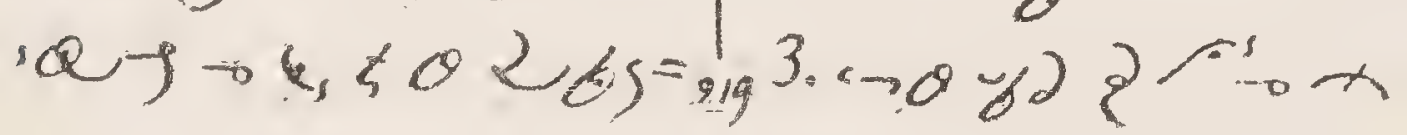

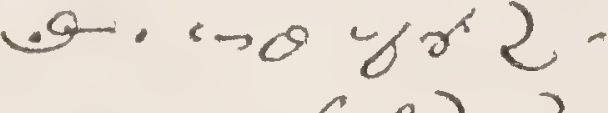

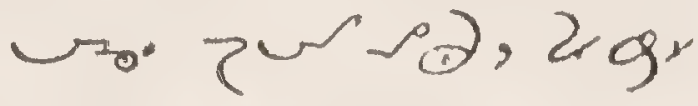

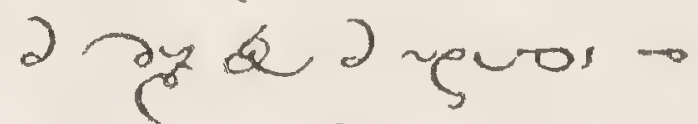

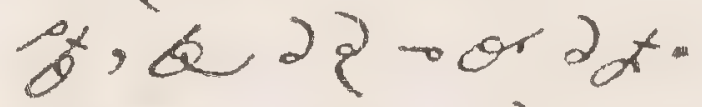
sor कर 90 का - 29 -

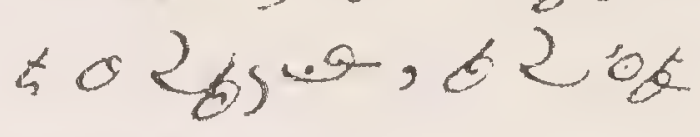
$1-65$.

$$
\rightarrow 2 \bar{\rho} \text {. }
$$

Sancta Mater.

id 6h 2-en?,

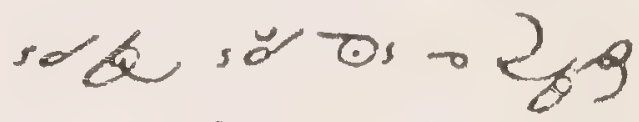
-or 2 or 2 .

Adoramuste, eth. x. y'cry - 2 , त9 95052 . = e d unguas. $2<, 2+2 \div z$.

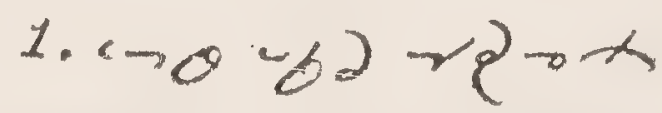

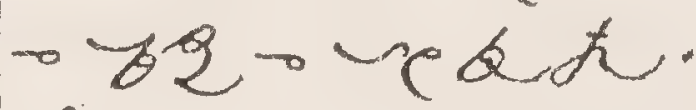

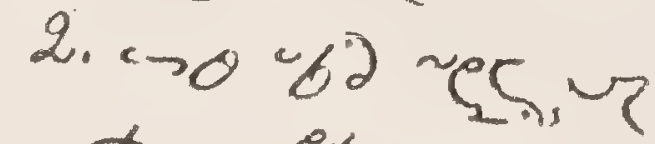
- $x-8$ - 8 . 


$$
\begin{aligned}
& \text { - द, मे. }
\end{aligned}
$$

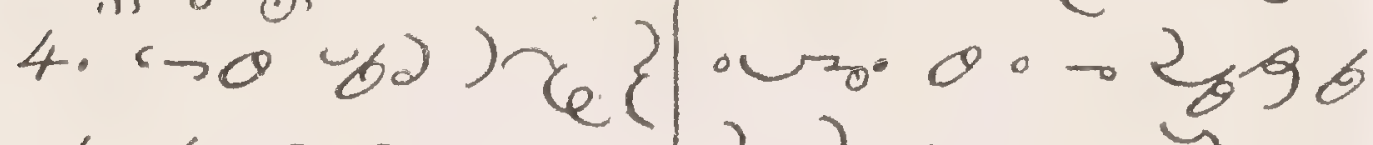

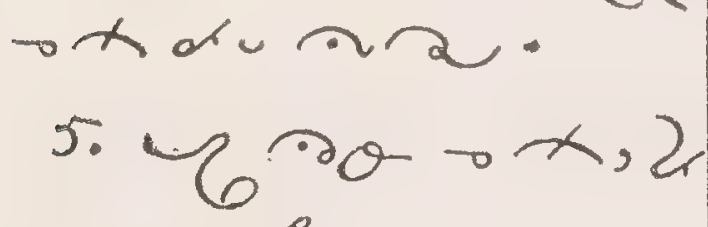

$$
\begin{aligned}
& \text { रे, } \rightarrow \text { रद }
\end{aligned}
$$

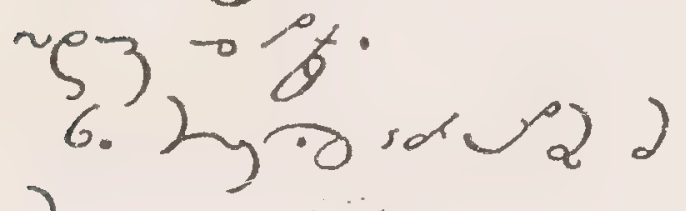

$$
\begin{aligned}
& \text { रon } \rightarrow \text { ix. }
\end{aligned}
$$

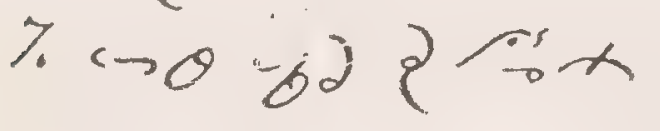

$$
\begin{aligned}
& \rightarrow 3,{ }^{\circ} \cdot
\end{aligned}
$$

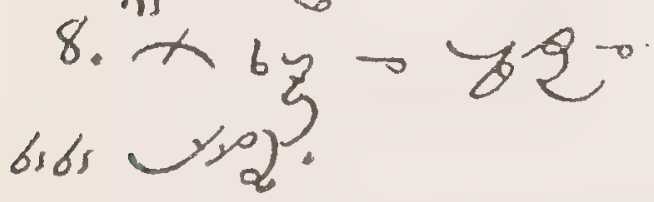

$$
\begin{aligned}
& \text { c.sary bot; } \\
& \text { गa } \rightarrow 2 \text { a d } \\
& \text { \& } 002705 . \\
& \text { un - } 2 \text { a'en } \\
& \text { a sor to nos } \\
& \text { Q }
\end{aligned}
$$

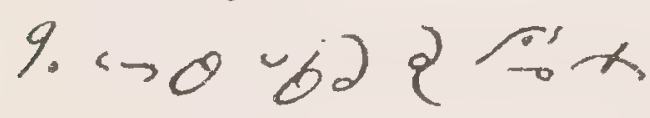$$
-3, \partial_{3} \text {. }
$$

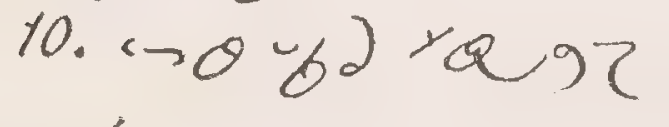$$
\rightarrow \text { 大。 }
$$

Pater. eq कुष. Ave o 8ry er. Gloria. $16-502+9$, -sog o $\}$, sog - w. $=-6060,100,6$ so \& son.

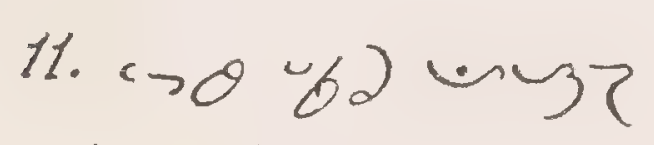

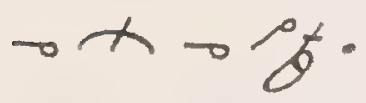

12. $-\rightarrow 0$. $62 \alpha+x$ $2 \rightarrow \infty$.

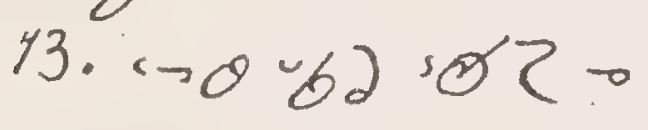
$x \rightarrow 20 \frac{8}{b}$

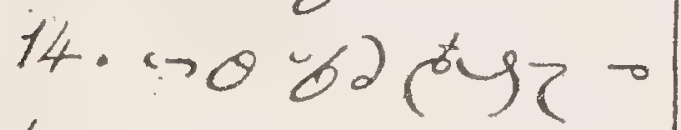
$x$. Prayerat each station $00-269$ be du Guard of Honor. Engagement. o $\propto$ ! -gor sos, og 几 -

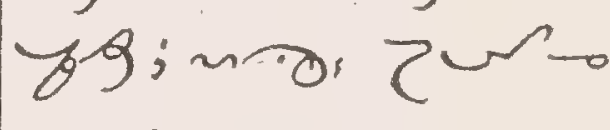
ज6; 769 ynd 2: ogr curos, Prayer at each station. er $6, \rightarrow 26 \partial 5, \rightarrow 2$

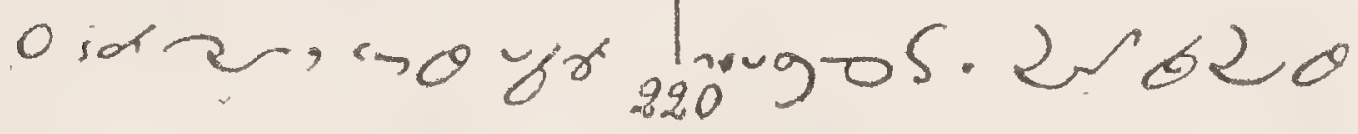


MANUAL

29

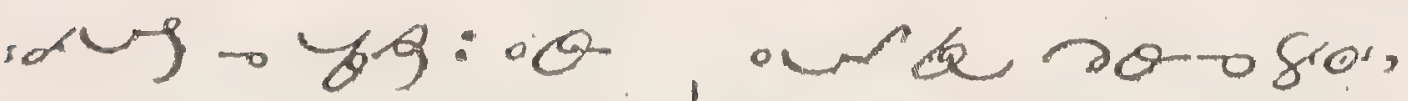

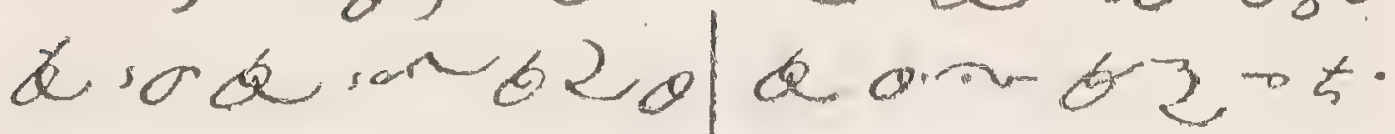
$60-05$ \& $22 \div \otimes:$

$-3-$ unce - o o को,e e. $62, y-2,9$

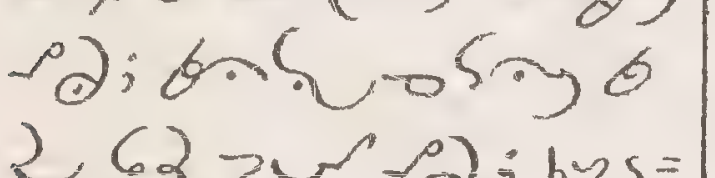
$2632(2) ; 6 y=$ गु此 6,00 asugos. Hymn.

$$
\text { "\%:-8, \& \& }
$$

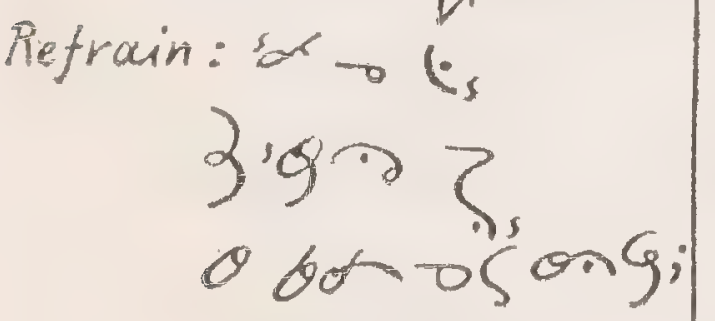
sd a sogofor -sOg o ior o ba;

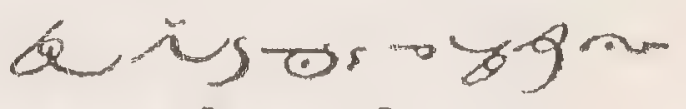
a 63 a dis. Other Hymns. 1. Holy Trinity. "3"ton"

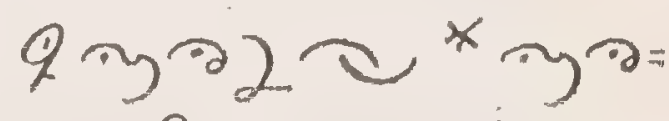
2. 9 , बrgma ¿\}, ट\},

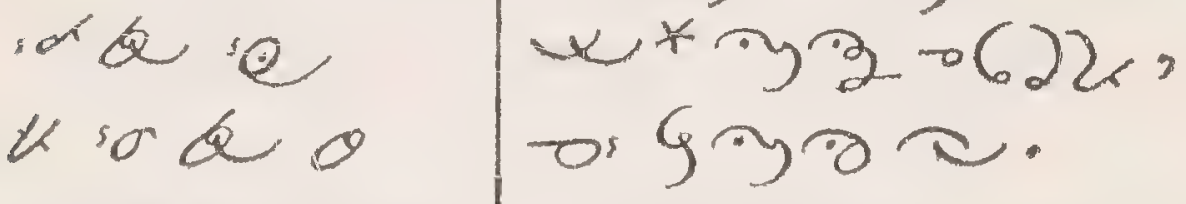
$-\varphi_{0} 2 \mathrm{e},-\xi_{0} 2$ ¿, m.2 2 , or $93=$ $\partial \approx$.$$
\text { सQgento }
$$$$
\begin{aligned}
& \text { गम2 } 29+0 \rightarrow 6= \\
& 0, x \rightarrow 6 \text { orgmo }
\end{aligned}
$$

$2 \operatorname{sic} e r g \mid c$.

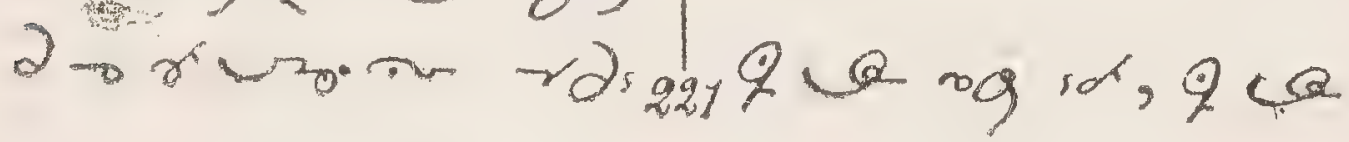


$\circ \operatorname{ros} \theta, q \log _{1}-\{\rightarrow 2-5 \%$

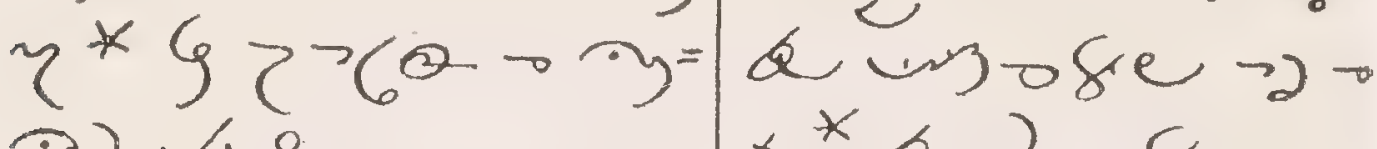
$\therefore 2 \mathrm{~s}$.

$\rightarrow 32 \mathrm{~kg} 2=$ $5, *$ acose 0 ryaa ${ }^{*} \rightarrow \sin$ Q xe $\rightarrow$ hy.

$0 \curvearrowright 0 \partial, 2$ $\rightarrow{ }^{*} \partial 2 \sim \sin$ $-0 x \rightarrow 6$.

2. Tesus-Christ.

$\rightarrow 2 y r \cdot \sim$ in $=$ $22 k 9<5$ $x \rightarrow \approx$.

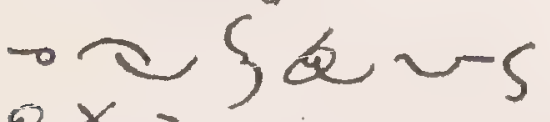
g्र 2 hon o .

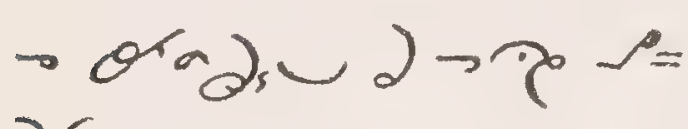
28.

$-2\} \partial$ m. C- oram-r sot $8, * \rightarrow w$.ne. Q $\rightarrow$.

-2 36 \%, 29 org $2, * 62 \times-022$ ar $\}_{5}$ $\rightarrow x^{2} d$ urgogex दर 2 - 2 . $\rightarrow x 2$. 022

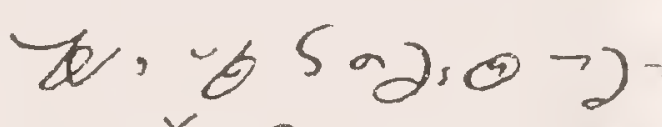
20 * lezan th 女 $\sim-\infty\},-6 \partial$ ? $\rightarrow$ or ?.

) irve on, wh, $\rightarrow \partial b x^{\circ}$

3. Christmas.

"C: 〜n-ijg" 1. vo $2 \sim 6$ $S-r$ sive eze int $\{\rightarrow 25$ \&, S $\rightarrow 0$ or $\zeta_{\text {. }}$ 163 wn' $10^{\circ} \mathrm{zm}{ }^{\circ}$ 
MANUAL

31

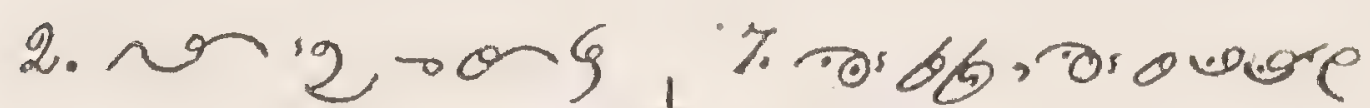

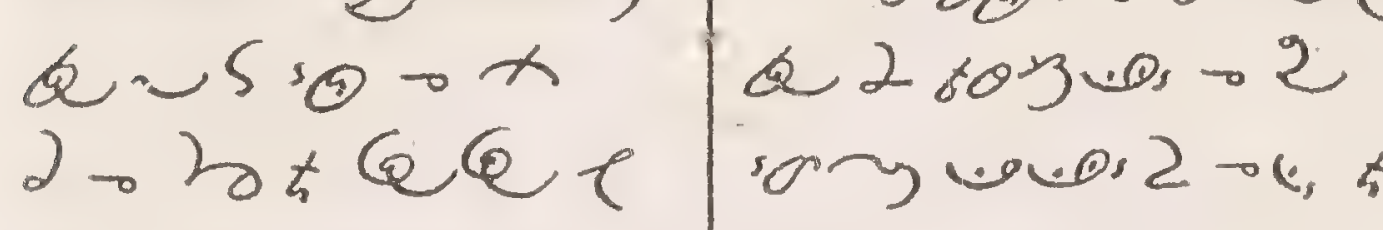

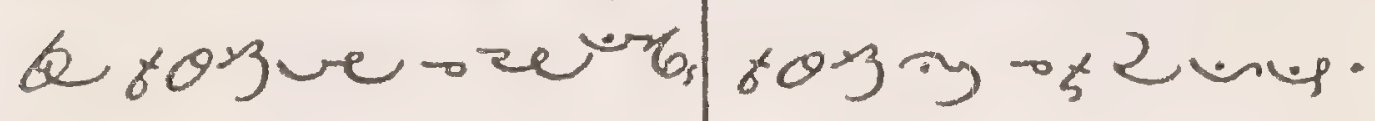

3. $639 z e-74$ 'g N, "g

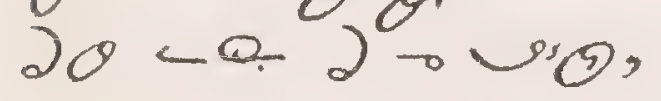
d'e $a \sim$ sere.

$4.022-6 e+$

ᄀo $x$ a $\sim \operatorname{an}\}$, 2 - 2 in 5 on 25 ,

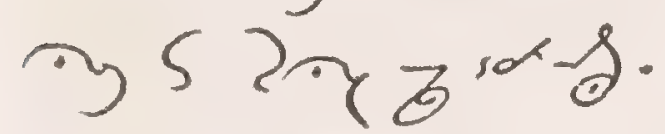

s. orugstas

बढ。g 告

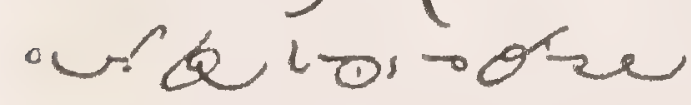

x a by. o. 2 .

6. - ro, sij-39,

女 toos $2 \pi y$

- 6 or e b2䒕

$-202,9-4,4$.
8.0 2u mas 02 ciedsots bofy $\rightarrow$ क 52 .

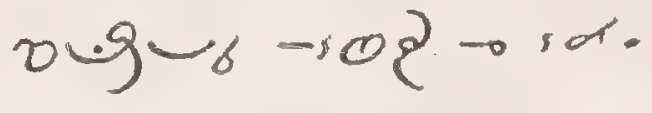

4. Passion Hymn.

"li ovis r ru"

1. $2 \curvearrowright \xi x$

का वर 202

- 3-2 9.0

$62 \lg -0 y$.

- 3 9 y 0 k bot

'0ग $463+5$,

63 क. ०

a byg vo $=2$.

(1)

2. (2.202) 36

$6350-29$.

or wox or os 2230 ing ons: 
$2-2-52 u$ -6 or 2603 , nob 20 Dra

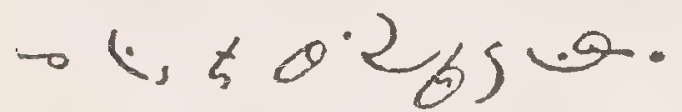

5. Other Passion Hymn. 1. b u b or x a d $20-g$

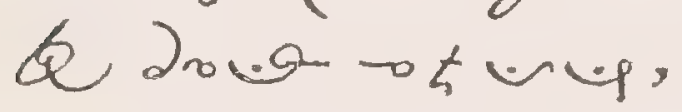
oor $2 x$. Refrain: otingojor -4, to 2 wu, "To is on wh,

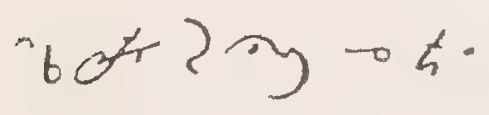

2. $20052-x$,

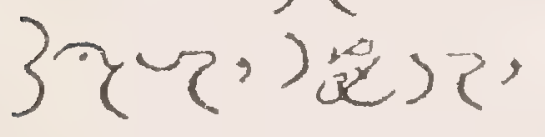
9ras 50 . whe cyur.0862. $0 x, \lambda \otimes 2 \times, 4$ 3. $6, \frac{\dot{y}}{86-2}-2$

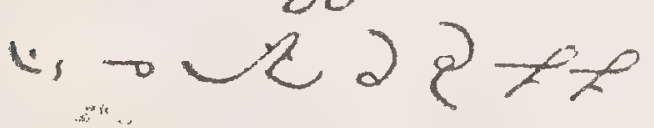
b 6 - o verz 28 b जro. on 062 .
4. reys or w व 682 - थर, कर शे शे द्रद द nug $=02$. $0 \times$ u.

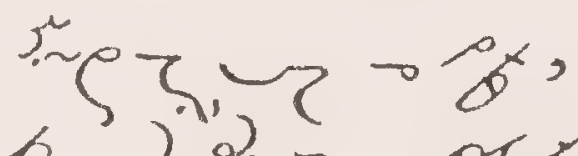
a d $2=0$ - or od दर - ? 2) yse ob ox 4 . 6. $2 \log ^{2}+x$ 62 늘 0

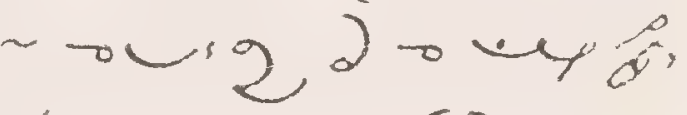
a sug $=62$.

$0 x, t$.

6. To The B. Virgin. "Cí d drod Gg," Refrunn: sol sinas gernofigervof.

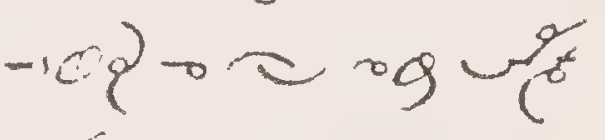
280 ze $=0$

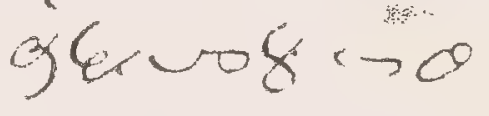

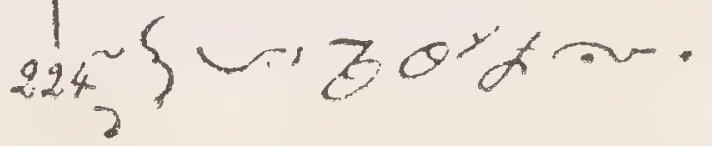


MANUAL

33

1. in Sinnortic

$$
\begin{aligned}
& -\approx \rho \text {. } \\
& \text { 2. } 0,2 \circ 9,2
\end{aligned}
$$

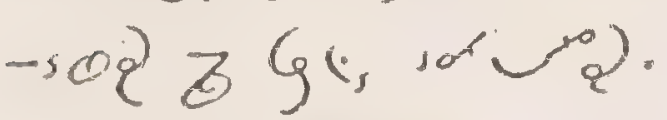

7. Maria Mater gratice.

$$
\text { 1.06八つOへo }
$$

dat bot or

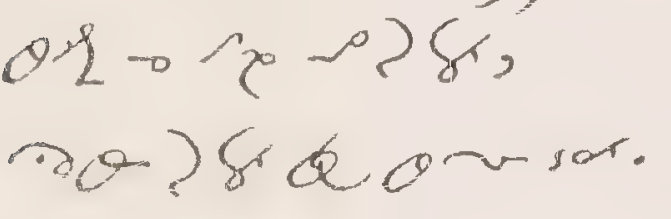

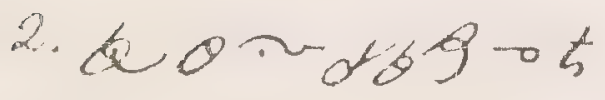
o sis aor

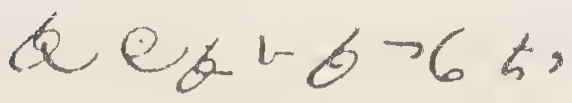
or 0 t 0 yg.

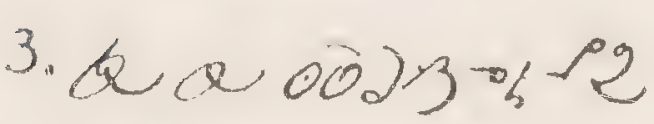

的2,

or $4 a-2$

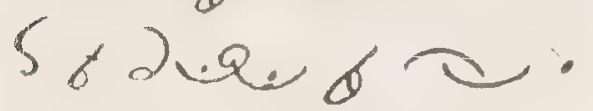

8. Purgatory."0, -"

1.uncsoound

nesurne,
-6 or 603 rin: Ot! $05 !$

-69ukezy

orepor

2. $\partial$ Dre, sot nan

sd a Doofi,

Gor od yern

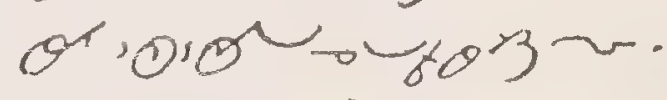

2n, 2n,

op 628 yusa

sct a $\log \log 9$.

9. Child's Funeral.

"

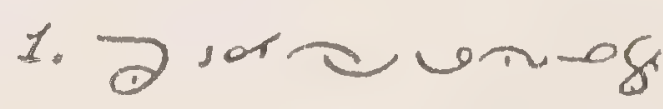

- und er 2 \& $x$;

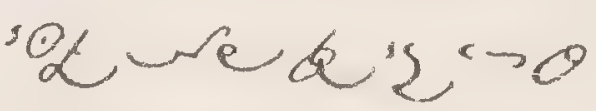

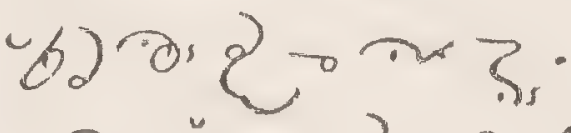

or $\check{\sim}-2_{6}-2$;

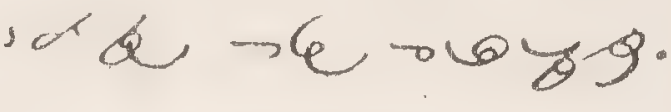

2. -6$\} \times 63$.

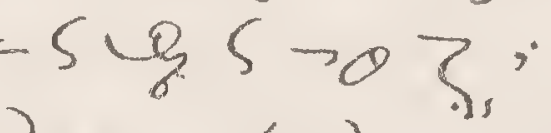

a te \& $2 \alpha d \rightarrow b$, 
31

SKWAMISH

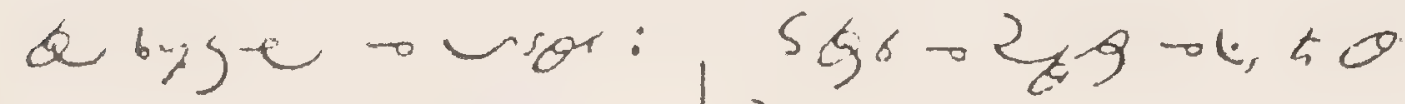

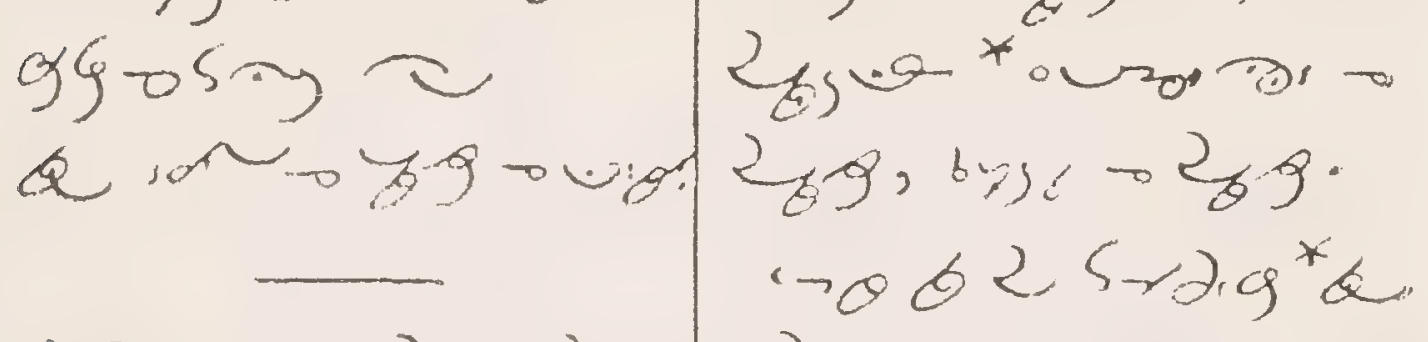

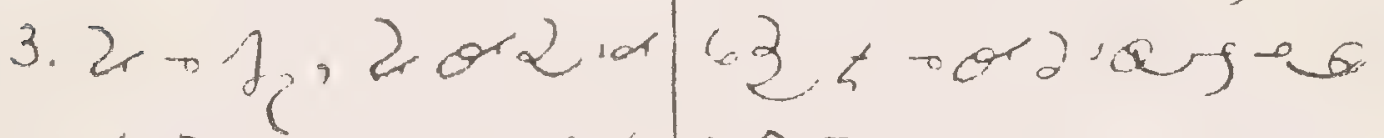

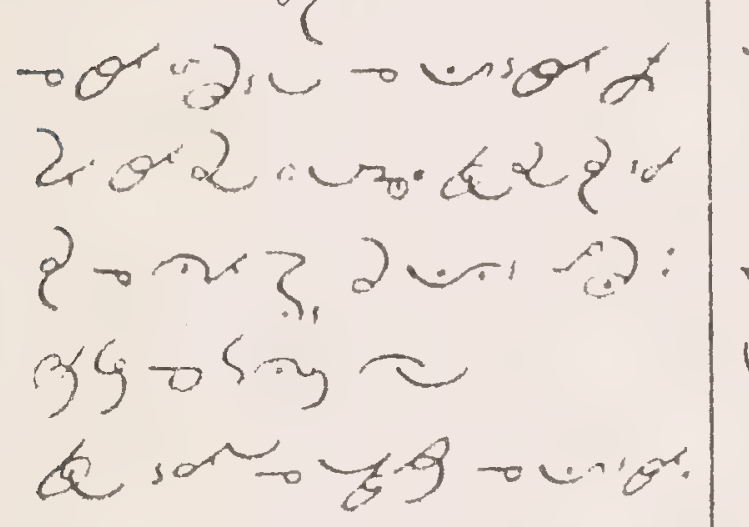

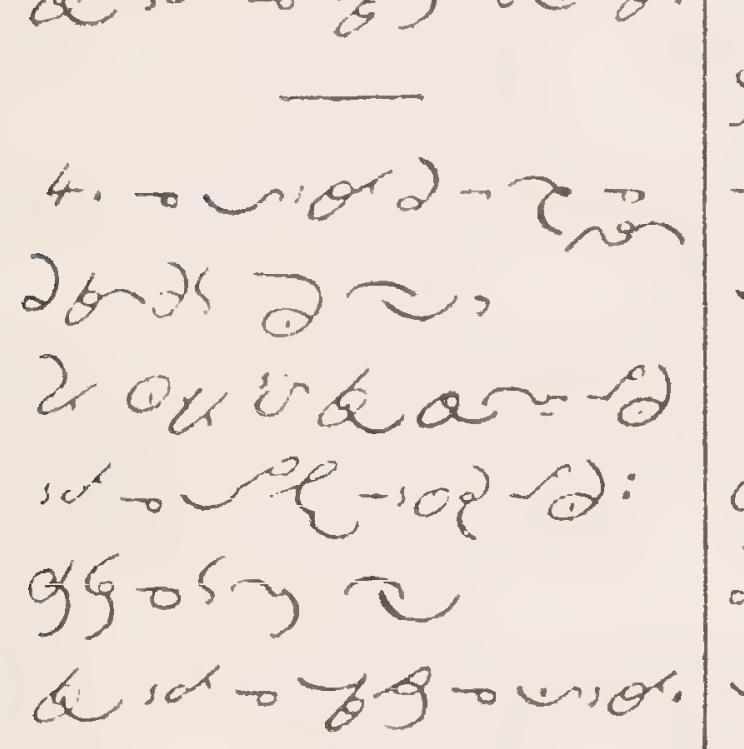

10. Miserere Mei Deus.

igy 0 , 2 rog

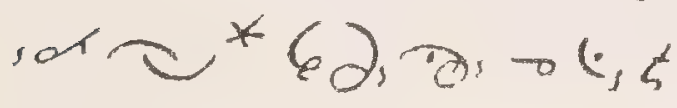

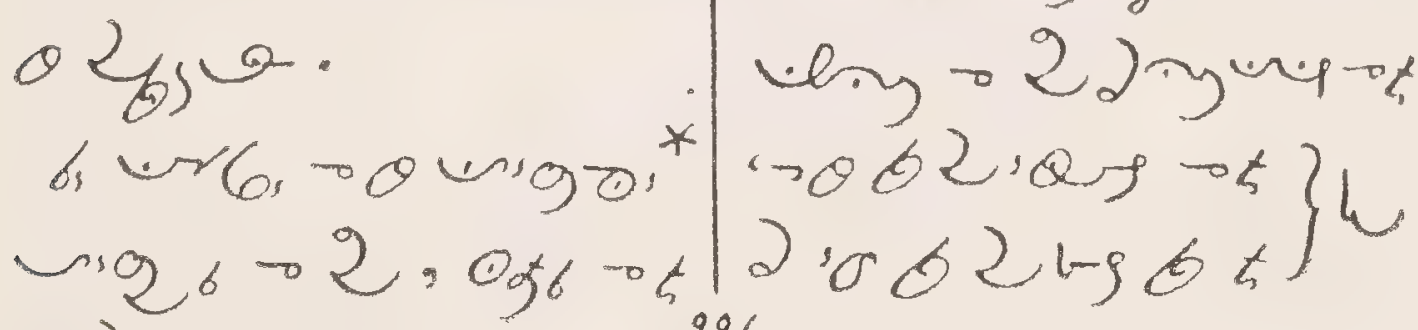
o 2 .
$2 \pi$

$40,4,2000$

$\left.2^{3}\right)^{*} \theta 26-3,9-$

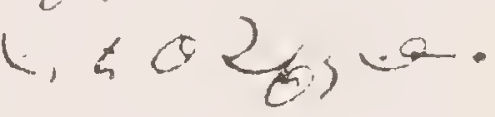

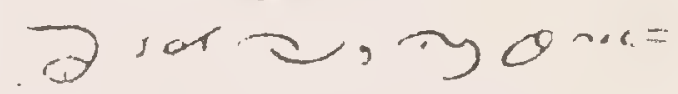

yol, ${ }^{x}$ y unys of

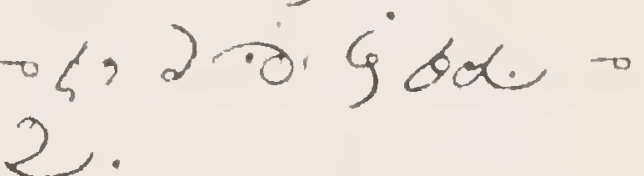

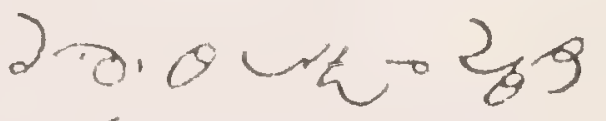

$2\}, * 9$, o $20 \cdots$

$y \rightarrow y 29.0 \%$

11. Contrition.

"L:S o 2 a."

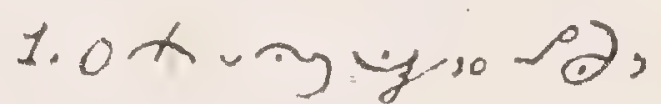

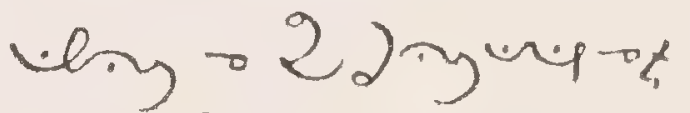

$\left.\begin{array}{l}-\theta b 2 \cdot a-50 \\ 2,0 b 2 \lg b t\end{array}\right\} \mathrm{L}$

0 or 2
द 9 in ota 
MANUAL

35

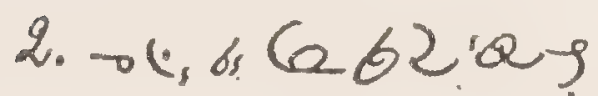

ryuoug os 2 ogsd $\xi$,

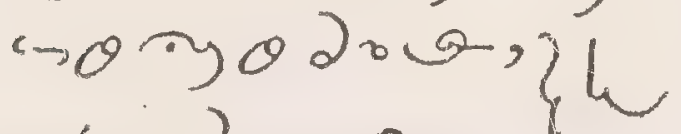

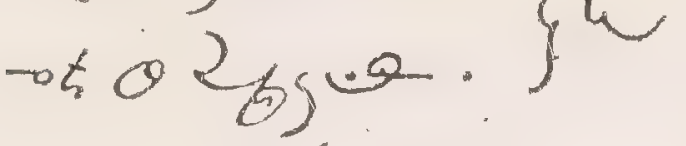

3. $02,8982.25 .2$,

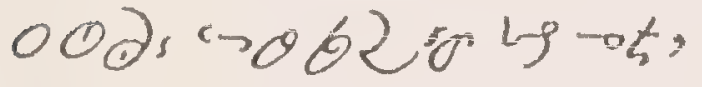

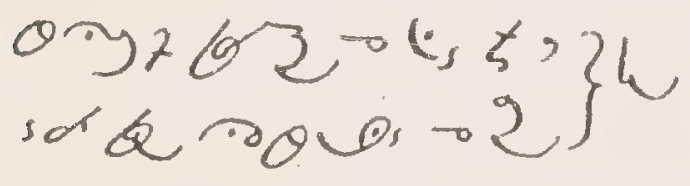

acor 2 .

12. Real Presence.

$\partial x \curvearrowright-\xi$

थल $\oplus$ :

(a) zunarara

$\partial \partial \partial \rightarrow \Theta$.

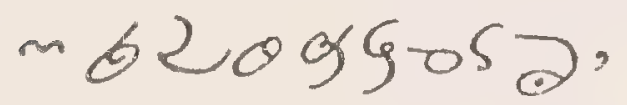

के, 22 - ew:

- 26 dotos a.

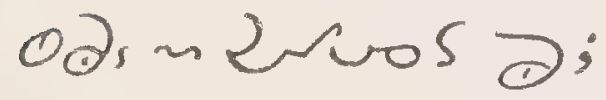

o vus - $289-$

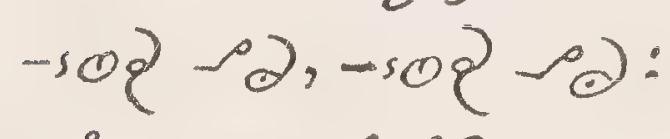

of पद तथ-

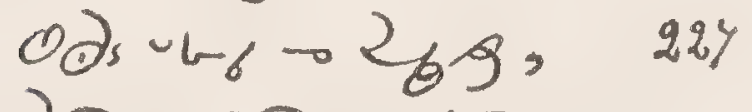

13. Before Communion.

"Ti O a d T 6 ."

1. Gury bid

$\operatorname{cod} \theta$,

a $x$,

utorasin the

noveryy,

orno gunt.

2. बभुदरन

- 2 sorgu,

वप्त ह

C bygud:

ory bot 2 .

3. 3 s sa to $9 r$

dry rewos 2 ,

ensy $\rightarrow 2$

ods $50.28-9$;

Dry $x+5$

O九。 0 to 2 un

dry unn 
14. During Communion. " $\angle$ : 1. $0 \operatorname{sos} x$

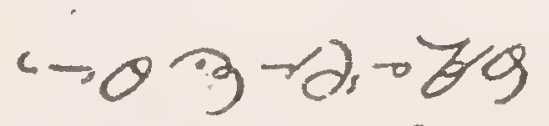

Oें य टथ" $62 \&+2\}:$ $\rightarrow 6$ or som os

$\rightarrow-4 k a 2,3$, $62 r+63 y$

- e. 6 हs.

$0 \operatorname{sos} x$

bro tr? 2yst:

$\because, \pi, 2 \theta ?$ कर कुज 64 .

2. Gs $2 x$ oraegesion, G'O 0 केष b2063ngt, $76,28 \rho 9$ करण - का

$2 \pi 2005$ उद पर यक

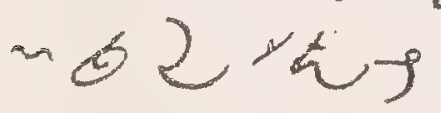

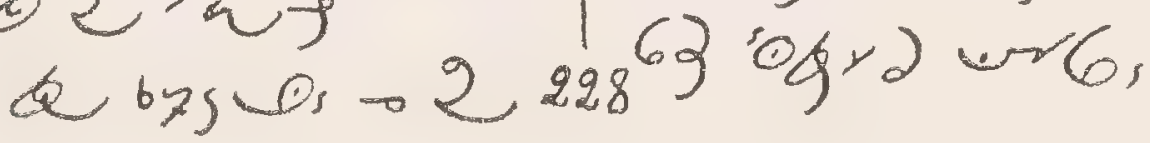

Q Ys.os

Q $\theta$ bof or $z$.

15. After Communion.

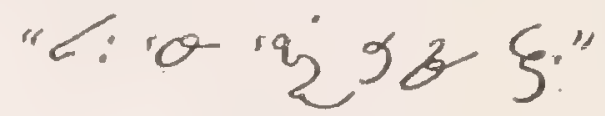

1. $0 \approx \xi x$

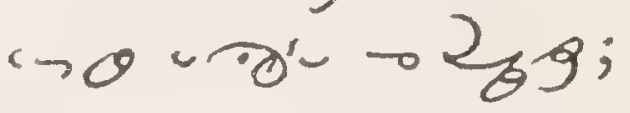

osdout, - oeny,

-oráar o. 2 .

- 269 obotos,

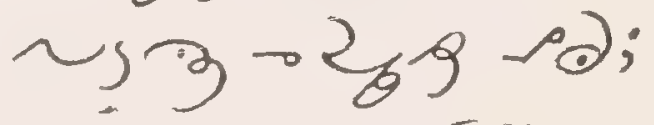

गे acus 0 .

ry $\theta$ ( $z^{\text {sor }} \rho$.

2. extos?

$\partial \operatorname{og} \operatorname{sot} x$;

वरक्ष

$63 \cos 2 \rightarrow 0,4 ;$

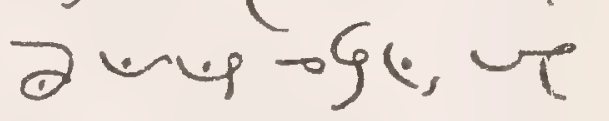

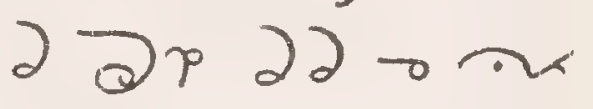

ar $)^{1 \alpha}+2$

3.०3 क्षिक 22 
74962063 ${ }^{3} 69+\infty \beta \rightarrow t$. un a dous-2

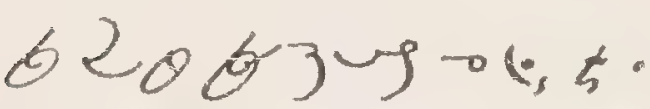
sd द व $40-79$ sक करण - 2 .

16. Before the Sermon.

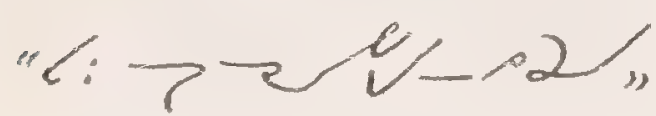

1. o eurys625.\%,

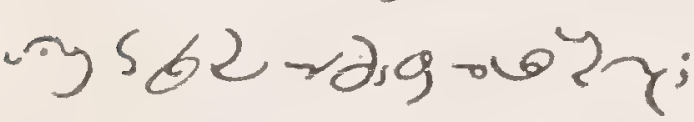

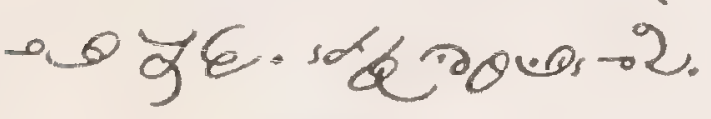

2.

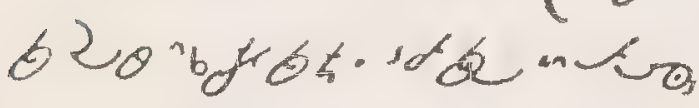
- 239620 vough

1\%. Sorrow for Sin.

$$
\begin{aligned}
& \text { "e var" } \\
& \text { od, Fy 20. } \\
& \text { - G e, t } \\
& 02 \operatorname{sg} \\
& \text { Gen 万abotr } \\
& \text { gue } 5 \text { id }
\end{aligned}
$$

$$
\begin{aligned}
& 02 \text { का. } \\
& \text { non } \\
& \rightarrow \text { - } \frac{\pi}{6} \\
& 56 \text { का } \\
& \text { - } 96,+02 \\
& \text { 30. Gsor } \\
& \text { dbosa } \\
& \text { o-w } 2 .
\end{aligned}
$$

18. Thanksgiving.

"lis sourysic

1.ony 9905

$$
\begin{aligned}
& 0 x, \\
& \text { ony g } 9 \text { os }
\end{aligned}
$$$$
\text { osolownes }
$$

यक -5 क्ष

$2 \pi+2 \rightarrow$

Q. 2603 .

$\because \operatorname{son} 72$

-

'69ro?

620345

2. $6>t \bar{c}>0$

$229010908-102-6$ 


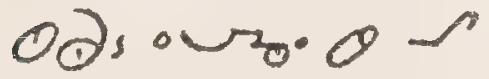

के खरs,

$\rightarrow 6$ or enogor

$-2 \cup-3$

$\cos \oplus$

Q Dेग

Q b उत-

ory 99 os

- sol $\theta \cup$.

19. Epiphiany. "fS. s. sol $(1,2) \rho \alpha$

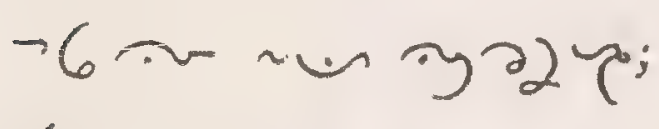

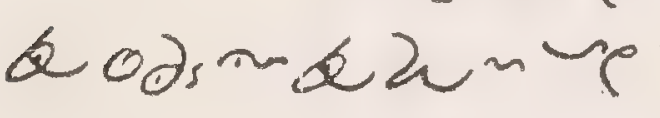
5 \& ing-o gee $63 x-2-62$

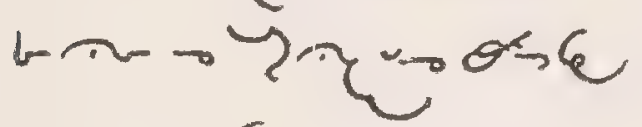
untorn - rgancr.

20. Magnificat. - gy a $a^{*} \circ 2 \tau^{\circ}$ is

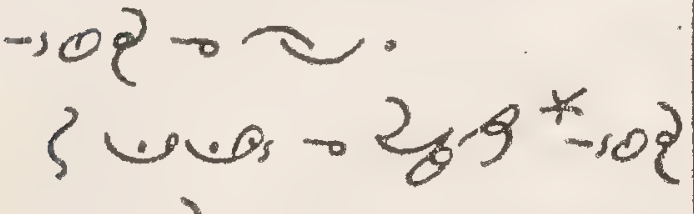

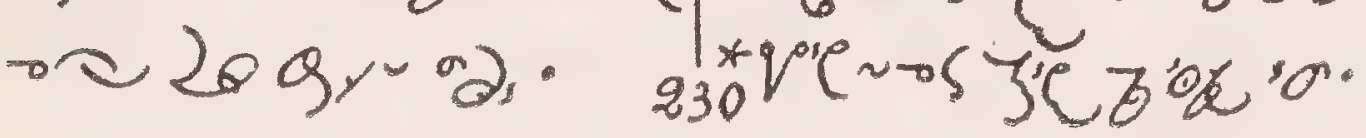

$-02 \cos d \tau$ -2, 9,2 e ge. rorendio do $7 \pi \rightarrow \operatorname{ur} 6, \rightarrow 1 d$ - 0502 .

$\rightarrow 2 \sim$ bsue, 699

$r-62, *-2.9$ sd.

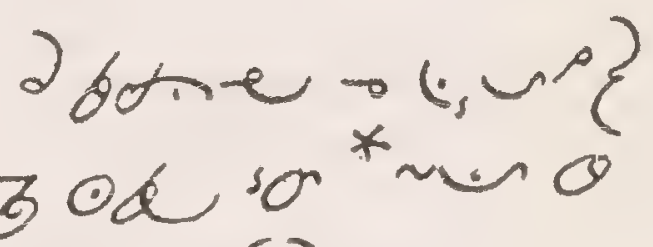
beft 062 .

2 bnsicer oos ar ${ }^{*} \partial \operatorname{tg} e \sim$ on $\sqrt{g}$.

diqe neray re *or a vese

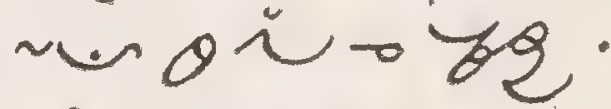
dure res ve w6r' ${ }^{*} \theta=$ ve nua or gq. dunse $\sim \operatorname{lo}_{0}-6$

万\{ दa mise. $3 \rightarrow Y r+v 00$ - 3 
To the Holy Family. Iot $62 \pi \rho x k$ ve $\rightarrow$ dure $\sim$ ?

ox.mos 2 wess. xaware -

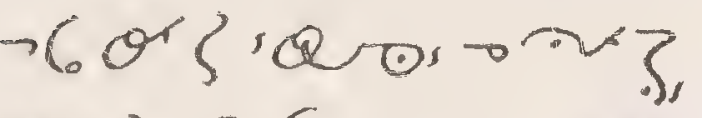

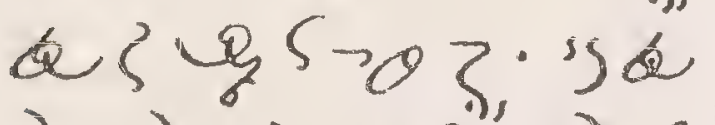
$\partial \theta-2+2020$

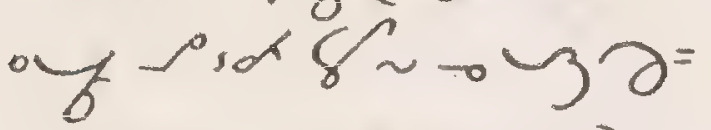
ज; $\rightarrow-g-10 \%$ 。 rst8 $\sim$ yan; d 209 byg 0 < rsing 25 bys -)e. unos yo ) i:sot a lor, sor

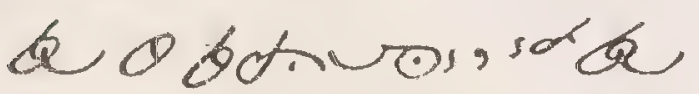
Qbusos, sar kes -) teg om bot क; sat कर्s?)

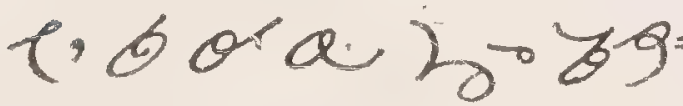

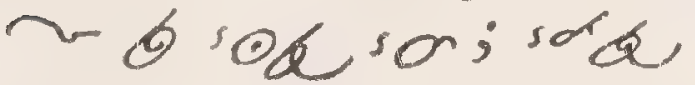
Lor o ( - orse -erd-2ror. is us a nर, 0 sक $82 \partial 2$ -

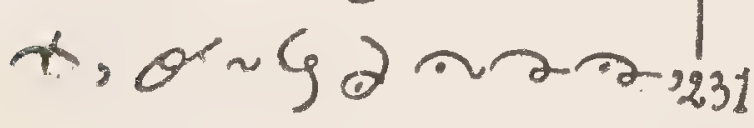

$0 \partial \eta \partial-\partial \partial v_{c}$ -2 2 0 ob $\rightarrow x$; n rsar $8 \mathrm{c}=0$

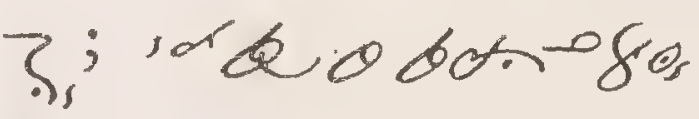
Q

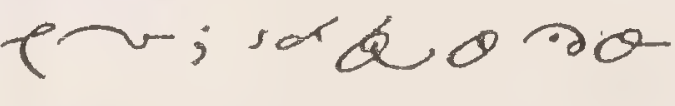
ofor d o a n óger siat 8 a $2=$ vos 9 96 - 
40

SKWAMISH

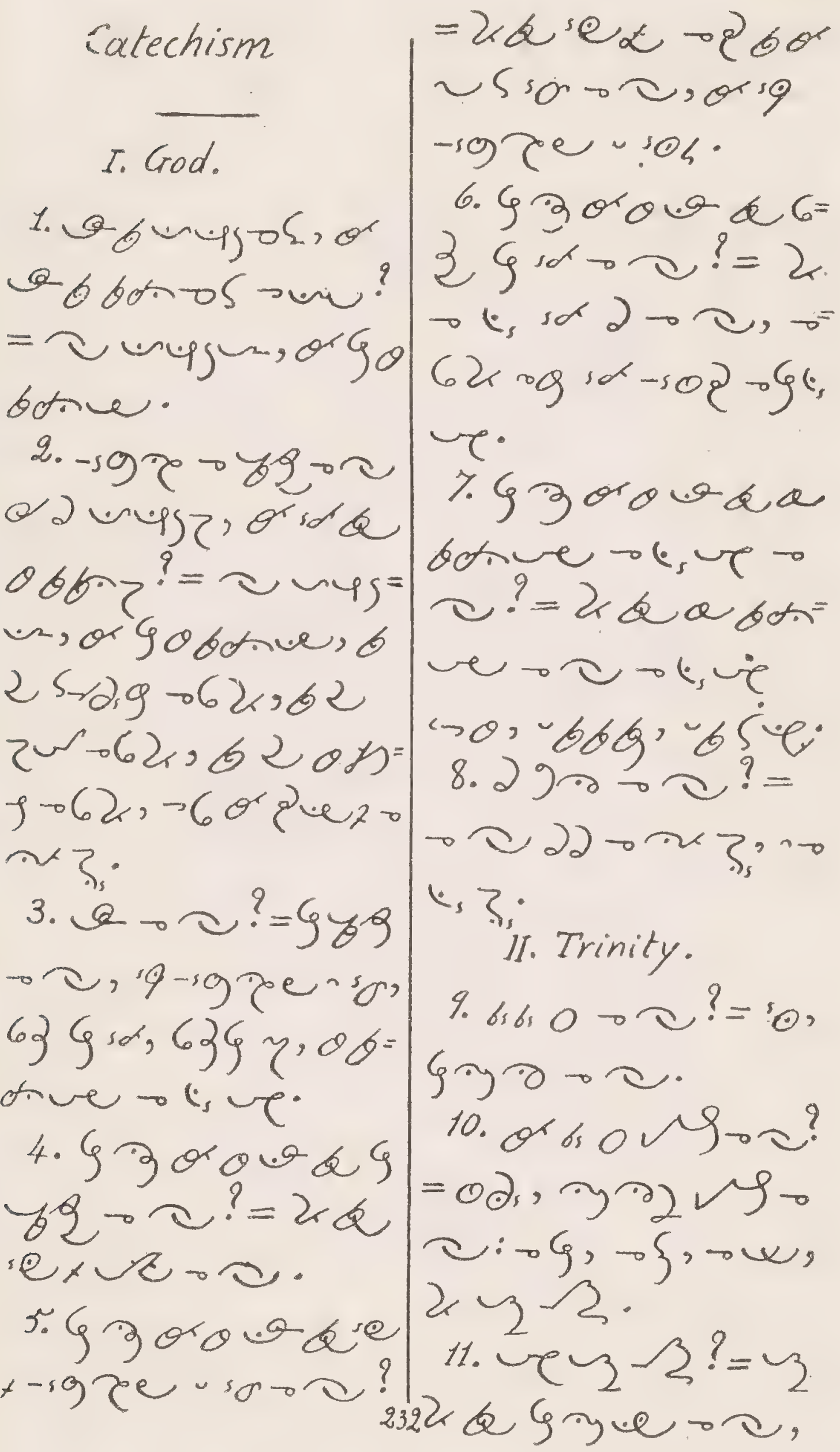


orngs 2 s.

12. $\rightarrow 9,20 \curvearrowright ?=$

Ods 0 द 2 巳.

13. $\rightarrow\{, 20 \approx ?=$

$02-\left\{\begin{array}{l}2 \\ 0 .\end{array}\right.$

14. कu, 20尺?

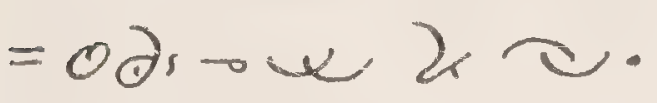

15. $\rightarrow \xi, \rightarrow \xi, \rightarrow \omega$,

गे 20 ¿? =

द, तु 2 ฯ, or

दलव

16. $93 \rightarrow \pi \partial 2 \mu$

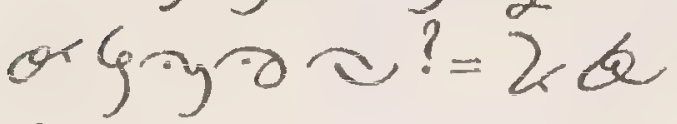

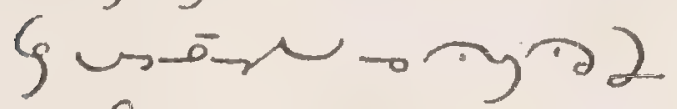

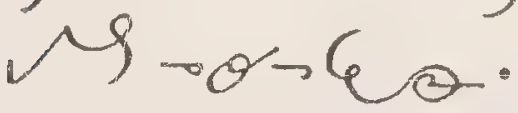

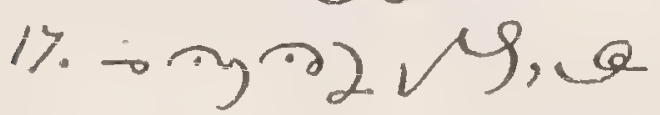

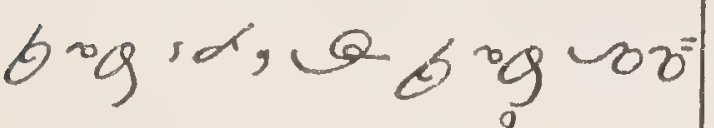

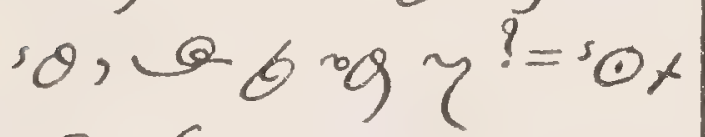

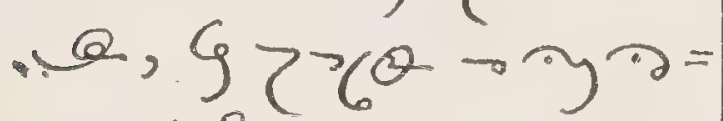
249.

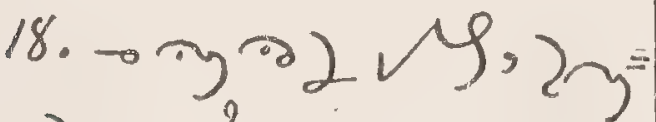
म)ae? = 0a, 2ry= 2) $a \theta$, oryd, $x_{x}$ xe $\rightarrow$ ) ry.
III. Jesus Christ.

19. )ry 00 - un ay

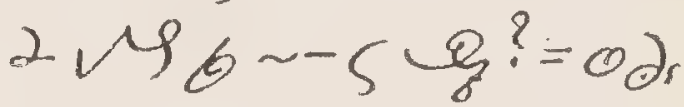

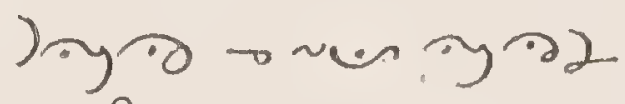

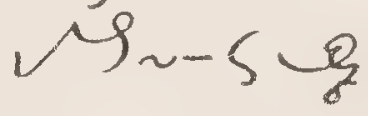

20. Q- u'ंग2

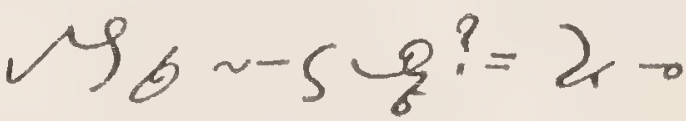

\section{$2 \xi$.}

21. $-9 \gamma_{2} \rightarrow \xi \varepsilon$

$\sim-5 \frac{g}{\sigma} \cdot=25$

bu-s of a ive

2 $\sim \operatorname{ora}_{2}, \alpha=$

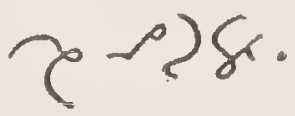

22. 29ra 2-le

$\rightarrow \infty\left\{\begin{array}{l}-\infty \sim \infty \\ \text { r }\end{array}\right.$

a) $\backsim=02 \xi .2$

ve o $\left.2 \sim \theta^{\circ}\right)_{5}$

$\alpha$ onsot 8 , o w enes \& $\rightarrow$.

23. - $2\left\{k \sim \times \frac{8}{6}\right.$

ग'次? = $\neg 6 ;-2\} 2 \xi, 0$ $\xi \curvearrowright$.

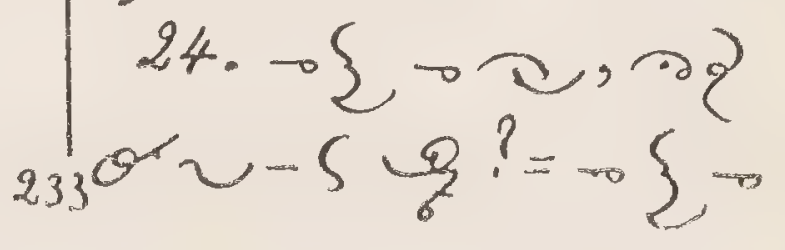




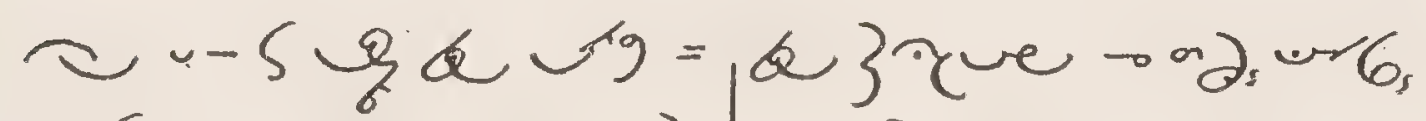
ofe $\rightarrow 204, \alpha z=\rightarrow \partial b \alpha$.

voge on ?.

$25 .-\{\rightarrow 2 \notin \sim$ $+g, a b 2$ ? = $\{\approx \approx+y, y d x$ 2 .

26. Q $0 x ?=0 x$ $2\{, 2 \partial 0 \%$. 2\%.- $9 x \cos 2$ w. ) ofie $\rightarrow x$ ? = $\rightarrow x$ 2. 5908 \& $2 x$ $2 \rightarrow \frac{8}{8}$.

28. $\rightarrow x \partial 2,0 \rightarrow$ )

$\rightarrow \partial 0 ?=\rightarrow \times \partial \cdots$ - 2 2 z 25 a $\rightarrow \rightarrow \partial v$. 29. $0 \times 女 \sim 59)$ $\Rightarrow$ गr, $\partial$ 's $\sim-\theta\}_{i} \cdot=-i x$

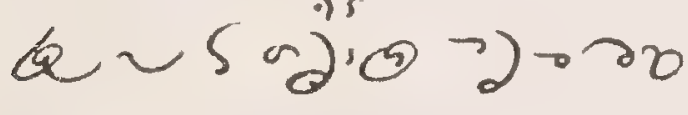
'egar ba几

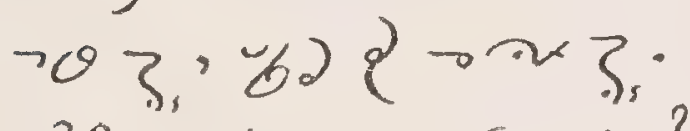
30. - ג60.25 ? ? ? $=0 \partial_{5}$ - $大 t_{2} s-0.3,234$

31. 2 cox-0r6, o 3rue? $=26$ que or zis sos $^{2}$ ur $\sigma_{1}$, or sare ris urg, $-502 \rightarrow$ in

IV. The Church.

32, Q $62 \gamma S_{5} \cup$. $\operatorname{er} 6,-Y)=x, 2$ og o r - sog - a

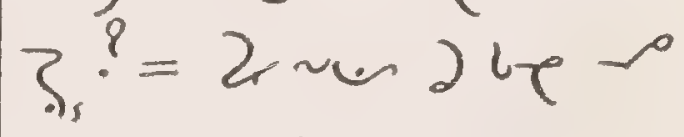
xordic $\rightarrow$ eq $=$ Eroto.

33. जe -96roor?= -96rod, 2u u

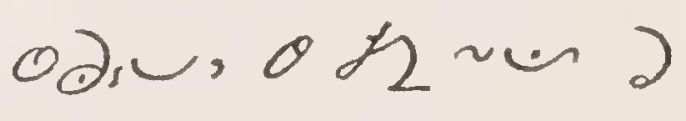

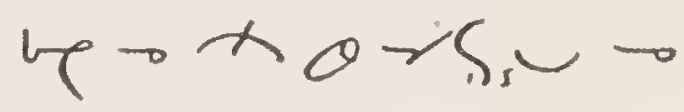
er 6 , 34. Quen a wo xorbi-leg $\cos ?=2 \rightarrow\{, \sim \rightarrow$ $\sim \longrightarrow$

35.4509 Ero-o.? $=$ 
the - g6ror, Go - vo, a - $x$ क 6 r वग; क्व है? 3 or $6:$

36. 2 . 9 क g6ror $6 \mathrm{co}_{0}$ ? = 2060?

$\Rightarrow+y, \rightarrow \Rightarrow-b$, $2 \cdot u \cdot e+x y=$ Eroto.

3\%. Q o orve $9=$ Eroter ox? =2. torna-gkror

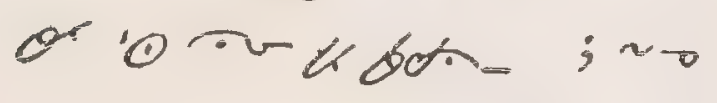
q. 2orve or: 0 bor.

38. - g6 ब0, $\theta=$ Si, ? ur $\sigma_{1}, \partial \varphi \theta$ $\sim_{0} \cdot ?=0 \partial_{3}-9 \varepsilon_{0}$ or urs o ardsue rro; 2 - $2 \boldsymbol{u}_{0}$ a g-gerom, ror Ga az..

39. a a 2 -5

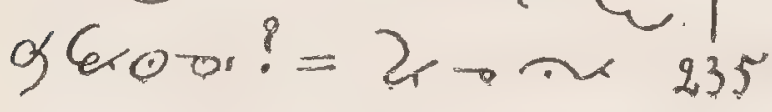

-89) we dare रथ. gkerom 62,2 ran. V.The lireed. 40. -sato a rasa m, हn कर

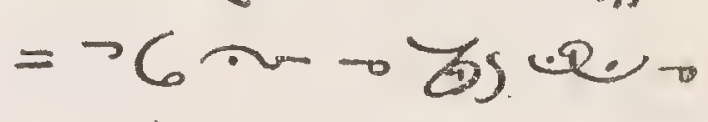
sotod $\}\{\rightarrow$. 4i. Q o rat $\mathcal{E}$ ? = 2. To, 000, o-e- olर

\section{e.}

42. 2906002,4 ? $=200230$ \%o¿ogens gुer. 43.sd a zuros

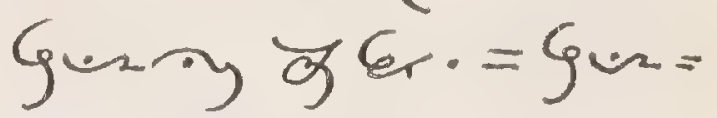
r $0 \approx \xi$, $44.2 \sin ^{\circ} 002$ $96 ?=G=2 y_{0}$ Odiw $\rightarrow$ gुer: 20 2. 2 - $S_{6},-y \partial u$ ure - C62000.u. 
44

SKW AMISH

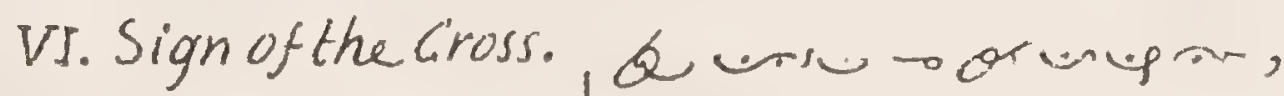

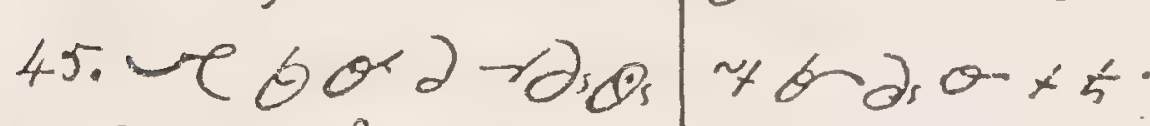

$-\{\{\rightarrow 2 ?=2 \alpha \quad 51.63$ or 2 to 2 a

2 ion. un worgk, -

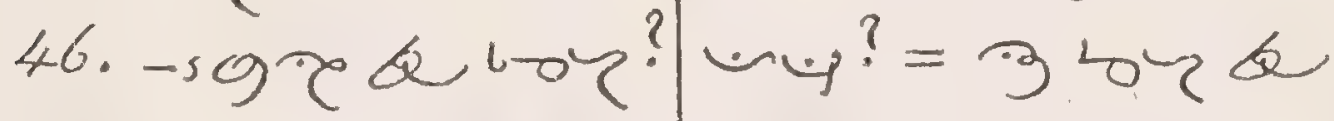

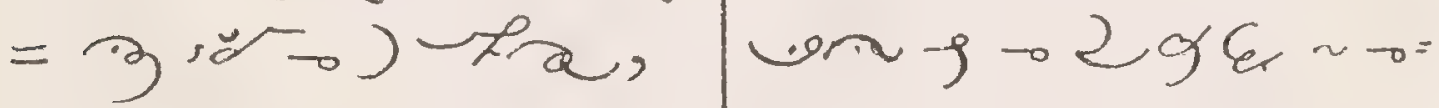

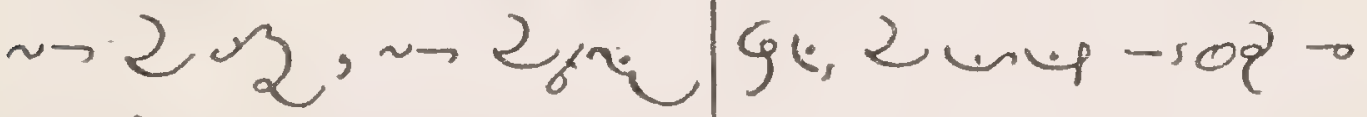

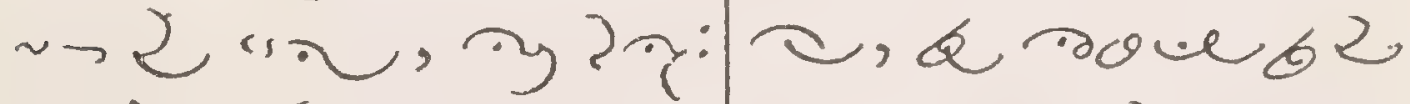

$\therefore \mathcal{2} \rightarrow \zeta, \sim \rightarrow\{, \sim$

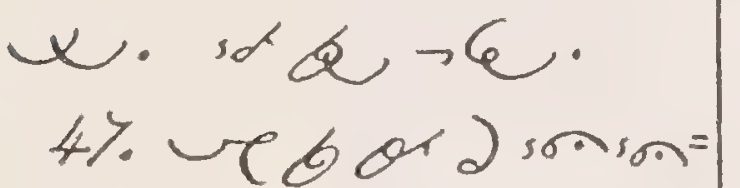

wark ioy? = क

sकin $-2-\beta, \sim \alpha$

$2-x \rightarrow x \partial-\not{d}$.

48. - sg ( od or $\sin =$

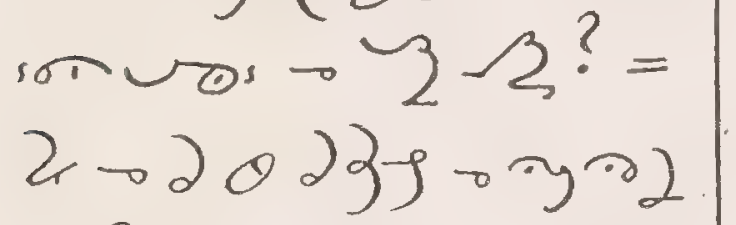

Y: $\rightarrow \xi, \rightarrow\},-w$.

49. - 97 bor 2 so $=$

mur 0 -

$\partial-\sigma^{2} \cdot=2 \theta \cdot 2$

289.9.

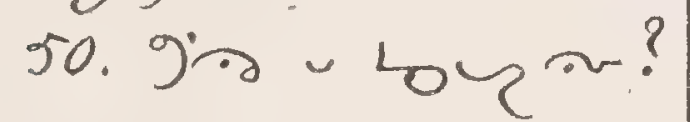

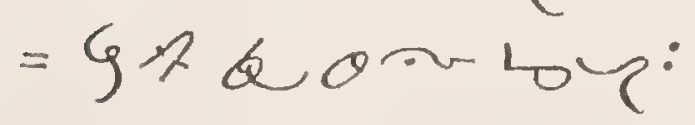

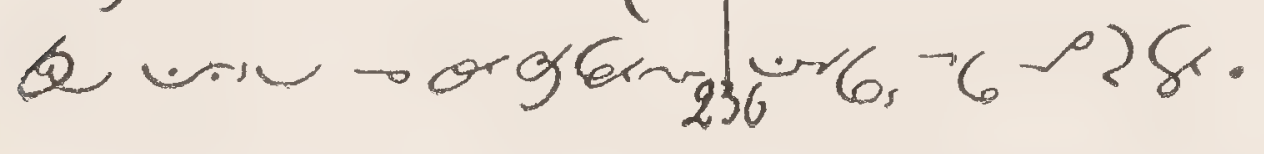

52.9302 5 7

Q 6 ind, 0,5 ? =

反 गove 2

et -5 .

VII. The Commandments.

53. $2900-6=0$

$z r \cdot r ?=2 \rightarrow \partial$

o $23-$ "g $\psi 2 \xi=$

54.-sgx $\sim 20$

$\alpha-9 y z-2$ ?

$=\omega 2 k+00$

grr 0 , or

Nh 0 a 0.89

aroras 
55. $\sim 209 \theta \rightarrow 6,96 .=84920$ $-Y r-96 r o r^{?}=$ r.

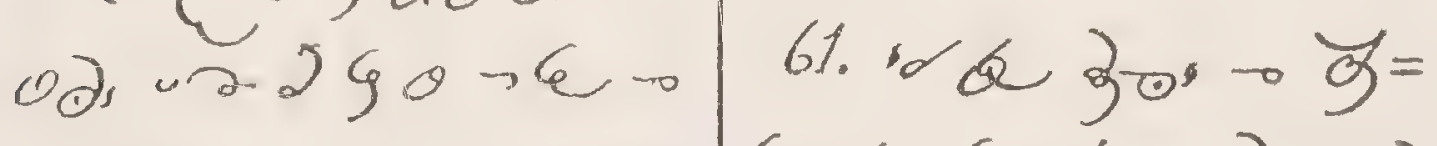

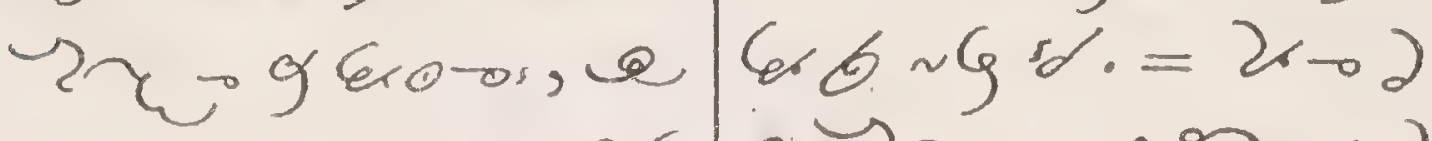

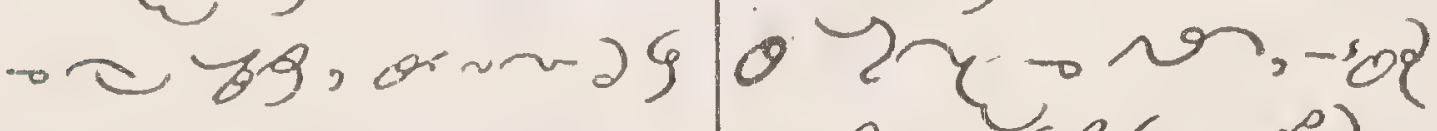

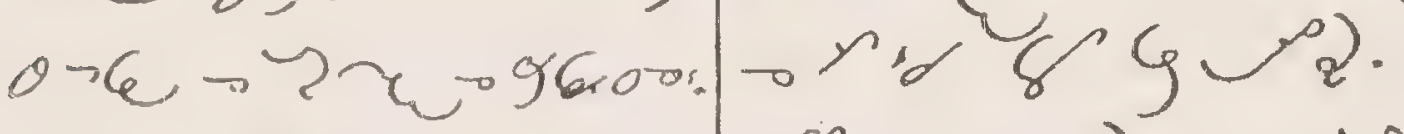

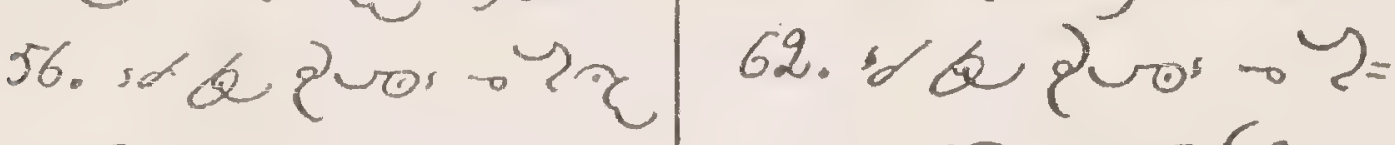

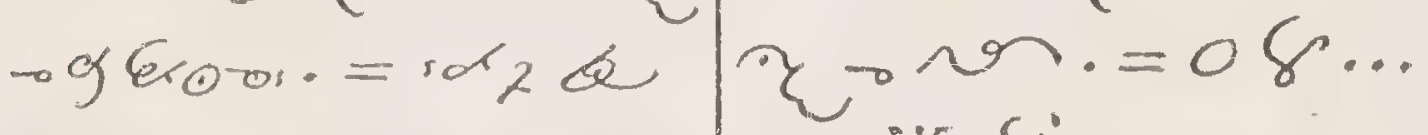

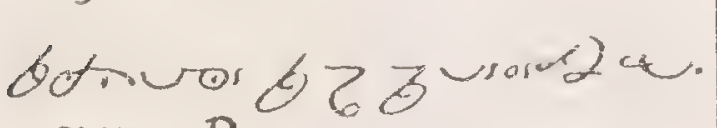
VIII. Prayer.

$5 \%$, Gopano, ormo ?

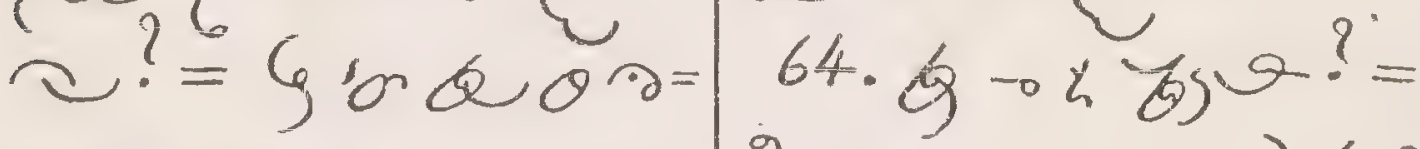

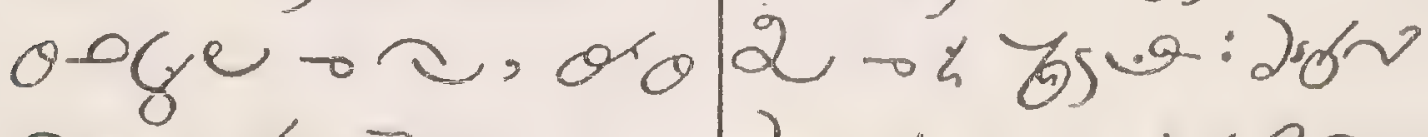

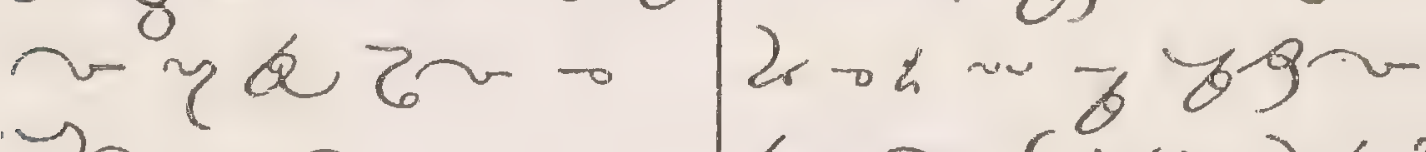
$58.402 \sim \sim 20$

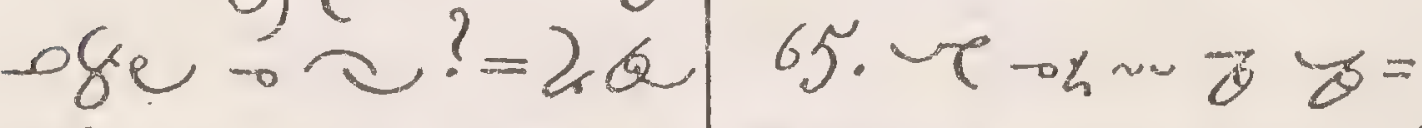

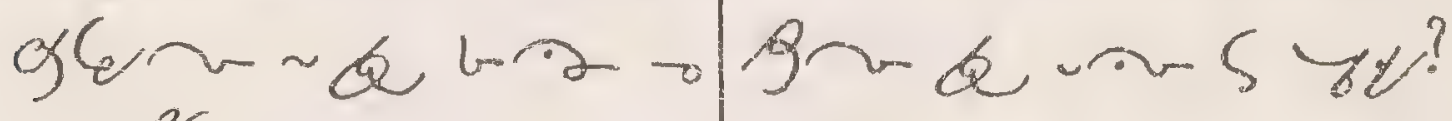

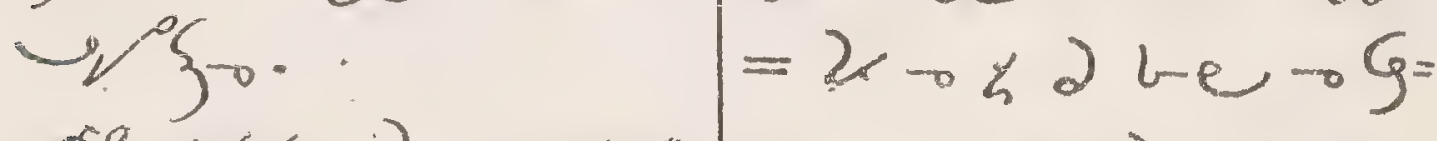

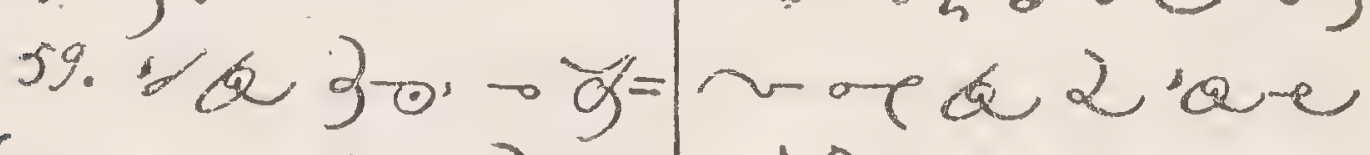

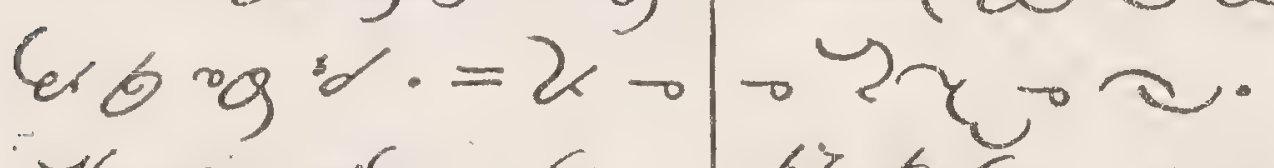
The ow - $x$.

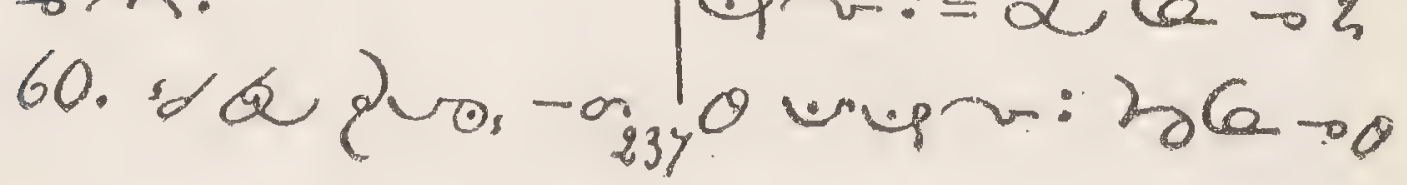




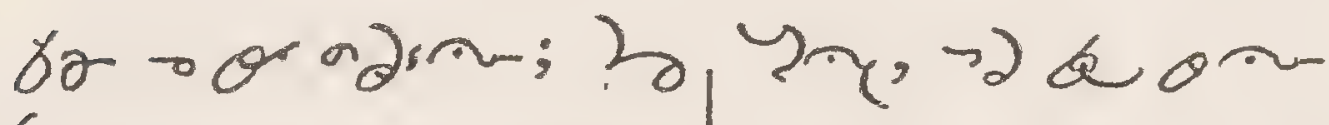

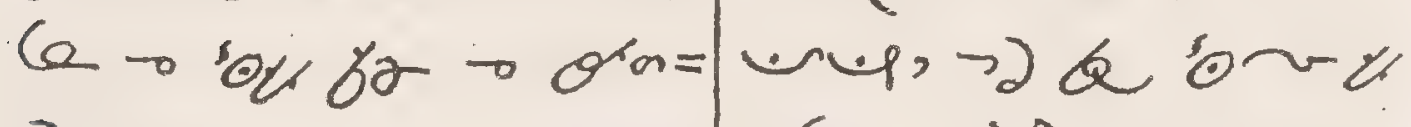
$\partial_{\text {r几n. }}$

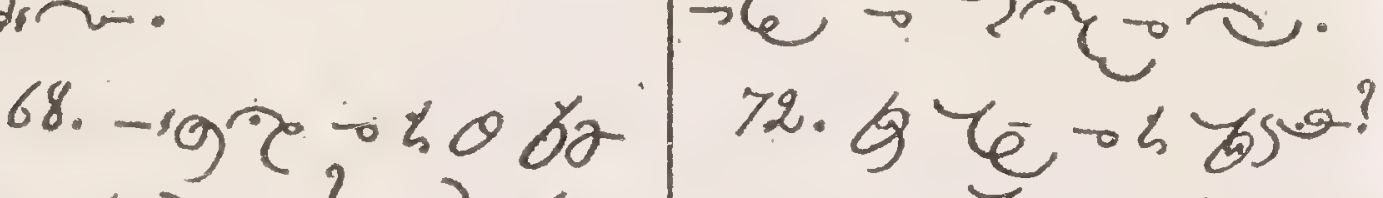

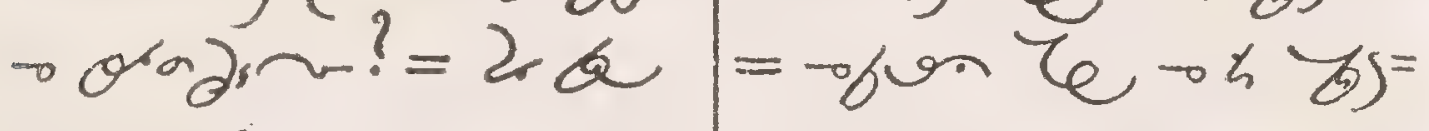

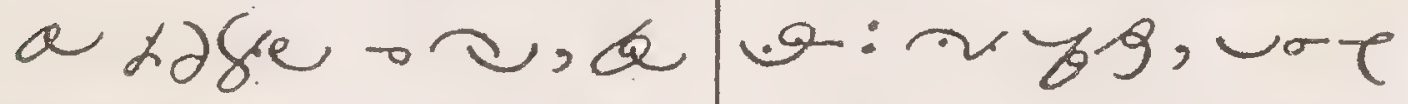

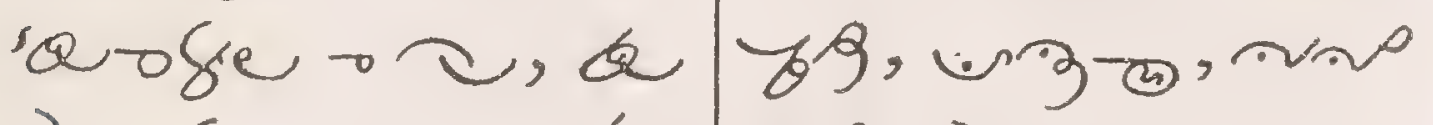

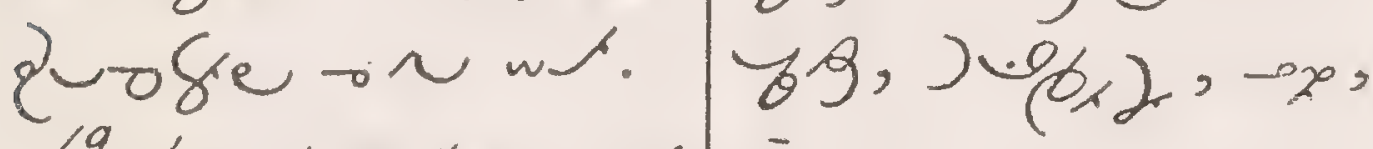
69. $g-4 \theta \sigma \sigma-\theta=\hat{e}$.

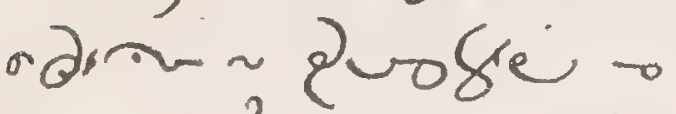

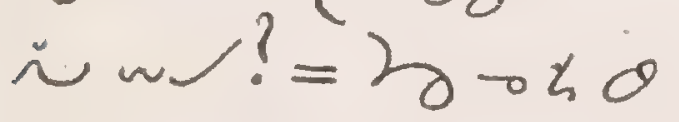
br 0 orar $\sim$ - bar $=$

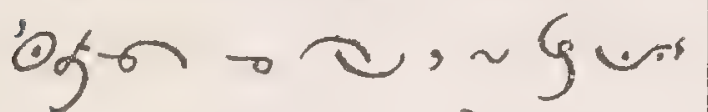

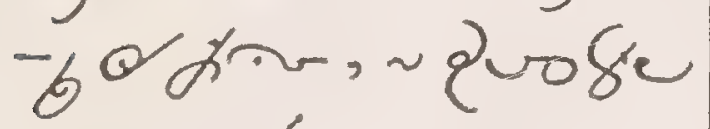
$\rightarrow \sim$ w.

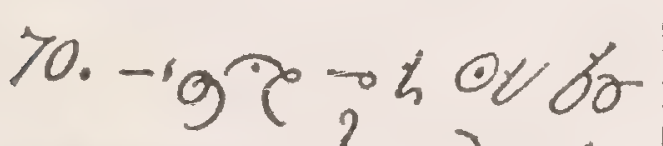

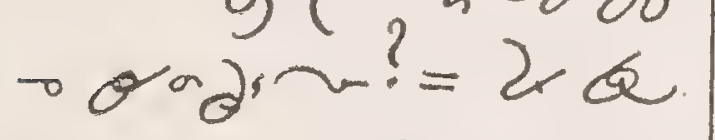
巴义'a力beっ or. परa Se.

71. $\rightarrow 92$ \& $5 \mathrm{~m}$ $y \cdot+b^{2}=-\partial \rightarrow u_{0}=$

X. Baptism.

73. 93 - 102 eer ofon $x$ ? $=2$ \& byse $-\theta=$ ๙25 - .

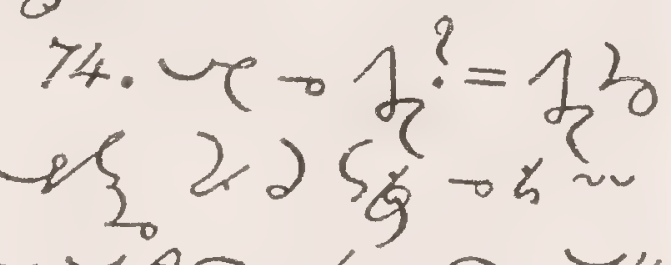

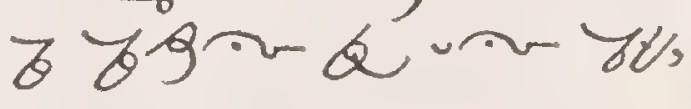

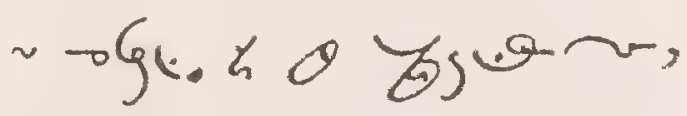
or $\sim 62$ 2 104

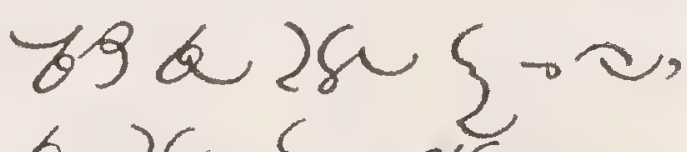

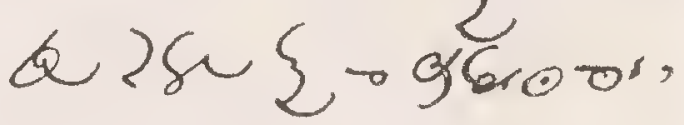
bisur.

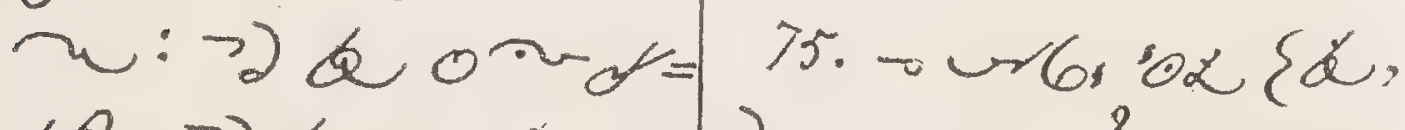

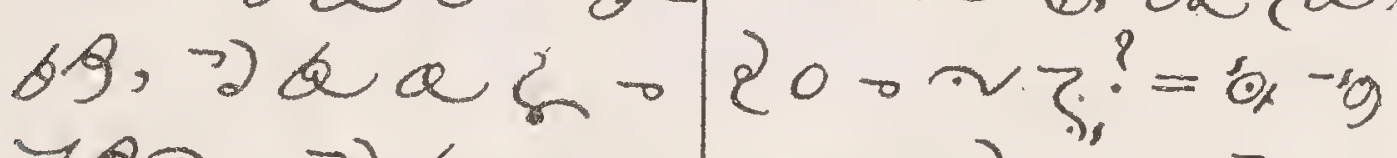

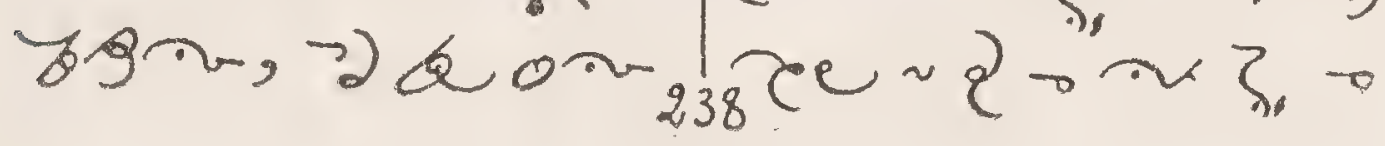




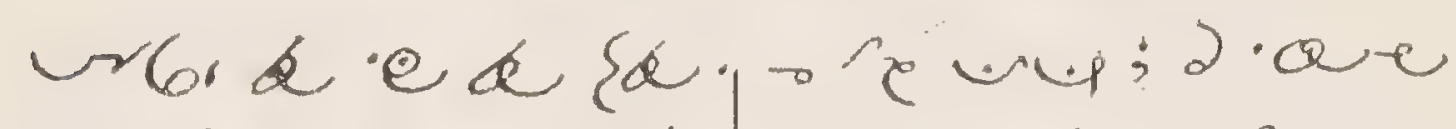

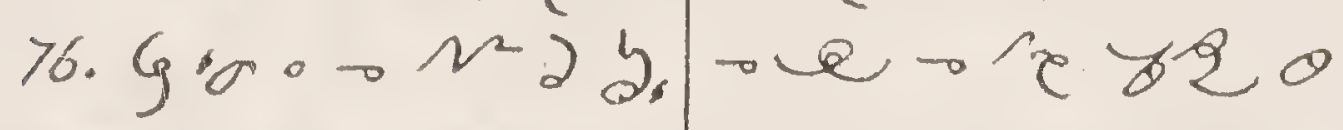
- or z c S g o

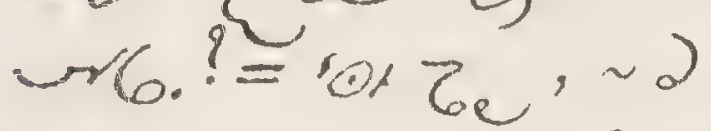
$\xi, r+\cdots 6, d s b=$ - 0 万人 y.

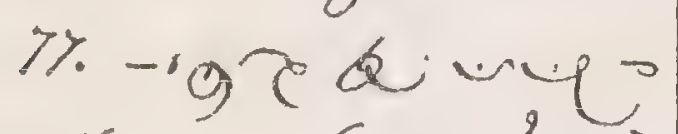
Non, b s see? $=2$ bree o y a d re

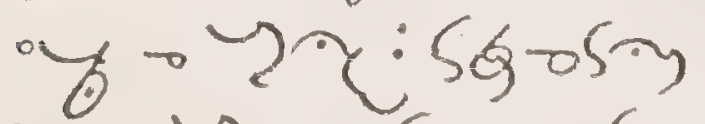
$a+2-\xi, \sim \cdots \xi$, $\sim$ -

78. -gredere - D b)ye d zun - $h_{1} ?=0$ ree $\theta 0=$ ane $\rightarrow$ Yर 0 - क; के गुल-कर

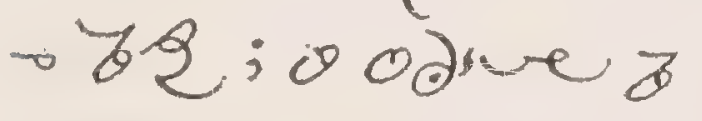

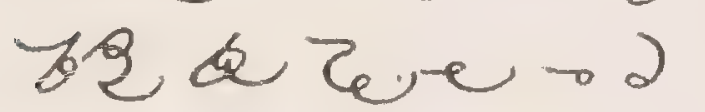
制 960 or. 79. v o a bere 0

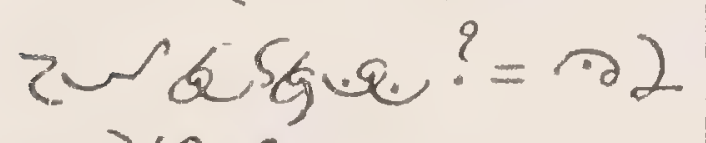

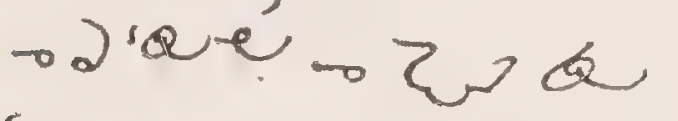
Sque,: a aee o ane wro." Xi. Confirmation. 81. The a ales? =

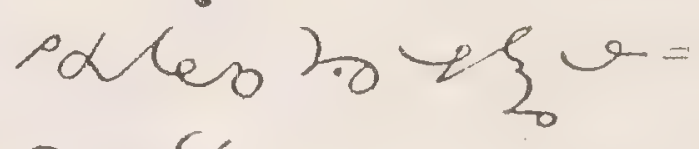
noteraw - for ex a rsogie dor

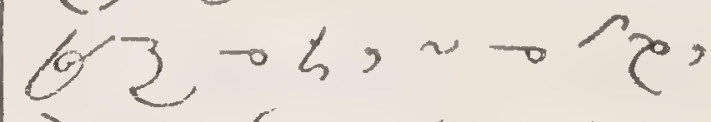
arobeder $\sim x$.

82. Q6 a $05=$ $\alpha e_{0} ?=9$ son $y$ ov ro sales. 83.-gק

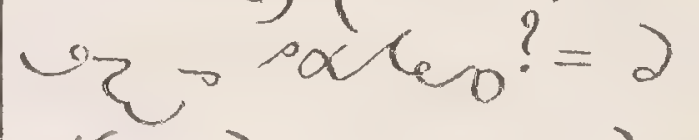

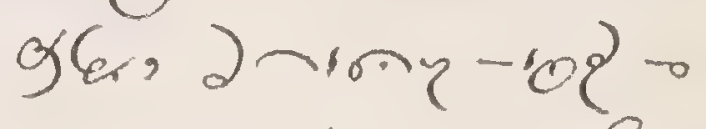

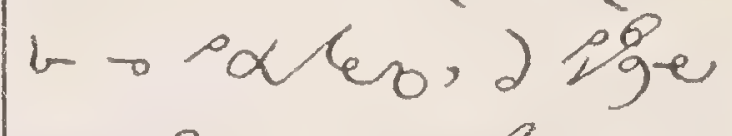

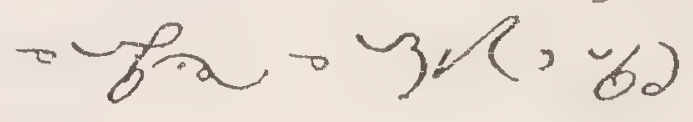
) gere.

84. -g p u z? a bersaker? $=2$ : be tho

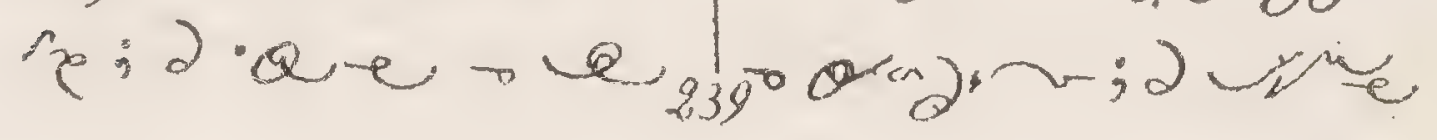


- 2 mecínors - Dra o or - 5$\} 0^{\circ}$ a, 0 b d y,a ‥

Xill. Holy Eucharist.

85. we $\rightarrow \oplus$ ? $=\oplus 2$

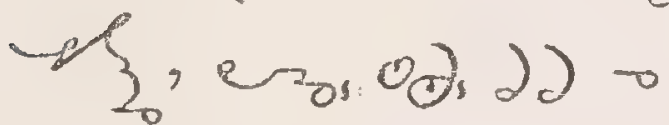

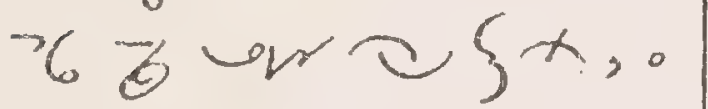

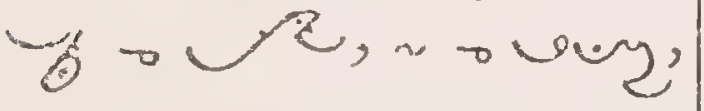
$\sim \rightarrow$ gad.

86. जe 2-e co? $=2 \cdot x: 2,2 y 2$ we $\rightarrow \theta$; d e d $y$.

87. -980 ecos $\theta=-x \theta$ -) ow $\mathcal{H}^{\prime}, 2$ be

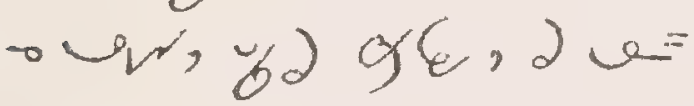

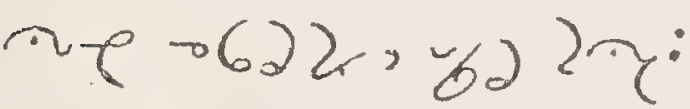
bor, , 3 कि, 2, ) 27 ; o e be o fre, doriec on, yd $9=$ C, a amp o 022 , od $27: b_{6}, y_{2}$

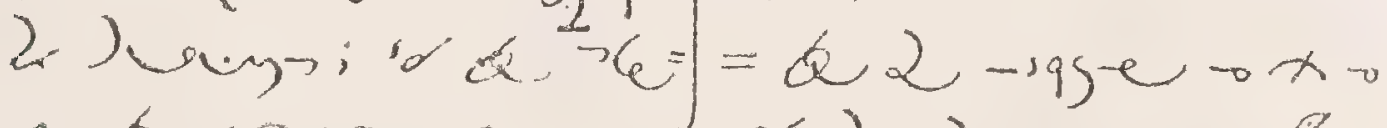

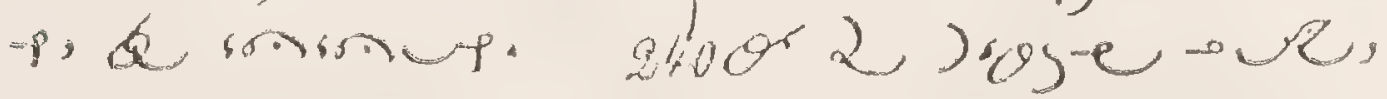

$83.62 \alpha-2 r \rightarrow x$ "2) $2,2 \rightarrow, 2) w=$ $y \rightarrow, \Rightarrow 60-\cdots \cdot e$ ? $=x^{2} 2-x " 2$ ) ox D) Jon ow;

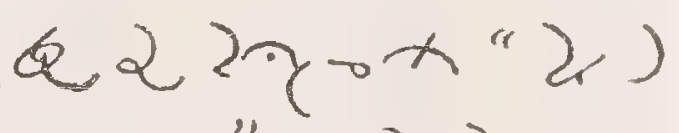
vys", 0.2 2 vey -x 2 ) bon o o . 89. द 2 lioje ox $\rightarrow$ w d e 22 , -og a werge

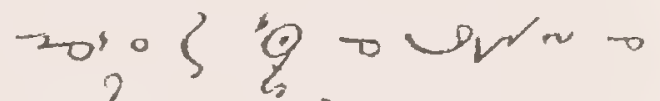
oj? = a 2 ) os e 0 $x-w^{2}$ Q 2 ,

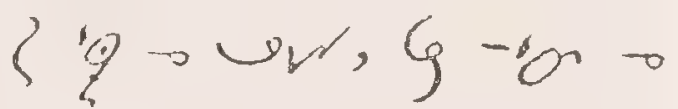

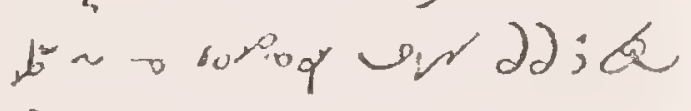
2 Joge ox o o a w w 5 क्ष

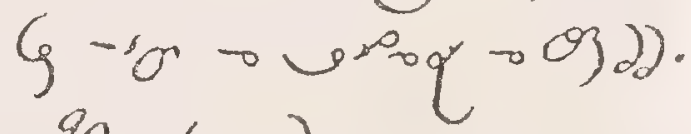
90. d 2 -iobe $x \rightarrow$ or 2 jose 2, d are $y /, 2 \circ 4-12$ ? 


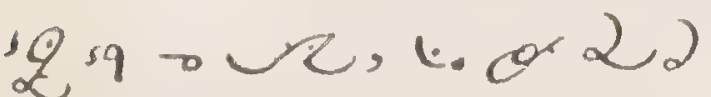
- or 2 ) ose a e 91. द. 2 2 $-x-50$ o our a kure, 9

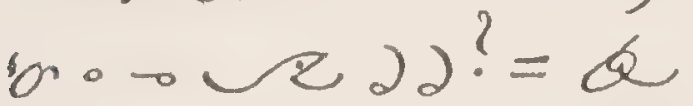
$2 \pi$ rox-s0p o er

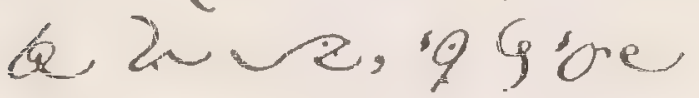
- र2ग, 262 。

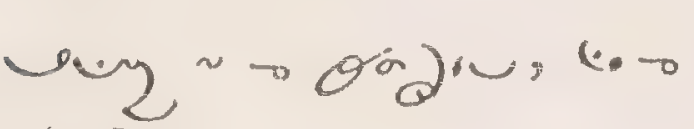
大ร.

$92.93 .+202=$ e $0 \Theta:=x \partial$ ere

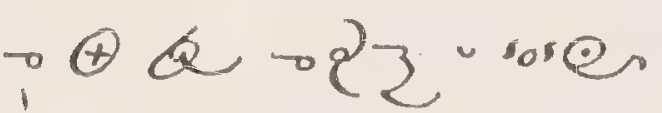

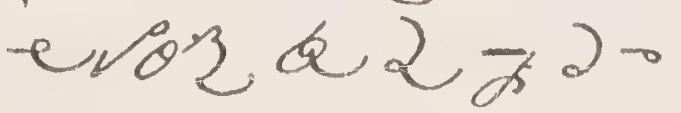

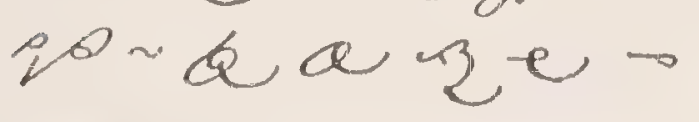
ongrn.

93. 23.) ion ard o

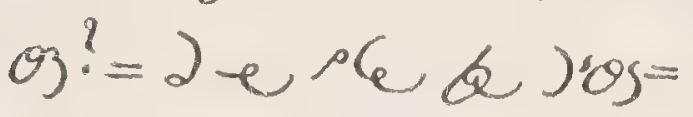
e-m.ver 2

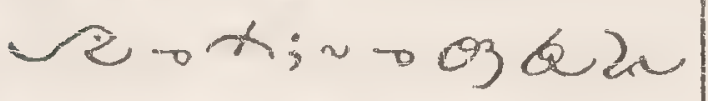
ay $0 x-302$ $v_{2} \div x$.

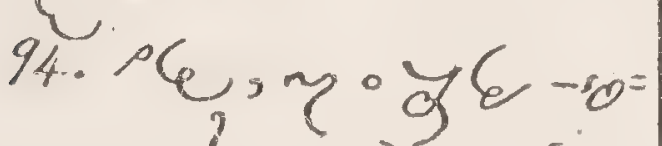

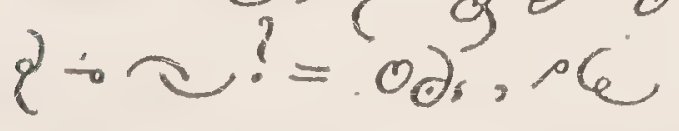

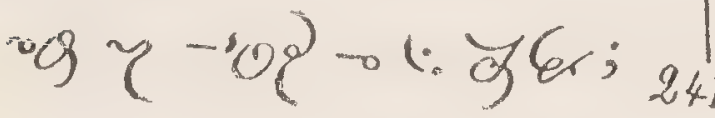

te the andor $\theta$

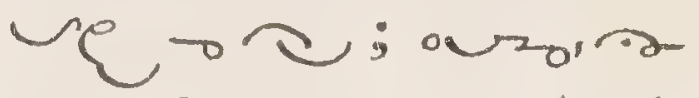
$0999-62,403=$ an $\sim 5 y_{1} \rightarrow e^{2} 2$ \%, $\rightarrow$. e 97 . 95. $56,0230-10 ?$ - य व एद od 3 -rog - N

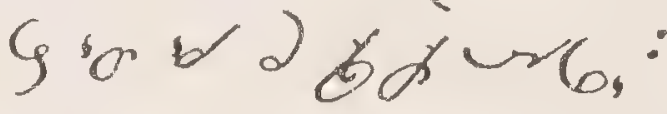
ur a e inut.

96.936096 a wer $x$-ord" $=$ 2 ? = \& $2 \oplus a$

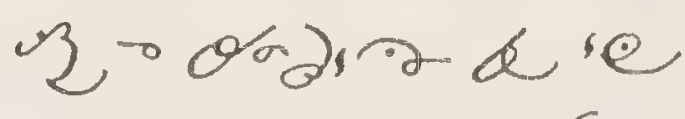

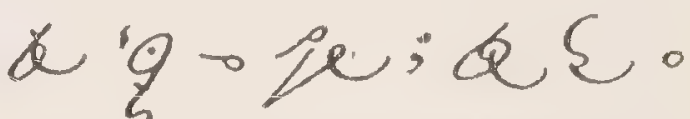
yop; a a ye

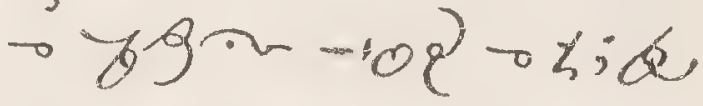
rsolie a on $\sigma=$ 之一他 bofer $x$.

97. $\oplus$, द० be byse य 4.0 t -62 ? $=$

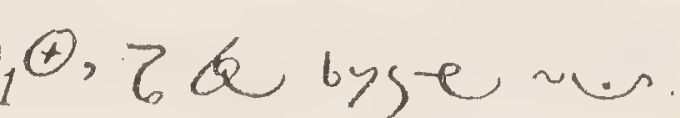




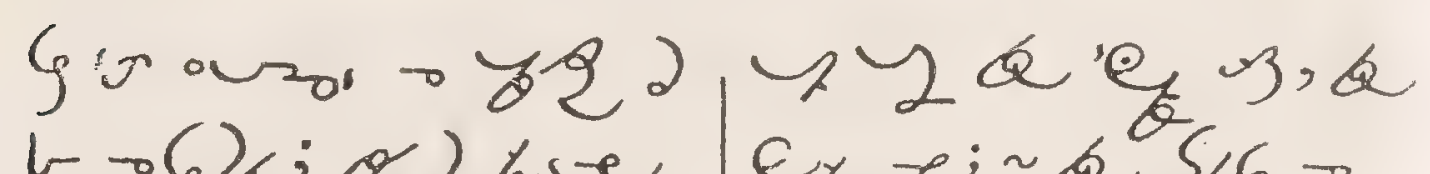

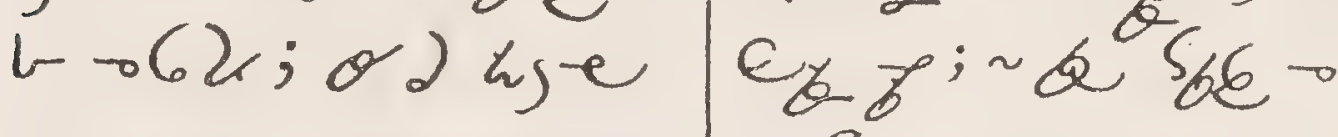

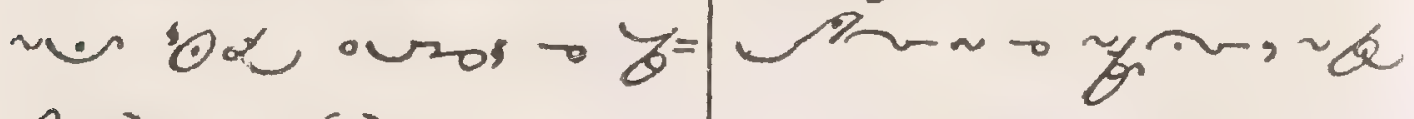

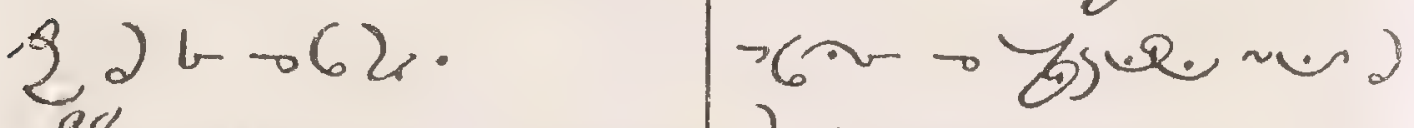

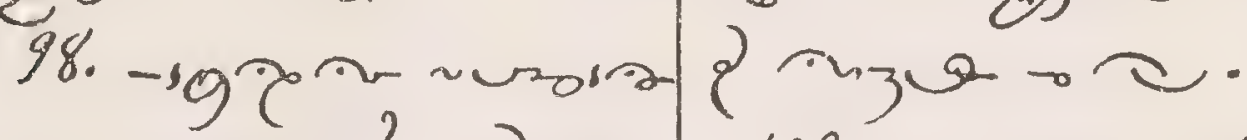

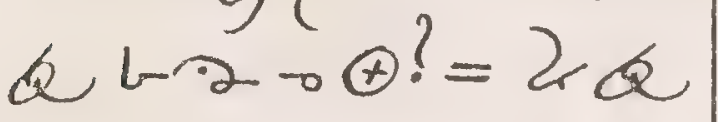
bysin-orara $\sim$ 102. -g?क दर

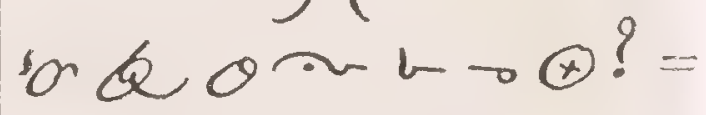
Pn.

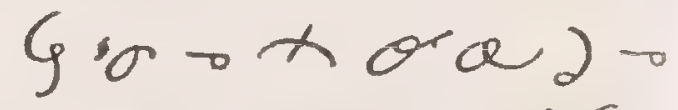

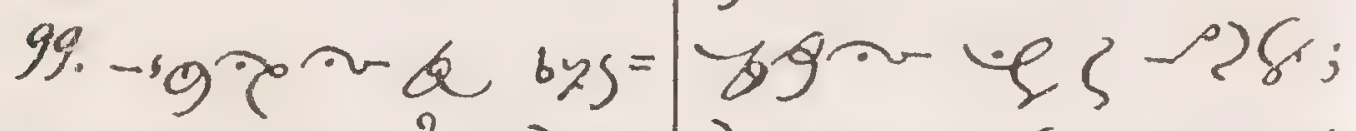

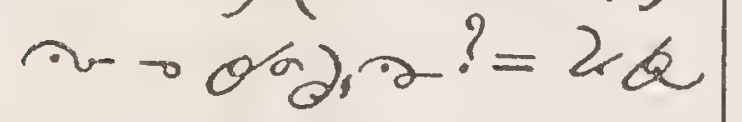

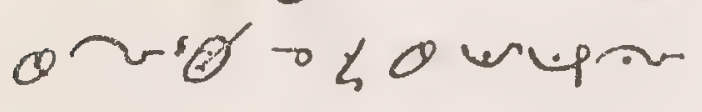

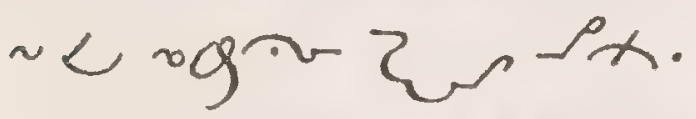
100. \%八 - 8 gr

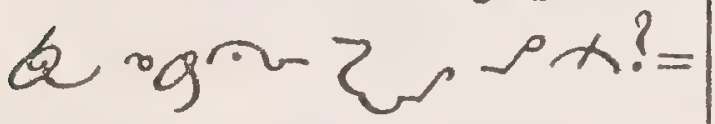

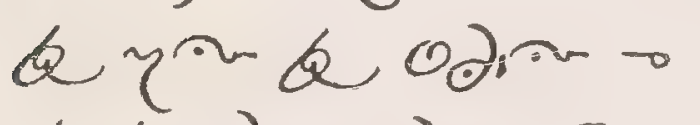
xद्व $200 \oplus$; द yथ

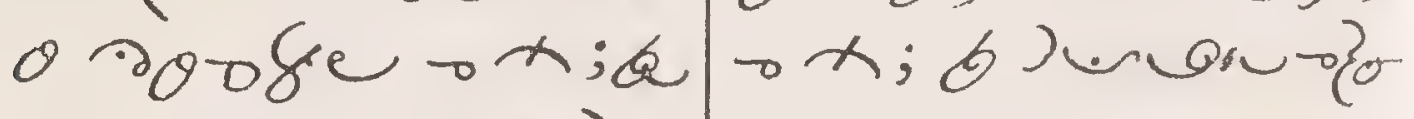

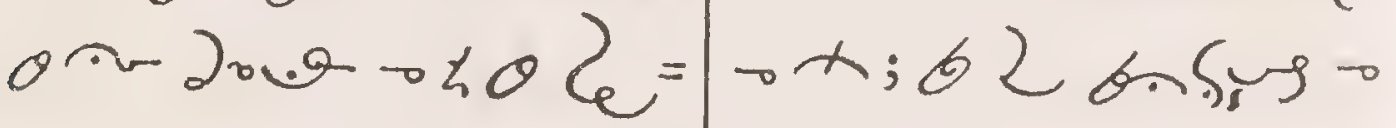

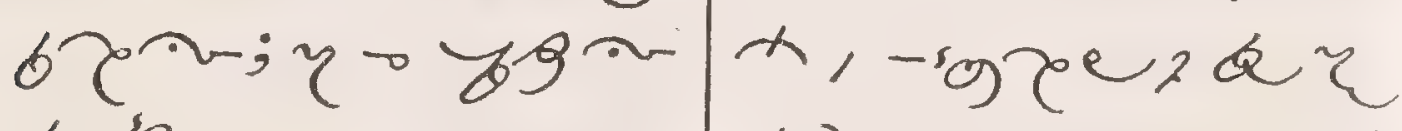

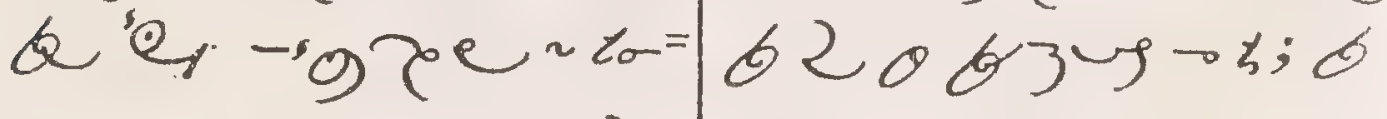

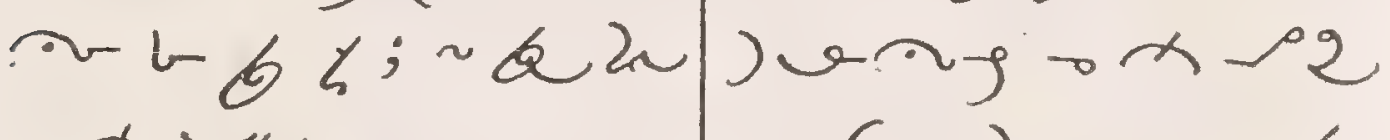
$\rightarrow x$ bban. $\quad$ oge.) unp; 0

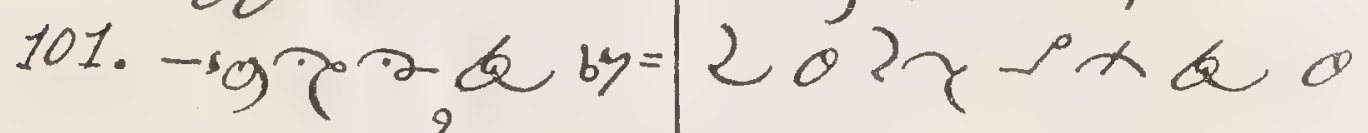

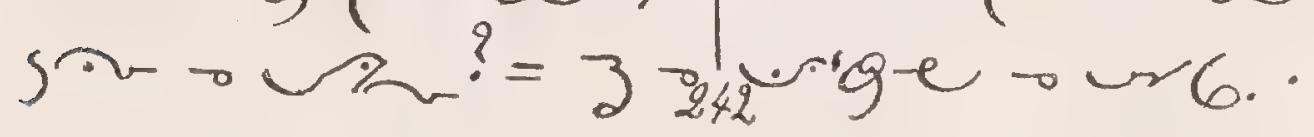


MANUAL

51

104. 023. T.8, 0 (1); Ob 0 - 22

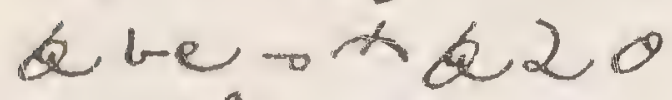
$20 \Theta^{?}=0 \partial .003$ -62 \&a b6- 82 binex 0 र2 0

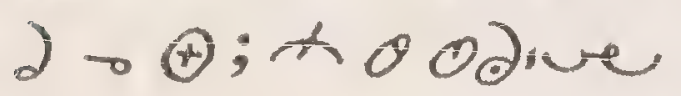
- obb- 8270 ra ene o ope. XIII. Penance.

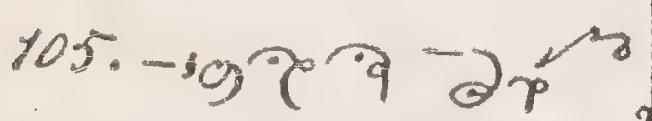
\& S la $\rightarrow$ thes? $=625 \mathrm{lg} \rightarrow \mathrm{th}$ ke, urin $\rightarrow$ ) in =

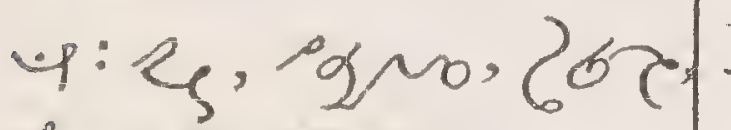

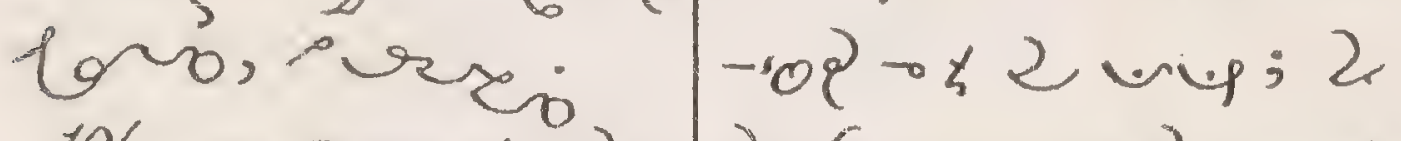

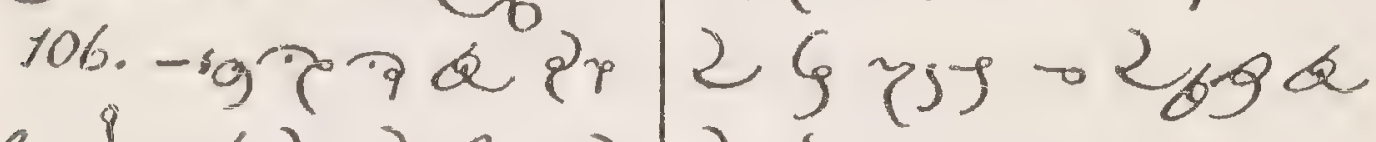

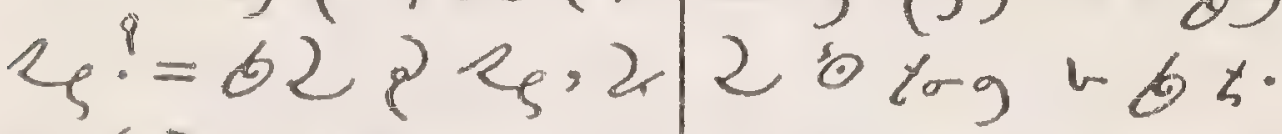

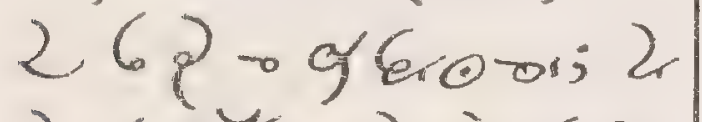
$26) \%(2 ; 26)$ 1. 2y: 262$) g 6$ - ed k-ge o $389 ; 2.26)=9$

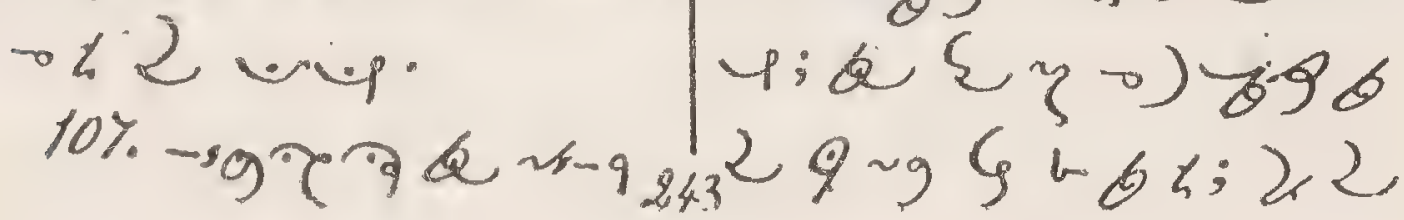

oh upp? = 62 $\sqrt{2}-g \rightarrow h 2$ enep; 2 2 gitg-g-gेz - $2 ; \sim-\alpha$ r - 960 osin a gon

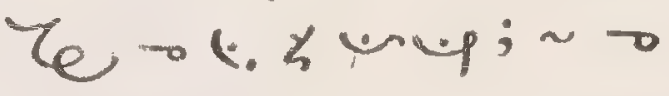
ungen oe; 2 ) Gionsass 1 ge 62 ra 6 Jus.

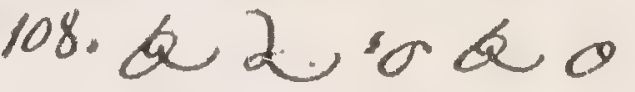
). or say

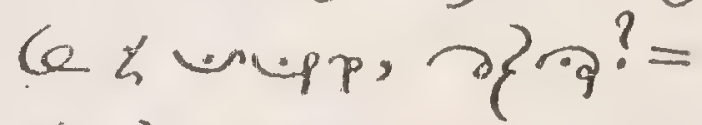
(द) 2020$) \cdot 5=$ Jastlye o z 6 क un. $2 \times 262009$ 10\%. - 97 平质 $20=$

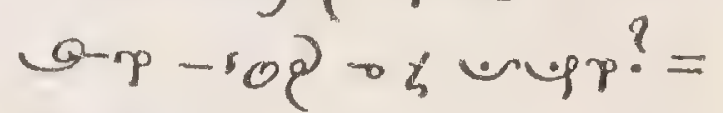
$2.29960 \approx b$ enum o que - - 2y - to2 $=$

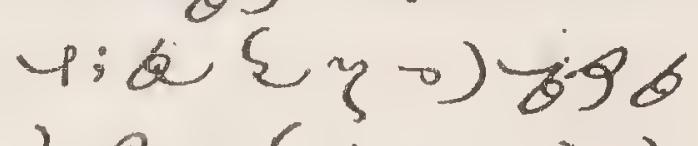




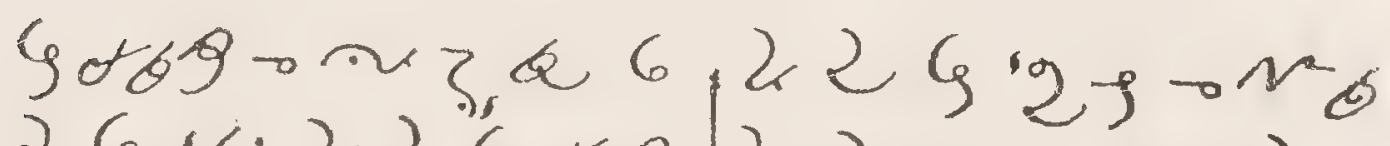

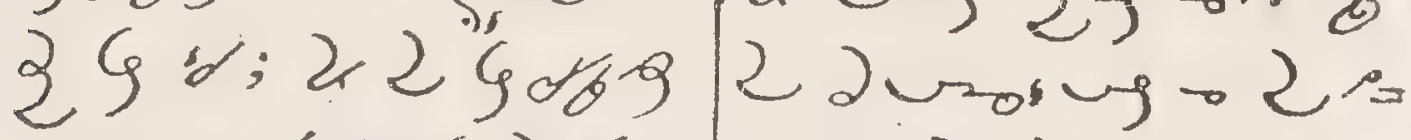
更

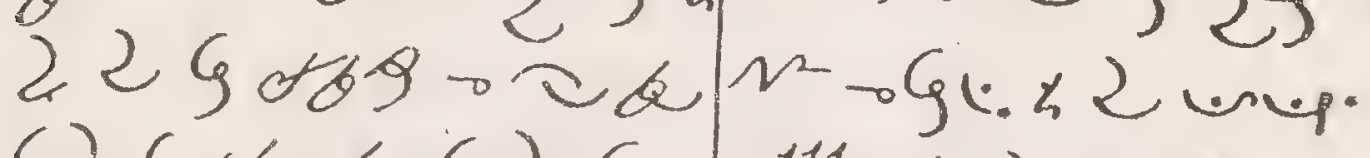
$629: 3629$ $60 ; 225+289$ $x$ a 63 ?

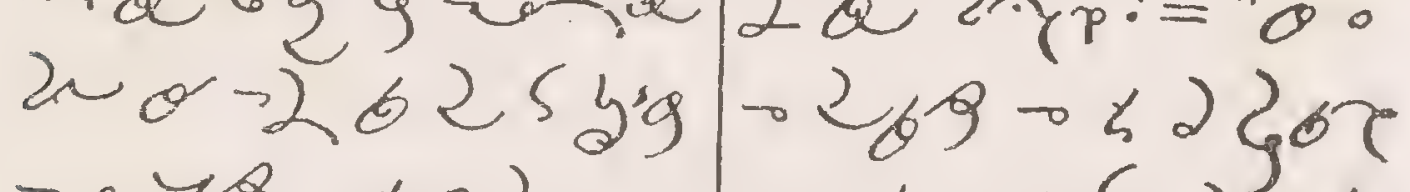

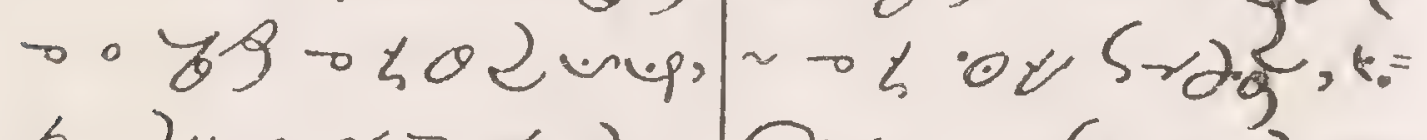

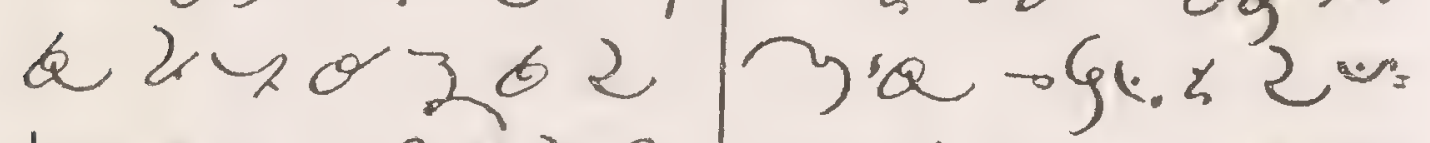
by $\sim 91-64$ 110. - 9 वर्व бरр - $w$ ? $=2 \sin y=$ - $2,2<\xi$ ion, $2.26 \cdot 2\{: " \circ) \xi$,

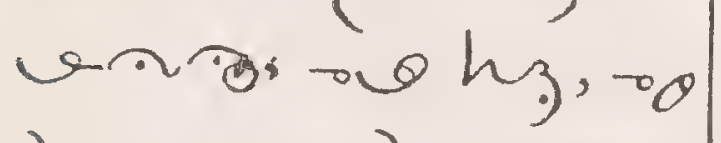
रथल 2 ; 360 $-\log -2 \cos 6$ - 20 of

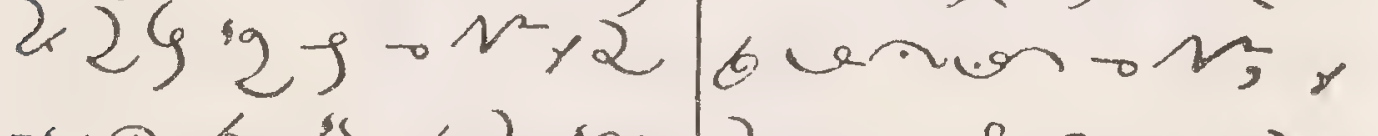

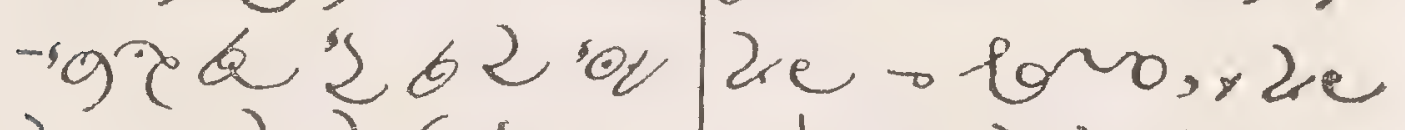
$667229,2,-0 h_{3} ; 22627$.

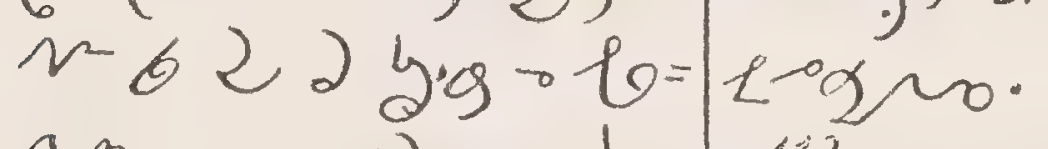

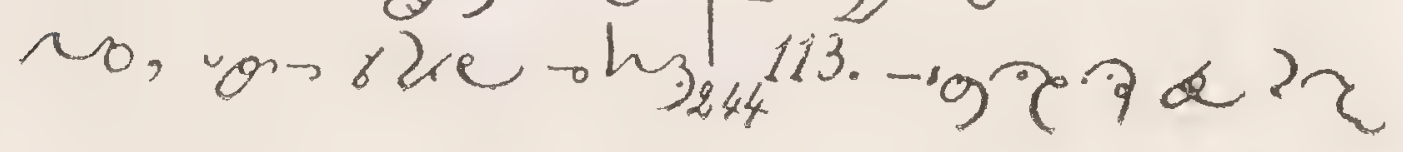


MANUAL

53

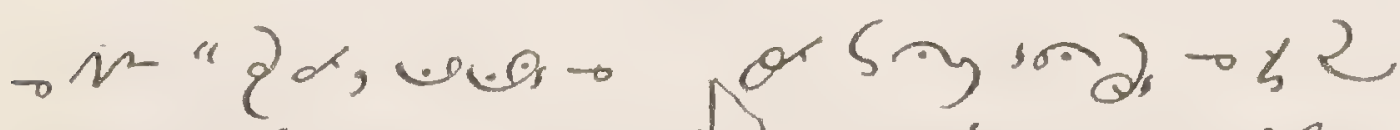

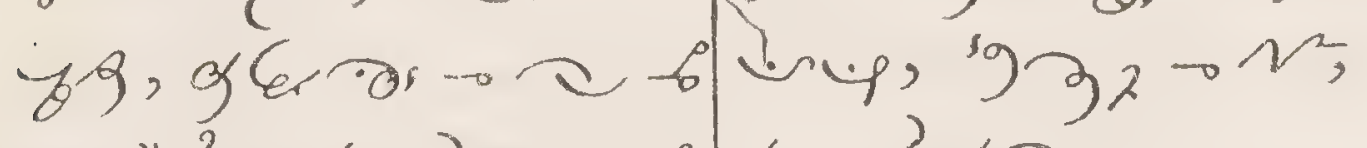

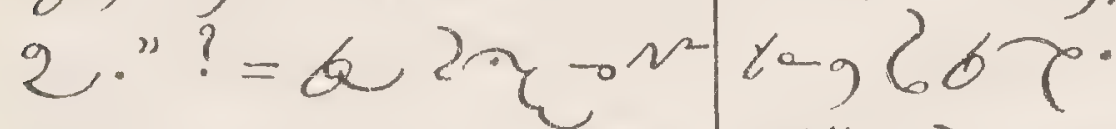

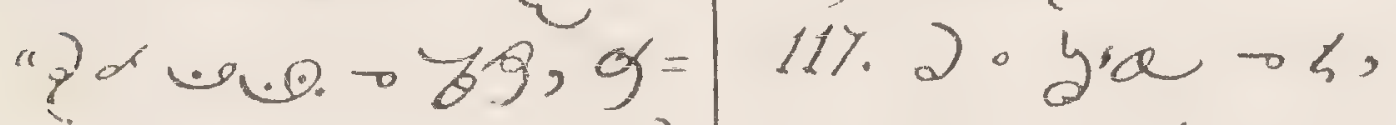

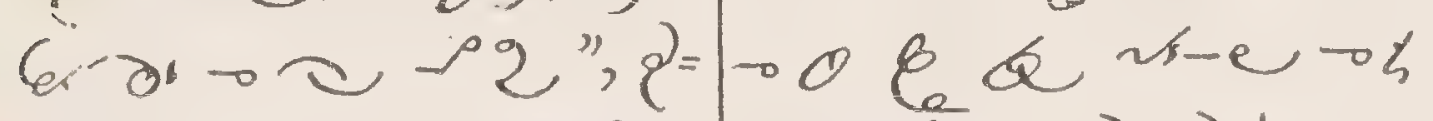

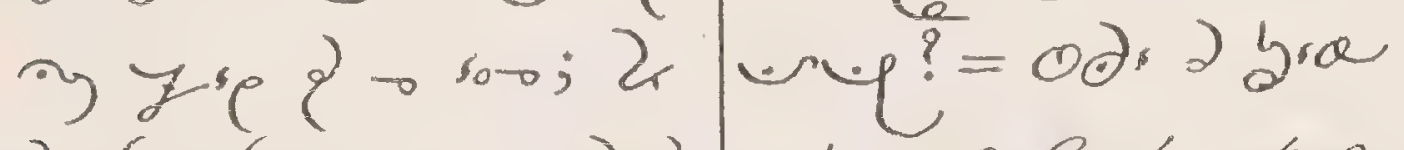

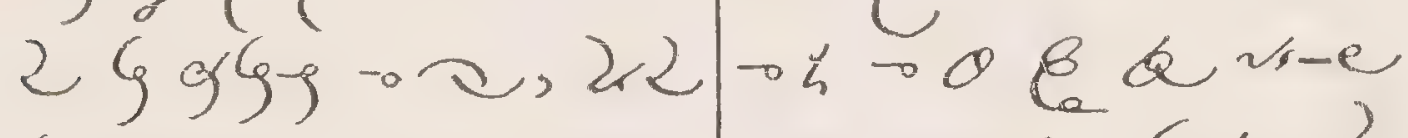

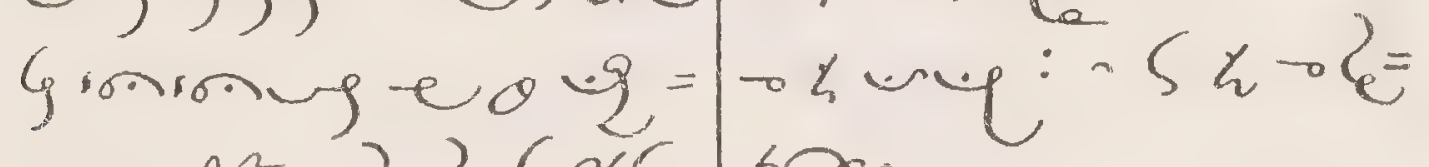

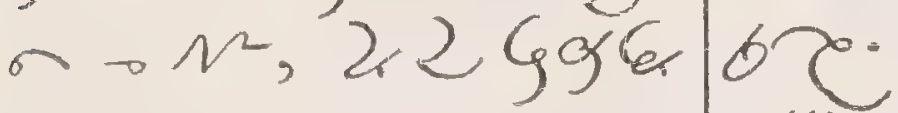

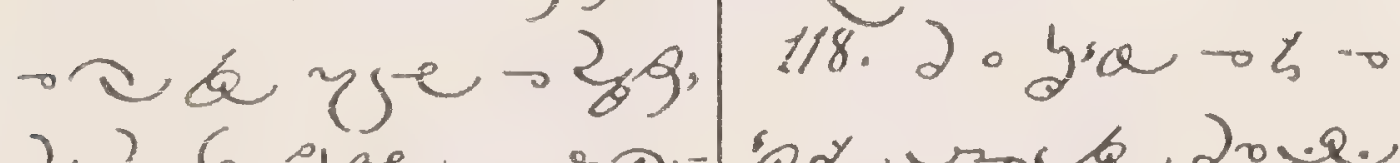

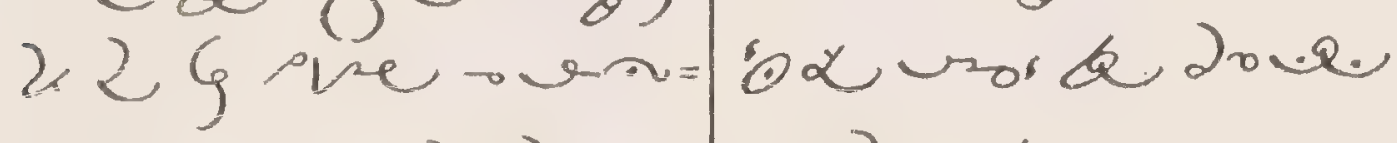

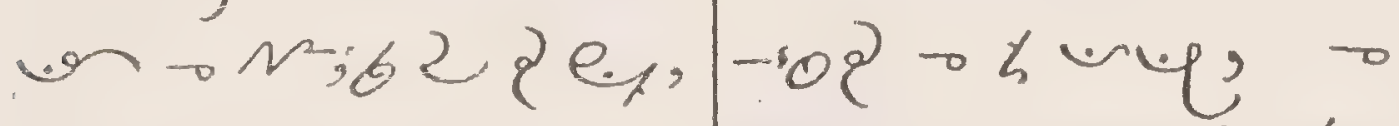

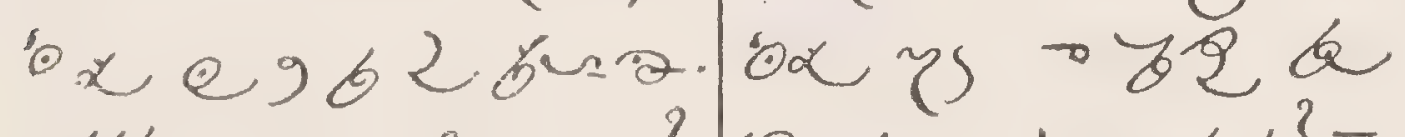

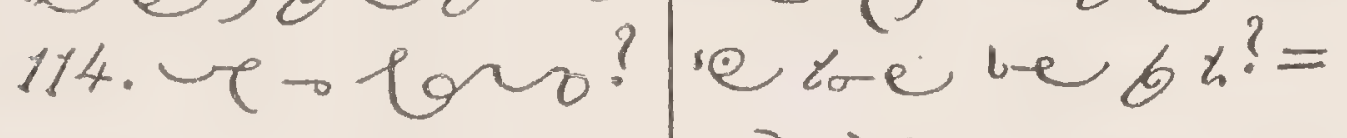

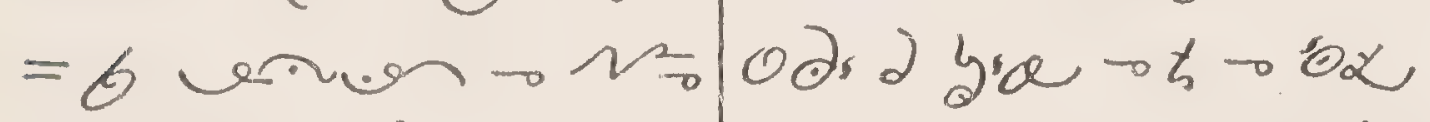

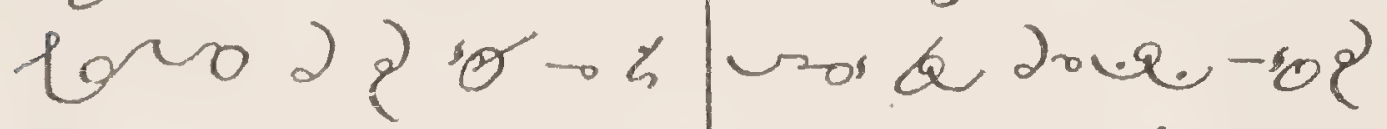

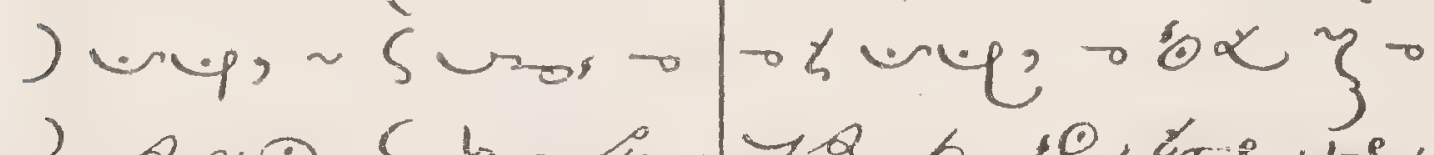

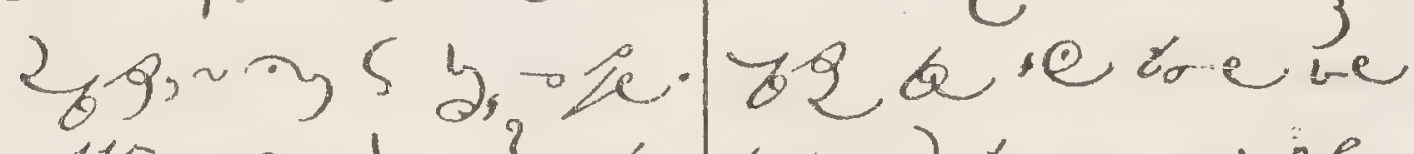

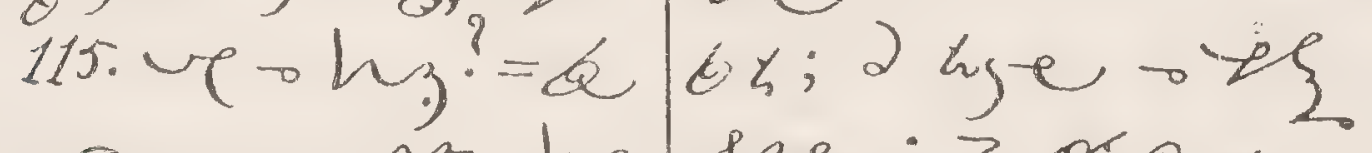

aren $\rightarrow r^{2}$ ohy lse: 6 or ex

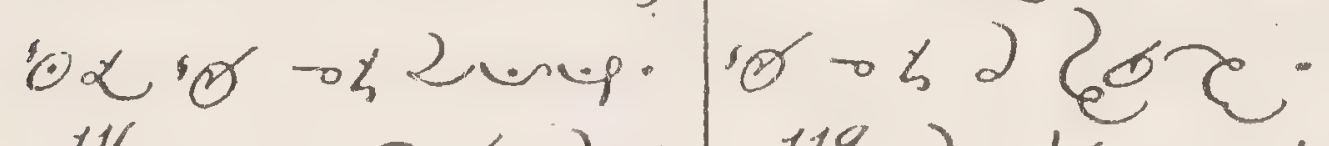

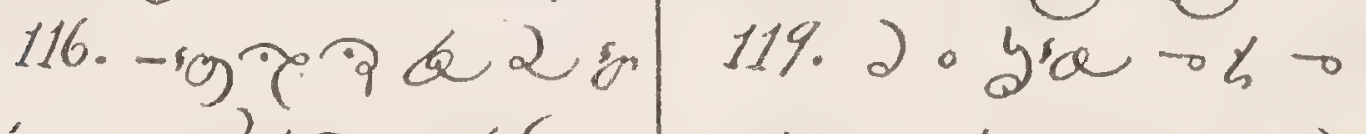

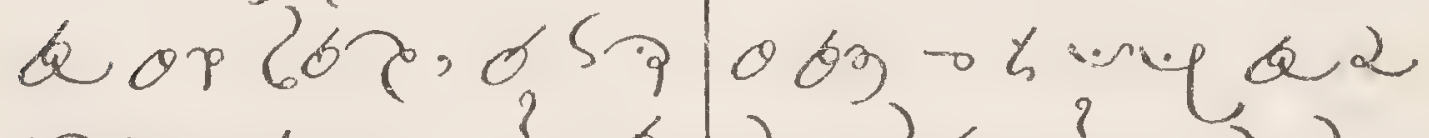

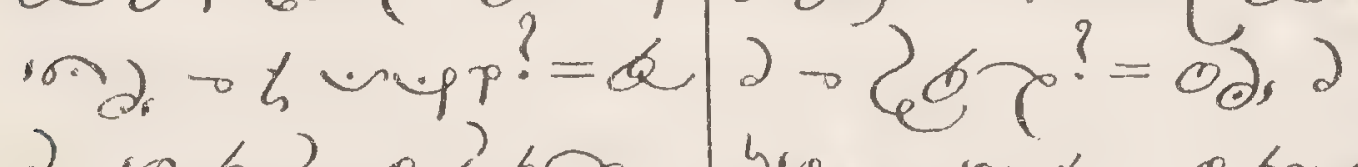

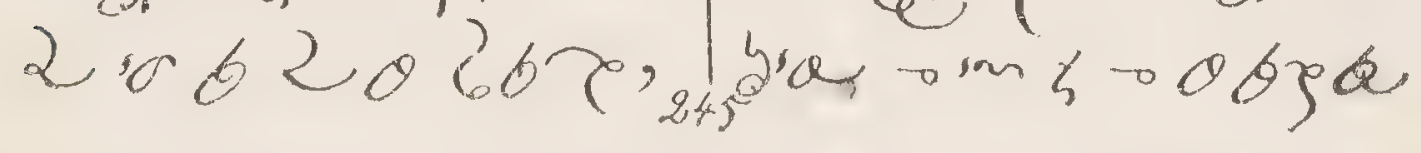




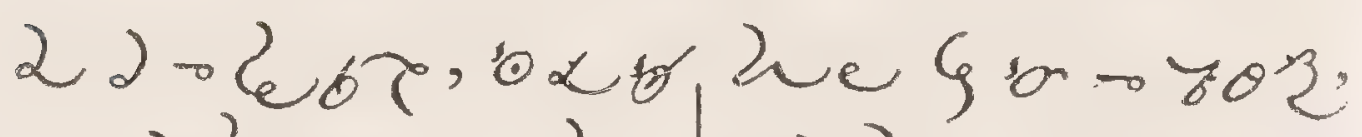

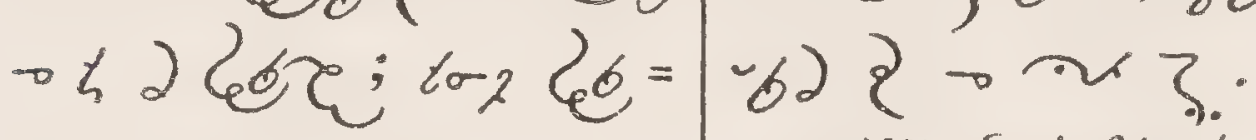
$\varepsilon \circ y+2$ bae. $120^{\circ} 280$ n -2 S $\partial \cdot a-\sim a\}=$

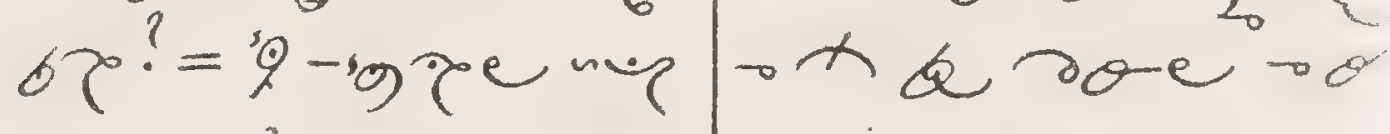

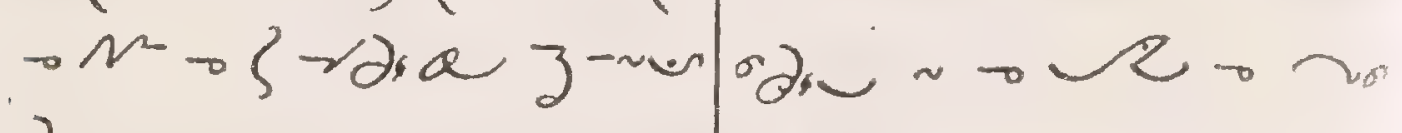
66 rie $\rightarrow$ x 62 or or. G 20 oge 0 r. $121.93+N \theta \theta$

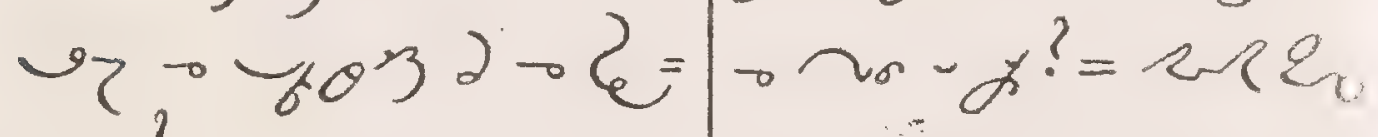
$b \gamma^{?}=\mu$ ar $-y^{\prime}=$ 疒小 or $-108+2 \div \%$ o ysan: $e$ ¿4890 6 - $40=$

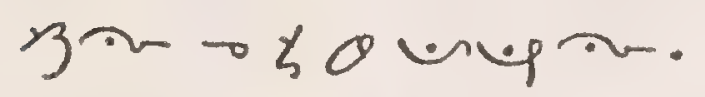
122. 2 क्ष $32-62$

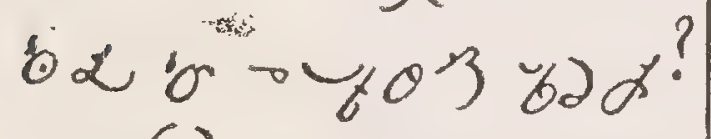
$=-62$ b2 s- $-6=$ oर w द 602.

123. (97:02 062

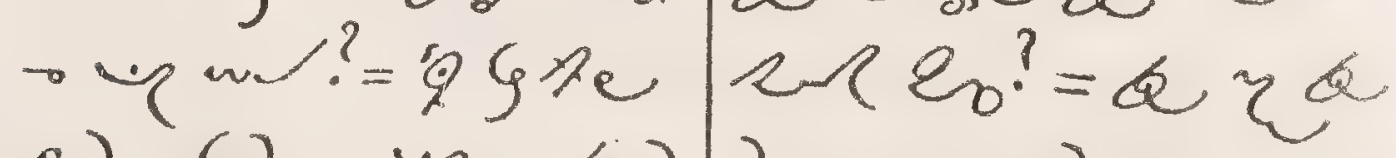

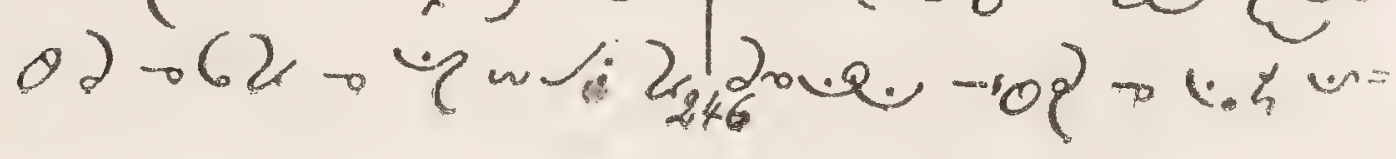

128. $2 \mathrm{R} 2,-\mathrm{s}_{0} \mathrm{~T}$ 6 are oral ro oraju $\rightarrow \mathrm{k}$ orezure; $\theta$ d $\zeta \rightarrow$ orar 0 of 0 र. 12\%. $2 \mathrm{c} \varepsilon_{0},-9 \%$ Qroe $\sim 2$. $\sim_{0}-\not{ }^{\prime}=\operatorname{se} \varepsilon_{0}$ is $-2 \div 20 y$ द $26 \cdot 2$ \&a:

130.-30? ग0. \& uas a be XIV. Ext. Unction. 12\%. $-2 \mathrm{c} \varepsilon_{0}$ ?

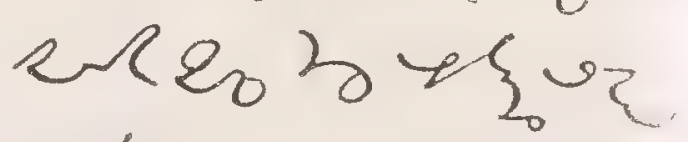


प, devar bory - ra a d o dr. 131. $-9 x-2 a$ $u_{2}-2 \varepsilon_{0} ?=n$ d ve $\Rightarrow$ gुger wer, d

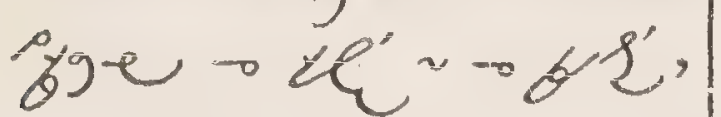
$\sim 6_{z}, i \rightarrow$ anz; $\sim 2, \sim, \sim \cdots$ nong, de unger ert.hula bon rh, a a b - \% a a بad. h, a a $2 r \rightarrow b, a$ a be $\rightarrow$ h, दa $2+4$.

$X V$. Holy orders.

132. ve o o ? =f b es a ter $x$ 2.2 by a w w

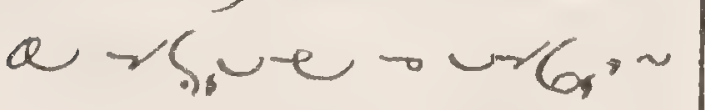

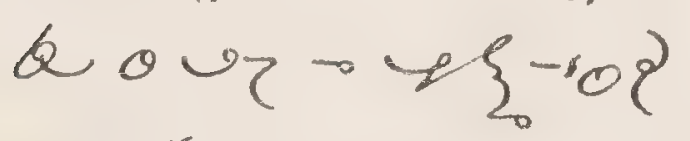
$-165^{\circ}$.

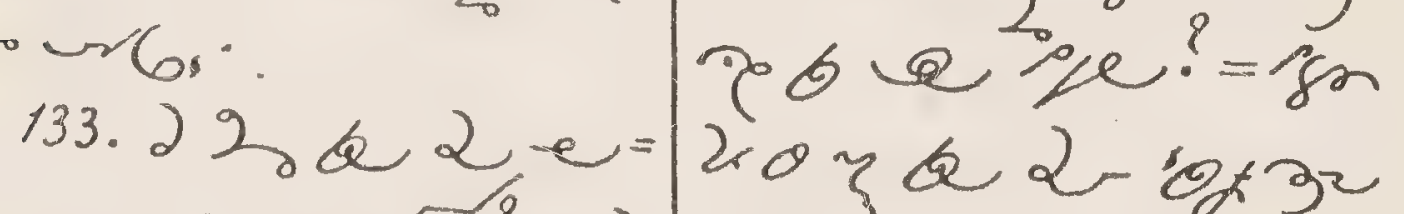

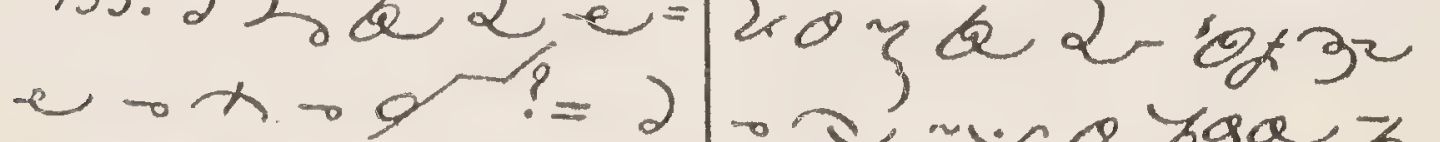

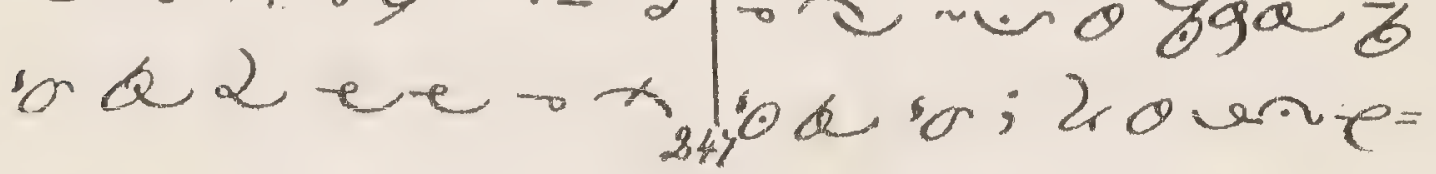

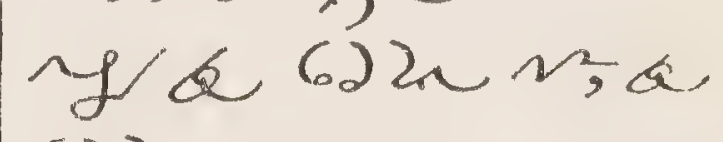
6) $2 x \sim$. 134. Q6 ? 09 ? $=G \cdot 0$ व $\leadsto$ $0 \gamma^{2} \rightarrow 9$ 135: $\alpha_{2}+3 \%$ $\rightarrow \& \varepsilon \sim ?=0$ my 8 \& $2 N$

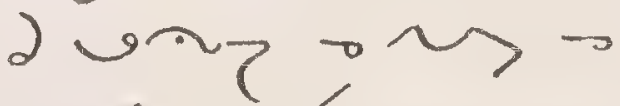
पर stad are o wh, " क a ?

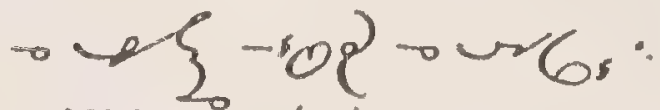
XVI.Matrimony. 136. $\mathrm{C} \cdot \mathrm{rm}$ ? sin to espere $-x 20$ bys 020

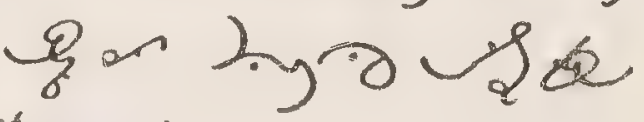
in b tora. 13\%. ef ism, $-19=$ . 


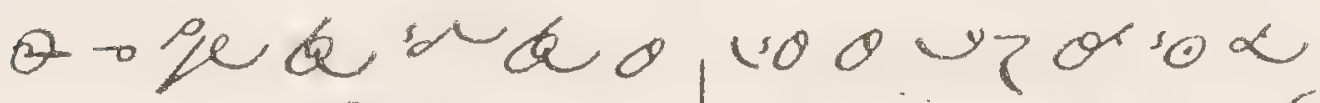

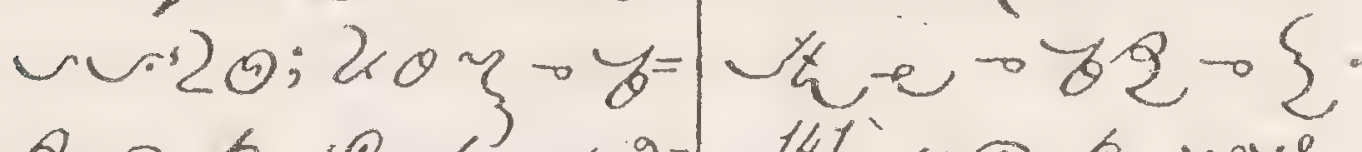

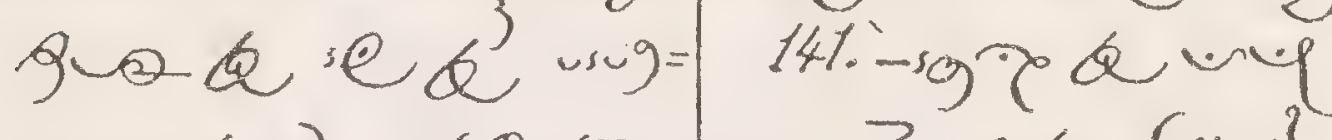

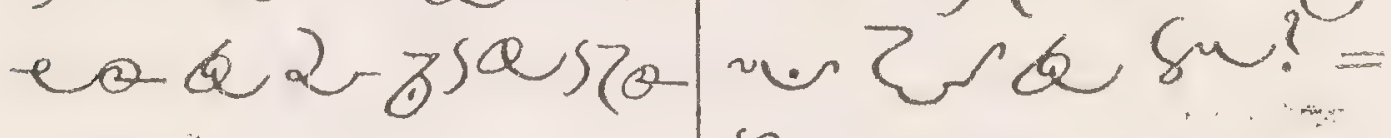
$\rightarrow$ 尺

138. -9 900 दर su? $=-2$ ras $s$ क-sgre riga $\sim 622$. $62 \%$ (Q) $\rightarrow$ r $; 2) \cup$ id 2 $\sim ; 0$ a g ger-so? Q a d r ver $\rightarrow r^{2}$

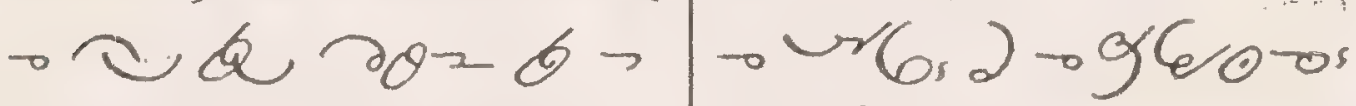

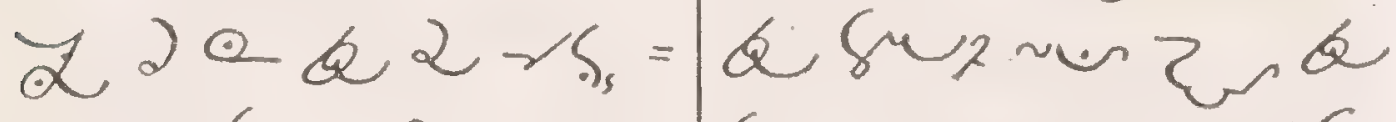
ve $\rightarrow$ L $\sim 2$ Q $\partial \theta=b-\breve{\alpha}$. 139. -s gro vous

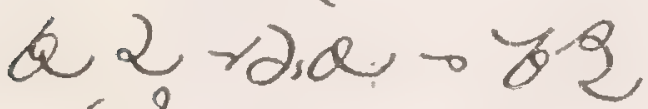
$-\{?=\log$ ad \{ - vasox-sgre $\rightarrow \gamma^{2}, 2$ - $20=$ e $-\{; a$ क 2 $b \cdot \partial x \rightarrow y r-2$.

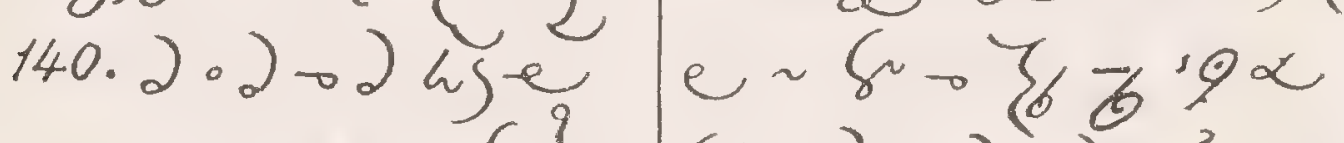

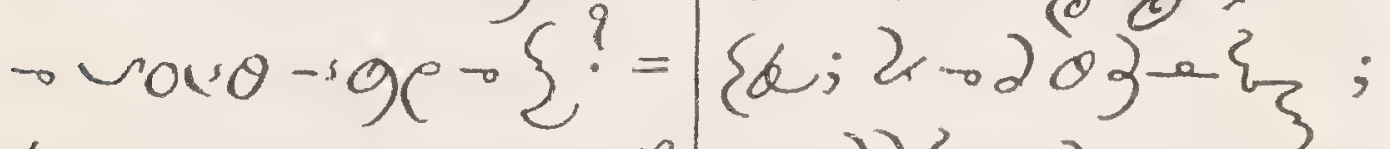

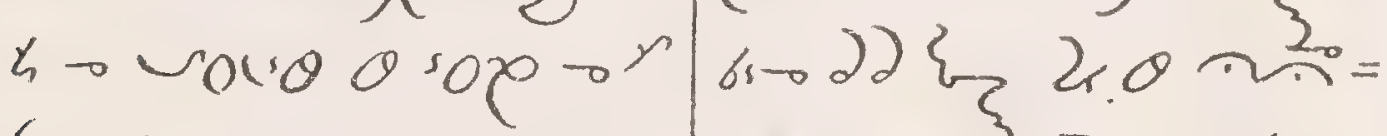

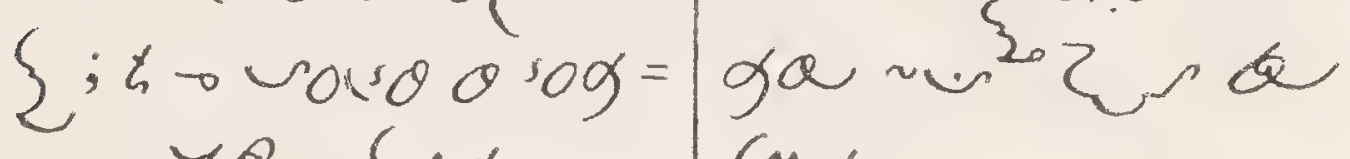

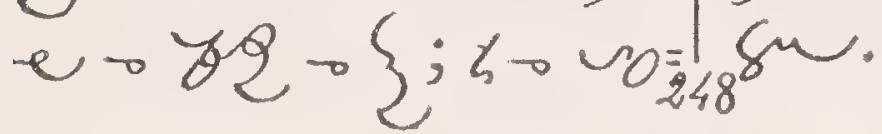


Sheshel

Manual 



\section{Shorilud duanual}

OR

\section{efrapers, fingmus}

\section{and Cattedisin}

In the Serhel deanguage

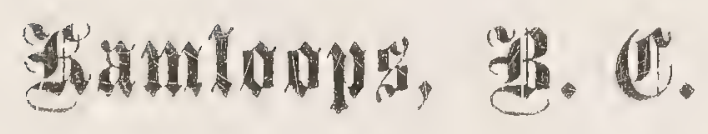

1896 

Shishel 2, 202 b2? Morning Prayers. $\begin{array}{lll}0 & 0 \\ 0 & 0 & 0\end{array}$

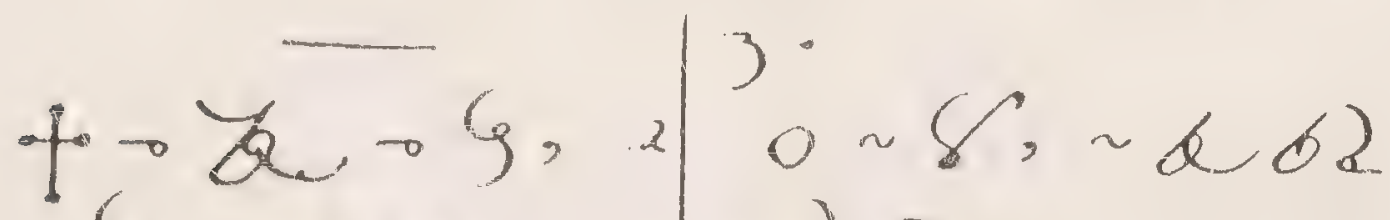

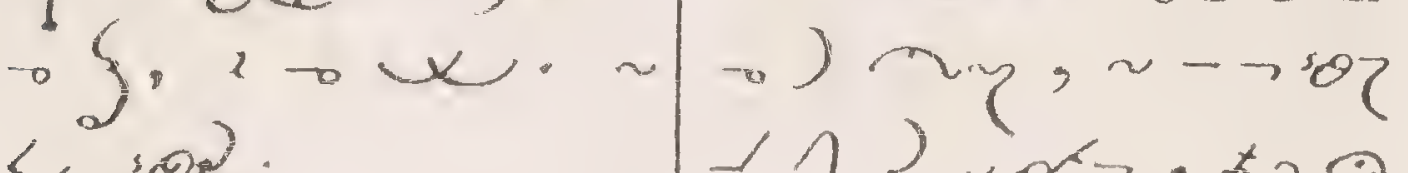
$<$, oृ?

\section{Offering of the day.}

Anury, be

. r.t. $22 y=$ chotho-be Q.

m.

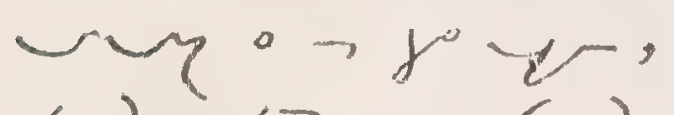
$\left.62 \pi \theta^{\circ}\right\} \div\{2$ rea, wor. bigor $x$.

02 are o 2 wo $\rightarrow$. voran, tol - - ) bis, $20.9,-3 \sim 5$. wars y 20 3629 .

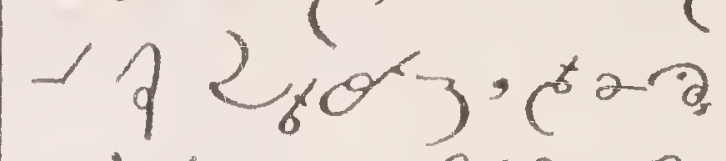
- y 5 , son, $\theta$ d) 2 - ar $a=$ $\because y<2 \times 10^{\circ} 0=$ D. $\rightarrow 5063$.

Pater. Uda 9

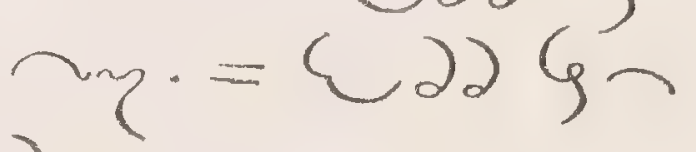

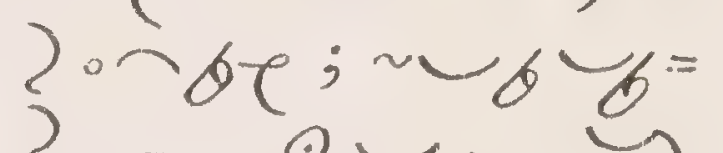
$z=-9 y \rightarrow y$ br $\sim ; \sim)_{6}$

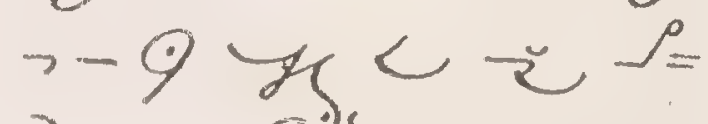

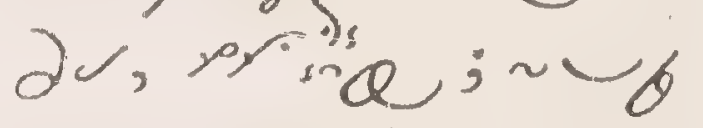
-9 y, ?o $\rightarrow Q_{0}$

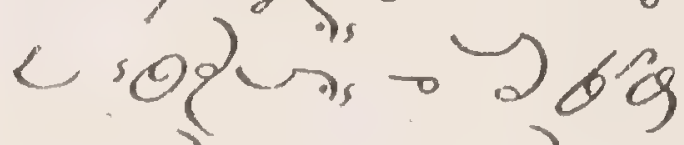
जoशे०- मु? ?

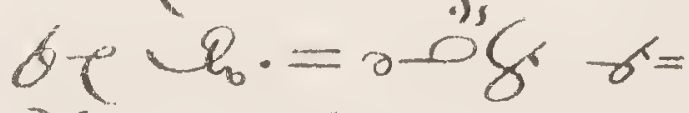

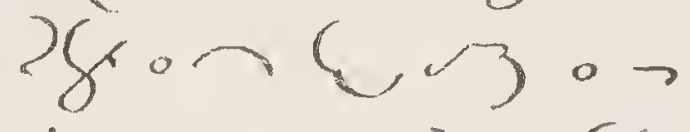

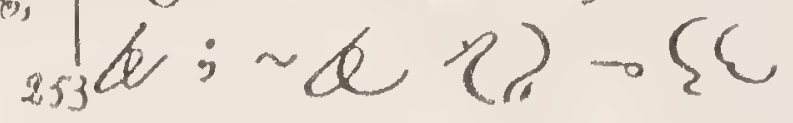


wea, woptoc

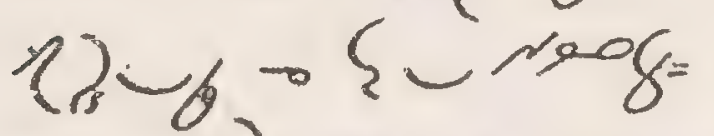
$\sim 02, y ; r^{\circ}$

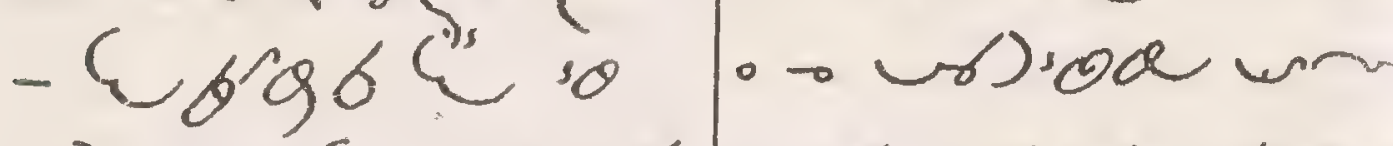

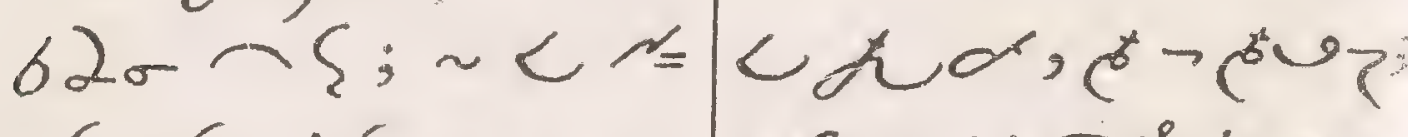
- Los $6 \mathrm{C}: 0$ re $=$ $\sigma \cdot \sim C A B$. Ave. o \&, صr= ro o qe, $\times 2$ $\rho 2 \mu,-\partial \mathcal{\mu} \mu_{\sim} \in$ $\cdot-1, \sim_{\xi}^{\infty}, g_{\alpha} \sim$ - 5$\}$ 2 $=\sim$ $8 \cdot 2-x, 2 y=$

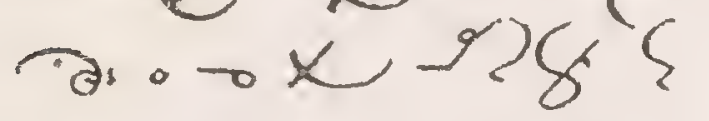

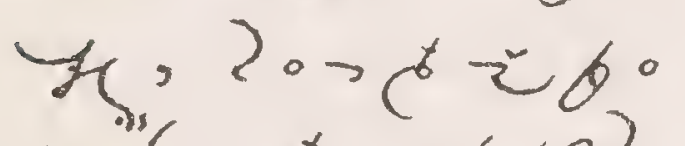
-C力. यL. credo. 000,0 n. $=\sigma 0 \partial \frown \frown x \xi$, rer.-que, $=$ 2 vinor be 2. $\left.\rightarrow Q_{0} ; \sigma 0 \partial \pi\right)$ $x,-\infty 2\}(2)$

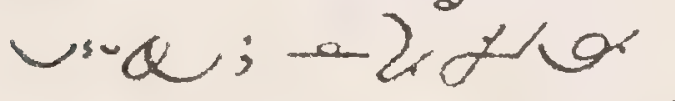
$6 \hat{Q}^{2} \cdot-2-28$,

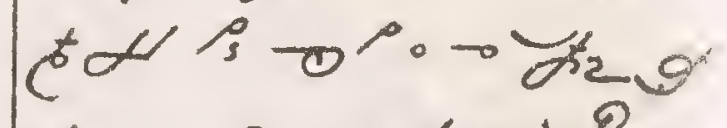
$t \rightarrow \omega_{0} \rightarrow b e^{Q_{0}}$ $\rightarrow ら \cdot \pi \sim d s q$ - $2 \xi \%$ r. - Qu一; 0 क - Sue o on Hs,

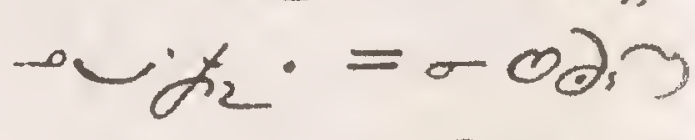
60, $2=80$ $\rightarrow \sim \pi, 3,-5-8$ o o SC rea; sowerse tक्या कर

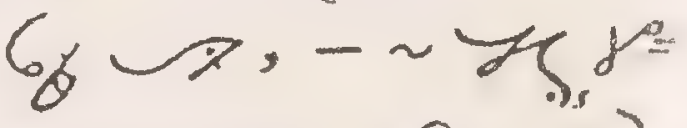

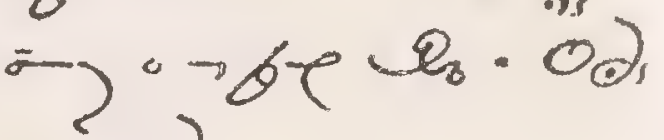
.

Confiteor. 10,0ry br

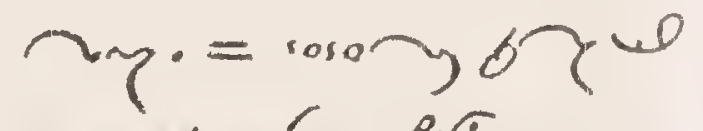
$\rightarrow x G$ Pिक

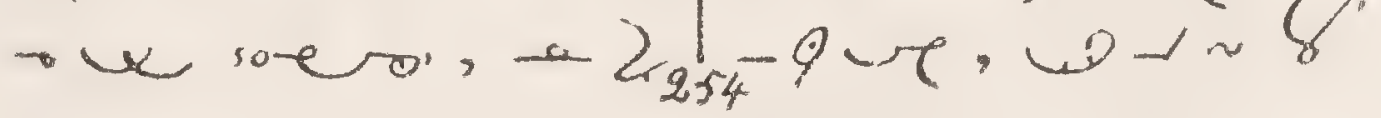


P), Q , GN, *, ery zw $\gamma, \omega-b e$, 1,0 -बर ग द, का न दर He, glokin. $\varepsilon, 8 \forall n$, $\{, \cdots$ un: $\simeq$, öary - vuruerar" o 2D (3) $89,-20$ () $8,9,-22-1$

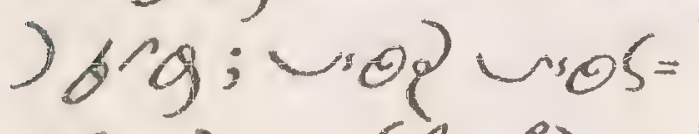

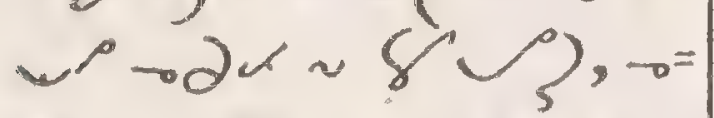

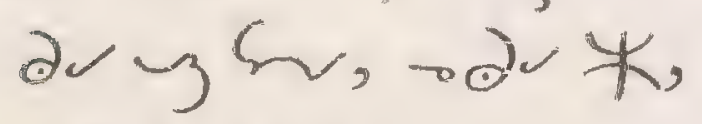
करो $y / 3 w / z r$, rat का मा: ) $9, x_{x \rightarrow x}$ Soro.

Misereatur. of vio= Los, x ryo $\rightarrow-$ que, $\tau_{1} \rightarrow\{(N=$

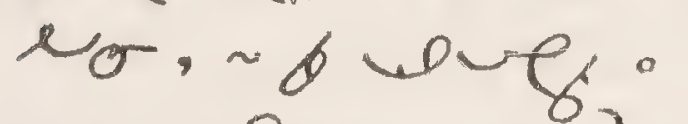
Dbe $\mathscr{T}_{0}=\sim<S O Q$ indulgentiam. vis: $\cos x, 94 \sim, 9 \alpha$

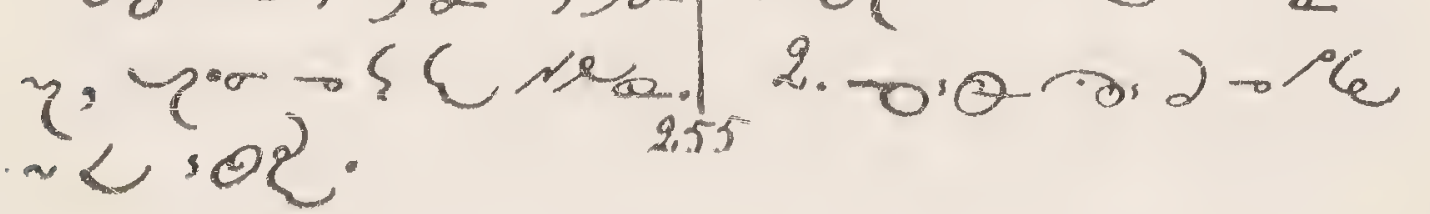

The Commandments.

$$
\begin{aligned}
& g-x<-x . \\
& 1 . \sim<-a-x .
\end{aligned}
$$

2. 00 . 590:

支, ‥

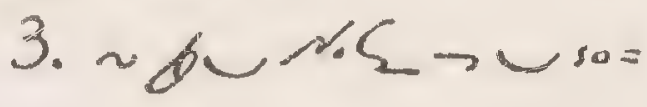
52.

$4 \cdot \sim b 62-92$ $s \rightarrow$

2.sedsor $\theta$.

6. sorrs 60-60.

\%.orar ver.

8.00 , tr. ofos.

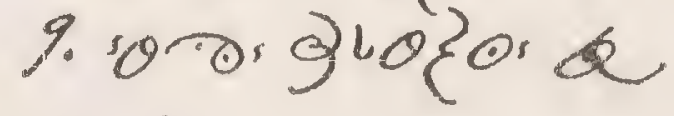
606 .

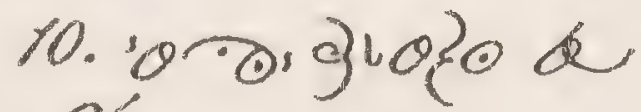
29?

Precepts of the Church.

$\rightarrow C \rightarrow$ fu $\rightarrow$ r.

0 - 1.

1. $\sim$ d $\cdot 5 \rightarrow \sim V$
Sosontods. तsop. - usosed. 
rat

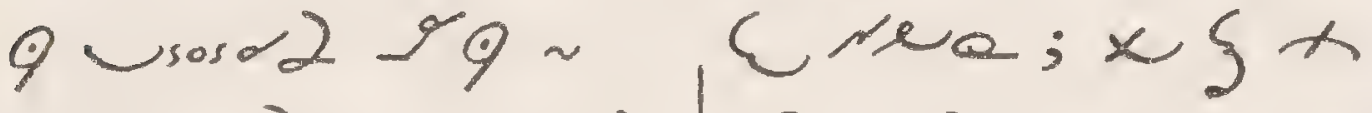

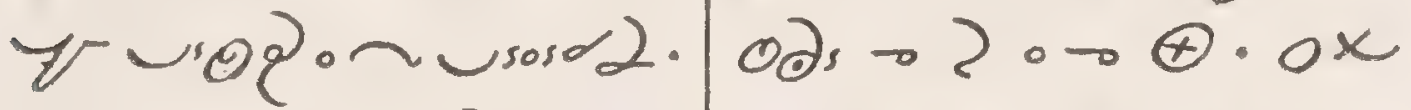

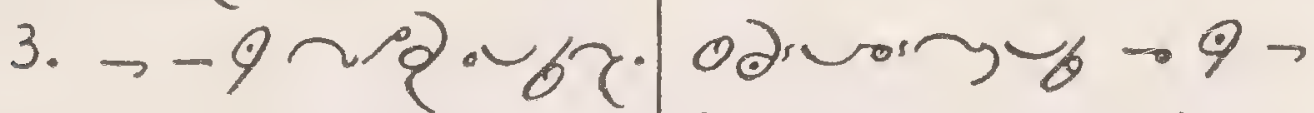

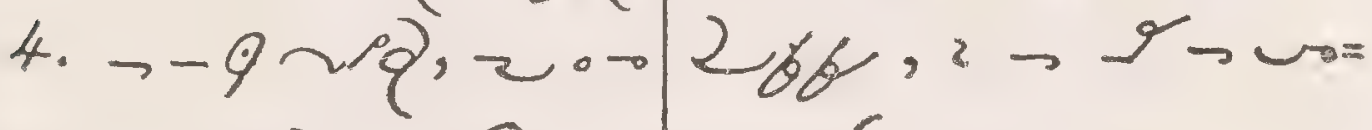

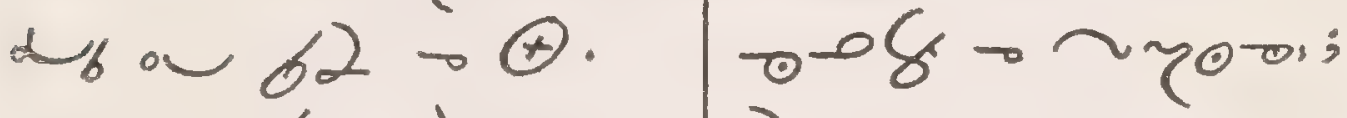

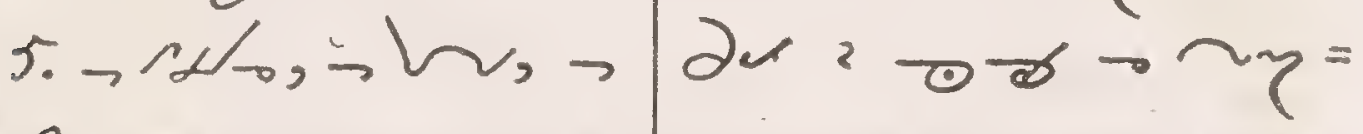

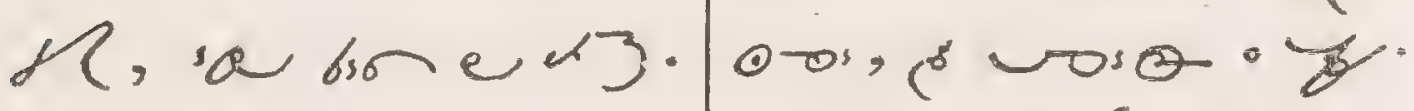

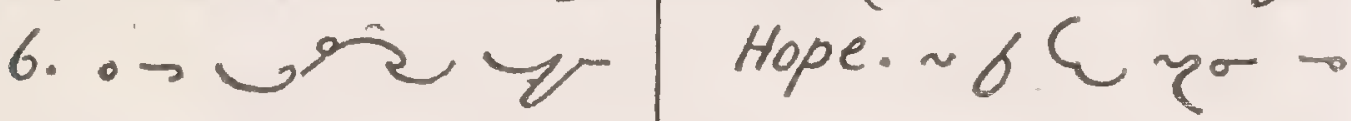

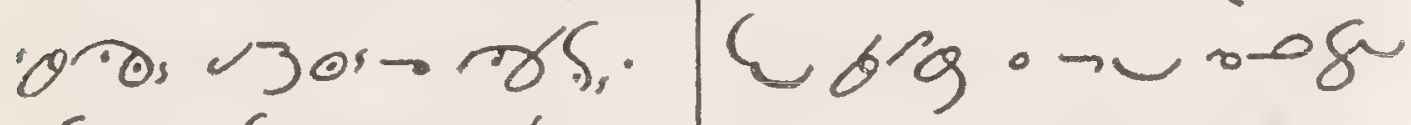
Seven Sacraments. tox $_{0}=0 x, 9 \alpha$ x< wurgo $\rightarrow$ sol d usos $\rightarrow$ ys;

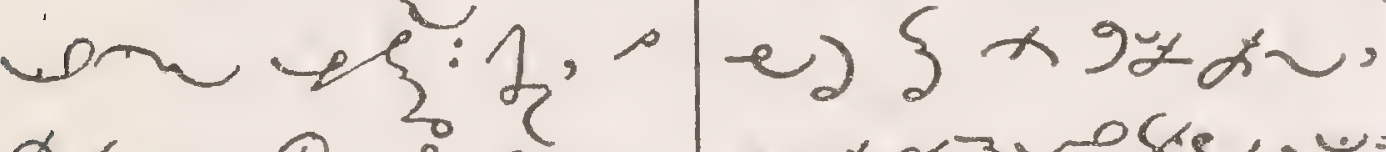

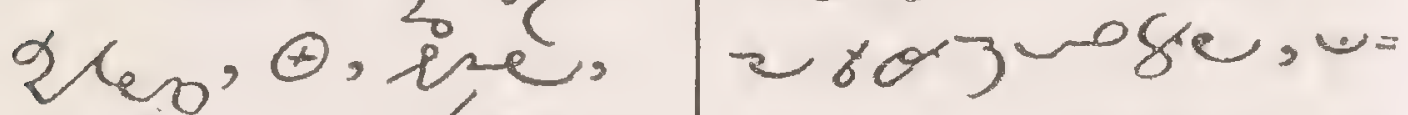
ak $\varepsilon_{0} \cdot 9$, Act of Faith. $~ 6 C$

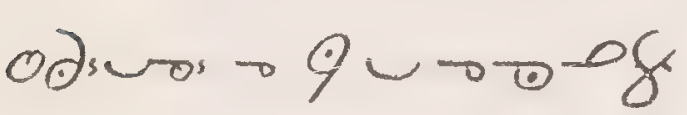
$\rightarrow \sim^{\circ} \sigma \cdot=0 \partial+$ ? - x, 2 a r o 0 br $e_{0} \rightarrow \pi, 2,3$ weror $\Lambda$ n? $\{y ; x G, x\{, x$

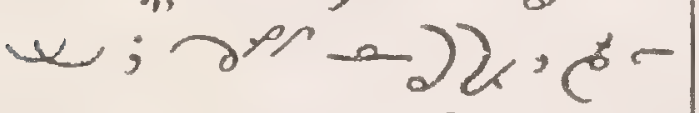

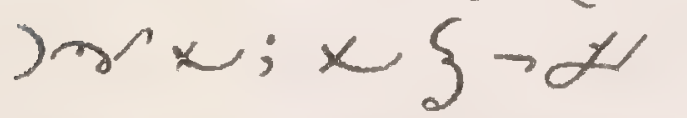

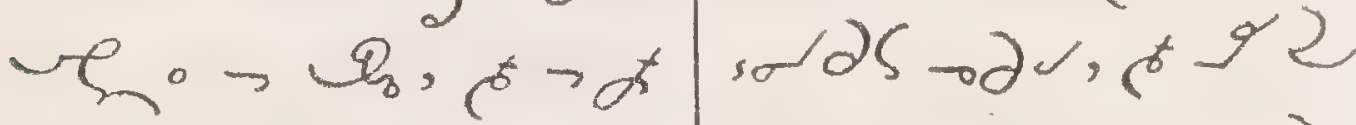

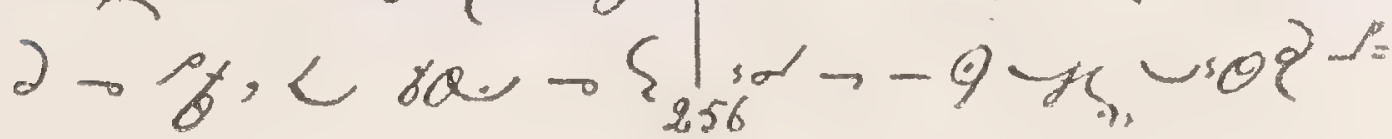




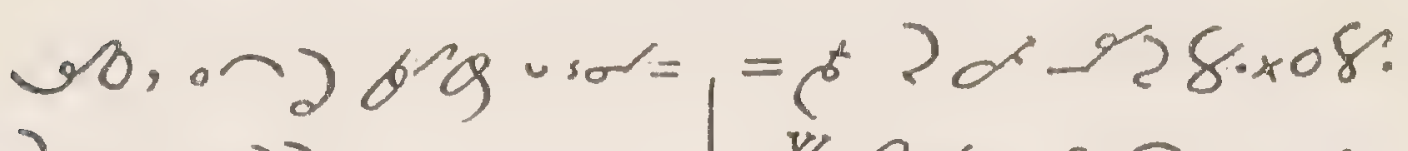
2.9 no- 2).

Contrition. wa N. $0 \times 2$ ? 2 so $98 \cdot=0$ oregl $\mathrm{rn}=0 \times$ war $\sim, c \delta 2 \sim y \rightarrow$ o- og $\rho x$. - $\{2$ rea: 20

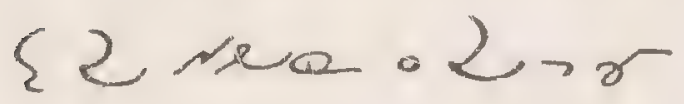
- be $D_{0} ; 2 \rightarrow\{2$ Nea went nry. $\cos$ wa $62 \rightarrow$ sorento

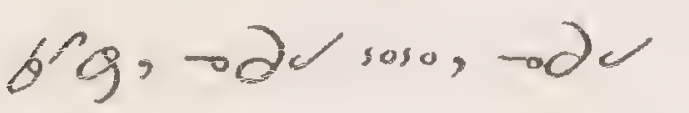
rive. song 62 - S, vis ) b 96 $25 \% 62 \frown 5 . \theta$

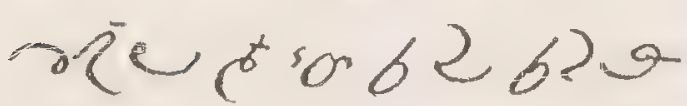
< s Unigne - 5 . $\sim$ b $6 q_{\text {w }}$ ow 0 . The Angrelus. Nay. 1. भिx मून क $\therefore r=r 2 x$ wal ig, ru whesog.0\%. 2. $=\sim 462 \operatorname{sog} 0-7$. 3. $-x \xi \rightarrow-0,0$ की

oremus. ox. fon -ej ye $\alpha 0$ रे beg; rond -0: $\delta=$ ard $\rightarrow$. $62 \wedge \rightarrow 2$ soy $1 x$ $\sim \rightarrow 2 \alpha \alpha^{2} d \rightarrow 0$.

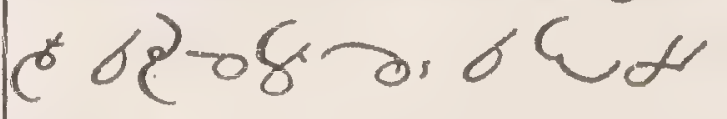
"t wn.obe $\hat{e}_{0}=\sim<\mathrm{s}^{\circ}$. Sub tuum. O 8 . O 6 , r a - Ju, $2 \alpha+\cdots x, .92$ rea $\alpha \mu \sim \alpha$ $\because \operatorname{cog} 6 \mathcal{C} \theta \alpha$ ze, bneras, of 98. 
Night Prayers.

Adoration. $O x, x=$

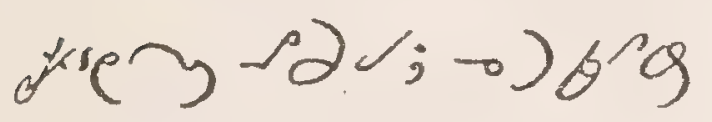
b2u $6-200 ; 1=$ vosing oad a su

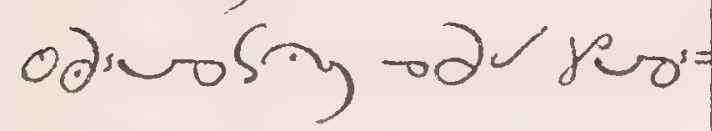

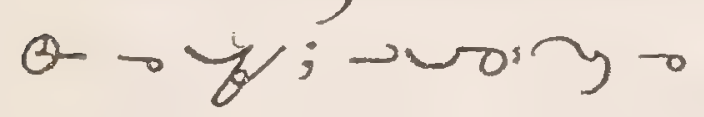
Joch $-\partial \alpha, 0-z$ eveor, os n a ris; solds刀y todo - 9) Jíg ponio o. - 62 人 。-e son;

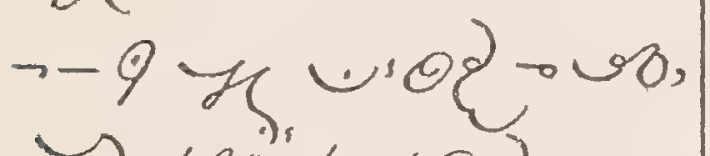

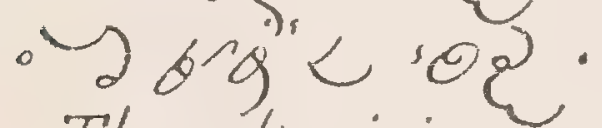

Thanksgiving. 0

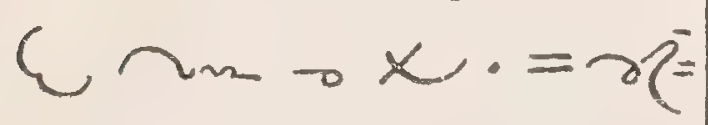
$y$ mmen $-\partial \alpha, x$, - 9 aर́un? ifd 9 6ol sord, है

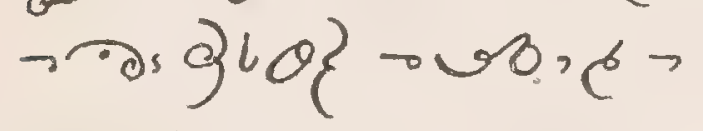
arder - tri wa or $\xi \alpha-\theta \partial \cdot 9 y c$

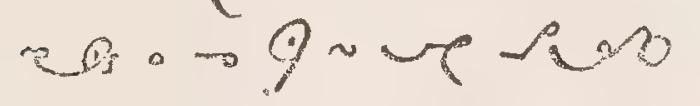
s. $-\partial \alpha \cdot \sim-6 \partial j 200$ be Yh, yen avo be mis ox. Prayer For Light. $6 c_{f}: 0 x<6=$ $=8-628 \cdot=c \cdot c$ opar elar, de ba $\rightarrow-f 3$ b' wing ore 2* S, o re $2 \% \omega^{\circ}=$ $6 \mathrm{sg}+52 \mathrm{Lu} ; \sim$ 6 aves 62 brom

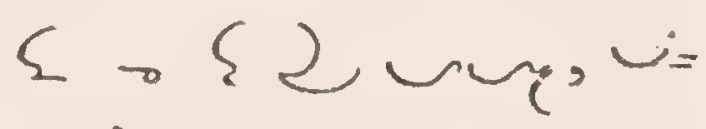

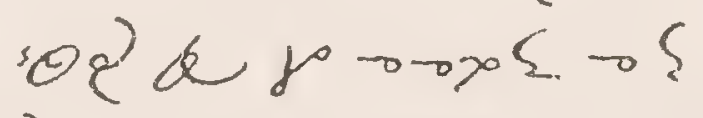
2un, < s $x=$ ex 9 ing ta 62

$\sim \sigma \ll \sigma \sim \varsigma C$ $\sim 20 \rightarrow \gamma y$.

Examination of Conscience. Contrition. 2a-62

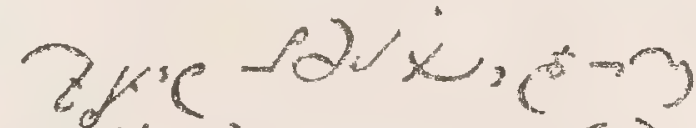$$
\text { 19. }
$$

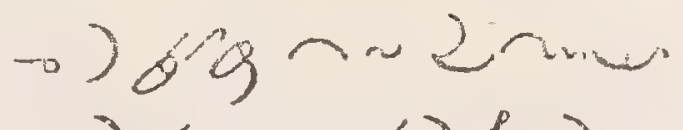
$\frown \xi$. 


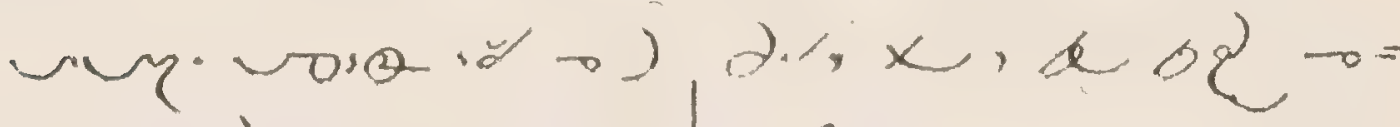

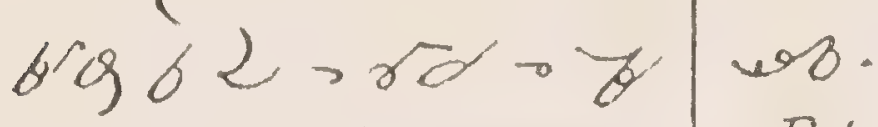

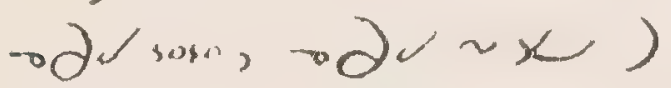

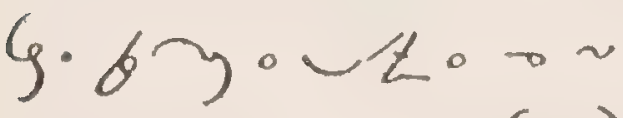

$e x, \infty \rightarrow\{2$

2)Diwion

sd, wok sion, e. $\rightarrow x<2 k$ $\eta \rightarrow b 9<\gamma 680=$ 3 .

Firm Purpose. $\sim \delta$ $\tau^{\circ} \rightarrow \operatorname{cog} \sigma 4$ $\theta+62-\xi=0$

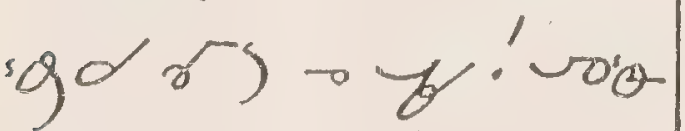
$\because$ - $) 6962-4$

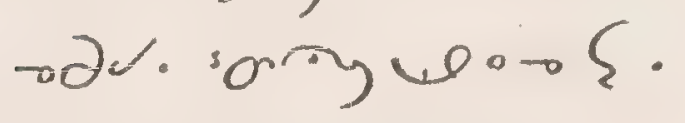

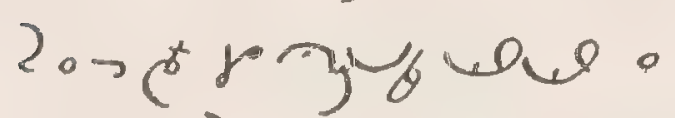

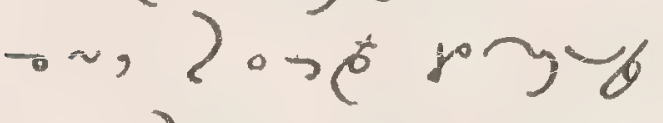
uson, e vun =

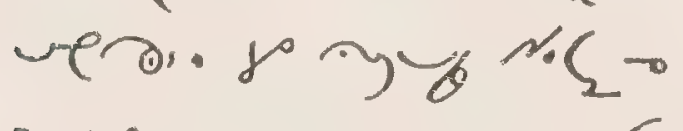
3 big c are as,

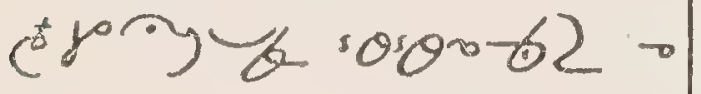
s; joy mas

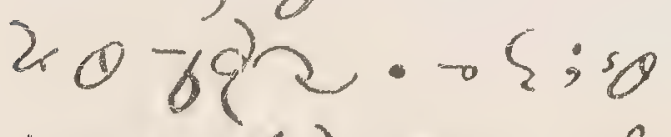
Pater (j) 9 -y. Ave. o $8 \mathrm{Cr}$. credo. rod. $>$ r. Confiterr. sosatyor... Night offering. $O x$ ek - as oर ca $62 d n d$ ae b2 solus $\rightarrow b \cdot 0 \sim$ $8(2 \rightarrow \partial \alpha)$ 几 ,

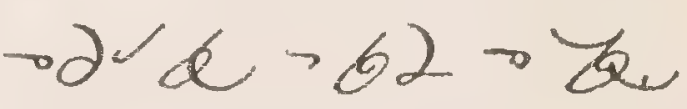
fo $\rightarrow x$ S som

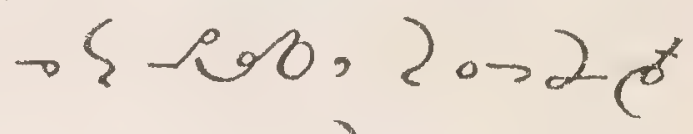
$26 \cdot 22 x \cdot \sim<$ sog.

Angelus. von.

\section{Preparation} for confession. x. $\rightarrow$ Q $N_{0} \rightarrow \sim_{\eta}=$ orbag $0 \rightarrow c^{6}=$

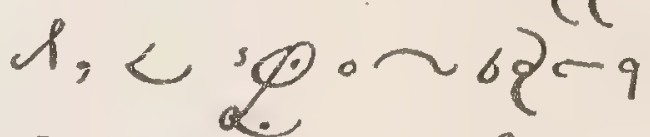
rbo, de wra 0 Hore ot any sount, de $w=$ 
68

SHESHEI

G.1.- Sun $=2 x 6 \operatorname{sic}_{6} 2 \alpha$

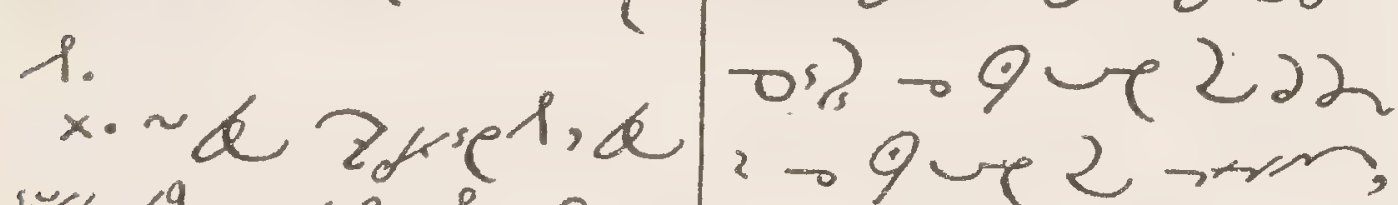

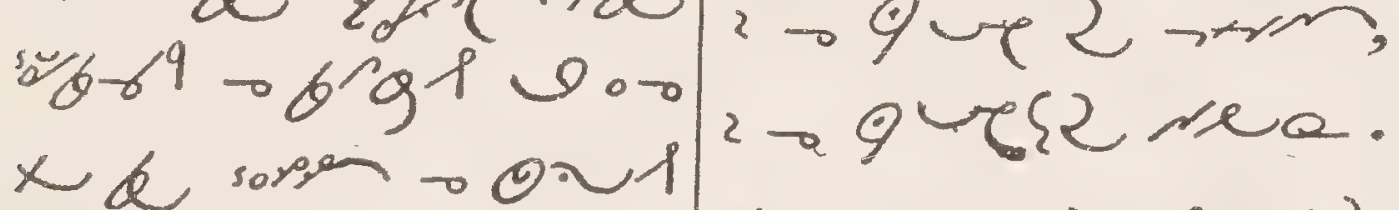

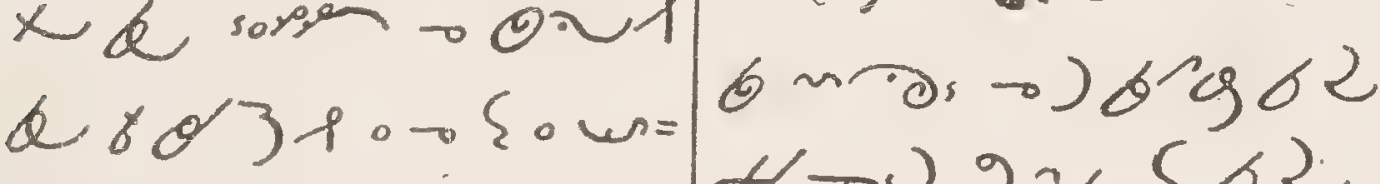
urt.

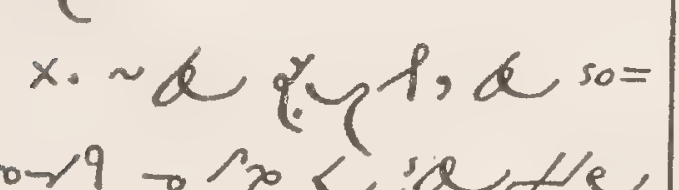
ard -rectide 12ptoruyt. 世. $<\sigma_{n=} \rightarrow f^{\prime} \cdot \sigma^{2} g 1$

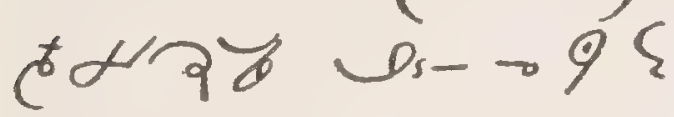
- vut. $=0 x ! \cos 6$ 2 os? b rod -

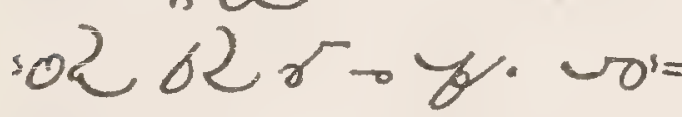
$0-62$ 0\% 0 s00, $y=$ seas or) n, $c^{+} \theta$

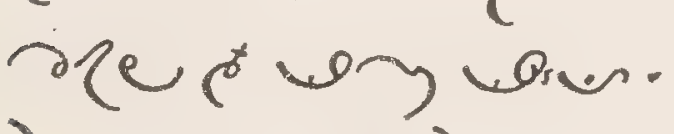

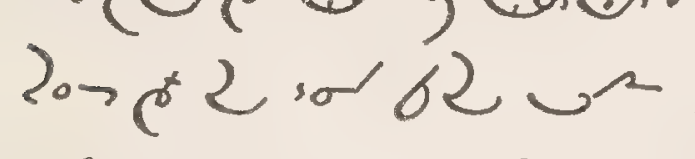
) n, 02 102 6 $2 \%$. 20न and a es qu,
माश 20 कर

res $\rightarrow$ y $y$, so $=$

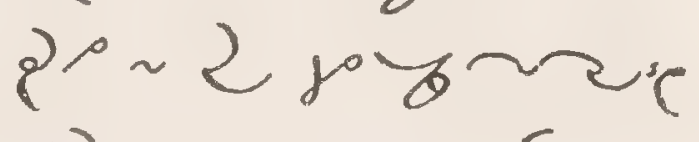

бट un $\frown 5$.

x. 2 6r bra od og 2 sosd 21 . x. d bracuec $\rightarrow$ רas 90 . m, o ve o loro, 0 ve o hy.

$x \cdot \sim$ b bra $78=$ 3 wersor. o a usoplusar. x. ㅏ bra o $417=$

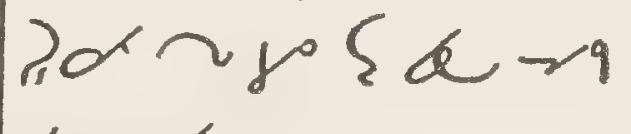
ord. x. $\sim \alpha \sim r a \rightarrow 5$ - vuyitoras. $260^{\circ}$ q novertar 


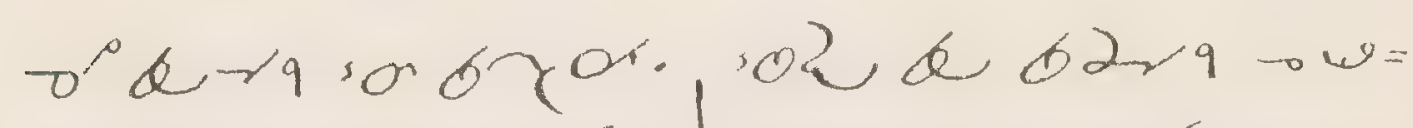

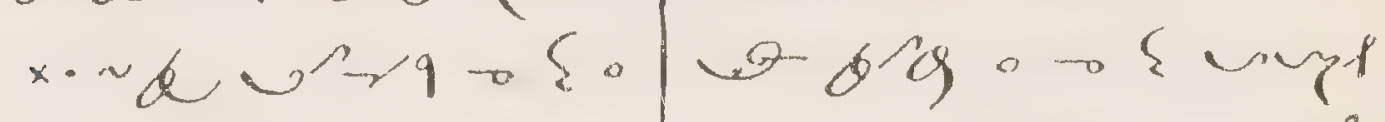

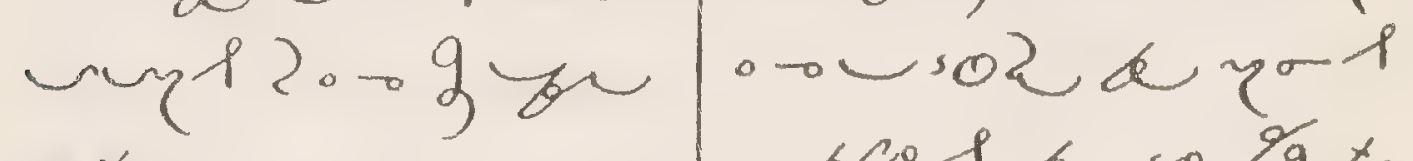
ox. - bigte so- $996=$

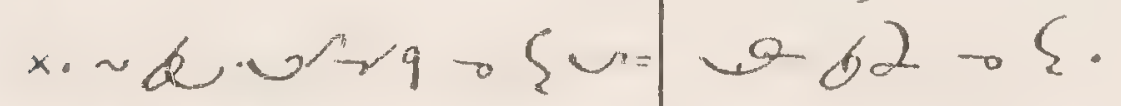
ret 200 - ose

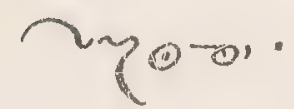
$x \cdot \sim d \sim y$, Lertonus= $x \cdot \sim d \sim r q \rightarrow\{\backsim=$ gra, of nuyt, nd 2 oren

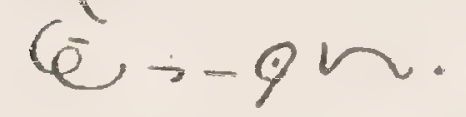

=begs, gris,

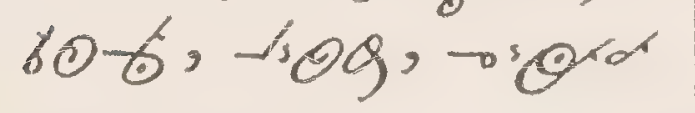
op, $e_{e}$.

$x \cdot \sim 6 \cup r 904$ woglas - unzuo = se $\rightarrow x$. 口f U:O ?R -

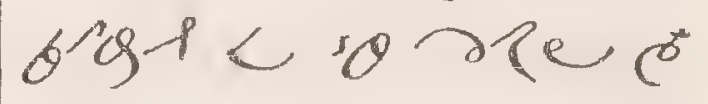
9 क $602 \rightarrow 5$.

$=0 x \sim d \alpha r=$ wo (2sogur o ) big o 52 rea; ox, bolnos - w supen o) b19

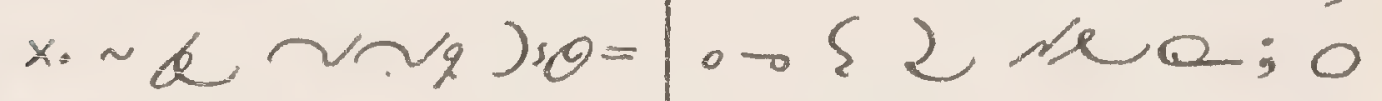
arg $\rightarrow$ surto $x, \sim$ d woubs.

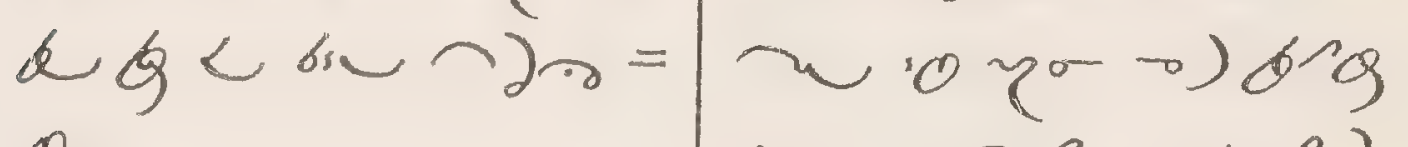
Q.

$x \cdot \sim 621>\gamma \rightarrow$ se, vergor chy bewarg gui $\cdots \frown-\{\rightarrow \phi, \theta q$.

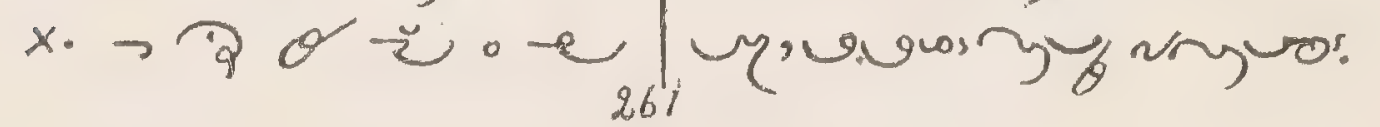


$0 \times \sim \alpha \cdots a_{0} \sim=, b+Q_{0} 0 \sim 9 \cdot c \partial=$

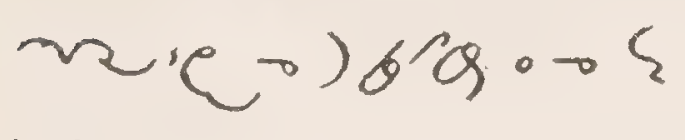
rus.

$0 x, \sim \frac{d}{a}$ os $\gamma^{\circ}$ रा 2 song, करs जy 0 - $5 \cdot 2006$ $\operatorname{sog}-1 \operatorname{sig}<\operatorname{se}$ $\operatorname{sos} 0 \mathrm{~d} 2 \rightarrow \xi$.

$0 x, \sim \alpha$ as $\theta$ $0-2_{6} \theta_{s} \rightarrow\{, \omega=$ wors $\mathrm{SC}^{2}, 0 \mathrm{~d} d$, $62 \sim y-\rho)$

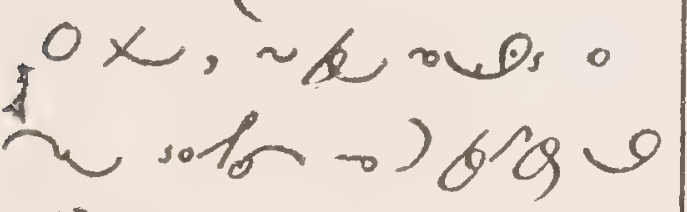
$\rho \partial, \sigma_{0 \rightarrow}-9 y_{\text {is }}$ First Consideration."

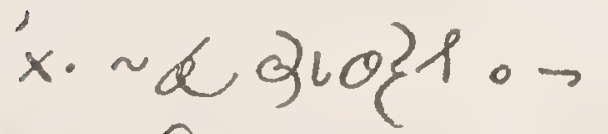
be $\varphi_{0}<2 \pi \sim \alpha$ $\partial \operatorname{sen} \rightarrow \operatorname{sig} 1$.

$=0 \partial \mathrm{d} \alpha 2 \approx \sim$ $\rightarrow \sigma r q_{0}: 0 \sim y_{s}$ $2 \circ \rightarrow Q_{0}, c^{\circ} \operatorname{arct}$ oor be h; - 2 U, - $\rightarrow$, Usops

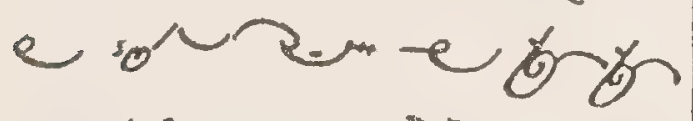
- brg - adr. a $2 \pi ; 0-8,20-2 \%=$

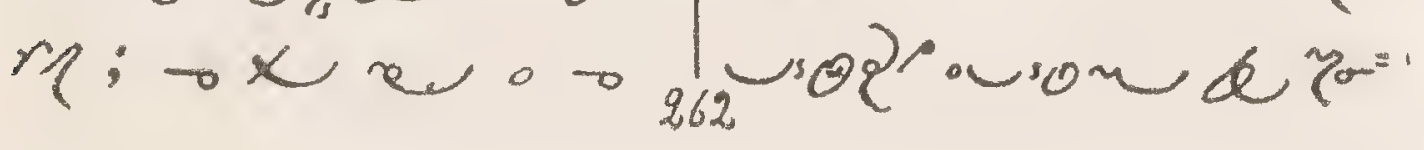
ree en as - Jvor?otoc, csa dectry Qs - ox) G.

$0 \partial$. - . 0 soug= ᄀ, u $\sim 25 \div$ व 0 火. 40$)$ b9 $\rightarrow$ भer ed $y,-\partial$ यु, $\partial x \cdot 20$ b2 $5-1),-\infty$ No o o g w 0 d. - or - 2 < sol. sn

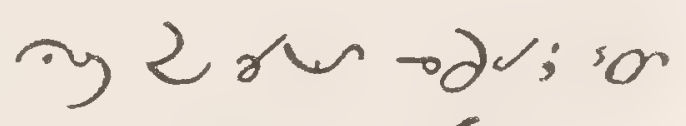
कy un $\frown$.

$x \cdot \rightarrow$ a wabt $a_{0}<2 \alpha \sim 2-5$ - vurargr. voge uso x de wat o sund 
MANUAL

71

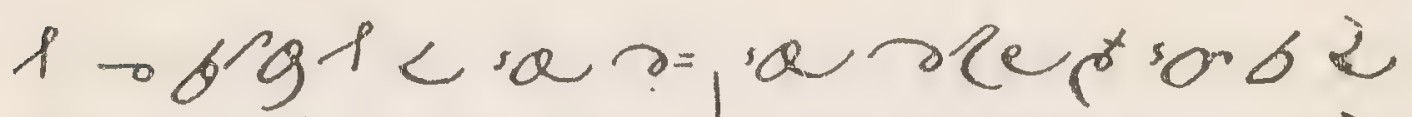

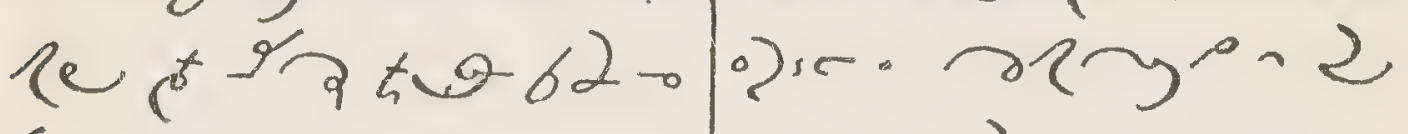
々.

$\operatorname{soc} 9 \cdot 2 \rightarrow \pi \rightarrow$ ze

x. 00, sका $62 \rightarrow-x \cdot 2 \rightarrow \leqslant 20$

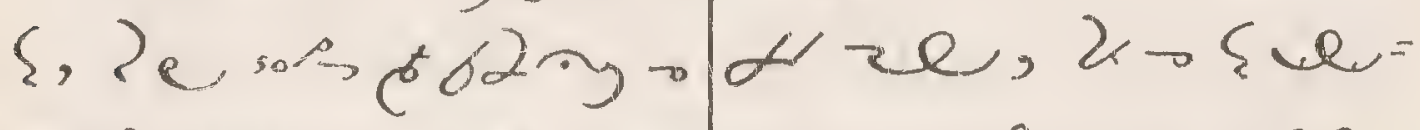

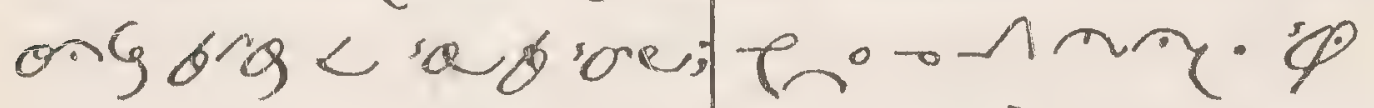

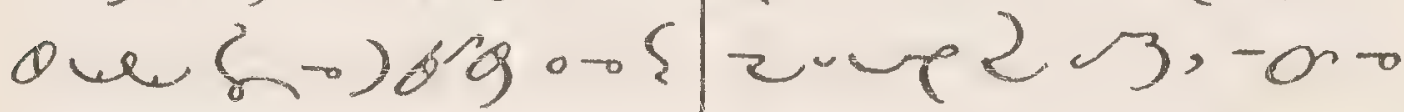

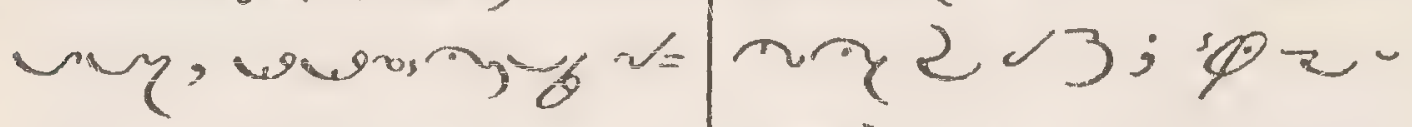

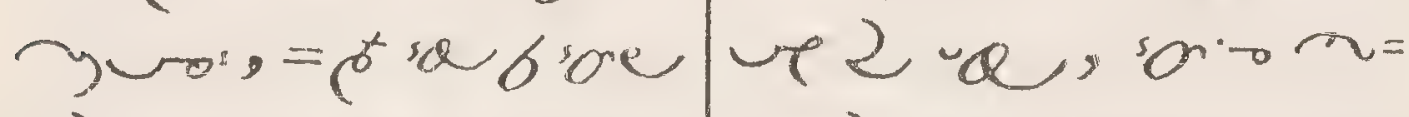

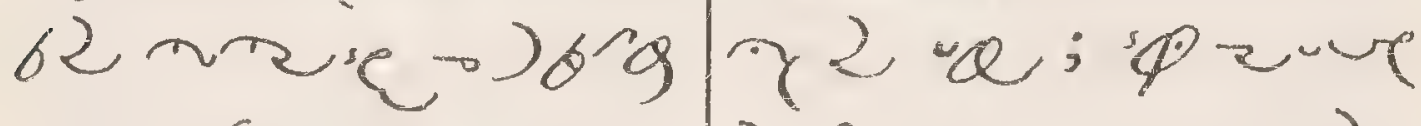

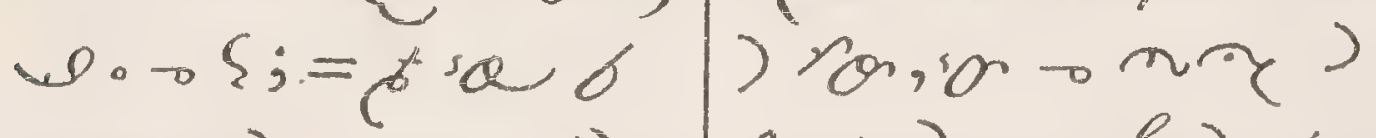

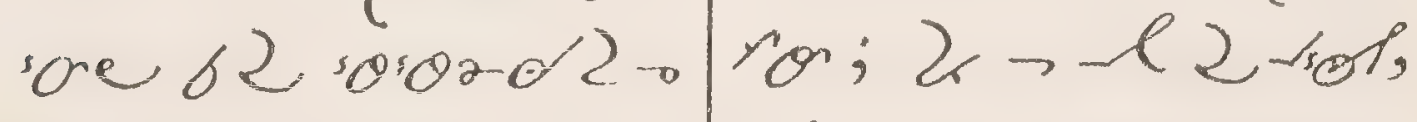

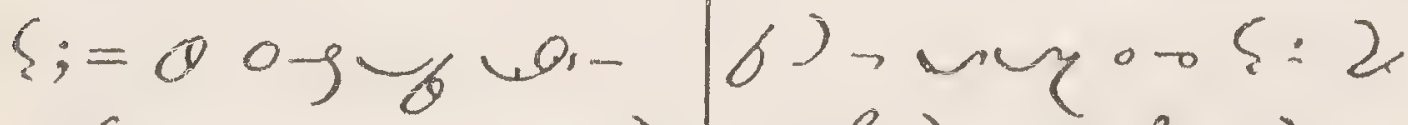

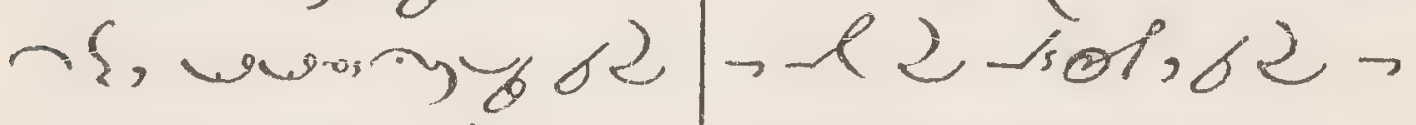

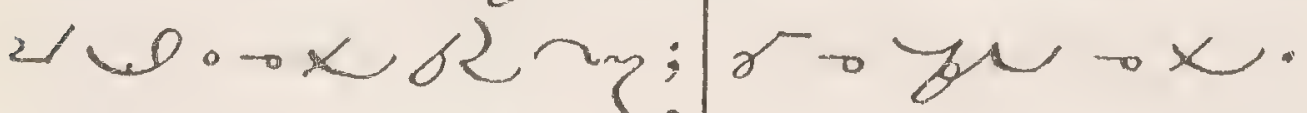

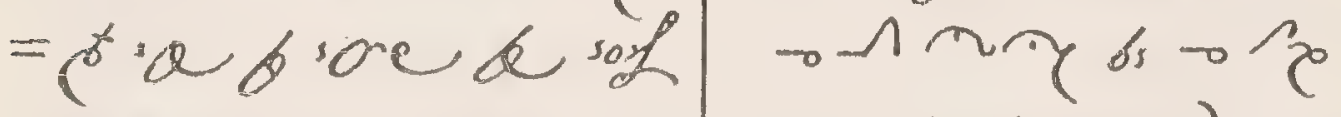

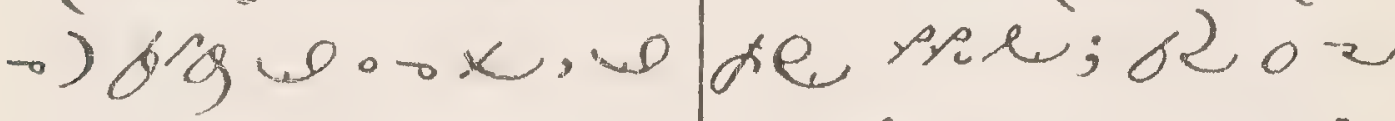

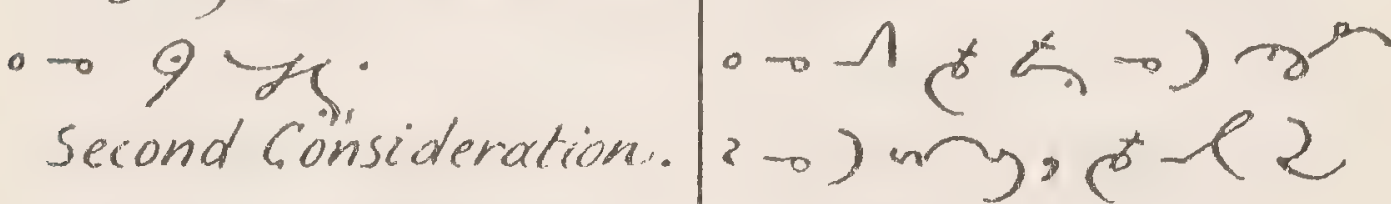

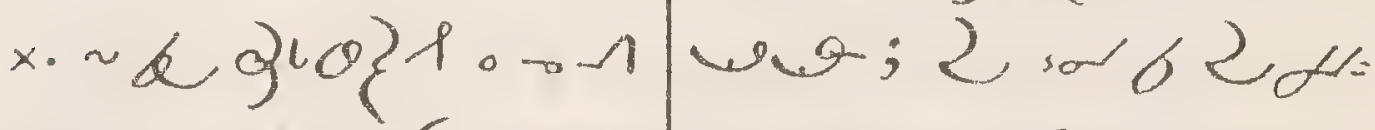

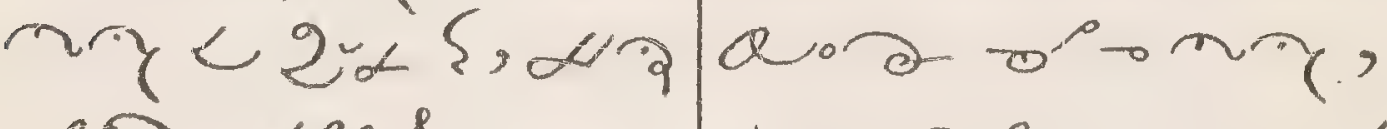

soph-bigt. हैa de - ox w

$=0 \partial \alpha<2, \alpha$ s. yuen.

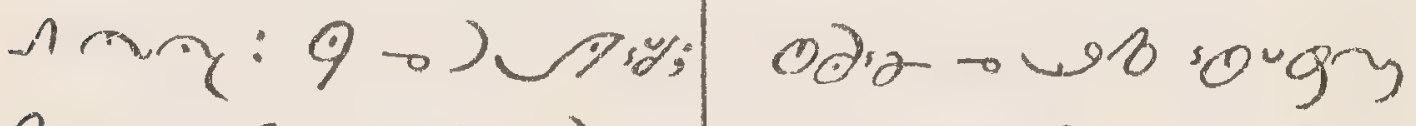

9 y, 9 y w w 2 ; 
3 de.0.0. 02 win $\circ \varepsilon \cdot 200$ हso $\theta>$ 69 62 - करen

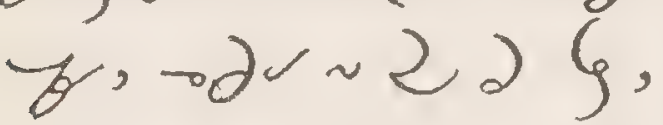
- $\partial x \cdot 20062$ ro) $n$; $\rightarrow$ gra - ) g9u- $2 \Omega \cdot 0$ $202 \cos +n$, r $\rightarrow-9 n \cdot \rightarrow 32$ $\rightarrow \partial+0$ b $9 ; 0-\infty$

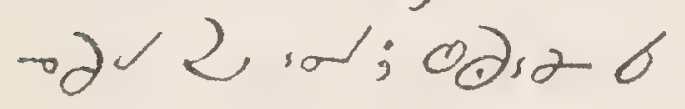
टाम

$$
x>9 \text {. } 0 \text { - } \rightarrow 1
$$

$n \rightarrow 2 \% 5 ; 2-5$ - unyt, era dsot, - Sungt, woge vionde ror -

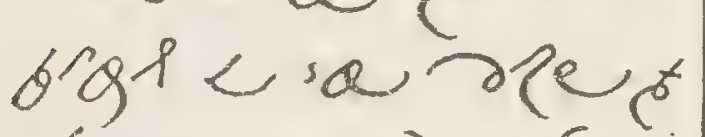
Iक और 62 - 5 . $x \cdot 0 \partial \mathrm{s}$. on 62 .

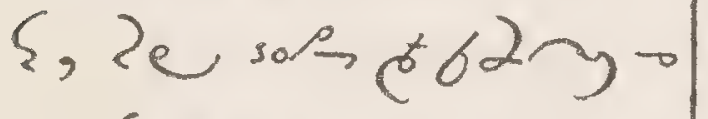
anc big < sa b $5=$ e; a versigo

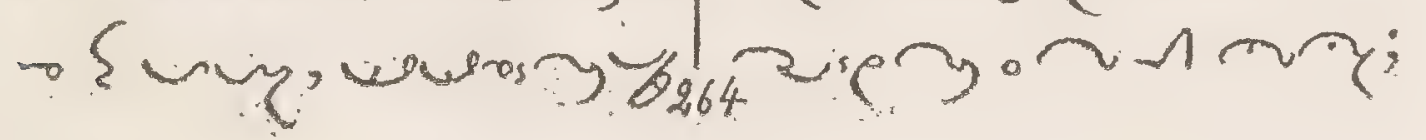

Nyur, $=t$ sa 6 to e 62 ars -10 iq wo. $5 ;=6$ sa Gsone bL sosord 2

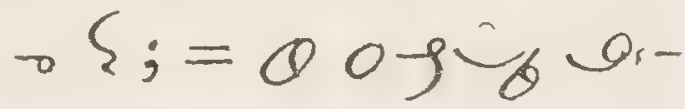
$-\delta$, werisy 6 $22100 x \sigma 2$ $\sim_{\eta} ;=b^{+} \cdot \sigma$ se d sof 0 ) b 9 . $\rightarrow x, w_{0} 09$ ys. Third Consideration. $x . \sim<9 i \theta) 1_{0}-0$ $x<2 \mu \sim 2,2 \alpha$ $y, \alpha 2$ sore $=6 g^{1}$. $=0 x, \rightarrow \partial \alpha 9 x$ $\sim, 9 u r,-\partial<c$ son -6$) \delta,-\partial \sim \cdots=$

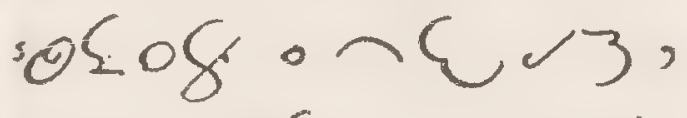
2 - 9 (u; só-

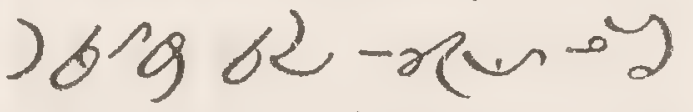
b, $2-2$ b9g; 0 $x,-\partial$ e - o ge $\sigma^{\prime \prime}-2 \partial$ bs, $0 \partial \alpha \omega=$

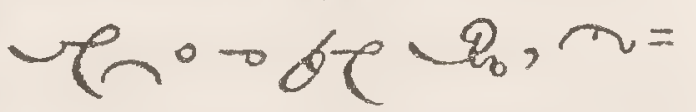
(2) 
, $\sim, \eta-0) \operatorname{sig} \cos \theta$ $962 \frown \varepsilon$.

x. $\rightarrow$ व $\theta_{s}-\rightarrow x<$ $2+\sim,<2 \pi ; 2$ $\rightarrow$ pereq, $2 \rightarrow$ soppto - big.o surt; wor ${ }^{\circ}$ arou de $\omega=$ wallos wey mperoudrot - bigt ua de

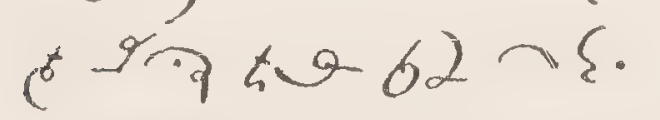

* 02 ar ind 62 - 5, 2e net b2) Goig lia dore; owe 50 ) 90 . un, wandy $\mathrm{s}=$

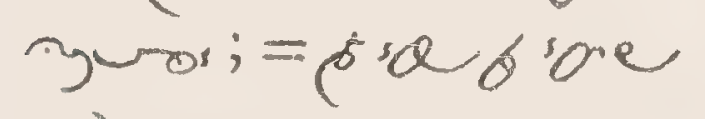
62 m2:e 0 sig w०० $\{$; = a bse $62 \operatorname{son} 022-\xi ;=$ $009 \gamma \cdot 0,-5, \omega=$ wormy r $622,0$. of. Fourth Consideration. $x$ \& giogl $\rho x$ <2\% ze, N sope $=$ 6igt.

$$
=0 \partial ;<2 \% x
$$

$\rightarrow x ;-x<o^{2} x d$

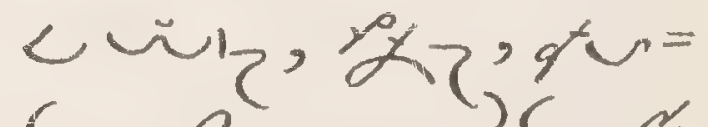
$\left.s^{2}, n^{\circ} \mathrm{C}, 3\right\}, N=$ $\left.s_{2} ; 2-0\right) \backsim \sim=$ L sarb ox; wor? soe os ox $62-$ wr $\rightarrow\{; 0 x, 2-1) n$

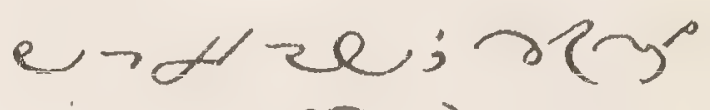
vice sor. ) bis. -52 la? dro cog sog o 2 vir $0 \curvearrowleft$ ? ox, 2 r on u=

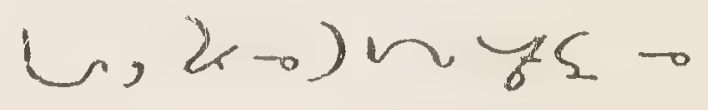
vis ay 629 $\rightarrow{ }^{\prime}=7 b^{\prime} \rightarrow 9$

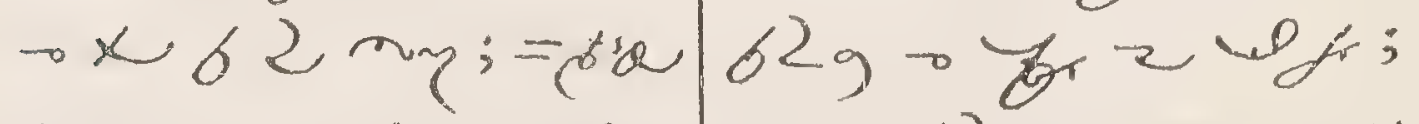

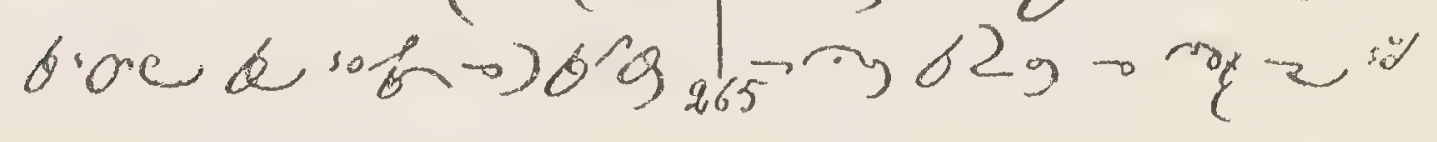




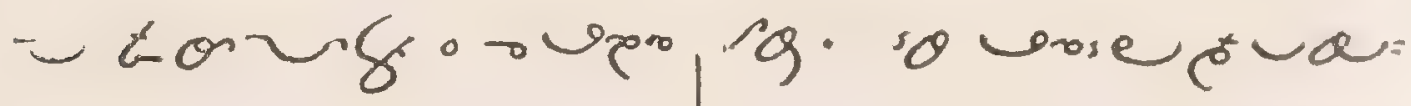
$\varepsilon-\partial \xi$.

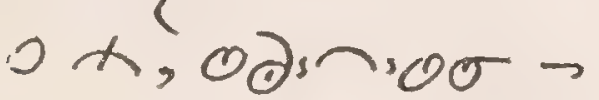

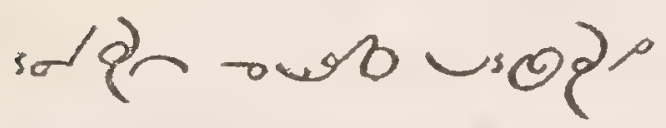

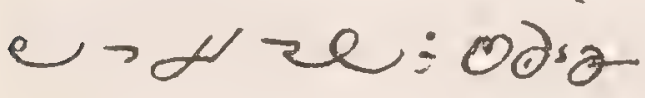
wo sougr $62=$

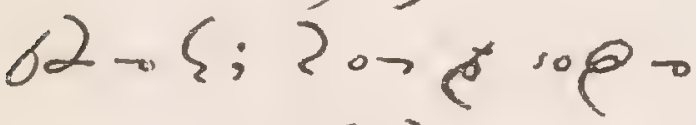
3690 < 2 rea;

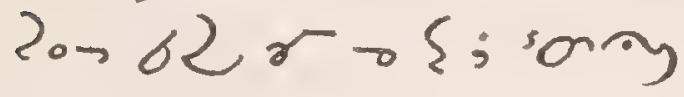
$62 \rightarrow \xi$.

$0 x \rightarrow 9$ bru red 2 of; $\rightarrow$ a U $\rightarrow$ a d ' $=0,60 \log _{2} \%$ wen, $\rightarrow$ कos sol $u_{2} \rightarrow a^{2} \rightarrow \pi$ गे so ₹ s'b- so है। $2 \alpha ; 2 \rightarrow) \sim \alpha=$ an. odig ow 0 ery be angor n; 2.0 62 \% ट reo. so dre

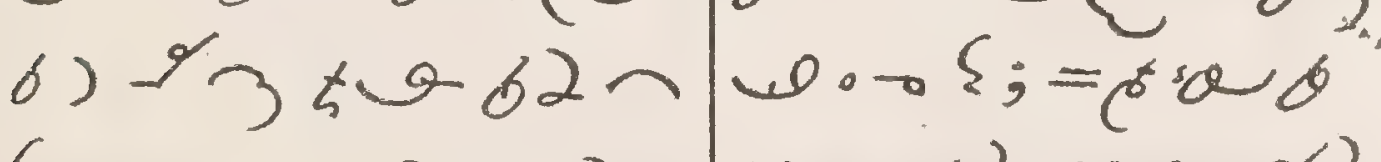

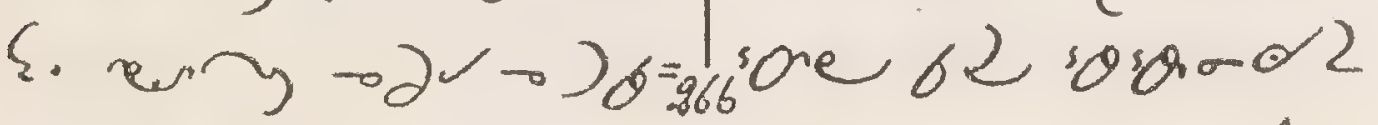

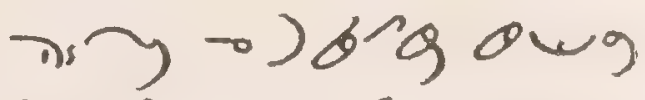
$310\}-\sim \varepsilon \cdot 0 \times 9$.

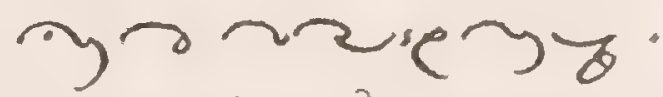
$206 \partial .9 \frown 5,0$, $910\} \circ \neg\{, \sim 66)$ Nm $\rightarrow x, \Omega_{6} \sigma$ anyor 0 ) 69 . $x$ - $\rightarrow$ as - o $x<$ $2 x$ sor. $2 \rightarrow 50$ unt ezodsor, sopeson de we

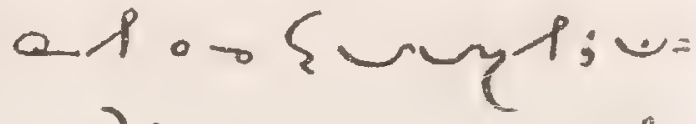
soperond rot - brgt uia de

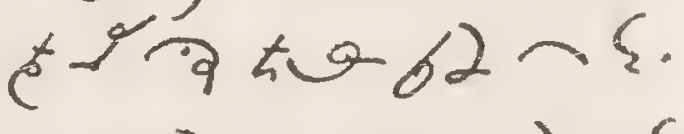
$x \cdot 0 \partial \alpha$ orn $62-k$

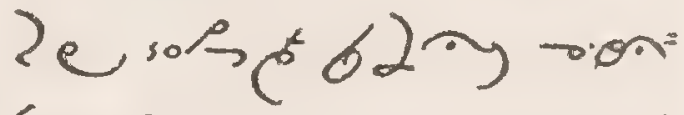
sog< sa ore;

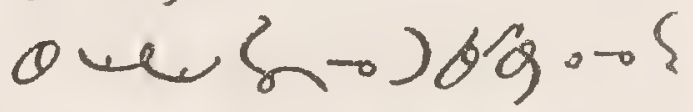
my, was $7 \sigma^{2}=$ ruros; $=6$ sa bse

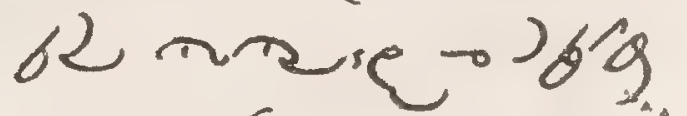

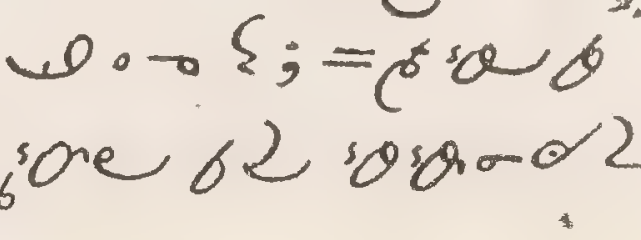


MANUAL

75

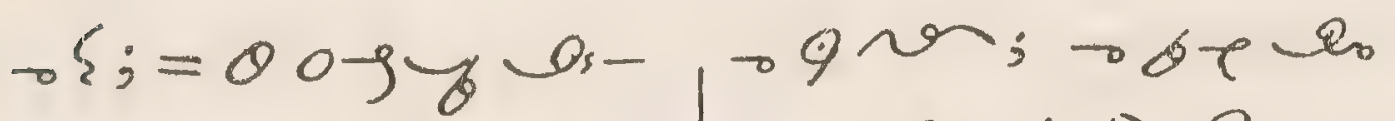

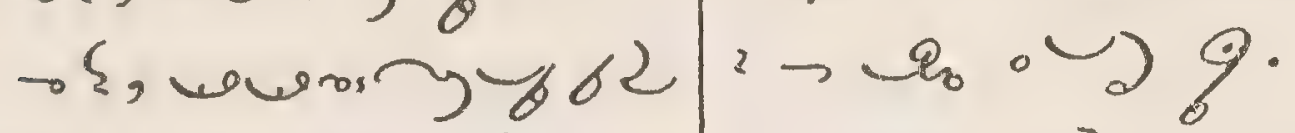

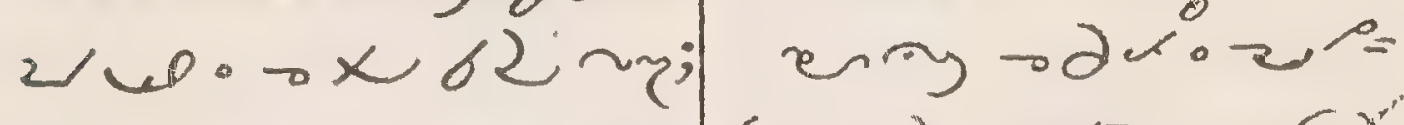

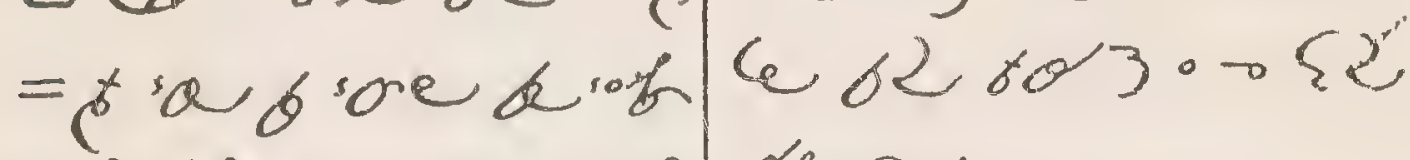
$\rightarrow) 6 r g 0 \rightarrow x, \omega$

$\rightarrow 9$ J s

Confiteor. soin s. 6 .

$n^{*}=q^{\circ}$

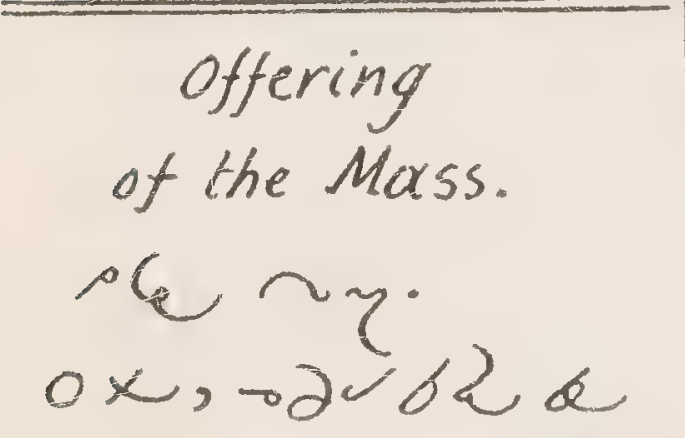

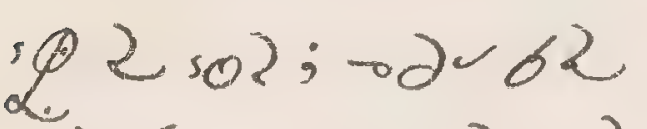

$825 ; \rightarrow 24602$

soug; worla ) $\mathrm{s}=$

reberrad。

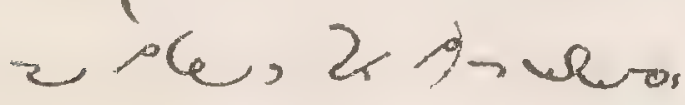

$\left.\rightarrow M^{2}\right)$ bq $<<2=$

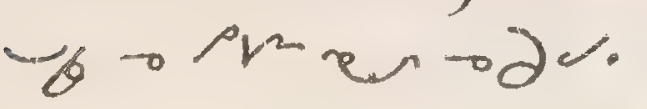

eng odso e ke

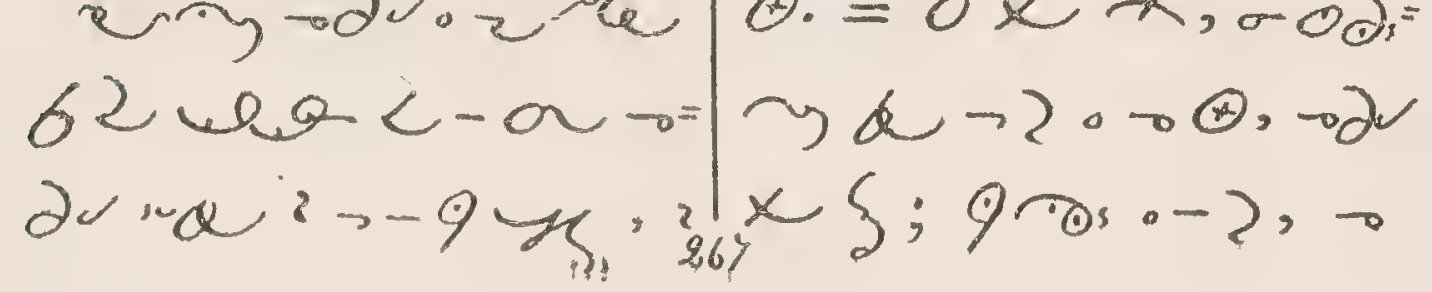

$$
\text { ming od z स }
$$

62 num -avos

- Que wor, : -

9 geres.

eny ral oz 56

d visoses avos

$r^{\circ}+380919 x=$

$662 \frown 5$.

ikar $\sim 0$ ) \&

oर 000 .

Prayers

for Communion.

Before Communion.

Adoration. bC 02:

vor $0<2$ ?

$\theta .=0 x x, \sigma 0 \partial=$ 
T, 2 $\rightarrow$ or, $2 \rightarrow U=$ U, - Nk, 62\%

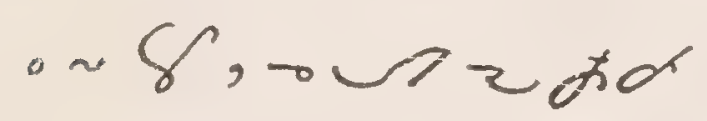

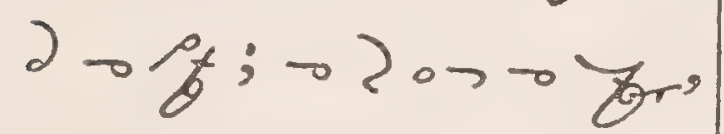
- bre r fred do s, $b-50$ - 34 cor -6 38.

Fumility. $\sigma k$ so obog $0 . \rightarrow x .=$ $0 x \alpha, \theta$ sos

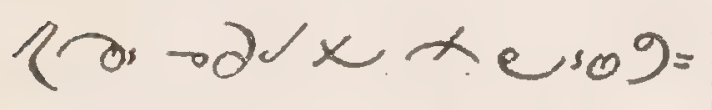

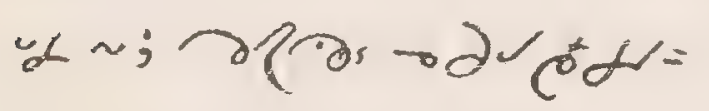
Ors o o b9; res? bew. r. - רry, ex a say w?

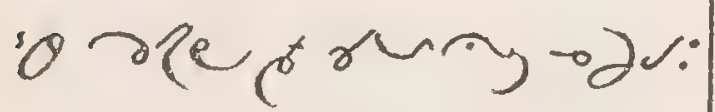
$0 x x$ odr m

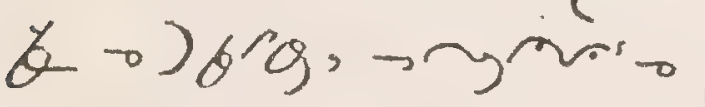
or , uspu r.y. Contrition. $\sigma \&$ we $=$ $\operatorname{sen}$. Oेs to ?०० 0 wry dr:ary o

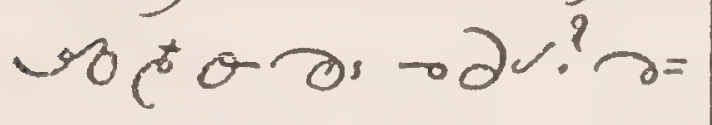
oर bou $\rightarrow \partial \alpha \cdot 2$ sol

ox $x, \ldots$ s sopo -) n. sozection $62100,62 \alpha+2$ -) n. $\rightarrow$ ry ro

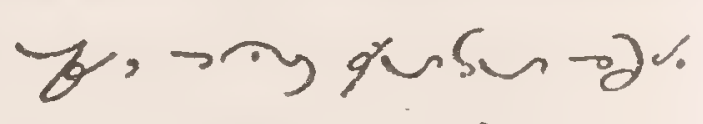
$0 x x$, a $\rightarrow 2$ sop, 20न $0 x \alpha, \operatorname{cs} \alpha$ and -sew $)$ (son $62,0 \partial \alpha \circ \rightarrow)$ ses Uop $\rightarrow$ by $\rightarrow$ ar; so 2 sale b2 ot tw - 20,62 then $02 \%$ Us - Jos ory.

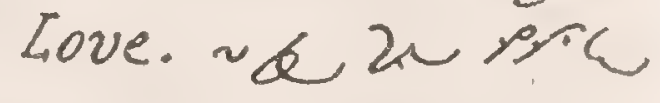
sol $\rightarrow x .=0 x x$, rifos sol $\rightarrow$ wo, ?

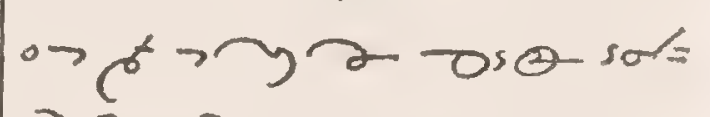
25 ods; arme sosoldg 20 ? 2 solras rol, $6<$, w, 'ge < $<; 2$,

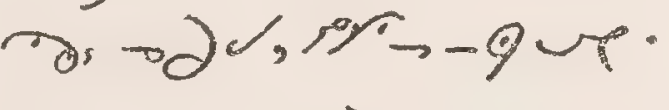
od, $x, \alpha, 2$ s

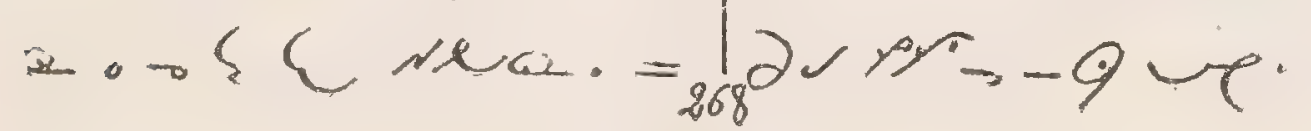




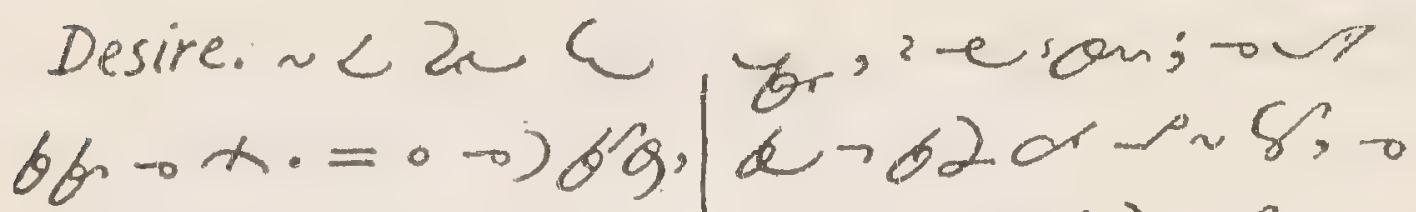

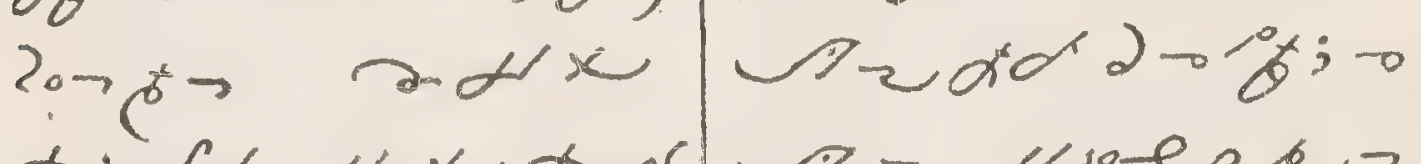

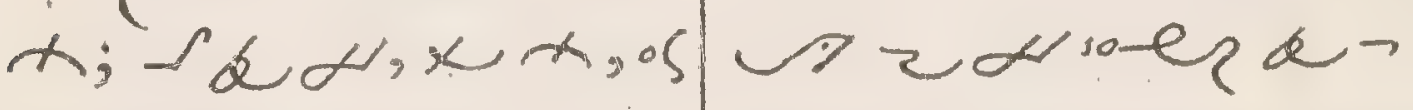

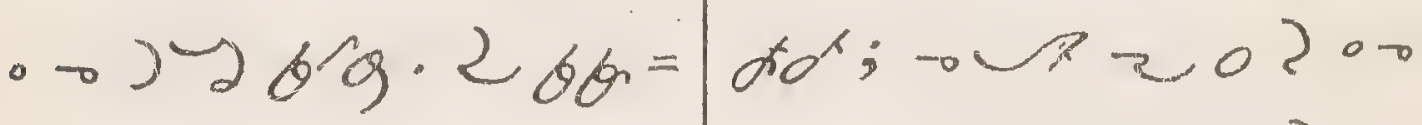

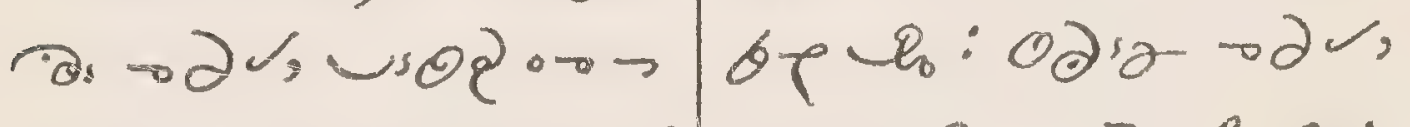

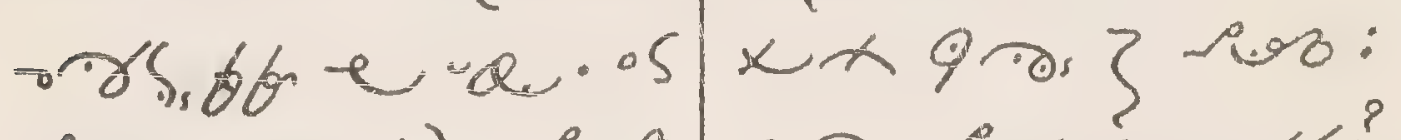

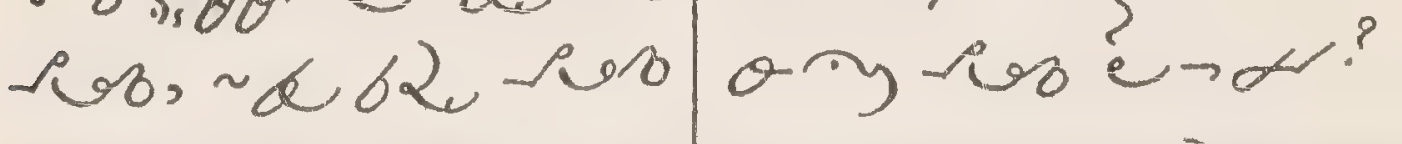

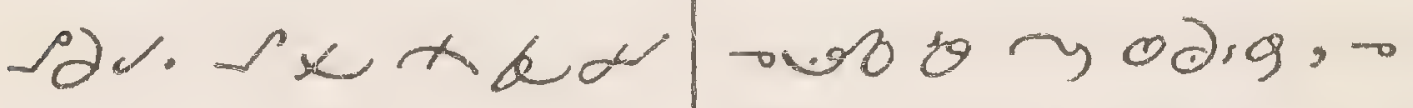
- ob, 62 s.0.0 少的

jysig ose Ln, a roe

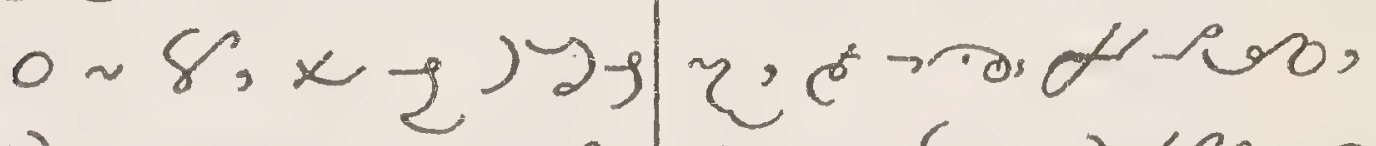

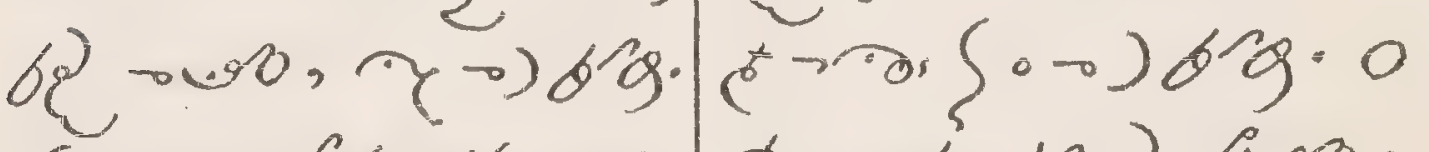

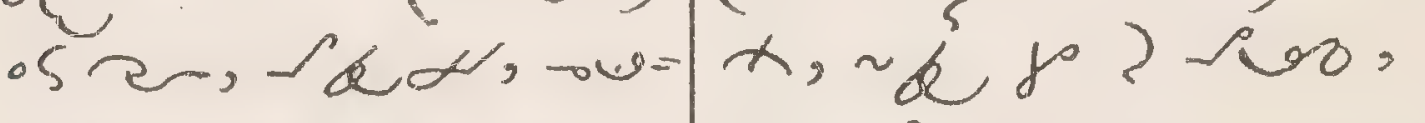

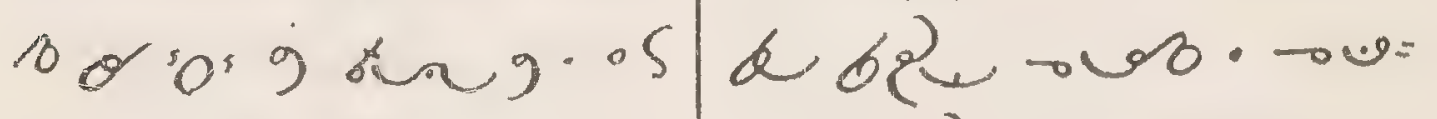
2, os, a $\alpha$. उ.06र ₹,

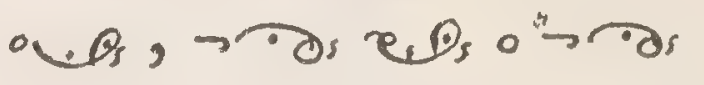

After Communion.

Adoration. ods $\mathrm{Z}\}$

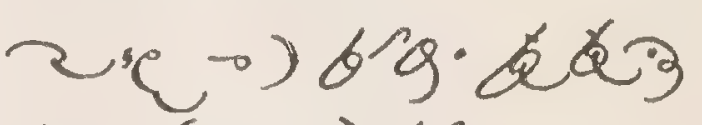
$b-507) 6 \hat{g}$, sa

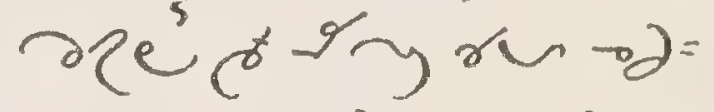
d. $0 x, 2 \operatorname{sol} 62$

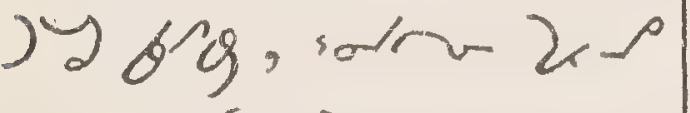

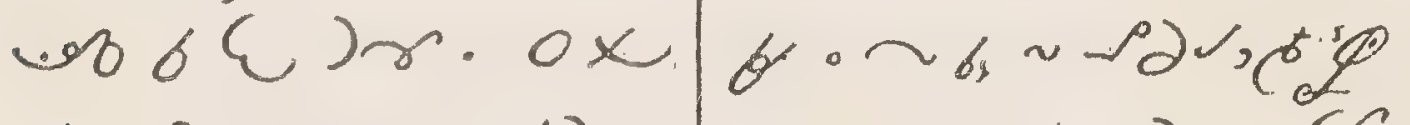

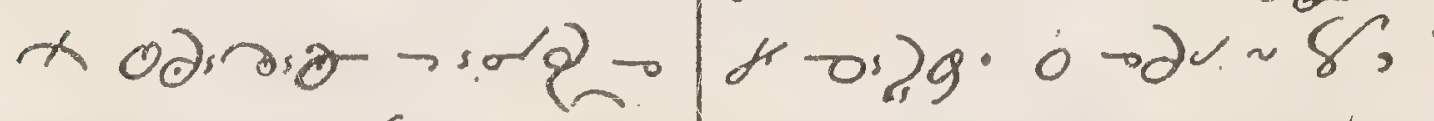

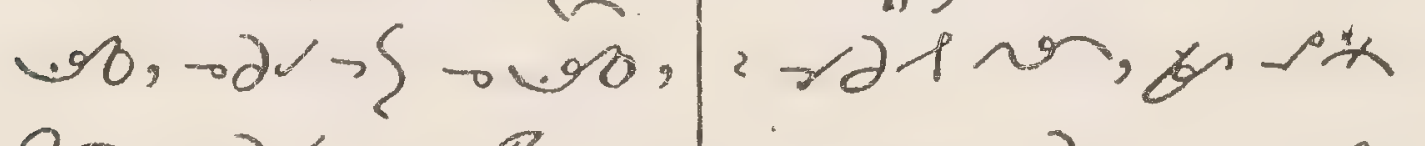

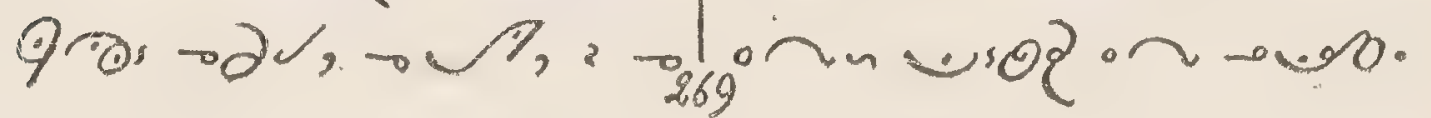


78

SHESHEL

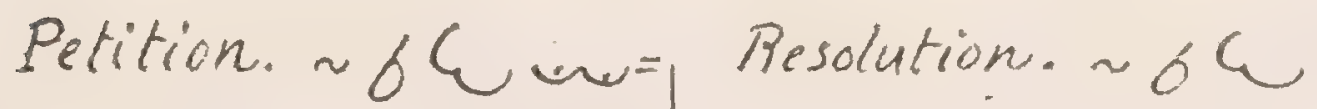

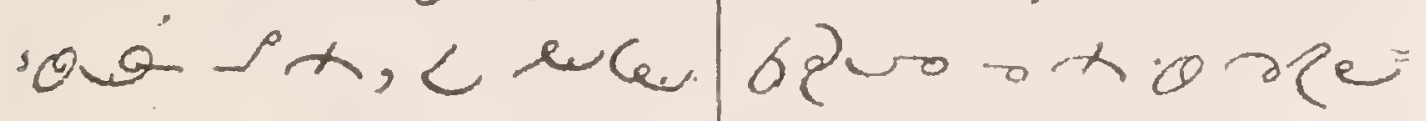
- Lbrg. =ox, b yesorokio= $\rightarrow \cos 0 \rightarrow \operatorname{s} g, \sim \operatorname{coc} \alpha 2 \rightarrow \xi=0 x$,

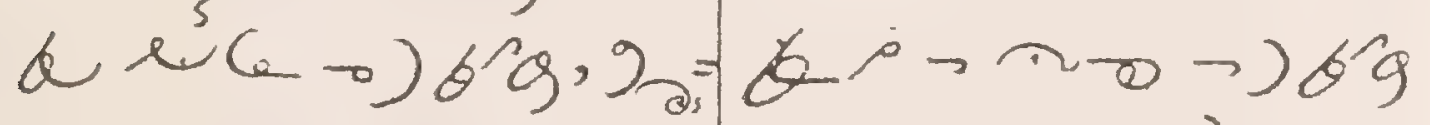

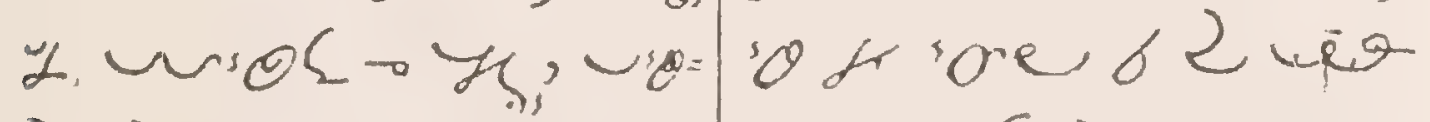

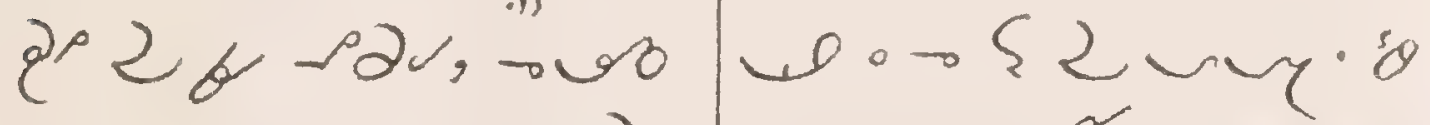

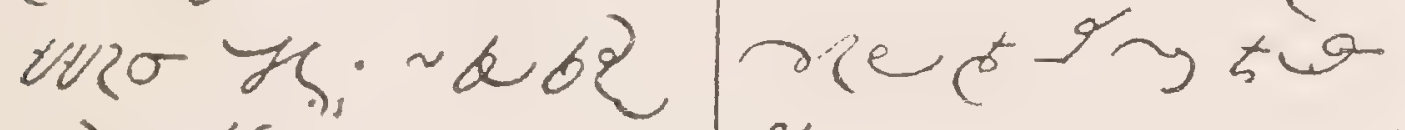

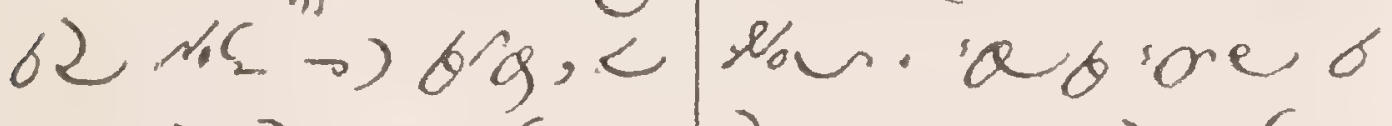
a sophe $\rightarrow \xi ; \sim 2 \sin \alpha 2-k \cdot 0$

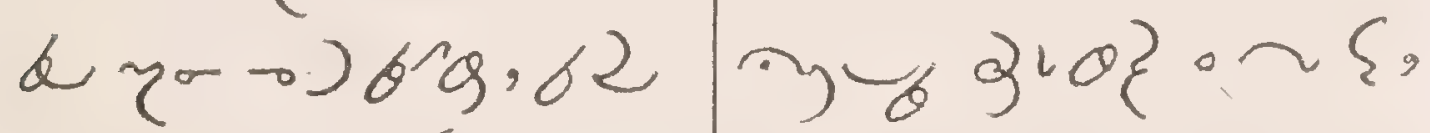

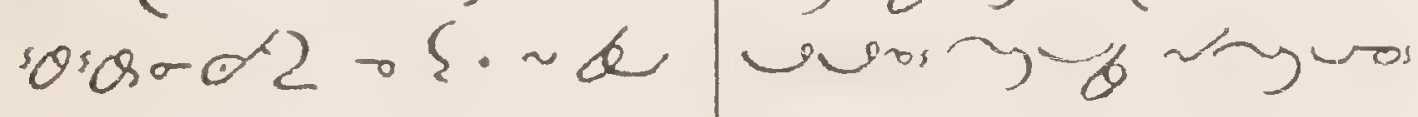

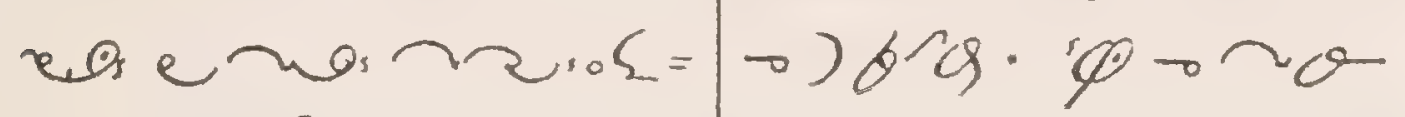

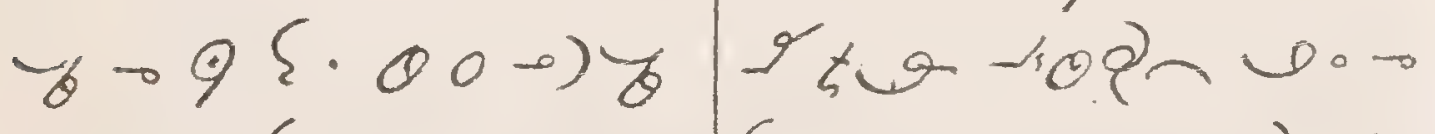

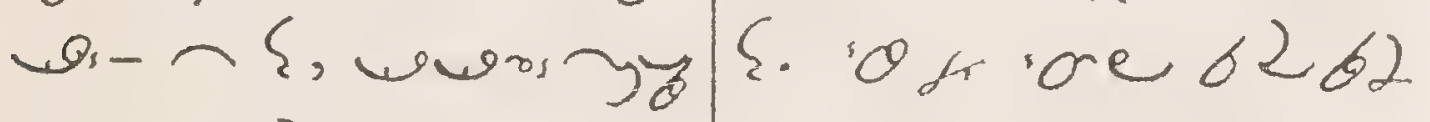

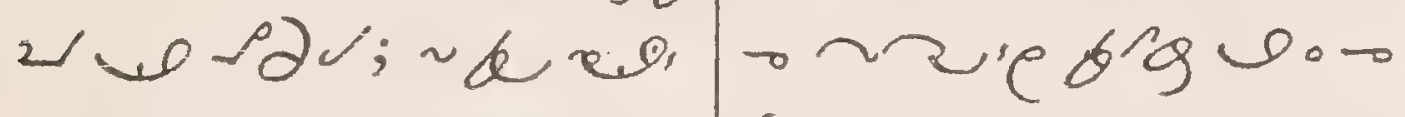

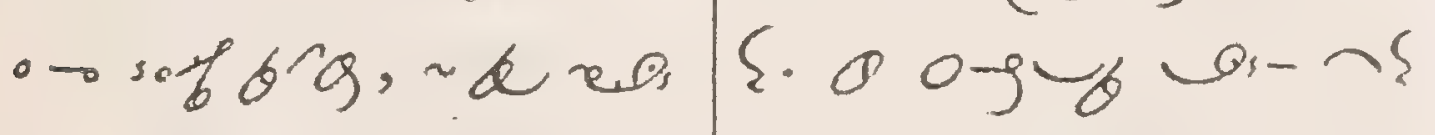
enso rea wo warisyber,

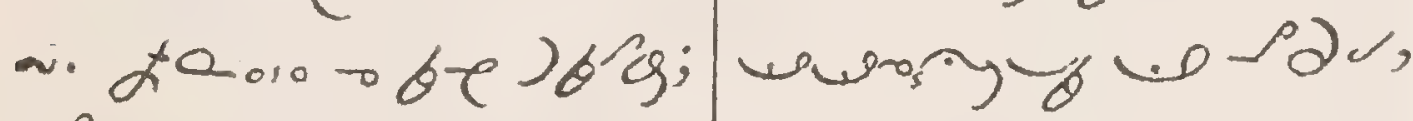

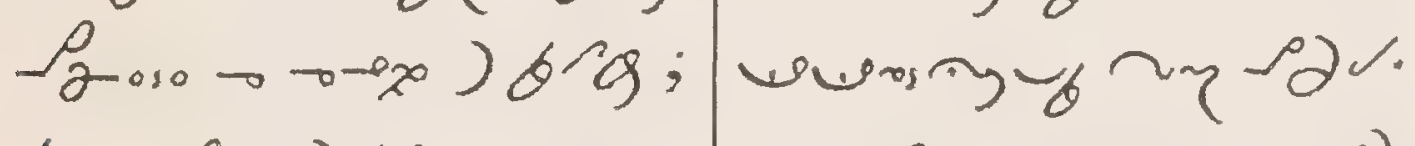

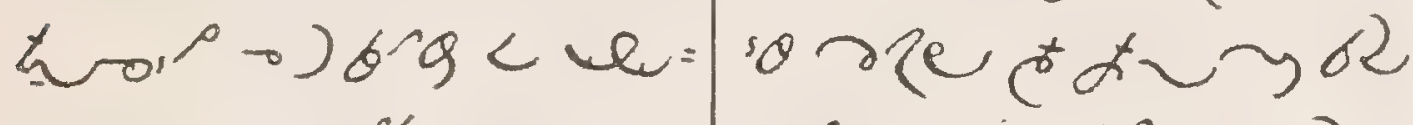

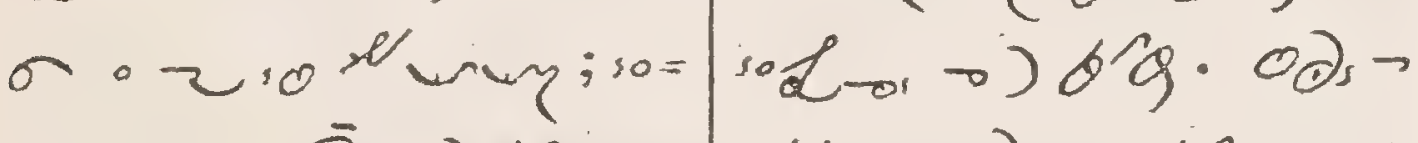

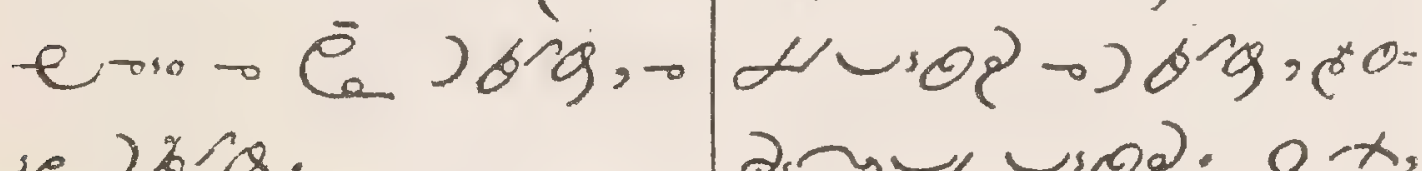
sers.

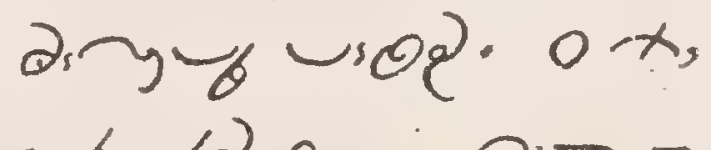

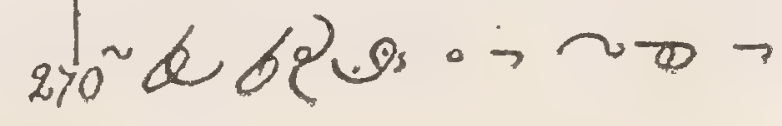




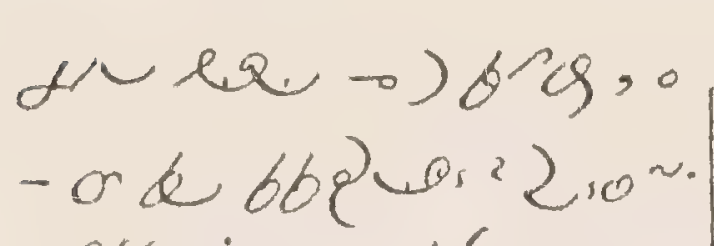
offering. $~ b C_{2}=$ x。 $(2 \partial)=0$ $x, 200 b c-\partial r 0=$

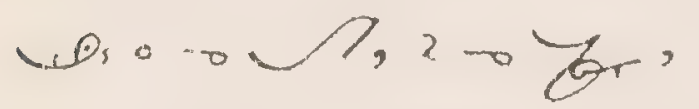

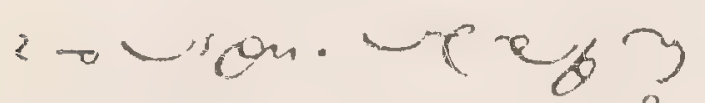

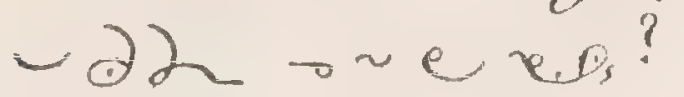
$\operatorname{err} 0-\pi / 29 l=$ Q) "a b.ce 62 so $=$ an;0d enry:-) bog, saly sor 62

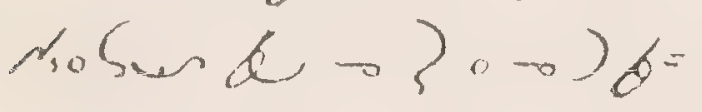
g. eng. on,

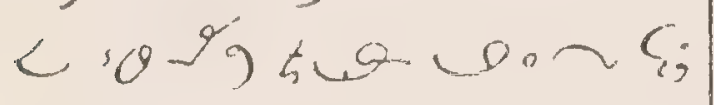
actioly $x \rightarrow$ ? 0 -

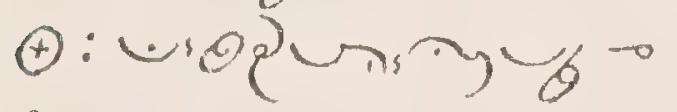
g) a $0 x, 2 \rightarrow-0$

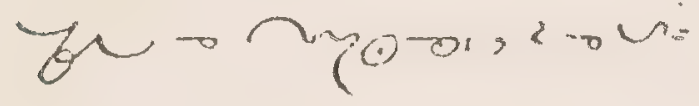
une

intercession. $\sigma C$ b.tote wiouso= $\log x_{0}=0 x \sim d$ rik $\rightarrow$ Y n noor,

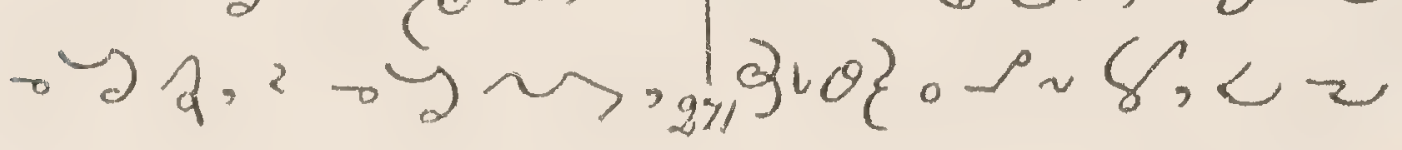

$2-2 x^{2} \cdot \sim 60$ $-(2) \leadsto, 2-c$

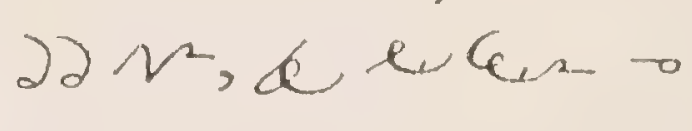

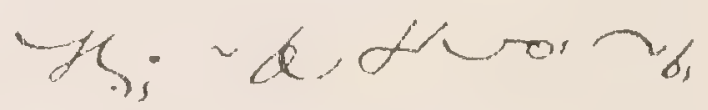

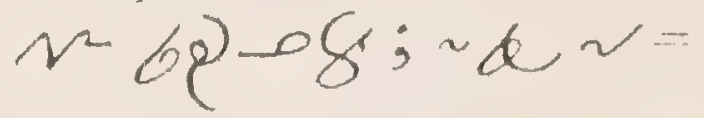

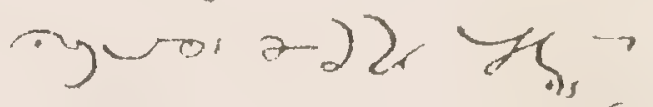

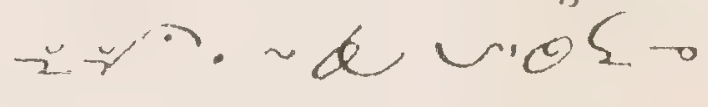
) von'm whod 2.ruenr; b2 $\Rightarrow \frown y^{2} \rightarrow \sin \rho$ $2 \operatorname{ten} 3,<2 \sigma^{\circ} 0=$ 100 d $\rightarrow 0^{\circ} 3-$.

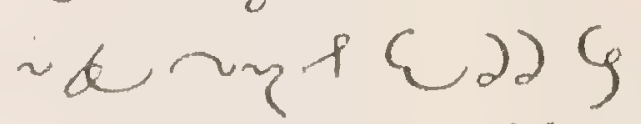
n, गु; $208 \sim 4$ 2; जल2-619A. é row yor.

The Rosary. I. Joyful mysteries. 1. yo Es a $\sim 6$

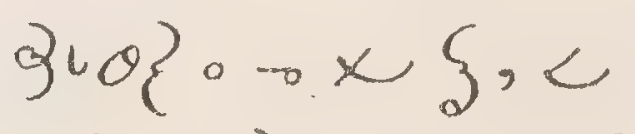

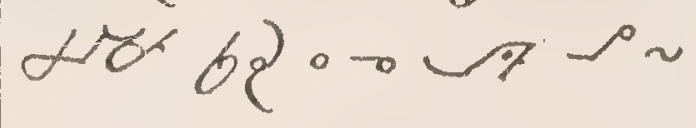
- We soe os.

2. we kr, 06 
80

SHESHEL

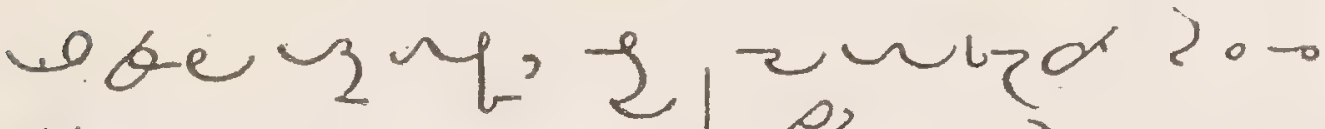

*. गे, हr, $6 \mathcal{L}$

अ

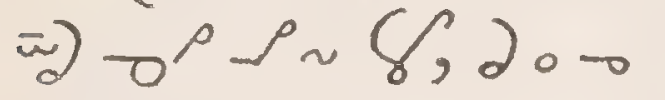

celor.

4. $\operatorname{Cor} \varepsilon r, \sim 6 C$

अण $\rho \sim \delta, \alpha>\omega$

- nyoors aer 0

$x \in$ px.

r. uner st, 0

$(9,0) \sim 8 k-$

b. 3 ar wer

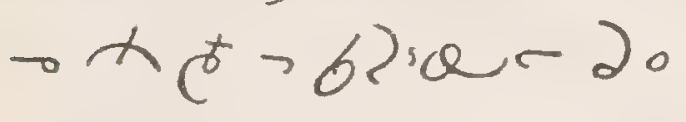

$\rightarrow$ ñor

Sorrowful Mysteries.

6. oped Ersio

$<960) \rightarrow(2) \cup=$

sud, 2 idd 20

$\rightarrow$ a , $x-y \rightarrow$

$b=v \varepsilon \cdot 0 e_{0}$.

7. ent es: ?

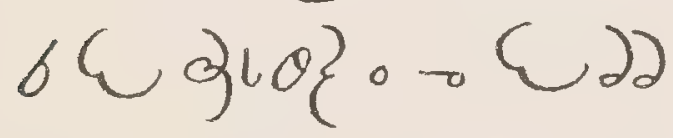

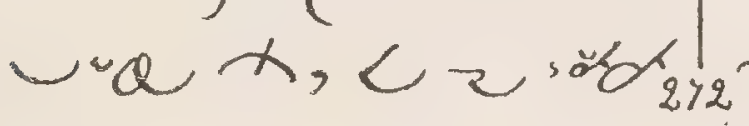

az - brua.

8. - ne हs: $\sim 6$

$(3406 \circ-(2) 2$

suce $x,<$ sad

ztourdo 0

रं०, है ح नैं

9. oust Er: :

bथ 940$\}-\varepsilon \partial$

iva $x, b=0$

$\partial_{7}<k, 6 \alpha_{e}-0$

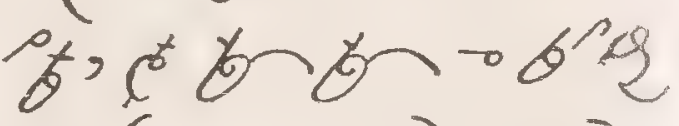

s sue - a $2<x$

dis, $<2 x d$

10.9 Er: 64

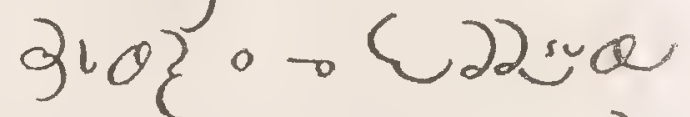

人, < 2 or 2 ?

$\rightarrow 62$, $c^{-} \rightarrow$ yor

f $\rightarrow u r^{2}-r^{e}$

- य 2 200 क

$\rightarrow$ a b $60 \sim$. Glorious Mysteries.

11. gd a o jo s ? 
una $x, \cup 2,0$ $\partial_{2} y c+\alpha i \alpha^{\circ}$

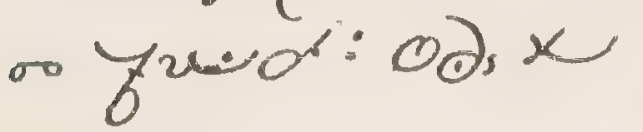
-2 .

12. $g \alpha z-27 \mathcal{E}=$ $r: \sim \sigma C$ 3ro $00 \mathrm{C}=$ d) wax, $\rightarrow$ ion Gadno y-und

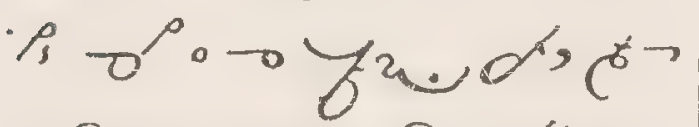

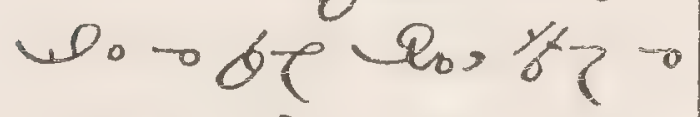

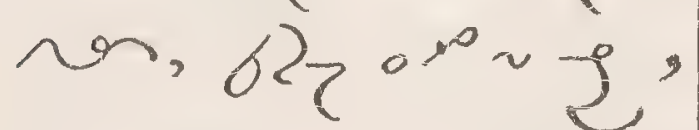

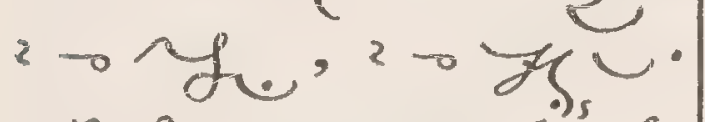
अ. $g^{2}$ - गे, है, $\sim 6(910\} \circ-(2) 2$ ua $x,<$ z, $\rightarrow$ th or rols $\rightarrow 2 \xi$, t 0 and $=$ a - wory ; unpura s tal. 14. $g_{\alpha 2} \rightarrow C_{Q} \varepsilon=$

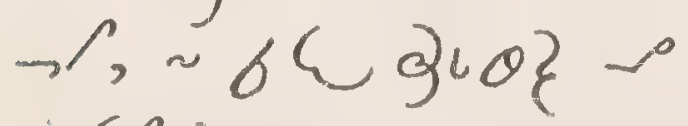

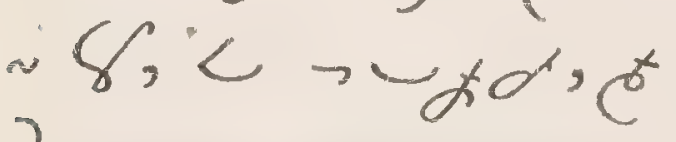
$20 x \rightarrow \rho_{10}=b^{\circ}$ vara oto be $y^{\prime} \mathrm{z}^{\circ}$. n
15. gat 2 - . . on ES: $\sim \sigma C .010$ ? $\rho_{\sim} \delta,<z$, s ?

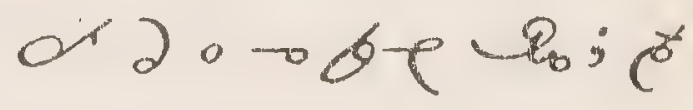
2小 $\rightarrow \rightarrow$ kon

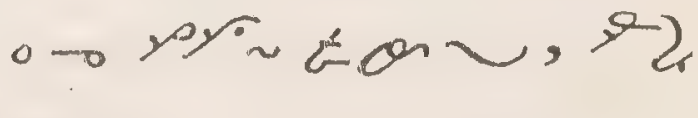
or $\sim 0-9 y_{s}$

Via Crucis.

Prep. Prayer. ox, odrar sor o ovo, unpar -av, a Nor os a $a$

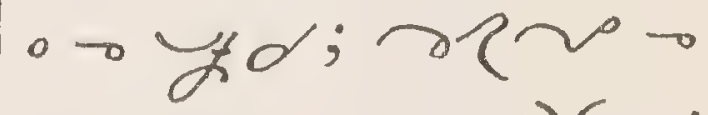

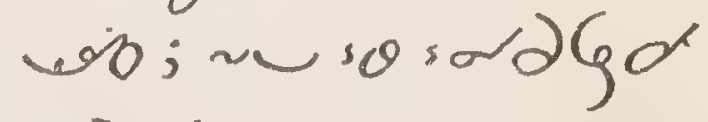
- वर, siy rd - ty, $\rightarrow$ ry qusur 0 व

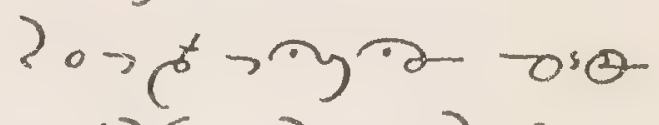
solas rad, 2ava

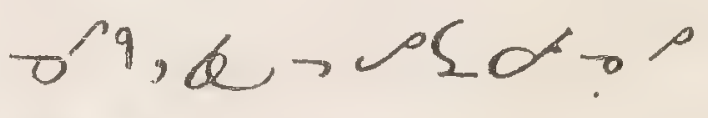
os $\alpha>\omega 0-\pi \alpha^{\circ}$

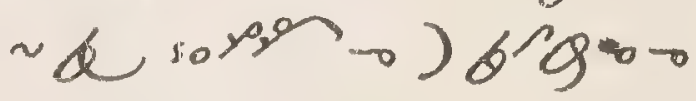
¿2 rea, c so 99 $62-5$. 273 जe ?. 
Sancta Mater.

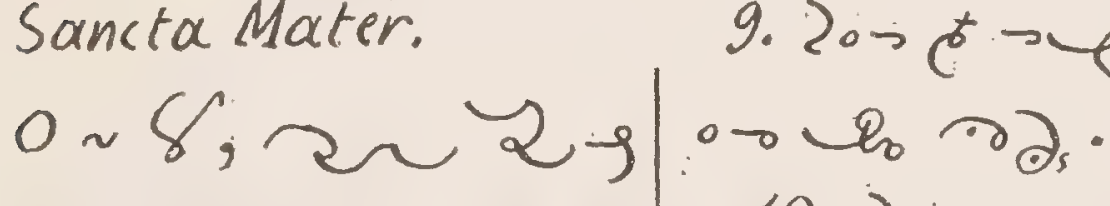

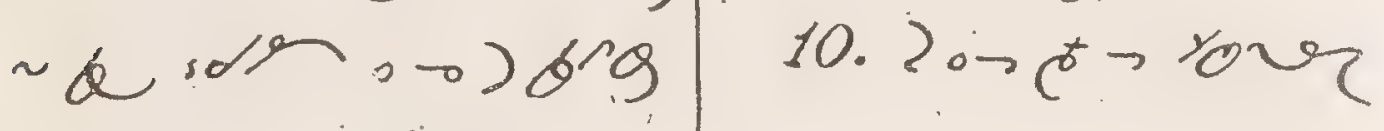

erös $\rho$.

Adoramuste. $z_{\text {grs }} \mathrm{C}=$

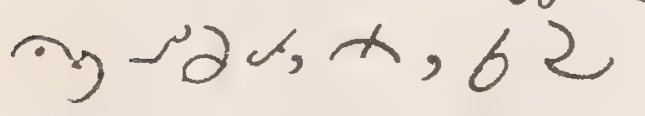
$\rightarrow$ 大.

11. $20 \rightarrow e \rightarrow \cup \sim 7$ $\rightarrow$ 吅西.

רin $-\partial \alpha, b>-0$

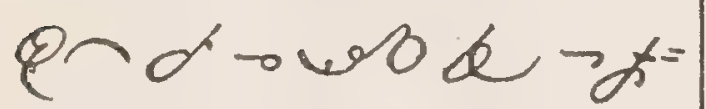
$\alpha 20 \rightarrow 0$.

1. $20 \rightarrow c=\cos \rho$ $x \rightarrow b^{\prime} q \rightarrow$ we 12. $20 \rightarrow$ t $\rightarrow+\alpha$ $20 \div 0$.

13. 20 $20 \mathrm{C}^{\circ} \mathrm{C}=$ $x$ o o o o

14. ?० है गण 2. 200 ह

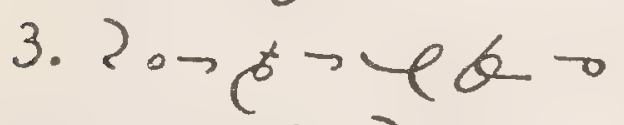
x。 $\rightarrow b_{0} \partial$ 4. $\operatorname{son} c \alpha x\}-x$ $\rightarrow 2$. $5.668 \rightarrow x 2$ - $-\frac{\rho}{6}$. 6.) $\partial \sim \infty_{5}^{\infty} \cup=$ $\alpha$. $\rightarrow x$.

At each Station.

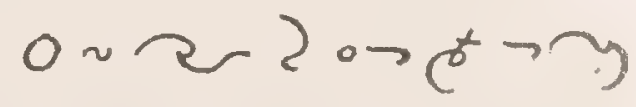

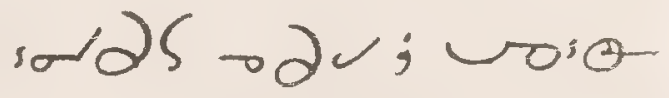
sष - ग $89 \alpha-80$ -y八 $\sim x ;$ ' $62+5,68 \cup-60$ Lse liog? - 5 , e. $-100<\gamma^{x}=$ $\partial S$ - $\partial \alpha$

$y_{1} 20 r c \rightarrow e b-$ x。禺 $\omega_{0}$.

8. x vare 0 . 2 $\rightarrow 600$ y)

Pater. $(\lambda \partial 2\} \cap y$. Ave. 08 กn. Gloria. Ho $0 \rightarrow G$, $\left.\lim _{2 / 4}\right\}, \omega \rightarrow 0_{0}=$ 
MANUAL

83

जor a borsords

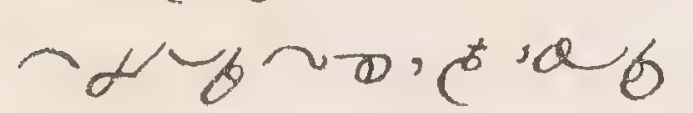
soe.

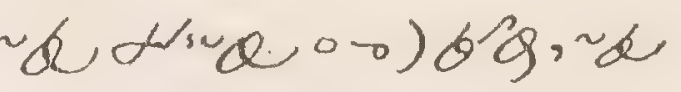
ba - oque kwarind

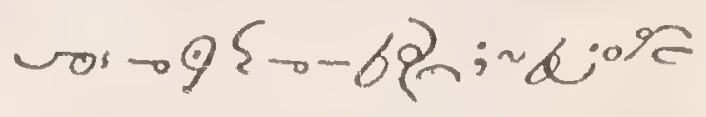

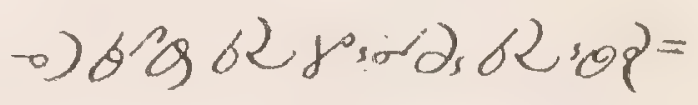

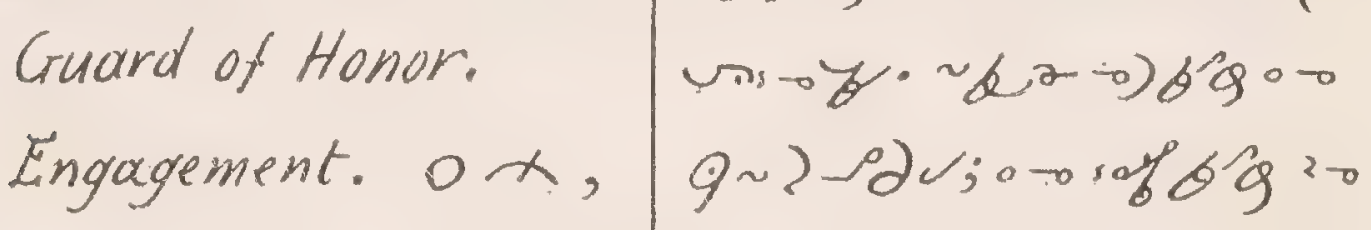

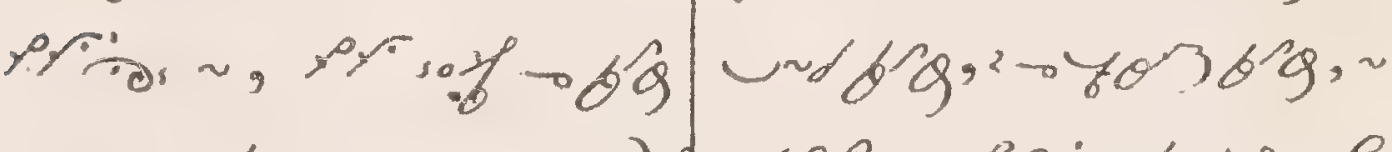

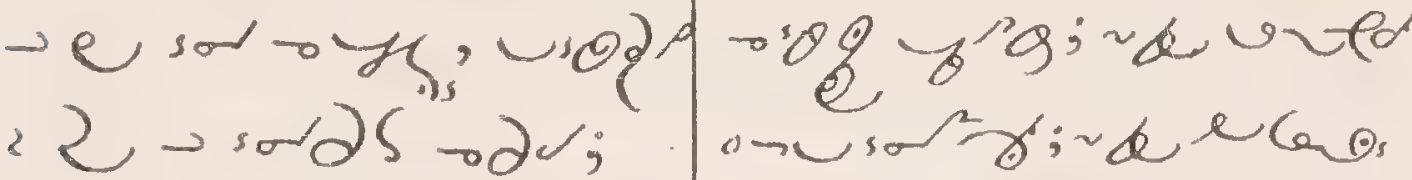

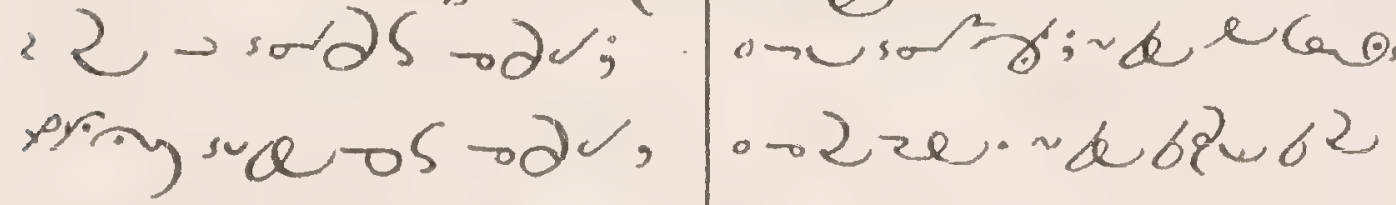

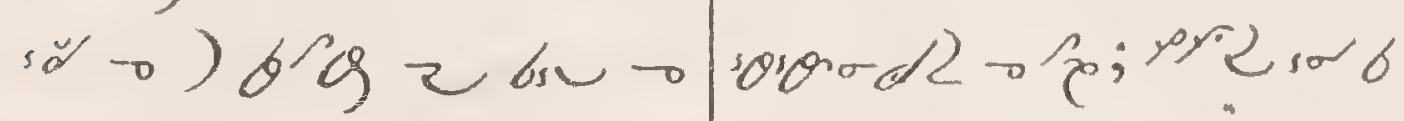
H, $2 \partial s$, ,

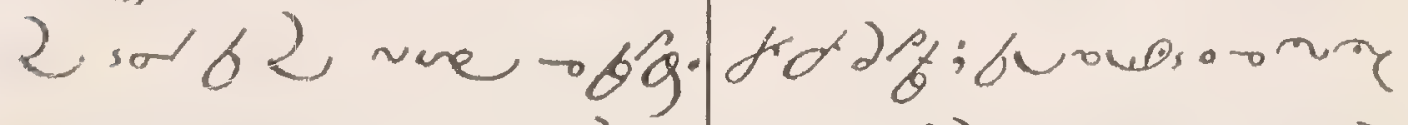

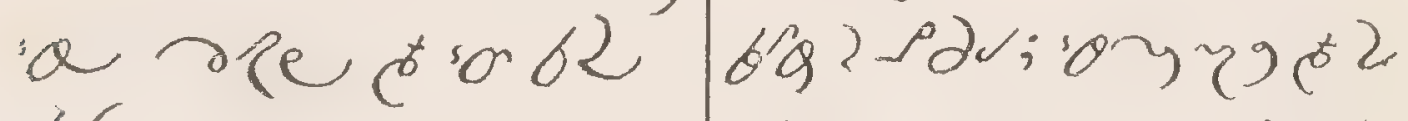

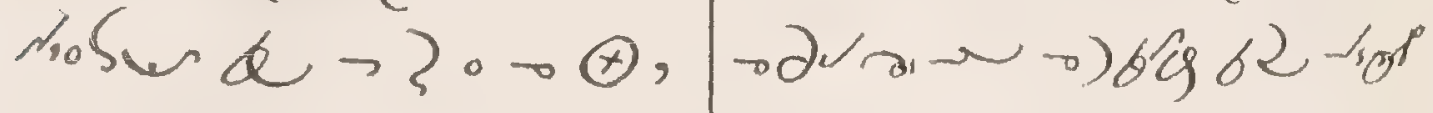

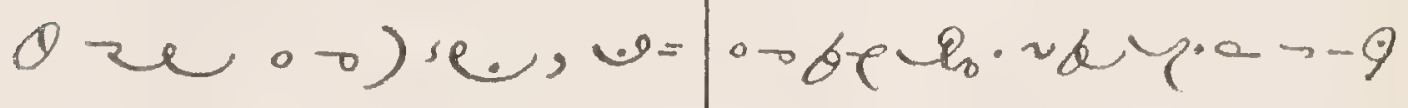

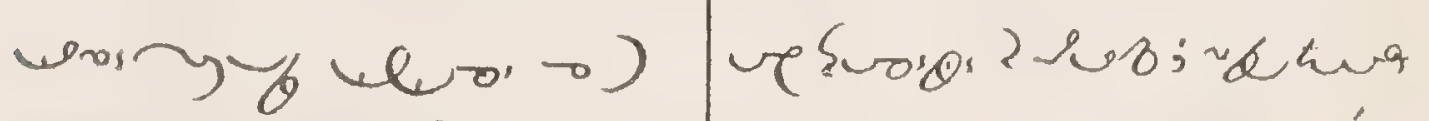

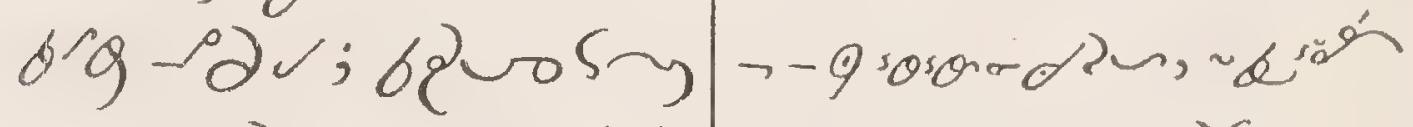

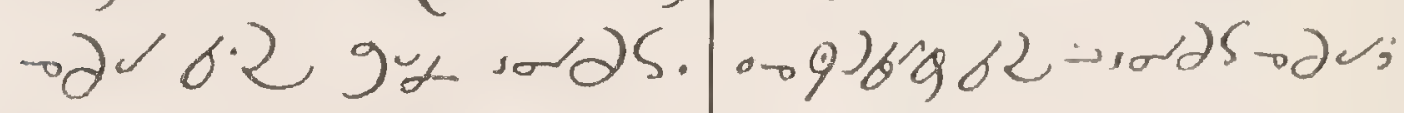

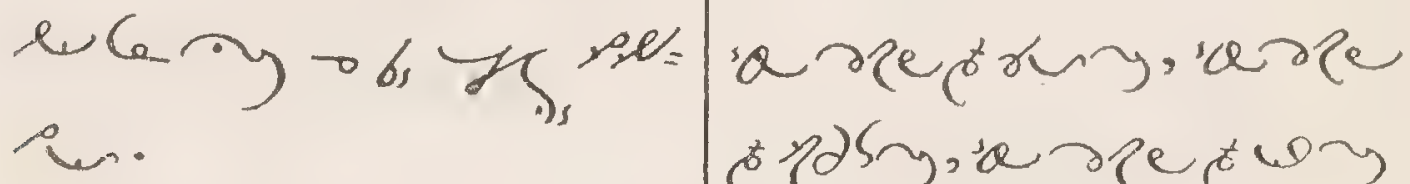

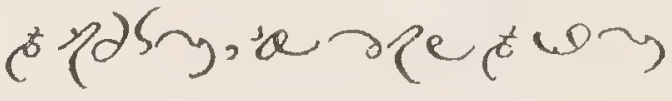

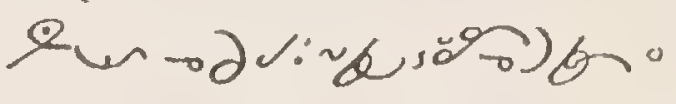

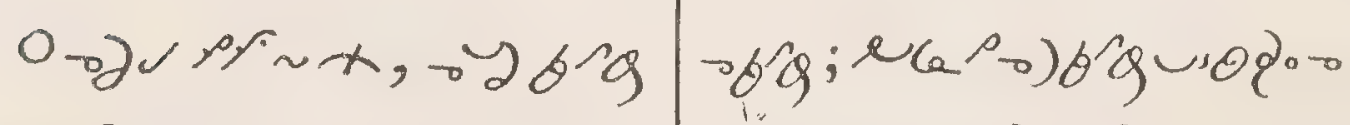

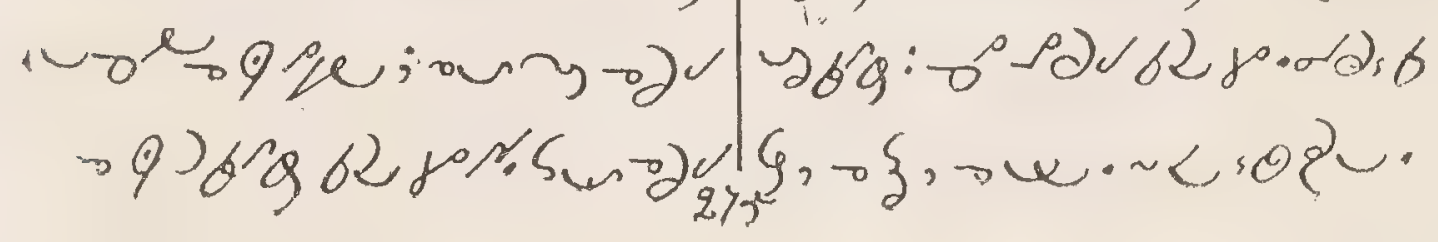


Shishel Hymns.

1. Adeste fideles.

$\sim$ be so.jot

bitolorg,

Sop sopo-bin.

- $2 \alpha^{\circ}$ deruarion

Soridges

Solidar

soridos x $\}$.

2. Venez Divin Messie.

$$
5 \times \xi
$$

bou voron wie

semsoros.

Se, Sp, sp.

spsuce srob " -0 -9 th, -0-8, id a t

3. Epiphanie." \&AS." " bCQ e.0. जor. a sisua

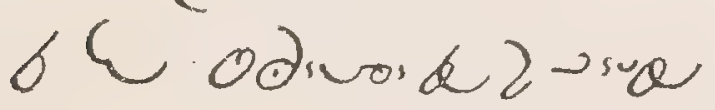
rawobege. gutc sor ez 62 a ravauroe mung od $x$ racosacu. 4. Vivat in excelsis.

ann o od $x x$,

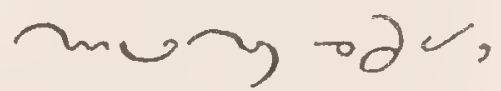
o toneregr: Notan $\rightarrow$ - or 2 - क्ष < 60308 $\rightarrow \sim \rightarrow) b^{\prime} \theta$ ( ) $-2 \mu \alpha$ जorरsin 6260.95 .

6. $0\{200$ $=628 v_{0 \rightarrow} \leqslant ;$

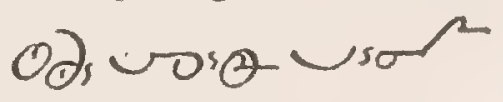
bL 000 be $a_{0}$; uoperos

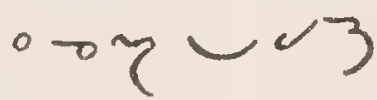
$\circ$ - 
MANUAL

85

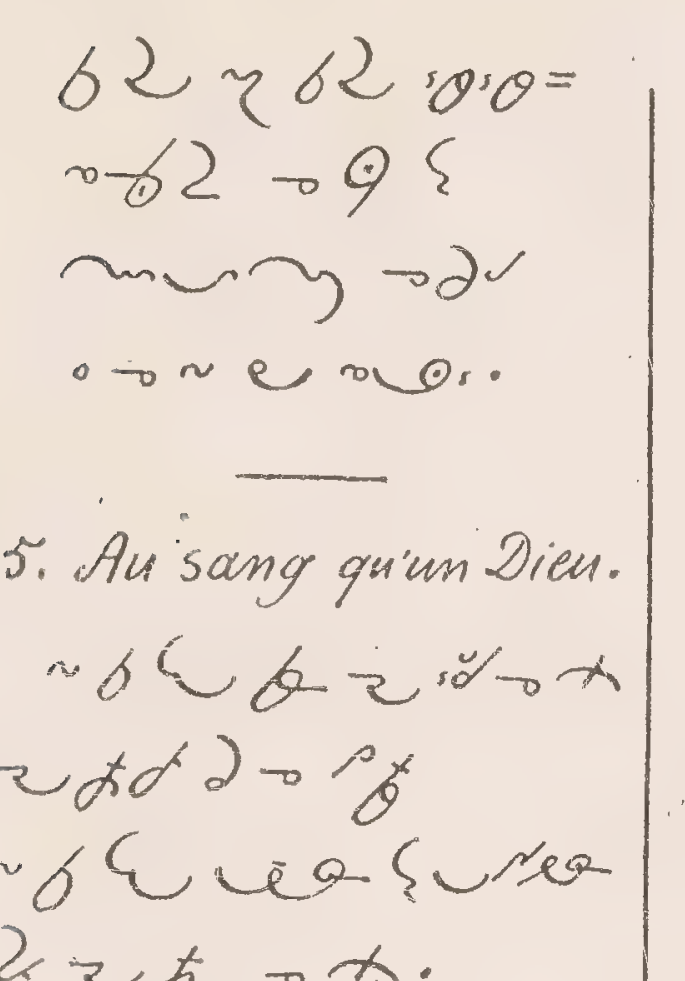

正二大。办。

Refrain.

oxiery

- 52 rea

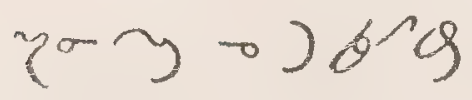

ron nuy o

$t \rightarrow \theta$ e

hura ro-gors;

$\rightarrow$ कर, $\rightarrow$ -

ter, 3rear.

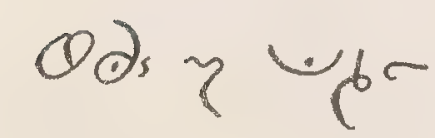

$\rightarrow y \rightarrow$ 次

b. - yefor $Q$

$\rightarrow$ Ods sor.
Sud sod $\rightarrow$ Cer

zt-oneror

so'g z b-iand

$\rightarrow$ guserd.

use - o g

$\rightarrow \omega 0 \rightarrow$ th

brivoge-ed

gara ourawore

voerorgy

- de 2 - 2

gy vore so

toe surea.

dorewworo"

-ousar of

d-esuresoly

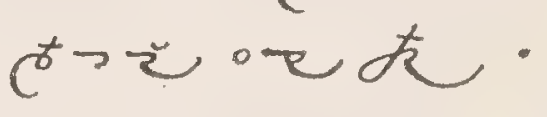

6. Sanie air.

ox

-कमकर 2 - 90 ;

aरकu< 4020

$2146262-2 \sim y$. 
86

SHESHEL

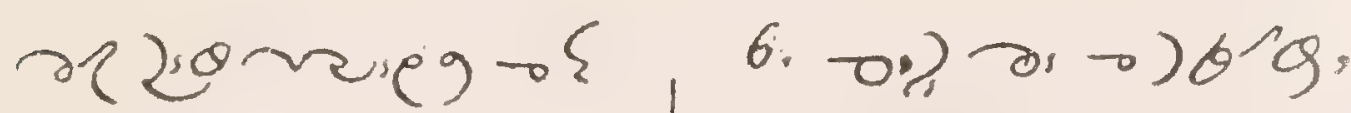
Lisiond 2

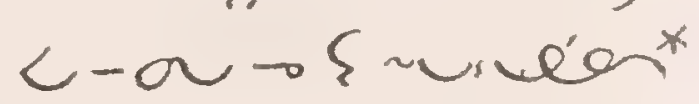

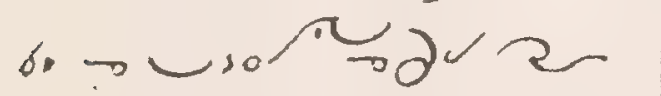
$.0 .6262 \rightarrow y^{2}$

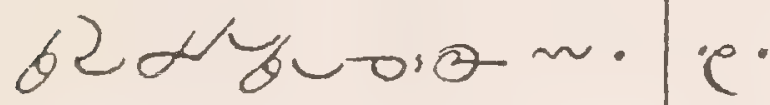

7. Miserere mei Deus.

unpovo, aurn

$\sim x, * 2,-9 \leqslant \varepsilon$

rea

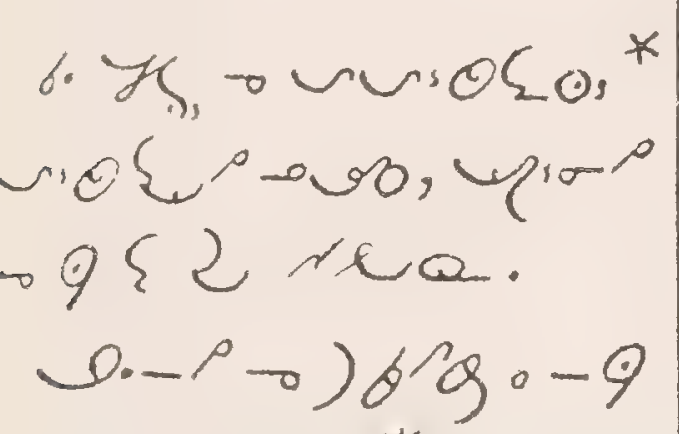

¿2 rea, * vo.0

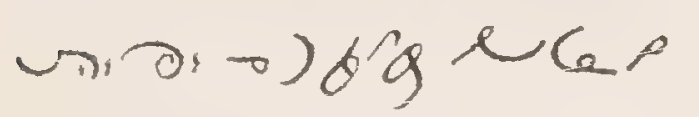

$\rightarrow)$ sig.

20न

$<2+562 \rightarrow r \rightarrow 2$

$\sim \gamma$.

$94,9-26.2$

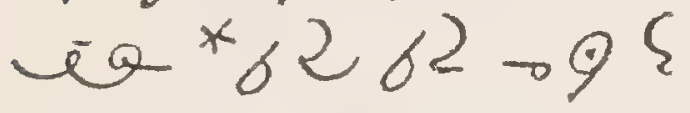

2 rea.

$\partial \partial \sim x, \rightarrow \neg 92=$

an, ${ }^{*} \rightarrow$ muar

म) $6=2.600+0.278$
8. O Bon Jesus, enfin..

$0 \dot{x}, \sim 6)=95$

$\operatorname{usprog}$ or 8 y;

$0 \partial s \rightarrow{ }^{\circ} \theta-\left\{\theta^{\wedge} 9\right\}$

- $\{$ crea,

$0 \sim 2$

$-6.00(r \rightarrow 2)$

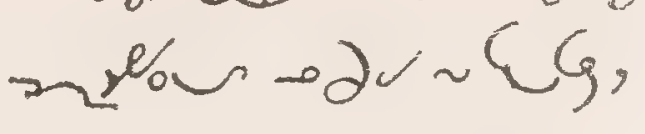

ग०
$\rightarrow$ कर $0 \sim 2 \sim$

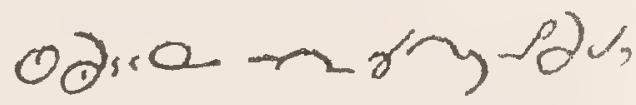

von sor be unots

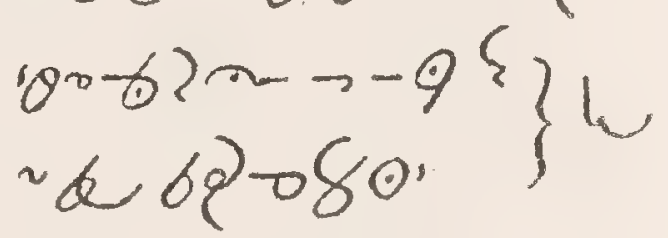

$0 \sim 2$ 


$$
\begin{aligned}
& 02 \text { s } \\
& \text { याँ } \\
& \circ 0 \text {. } \\
& 2 \text { rea; } \\
& 0 \partial \\
& \text { याe } \\
& 62+\% \\
& \text { - } x \text {. } \\
& 2 p^{p} \\
& 02 \text { sorea } \\
& \mathrm{\gamma}_{2} \\
& . \check{6} 69 \\
& \text { प.Q? } \\
& 50 \times 97 \\
& \text { - } \\
& \leftrightarrow \frown \infty \text {. } \\
& \text { iog } \\
& 1009 \\
& \text { क्षे? } \\
& \text { गुरक्ष } \\
& \text { जo? } \\
& \text { iog } 9 \\
& \text { or } 0 \text {. } \\
& 9 \rightarrow 1 n \gamma \\
& 22 \text { to. } \\
& 6262 \cdot 5 \\
& \text { veors } \\
& x \text {. } \\
& \text { ०), } \\
& \text { owore } \\
& 620 \\
& \text { - } 1 n r \text {. } \\
& \text { otsor. }
\end{aligned}
$$


88

SHESHEL

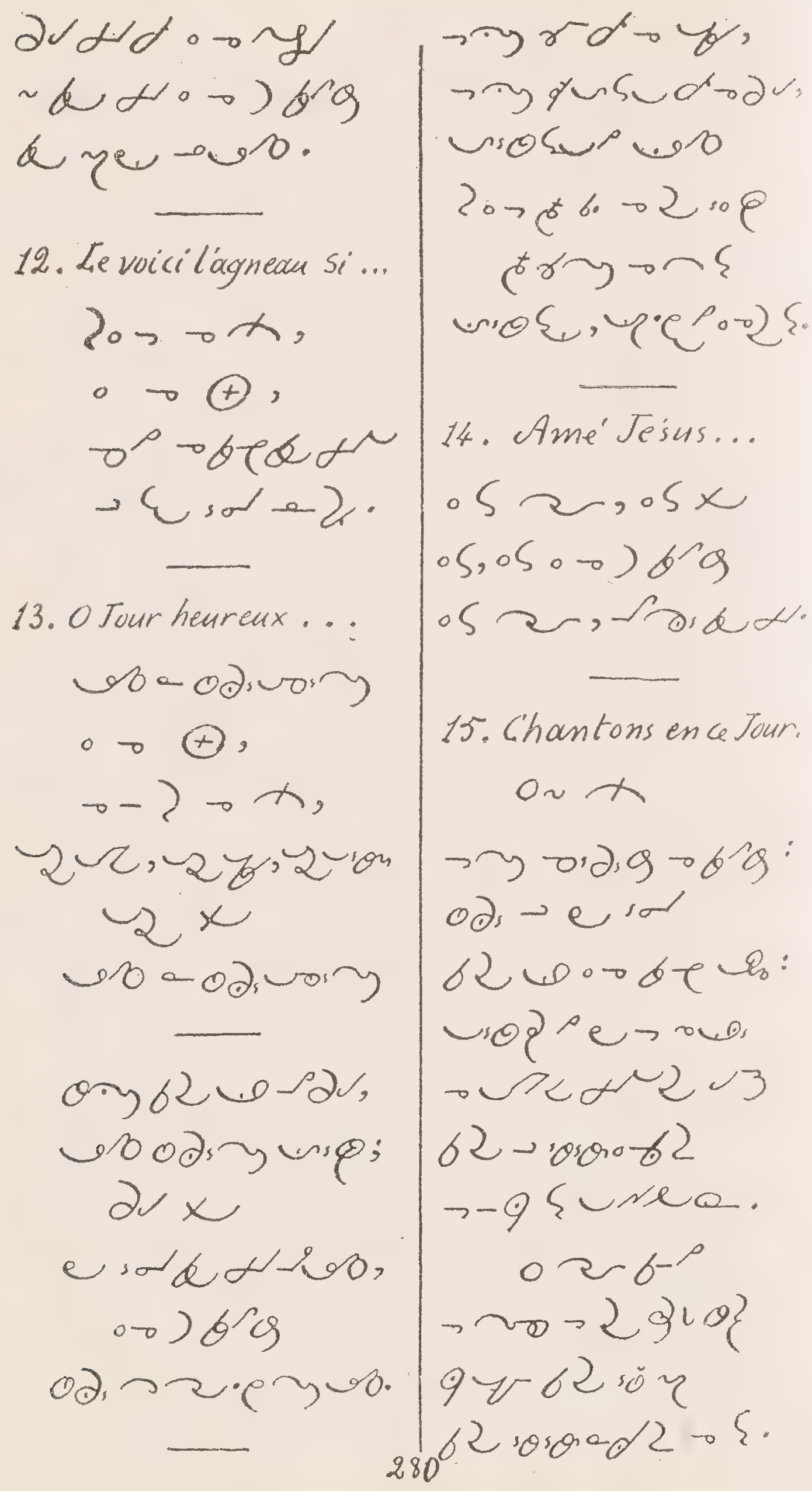


MANUAL

89

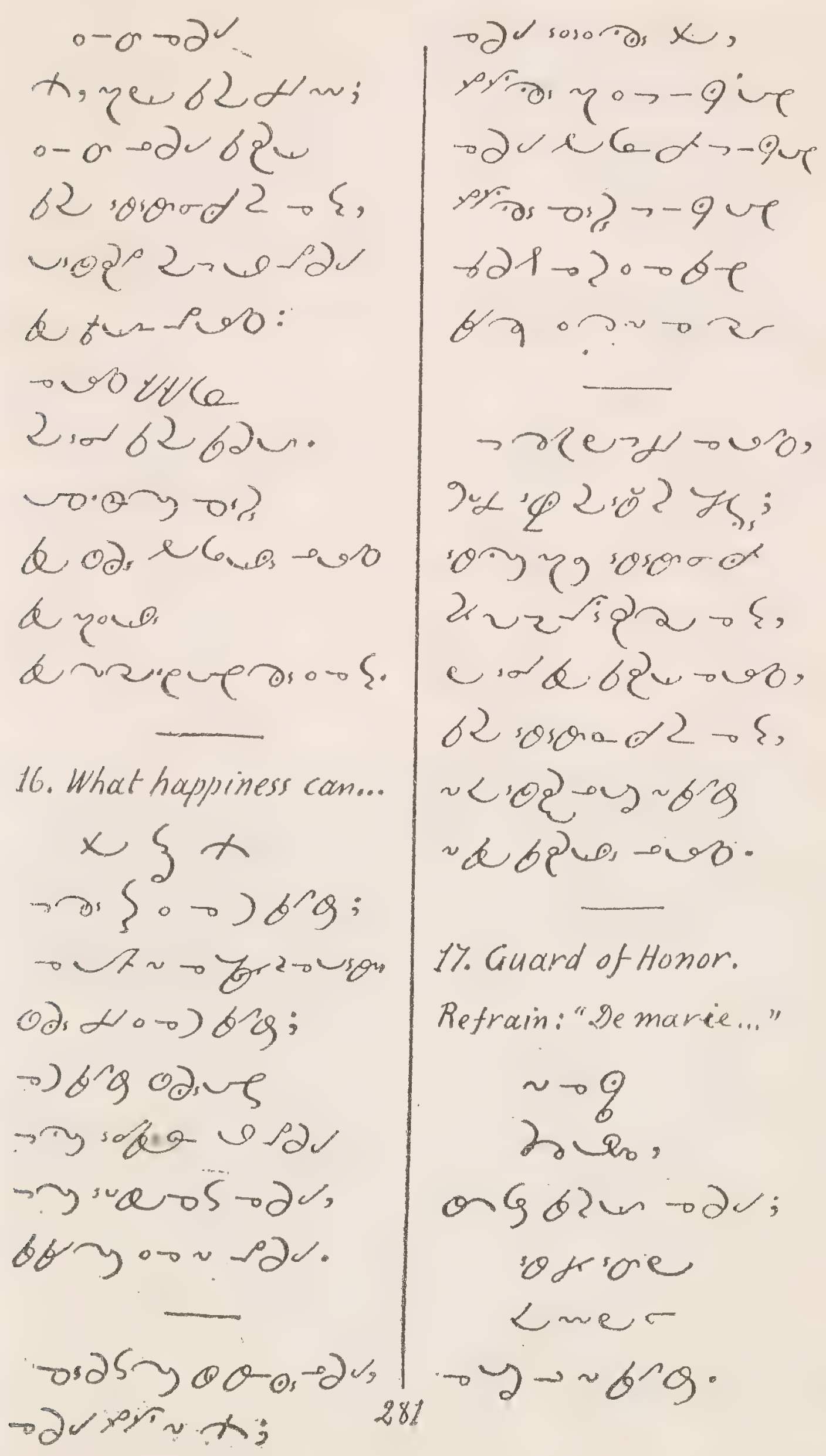


90

SHESHEL

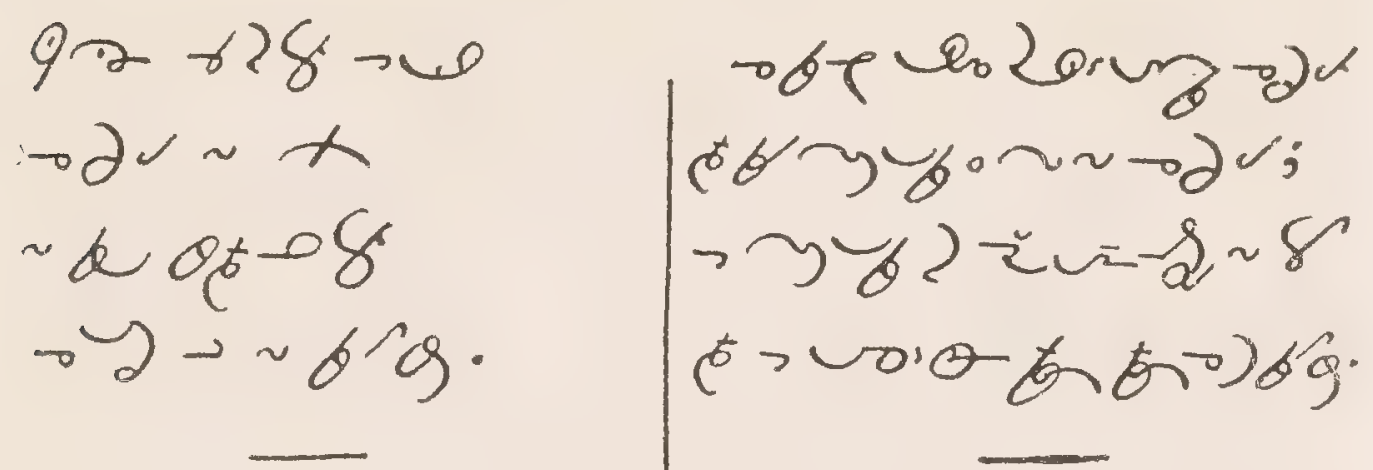

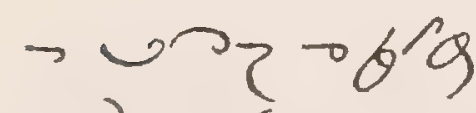

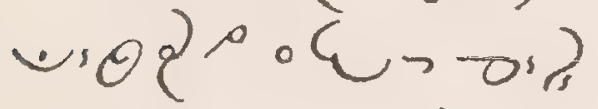

- e sat do bo

oर osenos.

$\sim \alpha$ fog -28 ,

o.- 2 bra

cist - cora

Whand big.

18. Divine Marie,...

$0 \sim 8$. 6 becer

62 y $62650-095$

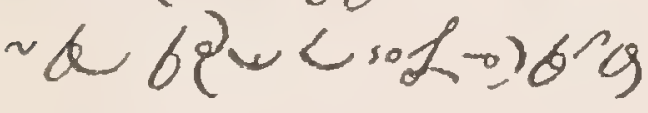

जo?

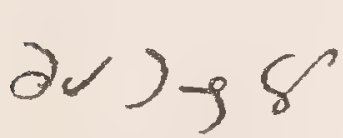

$-2 \mathrm{so}$

$6200-60$

$\sim \rho_{0}$.
19. Devant vous, esprits.

1. $20 \times 0.08$

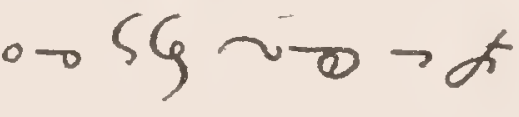

de e sorecsou? ?

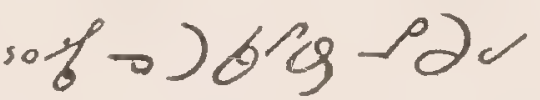

प

2. $\sigma\{x p \sim$

Wdugor $Q_{0}$,

च《之क) Dor

टथk $-5 \zeta \sim_{0} \alpha^{\circ}$

muning $x$

$\sim 0 \operatorname{sig} \rightarrow 5 g$.

3. $2 x-y \sim 2$ zion

-uion -5 3 st.

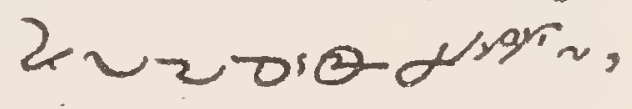

tworob $e_{0}$ :

imun $x$

$282 \sim 0699-59$. teloorb-e 
4. 59 usoporon -6 ) $x$

sofroce curidu n जर 9 - a

mury $x$

$\sim \circ \sec ^{2} \rightarrow 59$.

20. An fond des brutlants.

r 30-overara,

$\rightarrow 2 \operatorname{sos} \theta, a n$ ing:

- Q \& \& reo',

जop? $(60)$

$$
0 \leqslant, O\} \text {, }
$$

wogolespur,

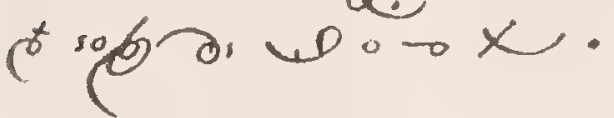

$0-6 \partial 12$ voro,

० 680801 ;

- $-0 \sim \sim \eta 01$

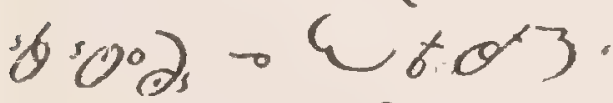

2noo, 2mo,

or. tafor, dob $p-g-28 \min \theta$
21. The Magnificat.

-2 an $\forall b 0 \div 0$ D. $0 \times$.

ᄀt大 0 ) bis ${ }^{*}<0$

o 0 ) 2 som.

- $\rightarrow$ os of) b9

. 102 the * "og

2 sabsore

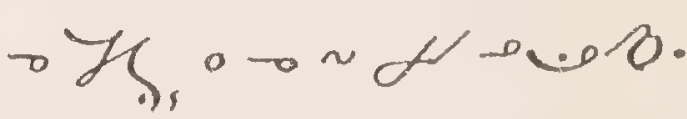

$0 \rightarrow$ t ree, woor.

$\eta-a, x_{2}-\boldsymbol{C}^{\circ} \sim$

ex yuscore :

q $2 g^{x}-\sim 2 \cdot c$

$0-2$.

$\rightarrow$ laver.usol

$4 z^{*} \rightarrow$ hina obe

$\rightarrow 6 / 2$.

$\rightarrow$ sirsa

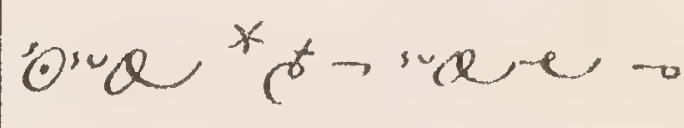

$\rightarrow \operatorname{sot}-6 / 2$.

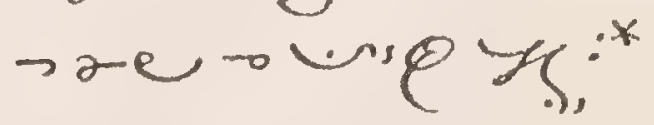

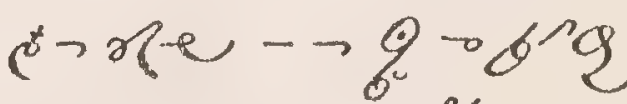

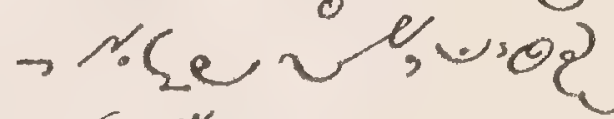

- 2 : * sol para 
enco orido бC Dód: ipoe $2-\rightarrow+4 \xi<5$ bione.

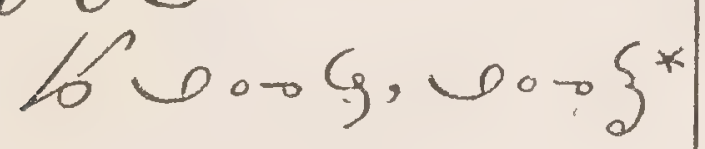
$00-3 \sim 9$

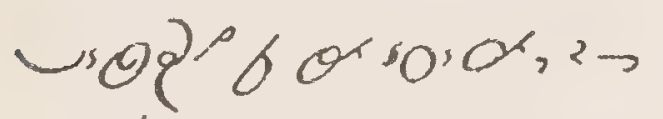
ก⿻; 2 2sabsoe $\sim \operatorname{sog} u$.

To the Holy Family.

ox.'- arsad - $=$

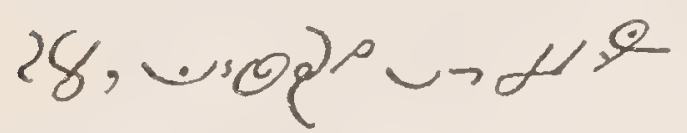
- be a, b- dd -

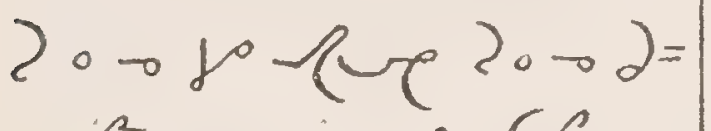
$\sigma^{2}, y i s \delta_{2}$ उ2, sof 0 bs Q⿻ duspece of o Ru, a rरver.

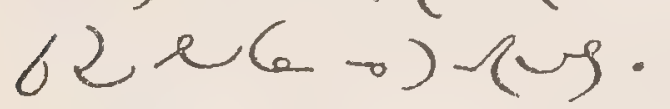
aino -0$)-(-9, \sim d$

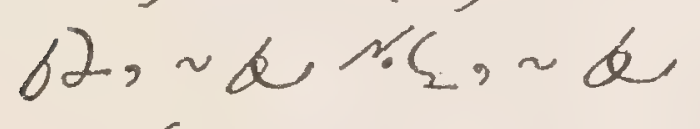
Nele, b w wor 0284

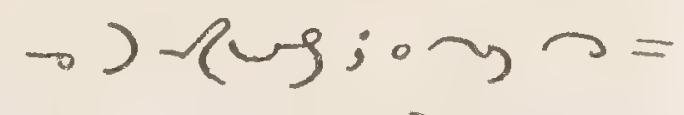

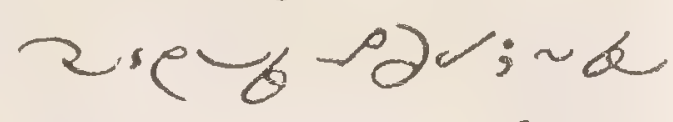
Wris or) pus $<$ yer zu- $<\theta=$

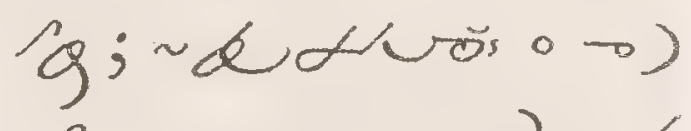
pus a wor $\alpha$ $\rightarrow y$ Reg 20-20

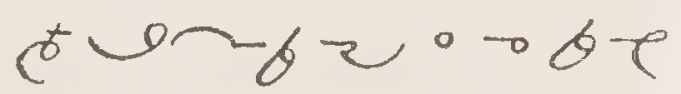
Q

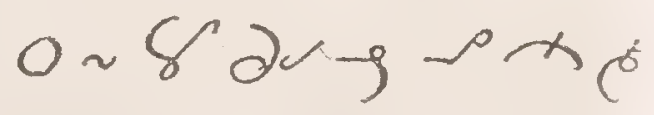
भo $\partial<-\rho, \sim \alpha$ $b-x<62 \omega$ La.. pug; 0 C b'g; $⿻ 60$ soguore o 公 $\sim$

$0 \rightarrow \partial \dot{j} 32, \partial$ $-6 \partial y-x<r_{0} L$ $\because x 2 \sim 820-$ Q pig 2 - $286 \mathrm{c}=0$ Ha ze, zus * Soct a sisox. $\measuredangle \sim r$. 
MANUAL

93

Gatechism.

r. God.

1. a clevkd's

or rosuron?

= be are lend

c a Mue.

2. $20-b^{\prime} q-x$

U2 eदs

zNhy? =xe=

Ged, $C^{t} \rightarrow$ Nese,

$62 \alpha$ ord, -2, 6

2 म sol') - 2, 62

मून-2, जiक?

$\sim<\cos 0$

be $-b_{0}$.

3. $a-0 x ?=0-$

b' 0 - x, s a 20

c son, $94 \sim, 92$

r. $6 \mathrm{~L}_{\mathrm{c}}>-9 \cup e$.

4. गरe ग००

$b=b^{\prime} q-x ?=$

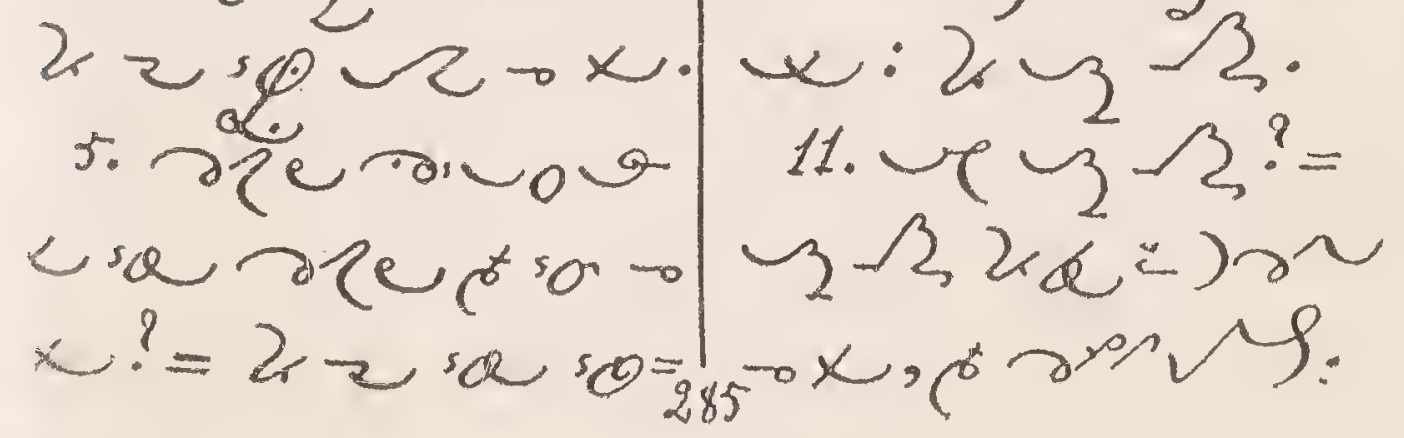

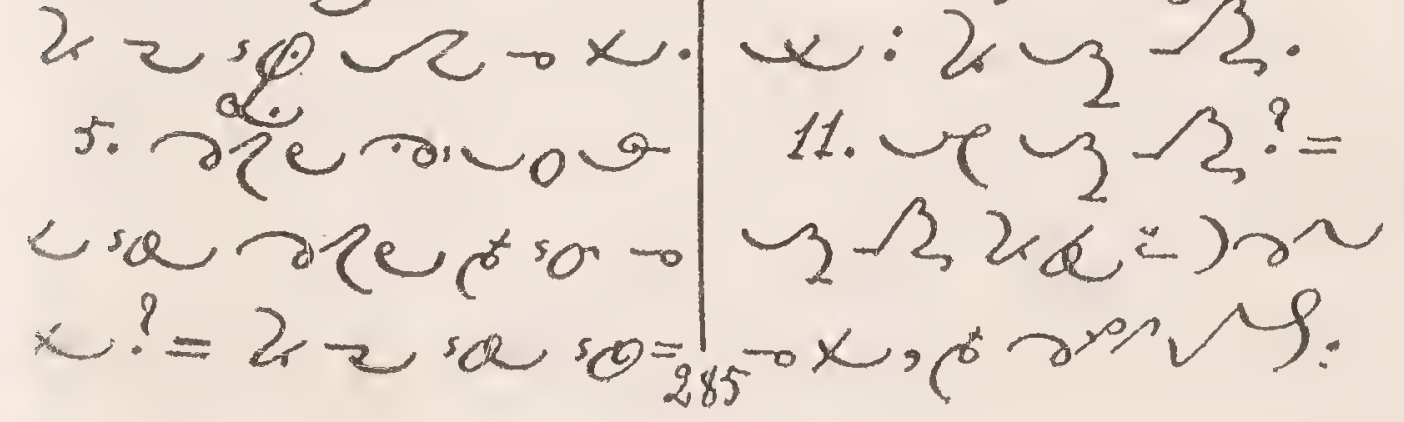

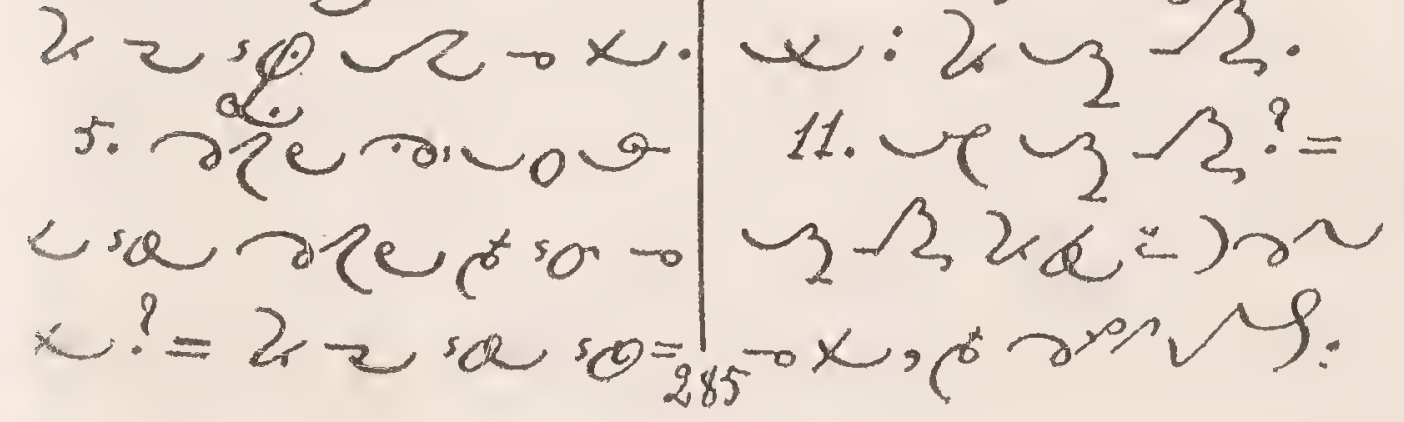

re a d sar

$x$.

6. rरerura

$<2 * \sim-x ?=2$

$-9 \sim 20-x,-2$

rovos-9ur

\% drerua

<or. $\rightarrow-9 \cup r$

$x ?=2-6 b^{\circ} \circ$

$x=-9 \times$ ?०

$\sim \sim$ or sos, $\sim \alpha=$

千 20 .

8. 63 $0 x ?=$

$x \rightarrow 200$ - $e_{0}, 2$

$-9 P_{0}$.

9. bod uso ox ? =

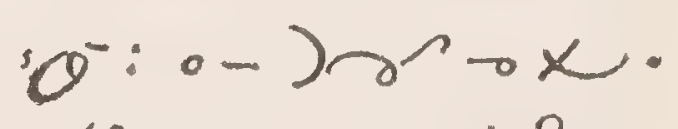

10. co bid o v9.

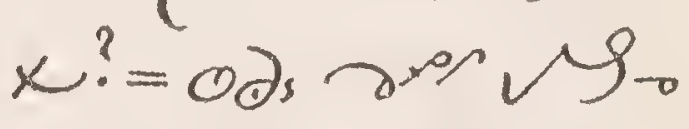

$x: \rightarrow\},-\{, \circ$

w: $2, \beta$.

11. $-3 / 3$ ?

$\Omega, 2(\alpha=)$
II. Trinity. 
12. $\rightarrow G, 2 \circ \times ?$ ? III. Jesus-Christ. $0 \partial s \rightarrow \xi, 2 x$. 13. $\rightarrow\{, 2 \circ x ?=$ $0 \partial s \rightarrow \sum_{2} 2 x$. 14. $\rightarrow x, 2 \circ x$ ? $=0 \partial s_{s}, 2 x$. 15. $\rightarrow \xi,-5, \rightarrow$ 火, 2 sp $x ?={ }^{\circ} \theta$ woqe: rerv, c $=2 r x$.

16. 0 - 20 \%

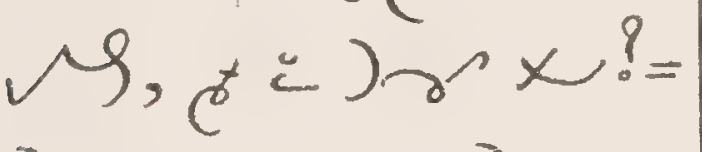
2 zenestr $\rightarrow \operatorname{ser}$ is orio $=$ 2े . 1\%. ory, $\theta$ $\sim \rho \sim, \sim \sim \rho \rho_{0}=$ $r k, \theta \sim r y ?=$

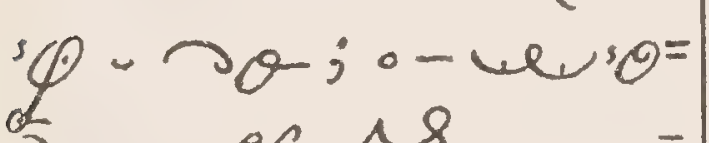
$\sum$ or ses. sogr

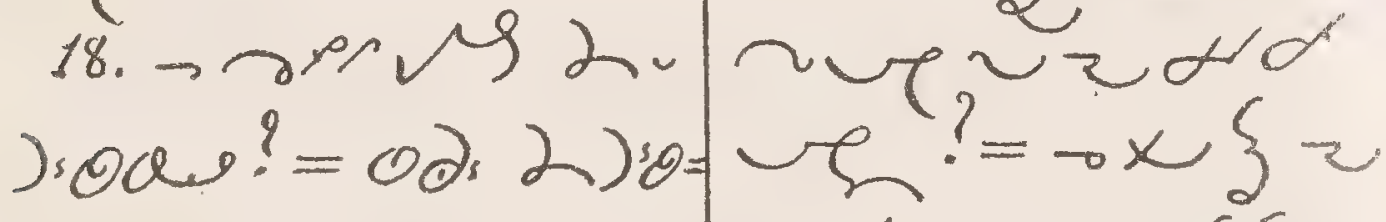
19. o or dry 几 hlor ? = $2 \rightarrow x \xi$. 20. $2 \overline{2}-x \xi, n$ $\rightarrow \alpha d \cup L_{0} \rightarrow Q_{0}$ ?

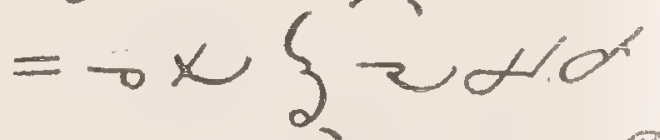
L $\rightarrow$ ode ove rcion suse? o) $22 . \rightarrow 200 \geq 6)=$ eorx $2 \rightarrow$ son? $=\rightarrow x$ $\xi=$ bdedive rovion $2 \rho$ s - W so-e os. $23.0 x\}<=0$

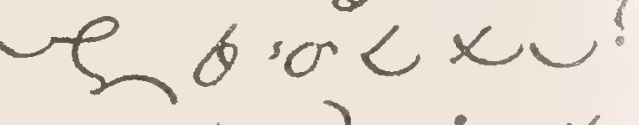
$=$ is visoge: $\rightarrow x$

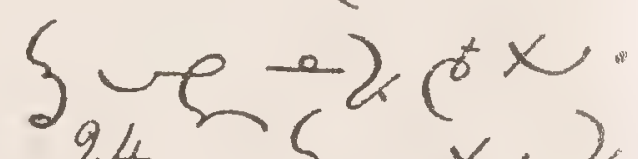
$24 .-50 x 2$ $a:-3 r, s u=\alpha d v h-068$ e $\rightarrow$ ara $\left.2860^{\rho} \circ\right\},<\sqrt{0}=$ 
Suo-be $a_{0}$.

$25.0 x \xi 6 z \theta$

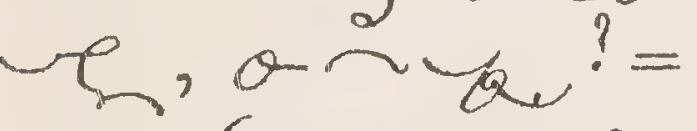
$\rightarrow x\}=$ or 大八质。

26. $\theta \rightarrow x ?=-x$ $2,\{, x>0-e$

2) $\partial 2 \simeq 20$ $680 x$ ? $=0 \cdot x$ .068 6 z 2 $-96$.

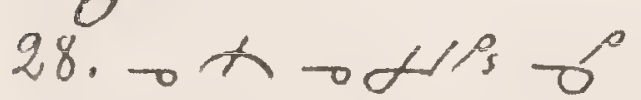

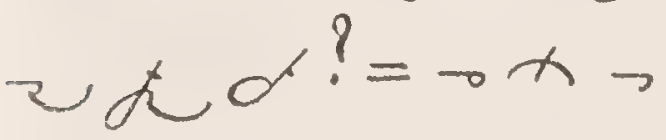
च० or

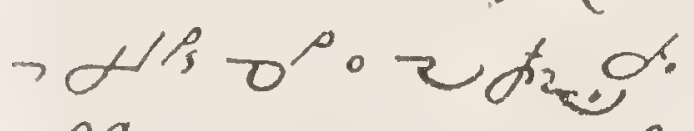

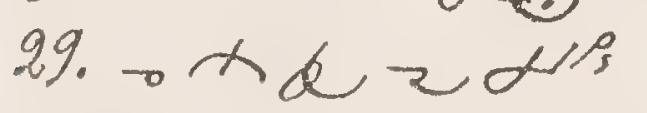
o 0 zing, $\rightarrow O^{s}$

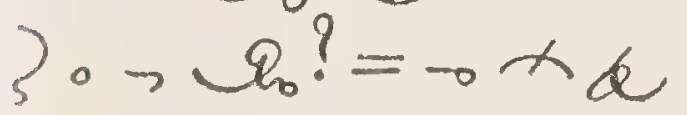
edrs osoztimor 《oydz? $\because a_{0} C^{t} \rightarrow 0_{0} \rightarrow$ be 9 . 30. 0 x $\%$ 名 $\mathrm{a}$ Ho $\rightarrow D_{0}$ ? = Oे 5 - 28
Q d b bur oori

$y_{s_{5}}{ }^{2} \rightarrow y_{2}$.

31. 2e px-

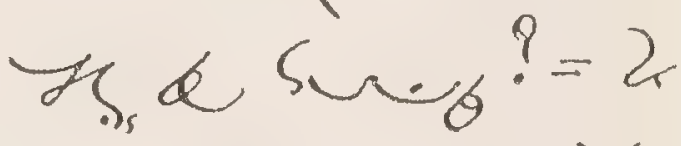

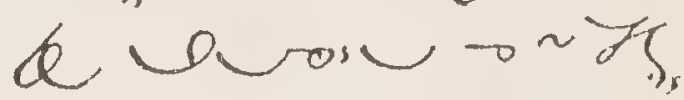
- - be $d_{0}$, है बर $\rightarrow\left\{y_{s} \circ \wedge \sim \gamma\right.$ IV. The Church.

32. $\theta$ O $=0$.

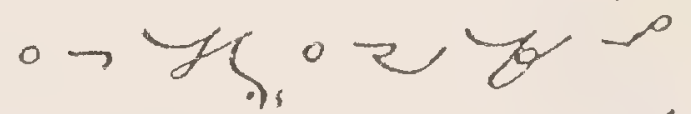
大, ze山० $\rightarrow$ wot $\omega_{0} ?=$ $22 \rightarrow-b^{2} e^{\rho x}$

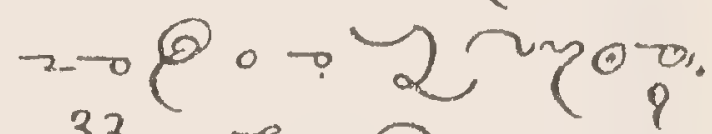
33. $\mathrm{x} \rightarrow \mathrm{n} 2 \mathrm{or}^{-\mathrm{s}}$ ?

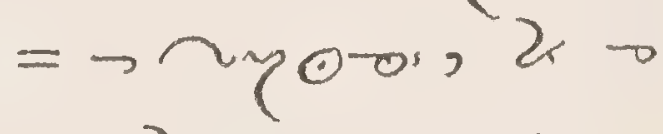
$\rightarrow 0$ dur $\rightarrow-\not 2$ $-6 \alpha e-\rho x-m$ $\rightarrow 2$ นn०. $34.00=-62 \mathrm{C}$ $\rho x \rightarrow 00.2$ حyo- $?=2 \rightarrow 7$, 20 $35.650 \rightarrow 2$ noor? 


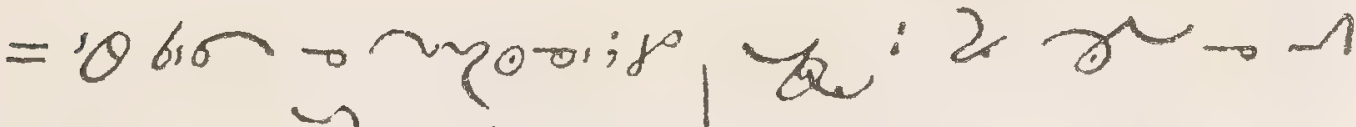
20, $2 \rightarrow x \sim n=$

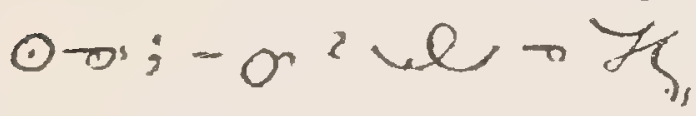
- o or $\varphi_{b}$.

36. 2 म लn०ल ose? $=2 \rightarrow 60 ?, 5$ $-N \mathrm{~g} /, \rho_{0}-C_{\text {, }}$ zuog y רחים

37. $\theta \frown$ vive D

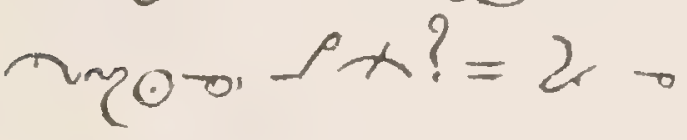
大 v"Q o 万Yor"

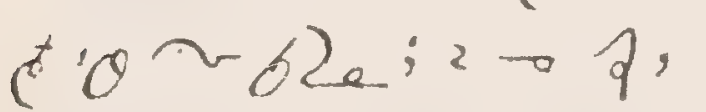
2unc - oo- b2a.

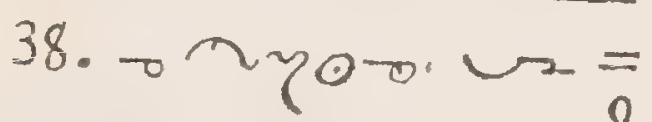
o o b a - a t ? $=02 \rightarrow n_{200}-v_{2}=$

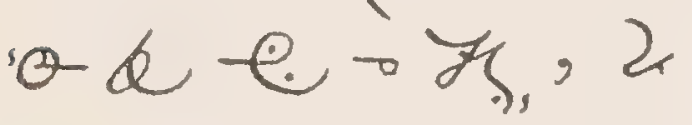
- $x$ u $\rightarrow$ =

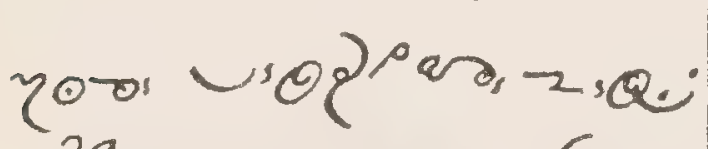
39. $\sigma$ ح页一个。

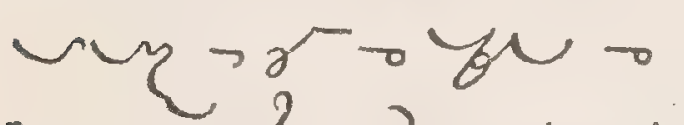
myorr? $=2 x+b+b=$ so, $-62 e-5 e$.

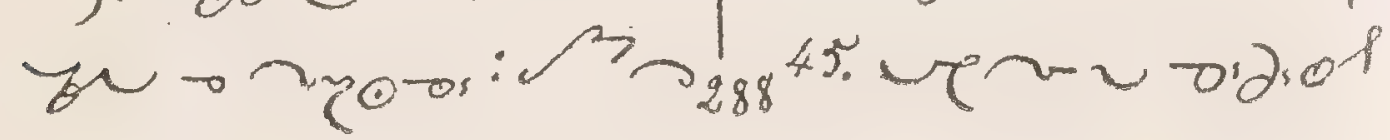




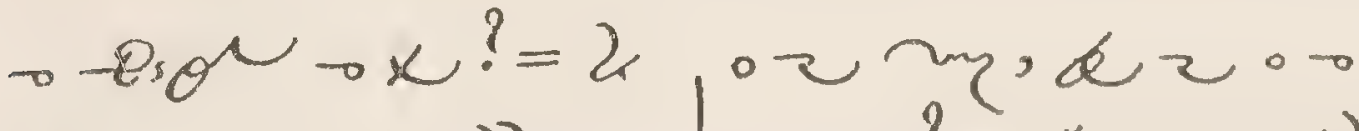

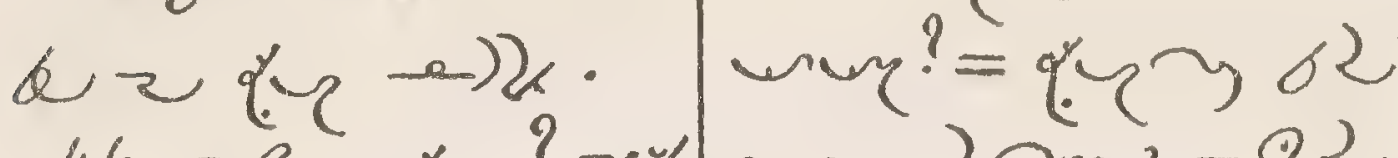
46. วle qu? =s

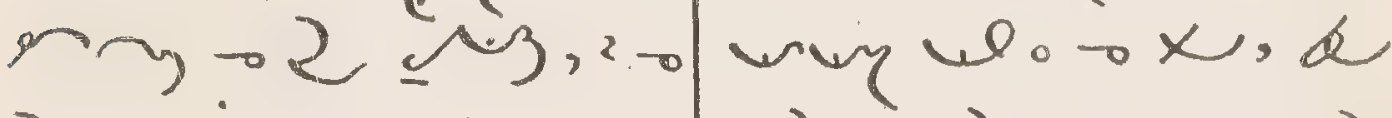

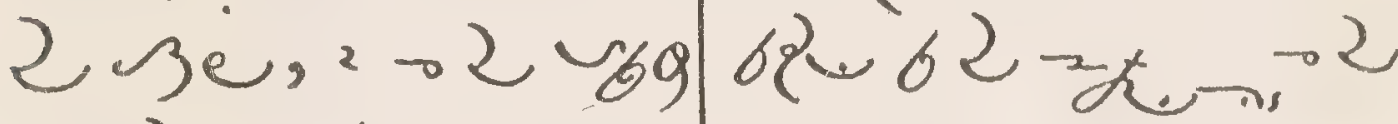
$2 \rightarrow 2 \sim / \mathrm{Sg}_{\mathrm{g}} \rightarrow 3$

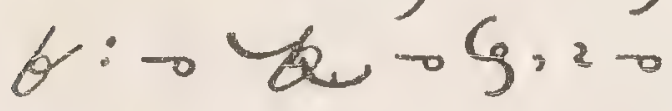
$\{, 2 \rightarrow W \cdot \sim<, 02$. 4\% $>\mathrm{sog}$ os $q^{?}=\log \rightarrow-z$ $20 x=\alpha d \partial_{0}$ of.

48. 22 2iog -2 $\beta ?=2-6 b^{-0 a}$ $\Rightarrow$ ope $\varphi$ o $\xi$, 0

$\{, \rightarrow \psi \cdot$

49. ग2र.06- $x$ \& $2 x d ?-g$ ? $=$ $262 \rightarrow \frac{9}{6} a$. 50. 34 है ky?

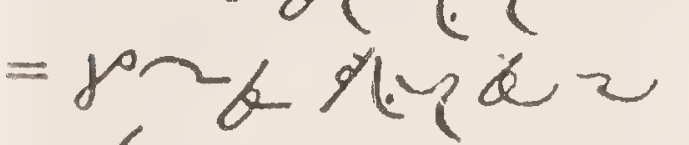
- L ح , \& z.0 - L vur. ovie

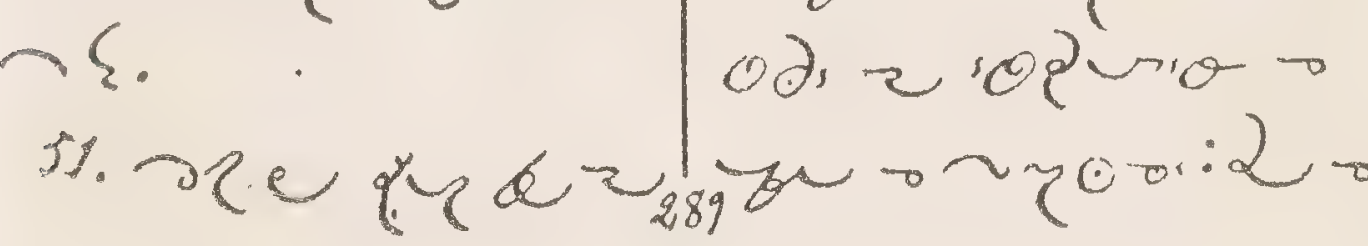

52. วरe \%क्र Qs - $\rightarrow\{$ ? = d 68 . $\rightarrow x 62 \cdot 0 b^{2} \mathrm{e} s$. VIJ. The Commandments.

53.6 20.08m= ' $\theta$ or $\rightarrow x$ ? = 2 - bovioe - 9 yc $0 x$.

54. - 9 y $0 x$ ? = L

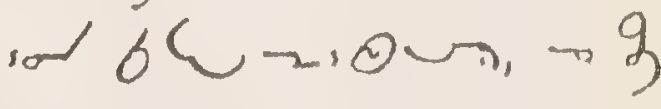

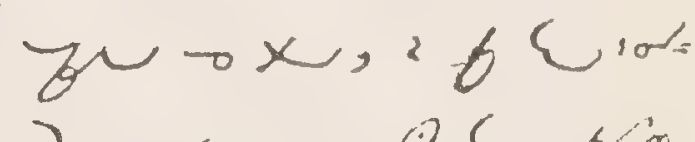
d ox.0.

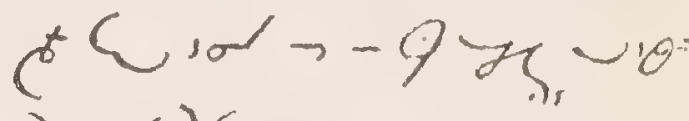
2 4$) 8$ 55. 2 र० wopm. - y - 万y०0 ? ods zopura 0 
xb9b4iogn, yod ton.

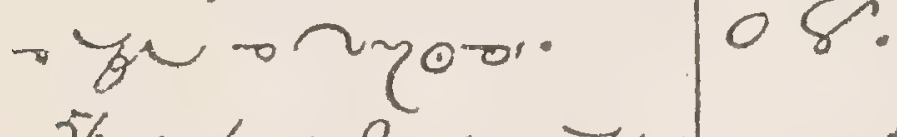
56. d wer tou IX. $\sin$.

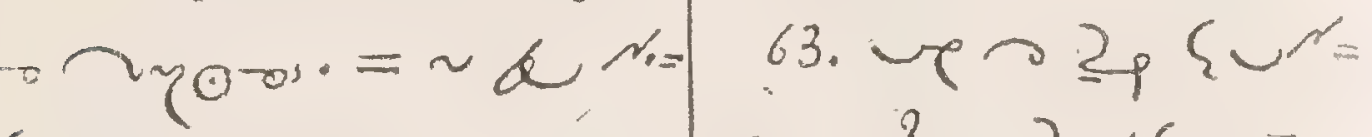
$\Sigma \rightarrow \sim y$.

VIJT. Prayer.

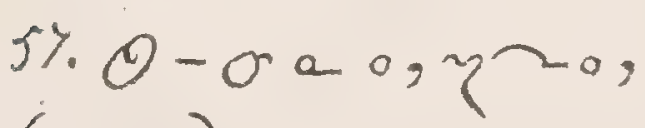
ea? = $2,4 \sigma 0$ zox $0 x$

$64 \cdot g \rightarrow$ \& viea!

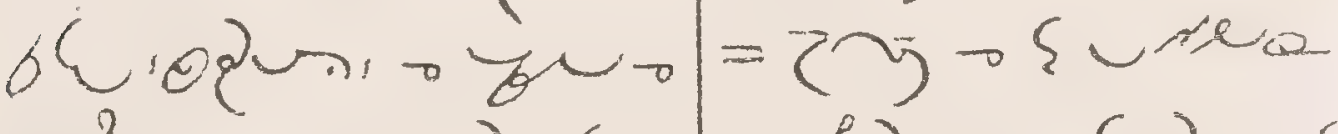
$x ?=-o b$ bedes - $x, 2<, 0$, $6<$ og n, $\rightarrow$ ar $x$. 58. $2 ?-68$ - 680 $. x ?=2 \delta \varepsilon \sim \eta$, :bC62 -

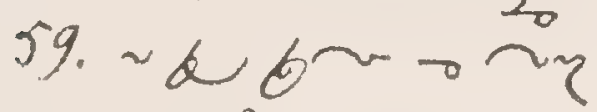
$\circ r^{\circ} \sim=2 \cdot r r$ $\sim \rho \operatorname{og} \sim \alpha$.

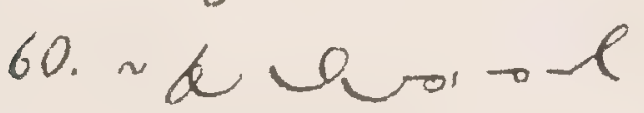
$n_{x}=(\cup 2) g \cap 0$ $\therefore b 0^{\circ}$ 61. $\sim$ d b $\rightarrow \sim 7$.

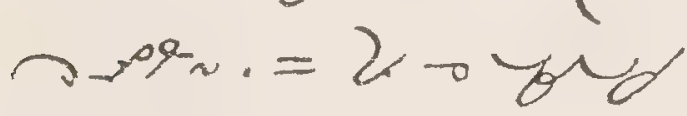
on wer $49-$ 入. $\sim f 2 \rightarrow \gamma\{20, k$ son $6 \& \alpha 5 \xi$, tod $2 x \rightarrow\{$ rea. $6 \%$ - $0520-6$ ion $6<\alpha S G ?=2$. $s \rightarrow b^{2} e d-\{\zeta A$ a zare-ar $x$.

66. $\curvearrowright 29 \sim 4$ $\rightarrow\{$ cree? $=2$ $\sim(2) v_{6906}$ $r+$ a 0 .

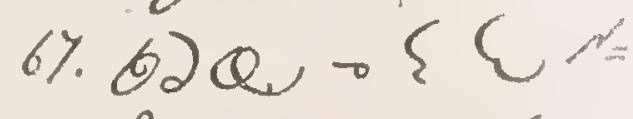
le? $=y^{-}\{c$ sea: has to - Eson; ta da -

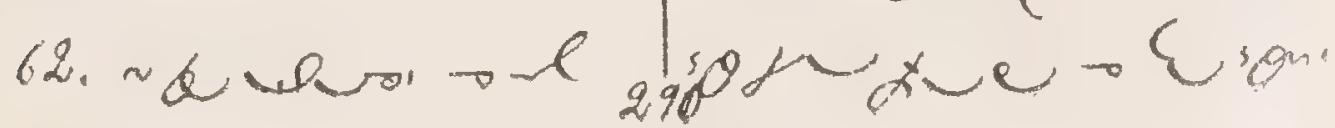


MANUAL

99

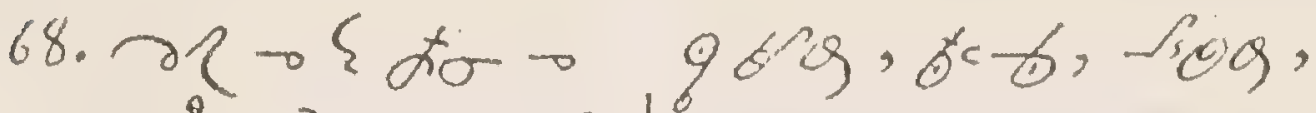

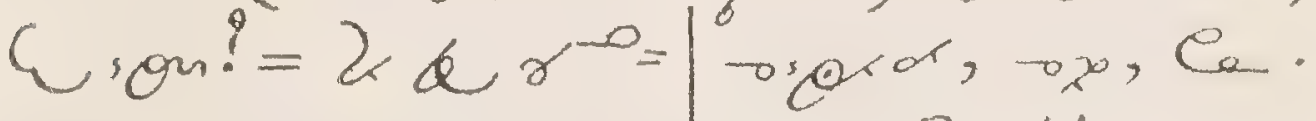
sox, e a $0=$ $X$. Baptism.

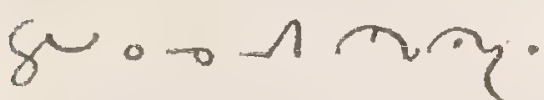

73. 22क $0<4=$

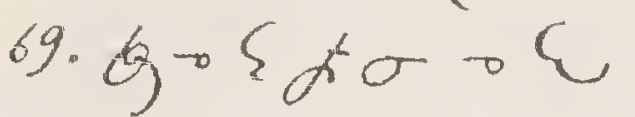

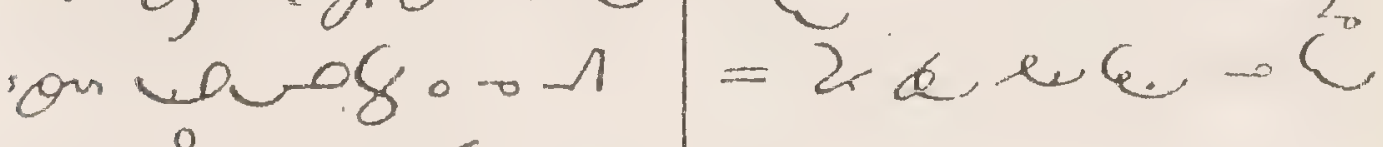

$\pi r^{?}=\gamma^{\circ} \rightarrow\left\{\alpha_{\sigma} \rightarrow\right.$ son.

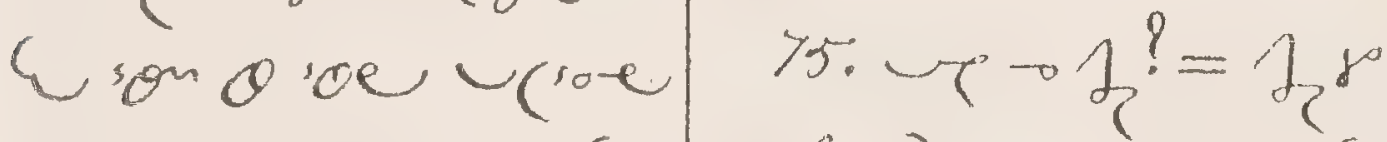

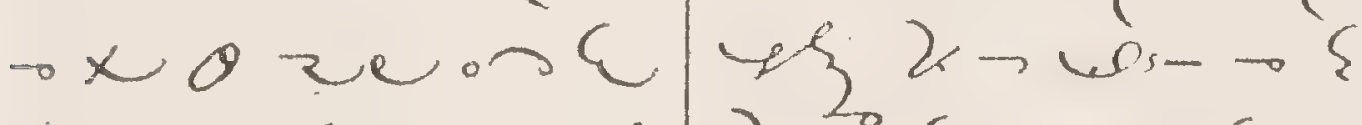

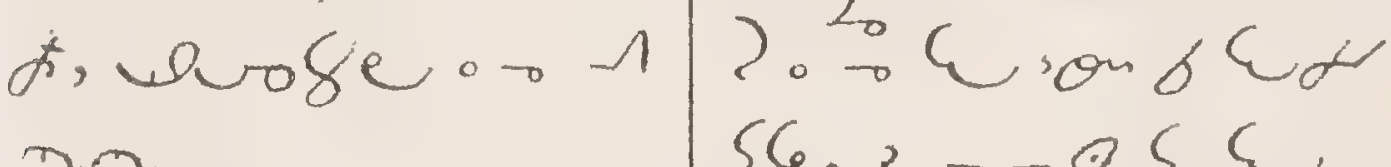

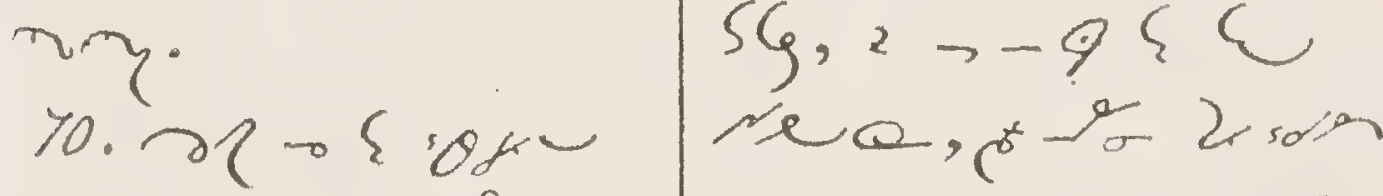

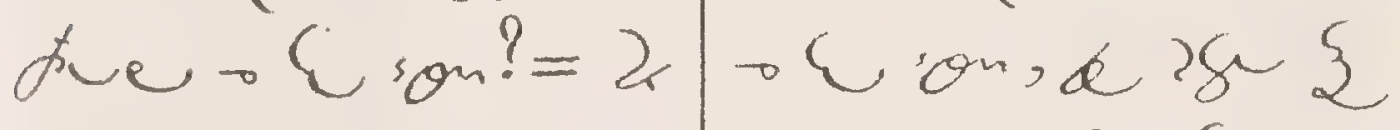

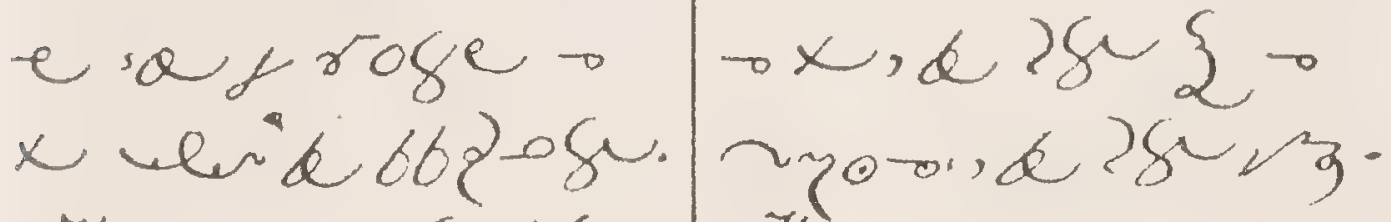

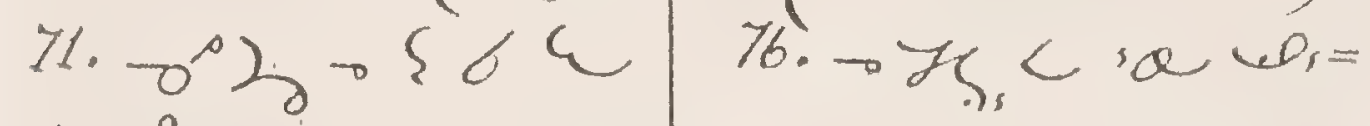

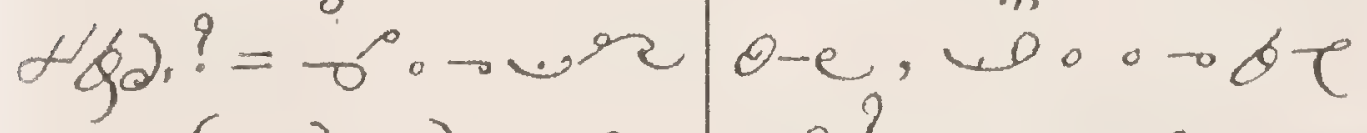

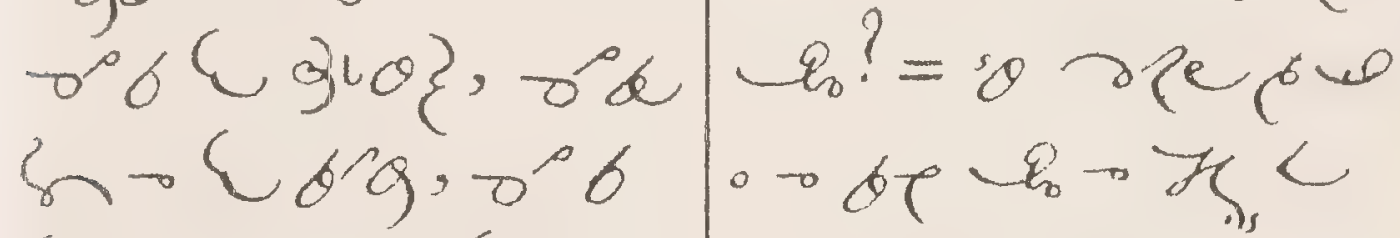
¿b6, ob Lw = "a woe.

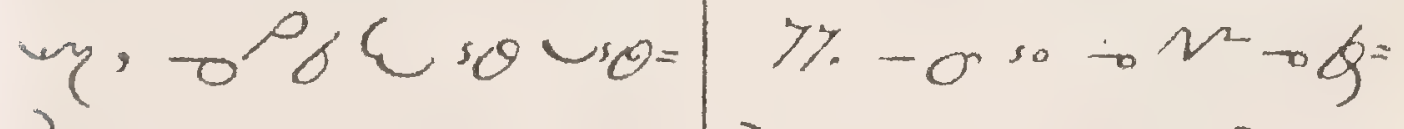

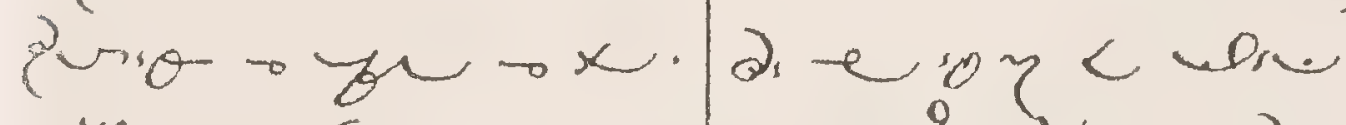

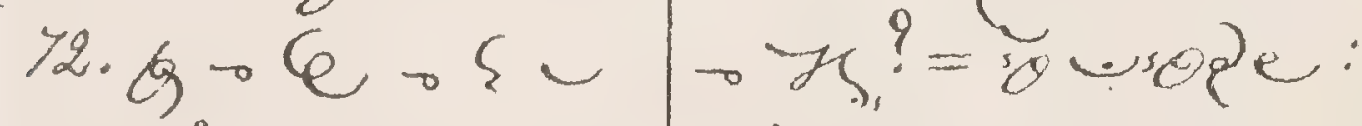

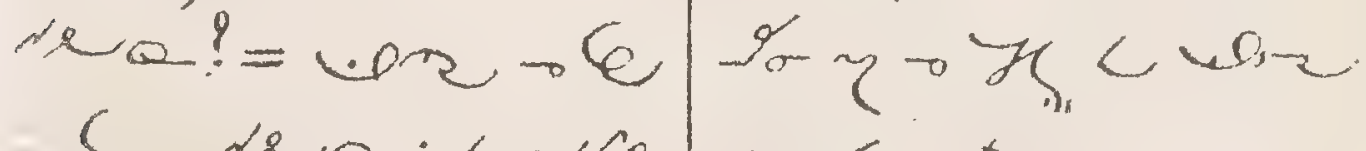

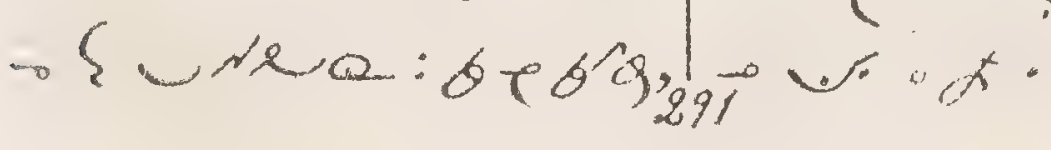


78. 206 - th, orpe? $=$ or e $^{\text {ro }}$ wa $\circ$ है - ane

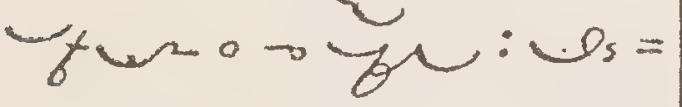

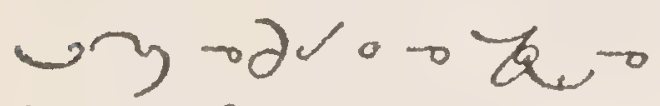
g $=0 \xi, 2, \psi$

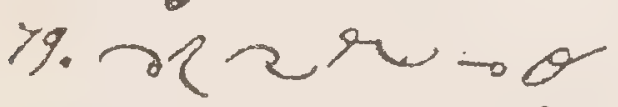
$\sin <$, r 2 ? = $\rightarrow \gamma \rightarrow 0 \partial$ जार y ox; - wer - $\{$ \xe; $\rightarrow 0=$ wax ore

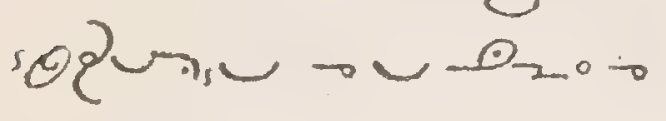
niner. 80. $4 \rightarrow$ re o $\mathrm{col}$ ¿ une? $=-\varepsilon$ sor CU,erar o re: ore ra; re - 2 - run; re oy reog $=$ ore ofy.

XI. Confirmation.

81. rop parer? = oderoys age

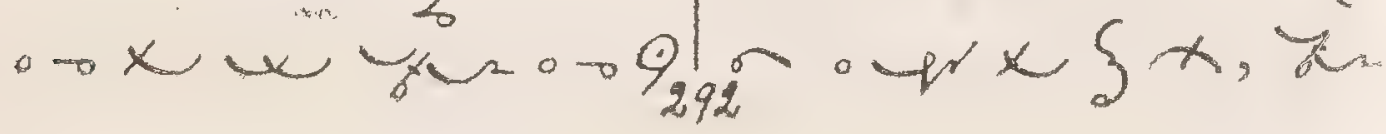

2 qu, rog bCised 2 . - $x \cdot e^{t} \rightarrow \sin 68 \mathrm{e}$ ocon s.

82. $\theta \frown \operatorname{2og} \circ \cdots$ aleo? = - orom arogoraker.

83. 2. 0 ? <aog o o che? $\sim \sim \sim_{\eta} \rightarrow \partial \omega$ - 62 opleo, -

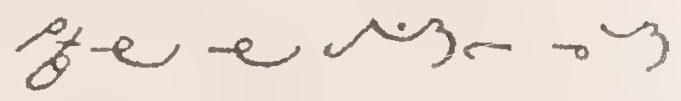
rer role. 84. 2- $-6-3 x$ Cso < $62 . \rightarrow=$ Qhe? = soee ¿to - Cion; ? Didia जo\& - tror - x; $\rightarrow$ bo < 62 ... XII. H. Eucharist.

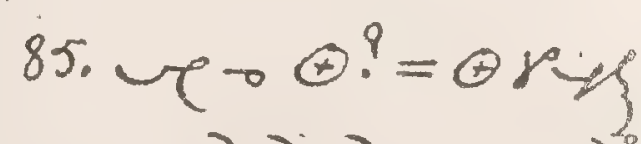

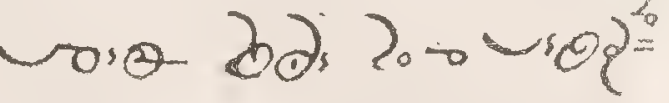

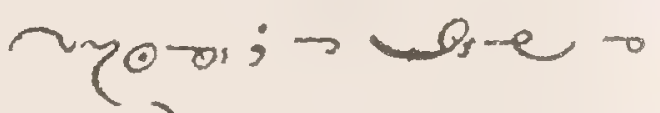




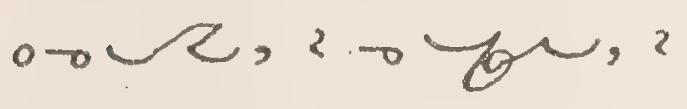
- vion.

86. o- seled o $\Theta ?=2 \rightarrow x,-\infty 22$ $c \rightarrow$ euc $-\oplus, d$ b.

8\%. ग2 0 x< $8=$ $\alpha_{0} \theta ?=x-3$ \%

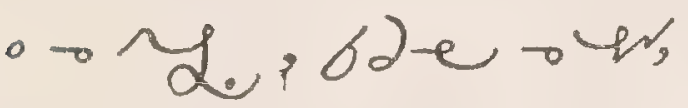

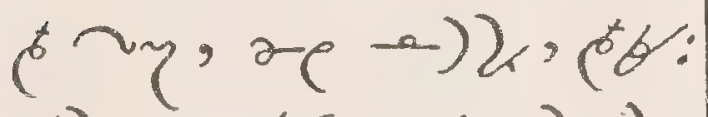
b) $\rightarrow$ क षैल भ, 22 $\rightarrow \mu l .06<2$.

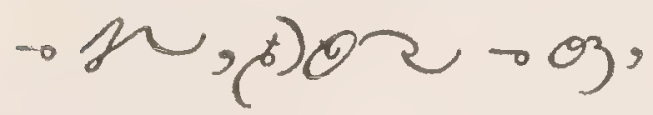

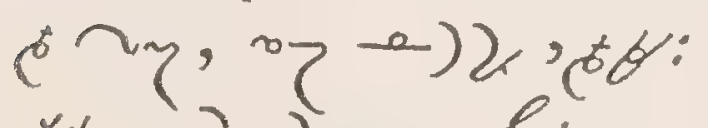

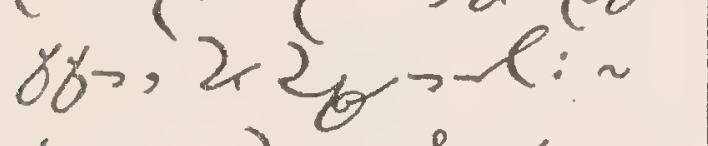
dusgus 1 , b so = des

88. $-y=x 22$ $\rightarrow, l, 26>-l$, wsog. o wur? = $-b \rightarrow x 2,2 u n$ ods $x \sim>\rightarrow, \rightarrow a=$ "garend; - y × 2 2, bu $0 x,-\operatorname{ang}<$ $03 \%$.
89. $2.075 x$ wer enc, 2 or $<2$, bu, wos $=$ Q : $0 \mathrm{C}_{6}, \mathrm{yer}_{2}$ og ? = $\log ^{\rho} x$. ere $x<2$,

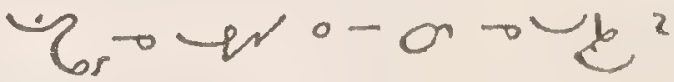
-usol ous? ? w nor $x-003<2$ be, $-2, \rightarrow 03,0-$ or o vag o o o 0 . 90. <2.1 10 2'sen our, $-2=$ e $\sim \mathcal{L}$, to so 1 . $\Omega ?=\left\langle 2 \Lambda_{2} \rho\right.$ $x-z$ sod ou , sor s. $2-z$ sow 91. $2 \operatorname{ang} r \rightarrow x \rightarrow$ we $\sim R, 0-\sigma$ s $\longrightarrow<-?$ ? $=<2$ $\operatorname{ang} \rho x \rightarrow$ yer wU, :o-oe $\sim 202 ; 0-9-2]$ gur wion: 9 $2,3 x \rightarrow$ ? 


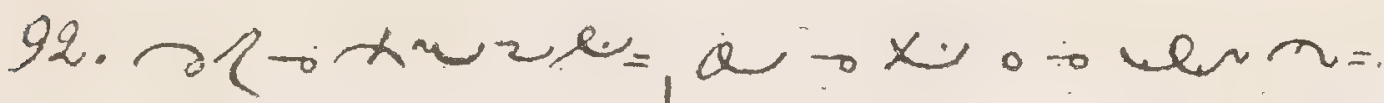

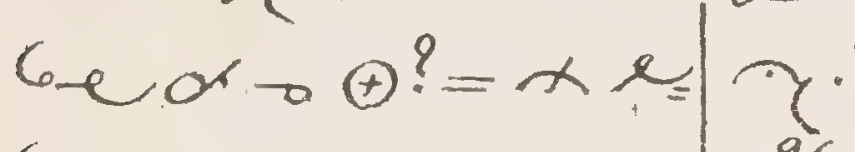

aet $\oplus$ Cing sase $\rightarrow$ zor $2, c$ $2 \operatorname{tat} 2 \rightarrow \frac{\theta}{6}, 2<$ $2 \sqrt{2}-\varepsilon-\varepsilon$ ion.

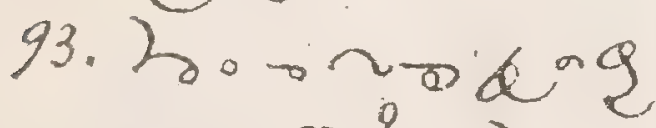
$\rightarrow \cos ^{2}-9,9=2$ z -

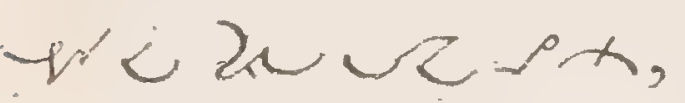
$\rightarrow 03<2$ 西为 oporims $\rho$. 94. vPG, r so $2 y$ U. $0 x ?=\smile \rho G$, sy $y \circ-9 \cap y: z$ pluson "urs x; vornim-2; Won $\left.6 \theta^{\circ}\right\} \backslash\{\varepsilon=$ wa; $\rightarrow x$ pe $2>-9$ ن2

$$
\text { 95. نPG } \rightarrow \text { - o't }
$$
- o vion? = Uí

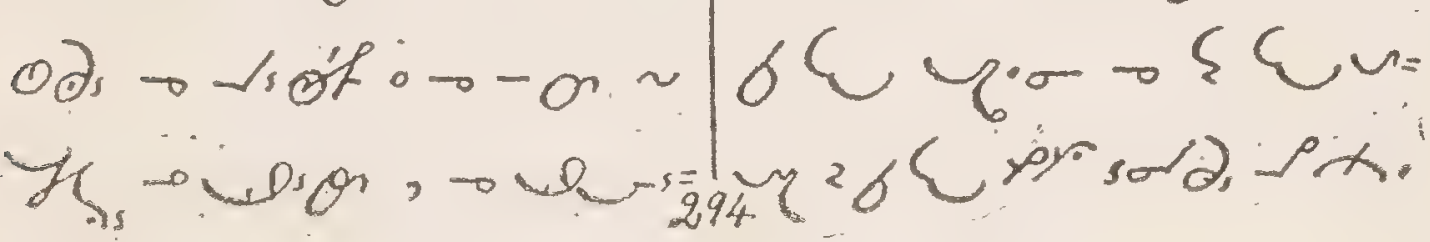

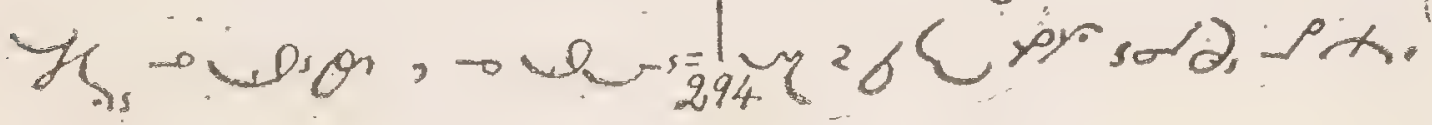

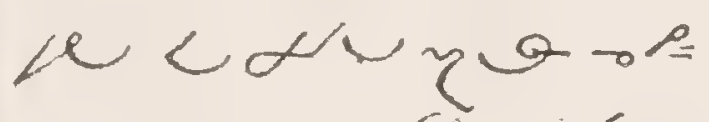

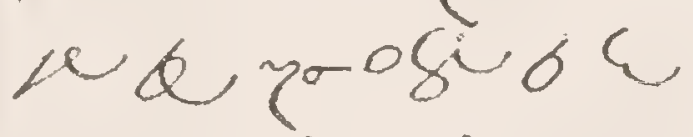

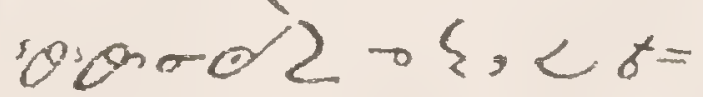
vogesx.

9\% $\rightarrow$ wod०<ek -) $2 \times 9-662^{?}=0$ wor< luk. on. 0 . कर $62-a ; c^{-}-$spe ? 0 wabe of 2 $-62-2 ;$

98. गर $6<62 \rightarrow \Theta^{?}=26$ Cele coson? $\rightarrow\{\cup>$.

99. 2-6 264 ele $\rightarrow$ son? = 2 
MANUAL

103

100. 2。

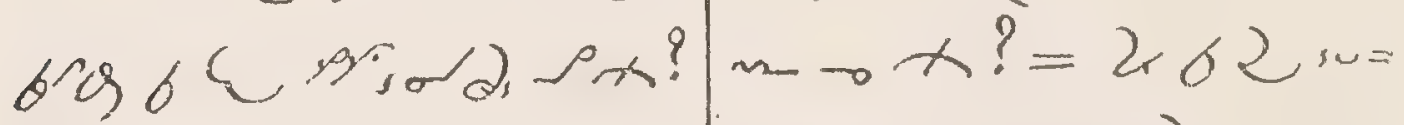

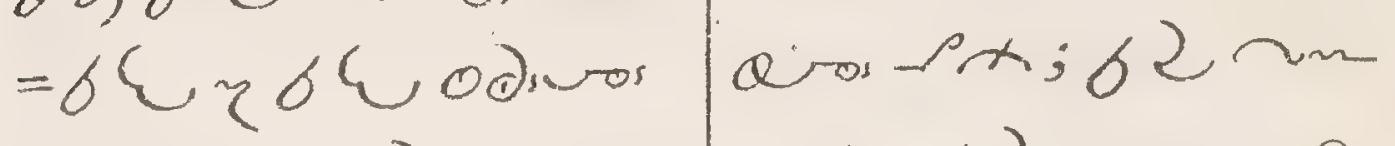

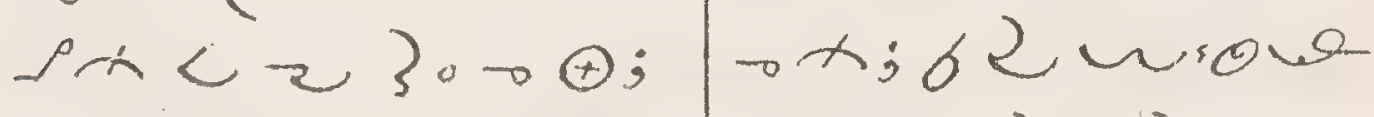

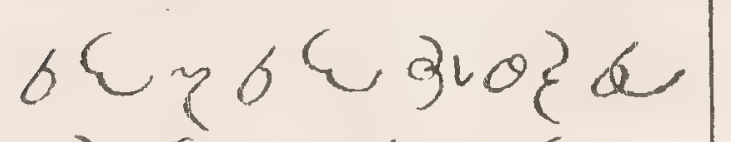
$62080 x ; 6 c$ sx: 62 68vos

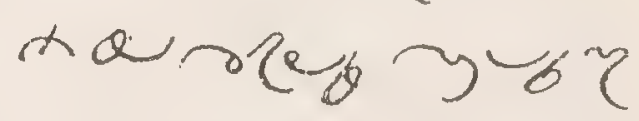

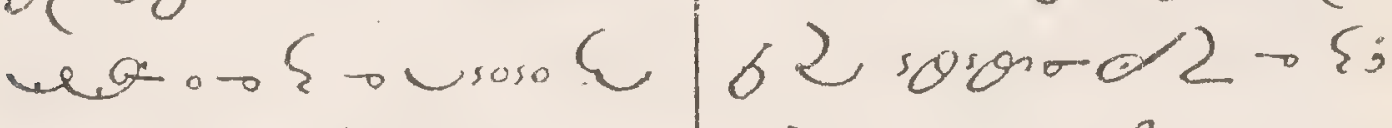

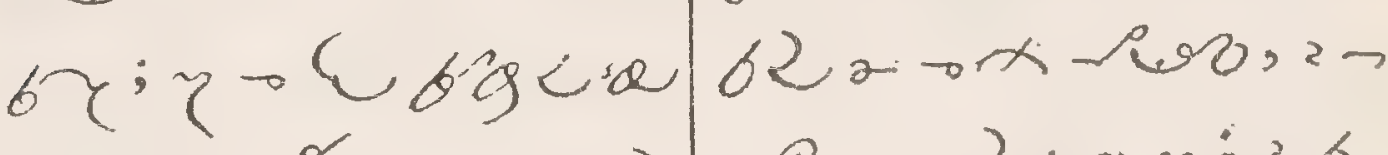

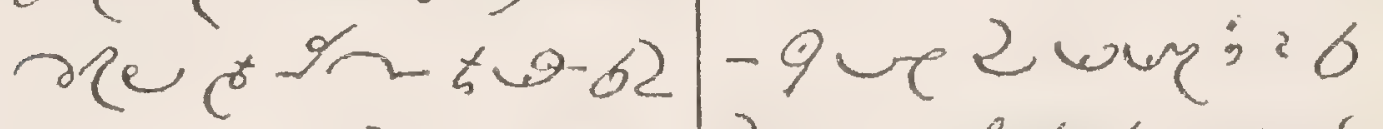

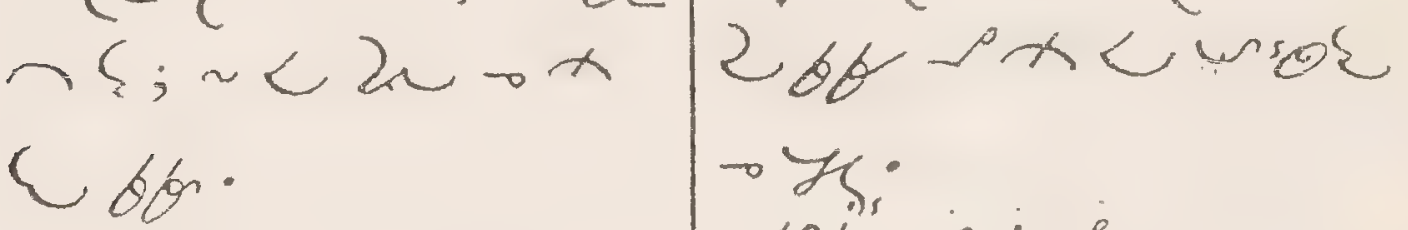

101. 2.05-6C 104. Sisto to iod é $\rightarrow C \backsim ?=0$ b2e $\rightarrow \oplus,-6 \phi^{2} \rightarrow$

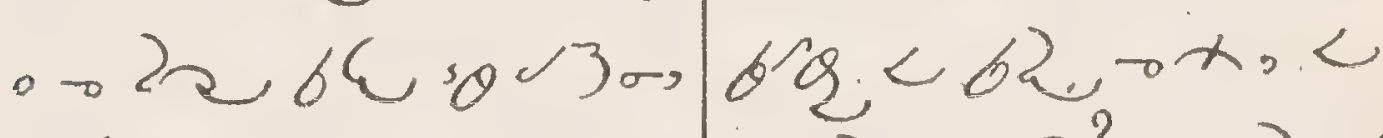

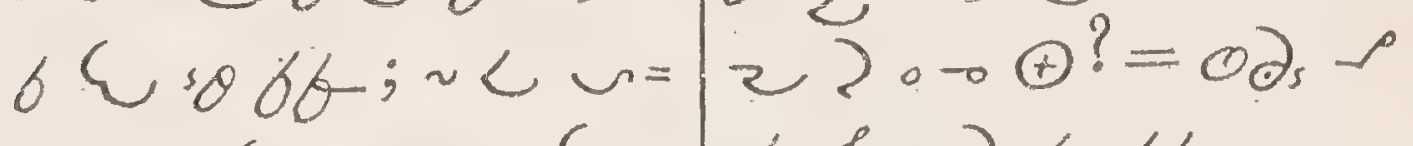

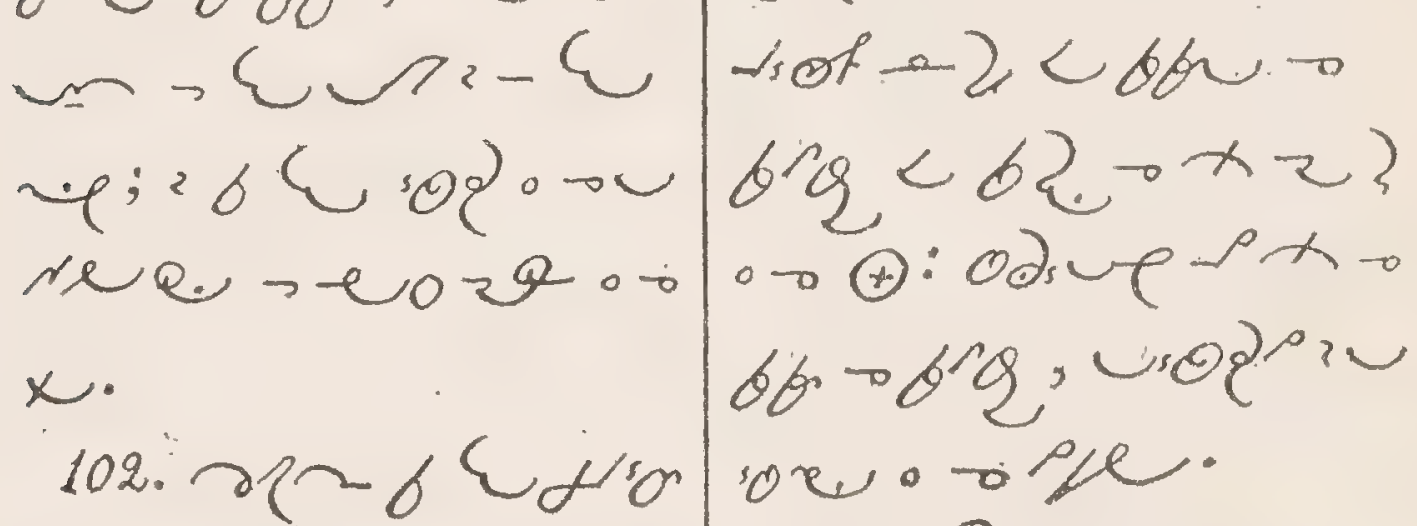
$62+\theta$ ? $=0-0-x$ XIII. Penance

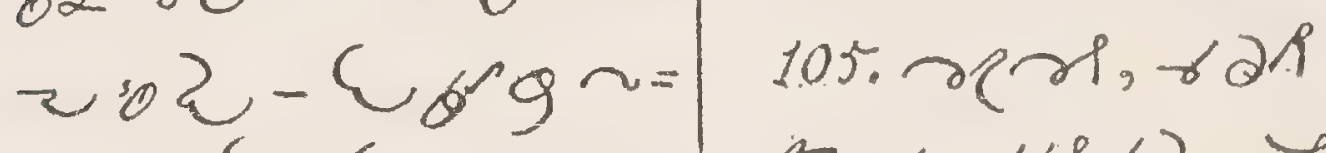
$-\theta-\{-2 \xi, 25$ अ, $=P \mathrm{C}^{\circ} \mathrm{O}^{\circ} \mathrm{\sigma C}$ we $?=62+62^{\circ}$

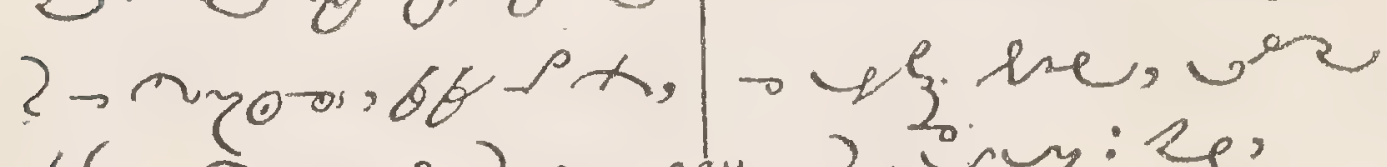

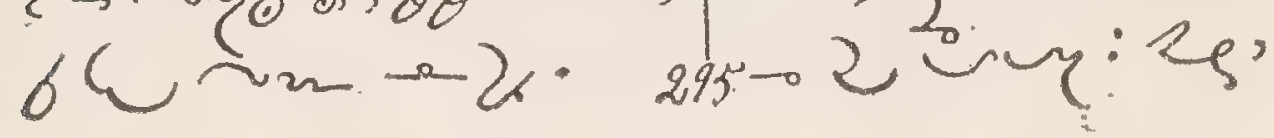




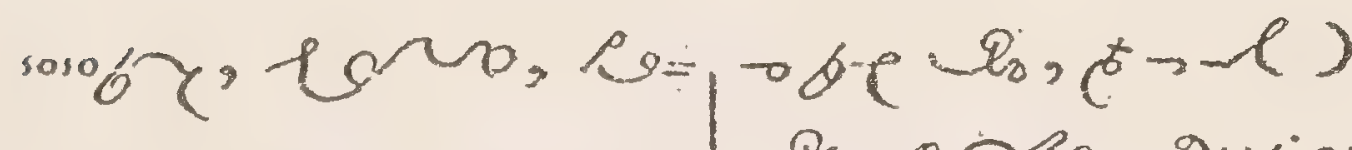
zero.

106. 2र 21 (6) $11=$ y? =62 o k, ह)

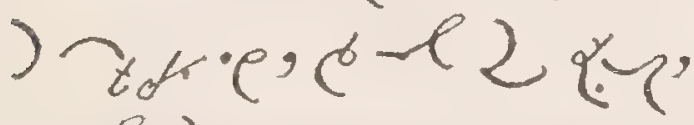
e t) nyotox

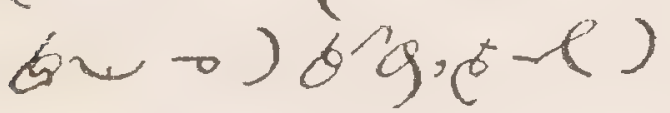
(no) n.

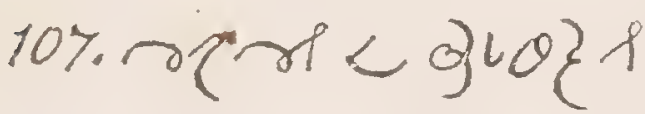

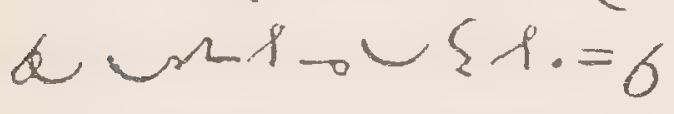

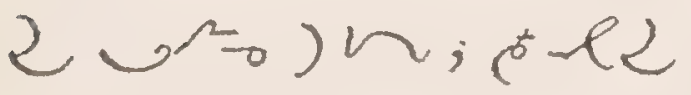
giog.ograt,

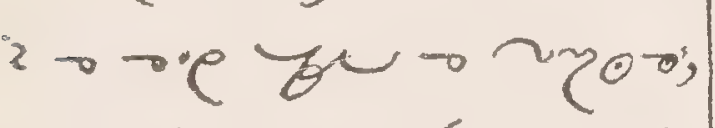
$\because$ orथ $n, 2 \rightarrow$ viver $\rightarrow$ $x, c-12.0002=$ o(bu 2 dra) n.

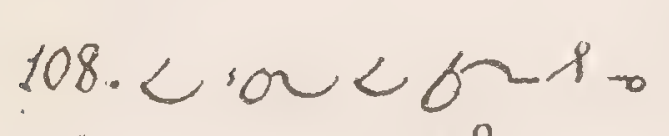

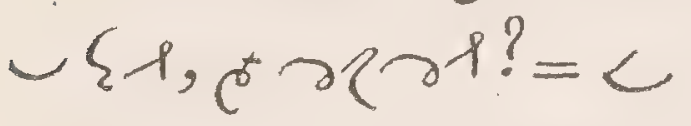
s 62 6r- $) \backsim$, e-l) nyotx< eveosq ro;

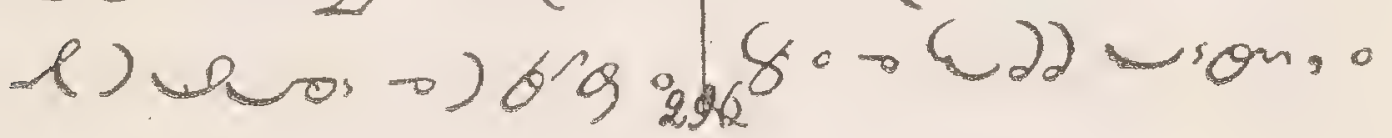

$109 .<\operatorname{soc} 310 \%+$ - to $0 . x_{0},<2 \alpha \sim$ (c) $2 x+=(t-\mu)$

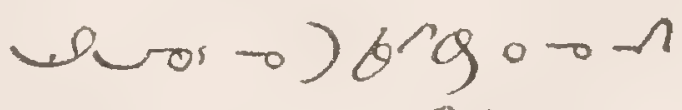
nr, tar s.os o dre $2 \alpha$ sक

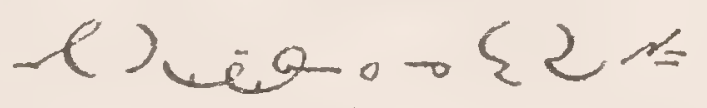
世; $369<.9962$ $\varsigma$.

110. L $a<0,0\} 1$ $\rightarrow$ ming $<2 \alpha 5$,

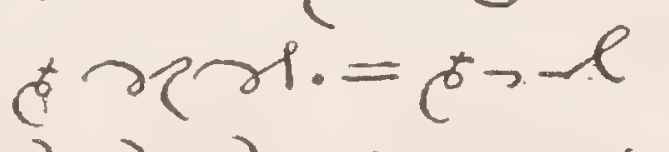
$29100<2 \pi \sim<$ $2 \div \gamma-x,-2 \varepsilon$ son, 2 viokog $\circ<+3 \sim \sim-9$ Cue;-2usolo. 


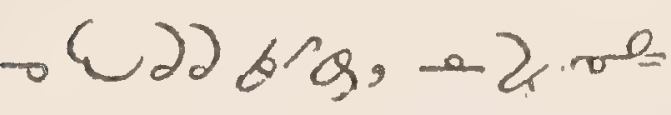
8०om $20-9 C$ $\tau \alpha_{0}-(22) \sigma^{\prime} g$, -2. vango-be $a_{0},-2$ and 00

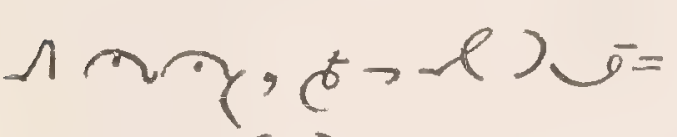
a. 0 र ree, $c \rightarrow-l 2 \eta a-0 \theta^{n} g$ Li 962 万. 111. Cs a < 310$\} 1$ $0 x<2-\alpha$

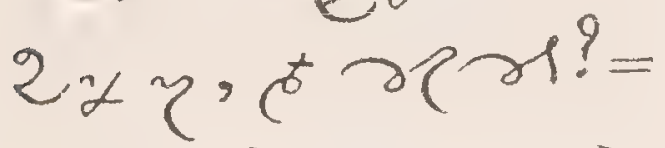
(t)- (a) as

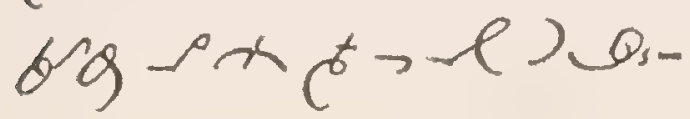
orpe $2 \alpha$ usp. - (l) los-o dre L2u:"zdod

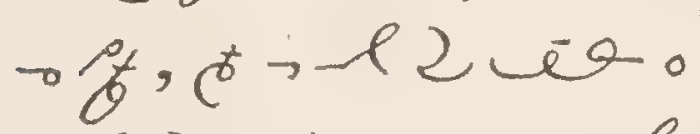
- K2 rea, to-l

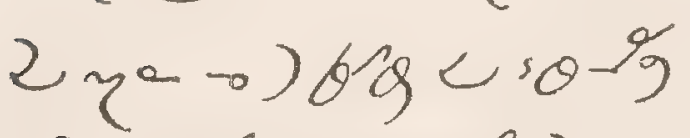
$62 \sim \varepsilon, c^{+}>-<2 m$ solen $u$, ta-l) n 0 os sosong br n. 112. arst b otso= sobr? = wary zure gist

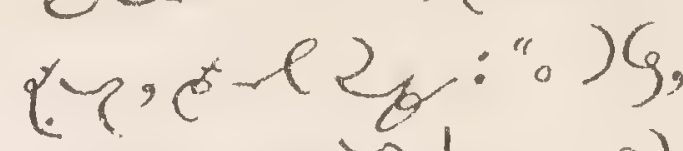

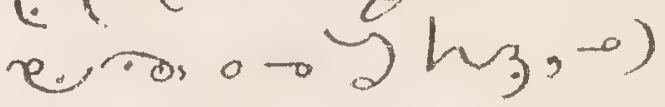
gurn; soson 36 ? Q $00 x$ xpryo- 0

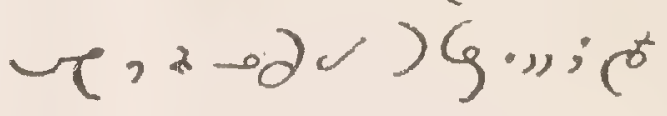
करण

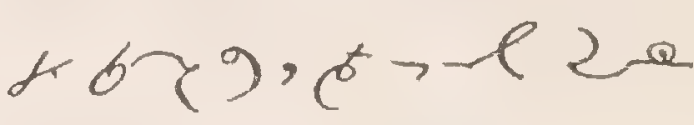
smoved ? end, r pelero loro, "pore - hz; - Noeverds

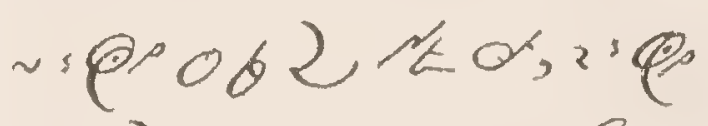
ob2 andicale टक (००) n. 113. Lson< ए - wor

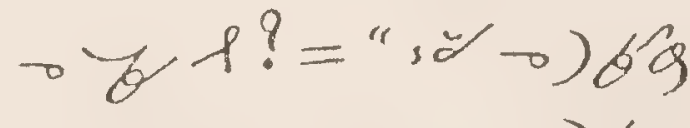
$\circ) \backsim-6$ sorred

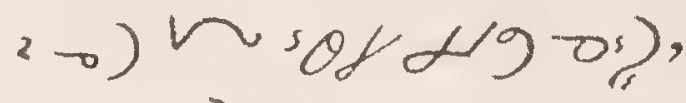
-9 $3-9)$, $\sim x<\operatorname{cis} 0$ o Qr, $2 \rightarrow \partial \alpha) \xi<$ 


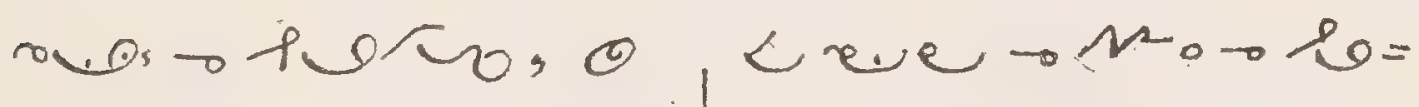
$\rightarrow$ usale.

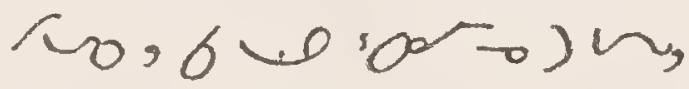

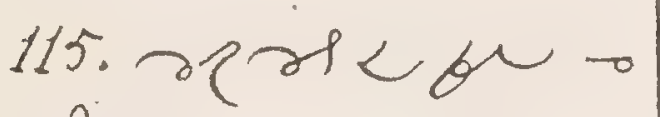
$r ?=) \circ \rho q \Omega \circ$ Nm- ) bs, $\alpha 3$ Q2. res. br $\rightarrow N^{2}, 62, \theta=$ $2-) \operatorname{sg} ; 020$ ? overorer? "Ppove retono, "Qsore o r w;

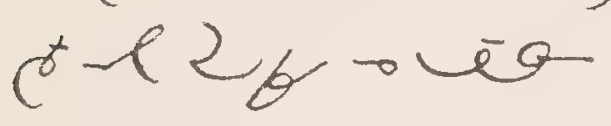
in. 116. अर्म< bu M" wPFto

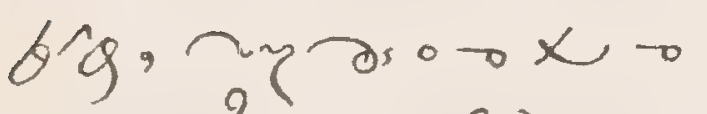
$.00 \cdot \cdots ?=t-l<\omega$

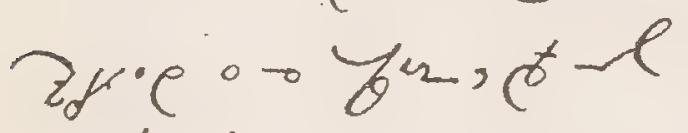
2大 tor 0.690 $x$, et 2 : 2 ? $=$ Q. $\rightarrow r^{2}, c^{2}-n_{n}$ $\circ \rightarrow x<$ ye. 0 ) $b=$ g, the rie or ver tra $r^{2}, \mathrm{l}$ (er), tsobso 3 rea. 118. or $\rightarrow \mathrm{h}_{3} ?=\mathrm{C}$ ve orsor ha, solus 0 -) $\backsim$. 119. $22 x,<\alpha 11$ or $\rightarrow n$, d w son sosoby? $=62 \alpha$

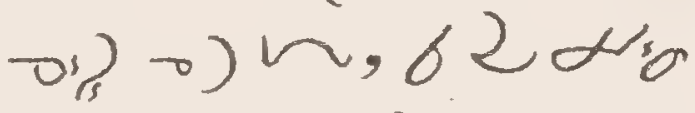
sosobr, ${ }^{2}, 2<-a$. r, $62 \% \circlearrowleft$ soso b. 120. $0 \rightarrow$ un th

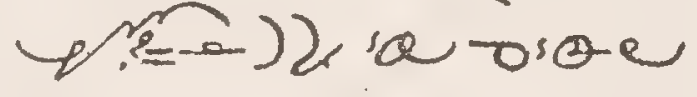

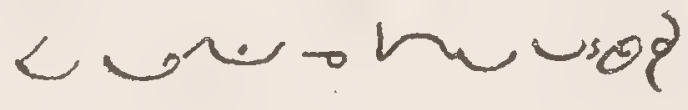

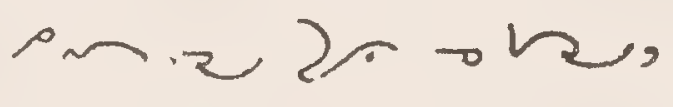
e) 2 ung 0,4 en. 121. a t t a in $\rightarrow \rightarrow n$ ef ? = -2 a ose $<$ we. ofudere,

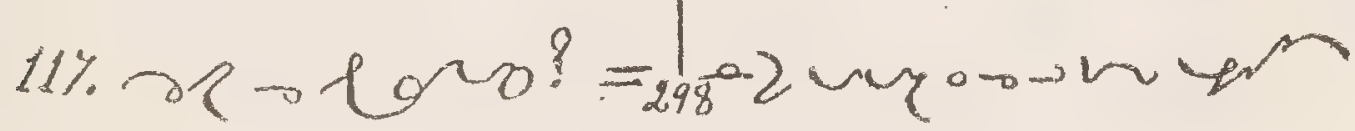


MANUAL

107

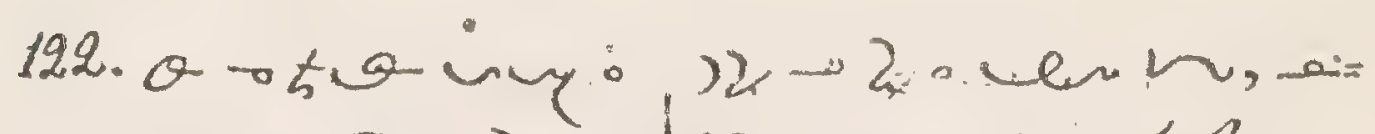
sosobr? $=-2<$ bm. pon 2 sosot, - $2, y^{5}-0$ of 25006 r. -2 vnown 123. - o o ro $\& 6 \theta \rightarrow N$ - odorasa 2.00 5030= $b \gamma^{?}=$ is berem

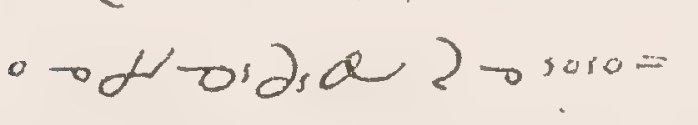
or; r-gunon -

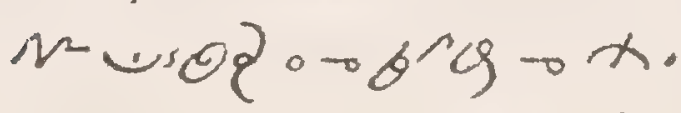
$32-40 \div 46$. 126. josac? - - ) $-46 ?=\operatorname{sirh}$ ? - $) 2 x-18 ;<2$

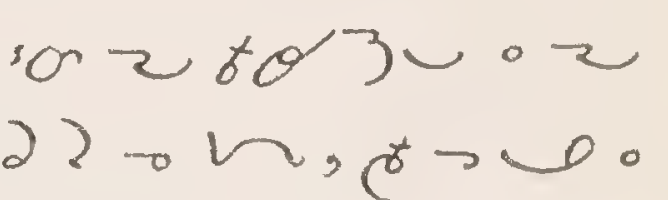
- be - a.

$X I V . E x t$. Unction.

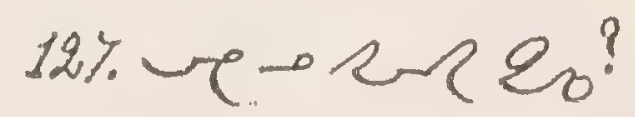
$=\operatorname{sic} \varepsilon_{0}$ je⿻ $x$ mund $<$ oque $\operatorname{son} 20 \Omega$. 124. propere? = to

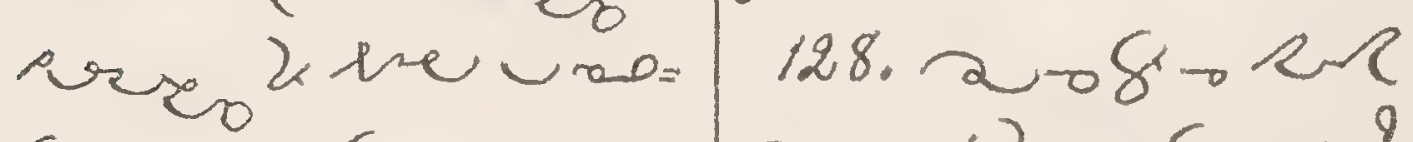

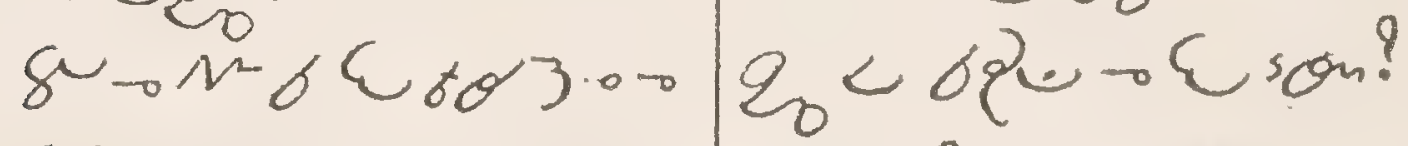

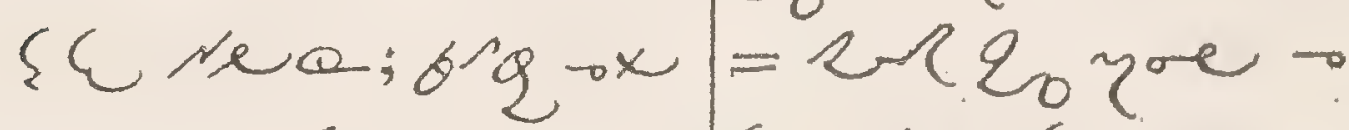

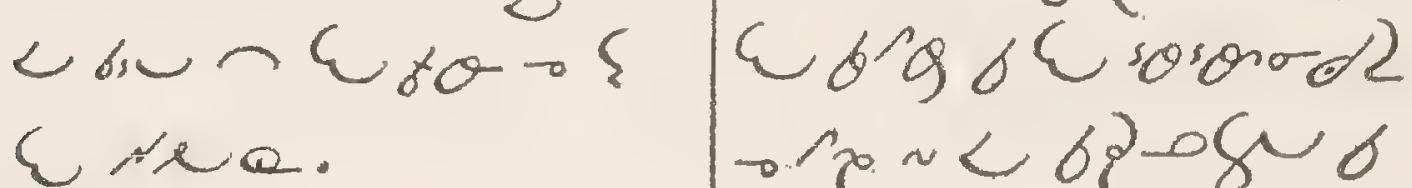
125. by-a) $2^{\circ} \varepsilon \sim \omega \hbar$. 0 oretoz० $-22 \curvearrowleft$ ? = - 24 129. adc onk

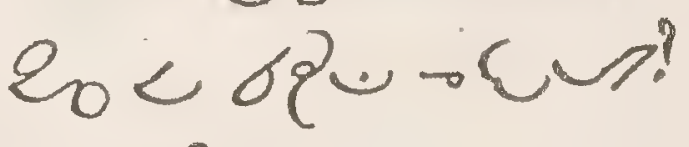

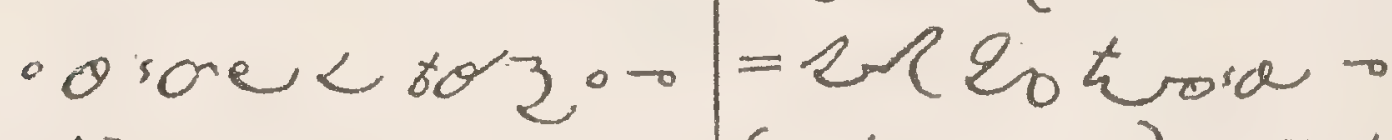

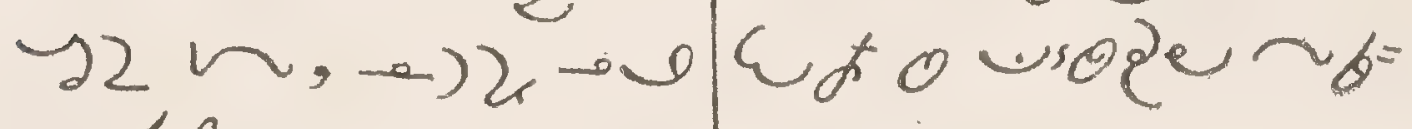

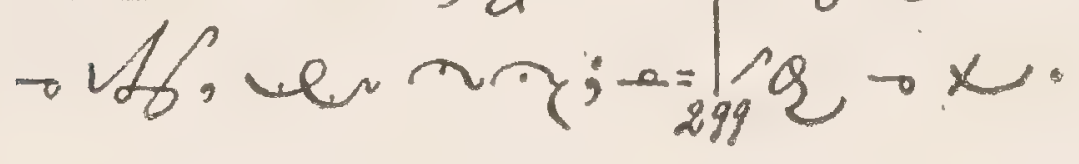


130. 206 $64 U_{=}=X V . H$. Orders.

G. $\mathrm{C}$ son' $6 \mathrm{C}$

$0.62 \rightarrow R \cdot \varepsilon_{0} ?=2$

132. $\partial \uparrow \rightarrow g^{-}:=0$ 9 ro $\rightarrow \rightarrow e_{0}=$ bदvegog $5 \mathrm{C}$ $e d \rightarrow x, 2 \pi i 26$ Nea, $26 \mathrm{C}$ sosot 06626 .

cme-e. $H_{\text {, }}, 2<\rightarrow-. \cup$ 131. 2- $864 \cdot \ln$ $\rightarrow$ T $90 \rightarrow H_{j}$

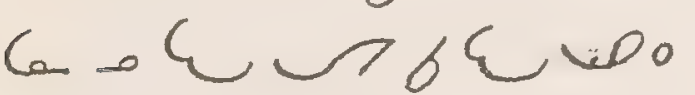
62 - $2 \pi \varepsilon_{0} ?=26$ 133. $\rightarrow$ re? Gepd $\rho$ of? =

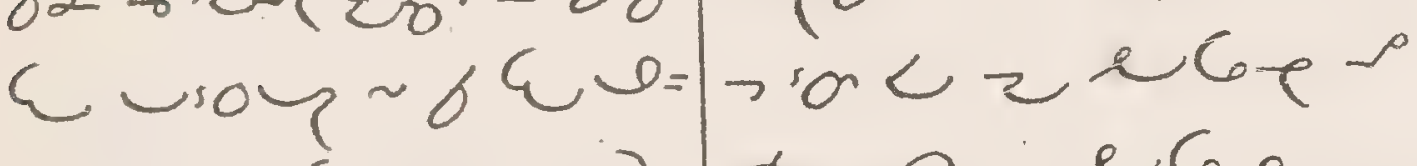
sor, $26 \varepsilon$ es 2 , 2.2 sose $02 \varepsilon_{0}$. $131^{\text {bis }} 2 \mathrm{Cl} \rightarrow \mathrm{M}$ Lrogurane? = b2e 0 soso Uiĕ, ofe 0 cine, r

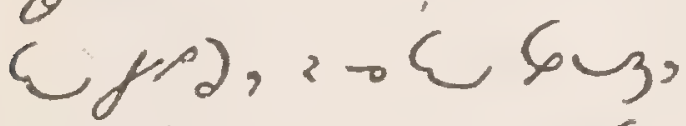

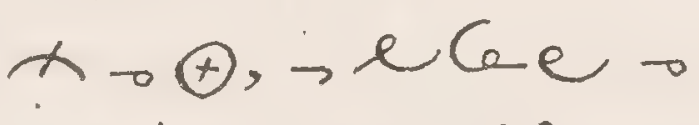
$y /() x^{2}, c$ c) $\sim$.

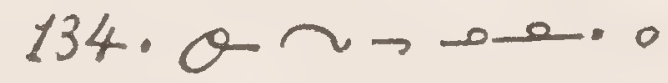
$\rightarrow q^{\prime} ?=0-\infty-\hat{q}$ $2 \leadsto$ - o. o. of. 135. 2e 20 $\because C v_{3}, 2 \rightarrow C$ re, $2, \varepsilon$ an, $y=$ wor ye: "थ Y $\rightarrow x$ o $\rightarrow \sim \sim r d$ - rul, r o yse, $=6 z^{2}, 2$ wez;

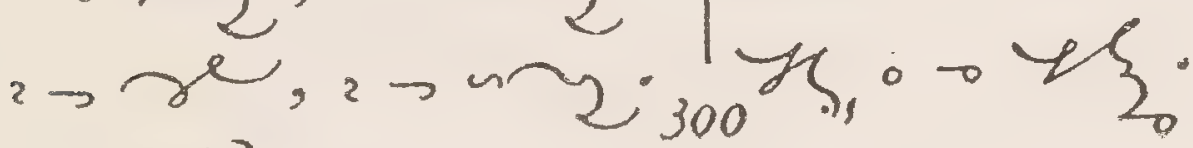
$\sim$ ज्षें,"

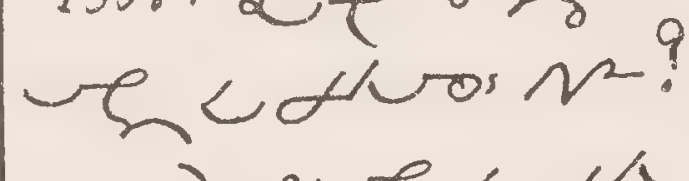

$=-$ hrech

$N^{2} \rightarrow \mathrm{e}^{\circ} \rightarrow \mathrm{m}>00$

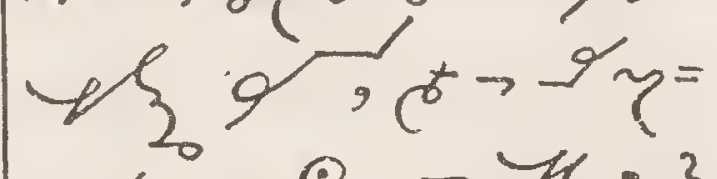
$r<-e-y_{s}, 2$ C2 I 2 . 0 
MANUAL

109

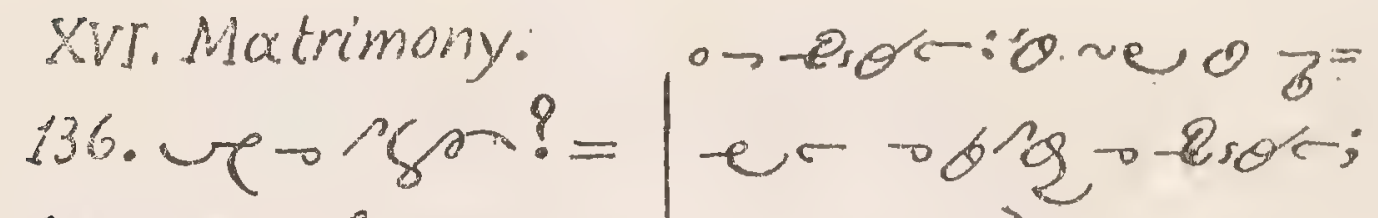

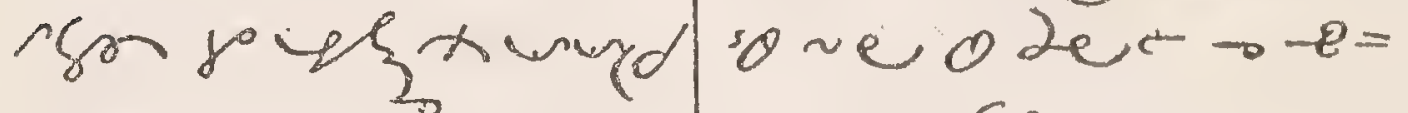

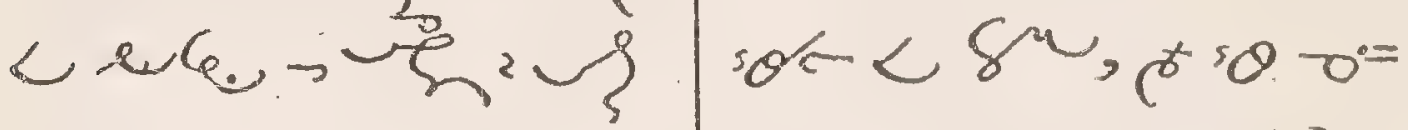

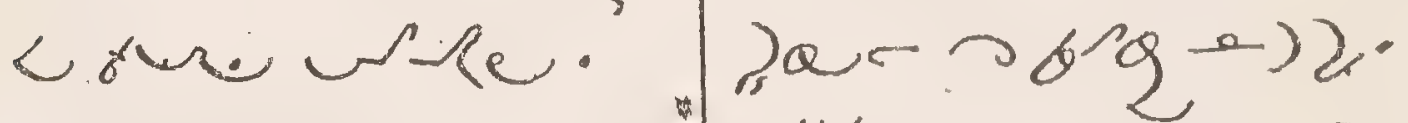

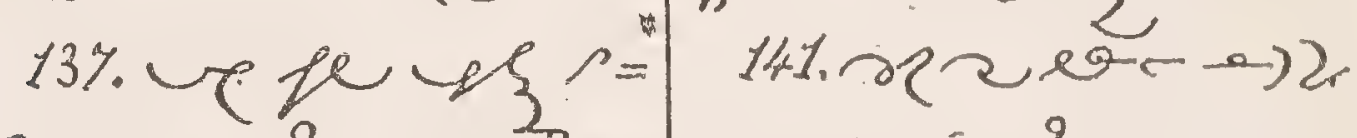

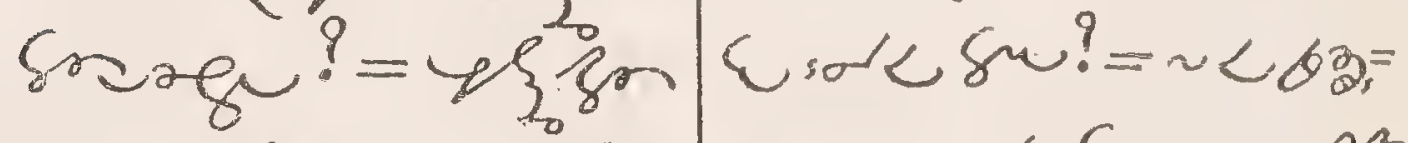

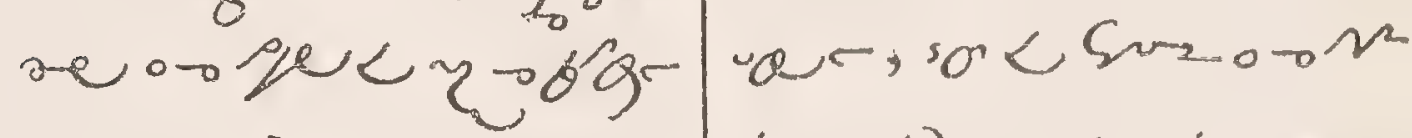

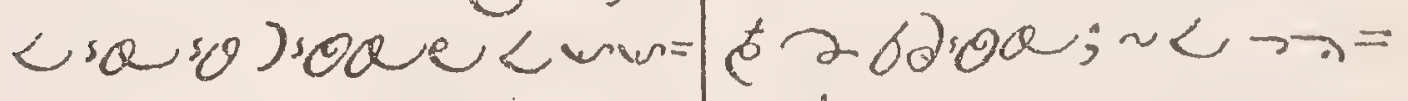

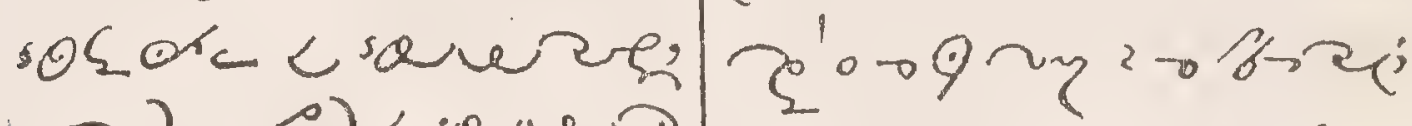

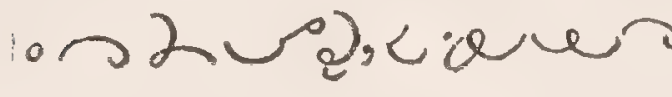

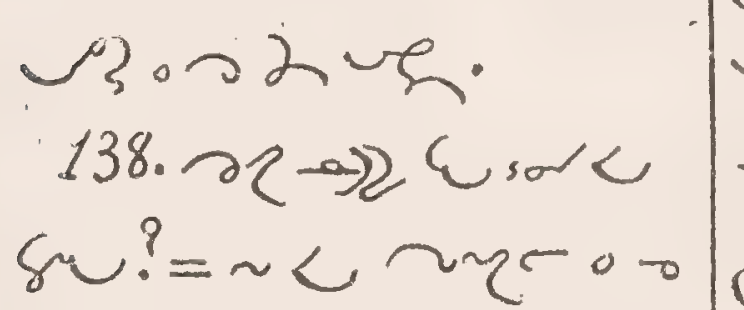
$x<\alpha($ or)

soe, 2 su: $\sim<$ Res z PDic.

139. गर 2le z

P.P. ? $=\sim<$ Les

$6 Q 0 \times \omega 0-\sec$

colber-big. esol.

140. $\partial 2>0=0$

+Ake z S.A.? =

som.oto onler
एथ< soseyre; $x$ sosd $2<$ bor

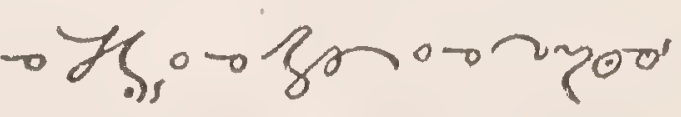

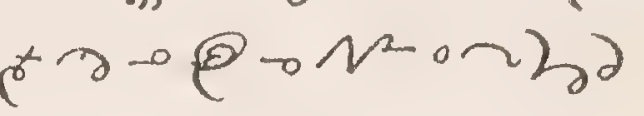

yr<iogic.

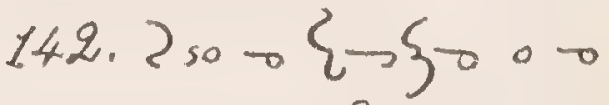

nno $\rho x ?=0$ कs?

$-g,-G, 2 u-\xi=2$

usor 0 ron.

142 , bis de of odo

$\circ\{\}_{0}=50$ usoe,

rogranor, zdi=

ox.esobu, e

dsax oz sorid.

$-$ 



\title{
Slawanten dthmual
}

\author{
OR
}

frowers, finmus

\section{and Cantedivint}

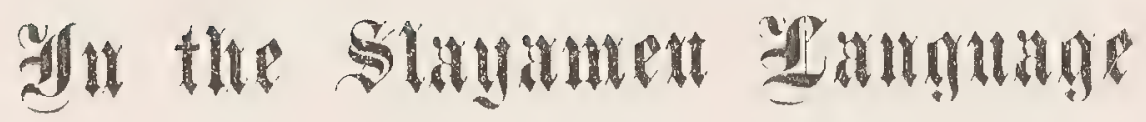

dianlooung, 题.

1896 



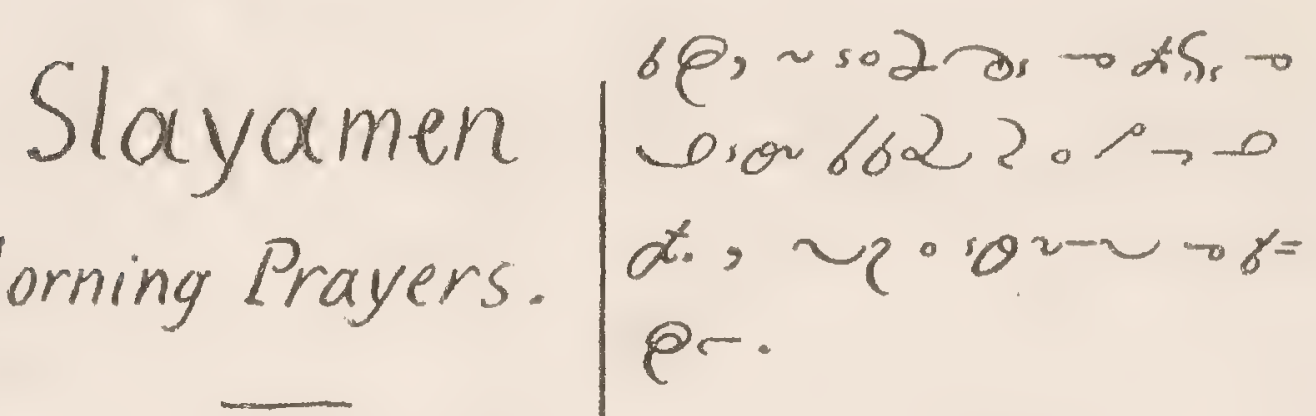

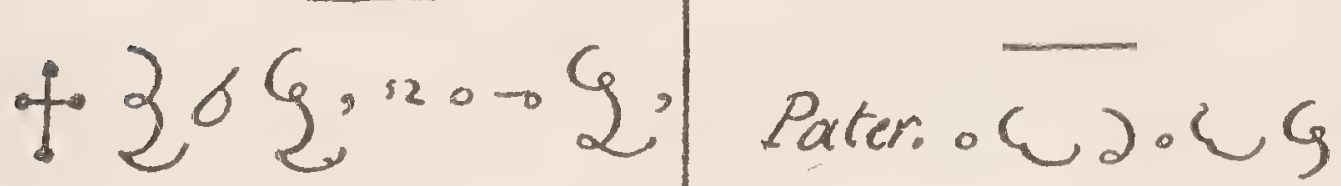

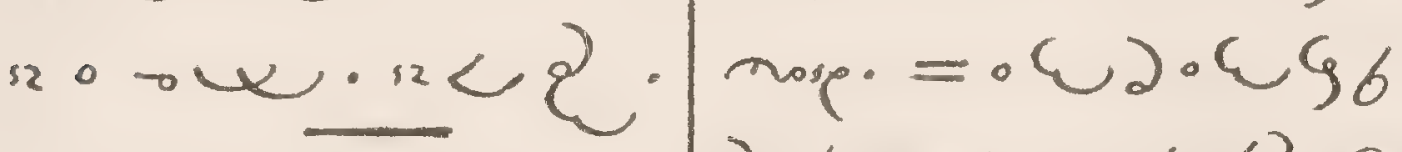

Offering of the day.

moun 122 ,

$-\cup^{\circ}=20,0-+\varphi$ der $\theta \sim b=\omega$ tip $\%$ moth. sodur.

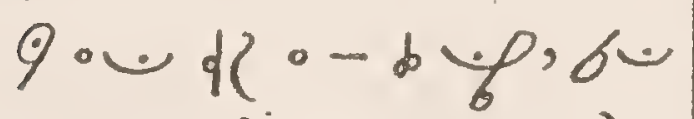

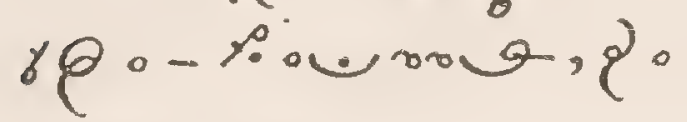
$-\cos 2 x$.

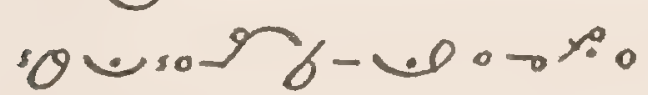
- b; 0.0y

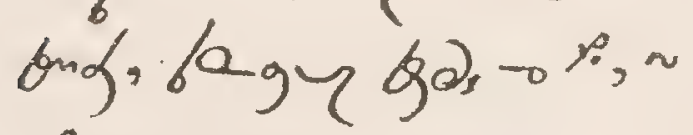
今̧⿻ 'quar éng. -4idb eb2?00d

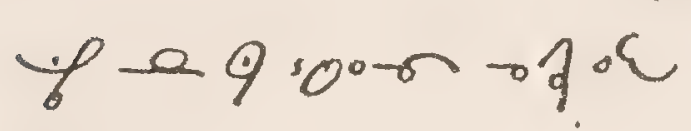
18 .

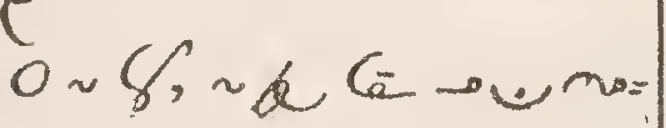
s, , 200 s00n of $0<$ $206 \sim 2-; 4<620$ 9 ats, oye 3 w.

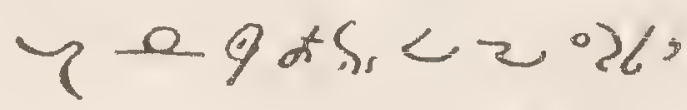
sul z; n eq ats. - $\rightarrow$ b. pvor - y. bug, porats 206 b. $=50208 \xi .6 \mathrm{C}$ $3 \cdot-4 ; \sim 6 c k$

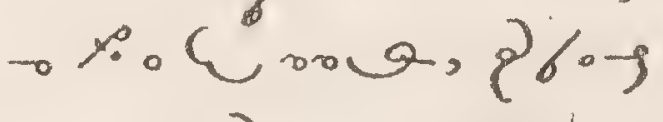

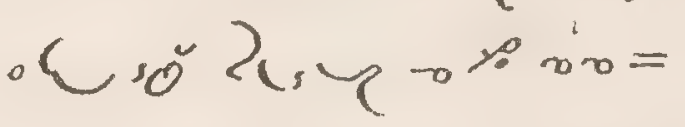
-og $=2.25 \% 50$ - C bing. be sola. - P; d wokos. 6 Csozer. 2 \&. Ave. of mose $=0$ Sindorafer >

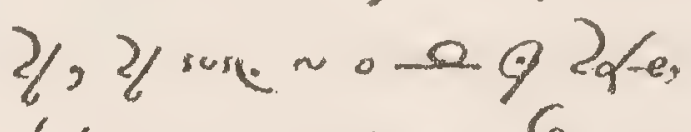
$562, a+y 0<52$. 
$=\sim 8,-9,7$, mosewos $\% 728$ \% 22 ob 4 क. $2<\%$. Credo. $-l_{d}$ no.mop. $=-6, n 0>6$, sul $9=$ co-9.9.6. $\sim-b_{0}, \sim 0 \neg b_{0} ; a / l_{s}=$ na, $x, 4600, \xi, 0<$ 2.4diburar Go - $2000, \sim 8$, wo orarib ras

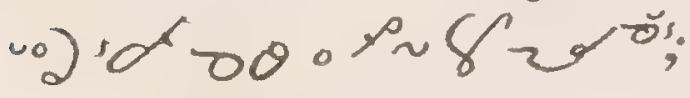
brosog buedre - wor 200\%

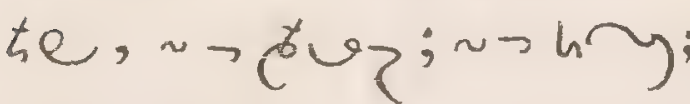

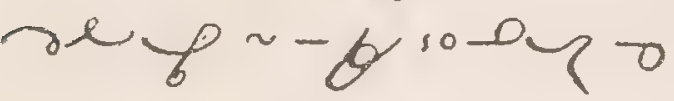
$\theta 0 \rightarrow 6 h e ; " \rightarrow \infty 0 / 0$

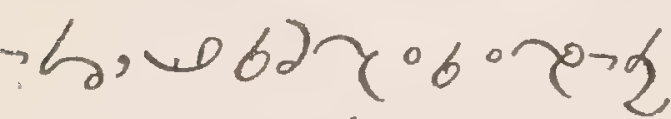
179 suse re a 9 r ooy<beno-e-

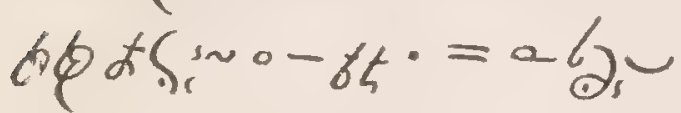
a w, on noseos tof,

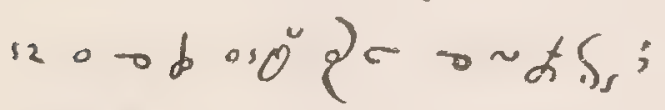
Q 2 - è $50 \bar{a} \rightarrow \%$

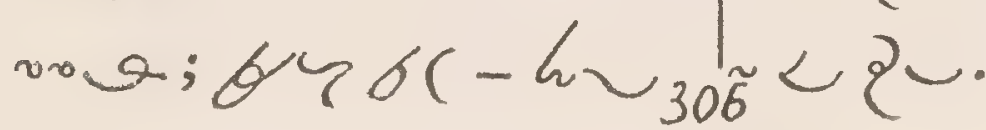

bhos; to $2 S_{s}$, bo 2. /uratz: a 2 . confiteor: sororiso mose $=\operatorname{sos} 036 \%$. 67 sus se, on- 9 ; $0_{0}, 8, v_{0}=362$ L, , $g / 3 w \sim 3 \gamma, 0.6-9$

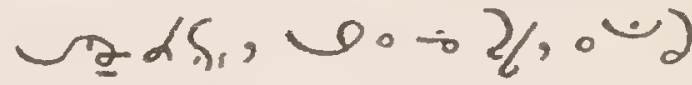

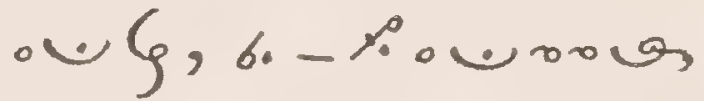

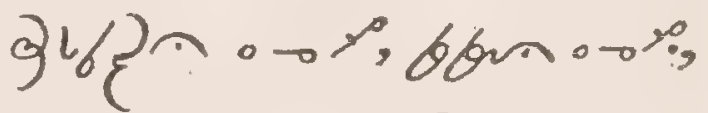

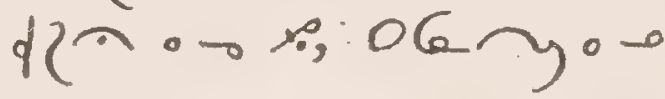

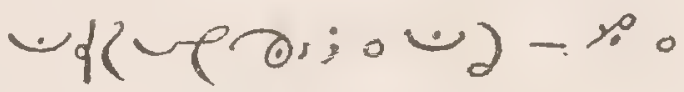

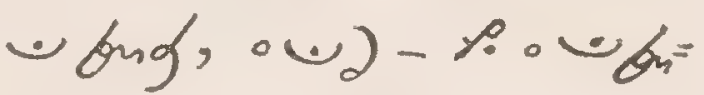
g, ०. $) \rightarrow p$ ovbug; 26 9 का i) $\sim 2,3, \partial_{9}$ H/3w/3r, 2q.2 सS, $\left.{ }^{2} \xi \circ \omega\right) \cdot u$ mose $9 \circ>02$. Misereatur. 28 of aS = $0 \delta \geqslant>$ suse res

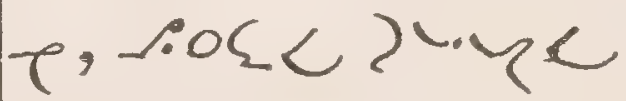

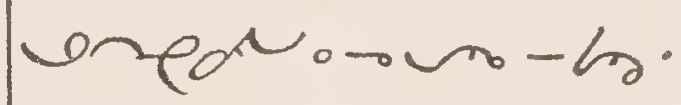


MANUAL

115

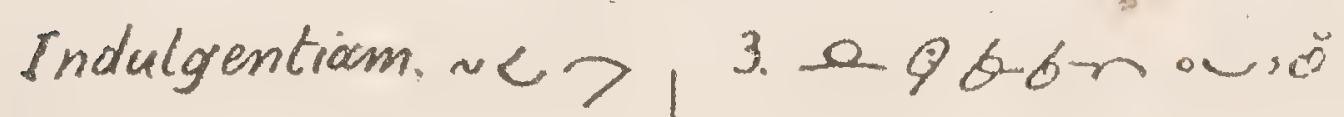

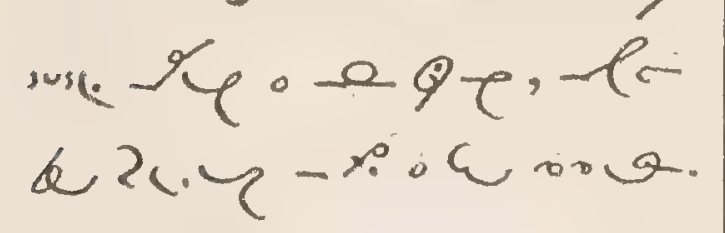

$\sim \mathcal{L}$.

The Commandments.

9 on, $>$.

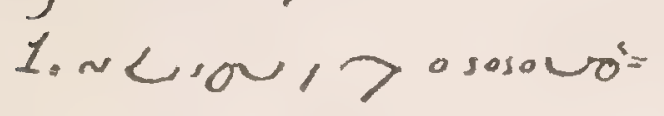

Q.

$2.50 \cdot 40-2,7$.

3. 2 b $82-5050 \% 2$

$4 \cdot n$ ab $62-29=0$

$\rightarrow \rho$.

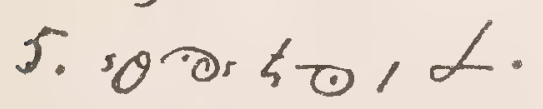

6. $3 \theta \cos 6 / 0 \theta \cdot$

r. so

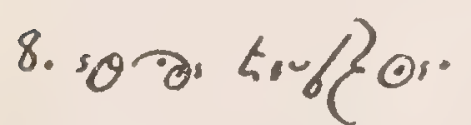

9.50 ar oi

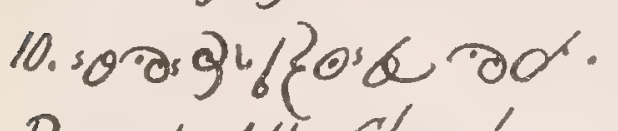

Precepts of the Church.

$\rightarrow$ on on nepos.

1.N a 62 - vg.

$0 \operatorname{sos} 0 \times 2$.

2. 0.00 .200049

$\operatorname{sos} \alpha 2, \circ \partial-\beta \circ \sim \varphi p$

$0 \rightarrow$ soses 2 .
6 ช。.

4. "e $966 n^{2}-\alpha_{1}$ e. ज्व

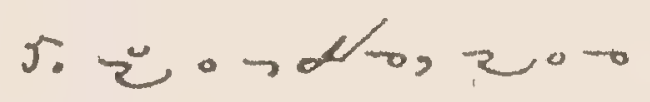

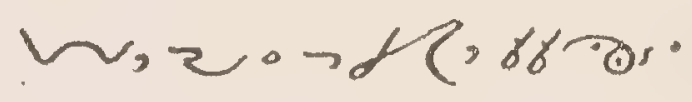

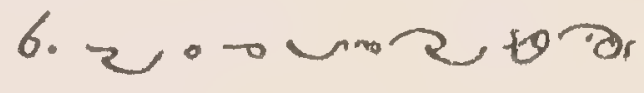

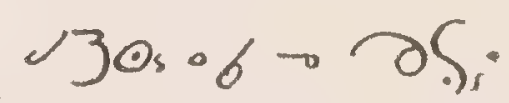

Seven Sacraments.

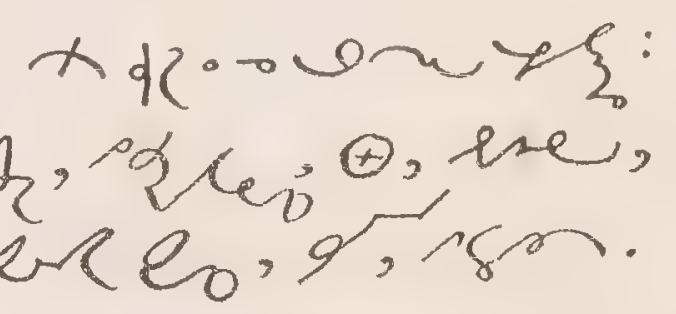

Act of Faith. $\sim \sigma C l=$

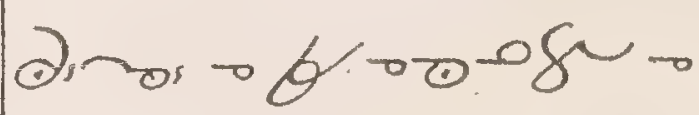

nepos. $=\delta_{s} 2_{1}>$,

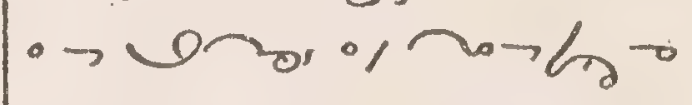

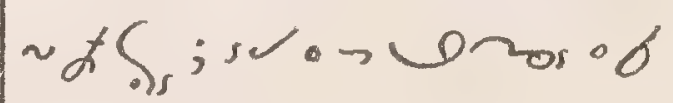

$\Lambda H_{0} \rightarrow x_{0} ;>\varphi$,

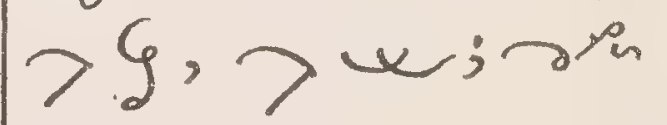

our, sc - -ibo $>$ >;

$7 \xi=b$ la $\rightarrow$ b.

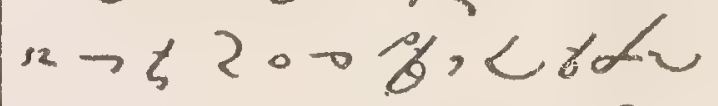

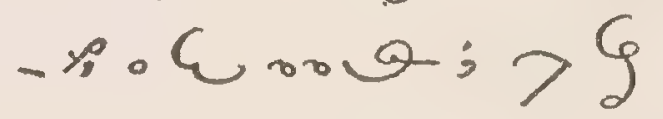

$\times \partial_{s}>200 \Theta ; 0,>$

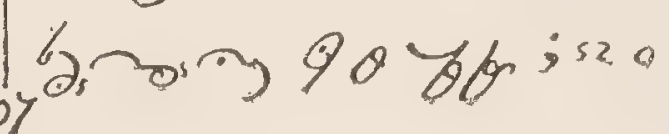


- 0008 rapor,

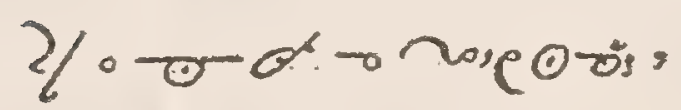

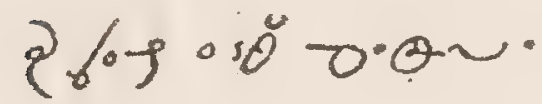
Hope. $6 C$ Ka (ibig. so) $08,>$. $=0,7$ jodsorea

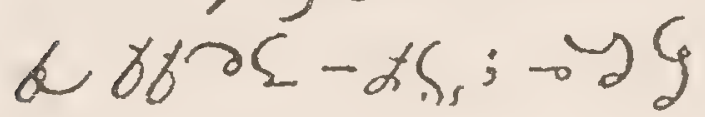

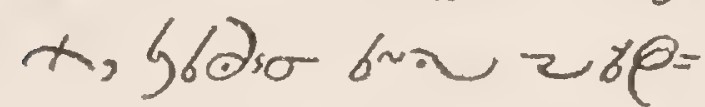
rogie g b p. . onga

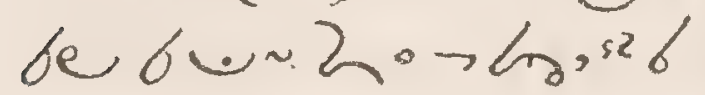
oousg a br so 20 of no-lo; 0 for $a^{2}=$ - 9 gure o bu.

Love. r $\sim>$ osuse. ०C, $=0,2560=$ कान sol $\mathrm{g}$, है। 9 - ex $\rightarrow \operatorname{sol} 252$; s2.

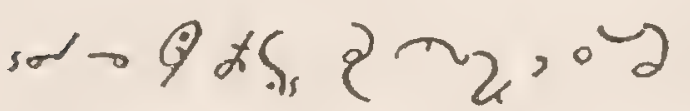
bing r.solosg ous. contrition. esan as = se. $=0,>$, wola - - שrorg;su-m

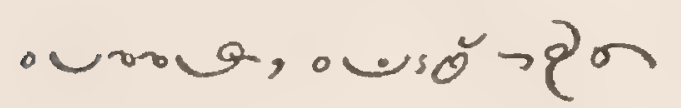

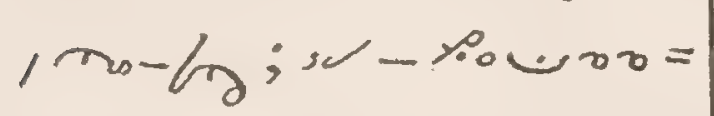

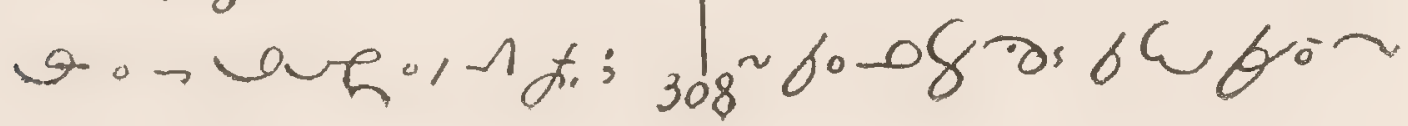

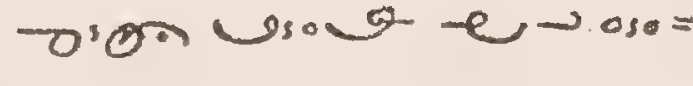
so, $\rightarrow$ bung, $\div z$, soso,

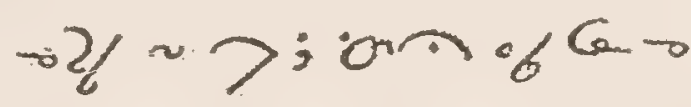
$\because ; 0$ ow tunghs ure bron G. cia rab r sow b2a, <sa -logan -y. a d bé 2a The Angelus. 9 . inn, 76 -a or $\sim S \cdot=8 \cdot 6$. 世 $12<2 \cup .=08 \ldots$ 2. $2,2 \varepsilon,>=\sim 2$ $6 \cdot g \circ \sigma_{0} \cdot=08 .$. 3. $>9 \& 2 \times-7 .=5$ 2 or $028 .=08$... X. 0, $>2$ roseo 0 ? $8 \cdot=6, b, a, n, \sim 6=$

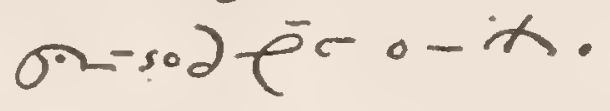
Oremus. 0,7b $b^{25}$ $\rightarrow$ fe $b=0<$ a n, $>b-0 \cos$ Log; Gajosoope x, s200 sot. 200 है, 
s an of $n-h=\sim$ ᄂ?

Sub tuum. of:

$08, \sim 2,2,2,1$,

> on ove orlin

a w.ocos ocisogo

ze; brumsirar to.

28.

Night Prayers.

Adoration. O, 7 ,

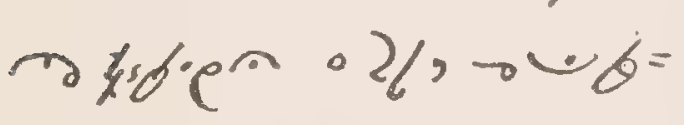
"gु62urenzon? - vosin $z-e^{2} ; \partial_{5}=$ vosin 2600 - 0.0 ty; w vor a ow ong

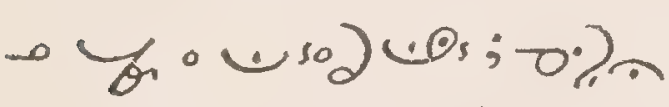
$\rightarrow$ suse ; soulds 2 ? - 0 q0e bugo borio 268人-eiónjerr

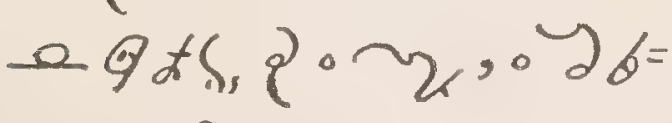
ng $<$.

Thankigiving, 6 crope $\%>\cdot=\partial \xi$, noso $?$ ?

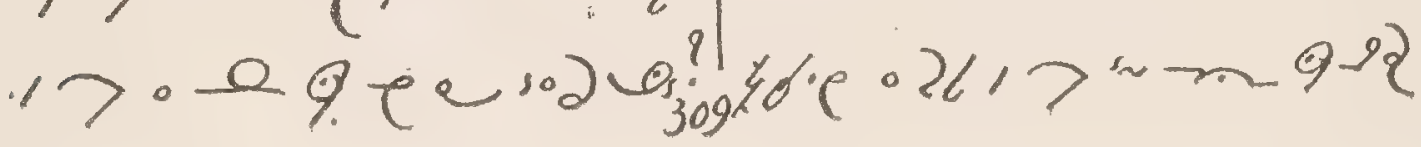

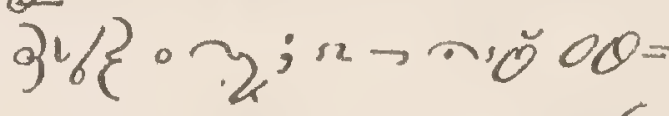
$0 ;$; doue; 9 yeroso $=$ Les a $9 \sim \mathrm{C}^{\prime \prime}$ dS, ; sor or 0.0.9०. bug b a dsom 2;:

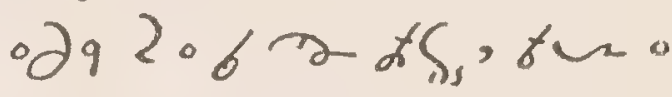
?. 6 < moon $>$. Prayer for hight. $\sim \sigma$

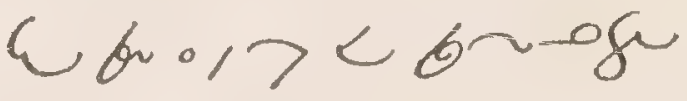
$28 \cdot=0,700026=$ $\checkmark 6 r, \sim b \quad b=0$.t. -vong, bdratren

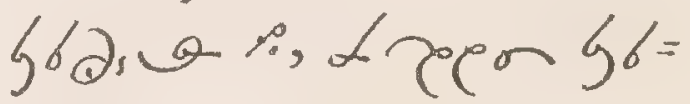
du osoug o row dर:

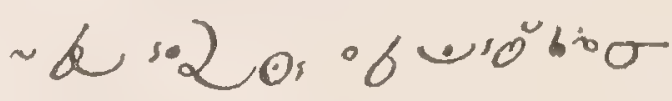

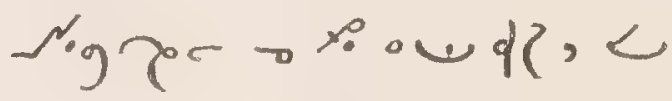
arper r buanco.

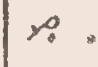

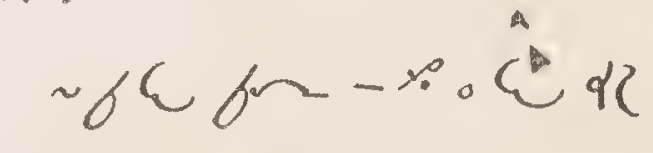
20 o o d . . . . Exam. of Conscience.

Contrition. 20, / 2

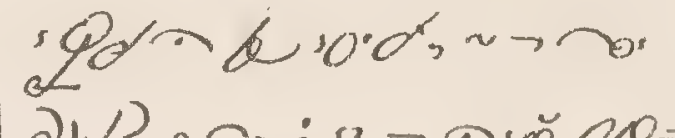


$\omega \rightarrow \phi 2.9-\% \cdot \omega d x$.

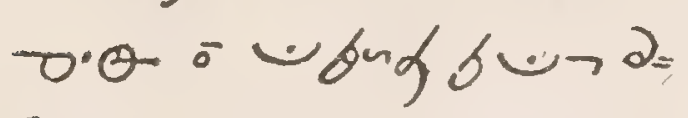

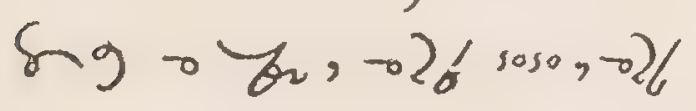
यद $\sim 70 \cdots 6.62$ so

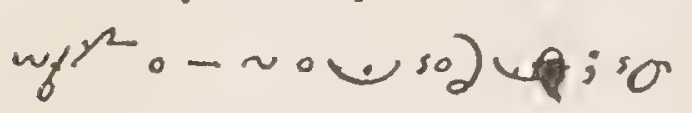
oroundiber

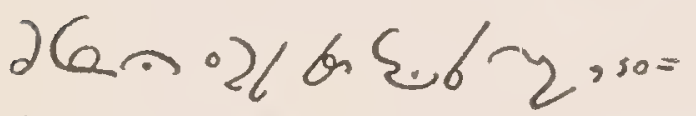

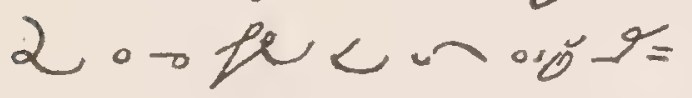

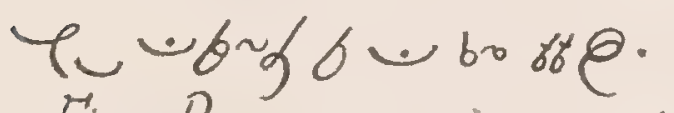
Firm Purpose. $z \omega 9=$

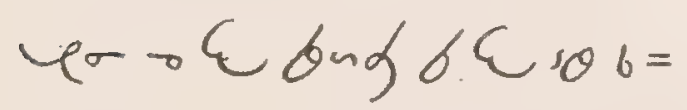

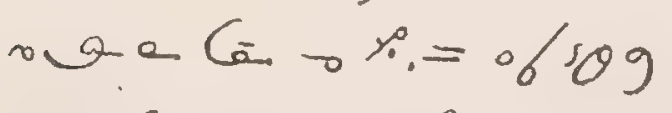

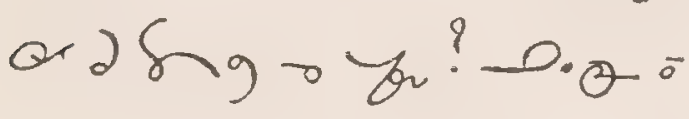
rongruss s/do 2: son of 0 .

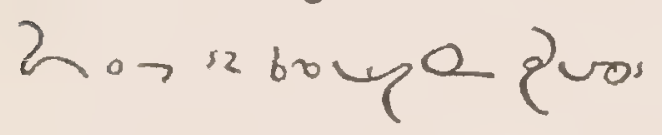
-Udl vers; brupo wer erougciare =

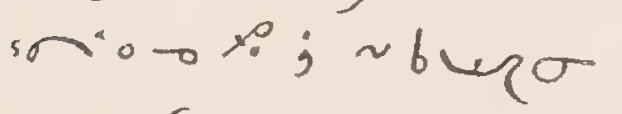
mrरe of agme or soge so 26 be momose .) $>$ a be 2 . Pater. oC ac 9 nare. Ave. of nose. Credo.

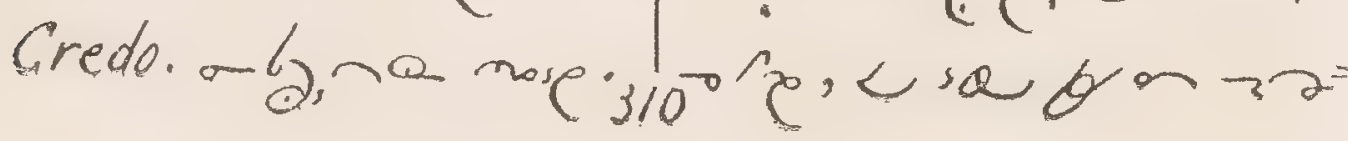

Confiteor. soso g o 6 .. Night offering. $0,7,=$ de zow inge

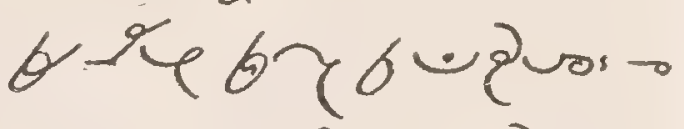
ta; $0 \sim 8,5202 \%$ Non, s20?/ - -3, bn० 170 ?. soma or.z; 200 乙2 चर०6-4. $\sim<$. Angelus. vo.

Preparation.

for Confession. $x \rightarrow 2 \theta 0-$ moseoro

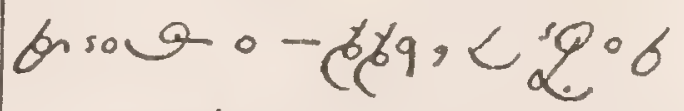
$m_{n+1} b b_{0},<$ wing or $129, \alpha<0,900 \%$ र29.

$x \sim<>y_{0} e_{9}<l=$ murop - bug 9017 , < osoleq - L几 q < b $q$ - 0 \%

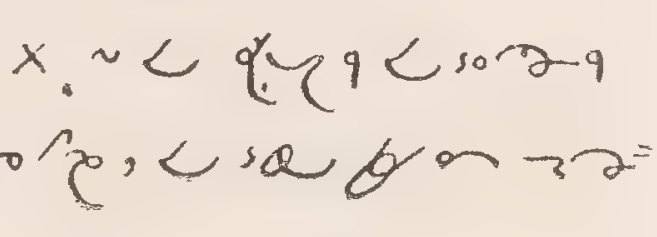




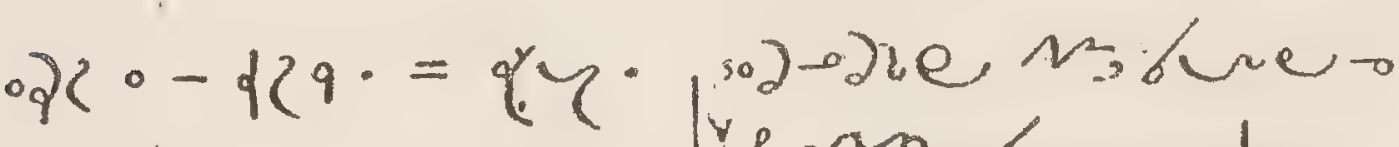

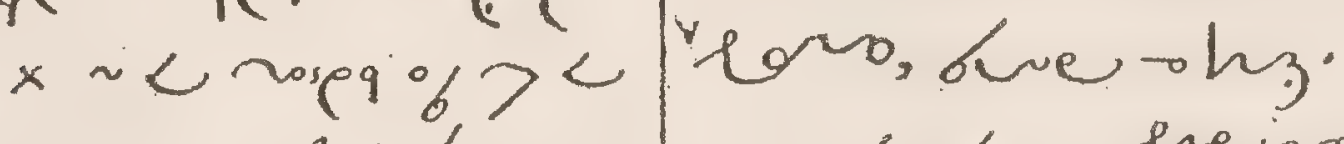

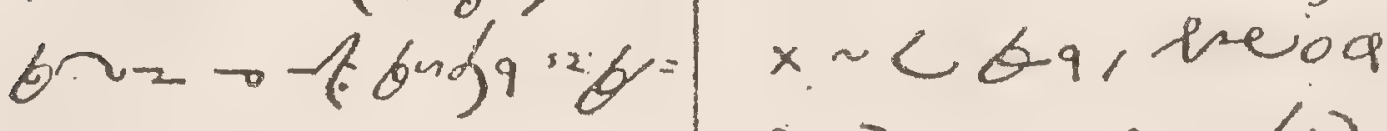

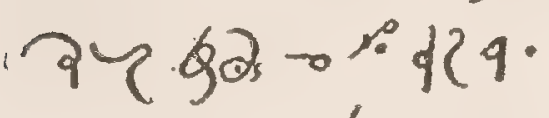
so) $-21, \rightarrow 2,669$

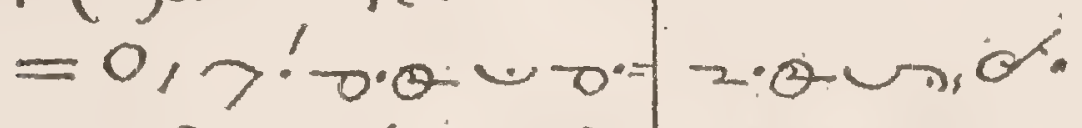
त) हn6oor yecoson,

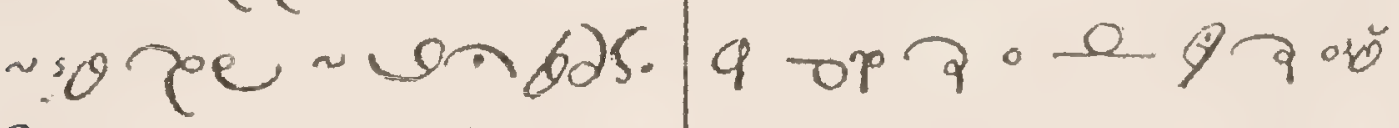

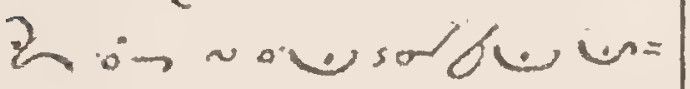
- ए以 , .

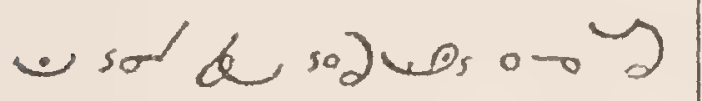
Le, "rontoon

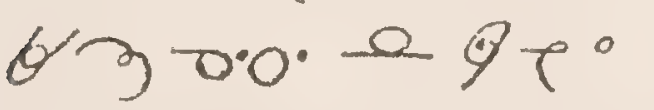

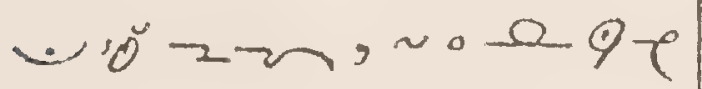
ascen.eg\%。

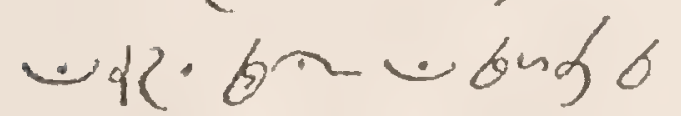
-6 0.0.6 56j, a - - e. 30 . 30 - 6 ;

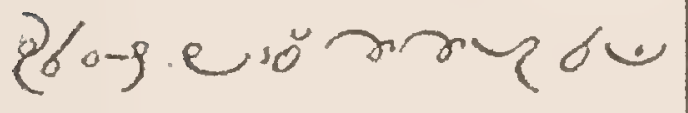
d $2 \circ \rightarrow \%$ $x \sim<60.62 \sim$

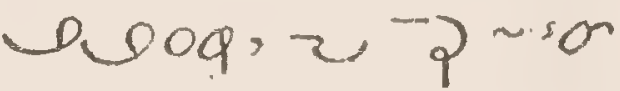
Cobroos. $x \sim<\sin t$ to $200 g$ on i> $x \sim$ L wing to d? 9 20- ore bu - noreor

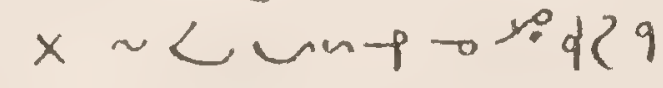
$2 \circ$ on 으 9 . $x \sim<u n+662$ q जo-d2uge, $>$. $x \sim 6,6 \sim d 6$ as e $9 \% 129662$ क 620 as. Q) $\operatorname{sos} 2 q^{\circ}$. $x \sim b, \operatorname{Cog}, \rightarrow x^{\circ} d x$

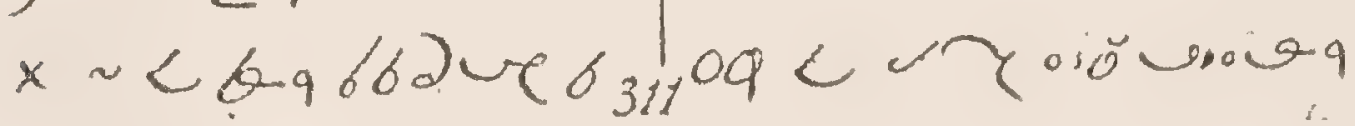




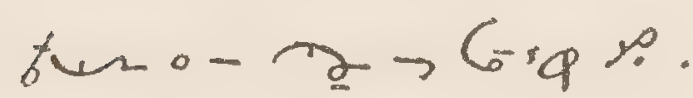

$x \rightarrow 2 \sim \tau$ -

< ware-bigh - o

rovg, os 0 , $2<$ Ko-q- bug $q<\operatorname{soq}$

brag Ca o ?

$x \sim<\operatorname{roseg} 0,><$

sodape ofsusogao$\because 429,06 \mathrm{~s}, \mathrm{~T}$ (c) - buga<ia per bo a 6 -

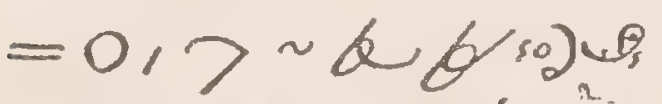

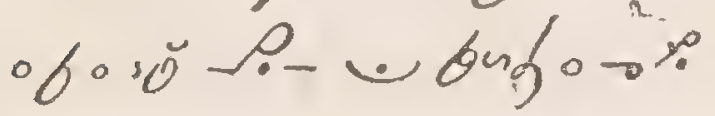
- vma; 97 ib $b$ sod es of o so oso.e buf $\rightarrow$ pow sover; $0,7 \sim d b$ solos - bo rea ronge sa per buvara $\rightarrow \%$.

$\times 0,7 \sim \alpha$ solos $\rightarrow$ ong bug, buron $\zeta$ oveng o to

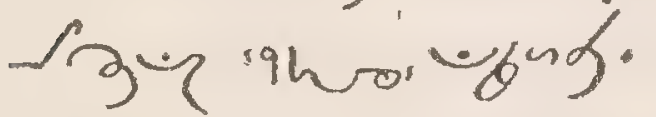

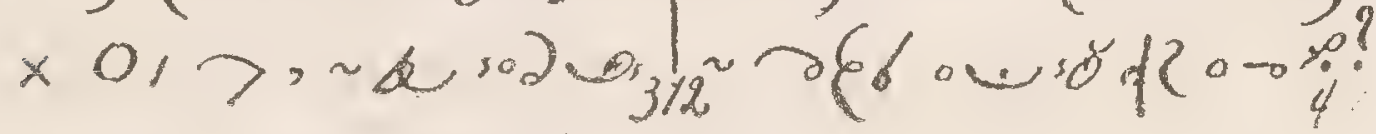

ofsuev ubug oto - $q$ ?

$\times 0,7 \sim d$ solos

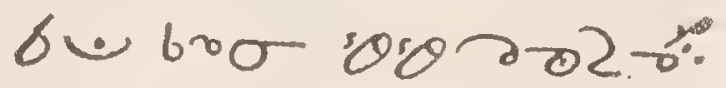
x 0/7, \& sodus, 60-g? $80, \div \%, \frac{3}{3}$ ren.? \& s. $2 /$.

$\times 0,7, \sim \alpha$ so $\omega_{5}$

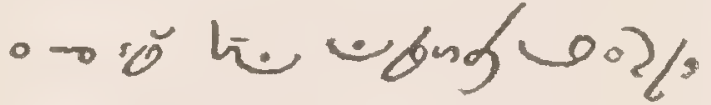

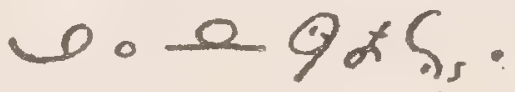
First Cionsideration. $x \sim<36\{9$ ० $\Rightarrow,<G b i v \sim, 6$ $=5,0<\cdot 562,0$ $\sim, \partial-\operatorname{lo}: \operatorname{mos} 2$ o $\rightarrow$ ba, $\sigma 0 \operatorname{son}, 126$ D.6ra-lm; ow

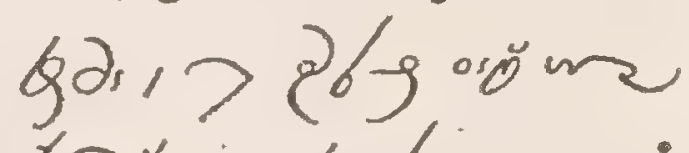
bot-ging oun: ious io 0.?son 6 bril $720 \%$ m baris. $\sim 26$.wog, क $\cos 0$ - bing 9 . 
MANUAL

121

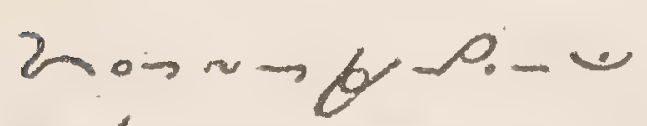

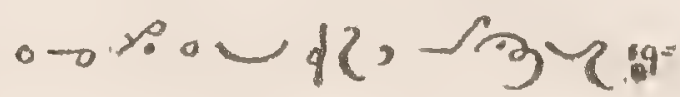

buturareans her:

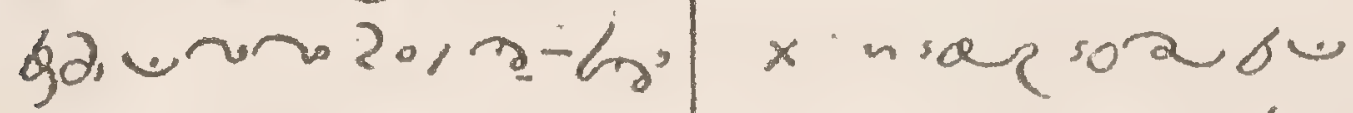

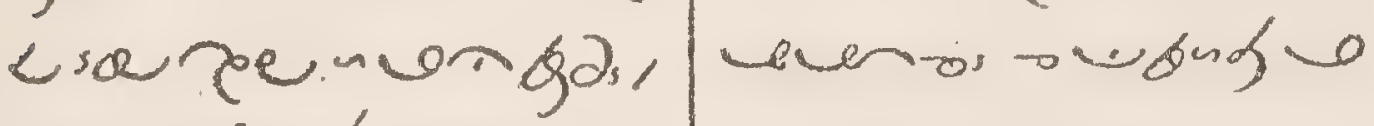

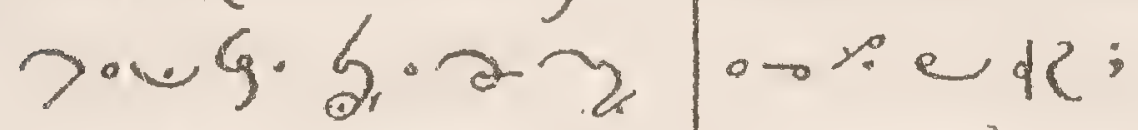

kif

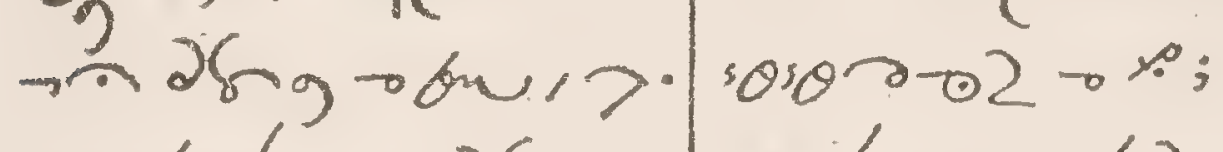

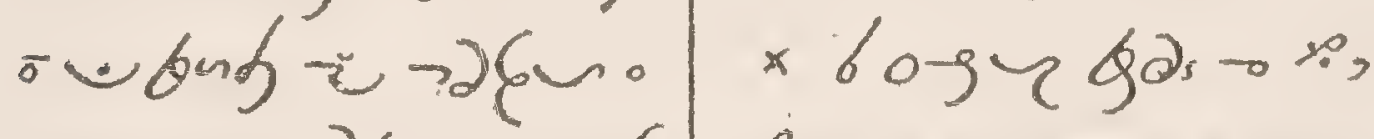

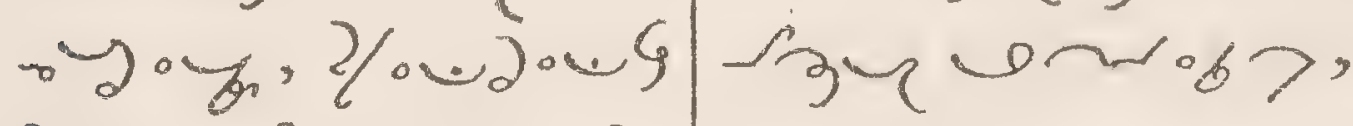

$\geqslant / 7 \cdot$ hon ob 2 d be nose;

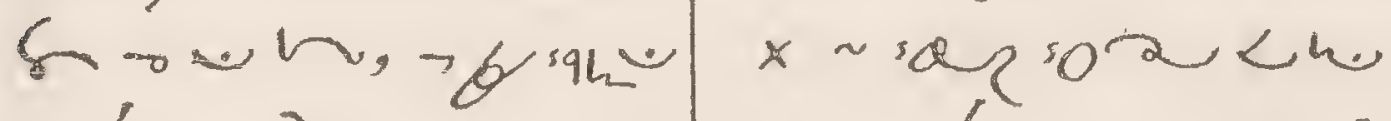

bug $0.2 ;$;

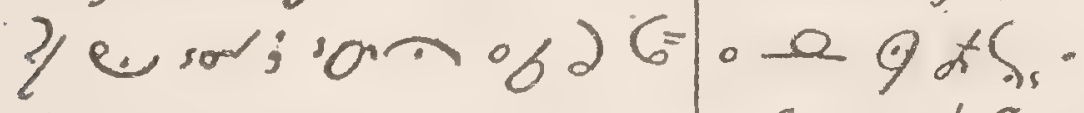

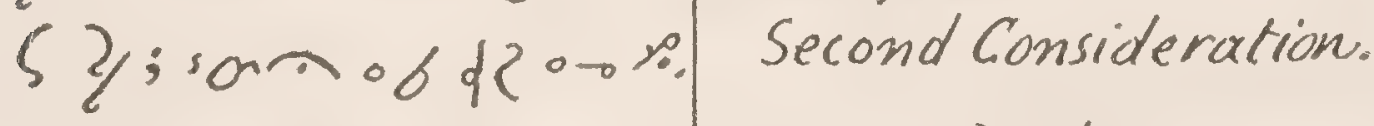

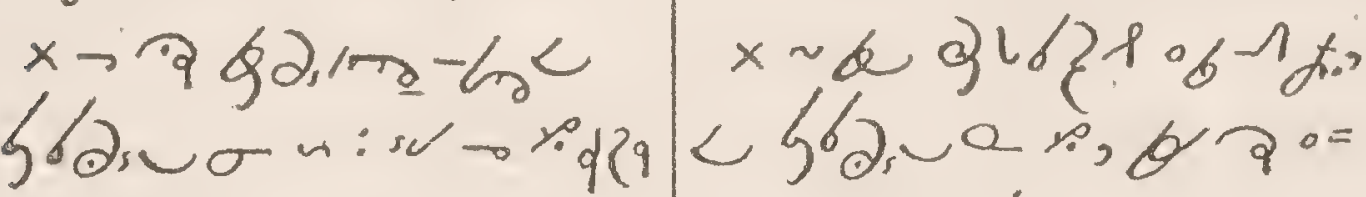

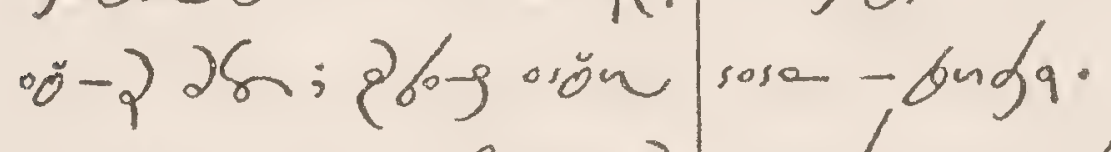

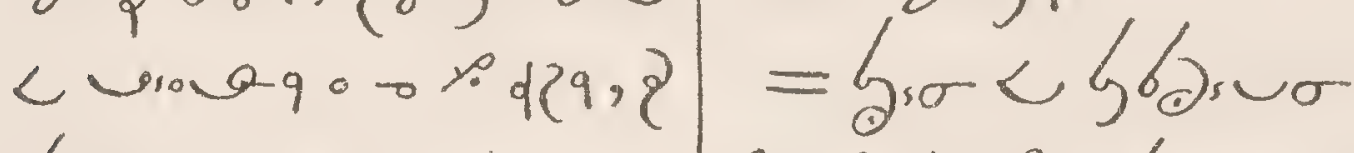

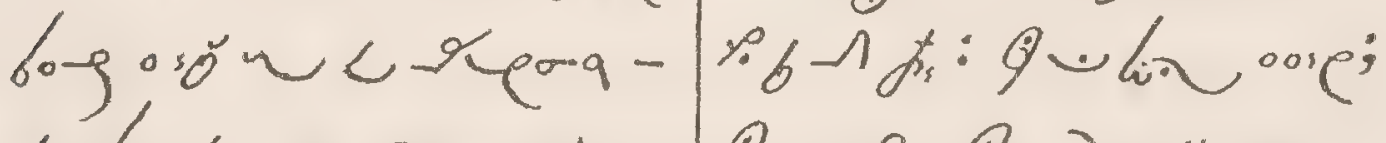

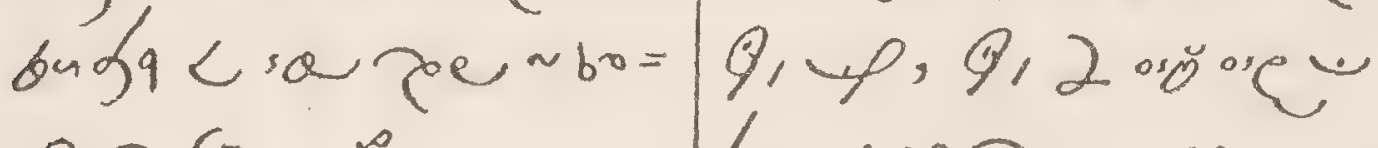

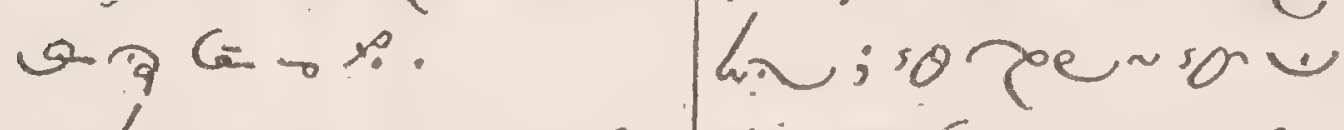

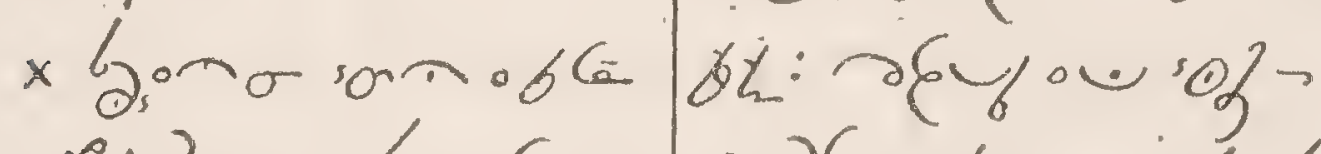

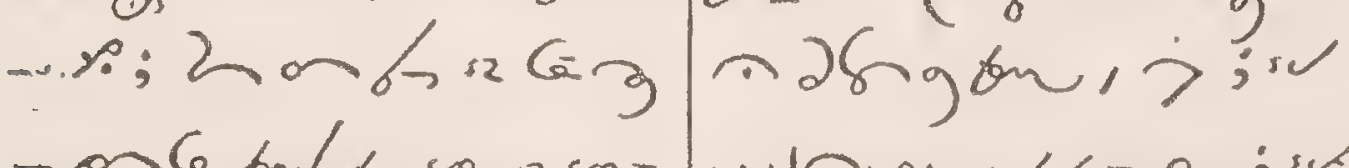

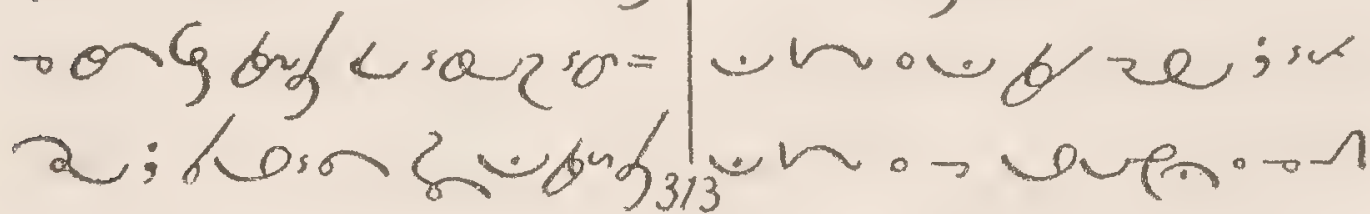


a.; 806 C me oboro e 3i;

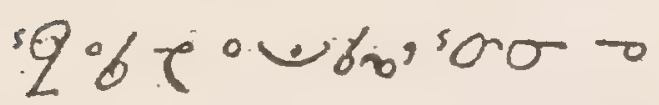

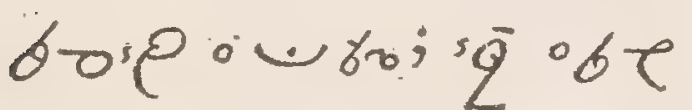
- 66,500 - 06 o: - 66 . sus ov 9 , $\rightarrow 2 \delta$ ibn, >is

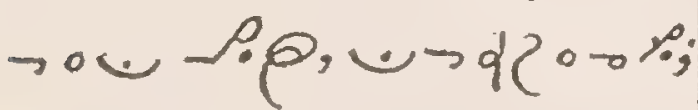
$\rightarrow$ sis b. 0 Pरn,

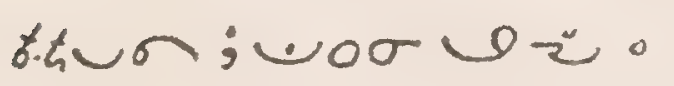

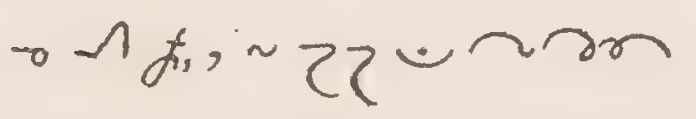

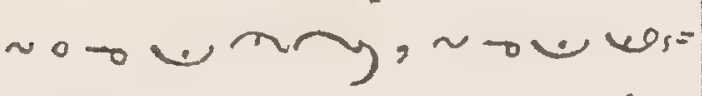
a; a vor bub $\alpha=$ a roe:17sque: 2.

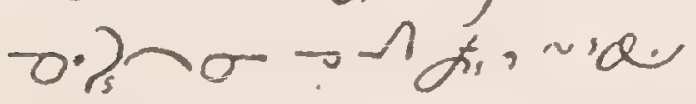
frs 3 of dर० $0 \rightarrow$ P. - ing ov

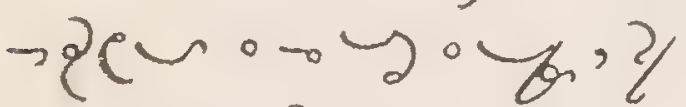

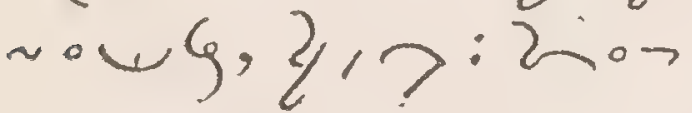
$\sim / 426$ bisumbung $0.2 /$

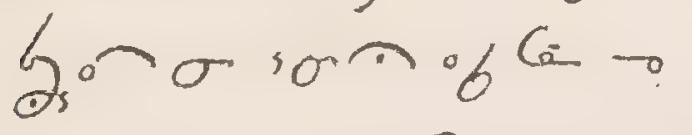

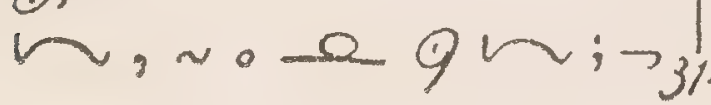

- о 20r:? rong; sordo \%: eso'; तosion $625 \%$ $x \rightarrow 9932, \sigma-1$ \%.,

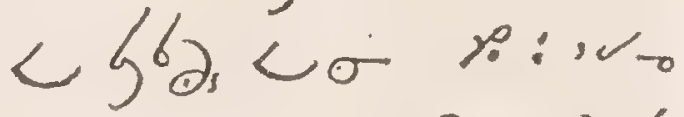

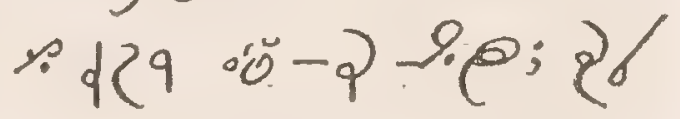
o-g0són< Usoga

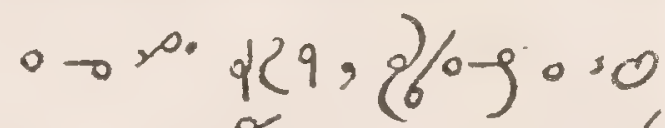

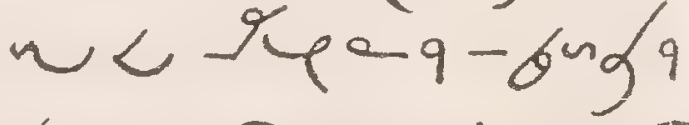
La de r bora $G \rightarrow \infty$. $\times$ bs on san ob $\rightarrow$ ong bin $/<\sec ^{5}=$ a; $/ 6$ on 2 ong oroudl, srac $9=$ hnos;

$x \sim \sin 6$ ven or o venge $\circ$ o

$x \sim \sec { }^{2} \cdot 60 \mathrm{~W}$

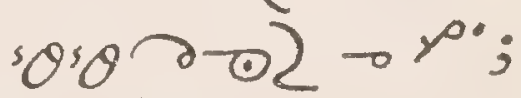
$\times 60-9 ? g \partial, \rightarrow \infty$, किय क्ष $?: 6$ 
MANUAL

1.2:3

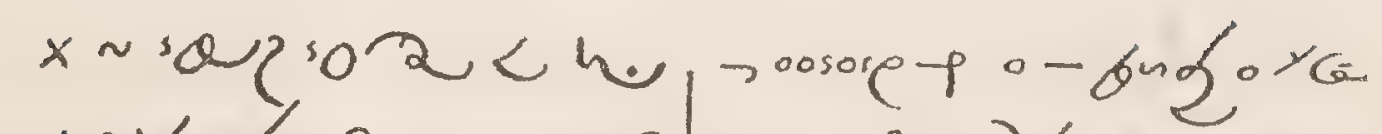
- ebugl 0017,0 9 tox; 9609 osón

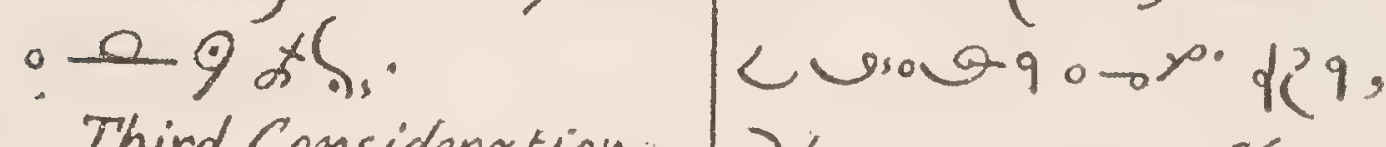
Third Consideration. $869 ; 0$;

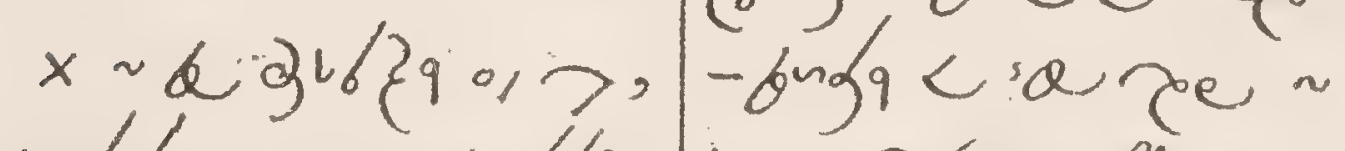

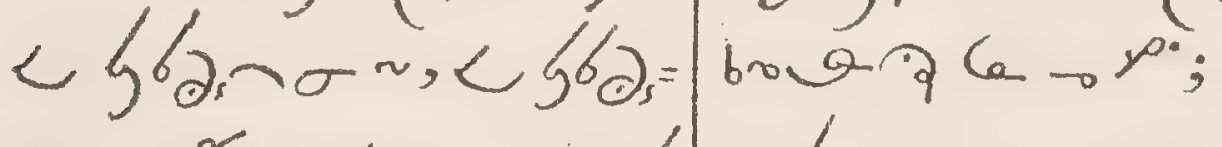

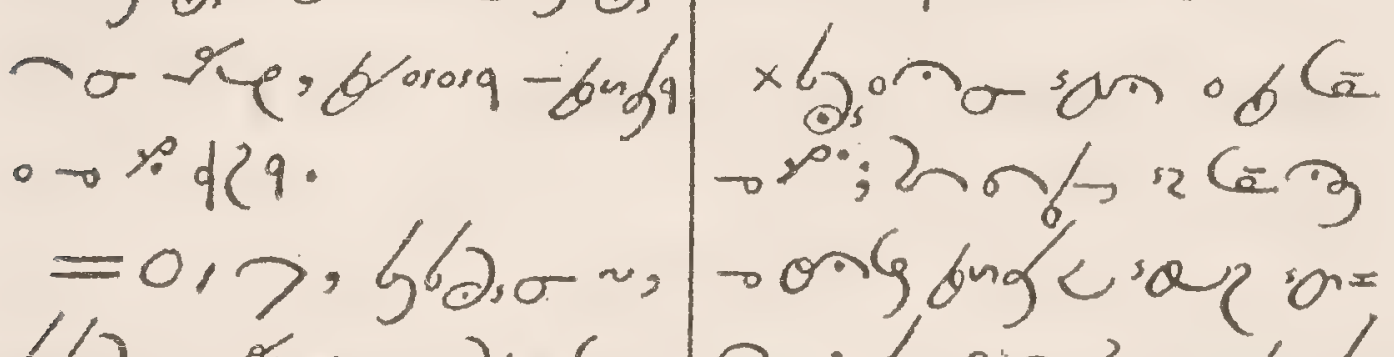

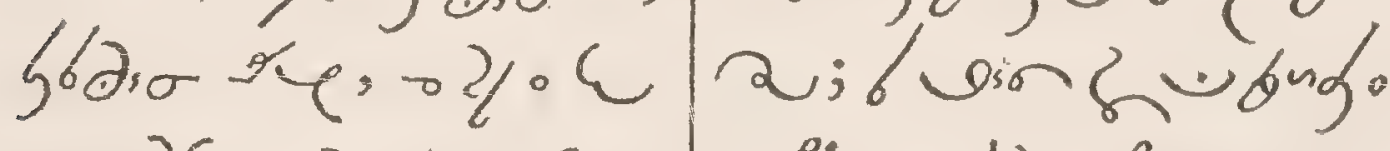

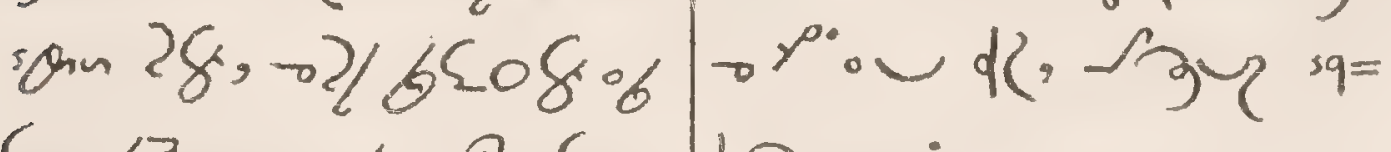
c $3,12 \circ 6-9 \circ c$ h⿻or;

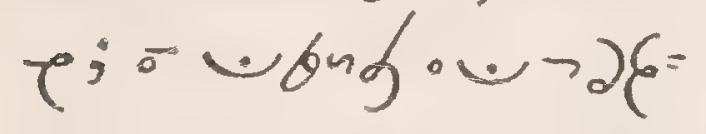

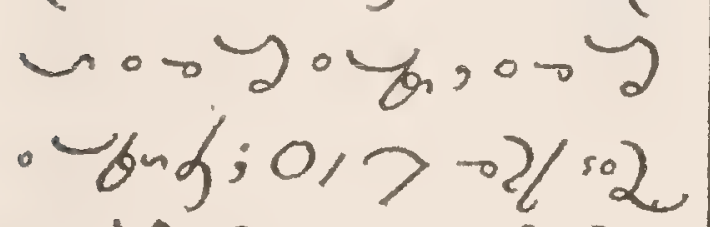

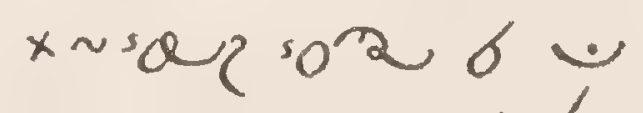
un os réfing $\circ \rightarrow \circ d$;

$\rightarrow$ a fe bor

cougt 2/vers.

$6 a-b, 2$ wer x जarso 26

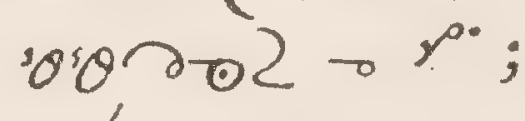

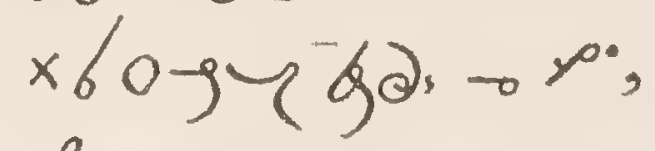

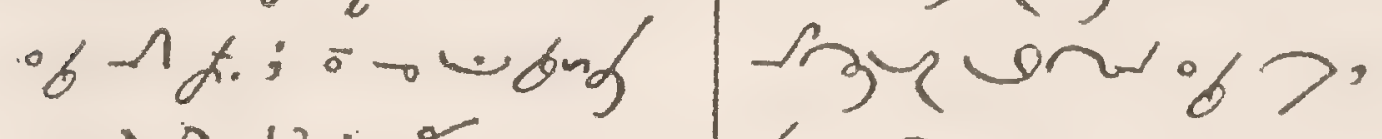

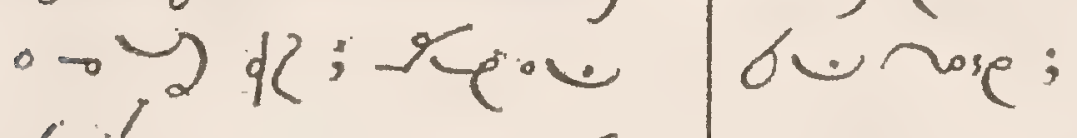

bing owsobage -

$x \sim$ sal sor $\rightarrow$ ving $0.1>, 6$ $x \rightarrow P Q \partial, 1 \geq<5=$ - 29 th. bue n, לb ds,e Fourth Consideration.

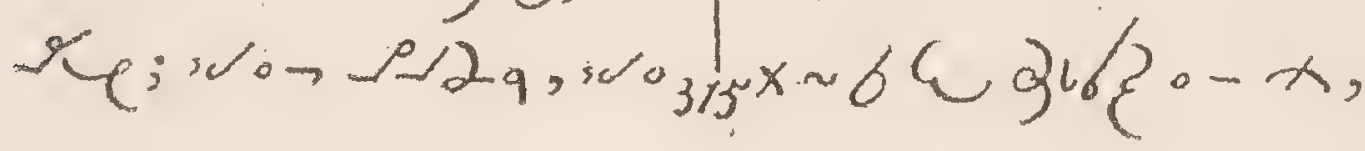


456 ivore ba osorg - bugfa.

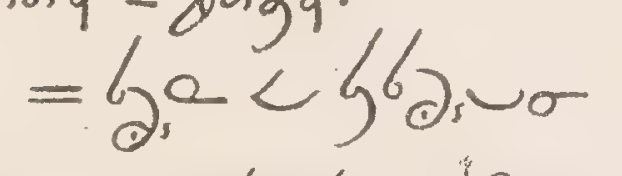

ze-tarie,

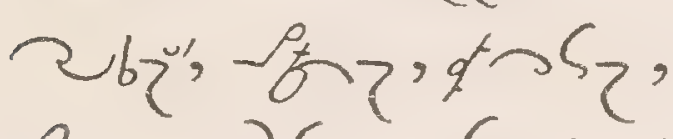
मि. 36, wh: : run osoleforeto x; z 2 osenos ox u力d oun oxionz sgos orn wong usodeso $3 \circ$ od on.

$$
\text { ox!'stern. }
$$

रdristowhom de or 2ukidist bras o whan,

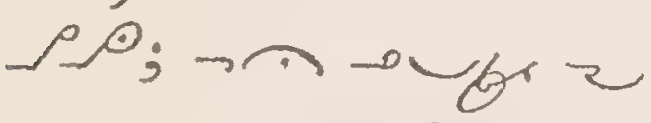
efx; $\rightarrow$ mble s द, zorez - - osóra, n sink.

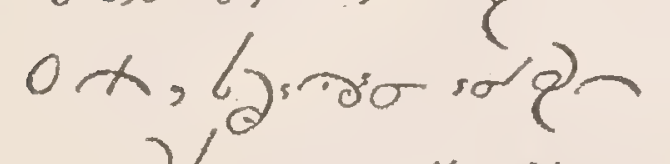

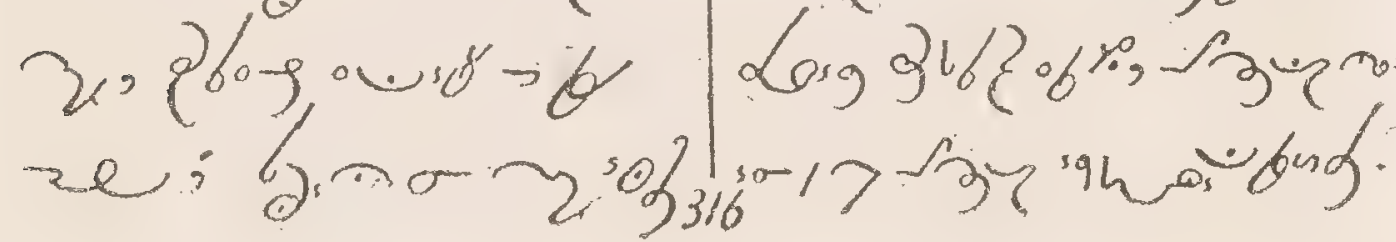

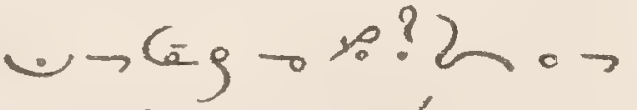

$$
\begin{aligned}
& \text { r e vong oro. }
\end{aligned}
$$

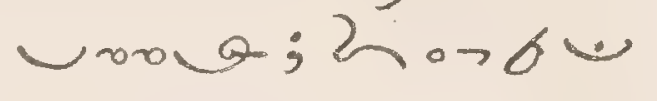

$$
\begin{aligned}
& \partial S \rightarrow \% \text {. } \\
& 0 \times \rightarrow \approx 6 \text { un } \\
& \text { zer 20-g; } \rightarrow \text { d }
\end{aligned}
$$

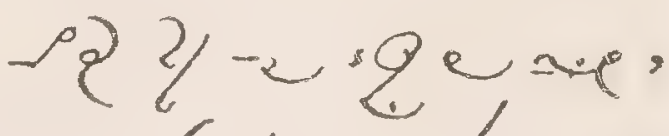

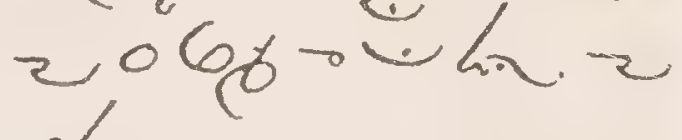

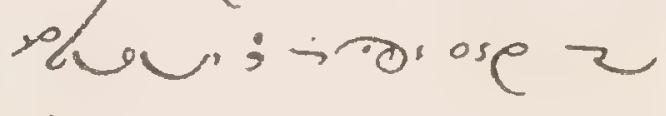

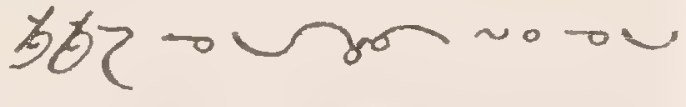

$$
\begin{aligned}
& \text { ר; ; } \rightarrow \text { os ose } \\
& \text { Proz-og; } 0.00 \\
& \text { ose } \sim \text { - } \\
& \text { notw; bariof } \\
& \text { ค, - }
\end{aligned}
$$

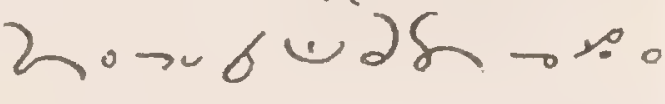

$$
\begin{aligned}
& \text { Ura;s pe } \sim 6=
\end{aligned}
$$

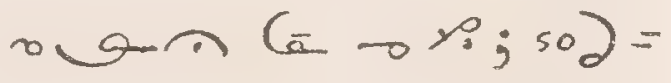

$$
\begin{aligned}
& \text { ur?/owbug: } \\
& \text { s } \theta \text { तse <orns } \\
& \text { wbug, busg } 316 \text { ? }
\end{aligned}
$$

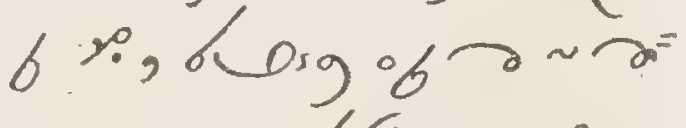

$$
\begin{aligned}
& \text { xus; sd / } 6 \log _{6} \text { : }
\end{aligned}
$$

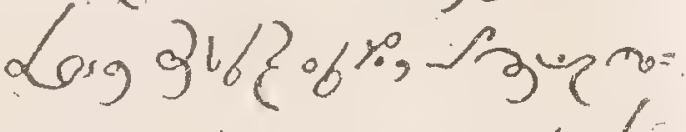


$x \rightarrow 280, x<562=$

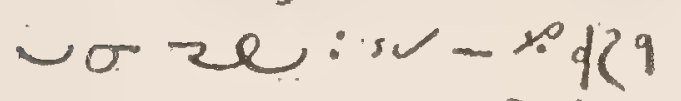

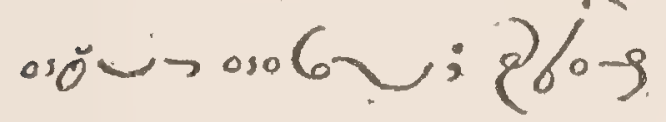

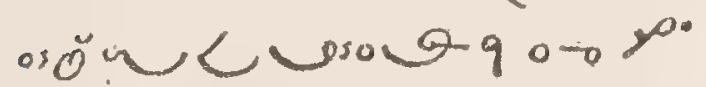
$d(9,869 ; 0$, one re -q-bugqusare $\sim$ ba向 $6 \rightarrow P^{\circ}$;

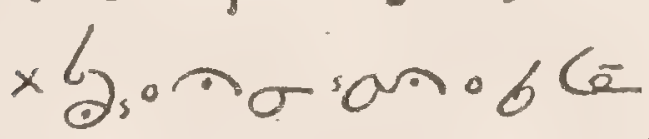

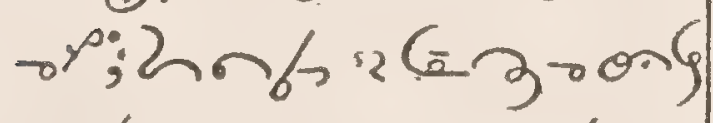
bugesararifin

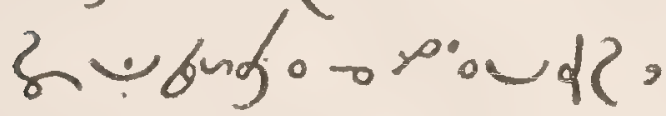
3y'ghnos; $x \sim \sin 6$ wentor refing

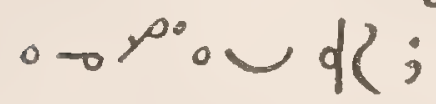

$x \sim \sec 20 \mathrm{C}$ $\cos \theta 0$ क 20 ;

$\times$ bo-gy gas o ro. sign on :6?, beras:

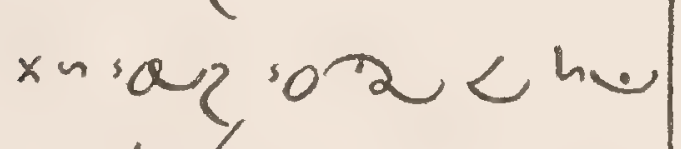
refong $60,7,6$ - 으 $9 \% S_{s}$.

Act of Ciontrition. wers a nape Confiteor. soson br nose 3is
Offering of the Mass. se rose.

$0,7,262 \cos <$

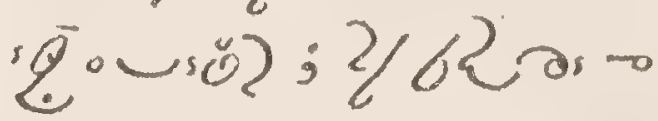

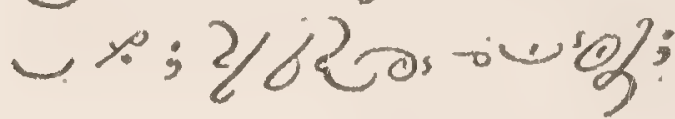

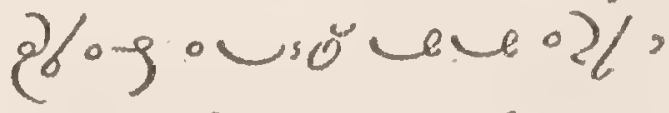
सी soluoure;s

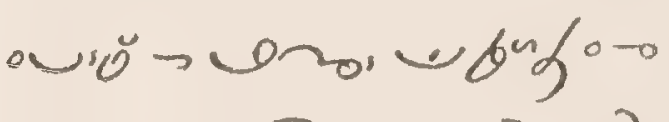

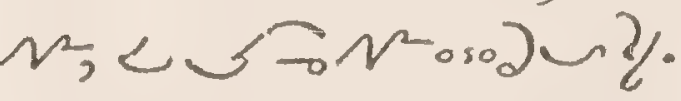
sodn 2/ O P 6

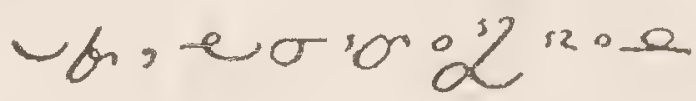
$9 \alpha h_{s}$, no- Q q non; -na-buatao $2 \theta$

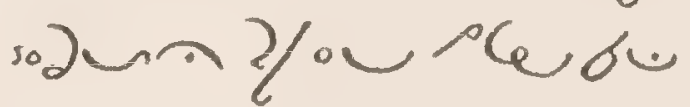
mon $2 / 0$ e $9 e^{\circ}$ so

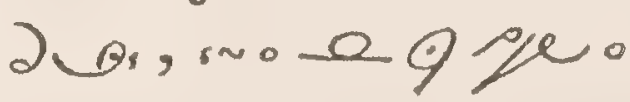
Uso) os; sodun? - pkrbe-x。

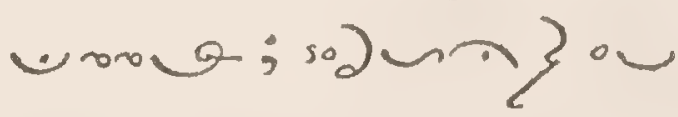

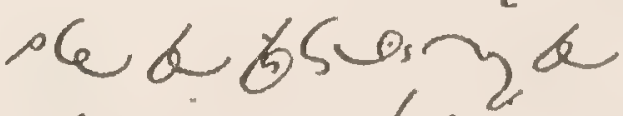
Tea vong 6 wsob = ag 6 o egng bevobre - tra. 
Prayers for Communion.

Before Ciommunion.

Adoration. $6 C C_{2}=$ vox 2 $2000=0$ >xíabre $>200 \Theta ;-2 / 1>62 ;$ Q वि $0-2$, - yei-7, -ye br. -yeson. b, 2 - $2 \cup \sqrt{7,},-$ ce or $00 \cdot r^{2} 8 ;-d$ or 20- $\% ; 0-2-2$ corbor 20-9y, e tpued ?8.

Humility. $\sigma Q$, ury $=$ ar $\rightarrow$ bug $\omega_{0}-x_{\text {. }}$ $=0,7 x !$ so. 0.0 s

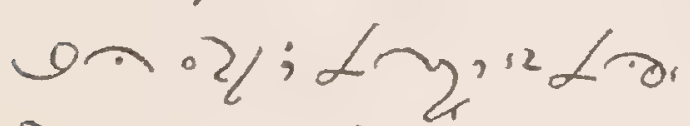
2): गदका, $2, \pi x$

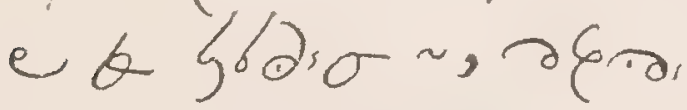

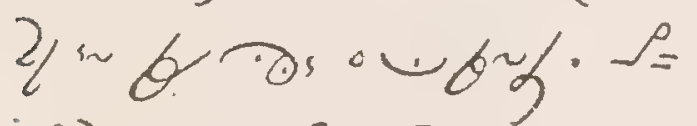

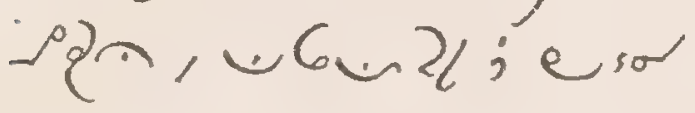
6. $0.62 n, 6$ oig of $20, " 26,60.0^{\circ} 6$ ?

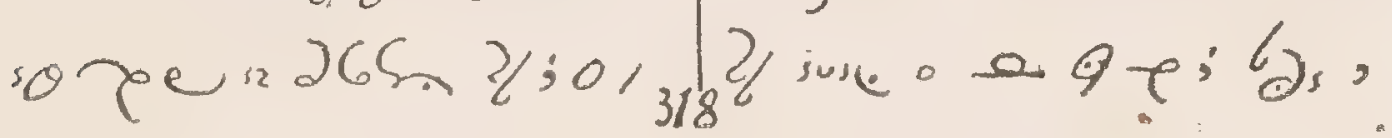

? $x$ bo woing ano 2. contrition. $\sim 6 C-\omega_{s 0}=$

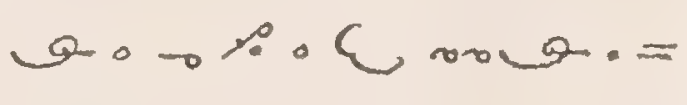
$0,7 \times 6, \mu-0$ un; so ree wow i- bub o.0 rwh;

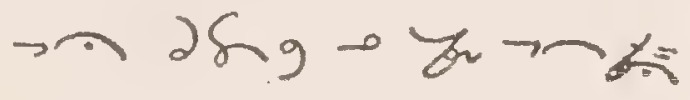
$\operatorname{sen} 2 ; 0,7 x, 60$ s.o七n; 几0?

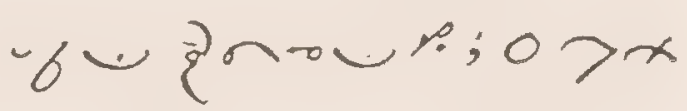

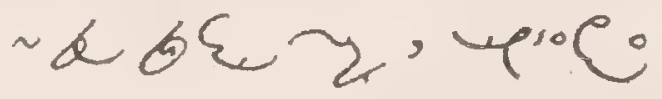
un; $\operatorname{son} 6 u 2 \%$. - bng?०obnge;

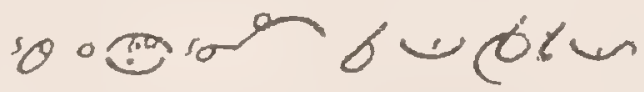
2; 6ய2 ? ? ज.bug o t y.

Love. $\longleftarrow \circlearrowleft x$. se. oc sol. =0, >x

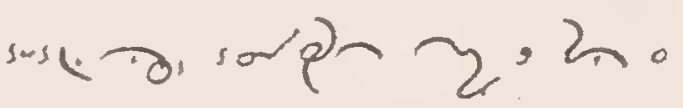
- 20.0 - sol $252 \%$

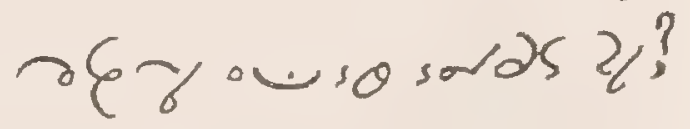

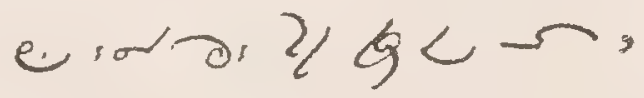

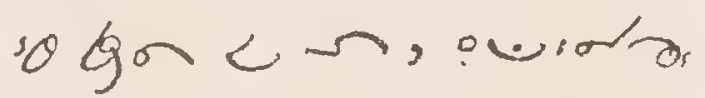


in $\therefore$ eq

Desire. $<x$ - $c_{6 t}=0 \cdot \operatorname{bug}, 2$ $0 \rightarrow \cdots+2 x$ $17 x 60$ bory; b.

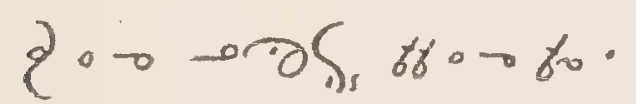

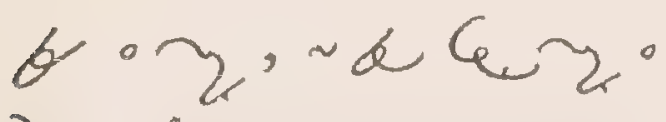

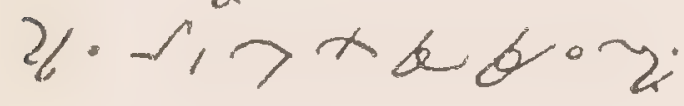
\% $6.0 .9 \cdot 60 ?$ $0.82,7,0 \times 25$ beग. 2, soo ung; brer, - a b. $50^{\circ}$

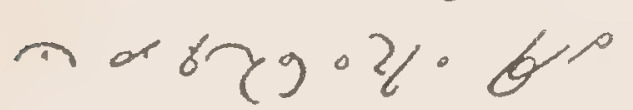
2orsab.

After Communion. Adorution. $y_{s} \partial \rightarrow$ , $70-r, x \rightarrow b \cdot b=$ - yougisoraraz $6 \varepsilon y, 0,>x, \frac{y}{2}=$ काa $\rightarrow$ ar $b \rightarrow 02, \phi \pi, \sigma$

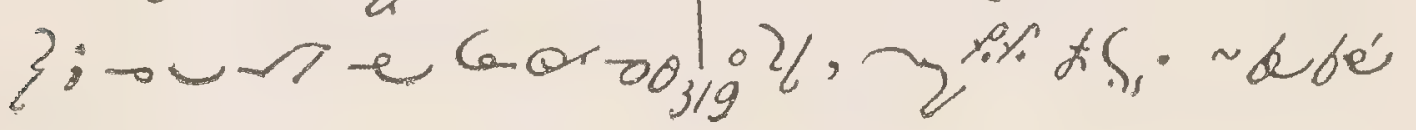

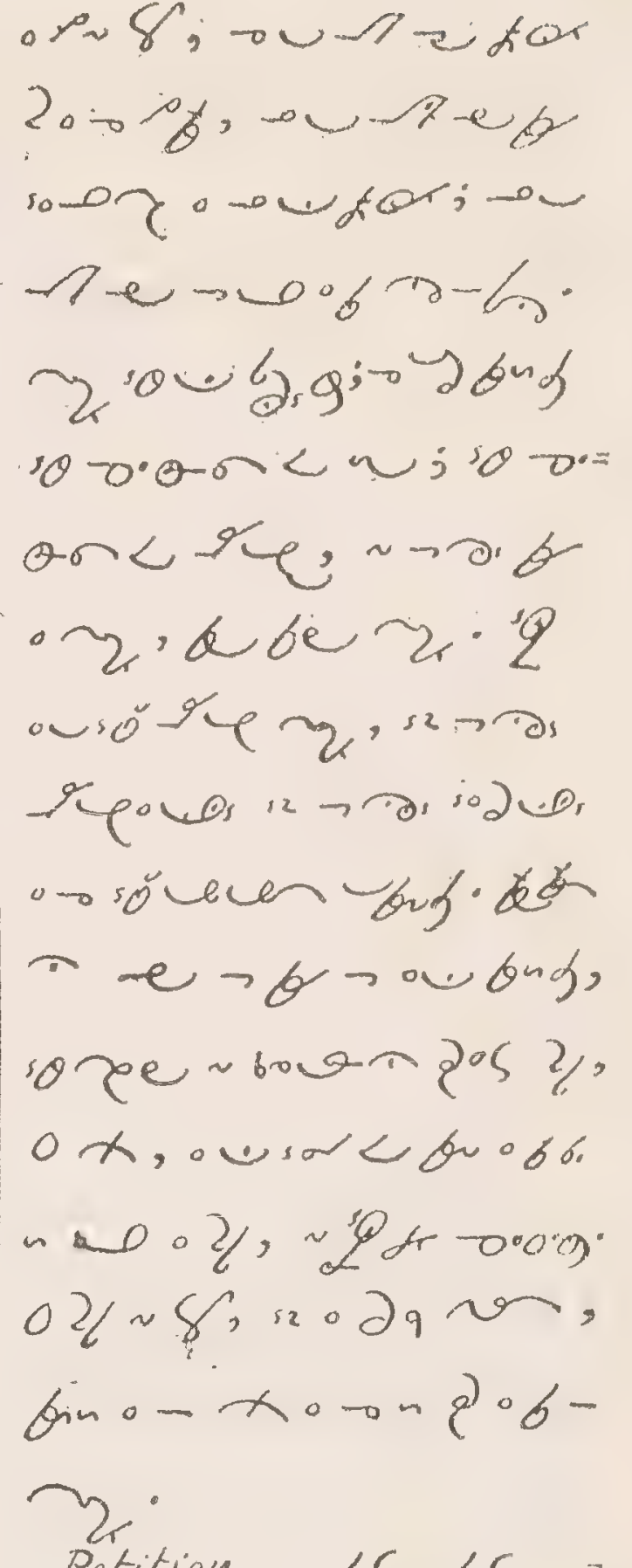

Petition. $~ 6 \&$ be $\rightarrow=$ $26 . x<$ coun $\rightarrow C$ bug. $=0 x, \rightarrow \infty$ zovong, nd rom

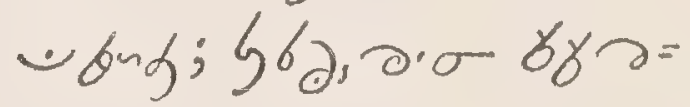

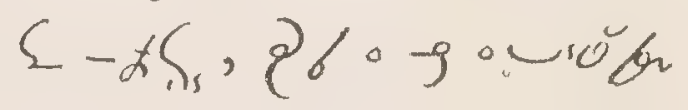


bunokebugt:a

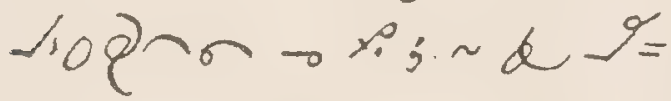
Towong owrora. 2 ori; r soderobe rrsin 0 Q Q⿱一𫝀口: $60 \mathrm{y}$

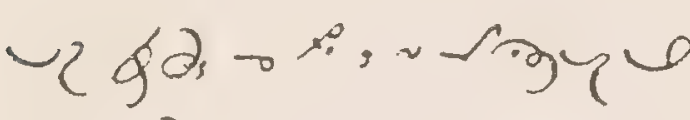
$\sim 02 / ; \sim \alpha, 100,0,00$

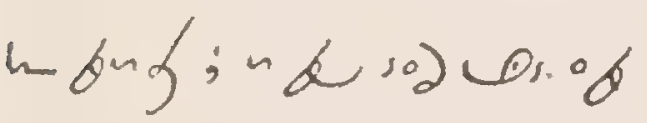
wo" Ke a loon. thof - 2 -ebyj; vorofung wan.

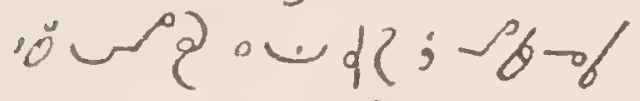

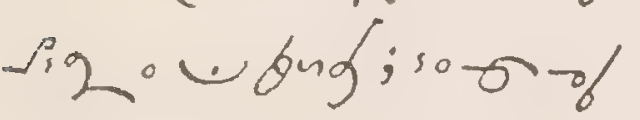

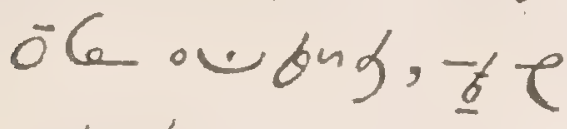
- ong. Resolution. a b f for $x$, or ree obso $9=$ eok sowar 2 $=0 \times b-2-20 y=$ ngl: sogrs a bu wa แ ree nbran $265 \%$; sक्ष s 2 s

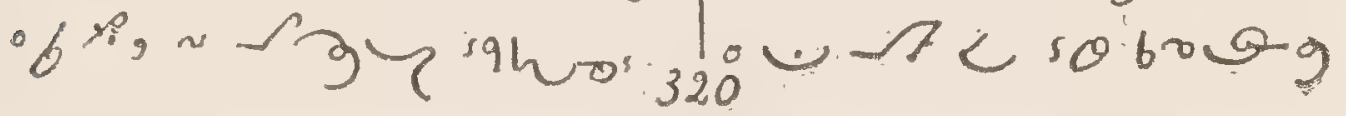

ubug. sq. $6 \alpha$ bre

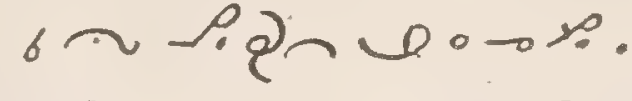

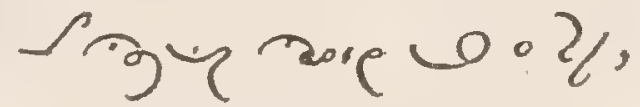
s 4 10 $2<02 \rightarrow 2=$ xing. 60 gry ba.

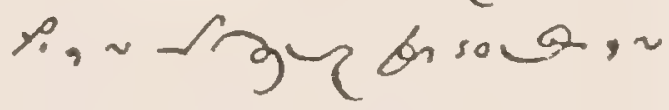
s उप nose $0.2 \% \cdot 50$.

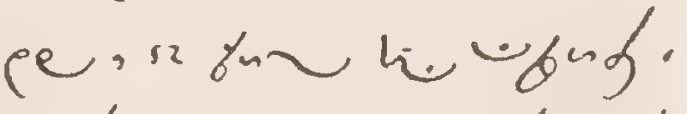

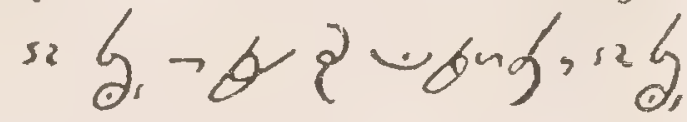
पद. Ox, य b - a-nangug. sona e bbe a wion. Offering. $6 \varepsilon$ so 2 大. 6 C n 2 h. $=0$ $x, 200-y^{2}=$ 102ug,ouvasson tr, s2o-uson:er T/Nonousoded.

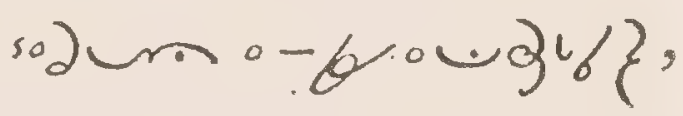
sor'ara crovi; solur.ebugbir? sо а «w.oke, e 220 - vifugisidan 
Worg; ranuet? $100 \Theta: 2 v 0$. 17,320 tose bn roser, 1200 dr ror.

Intercession. $~ b C$ bu. $-x .66 \operatorname{cog} 0 .=0 x$ $\sim k$ w.ok to nospos $120 \rightarrow y$ वे, 1200 - 20 $120 \rightarrow 2 \cdot N \cdot \sim d 6 \sigma$

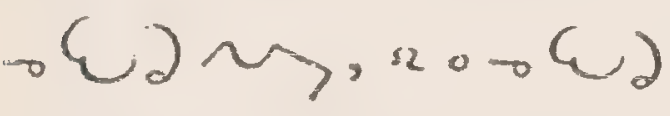
$m,<\sim 6 w-\not S_{1}$ "kberos b. $2<$ bogg. $\sim \alpha$ squm $\frac{a}{20}$

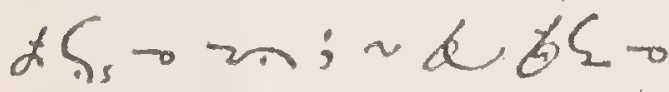
- mono- 0 son, 662 2 : $\rightarrow 0$ t. : Cag . mose, 0 os 0 on 0 ?

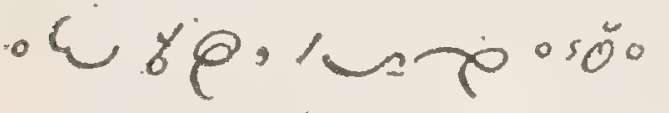
2u- ${ }^{\prime} \rho-$

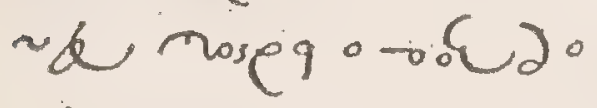
\&G Ros, ग2; s 000 snop, ad; dying d

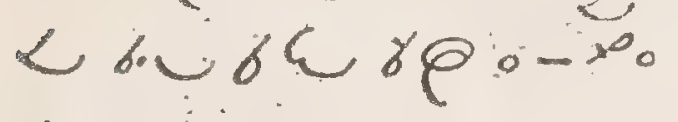
Cors.

\section{The Rosary.}

S. Joytul Mysteries.

1. suse $\sum r: \sim 6<\%=$ $16 \% \circ>9<6 \sim 6_{0}=$ 8 og a $8,-k$ - osenor.

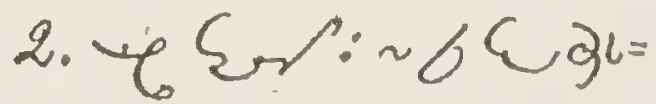

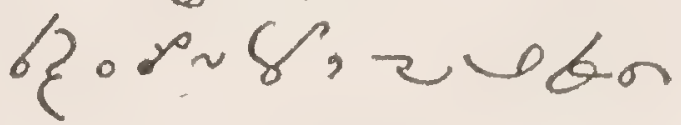
z $\{, \alpha\} *$.

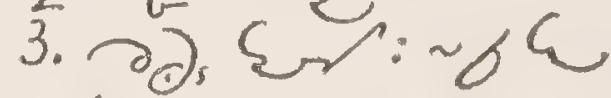
9l6? - $x=6-0$ do

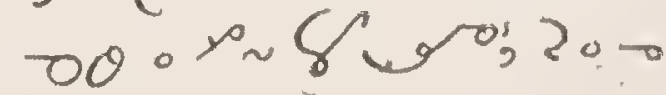
celo-os. 4. Cor Sor: 06 3प6 ० 28500 moses., so) $-e,>9$ o- $x$.

5. vonger: 26

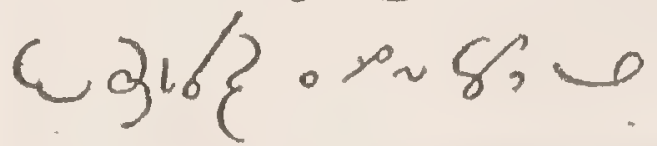
$\sim 3$ an wn-r ox $\therefore \rightarrow$ Gon 2or nasedr. IS. Sorrowful.

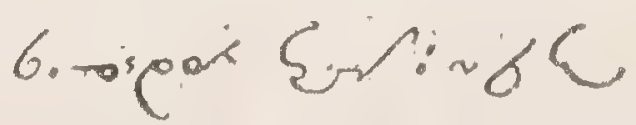

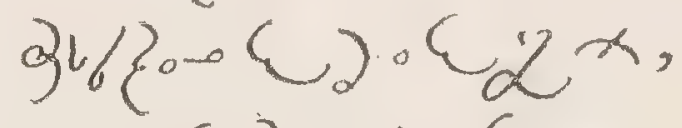
$32,20006200 y \cdot \xi:=$ 


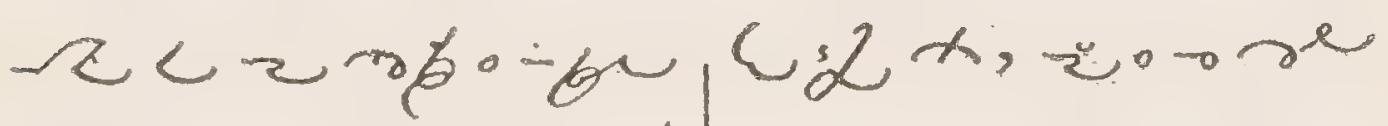

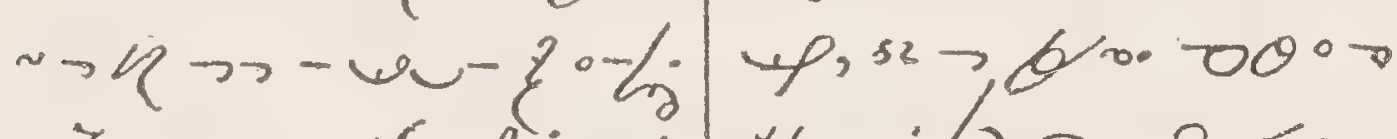
\% on wor: 6 $\varepsilon 36\} \cdot \varepsilon \partial \cdot \varepsilon$ $x, 2 \operatorname{ascor} z$ wifar

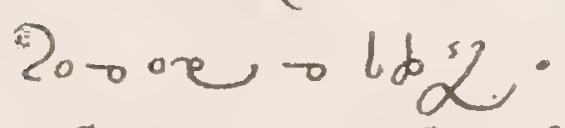
8. -rudkr: $C 36\} \circ C_{20} \varepsilon$ $x, \operatorname{ascos} z-0,-0,8$.

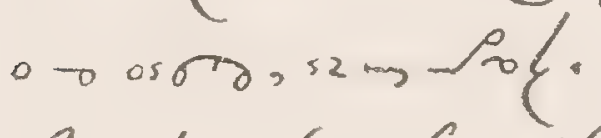

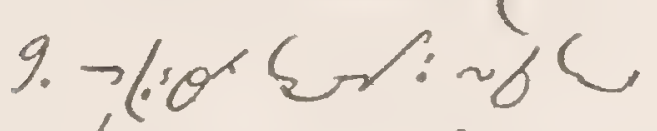

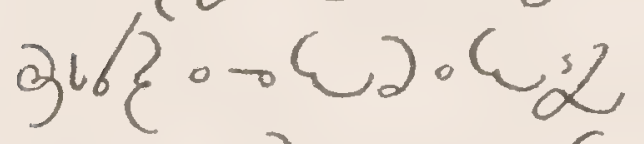
$x, z 2 c \varkappa, a_{n}$

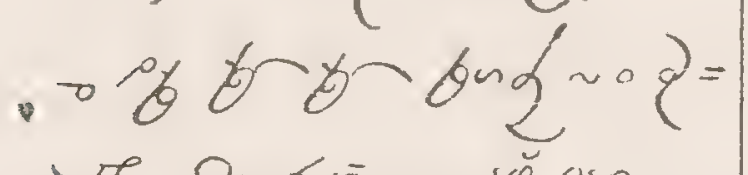

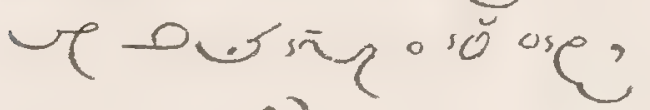

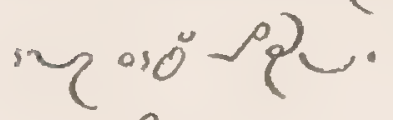
10. gor s: 66 $3463002 \cdot c 2 x$ $\varphi \rightarrow g \circ z^{-1}, \sim \infty=$

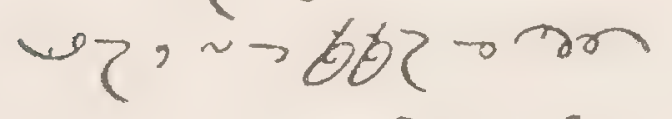

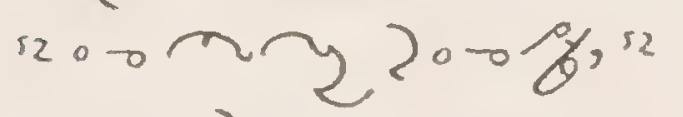
$\rightarrow$ t. breon

115. Glorious.

11. gos $=0$ od $\mathcal{L}$ :

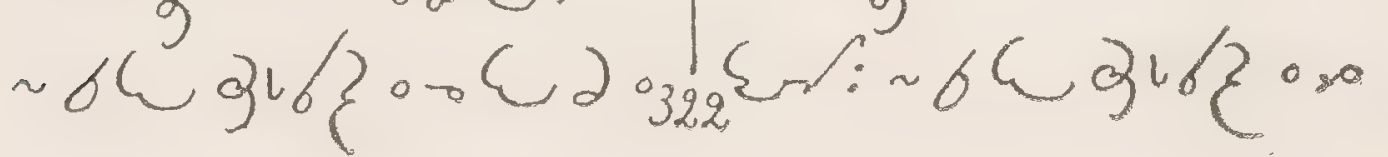

$14 \cdot g \cdot \alpha s 20$ Ce Er: " $6496,30 \times 2$ ?

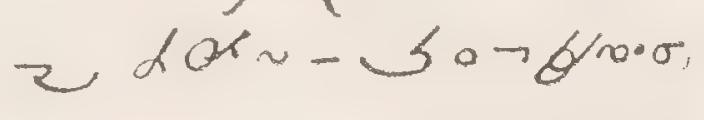
“awor, a-bas twothen.

15. $g \alpha \sim 0 \rightarrow$ Nor 13. gor s2 $0-0 \partial_{2} \varepsilon=$ $r: \sim 6<34 / 2 \circ$

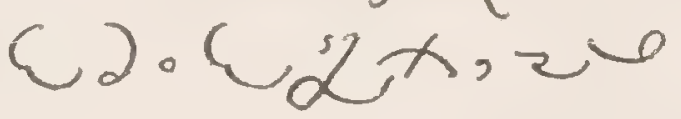
$6208.6 \cdot 2-2$ $29, b$ voon $\rightarrow$ - $y / 2$ guson oon. 12. gor 120 w Er:

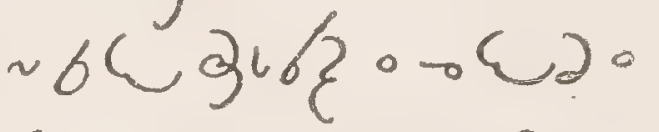

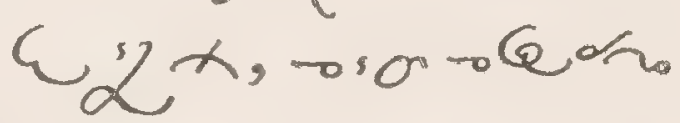
U, se $\rightarrow$ bro $0 \theta \cdots$

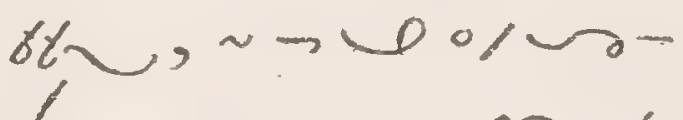

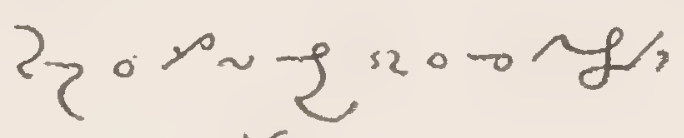
$\therefore 2$ c o o th Sis. 
$\sim 8,=2 \cos 206$

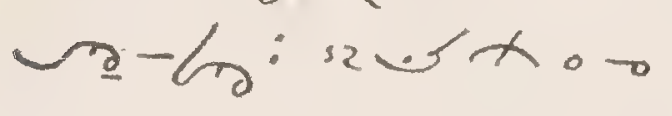
Cer orous a c Cé, D.r sus noe Qdx;

Via Crucis.

Prep. Prayer. ox, '厶.

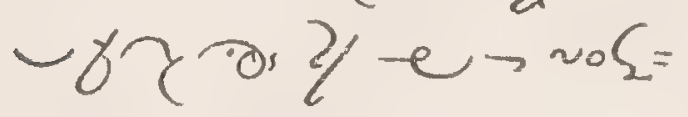
$\alpha \rightarrow g$, , e lo

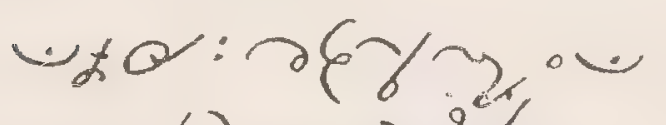

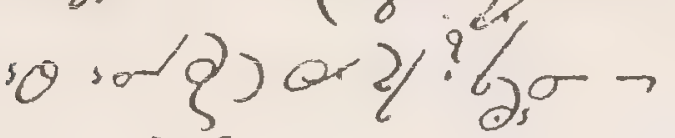
bor 250 - thin स्usu 2/; honu :

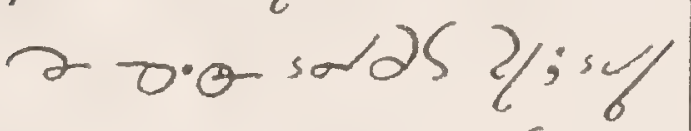
- es D a volor $\rightarrow \%$, e, $000 y=$ a: b osose r.e by fy - ox ow wa a be sobrag Ga or.

wou noe

Sancta Mater.

$0 \sim 8,2-2,9$ ra dx owbing $\rightarrow$ io osoté
Adoramus. Syoset. 2/, $x, \sim$ o cosou 2/; dxary a dor? $0 \rightarrow \infty$.

1.2 ำ $0.80-$ x力如反一⿻上丨

2.20 z s vegue ox $0 \rightarrow$ of

3. $20 z \sim-6 / 2$ -xodrarar. 4. 万० $20-$ d $/ 20-$ $x+2$

5. 760 - o. 드 $\rightarrow$ g

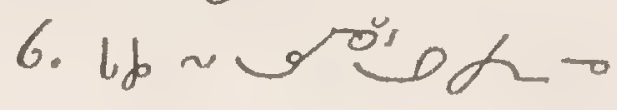
x.

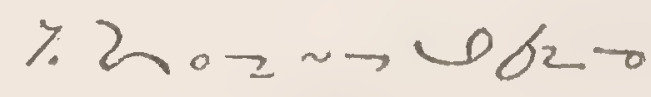
大. - b ब.

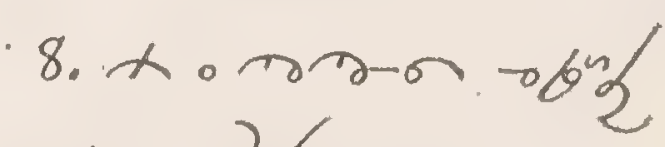
- grou 2fe.

$9.20212-662$ -x.h dos. $10.20212-260$ $\rightarrow$.

$311.20 \geq \sim-86 z ?$ 
$-0 \%$.

$12.20=12-y \rightarrow x$

$20 \rightarrow \infty$.

13. 2 ? ב $12-50007$

$\rightarrow x-000 \div 8$.

$14.20 z=-6 a z$ ox.

Prayer at ea. Station. on 2, 2, ב \% o.0 sold $2 / 0.0$ - bug e $728 \mathrm{~g}$. ton, $>\cdot{ }^{\circ} \rightarrow 0.06$

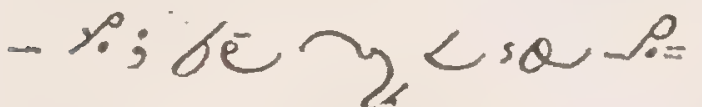
री 0 bra sat? $2 /$.

Pater. oca acs mase. Ave. of rore.

Gloria. fo borg, $\omega_{0.5}, \sigma_{0}-$ $=2 \cdot b \cdot 0 \cdot 0$, $20-3$

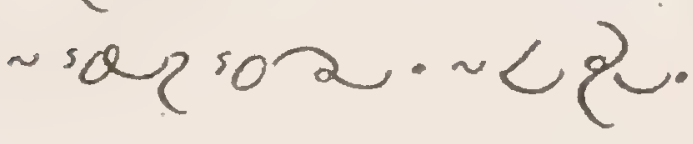

Guard of Honor.

Engagément. Ox, supe

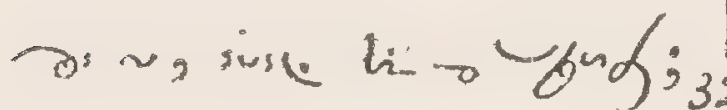

-eesal 9 atsis $; 8$ og

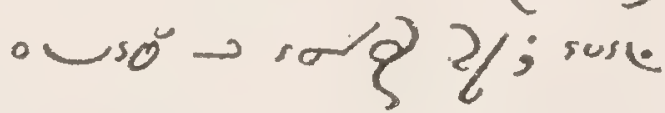
- 2 दे 2; - wong च $6.5 S_{s} \rightarrow 22 S$, $\rightarrow$-2ये; ousar vorma r fong:" ree noc wook y,es200 $\otimes \cdot 6$

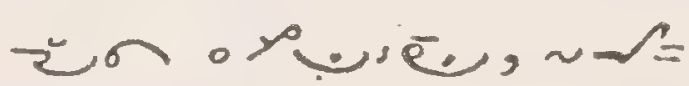
3y aror enong. ग/; bovosia- 2/6

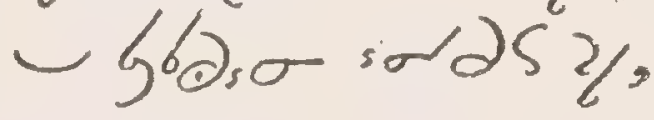

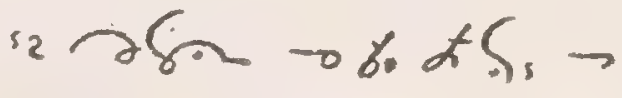
sed.

Hymns.

1. Vivat in Excelsis.

$$
\begin{aligned}
& \text { rosoung } 02 /, \\
& >x, \\
& \text { rosoung o?/ } \\
& \text { orne sodus; }
\end{aligned}
$$

bo' os:?

$\rightarrow$ क. रे 20. $8 \varphi \operatorname{sig} 0^{\circ}$ 


$$
\begin{aligned}
& 0.2 / x \\
& 26-2.0-6 \\
& 6+2-9 \%
\end{aligned}
$$

2. Au sang quin Dien.

$$
\text { noposen }
$$$$
\text { 2tor } 20+\frac{6}{6} \text {, }
$$$$
\text { s } 9 \text { \% }
$$

$2 / 9=4 x$

$$
\text { Refram: }
$$

$0 \times 6.06909$

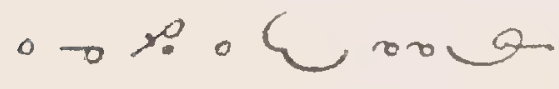

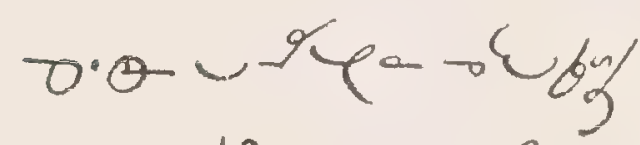
worg $d$ o tho

$$
r e x-x
$$

Qrome otori Phier 2 sonyer.-28nidro rever $\rightarrow$ tosohe b. 0 bobrota bio osa os.

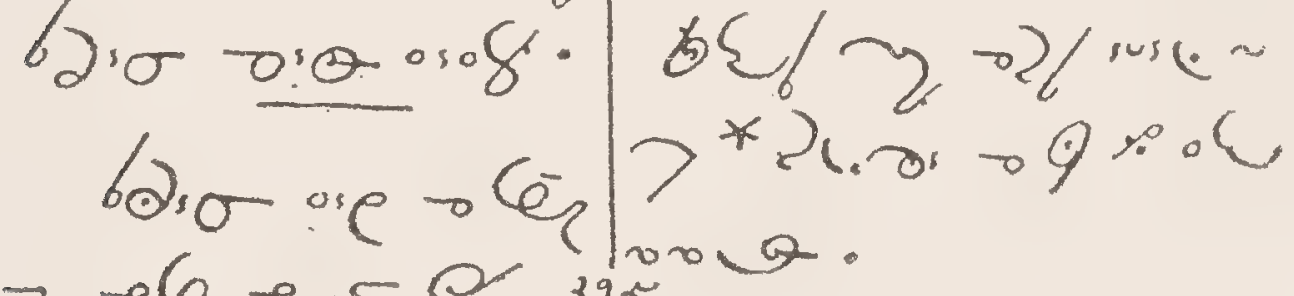

. 5 - krod

zonste.

ise $-6+\frac{\theta}{6}$

$\rightarrow$ orded oin

$60 \%$ o. 010

graroia o.

b6 $6-0 \rightarrow 0$

- ror $\sim 0$ m

$\zeta 6 \sigma_{0} 0$ oso 65

the

$\theta \rightarrow 6 / 2000$

$0-0 \Omega^{2} \rho$;

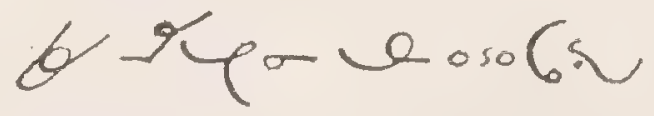

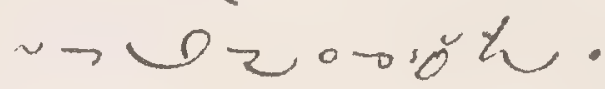

OX so Os Ca ro,

or 2 . Unow;

bo,a o a bing.

-

3. Miserere mei Deus. 


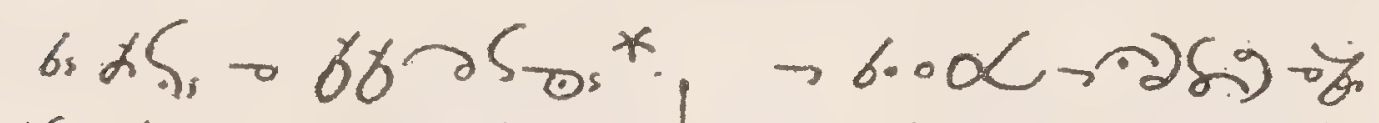

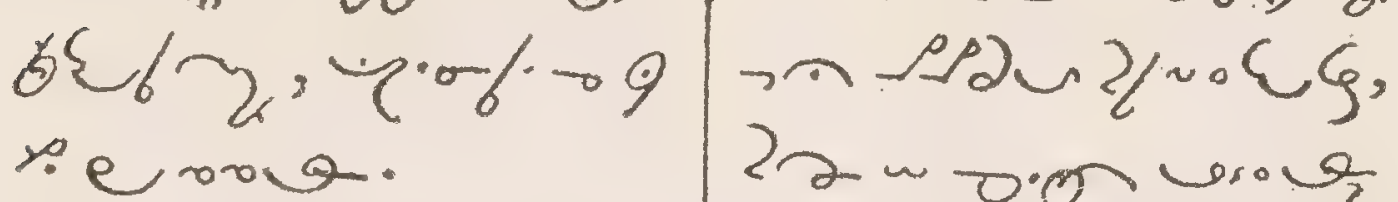

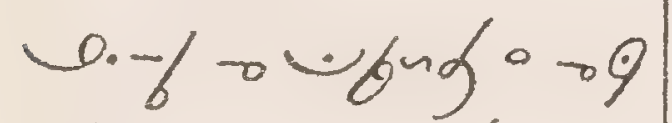
C.0.erg, $0^{\circ}=$

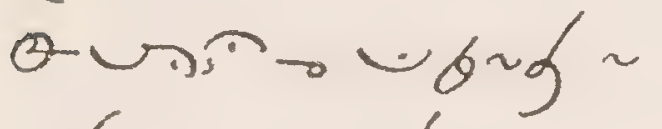
ale rogng.

20 $\sim 2 \theta 0_{0} 0=$

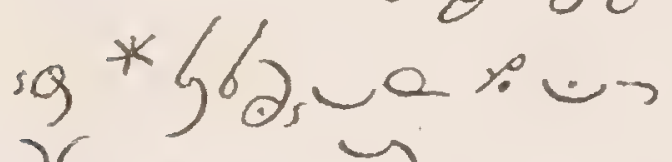
2द $9 \sim 2.4$. $9 \varphi, 926 . \div$ Giog*6.62 0 q Perag.

$-2 / \sim 7 \rightarrow \Omega-2=$

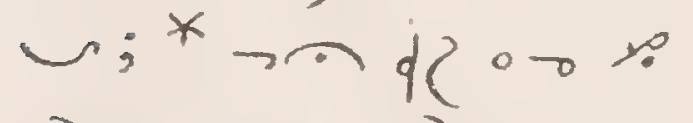

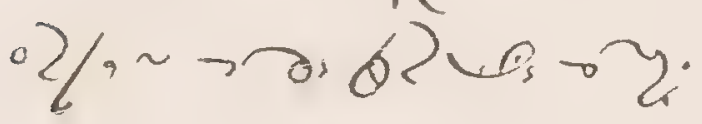
$0 \%$ a s o you

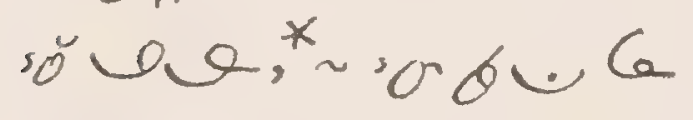
- te. worte.

4. O Bon Tesus. ox, 65.080. 28

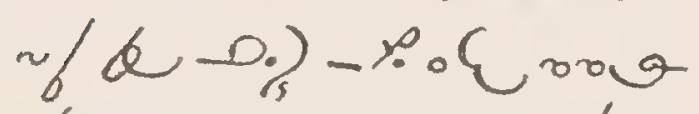
$\partial_{s} \rightarrow$ P. 0 \&bugh - or \& rogal $0 \sim 2$. 2a o.0 wouk

$$
0 \sim 2 r .
$$

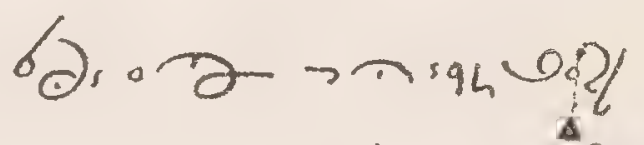

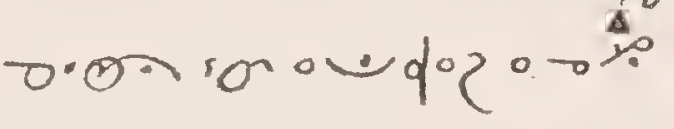

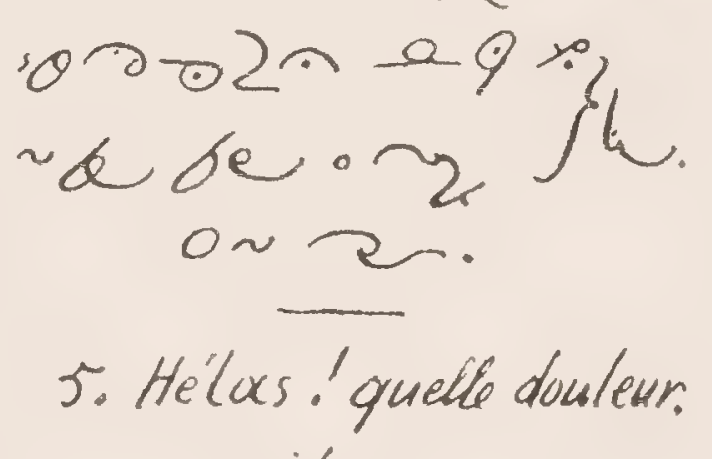
$\%$ के $\rightarrow$ anso

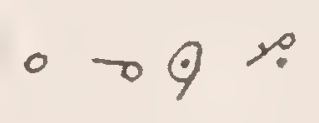$$
\text { osuo erow }
$$$$
\neg \text { คื, }
$$$$
\rightarrow 2 \mathrm{~d}
$$

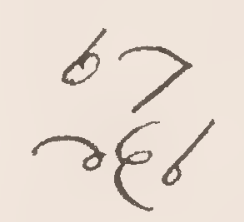

$$
\begin{gathered}
-x \rightarrow t r g \\
\rightarrow 0 . d
\end{gathered}
$$$$
\rightarrow \text { 以. }
$$

$$
\text { \%) }
$$


$\operatorname{sog} \theta$

नि0.0.9

- oveng.<smiles>[TeH]</smiles>

ज्व स्sक

.628

- on, 7

26

यigl-j

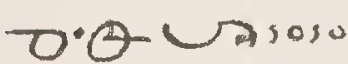

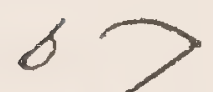

396

now-e

$\operatorname{cod} d x$

oo to

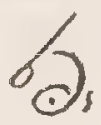

sevele

$\rightarrow$ ค

- $1 \wedge \not{0}$

6.0 Filii et filia.

$$
\text { O क力s, o:? }
$$

suse $\sim-y$ o s ep

Now 0 .

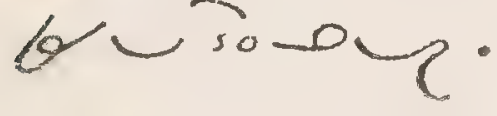

defo, defo,den.

wher benga

Cis soso o $x$

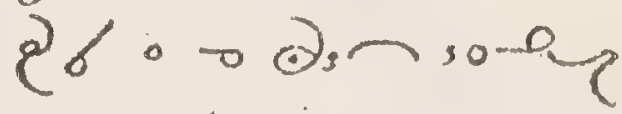

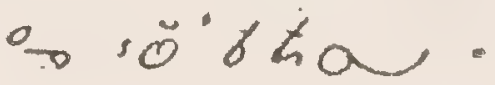

\%. Veni Creator.

by 2 ?

20 for. or $y$

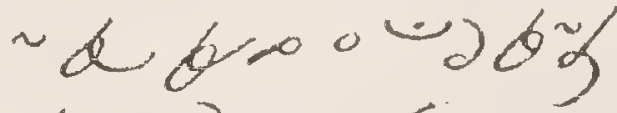

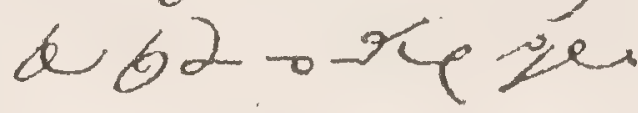

8. Le voici l'agneau.

$200 \rightarrow$ t

$00 \Theta$

001य6.

"bl by -

9. 0 Jour heureux.

d.

$\circ \rightarrow \Theta$

$\because 20 x$

2.226rion

3,7

${ }_{32,} \alpha_{0}$, vor $\sigma \sigma \sim_{2}$. 
1.36

SLAYAMEN

$\alpha \rightarrow \sim \cos$.

2. $2, a \operatorname{sog} 6 ?$

2,1
$\sin \theta \cdot 2$

verbang.

$\rightarrow 259-5$

यक्षsय?.

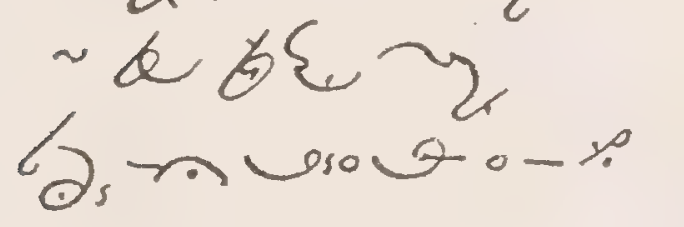

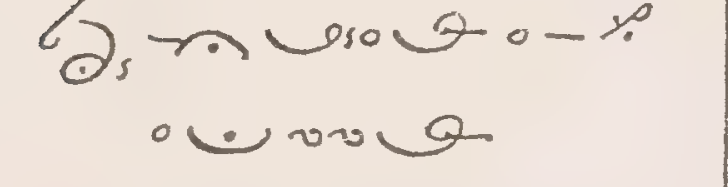

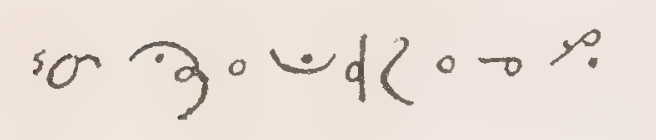

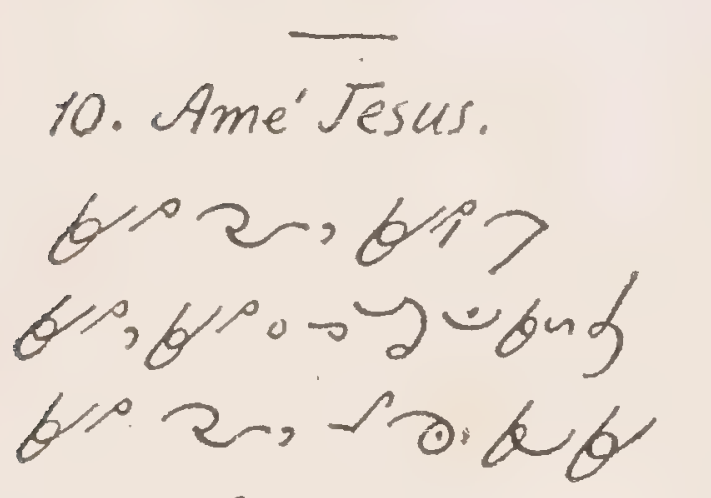

11. Chantons encejour.

o $x$

$\rightarrow$ - b >.d.9-bug:

bsaesal

de

(6-9 $\simeq 7302$ w 328
- Pbiburas

$\rightarrow \operatorname{sos} \theta \partial 2$

- \%. unal.

0 2- ba

$\left.-2 b_{0}-916\right\}$,

bेन

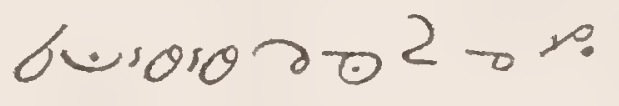

on $-2 / 0 \sim x$

rebub o

$30-2 / 6$ be

busosare $\rightarrow$

$26 \rightarrow 0-6 u$

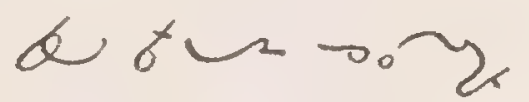

$\sim \alpha 20 \sim 2$

bubrore

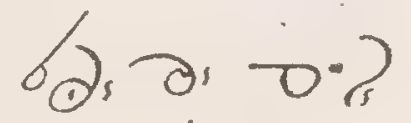

\& $b_{0}, 2 \sigma_{0}^{0,2}$

d reos.

kraveror\%

12. What happiness...

$>9 x$

$\rightarrow$ or bu० : - by 
$\partial_{s} \partial>b \rightarrow 0-0$ bug 13. Guard of Honor. refing bas, $\rightarrow$ ing $0.2 /$, $\rightarrow 2$-OS $\rightarrow 2 /$, bb: $: 0$ - $02 /$.

$0.25 \% 66$ o. $2 /$ -2/ suse $\sim$, $-2 / 1000$ का sus a r a rapel

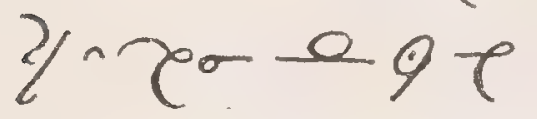

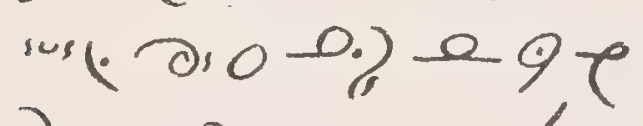
$\partial_{9} 0-2012-6$ क $2 \circ 6 \sim 0 x$.

$$
\rightarrow \partial \varepsilon^{\circ} \rightarrow b^{\circ} \geqslant
$$
b. sor Kegusotot

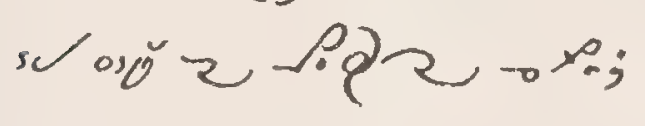
esa 6 be $\rightarrow$ ? bow sos a 2 2. $\sim 2, \rightarrow-2 \sim$ bug "b be o o 2 .
Refrain "De Marie..."

$$
20 \%
$$$$
\text { ong } 624 ?
$$$$
\text { sofio } 2
$$

$$
\begin{aligned}
& \text { Lomat } \\
& \text { oy r bing }
\end{aligned}
$$

$92028 \rightarrow 0$

$-2 / \sim x$

$\sim d 6,08$

$\rightarrow-2 \sim$ bus.

$\rightarrow$ He roung

2)

\% bo ogos $b^{2} \operatorname{sos} \theta \partial-2 \rightarrow$

$\sim$ d pog 28 , Uow ong, $\sim$ b i juorriog be borbons. 
14. Devant vous, esprits.

b> sod-ofo.

0 - $>19$

socsale bi ion

unorartat

k $\rightarrow$ gung o?

$\sim<$ \& I butg.

$-g x \operatorname{sus} 2$

bde $h$ or

vert 200 of,

curerat

rosoun i 7

$\sim<-y$ ong.

st $y \sim$ J $010 \mathrm{n}$

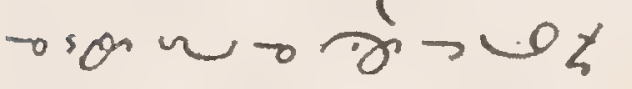

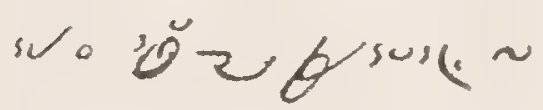

$\sim$ oro- $0-5$

rosoun,$>$

$\sim \varepsilon q-2$ ong.

- o $2 \cdot b u$ on

$\rightarrow b^{2} \mathrm{~m} 17$

sot sor $2<<402 / 330$ $\left.\sim \cos ()^{\circ}\right)$

nosom? $>$

$\sim C_{2} \rightarrow$ o ong.

15. Au fond des brülants.

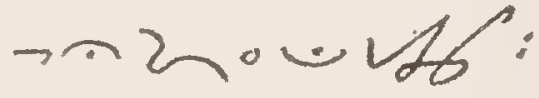

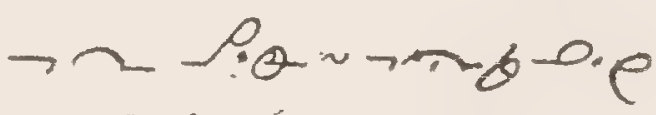

eQ906rong

8600.4660

$$
0 \% 0 \%,
$$

- voug soso 2

Pool 0 \%

O $\partial_{q} \circ$ rode $q$

$\sim 6.0008 .9$

sch on o majeq

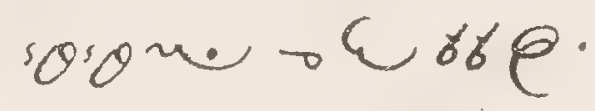

$$
\text { 的的, 品, }
$$

mo-o8a.0 9 b

$\sim 5089.28$. 
Slayomen Gatechism. I. God.

1. Lou reenot, $2 / 2$ 6 w.oburo- 2 ? $=$

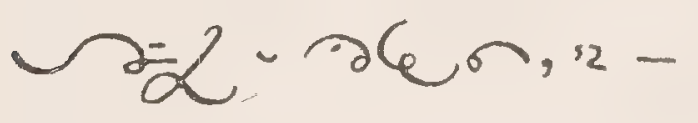
nosun.

2. $2 \xi b \cos 6>a$ -rene "s wookun? $=>$ dee ${ }^{2}, n=$ ohen, bi b o.0. one, betros Dow, oLbdL Dove glo go, $=$ eroburb. 3. $46>^{?} ?=0-6 \sigma^{\circ}$, 7, :o de.s; $56=$ 2.a , j60, a gro; b2n-9e. 4. $\partial$ ge no - - bj ' $\rangle^{?}=\mathrm{N}$ (2)-7.

5. a e e.o ane 'a pee \& on 7 ? = N-carane ${ }^{\prime}$ so $17 \operatorname{ser}^{2}$ b.
6. गदerogere 562,0 $\sim 17 ?=$ Ne qu2067:$\sim 090$

\% reeione 62 eq $q 5,7^{?}=n$ $-626>-99+2$ $0 \rightarrow, 12 \circ 6 \cdot 0 \cdot d, 1006$ $\operatorname{mon} \theta$.

$8.6767^{?}=176$ 2 \% $2-\frac{1}{4}, 20-$ 96.

\section{JI. Trinity.}

g. fion so, $7=10$; boa, 7 .

10. boun so $\sqrt{9,}>^{?} \cdot=$ Q $a_{5} ;$; G, of, ow: wis $\Omega$.

11. $\tau^{\rightarrow}-2-\beta ?=-3$ $\beta$, sh- whe tbe 17 , sing. 12. $-G, 120,7$ ? = 分, og, $>$. 
$-\xi, 5,17$ 14. 0 W, $320,17^{?}=$ 金, ow, 只, 15. $\circ$ G, $\rightarrow \xi,-\infty$,

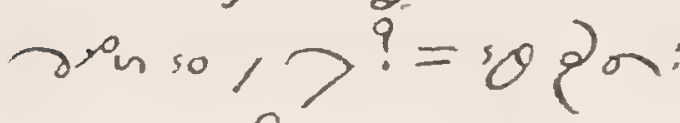
2is $19,32-1602$, $>$.

16. $\partial \xi \cdot 0 \mathrm{~s}^{\circ}$ r, $12-1600,7 ?=$ uzendortor $=$

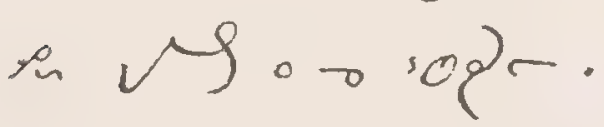
1\% o o sus 4 , b

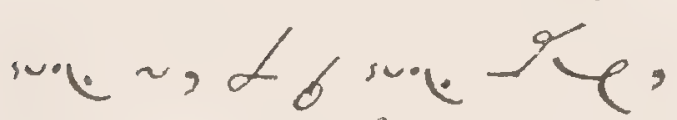

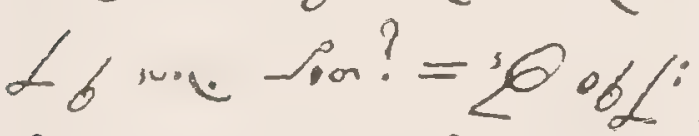
$\eta 0 \sin ^{2} \varphi_{0} \rightarrow 0^{\circ}=$ शि.

18. osise, 2. )$\cdot 0 \alpha \cdot=\alpha_{0} ; \alpha$. ) 0 L: - 1 is, a ve $\rightarrow$ ld.

III. Jesus Christ.

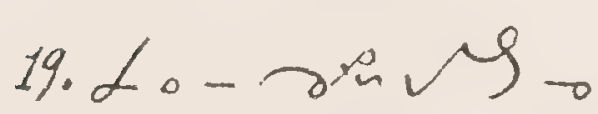
bd $\zeta d ?=\cdots,>\xi$. 20. $2 \xi 17 \xi k 6$

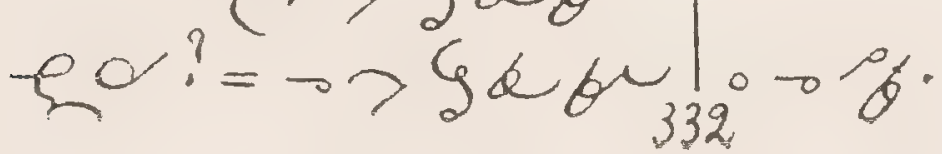

לo, arean - oson z. ?8.

22. sy $\rightarrow$ ordel? $\xi-\Omega$ : 0 son? $=0>$ g, hee 0 टnorom $20 x$ $\sim \delta$, ow. or z23. $0>$ gab h, - 2 : 0 < ? $=10 \%=$ e: $\rightarrow>g, h^{-\mu}$ : 7 . $24 \cdot-2,>=1$ .0 er दd? $=-\xi$ 17 a bu hax 800 orm, w anof :firdot 25. - $1>x$ b $5 \alpha, \alpha^{2} 2^{?}=-2$ $1>$ a d cá $x$ 62. 26. $\angle 0 x ?=0 x$, U, $>-b d h$ 2x. $+1,0-0$ \& $0 x ?=$ -x xof et? 
28. 万人- 0 - 600 - oblue? = - 2 six zosery, noothe. 29.0大二⿺辶0 $00^{\circ}$ - the, - $2 \cdot 0.200$ 品? $=6$, ox oo-othe, ceano f - 0 o, volo.

30. ox boverg

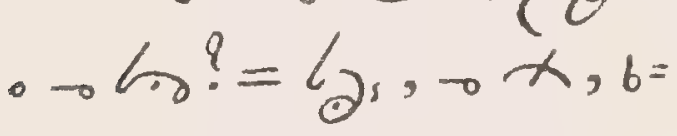
are $\theta 0$ onch

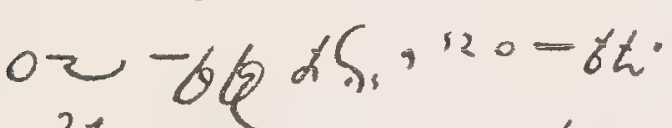
31. 2er:ox-

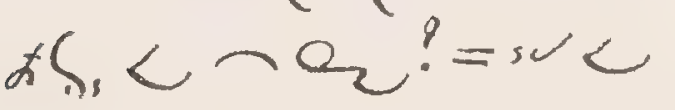
avd outh. ob - bo, s varia or $\not S_{s} \circ \sigma \wedge d s$ IV. The Church.

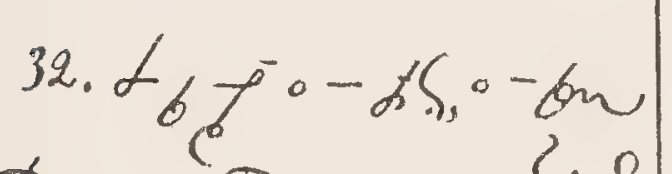
$x,-$ sine o- $\} 0$ $\% \sim s-b ?=-a)>6=$ er人एo-

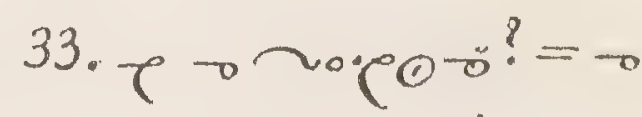
حopoor, sto - bs, decheo-x- bo $-\alpha S_{s}$. $34 . L_{6} 6 c^{6} \cdot-x-e^{\circ}$

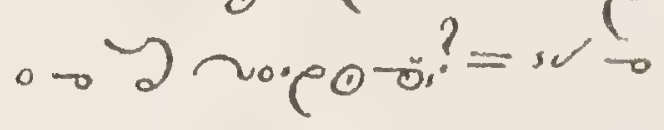
क, $12000 \mathrm{M}, 1200 \mathrm{~N}$.

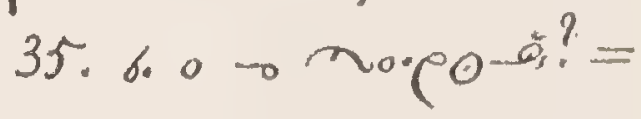

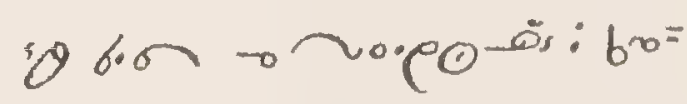
a - $0.02 \lambda \sim{ }^{\circ} \mathrm{C}^{\circ}$

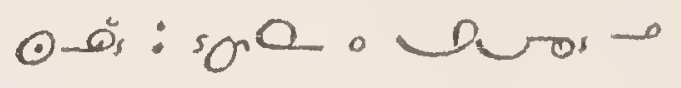

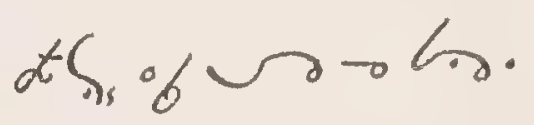

36. - $0 . \theta ?=-4060$ ? Do०० $y /, 0000$ b., $0.0-2 x$ حo.eo-s.

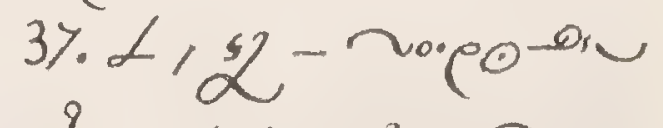
$x ?=\cdot x+02 \div \sim \sim^{\circ} \mathrm{C}=$

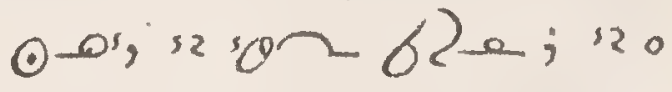

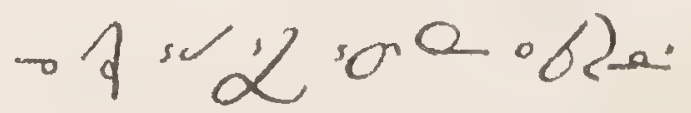

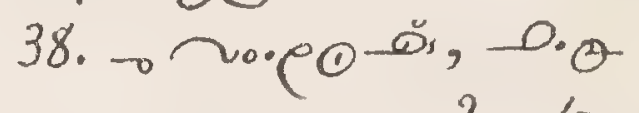

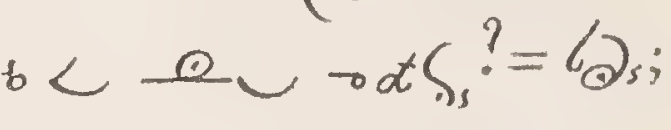

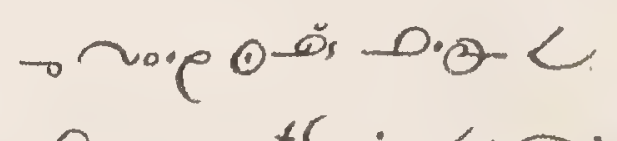
Q⿻ ot $h_{s}:$ is, $>w$ 


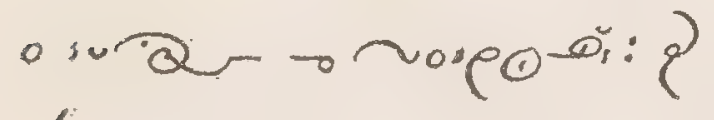
bo-g os 0.2 .

$39.263 e-\%+0 k$ - d ke-bu - ino $=$

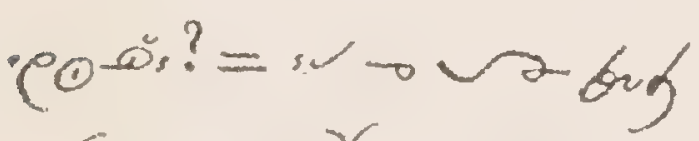
- Ge - 25n- bu

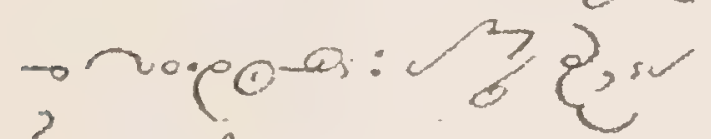
\{ $01 \%$ v. The lireed. 40. 26ol man, "wh "óvats? $=6(2-3)-d t \rightarrow \infty$ low 22. $41.2-6 \cdots$ tion 6 $7^{?}=\cdot-\omega \cdot \theta-\theta 0=$ vor, a prow - bu $1>$

42.400 .000 ?

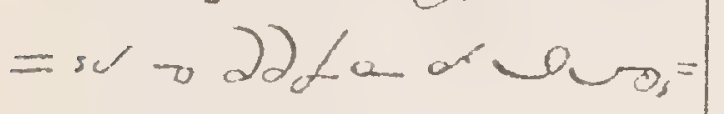
$v$ or a das core.

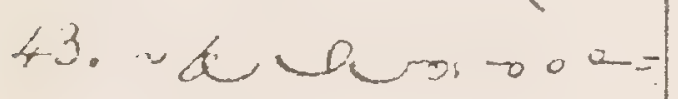

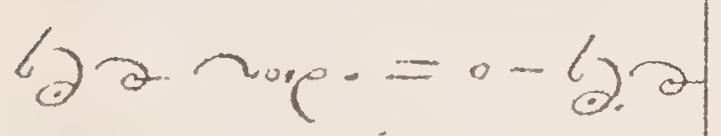
7950.96.

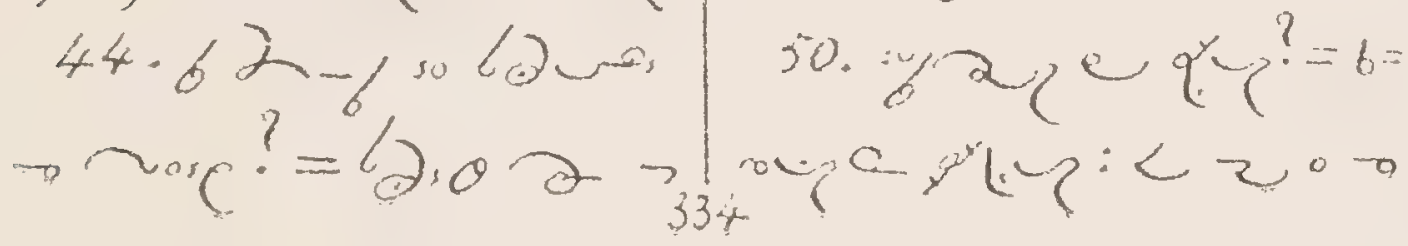

Cymorg to nope isu $\checkmark>$. 0 , s.gent $>$ obe laiv. VT. Sign of the Cross.

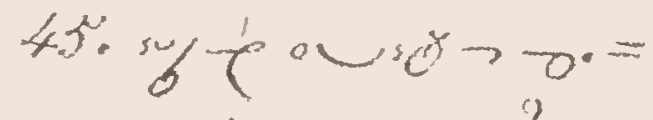
$0.0 \cdot 0$ lison $1.7^{?}=$

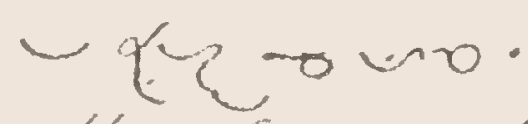
46. गद्धes क्ष? =

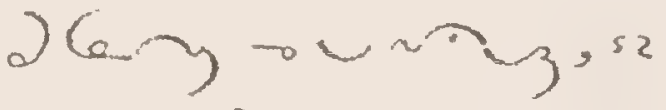

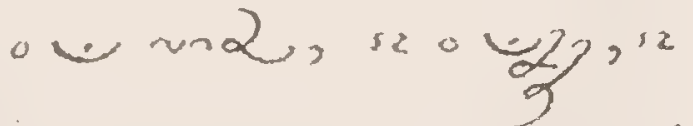
- wor $269,+20.9, n$ ow. $u<\varepsilon$. $4 \%$ Co.0.erer? $=0.0 \Omega-3-3,120$ -x 2 to 200 g. $48.260-0.00$ $-3-3 ?=-1022<0=$

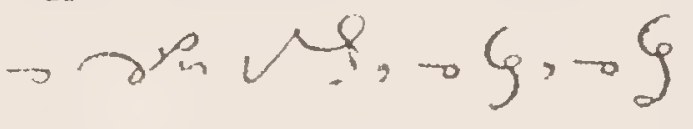
- w.

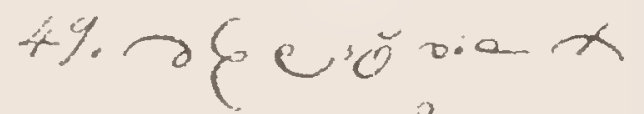

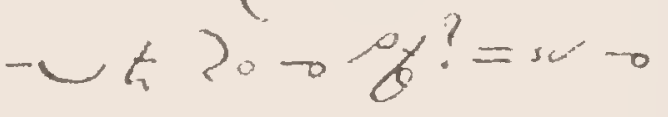
$\varepsilon \rightarrow$ 乎的. 50.

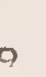
(n) 


\section{MANUAL}

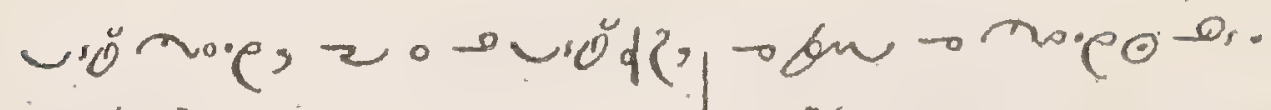
$\omega 6$ bog $0 \%$

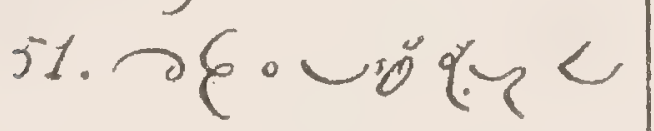

zonsomas, <z

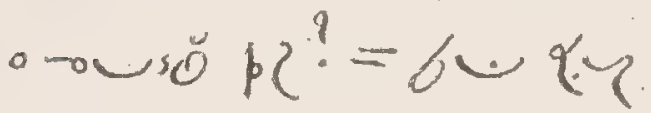
$6 \cdot \log _{2} \rightarrow-\log ^{2} 0 \mathrm{e}$ q०खार $906>\cdot 6$

o. d d

52. गदeध

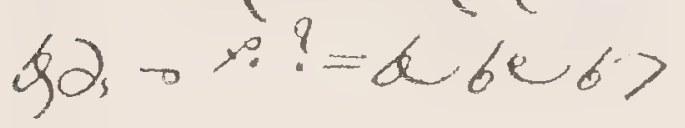
be. $\mathrm{Cug}_{\mathrm{g}} \rightarrow \mathrm{P}$.

VIt. The Commandmients.

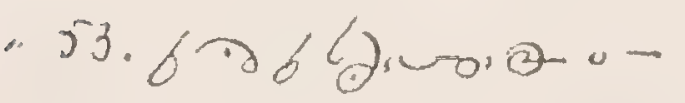
En $6>^{?}=\omega-22 / 2$ $\circ \rightarrow g$ bir $>$.

s4. $\partial \mathrm{C}^{\mathrm{a}} \sim 2.0 \mathrm{~m}$ - 9 on $17^{?}=0$ C oc z.ous, ag bu $1>, 0<\operatorname{sos} 2, p$ $0-9 \cdot \varepsilon$ ong, $a \cdot \varepsilon_{s}$ e日ts, $2 \circ$ \&.

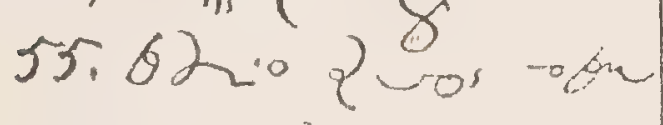
$\rightarrow m_{0.00}{ }^{\prime} ?=b_{1} \cdot \theta$

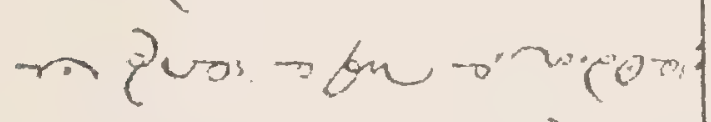
56.0 d c o d o bu - nopeor $=$ ins,

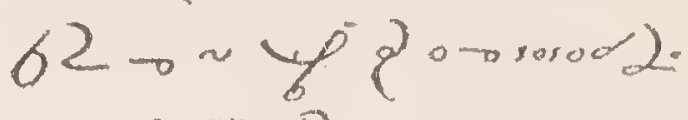
VIII. Prayer. ry. $6-102$.

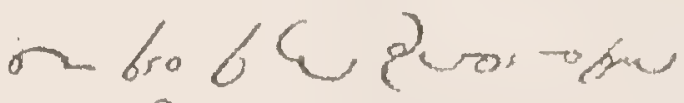
$17 ?=006600$ 以>. \& 2) var bui, ? 58. a kas googe $17 ?=$ s 6 a no.

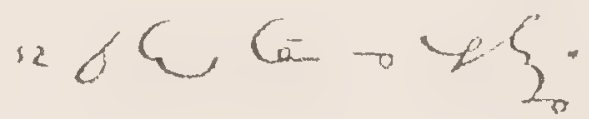
$5 \% \sim$ \& 226 ino.e. $=s+$ mo.e $\operatorname{cog}$ - 0 ג.'

60. * a sos 6 ?

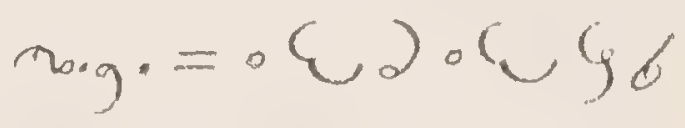
$2.6 \cup 2 \cdots$ 61.12 6 bos 226 $\sim$ no.g = a to bue ro

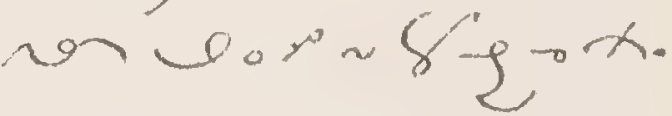
62. - b var--bu

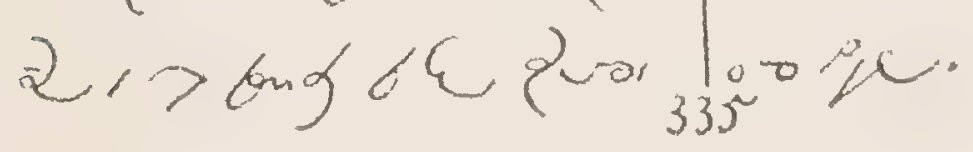




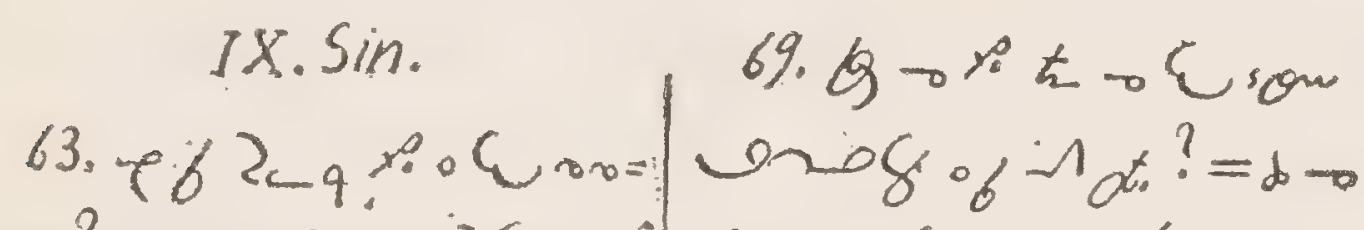
$\omega ?=\pi-\varepsilon+2 \delta \rightarrow 0$ on 17 .

$64 . \mathrm{g} \rightarrow \% \circ \varepsilon$ rova?

$=$ us o yrow:

"2now:Crowa 2 - $4 \operatorname{son} 6$ U for: s2 $n_{0}-r_{0}<$ rma. $65.0 .2500 \mathrm{C}$

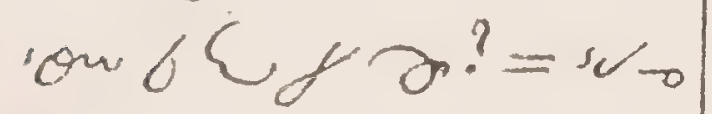

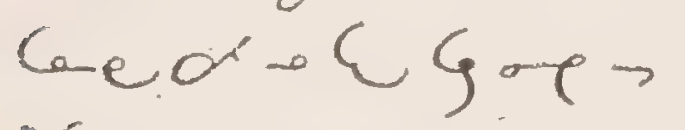
ase-on $1>$.

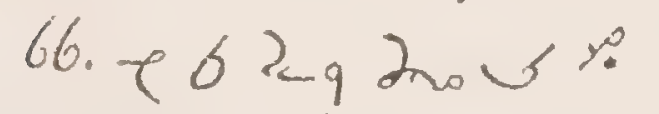

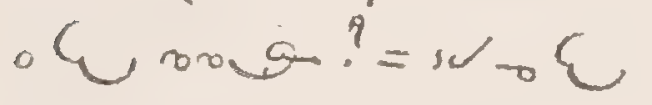
Lo gug $6 \mathrm{c} 2 \mathrm{~h}$. om 17 .

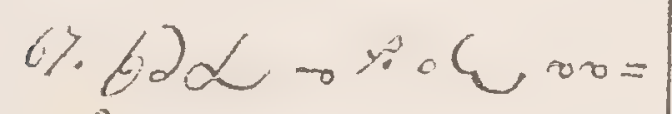
$Q ?=\omega G+\% \circ \mathcal{C}$ row: DroL ričt - Lion;is Jor $\alpha$ racke ok com.

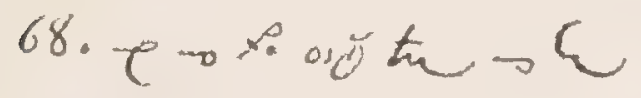
sow? = w $00^{\circ}$ '.

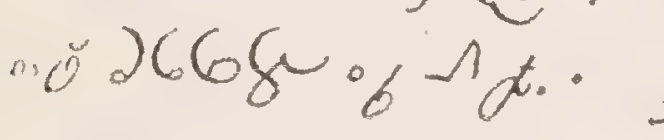

r.t. Usont soe

yeer 7 bre of Et, onoge of $n$ t..

$70.2 \varepsilon+20$ sofros tha - Cuson? = wi ser $2668 \mathrm{~s}$, $\rightarrow-6<660-0$.

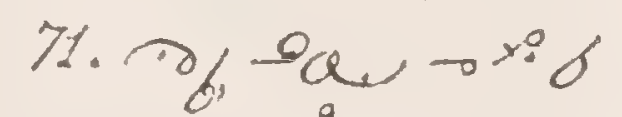
ce $660, ?=0000$ wn: $006\{96$; 006र- L bng; 00 oc be; 00 oc di;

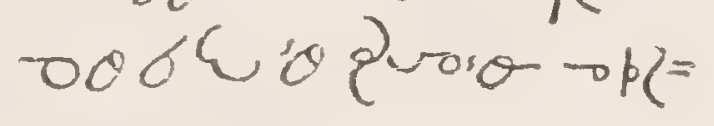

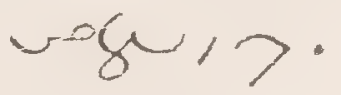

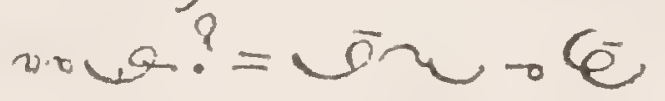
- pocraviso but $9 \operatorname{cong}$, thens,

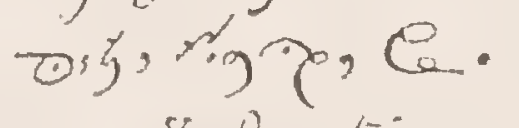
X. Baplis:n.

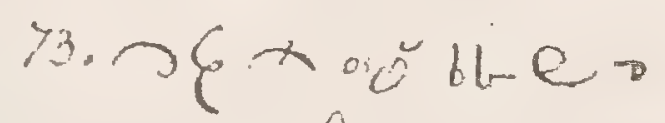
sin $y !=w C=$ $22 . g-G, \cdots \circ$ 
$=02-C$ sion. 2nm

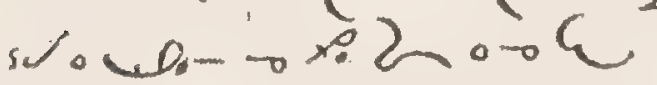
son bC gri, : 60

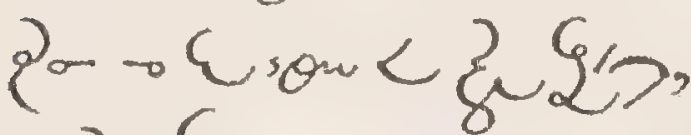
टद्यु क n. \& 12 .

76. $\rightarrow$ ot $6,<; \theta, 0, \theta=$ 几,, so \% vad? $=$

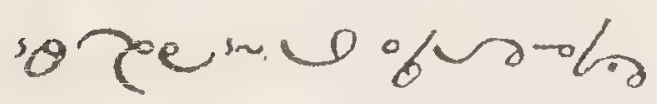
-oth c savion.

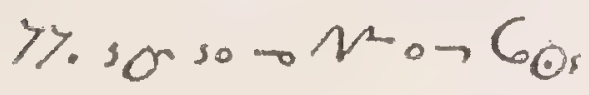

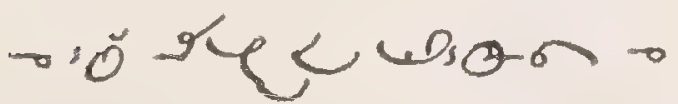

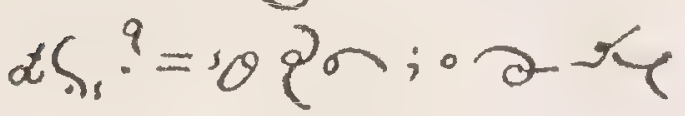
$-d h_{1}<\omega_{s}-\cdots \partial$ ob, t.

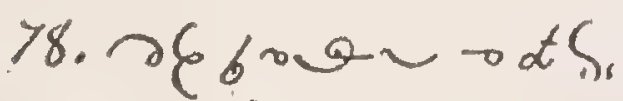
o wen? = 6ren os

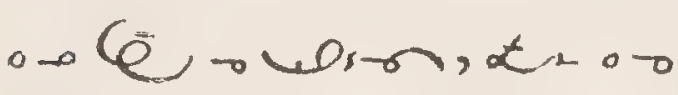

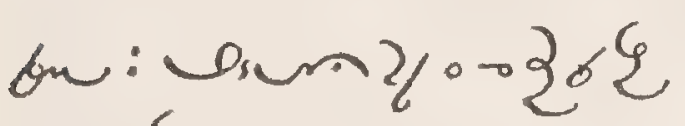
200

79. $\partial 66 a-\cdots 0.0 \%$ <तN

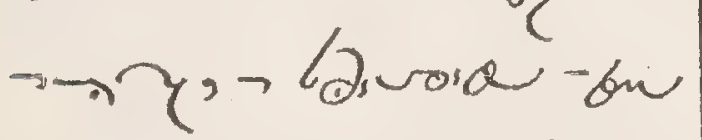

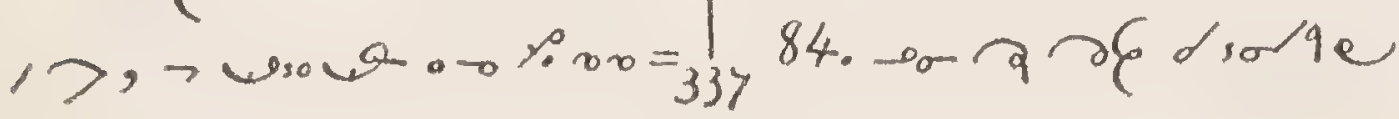

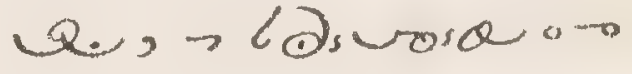
त. $\mathrm{Co}$.

80.662 s n sol L wisen?=re $\rightarrow 2 \sin ;-2 \sin \rightarrow 0$

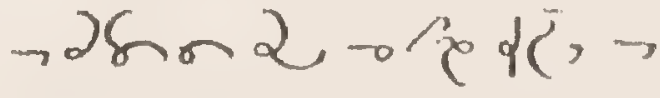
$\sin 2 \rightarrow c \cos )^{\circ} 0=$ $102 \cup-0.06$.

$X I$. Confirmation.

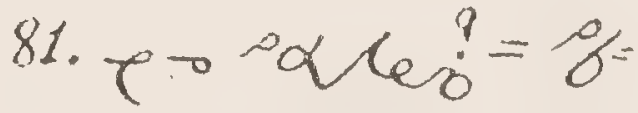
neo de 100 - geis w, tho-9. J fe, (6) 56008 besosom= -20 coger brovas x. $\left.82 \cdot f^{\circ}\right)+\cdot 240_{0} ?=$ sorom, osolecood 83. $2 \xi$ om<sola so-osker:=m

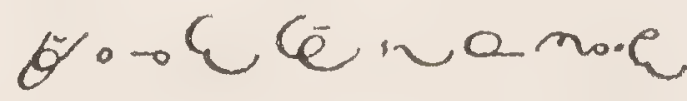
watau-gor

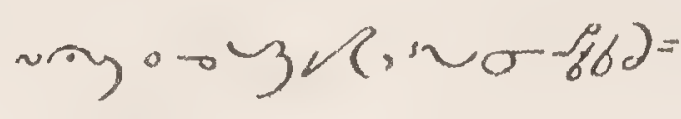
$-80$. bug zice as.- 


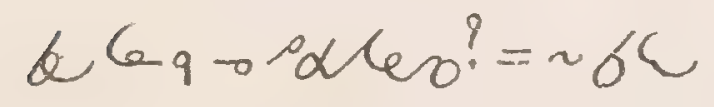

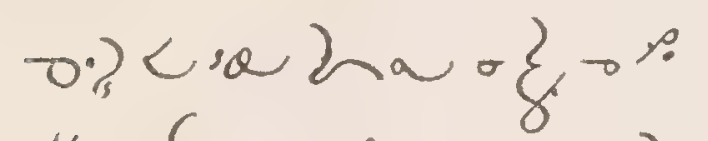

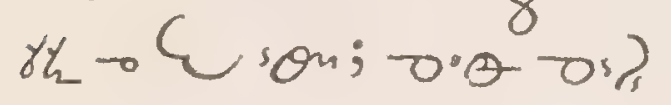

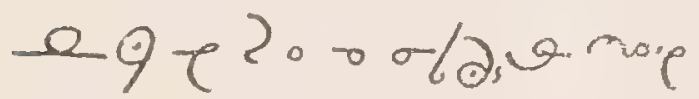
sza on $>d \bar{L}-\cos \theta$

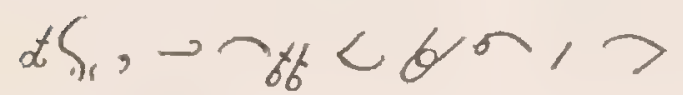
w. \&.

XII. Holy Eucharist.

85. $\tau^{-0} \Theta ?=\Theta \alpha \varphi \xi$ $0.0-\partial_{1}, 2,>0-8 \circ \theta^{\circ}$ er; $>$ gx, twror. 120.0 bu, s2 orson. 86. $\angle 6=>6$ or $0 \rightarrow ?=$ sx; $22,12 \rightarrow n$ nee -

8\%. गद大. $D \oplus ?=x / 3$ bus.0 If, is see o er, sis noops $\rightarrow$ sode Oue,

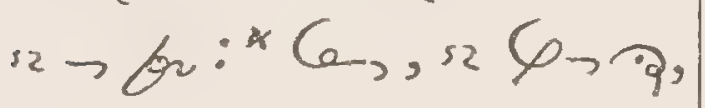
se $M_{3, x} \circ<\bar{a}$ - gr, s $\rightarrow$ bite oroj ny<eros $q<\operatorname{sog} u \cdot x$ 88. wow $九$, * , e 7 , stew brax p so $-d \bar{\zeta}^{-} ?=$ - bux: xs e D, D,

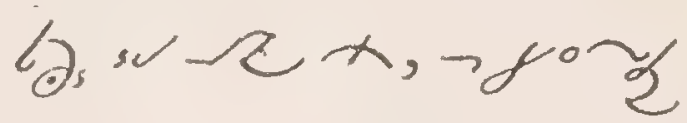
cure; von $x$ "saw br , la, w or x; $\rightarrow$ gon $2<03$. 89. 2 on $6 \gamma^{\sigma} x$ -

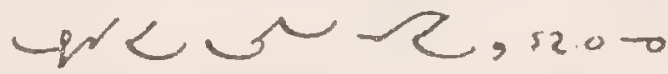
oु< rear, 0.0.

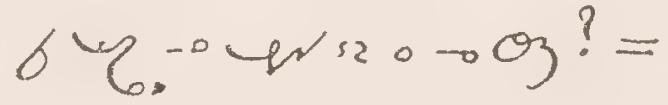
zonferx mere

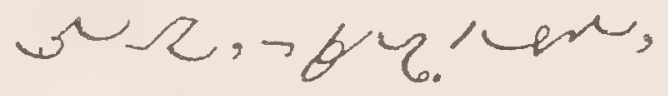

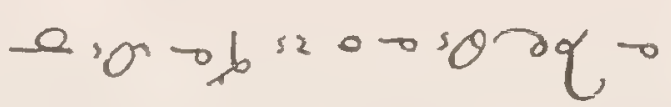
yrop; z on feo- $x$ 。

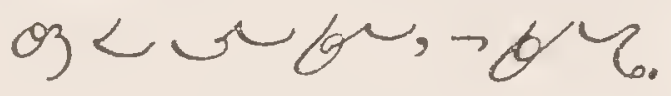

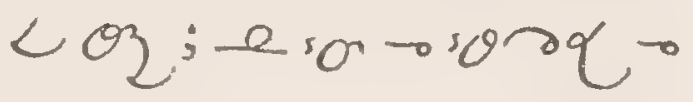
Oु०2.

90. च уॅन $0-x>0$ so on 2 - 2, $\rightarrow 102 e$ $\rightarrow \mathcal{L}$, , soy 0 2? = चทँन ${ }^{\circ-x} \rightarrow$ \%ั0 $=$ Lu- 2 , is fipe $\Omega \times,-9.0 \% 2 \rightarrow 0$

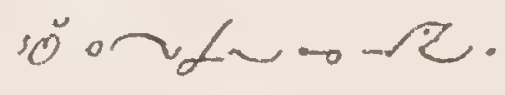
11. 20 26 0 - $x$ - 


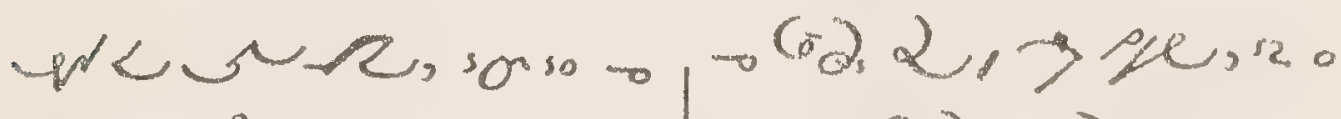

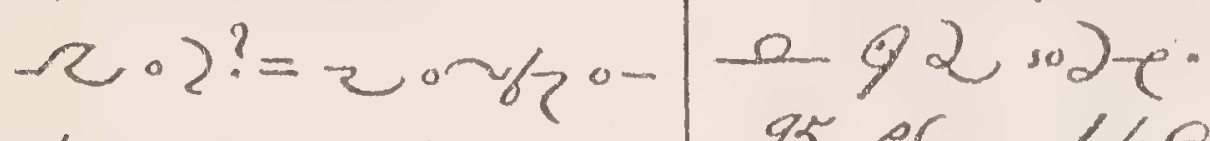

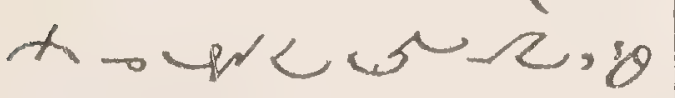

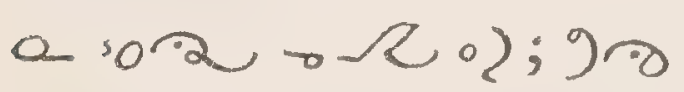
-26 be, iz a ron: 9 $2 \times 2$.

92. $\partial \xi \pitchfork .0 \%$ - $\partial \mathrm{ae}$ $-\oplus ?=x \sim \partial \mathrm{Ge}_{\mathrm{e}} \oplus \oplus$,

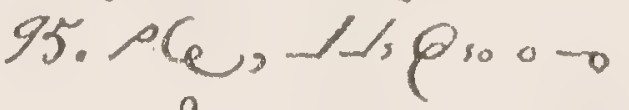

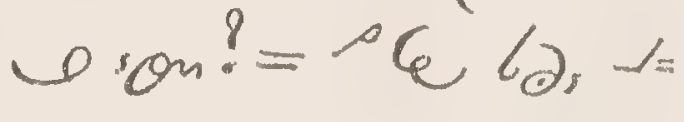
tiporsor $\sim \alpha h_{s} \rightarrow \omega$ son; on voral $7^{\alpha}$ $0 \rightarrow 0$. 96. गदesŏ as z Luperen "os sosen=

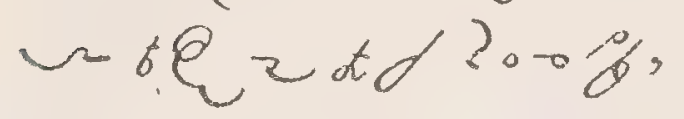
"2<2uJuron Lion.

93.1260 o- रो व.०० abe ormatere

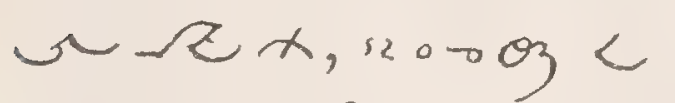
$\sim$ frx? $=20.0^{\circ} \mathrm{C}$ Lonteorno er, LیR木, 120003 जbux:0000\% sode $x$.

94. PG, ws so noop? = Ph us tho eqrap sh s. (2) 170 on nose aw; is o U 3 - $0-x \rightarrow$ Lion? $=s-\oplus \cdot 0 \cdot \sqrt{2}-C$ son, cia vanate

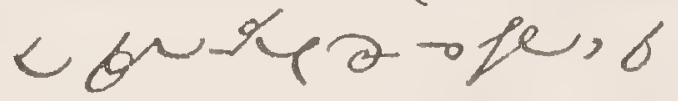

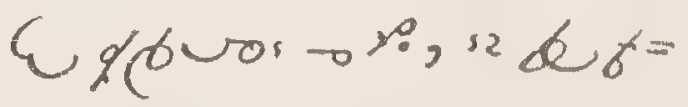
woge o. 0 . 9\%. $\oplus, 8$. $0<$ w nlae Q) $\mathrm{ar} \rightarrow$ 6G? $=\otimes$ ? Lwree rosor to

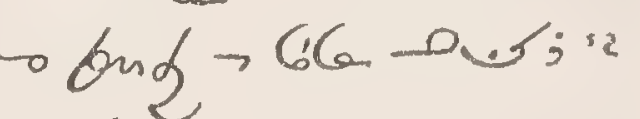
$\rightarrow$ Pile o sio o oiae - bung $\rightarrow$ G Ca - G. 98. $36-n z \cdot 0 m$ bC $6-0 ?=346$ CwวG ० \& $\Omega$.

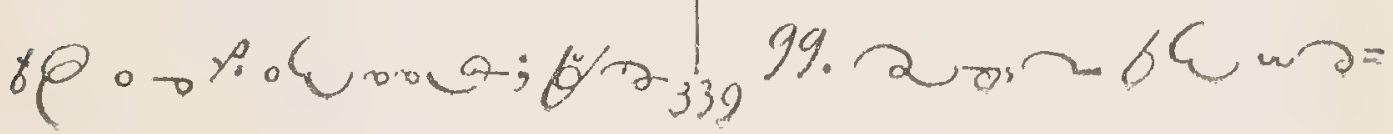




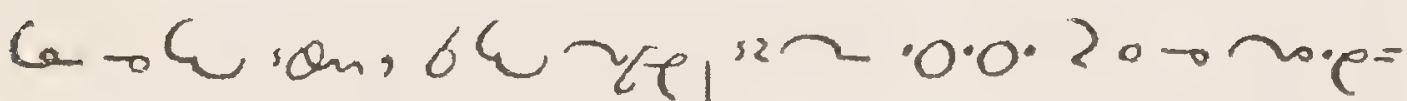

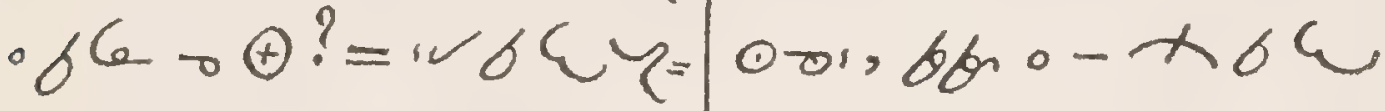
.a 2 Q 9 \% L rant; mo.a eus.

" $6<$ sus solas $x$. 100. avorn to $\mathcal{C}$ by bC suse soras $x ?=\delta$ \& थर००ब;64\% bद $34,6<6000$ x; 64 son $00 \%$

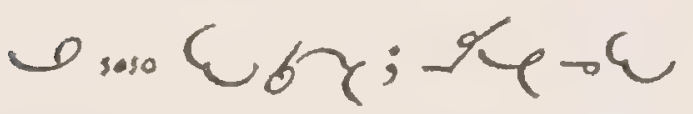
103. $2 \xi 264 \operatorname{mos}$ ox? = su $b 4 \alpha-x$, oc nosa $\sigma<6=$

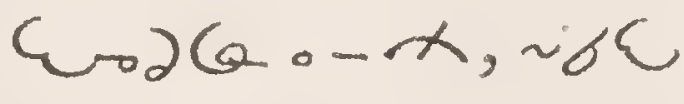
gurt of a ge oc

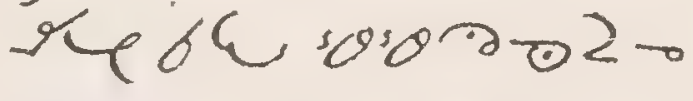
$\therefore, \sim 6 \varepsilon$ so $2 x \circ 2^{\prime 2}$ - Q $9 \cdot \cdots+2,66$ bug $<$ se $\partial e^{52}$ bo $=$ an 6 o $x \cdot C_{66}$.

101. Duor $6<$ c $=$ $\partial C_{n}-C \Omega ?=0 \theta$ $0-x<\not L \cup-2 S_{5}$. 104. d1:0 so tosote ae 00,66 obut< le $x \geq 2000$ ? $=b_{2},-1 s 0 e-66-\operatorname{bun} 2$ - 62 so al bC 3, so गद bC b6,

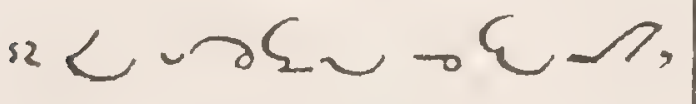

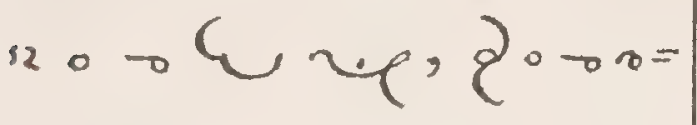
an mus? 102. $\partial \xi 264$ b'so $G \rightarrow \Theta$ ? = so $\times 0.0$ $2-\varepsilon$ bugh, $<6 \sim x=200$

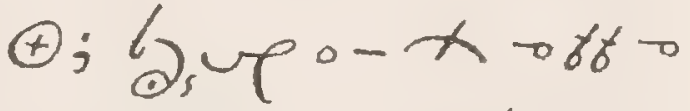
bund 26 o g os sole - fe.

\section{Penance.}

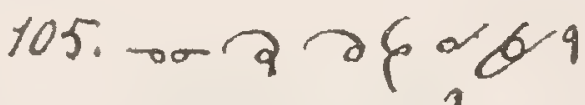
6 - If he? = $\theta$ cat $\rightarrow$ hes, no $=$

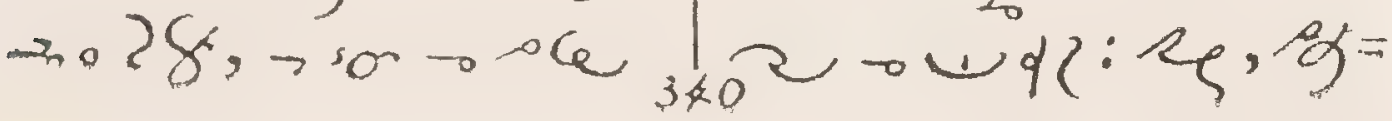




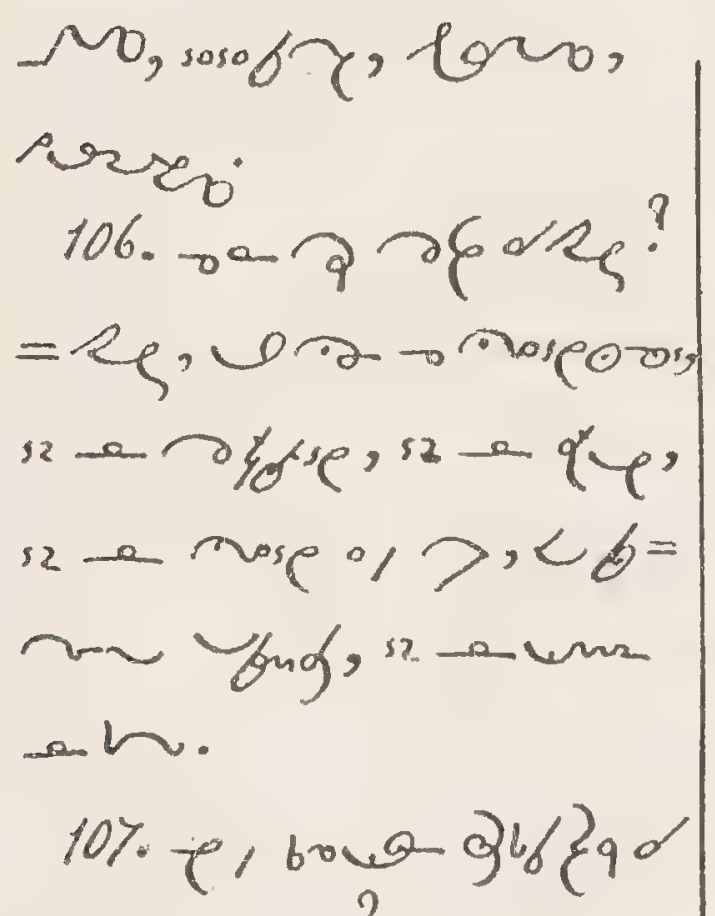
ungonsq? = su ca 946 \& 69 gow 17,320 - re on tospors s2.e vann, eqn, s2o oudre

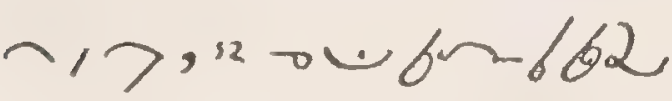

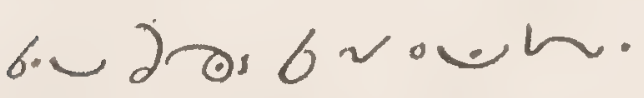
108. sou but or:9, " $2 \xi a ?=$ son, br e.un, sa-ecto og ro: ososor Yougo

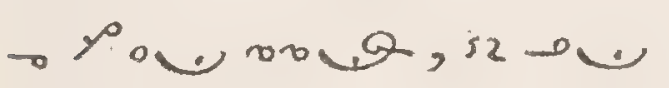
Ke - vbugtia $a=$

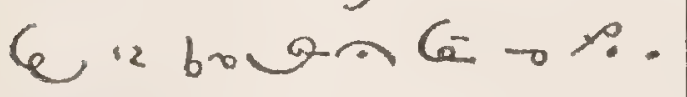

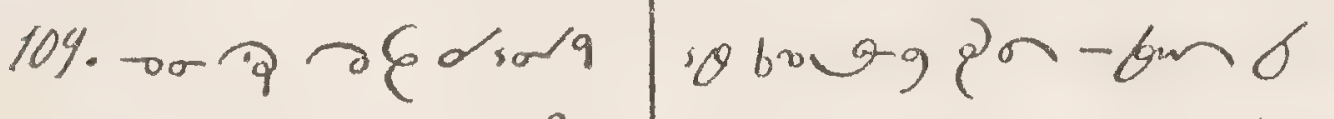

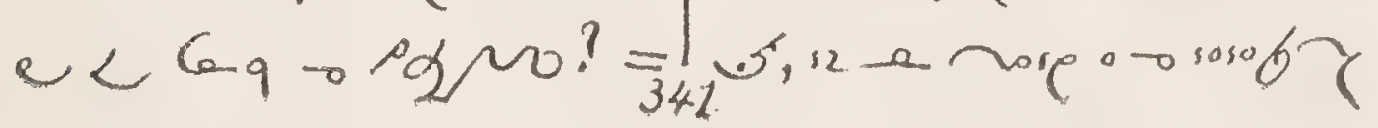


noth aquo.

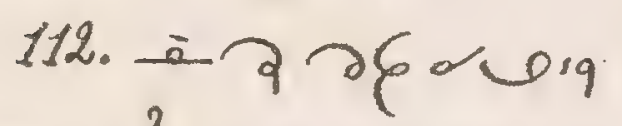

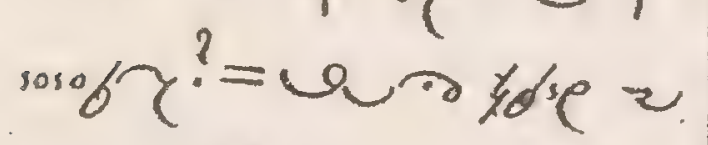

$=0 N^{2}, 12-\sin 2$ $12-\cup 60: " \circ \sigma \in \zeta$, so $)=$

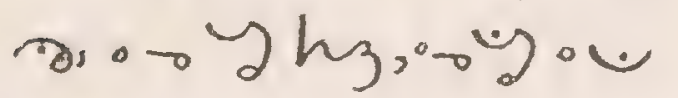
den; sosory 6 ? 6017 rot.

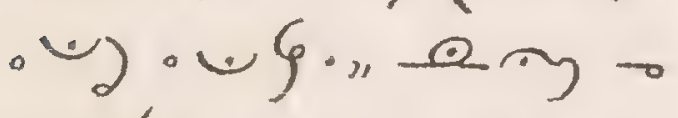
m6gn-.0.8867 ); बतु $0 N, 0002$ e, bpe, bre o $1=$ oro, fue ohy; enoposere the 0,66222 . 662 wyt, eng omo-en. 1/3. 匕 i n 2 eq om onig, $2 /$ bo bug? =" ob - butgoroc

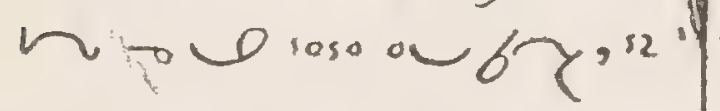

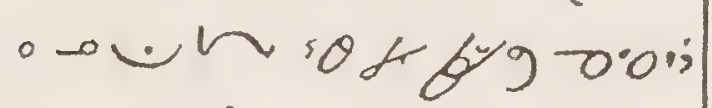
ค9 贯, 90un; 1217 b the $12{ }^{2}$ 36salos. ono. besato.

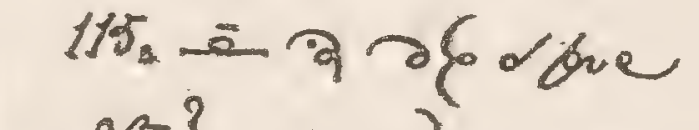
$-N^{2} ?=n_{0} \cdot 2-00$ bu - $M, \sim<6062 \%$

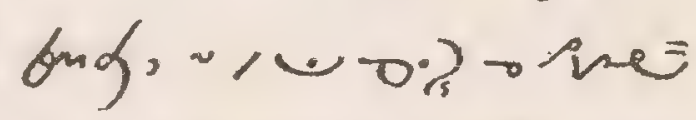
$\varepsilon, \sim<0.00102$ Lee, bue obro, bue ohy;n - even - tod rapo.

116. ia a $\partial 6$ ore - Ne "ef wimuda bug, ser ar $2, ?=6 n$

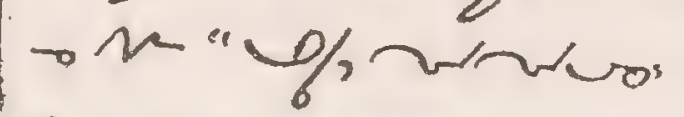
kibng, are ar Z", "2 0 ers Yonse, >, .

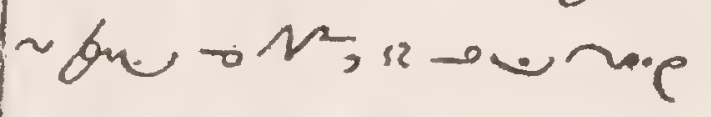
$1>$ br ken overy

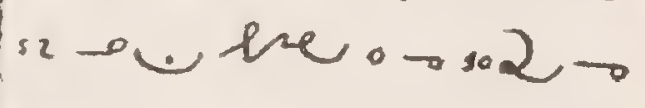
$m, 1200-f, 12$ sob. -urana.

11\%. $2 \xi-\operatorname{lon} 0 ?=<$ :2, - 20000,6 son-ch, bron cong, bra- -6 342 qe. 


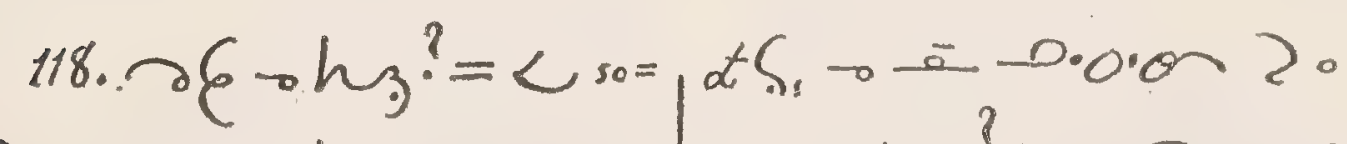
$2 \rightarrow m_{0}+h_{3}, 60^{\circ} \theta=$ sosob ${ }^{?}=$ so pee ${ }^{2}$ $\sin \operatorname{sic} 2 \omega n$. bbae $\rightarrow N^{2}-\alpha S_{r}-0$ 119. $=9 \partial \xi \alpha b q-0$ o. onsqdso ausor 66rog? = c son 6r, $\sim$ g $30.0 \cdot-$

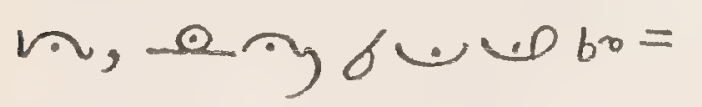
an sosob?.

120. Lo dर००-n yr? $=\operatorname{aucs} \theta 0^{\circ}=$ e 0.0 .00200 sosog $=$ $\gamma:$ debigt o.0ั\% be

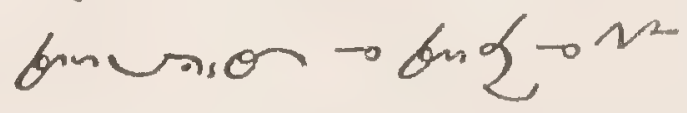
$00=0.0 .0$. 200 soso $=$ br.

124. ว 6 - ozs? =

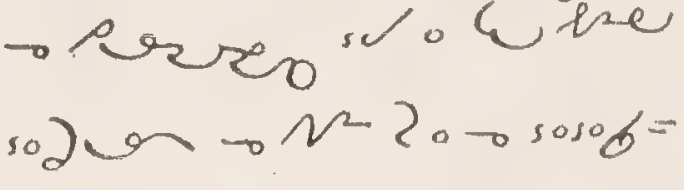

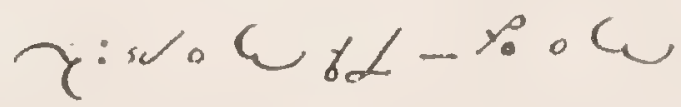
Bo L cune to th

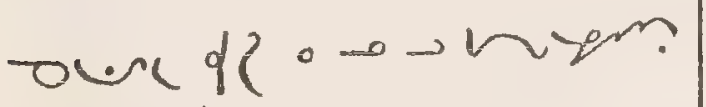
one $2 \sin ,><$ boul

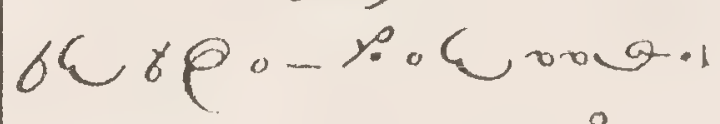
124 bis. of $\rightarrow$ he? $=$ $\rightarrow$ he dlo- beré ' $0.6>$ $125.6 x y-0 x<$ 122. L, bo a dर 0 क $\rightarrow$ ? ny 2 200 sosoby?

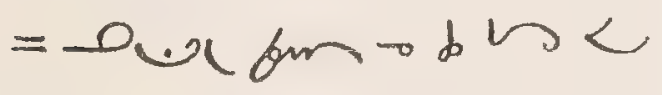

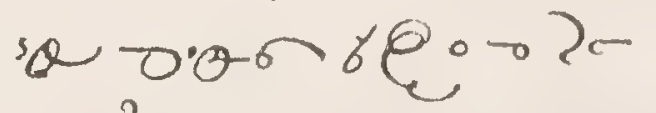
$n ?=-u$ a 0.00

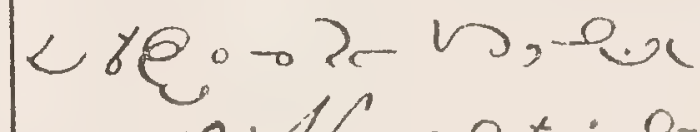

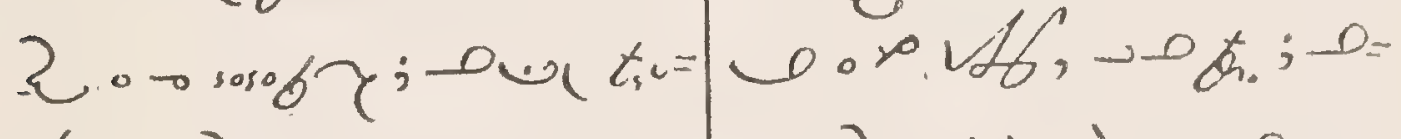

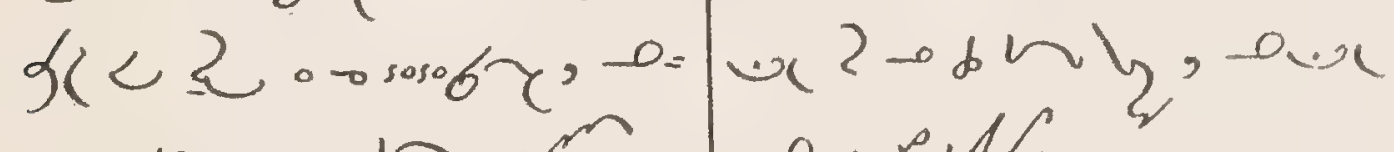

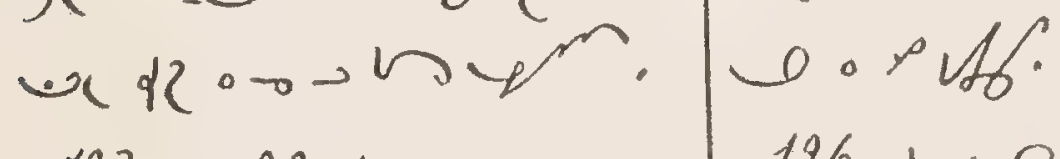

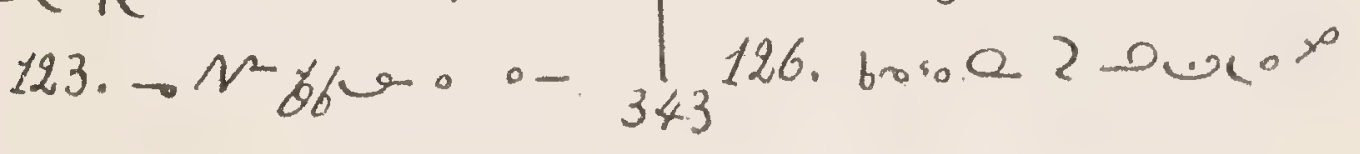










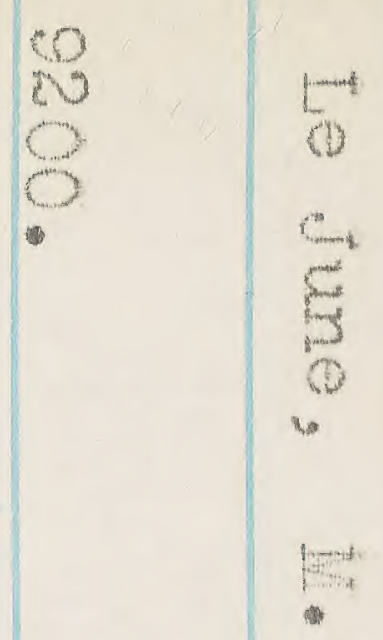

1

ras
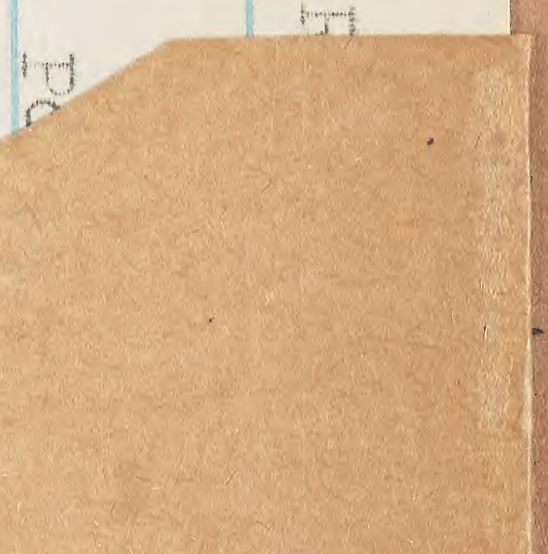


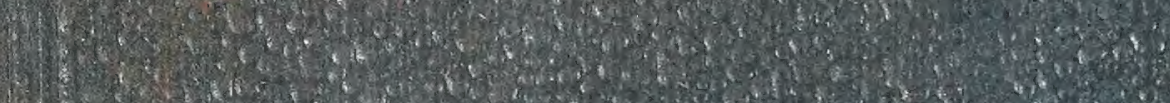

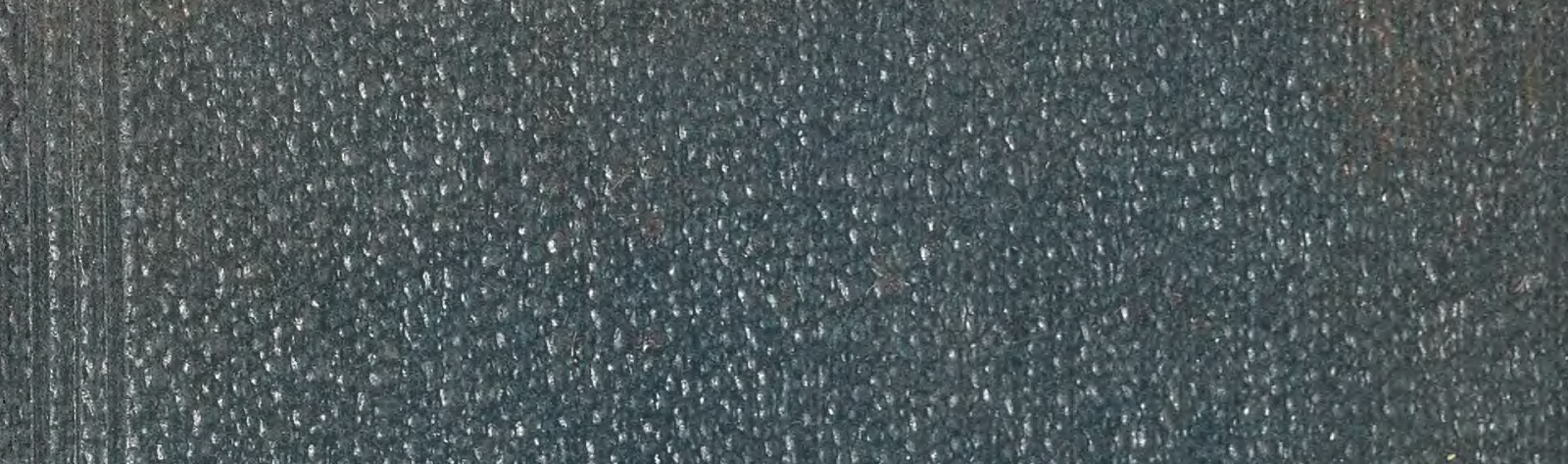

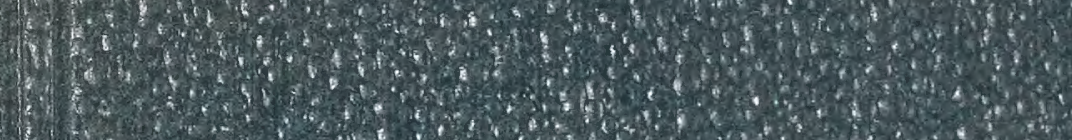

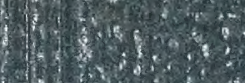

16. (1)

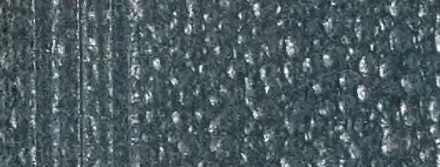

19x

Dinging 
SMITHSONIAN INSTITUTION LIBRARIES

||||||||||||||||||||||||||

$\begin{array}{llll}3 & 9088 & 00581 & 2946\end{array}$ 